
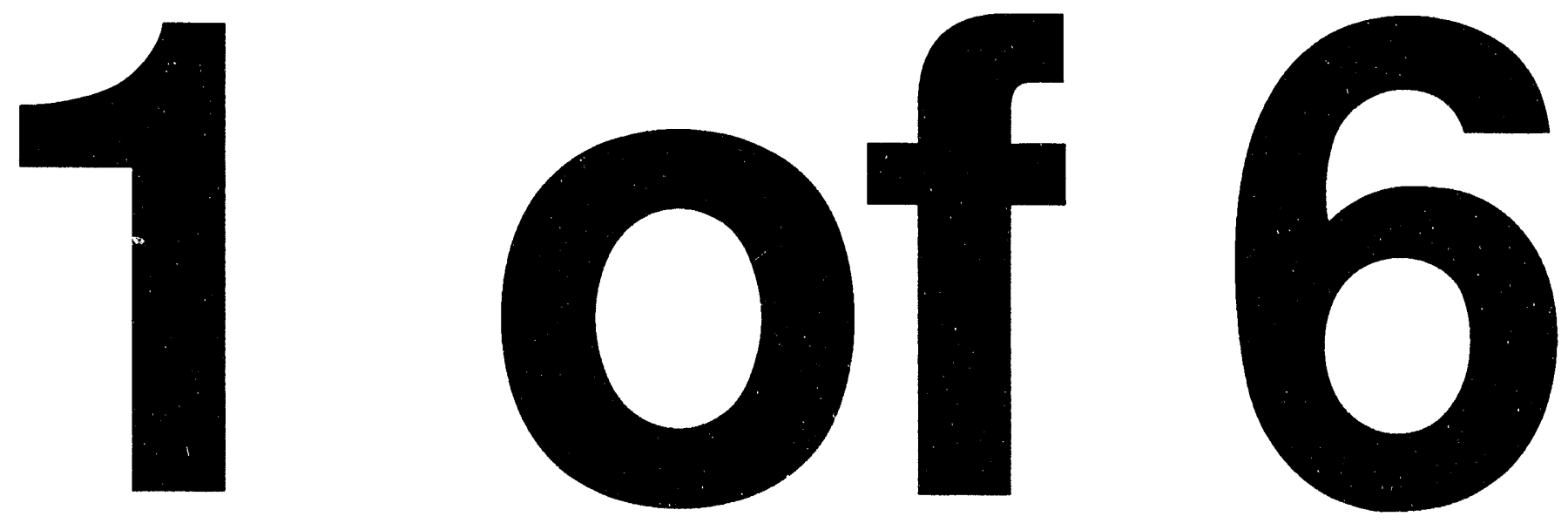


\title{
CALENDAR YEAR 1993 GROUNDWATER QUALITY REPORT FOR THE BEAR CREEK HYDKOGEOLOGIC REGIME, Y-12 PLANT, OAK RIDGE, TENNESSEE
}

\begin{abstract}
1993 Groundwater and Surface Water Quality Data and Calculated Rate of Contaminant Migration
\end{abstract}

\author{
February 1994
}

Prepared by

HSW ENVIRONMENTAL CONSULTANTS, INC.

Under Purchase Order 70Y-EAQ10C

for

Environmental Management Department

Health, Safety, Environment, and Accountability Organization

Oak Ridge Y-12 Plant

Oak Ridge, Tennessee 37831

Managed By

MARTIN MARIETTA ENERGY SYSTEMS, INC. for the U.S. Department of Energy Under Contract No. DE-AC05-84OR21400 


\section{DISCLAIMER}

This report was prepared as an account of work sponsored by an agency of the United States Government. Neither the United States Government nor any agency thereof, nor any of their employees, makes any warranty, express or implied, or assumes any legal liability or responsibility for the accuracy, completeness, or usefulness of any information, apparatus, product, or process disclosed, or represents that its use would not infringe privately owned rights. Reference herein to any specific commercial product, process, or service by trade name, trademark, manufacturer, or otherwise does not necessarily constitute or imply its endorsement, recommendation, or favoring by the United States Government or any agency thereof. The views and opinions of authors expressed herein do not necessarily state or reflect those of the United States Government or any agency thereof. 


\section{TABLE OF CONTENTS}

Section

Page

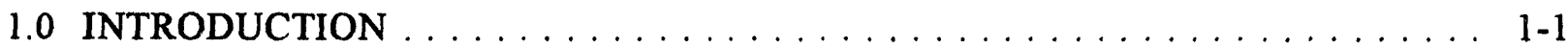

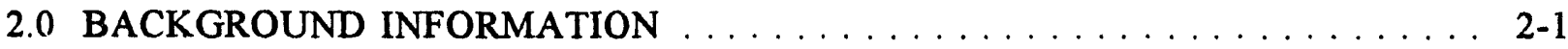

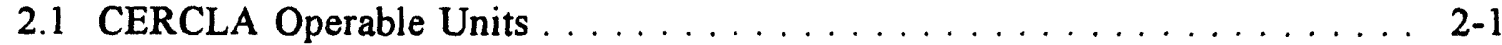

2.1.1 Bear Creek Operable Unit $01 \ldots \ldots \ldots \ldots . \ldots \ldots$. . . . . . . . . .

2.1.1.1 S-3 Site . . . . . . . . . . . . . . . . . 2-3

2.1.1.2 Oil Landfarm Waste-Management Area . . . . . . . . . . 2-4

2.1.1.3 Bear Creek Burial Grounds Waste-Management

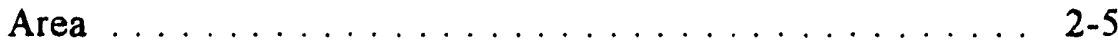

2.1.2 Bear Creek Operable Unit $02 \ldots \ldots \ldots \ldots \ldots$ 2-6

2.1.2.1 Spoil Area I . . . . . . . . . . . . . . . . . . 2-6

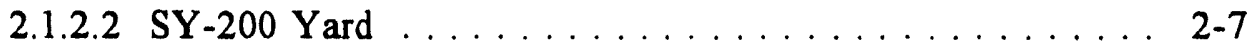

2.1.2.3 Rust Spoil Area . . . . . . . . . . . . . . . . . . . . . 2-7

2.1.3 Bear Creek Operable Unit $04 \ldots \ldots \ldots$. . . . . . . . . . . . . .

2.2 Above Grade Low Level Waste Storage Facility . . . . . . . . . . . . . . 2-8

3.01993 GROUNDWATER PRUTECTION PROGRAM $\ldots \ldots \ldots \ldots \ldots \ldots \ldots \ldots$

3.1 Monitoring Programs $\ldots \ldots \ldots \ldots \ldots \ldots \ldots \ldots \ldots \ldots \ldots$ 3-1

3.1.1 RCRA Compliance Monitoring . . . . . . . . . . . . . 3-1

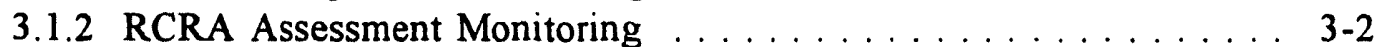

3.1.3 Exit Pathway Monitoring . . . . . . . . . . . . . . . . 3-2

3.1.4 Surveillance and Maintenance Monitoring . . . . . . . . . 3-2

3.1.5 Best-Management Practice Monitoring . . . . . . . . . . . . 3-3

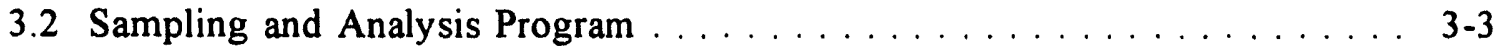

3.2.1 Sampling Locations . . . . . . . . . . . . . . . . . . . . . . . . 3-3

3.2.2 Sampling Frequency . . . . . . . . . . . . . . . . . . . 3-4

3.2.3 Sample Collection ... . . . . . . . . . . . . . . . . . . 3-4

3.2.4 Quality Assurance/Quality Control Sampling . . . . . . . . . . 3-5

3.2 .5 Laboratory Analysis . . . . . . . . . . . . . . . . . . . . . . 3-6

3.2 .6 Data Management ... . . . . . . . . . . . . . . . 3-6

3.2 .7 Analytical Results . . . . . . . . . . . . . . . . . . 3-7

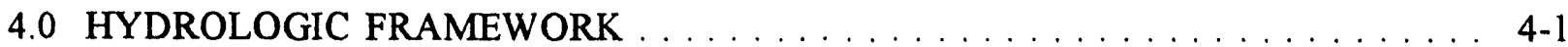

4.1 Geology . . . . . . . . . . . . . . . . . . . . . . . . . 4 4-2

4.2 Groundwater System . . . . . . . . . . . . . . . . . . 4-3

4.2.1 Stormflow Zone ... . . . . . . . . . . . . . . . . . 4-4

4.2 .2 Vadose Zone . . . . . . . . . . . . . . . . . . . . 4 4-5

4.2.3 Groundwater Zone . . . . . . . . . . . . . . . . . . . . . 4-5

4.2.3.1 Water Table Interval . . . . . . . . . . . . . . . . . 4 4-6

4.2.3.2 Shallow Interval . . . . . . . . . . . . . . . . . . . 4 4-6

4.2.3.3 Intermediate Interval . . . . . . . . . . . . . . . . 4-7

4.2.3.4 Deep Interval . . . . . . . . . . . . . . . . . . 4-8

4.2 .4 Aquiclude $\ldots \ldots \ldots \ldots \ldots \ldots \ldots \ldots \ldots . \ldots \ldots . \ldots \ldots$ 


\section{TABLE OF CONTENTS (cont'd)}

Section

4.2.5 Groundwater Flow Directions . . . . . . . . . . . . . . . . 4-9 . . . . . . . . . . . .

4.2 .6 Contaminant Transport . . . . . . . . . . . . . . . . 4 4-10

4.2.6.1 Quantitative Estimates . . . . . . . . . . . . . . 4-11

4.2.6.2 Qualitative Estimates . . . . . . . . . . . . . . . . 4-12

4.3 Surface-Water System . . . . . . . . . . . . . . . . . . . 4-13

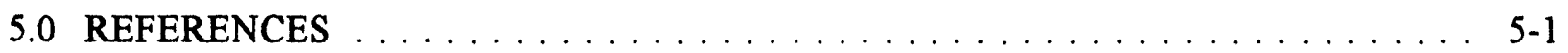

Appendices
A Figures
B Tables
C Monitoring-Well Construction Details
D Sampling Sequence
E Groundwater and Surface-Water Quality Data
F Field Duplicate Data
G Ion-Charge Balance Calculations
H Trace Metal Data Summary
I QA/QC Data Summaries
J Validated Results of Volatile Organic Compound Analyses
K Radiochemical Data Summary
L Annual Average Nitrate (as N) Concentrations
M Groundwater Elevations 


\section{LIST OF FIGURES}

Figure

Page

$1 \quad$ Regional Location of the Y-12 Plant $\ldots \ldots \ldots \ldots \ldots \ldots \ldots \ldots$

2 Hydrogeologic Regimes at the $\mathrm{Y}-12$ Plant $\ldots \ldots \ldots \ldots \ldots \ldots \ldots$ A-2

3 Bear Creek Valley Waste-Management Sites $\ldots \ldots \ldots \ldots \ldots \ldots \ldots$

4 Components of the Oil Landfarm Waste-Management Area . . . . . . . . . . A-4

5 Components of the Bear Creek Burial Grounds Waste-Management Area . . . . A A-5

6 Location of Monitoring Wells Sampled During $1993 \ldots \ldots \ldots \ldots \ldots$

7 Location of Springs and Surface Water Stations Sampled During $1993 \ldots \ldots$. . . A-7

8 Topography and Bedrock Geology in the Bear Creek Hydrogeologic Regime . . A-8

9 Schematic Profile of Hydrostratigraphic Units in the Bear Creek Hydrogeologic Regime . . . . . . . . . . . . . . . . . . A A-9

10 Groundwater Elevations in the Water-Table Interval $\ldots \ldots \ldots \ldots \ldots \ldots \ldots$

11 Groundwater Elevations in the Shallow and Intermediate Bedrock Intervals . . . A-11 


\section{LIST OF TABLES}

Table

Page

1 Operable Units Established for the Bear Creek Hydrogeologic Regime . . . . . . . B-1

2 Monitoring Wells, Springs, and Surface Water Stations Sampled During 1993 . . B-2

3 General Construction Information for Monitoring Wells Sampled During 1993 . . B-7

$4 \quad$ Analytical Parameters for Groundwater and Surface Water Samples

Collected During $1993 \ldots \ldots \ldots \ldots \ldots \ldots \ldots$. . . . . . . . . . . . . . . . . . . . 


\section{LIST OF ACRONYMS}

$\begin{array}{ll}\text { ARAR } & \text { Appropriate, Relevant, and Applicable Requirement } \\ \text { BCV } & \text { Bear Creek Valley } \\ \text { BCK } & \text { Bear Creek Kilometer } \\ \text { CERCLA } & \text { Comprehensive Environmental Response, Compensation, and Liability Act } \\ \text { CY } & \text { Calendar Year } \\ \text { DOE } & \text { U.S. Department of Energy } \\ \text { EMD } & \text { Environmental Monitoring Database } \\ \text { EPA } & \text { U.S. Environmental Protection Agency } \\ \text { FFA } & \text { Federal Facility Agreement } \\ \text { GWPP } & \text { Groundwater Protection Program } \\ \text { HSEA } & \text { Health, Safety, Environment, and Accountability (Organization) } \\ \text { ORNL } & \text { Oak Ridge National Laboratory } \\ \text { ORR } & \text { Oak Ridge Reservation } \\ \text { OU } & \text { Operable Unit } \\ \text { PCPA } & \text { Post-Closure Permit Application } \\ \text { QA/QC } & \text { Quality Assurance/Quality Control } \\ \text { RCRA } & \text { Resource Conservation and Recovery Act } \\ \text { RIFS } & \text { Remedial Investigation/Feasibility Study } \\ \text { SWMU } & \text { Solid Waste-Management Unit } \\ \text { TDEC } & \text { Tennessee Department of Environment and Conservation } \\ \text { TSD } & \text { Treatment, Storage, and Disposal (Unit) } \\ \text { VOC } & \text { Volati Organic Compound } \\ \text { WMA } & \text { Wast. Management Area }\end{array}$



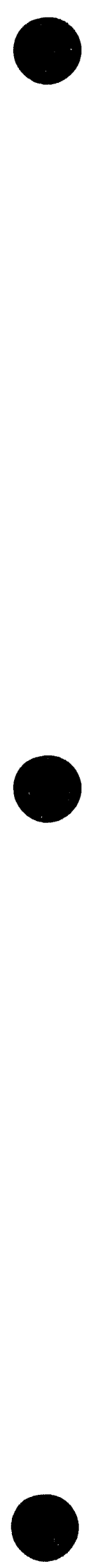


\subsection{INTRODUCTION}

This report contains groundwater and surface-water quality data obtained during the 1993 calendar year (CY) at several hazardous and non-hazardous waste-management facilities associated with the U.S. Department of Energy (DOE) Y-12 Plant located on the DOE Oak Ridge Reservation (ORR) southeast of Oak Ridge, Tennessee (Figure 1). These sites are located southwest of the Y-12 Plant complex within the Bear Creek Hydrogeologic Regime (Bear Creek Regime), which is one of three regimes defined for the purposes of groundwater and surface-water quality monitoring at the Y-12 Plant. The Environmental Management Department of the Y-12 Plant Health, Safety, Environment, and Accountability (HSEA) Organization manages the monitoring activities in each regime as part of the Y-12 Plant Groundwater Protection Program (GWPP).

The annual groundwater and surface water ieport for the Bear Creek Regime is completed in two-parts; Part 1 (this report) containing the groundwater and surface-water quality data and Part 2 containing a detailed evaluation of the data. The primary purpose of this report is to serve as a reference for the groundwater and surface-water quality data obtained each year under the lead of the Y-12 Plant GWPP. However, because it contains information needed to comply with Resource Conservation and Recovery Act (RCRA) interim status assessment monitoring reporting requirements, this report is submitted to the Tennessee Department of Health and Environment (TDEC) by the RCRA reporting deadline (March 1 of the following CY). Part 2 of the annual groundwater report, to be issued mid-year, will contain a regime-wide evaluation of groundwater and surface water quality, present the findings and status of ongoing hydrogeologic studies, describe changes in monitoring priorities, and present planned modifications to the groundwater and surface water sampling and analysis activities. 


\subsection{BACKGROUND INFORMATION}

Located west of the Y-12 Plant in Bear Creek Valley (BCV), the Bear Creek Regime is bound to the north by Pine Ridge and to the south by Chestnut Ridge (directions in this report are in reference to the Y-12 Plant grid system). The regime encompasses the portion of BCV extending from a surface water and shallow groundwater divide at the west end of the Y-12 Plant to the western boundary of the Bear Creek watershed (Figure 2).

Groundwater and surface-water quality monitoring in the Bear Creek Ridge Regime during CY 1993 was performed at two classes of waste-management facilities:

sites scheduled for investigation under the Comprehensive Environmental Response, Compensation, and Liability Act (CERCLA); and

a low level radioactive waste storage facility.

A brief description of the waste-management facilities in the regime, including waste inventories and disposal histories, and a discussion of the regulatory status and groundwater monitoring history of each site, are provided in the following sections.

\subsection{CERCLA Operable Units}

In 1984, the U.S. Environmental Protection Agency (EPA) and the TDEC determined that several of the waste-management sites at the Y-12 Plant were hazardous waste treatment, storage, and disposal (TSD) units subject to regulation under RCRA. Three of the TSD units are located in the Bear Creek Regime; the S-3 Site, the Oil Landfarm waste-management area (WMA), and the Bear Creek Burial Grounds WMA. These sites were granted RCRA interim status until operating or post-closure RCRA permits were obtained by DOE, and RCRA Post-Closure Permit Applications (PCPAs) for these sites were initially submitted to the TDEC in 1987. Also in 1987, Spoil Area I, the Rust Spoil Area, and the SY-200 Yard were identified as solid waste-management units (SWMUs) subject to regulation under section 3004(u) of RCRA (Welch et a․ 1987).

In November 1989, the ORR was added to the National Priority List and in January 1992, the DOE, the EPA, and the TDEC negotiated a Federal Facility Agreement (FFA) regarding 
environmental restoration at the ORR under CERCLA. This agreement changed the regulatory status of the RCRA TSD units and SWMUs in the Bear Creek Regime to CERCLA Operable Units (OUs). As such, the DOE appealed the applicability of RCRA post-closure care, corrective action, and groundwater monitoring requirements to CERCLA OUs.

In April 1993, the DOE, TDEC, and Martin Marietta Energy Systems, Inc. signed an Agreed Order for a post-closure permit for the S-3 Site, thereby resolving the appeal and formally agreeing to proceed with CERCLA as a lead regulatory program with RCRA as an appropriate, relevant, and applicable requirement (ARAR). Under this agreement, RCRA will be applied as an ARAR to the extent that post-closure maintenance and care of former TSD facilities will be conducted in compliance with the terms of RCRA post-closure permits. Groundwater monitoring is to be integrated with the CERCLA remedial investigation/feasibility study (RI/FS) programs with RCRA corrective action deferred to CERCLA. Groundwater monitoring data reporting will comply with RCRA post-closure permit conditions as well as RI/FS requirements.

Two : „es of OUs were established under the FFA; source control OUs and integrator OUs. Source control OUs are individual or groups of waste sites for which a RI/FS will be performed, including any interim actions and/or remedial actions. Integrator OUs encompass groundwater and surface water, and are considered separate from source control OUs that contribute to the contamination of these media. Integrator OUs were established because available data showed that contaminants from multiple sources had intermingled in the groundwater system and migrated through a common exit-pathway (U.S. Department of Energy 1992).

The FFA established three source control OUs and one integrator OU that lie within the Bear Creek Regime (Table 1). Source control OUs 01 and 02 include all of the wastemanagement sites in the regime. The third source control OU encompasses contaminated sediments within the Bear Creek flood plain, and has no groundwater component. Groundwater and surface water in the regime comprise the integrator OU (Bear Creek OU 04).

\subsubsection{Bear Creek Operable Unit 01}

Bear Creek OU 01 is a source control OU comprised of the three former RCRA TSD units in the regime; the S-3 Site, the Oil Landfarm WMA, and the Bear Creek Burial Grounds 
WMA (Table 1). An RI work plan for this OU has been prepared and reviewed by the EPA (Region IV) and the TDEC (U.S. Department of Energy 1993a). Efforts to address EPA and TDEC comments on the work plan are currently in progress.

\subsubsection{S-3 Site}

The S-3 Site is next to the west end of the Y-12 Plant (Figure 3), just west of the groundwater flow divide separating the Bear Creek Regime from the Upper East Fork Poplar Creek Hydrogeologic Regime (East Fork Regime). Formerly called the S-3 Ponds, the site was constructed in 1951 and consisted of four unlined ponds covering an area of roughly $400 \mathrm{x}$ 400 feet $(\mathrm{ft}$ ). Each pond was about $17 \mathrm{ft}$ deep (excavations did not extend into bedrock), and had a storage capacity of about 2.5 million gallons (Geraghty \& Miller, Inc. 1988a).

Wastes discharged into the ponds consisted primarily of nitric and other acids, nitrate wastes, pickling and plating wastes, machine coolants, caustic solutions, depleted uranium in nitric acid solution, technetium in raffinate and condensate, and miscellaneous liquid wastes (mop waters) associated with routine clean-up and operations at the Y-12 Plant. Most of the acid wastes were discharged into the S-3 Ponds from a pipeline (the Nitric Acid Pipeline) connected to operations within Buildings 9212, 9206, and 9998 (the H-1 Foundry) located in the west central section of the Y-12 Plant complex. A small section of the Nitric Acid Pipeline lies within the westernmost part of the Bear Creek Regime. The remainder of the pipeline lies east of the groundwater and surface water divide separating the Bear Creek Regime from the East Fork Regime.

Waste disposal at the S-3 Ponds ceased in 1984 and in 1988 the site was closed as a landfill in accordance with a closure plan approved by the TDEC (Martin Marietta Energy Systems 1988a). When closed, the ponds contained 2 to $5 \mathrm{ft}$ of sludge produced during in-situ denitrification and neutralization of wastewater in the ponds, and a small volume of contaminated sediments removed from Bear Creek downstream of the site. The sludge was not leachable in weak acid and water (Geraghty \& Miller, Inc. 1988a). During closure, the sludge and sediments were stabilized with coarse aggregate and the ponds were covered with a low-permeability cap. Construction of an asphalt parking lot over the cap completed final closure of the site, which was certified by the TDEC on November $15,1990$. 
The TDEC issued a RCRA post-closure permit for the S-3 Site in April 1993. Negotiations with the TDEC regarding modifications to the permit and the effective date of the permit are currently in progress.

\subsubsection{Oil Landfarm Waste-Management Area}

The Oil Landfarm WMA is approximately one mile west of the Y-12 Plant (Figure 3), and consists of the Oil Landfarm, the Boneyard, the Burnyard, the Chemical Storage Area, and the Sanitary Landfill I. Figure 4 illustrates the components of the Oil Landfarm WMA.

The Oil Landfarm consisted of three areas where waste oils and coolants were applied to nutrient-adjusted soil during the dry months of the year (April to October) to enhance biodegradation. These oils and coolants contained beryllium compounds, depleted uranium, polychlorinated biphenyls, and spent solvents. Approximately one million gallons of waste oil were applied to soils at the site between 1973 and 1982 (Geraghty \& Miller, Inc. 1988b). In 1989, the site was covered with a low-permeability cap in accordance with a TDEC-approved closure plan (Martin Marietta Energy Systems, Inc. 1988b). The TDEC certified final closure of the Oil Landfarm on December 15, 1990.

The Boneyard was used for the disposal of magnesium chips and construction debris (e.g., concrete) in unlined shallow trenches. The magnesium chips were placed in the trenches and burned, and residues were covered with soil and compacted. Filled trenches were covered with top soil and seeded with grass (Geraghty \& Miller, Inc. 1988b).

The Burnyard consisted of two unlined trenches about $300 \mathrm{ft}$ long by $40 \mathrm{ft}$ wide. Between 1943 and 1968, various types of refuse (including pesticide containers, metal shavings, solvents, oils, and laboratory chemicals) were burned in the trenches. Some residues may have been buried in the Boneyard (Geraghty \& Miller, Inc. 1988b).

The Chemical Storage Area was constructed on top of the Burnyard and used as an area for releasing compressed gas from cylinders with leaking or damaged valves, and for disposal of reactive or explosive laboratory chemicals. Gas in the damaged cylinders was allowed to leak into the atmosphere or was bled off; corrosive gases were bled through neutralizing slurries. Laboratory chemicals disposed at the site included acids, bases, organics, water-reactive compounds, and shock-sensitive compounds such as picric acid. The chemicals were handled to 
induce the expected reaction or explosion, and remaining liquids were discharged into a small unlined surface impoundment (Geraghty \& Miller, Inc. 1988b).

Sanitary Landfill I is south of the Oil Landfarm next to Bear Creek (Figure 4). The TDEC permitted the site for disposal of non-hazardous wastes generated at the Y-12 Plant, including paper, cardboard, plastics, rubber, wood, brush, organic refuse, textile products, and asphalt roofing materials. Waste disposal at the site started in 1968 and the site contained about 105,000 tons of waste when disposal activities ceased in 1982 (Geraghty \& Miller, Inc. 1988b). In 1983, the site was graded and capped in accordance with a TDEC-approved closure plan (Bailey 1983).

As noted previously, a RCRA Part B PCPA for the Oil Landfarm WMA was submitted to the TDEC in 1987. The PCPA for the site is currently being revised to reflect the signed Agreed Order and the provisions contained in the post-closure permit issued by the TDEC for the S-3 Site.

\subsubsection{Bear Creek Burial Grounds Waste-Management Area}

The Bear Creek Burial Grounds WMA, located approximately two miles west of the Y-12 Plant (Figure 3), is comprised of several waste disposal units designated Burial Grounds A (North and South), B, C, D, E, and J, and two ponds designated Oil Retention Ponds No. 1 and 2 (Figure 5). Each disposal unit consisted of multiple trenches excavated 14 to $25 \mathrm{ft}$ below grade that were used for disposal of liquid and/or solid wastes. Perforated stand-pipes installed vertically in some trenches were used for liquid waste disposal; rock and gravel were backfilled around the standpipes for support and to maximize the drainage rate. Oil Retentior Ponds No. 1 and 2 were constructed to collect oils seeping from disposal trenches in Burial Ground A South, and A North, respectively (Figure 5).

Burial Grounds A (North and South) and C primarily received liquid wastes that generally consisted of waste oils and coolants, spent solvents, and mop waters. Solid wastes disposed at the site included salts, metals (primarily beryllium and uranium) and metal oxides, metal saw-fines, and asbestos. Hazardous waste disposal activities ceased in 1981 (Geraghty \& Miller, Inc. 1988c). 
Burial Grounds A (North and South) and C, and the Oil Retention Ponds were closed in 1988 and 1989 in accordance with TDEC-approved closure plans (Martin Marietta Energy Systems, Inc. 1988c and 1988d). The TDEC certified final closure of Burial Ground A (North and South) on December 15, 1989. Certification of final closure of Burial Ground C was requested from the TDEC on December 27, 1990. The TDEC certified final closure of Oil Retention Ponds No. 1 and 2 on November 15, 1990, and December 11, 1990, respectively.

As noted previously, a RCRA Part B PCPA for the Bear Creek Burial Grounds WMA was submitted to the TDEC in 1987. The PCPA for the site is currently being revised to reflect Agreed Order and provisions contained in the post-closure permit issued by the TDEC for the S-3 Site.

\subsubsection{Bear Creek Operable Unit 02}

Bear Creek OU 02 is a source control OU comprised of three former RCRA SWMUs; Spoil Area I, the SY-200 Yard, and the Rust Spoil Area (Table 1). The RI work plan for this OU (U.S. Department of Energy 1993b) has been approved by the EPA and the TDEC. Field work for the RI was completed in December 1993 and included one round of groundwater sampling from monitoring wells located at each of the sites.

\subsubsection{Spoil Area I}

Spoil Area I is located on the northern slope of Chestnut Ridge, west of the Y-12 Plant near the intersection of Old Bear Creek Road and West Patrol Road (Figure 3). The five-acre site has been used since about 1980 for disposal of non-radioactive construction debris. The TDEC permitted the site in 1986 (Permit No. DML011030012) for disposal of rubble and other non-combustible, stable solid wastes (Tennessee Department of Health and Environment 1986).

The site has received approximately 100,000 cubic yards of debris, including asphalt, brick, concrete, roofing materials, brush, steel rebar, rock, and tile (Battelle Columbus Division 1989a). 


\subsubsection{SY-200 Yand}

The SY-200 Yard is located south of Bear Creek near the base of the northern slope of Chestnut Ridge, approximately one-half mile west of the Y-12 Plant (Figure 3). Operated from the 1950s to 1986, the SY-200 Yard was a $300 \times 200 \mathrm{ft}$ gravel-covered area used for temporary storage of equipment, machinery, and miscellaneous items. Records indicate that waste materials were not disposed or stored at the site (Geraghty \& Miller, Inc. 1989a).

\subsubsection{Rust Spoil Area}

The Rust Spoil Area is a 5.4-acre site located west of the SY-200 Yard (Figure 3) that was used between 1975 and 1983 for disposal of solid wastes (spoil) generated during various renovation, maintenance, and construction operations at the Y-12 Plant. Waste materials were primarily non-radioactive soil fill, masonry, and concrete with steel rebar, but also may have included materials containing solvents, asbestos, mercury, and uranium (Battelle Columbus Division 1989b). The total waste volume is estimated at less than 100,000 cubic yards. Closure of the site was completed in 1984 in accordance with a TDEC-approved closure plan (MCI Consulting Engineers 1983).

\subsubsection{Bear Creek Operable Unit 04}

Bear Creek OU 04 is the integrator OU encompassing groundwater and surface water in the regime. Since 1990, groundwater and surface-water quality monitoring in the regime has been performed in accordance with the technical approach described in the Comprehensive Groundwater Monitoring Plan for the Department of Energy Y-12 Plant (Comprehensive Monitoring Plan; Geraghty \& Miller, Inc. 1990), which defined the following monitoring objectives:

\footnotetext{
monitor at the plume boundaries for evidence of lateral contaminant migration; determine the maximum depth of groundwater contamination to the extent necessary for the evaluation and selection of remedial alternatives;
} 
focus well installation and monitoring efforts on the Maynardville Limestone - the primary avenue for contaminant migration in groundwater;

perform regular sampling of surface water in Bear Creek;

define and coordinate studies to determine the hydrogeologic characteristics of the flow system in the Bear Creek Regime;

perform all sampling activities in accordance with RCRA assessment monitoring protocols (i.e., quarterly sampling frequency) to ensure continued regulatory compliance; and

report all the monitoring results in a single regime-wide groundwater quality assessment report.

Although some objectives of this technical approach, such as following RCRA assessment monitoring protocols, are less applicable because of changing regulatory developments, implementation of this approach has provided a substantial portion of the groundwater and surface-water quality data needed to effectively characterize the extent of contamination in the Bear Creek Regime. This is reflected by the relatively limited groundwater monitoring activities planned for Bear Creek OU 04. The approved RI work plan for the OU specifies quarterly monitoring of 25 wells for one year (U.S. Department of Energy 1993c). These sampling activities are scheduled to begin in March 1994.

\subsection{Above Grade Low Level Waste Storage Facility}

The Above Grade Low Level Waste Storage Facility is located west of the Oil Landfarm WMA (Figure 4). An access road divides the facility into a northern and southern section. Construction of the northern section of the facility was completed in the second quarter of 1993, but only the northern section is currently in operation. This section consists of six, $52 \times 171 \mathrm{ft}$ prefabricated rubber buildings engineered with concrete, slab-on-grade foundations with floor dikes as secondary containment. The facility is used to store low level radioactive solid wastes including construction/demolition debris, process materials, rubbish, sludge and soil, and beryllium in drums and B-25 boxes. 


\subsection{GROUNDWATER PROTECTION PROGRAM}

The following sections describe the groundwater and surface-water sampling activities performed during CY 1993 under the lead of the Y-12 Plant GWPP. Included are brief descriptions of the monitoring programs for which the sampling was performed, as well as details regarding sampling locations and frequencies, sample collection procedures, analytical parameters, quality assurance/quality control (QA/QC) sampling, and data management.

\subsection{Monitoring Programs}

Groundwater and surface-water sampling activities during 1993 addressed the purposes of four monitoring programs: (1) RCRA Compliance Monitoring, (2) RCRA Assessment Monitoring, (3) Exit-Pathway Monitoring, (4) Surveillance and Maintenance Monitoring, and (5) Best-Management Practice Monitoring. Each of these monitoring programs is implemented within the context of the technical approach contained in the Comprehensive Monitoring Plan. However, in light of the increase in regulatory requirements, an increasing number of wells are sampled to meet multiple programmatic goals.

\subsubsection{RCRA Compliance Monitoring}

As noted in the preceding section, RCRA compliance monitoring for the S-3 site was initiated in September 1993 in accordance with the Agreed Order signed in April 1993. This program currently involves semi-annual collection of groundwater samples from a network of 23 compliance wells (Table 2) located throughout the Bear Creek Regime, and analysis of the samples for the parameters specified in the post closure permit. Analytical results for the compliance wells are included in Appendix E. An evaluation of groundwater flow directions, which also is a permit condition, is provided in Section 4.2.5.

The modifications to the post-closure permit issued for the S-3 Site by the TDEC in April 1993 contain additional monitoring requirements that are currently being negotiated. The well network specified for compliance monitoring will likely change pending resolution of these negotiations. 


\subsubsection{RCRA Assessment Monitoring}

Quarterly RCRA Assessment Monitoring was continued during 1993 and initially included 47 monitoring wells, most located at the Oil Landfarm and Bear Creek Burial Grounds WMAs. In the second quarter of 1993, however, 39 of these wells were shifted to the Surveillance and Maintenance Monitoring program managed by the Y-12 Plant Environmental Restoration Program. Further reduced emphasis on assessment monitoring is expected and the program will be discontinued entirely when post-closure permits for these sites are issued by the TDEC.

\subsubsection{Exit Pathway Monitoring}

In June 1990, the DOE revised Order 5400.1 regarding environmental protection standards for DOE facilities. This order required monitoring of groundwater that is or could be affected by operations at DOE facilities to demonstrate compliance with DOE requirements and applicable Federal, State, and local laws and regulations. Compliance with most of the DOE Order 5400.1 requirements was achieved through continued implementation of ongoing monitoring programs required under TDEC and EPA regulations. However, the order did impose additional requirements for monitoring groundwater quality where contamination was most likely to be transported beyond the ORR boundaries.

Exit Pathway Monitoring is implemented under the Y-12 Plant GWPP to comply with the DOE Order 5400.1 requirements. In 1993, Exit Pathway Monitoring involved quarterly sampling of groundwater from 22 monitoring wells completed in the Maynardville Limestone and from 5 springs discharging to Bear Creek, and surface water from 5 locations along Bear Creek (Table 2). Six of the exit pathway wells are also used for RCRA Compliance Monitoring.

\subsubsection{Surveillance and Maintenance Monitoring}

As noted in Section 3.1.2, beginning in the second quarter of 1993, 39 wells previously used for RCRA Assessment Monitoring were shifted to the Surveillance and Maintenance Monitoring program managed by the Y-12 Plant Environmental Restoration Program (Table 2). The wells are located near the closed and capped source control OUs, and are sampled quarterly 
to provide data needed to evaluate cap effectiveness in terms of water-quality trends over time as well as provide data on the extent of contamination within the Bear Creek Regime. The Y-12 Plant GWPP will implement the monitoring requirements of the Surveillance and Maintenance Monitoring program in the future.

\subsubsection{Best-Management Practice Monitoring}

Groundwater samples were collected during CY 1993 from 3 monitoring wells at the Above Grade Low Level Storage Facility as a best-management practice of the Y-12 Plant GWPP (Table 2). Groundwater monitoring as a best-management practice at this site was requested by the TDEC and has been performed since 1991.

\subsection{Sampling and Analysis Program}

The following sections describe the groundwater and surface water sampling and analyses activities performed during CY 1993. Included are descriptions of the sampling locations, frequency, and procedures, analytical parameters, quality assurance/quality control (QA/QC) sampling, and data management.

\subsubsection{Sampling Locations}

Groundwater samples were collected from the 89 monitoring wells listed in Table 2. Locations of these monitoring wells are shown on Figure 6, and data regarding the elevation of the screened or open hole interval, the aquifer zone, and the bedrock formation monitored by the wells are provided in Table 3. Detailed well construction information is contained in Appendix C.

Samples of groundwater discharging to Bear Creek from five springs were collected during 1993. Three of these springs are located near the waste disposal sites in the regime; one (SS-1) in the upper reaches of Bear Creek just downstream of the S-3 Site and two (SS-4 and SS-5) located south of the Bear Creek Burial Grounds WMA (Figure 7). The other two springs (SS-6 and SS-8) are located a few miles west of the Bear Creek Burial Grounds WMA. 
Surface water samples were collected along Bear Creek at two background stations and three downstream stations (Figure 7). The background stations (NT-01 and NT-13) are in northern tributaries to Bear Creek that have not been affected by waste-management activities in the Bear Creek Regime. Sampling locations along Bear Creek are designated by the distance (in kilometers) from the confluence of Bear Creek and East Fork Poplar Creek. The three locations sampled during 1993 were Bear Creek Kilometer (BCK) 0.63, BCK 4.55, and BCK 9.40.

\subsubsection{Sampling Frequency}

Groundwater and surface water samples were collected during each quarter of 1993. First through fourth quarter sampling events were performed from January 28 to March 30, April 16 to June 27, July 1 to September 29, and October 6 to January 29, respectively.

Groundwater samples were collected during each quarter from each spring and surface-water sampling station and from all but 10 of the monitoring wells. One well (GW-052) at the Bear Creek Burial Grounds WMA was dry during the third and fourth quarters. Well GW-063 at the Oil Landfarm WMA was sampled only once because it was plugged and abandoned on May 7, 1993. Only one groundwater sample was collected from 7 of the wells used for RCRA Compliance Monitoring; GW-042, GW-079, GW-080, GW-162, and GW-342 at the Bear Creek Burial Grounds WMA and wells GW-613 and GW-614 at the S-3 Site. Semi-annual sampling of these wells was initiated in the fourth quarter of 1993 after they were designated as compliance wells in the RCRA post-closure permit for the S-3 Site. After installation in April 1993, well GW-800 at the Oil Landfarm WMA was sampled during the second, third, and fourth quarters of 1993.

\subsubsection{Sample Collection}

Groundwater samples were collected by personnel from the Oak Ridge K-25 Site Sampling and Environmental Support Department; personnel from the Y-12 Plant Compliance Monitoring Section of the HSEA Organization collected the surface-water samples. Pre-defined sampling sequences were followed to minimize the potential for cross-contamination. Monitoring wells were sampled during each quarterly event in the sequences listed in 
Appendix D, which generally reflect sampling from least contaminated wells to the most contaminated wells at a site or in a sampling group (a series of monitoring wells grouped for sampling and data-tracking purposes). Samples from the springs and surface water stations were collected from the farthest downstream (least contaminated) location (BCK 0.63) to the farthest upstream (most contaminated) location (Spring SS-1).

Groundwater sampling was performed in accordance with the most recent version of the Standard Operating Procedure 8102 approved by the Y-12 Plant GWPP Manager. Descriptions of the field methods and procedures used to collect the groundwater and surface water samples are contained in Environmental Surveillance Procedures Quality Control Program, Martin Marietta Energy Systems, Inc. (Kimbrough et al. 1987). The EPA (Region IV) and the TDEC have approved these methods and procedures (U.S. Environmental Protection Agency 1988a).

\subsubsection{Quality Assurance/Quality Control Sampling}

Quality assurance/quality control samples included laboratory blanks, trip blanks, equipment rinsates, and duplicate groundwater samples. A total of 142 laboratory blanks, 197 trip blanks, 61 equipment rinsate samples, and 37 duplicate samples were analyzed during 1993. Similar numbers of QA/QC samples were analyzed during each quarterly sampling event: 31 to 42 laboratory blanks, 41 to 55 trip blanks, 14 to 16 equipment rinsates, and 9 to 10 duplicate samples.

Laboratory blanks were prepared using deionized water and analyzed along with the groundwater and surface-water samples. Trip blanks were prepared in the laboratory using deionized water and transported unopened in coolers containing groundwater and/or surface-water samples to be analyzed for volatile organic compounds (VOCs). Equipment rinsates were obtained from the deionized water used to decontaminate the groundwater sampling equipment. These samples were collected after a sampling team had completed sampling at a site or finished a sampling group (typically no more than 10 wells grouped for sampling and data-tracking purposes). If more than one team collected samples at a site or within a sampling group, each collected an equipment rinsate sample. Duplicate groundwater and surface water samples were collected at a frequency of at least one per sampling group, or one for every 10 monitoring wells, whichever was greater. 


\subsubsection{Laboratory Analysis}

All groundwater and surface water samples were analyzed for the suite of parameters and constituents listed on Table 4. The analytical parameters and constituents included:

trace metals and principal ions (total and dissolved concentrations);

V VOCs;

- gross alpha activity and gross beta activity;

- total suspended solids, total dissolved solids, and turbidity;

- field and laboratory determinations of $\mathrm{pH}$ and specific conductance; and

- field determinations of temperature, dissolved oxygen, and oxidation-reduction potential.

.jamples from wells with elevated gross alpha and/or gross beta activity, as determined from CY 1992 data, were analyzed for selected radionuclides. The K-25 Analytical Chemistry Department nerformed mist of the laboratory analyses; radiochemical analyses were performed by the Oak Ridge National Laboratory (ORNL) Chemistry Division.

All of the laboratory blanks, trip blanks, and equipment rinsate samples were analyzed for the suite of VOCs listed on Table 4. Selected equipment rinsate samples also were analyzed for trace metals (Inductively Coupled Plasma analyses), nitrate (as $N$ ), and gross alpha and gross beta activity. Duplicate groundwater samples were analyzed for the constituents and parameters specified for the well from which the duplicate sample was collected.

\subsubsection{Data Management}

Analytical results obtained by the K-25 and ORNL laboratories were verified, entered into the Y-12 Plant Environmental Monitoring Database (EMD), and electronically processed through several data evaluation procedures. Brief descriptions of the data verification/entry/evaluation procedures follow; details are provided in Data Verfication and Evaluation Techniques for Groundwater Monitoring Programs (Mercier and Turner 1990).

Analytical results obtained from each quarterly sampling event were either downloaded directly into the EMD from data files provided by the analytical laboratory or were manually input from hardcopy uucumentation (laboratory reports and field data sheets). For manually input data, a double-entry procedure was followed whereby the hardcopy data were input twice into separate data files. A computer program then compared the two input data files and identified 
discrepancies, which were then accounted for and corrected. When all data from the quarterly sampling event were in the EMD, a computer program then verified that the parameter names were correct, correct units were reported, and that the results reported for each parameter were within an expected range or were valid numeric values. As a final step in the data entry/verification process, printouts of the data were visually checked against the laboratory reports and field data sheets for completeness and obvious data outliers. The appropriate laboratory was contacted to resolve incomplete data transfers, discrepancies between electronic and hardcopy versions of the data, and questions regarding data outliers.

Upon completion of the data verification and entry into the EMD, a computer program was used to scan the monitoring data, flag questionable or suspect data based on pre-defined criteria, and prepared a report for transmittal to the appropriate laboratory. This data evaluation program calculated ion-charge balances for each sample, identified changes in analytical detection limits, validated analytical results for VOCs using the QA/QC sample data in accordance with an EPA validation procedure (U.S. Environmental Protection Agency 1988b). Questionable or suspect data were reported to the appropriate analytical laboratory. Errors in the data were corrected and questionable results unresolved by the appropriate laboratory were flagged.

\subsubsection{Analytical Results}

The groundwater and surface-water quality data obtained during 1993 are contained in Appendices $\mathrm{E}$ through $\mathrm{L}$. The analytical results for each groundwater and surface water sample are contained in Appendix E; results for duplicate groundwater and surface water samples are presented in Appendix F. Ion-charge balances for each groundwater and surface water sample are presented in Appendix G. Appendix H contains trace metal results that exceed Maximum Contaminant Levels or background screening levels. Analytical results for the QA/QC samples are summarized in Appendix I. A summary of the validated analytical results for VOCs is provided in Appendix $\mathrm{J}$, and radiochemical data are summarized in Appendix K. Annual average concentrations of nitrate (as N) (a primary groundwater contaminant in the Bear Creek Regime) determined for each well, spring, and surface water station are provided in Appendix L. 


\subsection{HYDROLOGIC FRAMEWORK}

This section contains a general description of the complex hydrogeologic system in the Bear Creek Regime. It provides sufficient descriptive detail needed for the purposes of RCRA reporting requirements regarding evaluation of groundwater flow directions, and determination of representative rates of groundwater flow and contaminant transport. The description is based on the conceptual framework and associated nomenclature presented in: Status Report-A Hydrologic Framework for the Oak Ridge Reservation (Solomon et al. 1992).

Groundwater in the Bear Creek Regime occurs in two broad units with fundamentally different hydrologic characteristics: the Knox Aquifer and the ORR Aquitards. The Knox Aquifer is comprised of the Knox Group and the underlying Maynardville Limestone of the Conasauga Group. The bulk permeability of the Knox Aquifer, which is due primarily to solution conduits, is ten times greater than that of the ORR Aquitards. The Maynardville Limestone formation is believed to be a drain for the flow system in the Bear Creek Regime and provides the primary pathways for migration of contaminants in groundwater.

The remaining Conasauga Group formations, the underlying Rome Formation, and the Chickamauga Group form the ORR Aquitards. In this report, the term "ORR Aquitards" refers only to the Conasauga Group (excluding the Maynardville Limestone), and the Rome Formation. Flow in the ORR Aquitards is controlled by short, intersecting fractures in a relatively impermeable matrix, and most groundwater flows through a thin zone near the water table. Most of the waste-management sites in the Bear Creek Regime are underlain by the ORR Aquitards, in which contaminant migration rates relative to groundwater flow velocities are reduced by diffusive exchange between matrix and fractures (Solomon et al. 1992).

Pine Ridge and Chestnut Ridge are the primary recharge areas for the groundwater system in the Bear Creek Regime. Annual recharge is greater for the Knox Aquifer (about 2.6 inches) than for the ORR Aquitards (about 0.8 inches), and it is possible that a portion of the recharge to each unit results from delayed unsaturated flow (Solomon et al. 1992). Groundwater in the ORR Aquitards generally flows from the recharge areas toward the Maynardville Limestone, which outcrops in BCV along the base of Chestmut Ridge and underlies Bear Creek in much of the regime. Groundwater in the Maynardville Limestone generally flows west along geologic strike. 


\subsection{Geology}

The geology in the area of the Y-12 Plant is generally characterized by sequences of southeast-dipping, fractured clastic (primarily shale and siltstone) and carbonate (limestone and dolostone) strata of Lower Cambrian to Lower Ordovician age (Figure 8). Bear Creek Valley is underlain by the interbedded limestone and shale formations of the Conasauga Group. Shale and siltstone beds of the underlying Rome Formation form Pine Ridge to the north, and the primarily dolostone strata of the overlying Knox Group form Chestnut Ridge to the south. Strike and dip of bedding in the Bear Creek Regime are generally $\mathrm{N} 55^{\circ} \mathrm{E}$ and $45^{\circ} \mathrm{SE}$, respectively.

Bedrock in the Bear Creek Regime is overlain by any of several materials, including alluvium, colluvium, man-made fill, fine-grained residuum from the weathering of the bedrock, saprolite (a transitional mixture of fine-grained residuum and bedrock remnants), and weathered bedrock. In many areas, the saprolite retains primary textural features of the bedrock, including fractures (Solomon et al. 1992). The overall thickness of these materials in BCV is typically less than $40 \mathrm{ft}$.

The Maynardville Limestone subcrops along the axis of $\mathrm{BCV}$ at the base of Chestnut Ridge and the underlying formations of the ORR Aquitards subcrop successively to the north toward Pine Ridge (Figure 8). The Maynardville Limestone consists of fine- to coarse-grained, thin-bedded to massive limestone and dolostone (King and Haase 1987). Information obtained during 1992 indicates that the thickness of the Maynardville Limestone varies from about 300 to $620 \mathrm{ft}$ and averages about $490 \mathrm{ft}$ (Shevenell et al. 1992)." Additionally, Shevenell et al. (1992) report, based on lithologic information obtained during installation of several exit-pathway monitoring wells, that most of the basal portion of the Maynardville Limestone (the Low Hollow member) is not present in an area southeast of the Bear Creek Burial Grounds WMA. This loss of stratigraphic section may reflect a facies change in the Maynardville, as suggested by Shevenell et al. (1992), or may be the result of structural displacement. Further investigation to explain the substantial thinning of the Maynardville Limestone in this area is ongoing.

Underlying formations of the Conasauga Group that comprise the ORR Aquitards are, from youngest to oldest, the Nolichucky Shale, Maryville Limestone, Rogersville Shale, Rutledge Limestone, and Pumpkin Valley Shale (Figure 8). The lithologic compositions of the Nolichucky Shale and Maryville Limestone are similar in the Bear Creek Regime. These formations consist 
primarily of between 350 and $550 \mathrm{ft}$ of massive to thin-bedded mudstone with interbedded limestone (King and Haase 1987). Beneath the Maryville Limestone are the massive mudstones of the Rogersville Shale and the interbedded limestone and shale of the Rutledge Limestone. The thickness of each of these formations ranges from 90 to $120 \mathrm{ft}$ (King and Haase 1987). The Pumpkin Valley Shale consists of 260 to $320 \mathrm{ft}$ of massive shale and mudstone with thin beds of siltstone and sandstone (King and Haase 1987).

The most pervasive structural features in the Bear Creek Regime are extensional, hybrid, and shear fractures (Solomon et al. 1992). Three major joint sets are evident in the Bear Creek Regime; one that roughly parallels bedding, one steeply dipping set that parallels geologic strike, and one steeply dipping set that trends perpendicular to strike (Dreier et al. 1987). Fracture densities ranging from about 1 to 60 per foot have been observed in rock outcrops near ORNL (Dreier et al. 1987; Sledz and Huff 1981). Most fractures are short, ranging from tenths of an inch to a few feet in length (Solomon et al. 1992). Within a fracture, groundwater may flow either downdip, laterally, or in both directions. Changes in flow direction may occur at fracture splits and intersections, and groundwater flow paths may locally resemble stairsteps in both plan and sectional views (Moore 1989).

Dissolution of carbonates along fractures, particularly in the Maynardville Limestone, has produced solution-enlarged features ranging from less than an inch to tens of feet in thickness. Shevenell et al. (1992) report that solution cavities occur throughout the Maynardville Limestone, but occur most frequently in the uppermost and lowermost of six stratigraphic zones defined by gamma-log signatures and core data. All water-bearing intervals in the uppermost zone occur at less than $235 \mathrm{ft}$ below the ground surface, and $22 \%$ of these intervals were identified as cavities. Water-bearing intervals in the lowermost zone occur at depths up to about $450 \mathrm{ft}$ below the ground surface, and $19 \%$ of these intervals were identified as cavities (Shevenell et al. 1992).

\subsection{Groundwater System}

Solomon et al. (1992) divide the groundwater flow system in both the Knox Aquifer and the ORR Aquitards into four subsystems: (1) the stormflow zone, (2) the vadose zone, (3) the groundwater zone (subdivided into water table, intermediate, and deep intervals), and (4) the aquiclude (Figure 9). The divisions are based on the amount of water transmitted by each 
subsystem (i.e., flux), which decreases with depth; the largest water flux occurs in the stormflow zone and the smallest in the aquiclude.

The groundwater flow system is vertically gradational with no discreet boundaries separating the subsystems. The stormflow zone occurs in regolith as does much of the vadose zone, although in some areas the vadose zone includes weathered and fresh rock. About $90 \%$ of subsurface flow occurs in the stormflow zone. In the groundwater zone, flow volumes decrease and solute residence times increase sharply with depth. The bulk (98\%) of groundwater flow occurs at shallow depths ( $<100 \mathrm{ft}$ below ground surface), and most (80\%) of this flow occurs in the water table interval. Below the water table interval, groundwater flow occurs along permeable fractures (or possibly fractured regions) within a relatively impermeable matrix. Variations in the number and aperture of the fractures within these regions occur along flow paths, and these differences determine lateral and vertical flow directions. Additionally, the vertical spacing between the permeable regions increases with depth, and permeable regions at depth are poorly connected in three dimensions (Solomon et al. 1992).

\subsubsection{Stormflow Zone}

Investigations in Bethel Valley and Melton Valley near ORNL show that groundwater occurs intermittently above the water table in the ORR Aquitards in a shallow "stormflow zone" that extends from ground surface to a depth of about $6 \mathrm{ft}$ (Moore 1989). Channels for lateral flow in the stormflow zone include macropores and mesopores, which are connected voids created by various processes, including biochanneling, cracking, and soil particle aggregation (Moore 1989). This zone is thicker and more permeable in forested areas than in grassy or brushy areas, and is more permeable near the land surface than at deeper levels (Moore 1989). However, a stormflow zone may not exist in areas that are not vegetated.

Water is present in the stormflow zone during, and for short periods after, significant rainfall events; some of the water is lost to evapotranspiration and recharge to the water table, and the remaining water discharges at nearby seeps, springs, and streams. Analysis of infiltrometer test results from Bethel Valley and Melton Valley suggest that the hydraulic conductivity of the stormflow zone averages about 29 feet per day (ft/day) near the top and $0.1 \mathrm{ft} /$ day near the base (Moore 1989). Lateral flow in the stormflow zone is intermittent, lasting 
a few days to a few weeks after precipitation. Flow is fairly rapid when the zone is full of water but relatively slow when nearly drained (Moore 1989).

\subsubsection{Vadose Zone}

The vadose zone occurs between the stormflow zone and the water table (Figure 9). The geometric mean depth to the water table in the ORR Aquitards is about $13 \mathrm{ft}$ (Moore 1989). The vadose zone is primarily composed of regolith. Moore (1989) estimates effective porosity (the porosity through which advective transport can occur) for regolith of 0.01 to 0.15 .

Water is added to the vadose zone by percolation from the stormflow zone and is removed by transpiration and recharge to the water table. The vadose zone is unsaturated except in the capillary fringe above the water table and within wetting fronts during periods of vertical percolation from the stormflow zone (Moore 1989). A geometric mean hydraulic conductivity of about $0.006 \mathrm{ft} /$ day was determined from analysis of infiltrometer tests in the vadose zone in Bethel Valley and Melton Valley (Moore 1989).

\subsubsection{Groundwater Zone}

Solomon et al. (1992) divide the saturated zone in the Knox Aquifer and ORR Aquitards into water table, intermediate, and deep intervals based on observed changes in hydrologic characteristics and groundwater geochemistry with depth (Figure 9). For the purposes of this report, the above subdivisions have been modified for the Bear Creek Regime to include a shallow interval between the water table and intermediate intervals. The boundary between the shallow and intermediate intervals is set at $100 \mathrm{ft}$ below ground surface, which generally corresponds with an aquifer subdivision noted by Moore (1989) in an evaluation of groundwater flow parameters, and is the depth at which a fairly abrupt change from calcium-magnesium-bicarbonate groundwater to sodium-bicarbonate groundwater typically occurs in the ORR Aquitards. Additionally, solution cavities in the Maynardville Limestone occur most frequently at depths less than $100 \mathrm{ft}$ below the ground surface, suggesting that the active karst flow system in the Maynardville Limestone is relatively shallow (Shevenell et al. 1992). 


\subsubsection{Water Table Interval}

A permeable interval 3 to $15 \mathrm{ft}$ thick usually occurs in the transitional horizon between regolith and unweathered bedrock, and the water table often occurs within this permeable interval (Solomon et al. 1992). The saturated thickness of this interval changes with cyclic variations in the water table elevation, and the interval may be nearly drained during seasonal water table declines. It is postulated that in the ORR Aquitards, the water table interval transmits more than $80 \%$ of the flow that occurs below the stormflow zone. Estimates of the permeability of the water-table zone are provided by results of hydraulic conductivity tests performed in wells completed in regolith below the water table. Analysis of these test results indicated a range of hydraulic conductivity of 0.03 to $3.25 \mathrm{ft}$ /day (Connell and Bailey 1989).

Results of geochemical dating studies of groundwater in the ORR Aquitards in Melton Valley generally support the concept of a groundwater zone that is most active near the water table and becomes increasingly sluggish with depth (Solomon et al. 1992). Analyses of ${ }^{3} \mathrm{H}:{ }^{3} \mathrm{He}$ ratios in groundwater samples collected from 1 to $6 \mathrm{ft}$ below the water table showed ages ranging from 0.09 to 7.23 years, and the presence of large amounts of ${ }^{4} \mathrm{He}$ in groundwater samples from greater depths (45 to $165 \mathrm{ft}$ below ground surface) suggest much greater residence times (Poreda et al. 1988).

\subsubsection{Shallow Interval}

The shallow interval of the groundwater zone extends from the bottom of the water table interval to depths of about $100 \mathrm{ft}$ in the ORR Aquitards (Figure 9), and from 160 to $325 \mathrm{ft}$ in the Knox Aquifer (Moore 1989). Groundwater in this zone is typically encountered under unconfined conditions in fractures and solution cavities. Enlarged fractures and cavities are the primary water-producing features and solute-transport pathways, and are supplied by seepage through fractures in the rock matrix. Fractures outnumber the cavities, are interconnected, and provide the continuity for groundwater flow paths (Moore 1989).

Analysis of hydraulic conductivity test results for wells completed at shallow depths in the ORR Aquitards and the Knox Aquifer generally shows two populations; an upper population with a geometric mean hydraulic conductivity of about $0.15 \mathrm{ft} /$ day and a lower population with 
a geometric mean hydraulic conductivity of about $0.0008 \mathrm{ft} /$ day (Moore 1989). The upper population represents more permeable, water-producing intervals and the lower population represents less permeable matrix intervals.

The hydraulic conductivity of the water-producing intervals at shallow depths in the Knox Aquifer is much greater owing to an interconnected network of solution cavities and solution-enlarged fractures. For instance, results of hydraulic conductivity tests for wells less than $100 \mathrm{ft}$ deep in the Maynardville Limestone, as summarized in Geraghty \& Miller, Inc. (1989b), range from 0.13 to $99 \mathrm{ft} /$ day and average about $22 \mathrm{ft} /$ day. Additionally, Shevenell et al. (1992) report that fractures and solution cavities in the Maynardville Limestone encountered during installation of several exit-pathway wells yield as much as 200 to 300 gallons per minute $(\mathrm{gpm})$, which is several orders-of-magnitude greater than the typical yield $(0.25 \mathrm{gpm}$; Solomon et al. 1992) of wells completed in the ORR Aquitards. Results of a dye tracer test in the Maynardville Limestone near the Bear Creek Burial Grounds WMA suggested (qualitatively) a conduit flow rate of about $25 \mathrm{ft} /$ day (Geraghty \& Miller, Inc. 1989c).

Results of tracer tests performed in the Bear Creek Regime and calculations of the fracture porosity in bedrock provide estimates of the effective porosity of the ORR Aquitards and the Maynardville Limestone. Tracer tests have yielded effective porosity values ranging from 0.002 to 0.004 (Law Engineering Testing Company 1983; Lozier et al. 1987). Moore (1989) calculated a fracture porosity ranging from 0.001 to 0.002 , based on estimates of fracture density and average fracture aperture for water-producing intervals at shallow depths in the Conasauga Group.

\subsubsection{Intermediate Interval}

Groundwater in the intermediate bedrock interval occurs in relatively permeable fractures (or possibly fractured intervals) within a relatively impermeable matrix. Review of data obtained with an electromagnetic borehole flowmeter suggest that the permeable regions commonly develop within a single layer of rock, and the average thickness of a permeable region (assuming an average dip of $35^{\circ}$ ) is less than $2 \mathrm{ft}$. The vertical spacing between permeable regions in the intermediate interval is believed to be more than $100 \mathrm{ft}$ (Solomon et al. 1992); a vertical spacing of $150 \mathrm{ft}$ is supported by results of matrix diffusion modeling of measured ${ }^{14} \mathrm{C}$ and ${ }^{3} \mathrm{H}$ activities 
(Toran et al. 1991). Results of straddle packer tests in wells completed in the intermediate interval of the ORR Aquitards at several locations in BCV and Melton Valley show a range of mean hydraulic conductivity of 0.006 to $0.016 \mathrm{ft} /$ day (Solomon et al. 1992).

\subsubsection{Deep Interval}

Identification of the deep bedrock interval is based on hydraulic and geochemical data for several deep (greater than $500 \mathrm{ft}$ ) monitoring wells and coreholes. Solomon et al. (1992) report that the boundary between the intermediate interval and the deep interval in BCV occurs at depths of about $325 \mathrm{ft}$ in the ORR Aquitards and $650 \mathrm{ft}$ in the Knox Aquifer. The boundary is based on lower hydraulic conductivity and unusually high hydraulic heads (estimated from packer tests), although use of hydraulic conductivity may not always be a reliable indicator (Solomon et al. 1992).

The transition from the intermediate interval to the deep interval in the ORR Aquitards is often indicated by a change from sodium-bicarbonate groundwater to sodium-chloride groundwater accompanied by a general increase in specific conductance and total dissolved solids. This change in groundwater geochemistry probably reflects longer residence times in the deep interval, and usually occurs at depths of more than $400 \mathrm{ft}$ below the ground surface (Solomon et al. 1992). However, no such change in groundwater geochemistry has been observed in the Knox Aquifer; calcium-magnesium-bicarbonate or calcium-sulfate grounc water occurs to depths of $1,000 \mathrm{ft}$ below the ground surface (Dreier et al. 1993).

Evaluation of data obtained from straddle packer tests and slug tests, and slow-recovery analyses, indicates that the geometric mean hydraulic conductivity of the deep interval in BCV is about $0.001 \mathrm{ft} /$ day (Solomon et al. 1992). Although the mean hydraulic conductivity is comparable to that of the intermediate interval, measured conductivities in the deep interval are as low as $2.83 \times 10^{-6} \mathrm{ft} /$ day (Solomon et al. 1992). The low conductivities may reflect reduced fracture apertures or increased fracture spacings (Solomon et al. 1992). 


\subsubsection{Aquiclude}

The aquiclude occurs below the deep groundwater interval and is generally marked by the presence of saline water with total dissolved solids of 40,000 to 300,000 milligrams per liter (mg/L) (Solomon et al. 1992). The water in the aquiclude is a sodium-, calcium-, and chloride-rich brine that is chemically similar to brines associated with major sedimentary basins (Solomon et al. 1992). In Melton Valley, saline water typically occurs at depths of about 600 to $700 \mathrm{ft}$ below the ground surface (Solomon et al. 1992).

Saline groundwater has not been encountered at depth in the Bear Creek Regime. However, several wells in BCV completed at depths of about 500 to $1,000 \mathrm{ft}$ below the ground surface monitor groundwater with total dissolved solids of several thousand $\mathrm{mg} / \mathrm{L}$ to several tens of thousand $\mathrm{mg} / \mathrm{L}$. The presence of water with relatively high total dissolved solids (as well as increased sodium and chloride concentrations) in the deep groundwater interval in BCV may represent a mixing zone or diffusion zone between the fresh and saline groundwater, suggesting that the aquiclude is most likely present at greater depth in BCV than in Melton Valley (Solomon et al. 1992).

\subsubsection{Groundwater Flow Directions}

Directions of groundwater flow in the Bear Creek Regime were evaluated from static water-level measurements obtained from 206 monitoring wells during January 1993 (the seasonally high water table) and 186 wells in August/September 1993 (the seasonally low water table); depths to water and water-level elevations for each well are tabulated in Appendix $\mathbf{M}$. Review of these data show that water level declines averaged about $5 \mathrm{ft}$ in water-table interval wells and about $7 \mathrm{ft}$ in bedrock (shallow and intermediate intervals) wells, with the largest decreases typically occurring in bedrock wells completed in the Maynardville Limestone. Additionally, the pattern of water level decrease was the same in all wells such that there were no significant changes in groundwater flow directions (i.e., no reversals of horizontal hydraulic gradients)

Groundwater elevations in the water table and bedrock (shallow and intermediate) intervals during the seasonally high and seasonally low groundwater flow conditions are 
illustrated on Figures 10 and 11 , respectively. On average, groundwater elevations in the water table interval were 16 to $18 \mathrm{ft}$ higher than in the bedrock. The groundwater surface in both the water table interval and bedrock is a subdued replica of surface topography, with steep gradients along the flanks of Pine Ridge and Chestnut Ridge and a gentle slope southwest down the axis of BCV. The horizontal hydraulic gradient averages about 0.046 across BCV (strike-normal) and about 0.012 along the axis of BCV (strike-parallel).

The overall direction of groundwater flow in the ORR Aquitards is south and southwest toward the Maynardville Limestone, suggesting that this formation is a hydrologic drain for the groundwater flow system in the regime. Groundwater in the Maynardville generally flows westerly parallel to strike.

\subsubsection{Contaminant Transport}

Quantitative and qualitative methods were used to estimate contaminant migration rates. Quantitative migration rates were estimated only for the water table and shallow intervals in the groundwater zone. Migration rates in the stormflow zone were not estimated because waste sources in the Bear Creek Regime lie below this zone. Estimates of migration rates in the intermediate and deep intervals also were not performed, but representative rates are probably as low as a few centimeters per year (Solomon et al. 1992). Qualitative transport rates were determined based on the extent of nitrate in the Bear Creek Regime assuming a constant input rate from a single so irce area.

The wide range of potential contaminant migration rates reported in the following sections demonstrate the uncertainty in determining a representative rate of solute transport for this groundwater flow system. Long term transport of solutes in dual porosity and/or highly heterogeneous media is a complex physical and chemical process that is not fully explained by calculations using the modified Darcy equation or qualitative estimates based on distances from source areas to plume boundaries. It is probable that at various times and under various conditions, migration rates throughout the given range occur; some contaminants such as nitrate move relatively rapidly through the flow system, while other more highly attenuated constituents move much more slowly. Moreover, even the highly mobile compounds will diffuse into smaller pores from the large conduits and later, under a reverse concentration gradient, will slowly diffuse 
back out. Thus, although the actual transport rates may vary over soveral orders of magnitude, the relatively limited extent of the contaminant plumes in the Bear Creek Regime suggests that slower transport rates may dominate and that attenuation processes such as matrix diffusion are an important controlling factor.

\subsubsection{Quantitative Eutimates}

Quantitative estimates of contaminant transport were based on the assumption that contaminants move with the groundwater unimpeded by various retardation processes. Factors which would reduce transport rates, such as matrix diffusion, degradation, sorption, and chemical precipitation, or factors which may increase transport, such as dispersion, were not explicitly considered. Free product migration, which may occur independent of groundwater flow rates and directions, also was not considered.

Assuming that saturated regolith and fractured bedrock can be treated as an equivalent porous medium, average groundwater flow rates can be estimated using the modified Darcy equation:

$$
\mathbf{v}=\mathbf{K} \mathbf{i} \mathbf{n}
$$

where $\mathbf{v}$ is the average linear velocity of the groundwater, $\mathbf{K}$ is the hydraulic conductivity of the medium, $\mathbf{i}$ is the hydraulic gradient, and $\mathbf{n}$ is the effective porosity. Although the modified Darcy equation can be used to calculate average flow velocities in fractured rock, the velocities in individual fractures may vary over orders of magnitude, depending on the fracture aperture and wall roughness (Freeze and Cherry 1979).

Using the range of hydraulic conductivities for regolith ( 0.03 to $3.25 \mathrm{ft} /$ day), effective porosities ranging from 0.001 to 0.15 , an average strike-parallel (down-valley) gradient of 0.011 , and an average strike-normal (cross-valley) gradient of 0.045 , calculated rates of solute transport in the water table interval range from 0.002 to $146.2 \mathrm{ft} /$ day. However, the bulk of the water mass in the water table interval resides within porous matrix zones between fractures, and diffusive exchange between matrix and fractures would reduce contaminant migration rates relative to calculated flow rates (Solomon ef al. 1992). 
Contaminant transport rates for the shallow interval in the ORR Aquitards were calculated using a geometric mean hydraulic conductivity of $0.0008 \mathrm{ft} /$ day for matrix intervals and $0.15 \mathrm{ft} /$ day for water-producing intervals, effective porosity values ranging from 0.001 to 0.004 , and an average strike-normal hydraulic gradient of 0.046 . Using these values, the rate of contaminant migration in the ORR Aquitards ranges from 0.0009 to $6.7 \mathrm{ft} /$ day.

Contaminant transport rates for the shallow interval in the Maynardville Limestone were calculated using the average hydraulic conductivity ( $22 \mathrm{ft} /$ day) obtained from well tests, effective porosity values ranging from 0.001 to 0.004 , and an average strike-parallel hydraulic gradient of 0.012. Using these values, the rate of contaminant migration in the Maynardville Limestone ranges from about 66 to $264 \mathrm{ft} /$ day. Relatively high transport rates in the Maynardville Limestone also are indicated by results of a dye-tracer study performed in the Bear Creek Regime which yielded a transport rate of about $200 \mathrm{ft} /$ day (Geraghty \& Miller, Inc. 1989d).

\subsubsection{Qualitative Estimates}

Qualitative estimates of contaminant migration rates in the Bear Creek Regime were based on the extent of the nitrate plume originating from the S-3 Site observed during CY 1990 (HSW Environmental Consultants, Inc. 1991). Unlike most of the other groundwater contaminants in the Bear Creek Regime, many of which have multiple source areas, the S-3 Site is the only significant nitrate source in the regime. Moreover, nitrate salts are highly soluble and generally move with no significant sorption or decay during transport (Geraghty \& Miller, Inc. 1989d).

The nitrate plume originating from the S-3 Site extends westward (along strike) about $3,650 \mathrm{ft}$ in the water table interval, approximately $2,000 \mathrm{ft}$ at shallow depths in the ORR Aquitards, and about 9,500 $\mathrm{ft}$ west at shallow depths in the Maynardville Limestone (HSW

Environmental Consultants, Inc. 1991). Given the 39 years between initial waste disposal at the S-3 Site in 1951 and the horizontal extent of the nitrate plume observed in 1990, the rate of nitrate transport ( $\mathrm{v}=$ distance/time) is about $0.3 \mathrm{ft} / \mathrm{day}$ in the water table interval, about $0.1 \mathrm{ft} / \mathrm{day}$ in the ORR Aquitards, and about $0.7 \mathrm{ft} / \mathrm{day}$ in the Maynardville Limestone. 


\subsection{Surface-Water System}

The surface-water system in the Bear Creek Regime consists of Bear Creek and its tributaries. The headwaters of Bear Creek are at the west end of the Y-12 Plant near the S-3 Site (Figure 7). From its headwaters, the creek flows west for approximately 4.5 miles, where it turns north through a gap in Pine Ridge and flows into East Fork Poplar Creek about 6 miles upstream of its confluence with the Clinch River.

Several sections of Bear Creek were modified since construction of the Y-12 Plant. The channel at the original headwaters was filled with rubble during construction of the S-3 Ponds and moved to its present location, and sections of the creek north of the Rust Spoil Area, east and south of the Oil Landfarm WMA, and south of the Bear Creek Burial Grounds WMA were rerouted. Spring SS-4, which discharges to Bear Creek south of the Bear Creek Burial Grounds WMA (Figure 7), may be a seepage face for groundwater moving through the buried original creek channel, which is located south of the present channel (Geraghty \& Miller, Inc. 1989c).

The highest flows in Bear Creek occur in response to precipitation during winter and spring. Low flows occur during late summer and early fall, when several tributaries become dry and parts of the main channel within the Bear Creek Regime are dry. After periods of rainfall, the first several hundred feet of the creek headwaters may contain water from surface runoff, but at other times the headwaters remain dry for about $700 \mathrm{ft}$ downstream of the S-3 Site. Flow in the creek during rainfall events is usually of short duration with rapid recession and no observed overbank flow (Geraghty \& Miller, Inc. 1987).

Along much of its course through the Bear Creek Regime, Bear Creek lies entirely within the outcrop area of the Maynardville Limestone, and to a large extent, dissolution along fractures controls the position of the channel (Geraghty \& Miller, Inc. 1987). Solution cavities have been encountered during drilling in the Maynardville near Bear Creek, indicating that they are an integral part of the Bear Creek flow system.

Shevenell et al. (1992) report evidence of hydraulic communication between subsurface conduits and springs discharging to Bear Creek observed during installation of several exit-pathway monitoring wells. For example, during installation of a well (GW-684), production of water from a fracture or cavity encountered at a depth of about $125 \mathrm{ft}$ below the ground surface had turbidity and flow rate impacts on one of two adjacent springs (SS-5) located about $100 \mathrm{ft}$ north (updip) of the well (Shevenell et al. 1992). Surprisingly, hydraulic connection between the other spring at this location and the Copper Ridge Dolomite of the Knox Group was 
indicated by turbidity impacts observed during installation of an exit-pathway well (GW-683) located about $250 \mathrm{ft}$ south of well GW-684 (Shevenell et al. 1992).

Because of the hydrologic interaction between the creek and the solution cavity system in the Maynardville Limestone, and possibly the Copper Ridge Dolomite, Bear Creek contains influent (losing) reaches where water flows from the creek to the groundwater system, and effluent (gaining) reaches where the creek receives groundwater discharge. A review of historical stream flow data performed as part of a dye-tracer study initiated to evaluate these hydrologic interactions indicated that Bear Creek loses flow during extreme low-flow conditions over much of its length in the Bear Creek Regime (Geraghty \& Miller, Inc. 1989c). During normal flow conditions, Bear Creek loses flow in its upper reaches but gains flow in its lower reaches downstream of the Bear Creek Burial Grounds WMA. Results of the dye test also produced conclusive evidence that the surface water lost through influent reaches of Bear Creek between the Oil Landfarm WMA and the Bear Creek Burial Grounds WMA discharges back into the creek from Spring SS-5 (Geraghty \& Miller, Inc. 1989c). 


\subsection{REFERENCES}

Bailey, J.K. 1983. Closure Plan for the Y-12 Centralized Sanitary Landfill I, Department of Energy Y-12 Plant, Oak Ridge, Tennessee. Prepared by Union Carbide Corporation, Nuclear Division (Y-IA 160) (Revised).

Battelle Columbus Division. 1989a. RCRA Facility Investigation Plan, Spoil Area I (D-107), Oak Ridge Y-12 Plant, Oak Ridge, Tennessee. Prepared for Martin Marietta Energy Systems, Inc. (Y/TS-363 R1).

Battelle Columbus Division. 1989b. RCRA Facility Investigation Plan, Rust Spoil Area (D-106), Oak Ridge Y-12 Plant, Oak Ridge, Tennessee. Prepared for Martin Marietta Energy Systems, Inc. (Y/TS-354 R1).

Connell, J.F. and Z.C. Bailey. 1989. Statistical and Simulation Analysis of HydraulicConductivity Data For Bear Creek and Melton Valleys, Oak Ridge Reservation, Tennessee. U.S. Geological Survey Water-Resources Investigations Report 89-4062.

Dreier, R.B., D.K. Solomon, and C.M. Beaudoin. 1987. Fracture Characterization in the Unsaturated Zone of a Shallow Land Burial Facility, in: Flow and Transport through Fractured Rock, American Geophysical Union Monograph 42.

Dreier, R.B., T.O. Early, and H.L. King. 1993. Results and Interpretations of Groundwater Data Obtained from Multiport-Instrumented Coreholes (GW-131 through GW-135), Fiscal Years 1990 and 1991. Martin Marietta Energy Systems, Inc. (Y/TS-803).

Freeze, R.A. and J.A. Cherry. 1979. Groundwater. Prentice-Hall, Inc., Eaglewood Cliffs, New Jersey, (pp. 408-409).

Geraghty \& Miller, Inc. 1987. Hydrogeologic Investigations of the S-3 Ponds Area at the Y-12 Plant. Prepared for Martin Marietta Energy Systems, Inc. (Y/SUB/87-002606C/18).

Geraghty \& Miller, Inc. 1988a. Post Closure Permit Application for the S-3 Ponds WasteManagement Area at the Y-12 Plant. Prepared for Martin Marietta Energy Systems, Inc. (Y/SUB/87-00206C/11R1).

Geraghty \& Miller, Inc. 1988b. Post Closure Permit Application for the Oil Landfarm Waste Management Area at the Y-12 Plant. Prepared for Martin Marietta Energy Systems, Inc. (Y/SUB/87-00206C/12).

Geraghty \& Miller, Inc. 1988c. Post Closure Permit Application for the Burial Grounds Waste Management Area at the Y-12 Plant. Prepared for Martin Marietta Energy Systems, Inc. (Y/SUB/87-00206C/12).

Geraghty \& Miller, Inc. 1989a. RCRA Facility Investigation Plan, SY-200 Yard, Oak Ridge Y-12 Plant, Oak Ridge, Tennessee. Prepared for Martin Marietta Energy Systems, Inc. (Y/TS-598). 
Geraghty \& Miller, Inc. 1989b. Development of Ground-Water Flow Models for the S-3 Waste Management Area, Y-12 Plant, Oak Ridge, Tennessee. Prepared for Martin Marietta Energy Systems, Inc. (Y/SUB/89-00206C/1).

Geraghty \& Miller, Inc. 1989c. Tracer Study of the Hydrologic System of Upper Bear Creek, Y-12 Plant, Oak Ridge, Tennessee. Prepared for Martin Marietta Energy Systems, Inc. (Y/SUB/89-00206C/4).

Geraghty \& Miller, Inc. 1989d. Development of Contaminant Transport Models for Four Constituents at the S-3 Site, Y-12 Plant, Oak Ridge, Tennessee. Prepared for Martin Marietta Energy Systems, Inc. (Y/SUB/89-00206C/3).

Geraghty \& Miller, Inc. 1990. Comprehensive Groundwater Monitoring Plan for the Department of Energy Y-12 Plant. Prepared for Martin Marietta Energy Systems, Inc. (Y/SUB/90$00206 \mathrm{C} / 5$ ).

HSW Environmental Consultants, Inc. 1991. Groundwater Quality Assessment for the Bear Creek Hydrogeologic Regime at the Y-12 Plant 1990: Data Interpretations and Proposed Program Modifications. Prepared for Martin Marietta Energy Systems, Inc. (Y/SUB/91YP507C/1 Part 2).

Kimbrough, C.W., L.W. Long, and L.W. McMahon (eds.). 1987. Environmental Surveillance Procedures Quality Control Program Martin Marietta Energy Systems, Inc. Prepared by Advanced Sciences, Inc. for the Environmental and Safety Activities Organization, Martin Marietta Energy Systems, Inc. (ESH/SUB/87-21706/1 Revision 1).

Jones, S.B., B.K. Harrington, and S.M. Field. 1992. Updated Subsurface Data Base for Bear Creek Valley, Chestnut Ridge, and Parts of Bethel Valley on the U.S. Department of Energy Oak Ridge Reservation. Martin Marietta Energy Systems, Inc. (Y/TS-881).

King, H.L., and C.S. Haase. 1987. Subsurface-Controlled Geological Maps for the Y-12 Plant and Adjacent Areas of Bear Creek Valley. Oak Ridge National Laboratory (ORNL/TM-10112).

Law Engineering Testing Company. 1983. Results of Ground-Water Monitoring Studies. Prepared for Union Carbide Corporation, Nuclear Division (Y/SUB/83-47936/1).

Lozier, W.B., C.A. Spiers, and R. Peerson. 1987. Aquifer Pump Tests with Tracers. Prepared for Martin Marietta Energy Systems, Inc. (ORNL/SUB/86-32136).

MCI Consulting Engineers, Inc. 1983. Closure Plan for Rust Engineering Y-12 Spoil Area, Oak Ridge, Tennessee. Prepared for Union Carbide Corporation, Nuclear Division. Included as Appendix B in RCRA Facility Investigation Plan, Rust Spoil Area (D-106), Oak Ridge Y-12 Plant, Oak Ridge, Tennessee.

Martin Marietta Energy Systems, Inc. 1988a. Revised RCRA Closure Plan for the S-3 Ponds. Prepared by the Y-12 Plant Environmental Management Department; Health, Safety, Environment, and Accountability Division (Y/TS-393). 
Martin Marietta Energy Systems, Inc. 1988b. Revised RCRA Closure Plan for the Oil Landfarm. Prepared by the Y-12 Plant Environmental Management Department; Health, Safety, Environment, and Accountability Division (Y/TS-394).

Martin Marietta Energy Systems, Inc. 1988c. Revised RCRA Closure Plan for the Bear Creek Burial Grounds. Prepared by the Y-12 Plant Environmental Management Department; Health, Safety, Environment, and Accountability Division (Y/TS-395).

Martin Marietta Energy Systems, Inc. 1988d. Revised RCRA Closure Plan for the Oil Retention Ponds. Prepared by the Y-12 Plant Environmental Management Department; Health, Safety, Environment, and Accountability Division (Y/TS-392).

Mercier, T.M. and R.R. Turner. 1990. Data Verification and Evaluation Techniques for Groundwater Monitoring Programs. Prepared by H\&R Technical Associates, Inc. for Martin Marietta Energy Systems, Inc. (Y/SUB/90-K523C/2).

Moore, G.K. 1989. Groundwater Parameters and Flow System Near Oak Ridge National Laboratory. Oak Ridge National Laboratory (ORNL/TM-11368).

Poreda, L.N., T.E. Cerling, and D.K. Solomon. 1988. Tritium and Helium Isotopes as Hydrologic Tracers in a Shallow Unconfined Aquifer. Journal of Hydrology (103:1-9).

Shevenell, L.A., R.B. Dreier, and W.K. Jago. 1992. 1992 Summary of Fiscal Years 1991 and 1992 Construction, Hydrologic, and Geologic Data Obtained from the Maynardville Limestone Exit-Pathway Monitoring Program. Martin Marietta Energy Systems, Inc. (Y/TS-814).

Sledz, J.J., and D.D. Huff. 1981. Computer Model for Determining Fractrre Porosity and Permeability in the Conasauga Group. Oak Ridge National Laboratory (ORNL/TM-7695).

Solomon, D.K., G.K. Moore, L.E. Toran, R.B. Dreier, and W.M. McMaster. 1992. Status Report - A Hydrologic Framework for the Oak Ridge Reservation. Oak Ridge National Laboratory (ORNL/TM-12026).

Tennessee Department of Health and Environment. 1986. Letter from T. Tiesler, Director of the Division of Solid Waste-Management, to J.L. Foutch, U.S. Department of Energy Oak Ridge Operations, January 13, 1986.

Toran, L.E., D.K. Solomon, W.M. McMaster, and C.M. Morrissey. 1991. Matrix Diffusion as a Mechanism to Explain Recent Tritium and old ${ }^{14} \mathrm{C}$ in Groundwater from Fractured Sedimentary Rock. EOS Transactions 72(17):111.

U.S. Department of Energy. 1992. Oak Ridge Reservation Site Management Plan for the Environmental Restoration Program. U.S. Department of Energy Oak Ridge Field Office (DOE/OR-100/R2). 
U.S. Department of Energy. 1993a. Remedial Investigation Work Plan for Bear Creek Operable Unit 1 (S-3 Ponds, Boneyard/Burnyard, Oil Landfarm, Sanitary Landfill I, and the Bear Creek Burial Grounds Including Oil Retention Ponds 1 and 2) at the Oak Ridge Y-12 Plant, Oak Ridge, Tennessee. (DOE/OR/01-1161\&D1).

U.S. Department of Energy. 1993b. Remedial Investigation Work Plan for Bear Creek Operable Unit 1 (Rust Spoil Area, SY-200 Yard, Spoil Area I) at the Oak Ridge Y-12 Plant, Oak Ridge, Tennessee. (DOE/OR/01-1060\&D3).

U.S. Department of Energy. 1993c. Remedial Investigation Work Plan for Bear Creek Operable Unit 1 (Shallow Groundwater in Bear Creek Valley) at the Oak Ridge Y-12 Plant, Oak Ridge, Tennessee. (DOE/OR/01-1161\&D1).

U.S. Environmental Protection Agency. 1988a. Letter from J.H. Scarbrough, Chief of the Region IV RCRA Branch, and T. Tiesler, Director of the Division of Solid WasteManagement, Tennessee Department of Health and Environment, to R.J. Spence, Y-12 Site Manager, U.S. Department of Energy - Oak Ridge Operations, November 18, 1988.

U.S. Environmental Protection Agency. 1988b. Laboratory Data Validation Functional Guidelines for Evaluating Organics Analyses. U.S. EPA, Office of Solid Waste.

Welch, S.H., C.S. Haase, C.W. Kimbrough, and T.M. Mercier. 1987. Solid Waste Management Unit Information for Y-12 Plant RCRA 3004(u) Facility Assessment, Volumes I, II, and III. Martin Marietta Energy Systems, Inc. (Y/TS-273). 
APPENDIX A

FIGURES 


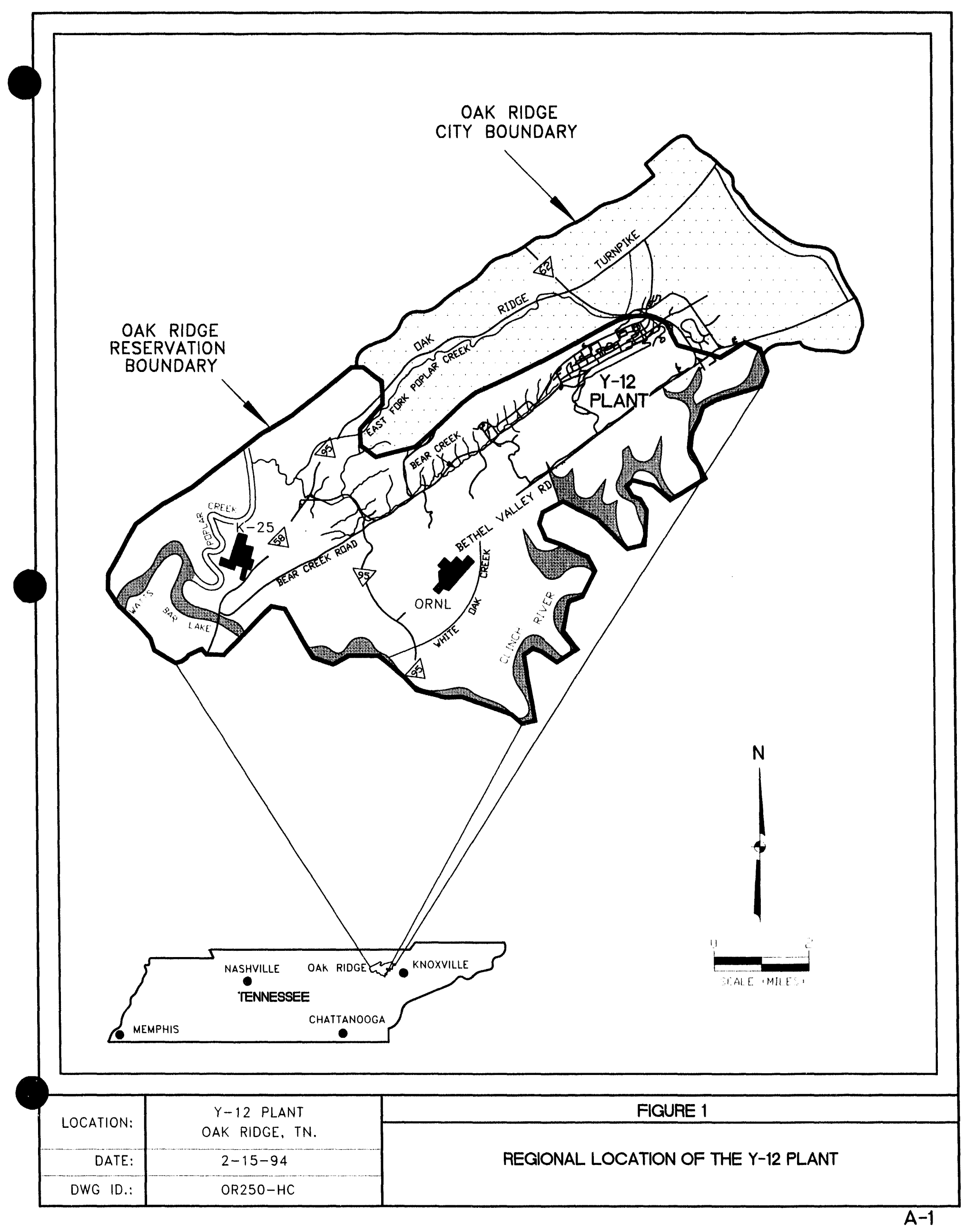




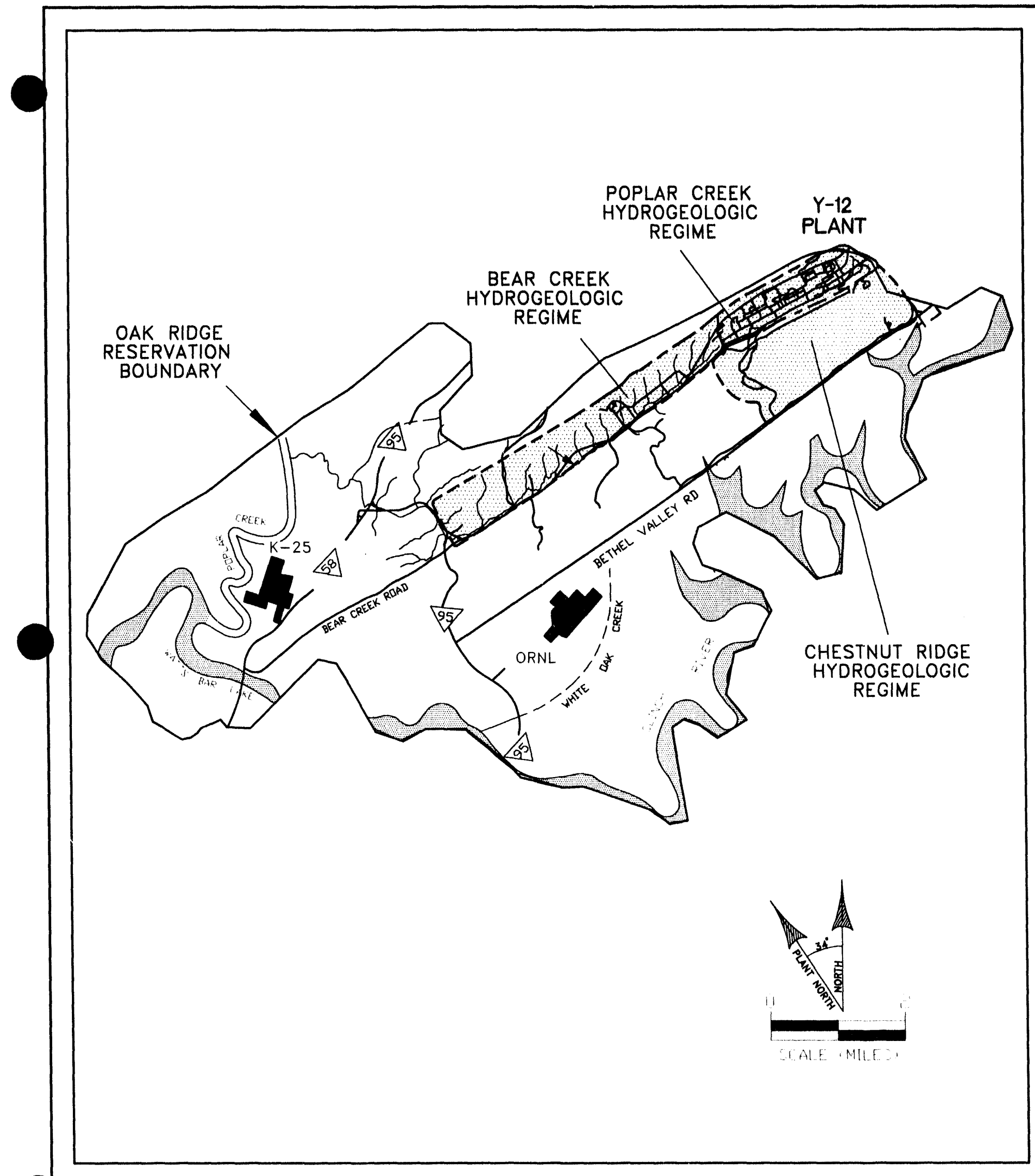

\begin{tabular}{|r|c|c|}
\hline LOCATION: & $\begin{array}{c}\text { Y-12 PLANT } \\
\text { OAK RIDGE, TN. }\end{array}$ & FIGURE 2 \\
\cline { 2 - 3 } DATE: & $2-19-94$ & $\begin{array}{c}\text { HYDROGEOLOGIC REGMES } \\
\text { AT THE Y-12 PLANT }\end{array}$ \\
\hline DWG ID.: & OR321-HC & P \\
\hline
\end{tabular}




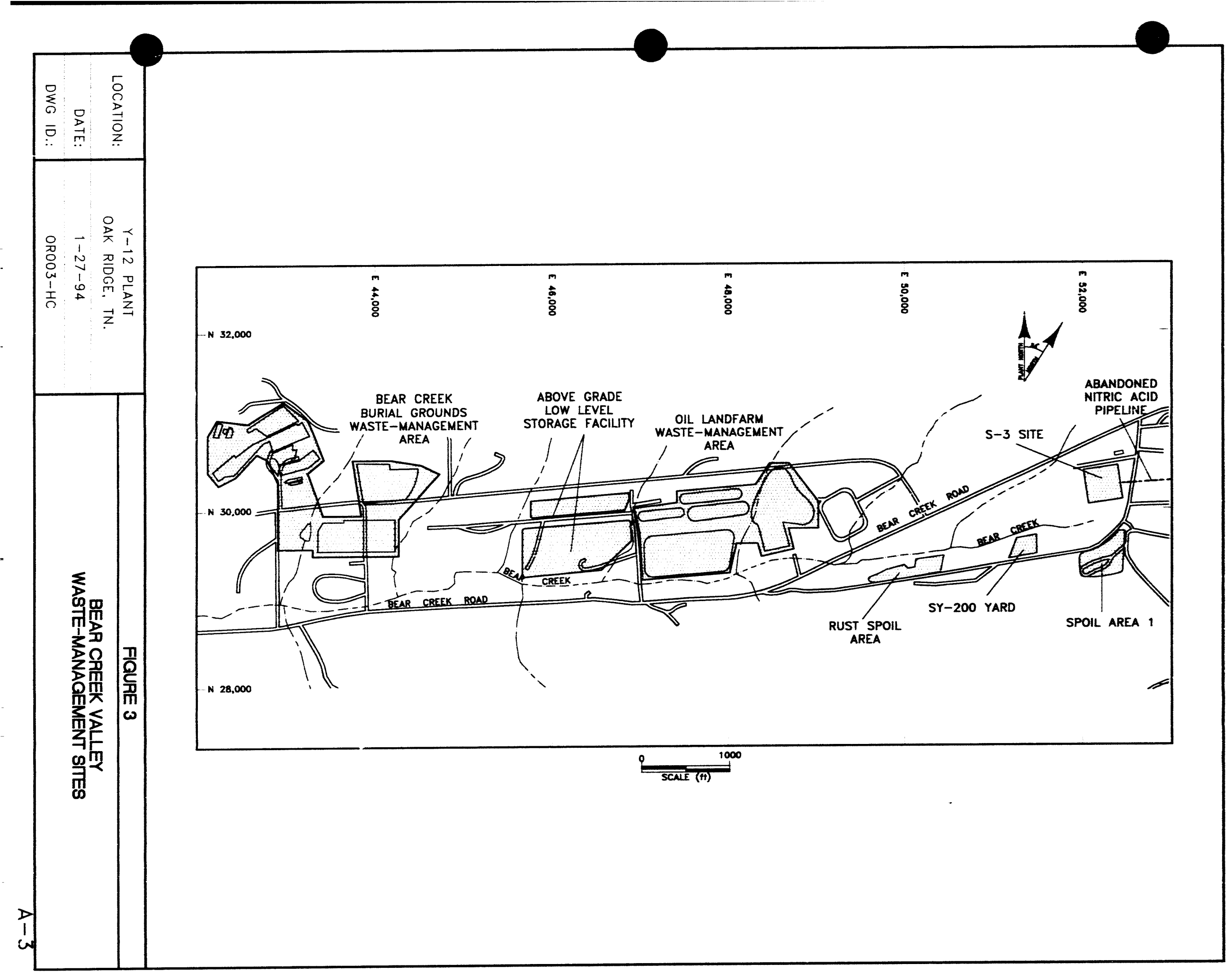




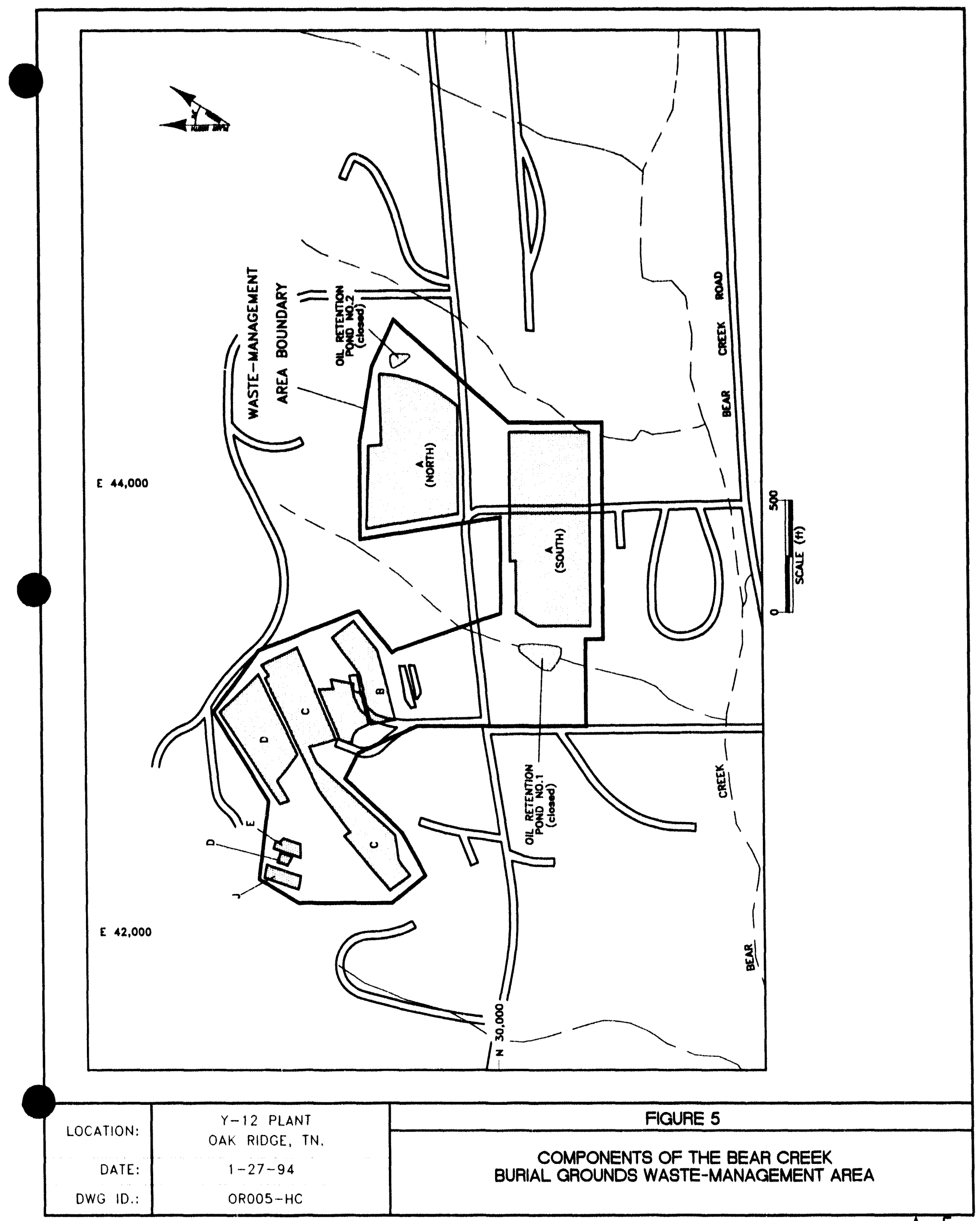




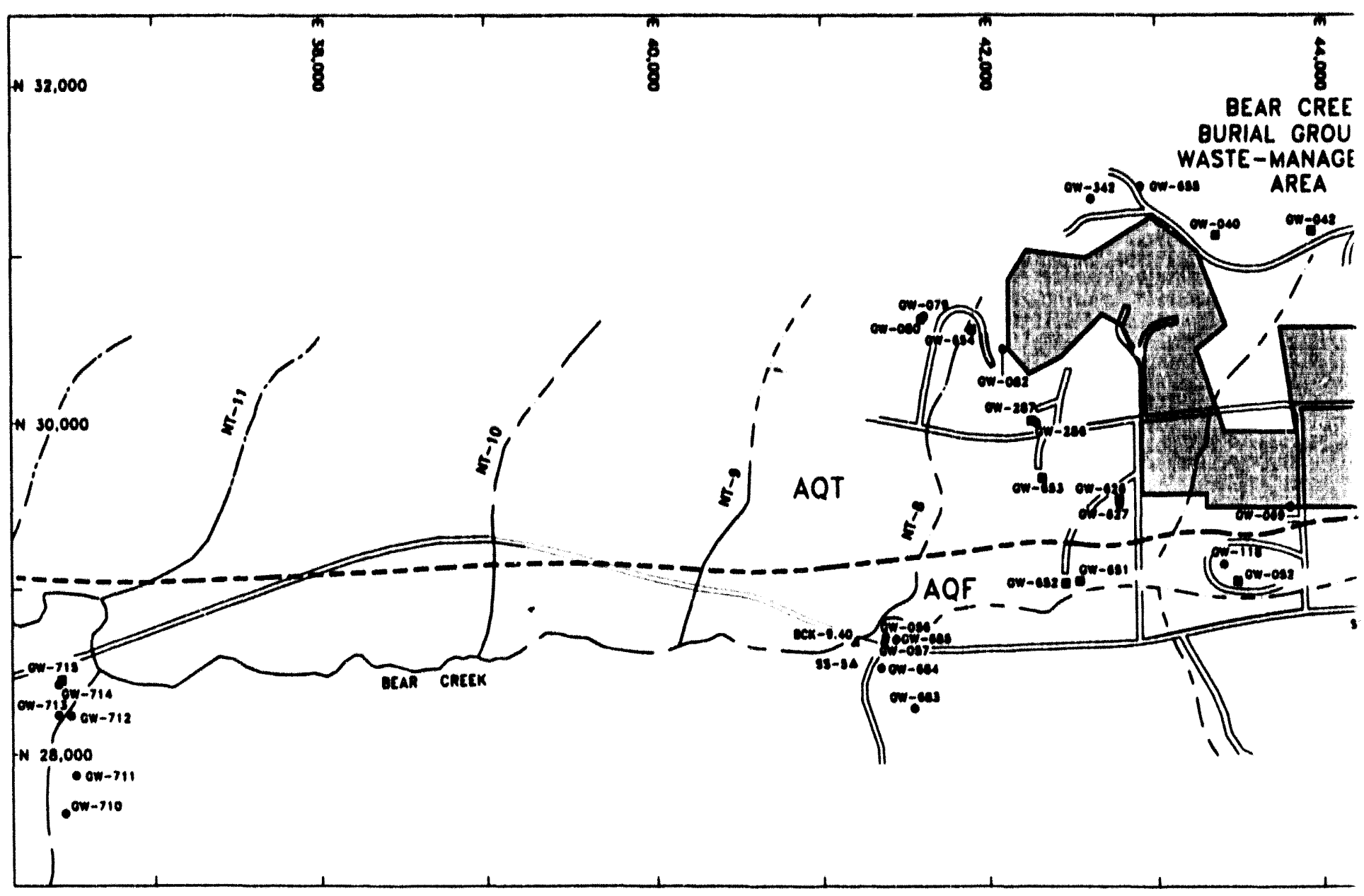

\section{EXPLANATION}

GW - D - Water Table Moniloring Well

GW - - Bedrock Monitoring Woll

SS -

BCK - $\triangle$ - Spring (SS) or Surface-Water (BCK or NT) Sampling Station

NT -

- - - - Surface Drainage Foalure

AQT - Oak Ridge Reservalion Aquitards

- _- - - Approximate Nollchucky Shale\Maynordville Limeslone Contact

AQF - Knox Aquiler 

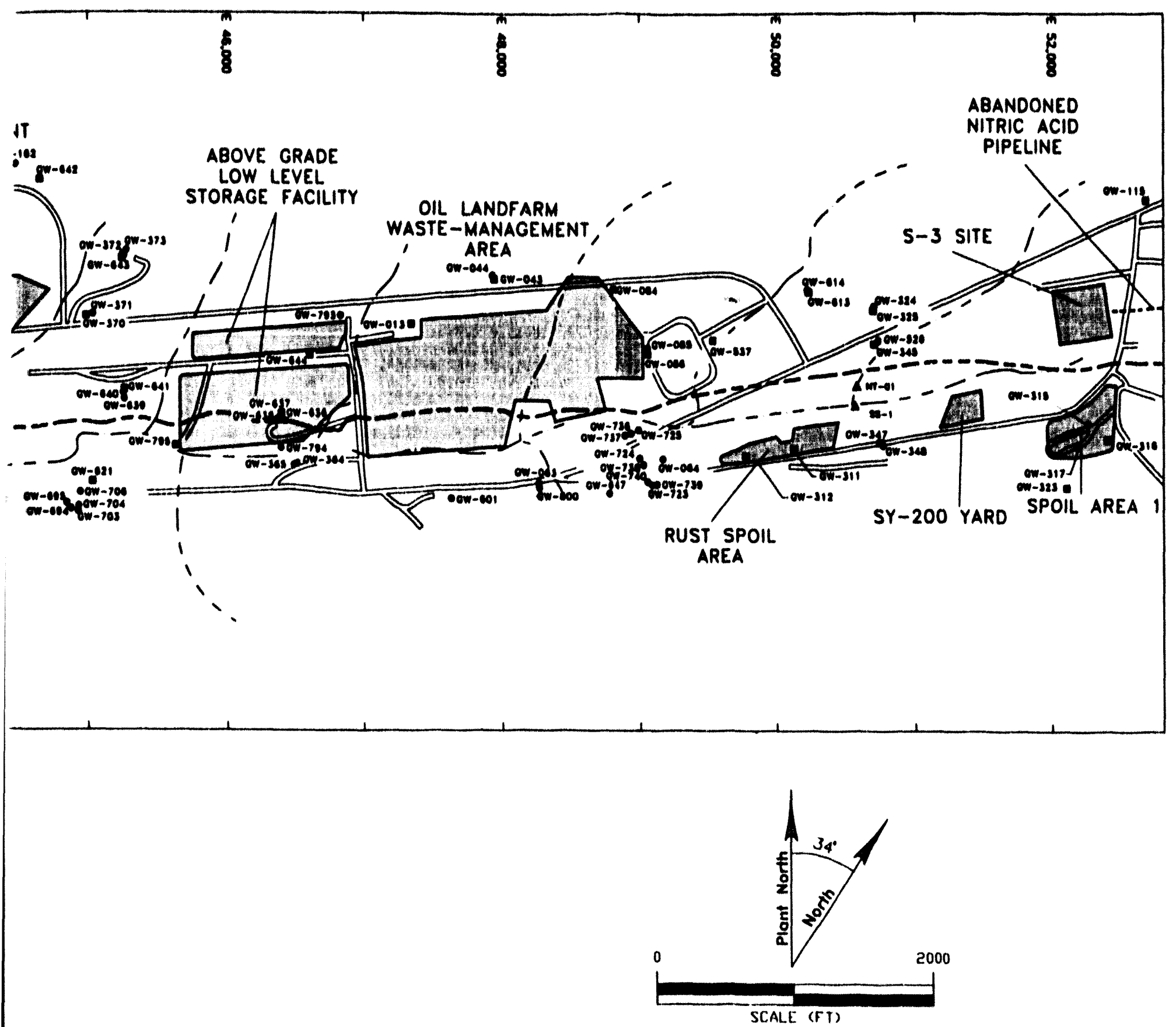

\begin{tabular}{|c|c|c|}
\hline \multirow{2}{*}{ LOCATION: } & $\begin{array}{c}\text { Y-12 PLANT } \\
\text { OAK RIDGE, TN. }\end{array}$ & FQURE 6 \\
\cline { 2 - 3 } DATE: & $2-18-94$ & LOCATION OF MONTORING WELLS \\
\hline DWG 10.: & OR $305-H C$ & SAMPLED DURING 1993
\end{tabular}




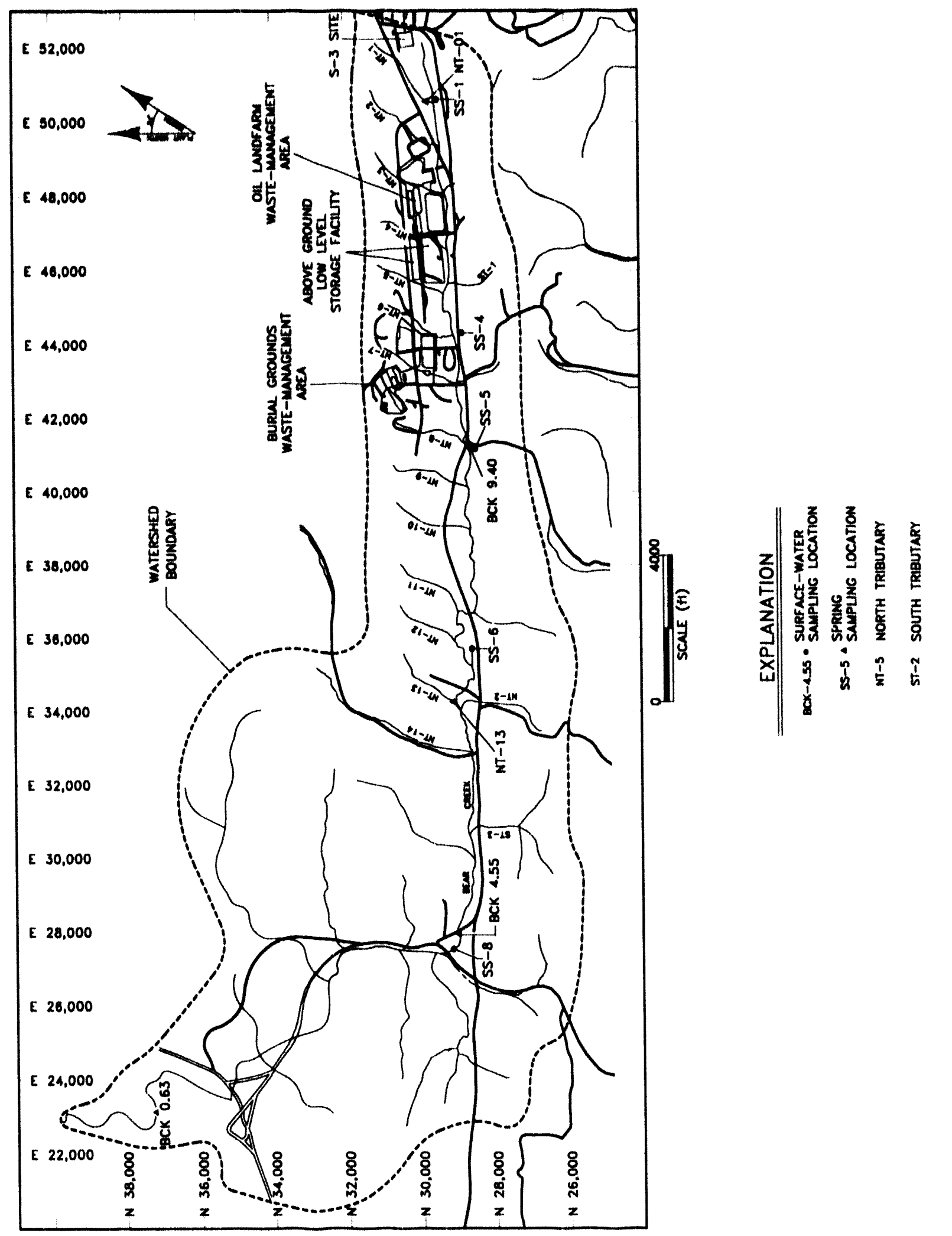

LOCATION:

DATE:

DWG ID.:
Y-12 PLANT

OAK RIDGE, TN.

1-27-94

ORO10-HC
FIQUPE 7

LOCATION OF SPFINGS AND SURFACE WATER STATIONS SAMPLED DURING 1993 


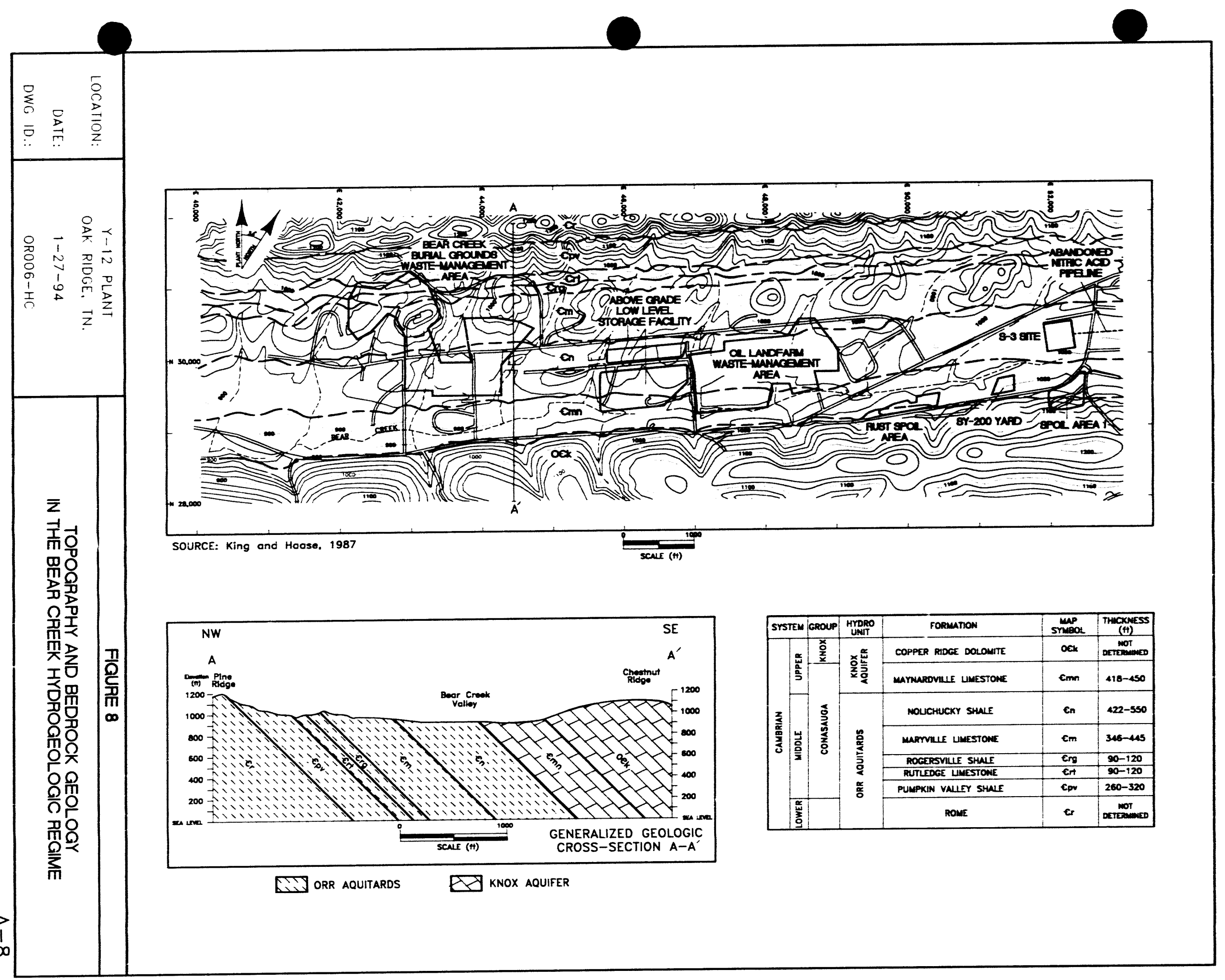




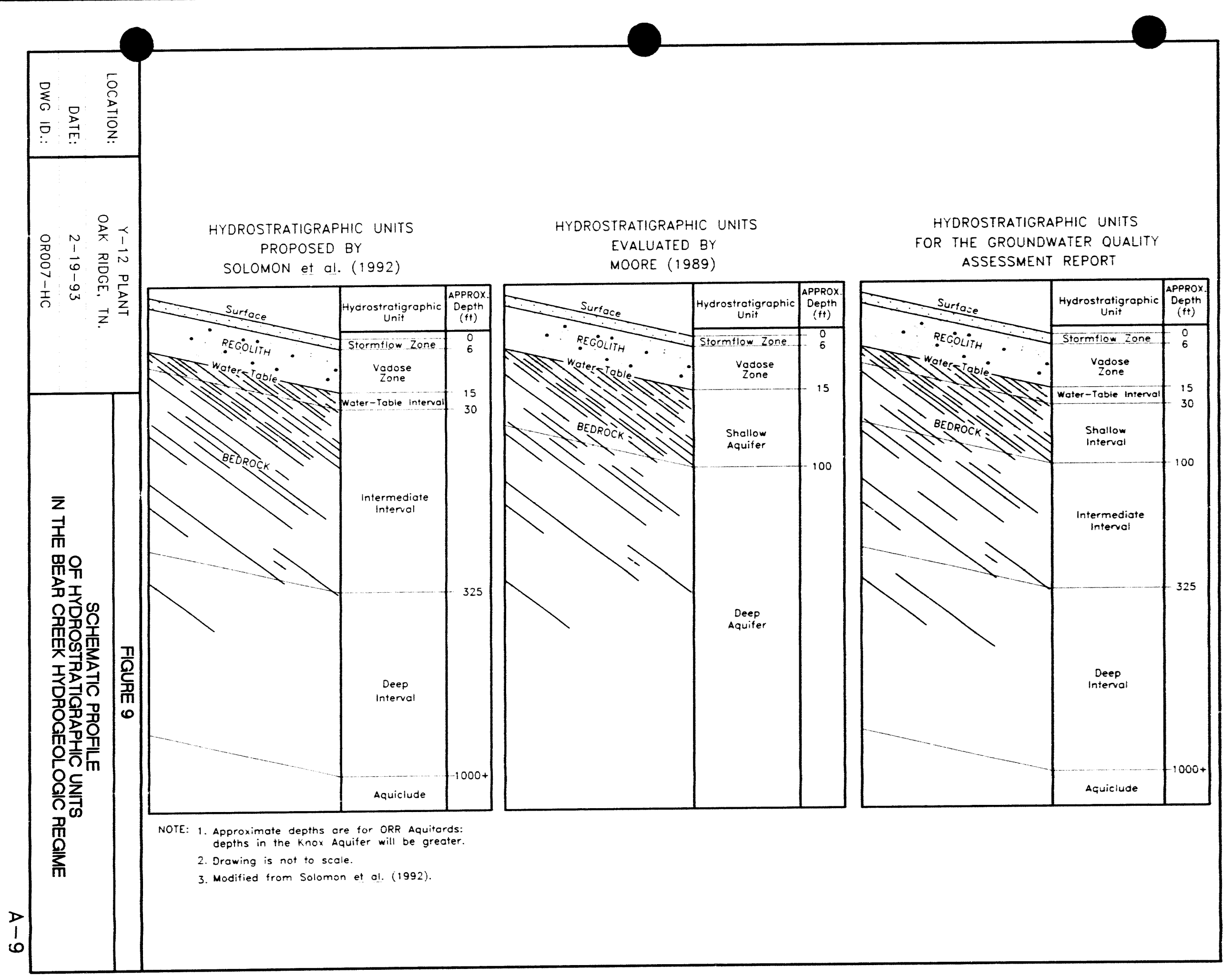



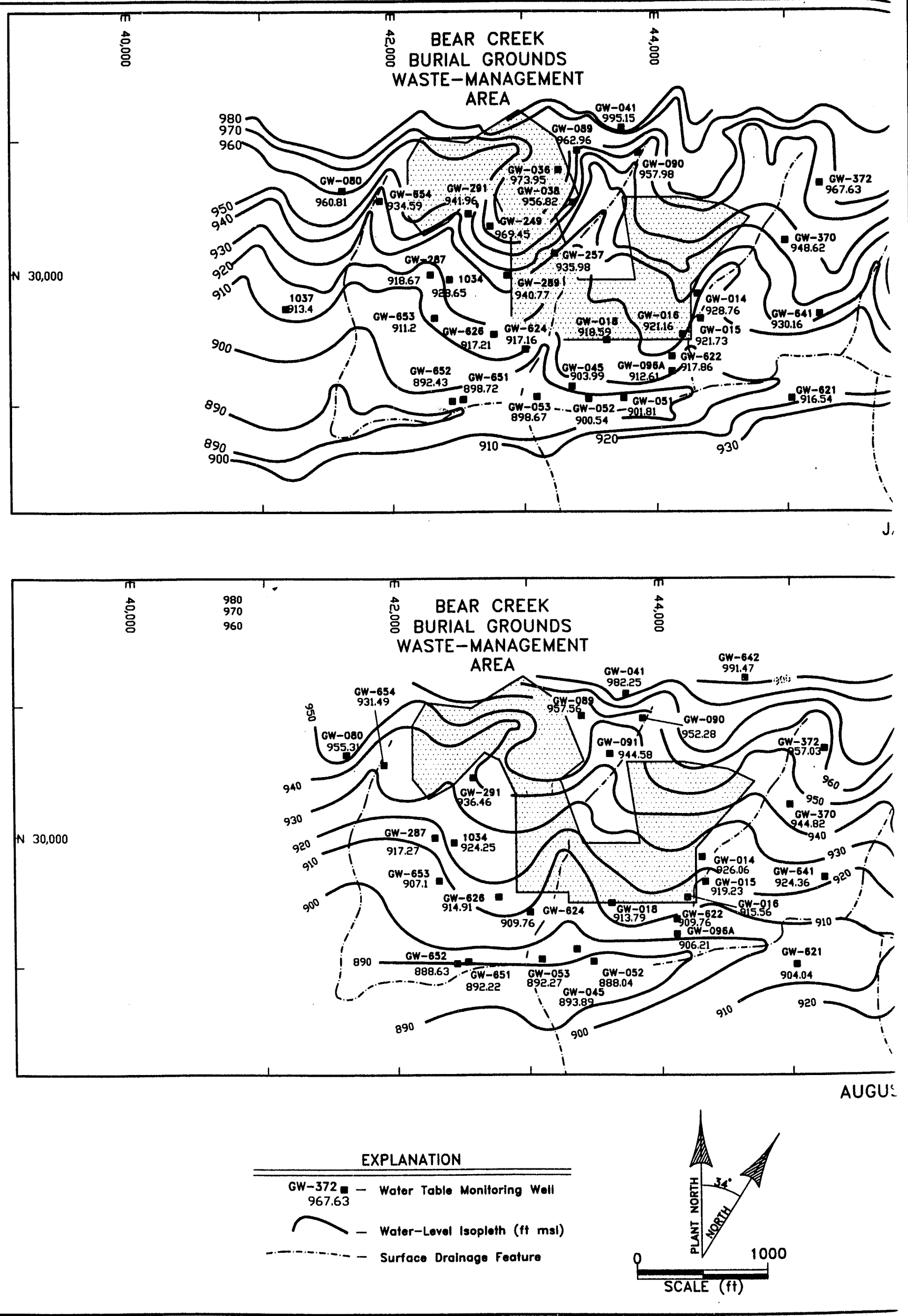


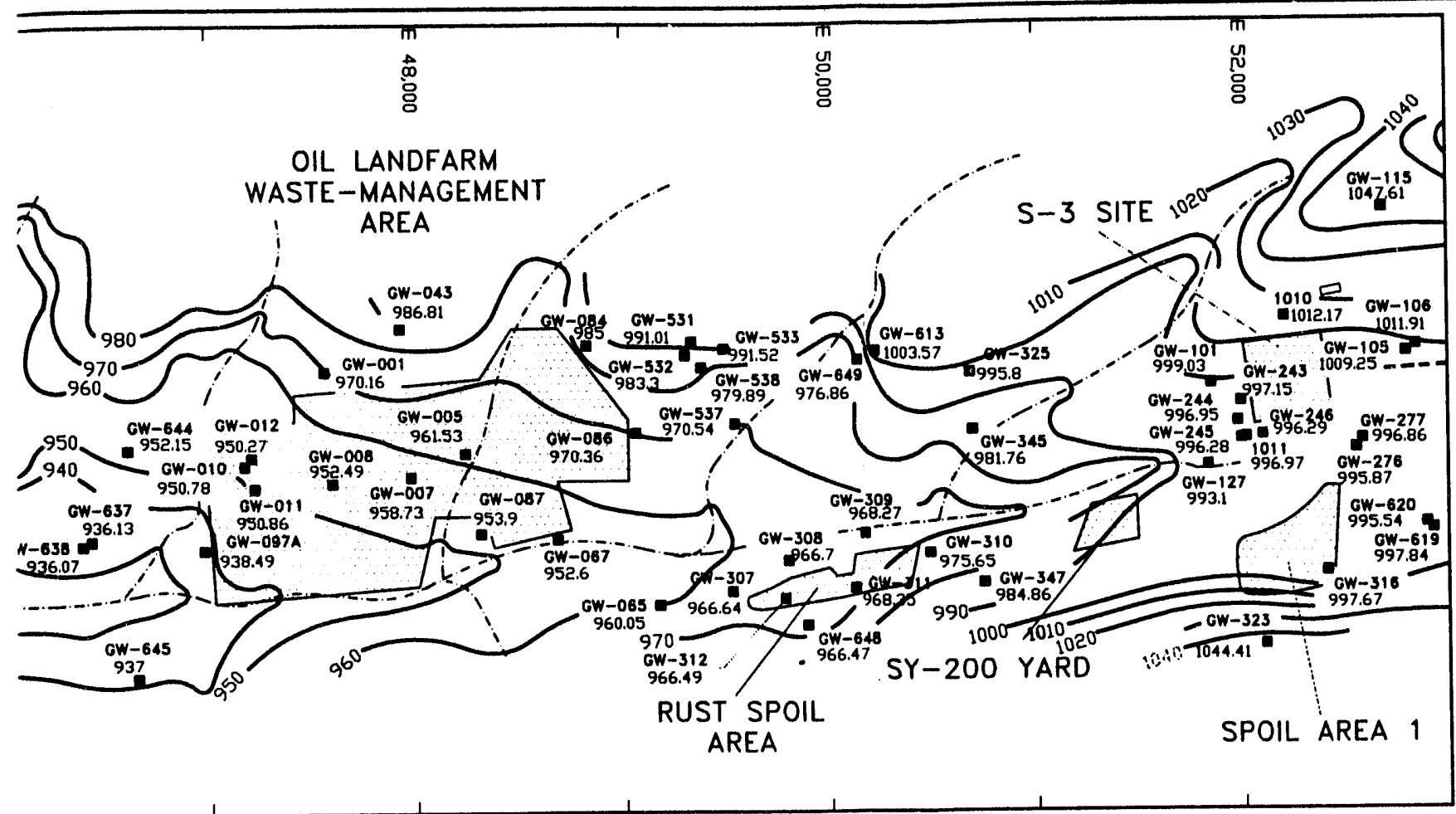

ARY $10-14,1993$
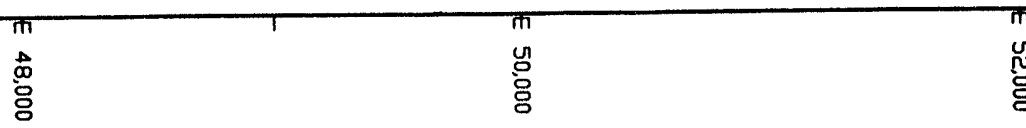

OIL LANDFARM

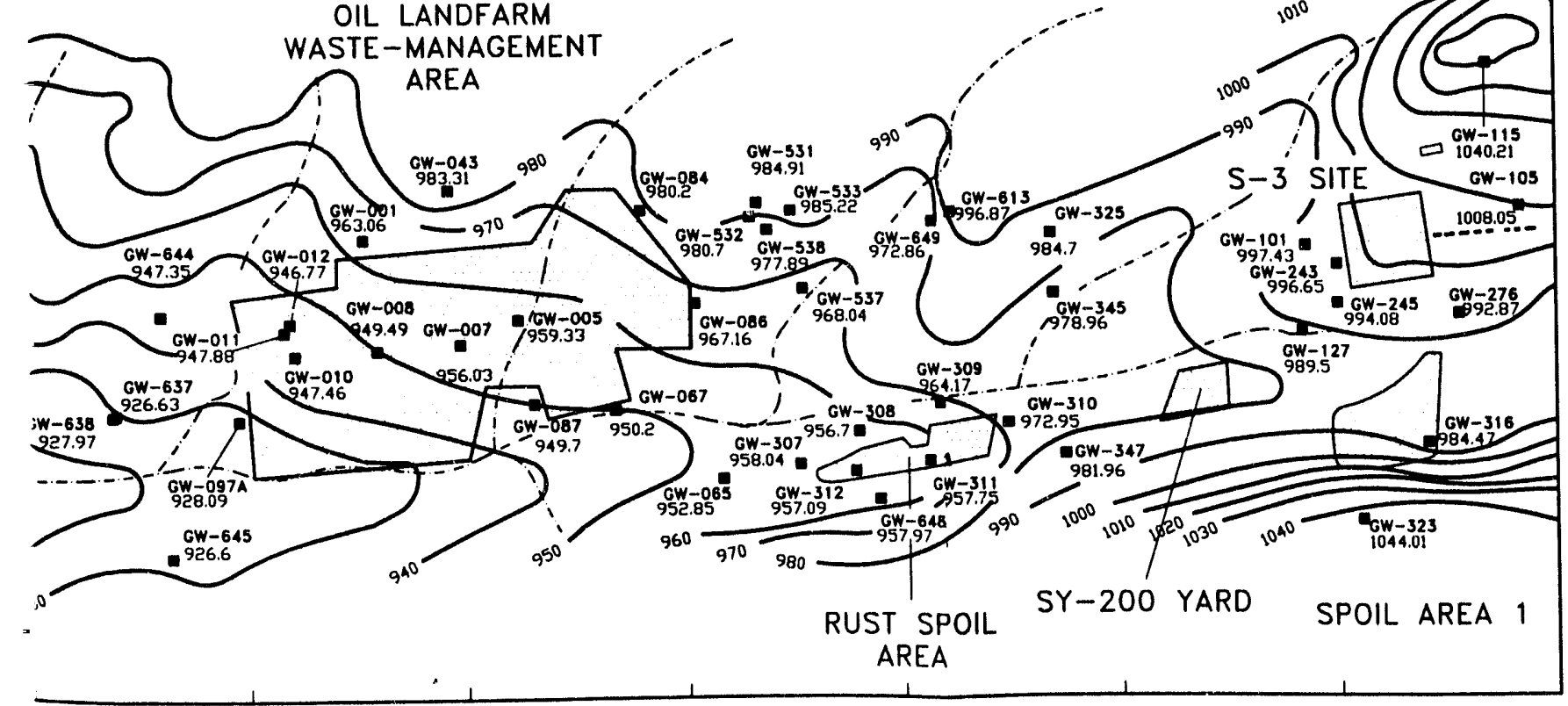

31 - SEPTEMBER 3, 1993

\begin{tabular}{|r|c|}
\hline LOCATION: & $\begin{array}{c}\text { Y-12 PLANT } \\
\text { OAK RIDGE, TN. }\end{array}$ \\
\hline DATE: & $2-18-94$ \\
\hline DWG ID.: & OR312-HC \\
\hline
\end{tabular}

FGURE 10

GROUNDWATER ELEVATIONS

IN THE WATER-TABLE INTERVAL 

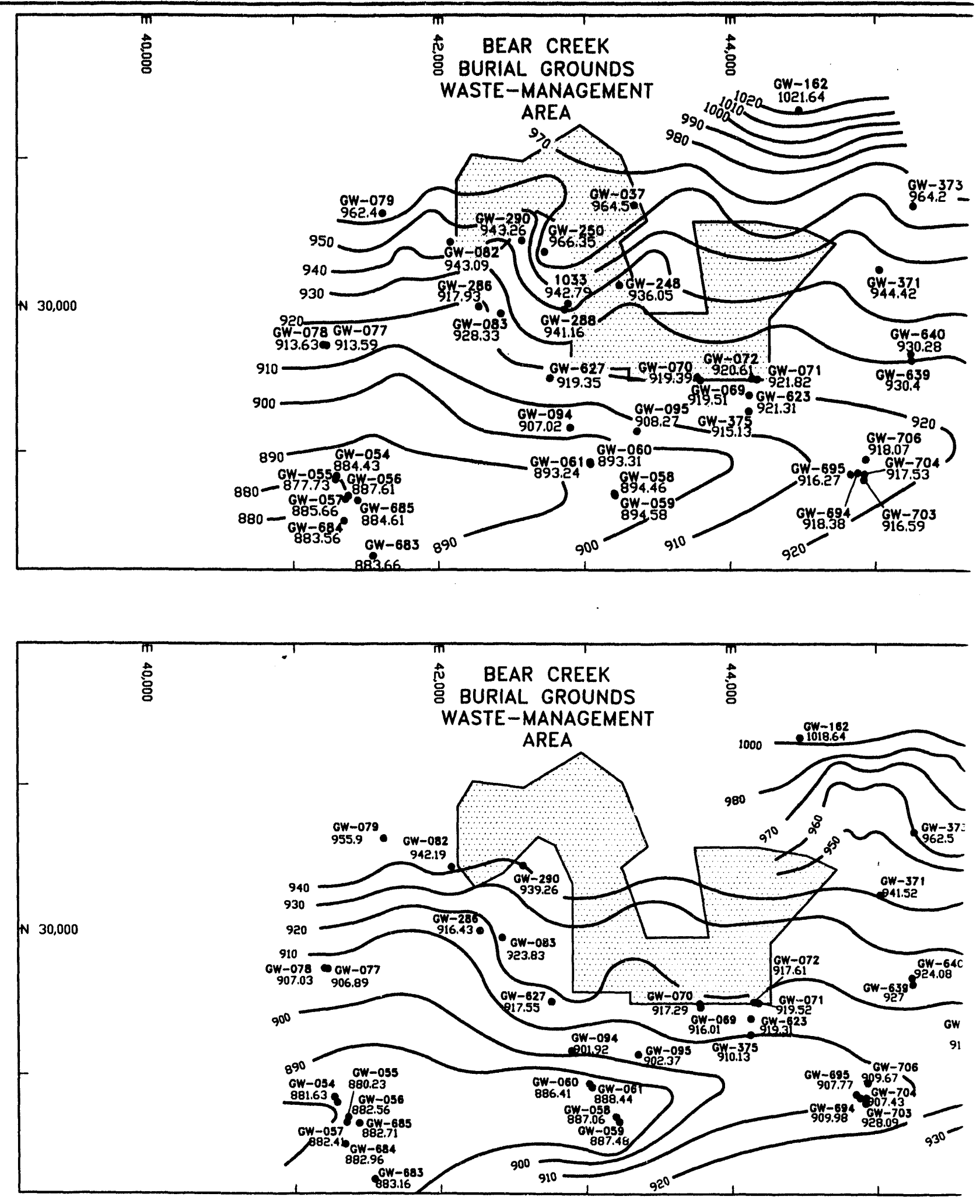

AU
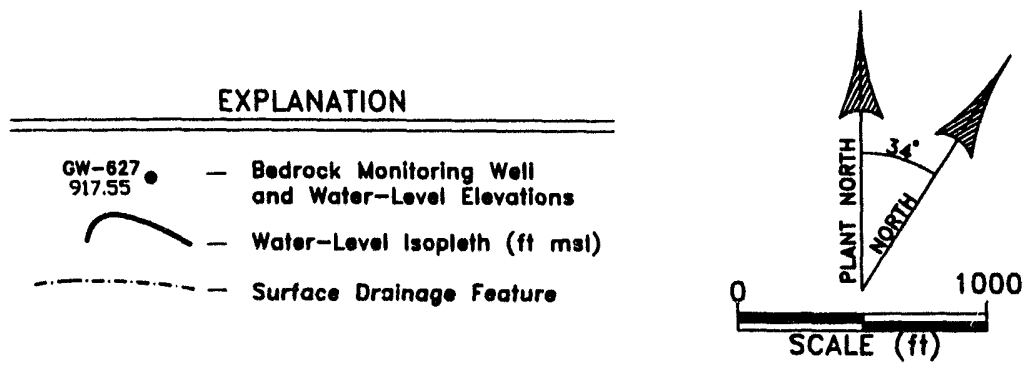


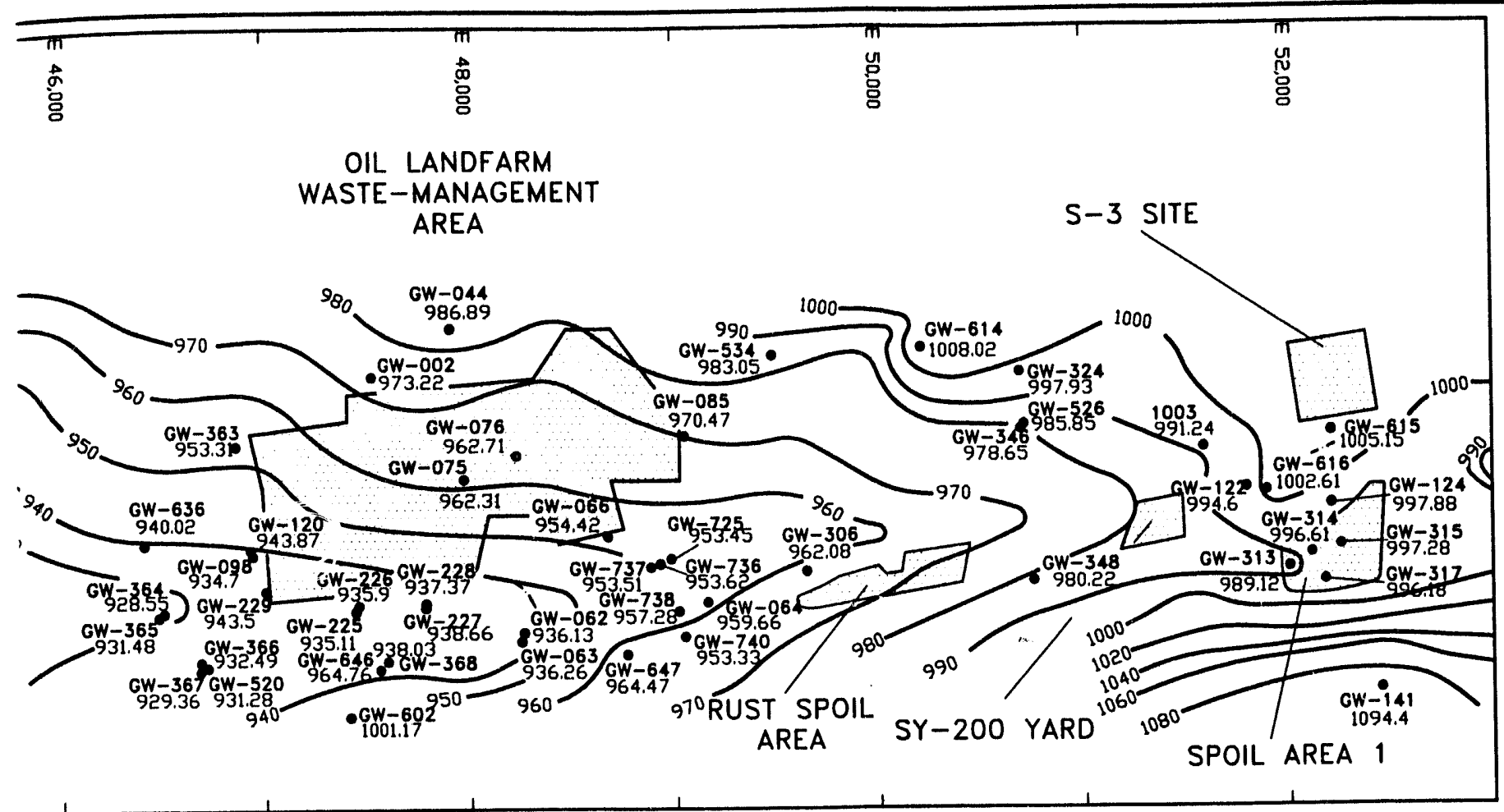

IANUARY $10-14,1993$

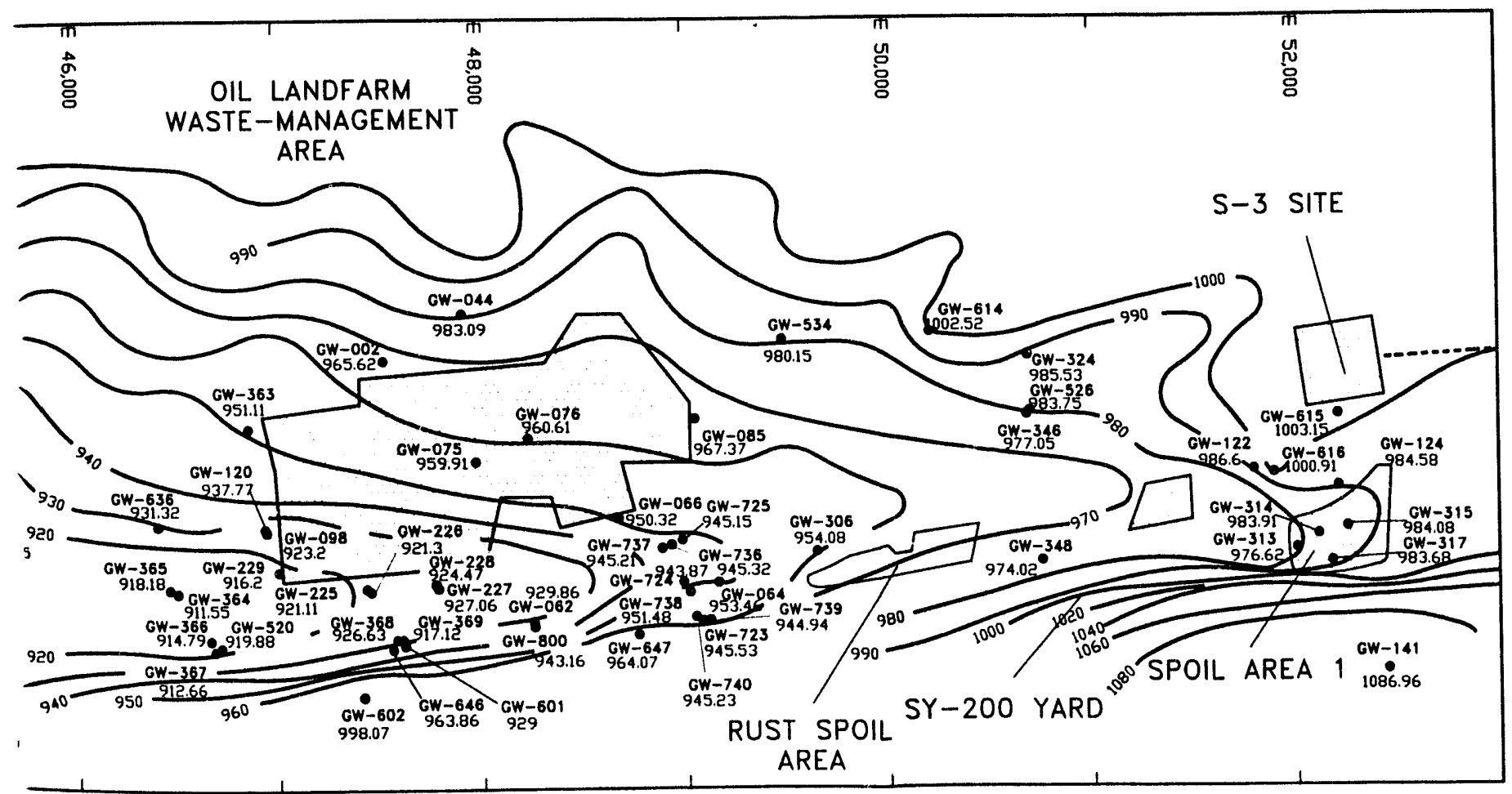

PT 31 - SEPTEMBER 3, 1993

\begin{tabular}{|r|c|}
\hline LOCATION: & $\begin{array}{c}\text { Y-12 PLANT } \\
\text { OAK RIDGE, TN. }\end{array}$ \\
\hline DATE: & $2-18-94$ \\
\hline DWG ID.: & OR313-HC \\
\hline
\end{tabular}

FGURE $\boldsymbol{n}$

GROUNDWATER ELEVATIONS IN THE SHALLOW AND INTERMEDIATE BEDROCK INTERVALS 


\section{APPENDIX B}

\section{TABLES}


Table 1. Operable Units Established for the Bear Creek Hydrogeologic Regime

Site Name

Regulatory Classification

Historical $^{1} \quad$ Current $^{2}$

S-3 Site

TSD Unit

BC OU 01

Oil Landfarm WMA
Oil Landfarm
TSD Unit
$\mathrm{BC}$ OU 01

Burnyard, Boneyard, and Hazardous Chemical

Storage Area

SWMU

BC OU 01

Sanitary Landfill I

SWMU

BC OU 01

Bear Creek Burial Grounds WMA

Burial Grounds A (North and South)

TSD Unit

BC OU 01

Burial Grounds C

TSD Unit

BC OU 01

Burial Grounds B, D, E, and J

SWMUs

BC OU 01

Oil Retention Pond No. 1

SWMU

BC OU 01

Oil Retention Pond No. 2

SWMU

BC OU 01

Spoil Area I

SWMU

BC OU 02

SY-200 Yard

SWMU

BC OU 02

Rust Spoil Area

SWMU

BC OU 02

Bear Creek Flood Plain Soils

N/A

BC OU 03

Bear Creek Groundwater, Surface Water, and

Creek Sediments

N/A

BC OU 04

Above Grade Low Level Storage Facility

N/A

N/A

\section{Notes:}

1 Regulatory status before 1992 Federal Facility Agreement.

TSD Unit - RCRA regulated land-based treatment, storage, or disposal unit.

SWMU - RCRA regulated solid waste management unit.

N/A - Not Applicable (not previously regulated as a separate unit or not currently regulated).

2 Modified from: Oak Ridge Reservation Site Management Plan for the Environmental Restoration Program (U.S. Department of Energy 1992).

BC OU 01 - Bear Creek Operable Unit 01 (Source Control OU)

BC OU 02 - Bear Creek Operable Unit 02 (Source Control OU)

BC OU 03 - Bear Creek Operable Unit 03 (Source Control OU)

BC OU 04 - Bear Creek Operable Unit 04 (Integrator OU) 
Table 2. Monitoring Wells, Springs, and Surface Water Stations Sampled During 1993

\begin{tabular}{|c|c|c|c|c|}
\hline $\begin{array}{c}\text { Monitoring } \\
\text { Program }\end{array}$ & $\begin{array}{c}\text { Sampling } \\
\text { Point }^{+}\end{array}$ & Location ${ }^{2}$ & $\begin{array}{l}\text { Quarters } \\
\text { Sampled }\end{array}$ & Notes \\
\hline \multicolumn{5}{|c|}{ RCRA Compliance Monitoring } \\
\hline & GW-040 & BG & 4 & Background/Upgradient Well \\
\hline & GW-042 & BG & 1 & Background/Upgradient Well \\
\hline & GW-043 & OLF & 4 & Background/Upgradient Well \\
\hline & GW-044 & OLF & 4 & Background/Upgradient Well \\
\hline & GW-079 & $\mathbf{B G}$ & 1 & Background/Upgradient Well \\
\hline & GW-080 & BG & 1 & Background/Upgradient Well \\
\hline & GW-084 & OLF & 4 & Background/Upgradient Well \\
\hline & GW-115 & S3 & 4 & Background/Upgradient Well \\
\hline & GW-162 & BG & 1 & Background/Upgradient Well \\
\hline & GW-324 & S3 & 4 & Background/Upgradient Well \\
\hline & GW-325 & S3 & 4 & Background/Upgradient Well \\
\hline & GW-342 & BG & 1 & Background/Upgradient Well \\
\hline & GW-372 & BG & 4 & Background/Upgradient Wel \\
\hline & GW-373 & BG & 4 & Background/Upgradient Well \\
\hline & GW-613 & S3 & 1 & Background/Upgradient Well \\
\hline & GW-614 & S3 & 1 & Background/Upgradient Well \\
\hline & GW-642 & BG & 4 & \\
\hline \multicolumn{5}{|c|}{ RCPA Assessment Monitoring } \\
\hline & GW-056 & $\mathrm{BG}$ & 4 & \\
\hline & GW-057 & $\mathrm{CMN}$ & 4 & \\
\hline & GW-063 & OLF & 1 & P \& A May 93 \\
\hline & GW-064 & OLF & 4 & \\
\hline & GW-311 & RS & 4 & \\
\hline & GW-312 & RS & 4 & \\
\hline & GW-317 & SPI & 4 & \\
\hline & GW-323 & SPI & 4 & \\
\hline
\end{tabular}

B-2 
Table 2 (cont'd)

\begin{tabular}{|c|c|c|c|c|}
\hline $\begin{array}{c}\text { Monitoring } \\
\text { Program }\end{array}$ & $\begin{array}{c}\text { Sampling } \\
\text { Point }^{\prime}\end{array}$ & Location ${ }^{2}$ & $\begin{array}{l}\text { Quarters } \\
\text { Sampled" }\end{array}$ & Notes \\
\hline \multicolumn{5}{|c|}{ Exit-Pathway Monitoring } \\
\hline & GW-710 & CMN & 4 & Compliance Monitoring Well \\
\hline & GW-711 & CMN & 4 & Compliance Monitoring Well \\
\hline & GW-712 & CMN & 4 & Compliance Monitoring Well \\
\hline & GW-713 & CMN & 4 & Compliance Monitoring Well \\
\hline & GW-714 & $\mathrm{CMN}$ & 4 & Compliance Monitoring Well \\
\hline & GW-715 & $\mathrm{CMN}$ & 4 & Compliance Monitoring Well \\
\hline & GW-683 & $\mathrm{CMN}$ & 4 & \\
\hline & $G W-684$ & $\mathrm{CMN}$ & 4 & \\
\hline & GW-685 & CMN & 4 & \\
\hline & GW-694 & CMN & 4 & \\
\hline & GW-695 & $\mathrm{CMN}$ & 4 & \\
\hline & GW-703 & $\mathrm{CMN}$ & 4 & \\
\hline & GW-704 & $\mathrm{CMN}$ & 4 & \\
\hline & GW-706 & $\mathrm{CMN}$ & 4 & \\
\hline & GW-723 & $\mathrm{CMN}$ & 4 & \\
\hline & GW-724 & $\mathrm{CMN}$ & 4 & \\
\hline & GW-725 & $\mathrm{CMN}$ & 4 & \\
\hline & GW-736 & $\mathrm{CMN}$ & 4 & \\
\hline & GW-737 & $\mathrm{CMN}$ & 4 & \\
\hline & GW-738 & $\mathrm{CMN}$ & 4 & \\
\hline & GW-739 & $\mathrm{CMN}$ & 4 & \\
\hline & GW-740 & $\mathrm{CMN}$ & 4 & \\
\hline & BCK 0.63 & $\mathrm{BC}$ & 4 & \\
\hline & SS-8 & $\mathrm{BC}$ & 4 & Spring \\
\hline & BCK 4.55 & $\mathrm{BC}$ & 4 & \\
\hline & NT-13 & $\mathrm{BCT}$ & 4 & Background Location \\
\hline & SS-6 & $\mathrm{BC}$ & 4 & Spring \\
\hline
\end{tabular}


Table 2 (cont'd)

\begin{tabular}{|c|c|c|c|c|}
\hline $\begin{array}{l}\text { Monitoring } \\
\text { Program }\end{array}$ & $\begin{array}{l}\text { Sampling } \\
\text { Point' }\end{array}$ & Location ${ }^{2}$ & $\begin{array}{l}\text { Quarten } \\
\text { Sampled" }\end{array}$ & Notes \\
\hline \multicolumn{5}{|c|}{ Exit-Pathway Monitoring (cont'd) } \\
\hline & BCK 9.40 & BC & 4 & \\
\hline & SS-5 & BC & 4 & Spring \\
\hline & SS-4 & BC & 4 & Spring \\
\hline & SS-1 & BC & 4 & Spring \\
\hline & NT-01 & BCT & 4 & Background Location \\
\hline \multicolumn{5}{|c|}{ Surveillance and Maintenance Monitoring } \\
\hline & GW-013 & OLF & 4 & \\
\hline & GW-052 & BG & 2 & Dry 3rd and 4th Quarter \\
\hline & GW-069 & BG & 4 & \\
\hline & GW-082 & BG & 4 & \\
\hline & GW-085 & OLF & 4 & \\
\hline & GW-086 & OLF & 4 & \\
\hline & GW-118 & BG & 4 & \\
\hline & GW-286 & BG & 4 & \\
\hline & GW-287 & BG & 4 & \\
\hline & GW-315 & SPI & 4 & \\
\hline & GW-316 & SPI & 4 & \\
\hline & GW-345 & S3 & 4 & \\
\hline & GW-347 & S3 & 4 & \\
\hline & GW-348 & S3 & 4 & \\
\hline & GW-364 & OLF & 4 & \\
\hline & GW-365 & OLF & 4 & \\
\hline & GW-370 & BG & 4 & \\
\hline & GW-371 & BG & 4 & \\
\hline & GW-526 & S3 & 4 & \\
\hline & GW-537 & OLF & 4 & \\
\hline
\end{tabular}


Table 2 (cont'd)

\begin{tabular}{|c|c|c|c|c|}
\hline $\begin{array}{c}\text { Monitoring } \\
\text { Program }\end{array}$ & $\begin{array}{c}\text { Sampling } \\
\text { Point' }\end{array}$ & Location ${ }^{2}$ & $\begin{array}{l}\text { Quarter } \\
\text { Sampled }\end{array}$ & Notes \\
\hline \multicolumn{5}{|c|}{ Survelliance and Malntenence Monitoring (cont'd) } \\
\hline & GW-601 & OLF & 4 & \\
\hline & GW-621 & BG & 4 & \\
\hline & GW.626 & BG & 4 & \\
\hline & GW-627 & BG & 4 & \\
\hline & GW.636 & OLF & 4 & \\
\hline & GW-637 & OLF & 4 & \\
\hline & GW-638 & OLF & 4 & \\
\hline & GW-639 & BG & 4 & \\
\hline & GW-640 & BG & 4 & \\
\hline & GW-641 & BG & 4 & \\
\hline & GW-643 & BG & 4 & \\
\hline & GW-644 & OLF & 4 & \\
\hline & GW-647 & OLF & 4 & \\
\hline & GW-651 & BG & 4 & \\
\hline & GW-652 & BG & 4 & \\
\hline & GW-653 & BG & 4 & \\
\hline & GW-654 & BG & 4 & \\
\hline & GW-655 & BG & 4 & \\
\hline & GW-800 & OLF & 3 & Well Installed Apr 93 \\
\hline \multicolumn{5}{|c|}{ Best-Management Practice Monitoring } \\
\hline & GW-793 & AGLLSF & 4 & \\
\hline & GW-794 & AGLLSF & 4 & \\
\hline & GW-795 & AGLLSF & 4 & \\
\hline
\end{tabular}


Table 2 (cont'd)

Notes:

1 See Table 3 and Appendix C for well construction details.

BCK - Bear Croek Kilometer

NT - Northem Tributary

2 AGLLSF - Above Grade Low Level Storage Facility

BC - Bear Creok

BCT - Bear Creok Tributary

BG - Bear Creek Burial Grounds WMA

CMN - Maynardville Limestone

OLF - Oil Landfarm WMA

RS - Rust Spoil Area

S3 - S-3 Site

SPI - Spoil Aron I

3 The number of quarterly sampling events in which samples were collected during CY 1993. 
Table 3. General Construction Information for Monitoring Wells Sampled During 1993

\begin{tabular}{|c|c|c|c|c|c|c|c|c|c|}
\hline \multirow{3}{*}{$\begin{array}{r}\begin{array}{r}\text { Well } \\
\text { No.' }\end{array} \\
\text { GW-013 }\end{array}$} & \multirow{3}{*}{$\frac{\text { Location }^{2}}{\text { OLF }}$} & \multicolumn{2}{|c|}{ Aquifer? } & \multicolumn{6}{|c|}{ Monitored Interval" } \\
\hline & & \multirow{2}{*}{$\frac{\text { Zone }}{\text { WT }}$} & \multirow{2}{*}{$\frac{\text { Unit }}{\text { Cn }}$} & \multicolumn{3}{|c|}{ Depth } & \multicolumn{3}{|c|}{ Devation } \\
\hline & & & & 6.0 & - & 14.0 & 956.3 & - & 948.3 \\
\hline GW-040' & BG & WT & Cpv & 21.0 & - & 29.0 & 983.1 & - & 975.1 \\
\hline GW-042' & BG & WT & Cpv & 13.4 & - & 28.2 & 986.6 & - & 971.8 \\
\hline GW-043' & OLF & WT & $\mathrm{Cm}$ & 10.0 & - & 40.0 & 999.8 & - & 969.8 \\
\hline GW-044" & OLF & BDR & $\mathrm{Cm}$ & 42.5 & - & 70.0 & 967.8 & - & 940.3 \\
\hline GW-052 & BG & WT & Cmn & 4.0 & - & 19.5 & 899.7 & - & 884.2 \\
\hline GW-056 & BG & BDR & $\mathrm{Cmn}$ & 49.1 & - & 55.2 & 837.6 & $\cdot$ & 831.5 \\
\hline GW-057 & EXP & BDR & $\mathrm{Cmn}$ & 17.5 & - & 22.8 & 869.3 & - & 864.0 \\
\hline GW-063 & OLF & BDR & $\mathrm{Cmn}$ & 27.7 & - & 32.7 & 931.2 & - & 926.2 \\
\hline GW-064 & OLF & BDR & $\mathrm{Cmn}$ & 46.8 & & 52.7 & 932.4 & - & 926.5 \\
\hline GW-069 & BG & BDR & $C_{n}$ & 79.0 & - & 99.2 & 845.3 & - & 825.1 \\
\hline GW-079" & BG & BDR & $\mathrm{Crg}$ & 49.9 & $\cdot$ & 64.9 & 927.2 & $\cdot$ & 912.2 \\
\hline GW-080' & BG & WT & Crg & 20.8 & $\cdot$ & 29.7 & 956.2 & $\cdot$ & 947.3 \\
\hline GW-082 & BG & BDR & $\mathrm{Cm}$ & 29.4 & - & 34.4 & 930.1 & - & 925.1 \\
\hline GW-084" & OLF & WT & $\mathrm{Cm}$ & 18.5 & - & 27.8 & 975.9 & $\cdot$ & 966.6 \\
\hline GW-085 & OLF & BDR & $\mathrm{Cn}$ & 48.4 & - & 58.8 & 931.4 & - & 921.0 \\
\hline GW-086 & OLF & WT & $\mathrm{Cn}$ & 21.0 & - & 29.6 & 958.5 & $\cdot$ & 949.9 \\
\hline GW-115' & S3 & WT & $\mathrm{Cm}$ & 37.6 & - & 53.0 & $1,014.3$ & $\cdot$ & 998.9 \\
\hline$G W-118^{b}$ & BG & BDR & $\mathrm{Cmn}$ & 525.0 & - & 575.0 & 384.4 & - & 334.4 \\
\hline$G W-162^{a, b}$ & BG & BDR & $\mathrm{Cpv}$ & 92.0 & - & 125.0 & 946.1 & $\cdot$ & 913.1 \\
\hline GW-286 & BG & BDR & $\mathrm{Cm}$ & 19.5 & - & 32.3 & 905.0 & $\cdot$ & 892.2 \\
\hline GW-287 & BG & WT & $\mathrm{Cm}$ & 5.6 & - & 12.5 & 919.0 & $\cdot$ & 912.1 \\
\hline GW-311 & RS & WT & $\mathrm{Cmn}$ & 25.6 & - & 40.3 & 970.8 & $\cdot$ & 956.1 \\
\hline GW-312 & RS & WT & $\mathrm{Cmn}$ & 29.6 & - & 41.0 & 964.5 & $\cdot$ & 953.1 \\
\hline GW-315 & SPI & BDR & Cmn & 90.0 & - & 104.0 & 954.8 & - & 940.8 \\
\hline
\end{tabular}


Table 3 (cont'd)

\begin{tabular}{|c|c|c|c|c|c|c|c|c|}
\hline \multirow{3}{*}{$\begin{array}{r}\begin{array}{r}\text { Well } \\
\text { No.' }\end{array} \\
\text { GW-316 }\end{array}$} & \multirow{3}{*}{$\begin{array}{l}\text { Location }^{2} \\
\text { SPI }\end{array}$} & \multicolumn{2}{|c|}{ Aquifer } & \multicolumn{5}{|c|}{ Monitored Interval } \\
\hline & & \multirow{2}{*}{$\frac{\text { Zone }}{\text { WT }}$} & \multirow{2}{*}{$\frac{\text { Unit }}{\text { Cmn }}$} & \multicolumn{3}{|c|}{ Depth } & \multicolumn{2}{|c|}{ Elevation } \\
\hline & & & & 67.5 & $\cdot$ & 80.0 & 977.2 & 964.7 \\
\hline GW-317 & SPI & BDR & $\mathrm{Cmn}$ & 117.0 & - & 132.0 & 943.8 & 928.8 \\
\hline GW-323 & SPI & WT & OCk & 94.0 & - & 108.0 & $1,033.4$ & - $1,019.4$ \\
\hline GW-324" & S3 & BDR & $\mathrm{Cn}$ & 66.5 & - & 80.0 & 934.9 & 921.4 \\
\hline$G W-325^{\circ}$ & S3 & WT & Cn & 5.1 & - & 17.9 & 995.4 & 982.6 \\
\hline GW-342" & BG & BDR & Cpv & 61.0 & - & 75.0 & $1,023.5$ & - $1,009.5$ \\
\hline GW-345 & S3 & WT & $\mathrm{Cn}$ & 15.2 & - & 26.3 & 979.7 & 968.6 \\
\hline GW-347 & S3 & WT & $\mathrm{Cmn}$ & 9.5 & - & 27.8 & 988.3 & 970.0 \\
\hline GW-348 & S3 & BDR & $\mathrm{Cmn}$ & 68.1 & - & 80.9 & 929.9 & 917.1 \\
\hline GW-364 & OLF & BDR & $\mathrm{Cmn}$ & 47.0 & - & 60.3 & 886.4 & 873.1 \\
\hline$G W-365^{b}$ & OLF & BDR & $\mathrm{Cmn}$ & 126.7 & - & 150.0 & 806.3 & 783.0 \\
\hline GW-370 & BG & WT & $\mathrm{Cm}$ & 21.1 & - & 33.1 & 936.8 & 924.8 \\
\hline GW-371 b & BG & BDR & $\mathrm{Cm}$ & 110.0 & - & 125.0 & 848.1 & 833.1 \\
\hline GW-372" & BG & WT & $\mathrm{Cm}$ & 39.8 & - & 51.6 & 940.1 & 928.3 \\
\hline$G W-373^{2, b}$ & BG & BDR & $\mathrm{Cm}$ & 123.0 & - & 158.0 & 857.8 & 822.8 \\
\hline$G W-526^{b}$ & S3 & BDR & $\mathrm{Cn}$ & 101.0 & - & 123.0 & 894.3 & 872.3 \\
\hline GW-537 & OLF & WT & $\mathrm{Cn}$ & 4.8 & - & 23.3 & 969.4 & 950.9 \\
\hline$G W-601^{b}$ & OLF & BDR & $\mathrm{Cmn}$ & 318.5 & - & 356.0 & 680.6 & 643.1 \\
\hline GW-613' & S3 & WT & $\mathrm{Cn}$ & 30.0 & - & 42.0 & 980.2 & 968.2 \\
\hline GW-614" & S3 & BDR & $\mathrm{Cn}$ & 71.6 & - & 90.2 & 937.8 & 919.2 \\
\hline GW-621 & BG & WT & $\mathrm{Cmn}$ & 23.3 & - & 40.5 & 899.8 & 882.6 \\
\hline GW-626 & BG & WT & $\mathrm{Cn}$ & 63.0 & - & 78.0 & 877.0 & 862.0 \\
\hline GW- $627^{\circ}$ & BG & BDR & $\mathrm{Cn}$ & 254.0 & - & 270.0 & 686.4 & 670.4 \\
\hline$G W-636^{b}$ & OLF & BDR & $\mathrm{Cn}$ & 98.5 & - & 117.0 & 839.6 & 821.1 \\
\hline GW-637 & OLF & WT & $\mathrm{Cn}$ & 14.5 & - & 27.5 & 923.8 & 910.8 \\
\hline GW-638 & OLF & WT & $\mathrm{Cn}$ & 2.5 & - & 11.5 & 935.6 & 926.6 \\
\hline
\end{tabular}

B-8 
Table 3 (cont'd)

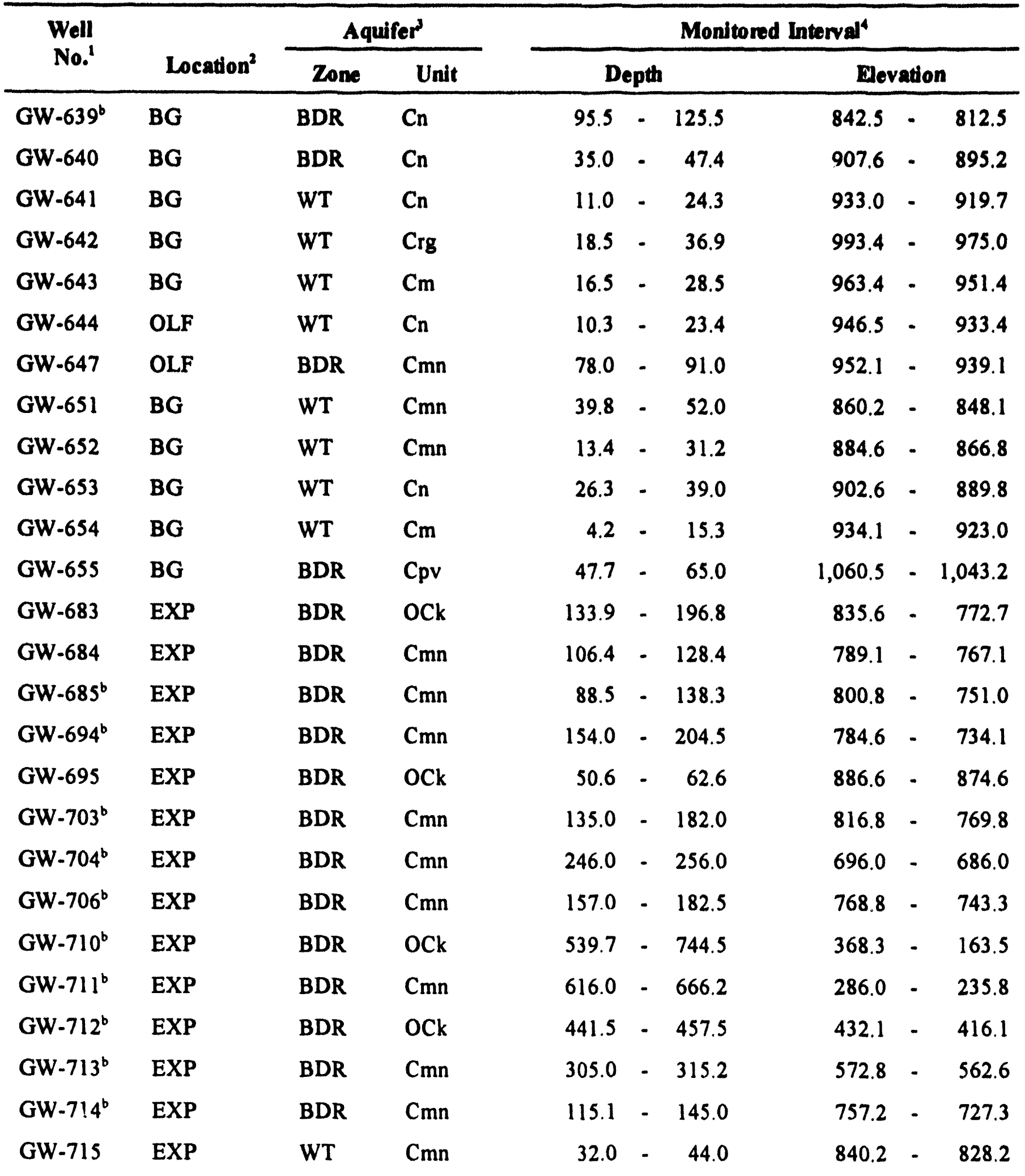


Table 3 (cont'd)

\begin{tabular}{|c|c|c|c|c|c|c|c|c|c|}
\hline \multirow{3}{*}{$\begin{array}{r}\begin{array}{r}\text { Well } \\
\text { No. }\end{array} \\
\text { GW-723b }\end{array}$} & \multirow{3}{*}{$\frac{\text { Location }^{2}}{\text { EXP }}$} & \multicolumn{2}{|c|}{ Aquifer ${ }^{3}$} & \multicolumn{6}{|c|}{ Monitored Interval ${ }^{4}$} \\
\hline & & \multirow{2}{*}{$\frac{\text { Zone }}{\text { BDR }}$} & \multirow{2}{*}{$\frac{\text { Unit }}{\text { Cmn }}$} & \multicolumn{3}{|c|}{ Depth } & \multicolumn{3}{|c|}{ Elevation } \\
\hline & & & & 340.6 & - & 444.5 & 678.7 & - & 574.8 \\
\hline$G W-724^{b}$ & EXP & BDR & $\mathrm{Cmn}$ & 289.6 & - & 301.6 & 687.0 & - & 675.0 \\
\hline$G W-725^{b}$ & EXP & BDR & $\mathrm{Cmn}$ & 132.5 & - & 142.5 & 825.8 & - & 815.8 \\
\hline GW-736 & EXP & BDR & Cmn & 92.0 & - & 105.0 & 865.6 & - & 852.6 \\
\hline GW-737 & EXP & BDR & Cmn & 79.0 & - & 89.5 & 878.5 & - & 868.0 \\
\hline GW-738 & EXP & BDR & $\mathrm{Cmn}$ & 63.5 & - & 88.0 & 916.9 & - & 892.4 \\
\hline$G W-739^{b}$ & EXP & BDR & $\mathrm{Cmn}$ & 289.2 & - & 320.0 & 731.5 & - & 700.7 \\
\hline GW $-740^{b}$ & EXP & BDR & $\mathrm{Cmn}$ & 165.6 & 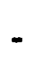 & 190.0 & 851.4 & - & 827.0 \\
\hline GW-793 & AGLLSF & BDR & $\mathrm{Cn}$ & 16.9 & - & 29.0 & 948.2 & - & 936.1 \\
\hline GW-794 & AGLLSF & BDR & $\mathrm{Cmn}$ & 25.8 & - & 39.3 & 905.1 & - & 891.6 \\
\hline GW-795 & AGLLSF & WT & $\mathrm{Cmn}$ & 7.5 & - & 20.1 & 915.4 & - & 902.8 \\
\hline GW-800 & OLF & BDR & OCk & 19.3 & - & 31.5 & 942.1 & - & 929.9 \\
\hline
\end{tabular}

Notes:

1 Well construction information compiled from: Updated Subsurface Data Base for Bear Creek Valley, Chestnut Ridge, and Parts of Bethel Valley on the U.S. Department of Energy Oak Ridge Reservation (Jones et al. 1993).

2 AGLLSF - Above Grade Low Level Storage Facility

BG - Bear Creek Burial Grounds WMA

EXP - Exit Pathway (Maynardville Limestone)

OLF - Oil Landfarm WMA

RS - Rust Spoil Area

S3 - S-3 Site

SPI - Spoil Area I

B- 10 
Table 3 (cont'd)

3 BDR - Bedrock (Shallow, Intermediate, and Deep Intervals)

WT - Water Table Interval

OCk - Knox Group

Cmn - Maynardville Limestone

Cn - Nolichucky Shale

Cm - Maryville Limestone

Crg - Rogersville Shale

Crt - Rutledge Limestone

Cpv - Pumpkin Valley Shale

$\mathrm{Cr} \quad-$ Rome Formation

4 Depth in feet below the ground surface.

Elevation in feet above mean sea level.

a Background/Upgradient monitoring well.

b Open borehole well construction. 
Table 4. Analytical Parameters for Groundwater and Surface Water Samples Collected During 1993

\begin{tabular}{|c|c|c|c|}
\hline $\begin{array}{c}\text { Sampling } \\
\text { Point }^{\mathbf{1}}\end{array}$ & Location $^{2}$ & $\begin{array}{l}\text { Analytical } \\
\text { Parameters }\end{array}$ & Notes \\
\hline GW-013 & OLF & STD $^{3}$ & \\
\hline GW-040 & BG & STD/CMP ${ }^{4}$ & CMP parameters analyzed 4th Qtr. sample only \\
\hline GW-042 & BG & CMP & \\
\hline GW-043 & OLF & STD/CMP & CMP parameters analyzed 4th Qtr. sample only \\
\hline GW-044 & OLF & STD/CMP & CMP parameters analyzed 4th Qtr. sample only \\
\hline GW-052 & BG & STD & \\
\hline GW-056 & BG & STD & \\
\hline GW-057 & $\mathrm{CMN}$ & STD & \\
\hline GW-063 & OLF & STD & \\
\hline GW-064 & OLF & STD & \\
\hline GW-069 & BG & STD & \\
\hline GW-079 & BG & CMP & \\
\hline GW-080 & BG & CMP & : \\
\hline GW-082 & BG & STD & \\
\hline GW-084 & OLF & STD/CMP & CMP parameters analyzed 4th Qtr. sample only \\
\hline GW-085 & OLF & STD & \\
\hline GW-086 & OLF & STD & \\
\hline GW-115 & S3 & STD/CMP & CMP parameters analyzed 4th Qtr. sample only \\
\hline GW-118 & BG & STD & \\
\hline GW-162 & BG & CMP & \\
\hline GW-286 & BG & STD & \\
\hline GW-287 & BG & STD & \\
\hline GW-311 & RS & STD & \\
\hline GW-312 & RS & STD & \\
\hline GW-315 & SPI & STD & \\
\hline
\end{tabular}

B-12 
Table 4. (cont'd.)

\begin{tabular}{|c|c|c|c|}
\hline $\begin{array}{c}\text { Sampling } \\
\text { Point }^{+}\end{array}$ & Location ${ }^{2}$ & $\begin{array}{l}\text { Analytical } \\
\text { Parameters }\end{array}$ & Notes \\
\hline GW-316 & SPI & STD & \\
\hline GW-317 & SPI & STD & \\
\hline GW-323 & SPI & STD & \\
\hline GW-324 & S3 & STD/CMP & CMP parameters analyzed 4th $Q$ tr. sample only \\
\hline GW-325 & S3 & STD/CMP & CMP parameters analyzed 4th Qtr. sample only \\
\hline GW-342 & BG & CMP & \\
\hline GW-345 & S3 & STD & \\
\hline GW-347 & S3 & STD & \\
\hline GW-348 & S3 & STD & \\
\hline GW-364 & OLF & STD & \\
\hline GW-365 & OLF & STD & \\
\hline GW-370 & BG & STD & \\
\hline GW-371 & BG & STD & \\
\hline GW-372 & BG & STD/CMP & CMP parameters analyzed 4th Qtr. sample only \\
\hline GW-373 & BG & STD/CMP & CMP parameters analyzed 4th Qtr. sample only \\
\hline GW-526 & S3 & STD & \\
\hline GW-537 & OLF & STD, BETA ${ }^{S}$ & \\
\hline GW-601 & OLF & STD & \\
\hline GW-613 & S3 & CMP & \\
\hline GW-614 & S3 & CMP & \\
\hline GW-621 & BG & STD & \\
\hline GW-626 & BG & STD & \\
\hline GW-627 & BG & STD & \\
\hline GW-636 & OLF & STD & \\
\hline GW-637 & OLF & STD & \\
\hline GW-638 & OLF & STD & \\
\hline
\end{tabular}


Table 4. (cont'd.)

\begin{tabular}{|c|c|c|c|}
\hline $\begin{array}{c}\text { Sampling } \\
\text { Point }^{3}\end{array}$ & Location ${ }^{2}$ & $\begin{array}{l}\text { Analytical } \\
\text { Parameters }\end{array}$ & Notes \\
\hline GW-639 & BG & STD & \\
\hline GW-640 & BG & STD & \\
\hline GW-641 & BG & STD & \\
\hline GW-642 & BG & STD/CMP & CMP parameters analyzed 4th Qtr. sample only \\
\hline GW-643 & BG & STD & \\
\hline GW-644 & OLF & STD & \\
\hline GW-647 & OLF & STD & \\
\hline GW-651 & $\mathrm{BG}$ & STD & \\
\hline GW-652 & $\mathbf{B G}$ & STD & \\
\hline GW-653 & $\mathrm{BG}$ & STD & \\
\hline GW-654 & $\mathbf{B G}$ & STD & \\
\hline GW-655 & $\mathrm{BG}$ & STD & \\
\hline GW-683 & $\mathrm{CMN}$ & STD & \\
\hline GW-684 & $\mathrm{CMN}$ & STD & \\
\hline GW-685 & $\mathrm{CMN}$ & STD & \\
\hline GW-694 & $\mathrm{CMN}$ & STD, BETA & \\
\hline GW-695 & $\mathrm{CMN}$ & STD & $\therefore$ \\
\hline GW-703 & $\mathrm{CMN}$ & STD & \\
\hline GW-704 & $\mathrm{CMN}$ & STD & \\
\hline GW-706 & $\mathrm{CMN}$ & STD, BETA & \\
\hline GW-710 & $\mathrm{CMN}$ & STD & \\
\hline GW-711 & $\mathrm{CMN}$ & STD & \\
\hline GW-712 & $\mathrm{CMN}$ & STD & \\
\hline GW-713 & $\mathrm{CMN}$ & STD & \\
\hline GW-714 & $\mathrm{CMN}$ & STD & \\
\hline$G W-715$ & $\mathrm{CMN}$ & STD & \\
\hline
\end{tabular}

B-14 
Table 4. (cont'd.)

\begin{tabular}{|c|c|c|c|}
\hline $\begin{array}{c}\text { Sampling } \\
\text { Point }^{1}\end{array}$ & Location ${ }^{2}$ & $\begin{array}{l}\text { Analytical } \\
\text { Parameters }\end{array}$ & Notes \\
\hline GW-723 & CMN & STD & \\
\hline GW-724 & $\mathrm{CMN}$ & STD & \\
\hline GW-725 & $\mathrm{CMN}$ & STD & \\
\hline GW-736 & CMN & STD & \\
\hline GW-737 & $\mathrm{CMN}$ & STD & \\
\hline GW-738 & $\mathrm{CMN}$ & STD & \\
\hline GW-739 & $\mathrm{CMN}$ & STD & \\
\hline GW-740 & $\mathrm{CMN}$ & STD & \\
\hline GW-793 & AGLLSF & STD & \\
\hline GW-794 & AGLLSF & STD, $\mathrm{U}^{6}$ & \\
\hline GW-795 & AGLLSF & STD, U & \\
\hline GW-800 & OLF & STD & \\
\hline NT-01 & BCT & STD, BETA & \\
\hline NT-13 & $\mathrm{BCT}$ & STD & \\
\hline SS-1 & $\mathrm{BC}$ & STD,BETA & \\
\hline SS-4 & $\mathrm{BC}$ & STD & \\
\hline SS-5 & BC & STD & \\
\hline SS-6 & $\mathrm{BC}$ & STD & \\
\hline SS-8 & $\mathrm{BC}$ & STD & \\
\hline \multicolumn{4}{|c|}{ BCK 0.63} \\
\hline & $\mathrm{BC}$ & STD & \\
\hline \multicolumn{4}{|c|}{ BCK 04.55} \\
\hline & $\mathrm{BC}$ & STD & \\
\hline \multicolumn{4}{|c|}{ BCK 09.40} \\
\hline & $\mathrm{BC}$ & STD & \\
\hline
\end{tabular}


Table 4. (cont'd.)

Notes:

1. BCK - Bear Creek Kilometer

GW - Monitoring Well

NT - Northern Tributary

SS - Spring

2. AGLLSF - Above Grade Low Level Storage Facility

BC - Bear Creek

BCT - Bear Creek Tributary

BG - Bear Creek Burial Grounds WMA

CMN - Maynardville Limestone

OLF - Oil Landfarm WMA

RS - Rust Spoil Area

S3 - S-3 Site

SPI - Spoil Area I

3. STD - Standard suite of analytical parameters; includes the following:

- Inductively Coupled Plasma (ICP) Metals

$\begin{array}{lll}\text { Aluminum } & \text { Chromium } & \text { Potassium } \\ \text { Antimony } & \text { Cobalt } & \text { Selenium } \\ \text { Arsenic } & \text { Copper } & \text { Silver } \\ \text { Barium } & \text { Iron } & \text { Sodium } \\ \text { Beryllium } & \text { Magnesium } & \text { Strontium } \\ \text { Boron } & \text { Manganese } & \text { Thorium } \\ \text { Cadmium } & \text { Molybdenum } & \text { Vanadium } \\ \text { Calcium } & \text { Nickel } & \text { Zinc }\end{array}$

- Atomic Absorption Spectroscopy Metals

Cadmium

Chromium

Lead

- Other Metals

Mercury (Cold Vapor Atomic Abosrption Spectroscopy)

Uranium (Flourometric Analysis)

- Volatile Organic Compounds

Acetone

Benzene

Bromodichloromethane

Bromoform

Bromomethane

1,2-Dichloropropane

2-Butanone

Carbon disulfide

cis-1,3-Dichloropropene

trans-1,3-Dichloropropene

Ethylbenzene

2-Hexanone

4-Methyl-2-pentanone

Methylene chloride 
Table 4. (cont'd.)

Volatile Organic Compounds (cont'd.)

Carbon tetrachloride

Chlorobenzene

Chlorodibromomethane

Chloroethane

Chloroform

Chloromethane

1,1-Dichloroethane

1,2-Dichloroethane

1,1-Dichloroethene

1,2-Dichloroethene

Styrene

1,1,2,2-Tetrachloroethane

Tetrachloroethene

Toluene

1,1,1-Trichloroethane

1,1,2-Trichloroethane

Trichloroethene

Vinyl acetate

Vinyl chloride

Xylenes

Anions

Alkalinity - Carbonate

Alkalinity - Bicarbonate

Nitrate (as $\mathrm{N}$ )

Chloride

Fluoride

Sulfate

- Radiological Parameters

Gross Alpha Activity

Gross Beta Activity

- Miscellaneous Parameters

$\mathrm{pH}$

Specific Conductance

Total Suspended Solids (TSS)

Total Dissolved Solids (TDS)

Turbidity

- Field Measurements

Static Water Level

$\mathrm{pH}$

Temperature

Dissolved Oxygen

Specific Conductance

Oxidation - Reduction Potential (REDOX)

4. CMP - Compliance Monitoring Parameters; includes the following:

- Standard suite of analytical parameters

- Americium ( $\left.{ }^{241} \mathrm{Am}\right)$

- Iodine $\left({ }^{129} \mathrm{I}\right)$

- Neptunium $\left({ }^{237} \mathrm{~Np}\right)$

- Plutonium $\left({ }^{238} \mathrm{Pu}\right)$

- Radium (total)

- Strontium (total)

- Technetium $\left({ }^{99} \mathrm{Tc}\right)$ 
Table 4. (cont'd.)

4. Compliance Monitoring Parameters (cont'd)

- Tritium $\left({ }^{3} \mathrm{H}\right)$

- Isotopic Uranium $\left({ }^{234} \mathrm{U},{ }^{233} \mathrm{U},{ }^{238} \mathrm{U}\right)$

5. BETA - Beta-emitting isotopes:

- Strontium (total)

- Technetium $\left({ }^{99} \mathrm{Tc}\right)$

- Tritium $\left({ }^{3} \mathrm{H}\right)$

6. U - Isotopic Uranium $\left({ }^{234} \mathrm{U},{ }^{235} \mathrm{U},{ }^{238} \mathrm{U}\right)$ 
APPENDIX C

MONITORING-WELL CONSTRUCTION DETAIIS 


\section{EXPLANATION}

\section{LOCATION:}

$\begin{array}{rll}\text { AGLLSF } & : & \text { Above Grade Low Level Storage Facility } \\ \text { BG } & : & \text { Bear Creek Burial Grounds WMA } \\ \text { EXP } & : & \text { Exit-Pathway Monitoring } \\ \text { OLF } & : & \text { Oil Landfarm WMA } \\ \text { RS } & - & \text { Rust Spoil Area } \\ \text { S3 } & : & \text { S-3 Site } \\ \text { SPI } & - & \text { Spoil Area I }\end{array}$

\section{CASING MATERIAL:}

$\begin{array}{rll}\text { PVC } & - & \text { Polyvinyl chloride } \\ \text { SS } & - & \text { Stainless Steel }\end{array}$

\section{MONITORED INTERVAL:}

$\begin{array}{rll}\text { BDR } & - & \text { Bedrock Interval } \\ \text { WT } & - & \text { Water-Table Interval } \\ \text { OCk } & - & \text { Knox Group } \\ \mathrm{CON} & - & \text { Conasauga Group (undifferentiated) } \\ \mathrm{Cmn} & - & \text { Maynardville Limestone } \\ \mathrm{Cn} & - & \text { Nolichucky Shale } \\ \mathrm{Cm} & - & \text { Maryville Limestone } \\ \mathrm{Crg} & - & \text { Rogersville Shale } \\ \mathrm{Crt} & - & \text { Rutledge Limestone } \\ \mathrm{Cpv} & - & \text { Pumpkin Valley Shale } \\ \mathrm{Cr} & - & \text { Rome Formation } \\ \mathrm{Top} & - & \text { Depth to top of filter pack or open-hole } \\ \mathrm{ottom} & - & \text { Depth to bottom of filter pack or open-hole } \\ \mathrm{sw} / .01 & - & \text { Spiral wound, } 0.01 \text { inch slot size } \\ \mathrm{pp} / .01 & - & \text { Perforaied pipe, } 0.01 \text { inch hole size }\end{array}$

\section{NOTES:}

Coordinates

Elevations

Depths

Casing Diameter Borehole Diameter Length
- Y-12 Plant grid system

- Feet above mean sea level

- Feet below ground surface

- Outside diameter, in inches

- Nominal diameter, in inches

- Feet

- Not applicable or not available

All data compiled from Jones et all. 1993. 
APPandX C

Monitoring-Well Construotion Detelle

\begin{tabular}{|c|c|c|c|c|c|c|c|}
\hline \multirow{2}{*}{$\begin{array}{l}\text { Woll No. } \\
\text { woetion }\end{array}$} & ON-013 & $0 N-040$ & $O W-042$ & ON-043 & ON-044 & ON-052 & ON-056 \\
\hline & ote & 80 & 80 & or: & OLF & $\mathbf{B a}$ & 80 \\
\hline rant coordinate & 47343 & 43358 & 43938 & 47932 & 47928 & 43483 & 41384 \\
\hline Morth Coordinate & 30174 & 31211 & 31137 & 30522 & 30526 & 29046 & 28698 \\
\hline eturvarions & $\cdot$ & $\cdot$ &. &. & - &. & - \\
\hline Measuring point & 964.52 & 1008.02 & 1003.39 & 1011.51 & 1013.49 & 906.04 & 890.71 \\
\hline surtace & 962.29 & 1004.11 & 999.95 & 1009.78 & 1010.3 & 903.71 & 886.65 \\
\hline Weathered Rock & 956.99 &. & 996.45 & 1008.78 & 1009.3 &. & - \\
\hline Freah Rook & 951.79 & $\cdot$ &. & 971.78 & 972.3 &. & 880.25 \\
\hline Top Mnitored Interval & 936.29 & 983.11 & 986.55 & 999.78 & 967.8 & 899.71 & 837.53 \\
\hline Bottom Monltored Interval & 948.29 & 975.11 & 971.75 & 969.78 & 940.3 & 884.21 & 831.45 \\
\hline BUREACE/CONDUCTOR CASIME & $\cdot$ &. &. &. &. & . & - \\
\hline Casing Dopth &. & $\cdot$ &. & . & $\cdot$ & $\cdot$ & 32 \\
\hline Casing Diameter & $\cdot$ & $\cdot$ & $\cdot$ & $\cdot$ & $\cdot$ & $\cdot$ & 4.5 \\
\hline Casing Materlal & $\cdot$ &. & . & $\cdot$ & unknown &. & PvC $/ 140$ \\
\hline WRLL CASTHO BORAHOLE &. &. &. &. &. &. & - \\
\hline Borahole Dopth & $33 . \dot{8}$ & 35 & 30 & 40 & $\dot{20}$ & $19 . \dot{5}$ & $55 . \dot{2}$ \\
\hline Borehole Dianoter & 6 & 6 & 6 & 6 & 4 & 6 & 1 \\
\hline Weathered Rock - Depth & 3.3 & $\cdot$ & 3.5 & 1 & 1 & $\cdot 1$ & - \\
\hline Frash Rock - Depth & 10.5 & $\cdot$ & . & 38 & 38 &. & 6.4 \\
\hline WELL castro & . &. &. &. &. &. & - \\
\hline Wall Casing Depth & 10.4 & 27.7 & 26.9 & 32.8 & 58 & 18.3 & 55.2 \\
\hline Well Casing Diameter & 2.37 & 2.37 & 2.37 & 2.37 & 2.37 & 2.37 & 2.37 \\
\hline Well Casling Material & $85 / 1304$ & $8 s / 1304$ & $s 5 / 1304$ & $8 s / \$ 304$ & $8 s / 1304$ & ss/ $/ 304$ & $55 / 1304$ \\
\hline MONITORED INTERVAL & $\cdot$ & . & $\cdot 1$ &. & . &. & • \\
\hline Aquifer zone & $\dot{w T}$ & $\dot{\omega r}$ & $\dot{w T}$ & $\dot{w r}$ & $\begin{array}{r}\dot{B} \\
\mathrm{BDR}\end{array}$ & $\dot{\text { wT }}$ & BDR \\
\hline Geologic Formation & $c n$ & Cpv & Cpv & $\mathrm{cm}$ & $\mathrm{Cm}$ & $\operatorname{comn}$ & $\mathrm{Cmn}$ \\
\hline Soreened or Open & Screen & Screen & screen & Screen & screan & Soraen & Screen \\
\hline Top - Depth & 6 & 21 & 13.4 & 10 & 42.5 & 4 & 49.1 \\
\hline Bottom - Depth & 14 & 29 & 28.2 & 40 & 70 & 19.5 & 55.2 \\
\hline Soreen Material & ss/aw/.01 & $\mathrm{ss} / \mathrm{sw} / .02$ & $\mathrm{ss} / \mathrm{sw} / .01$ & $\mathrm{ss} / \mathrm{sw} / .01$ & $s s / \mathrm{sw} / .01$ & $\mathrm{ss} / \mathrm{sw} / .01$ & $88 / 8 w / .01$ \\
\hline Screen Length & 2.0 & 2.0 & 2.0 & 10.0 & 10.0 & 5.0 & 2.0 \\
\hline Open-Hole Length & $\cdot$ & &. &. &. & $\cdot$ & - \\
\hline Open-Hole Diameter & $\cdot$ & &. & . & $\cdot$ & - & • \\
\hline
\end{tabular}

(CONTINURD) 
APPENDIX C

Monitoring-Well Conatruotion Detall.

\begin{tabular}{|c|c|c|c|c|c|c|c|}
\hline \multirow{2}{*}{$\begin{array}{l}\text { woll No. } \\
\text { Lootion }\end{array}$} & OW-057 & ON-063 & Ow-064 & ON-069 & ow-079 & OW-080 & ew-082 \\
\hline & $\mathbf{E x P}$ & orf & orr & BO & $\mathbf{B O}$ & $\mathbf{B G}$ & $B O$ \\
\hline East Coordinate & 41380 & 48257 & 49168 & 43803 & 41619 & 41622 & 42090 \\
\hline Morth Coordinate & 28688 & 29016 & 29195 & 29490 & 30632 & 30624 & 30434 \\
\hline ELVATrons & • & • & - & $\cdot$ & - & $\cdot$ & • \\
\hline Measuring Point & 889.36 & 961.93 & 981.56 & 927.01 & 980.5 & 980.31 & 962.49 \\
\hline surface & 886.83 & 958.89 & 979.17 & 924.25 & 977.14 & 977.03 & 959.49 \\
\hline Weathered Rock & & - & - & 921.25 & 973.14 & 973.53 & 952.49 \\
\hline Freah Rock & 883.43 & 942.19 & 944.47 & 900.25 & 950.64 & 953.53 & 936.49 \\
\hline Top Monitored Interval & 869.33 & 931.19 & 932.37 & 845.25 & 927.24 & 956.23 & 930.09 \\
\hline Bottom monitored Interval & 864.03 & 926.19 & 926.47 & 825.05 & 912.24 & 947.33 & 925.09 \\
\hline SURAACE/CONDUCTOR CASIMG & . & . & $\cdot$ & $\cdot$ & • & $\cdot$ & - \\
\hline Caulng Depth & 14 & $\bullet$ & $\cdot$ & $\cdot$. & $\cdot$ & $\cdot \dot{.}$ & $2 \dot{5}$ \\
\hline Caning Diameter & 4.5 & $\cdot$ & . & $\cdot$ & . & $\cdot$ & 6.5 \\
\hline Casing Material & Pvc/140 & unknown & unknown & unknown & . &. & $\mathrm{PVC} / \$ 40$ \\
\hline WLL CASING BORRHOLE & . &. & . &. & - &. & • \\
\hline Box ehole Depth & 25 & $\dot{35}$ & ${ }_{57}$ & 100 & 65 & 30 & 35 \\
\hline Borehole Diameter & 4 & 4 & 4 & 7.88 & 6.5 & 6.5 & 4 \\
\hline Weathered Rock - Depth & - & $\cdot$ & . & 3 & 4 & 3.5 & 7 \\
\hline Presh Rock - Depth & 3.4 & 16.7 & 34.7 & 24 & 26.5 & 23.5 & 23 \\
\hline WELL CASINO & - & . & - & $\cdot$ & - & . & • \\
\hline Well Casing Depth & 22.8 & 32.7 & 52.7 & 99.2 & 64.9 & 29.7 & 34.4 \\
\hline Well Casing Diameter & 2.37 & 2.37 & 2.37 & 2.37 & 2.37 & 2.37 & 2.37 \\
\hline Well Casing Material & $s 8 / \$ 304$ & Ss $/ 1304$ & ss $/ 1304$ & $85 / 1304$ & $s s / 1304$ & ss/1304 & ss//304 \\
\hline MONITORED INTERVAL & - &. & $\dot{\varphi}$ & $\cdot$ & $\bullet$ & $\cdot$ & • \\
\hline Aguifer zone & BDR & BDR & BDR & BDR & BDR & $\dot{w T}$ & BDR \\
\hline Geologic Formation & Cmn & $\mathrm{cmn}$ & $\mathrm{cmn}$ & $c_{n}$ & crg & $\operatorname{crg}$ & $a n$ \\
\hline Screened or Open & Screen & Screen & Screen & Screen & Screen & Screen & Screen \\
\hline Top - Depth & 17.5 & 27.7 & 46.8 & 79 & 49.9 & 20.8 & 29.4 \\
\hline Bottom - Depth & 22.8 & 32.7 & 52.7 & 99.2 & 64.9 & 29.7 & 34.4 \\
\hline 8creen Material & $\mathrm{ss} / \mathrm{sw} / .01$ & $\mathrm{sS} / \mathrm{ow} / .01$ & $\mathrm{ss} / \mathrm{sw} / .01$ & ss/ow/.01 & $s s / 8 w / .01$ & $\mathrm{ss} / \mathrm{sw} / .01$ & ss/sw/.01 \\
\hline Scraen Length & 2.0 & 5.0 & 2.0 & 10.2 & 5.0 & 5.0 & 5.0 \\
\hline Open-Hole Length & • & $\cdot$ & . & $\cdot$ & . & - & . \\
\hline Open-Hole Diameter & . & 1 & 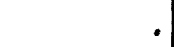 &. & 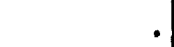 & . & \\
\hline
\end{tabular}

(CONTINUED) 
APPENDXX C

Monltoring-Well Construction Detalle

\begin{tabular}{|c|c|c|c|c|c|c|c|}
\hline \multirow{2}{*}{$\begin{array}{l}\text { well No. } \\
\text { Looation }\end{array}$} & GW-084 & aw-085 & QW-086 & OW-113 & $0 w-118$ & ON-162 & $O W-286$ \\
\hline & orf & orF & orf & 83 & $B G$ & BO & Bo \\
\hline Rast Coordinate & 48801 & 49058 & 49043 & 52685 & 43404 & 44480.91 & 42287 \\
\hline Worth Coordinate & 30421 & 30002 & 29993 & 31073 & 29147 & 31330.75 & 29993 \\
\hline ELEVATIONS &. & $\cdot$ & $\cdot$ & - & $\cdot$ & $\cdot 1$ & - \\
\hline Moasuring Point & 996.6 & 982.87 & 982.76 & 1054.41 & 912.39 & 1040.44 & 926.83 \\
\hline surface & 994.37 & 979.82 & 979.5 & 1051.92 & 909.37 & 1038.06 & 924.52 \\
\hline Weathered Rock & 990.37 & 977.82 & 971.5 & $\cdot$ & &. & 919.52 \\
\hline Freeh Rook & & 939.82 &. & $\cdot 1$ & 894.37 & 1026.06 & 914.52 \\
\hline Top Monitored Interval & 975.87 & 931.42 & 958.5 & 1014.32 & 384.37 & 946.06 & 905.02 \\
\hline Bottom Monitored Interval & 966.57 & 921.02 & 949.9 & 998.92 & 334.37 & 913.06 & 892.22 \\
\hline SURPACE/CONDUCTOR CASING & $\cdot$ & $\cdot$ & $\cdot$ & $\cdot$ & $\cdot$ & $\cdot$ & - \\
\hline Casing Dopth & $\cdot$ & $\cdot$ & $\cdot$ &. & 31 & 26 & 14.2 \\
\hline Casing Diamater & $\cdot$ & $\cdot$ & $\cdot$ &. & 10.75 & 10.75 & 10.75 \\
\hline Casing Material & $\cdot$ & unknown & $\cdot$ & $\cdot$ & $\cdot$ & $\cdot$ & - \\
\hline WRLX CA8ING BOREHOLE &. & $\cdot$ & $\cdot$ & $\cdot$ & $\cdot$ & $\cdot$ & - \\
\hline Borehole Depth & 34 & $6 \dot{2}$ & 33.5 & $\dot{53}$ & 525 & $\dot{92}$ & $32 . \dot{3}$ \\
\hline Borahole Diamoter & 6.5 & 4 & 4 & $\cdot$ & 9.87 & 10 & 9 \\
\hline Weathered Rock - Depth & 4 & 2 & 8 & $\cdot$ & $\cdot$ & $\cdot$ & 5 \\
\hline Fresh Rock - Depth & $\cdot$ & 40 & $\cdot$ & $\cdot$ & 15 & 12 & 10 \\
\hline WELL CASING & $\cdot$ & $\cdot$ & $\cdot$ & $\cdot$ & - & $\cdot$ & - \\
\hline Well Casing Depth & 27.8 & 58.8 & 29.6 & 53 & $52 \dot{0}$ & $\dot{92}$ & 32 \\
\hline Well Casing Diameter & 2.37 & 2.37 & 2.37 & 2.37 & 4.5 & 6.62 & 4.5 \\
\hline Well Casing Material & $s s / 1304$ & ss $/ 1304$ & ss/1304 & ss $/ \$ 304$ & Steel/F25 & $s t=1 / 725$ & $5 s / 304$ \\
\hline MONITORED INTERVAL. & . &. &. & $\cdot$ & $\cdot$ & $\cdot$ & • \\
\hline Aquifer zone & $\dot{w r}$ & BDR & $\dot{w r}$ & $\dot{\text { WT }}$ & BDR & BDR & BDR \\
\hline Ceologic Formation & $\mathrm{cm}$ & $\mathrm{Cn}$ & $\mathrm{Cn}$ & $\mathrm{Cm}$ & $\mathrm{cn}$ & Cpv & $\mathrm{cm}$ \\
\hline Screened or Open & Screen & Screen & Screen & screan & Open & Open & Screen \\
\hline Top - Depth & 18.5 & 48.4 & 21 & 37.6 & 525 & 92 & 19.5 \\
\hline Bottom - Depth & 27.8 & 58.8 & 29.6 & 53 & 575 & 125 & 32.3 \\
\hline Screen Material & ss/sw/.01 & $\mathrm{ss} / \mathrm{sw} / .01$ & ss/ow/.01 & $\mathrm{ss} / \mathrm{sw} / .01$ & $\cdot$ &. & $\mathrm{ss} / \mathrm{sw} / .01$ \\
\hline Screen Length & 5.0 & 5.0 & 5.0 & 10.0 & $\cdot$ & $\cdot$ & 10.0 \\
\hline Open-Hole Length & $\cdot$ & - & $\cdot$ & $\cdot$ & 50 & 33 & • \\
\hline Open-Hole Diametor & - & - & $\cdot$ & $\cdot$ & 4 & 6 & - \\
\hline
\end{tabular}


APPENDIX C

Monitoring-Well Construction Details

\begin{tabular}{|c|c|c|c|c|c|c|c|}
\hline Well No. & $G W-287$ & $G w-311$ & $G W-312$ & GW-315 & GW-316 & GW-317 & $G W-323$ \\
\hline Location & BG & RS & RS & SPI & SPI & SPI & SPI \\
\hline East Coordinate & 42288 & 50126 & 49778 & 52268 & 52412 & 52192 & 52106 \\
\hline North Coordinate & 29989 & 29267 & 29216 & 29455 & 29336 & 29285 & 28985 \\
\hline ELEVATIONS & $\cdot$ & $\cdot$ & • & $\cdot$ & $\cdot$ & $\cdot$ & • \\
\hline Measuring Point & 926.87 & 999.35 & 996.49 & 1047.28 & 1047.17 & 1062.98 & 1130.11 \\
\hline Surface & 924.6 & 996.43 & 994.13 & 1044.84 & 1044.73 & 1060.8 & 1127.41 \\
\hline Weathered Rock & 920.1 & 956.13 & - & 990.84 & 989.73 & $\cdot$ & 1052.41 \\
\hline Fresh Rock & 913.6 & $\cdot$ & - & 973.84 &. & 1012.8 & - \\
\hline Top Monitored Interval & 919 & 970.83 & 964.53 & 954.84 & 977.23 & 943.8 & 1033.41 \\
\hline Bottom Monitored Interval & 912.1 & 956.13 & 953.13 & 940.84 & 964.73 & 928.8 & 1019.41 \\
\hline SURFACE/CONDUCTOR CASING & $\cdot$ & $\cdot$ & - & $\cdot$ & $\cdot$ & . & - \\
\hline Casing Depth & $\cdot$ & $\cdot \dot{1}$ & $\cdot$ & 84.4 & 54.7 & $49 . \dot{3}$ & $\dot{93}$ \\
\hline Casing Diameter & $\cdot$ & $\cdot$ & - & 10.75 & 10.75 & 10.75 & 10.75 \\
\hline Casing Material & $\cdot$ & $\cdot$ & - & $\cdot$ & $\cdot$ & $\cdot$ & - \\
\hline WEIL CASIMG BOREHOLE & $\cdot$ & $\cdot$ & - & $\cdot$ & $\cdot$ & $\cdot$ & - \\
\hline Borehole Depth & 12.5 & 40.3 & ${ }_{41}^{\circ}$ & 104 & 80 & 132 & 108 \\
\hline Borehole Diameter & 9 & 10 & 12 & 10 & 10 & 10 & 10 \\
\hline Weathered Rock - Depth & 4.5 & 40.3 & - & 54 & 55 & . & 75 \\
\hline Fresh Rock - Depth & 11 & $\cdot$ & - & 71 & $\cdot$ & 48 & - \\
\hline WELL CASING & $\cdot$ &. & . &. & $\cdot$ & $\cdot$ & - \\
\hline Well Casing Depth & $12 . \dot{3}$ & 40.3 & $40 . \dot{5}$ & 103.3 & 79.4 & $130 . \dot{5}$ & 107 \\
\hline Well Casing Diameter & 4.5 & 4.5 & 4.5 & 4.5 & 4.5 & 4.5 & 4.5 \\
\hline Well Casing Material & $\mathrm{SS} / \$ 304$ & SS $/ \$ 304$ & Ss $/ \$ 304$ & $\mathrm{sS} / \# 304$ & SS $/ \$ 304$ & ss $/ * 304$ & Ss $/ \$ 304$ \\
\hline MONITORED INTERVAL & $\cdot$ & $\cdot$ & $\bullet$ & $\cdot$ & $\cdot$ & $\cdot$ & • \\
\hline Aquifer zone & $\dot{\mathrm{wT}}$ & WT & $\dot{\text { WT }}$ & BDR & พั & BDR & $\dot{\omega T}$ \\
\hline Geologic Formation & $\mathrm{cm}$ & $\mathrm{Cmn}$ & $\mathrm{Cmn}$ & $C m n$ & $\operatorname{cmn}$ & $\operatorname{comn}$ & ock \\
\hline Screened or Open & Screen & Screen & Screen & Screen & Screen & Screen & Screen \\
\hline Top - Depth & 5.6 & 25.6 & 29.6 & 90 & 67.5 & 117 & 94 \\
\hline Bottom - Depth & 12.5 & 40.3 & 41 & 104 & 80 & 132 & 108 \\
\hline Screen Material & $\mathrm{ss} / \mathrm{sw} / .01$ & $\mathrm{ss} / \mathrm{sw} / .01$ & ss/sw/.01 & $\mathrm{ss} / \mathrm{sw} / .01$ & $\mathrm{ss} / \mathrm{sw} / .01$ & $\mathrm{ss} / \mathrm{sw} / .01$ & $\mathrm{ss} / \mathrm{sw} / .01$ \\
\hline Screen Length & 5.0 & 10.6 & 10.0 & 10.0 & 10.7 & 11.0 & 10.5 \\
\hline Open-Hole Length & - & - & - & - & $\cdot$ & - & - \\
\hline Open-Hole Diameter & $\cdot$ & . & - & - & . & - & - \\
\hline
\end{tabular}

(CONTINUED) 
APPENDIX C

Monitoring-Well Construction Detalis

\begin{tabular}{|c|c|c|c|c|c|c|c|}
\hline \multirow{2}{*}{$\begin{array}{l}\text { Well No. } \\
\text { Location }\end{array}$} & $G W-324$ & $G W-325$ & $G W-342$ & $G W-345$ & GW-347 & $G W-348$ & GW-364 \\
\hline & s3 & s3 & BG & s3 & $\mathbf{s 3}$ & s3 & oLf \\
\hline Bast Coordinate & 50700 & 50689 & 42608.87 & 50700 & 50750 & 50763 & 46508 \\
\hline North Coordinate & 30307 & 30306 & 31326.9 & 30029 & 29292 & 29294 & 29152 \\
\hline ELEVATIONS & . & $\cdot 1$ & $\cdot$ & . & $\cdot$ & $\cdot$ & • \\
\hline Measuring Point & 1003.83 & 1003 & 1087.1 & 999.46 & 1001.56 & 1000.12 & 935.95 \\
\hline Surface & 1001.44 & 1000.53 & 1084.49 & 994.93 & 997.76 & 998 & 933.39 \\
\hline Weathered Rock & . & . & 1064.49 & 985.93 & 971.26 & 962.2 & 911.39 \\
\hline Fresh Rock & 982.44 & $\cdot$ & 1057.49 & 968.63 & $\cdot$ & 961.4 & 903.39 \\
\hline Top Monitored Interval & 934.94 & 995.43 & 1023.49 & 979.73 & 988.26 & 929.9 & 886.39 \\
\hline Bottom Monitored Interval & 921.44 & 982.63 & 1009.49 & 968.63 & 969.96 & 917.1 & 873.09 \\
\hline SURFACE/CONDUCTOR CASING & . &. &. & . & $\cdot$ & • & • \\
\hline Casing Depth & $\dot{25}$ & $\cdot$ & 36 & $\dot{\bullet}$ & $\cdot \dot{1}$ & $46 . \dot{4}$ & 30 \\
\hline Casing Diameter & 10.75 & $\cdot$ & 10.75 & - & $\cdot$ & 10.75 & 11 \\
\hline Casing Material & - & $\cdot$ & $\cdot$ & - &. & unknown & unknown \\
\hline WELL CASING BOREHOLE & - & $\cdot$ & $\cdot$ & $\cdot$ & $\cdot$ & $\cdot$ & • \\
\hline Borehole Depth & 80 & 17.9 & 75 & 26.3 & 27.8 & 80.9 & 60.3 \\
\hline Borehole Diameter & 10 & 10 & 9.5 & 14.5 & 9.5 & 9.5 & 10.6 \\
\hline Weathered Rock - Depth & - & $\cdot$ & 20 & 9 & 26.5 & 35.8 & 22 \\
\hline Fresh Rock - Depth & 19 & $\cdot$ & 27 & 26.3 &. & 36.6 & 30 \\
\hline WEIL CASING & - & . & $\cdot$ &. & . &. & . \\
\hline Well Casing Depth & $\dot{79}$ & 17.3 & 75 & 26.3 & $27 . \dot{8}$ & 80.9 & 60.3 \\
\hline Well Casing Diameter & 4.5 & 4.5 & 4.5 & 4.5 & 4.5 & 4.5 & 4.5 \\
\hline Well Casing Material & SS $/ 1304$ & Ss $/ \$ 304$ & ss $/ \$ 304$ & ss $/ 1304$ & ss $/ \$ 304$ & $\mathrm{ss} / 1304$ & ss $/ \$ 304$ \\
\hline MONITORED INTERVAL & • & $\cdot$ & $\cdot$ & . & - &. & • \\
\hline Aquifer Zone & BDR & wr & BDR & wT & $\dot{\omega T}$ & $\mathrm{BDR}$ & BDR \\
\hline Geologic Formation & Cn & $\mathrm{Cn}$ & Cpv & $\mathrm{cn}$ & Cmn & $\mathrm{cmn}$ & $\operatorname{Cmn}$ \\
\hline Screened or Open & Ssreen & Screen & Screen & Screen & Screen & Screen & Screen \\
\hline Top - Depth & 66.5 & 5.1 & 61 & 15.2 & 9.5 & 68.1 & 47 \\
\hline Bottom - Depth & 80 & 17.9 & 75 & 26.3 & 27.8 & 80.9 & 60.3 \\
\hline Screen Material & SS/EW/.01 & SS/sw/.01 & SS/sw/.01 & $\mathrm{ss} / \mathrm{sw} / .01$ & $\mathrm{ss} / \mathrm{sw} / .01$ & $\mathrm{ss} / \mathrm{sw} / .01$ & SS/sw/.01 \\
\hline Screen Length & 10.5 & 10.0 & 10.0 & 10.0 & 15.8 & 10.4 & 10.0 \\
\hline Open-Hole Length & • & $\cdot$ & . & 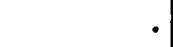 & $\cdot$ & $\cdot$ & • \\
\hline Open-Hole Diameter & 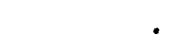 & . & . & . & . & $\cdot$ & • \\
\hline
\end{tabular}


APPENDIX $C$

Monitoring-Well Construction Details

\begin{tabular}{|c|c|c|c|c|c|c|c|}
\hline \multirow{2}{*}{$\begin{array}{l}\text { Well No. } \\
\text { Location }\end{array}$} & $G W-365$ & $G w-370$ & GW-371 & GW-372 & GW-373 & GW-526 & GW-537 \\
\hline & OLF & BG & BG & BG & BG & $\mathbf{s 3}$ & orf \\
\hline East Coordinate & 46490 & 44989 & 45036 & 45257 & 45271 & 50708 & 49539.23 \\
\hline North Coordinate & 29150 & 30241 & 30245 & 30673 & 30679 & 30033 & 30057.19 \\
\hline ELEVATIONS & $\cdot$ & $\cdot$ & $\cdot$ & $\cdot$ & $\cdot$ & $\cdot$ & • \\
\hline Measuring Point & 935.58 & 960.62 & 960.22 & 983.53 & 982.8 & 997.45 & 976.24 \\
\hline Surface & 933.03 & 957.94 & 958.13 & 979.87 & 980.75 & 995.34 & 974.19 \\
\hline Weathered Rock & 922.03 & 946.94 & 950.63 & 965.37 & 959.75 & 991.84 & 959.29 \\
\hline Fresh Rock & 918.03 & 932.94 & 933.13 & 932.87 & 905.75 & 971.74 & • \\
\hline Top Monitored Interval & 806.33 & 936.84 & 848.13 & 940.07 & 857.75 & 894.34 & 969.39 \\
\hline Bottom Monitored Interval & 783.03 & 924.84 & 833.13 & 928.27 & 822.75 & 872.34 & 950.89 \\
\hline SURFACE/CONDUCTOR CASING & $\cdot$ & $\cdot$ & $\cdot$ & $\cdot$ & $\cdot$ & $\cdot$ & • \\
\hline Casing Depth & 18.5 & $\cdot$ & 36 & $\cdot$ & 34.5 & 23.6 & • \\
\hline Casing Diameter & 11.75 &. & 10.75 & $\cdot$ & 10.75 & 10.75 & • \\
\hline Casing Material & unknown & $\cdot$ & unknown & $\cdot$ & unknown & unknown & • \\
\hline WELL CASING BOREHOLE & $\cdot$ & $\cdot$ & $\cdot$ &. & . & $\cdot$ & • \\
\hline Borehole Depth & 126.7 & $33 . \dot{1}$ & 110 & $51 . \dot{6}$ & $12 \dot{3}$ & 101 & 24.5 \\
\hline Borehole Diameter & 10.75 & 9.5 & 9.5 & 9.5 & 9.5 & 9.5 & 8.75 \\
\hline Weathered Rock - Depth & 11 & 11 & 7.5 & 14.5 & 21 & 3.5 & 14.9 \\
\hline Fresh Rock - Depth & 15 & 25 & 25 & 47 & 75 & 23.6 & • \\
\hline WELI CASING & $\cdot$ & $\cdot$ & $\cdot$. & $\cdot$ & • & $\cdot$ & $\cdot$ \\
\hline Well Casing Depth & 126.7 & 33.1 & 110 & 51.6 & 123 & 101 & 23.3 \\
\hline Well Casing Diameter & 6.62 & 4.5 & 6.37 & 4.5 & 6.62 & 6.62 & 4.5 \\
\hline Well Casing Material & Steel/F25 & SS/\$304 & Steel/F25 & SS $/ \$ 304$ & Steel/F25 & Steel/F25 & SS $/ \$ 304$ \\
\hline MONITORED INTERVAL & $\cdot$ & . & $\cdot$ & $\cdot$ & $\cdot$ & $\cdot$ & • \\
\hline Aquifer Zone & BDR & WT & BDR & WT & BDR & BDR & พั \\
\hline Geologic Formation & $\operatorname{comn}$ & $\mathrm{cm}$ & $\mathrm{cm}$ & $\mathrm{cm}$ & $\mathrm{Cm}$ & $\mathrm{Cn}$ & $\mathrm{Cn}$ \\
\hline Screened or open & Open & Screen & Open & Screen & Open & Open & Screen \\
\hline Top - Depth & 126.7 & 21.1 & 110 & 39.8 & 123 & 101 & 4.8 \\
\hline Bottom - Depth & 150 & 33.1 & 125 & 51.6 & 158 & 123 & 23.3 \\
\hline Screen Material & $\cdot$ & $\mathrm{ss} / \mathrm{sw} / .01$ & $\cdot$ & $\mathrm{ss} / \mathrm{sw} / .01$ & - & • & ss/sw/.01 \\
\hline Screen Length & $\cdot$ & 10.0 & $\cdot$ & 10.0 & - & - & 15.0 \\
\hline Open-Hole Length & 23.3 & $\cdot$ & 15 & $\cdot$ & 35 & 22 & • \\
\hline Open-Hole Diameter & 6 & $\cdot 1$ & 6 & $\cdot$ & 6 & 6.1 & • \\
\hline
\end{tabular}

(CONTINUED) 
APPENDIX C

Monitoring-Well Construction Details

\begin{tabular}{|c|c|c|c|c|c|c|c|}
\hline \multirow{2}{*}{$\begin{array}{l}\text { Well No. } \\
\text { Location }\end{array}$} & $G W-601$ & GW-613 & GW-614 & $G W-621$ & GW-626 & GW-627 & $G W-636$ \\
\hline & OLF & s3 & s3 & BG & BG & BG & OLF \\
\hline East Coordinate & 47629.49 & 50231.77 & 50223.05 & 45032.91 & 42772.01 & 42774.1 & 46412.64 \\
\hline North Coordinate & 28902.55 & 30406.77 & 30425.77 & 29023.3 & 29535.32 & 29505.05 & 29482.3 \\
\hline ELBVATIONS & - & - & - & - &. & - & . \\
\hline Measuring Point & 1002.2 & 1013.37 & 1012.22 & 925.24 & 942.61 & 942.85 & 941.92 \\
\hline Surface & 999.09 & 1010.17 & 1009.39 & 923.07 & 939.95 & 940.39 & 938.08 \\
\hline Weathered Rock & 991.09 & 995.17 & 996.89 & 901.07 & 937.95 & 937.39 & 930.08 \\
\hline Fresh Rock & 945.09 & - & 958.59 & . & 875.95 & 897.39 & 922.08 \\
\hline Top Monitored Interval & 680.59 & 980.17 & 937.79 & 899.77 & 876.95 & 686.39 & 839.58 \\
\hline Bottom Monitored Interval & 643.09 & 968.17 & 919.19 & 882.57 & 861.95 & 670.39 & 821.08 \\
\hline SURFACE/CONDUCTOR CASING & • & • & $\cdot$ & • &. & $\cdot$ & • \\
\hline Casing Depth & 41.4 & 16.8 & $44 . \dot{4}$ & 24 & 62.5 & 47.5 & 23 \\
\hline Casing Diameter & 11.75 & 10.75 & 10.75 & 10.75 & 11.75 & 11.75 & 11.75 \\
\hline Casing Material & steel & steel & steel & Steel & steel & Steel & Steel \\
\hline WELI CASING BOREHOLE & • & • & $\cdot$ & • & $\cdot$ & . & • \\
\hline Borehole Depth & 319 & $\dot{42}$ & $90 . \dot{2}$ & 43 & $\dot{78}$ & $25 \dot{\bullet}$ & 99 \\
\hline Borehole Diameter & 10.625 & 9.5 & 9.5 & 7.875 & 9.5 & 10.625 & 10.75 \\
\hline Weathered Rock - Depth & 8 & 15 & 12.5 & 22 & 2 & 3 & 8 \\
\hline Fresh Rock - Depth & 54 & • & 50.8 & - & 64 & 43 & 16 \\
\hline WELL CASIME & - & • & $\bullet$ & • &. & $\cdot$ & • \\
\hline Well Casing Depth & $318 . \dot{5}$ & $\dot{42}$ & $90 . \dot{2}$ & $40 . \dot{5}$ & $\ddot{78}$ & 254 & 98.5 \\
\hline Well Casing Diameter & 7 & 4.5 & 4.5 & 4.5 & 4.5 & 7 & 7 \\
\hline Well Casing Material & Steel & SS $/ \$ 304$ & Ss/ $/ 304$ & SS $/ 1304$ & Ss $/ 1304$ & Steel & Steel \\
\hline MONITORED INTERVAL & $\cdot$ & • & $\dot{\bullet}$ & • & $\cdot$ & $\cdot$ & • \\
\hline Aguifer zone & BDR & $\dot{W T}$ & ${ }_{\mathrm{BDR}}^{\bullet}$ & $\dot{\text { WT }}$ & $\dot{w r}$ & $\dot{B D R}$ & BDF \\
\hline Geologic Formation & $\mathrm{cmn}$ & $\mathrm{Cn}$ & $\mathrm{cn}$ & $\mathrm{cms}$ & $\mathrm{Cn}$ & $\mathrm{Cn}$ & $\mathrm{cr}$ \\
\hline Screened or Open & Open & Screen & Screen & Screen & Screen & Open & Open \\
\hline Top - Depth & 318.5 & 30 & 71.6 & 23.3 & 63 & 254 & 98.5 \\
\hline Bottom - Depth & 356 & 42 & 90.2 & 40.5 & 78 & 270 & 117 \\
\hline Screen Material & - & $\mathrm{ss} / \mathrm{sw} / .01$ & Ss/sw/.01 & ss/sw/.01 & $\mathrm{ss} / \mathrm{sw} / .01$ &. & . \\
\hline Screen Length & - & 10.3 & 15.0 & 15.7 & 10.0 & . & . \\
\hline Open-Hole Length & 37.5 & - & . & . &. & 16 & 18.5 \\
\hline Open-Hole Diameter & 6.25 & - & - & - & $\cdot$ & 6.25 & 6.25 \\
\hline
\end{tabular}

(CONTINUED) 
APPENDIX $C$

Monitoring-Well Construction Detalls

\begin{tabular}{|c|c|c|c|c|c|c|c|}
\hline We11 No. & $G W-637$ & $G W-638$ & Gw-639 & $6 w-640$ & $G w-641$ & $G w-642$ & $G W-643$ \\
\hline Iocation & OLF & OLF & BG & BG & BQ & BG & BG \\
\hline Bast Coordinate & 46402.39 & 46388.51 & 45259.76 & 45252.92 & 45250.72 & 44654.05 & 45253.26 \\
\hline North Coordinate & 29492.81 & 29485.32 & 29626.12 & 29670.1 & 29685.73 & 31215.27 & 30656.47 \\
\hline ETEVATIONS & . & $\cdot$ & - & - & - & - & - \\
\hline Measuring Point & 941.83 & 941.77 & 940.7 & 945.38 & 946.66 & 1014.67 & 982.42 \\
\hline Surface & 938.26 & 938.14 & 937.98 & 942.59 & 944.01 & 1011.93 & 979.9 \\
\hline Weathered Rock & 928.26 & 930.14 & 934.98 & 939.59 & 933.01 & 1010.93 & 979.4 \\
\hline Fresh Rock & & $\cdot$ & 917.98 & 917.59 & - & 991.93 & - \\
\hline Top Nonitored Interval & 923.76 & 935.64 & $842 \cdot 48$ & 907.59 & 933.01 & 993.43 & 963.4 \\
\hline Bottom Monitored Interval & 910.76 & 926.64 & 812.48 & 895.19 & 919.71 & 975.03 & $951 \cdot 4$ \\
\hline SURFACE/CONDUCTOR CASING & $\cdot$ & $\cdot$ & - & - & - & $\cdot$ & - \\
\hline Casing Depth & 15 & . & 31 & 35.5 & - & 21.5 & - \\
\hline Casing Diameter & 10.75 & $\cdot$ & 11 & 10.75 & - & 10.75 & - \\
\hline Casing Material & Steel & $\cdot$ & Steel & Steel & - & Steel & - \\
\hline WELL CASING BORBHOLE & $\cdot$ & $\cdot$ & $\cdot$ & $\cdot$ & - & - & - \\
\hline Borehole Depth & 27.5 & 12 & 95.5 & 47.4 & 24.3 & 36.9 & 29.3 \\
\hline Borehole Diameter & 10 & 10 & 10 & 9.5 & 9.5 & 9.5 & 14 \\
\hline Weathered Rock - Depth & 10 & 8 & 3 & 3 & 11 & 1 & 0.5 \\
\hline Eresh Rock - Depth & $\therefore$ & $\cdot$ & 20 & 25 & $\cdot$ & 20 & - \\
\hline WELL CASING & $\cdot$ & $\cdot$ & $\cdot$ & $\cdot$ & $\cdot$ & - & - \\
\hline well Casing Depth & 27.5 & 11.5 & 95.5 & 47.4 & 24.3 & 36.9 & 28.5 \\
\hline Well Casing Diameter & 4.5 & 4.5 & 7 & 4.5 & 4.5 & 4.5 & 4.5 \\
\hline Well Casing Material & SS/\$304 & $s s / \$ 304$ & steel & SS $/ 304$ & SS/ $/ 304$ & ss $/ 304$ & ss/\$304 \\
\hline MONITORED INTERVAL & $\cdot$ & $\cdot$ & $\cdot$ & $\cdot$ & $\cdot$ & $\cdot$ & - \\
\hline Aquifer Zone & WT & WT & BDR & BDR & WT & WT & WT \\
\hline Geologic Formation & $\mathrm{Cn}$ & $\mathrm{Cn}$ & $\mathrm{Cn}$ & $\mathrm{Cn}$ & $\mathrm{Cn}$ & Crg & $\mathrm{Cm}$ \\
\hline Screened or Open & Screen & Screen & Open & Screen & Screen & Screen & Screen \\
\hline Top - Depth & 14.5 & 2.5 & 95.5 & 35 & 11 & 18.5 & 16.5 \\
\hline Bottom - Depth & 27.5 & 11.5 & $125 \cdot 5$ & 47.4 & 24.3 & 36.9 & 28.5 \\
\hline Screen Material & $\mathrm{SS} / \mathrm{sw} / .01$ & $\mathrm{ss} / \mathrm{sw} / .01$ & - & SS/Ew/.01 & SS/8w/.01 & $\mathrm{ss} / \mathrm{sw} / .01$ & $\mathrm{ss} / \mathrm{sw} / .01$ \\
\hline Screen Length & 10.0 & 5.0 & - & 11.1 & 10.4 & 16.2 & 10.6 \\
\hline Open-Hole Length & $\cdot$ & - & 30 & - & $\cdot$ & - & - \\
\hline Open-Hole Diameter & - & $\cdot$ & 6.25 & $\cdot$ & $\cdot$ & - & - \\
\hline
\end{tabular}

( CONTINUED) 
APPENDIX C

Monitoring-Well Conetruction Detalla

\begin{tabular}{|c|c|c|c|c|c|c|c|}
\hline \multirow{2}{*}{$\begin{array}{l}\text { Well No. } \\
\text { Location }\end{array}$} & $G w-644$ & GW-647 & $G W-651$ & $G W-652$ & $6 w-653$ & $G W-654$ & $G w-655$ \\
\hline & OLF & OLF & BG & BG & BG & BG & BG \\
\hline Rast Coordinate & 46608.69 & 48770.23 & 42535.39 & 42452.49 & 42317.29 & 41907.28 & 42907.49 \\
\hline North Coordinate & 29945.31 & 28940.89 & 29042.93 & 29029.46 & 29660.39 & 30548.76 & 31403.07 \\
\hline ELEVATIONS & . & . &. & . & . & . & - \\
\hline Measuring Point & 959.75 & 1033.27 & 903.12 & 900.83 & 931.6 & 940.79 & 1111.42 \\
\hline Surface & 956.78 & 1030.14 & 900.05 & 897.98 & 928.85 & 938.26 & 1108.2 \\
\hline Weathered Rock & 953.58 & 1020.14 & 892.55 & 888.48 & 925.35 & 934.26 & 1099.2 \\
\hline Fresh Rock & 940.28 & 980.14 & $\cdot$ & . &. &. & 1061.2 \\
\hline Top Monitored Interval & 946.48 & 952.14 & 860.25 & 884.58 & 902.55 & 934.06 & 1060.5 \\
\hline Bottom Monitored Interval & 933.38 & 939.14 & 848.05 & 866.78 & 889.85 & 922.96 & 1043.2 \\
\hline SURFACE/CONDUCTOR CASING &. & $\cdot$ &. & $\cdot$ & $\cdot$ &. & - \\
\hline Casing Depth & $\cdot$ & $15 . \dot{5}$ & 39 & $12 . \dot{6}$ & $\cdot$ & $\cdot$ & • \\
\hline Casing Diameter &. & 10.75 & 10.75 & 10.75 &. & $\cdot$ & • \\
\hline Casing Material & $\cdot$ & Steel & Steel & Steel & $\cdot$ & $\cdot$ & - \\
\hline WELI CASING BOREHOLE & $\cdot$ & $\cdot$ & • & . & $\cdot$ & $\cdot$ & - \\
\hline Borehole Depth & 23.4 & 91 & 52 & 31.2 & 39 & 15.5 & 65 \\
\hline Borehole Diameter & 9.5 & 9.5 & 9.5 & 9.5 & 9.5 & 9.5 & 9.5 \\
\hline Weathered Rock - Depth & 3.2 & 10 & 7.5 & 9.5 & 3.5 & 4 & 9 \\
\hline Fresh Rock - Depth & 16.5 & 50 & $\cdot$ &. & $\cdot$ & $\cdot$ & 47 \\
\hline WELL CASING & $\cdot$ & $\cdot$ & . &. &. &. & • \\
\hline Well Casing Depth & 23.4 & $\dot{91}$ & $\dot{52}$ & 31.2 & 39 & $15 \cdot 3$ & $\dot{65}$ \\
\hline Well Casing Diameter & 4.5 & 4.5 & 4.5 & 4.5 & 4.5 & 4.5 & 4.5 \\
\hline Well Casing Material & $S S / \$ 304$ & SS $/ \$ 304$ & $\mathrm{SS} / \# 304$ & ss $/ 1304$ & ss $/ \$ 304$ & SS $/ \$ 304$ & $s 5 / \$ 304$ \\
\hline MONITORED INTERVAL & $\cdot$ & $\cdot$ & - & $\cdot$ & . & $\cdot$ & • \\
\hline Aquifer zone & $\dot{\mathrm{wT}}$ & BDR & $\dot{\omega T}$ & $\dot{\mathrm{wr}}$ & $\dot{\omega r}$ & $\dot{w r}$ & BDR \\
\hline Geologic Formation & $\mathrm{Cn}$ & $\mathrm{cmn}$ & $\mathrm{cmn}$ & $\mathrm{Cmn}$ & $\mathrm{Cn}$ & $\mathrm{Cm}$ & Cpv \\
\hline Screened or Open & Screen & Screen & Screen & Screen & Screen & Screen & Screen \\
\hline Top - Depth & 10.3 & 78 & 39.8 & 13.4 & 26.3 & 4.2 & 47.7 \\
\hline Bottom - Depth & 23.4 & 91 & 52 & 31.2 & 39 & 15.3 & 65 \\
\hline Screen Material & $\mathrm{ss} / \mathrm{sw} / .01$ & $\mathrm{ss} / \mathrm{sw} / .01$ & $\mathrm{ss} / \mathrm{sw} / .01$ & ss/sw/.01 & $\mathrm{ss} / \mathrm{sw} / .01$ & $\mathrm{ss} / \mathrm{sw} / .01$ & ss/sw/.01 \\
\hline Screen Length & 10.3 & 20.0 & 10.0 & 16.2 & 10.0 & 10.0 & 15.9 \\
\hline Open-Hole Length & $\cdot$ & $\cdot$ & $\cdot$ & - & $\cdot$ & $\cdot$ & . \\
\hline Open-Hole Diameter & . & $\cdot$ & . & . & $\cdot$ &. & • \\
\hline
\end{tabular}

(CONTINUED) 
APPENDIX C

Monitoring-Well Construction Detalls

\begin{tabular}{|c|c|c|c|c|c|c|c|}
\hline Well No. & $G W-683$ & $G w-684$ & OW-685 & $6 w-694$ & OW-695 & $6 w-703$ & OW-704 \\
\hline Location & EXP & EXP & $\mathbf{E X P}$ & $\mathbf{E X P}$ & $\mathbf{E X P}$ & $\mathbf{E X P}$ & $\mathbf{8 X P}$ \\
\hline Bast Coordinate & 41552.33 & 41353.53 & 41447.78 & 44893.28 & 44868.3 & 44930.51 & 44934.98 \\
\hline North Coordinate & 28281.78 & 28524.52 & 28667.4 & 28844.77 & 28844.73 & 28806.34 & 28844.67 \\
\hline ELEVATIONS & - & - & - & - & $\cdot$ & $\cdot$ & $\cdot$ \\
\hline Measuring Point & 972.06 & 898.66 & 891.51 & 941.38 & 939.37 & 954.69 & 944.73 \\
\hline surface & 969.45 & 895.53 & 889.28 & 938.58 & 937.22 & 951.8 & 941.99 \\
\hline Weathered Rock & 947.45 & $\cdot$ & 884.28 & 927.58 & 931.22 & 944.8 & 925.99 \\
\hline Fresh Rock & 943.45 & 886.53 & 873.78 & 917.58 & 919.22 & 941.8 & 918.99 \\
\hline Top Monitored Interval & 835.55 & 789.13 & 800.78 & 784.58 & 886.62 & 816.8 & 695.99 \\
\hline Bottom Monitored Interval & 772.65 & 767.13 & 750.98 & 734.08 & 874.62 & 769.8 & 685.99 \\
\hline SURPACE/CONDUCTOR CASING & $\cdot$ & $\cdot$ & $\bullet$ & $\cdot$ & $\cdot$ & $\cdot$ & $\cdot \dot{ }$ \\
\hline Casing Depth & 82 & 87 & $\cdot$ & 25.8 & 22.5 & $\cdot \dot{ }$ & $2 \dot{1}$ \\
\hline Casing Diameter & 11.75 & 11.75 & . & 23.75 & 11.75 & - & 11.75 \\
\hline Casing Material & Steel & Steel & • & Steel & steel & $\cdot$ & steel \\
\hline WELL CASING BOREHOLE & $\cdot \dot{ }$ & $\cdot$ & $\cdot$ &. &. & $\cdot$ & $\cdot$ \\
\hline Borehole Depth & 197.5 & 129.6 & $88 . \dot{5}$ & 154 & 62.6 & 135 & $24 \dot{ }$ \\
\hline Borehole Diameter & 10.625 & 10.5 & 10.625 & 10.6 & 9.875 & 10.625 & 10.625 \\
\hline Weathered Rock - Depth & 22 & $\cdot$ & 5 & 11 & 6 & 7 & 16 \\
\hline Fresh Rock - Depth & 26 & 9 & 15.5 & 21 & 18 & 10 & 23 \\
\hline WELL CASING & $\cdot$ & $\cdot$ & . & . & $\cdot$ & - & - \\
\hline Well Casing Depth & $197 . \dot{1}$ & 128.8 & $88 . \dot{3}$ & 153 & $62 . \dot{6}$ & $133.8^{\circ}$ & 244.5 \\
\hline well Casing Diameter & 4.5 & 4.5 & 7 & 7 & 4.5 & 7 & 7 \\
\hline Well Casing Material & ss $/ \$ 304$ & SS $/ \$ 304$ & Steel/F25 & Steel/F25 & SS $/: 304$ & Steel/F25 & Steel/F25 \\
\hline MONITORED INTERVAL & $\cdot 1$ & $\cdot$ & . &. & $\cdot$ & - & - \\
\hline Aquifer zone & BDR & BDR & BDR & BDR & $\begin{array}{r}\dot{B} \\
\mathrm{BDR}\end{array}$ & BDR & BDR \\
\hline $\begin{array}{l}\text { Aquifer zone } \\
\text { Geologic Formation }\end{array}$ & ock & $\mathrm{Cmn}$ & $\mathrm{cmn}$ & $\mathrm{cmn}$ & ock & $\mathrm{cmn}$ & $\mathrm{Cmn}$ \\
\hline Screened or Open & Screen & Screen & Open & Open & Screen & Open & Open \\
\hline Top - Depth & 133.9 & 106.4 & 88.5 & 154 & 50.6 & 135 & 246 \\
\hline Bottom - Depth & 196.8 & 128.4 & 138.3 & 204.5 & 62.6 & 182 & 256 \\
\hline Screen Material & $\mathrm{ss} / \mathrm{pp} / .01$ & $\mathrm{ss} / \mathrm{pp} / .01$ & $\cdot$ & $\cdot$ & ss/sw/.01 & - & - \\
\hline Screen Length & 50.8 & 14.6 & $\cdot 1$ & $\cdot$ & 10.0 & - & - \\
\hline Open-Hole Length & $\cdot$ & $\cdot$ & 49.8 & 50.5 & $\cdot$ & 47 & 10 \\
\hline Open-Hole Diameter & $\cdot$ & $\bullet$ & 6.625 & 6.25 & $\cdot$ & 6.25 & 6.5 \\
\hline
\end{tabular}

(CONTINUED) 
APPENDIX C

Monitoring-Well Construction Details

\begin{tabular}{|c|c|c|c|c|c|c|c|}
\hline \multirow{2}{*}{$\begin{array}{l}\text { Well No. } \\
\text { Location }\end{array}$} & 6w-706 & $0 w-710$ & $6 W-711$ & $6 W-712$ & $6 W-713$ & GW-714 & $G W-715$ \\
\hline & $\mathbf{E x p}$ & EXP & EXP & EXP & $\operatorname{ExP}$ & $\mathbf{E X P}$ & $\mathbf{E X P}$ \\
\hline Bast Coordinate & 44943.63 & 36470.81 & 36535.38 & 36506.87 & 36434.4 & 36435.09 & 36453.11 \\
\hline North Coordinate & 28946.41 & 27645.05 & 27872.8 & 28232.52 & 28235.95 & 28421.56 & 28424.58 \\
\hline ELEVATIONS & - & $\cdot$ & . & $\cdot$ & $\cdot$ & $\cdot$ & $\cdot$ \\
\hline Measuring Point & 928.67 & 911.26 & 905.2 & 877.09 & 880.63 & 875.08 & 874.72 \\
\hline Surface & 925.78 & 908.03 & 901.96 & 873.61 & 877.83 & 872.3 & 872.17 \\
\hline Weathered Rock & 908.78 & $\cdot$ & 886.96 & 861.61 & 851.03 & 845.3 & 838.17 \\
\hline Fresh Rock & 898.78 & 904.53 & 881.46 & 807.61 & 814.03 & 837.3 & • \\
\hline Top Monitored Interval & 768.78 & 368.33 & 285.96 & 432.11 & 572.83 & 757.2 & 840.17 \\
\hline Bottom Monitored Interval & 743.28 & 163.53 & 235.76 & 416.11 & 562.63 & 727.3 & 828.17 \\
\hline SURFACE/CONDUCTOR CASING & $\cdot$ & $\cdot$ & $\cdot$ & $\cdot$ & $\cdot 1$ & $\cdot$ & . \\
\hline Casing Depth & 40.3 & 83.4 & 17 & 44.8 & 50 & 40.5 & 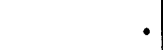 \\
\hline Casing Diameter & 11.75 & 11.75 & 11.75 & 11.75 & 11.75 & 11.75 & 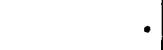 \\
\hline Casing Material & steel & Steel & Steel & Steel & $\cdot$ & steel & - \\
\hline WELL CASIMG BOREHOLE & - & $\cdot$ & $\cdot$ & $\cdot$ & $\cdot$ & $\cdot$ & - \\
\hline Borehole Depth & 157 & 539.7 & 616 & 441.5 & 305 & 115.1 & 44.6 \\
\hline Borehole Dianieter & 10.6 & 10.6 & 10.6 & 10.6 & 10.6 & 10.6 & 10.6 \\
\hline Weathered Rock - Depth & 17 & $\cdot$ & 15 & 12 & 26.8 & 27 & 34 \\
\hline Fresh Rock - Depth & 27 & 3.5 & 20.5 & 66 & 63.8 & 35 & • \\
\hline WRLL CASING & - &. & $\cdot$ & $\cdot$ & $\cdot$ & $\cdot$ & - \\
\hline Well Casing Depth & $156 . \dot{1}$ & 539.7 & 616 & $451 . \dot{5}$ & 305 & 113.8 & 33.1 \\
\hline Well Casing Diameter & 7 & 7 & 7 & 7 & 7 & 7 & 4.25 \\
\hline Well Casing Material & Steel/F25 & Steel & Steel & Steel & Steel/F25 & Steel & Ss $/ \$ 304$ \\
\hline MONITORED INTERVAL & $\cdot$ &. & $\cdot$ &. & $\cdot$ & $\cdot$ & • \\
\hline Aquifer Zone & BDR & BDR & BDR & ISDR & BDR & BDR & $\dot{\text { WT }}$ \\
\hline Geologic Formation & $\operatorname{cman}$ & ock & $\operatorname{cmn}$ & ock & $\operatorname{cmn}$ & $\mathrm{cmn}$ & $\mathrm{Cmn}$ \\
\hline Screened or Open & Open & Open & Open & oren & Open & Open & Screen \\
\hline Top - Depth & 157 & 539.7 & 616 & 441.5 & 305 & 115.1 & 32 \\
\hline Bottom - Depth & 182.5 & 744.5 & 666.2 & 457.5 & 315.2 & 145 & 44 \\
\hline Screen Material & $\cdot$ & $\cdot$ & $\cdot$ & $\cdot$ & $\cdot$ & $\cdot$ & ss/sw/.01 \\
\hline Screen Length & $\cdot$ & $\cdot$ & $\cdot$ &. & $\cdot$ & . & 9.9 \\
\hline Open-Hole Length & 25.5 & 204.8 & 50.2 & 16 & 10.2 & 29.9 & • \\
\hline Open-Hole Diameter & 6.25 & 6.25 & 6.25 & 6.25 & 6.25 & 6.25 & . \\
\hline
\end{tabular}


APpENDIX C

Monitoring-Well Construction Detal1s

\begin{tabular}{|c|c|c|c|c|c|c|c|}
\hline We11 No. & $6 w-723$ & OW-724 & $G w-725$ & $G W-736$ & $6 w-737$ & $G W-738$ & $6 w-739$ \\
\hline Iocation & $\mathbf{E X P}$ & $\mathbf{E X P}$ & EXP & $\mathbf{E X P}$ & $\mathbf{E X P}$ & $\mathbf{B X P}$ & $\mathbf{E X P}$ \\
\hline Bast Coordinate & 49088.55 & 48995.17 & 48989.13 & 48936 & 48890 & 49026 & 49126 \\
\hline North Coordinate & 29006.44 & 29198.24 & 29405.49 & 29381 & 29365 & 29150 & 29010 \\
\hline EIEVATIONS & - & . & . & - & . &. & - \\
\hline Mageuring Point & 1022.23 & 979.27 & 961.05 & 960.12 & 959.91 & 983.08 & 1023.74 \\
\hline Surface & 1019.31 & 976.62 & 958.26 & 957.55 & 957.5 & 980.36 & 1020.66 \\
\hline Weathered Rock & 992.61 & 943.12 & 944.26 & 950.05 & . & 968.36 & 986.66 \\
\hline Prash Rock & 989.61 & 936.62 & 940.76 & 945.55 & 943.5 & 965.26 & 978.66 \\
\hline Top Monitored Interval & 678.71 & 687.02 & $825 \cdot 76$ & 865.55 & 878.5 & 916.86 & 731.46 \\
\hline Bottom Monitored Interval & 574.81 & 675.02 & 815.76 & 852.55 & 868 & 892.36 & 700.66 \\
\hline SURPACE/CONDUCTOR CASING & $\cdot$ & $\cdot$ & $\cdot$ & $\bullet$ & $\cdot$ & $\cdot$ & • \\
\hline Casing Depth & 39 & 40 & 21 & 21.5 & 22 & 16.5 & 60.3 \\
\hline Casing Diameter & 11.75 & 11.75 & $11 \cdot 75$ & $11 \cdot 75$ & 11.75 & 11.75 & 11.75 \\
\hline Casing Material & steel & Steel & Steel & . & - & - & • \\
\hline WBLE CASING BOREHOLE & - & - & . & . & . & $\cdot$ & - \\
\hline Borehole Depth & 340.6 & 289.6 & $132 \cdot 5$ & $102 \cdot 5$ & 89.5 & 90.1 & 289.2 \\
\hline Borehole Diameter & 10.6 & 10.6 & 10.6 & 10.6 & 10.6 & 10.6 & 10.6 \\
\hline Weathered Rock - Depth & 26.7 & 33.5 & 14 & 7.5 & $\cdot$ & 12 & 34 \\
\hline Fresh Rock - Depth & 29.7 & 40 & $17 \cdot 5$ & 12 & 14 & 15.1 & 42 \\
\hline WBIL CASING & . & . &. & . & $\cdot$ & $\cdot$ & - \\
\hline Well Casing Depth & 340.6 & 289.6 & 132.5 & 102.5 & 89.5 & 87.5 & 289.2 \\
\hline Well Casing Diameter & 7 & 7 & 7 & 4.5 & 4.5 & 4.5 & 7 \\
\hline Well Casing Material & steel & Steel & steel & SS $/ \$ 304$ & ss $/ \$ 304$ & $\mathrm{ss} / \$ 304$ & steel \\
\hline IITORED INTERVAL & $\cdot$ & - & . & - & $\cdot$ & . & - \\
\hline ifer zone & BDR & BDR & BDR & BDR & BDR & BDR & BDR \\
\hline veologic Formation & $\operatorname{cmn}$ & $\operatorname{cmn}$ & $\mathrm{Cmn}$ & Cmn & $\mathrm{Cmn}$ & $\operatorname{cmn}$ & $\mathrm{Cmn}$ \\
\hline Screened or Open & Open & Open & Open & Screen & Screen & Screen & Open \\
\hline Top - Depth & 340.6 & 289.6 & $132 \cdot 5$ & 92 & 79 & 63.5 & 289.2 \\
\hline Bottom - Depth & 444.5 & 301.6 & $142 \cdot 5$ & 105 & 89.5 & 88 & 320 \\
\hline Screen Material & . & - & $\cdot$ & $\mathrm{sS} / \mathrm{sw} / .01$ & $\mathrm{ss} / \mathrm{sw} / .01$ & $\mathrm{ss} / \mathrm{sw} / .01$ & - \\
\hline Screen Length & - & - & $\cdot$ & 10.1 & 10.1 & 20.2 & • \\
\hline Open-Hole Length & 103.9 & 12 & 10 & . & . &. & 30.8 \\
\hline Open-Hole Diameter & 6.25 & 6.25 & 6.25 & . & - & . & 6.25 \\
\hline
\end{tabular}

(CONTINUED) 
APPEKDIX C

Monitoring-Well Conmtruction Detalle

\begin{tabular}{|c|c|c|c|c|c|}
\hline Wa11 No. & OW-740 & OW-793 & GW-794 & GW-795 & $G W-800$ \\
\hline Location & $\operatorname{Exp}$ & AOLLSF & AGLLSF & AGLLSF & oLf \\
\hline Bast Coordinate & 49055 & 46831.52 & 46392.92 & 45630.06 & 48260.3 \\
\hline North Coordinate & 29027 & 30235.82 & 29273.17 & 29286.69 & 28981.6 \\
\hline ELEVATIONS & - & $\cdot$ & - & • & • \\
\hline Measuring Point & 1019.63 & 965.58 & 931.55 & 923.57 & 964.36 \\
\hline surface & 1016.95 & 965.05 & 930.91 & 922.92 & 961.4 \\
\hline Weathered Rock & 978.85 & 954.05 & 927.41 & 917.92 & $\cdot$ \\
\hline Preah Rock & 971.85 & 951.05 & 917.61 & 910.92 & - \\
\hline Top Monitored Interval & 851.35 & 948.15 & 905.11 & 915.42 & 942.1 \\
\hline Bottom Monltored Interval & 826.95 & 936.05 & 891.61 & 902.82 & 929.9 \\
\hline SURFACE/CONDUCTOR CASING & $\cdot$ & $\cdot$ & • & • & $\cdot$ \\
\hline Casing Depth & 46.9 & $\cdot$ & $\cdot$ & - & $\cdot$ \\
\hline Casing Diameter & 11.75 & $\cdot$ & • & - & $\cdot$ \\
\hline Casing Material & $\cdot$ & $\cdot 1$ & - & - & $\cdot$ \\
\hline WELL CASING BORBHOLE & $\cdot$ & $\cdot$ & $\cdot$ & • & $\cdot$ \\
\hline Borehole Depth & 165.6 & 29 & 39.3 & 20.1 & 32 \\
\hline Borehole Diameter & 10.6 & 9.9 & 9.9 & 9.9 & 10.63 \\
\hline Weathered Rock - Depth & 38.1 & 11 & 3.5 & 5 & $\cdot$ \\
\hline Fresh Rock - Depth & 45.1 & 14 & 13.3 & 12 & $\cdot$ \\
\hline WELI CASING & $\cdot$ & $\cdot$ & $\cdot$ & $\cdot$ & $\cdot$ \\
\hline Well Casing Depth & 165.6 & 18.4 & 28.7 & 9.8 & 29.9 \\
\hline Well Casing Diameter & 7 & 4.5 & 4.5 & 4.5 & 4.5 \\
\hline Well Casing Material & Steel & ss $/ \$ 304$ & ss $/: 304$ & ss $/ \$ 304$ & ss $/ \$ 304$ \\
\hline MONITORED INTERVAL & $\cdot$ & $\cdot$ & $\cdot$ & $\cdot$ & - \\
\hline Aquifer Zone & $\mathrm{BDR}$ & $\mathrm{BDR}$ & $\mathrm{BDR}$ & wT & BDR \\
\hline Geologic Formation & $\mathrm{cmn}$ & $\mathrm{cn}$ & Cmn & $\mathrm{Cmn}$ & ock \\
\hline Screened or Open & Open & Screen & Screen & Screen & Screen \\
\hline Top - Depth & 165.6 & 16.9 & 25.8 & 7.5 & 19.3 \\
\hline Bottom - Depth & 190 & 29 & 39.3 & 20.1 & 31.5 \\
\hline Screen Material & $\cdot$ & ss/sw/.01 & SS/sw/.01 & ss/sw/.01 & $\mathrm{ss} / \mathrm{sw} / .01$ \\
\hline Screen Length & $\cdot$ & 9.9 & 10.0 & 9.9 & 10.0 \\
\hline Open-Hole Length & 24.4 & $\cdot$ & $\cdot$ & $\cdot$ & - \\
\hline Open-Hole Diameter & 6.25 & - & - & $\cdot$ & - \\
\hline
\end{tabular}


II 


\section{EXPLANATION}

SAMPLING POINT:

$$
\begin{aligned}
\text { BCK } & \text { - Bear Creek Kilometer } \\
\text { GW } & - \text { Monitoring Well } \\
\text { NT } & \text { - North Tributary to Bear Creek } \\
\text { SS } & - \text { Spring }
\end{aligned}
$$

\section{LOCATION:}

$$
\begin{aligned}
\text { AGLLSF } & \text { - } \\
\text { BG } & \text { - } \text { Bear Creek Brade Low Level Storage Facility } \\
\text { EXP } & - \text { Exit-Pathway Monitoring } \\
\text { OLF } & - \text { Oil Landfarm WMA } \\
\text { RS } & - \text { Rust Spoil Area } \\
\text { S3 } & - \text { S-3 Site } \\
\text { SPI } & - \text { Spoil Area I }
\end{aligned}
$$


APPENDIX D

sampling sequence

\begin{tabular}{|c|c|c|c|}
\hline $\begin{array}{l}\text { Sampling } \\
\text { Point }\end{array}$ & Iocation & Date & TIme \\
\hline \multicolumn{4}{|c|}{ First Quartor 1993} \\
\hline$O W-115$ & 83 & $01 / 28 / 93$ & $11: 45$ \\
\hline$O W-324$ & 83 & $01 / 29 / 93$ & $12: 11$ \\
\hline$G W-325$ & 83 & $02 / 01 / 93$ & $10: 30$ \\
\hline OW -347 & 83 & $02 / 01 / 93$ & 12,30 \\
\hline$O N-348$ & 83 & $02 / 01 / 93$ & $14: 10$ \\
\hline ow-323 & SPI & $02 / 02 / 93$ & $10: 30$ \\
\hline$G W-317$ & SPI & $02 / 02 / 93$ & 13,00 \\
\hline$G K-316$ & SPI & $02 / 02 / 93$ & $15: 00$ \\
\hline$G W-345$ & $\mathbf{8 3}$ & $02 / 03 / 93$ & $10: 00$ \\
\hline$G W-526$ & $\mathbf{8 3}$ & $02 / 03 / 93$ & $13: 30$ \\
\hline GW-315 & SPI & $02 / 03 / 93$ & $15: 09$ \\
\hline OW-043 & OLF & $02 / 04 / 93$ & $10: 00$ \\
\hline$G W-044$ & OLF & $02 / 04 / 93$ & $10: 45$ \\
\hline GW-084 & OLF & $02 / 04 / 93$ & $11: 31$ \\
\hline GW-086 & OLF & $02 / 05 / 93$ & $10: 34$ \\
\hline GW-655 & BG & $02 / 05 / 93$ & $11: 45$ \\
\hline$G W-311$ & RS & $02 / 08 / 93$ & $11: 45$ \\
\hline $6 w-642$ & BG & $02 / 08 / 93$ & $11: 45$ \\
\hline$G W-312$ & Rs & $02 / 08 / 93$ & $14: 30$ \\
\hline$G W-647$ & OLF & $02 / 08 / 93$ & $14: 35$ \\
\hline$G W-064$ & OLF & $02 / 09 / 93$ & $10: 29$ \\
\hline$G W-372$ & BG & $02 / 09 / 93$ & 12,55 \\
\hline GW-740 & $\operatorname{ExP}$ & $02 / 09 / 93$ & $15: 09$ \\
\hline GW-739 & EXP & $02 / 10 / 93$ & $7: 45$ \\
\hline$G W-724$ & EXP & $02 / 10 / 93$ & $9: 45$ \\
\hline GW-738 & $\mathbf{E X P}$ & $02 / 10 / 93$ & 11,55 \\
\hline GW-736 & EXP & $02 / 11 / 93$ & $10: 26$ \\
\hline$G W-373$ & BG & $02 / 11 / 93$ & $11: 00$ \\
\hline GW-085 & OLF & $02 / 11 / 93$ & $11: 03$ \\
\hline GW-737 & EXP & $02 / 11 / 93$ & $12: 40$ \\
\hline$G W-537$ & OLF & $02 / 11 / 93$ & $13: 34$ \\
\hline$G W-723$ & EXP & $02 / 12 / 93$ & $13: 37$ \\
\hline GW-725 & EXP & $02 / 12 / 93$ & $13: 55$ \\
\hline GW -643 & BG & $02 / 16 / 93$ & $10: 04$ \\
\hline GW-371 & BG & $02 / 16 / 93$ & $14: 30$ \\
\hline GW -370 & BG & $02 / 17 / 93$ & $11: 50$ \\
\hline GW-641 & BG & $02 / 22 / 93$ & $12: 45$ \\
\hline$G W-640$ & BG & $02 / 22 / 93$ & $13: 50$ \\
\hline GW -063 & OLF & $02 / 23 / 93$ & $11: 00$ \\
\hline$G W-654$ & BG & $02 / 24 / 93$ & $11: 15$ \\
\hline$G W-287$ & BG & $02 / 24 / 93$ & $13: 00$ \\
\hline GW-639 & BG & $02 / 24 / 93$ & $13: 26$ \\
\hline$G W-286$ & BG & $02 / 25 / 93$ & $10: 45$ \\
\hline GW-793 & AGLISF & $02 / 26 / 93$ & $10: 32$ \\
\hline$G W-652$ & BG & $03 / 01 / 93$ & $10: 39$ \\
\hline
\end{tabular}


APPENDIX D

sampling sequence

\begin{tabular}{|c|c|c|c|}
\hline $\begin{array}{l}\text { Bampling } \\
\text { Point }\end{array}$ & Location & Date & Time \\
\hline$c w-651$ & $\mathrm{BC}$ & $03 / 01 / 93$ & $12: 30$ \\
\hline OW-794 & AGLL8F & $03 / 01 / 93$ & $13: 00$ \\
\hline$G W-653$ & BO & $03 / 01 / 93$ & $13: 28$ \\
\hline ON-795 & AOLLsF & $03 / 02 / 93$ & $10: 21$ \\
\hline OW-685 & $\operatorname{Exp}$ & $03 / 02 / 93$ & $14: 15$ \\
\hline$C W-684$ & $\mathbf{E X P}$ & $03 / 03 / 93$ & $12: 25$ \\
\hline CW-069 & $\mathbf{B C}$ & $03 / 03 / 93$ & $14: 16$ \\
\hline$a W-056$ & BG & $03 / 04 / 93$ & $9: 56$ \\
\hline Ow-118 & $\mathrm{BC}$ & $03 / 04 / 93$ & $11: 30$ \\
\hline ow-057 & EXP & $03 / 04 / 93$ & $11: 56$ \\
\hline GW-683 & EXP & $03 / 04 / 93$ & $15: 01$ \\
\hline $\mathrm{BCK}-00.63$ & EXP & $03 / 09 / 93$ & 7,55 \\
\hline$B C K-04.55$ & EXP & $03 / 09 / 93$ & $8: 12$ \\
\hline $88-8$ & Exp & $03 / 09 / 93$ & $8: 22$ \\
\hline$N T-13$ & $\mathbf{P X P}$ & $03 / 09 / 93$ & $8: 55$ \\
\hline $88-6$ & EXP & $03 / 09 / 93$ & $9: 02$ \\
\hline BCK-09.40 & EXP & $03 / 09 / 93$ & $9: 24$ \\
\hline $88-5$ & $\mathbf{E x P}$ & $03 / 09 / 93$ & $9: 30$ \\
\hline $88-4$ & EXP & $03 / 09 / 93$ & $9: 50$ \\
\hline$N T-01$ & EXP & $03 / 09 / 93$ & $10: 04$ \\
\hline $88-1$ & EXP & $03 / 09 / 93$ & $10: 26$ \\
\hline$G W-627$ & $\mathbf{B G}$ & $03 / 09 / 93$ & $13: 10$ \\
\hline$O W-626$ & BG & $03 / 10 / 93$ & $10: 14$ \\
\hline$G W-082$ & BG & $03 / 10 / 93$ & $14: 25$ \\
\hline ON -712 & $\mathbf{E X P}$ & $03 / 18 / 93$ & $7: 02$ \\
\hline OW -713 & EXP & $03 / 19 / 93$ & $13: 59$ \\
\hline GN-621 & BG & $03 / 20 / 93$ & $8: 42$ \\
\hline GW-711 & EXP & $03 / 20^{\circ} / 93$ & $9: 04$ \\
\hline oพ-703 & Exp & $03 / 20 / 93$ & $13: 20$ \\
\hline$O W-714$ & EXP & $03 / 21 / 93$ & $8: 00$ \\
\hline GW-715 & EXP & $03 / 21 / 93$ & $8: 59$ \\
\hline GW-710 & EXP & $03 / 21 / 93$ & $14: 50$ \\
\hline GW-704 & EXP & $03 / 22 / 93$ & $10: 42$ \\
\hline GW-694 & EXP & $03 / 23 / 93$ & $13: 41$ \\
\hline GW-706 & EXP & $03 / 23 / 93$ & $13: 45$ \\
\hline GW-695 & EXP & $03 / 24 / 93$ & $13: 30$ \\
\hline OW-644 & OLF & $03 / 25 / 93$ & $10: 02$ \\
\hline$G W-0.2$ & BG & $03 / 25 / 93$ & $10: 15$ \\
\hline GW-637 & OLF & $03 / 25 / 93$ & $11: 05$ \\
\hline$G W-638$ & OLF & $03 / 25 / 93$ & $13: 09$ \\
\hline GW -040 & BG & $03 / 25 / 93$ & $14: 00$ \\
\hline$G W-6.36$ & OLF & $03 / 26 / 93$ & $15: 12$ \\
\hline$G K-364$ & OLF & $03 / 27 / 93$ & $10: 30$ \\
\hline GW-365 & OLF & $03 / 28 / 93$ & $10: 03$ \\
\hline$G W-601$ & OLF & $03 / 28 / 93$ & $13: 30$ \\
\hline GW-013 & OLE & $03 / 29 / 93$ & $11: 15$ \\
\hline
\end{tabular}




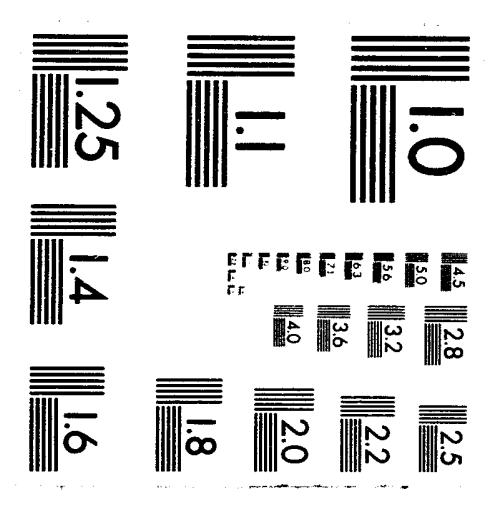



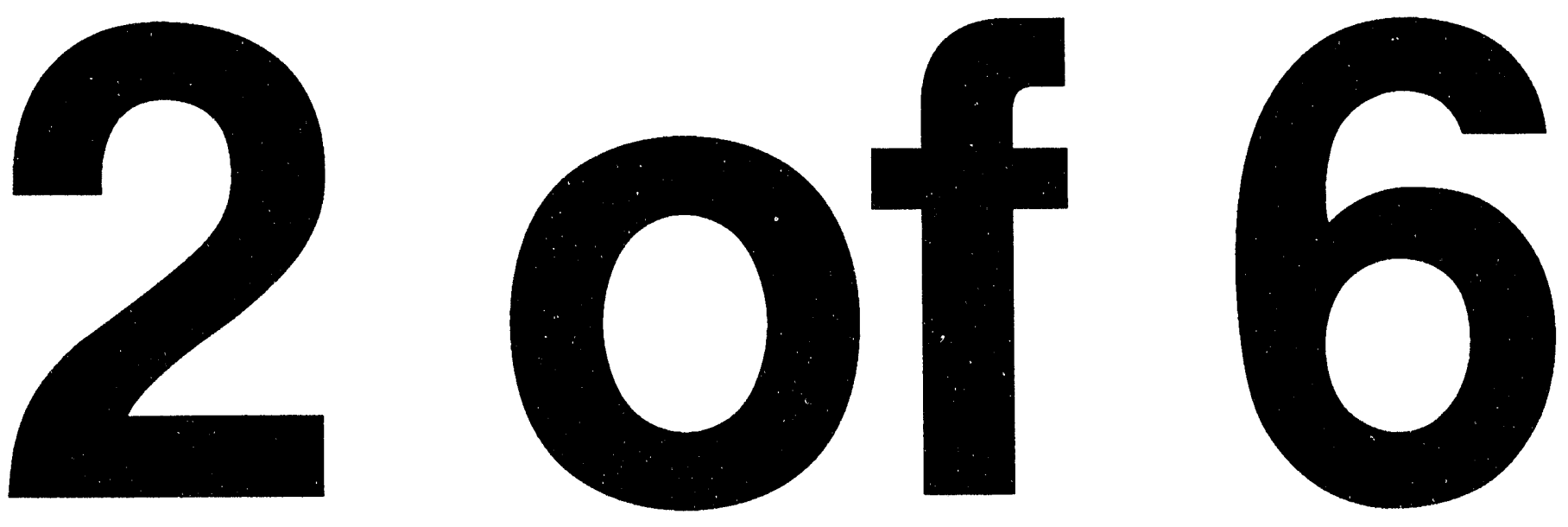
APPENDIX D

Sampling sequence

\begin{tabular}{llll}
\hline $\begin{array}{c}\text { Sampling } \\
\text { Point }\end{array}$ & Location & Date & Time \\
\hline
\end{tabular}

Second Quarter 1993

\begin{tabular}{|c|c|c|c|}
\hline$G W-654$ & BG & $04 / 16 / 93$ & $11: 15$ \\
\hline$G W-287$ & BG & $04 / 16 / 93$ & $12: 45$ \\
\hline$G W-286$ & BG & $04 / 19 / 93$ & $11: 15$ \\
\hline$G W-652$ & BG & $04 / 19 / 93$ & $12: 10$ \\
\hline$G W-651$ & BG & $04 / 19 / 93$ & $14: 00$ \\
\hline$G W-653$ & BG & $04 / 20 / 93$ & $12: 13$ \\
\hline GW-069 & BG & $04 / 21 / 93$ & $11: 31$ \\
\hline GW-118 & BG & $04 / 21 / 93$ & $12: 15$ \\
\hline$G W-685$ & EXP & $04 / 21 / 93$ & $12: 30$ \\
\hline GW-082 & BG & $04 / 21 / 93$ & $13: 44$ \\
\hline$G W-684$ & EXP & $04 / 22 / 93$ & $10: 57$ \\
\hline GW-056 & BG & $04 / 22 / 93$ & $12: 34$ \\
\hline$G W-057$ & EXP & $04 / 22 / 93$ & $13: 02$ \\
\hline$G W-683$ & EXP & $04 / 23 / 93$ & $12: 16$ \\
\hline GW-627 & BG & $04 / 23 / 93$ & $13: 40$ \\
\hline GW-626 & BG & $04 / 26 / 93$ & $10: 48$ \\
\hline GW-052 & BG & $04 / 26 / 93$ & $13: 40$ \\
\hline$G W-712$ & EXP & $04 / 28 / 93$ & $13: 10$ \\
\hline$G W-711$ & EXP & $04 / 29 / 93$ & $9: 52$ \\
\hline GW-714 & EXP & $04 / 29 / 93$ & $16: 20$ \\
\hline GW-715 & EXP & $04 / 29 / 93$ & $17: 16$ \\
\hline GW-713 & EXP & $04 / 30 / 93$ & $11: 00$ \\
\hline GW-710 & EXP & $04 / 30 / 93$ & $13: 35$ \\
\hline $\mathrm{BCK}-00.63$ & EXP & $05 / 03 / 93$ & $8: 25$ \\
\hline BCK -04.55 & EXP & $05 / 03 / 93$ & $8: 40$ \\
\hline ss- 8 & EXP & $05 / 03 / 93$ & $8: 45$ \\
\hline NT-13 & EXP & $05 / 03 / 93$ & $9: 08$ \\
\hline ss-6 & EXP & $05 / 03 / 93$ & $9: 20$ \\
\hline BCK -09.40 & EXP & $05 / 03 / 93$ & $9: 35$ \\
\hline SS-5 & EXP & $05 / 03 / 93$ & $9: 43$ \\
\hline ss - 4 & EXP & $05 / 03 / 93$ & $10: 20$ \\
\hline $\mathrm{NT}-01$ & EXP & $05 / 03 / 93$ & $10: 32$ \\
\hline ss-1 & EXP & $05 / 03 / 93$ & $10: 55$ \\
\hline$G W-043$ & OLF & $05 / 10 / 93$ & $11: 45$ \\
\hline$G W-655$ & BG & $05 / 10 / 93$ & $12: 00$ \\
\hline$G W-044$ & OLF & $05 / 10 / 93$ & $12: 09$ \\
\hline$G W-084$ & OLF & $05 / 10 / 93$ & $12: 40$ \\
\hline$G W-086$ & OLF & $05 / 11 / 93$ & $9: 30$ \\
\hline$G W-040$ & BG & $05 / 11 / 93$ & $10: 00$ \\
\hline$G W-311$ & RS & $05 / 11 / 93$ & $10: 45$ \\
\hline GW-312 & RS & $05 / 11 / 93$ & $12: 15$ \\
\hline GW-642 & BG & $05 / 11 / 93$ & $13: 20$ \\
\hline$G W-800$ & OLF & $05 / 11 / 93$ & $13: 45$ \\
\hline$G W-085$ & OLF & $05 / 12 / 93$ & $12: 45$ \\
\hline
\end{tabular}


APPENDIX D

sampling sequence

\begin{tabular}{|c|c|c|c|}
\hline $\begin{array}{l}\text { Sampling } \\
\text { Point }\end{array}$ & Location & Date & Time \\
\hline$G W-064$ & OLF & $05 / 12 / 93$ & $13: 52$ \\
\hline GW-537 & OLF & $05 / 12 / 93$ & $14: 36$ \\
\hline$G W-373$ & BG & $05 / 14 / 93$ & $10: 03$ \\
\hline$G W-647$ & OLF & $05 / 17 / 93$ & $11: 15$ \\
\hline$G W-740$ & EXP & $05 / 17 / 93$ & $18: 15$ \\
\hline GW-723 & EXP & $05 / 18 / 93$ & $9: 45$ \\
\hline$G W-724$ & $\operatorname{EXP}$ & $05 / 18 / 93$ & $9: 48$ \\
\hline GW-739 & EXP & $05 / 18 / 93$ & $10: 00$ \\
\hline GW-738 & EXP & $05 / 18 / 93$ & $11: 50$ \\
\hline GW-736 & EXP & $05 / 18 / 93$ & $14: 45$ \\
\hline$G W-737$ & EXP & $05 / 19 / 93$ & $10: 38$ \\
\hline$G W-372$ & BG & $05 / 19 / 93$ & $12: 40$ \\
\hline$G W-643$ & BG & $05 / 19 / 93$ & $13: 20$ \\
\hline GW-725 & EXP & $05 / 19 / 93$ & $14: 00$ \\
\hline GW-371 & BG & $05 / 21 / 93$ & $12: 40$ \\
\hline$G W-370$ & BG & $05 / 21 / 93$ & $13: 45$ \\
\hline$G W-641$ & BG & $05 / 24 / 93$ & $10: 20$ \\
\hline$G W-640$ & BG & $05 / 24 / 93$ & $11: 17$ \\
\hline$G W-639$ & BG & $05 / 24 / 93$ & $16: 3 n$ \\
\hline$G W-793$ & AGLISF & $05 / 25 / 93$ & $9: 16$ \\
\hline GW-115 & s3 & $05 / 25 / 93$ & $9: 22$ \\
\hline$G W-794$ & AGLLSF & $05 / 25 / 93$ & $11: 00$ \\
\hline$G W-324$ & s3 & $05 / 25 / 93$ & $11: 30$ \\
\hline GW-795 & AGLLSF & $05 / 25 / 93$ & $12: 56$ \\
\hline$G W-325$ & s3 & $05 / 25 / 93$ & $13: 00$ \\
\hline$G W-347$ & s3 & $05 / 27 / 93$ & $9: 42$ \\
\hline$G W-348$ & s3 & $05 / 27 / 93$ & $13: 17$ \\
\hline GW-323 & SPI & $05 / 28 / 93$ & $10: 45$ \\
\hline GW-317 & SPI & $05 / 28 / 93$ & $13: 40$ \\
\hline$G W-316$ & SPI & $06 / 01 / 93$ & $11: 05$ \\
\hline$G W-345$ & s3 & $06 / 01 / 93$ & $14: 00$ \\
\hline$G W-621$ & BG & $06 / 02 / 93$ & $9: 50$ \\
\hline GW-526 & s3 & $06 / 03 / 93$ & $9: 10$ \\
\hline GW-703 & EXP & $06 / 03 / 93$ & $10: 30$ \\
\hline GW-315 & SPI & $06 / 03 / 93$ & $10: 46$ \\
\hline GW -704 & EXP & $06 / 04 / 93$ & $14: 00$ \\
\hline$G W-694$ & EXP & $06 / 06 / 93$ & $14: 12$ \\
\hline GW-695 & EXP & $06 / 09 / 93$ & $11: 27$ \\
\hline GW-706 & EXP & $06 / 11 / 93$ & $13: 32$ \\
\hline$G W-013$ & OLF & $06 / 17 / 93$ & $11: 20$ \\
\hline GW- 644 & Ö̈F & $06 / 25 / 93$ & $9: 38$ \\
\hline$G W-637$ & OLF & $06 / 26 / 93$ & $9: 50$ \\
\hline$G W-638$ & OLF & $06 / 26 / 93$ & $11: 20$ \\
\hline$G W-636$ & OLF & $06 / 27 / 93$ & $10: 20$ \\
\hline$G W-364$ & OLF & $06 / 27 / 93$ & $12: 20$ \\
\hline$G W-601$ & OLF & $06 / 27 / 93$ & $14: 15$ \\
\hline$G W-365$ & OLF & $06 / 27 / 93$ & $14: 25$ \\
\hline
\end{tabular}


APPENDIX D

sampling sequence

\begin{tabular}{|c|c|c|c|}
\hline $\begin{array}{l}\text { Sampling } \\
\text { Point }\end{array}$ & Location & Date & Time \\
\hline
\end{tabular}

Third Quarter 1993

\begin{tabular}{|c|c|c|c|}
\hline$G W-655$ & BG & $07 / 01 / 93$ & $10: 40$ \\
\hline$G W-654$ & BG & $07 / 01 . / 93$ & $11: 40$ \\
\hline$G W-642$ & BG & $07 / 01 / 93$ & $13: 30$ \\
\hline GW-373 & BG & $07 / 02 / 93$ & $9: 30$ \\
\hline GW-040 & BG & $07 / 02 / 93$ & $10: 45$ \\
\hline GW-372 & BG & $07 / 02 / 93$ & $12: 45$ \\
\hline$G W-643$ & BG & $07 / 08 / 93$ & $9: 03$ \\
\hline$G W-056$ & BG & $07 / 09 / 93$ & $11: 40$ \\
\hline$G W-057$ & EXP & $07 / 09 / 93$ & $12: 30$ \\
\hline GW -371 & BG & $07 / 09 / 93$ & $13: 00$ \\
\hline$G W-287$ & BG & $07 / 10 / 93$ & $9: 20$ \\
\hline GW-370 & BG & $07 / 10 / 93$ & $9: 45$ \\
\hline$G W-286$ & BG & $07 / 10 / 93$ & $11: 00$ \\
\hline$G W-641$ & BG & $07 / 10 / 93$ & $11: 15$ \\
\hline$G W-652$ & BG & $07 / 10 / 93$ & $11: 47$ \\
\hline$G W-640$ & BG & $07 / 10 / 93$ & $12: 20$ \\
\hline$G W-651$ & BG & $07 / 10 / 93$ & $13: 30$ \\
\hline$G W-653$ & BG & $07 / 10 / 93$ & $14: 14$ \\
\hline GW-639 & BG & $07 / 11 / 93$ & $11: 30$ \\
\hline$G W-685$ & $\operatorname{EXP}$ & $07 / 11 / 93$ & $12: 45$ \\
\hline GW-793 & AGLLSF & $07 / 11 / 93$ & $13: 00$ \\
\hline GW-794 & AGLLSF & $07 / 11 / 93$ & $14: 30$ \\
\hline$G W-684$ & EXP & $07 / 11 / 93$ & $15: 15$ \\
\hline GW-795 & AGLLSF & $07 / 12 / 93$ & $11: 20$ \\
\hline$G W-683$ & EXP & $07 / 12 / 93$ & $14: 40$ \\
\hline$G W-069$ & BG & $07 / 25 / 93$ & $10: 44$ \\
\hline GW-118 & BG & $07 / 25 / 93$ & $12: 00$ \\
\hline$G W-082$ & BG & $07 / 25 / 93$ & $12: 31$ \\
\hline$G W-627$ & BG & $07 / 28 / 93$ & $12: 34$ \\
\hline GW-712 & EXP & $07 / 29 / 93$ & $11: 04$ \\
\hline GW-711 & EXP & $07 / 30 / 93$ & $11: 09$ \\
\hline GW-713 & $\operatorname{EXP}$ & $07 / 31 / 93$ & $12: 17$ \\
\hline GW-714 & EXP & $08 / 01 / 93$ & $12: 30$ \\
\hline GW-710 & EXF & $08 / 01 / 93$ & $13: 15$ \\
\hline GW -715 & EXP & $08 / 01 / 93$ & $14: 06$ \\
\hline$G W-626$ & BG & $08 / 02 / 93$ & $11: 11$ \\
\hline $\mathrm{BCK}-00.63$ & EXP & $08 / 16 / 93$ & $8: 00$ \\
\hline $\mathrm{BCK}-09.40$ & $\operatorname{EXP}$ & $08 / 16 / 93$ & $8: 00$ \\
\hline $\mathrm{BCK}-04.55$ & $\operatorname{EXP}$ & $08 / 16 / 93$ & $8: 40$ \\
\hline ss-8 & EXP & $08 / 16 / 93$ & $8: 50$ \\
\hline NT-13 & EXP & $08 / 16 / 93$ & $9: 20$ \\
\hline ss-6 & EXP & $08 / 16 / 93$ & $9: 40$ \\
\hline ss -5 & EXP & $08 / 16 / 93$ & $10: 06$ \\
\hline ss -4 & EXP & $08 / 16 / 93$ & $10: 35$ \\
\hline
\end{tabular}


APPENDIX D

Sampling sequence

\begin{tabular}{|c|c|c|c|}
\hline $\begin{array}{l}\text { Sampling } \\
\text { Point }\end{array}$ & Location & Date & Time \\
\hline ss-1 & EXP & $08 / 16 / 93$ & $11: 05$ \\
\hline$N T-01$ & EXP & $08 / 16 / 93$ & $11: 22$ \\
\hline GW-115 & s3 & $09 / 11 / 93$ & $9: 07$ \\
\hline GW-043 & OLF & $09 / 11 / 93$ & $10: 30$ \\
\hline GW-324 & s3 & $09 / 11 / 93$ & $11: 10$ \\
\hline$G W-044$ & OLF & $09 / 11 / 93$ & $12: 00$ \\
\hline GW-084 & OLF & $09 / 11 / 93$ & $13: 00$ \\
\hline$G W-325$ & s3 & $09 / 11 / 93$ & $13: 35$ \\
\hline$G W-064$ & OLF & $09 / 11 / 93$ & $14: 45$ \\
\hline$G W-347$ & s3 & $09 / 12 / 93$ & $9: 23$ \\
\hline GW-086 & OLF & $09 / 12 / 93$ & $11: 00$ \\
\hline$G W-348$ & s3 & $09 / 12 / 93$ & $11: 20$ \\
\hline$G W-311$ & RS & $09 / 12 / 93$ & $12: 30$ \\
\hline GW-312 & RS & $09 / 12 / 93$ & $14: 00$ \\
\hline GW-323 & SPI & $09 / 12 / 93$ & $14: 25$ \\
\hline GW-317 & SPI & $09 / 13 / 93$ & $12: 00$ \\
\hline$G W-316$ & SPI & $09 / 13 / 93$ & $13: 45$ \\
\hline$G W-800$ & OLF & $09 / 14 / 93$ & $9: 15$ \\
\hline$G W-345$ & s3 & $09 / 14 / 93$ & $9: 58$ \\
\hline GW-085 & OLF & $09 / 14 / 93$ & $10: 30$ \\
\hline GW-537 & OLF & $09 / 14 / 93$ & $11: 20$ \\
\hline$G W-526$ & s3 & $09 / 14 / 93$ & $14: 02$ \\
\hline GW-315 & SPI & $09 / 14 / 93$ & $15: 43$ \\
\hline GW-013 & OLF & $09 / 17 / 93$ & $10: 30$ \\
\hline$G W-644$ & OLF & $09 / 17 / 93$ & $11: 18$ \\
\hline GW-637 & OLF & $09 / 17 / 93$ & $12: 55$ \\
\hline GW-638 & OLF & $09 / 17 / 93$ & $14: 05$ \\
\hline GW-636 & OLF & $09 / 18 / 93$ & $13: 10$ \\
\hline$G W-364$ & OLF & $09 / 19 / 93$ & $9: 30$ \\
\hline GW-601 & OLF & $09 / 19 / 93$ & $13: 00$ \\
\hline$G W-365$ & OLF & $09 / 19 / 93$ & $15: 45$ \\
\hline GW-621 & BG & $09 / 20 / 93$ & $11: 10$ \\
\hline GW-703 & $\operatorname{EXP}$ & $09 / 20 / 93$ & $16: 00$ \\
\hline GW-704 & EXP & $09 / 22 / 93$ & $13: 23$ \\
\hline GW-694 & EXP & $09 / 22 / 93$ & $13: 30$ \\
\hline GW-695 & EXP & $09 / 22 / 93$ & $15: 30$ \\
\hline GW-706 & EXP & $09 / 23 / 93$ & $17: 00$ \\
\hline GW-647 & OLF & $09 / 25 / 93$ & $10: 20$ \\
\hline GW-739 & EXP & $09 / 27 / 93$ & $13: 20$ \\
\hline GW-740 & $\operatorname{EXP}$ & $09 / 27 / 93$ & $14: 55$ \\
\hline GW-738 & EXP & $09 / 27 / 93$ & $16: 48$ \\
\hline GW-723 & EXP & $09 / 27 / 93$ & $17: 30$ \\
\hline GW-736 & EXP & $09 / 28 / 93$ & $11: 13$ \\
\hline GW -725 & EXP & $09 / 28 / 93$ & $13: 15$ \\
\hline GW-737 & EXP & $09 / 28 / 93$ & $13: 27$ \\
\hline$G W-724$ & EXP & $09 / 29 / 93$ & $14: 15$ \\
\hline
\end{tabular}


APPENDIX D

sampling sequence

\begin{tabular}{|c|c|c|c|}
\hline $\begin{array}{l}\text { Sampling } \\
\text { Point }\end{array}$ & Location & Date & Time \\
\hline
\end{tabular}

Fourth Quarter 1993

\begin{tabular}{|c|c|c|c|}
\hline$G W-655$ & BG & $10 / 06 / 93$ & $13: 00$ \\
\hline$G W-654$ & BG & $10 / 07 / 93$ & $10: 35$ \\
\hline$G W-342$ & BG & $10 / 07 / 93$ & $11: 50$ \\
\hline GW-287 & BG & $10 / 07 / 93$ & $11: 55$ \\
\hline$G W-286$ & BG & $10 / 07 / 93$ & $13: 15$ \\
\hline GW-056 & BG & $10 / 09 / 93$ & $11: 00$ \\
\hline GW-057 & EXP & $10 / 09 / 93$ & $11: 29$ \\
\hline$G W-040$ & BG & $10 / 09 / 93$ & $11: 50$ \\
\hline GW-651 & BG & $10 / 10 / 93$ & $10: 30$ \\
\hline GW-162 & BG & $10 / 10 / 93$ & $11: 05$ \\
\hline GW-069 & BG & $10 / 10 / 93$ & $11: 10$ \\
\hline$G W-653$ & BG & $10 / 10 / 93$ & $11: 20$ \\
\hline$G W-642$ & BG & $10 / 10 / 93$ & $12: 00$ \\
\hline GW-118 & BG & $10 / 10 / 93$ & $12: 15$ \\
\hline$G W-080$ & BG & $10 / 10 / 93$ & $13: 45$ \\
\hline$G W-373$ & BG & $10 / 11 / 93$ & $13: 53$ \\
\hline$G W-685$ & $\operatorname{EXP}$ & $10 / 11 / 93$ & $16: 10$ \\
\hline GW-372 & BG & $10 / 12 / 93$ & $10: 30$ \\
\hline$G W-684$ & EXP & $10 / 12 / 93$ & $11: 32$ \\
\hline$G W-643$ & BG & $10 / 12 / 93$ & $11: 45$ \\
\hline$G W-683$ & EXP & $10 / 12 / 93$ & $16: 02$ \\
\hline GW-371 & BG & $10 / 13 / 93$ & $14: 00$ \\
\hline GW-370 & BG & $10 / 14 / 93$ & $11: 30$ \\
\hline$G W-641$ & BG & $10 / 14 / 93$ & $13: 00$ \\
\hline GW-640 & BG & $10 / 14 / 93$ & $13: 59$ \\
\hline$G W-639$ & BG & $10 / 15 / 93$ & $13: 00$ \\
\hline GW-793 & AGLLSF & $10 / 18 / 93$ & $12: 09$ \\
\hline GW-079 & BG & $10 / 18 / 93$ & $13: 15$ \\
\hline GW-794 & AGLLSF & $10 / 18 / 93$ & $13: 30$ \\
\hline$G W-082$ & BG & $10 / 18 / 93$ & $13: 50$ \\
\hline$G W-652$ & BG & $10 / 18 / 93$ & $13: 51$ \\
\hline GW-795 & AGLLSF & $10 / 18 / 93$ & $14: 05$ \\
\hline$G W-044$ & OLF & $10 / 19 / 93$ & $12: 30$ \\
\hline$G W-115$ & s3 & $10 / 19 / 93$ & $12: 39$ \\
\hline$G W-043$ & OLF & $10 / 19 / 93$ & $13: 30$ \\
\hline GW-324 & s3 & $10 / 19 / 93$ & $14: 41$ \\
\hline GW-084 & OLF & $10 / 20 / 93$ & $10: 00$ \\
\hline$G W-613$ & s3 & $10 / 20 / 93$ & $10: 04$ \\
\hline$G W-064$ & OLF & $10 / 20 / 93$ & $11: 10$ \\
\hline GW-614 & s3 & $10 / 20 / 93$ & $12: 17$ \\
\hline$G W-627$ & BG & $10 / 20 / 93$ & $12: 54$ \\
\hline GW- 626 & BG & $10 / 20 / 93$ & $14: 23$ \\
\hline GW-086 & OLF & $10 / 21 / 93$ & $9: 36$ \\
\hline GW-311 & RS & $10 / 21 / 93$ & $11: 30$ \\
\hline
\end{tabular}


APPENDIX D

Sampling sequence

\begin{tabular}{|c|c|c|c|}
\hline $\begin{array}{l}\text { Sampling } \\
\text { point }\end{array}$ & Location & Date & Time \\
\hline$G W-325$ & s3 & $10 / 21 / 93$ & $13: 30$ \\
\hline$G W-312$ & RS & $10 / 21 / 93$ & $13: 45$ \\
\hline$G W-800$ & OLF & $10 / 22 / 93$ & $9: 15$ \\
\hline$G W-347$ & s3 & $10 / 22 / 93$ & $10: 20$ \\
\hline$G W-085$ & OLF & $10 / 22 / 93$ & $10: 40$ \\
\hline$G W-348$ & s3 & $10 / 22 / 93$ & $11: 43$ \\
\hline$G W-537$ & OLF & $10 / 22 / 93$ & $11: 55$ \\
\hline$G W-323$ & SPI & $10 / 23 / 93$ & $9: 50$ \\
\hline GW-317 & SPI & $10 / 24 / 93$ & $10: 35$ \\
\hline$G W-316$ & SPI & $10 / 24 / 93$ & $12: 20$ \\
\hline$G W-345$ & s3 & $10 / 26 / 93$ & $9: 45$ \\
\hline$G W-526$ & s3 & $10 / 26 / 93$ & $13: 35$ \\
\hline GW-315 & SPI & $10 / 27 / 93$ & $9: 58$ \\
\hline GW-712 & EXP & $11 / 06 / 93$ & $12: 52$ \\
\hline GW-711 & EXP & $11 / 07 / 93$ & $12: 41$ \\
\hline BCK -00.63 & EXP & $11 / 08 / 93$ & $9: 05$ \\
\hline $\mathrm{BCK}-04.55$ & EXP & $11 / 08 / 93$ & $9: 15$ \\
\hline ss- 8 & EXP & $11 / 08 / 93$ & $9: 27$ \\
\hline$N T-13$ & EXP & $11 / 08 / 93$ & $10: 10$ \\
\hline ss-6 & $\operatorname{EXP}$ & $11 / 08 / 93$ & $10: 25$ \\
\hline BCK -09.40 & $\operatorname{EXP}$ & $11 / 08 / 93$ & $10: 33$ \\
\hline ss-5 & EXP & $11 / 08 / 93$ & $10: 41$ \\
\hline ss-4 & EXP & $11 / 08 / 93$ & $10: 50$ \\
\hline ss-1 & EXP & $11 / 08 / 93$ & $11: 37$ \\
\hline$N T-01$ & EXP & $11 / 08 / 93$ & $12: 00$ \\
\hline GW-713 & $\operatorname{EXP}$ & $11 / 09 / 93$ & $13: 30$ \\
\hline GW-714 & EXP & $11 / 09 / 93$ & $13: 40$ \\
\hline GW-710 & EXP & $11 / 09 / 93$ & $14: 00$ \\
\hline GW-715 & EXP & $11 / 11 / 93$ & $10: 30$ \\
\hline$G W-647$ & OLF & $12 / 10 / 93$ & $11: 00$ \\
\hline GW-740 & EXP & $12 / 11 / 93$ & $14: 17$ \\
\hline GW-739 & EXP & $12 / 13 / 93$ & $14: 12$ \\
\hline GW-723 & EXP & $12 / 14 / 93$ & $8: 15$ \\
\hline GW-738 & $\operatorname{EXP}$ & $12 / 14 / 93$ & $11: 00$ \\
\hline GW-724 & $\operatorname{EXP}$ & $12 / 14 / 93$ & $13: 00$ \\
\hline GW -725 & EXP & $12 / 15 / 93$ & $12: 36$ \\
\hline GW-736 & EXP & $12 / 15 / 93$ & $15: 22$ \\
\hline GW-737 & EXP & $12 / 15 / 93$ & $17: 37$ \\
\hline$G W-644$ & OIF & $12 / 18 / 93$ & $8: 49$ \\
\hline$G W-637$ & OIF & $12 / 18 / 93$ & $9: 30$ \\
\hline$G W-638$ & OLF & $12 / 18 / 93$ & $10: 30$ \\
\hline$G W-364$ & OLF & $12 / 18 / 93$ & $12: 30$ \\
\hline$G W-636$ & OLF & $12 / 18 / 93$ & $15: 14$ \\
\hline GW-013 & OLF & $12 / 19 / 93$ & $9: 50$ \\
\hline$G W-365$ & OLF & $12 / 19 / 93$ & $11: 55$ \\
\hline$G W-621$ & BG & $12 / 20 / 93$ & $11: 18$ \\
\hline GW-703 & EXP & $12 / 21 / 93$ & $9: 55$ \\
\hline
\end{tabular}


APPENDIX D

Sampling sequence

\begin{tabular}{llll}
\hline $\begin{array}{c}\text { Sampling } \\
\text { Point }\end{array}$ & Location & Date & Time \\
\hline & EXP & $12 / 21 / 93$ & $12: 05$ \\
GW-695 & EXP & $12 / 22 / 93$ & $14: 30$ \\
GW-704 & EXP & $12 / 28 / 93$ & $14: 00$ \\
GW-694 & EXP & $12 / 28 / 93$ & $15: 30$ \\
GW-706 & BG & $12 / 29 / 93$ & $10: 50$ \\
GW-042 & OLF & $12 / 21 / 94$ & $15: 25$ \\
GW-601 & & &
\end{tabular}


APPENDIX E

GROUNDWATER AND SURFACE-WATER QUALTY DATA 


\section{EXPLANATION}

\section{SAMPLING POINT:}

$$
\begin{array}{rll}
\text { BCK } & \text { - } & \text { Bear Creek Kilometer } \\
\text { GW } & - \text { Monitoring Well } \\
\text { NT } & - & \text { North Tributary to Bear Creek } \\
\text { SS } & - \text { Spring }
\end{array}
$$

\section{LOCATION:}

$$
\begin{aligned}
\text { AGLLSF } & \text { - Above Grade Low Level Storage Facility } \\
\text { BG } & - \text { Bear Creek Burial Grounds WMA } \\
\text { EXP } & - \text { Exit-Pathway Monitoring } \\
\text { OLF } & - \text { Oil Landfarm WMA } \\
\text { RS } & - \text { Rust Spoil Area } \\
\text { S3 } & - \text { S-3 Site } \\
\text { SPI } & - \text { Spoil Area I }
\end{aligned}
$$

\section{ALL DATA:}

- Not Analyzed

\section{VOLATILE ORGANIC COMPOUNDS:}

All results in micrograms per Liter (ug/L).

$U$ - Compound not detected at the reported minimum attainable detection limit.

METALS, MAJOR IONS, MISCELLANEOUS, AND RADIOCHEMICAL PARAMETERS:

All analyses are by Inductively Coupled Plasma (ICP) Spectroscopy unless otherwise noted.

Bicarbonate and carbonate alkalinity reported as $\mathrm{CaCO}_{3}$.

$<\quad$ - Compound analyzed but not detected at the reported minimum attainable detection limit

$\mathrm{mg} / \mathrm{L}$ - milligrams per Liter

AAS - Atomic Absorption Spectroscopy

CVAA - Cold Vapor Atomic Absorption

DIS - Dissolved Concentration (Filtered Sample)

TOT - Total Concentration (Unfiltered Sample) 
Fluor - Fluorometric

TDS - Total Dissolved Solids

TSS - Total Suspended Solids

Sp. Cond. - Specific Conductance

ppm - parts per million

umho/cm - micromhos per centimeter

NTU - Nephelometric Turbidity Unit

$\mathrm{mV}$ - millivolts

$\mathrm{pCi} / \mathrm{L}$ - picoCuries per liter

$\mathrm{Bq} / \mathrm{L}$ - Becquerels per liter

$\mathrm{CE}+\%$ - Counting Error (two standard deviations) 
APPENDIX E.1

TRACE METALS AND MAJOR IONS 


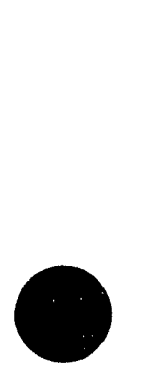

•

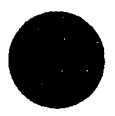


APPENDIX E.1

Groundwater and Surface-Water Quality Data, 1993

\begin{tabular}{|c|c|c|c|c|c|c|c|c|}
\hline \multirow{4}{*}{$\begin{array}{l}\text { Sampling Point } \\
\text { Location } \\
\text { - } \\
\text { Date Sampled }\end{array}$} & \multicolumn{8}{|c|}{ GW-013 } \\
\hline & \multicolumn{8}{|c|}{ OLF } \\
\hline & \multicolumn{2}{|c|}{$03 / 29 / 93$} & \multicolumn{2}{|c|}{$06 / 17 / 93$} & \multicolumn{2}{|c|}{$09 / 17 / 93$} & \multicolumn{2}{|c|}{$12 / 19 / 93$} \\
\hline & TOT & DIS & TOT & DIS & TOT & DIS & TOT & DIS \\
\hline METALS $(m g / L)$ & - & $\cdot$ & - & - & - & - & - & - \\
\hline Aluminum & $<0.02$ & $<0.02$ & $<0.02$ & 0.022 & 0.033 & 0.041 & 0.066 & $<0.02$ \\
\hline Antimony & $<0.05$ & $<0.05$ & $<0.05$ & $<0.05$ & $<0.05$ & $<0.05$ & $<0.05$ & $<0.05$ \\
\hline Arsenic & $<0.05$ & $<0.05$ & $<0.05$ & $<0.05$ & $<0.05$ & $<0.05$ & $<0.05$ & $<0.05$ \\
\hline Barium & 0.48 & 0.46 & 0.45 & 0.46 & 0.56 & 0.57 & 0.51 & 0.5 \\
\hline Beryllium & $<0.0003$ & $<0.0003$ & $<0.0003$ & $<0.0003$ & $<0.0003$ & $<0.0003$ & $<0.0003$ & $<0.0003$ \\
\hline Boron & 0.014 & 0.018 & 0.012 & 0.013 & 0.027 & 0.037 & 0.014 & 0.023 \\
\hline Cadmiun (AAS) & $<0.002$ & $<0.002$ & $<0.002$ & $<0.002$ & $<0.002$ & $<0.002$ & $<0.002$ & $<0.002$ \\
\hline Cadmium & $<0.003$ & $<0.003$ & $<0.003$ & $<0.003$ & $<0.003$ & $<0.003$ & $<0.003$ & $<0.003$ \\
\hline Chromium (AAS) & $<0.01$ & $<0.01$ & $<0.01$ & $<0.01$ & $<0.01$ & $<0.01$ & $<0.01$ & $<0.01$ \\
\hline Chromium & $<0.01$ & $<0.01$ & $<0.01$ & $<0.01$ & $<0.01$ & $<0.01$ & $<0.01$ & $<0.01$ \\
\hline Cobalt & $<0.005$ & $<0.005$ & $<0.005$ & $<0.005$ & $<0.005$ & $<0.005$ & $<0.005$ & $<0.005$ \\
\hline Copper & 0.0057 & $<0.004$ & $<0.004$ & $<0.004$ & $<0.004$ & $<0.004$ & $<0.004$ & $<0.004$ \\
\hline Iron & 2.3 & 1.1 & 1.4 & 1.2 & 1.5 & 1.4 & 1.1 & 0.55 \\
\hline Lead (AAS) & $<0.004$ & $<0.004$ & $<0.004$ & $<0.004$ & $<0.004$ & $<0.004$ & $<0.004$ & $<0.004$ \\
\hline Mercury (CVAA) & $<0.0002$ & $<0.0002$ & $<0.0002$ & $<0.0002$ & $<0.0002$ & $<0.0002$ & $<0.0002$ & $<0.0002$ \\
\hline Molybdenum & $<0.01$ & $<0.01$ & $<0.01$ & $<0.01$ & $<0.01$ & $<0.01$ & $<0.01$ & $<0.01$ \\
\hline Nickel & $<0.01$ & $<0.01$ & $<0.01$ & $<0.01$ & $<0.01$ & $<0.01$ & $<0.01$ & $<0.01$ \\
\hline Selenium & $<0.05$ & $<0.05$ & 0.058 & $<0.05$ & $<0.05$ & $<0.05$ & $<0.05$ & $<0.05$ \\
\hline Silver & $<0.006$ & $<0.006$ & $<0.006$ & $<0.006$ & $<0.006$ & $<0.006$ & $<0.006$ & $<0.006$ \\
\hline Strontium & 0.19 & 0.19 & 0.18 & 0.2 & 0.23 & 0.23 & 0.2 & 0.2 \\
\hline Thorium & $<0.2$ & $<0.2$ & $<0.2$ & $<0.2$ & $<0.2$ & $<0.2$ & $<0.2$ & $<0.2$ \\
\hline Uranium (Fluor) & $<0.001$ & $<0.001$ & $<0.001$ & $<0.001$ & $<0.001$ & $<0.001$ & $<0.001$ & $<0.001$ \\
\hline Vanadium & $<0.005$ & $<0.005$ & $<0.005$ & $<0.005$ & $<0.005$ & $<0.005$ & $<0.005$ & $<0.005$ \\
\hline zinc & $<0.002$ & 0.0036 & 0.0025 & 0.0038 & 0.0074 & 0.0096 & 0.0048 & 0.003 \\
\hline MAJOR IONS $(\mathrm{mg} / \mathrm{L})$ & $\cdot$ & $\cdot$ & . & $\cdot$ & . & - & $\cdot$ & - \\
\hline Alkalinity-нCO3 & 299 &. & 292 & $\cdot$ & 284 &. & 310 & - \\
\hline Alkalinity-CO3 & $<1$ & - & $<1$ & . & $<1$ & . & $<1$ & - \\
\hline Calcium & 92 & 91 & 95 & 96 & 110 & 110 & 100 & 100 \\
\hline Chloride & 4.3 & - & 4.4 & . & 4 & - & 5 & - \\
\hline Fluoride & $<0.1$ & - & $<0.1$ & - & $<0.1$ & . & $<0.1$ & - \\
\hline Magnesium & 12 & 11 & 12 & 12 & 13 & 13 & 12 & 12 \\
\hline Manganese & 1.1 & 1 & 1.1 & 1.1 & 1.3 & 1.3 & 1.2 & 1.1 \\
\hline Nitrate-N & $<0.2$ & - & $<0.2$ & . & $<0.2$ & - & $<0.2$ & - \\
\hline Potassium & 1.6 & 1.8 & 1.5 & 1.2 & 1.6 & 1.7 & 1.6 & 1.7 \\
\hline Sodium & 3.7 & 3.7 & 3.4 & 3.6 & 4.4 & 4.5 & 3.9 & 4.1 \\
\hline Sulfate & 4 & - & 5.9 & - & 4 & - & 3 & - \\
\hline
\end{tabular}

(CONTINUED) 
APPENDIX E. 1

Groundwater and Surface-Water Quality Data, 1993

\begin{tabular}{|c|c|c|c|c|c|c|c|c|}
\hline \multirow{4}{*}{$\begin{array}{l}\text { Sampling Point } \\
\text { Location } \\
\text { Date Sampled }\end{array}$} & \multicolumn{8}{|c|}{$G W-040$} \\
\hline & \multicolumn{8}{|c|}{ BG } \\
\hline & \multicolumn{2}{|c|}{$03 / 25 / 93$} & \multicolumn{2}{|c|}{$05 / 11 / 93$} & \multicolumn{2}{|c|}{$07 / 02 / 93$} & \multicolumn{2}{|c|}{$10 / 09 / 93$} \\
\hline & TOT & DIS & TOT & DIS & TOT & DIS & TOT & DIS \\
\hline METALS (mg/I) & $\cdot$ & $\cdot$ & $\cdot$ & $\cdot$ & $\cdot$ & $\cdot$ & $\cdot$ & • \\
\hline Aluminum & 23 & 0.027 & 15 & 0.054 & 11 & 0.025 & 13 & 0.27 \\
\hline Antimony & $<0.05$ & $<0.05$ & $<0.05$ & $<0.05$ & $<0.05$ & $<0.05$ & $<0.05$ & $<0.05$ \\
\hline Arsenic & 0.057 & $<0.05$ & $<0.05$ & $<0.05$ & $<0.05$ & $<0.05$ & $<0.05$ & $<0.05$ \\
\hline Barium & 0.35 & 0.036 & 0.19 & 0.035 & 0.17 & 0.039 & 0.2 & 0.034 \\
\hline Beryllium & 0.0018 & $<0.0003$ & 0.00068 & $<0.0003$ & 0.00047 & $<0.0003$ & 0.00048 & $<0.0003$ \\
\hline Boron & 0.033 & 0.022 & 0.05 & 0.03 & 0.035 & 0.017 & 0.037 & 0.021 \\
\hline Cadmium (AAS) & $<0.002$ & $<0.002$ & $<0.002$ & $<0.002$ & $<0.002$ & $<0.002$ & 0.031 & 0.0041 \\
\hline Cadmium & $<0.003$ & $<0.003$ & $<0.003$ & $<0.003$ & 0.0038 & $<0.003$ & 0.033 & 0.0044 \\
\hline Chromium (AAS) & 0.22 & $<0.01$ & 0.025 & $<0.01$ & 0.05 & $<0.01$ & 0.12 & $<0.01$ \\
\hline Chromium & 0.049 & $<0.01$ & 0.034 & 0.012 & 0.067 & $<0.01$ & 0.075 & $<0.01$ \\
\hline Cobalt & 0.018 & $<0.005$ & 0.01 & 0.0062 & 0.011 & $<0.005$ & 0.015 & 0.0065 \\
\hline Copper & 0.014 & $<0.004$ & 0.013 & 0.0043 & 0.017 & $<0.004$ & 0.054 & $<0.004$ \\
\hline Iron & 33 & 0.15 & 16 & 0.14 & 19 & 0.14 & 22 & 0.54 \\
\hline Lead (AAS) & 0.04 & $<0.004$ & 0.011 & $<0.004$ & 0.028 & $<0.004$ & 0.039 & $<0.004$ \\
\hline Mercury (CVAA) & $<0.0002$ & $<0.0002$ & $<0.0002$ & $<0.0002$ & 0.00027 & 0.00026 & $<0.0002$ & $<0.0002$ \\
\hline Molybdenum & $<0.01$ & $<0.01$ & $<0.01$ & $<0.01$ & $<0.01$ & $<0.01$ & $<0.01$ & $<0.01$ \\
\hline Nickel & 0.053 & 0.019 & 0.045 & 0.023 & 0.051 & $<0.01$ & 0.046 & 0.01 \\
\hline Selenium & $<0.05$ & $<0.05$ & $<0.05$ & $<0.05$ & $<0.05$ & $<0.05$ & $<0.05$ & $<0.05$ \\
\hline Silver & $<0.006$ & $<0.006$ & $<0.006$ & $<0.006$ & $<0.006$ & $<0.006$ & $<0.006$ & $<0.006$ \\
\hline strontium & 0.039 & 0.017 & 0.029 & 0.016 & 0.026 & 0.015 & 0.028 & 0.018 \\
\hline Thorium & $<0.2$ & $<0.2$ & $<0.2$ & $<0.2$ & $<0.2$ & $<0.2$ & $<0.2$ & $<0.2$ \\
\hline Uranium (F'luor) & $<0.001$ & 0.001 & $<0.001$ & $<0.001$ & 0.001 & 0.001 & $<0.001$ & $<0.001$ \\
\hline Vanadium & 0.035 & $<0.005$ & 0.018 & $<0.005$ & 0.019 & $<0.005$ & 0.026 & $<0.005$ \\
\hline zinc & 0.075 & 0.0069 & 0.037 & 0.0079 & 0.057 & 0.014 & 0.062 & 0.0097 \\
\hline MAJOR IONS $(\mathrm{mg} / \mathrm{L})$ & $\cdot$ & . & . & . & . &. & . & . \\
\hline Alkalinity-HCO3 & 38 & $\cdot$ & 37 & $\cdot$ & $4 \dot{1}$ & $\cdot$ & 38 & . \\
\hline Alkalinity- $\mathrm{CO} 3$ & $<1$ & - & $<1$ & - & $<1$ & . & $<1$ & . \\
\hline Calcium & 7.6 & 6.6 & 6.6 & 5.9 & 7.6 & 5.6 & 8.6 & 7.6 \\
\hline Chloride & 2.7 & . & 2.2 & - & 1.9 & . & 2 & . \\
\hline Fluoride & 0.4 & - & 0.3 & - & 0.3 & . & 0.3 & . \\
\hline Magnesium & 11 & 6.1 & 8.7 & 6.2 & 9.3 & 6.7 & 9.9 & 7.2 \\
\hline Manganese & 1.4 & 1 & 1.2 & 1.1 & 1.3 & 1.1 & 1.4 & 1.3 \\
\hline Nitrate-N & $<0.2$ & - & $<0.2$ & - & $<0.2$ & - & $<0.2$ & . \\
\hline Potassium & 8.6 & 1.6 & 6.9 & 1.7 & 5.6 & 2.1 & 6.7 & 1.8 \\
\hline Sodium & 10 & 11 & 11 & 11 & 12 & 12 & 12 & 12 \\
\hline Sulfate & 30 & . & 30 & - & 31 & - & 29 & 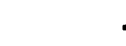 \\
\hline
\end{tabular}

(CONTINUED) 
APRENDIX $\mathrm{E} .1$

Groundwater and Surface-water Quality Data, 1993

\begin{tabular}{|c|c|c|c|c|c|c|c|c|}
\hline \multirow{4}{*}{$\begin{array}{l}\text { Sampling Point } \\
\text { Location } \\
\text { Date Sampled }\end{array}$} & \multicolumn{2}{|c|}{$G W-042$} & \multicolumn{6}{|c|}{ GW-043 } \\
\hline & \multicolumn{2}{|c|}{ BG } & \multicolumn{6}{|c|}{ OLF } \\
\hline & \multicolumn{2}{|c|}{$12 / 29 / 93$} & \multicolumn{2}{|c|}{$02 / 04 / 93$} & \multicolumn{2}{|c|}{$05 / 10 / 93$} & \multicolumn{2}{|c|}{$09 / 11 / 93$} \\
\hline & TOT & DIS & TOT & DIS & TOT & DIS & TOT & DIS \\
\hline METALS $(\mathrm{mg} / \mathrm{L})$ & $\cdot$ & $\cdot$ & . & $\cdot$ & $\cdot$ & $\cdot$ & . & • \\
\hline Aluminum & $<0.02$ & $<0.02$ & 0.33 & $<0.02$ & 0.46 & $<0.02$ & 3.5 & $<0.02$ \\
\hline Antimony & $<0.05$ & $<0.05$ & $<0.05$ & $<0.05$ & $<0.05$ & $<0.05$ & $<0.05$ & $<0.05$ \\
\hline Arsenic & $<0.05$ & $<0.05$ & $<0.05$ & $<0.05$ & $<0.05$ & $<0.05$ & $<0.05$ & $<0.05$ \\
\hline Barium & 0.11 & 0.088 & 0.022 & 0.019 & 0.015 & 0.013 & 0.026 & 0.014 \\
\hline Beryllium & $<0.0003$ & $<0.0003$ & $<0.0003$ & $<0.0003$ & $<0.0003$ & $<0.0003$ & $<0.0003$ & $<0.0003$ \\
\hline Boron & 0.033 & 0.042 & 0.024 & 0.018 & 0.03 & 0.031 & 0.011 & 0.012 \\
\hline Cadmium (AAS) & 0.066 & 0.077 & $<0.002$ & 0.0021 & $<0.002$ & $<0.002$ & 0.0078 & 0.0069 \\
\hline Cadmium & 0.062 & 0.082 & $<0.003$ & $<0.003$ & $<0.003$ & $<0.003$ & 0.0089 & 0.0066 \\
\hline Chromium (AAS) & $<0.01$ & $<0.01$ & $<0.01$ & $<0.01$ & $<0.01$ & $<0.01$ & 0.012 & $<0.01$ \\
\hline Chromium & $<0.01$ & $<0.01$ & $<0.01$ & $<0.01$ & $<0.01$ & 0.013 & 0.01 & $<0.01$ \\
\hline Cobalt & $<0.005$ & $<0.005$ & $<0.005$ & $<0.005$ & $<0.005$ & $<0.005$ & $<0.005$ & $<0.005$ \\
\hline Copper & 0.011 & 0.037 & 0.1 & $<0.004$ & $<0.004$ & $<0.004$ & 0.0061 & 0.004 \\
\hline Iron & 0.93 & 0.54 & 0.26 & $<0.005$ & 0.73 & $<0.005$ & 2.6 & 0.0089 \\
\hline Lead (AAS) & 0.0047 & $<0.004$ & 0.01 & $<0.004$ & $<0.004$ & $<0.004$ & $<0.004$ & $<0.004$ \\
\hline Mercury (CVAA) & $<0.0002$ & $<0.0002$ & $<0.0002$ & $<0.0002$ & $<0.0002$ & $<0.0002$ & $<0.0002$ & $<0.0002$ \\
\hline Mol ybdenum & $<0.01$ & $<0.01$ & $<0.01$ & $<0.01$ & $<0.02$ & $<0.01$ & $<0.01$ & $<0.01$ \\
\hline Nickel & $<0.01$ & $<0.01$ & $<0.01$ & $<0.01$ & $<0.01$ & 0.023 & $<0.01$ & $<0.01$ \\
\hline Selenium & $<0.05$ & $<0.05$ & $<0.05$ & $<0.05$ & $<0.05$ & $<0.05$ & $<0.05$ & $<0.05$ \\
\hline Silver & $<0.006$ & $<0.006$ & $<0.006$ & $<0.006$ & $<0.006$ & $<0.006$ & 0.0074 & $<0.006$ \\
\hline Strontium & 0.014 & 0.011 & 0.041 & 0.039 & 0.025 & 0.027 & 0.028 & 0.026 \\
\hline Thorium & $<0.2$ & $<0.2$ & $<0.2$ & $<0.2$ & $<0.2$ & $<0.2$ & $<0.2$ & $<0.2$ \\
\hline Uranium (Fluor) & $<0.001$ & $<0.001$ & $<0.001$ & $<0.001$ & $<0.001$ & $<0.001$ & $<0.001$ & $<0.001$ \\
\hline Vanadium & $<0.005$ & $<0.005$ & $<0.005$ & $<0.005$ & $<0.005$ & $<0.005$ & $<0.005$ & $<0.005$ \\
\hline zinc & 0.043 & 0.08 & 0.057 & 0.014 & 0.011 & 0.0065 & 0.015 & 0.009 \\
\hline MAJOR IONS $(\mathrm{mg} / \mathrm{I})$ & . & . &. & . &. &. & . & • \\
\hline Alkalinity-HCO3 & 25 & $\cdot$ & $\dot{75}$ & $\cdot$ & $\dot{52}$ & $\cdot$ & $\dot{62}$ & • \\
\hline Alkalinity-CO3 & $<1$ & . & $<1$ & . & $<1$ &. & $<1$ & . \\
\hline Calcium & 3.2 & 2.5 & 22 & 21 & 14 & 14 & 15 & 15 \\
\hline Chloride & 1.5 & . & 1.1 & . & 1.6 & . & 1.1 & . \\
\hline Fluoride & 0.1 & . & 0.1 & . & $<0.1$ & . & $<0.1$ & . \\
\hline Magnesium & 4 & 2.8 & 3.9 & 3.7 & 2,4 & 2.4 & 3.5 & 2.9 \\
\hline Manganese & 0.25 & 0.17 & 0.0045 & 0.002 & 0.0086 & 0.0014 & 0.033 & 0.0023 \\
\hline Nitrate-N & $<0.2$ & • & $<0.2$ & . & $<0.2$ & . & $<0.2$ & • \\
\hline Potassium & 0.83 & $<0.6$ & 0.85 & 0.77 & 0.6 & 0.68 & 1.1 & 0.7 \\
\hline Sodium & 3 & 2.2 & 6.6 & 6.6 & 6.5 & 7.2 & 6.8 & 6.7 \\
\hline sulfate & 4.8 & . & 11 & . & 7.8 & . & 12.3 & • \\
\hline
\end{tabular}

(CONTINUED) 
APPENDIX E.1

Groundwater and Surface-Water Quality Data, 1993

\begin{tabular}{|c|c|c|c|c|c|c|c|c|}
\hline \multirow{4}{*}{$\begin{array}{l}\text { Sampling Point } \\
\text { Location } \\
\text { Date Sampled }\end{array}$} & \multicolumn{2}{|c|}{ GW-043 } & \multicolumn{6}{|c|}{$G W-044$} \\
\hline & \multicolumn{2}{|c|}{ OLF } & \multicolumn{6}{|c|}{ OLF } \\
\hline & \multicolumn{2}{|c|}{$10 / 19 / 93$} & \multicolumn{2}{|c|}{$02 / 04 / 93$} & \multicolumn{2}{|c|}{$05 / 10 / 93$} & \multicolumn{2}{|c|}{$09 / 11 / 93$} \\
\hline & TOT & DIS & TOT & DIS & TOT & DIS & TOT & DIS \\
\hline MRTALS (mg/L) & $\cdot$ & • & . & - & $\cdot$ & . & - & $\cdot$ \\
\hline Aluminum & 1.4 & $<0.02$ & 0.047 & 0.047 & 0.041 & $<0.02$ & 0.027 & $<0.02$ \\
\hline Antimony & $<0.05$ & $<0.05$ & $<0.05$ & $<0.05$ & $<0.05$ & $<0.05$ & $<0.05$ & $<0.05$ \\
\hline Arsenic & $<0.05$ & $<0.05$ & $<0.05$ & $<0.05$ & $<0.05$ & $<0.05$ & $<0.05$ & $<0.05$ \\
\hline Barium & 0.023 & 0.018 & 0.24 & 0.22 & 0.23 & 0.23 & 0.23 & 0.23 \\
\hline Beryllium & 0.00042 & $<0.0003$ & $<0.0003$ & $<0.0003$ & $<0.0003$ & $<0.0003$ & $<0.0003$ & $<0.0003$ \\
\hline Boron & 0.02 & 0.016 & 0.009 & 0.017 & 0.017 & 0.028 & 0.012 & 0.0083 \\
\hline Cadmium (AAS) & 0.002 & 0.0064 & $<0.002$ & $<0.002$ & $<0.002$ & $<0.002$ & $<0.002$ & $<0.002$ \\
\hline Cadmium & $<0.003$ & 0.0041 & $<0.003$ & $<0.003$ & $<0.003$ & $<0.003$ & $<0.003$ & $<0.003$ \\
\hline Chromium (AAS) & $<0.01$ & $<0.01$ & $<0.01$ & $<0.01$ & $<0.01$ & $<0.01$ & $<0.01$ & $<0.01$ \\
\hline Chromium & $<0.01$ & $<0.01$ & $<0.01$ & $<0.01$ & $<0.01$ & $<0.01$ & $<0.01$ & $<0.01$ \\
\hline Cobalt & $<0.005$ & $<0.005$ & $<0.005$ & $<0.005$ & $<0.005$ & $<0.005$ & $<0.005$ & $<0.005$ \\
\hline Copper & 0.012 & $<0.004$ & $<0.004$ & $<0.004$ & $<0.004$ & $<0.004$ & 0.0059 & 0.0046 \\
\hline Iron & 1 & 0.011 & 0.061 & $<0.005$ & 0.027 & 0.01 & 0.024 & $<0.005$ \\
\hline Lead (AAS) & $<0.004$ & $<0.004$ & 0.015 & $<0.004$ & $<0.004$ & $<0.004$ & $<0.004$ & $<0.004$ \\
\hline Mercury (CVAA) & $<0.0002$ & $<0.0002$ & $<0.0002$ & $<0.0002$ & $<0.0002$ & $<0.0002$ & $<0.0002$ & $<0.0002$ \\
\hline Mol ybdenum & $<0.01$ & $<0.01$ & $<0.01$ & $<0.01$ & $<0.01$ & $<0.01$ & $<0.01$ & $<0.01$ \\
\hline Nickel & $<0.01$ & $<0.01$ & $<0.01$ & $<0.01$ & $<0.01$ & $<0.01$ & $<0.01$ & $<0.01$ \\
\hline Selenium & $<0.05$ & $<0.05$ & $<0.05$ & $<0.05$ & $<0.05$ & $<0.05$ & $<0.05$ & $<0.05$ \\
\hline silver & $<0.006$ & $<0.006$ & $<0.006$ & $<0.006$ & $<0.006$ & $<0.006$ & $<0.006$ & $<0.006$ \\
\hline strontium & 0.036 & 0.035 & 0.088 & 0.082 & 0.074 & 0.074 & 0.076 & 0.076 \\
\hline Thorium & $\leqslant 0.2$ & $<0.2$ & $<0.2$ & $<0.2$ & $<0.2$ & $<0.2$ & $<0.2$ & $<0.2$ \\
\hline Uranium (Fluor) & $<0.001$ & $<0.001$ & $<0.001$ & $<0.001$ & $<0.001$ & $<0.001$ & $<0.001$ & $<0.001$ \\
\hline Vanadium & $<0.005$ & $<0.005$ & $<0.005$ & $<0.005$ & $<0.005$ & $<0.005$ & $<0.005$ & $<0.005$ \\
\hline zine & 0.015 & 0.0094 & 0.0072 & 0.014 & 0.0041 & 0.011 & 0.009 & 0.013 \\
\hline MAJOR IONS (mg/L) &. &. &. &. & $\cdot$ & $\cdot$ & $\cdot$ & $\cdot$ \\
\hline Alkalinity-HCO3 & $\dot{62}$ &. & 143 & $\cdot 1$ & $\begin{array}{r}\circ \\
128\end{array}$ & $\cdot$ & $\begin{array}{r}135 \\
\end{array}$ & $0^{\circ}$ \\
\hline Alkalinity- $\mathrm{CO} 3$ & $<1$ &. & $<1$ &. & $<1$ & $\cdot$ & $<1$ & . \\
\hline Calcium & 18 & 18 & 51 & 48 & 45 & 45 & 50 & 49 \\
\hline Chloride & 1 & . & 1.1 & $\cdot$ & 1.2 & $\cdot$ & 1 & 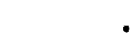 \\
\hline Fluorlie & $<0.1$ & . & 0.1 & . & $<0.1$ & $\cdot$ & 0.1 & 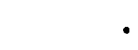 \\
\hline Magnesium & 3.6 & 3.3 & 3.4 & 3.2 & 2.9 & 2.9 & 3.2 & 3.2 \\
\hline Manganese & 0.021 & $<0.001$ & 0.049 & 0.002 & 0.016 & 0.0027 & 0.015 & 0.0018 \\
\hline Nitrate-N & $<0.2$ & . & $<0.2$ & $\cdot$ & $<0.2$ & $\cdot$ & $<0.2$ & 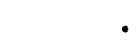 \\
\hline Potassium & 1.1 & 0.7 & 0.71 & 0.74 & $<0.6$ & 0.6 & $<0.6$ & $<0.6$ \\
\hline Sodium & 7 & 7.1 & 4.9 & 4.6 & 4.7 & 4.8 & 4.7 & 4.7 \\
\hline Sulfate & 9 & . & 9.1 &. & 8.5 & $\cdot$ & 6.7 & • \\
\hline
\end{tabular}

(CONTINUED) 
APPENDIX E.1

Groundwater and Surface-water Quality Data, 1993

\begin{tabular}{|c|c|c|c|c|c|c|c|c|}
\hline \multirow{4}{*}{$\begin{array}{l}\text { Sampling Point } \\
\text { Location } \\
\text { Date Sampled }\end{array}$} & \multicolumn{2}{|c|}{$G W-044$} & \multicolumn{4}{|c|}{ GW-052 } & \multicolumn{2}{|c|}{ GW-056 } \\
\hline & \multicolumn{2}{|c|}{ OLF } & \multicolumn{4}{|c|}{ BG } & \multirow{2}{*}{\multicolumn{2}{|c|}{$\frac{B G}{03 / 04 / 93}$}} \\
\hline & \multicolumn{2}{|c|}{$10 / 19 / 93$} & \multicolumn{2}{|c|}{$03 / 25 / 93$} & \multicolumn{2}{|c|}{$04 / 26 / 93$} & & \\
\hline & TOT & DIS & TOT & DIS & TOT & DIS & TOT & DIS \\
\hline $\operatorname{METALS}(\mathrm{mg} / \mathrm{L})$ & $\cdot$ & $\cdot$ & $\cdot$ & . & . & $\cdot$ & $\cdot$ & • \\
\hline Aluminum & $<0.02$ & $<0.02$ & 0.27 & $<0.02$ & $3 . \dot{5}$ & $0.02 \dot{2}$ & 0.16 & $<0.02$ \\
\hline Antimony & $<0.05$ & $<0.05$ & $<0.05$ & $<0.05$ & $<0.05$ & $<0.05$ & $<0.05$ & $<0.05$ \\
\hline Arsenic & $<0.05$ & $<0.05$ & $<0.05$ & $<0.05$ & $<0.05$ & $<0.05$ & $<0.05$ & $<0.05$ \\
\hline Barium & 0.23 & 0.23 & 0.063 & 0.056 & 0.089 & 0.057 & 0.21 & 0.16 \\
\hline Beryllium & $<0.0003$ & $<0.0003$ & 0.0005 & 0.00075 & $<0.0003$ & $<0.0003$ & 0.00049 & $<0.0003$ \\
\hline Boron & 0.0078 & 0.0099 & 0.034 & 0.027 & 0.045 & 0.039 & 0.059 & 0.032 \\
\hline Cadmium (AAS) & $<0.002$ & $<0.002$ & $<0.002$ & $<0.002$ & 0.033 & 0.0022 & $<0.002$ & $<0.002$ \\
\hline Cadmium & $<0.003$ & $<0.003$ & $<0.003$ & $<0.003$ & 0.026 & $<0.003$ & $<0.003$ & $<0.003$ \\
\hline Chromium (AAS) & $<0.01$ & $<0.01$ & $<0.01$ & $<0.01$ & 0.018 & $<0.01$ & 0.01 & $<0.01$ \\
\hline Chromium & $<0.01$ & $<0.01$ & $<0.01$ & $<0.01$ & 0.018 & $<0.01$ & $<0.01$ & $<0.01$ \\
\hline Cobalt & $<0.005$ & $<0.005$ & $<0.005$ & $<0.005$ & $<0.005$ & $<0.005$ & $<0.005$ & $<0.005$ \\
\hline Copper & $<0.004$ & 0.0045 & $<0.004$ & $<0.004$ & 0.0066 & $<0.004$ & $<0.004$ & $<0.004$ \\
\hline Iron & 0.005 & $<0.005$ & 0.44 & $<0.005$ & 3.4 & 0.018 & 1.2 & 0.029 \\
\hline Lead (AAS) & $<0.004$ & $<0.004$ & 0.0079 & $<0.004$ & 0.026 & $<0.004$ & $<0.004$ & $<0.004$ \\
\hline Mercury (CVAA) & $<0.0002$ & $<0.0002$ & $<0.0002$ & $<0.0002$ & $<0.0002$ & $<0.0002$ & $<0.00022$ & $<0.00022$ \\
\hline Mol ybdenum & $<0.01$ & $<0.01$ & $<0.01$ & $<0.01$ & $<0.01$ & $<0.01$ & $<0.01$ & $<0.01$ \\
\hline Nickel & $<0.01$ & 0.011 & 0.012 & $<0.01$ & 0.019 & $<0.01$ & $<0.01$ & $<0.01$ \\
\hline Selenium & $<0.05$ & $<0.05$ & $<0.05$ & $<0.05$ & $<0.05$ & $<0.05$ & $<0.05$ & $<0.05$ \\
\hline Silver & $<0.006$ & $<0.006$ & $<0.006$ & $<0.006$ & $<0.006$ & $<0.006$ & $<0.006$ & $<0.006$ \\
\hline strontium & 0.077 & 0.078 & 0.11 & 0.1 & 0.12 & 0.11 & 0.2 & 0.16 \\
\hline Thorium & $<0.2$ & $<0.2$ & $<0.2$ & $<0.2$ & $<0.2$ & $<0.2$ & $<0.2$ & $<0.2$ \\
\hline Uranium (Fluor) & $<0.001$ & $<0.001$ & 0.042 & 0.043 & 0.064 & 0.046 & 0.004 & 0.004 \\
\hline Vanadium & $<0.005$ & $<0.005$ & $<0.005$ & $<0.005$ & $<0.005$ & $<0.005$ & $<0.005$ & $<0.005$ \\
\hline zinc & 0.017 & 0.0071 & 0.0066 & 0.0076 & 0.044 & 0.0069 & 0.013 & 0.0049 \\
\hline MAJOR IONS $(\mathrm{mg} / \mathrm{L}$ ) & . & . & . & - & . & - & . & • \\
\hline Alkalinity-HCO3 & 141 & $\cdot$ & 105 & $\cdot$ & ${ }_{115}$ & $\cdot$ & 217 & • \\
\hline Alkalinity- $\mathrm{CO} 3$ & $<1$ &. & $<1$ & . & $<1$ & . & $<1$ & . \\
\hline Calcium & 49 & 49 & 49 & 47 & 53 & 47 & 120 & 92 \\
\hline Chloride & 1 & . & 18 & . & 18.5 & $\cdot$ & 229 & · \\
\hline Fluoride & $<0.1$ & $\cdot$ & 0.3 & . & 0.4 & . & $<0.1$ & . \\
\hline Magnesium & 3.3 & 2.9 & 7 & 6.9 & 8.2 & 7.7 & 25 & 21 \\
\hline Manganese & 0.0034 & $<0.001$ & 0.012 & $<0.001$ & 0.084 & 0.0019 & 0.43 & 0.29 \\
\hline Nitrate-N & $<0.2$ & . & 6.3 & . & 12.06 & . & $<0.2$ & . \\
\hline Potassium & 0.67 & 0.64 & 1.5 & 1.5 & 2.5 & 1.7 & 2.7 & 3.1 \\
\hline Sodium & 4.7 & 4.6 & 9.9 & 9.9 & 9.5 & 9.3 & 140 & 99 \\
\hline sulfate & 8 & . & 20 & - & 18.7 & . & 26 & . \\
\hline
\end{tabular}

(CONTINUED) 
APPENDIX E.1

Groundwater and Surface-Water Qual1ty Data, 1993

\begin{tabular}{|c|c|c|c|c|c|c|c|c|}
\hline \multirow{4}{*}{$\begin{array}{l}\text { Sampling Point } \\
\text { Location } \\
\text { Date Sampled }\end{array}$} & \multicolumn{6}{|c|}{ GW-056 } & \multicolumn{2}{|c|}{$G W-057$} \\
\hline & \multicolumn{6}{|c|}{ BG } & \multirow{2}{*}{\multicolumn{2}{|c|}{$\frac{\text { EXP }}{03 / 04 / 93}$}} \\
\hline & \multicolumn{2}{|c|}{$04 / 22 / 93$} & \multicolumn{2}{|c|}{$07 / 09 / 93$} & \multicolumn{2}{|c|}{$10 / 09 / 93$} & & \\
\hline & TOT & DIS & TOT & DIS & TOT & DIS & TOT & DIS \\
\hline METALS (mg/L) & - & - & - & - & - & - & • & . \\
\hline Aluminum & 0.12 & 0.032 & 0.17 & $<0.02$ & 0.17 & 0.024 & 0.099 & $<0.02$ \\
\hline Antimony & $<0.05$ & $<0.05$ & $<0.05$ & $<0.05$ & $<0.05$ & $<0.05$ & $<0.05$ & $<0.05$ \\
\hline Arsenic & $<0.05$ & $<0.05$ & $<0.05$ & $<0.05$ & $<0.05$ & $<0.05$ & $<0.05$ & $<0.05$ \\
\hline Barium & 0.16 & 0.16 & 0.16 & 0.16 & 0.16 & 0.15 & 0.13 & 0.12 \\
\hline Beryllium & $<0.0003$ & $<0.0003$ & $<0.0003$ & $<0.0003$ & $<0.0003$ & $<0.0003$ & $<0.0003$ & $<0.0003$ \\
\hline Boron & 0.032 & 0.043 & 0.017 & 0.03 & 0.038 & 0.038 & 0.075 & 0.028 \\
\hline Cedmium (AAS) & $<0.002$ & $<0.002$ & $<0.002$ & $<0.002$ & $<0.002$ & $<0.002$ & $<0.002$ & $<0.002$ \\
\hline Cadmium & $<0.003$ & $<0.003$ & $<0.003$ & $<0.003$ & 0.0035 & $<0.003$ & $<0.003$ & $<0.003$ \\
\hline Chromium (AAS) & 0.23 & $<0.01$ & 0.016 & $<0.01$ & 0.092 & $<0.01$ & 0.028 & $<0.01$ \\
\hline Chromium & 0.12 & $<0.01$ & 0.011 & $<0.01$ & 0.089 & $<0.01$ & $<0.01$ & $<0.01$ \\
\hline Cobalt & $<0.005$ & $<0.005$ & $<0.005$ & $<0.005$ & $<0.005$ & $<0.005$ & $<0.005$ & $<0.005$ \\
\hline Copper & $<0.004$ & $<0.004$ & $<0.004$ & 0.005 & $<0.004$ & $<0.004$ & $<0.004$ & $<0.004$ \\
\hline Iron & 1.6 & 0.12 & 1.4 & 1 & 1.5 & 0.8 & 0.14 & $<0.005$ \\
\hline Lead (AAS) & 0.0063 & $<0.004$ & $<0.004$ & $<0.004$ & $<0.004$ & $<0.004$ & $<0.004$ & $<0.004$ \\
\hline Mercury (CVAA) & $<0.0002$ & $<0.0002$ & 0.00021 & 0.00024 & $<0.0002$ & $<0.0002$ & $<0.00022$ & $<0.00022$ \\
\hline Molybdenum & $<0.01$ & $<0.01$ & $<0.01$ & $<0.01$ & $<0.0:$ & $<0.01$ & $<0.01$ & $<0.01$ \\
\hline Nickel & 0.22 & 0.2 & 0.031 & 0.026 & 0.049 & 0.031 & $<0.01$ & $<0.01$ \\
\hline Selenium & $<0.05$ & $<0.05$ & $<0.05$ & $<0.05$ & $<0.05$ & $<0.05$ & $<0.05$ & $<0.05$ \\
\hline Silver & $<0.006$ & $<0.006$ & $<0.006$ & $<0.006$ & $<0.006$ & $<0.006$ & $<0.006$ & $<0.006$ \\
\hline strontium & 0.16 & 0.16 & 0.18 & 0.17 & 0.18 & 0.16 & 0.16 & 0.16 \\
\hline Thorium & $<0.2$ & $<0.2$ & $<0.2$ & $<0.2$ & $<0.2$ & $<0.2$ & $<0.2$ & $<0.2$ \\
\hline Urantum (Fluor) & 0.003 & 0.004 & 0.005 & 0.005 & 0.007 & 0.005 & 0.002 & 0.002 \\
\hline Vanadium & $<0.005$ & $<0.005$ & $<0.005$ & $<0.005$ & $<0.005$ & $<0.005$ & $<0.005$ & $<0.005$ \\
\hline zinc & 0.014 & 0.008 & 0.01 & 0.019 & 0.011 & 0.008 & 0.043 & 0.024 \\
\hline MAJOR IONS (mg/L) & $\cdot$ & $\cdot$ & . & $\cdot$ & $\cdot$ & $\cdot$ & $\cdot$ & · \\
\hline Alkalinity-HCO3 & $22 \dot{0}$ & $\cdot$ & $\begin{array}{r}\bullet \\
275\end{array}$ & $\cdot$ & 314 & $\cdot$ & 208 & • \\
\hline Alkalinity-CO3 & $<1$ & $\cdot$ & $<1$ & $\cdot$ & $<1$ &. & $<1$ & . \\
\hline Calcium & 97 & 97 & 110 & 110 & 110 & 110 & 89 & 88 \\
\hline Chloride & 301 & $\cdot$ & 187 & $\cdot$ & 108 & . & 341 & 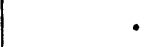 \\
\hline Fluoride & $<0.1$ & $\cdot$ & 0.1 & $\cdot$ & $<0.1$ & $\cdot$ & $<0.1$ & 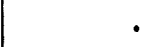 \\
\hline Magnesium & 19 & 20 & 23 & 22 & 22 & 21 & 17 & 17 \\
\hline Manganese & 0.13 & 0.057 & 0.48 & 0.44 & 0.6 & 0.51 & 0.051 & 0.04 \\
\hline Nitrate-N & 0.3 & . & $<0.2$ &. & $<0.2$ & . & 0.28 & \\
\hline Potassium & 1.9 & 1.8 & 2.2 & 1.9 & 2.1 & 1.9 & 1.9 & 1.7 \\
\hline Sodium & 130 & 140 & 75 & 73 & 55 & 52 & 130 & 120 \\
\hline Sulfate & 26 & $\cdot$ & 26 & . & 36 & $\cdot$ & 24 & - \\
\hline
\end{tabular}

(CONTINUED) 
APPENDIX B.1

Groundwater and Surface-Water Quality Data, 1993

\begin{tabular}{|c|c|c|c|c|c|c|c|c|}
\hline \multirow{4}{*}{$\begin{array}{l}\text { Sampling Point } \\
\text { Iocation } \\
\text { Date Sampled }\end{array}$} & \multicolumn{6}{|c|}{ GW-057 } & \multicolumn{2}{|c|}{$G w-063$} \\
\hline & \multicolumn{6}{|c|}{ EXP } & \multirow{2}{*}{\multicolumn{2}{|c|}{$\frac{\text { OLF }}{02 / 23 / 93}$}} \\
\hline & \multicolumn{2}{|c|}{$04 / 22 / 93$} & \multicolumn{2}{|c|}{$07 / 09 / 93$} & \multicolumn{2}{|c|}{$10 / 09 / 93$} & & \\
\hline & TOT & DIS & TOT & DIS & TOT & DIS & TOT & DIS \\
\hline METALS (mg/L) & . & - & . & $\cdot 1$ & • & $\cdot$ &. & ${ }^{*}$ \\
\hline Aluminum & $<0.02$ & 0.048 & 0.071 & 0.024 & 0.034 & 0.024 & 0.19 & $<0.02$ \\
\hline Antimony & $<0.05$ & $<0.05$ & $<0.05$ & $<0.05$ & $<0.05$ & $<0.05$ & $<0.05$ & $<0.05$ \\
\hline Arsenic & $<0.05$ & $<0.05$ & $<0.05$ & $<0.05$ & $<0.05$ & $<0.05$ & $<0.05$ & $<0.05$ \\
\hline Barium & 0.1 & 0.099 & 0.12 & 0.11 & 0.12 & 0.12 & 0.079 & 0.074 \\
\hline Beryllium & $<0.0003$ & $<0.0003$ & $<0.0003$ & $<0.0003$ & $<0.0003$ & $<0.0003$ & $<0.0003$ & $<0.0003$ \\
\hline Boron & 0.024 & 0.027 & 0.014 & 0.0098 & 0.032 & 0.034 & 0.02 & 0.0075 \\
\hline Cadmium (AAS) & $<0.002$ & $<0.002$ & $<0.002$ & $<0.002$ & $<0.002$ & $<0.002$ & $<0.002$ & $<0.002$ \\
\hline Cadmium & $<0.003$ & $<0.003$ & $<0.003$ & $<0.003$ & $<0.003$ & 0.0043 & $<0.003$ & $<0.003$ \\
\hline Chromium (AAS) & 0.031 & $<0.01$ & 0.019 & $<0.01$ & 0.016 & $<0.01$ & $<0.01$ & $<0.01$ \\
\hline Chromium & $<0.01$ & $<0.01$ & 0.017 & $<0.01$ & $<0.01$ & $<0.01$ & $<0.01$ & $<0.01$ \\
\hline Cobalt & $<0.005$ & $<0.005$ & $<0.005$ & $<0.005$ & $<0.005$ & $<0.005$ & $<0.005$ & $<0.005$ \\
\hline Copper & $<0.004$ & $<0.004$ & $<0.004$ & $<0.004$ & $<0.004$ & $<0.004$ & 0.0062 & $<0.004$ \\
\hline Iron & 0.086 & 0.013 & 0.22 & 0.11 & 0.066 & 0.012 & 0.31 & $<0.005$ \\
\hline Lead (AAS) & $<0.004$ & $<0.004$ & $<0.004$ & $<0.004$ & $<0.004$ & $<0.004$ & 0.005 & $<0.004$ \\
\hline Mercury (CVAA) & $<0.0002$ & $<0.0002$ & 0.00022 & 0.00024 & $<0.0002$ & $<0.0002$ & $<0.0002$ & $<0.0002$ \\
\hline Molybdenum & $<0.01$ & $<0.01$ & $<0.01$ & $<0.01$ & $<0.01$ & $<0.01$ & $<0.01$ & $<0.01$ \\
\hline Nickel & 0.012 & $<0.01$ & $<0.01$ & $<0.01$ & $<0.01$ & $<0.01$ & $<0.01$ & $<0.01$ \\
\hline Selenium & $<0.05$ & $<0.05$ & $<0.05$ & $<0.05$ & $<0.05$ & $<0.05$ & $<0.05$ & $<0.05$ \\
\hline silver & $<0.006$ & $<0.006$ & $<0.006$ & $<0.006$ & $<0.006$ & $<0.006$ & $<0.006$ & $<0.006$ \\
\hline strontium & 0.16 & 0.15 & 0.19 & 0.19 & 0.21 & 0.21 & 0.066 & 0.077 \\
\hline Thorium & $<0.2$ & $<0.2$ & $<0.2$ & $<0.2$ & $<0.2$ & $<0.2$ & $<0.2$ & $<0.2$ \\
\hline Uranium (Fluor) & 0.002 & 0.002 & 0.002 & 0.002 & 0.002 & 0.002 & 0.001 & 0.001 \\
\hline Vanadium & $<0.005$ & $<0.005$ & $<0.005$ & $<0.005$ & $<0.005$ & $<0.005$ & $<0.005$ & $<0.005$ \\
\hline zinc & 0.013 & 0.014 & 0.015 & 0.011 & 0.015 & 0.017 & 0.0095 & 0.0027 \\
\hline MAJOR IONS $(\mathrm{mg} / \mathrm{L})$ & - & . & . & . &. & . &. & . \\
\hline Alkal inlty-HCO3 & 218 & . & 266 &. & 302 & . & 228 & • \\
\hline Alkalinity-CO3 & $<1$ & . & $<1$ & . & $<1$ & . & $<1$ & . \\
\hline Calcium & 89 & 88 & 110 & 110 & 120 & 120 & 61 & 60 \\
\hline Chloride & 130 & . & 185 & . & 133 & . & 4.6 & • \\
\hline Fluoride & 0.1 & . & 0.1 & . & $<0.1$ & . & 0.1 & . \\
\hline Magnesium & 16 & 16 & 24 & 24 & 22 & 22 & 19 & 18 \\
\hline Manganese & 0.049 & 0.041 & 0.33 & 0.32 & 0.24 & 0.23 & 0.013 & $<0.001$ \\
\hline Nitrate-N & 0.27 & . & $<0.2$ & . & 0.3 & - & 1.8 & ${ }^{\circ}$ \\
\hline Potassium & 1.5 & 1.4 & 1.8 & 2.3 & 1.9 & 1.9 & 1.5 & 1.9 \\
\hline Sodium & 61 & 60 & 77 & 76 & 73 & 72 & 3 & 3.1 \\
\hline sulfate & 26 & . & 29 & . & 42 & . & 9.1 & 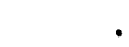 \\
\hline
\end{tabular}

(CONTINURD) 
APPENDIX E. 1

\begin{tabular}{|c|c|c|c|c|c|c|c|c|}
\hline \multirow{4}{*}{$\begin{array}{l}\text { Sampling Polnt } \\
\text { Location } \\
\text { Date Sampled }\end{array}$} & \multicolumn{8}{|c|}{$G W-064$} \\
\hline & \multicolumn{8}{|c|}{ OLF } \\
\hline & \multicolumn{2}{|c|}{$02 / 09 / 93$} & \multicolumn{2}{|c|}{$05 / 12 / 93$} & \multicolumn{2}{|c|}{$09 / 11 / 93$} & \multicolumn{2}{|c|}{$10 / 20 / 93$} \\
\hline & TOT & Drs & TOT & DIs & TOT & Drs & TOT & DIS \\
\hline METALS (mg/L) & - & - & - & - & - & • & - & • \\
\hline Aluminum & 0.56 & 0.16 & 0.45 & 0.032 & 0.23 & $<0.02$ & 0.88 & $<0.02$ \\
\hline Antimony & $<0.05$ & $<0.05$ & $<0.05$ & $<0.05$ & $<0.05$ & $<0.05$ & $<0.05$ & $<0.05$ \\
\hline Arsenic & $<0.05$ & $<0.05$ & $<0.05$ & $<0.05$ & $<0.05$ & $<0.05$ & $<0.05$ & $<0.05$ \\
\hline Barium & 0.05 & 0.046 & 0.05 & 0.049 & 0.047 & 0.045 & 0.053 & 0.049 \\
\hline Beryllium & $<0.0003$ & $<0.0003$ & $<0.0003$ & $<0.0003$ & $<0.0003$ & $<0.0003$ & $<0.0003$ & $<0.0003$ \\
\hline Boron & 0.029 & 0.036 & 0.027 & 0.028 & 0.036 & 0.012 & 0.033 & 0.038 \\
\hline Cadmium (AAS) & $<0.002$ & $<0.002$ & $<0.002$ & $<0.002$ & $<0.002$ & $<0.002$ & $<0.002$ & $<0.002$ \\
\hline Cadmium & $<0.003$ & $<0.003$ & $<0.003$ & $<0.003$ & $<0.003$ & $<0.003$ & $<0.003$ & $<0.003$ \\
\hline Chromiun (AAS) & $<0.01$ & $<0.01$ & $<0.01$ & $<0.01$ & $<0.01$ & $<0.01$ & $<0.01$ & $<0.01$ \\
\hline Chromium & $<0.01$ & $<0.01$ & $<0.01$ & $<0.01$ & $<0.01$ & $<0.01$ & $<0.01$ & $<0.01$ \\
\hline Cobalt & $<0.005$ & $<0.005$ & $<0.005$ & $<0.005$ & $<0.005$ & $<0.005$ & $<0.005$ & $<0.005$ \\
\hline Copper & $<0.004$ & $<0.004$ & $<0.004$ & $<0.004$ & 0.004 & 0.0053 & 0.0068 & $<0.004$ \\
\hline Iron & 0.48 & $<0.005$ & 0.41 & $<0.005$ & 0.2 & $<0.005$ & 0.69 & $<0.005$ \\
\hline Lead (MAS) & $<0.004$ & $<0.004$ & $<0.004$ & $<0.004$ & $<0.004$ & $<0.004$ & $<0.004$ & $<0.004$ \\
\hline Mercury (CVAA) & $<0.0002$ & $<0.0002$ & $<0.0002$ & $<0.0002$ & $<0.0002$ & $<0.0002$ & $<0.0002$ & $<0.0002$ \\
\hline Mol ybdenum & $<0.01$ & $<0.01$ & $<0.01$ & $<0.01$ & $<0.01$ & $<0.01$ & $<0.01$ & $<0.01$ \\
\hline Nlckel & $<0.01$ & $<0.01$ & $<0.01$ & $<0.01$ & $<0.01$ & $<0.01$ & $<0.01$ & $<0.01$ \\
\hline Selentum & $<0.05$ & $<0.05$ & $<0.05$ & $<0.05$ & 0.074 & $<0.05$ & $<0.05$ & $<0.05$ \\
\hline sliver & $<0.006$ & $<0.006$ & $<0.006$ & $<0.006$ & $<0.006$ & $<0.006$ & $<0.006$ & $<0.006$ \\
\hline strontium & 0.11 & 0.11 & 0.11 & 0.12 & 0.11 & 0.1 & 0.12 & 0.12 \\
\hline Thorium & $<0.2$ & $<0.2$ & $<0.2$ & $<0.2$ & $<0.2$ & $<0.2$ & $<0.2$ & $<0.2$ \\
\hline Uranium (Fluor) & $<0.001$ & 0.001 & $<0.001$ & 0.001 & 0.001 & 0.001 & 0.001 & 0.001 \\
\hline Vanadium & $<0.005$ & $<0.005$ & $<0.005$ & $<0.005$ & $<0.005$ & $<0.005$ & $<0.005$ & $<0.005$ \\
\hline zine & 0.0055 & 0.0039 & 0.009 & 0.0052 & 0.019 & 0.012 & 0.011 & 0.005 \\
\hline MAJOR IONS $(\mathrm{mg} / \mathrm{L})$ & . & $\cdot$ & . & . & $\cdot$ & $\cdot$ & $\cdot$ & • \\
\hline & 286 & $\cdot$ &. & $\cdot$ & . & $\cdot$ & $\dot{0}$ & $\cdot$ \\
\hline $\begin{array}{l}\text { Alkalinity }-\mathrm{HCO} 3 \\
\text { Alkalinity- } \mathrm{CO} 3\end{array}$ & $\begin{array}{r}280 \\
<1\end{array}$ & $\cdot$ & $\begin{array}{r}285 \\
<1\end{array}$ &. & $\begin{array}{r}171 \\
<1\end{array}$ &. & $\begin{array}{r}293 \\
<1\end{array}$ & $\cdot$ \\
\hline Calcium & 110 & 100 & 110 & 110 & 120 & 110 & 120 & $120^{\circ}$ \\
\hline Chloride & 11 &. & 12 &. & 11.3 &. & 15 & . \\
\hline Fluorida & $<0.1$ &. & $<0.1$ &. & $<0.1$ & . & $<0.1$ & . \\
\hline Magnesiun & 16 & 16 & 17 & 17 & 17 & 16 & 16 & 16 \\
\hline Manganese & 0.021 & 0.0026 & 0.015 & 0.0014 & 0.0067 & 0.0021 & 0.024 & $<0.001$ \\
\hline Nitrate-N & 8.3 & $\cdot$ & 4.7 & $\cdot$ & 7.67 & . & 9 & . \\
\hline Potaseium & 2.1 & 1.9 & 1.7 & 2 & 2.1 & 5 & 2.3 & 1.8 \\
\hline Sodium & 4.8 & 4.7 & 4.5 & 4.6 & 4.8 & 4.8 & 5.1 & 5.1 \\
\hline sulfate & 25 & . & 17 & . & 23.2 & . & 25 & . \\
\hline
\end{tabular}


APPENDIX E.1

Groundwater and Surface-water Quality Data, 1993

\begin{tabular}{|c|c|c|c|c|c|c|c|c|}
\hline \multirow{4}{*}{$\begin{array}{l}\text { 8aropling Point } \\
\text { Location } \\
\text { Date Sampled }\end{array}$} & \multicolumn{8}{|c|}{ OW-069 } \\
\hline & \multicolumn{8}{|c|}{ BG } \\
\hline & \multicolumn{2}{|c|}{$03 / 03 / 93$} & \multicolumn{2}{|c|}{$04 / 21 / 93$} & \multicolumn{2}{|c|}{$07 / 25 / 93$} & \multicolumn{2}{|c|}{$10 / 10 / 93$} \\
\hline & TOT & DIs & TOT & DIs & TOT & DIs & Tor & DIs \\
\hline Merrars $(m g / L)$ & $\cdot$ & $\cdot$ & $\cdot$ & $\cdot$ & $\cdot$ & $\cdot$ & $\cdot$ & - \\
\hline Aluminum & $<0.02$ & 0.078 & $<0.02$ & $<0.02$ & $<0.02$ & $<0.02$ & $<0.02$ & $<0.02$ \\
\hline Nntimony & $<0.05$ & $<0.05$ & $<0.05$ & $<0.05$ & $<0.05$ & $<0.05$ & $<0.05$ & $<0.05$ \\
\hline Arsentc & $<0.05$ & $<0.05$ & $<0.05$ & $<0.05$ & $<0.05$ & $<0.05$ & $<0.05$ & $<0.05$ \\
\hline Barium & 0.35 & 0.35 & 0.36 & 0.36 & 0.41 & 0.41 & 0.44 & 0.41 \\
\hline Bary11ium & $<0.0003$ & $<0.0003$ & $<0.0003$ & $<0.0003$ & $<0.0003$ & $<0.0003$ & $<0.0003$ & $<0.0003$ \\
\hline Boron & 0.11 & 0.1 & 0.1 & 0.089 & 0.11 & 0.092 & 0.11 & 0.1 \\
\hline Cadmium (AMS) & $<0.002$ & $<0.002$ & $<0.002$ & $<0.002$ & $<0.002$ & $<0.002$ & $<0.002$ & $<0.002$ \\
\hline Cadmium & $<0.003$ & $<0.003$ & $<0.003$ & $<0.003$ & $<0.003$ & $<0.003$ & $<0.003$ & $<0.003$ \\
\hline Chromium (AAS) & $<0.01$ & $<0.01$ & $<0.01$ & $<0.01$ & $<0.01$ & $<0.01$ & $<0.01$ & $<0.01$ \\
\hline Chromium & $<0.01$ & $<0.01$ & $<0.01$ & $<0.01$ & $<0.01$ & $<0.01$ & $<0.01$ & $<0.01$ \\
\hline Cobalt & $<0.005$ & $<0.005$ & $<0.005$ & $<0.005$ & $<0.005$ & $<0.005$ & $<0.005$ & $<0.005$ \\
\hline Copper & $<0.004$ & $<0.004$ & 0.0065 & $<0.004$ & 0.0074 & $<0.004$ & $<0.004$ & $<0.004$ \\
\hline Iron & 0.011 & 0.025 & 0.014 & $<0.005$ & 0.047 & 0.012 & 0.02 & 0.013 \\
\hline Lead (MAS) & $<0.004$ & $<0.004$ & $<0.004$ & $<0.004$ & $<0.004$ & $<0.004$ & $<0.004$ & $<0.004$ \\
\hline Mercury (CVMA) & $<0.0002$ & $<0.0002$ & $<0.0002$ & $<0.0002$ & $<0.0002$ & 0.0002 & $<0.0002$ & $<0.0002$ \\
\hline Mol ybdenum & $<0.01$ & $<0.01$ & $<0.01$ & $<0.01$ & $<0.01$ & $<0.01$ & $<0.01$ & $<0.01$ \\
\hline Nickel & $<0.01$ & $<0.01$ & $<0.01$ & $<0.01$ & $<0.01$ & $<0.01$ & $<0.01$ & $<0.01$ \\
\hline selentum & $<0.05$ & $<0.05$ & $<0.05$ & $<0.05$ & $<0.05$ & $<0.05$ & $<0.05$ & $<0.05$ \\
\hline silver & $<0.006$ & $<0.006$ & $<0.006$ & $<0.006$ & $<0.006$ & $<0.006$ & $<0.006$ & $<0.006$ \\
\hline strontium & 0.94 & 0.92 & 0.83 & 0.83 & 0.91 & 0.9 & 0.99 & 0.93 \\
\hline Thorium & $<0.2$ & $<0.2$ & $<0.2$ & $<0.2$ & $<0.2$ & $<0.2$ & $<0.2$ & $<0.2$ \\
\hline Uranium (Fluor) & $<0.001$ & 0.003 & $<0.001$ & $<0.001$ & $<0.001$ & $<0.001$ & $<0.001$ & 0.001 \\
\hline Vanadium & $<0.005$ & $<0.005$ & $<0.005$ & $<0.005$ & $<0.005$ & $<0.005$ & $<0.005$ & $<0.005$ \\
\hline zinc & 0.0048 & 0.0086 & 0.0081 & 0.0034 & 0.0093 & 0.0047 & 0.0055 & 0.0049 \\
\hline MuNOR IOKS $(\mathrm{mg} / \mathrm{L}$ ) &. & . & $\cdot$ & $\cdot$ & $\cdot$ & $\cdot$ & $\cdot$ & • \\
\hline Alkalinity-HCO3 & 111 &. & 111 &. & $13 i$ & . & 132 & • \\
\hline Alkalinity-CO3 & 20 &. & 10 &. & 6 &. & 8 & . \\
\hline Calcium & 6.6 & 7.5 & 8.7 & 8.6 & 11 & 11 & 15 & 12 \\
\hline Chloride & 39 & $\cdot$ & 36 & $\cdot$ & 39 &. & 48.8 & • \\
\hline Eluoride & 0.1 & $\cdot$ & 0.1 & $\cdot$ & $<0.1$ & $\cdot$ & 0.1 & • \\
\hline Magnesium & 5.6 & 5.6 & 5.2 & 5.3 & 6.1 & 6 & 6.5 & 6.2 \\
\hline Manganese & $<0.001$ & 0.048 & 0.0017 & 0.0026 & 0.0039 & 0.0049 & 0.006 & 0.0045 \\
\hline Nitrate-N & $<0.2$ &. & $<0.2$ & . & $<0.2$ &. & 0.26 & • \\
\hline Potase ium & 4.3 & 4.2 & 4.7 & 4.8 & 4.6 & 4.8 & 4.2 & 4.4 \\
\hline sodium & 61 & 59 & 58 & 57 & 63 & 63 & 65 & 64 \\
\hline sulfate & 1.9 & $\cdot$ & 7.8 & . & 3.9 & $\cdot$ & 2.3 & . \\
\hline
\end{tabular}

(CONT INUED) 
APPENDIX $\mathrm{B} .1$

\begin{tabular}{|c|c|c|c|c|c|c|c|c|}
\hline \multirow{4}{*}{$\begin{array}{l}\text { sampling polnt } \\
\text { Looation } \\
\text { Date sampled }\end{array}$} & \multicolumn{2}{|c|}{ cw-079 } & \multicolumn{2}{|c|}{ GW-080 } & \multicolumn{4}{|c|}{$a w-082$} \\
\hline & \multicolumn{2}{|c|}{ BG } & \multicolumn{2}{|c|}{ BG } & \multicolumn{4}{|c|}{ BC } \\
\hline & \multicolumn{2}{|c|}{$10 / 18 / 93$} & \multicolumn{2}{|c|}{$10 / 10 / 93$} & \multicolumn{2}{|c|}{$03 / 10 / 93$} & \multicolumn{2}{|c|}{$04 / 21 / 93$} \\
\hline & TOT & DIs & TOT & DIS & TOT & DIS & TOT & DIS \\
\hline METALS (mg/L) & . & $\cdot$ & . & $\cdot$ & - & . & . & - \\
\hline Aluminum & 0.036 & $<0.02$ & 3.5 & $<0.02$ & 0.74 & $<0.02$ & 0.11 & 0.022 \\
\hline Antimony & $<0.05$ & $<0.05$ & $<0.05$ & $<0.05$ & $<0.05$ & $<0.05$ & $<0.05$ & $<0.05$ \\
\hline Areanic & $<0.05$ & $<0.05$ & $<0.05$ & $<0.05$ & $<0.05$ & $<0.05$ & $<0.05$ & $<0.05$ \\
\hline Barium & 0.17 & 0.15 & 0.059 & 0.023 & 0.4 & 0.37 & 0.35 & 0.35 \\
\hline Beryl11um & $<0.0003$ & $<0.0003$ & $<0.0003$ & $<0.0003$ & 0.0005 & 0.00038 & $<0.0003$ & $<0.0003$ \\
\hline Boron & $<0.004$ & 0.0067 & 0.018 & 0.021 & 0.011 & 0.017 & 0.0083 & 0.0088 \\
\hline Cadmium (AAS) & $<0.002$ & $<0.002$ & $<0.002$ & $<0.002$ & $<0.002$ & $<0.002$ & $<0.002$ & $<0.002$ \\
\hline Cadmium & $<0.003$ & $<0.003$ & .0 .003 & $<0.003$ & $<0.003$ & $<0.003$ & $<0.003$ & $<0.003$ \\
\hline Chromium (AAS) & $<0.01$ & $<0.01$ & 0.012 & $<0.01$ & $<0.01$ & $<0.01$ & $<0.01$ & $<0.01$ \\
\hline Chromium & $<0.01$ & $<0.01$ & $<0.01$ & $<0.01$ & $<0.01$ & $<0.01$ & $<0.01$ & $<0.01$ \\
\hline Cobalt & $<0.005$ & $<0.005$ & 0.0053 & $<0.005$ & $<0.005$ & $<0.005$ & $<0.005$ & $<0.005$ \\
\hline Copper & $<0.004$ & $<0.004$ & 0.01 & 0.0075 & $<0.004$ & $<0.004$ & $<0.004$ & $<0.004$ \\
\hline Iron & 0.073 & $<0.005$ & 3.5 & 0.24 & 0.7 & 0.0065 & 0.082 & 0.009 \\
\hline Load (AAS) & $<0.004$ & $<0.004$ & $<0.004$ & $<0.004$ & $<0.004$ & $<0.004$ & $<0.004$ & $<0.004$ \\
\hline Mercury (CVMA) & $<0.0002$ & $<0.0002$ & $<0.0002$ & $<0.0002$ & $<0.0002$ & $<0.0002$ & $<0.0002$ & $<0.0002$ \\
\hline Nol ybdenum & $<0.01$ & $<0.01$ & $<0.01$ & $<0.01$ & $<0.01$ & $<0.01$ & $<0.01$ & $<0.01$ \\
\hline N1okel & $<0.01$ & $<0.01$ & 0.027 & 0.017 & $<0.01$ & $<0.01$ & $<0.01$ & $<0.01$ \\
\hline Selenium & $<0.05$ & $<0.05$ & $<0.05$ & $<0.05$ & $<0.05$ & $<0.05$ & $<0.05$ & $<0.05$ \\
\hline silver & $<0.006$ & $<0.006$ & $<0.006$ & $<0.006$ & $<0.006$ & $<0.006$ & $<0.006$ & $<0.006$ \\
\hline strontlum & 0.13 & 0.14 & 0.018 & 0.016 & 0.13 & 0.13 & 0.1 & 0.1 \\
\hline Thorium & $<0.2$ & $<0.2$ & $<0.2$ & $<0.2$ & $<0.2$ & $<0.2$ & $<0.2$ & $<0.2$ \\
\hline Uranium (rluor) & $<0.001$ & $<0.001$ & $<0.001$ & $<0.001$ & $<0.001$ & $<0.001$ & $<0.001$ & $<0.001$ \\
\hline Vanadium & $<0.005$ & $<0.005$ & 0.0056 & $<0.005$ & $<0.005$ & $<0.005$ & $<0.005$ & $<0.005$ \\
\hline zinc & 0.0099 & 0.0062 & 0.016 & 0.017 & 0.0054 & 0.0065 & 0.0065 & 0.0052 \\
\hline MAJOR IONS (mg/L) & . & $\cdot$ & . & . & . & . & . & . \\
\hline Alkalinity-HCO3 & 103 &. & 43 &. & $181^{\circ}$ & . & 160 & • \\
\hline Alkalinity-CO3 & $<1$ &. & $<1$ &. & $<1$ & . & $<1$ & . \\
\hline Calcium & 34 & 24 & 4 & 4.4 & 68 & 66 & 57 & 57 \\
\hline Chloride & 1.1 & $\cdot$ & 2 & . & 13.1 & - & 7.3 & • \\
\hline Fluoride & 0.1 &. & 0.1 & . & 0.2 & . & 0.2 & $\cdot$ \\
\hline Magnes I um & 3.7 & 4.5 & 4.8 & 4.9 & 6.8 & 6.5 & 6.5 & 6.4 \\
\hline Manganese & 0.047 & 0.014 & 0.072 & 0.085 & 0.14 & 0.11 & 0.063 & 0.064 \\
\hline Nitrate-N & $<0.2$ &. & $<0.2$ & . & $<0.2$ & . & $<0.2$ & • \\
\hline Potaselum & 1.5 & 2.7 & 2.5 & 2.7 & 1.2 & 1.2 & 1.2 & 1.5 \\
\hline Sodium & 4.1 & 5.4 & 14 & 14 & 4.6 & 4.5 & 4.3 & 4.4 \\
\hline Sulfate & 6.9 & . & 21 & . & 7.2 & . & 13 & . \\
\hline
\end{tabular}

(CONTINUED) 
APPENDIX B.1

Groundwater and surface-Water Quality Data, 1993

\begin{tabular}{|c|c|c|c|c|c|c|c|c|}
\hline \multirow{4}{*}{$\begin{array}{l}\text { sampling Point } \\
\text { Looation } \\
\text { Date sampled }\end{array}$} & \multicolumn{4}{|c|}{$G W-082$} & \multicolumn{4}{|c|}{ OW-084 } \\
\hline & \multicolumn{4}{|c|}{86} & \multicolumn{4}{|c|}{ orf } \\
\hline & \multicolumn{2}{|c|}{$07 / 25 / 93$} & \multicolumn{2}{|c|}{$10 / 18 / 93$} & \multicolumn{2}{|c|}{$02 / 04 / 93$} & \multicolumn{2}{|c|}{$05 / 10 / 93$} \\
\hline & TOT & DIs & TOT & DIs & TOT & DIs & TOT & DIs \\
\hline METALs $(m g / L)$ & - & . & . & - & - & $\cdot$ & . & - \\
\hline Aluminum & 0.28 & $<0.02$ & 0.02 & $<0.02$ & 1 & $<0.02$ & 1.5 & 0.037 \\
\hline Antimony & $<0.05$ & $<0.05$ & $<0.05$ & $<0.05$ & $<0.05$ & $<0.05$ & $<0.05$ & $<0.05$ \\
\hline Arsenic & $<0.05$ & $<0.05$ & $<0.05$ & $<0.05$ & $<0.05$ & $<0.05$ & $<0.05$ & $<0.05$ \\
\hline Bartium & 0.42 & 0.4 & 0.4 & 0.38 & 0.17 & 0.15 & 0.17 & 0.15 \\
\hline Beryl11um & $<0.0003$ & $<0.0003$ & $<0.0003$ & $<0.0003$ & 0.00033 & 0.00071 & $<0.0003$ & $<0.0003$ \\
\hline Boron & 0.01 & 0.015 & 0.02 & 0.015 & 0.011 & 0.02 & 0.016 & 0.014 \\
\hline Cadmium (MAS & $<0,002$ & $<0.002$ & $<0.002$ & $<0.002$ & $<0.002$ & $<0.002$ & $<0.002$ & $<0.002$ \\
\hline Cadmium & $<0.003$ & $<0.003$ & $<0.003$ & $<0.003$ & $<0.003$ & $<0.003$ & $<0.003$ & $<0.003$ \\
\hline Chromium (MAS) & $<0.01$ & $<0.01$ & $<0.01$ & $<0.01$ & $<0.01$ & $<0.01$ & $<0.01$ & $<0.01$ \\
\hline Chromium & $<0.01$ & $<0.01$ & $<0.01$ & $<0.01$ & $<0.01$ & $<0.01$ & $<0.01$ & 0.012 \\
\hline Cobalt & $<0.005$ & $<0.005$ & $<0.005$ & $<0.005$ & $<0.005$ & $<0.005$ & $<0.005$ & $<0.005$ \\
\hline Copper & 0.005 & 0.0049 & $<0.004$ & $<0.004$ & $<0.004$ & $<0.004$ & $<0.004$ & 0.0087 \\
\hline Iron & 0.26 & 0.017 & 0.036 & 0.0088 & 1 & $<0.005$ & 1.7 & 0.012 \\
\hline Lead (MAS) & $<0.004$ & $<0.004$ & $<0.004$ & $<0.004$ & $<0.004$ & $<0.004$ & $<0.004$ & $<0.004$ \\
\hline Mercury (CVMA) & $<0.0002$ & $<0.0002$ & $<0.0002$ & $<0.0002$ & $<0.0002$ & $<0.0002$ & $<0.0002$ & $<0.0002$ \\
\hline Mol ybdenum & $<0.01$ & $<0.01$ & $<0.01$ & $<0.01$ & $<0.01$ & $<0.01$ & $<0.01$ & $<0.01$ \\
\hline Nickel & 0.012 & 0.018 & $<0.01$ & $<0.01$ & $<0.01$ & $<0.01$ & $<0.01$ & 0.021 \\
\hline Selenium & $<0.05$ & $<0.05$ & $<0.05$ & $<0.05$ & $<0.05$ & $<0.05$ & $<0.05$ & $<0.05$ \\
\hline Silver & $<0.006$ & $<0.006$ & $<0.006$ & $<0.006$ & $<0.006$ & $<0.006$ & $<0.006$ & $<0.006$ \\
\hline Strontlum & 0.12 & 0.12 & 0.12 & 0.11 & 0.082 & 0.078 & 0.079 & 0.078 \\
\hline Thorium & $<0.2$ & $<0.2$ & $<0.2$ & $<0.2$ & $<0.2$ & $<0.2$ & $<0.2$ & $<0.2$ \\
\hline Uranium (Fluor) & $<0.001$ & $<0.001$ & $<0.001$ & $<0.001$ & $<0.001$ & $<0.001$ & $<0.001$ & $<0.001$ \\
\hline Vanadium & $<0.005$ & $<0.005$ & $<0.005$ & $<0.005$ & $<0.005$ & $<0.005$ & $<0.005$ & $<0.005$ \\
\hline Zine & 0.018 & 0.056 & 0.0031 & 0.0052 & 0.0072 & 0.0068 & 0.0099 & 0.0073 \\
\hline MAJOR IONS $(\mathrm{mg} / \mathrm{L})$ & $\cdot$ & $\cdot$ & $\cdot$ & $\cdot$ & $\cdot$ & . & 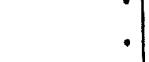 & $\cdot$ \\
\hline Alkalinity-HCO3 & $\begin{array}{r}\cdot \\
176\end{array}$ & $\cdot$ & 173 & $\cdot$ & $\begin{array}{r}\circ \\
149\end{array}$ &. & 149 & . \\
\hline Alkalinity-CO3 & $<1$ &. & $<1$ & $\cdot$ & $<1$ & - & $<1$ & . \\
\hline Calcium & 65 & 63 & 66 & 60 & 54 & 50 & 54 & 53 \\
\hline Chlortde & 17 & . & 17.6 & . & $<1$ & . & $<1$ & . \\
\hline Fluoride & 0.2 & . & 0.2 & . & 0.1 & . & $<0.1$ & . \\
\hline Magnesium & 7.6 & 7.4 & 7.5 & 7 & 4.3 & 3.7 & 4 & 3.6 \\
\hline Manganese & 0.11 & 0.087 & 0.12 & 0.11 & 0.04 & 0.0077 & 0.077 & 0.0073 \\
\hline Nitrate-N & $<0.2$ & . & $<0.2$ & . & $<0.2$ & . & $<0.2$ & . \\
\hline Potassium & 1.7 & 1.5 & 1.7 & 2.1 & 1.3 & 1.5 & 0.98 & 0.61 \\
\hline sodium & 4.8 & 4.7 & 4.7 & 4.5 & 5.4 & 5.4 & 5.1 & 5.2 \\
\hline Sulfate & 7.2 & . & 6.8 & . & 8.9 & . & 8.1 & 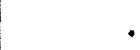 \\
\hline
\end{tabular}

(CONTINURD) 
APPENDIX E.1

Groundwater and Surface-Water Quality Data, 1993

\begin{tabular}{|c|c|c|c|c|c|c|c|c|}
\hline \multirow{4}{*}{$\begin{array}{l}\text { Sampling Point } \\
\text { Iocation } \\
\text { Date sampled }\end{array}$} & \multicolumn{4}{|c|}{$G W-084$} & \multicolumn{4}{|c|}{ GW-085 } \\
\hline & \multicolumn{4}{|c|}{ OLF } & \multicolumn{4}{|c|}{ OLF } \\
\hline & \multicolumn{2}{|c|}{$09 / 11 / 93$} & \multicolumn{2}{|c|}{$10 / 20 / 93$} & \multicolumn{2}{|c|}{$02 / 11 / 93$} & \multicolumn{2}{|c|}{$05 / 12 / 93$} \\
\hline & TOT & DIS & TOT & DIS & TOT & DIS & TOT & DIS \\
\hline METAus (mg/L) & - & - & $\cdot$ & $\cdot$ & $\cdot$ & $\cdot 1$ & - & • \\
\hline Aluminum & 1.3 & $<0.02$ & 1.5 & $<0.02$ & 0.13 & $<0.02$ & 0.18 & 0.097 \\
\hline Antimony & $<0.05$ & $<0.05$ & $<0.05$ & $<0.05$ & $<0.05$ & $<0.05$ & $<0.05$ & $<0.05$ \\
\hline Arsenio & $<0.05$ & $<0.05$ & $<0.05$ & $<0.05$ & $<0.05$ & $<0.05$ & $<0.05$ & $<0.05$ \\
\hline Barium & 0.17 & 0.16 & 0.17 & 0.16 & 1 & 0.95 & 1.2 & 1.1 \\
\hline Bary 111 um & $<0.0003$ & $<0.0003$ & $<0.0003$ & $<0.0003$ & $<0.0003$ & $<0.0003$ & $<0.0003$ & $<0.0003$ \\
\hline Boron & 0.026 & 0.019 & 0.012 & 0.008 & 0.041 & 0.021 & 0.039 & 0.035 \\
\hline Cadmium (AAS) & $<0.002$ & $<0.002$ & $<0.002$ & $<0.002$ & $<0.002$ & $<0.002$ & $<0.002$ & $<0.002$ \\
\hline Cadmium & $<0.003$ & $<0.003$ & $<0.003$ & $<0.003$ & $<0.003$ & $<0.003$ & $<0.003$ & $<0.003$ \\
\hline Chromlum (AAS) & $<0.01$ & $<0.01$ & $<0.01$ & $<0.01$ & $<0.01$ & $<0.01$ & $<0.01$ & $<0.01$ \\
\hline Chromium & $<0.01$ & $<0.01$ & $<0.01$ & $<0.01$ & $<0.01$ & $<0.01$ & $<0.01$ & $<0.01$ \\
\hline Cobalt & $<0.005$ & $<0.005$ & $<0.005$ & $<0.005$ & $<0.005$ & $<0.005$ & $<0.005$ & $<0.005$ \\
\hline Copper & 0.0042 & $<0.004$ & $<0.004$ & $<0.004$ & $<0.004$ & $<0.004$ & $<0.004$ & $<0.004$ \\
\hline Iron & 1.5 & 0.0087 & 1.7 & $<0.005$ & 0.077 & 0.044 & 0.078 & 0.006 \\
\hline Lead (AAS) & $<0.004$ & $<0.004$ & $<0.004$ & $<0.004$ & $<0.004$ & $<0.004$ & $<0.004$ & $<0.004$ \\
\hline Mercury (CVAM) & $<0.0002$ & $<0.0002$ & $<0.0002$ & $<0.0002$ & $<0.0002$ & $<0.0002$ & $<0.0002$ & $<0.0002$ \\
\hline Molybdenum & $<0.01$ & $<0.01$ & $<0.01$ & $<0.01$ & $<0.01$ & $<0.01$ & $<0.01$ & $<0.01$ \\
\hline Nickel & $<0.01$ & $<0.01$ & $<0.01$ & $<0.01$ & $<0.01$ & $<0.01$ & $<0.01$ & 0.012 \\
\hline Selenium & $<0.05$ & $<0.05$ & $<0.05$ & $<0.05$ & $<0.05$ & 0.089 & $<0.05$ & $<0.05$ \\
\hline silver & $<0.006$ & $<0.006$ & $<0.006$ & $<0.006$ & $<0.006$ & $<0.006$ & $<0.006$ & $<0.006$ \\
\hline strontium & 0.077 & 0.075 & 0.08 & 0.077 & 0.75 & 0.71 & 0.94 & 0.9 \\
\hline Thorium & $<0.2$ & $<0.2$ & $<0.2$ & $<0.2$ & $<0.2$ & $<0.2$ & $<0.2$ & $<0.2$ \\
\hline Uranium (Fluor) & $<0.001$ & 0.001 & $<0.001$ & $<0.001$ & $<0.001$ & $<0.001$ & $<0.001$ & $<0.001$ \\
\hline Vanadium & $<0.005$ & $<0.005$ & $<0.005$ & $<0.005$ & $<0.005$ & $<0.005$ & $<0.005$ & $<0.005$ \\
\hline zinc & 0.011 & 0.0071 & 0.0099 & 0.012 & 0.036 & 0.032 & 0.045 & 0.038 \\
\hline MAJOR IONS (mg/L) & $\cdot$ & $\cdot$ & - &. &. & $\cdot$ & . & $\bullet$ \\
\hline Alkalinity-HCO3 & 153 & $\cdot$ & $15 \dot{3}$ & $\cdot$ & 123 & $\cdot$ & 124 & $\bullet$ \\
\hline Alkalinity- $\mathrm{CO} 3$ & $<1$ &. & $<1$ &. & $<1$ &. & $<1$ & . \\
\hline Calcium & 59 & 56 & 58 & 55 & 320 & 340 & 440 & 410 \\
\hline Chloride & $<1$ & . & 1 & . & 16 & . & 16 & $\bullet$ \\
\hline Fluoride & 0.1 & . & 0.1 & . & 0.2 &. & $<0.1$ & - \\
\hline Magnes I um & 4.3 & 3.8 & 4 & 3.5 & 21 & 22 & 26 & 25 \\
\hline Manganese & 0.074 & 0.0059 & 0.077 & 0.0063 & 0.0061 & 0.0026 & 0.0061 & 0.013 \\
\hline Nitrate-N & $<0.2$ & . & $<0.2$ & - & 2139 & . & 240 & $\cdot$ \\
\hline Potassium & 0.87 & $<0.6$ & 1.5 & 0.73 & 2 & 1.7 & 2.3 & 2.1 \\
\hline Sodium & 5.4 & 5.4 & 5.5 & 5.4 & 12 & 14 & 13 & 12 \\
\hline sulfate & 7.8 & - & 7 & • & 11 & . & 7.7 & . \\
\hline
\end{tabular}

(CONTINUED) 
APPENDIX E.1

Groundwater and Surface-Water Quality Data, 1993

\begin{tabular}{|c|c|c|c|c|c|c|c|c|}
\hline \multirow{4}{*}{$\begin{array}{l}\text { Sampling point } \\
\text { Location } \\
\text { Date sampled }\end{array}$} & \multicolumn{4}{|c|}{ GW-085 } & \multicolumn{4}{|c|}{$0 w-086$} \\
\hline & \multicolumn{4}{|c|}{ OLF } & \multicolumn{4}{|c|}{ OLF } \\
\hline & \multicolumn{2}{|c|}{$09 / 14 / 93$} & \multicolumn{2}{|c|}{$10 / 22 / 93$} & \multicolumn{2}{|c|}{$02 / 05 / 93$} & \multicolumn{2}{|c|}{$05 / 11 / 93$} \\
\hline & TOT & DIS & TOT & DIS & TOT & DIS & Tor & Drs \\
\hline METALS (mg/L) & $\cdot$ & . & $\cdot$ & - & - & . & . &. \\
\hline Aluminum & $<0.02$ & $<0.02$ & 0.066 & 0.069 & 16 & 0.12 & 9.4 & 0.06 \\
\hline Antimony & $<0.05$ & $<0.05$ & $<0.05$ & $<0.05$ & $<0.05$ & $<0.05$ & $<0.05$ & $<0.05$ \\
\hline Arsenic & $<0.05$ & $<0.05$ & $<0.05$ & $<0.05$ & $<0.05$ & $<0.05$ & $<0.05$ & 0.072 \\
\hline Barium & 1.3 & 1.3 & 1.3 & 1.3 & 0.32 & 0.14 & 0.39 & 0.14 \\
\hline Beryllium & $<0.0003$ & $<0.0003$ & $<0.0003$ & $<0.0003$ & 0.0011 & $<0.0003$ & 0.00063 & $<0.0003$ \\
\hline Boron & 0.02 & 0.015 & 0.041 & 0.039 & 0.034 & 0.025 & 0.021 & 0.013 \\
\hline Cadmium (AAS) & $<0.002$ & $<0.002$ & $<0.002$ & $<0.002$ & $<0.002$ & $<0.002$ & $<0.002$ & $<0.002$ \\
\hline Cadmium & $<0.003$ & $<0.003$ & $<0.003$ & $<0.003$ & $<0.003$ & $<0.003$ & $<0.003$ & $<0.003$ \\
\hline Chromium (AAS) & $<0.01$ & $<0.01$ & $<0.01$ & $<0.01$ & 0.031 & $<0.01$ & 0.062 & $<0.01$ \\
\hline Chromium & $<0.01$ & $<0.01$ & $<0.01$ & $<0.01$ & 0.027 & $<0.01$ & 0.014 & $<0.01$ \\
\hline Cobalt & $<0.005$ & $<0.005$ & $<0.005$ & $<0.005$ & 0.012 & $<0.005$ & 0.0072 & $<0.005$ \\
\hline Copper & 0.0064 & $<0.004$ & $<0.004$ & $<0.004$ & 0.0097 & $<0.004$ & 0.011 & $<0.004$ \\
\hline Iron & 0.035 & $<0.005$ & 0.037 & $<0.005$ & 16 & 0.1 & 8.9 & $<0.005$ \\
\hline Lead (AAS) & $<0.004$ & $<0.004$ & $<0.004$ & $<0.004$ & 0.0095 & $<0.004$ & 0.016 & $<0.004$ \\
\hline Mercury (CVAA) & $<0.0002$ & $<0.0002$ & $<0.0002$ & $<0.0002$ & $<0.0002$ & $<0.0002$ & $<0.0002$ & $<0.0002$ \\
\hline Mol ybdenum & $<0.01$ & $<0.01$ & $<0.01$ & $<0.01$ & $<0.01$ & $<0.01$ & $<0.01$ & $<0.01$ \\
\hline Nickel & $<0.01$ & $<0.01$ & $<0.01$ & $<0.01$ & 0.014 & $<0.01$ & 0.023 & 0.016 \\
\hline Selenium & 0.065 & 0.11 & $<0.05$ & $<0.05$ & $<0.05$ & $<0.05$ & $<0.05$ & $<0.05$ \\
\hline Silver & $<0.006$ & $<0.006$ & $<0.006$ & $<0.006$ & $<0.006$ & $<0.006$ & $<0.006$ & $<0.006$ \\
\hline Strontium & 0.97 & 0.97 & 0.95 & 0.96 & 0.092 & 0.082 & 0.094 & 0.083 \\
\hline Thorium & $<0.2$ & $<0.2$ & $<0.2$ & $<0.2$ & $<0.2$ & $<0.2$ & $<0.2$ & $<0.2$ \\
\hline Uranium (Fluor) & 0.002 & $\cdot$ & $<0.001$ & $<0.001$ & $<0.001$ & 0.001 & $<0.001$ & $<0.001$ \\
\hline Vanadium & $<0.005$ & $<0.005$ & $<0.005$ & $<0.005$ & 0.018 & $<0.005$ & 0.01 & $<0.005$ \\
\hline Zinc & 0.07 & 0.059 & 0.035 & 0.03 & 0.095 & 0.061 & 0.083 & 0.04 \\
\hline MAJOR IONS $(\mathrm{mg} / \mathrm{L})$ & $\cdot$ &. & . &. & . &. &. & . \\
\hline Alkalinity-HCO3 & 128 & $\cdot$ & 121 & $\cdot$ & 96 & $\cdot$ & 95 & . \\
\hline Alkalinity- $\mathrm{CO} 3$ & $<1$ & $\cdot$ & $<1$ &. & $<1$ &. & $<1$ & . \\
\hline Calcium & 450 & 440 & 390 & 410 & 41 & 39 & 44 & 41 \\
\hline Chloride & 14 & $\cdot$ & 15 & $\cdot$ & 1.4 & $\cdot$ & 1.8 & $\cdot$ \\
\hline Fluoride & $<0.1$ & $\cdot$ & 0.1 & $\cdot$ & $<0.1$ & $\cdot$ & $<0.1$ & . \\
\hline Magnesium & 29 & 28 & 27 & 27 & 6.6 & 2.9 & 5 & 2.9 \\
\hline Manganese & 0.0059 & 0.0063 & 0.0069 & 0.0055 & 0.61 & 0.011 & 0.38 & 0.0069 \\
\hline Nitrate-N & 304 & $\cdot$ & 312.6 & $\cdot$ & 4.5 & $\cdot$ & 3.1 & . \\
\hline Potassium & 2.3 & 2.6 & 2.4 & 2.4 & 6.8 & 1.1 & 4.4 & 1 \\
\hline Sodium & 13 & 13 & 14 & 14 & 5.1 & 5.1 & 5.1 & 5.1 \\
\hline Sulfate & 11 & . & $<10$ &. & 14 &. & 15 & . \\
\hline
\end{tabular}

(CONTINUED) 
APPENDIX E.1

Groundwater and Surface-Water Quality Data, 1993

\begin{tabular}{|c|c|c|c|c|c|c|c|c|}
\hline \multirow{4}{*}{$\begin{array}{l}\text { Sampling Point } \\
\text { Location } \\
\text { Date Sampled }\end{array}$} & \multicolumn{4}{|c|}{ GW-086 } & \multicolumn{4}{|c|}{ GW-115 } \\
\hline & \multicolumn{4}{|c|}{ OLF } & \multicolumn{4}{|c|}{ s3 } \\
\hline & \multicolumn{2}{|c|}{$09 / 12 / 93$} & \multicolumn{2}{|c|}{$10 / 21 / 93$} & \multicolumn{2}{|c|}{$01 / 28 / 93$} & \multicolumn{2}{|c|}{$05 / 25 / 93$} \\
\hline & TOT & DIS & TOT & DIS & TOT & DIS & TOT & DIS \\
\hline METALS (mg/L) & $\cdot$ & - & $\cdot$ & - & - & - & $\cdot$ & - \\
\hline Aluminum & 22 & $<0.02$ & 22 & 0.022 & 0.044 & 0.025 & 0.07 & $<0.02$ \\
\hline Antimony & $<0.05$ & $<0.05$ & $<0.05$ & $<0.05$ & $<0.05$ & $<0.05$ & $<0.05$ & $<0.05$ \\
\hline Arsenic & $<0.05$ & $<0.05$ & $<0.05$ & $<0.05$ & $<0.05$ & $<0.05$ & $<0.05$ & $<0.05$ \\
\hline Barium & 0.42 & 0.14 & 0.49 & 0.14 & 0.24 & 0.24 & 0.24 & 0.24 \\
\hline Beryllium & 0.0012 & $<0.0003$ & 0.0015 & $<0.0003$ & $<0.0003$ & $<0.0003$ & $<0.0003$ & $<0.0003$ \\
\hline Boron & 0.021 & 0.022 & 0.021 & 0.006 & 0.028 & 0.018 & 0.016 & 0.03 \\
\hline Cadmium (AAS) & $<0.002$ & $<0.002$ & $<0.002$ & $<0.002$ & $<0.002$ & $<0.002$ & $<0.002$ & $<0.002$ \\
\hline Cadmium & 0.0078 & $<0.003$ & 0.0061 & $<0.003$ & 0.0048 & $<0.003$ & $<0.003$ & $<0.003$ \\
\hline Chromium (AAS) & 0.035 & $<0.01$ & 0.044 & $<0.01$ & $<0.01$ & $<0.01$ & $<0.01$ & $<0.01$ \\
\hline Chromium & 0.04 & $<0.01$ & 0.043 & $<0.01$ & $<0.01$ & $<0.01$ & $<0.01$ & $<0.01$ \\
\hline Cobalt & 0.019 & $<0.005$ & 0.026 & $<0.005$ & $<0.005$ & $<0.005$ & $<0.005$ & $<0.005$ \\
\hline Copper & 0.027 & 0.019 & 0.033 & $<0.004$ & $<0.004$ & $<0.004$ & 0.0043 & 0.03 \\
\hline Iron & 28 & $<0.005$ & 32 & $<0.005$ & 0.24 & 0.18 & 0.44 & 0.44 \\
\hline Lead (AAS) & 0.0089 & $<0.004$ & 0.014 & $<0.004$ & $<0.004$ & $<0.004$ & $<0.004$ & $<0.004$ \\
\hline Mercury (CVAA) & $<0.0002$ & $<0.0002$ & $<0.0002$ & $<0.0002$ & $<0.0002$ & $<0.0002$ & $<0.0002$ & $<0.0002$ \\
\hline Mol ybdenum & $<0.01$ & $<0.01$ & $<0.01$ & $<0.01$ & $<0.01$ & $<0.01$ & $<0.01$ & $<0.01$ \\
\hline Nickel & 0.041 & $<0.01$ & 0.048 & $<0.01$ & 0.012 & $<0.01$ & $<0.01$ & 0.019 \\
\hline Selenium & $<0.05$ & $<0.05$ & $<0.05$ & $<0.05$ & $<0.05$ & $<0.05$ & $<0.05$ & $<0.05$ \\
\hline silver & $<0.006$ & $<0.006$ & $<0.006$ & $<0.006$ & $<0.006$ & $<0.006$ & $<0.006$ & $<0.006$ \\
\hline strontium & 0.088 & 0.076 & 0.09 & 0.073 & 0.11 & 0.11 & 0.12 & 0.12 \\
\hline Thorium & $<0.2$ & $<0.2$ & $<0.2$ & $<0.2$ & $<0.2$ & $<0.2$ & $<0.2$ & $<0.2$ \\
\hline Uranium (Fluor) & 0.001 & 0.001 & 0.001 & $<0.001$ & $<0.001$ & $<0.001$ & $<0.001$ & $<0.001$ \\
\hline Vanadium & 0.027 & $<0.005$ & 0.036 & $<0.005$ & $<0.005$ & $<0.005$ & $<0.005$ & $<0.005$ \\
\hline zine & 0.16 & 0.094 & 0.13 & 0.033 & $<0.002$ & $<0.002$ & 0.0068 & 0.049 \\
\hline MAJOR IONS $(\mathrm{mg} / \mathrm{L})$ & $\cdot$ & $\cdot$ & - & $\cdot$ & $\cdot$ & $\cdot$ & $\cdot$ & • \\
\hline Alkalinity-HCO3 & $\dot{91}$ & $\cdot$ & $\dot{92}$ & $\cdot \dot{ }$ & 212 & $\cdot \dot{.}$ & 224 & $\bullet$ \\
\hline Alkalinity-CO3 & $<2$ & $\cdot$ & $<1$ & $\cdot$ & $<1$ & . & $<1$ & • \\
\hline Calcium & 43 & 42 & 43 & 37 & 71 & 69 & 69 & 69 \\
\hline Chloride & 1.3 & $\cdot$ & 2 & - & 3.5 & $\cdot$ & 4.1 & - \\
\hline Fluoride & $<0.1$ & $\cdot$ & $<0.1$ & $\cdot$ & 0.1 & $\cdot$ & 0.1 & • \\
\hline Magnesium & 9.1 & 3.1 & 9.9 & 2.9 & 9.5 & 9.5 & 11 & 11 \\
\hline Manganese & 1 & 0.0076 & 1.1 & 0.0041 & 0.097 & 0.092 & 0.3 & 0.3 \\
\hline Nitrate-N & 4.97 &. & 5.6 & . & $<0.2$ &. & $<0.2$ & . \\
\hline Potassium & 7.9 & 0.93 & 7.5 & 1.1 & 1.8 & 2 & 1.6 & 2.1 \\
\hline Sodium & 5.3 & 5.2 & 5.4 & 5.1 & 8.9 & 8.8 & 9.7 & 9.8 \\
\hline Sulfate & 10.9 & . & 9 & . & 18 & . & 17.9 & - \\
\hline
\end{tabular}

(CONTINUED) 
APPENDIX E.1

Groundwater and Surface-Water Quality Data, 1993

\begin{tabular}{|c|c|c|c|c|c|c|c|c|}
\hline \multirow{4}{*}{$\begin{array}{l}\text { Sampling Point } \\
\text { Location } \\
\text { Date Sampled }\end{array}$} & \multicolumn{4}{|c|}{ GW-115 } & \multicolumn{4}{|c|}{$G W-118$} \\
\hline & \multicolumn{4}{|c|}{ s3 } & \multicolumn{4}{|c|}{ BG } \\
\hline & \multicolumn{2}{|c|}{$09 / 11 / 93$} & \multicolumn{2}{|c|}{$10 / 19 / 93$} & \multicolumn{2}{|c|}{$03 / 04 / 93$} & \multicolumn{2}{|c|}{$04 / 21 / 93$} \\
\hline & TOT & DIS & TOT & DIS & TOT & DIS & TOT & DIS \\
\hline METALS (mg/L) & $\cdot$ &. & $\cdot$ & $\cdot$ & - & $\cdot$ & $\cdot$ & • \\
\hline Aluminum & $<0.02$ & $<0.02$ & 0.034 & $<0.02$ & $<0.2$ & $<0.2$ & $<0.2$ & $<0.2$ \\
\hline Antimony & $<0.05$ & $<0.05$ & $<0.05$ & $<0.05$ & $<0.5$ & $<0.5$ & $<0.5$ & $<0.5$ \\
\hline Arsenic & $<0.05$ & $<0.05$ & $<0.05$ & $<0.05$ & $<0.5$ & $<0.5$ & $<0.5$ & $<0.5$ \\
\hline Barium & 0.25 & 0.24 & 0.26 & 0.24 & 0.11 & 0.12 & 0.26 & 0.26 \\
\hline Beryllium & $<0.0003$ & $<0.0003$ & $<0.0003$ & $<0.0003$ & 0.0038 & $<0.003$ & $<0.003$ & $<0.003$ \\
\hline Boron & 0.026 & 0.017 & 0.015 & 0.024 & 0.22 & 0.17 & 0.2 & 0.57 \\
\hline Cadmium (AAS) & $<0.002$ & $<0.002$ & $<0.002$ & $<0.002$ & $<0.002$ & $<0.002$ & $<0.002$ & $<0.002$ \\
\hline Cadmium & $<0.003$ & $<0.003$ & $<0.003$ & $<0.003$ & $<0.03$ & $<0.03$ & $<0.03$ & $<0.03$ \\
\hline Chromium (AAS) & $<0.01$ & $<0.01$ & $<0.01$ & $<0.01$ & $<0.01$ & $<0.01$ & $<0.01$ & $<0.01$ \\
\hline Chromium & $<0.01$ & $<0.01$ & $<0.01$ & $<0.01$ & $<0.1$ & $<0.1$ & $<0.1$ & $<0.1$ \\
\hline Cobalt & $<0.005$ & $<0.005$ & $<0,005$ & $<0.005$ & $<0.05$ & $<0.05$ & $<0.05$ & $<0.05$ \\
\hline Copper & $<0.004$ & $<0.004$ & 0.0046 & $<0.004$ & $<0.04$ & $<0.04$ & $<0.04$ & $<0.04$ \\
\hline Iron & 0.4 & 0.35 & 0.34 & 0.28 & 12 & $<0.05$ & 19 & $<0.05$ \\
\hline Lead (AAS) & $<0.004$ & $<0.004$ & $<0.004$ & $<0.004$ & 0.028 & $<0.004$ & 0.017 & $<0.004$ \\
\hline Mercury (CVAA) & $<0.0002$ & $<0.0002$ & $<0.0002$ & $<0.0002$ & $<0.0002$ & $<0.0002$ & $<0.0002$ & $<0.0002$ \\
\hline Molybdenum & $<0.01$ & $<0.01$ & $<0.01$ & $<0.01$ & $<0.1$ & $<0.1$ & $<0.1$ & $<0.1$ \\
\hline Nickel & $<0.01$ & $<0.01$ & $<0.01$ & $<0.01$ & $<0.1$ & $<0.1$ & $<0.1$ & $<0.1$ \\
\hline Selenium & $<0.05$ & $<0.05$ & $<0.05$ & $<0.05$ & $<0.5$ & $<0.5$ & $<0.5$ & $<0.5$ \\
\hline silver & $<0.006$ & $<0.006$ & $<0.006$ & $<0.006$ & $<0.06$ & $<0.06$ & $<0.06$ & $<0.06$ \\
\hline Strontium & 0.12 & 0.12 & 0.13 & 0.12 & 0.93 & 1.1 & 1.6 & 2.7 \\
\hline Thorium & $<0.2$ & $<0.2$ & $<0.2$ & $<0.2$ & $<2$ & $<2$ & $<2$ & $<2$ \\
\hline Uranium (Fluor) & 0.001 & $<0.001$ & $<0.001$ & 0.001 & $<0.001$ & $<0.001$ & $<0.001$ & $<0.001$ \\
\hline Vanadium & $<0.005$ & $<0.005$ & $<0.005$ & $<0,005$ & $<0.05$ & $<0.05$ & $<0.05$ & $<0.05$ \\
\hline Zine & $<0.002$ & 0.0033 & 0.0036 & $<0.002$ & 0.03 & $<0.02$ & 0.025 & 0.042 \\
\hline MAJOR IONS (mg/L) &. &. & . &. &. & . & . & - \\
\hline Alkalinity-HCO3 & 235 & $\cdot$ & $23 \dot{5}$ & $\cdot$ & $\dot{99}$ & $\cdot$ & $\dot{71}$ & • \\
\hline Alkalinity-CO3 & $<1$ & . & $<1$ & . & 152 & . & 176 & - \\
\hline Calcium & 74 & 72 & 71 & 70 & 2 & 2.4 & 2.9 & 3 \\
\hline Chloride & 3.9 &. & 4 & . & 3220 & . & 3060 & - \\
\hline Fluoride & $<0.1$ & . & $<0.1$ &. & 2.7 & . & 1.7 & • \\
\hline Magnesium & 11 & 11 & 12 & 12 & 1.3 & 1.8 & 4.1 & 4.6 \\
\hline Manganese & 0.31 & 0.29 & 0.31 & 0.29 & 0.36 & $<0.01$ & 0.23 & $<0.02$ \\
\hline Nitrate-N & $<0.2$ & . & $<0.2$ &. & $<23$ & . & $<0.2$ & . \\
\hline Potassium & 1.8 & 2.8 & 2 & 1.8 & 26 & 24 & 21 & 22 \\
\hline Sodium & 10 & 10 & 11 & 9.8 & 2100 & 2000 & 1600 & 1800 \\
\hline Sulfate & 15.8 & . & 15 & . & $<100$ & . & $<1$ & - \\
\hline
\end{tabular}

(CONTINUED) 
APRENDIX E. 1

Groundwater and Surface-Water Quality Data, 1993

\begin{tabular}{|c|c|c|c|c|c|c|c|c|}
\hline \multirow{4}{*}{$\begin{array}{l}\text { Sampling Point } \\
\text { - } \\
\text { Location } \\
\text { - } \\
\text { Date Sampled }\end{array}$} & \multicolumn{4}{|c|}{ GW-118 } & \multicolumn{2}{|c|}{ GW-162 } & \multicolumn{2}{|c|}{$G W-286$} \\
\hline & \multicolumn{4}{|c|}{ BG } & \multicolumn{2}{|c|}{ BG } & \multicolumn{2}{|c|}{ BG } \\
\hline & \multicolumn{2}{|c|}{$07 / 25 / 93$} & \multicolumn{2}{|c|}{$10 / 10 / 93$} & \multicolumn{2}{|c|}{$10 / 10 / 93$} & \multicolumn{2}{|c|}{$02 / 25 / 93$} \\
\hline & TOT & DIS & TOT & DIS & TOT & DIS & TOT & DIS \\
\hline METACS (mg/L) & $\cdot$ & $\cdot$ & $\cdot$ & $\cdot$ & $\cdot$ & - & - & - \\
\hline Aluminum & $<0.2$ & $<0.2$ & $<0.1$ & $<0.1$ & 0.34 & $<0.02$ & 0.072 & $<0.02$ \\
\hline Antimony & $<0.5$ & $<0.5$ & $<0.25$ & $<0.25$ & $<0.05$ & $<0.05$ & $<0.05$ & $<0.05$ \\
\hline Arsenic & $<0.5$ & $<0.5$ & $<0.25$ & $<0.25$ & $<0.05$ & $<0.05$ & 0.053 & $<0.05$ \\
\hline Barium & $<0.01$ & 0.017 & 0.0058 & $<0.005$ & 0.31 & 0.23 & 0.32 & 0.32 \\
\hline Beryllium & $<0.003$ & $<0.003$ & $<0.0015$ & $<0.0015$ & $<0.0003$ & $<0.0003$ & $<0.0003$ & $<0.0003$ \\
\hline Boron & 0.15 & 0.15 & 0.13 & 0.13 & 0.045 & 0.039 & 0.04 & 0.038 \\
\hline Cadmium (AAS) & $<0.002$ & $<0.002$ & $<0.002$ & $<0.002$ & $<0.002$ & $<0.002$ & $<0.002$ & $<0.002$ \\
\hline Cadmium & $<0.03$ & $<0.03$ & $<0.015$ & $<0.015$ & $<0.003$ & $<0.003$ & $<0.003$ & $<0.003$ \\
\hline Chromium (AAS) & $<0.01$ & $<0.01$ & $<0.01$ & $<0.01$ & $<0.01$ & $<0.01$ & $<0.01$ & $<0.01$ \\
\hline Chromium & $<0.1$ & $<0.1$ & $<0.05$ & $<0.05$ & $<0.01$ & $<0.01$ & $<0.01$ & $<0.01$ \\
\hline Cobalt & $<0.05$ & $<0.05$ & $<0.025$ & $<0.025$ & 0.0052 & $<0.005$ & $<0.005$ & $<0.005$ \\
\hline Copper & $<0.04$ & $<0.04$ & $<0.02$ & $<0.02$ & 0.0046 & $<0.004$ & $<0.004$ & $<0.004$ \\
\hline Iron & 14 & $<0.05$ & 25 & 0.046 & 15 & 0.0086 & 0.098 & $<0.005$ \\
\hline Lead (AAS) & 0.0069 & $<0.004$ & $<0.004$ & $<0.004$ & $<0.004$ & $<0.004$ & $<0.004$ & $<0.004$ \\
\hline Mercury (CVAA) & $<0.0002$ & 0.00021 & $<0.0002$ & $<0.0002$ & $<0.0002$ & $<0.0002$ & $<0.0002$ & $<0.0002$ \\
\hline Molybdenum & $<0.1$ & $<0.1$ & $<0.05$ & 0.054 & $<0.01$ & $<0.01$ & $<0.01$ & $<0.01$ \\
\hline Nickel & $<0.1$ & $<0.1$ & $<0.05$ & $<0.05$ & $<0.01$ & $<0.01$ & $<0.01$ & $<0.01$ \\
\hline Selenium & $<0.5$ & $<0.5$ & $<0.25$ & $<0.25$ & $<0.05$ & $<0.05$ & $<0.05$ & $<0.05$ \\
\hline Silver & $<0.06$ & $<0.06$ & $<0.03$ & $<0.03$ & $<0.006$ & $<0.006$ & $<0.006$ & $<0.006$ \\
\hline strontium & 0.3 & 0.26 & 0.2 & 0.19 & 0.66 & 0.63 & 0.11 & 0.11 \\
\hline Thorium & $<2$ & $<2$ & $<1$ & $<1$ & $<0.2$ & $<0.2$ & $<0.2$ & $<0.2$ \\
\hline Uranium (Fluor) & $<0.001$ & $<0.001$ & $<0.001$ & $<0.001$ & 0.001 & $<0.001$ & 0.001 & $<0.001$ \\
\hline Vanadium & $<0.05$ & $<0.05$ & $<0.025$ & $<0.025$ & 0.0059 & $<0.005$ & $<0.005$ & $<0.005$ \\
\hline zinc & 0.037 & $<0.02$ & 0.015 & 0.012 & 0.014 & 0.0025 & 0.0075 & $<0.002$ \\
\hline MAJOR IONS $(\mathrm{mg} / \mathrm{L})$ & $\cdot$ & - & - & - & - & - & - & - \\
\hline Alkalinity- $\mathrm{HCO} 3$ & 43 & - & 49 & - & 115 & - & 140 & - \\
\hline Alkalinity-CO3 & 194 & $\cdot$ & 192 & - & $<1$ & - & $<1$ & - \\
\hline Calcium & 1.3 & 1.3 & 1 & 0.94 & 30 & 27 & 49 & 49 \\
\hline Chloride & 3910 & $\cdot$ & 3160 & $\cdot$ & 3 & - & 1.2 & - \\
\hline Fluoride & 1.5 & - & 1.2 & - & 0.1 & - & 0.1 & - \\
\hline Magnesium & 0.33 & 0.58 & 0.041 & 0.035 & 4.7 & 4.4 & 4.7 & 4.6 \\
\hline Manganese & 0.79 & 0.014 & 0.93 & $<0.005$ & 0.15 & 0.073 & 0.02 & 0.017 \\
\hline Nitrate-N & $<0.2$ & - & $<22.6$ & - & $<0.2$ & - & $<0.2$ & - \\
\hline Potassium & 31. & 34 & 28 & 26 & 3.5 & 3.1 & 0.93 & 0.7 \\
\hline Sodium & 2000 & 1900 & 2000 & 1900 & 15 & 15 & 5.1 & 5 \\
\hline Sulfate & $<1$ & - & $<100$ & - & 8.6 & - & 6.1 & - \\
\hline
\end{tabular}

(CONTINUED) 
APPENDIX $\mathrm{E} .1$

Groundwater and Surface-Water Quality Data, 1993

\begin{tabular}{|c|c|c|c|c|c|c|c|c|}
\hline \multirow{4}{*}{$\begin{array}{l}\text { Sampling Point } \\
\text { Location } \\
\text { - } \\
\text { Date Sampled }\end{array}$} & \multicolumn{6}{|c|}{ GW-286 } & \multicolumn{2}{|c|}{$G W-287$} \\
\hline & \multicolumn{6}{|c|}{ BG } & \multicolumn{2}{|c|}{ BG } \\
\hline & \multicolumn{2}{|c|}{$04 / 19 / 93$} & \multicolumn{2}{|c|}{$07 / 10 / 93$} & \multicolumn{2}{|c|}{$10 / 07 / 93$} & \multicolumn{2}{|c|}{$02 / 24 / 93$} \\
\hline & TOT & DIS & TOT & DIS & TOT & DIS & Tor & DIS \\
\hline METALS (mg/L) & - & - & $\cdot$ & • & - & $\cdot$ & - & - \\
\hline Aluminum & 0.11 & $<0.02$ & 0.034 & $<0.02$ & 0.024 & 0.024 & 0.3 & 0.045 \\
\hline Antimony & $<0.05$ & $<0.05$ & $<0.05$ & $<0.05$ & $<0.05$ & $<0.05$ & $<0.05$ & $<0.05$ \\
\hline Arsenic & $<0.05$ & $<0.05$ & $<0.05$ & $<0.05$ & $<0.05$ & $<0.05$ & $<0.05$ & $<0.05$ \\
\hline Barium & 0.29 & 0.28 & 0.3 & 0.3 & 0.31 & 0.31 & 0.13 & 0.12 \\
\hline Beryllium & $<0.0003$ & $<0.0003$ & $<0.0003$ & $<0.0003$ & $<0.0003$ & $<0.0003$ & 0.0023 & $<0.0003$ \\
\hline Boron & 0.012 & 0.033 & 0.011 & 0.014 & 0.014 & 0.014 & 0.025 & 0.012 \\
\hline Cadmium (AAS) & $<0.002$ & $<0.002$ & $<0.002$ & $<0.002$ & $<0.002$ & $<0.002$ & $<0.002$ & $<0.002$ \\
\hline Cadmium & $<0.003$ & $<0.003$ & $<0.003$ & $<0.003$ & $<0.003$ & $<0.003$ & $<0.003$ & $<0.003$ \\
\hline Chromium (AAS) & $<0.01$ & $<0.01$ & $<0.01$ & $<0.01$ & $<0.01$ & $<0.01$ & $<0.01$ & $<0.01$ \\
\hline Chromium & $<0.01$ & $<0.01$ & $<0.01$ & $<0.01$ & $<0.01$ & $<0.01$ & $<0.01$ & $<0.01$ \\
\hline Cobalt & $<0.005$ & $<0.005$ & $<0.005$ & $<0.005$ & $<0.005$ & $<0.005$ & $<0.005$ & $<0.005$ \\
\hline Copper & 0.0047 & $<0.004$ & $<0.004$ & $<0.004$ & $<0.004$ & 0.0077 & $<0.004$ & $<0.004$ \\
\hline Iron & 0.097 & 0.0073 & 0.033 & 0.012 & 0.026 & 0.0054 & 0.36 & $<0.005$ \\
\hline Lead (AAS) & $<0.004$ & $<0.004$ & $<0.004$ & $<0.004$ & $<0.004$ & $<0.004$ & $<0.004$ & $<0.004$ \\
\hline Mercury (CVAA) & $<0.0002$ & $<0.0002$ & $<0.0002$ & $<0.0002$ & $<0.0002$ & $<0.0002$ & $<0.0002$ & $<0.0002$ \\
\hline Mol ybdenum & $<0.01$ & $<0.01$ & $<0.01$ & $<0.01$ & $<0.01$ & $<0.01$ & $<0.01$ & $<0.01$ \\
\hline Nickel & $<0.01$ & $<0.01$ & $<0.01$ & $<0.01$ & $<0.01$ & $<0.01$ & $<0.01$ & $<0.01$ \\
\hline Selenium & $<0.05$ & $<0.05$ & $<0.05$ & $<0.05$ & $<0.05$ & $<0.05$ & $<0.05$ & $<0.05$ \\
\hline silver & $<0.006$ & $<0.006$ & $<0.006$ & $<0.006$ & $<0.006$ & $<0.006$ & $<0.006$ & $<0.006$ \\
\hline strontium & 0.095 & 0.093 & 0.098 & 0.098 & 0.11 & 0.1 & 0.081 & 0.079 \\
\hline Thorium & $<0.2$ & $<0.2$ & $<0.2$ & $<0.2$ & $<0.2$ & $<0.2$ & $<0.2$ & $<0.2$ \\
\hline Uranium (Fluor) & $<0.001$ & $<0.001$ & $<0.001$ & $<0.001$ & 0.001 & $<0.001$ & $<0.001$ & $<0.001$ \\
\hline Vanadium & $<0.005$ & $<0.005$ & $<0.005$ & $<0.005$ & $<0.005$ & $<0.005$ & $<0.005$ & $<0.005$ \\
\hline zinc & 0.022 & 0.023 & 0.0074 & 0.007 & 0.011 & 0.017 & 0.019 & 0.018 \\
\hline MAJOR IONS $(\mathrm{mg} / \mathrm{L})$ & • & . & - & . & - & . & $\cdot$ & - \\
\hline Alkalinity-HCO3 & 135 & $\cdot$ & 141 & . & 142 & $\cdot$ & 120 & • \\
\hline Alkalinity-CO3 & $<1$ & . & $<1$ & - & $<1$ & . & $<1$ & - \\
\hline calcium & 44 & 44 & 47 & 47 & 47 & 47 & 38 & 38 \\
\hline Chloride & 1.4 & . & 1.5 & - & 2 & - & 2.6 & - \\
\hline Fluoride & 0.1 & . & 0.2 & - & 0.1 & - & 0.2 & - \\
\hline Magnesium & 4.2 & $4 \cdot 1$ & 4.5 & 4.6 & 4.6 & 4.5 & 5.7 & 5.7 \\
\hline Manganese & 0.016 & 0.013 & 0.017 & 0.016 & 0.014 & 0.014 & 0.035 & 0.0093 \\
\hline Nitrate-N & $<0.2$ &. & $<0.2$ & - & $<0.2$ & . & $<0.2$ & - \\
\hline Potassium & 0.89 & 0.96 & 0.62 & 0.75 & 0.77 & 0.71 & 1.3 & 1.2 \\
\hline Sodium & 4.6 & 4.5 & 4.7 & 4.7 & 4.9 & 4.8 & 3.7 & 3.9 \\
\hline Sulfate & 6.2 & . & 7 & . & 6 & . & 8.2 & - \\
\hline
\end{tabular}

(CONTINUED) 
APPENDIX $\mathrm{B} .1$

Groundwater and Surface-Water Quallty Data, 1993

\begin{tabular}{|c|c|c|c|c|c|c|c|c|}
\hline \multirow{4}{*}{$\begin{array}{l}\text { Sampling Point } \\
\text { Location } \\
\text { Date Sampled }\end{array}$} & \multicolumn{6}{|c|}{ GW-287 } & \multicolumn{2}{|c|}{$6 w-312$} \\
\hline & \multicolumn{6}{|c|}{ BG } & \multirow{2}{*}{\multicolumn{2}{|c|}{$\frac{\text { RS }}{02 / 08 / 93}$}} \\
\hline & \multicolumn{2}{|c|}{$04 / 16 / 93$} & \multicolumn{2}{|c|}{$07 / 10 / 93$} & \multicolumn{2}{|c|}{$10 / 07 / 93$} & & \\
\hline & TOT & DIS & TOT & DIS & TOT & DIs & TOT & Drs \\
\hline METALS (mg/L) & $\cdot$ & . & $\cdot$ & $\cdot$ & $\cdot$ & $\cdot$ & • & - \\
\hline Aluminum & 0.064 & $<0.02$ & 0.22 & $<0.02$ & 0.34 & $<0.02$ & $\dot{2}$ & 0.31 \\
\hline Antimony & $<0.05$ & $<0.05$ & $<0.05$ & $<0.05$ & $<0.05$ & $<0.05$ & $<0.05$ & $<0.05$ \\
\hline Arsenic & $<0.05$ & $<0.05$ & $<0.05$ & $<0.05$ & $<0.05$ & $<0.05$ & 0.07 & $<0.05$ \\
\hline Barium & 0.12 & 0.11 & 0.12 & 0.12 & 0.15 & 0.13 & 0.024 & 0.019 \\
\hline Beryllium & $<0.0003$ & $<0.0003$ & $<0.0003$ & $<0.0003$ & $<0.0003$ & $<0.0003$ & $<0.0003$ & $<0.0003$ \\
\hline Boron & 0.016 & 0.01 & 0.014 & 0.0077 & 0.015 & 0.013 & 0.018 & 0.026 \\
\hline Cadmium (AAS) & $<0.002$ & $<0.002$ & 0.0048 & 0.0051 & 0.0049 & 0.0035 & $<0.002$ & $<0.002$ \\
\hline Cadmium & $<0.003$ & $<0.003$ & 0.0041 & 0.0041 & 0.0074 & 0.005 & 0.0031 & $<0.003$ \\
\hline Chromium (AAS) & $<0.01$ & $<0.01$ & $<0.01$ & $<0.01$ & $<0.01$ & $<0.01$ & 0.01 & $<0.01$ \\
\hline Chromium & $<0.01$ & $<0.01$ & $<0.01$ & $<0.01$ & $<0.01$ & $<0.01$ & $<0.01$ & $<0.01$ \\
\hline Cobalt & $<0.005$ & $<0.005$ & $<0.005$ & $<0.005$ & $<0.005$ & $<0.005$ & $<0.005$ & $<0.005$ \\
\hline Copper & 0.0096 & 0.0072 & 0.0046 & 0.0058 & $<0.004$ & $<0.004$ & 0.014 & $<0.004$ \\
\hline Iron & 0.053 & $<0.005$ & 0.25 & $<0.005$ & 0.44 & $<0.005$ & 2.9 & $<0.005$ \\
\hline Lead (AAS) & $<0.004$ & $<0.004$ & $<0.004$ & $<0.004$ & $<0.004$ & $<0.004$ & 0.024 & $<0.004$ \\
\hline Mercury (CVAA) & $<0.0002$ & $<0.0002$ & $<0.0002$ & $<0.0002$ & $<0.0002$ & $<0.0002$ & $<0.0002$ & $<0.0002$ \\
\hline Molybdenum & $<0.01$ & $<0.01$ & $<0.01$ & $<0.01$ & $<0.01$ & $<0.01$ & $<0.01$ & $<0.01$ \\
\hline Nickel & $<0.01$ & $<0.01$ & $<0.01$ & $<0.01$ & $<0.01$ & $<0.01$ & $<0.01$ & $<0.01$ \\
\hline Selenium & $<0.05$ & $<0.05$ & $<0.05$ & $<0.05$ & $<0.05$ & $<0.05$ & $<0.05$ & $<0.05$ \\
\hline silver & $<0.006$ & $<0.006$ & 0.017 & $<0.006$ & 0.023 & $<0.006$ & $<0.006$ & $<0.006$ \\
\hline Strontium & 0.072 & 0.07 & 0.068 & 0.064 & 0.083 & 0.076 & 0.071 & 0.082 \\
\hline Thorium & $<0.2$ & $<0.2$ & $<0.2$ & $<0.2$ & $<0.2$ & $<0.2$ & $<0.2$ & $<0.2$ \\
\hline Uranium (Fluor) & $<0.001$ & $<0.001$ & $<0.001$ & $<0.001$ & $<0.001$ & $<0.001$ & 0.001 & 0.001 \\
\hline Vanadium & $<0.005$ & $<0.005$ & $<0.005$ & $<0,005$ & $<0.005$ & $<0.005$ & $<0.005$ & $<0.005$ \\
\hline zine & 0.063 & 0.055 & 0.011 & 0.014 & 0.02 & 0.02 & 0.025 & 0.0041 \\
\hline MAJOR IONS $(\mathrm{mg} / \mathrm{L})$ & $\cdot$ &. &. & . & . &. &. & • \\
\hline Alkalinity-HCO3 & $\begin{array}{r}\cdot \dot{ } \\
115\end{array}$ & $\cdot$ & 125 & . & 137 & . & 226 & • \\
\hline Alkalinity- $\mathrm{CO} 3$ & $<1$ &. & $<1$ & . & $<1$ & . & $<1$ & • \\
\hline Calcium & 37 & 36 & 36 & 34 & 45 & 40 & 80 & 88 \\
\hline Chloride & 2.8 & $\cdot$ & 2.6 & . & 3 & $\cdot$ & 2 & • \\
\hline Fluoride & 0.1 &. & 0.2 & . & 0.2 & $\cdot$ & $<0.1$ & • \\
\hline Magnesium & 5.5 & 5.4 & 5.5 & 5.4 & 4.9 & 5.3 & 5.8 & 5.3 \\
\hline Manganese & 0.0089 & 0.005 & 0.018 & 0.0069 & 0.032 & 0.0039 & 0.089 & 0.0028 \\
\hline witrate-N & $<0.2$ & & $<0.2$ & - & $<0.2$ & $\cdot$ & 0.42 & • \\
\hline Potassium & 0.85 & 0.88 & 1.1 & 1.1 & 1.2 & 1.3 & 1.9 & 1.7 \\
\hline Sodium & 3.6 & 3.6 & 3.6 & 3.5 & 4.1 & 4.5 & 1.9 & 2 \\
\hline Sulfate & 11 & . & 6.4 & . & 7 &. & 4.4 & $\cdot$ \\
\hline
\end{tabular}

(CONT INUED) 
APPENDIX E.1

Oroundwater and Surface-Water Quality Data, 1993

\begin{tabular}{|c|c|c|c|c|c|c|c|c|}
\hline \multirow{4}{*}{$\begin{array}{l}\text { Sumpling point } \\
\text { Iocation } \\
\text { Date sampled }\end{array}$} & \multicolumn{6}{|c|}{ ow-311 } & \multicolumn{2}{|c|}{$o w-312$} \\
\hline & \multicolumn{6}{|c|}{ R8 } & \multirow{2}{*}{\multicolumn{2}{|c|}{$\frac{\text { Rs }}{02 / 08 / 93}$}} \\
\hline & \multicolumn{2}{|c|}{$05 / 11 / 93$} & \multicolumn{2}{|c|}{$09 / 12 / 93$} & \multicolumn{2}{|c|}{$10 / 21 / 93$} & & \\
\hline & TOT & DIS & Tor & Drs & Tor & Drs & Tot & Drs \\
\hline Metals (mg/L) & $\cdot$ & $\cdot$ & $\cdot$ & - & $\cdot$ & $\cdot 1$ & $\cdot 1$ & • \\
\hline Aluminum & 0.05 & 0.054 & 0.13 & 0.021 & 0.24 & $<0.02$ & 6.2 & 2.8 \\
\hline Antimony & $<0.05$ & $<0.05$ & $<0.05$ & $<0.05$ & $<0.05$ & $<0.05$ & $<0.05$ & $<0.05$ \\
\hline Areenic & $<0.05$ & $<0.05$ & $<0.05$ & $<0.05$ & $<0.05$ & $<0.05$ & $<0.05$ & $<0.05$ \\
\hline Bartum & 0.017 & 0.016 & 0.023 & 0.023 & 0.019 & 0.021 & 0.034 & 0.039 \\
\hline Beryllium & 0.0003 & $<0.0003$ & $<0.0003$ & $<0.0003$ & $<0.0003$ & $<0.0003$ & $<0.0003$ & $<0.0003$ \\
\hline Boron & 0.022 & 0.013 & 0.02 & 0.018 & 0.015 & 0.016 & 0.014 & 0.016 \\
\hline Cadmium (AAS) & $<0.002$ & $<0.002$ & $<0.002$ & $<0.002$ & 0.0022 & $<0.002$ & $<0.002$ & $<0.002$ \\
\hline Cadmium & $<0.003$ & $<0.003$ & $<0.003$ & $<0.003$ & $<0.003$ & $<0.003$ & $<0.003$ & $<0.003$ \\
\hline Chromium (is) & $<0.01$ & $<0.01$ & $<0.01$ & $<0.01$ & $<0.01$ & $<0.01$ & 0.015 & 0.01 \\
\hline Chromium & 0.029 & 0.015 & $<0.01$ & $<0.01$ & $<0.01$ & $<0.01$ & 0.011 & $<0.01$ \\
\hline Cobalt & $<0.005$ & $<0.005$ & $<0.005$ & $<0.005$ & $<0.005$ & $<0.005$ & $<0.005$ & $<0.005$ \\
\hline Copper & $<0.004$ & $<0.004$ & 0.0046 & $<0.004$ & $<0.004$ & 0.0042 & 0.0046 & $<0.004$ \\
\hline Iron & 0.028 & $<0.005$ & 0.42 & $<0.005$ & 0.27 & $<0.005$ & 2 & $<0.005$ \\
\hline Lead (AAS) & $<0.004$ & $<0.004$ & $<0.004$ & $<0.004$ & $<0.004$ & $<0.004$ & 0.014 & $<0.004$ \\
\hline Mercury (CVAA) & $<0.0002$ & $<0.0002$ & $<0.0002$ & $<0.0002$ & $<0.0002$ & $<0.0002$ & $<0.0002$ & $<0.0002$ \\
\hline Mol ybdenum & $<0.01$ & $<0.01$ & $<0.01$ & $<0.01$ & $<0.01$ & $<0.01$ & $<0.01$ & $<0.01$ \\
\hline Nickel & 0.031 & 0.014 & $<0.01$ & $<0.01$ & $<0.01$ & $<0.01$ & 0.011 & $<0.01$ \\
\hline Selenium & $<0.05$ & $<0.05$ & $<0.05$ & $<0.05$ & $<0.05$ & $<0.05$ & $<0.05$ & $<0.05$ \\
\hline silver & $<0.006$ & $<0.006$ & $<0.006$ & $<0.006$ & $<0.006$ & $<0.006$ & $<0.006$ & $<0.006$ \\
\hline Strontium & 0.07 & 0.07 & 0.065 & 0.065 & 0.067 & 0.064 & 0.44 & 0.44 \\
\hline Thorium & $<0.2$ & $<0.2$ & $<0.2$ & $<0.2$ & $<0.2$ & $<0.2$ & $<0.2$ & $<0.2$ \\
\hline Uranilum (Fluor) & 0.001 & 0.001 & 0.001 & 0.001 & 0.001 & $<0.001$ & $<0.001$ & $<0.001$ \\
\hline Vanadium & $<0.005$ & $<0.005$ & $<0.005$ & $<0.005$ & $<0.005$ & $<0.005$ & 0.013 & $<0.005$ \\
\hline 2 inc & 0.028 & 0.029 & 0.06 & 0.072 & 0.032 & 0.037 & 0.026 & 0.008 \\
\hline MAJOR IONS $(\mathrm{mg} / \mathrm{L})$ &. & . &. & . & . &. &. & . \\
\hline Alkalinity-HCO3 & 214 & . & 210 & . & 209 &. & $\dot{<}$ & . \\
\hline Alkalinity- $\mathrm{CO} 3$ & $<1$ & . & $<1$ & . & $<1$ &. & 50 & . \\
\hline Calcium & 79 & 78 & 82 & 82 & 81 & 76 & 170 & 150 \\
\hline Chloride & 2.5 & . & 2.7 & . & 3 &. & 1.3 & . \\
\hline Fluoride & $<0.1$ & . & $<0.1$ & - & $<0.1$ &. & 0.2 & . \\
\hline Magnesium & 5.2 & 5.1 & 4.8 & 4.8 & 4.4 & 4.7 & 2 & 0.2 \\
\hline Manganese & 0.0025 & 0.0029 & 0.013 & 0.0055 & 0.007 & 0.0019 & 0.035 & 0.0058 \\
\hline Nitrate-N & 0.5 & . & 0.54 & . & 0.5 &. & $<0.2$ & . \\
\hline Potasaium & 1.5 & 1.4 & 1.2 & 0.77 & 1.3 & 1 & 10 & 10 \\
\hline Sodium & 1.8 & 1.8 & 1.6 & 1.5 & 1.6 & 1.6 & 9.9 & 9.4 \\
\hline Sulfate & 9.8 & . & 3.8 & . & 3 & . & 5.7 & • \\
\hline
\end{tabular}

(CONTINUED) 
APPBNDIX 0.1

Groundwater and surface-Water Quality Data, 1993

\begin{tabular}{|c|c|c|c|c|c|c|c|c|}
\hline \multirow{4}{*}{$\begin{array}{l}\text { 8ampling point } \\
\text { Location } \\
\text { Date sampled }\end{array}$} & \multicolumn{6}{|c|}{$0 w-312$} & \multicolumn{2}{|c|}{ - $O W-315$} \\
\hline & \multicolumn{6}{|c|}{ R8 } & \multirow{2}{*}{\multicolumn{2}{|c|}{$\frac{8 P I}{02 / 03 / 93}$}} \\
\hline & \multicolumn{2}{|c|}{$05 / 11 / 93$} & \multicolumn{2}{|c|}{$09 / 12 / 93$} & \multicolumn{2}{|c|}{$10 / 21 / 93$} & & \\
\hline & Tor & DIs & TOT & DI8 & TOT & DI8 & TOT & DIs \\
\hline Hatrals (mg/L) & $\cdot$ & . & . & - & - & - & . & - \\
\hline Aluminum & 4 & 3.9 & 3.6 & 3.3 & 3.7 & 3.7 & $<0.02$ & $<0.02$ \\
\hline Antimony & $<0.05$ & $<0.05$ & $<0.05$ & $<0.05$ & $<0.05$ & $<0.05$ & $<0.05$ & $<0.03$ \\
\hline Arsenic & $<0.05$ & $<0.05$ & $<0.05$ & $<0.05$ & $<0.05$ & $<0.05$ & $<0.05$ & $<0.05$ \\
\hline Bartum & 0.028 & 0.024 & 0.025 & 0.041 & 0.033 & 0.033 & 0.041 & 0.04 \\
\hline Bary111um & $<0.0003$ & $<0.0003$ & $<0.0003$ & $<0.0003$ & $<0.0003$ & 0.0027 & $<0.0003$ & $<0.0003$ \\
\hline Boron & 0.013 & 0.012 & 0.0047 & 0.024 & 0.0052 & 0.0081 & 0.012 & 0.011 \\
\hline Cadmilum (AAS) & $<0.002$ & $<0.002$ & $<0.002$ & $<0.002$ & $<0.002$ & $<0.002$ & $<0.002$ & $<0.002$ \\
\hline Cadmium & $<0.003$ & $<0.003$ & $<0.003$ & $<0.003$ & $<0.003$ & $<0.003$ & $<0.003$ & $<0.003$ \\
\hline Chromium (MAS) & 0.013 & 0.012 & 0.011 & $<0.01$ & 0.011 & 0.01 & $<0.01$ & $<0.01$ \\
\hline Chromlum & 0.024 & 0.016 & 0.023 & 0.017 & 0.015 & 0.016 & $<0.01$ & $<0.01$ \\
\hline Cobalt & $<0.005$ & $<0.005$ & $<0.005$ & $<0.005$ & $<0.005$ & $<0.005$ & $<0.005$ & $<0.005$ \\
\hline Copper & 0.008 & $<0.004$ & 0.016 & 0.0094 & $<0.004$ & 0.0042 & $<0.004$ & $<0.004$ \\
\hline Iron & 0.025 & $<0.005$ & 0.76 & 0.03 & 0.036 & 0.035 & $<0.005$ & $<0.005$ \\
\hline Lead (MAS) & $<0.004$ & $<0.004$ & $<0.004$ & $<0.004$ & $<0.004$ & $<0.004$ & $<0.004$ & $<0.004$ \\
\hline Mercury (CVAA) & $<0.0002$ & $<0.0002$ & $<0.0002$ & $<0.0002$ & $<0.0002$ & $<0.0002$ & $<0.0002$ & $<0.0002$ \\
\hline Mol ybdenum & $<0.01$ & $<0.01$ & $<0.01$ & $<0.01$ & $<0.01$ & 0.011 & $<0.01$ & $<0.01$ \\
\hline Nickel & 0.014 & $<0.01$ & $<0.01$ & $<0.01$ & $<0.01$ & $<0.01$ & $<0.01$ & $<0.01$ \\
\hline Selenium & $<0.05$ & $<0.05$ & $<0.05$ & 0.056 & $<0.05$ & $<0.05$ & $<0.05$ & $<0.05$ \\
\hline silver & $<0.006$ & $<0.006$ & $<0.006$ & $<0.006$ & $<0.006$ & $<0.006$ & $<0.006$ & $<0.006$ \\
\hline strontium & 0.44 & 0.4 & 0.38 & 0.51 & 0.46 & 0.42 & 0.14 & 0.14 \\
\hline Thorlum & $<0.2$ & $<0.2$ & $<0.2$ & $<0.2$ & $<0.2$ & $<0.2$ & $<0.2$ & $<0.2$ \\
\hline Uranium (Fluor) & $<0.001$ & $<0.001$ & 0.001 & 0.001 & $<0.001$ & $<0.001$ & 0.001 & 0.001 \\
\hline Vanadium & 0.0062 & 0.0078 & 0.0054 & $<0.005$ & 0.0056 & 0.0082 & $<0.005$ & $<0.005$ \\
\hline zinc & 0.037 & 0.03 & 0.086 & 0.053 & 0.033 & 0.031 & 0.0085 & 0.0064 \\
\hline MAJOR IONS (mg/L) & $\cdot$ & $\cdot$ & . & $\cdot$ & $\cdot$ &. & $\cdot$ & - \\
\hline Alkalinity-HCO3 & $<1$ & $\cdot$ & $<1$ & $\cdot$ & $<1$ & $\cdot$ & 179 & . \\
\hline Alkalinity- $\mathrm{CO} 3$ & 52 & . & 48 & $\cdot$ & 50 & $\cdot$ & $<1$ & • \\
\hline Calcium & 160 & 140 & 160 & 200 & 170 & 150 & 89 & 86 \\
\hline Chloride & 1.9 &. & 1.3 & $\cdot$ & 1 &. & 11 & - \\
\hline Fluoride & 0.2 & $\cdot$ & 0.2 & $\cdot$ & 0.2 & . & 0.1 & - \\
\hline Magnesium & 0.036 & $<0.003$ & 0.42 & 0.025 & 0.019 & 0.023 & 8.1 & 7.9 \\
\hline Manganese & 0.0015 & 0.0019 & 0.01 & $<0.001$ & 0.001 & 0.0039 & $<0.001$ & $<0.001$ \\
\hline Nitrate-N & 0.26 &. & 0.26 & $\cdot$ & 0.2 & - & 7.8 & - \\
\hline Potassium & 9.6 & 8.7 & 9.6 & 9 & 11 & 9.8 & 3.5 & 3.4 \\
\hline Sodium & 8.2 & 9.5 & 16 & 16 & 14 & 13 & 6.3 & 6.5 \\
\hline Sultate & 9.9 &. & 3.9 & $\cdot$ & 6 & $\cdot$ & 38 & . \\
\hline
\end{tabular}

(CONTINUED) 
APPLNDIX $\mathbf{3} \cdot 1$

Groundwater and surteoe-Water Quallty Duta, 1993

\begin{tabular}{|c|c|c|c|c|c|c|c|c|}
\hline \multirow{4}{*}{$\begin{array}{l}\text { ganpling Dolnt } \\
\text { Looation } \\
\text { Date sampled }\end{array}$} & \multicolumn{6}{|c|}{$a N-315$} & \multicolumn{2}{|c|}{$0 x-316$} \\
\hline & \multicolumn{6}{|c|}{ 8PI } & \multicolumn{2}{|c|}{ 8PI } \\
\hline & \multicolumn{2}{|c|}{$06 / 03 / 93$} & \multicolumn{2}{|c|}{$09 / 14 / 93$} & \multicolumn{2}{|c|}{$10 / 27 / 93$} & \multicolumn{2}{|c|}{$02 / 02 / 93$} \\
\hline & $\operatorname{sos}$ & Dts & $\operatorname{rot}$ & DI8 & $20 T$ & DI8 & $\operatorname{Tox}$ & DI8 \\
\hline Mrncs (mg/L) & $\cdot$ & $\cdot$ & - & - & - & - & - & - \\
\hline Nluminum & 0.074 & $<0.02$ & $<0.02$ & $<0.02$ & $<0.02$ & $<0.02$ & 0.023 & $<0.02$ \\
\hline Ant Lmony & $<0.05$ & $<0.05$ & $<0.05$ & $<0.05$ & $<0.05$ & $<0.05$ & $<0.05$ & $<0.05$ \\
\hline Areendo & $<0.05$ & $<0.08$ & $<0.05$ & $<0.0 s$ & $<0.05$ & $<0.0 s$ & $<0.05$ & $<0.05$ \\
\hline Barium & 0.041 & 0.041 & 0.046 & 0.047 & 0.058 & 0.056 & 0.012 & 0.012 \\
\hline Bery 1114 m & $<0.0003$ & $<0.0003$ & $<0.0003$ & $<0.0003$ & $<0.0003$ & $<0.0003$ & $<0.0003$ & $<0.0003$ \\
\hline Boron & 0.04 & 0.012 & 0.014 & 0.012 & 0.012 & 0.014 & 0.013 & $<0.004$ \\
\hline Cadmium (MAs) & $<0.002$ & $<0.002$ & $<0.002$ & $<0.002$ & $<0.002$ & $<0.002$ & $<0.002$ & $<0.002$ \\
\hline CadmLum & $<0.003$ & $<0.003$ & $<0.003$ & $<0.003$ & $<0.003$ & $<0.003$ & $<0.003$ & $<0.003$ \\
\hline Chromium (MA) & $<0.01$ & $<0.01$ & $<0.01$ & $<0.01$ & $<0.01$ & $<0.01$ & $<0.01$ & $<0.01$ \\
\hline Chromium & $<0.01$ & $<0.01$ & $<0.01$ & $<0.01$ & $<0.01$ & $<0.01$ & $<0.01$ & $<0.01$ \\
\hline Cobalt & $<0.005$ & $<0.005$ & $<0.005$ & $<0.005$ & $<0.005$ & $<0.005$ & $<0.005$ & $<0.005$ \\
\hline Copper & $<0.004$ & $<0.004$ & $<0.004$ & 0.0047 & $<0.004$ & $<0.004$ & 0.0071 & $<0.004$ \\
\hline Iron & $<0.005$ & $<0.005$ & 0.031 & $<0.005$ & 0.026 & $<0.005$ & 0.87 & $<0.005$ \\
\hline Lead (MAs) & $<0.004$ & $<0.004$ & $<0.008$ & $<0.008$ & $<0.004$ & $<0.004$ & $<0.004$ & $<0.004$ \\
\hline Marcury (CVMA) & $<0.0002$ & $<0.0002$ & $<0.0002$ & $<0.0002$ & $<0.0002$ & $<0.0002$ & $<0.0002$ & $<0.0002$ \\
\hline molybdenum & $<0.01$ & $<0.01$ & $<0.01$ & $<0.01$ & $<0.01$ & $<0.01$ & $<0.01$ & $<0.01$ \\
\hline Nlckel & $<0.01$ & $<0.01$ & $<0.01$ & $<0.01$ & $<0.01$ & $<0.01$ & $<0.01$ & $<0.01$ \\
\hline selentum & $<0.05$ & $<0.05$ & $<0.05$ & $<0.05$ & $<0.05$ & $<0.05$ & $<0.05$ & $<0.05$ \\
\hline s1lver & $<0.006$ & $<0.006$ & $<0.006$ & $<0.006$ & $<0.006$ & $<0.006$ & $<0.006$ & $<0.006$ \\
\hline strontiun & 0.14 & 0.14 & 0.15 & 0.15 & 0.17 & 0.17 & 0.023 & 0.024 \\
\hline Thor I un & $<0.2$ & $<0.2$ & $<0.2$ & $<0.2$ & $<0.2$ & $<0.2$ & $<0.2$ & $<0.2$ \\
\hline Uraniun (Fluor) & 0.001 & 0.001 & 0.001 & 0.001 & 0.002 & 0.001 & $<0.001$ & 0.001 \\
\hline VanadLum & $<0.005$ & $<0.005$ & $<0.005$ & $<0.005$ & $<0.005$ & $<0.005$ & $<0.005$ & $<0.005$ \\
\hline zinc & 0.0034 & 0.0047 & 0.0066 & 0.011 & 0.015 & 0.013 & 0.049 & 0.054 \\
\hline MnJOR IONS (mg/L) & $\cdot$ & - & $\cdot$ & $\cdot$ & - & . & . & - \\
\hline Alkalinity-HCO3 & 216 & $\cdot$ & 229 & $\cdot$ & 218 & $\cdot$ & 191 & • \\
\hline Alkalinity-co3 & $<1$ & . & $<1$ & $\cdot$ & $<1$ & - & $<1$ & - \\
\hline Calclum & 89 & 88 & 100 & 100 & 100 & 100 & 42 & 41 \\
\hline Chlor Ide & 8.5 & $\cdot$ & 8.2 & . & 13 & - & 1.6 & - \\
\hline Fluorlde & $<0.1$ & . & $<0.1$ & . & $<0.1$ & $\cdot$ & 0.1 & - \\
\hline Magnes Lum & 9.3 & 9.2 & 11 & 11 & 10 & 10 & 21 & 21 \\
\hline Manganee & $<0.001$ & 0.0014 & 0.0012 & 0.0017 & 0.0012 & 0.0012 & 0.005 & 0.0018 \\
\hline Nitrate-N & 8.9 & . & 15 & . & 19.8 & - & 0.46 & - \\
\hline Potassium & 3 & 2.9 & 3 & 3 & 3.6 & 3.6 & $2 \cdot 3$ & 2.4 \\
\hline Sodium & 5.1 & 5.1 & 5.7 & 5.7 & 7.1 & 6.9 & 2 & 2.2 \\
\hline sulfate & 27 & . & 22 & . & 20 & - & 3.7 & • \\
\hline
\end{tabular}

(CONTINUED) 
APPENDIX 1.1

Oroundwater and gurface-Wator Qual1ty Data, 1993

\begin{tabular}{|c|c|c|c|c|c|c|c|c|}
\hline \multirow{4}{*}{$\begin{array}{l}\text { sanpling polnt } \\
\text { Location } \\
\text { Date sampled }\end{array}$} & \multicolumn{6}{|c|}{ ON-316 } & \multicolumn{2}{|c|}{ an-317 } \\
\hline & \multicolumn{6}{|c|}{ 8PI } & \multirow{2}{*}{\multicolumn{2}{|c|}{$\frac{8 P 1}{02 / 02 / 93}$}} \\
\hline & \multicolumn{2}{|c|}{$06 / 01 / 93$} & \multicolumn{2}{|c|}{$09 / 13 / 93$} & \multicolumn{2}{|c|}{$10 / 24 / 93$} & & \\
\hline & $20 x$ & Drs & $20 T$ & Dis & Tor & Drs & nor & Drs \\
\hline nerals (mg/L) & $\cdot$ & $\cdot$ & $\cdot$ & $\cdot$ & $\cdot$ & $\cdot$ & - & $\cdot$ \\
\hline Aluminum & 0.054 & $<0.02$ & 0.22 & $<0.02$ & $<0.02$ & $<0.02$ & 0.036 & $<0.02$ \\
\hline Antimony & $<0.03$ & $<0.05$ & $<0.05$ & $<0.08$ & $<0.05$ & $<0.05$ & $<0.05$ & $<0.08$ \\
\hline Arwenle & $<0.08$ & $<0.05$ & $<0.05$ & $<0.05$ & $<0.05$ & $<0.05$ & $<0.05$ & $<0.08$ \\
\hline Bariun & 0.012 & 0.014 & 0.014 & 0.013 & 0.012 & 0.012 & 0.018 & 0.016 \\
\hline Bery111um & $<0,0003$ & $<0.0003$ & $<0.0003$ & $<0.0003$ & $<0.0003$ & $<0.0003$ & 0.00035 & $<0.0003$ \\
\hline Boron & 0.026 & 0.027 & $<0.004$ & 0.0081 & 0.0046 & 0.0081 & 0.0067 & 0.011 \\
\hline Cadming (MB) & $<0.002$ & $<0.002$ & $<0.002$ & $<0.002$ & $<0.002$ & $<0.002$ & $<0.002$ & $<0.002$ \\
\hline Cadmi un & $<0.003$ & $<0.003$ & $<0.003$ & $<0.003$ & $<0.003$ & $<0.003$ & $<0.003$ & $<0.003$ \\
\hline Chromilum (MAs) & $<0.01$ & $<0.01$ & $<0.01$ & $<0.01$ & $<0.01$ & $<0.01$ & $<0.01$ & $<0.01$ \\
\hline Chromiun & 0.024 & $<0.01$ & $<0.01$ & $<0.01$ & $<0.01$ & $<0.01$ & $<0.01$ & $<0.01$ \\
\hline Cobalt & $<0.00 s$ & $<0.005$ & $<0.005$ & $<0.005$ & $<0.00 s$ & $<0.005$ & $<0.005$ & $<0.005$ \\
\hline Copper & $<0.004$ & $<0.004$ & $<0.004$ & $<0.004$ & $<0.004$ & $<0.004$ & 0.0042 & $<0.004$ \\
\hline Iron & 0.048 & $<0.005$ & 0.3 & 0.012 & 0.018 & $<0.005$ & 0.02 & $<0.00 s$ \\
\hline Laad (MA8) & $<0.004$ & $<0.004$ & $<0.004$ & $<0.004$ & $<0.004$ & $<0.004$ & $<0.004$ & $<0.004$ \\
\hline Meroury (CVMA) & $<0.0002$ & $<0.0002$ & $<0.0002$ & $<0.0002$ & $<0.0002$ & $<0.0002$ & $<0.0002$ & $<0.0002$ \\
\hline Nolybdanum & $<0.01$ & $<0.01$ & $<0.01$ & $<0.01$ & $<0.01$ & $<0.01$ & $<0.01$ & $<0.01$ \\
\hline Hickel & 0.03 & 0.012 & $<0.01$ & $<0.01$ & $<0.01$ & $<0.01$ & $<0.01$ & $<0.01$ \\
\hline Belentum & $<0.05$ & $<0.05$ & $<0.05$ & $<0.05$ & $<0.05$ & $<0.05$ & $<0.05$ & $<0.05$ \\
\hline 8ilver & $<0.006$ & $<0.006$ & 0.0065 & $<0.006$ & $<0.006$ & $<0.006$ & $<0.006$ & $<0.006$ \\
\hline strontlum & 0.025 & 0.026 & 0.024 & 0.024 & 0.024 & 0.024 & 0.034 & 0.034 \\
\hline Thorium & $<0.2$ & $<0.2$ & $<0.2$ & $<0.2$ & $<0.2$ & $<0.2$ & $<0.2$ & $<0.2$ \\
\hline Uranium (rluor) & $<0.001$ & $<0.001$ & 0.001 & $<0.001$ & 0.001 & 0.001 & 0.001 & 0.001 \\
\hline vanadium & $<0.005$ & $<0.005$ & $<0.005$ & $<0.005$ & $<0.005$ & $<0.005$ & $<0.005$ & $<0.005$ \\
\hline $2 \operatorname{lnc}$ & 0.02 & 0.015 & 0.029 & 0.02 & 0.015 & 0.018 & 0.012 & 0.011 \\
\hline MuJOR IONS (mg/L) & $\cdot$ & $\cdot$ & $\cdot$ & $\cdot$ & $\cdot$ & - & . & - \\
\hline Alkalinity-HCO3 & 197 & $\cdot$ & 209 & $\cdot$ & 198 & $\cdot$ & 206 & . \\
\hline Alkalinity-CO3 & $<1$ & $\cdot$ & $<1$ & $\cdot$ & $<1$ & - & $<1$ & - \\
\hline Calcium & 44 & 45 & 45 & 45 & 44 & 41 & 46 & 46 \\
\hline Chloride & 1.4 & $\cdot$ & 1.5 & . & 2 & - & $<1$ & - \\
\hline Fluoride & $<0.1$ & $\cdot$ & $<0.1$ & - & $<0.1$ & . & 0.1 & - \\
\hline Magnesium & 24 & 24 & 23 & 23 & 24 & 23 & 20 & 20 \\
\hline Mangenese & 0.0065 & 0.0043 & 0.01 & 0.0015 & 0.0012 & $<0.001$ & 0.0017 & $<0.001$ \\
\hline Nitrate-N & 0.33 & $\cdot$ & 0.3 & - & 0.35 & . & 0.26 & - \\
\hline Potase 1 um & 1.4 & 2 & 2.3 & 1.9 & 2.1 & 2.2 & 1.6 & 1.4 \\
\hline Sodium & 1.1 & 1.4 & 3.2 & 2.5 & 1.2 & 1.3 & 6.2 & 3.4 \\
\hline sulfate & 2.1 & & 3.8 & . & 3.8 & - & 5.2 & • \\
\hline
\end{tabular}

(CONTINUED) 
Appundix 8.1

Groundwater and surfaoe-Water Quallty Data, 1993

\begin{tabular}{|c|c|c|c|c|c|c|c|c|}
\hline \multirow{4}{*}{$\begin{array}{l}\text { Dempling polnt } \\
\text { Lootion } \\
\text { Date sampled }\end{array}$} & \multicolumn{6}{|c|}{$0 N-317$} & \multicolumn{2}{|c|}{$0 w-323$} \\
\hline & \multicolumn{6}{|c|}{ spt } & \multirow{2}{*}{\multicolumn{2}{|c|}{$\frac{0 P 1}{02 / 02 / 93}$}} \\
\hline & \multicolumn{2}{|c|}{$05 / 28 / 93$} & \multicolumn{2}{|c|}{$09 / 13 / 93$} & \multicolumn{2}{|c|}{$10 / 24 / 93$} & & \\
\hline & ros & Drs & $20 r$ & DI8 & ror & DIs & nor & Dr8 \\
\hline marace $(m g / L)$ & - & $\cdot$ & $\cdot$ & $\cdot$ & $\cdot$ & $\cdot 1$ & $\cdot 1$ & • \\
\hline Aluninum & $<0.02$ & $<0.02$ & 0.022 & $<0.02$ & 0.032 & $<0.02$ & 0.023 & $<0.02$ \\
\hline Ant inony & $<0.05$ & $<0.05$ & $<0.05$ & $<0.05$ & $<0.05$ & $<0.05$ & $<0.05$ & $<0.03$ \\
\hline Areenle & $<0.05$ & $<0.05$ & $<0.0 s$ & $<0.08$ & $<0.08$ & $<0.05$ & $<0.05$ & $<0.05$ \\
\hline Barliun & 0.017 & 0.016 & 0.017 & 0.017 & 0.017 & 0.018 & 0.36 & 0.36 \\
\hline Deryl11um & $<0.0003$ & $<0.0003$ & $<0.0003$ & $<0.0003$ & $<0.0003$ & $<0.0003$ & $<0.0003$ & $<0.0003$ \\
\hline soron & 0.02 & 0.016 & 0.0068 & 0.014 & 0.0055 & 0.0057 & 0.0079 & 0.0058 \\
\hline Cadal un (MAs) & $<0.002$ & $<0,002$ & $<0.002$ & $<0.002$ & $<0.002$ & $<0.002$ & $<0.002$ & $<0.002$ \\
\hline Cadnitun & $<0.003$ & $<0.003$ & $<0.003$ & $<0.003$ & $<0.003$ & $<0.003$ & $<0.003$ & $<0.003$ \\
\hline Chromlum (Ms) & $<0.01$ & $<0.01$ & $<0.01$ & $<0.01$ & $<0.01$ & $<0.01$ & $<0.01$ & $<0.01$ \\
\hline Chromilum & $<0.01$ & $<0.01$ & $<0.01$ & $<0.01$ & $<0.01$ & $<0.01$ & $<0.01$ & $<0.01$ \\
\hline Cobalt & $<0.005$ & $<0.005$ & $<0.005$ & $<0.005$ & $<0.00 s$ & $<0.005$ & $<0.005$ & $<0.005$ \\
\hline copper & $<0.004$ & $<0.004$ & 0.0048 & 0.0091 & $<0.004$ & $<0.004$ & $<0.004$ & $<0.004$ \\
\hline Iron & 0.012 & $<0.005$ & 0.077 & $<0.00 s$ & 0.036 & $<0.005$ & 0.1 & $<0.005$ \\
\hline Load (MA8) & $<0.004$ & $<0.004$ & $<0.004$ & $<0.004$ & $<0.004$ & $<0.004$ & $<0.004$ & $<0.004$ \\
\hline Mercury (CVM) & $<0.0002$ & $<0.0002$ & $<0.0002$ & $<0.0002$ & $<0.0002$ & $<0.0002$ & $<0.0002$ & $<0.0002$ \\
\hline Mol Ybdenum & $<0.01$ & $<0.01$ & $<0.01$ & $<0.01$ & $<0.01$ & $<0.01$ & $<0.01$ & $<0.01$ \\
\hline nickel & $<0.01$ & 0.013 & $<0.01$ & $<0.01$ & $<0.01$ & $<0.01$ & $<0.01$ & $<0.01$ \\
\hline solenium & $<0.05$ & $<0.05$ & $<0.05$ & $<0.05$ & $<0.05$ & $<0.05$ & $<0.05$ & $<0.05$ \\
\hline stiver & $<0.006$ & $<0.006$ & $<0.006$ & $<0.006$ & $<0.006$ & $<0.006$ & $<0.006$ & $<0.006$ \\
\hline strontiun & 0.03 & 0.03 & 0.062 & 0.064 & 0.032 & 0.033 & 0.019 & 0.02 \\
\hline Thoriun & $<0.2$ & $<0.2$ & $<0.2$ & $<0.2$ & $<0.2$ & $<0.2$ & $<0.2$ & $<0.2$ \\
\hline Urantum (rluor) & $<0.001$ & $<0.001$ & 0.001 & 0.001 & 0.006 & $<0.001$ & $<0.001$ & $<0.001$ \\
\hline Vanadium & $<0.005$ & $<0.005$ & $<0.00 s$ & $<0.005$ & $<0.005$ & $<0.005$ & $<0.005$ & $<0.005$ \\
\hline $8 \sin 0$ & 0.011 & 0.016 & 0.012 & 0.017 & 0.01 & 0.012 & 0.013 & 0.024 \\
\hline MWOR IOHS (mg/h) & . &. &. & . & . &. &. & . \\
\hline Alkalinity-HCO3 & 197 &. & $22 \dot{1}$ &. & 197 &. & 212 & . \\
\hline Alkalinity-CO3 & $<1$ & . & $<1$ &. & $<1$ &. & $<1$ & . \\
\hline Calcium & 46 & 45 & 32 & 50 & 43 & 42 & 42 & 42 \\
\hline Chloride & $<1$ &. & $<1$ & . & 1.1 & . & $<1$ & • \\
\hline Fluoride & $<0.1$ & $\cdot$ & $<0.1$ & . & $<0.1$ & . & $<0.1$ & . \\
\hline Magnealum & 20 & 20 & 24 & 24 & 21 & 21 & 25 & 23 \\
\hline Manganese & 0.0011 & 0.0011 & 0.0021 & $<0.001$ & 0.0012 & $<0.001$ & 0.0015 & 0.0018 \\
\hline Nitrate-N & $<0.2$ & $\cdot$ & $<0.2$ & $\cdot$ & 0.2 & . & $<0.2$ & - \\
\hline Potansium & 0.87 & 0.87 & 1.1 & 1.3 & 0.9 & 0.88 & 1.3 & 1.5 \\
\hline Sodium & 4.1 & 4.4 & 1.8 & 1.8 & 4.7 & 6.9 & 0.9 & 1 \\
\hline sultate & 3.6 & . & 3.3 & . & 3.9 &. & 1.9 & . \\
\hline
\end{tabular}

(CONTINUED) 
APpendix 1.1

\begin{tabular}{|c|c|c|c|c|c|c|c|c|}
\hline \multirow{4}{*}{$\begin{array}{l}\text { sampling polnt } \\
\text { Location } \\
\text { Date sempled }\end{array}$} & \multicolumn{6}{|c|}{ ON-323 } & \multicolumn{2}{|c|}{$0 N-324$} \\
\hline & \multicolumn{6}{|c|}{ 8PI } & \multirow{2}{*}{\multicolumn{2}{|c|}{$\frac{83}{01 / 29 / 93}$}} \\
\hline & \multicolumn{2}{|c|}{$05 / 28 / 93$} & \multicolumn{2}{|c|}{$09 / 12 / 93$} & \multicolumn{2}{|c|}{$10 / 23 / 93$} & & \\
\hline & ror & Drs & ror & D18 & ror & Drs & Tor & Drs \\
\hline narats $(\mathrm{mg} / \mathrm{L})$ & - & $\cdot 1$ & - & . & - & $\cdot 1$ & • & - \\
\hline Aluminus & $<0.02$ & 0.043 & $<0.02$ & $<0.02$ & $<0.02$ & $<0.02$ & $<0.02$ & $<0.02$ \\
\hline Ant Lmony & $<0.08$ & $<0.05$ & $<0.05$ & $<0.05$ & $<0.05$ & $<0.05$ & $<0.05$ & $<0.05$ \\
\hline Arsente & $<0.05$ & $<0.05$ & $<0.05$ & $<0.05$ & $<0.05$ & $<0.08$ & $<0.08$ & $<0.03$ \\
\hline Buriun & 0.36 & 0.37 & 0.34 & 0.34 & 0.39 & 0.39 & 0.2 & 0.2 \\
\hline Dary 111 un & $<0.0003$ & $<0.0003$ & $<0.0003$ & $<0.0003$ & $<0.0003$ & $<0.0003$ & $<0.0003$ & $<0.0003$ \\
\hline Doron & 0.049 & 0.026 & 0.014 & $<0.004$ & 0.007 & 0.0058 & 0.01 & 0.014 \\
\hline Cadnitum (MAs) & $<0.002$ & $<0.002$ & $<0.002$ & $<0,002$ & $<0.002$ & $<0.002$ & $<0.002$ & $<0.002$ \\
\hline cadmiun & $<0.003$ & 0.0048 & $<0.003$ & $<0.003$ & $<0.003$ & $<0.003$ & $<0.003$ & $<0.003$ \\
\hline Chromiun (MS) & $<0.01$ & $<0.01$ & $<0.01$ & $<0.01$ & $<0.01$ & $<0.01$ & $<0.01$ & $<0.01$ \\
\hline Chromlun & $<0.01$ & $<0.01$ & $<0.01$ & $<0.01$ & $<0.01$ & $<0.01$ & $<0.01$ & $<0.01$ \\
\hline Cobalt & $<0.005$ & $<0.003$ & $<0.005$ & $<0.005$ & $<0.00 s$ & $<0.005$ & $<0.005$ & $<0.00 s$ \\
\hline Copper & $<0.004$ & $<0.004$ & $<0.004$ & $<0.004$ & 0.0052 & $<0.004$ & $<0.004$ & $<0.004$ \\
\hline Iron & $<0.005$ & $<0.005$ & 0.0053 & $<0.005$ & $<0.005$ & $<0.005$ & 0.18 & 0.032 \\
\hline Load (MAB) & $<0.004$ & $<0.004$ & $<0.004$ & $<0.004$ & $<0.004$ & $<0.004$ & $<0.004$ & $<0.004$ \\
\hline Meroury (CVMA) & $<0.0002$ & $<0.0002$ & $<0.0002$ & $<0.0002$ & $<0.0002$ & $<0.0002$ & $<0.0002$ & $<0.0002$ \\
\hline Nolybdenum & $<0.01$ & $<0.01$ & $<0.01$ & $<0.01$ & $<0.01$ & $<0.01$ & $<0.01$ & $<0.01$ \\
\hline nlokel & $<0.01$ & 0.013 & $<0.01$ & $<0.01$ & $<0.01$ & $<0.01$ & $<0.01$ & $<0.01$ \\
\hline Eelenium & $<0.05$ & $<0.05$ & 0.063 & 0.055 & $<0.05$ & $<0.05$ & $<0.05$ & $<0.05$ \\
\hline stlver & $<0.006$ & $<0.006$ & $<0.006$ & $<0.006$ & $<0.006$ & $<0.006$ & $<0.006$ & $<0.006$ \\
\hline strontlun & 0.019 & 0.02 & 0.028 & 0.018 & 0,022 & 0.021 & 0.21 & 0.21 \\
\hline Thorlum & $<0.2$ & $<0.2$ & $<0.2$ & $<0.2$ & $<0.2$ & $<0.2$ & $<0.2$ & $<0.2$ \\
\hline Uranlum (Fluor) & $<0.001$ & $<0.001$ & $<0.001$ & $<0.001$ & $<0.001$ & $<0.001$ & $<0.001$ & $<0.001$ \\
\hline vanadium & $<0.005$ & $<0.005$ & $<0.005$ & $<0.005$ & $<0.005$ & $<0.005$ & $<0.005$ & $<0.005$ \\
\hline Ine & 0.009 & 0.0086 & 0.022 & 0.023 & 0.0081 & 0.0086 & 0.0059 & 0.0068 \\
\hline MwOR IONs $(\mathrm{mg} / \mathrm{L})$ &. &. & $\cdot$ & . & $\cdot$ & $\cdot$ & $\cdot$ & - \\
\hline Alkalinity- $\mathrm{HCO}$ & 207 & $\cdot$ & $20 \dot{3}$ & $\cdot$ & 215 & $\cdot$ & 100 & • \\
\hline Alkalinity-CO3 & $<1$ & $\cdot$ & $<1$ & . & $<1$ & $\cdot$ & $<1$ & . \\
\hline Calolum & 43 & 44 & 43 & 42 & 42 & 42 & 28 & 28 \\
\hline Chloride & 1.1 &. & 1.1 & . & 1 & $\cdot$ & 1.3 & • \\
\hline Fluoride & $<0.1$ & $\cdot$ & $<0.1$ &. & $<0.1$ & $\cdot$ & 0.1 & 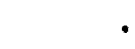 \\
\hline Magnes ium & 25 & 26 & 25 & 25 & 27 & 27 & 3.2 & 5.2 \\
\hline Manganase & 0.001 & 0.001 & $<0.001$ & 0.0012 & $<0.001$ & $<0.001$ & 0.016 & 0.015 \\
\hline Nitrate-N & $<0.2$ & $\cdot$ & $<0.2$ & $\cdot$ & $<0.2$ & $\cdot$ & $<0.2$ & 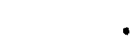 \\
\hline Potaselum & 1.1 & 1.2 & 1.3 & 0.98 & 1.3 & 1.2 & 0.96 & 1.1 \\
\hline sodium & 1.4 & 1.6 & 1.4 & 1.4 & 1.9 & 1.9 & 4.8 & s \\
\hline sulfate & 2.2 & . & 2.5 & $\cdot$ & 2 & $\cdot$ & 9.3 & $\cdot$ \\
\hline
\end{tabular}

(CONTINURD) 
APPENDTX $\mathbf{3 . 1}$

Groundwater and surfuce-Water Quallty Date, 1993

\begin{tabular}{|c|c|c|c|c|c|c|c|c|}
\hline \multirow{4}{*}{$\begin{array}{l}\text { Bampling Polnt } \\
\text { Lootion } \\
\text { - } \\
\text { Dete sempled }\end{array}$} & \multicolumn{6}{|c|}{$a v-324$} & \multicolumn{2}{|c|}{$a w-325$} \\
\hline & \multicolumn{6}{|c|}{83} & \multicolumn{2}{|c|}{83} \\
\hline & \multicolumn{2}{|c|}{$05 / 25 / 93$} & \multicolumn{2}{|c|}{$09 / 11 / 93$} & \multicolumn{2}{|c|}{$10 / 19 / 93$} & \multicolumn{2}{|c|}{$02 / 01 / 93$} \\
\hline & 202 & DIs & ror & DIs & ror & Drs & $\operatorname{tox}$ & DIs \\
\hline mancs $(\mathrm{mg} / \mathrm{L})$ & - & $\cdot$ & $\cdot$ & $\cdot 1$ & $\cdot$ & $\cdot$ & $\cdot$ & - \\
\hline Adualnum & $<0.02$ & $<0.02$ & $<0.02$ & $<0.02$ & $<0.02$ & $<0.02$ & 0.31 & $<0.02$ \\
\hline Ant loony & $<0.05$ & $<0.05$ & $<0.05$ & $<0.03$ & $<0.05$ & $<0.05$ & $<0.05$ & $<0.05$ \\
\hline Areente & $<0.05$ & $<0.05$ & $<0.05$ & $<0.05$ & $<0.05$ & $<0.05$ & $<0.05$ & $<0.05$ \\
\hline Bartum & 0.2 & 0.2 & 0.21 & 0.21 & 0.22 & 0.22 & 0.045 & 0.054 \\
\hline Bery111um & $<0.0003$ & $<0.0003$ & $<0.0003$ & $<0.0003$ & $<0.0003$ & $<0,0003$ & $<0.0003$ & $<0.0003$ \\
\hline Doron & 0.011 & 0.012 & 0.02 & 0.017 & 0.0084 & 0.011 & 0.0051 & 0.013 \\
\hline Cedmlun (MAs) & $<0.002$ & $<0.002$ & $<0.002$ & $<0.002$ & $<0.002$ & $<0.002$ & $<0.002$ & $<0.002$ \\
\hline Cadiatum & $<0.003$ & $<0.003$ & $<0.003$ & $<0,003$ & $<0.003$ & $<0.003$ & $<0.003$ & $<0.003$ \\
\hline Chronlum (MA) & $<0.01$ & $<0.01$ & $<0.01$ & $<0.01$ & $<0.01$ & $<0.01$ & $<0.01$ & $<0.01$ \\
\hline Chronlum & $<0.01$ & $<0.01$ & $<0.01$ & $<0.01$ & $<0.01$ & $<0.01$ & $<0.01$ & $<0.01$ \\
\hline Cobalt & $<0.00 s$ & $<0.005$ & $<0.005$ & $<0.005$ & $<0.005$ & $<0.005$ & $<0.005$ & $<0.005$ \\
\hline copper & 0.0048 & $<0.004$ & 0.0067 & $<0.004$ & $<0.004$ & $<0.004$ & 0.0049 & $<0.004$ \\
\hline Iron & 0.076 & 0.057 & 0.065 & 0.071 & 0.074 & 0.062 & 0.31 & 0.0069 \\
\hline Lead (Ans) & $<0.004$ & $<0.004$ & $<0.004$ & $<0.004$ & $<0.004$ & $<0.004$ & $<0.004$ & $<0.004$ \\
\hline Meroury (CVMA) & $<0.0002$ & $<0.0002$ & $<0.0002$ & $<0.0002$ & $<0.0002$ & $<0.0002$ & $<0.0002$ & $<0.0002$ \\
\hline Molybdenua & $<0.01$ & $<0.01$ & $<0.01$ & $<0.01$ & $<0.01$ & $<0.01$ & $<0.01$ & $<0.01$ \\
\hline nlekel & $<0.01$ & $<0.01$ & $<0.01$ & $<0.01$ & $<0.01$ & $<0.01$ & $<0.01$ & $<0.01$ \\
\hline selentum & $<0.05$ & $<0.05$ & $<0.05$ & $<0.05$ & $<0.05$ & $<0.05$ & $<0.05$ & $<0.05$ \\
\hline 811ver & $<0.006$ & $<0.006$ & $<0.006$ & $<0.006$ & $<0.006$ & $<0.006$ & $<0.006$ & $<0.006$ \\
\hline stront Iun & 0.22 & 0.22 & 0.23 & 0.23 & 0.25 & 0.25 & 0.01 & 0.012 \\
\hline Thorlum & $<0.2$ & $<0.2$ & $<0.2$ & $<0.2$ & $<0.2$ & $<0.2$ & $<0.2$ & $<0.2$ \\
\hline Urantum (rluor) & $<0.001$ & $<0.001$ & $<0.001$ & $<0.001$ & $<0.001$ & $<0.001$ & $<0.001$ & $<0.001$ \\
\hline Vanadium & $<0.005$ & $<0.005$ & $<0.005$ & $<0.005$ & $<0.005$ & $<0.005$ & $<0.005$ & $<0.005$ \\
\hline 2lne & 0.0072 & 0.0052 & 0.0042 & 0.0023 & 0.0044 & 0.0034 & 0.077 & 0.17 \\
\hline MWOR IONS (mg/L) & $\cdot$ & . & - & . & . & . & - & • \\
\hline Alkalinity-HCO3 & 102 & $\cdot$ & 104 & $\cdot$ & 103 & $\cdot$ & 9 & $\bullet$ \\
\hline Alkalinity-co3 & $<1$ &. & $<1$ & - & $<1$ & - & $<1$ & - \\
\hline Calolun & 28 & 29 & 30 & 31 & 29 & 30 & $2 \cdot 3$ & 2.6 \\
\hline Chloride & 1 & $\cdot$ & $<1$ & . & 1 & - & 1.6 & - \\
\hline Fluoride & 0.1 & . & $<0.1$ &. & $<0.1$ & - & $<0.1$ & - \\
\hline Magnen Lum & 5.5 & 5.5 & 5.6 & 5.7 & 5.9 & 6 & 1.4 & 1.5 \\
\hline Manganese & 0.016 & 0.016 & 0.016 & 0.016 & 0.017 & 0.018 & 0.023 & 0.035 \\
\hline Nitrate-N & $<0.2$ & $\cdot$ & $<0.2$ & - & $<0.2$ & - & $<0.2$ & - \\
\hline Potaseium & $<0.6$ & $<0.6$ & 1 & 0.82 & 0.9 & 0.84 & 0.7 & 1.3 \\
\hline sodlum & 4.8 & 4.9 & 5.2 & 5.2 & $5 \cdot 3$ & $5 \cdot 3$ & 2.8 & 3.6 \\
\hline sulfate & 9.3 & $\cdot$ & 7.4 & - & 7 & - & 7.5 & • \\
\hline
\end{tabular}

(CONTINUED) 
APPENDIX E.1

Groundwater and surface-Water Quality Data, 1993

\begin{tabular}{|c|c|c|c|c|c|c|c|c|}
\hline \multirow{4}{*}{$\begin{array}{l}\text { sampling polnt } \\
\text { Location } \\
\text { Date sampled }\end{array}$} & \multicolumn{6}{|c|}{ OW-325 } & \multicolumn{2}{|c|}{$G w-342$} \\
\hline & \multicolumn{6}{|c|}{83} & \multirow{2}{*}{\multicolumn{2}{|c|}{$\frac{B C}{10 / 07 / 93}$}} \\
\hline & \multicolumn{2}{|c|}{$05 / 25 / 93$} & \multicolumn{2}{|c|}{$09 / 11 / 93$} & \multicolumn{2}{|c|}{$10 / 21 / 93$} & & \\
\hline & TOT & Drs & TOT & DIs & TOT & DI8 & TON & DI8 \\
\hline Horars $(\mathrm{mg} / \mathrm{L})$ & $\cdot$ & $\cdot 1$ &. & $\cdot 1$ & $\cdot 1$ & $\cdot 1$ & $\cdot$ & \\
\hline Aluninum & $i$ & $<0.02$ & $5 . \dot{2}$ & $<0.02$ & 44 & 0.042 & 0.59 & $<0.02$ \\
\hline Antimony & $<0.05$ & $<0.05$ & $<0.05$ & $<0.05$ & $<0.05$ & $<0.05$ & $<0.05$ & $<0.05$ \\
\hline Arsenic & $<0.05$ & $<0.05$ & $<0.05$ & $<0.05$ & $<0.05$ & $<0.05$ & $<0.05$ & $<0.05$ \\
\hline Bartum & 0.055 & 0.049 & 0.065 & 0.04 & 0.29 & 0.035 & 0.19 & 0.15 \\
\hline Baryl11um & $<0.0003$ & $<0.0003$ & $<0.0003$ & $<0.0003$ & 0.0014 & $<0.0003$ & $<0.0003$ & $<0.0003$ \\
\hline Boron & 0.015 & 0.019 & 0.0091 & 0.014 & 0.014 & 0.0071 & 0.006 & 0.012 \\
\hline Cadmium (MAS) & $<0.002$ & $<0.002$ & $<0.002$ & $<0.002$ & $<0.002$ & $<0.002$ & $<0.002$ & $<0.002$ \\
\hline Cadmium & $<0.003$ & $<0.003$ & 0.0041 & $<0.003$ & $<0.003$ & $<0.003$ & $<0,003$ & $<0.003$ \\
\hline Chromtum (AAS) & $<0.01$ & $<0.01$ & 0.011 & $<0.01$ & 0.077 & $<0.01$ & $<0.01$ & $<0.01$ \\
\hline Chromiun & $<0.01$ & $<0.01$ & 0.011 & $<0.01$ & 0.098 & $<0.01$ & $<0.01$ & $<0.01$ \\
\hline cobalt & $<0.005$ & $<0.005$ & $<0.005$ & $<0.005$ & 0.021 & $<0.005$ & $<0.005$ & $<0.005$ \\
\hline Copper & 0.0059 & 0.0084 & 0.015 & 0.012 & 0.098 & $<0.004$ & $<0.004$ & $<0.004$ \\
\hline Iron & 0.87 & $<0.005$ & 4.2 & 0.24 & 46 & 0.016 & 2.4 & 1.2 \\
\hline Laad (MAS) & $<0.004$ & $<0.004$ & $<0.004$ & $<0.004$ & 0.042 & $<0.004$ & $<0.004$ & $<0.004$ \\
\hline Marcury (CVAM) & $<0.0002$ & $<0.0002$ & $<0.0002$ & $<0.0002$ & $<0.0002$ & $<0.0002$ & $<0.0002$ & $<0.0002$ \\
\hline Mal ybdenum & $<0.01$ & $<0.01$ & $<0.01$ & $<0.01$ & $<0.01$ & $<0.01$ & $<0.01$ & $<0.01$ \\
\hline Nickel & $<0.01$ & 0.02 & $<0.01$ & $<0.01$ & 0.061 & $<0.01$ & $<0.01$ & $<0.01$ \\
\hline Selenium & $<0.05$ & $<0.05$ & $<0.05$ & $<0.05$ & $<0.05$ & $<0.05$ & $<0.05$ & $<0.05$ \\
\hline silver & $<0.006$ & $<0.006$ & $<0.006$ & $<0.006$ & $<0.006$ & $<0.006$ & $<0.006$ & $<0.006$ \\
\hline strontium & 0.011 & 0.011 & 0.011 & 0.0094 & 0.023 & 0.0097 & 0.028 & 0.026 \\
\hline Thorium & $<0.2$ & $<0.2$ & $<0.2$ & $<0.2$ & $<0.2$ & $<0.2$ & $<0.2$ & $<0.2$ \\
\hline Uranium (Fluor) & $<0.001$ & $<0.001$ & $<0.001$ & $<0.001$ & 0.002 & $<0.001$ & $<0.001$ & $<0.001$ \\
\hline Vanadium & $<0.005$ & $<0.005$ & 0.0065 & $<0.005$ & 0.058 & $<0.005$ & $<0.005$ & $<0.005$ \\
\hline zine & 0.078 & 0.13 & 0.095 & 0.17 & 0.094 & 0.0051 & 0.0074 & 0.005 \\
\hline MMJOR IONS $(\mathrm{mg} / \mathrm{L})$ &. &. & $\cdot$ & $\cdot$ & . &. & . & \\
\hline Alkalinity-HCO3 & $\dot{8}$ &. & $1 \dot{2}$ &. & $\dot{9}$ &. & $3 \dot{8}$ & \\
\hline Alkalinity-CO3 & $<1$ &. & $<1$ &. & $<1$ &. & $<1$ & \\
\hline Calcium & 2.4 & 2.4 & 3.4 & 3.5 & 5.1 & 3.2 & 3.9 & 3.6 \\
\hline Chloride & 1.6 &. & 1.7 &. & 2 &. & $<1$ & \\
\hline Fluoride & $<0.1$ & . & $<0.1$ & $\cdot$ & $<0.1$ & $\cdot$ & 0.2 & \\
\hline Magnes I um & 1.4 & 1.2 & 1.6 & 1.1 & 5.7 & 1.2 & 5.5 & 5.1 \\
\hline Manganese & 0.026 & 0.021 & 0.062 & 0.033 & 0.35 & 0.055 & 0.29 & 0.27 \\
\hline Nitrate-N & $<0.2$ & . & $<0.2$ &. & $<0.2$ &. & $<0.2$ & \\
\hline Potassium & 0.71 & $<0,6$ & 1.7 & 0.85 & 6 & 0.69 & 1.7 & 2.2 \\
\hline sodium & 1.5 & 1.6 & 1.4 & 1.4 & 1.5 & 1.5 & 5.6 & 5.6 \\
\hline Sulfate & 5.1 & . & 3 & . & 3 & . & 6 & \\
\hline
\end{tabular}

(CONTINUED) 
Groundwater and Surface-Water Quality Data, 1993

\begin{tabular}{|c|c|c|c|c|c|c|c|c|}
\hline \multirow{4}{*}{$\begin{array}{l}\text { Sampling Point } \\
\text { Location } \\
\text { Date sampled }\end{array}$} & \multicolumn{8}{|c|}{ OW-345 } \\
\hline & \multicolumn{8}{|c|}{83} \\
\hline & \multicolumn{2}{|c|}{$02 / 03 / 93$} & \multicolumn{2}{|c|}{$06 / 01 / 93$} & \multicolumn{2}{|c|}{$09 / 14 / 93$} & \multicolumn{2}{|c|}{$10 / 26 / 93$} \\
\hline & TOT & Drs & TOT & DIs & TOT & Drs & TOT & DIs \\
\hline MBTALS (mg/L) & $\cdot$ & $\cdot 1$ & $\cdot$ & . & $\cdot 1$ & . & $\cdot$ & • \\
\hline Aluminum & 0.13 & 0.026 & 0.054 & $<0.02$ & 0.086 & $<0.02$ & $<0.2$ & $<0.2$ \\
\hline Antimony & $<0.05$ & $<0.05$ & $<0.05$ & $<0.05$ & $<0.05$ & $<0.05$ & $<0.5$ & $<0.5$ \\
\hline Arsenic & $<0.05$ & $<0.05$ & $<0.05$ & $<0.05$ & $<0.05$ & $<0.05$ & $<0.5$ & $<0.5$ \\
\hline Barlum & 0.043 & 0.042 & 0.071 & 0.068 & 0.19 & 0.17 & 13 & 13 \\
\hline Beryllium & $<0.0003$ & $<0.0003$ & $<0.0003$ & $<0.0003$ & 0.00075 & $<0.0003$ & $<0.003$ & $<0.003$ \\
\hline Boron & 0.017 & 0.011 & 0.028 & 0.017 & 0.016 & 0.014 & 0.2 & 0.21 \\
\hline Cadmium (AAS) & $<0.002$ & $<0.002$ & $<0.002$ & $<0.002$ & $<0.002$ & $<0.002$ & $<0.002$ & $<0.002$ \\
\hline Cadmium & $<0.003$ & $<0.003$ & $<0.003$ & $<0.003$ & $<0.003$ & $<0.003$ & $<0.03$ & $<0.03$ \\
\hline Chromium (AAS) & $<0.01$ & $<0.01$ & $<0.01$ & $<0.01$ & $<0.01$ & $<0.01$ & $<0.01$ & $<0.01$ \\
\hline Chromium & $<0.01$ & $<0.01$ & $<0.01$ & $<0.01$ & $<0.01$ & $<0.01$ & $<0.1$ & $<0.1$ \\
\hline Cobalt & $<0.005$ & $<0.005$ & $<0.005$ & $<0.005$ & $<0.005$ & $<0.005$ & $<0.05$ & $<0.05$ \\
\hline Copper & $<0.004$ & 0.019 & $<0.004$ & $<0.004$ & 0.083 & 0.012 & $<0.04$ & $<0.04$ \\
\hline Iron & 0.16 & 0.0068 & 0.072 & $<0.005$ & 0.22 & 0.013 & 15 & $<0.05$ \\
\hline Lead (AAS) & $<0.004$ & $<0.004$ & $<0.004$ & $<0.004$ & $<0.004$ & $<0.004$ & $<0.004$ & $<0.004$ \\
\hline Mercury (CVAA) & $<0.0002$ & $<0.0002$ & $<0.0002$ & $<0.0002$ & $<0.0002$ & $<0.0002$ & $<0.0002$ & $<0.0002$ \\
\hline Molybdenum & $<0.01$ & $<0.01$ & $<0.01$ & $<0.01$ & $<0.01$ & $<0.01$ & $<0.1$ & $<0.1$ \\
\hline Nickel & $<0.01$ & $<0.01$ & 0.016 & 0.015 & $<0.01$ & $<0.01$ & $<0.1$ & $<0.1$ \\
\hline Selenium & $<0.05$ & $<0.05$ & $<0.05$ & $<0.05$ & $<0.05$ & $<0.05$ & $<0.5$ & $<0.5$ \\
\hline silver & $<0.006$ & $<0.006$ & $<0.006$ & $<0.006$ & $<0.006$ & $<0.006$ & $<0.06$ & $<0.06$ \\
\hline strontium & 0.024 & 0.023 & 0.044 & 0.044 & 0.12 & 0.11 & 16 & 15 \\
\hline Thorium & $<0.2$ & $<0.2$ & $<0.2$ & $<0.2$ & $<0.2$ & $<0.2$ & $<2$ & $<2$ \\
\hline Uranium (Fluor) & $<0.001$ & $<0.001$ & $<0.001$ & $<0.001$ & 0.002 & 0.002 & $<0.001$ & $<0.001$ \\
\hline Vanadium & $<0.005$ & $<0.005$ & $<0.005$ & $<0.005$ & $<0.005$ & $<0.005$ & $<0.05$ & $<0.05$ \\
\hline zinc & 0.029 & 0.051 & 0.026 & 0.029 & 0.022 & 0.02 & 0.039 & $<0.02$ \\
\hline MAJOR IONS (mg/L) & $\cdot$ & $\cdot$ & • & $\cdot$ & $\cdot$ & $\cdot$ & $\cdot$ & $\bullet$ \\
\hline Alkalinity-HCO3 & 20 & $\cdot$ & 24 & $\cdot$ & $\dot{27}$ & $\cdot$ & $\dot{22}$ & • \\
\hline Alkalinity-CO3 & $<1$ &. & $<1$ &. & $<1$ &. & $<1$ & . \\
\hline Calcium & 5.1 & 4.9 & 11 & 11 & 35 & 33 & 130 & 130 \\
\hline Chloride & 4.7 & $\cdot$ & 4.8 & . & 5.4 & . & 7 & • \\
\hline Fluoride & $<0.1$ & $\cdot$ & $<0.1$ & . & $<0.1$ & . & $<0.1$ & • \\
\hline Magnesium & 1.8 & 1.7 & 2.6 & 2.6 & 6 & 5.6 & 53 & 52 \\
\hline Manganese & 0.04 & 0.035 & 0.033 & 0.031 & 0.066 & 0.057 & 0.06 & 0.027 \\
\hline Nitrate-N & 7.7 &. & 3.4 &. & 20 & . & 66 & . \\
\hline Potassium & 2 & 2.1 & 3.4 & 3.3 & 6.3 & 6.7 & 22 & 17 \\
\hline Sodium & 6.8 & 7 & 6.8 & 6.9 & 8.1 & 7.9 & 1600 & 1600 \\
\hline Sulfate & 12 & $\cdot$ & 8.5 & . & 14 & . & 9 & • \\
\hline
\end{tabular}

(CONTINUED) 
APPENDIX E. 1

Groundwater and Surface-Water Quality Data, 1993

\begin{tabular}{|c|c|c|c|c|c|c|c|c|}
\hline \multirow{4}{*}{$\begin{array}{l}\text { Sampling Point } \\
\text { Location } \\
\text { Date Sampled }\end{array}$} & \multicolumn{8}{|c|}{$G W-347$} \\
\hline & \multicolumn{8}{|c|}{ s3 } \\
\hline & \multicolumn{2}{|c|}{$02 / 01 / 93$} & \multicolumn{2}{|c|}{$05 / 27 / 93$} & \multicolumn{2}{|c|}{$09 / 12 / 93$} & \multicolumn{2}{|c|}{$10 / 22 / 93$} \\
\hline & TOT & DIS & TOT & DIS & TOT & DIS & TOT & DIS \\
\hline METALS (mg/L) & $\cdot$ & - & $\cdot$ & $\cdot 1$ & $\cdot$ & - & - & • \\
\hline Aluminum & 0.16 & $<0.02$ & 0.072 & $<0.02$ & 0.68 & $<0.02$ & 1.1 & $<0.02$ \\
\hline Ant imony & $<0.05$ & $<0.05$ & $<0.05$ & $<0.05$ & $<0.05$ & $<0.05$ & $<0.05$ & $<0.05$ \\
\hline Arsenic & $<0.05$ & $<0.05$ & $<0.05$ & $<0.05$ & $<0.05$ & $<0.05$ & $<0.05$ & $<0.05$ \\
\hline Barium & 0.0092 & 0.0094 & 0.012 & 0.01 & 0.013 & 0.012 & 0.01 & 0.0082 \\
\hline Beryllium & $<0.0003$ & $<0.0003$ & $<0.0003$ & $<0.0003$ & 0.00036 & $<0.0003$ & $<0.0003$ & $<0.0003$ \\
\hline Boron & $<0.004$ & $<0.004$ & 0.011 & 0.0092 & 0.015 & $<0.004$ & $<0.004$ & $<0.004$ \\
\hline Cadmium (AAS) & $<0.002$ & $<0.002$ & $<0.002$ & $<0.002$ & $<0.002$ & $<0.002$ & $<0.002$ & $<0.002$ \\
\hline Cadmium & $<0.003$ & $<0.003$ & $<0.003$ & $<0.003$ & $<0.003$ & $<0.003$ & $<0.003$ & $<0.003$ \\
\hline Chromium (AAS) & $<0.01$ & $<0.01$ & $<0.01$ & $<0.01$ & 0.018 & 0.012 & $<0.01$ & $<0.01$ \\
\hline Chromium & $<0.01$ & $<0.01$ & $<0.01$ & $<0.01$ & 0.015 & 0.01 & $<0.01$ & $<0.01$ \\
\hline Cobalt & $<0.005$ & $<0.005$ & $<0.005$ & $<0.005$ & $<0.005$ & $<0.005$ & $<0.005$ & $<0.005$ \\
\hline Copper & $<0.004$ & $<0.004$ & $<0.004$ & $<0.004$ & 0.016 & $<0.004$ & $<0.004$ & $<0.004$ \\
\hline Iron & 0.28 & $<0.005$ & 0.14 & $<0.005$ & 1.6 & $<0.005$ & 1.9 & $<0.005$ \\
\hline Lead (AAS) & $<0.004$ & $<0.004$ & $<0.004$ & $<0.004$ & 0.0073 & $<0.004$ & 0.007 & $<0.004$ \\
\hline Mercury (CVAA) & $<0.0002$ & $<0.0002$ & $<0.0002$ & $<0.0002$ & $<0.0002$ & $<0.0002$ & $<0.0002$ & $<0.0002$ \\
\hline Mol ybdenum & $<0.01$ & $<0.01$ & $<0.01$ & $<0.01$ & $<0.01$ & $<0.01$ & $<0.01$ & $<0.01$ \\
\hline Nickel & $<0.01$ & $<0.01$ & $<0.01$ & 0.017 & $<0.01$ & $<0.01$ & $<0.01$ & $<0.01$ \\
\hline Selenium & $<0.05$ & $<0.05$ & $<0.05$ & $<0.05$ & $<0.05$ & $<0.05$ & $<0.05$ & $<0.05$ \\
\hline Silver & $<0.006$ & $<0.006$ & $<0.006$ & $<0.006$ & $<0.006$ & $<0.006$ & $<0.006$ & $<0.006$ \\
\hline Strontium & 0.019 & 0.02 & 0.029 & 0.025 & 0.057 & 0.059 & 0.025 & 0.021 \\
\hline Thorium & $<0.2$ & $<0.2$ & $<0.2$ & $<0.2$ & $<0.2$ & $<0.2$ & $<0.2$ & $<0.2$ \\
\hline Uranium (Fluor) & $<0.001$ & $<0.001$ & $<0.001$ & $<0.001$ & 0.001 & 0.001 & $<0.001$ & $<0.001$ \\
\hline Vanadium & $<0.005$ & $<0.005$ & $<0.005$ & $<0.005$ & $<0.005$ & $<0.005$ & $<0.005$ & $<0.005$ \\
\hline zine & 0.014 & 0.032 & 0.025 & 0.014 & 0.015 & 0.0065 & 0.018 & 0.0064 \\
\hline MAJOR IONS $(\mathrm{mg} / \mathrm{L})$ & $\cdot$ & $\cdot$ & $\cdot \dot{.}$ & $\cdot$ & $\cdot$ & $\cdot$ & $\cdot$ & • \\
\hline Alkalinity-HCO3 & 74 & $\cdot$ & ${ }_{83}$ & $\cdot$ & 156 & $\cdot$ & $\dot{93}$ & - \\
\hline Alkalinity-CO3 & $<1$ &. & $<1$ &. & $<1$ &. & $<1$ & 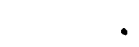 \\
\hline Calcium & 23 & 24 & 31 & 28 & 57 & 58 & 28 & 23 \\
\hline Chloride & $<1$ & . & $<1$ & $\cdot$ & $<1$ & $\cdot$ & $<1$ & • \\
\hline Fluoride & $<0.1$ & . & $<0.1$ &. & 0.1 & . & 0.1 & $\bullet$ \\
\hline Megnesium & 1.8 & 1.9 & 2.4 & 2.1 & 4.9 & 5.1 & 2 & 1.8 \\
\hline Manganese & 0.0091 & 0.0023 & 0.01 & 0.0049 & 0.027 & 0.0017 & 0.026 & 0.0016 \\
\hline Nitrate-N & $<0.2$ & . & $<0.2$ & . & $<0.2$ & . & $<0.2$ & $\cdot$ \\
\hline Potassium & 3.6 & 3.4 & 3.2 & 3.2 & 3.8 & 3.9 & 3.3 & 2.9 \\
\hline Sodium & 0.86 & 0.87 & 1.1 & 0.98 & 1.1 & 1.1 & 0.65 & 0.63 \\
\hline Sulfate & 1.1 & . & 1.4 & . & 6.2 &. & 3 & • \\
\hline
\end{tabular}

(CONTINUED) 
APPENDIX E.1

Groundwater and Surface-water Quality Data, 1993

\begin{tabular}{|c|c|c|c|c|c|c|c|c|}
\hline \multirow{4}{*}{$\begin{array}{l}\text { Sampling Point } \\
\text { Location } \\
\text { Date Sampled }\end{array}$} & \multicolumn{8}{|c|}{ GW-348 } \\
\hline & \multicolumn{8}{|c|}{ s3 } \\
\hline & \multicolumn{2}{|c|}{$02 / 01 / 93$} & \multicolumn{2}{|c|}{$05 / 27 / 93$} & \multicolumn{2}{|c|}{$09 / 12 / 93$} & \multicolumn{2}{|c|}{$10 / 22 / 93$} \\
\hline & TOT & DIS & TOT & DIs & TOT & DIS & TOT & DIS \\
\hline $\operatorname{MBTALS}(\mathrm{mg} / \mathrm{L}$ ) &. & $\cdot$ & . & $\cdot$ & . & - & $\cdot 1$ & $\theta$ \\
\hline Aluminum & 0.023 & $<0.02$ & 0.045 & $<0.02$ & $<0.02$ & $<0.02$ & 0.11 & $<0.02$ \\
\hline Antimony & $<0.05$ & $<0.05$ & $<0.05$ & $<0.05$ & $<0.05$ & $<0.05$ & $<0.05$ & $<0.05$ \\
\hline Arsenic & $<0.05$ & $<0.05$ & $<0.05$ & $<0.05$ & $<0.05$ & $<0.05$ & $<0.05$ & $<0.05$ \\
\hline Barium & 0.083 & 0.083 & 0.087 & 0.087 & 0.09 & 0.089 & 0.095 & 0.093 \\
\hline Beryllium & $<0.0003$ & $<0.0003$ & $<0.0003$ & $<0.0003$ & $<0.0003$ & $<0.0003$ & $<0.0003$ & $<0.0003$ \\
\hline Boron & $<0.004$ & $<0.004$ & 0.014 & 0.014 & 0.014 & 0.018 & 0.0054 & 0.0065 \\
\hline Cadmium (AAS) & $<0.002$ & $<0.002$ & $<0.002$ & $<0.002$ & $<0.002$ & $<0.002$ & $<0.002$ & $<0.002$ \\
\hline Cadmium & $<0.003$ & $<0.003$ & $<0.003$ & $<0.003$ & $<0.003$ & $<0.003$ & $<0.003$ & $<0.003$ \\
\hline Chromium (AAS) & $<0.01$ & $<0.01$ & $<0.01$ & $<0.01$ & $<0.01$ & $<0.01$ & $<0.01$ & $<0.01$ \\
\hline Chromium & $<0.01$ & $<0.01$ & $<0.01$ & $<0.01$ & $<0.01$ & $<0.01$ & $<0.01$ & $<0.01$ \\
\hline Cobalt & $<0.005$ & $<0.005$ & $<0.005$ & $<0.005$ & $<0.005$ & $<0.005$ & $<0.005$ & $<0.005$ \\
\hline Copper & $<0.004$ & $<0.004$ & $<0.004$ & $<0.004$ & $<0.004$ & $<0.004$ & $<0.004$ & $<0.004$ \\
\hline Iron & 0.047 & $<0.005$ & 0.011 & $<0.005$ & 0.02 & $<0.005$ & 0.038 & $<0.005$ \\
\hline Lead (AAS) & $<0.004$ & $<0.004$ & $<0.004$ & $<0.004$ & $<0.004$ & $<0.004$ & $<0.004$ & $<0.004$ \\
\hline Mercury (CVAA) & $<0.0002$ & $<0.0002$ & $<0.0002$ & $<0.0002$ & $<0.0002$ & $<0.0002$ & $<0.0002$ & $<0.0002$ \\
\hline Molybdenum & $<0.01$ & $<0.01$ & $<0.01$ & $<0.01$ & $<0.01$ & $<0.01$ & $<0.01$ & $<0.01$ \\
\hline Nickel & $<0.01$ & $<0.01$ & $<0.01$ & $<0.01$ & $<0.01$ & $<0.01$ & $<0.01$ & $<0.01$ \\
\hline Selenium & $<0.05$ & $<0.05$ & $<0.05$ & $<0.05$ & $<0.05$ & $<0.05$ & $<0.05$ & $<0.05$ \\
\hline Silver & $<0.006$ & $<0.006$ & $<0.006$ & $<0.006$ & $<0.006$ & $<0.006$ & $<0.006$ & $<0.006$ \\
\hline Strontium & 0.087 & 0.087 & 0.095 & 0.095 & 0.096 & 0.096 & 0.1 & 0.099 \\
\hline Thorium & $<0.2$ & $<0.2$ & $<0.2$ & $<0.2$ & $<0.2$ & $<0.2$ & $<0.2$ & $<0.2$ \\
\hline Uranium (Fluor) & 0.001 & 0.001 & 0.001 & 0.001 & 0.001 & 0.004 & 0.001 & 0.001 \\
\hline Vanadium & $<0.005$ & $<0.005$ & $<0.005$ & $<0.005$ & $<0.005$ & $<0.005$ & $<0.005$ & $<0.005$ \\
\hline zinc & 0.0051 & 0.0096 & 0.0058 & 0.011 & 0.0037 & 0.0022 & 0.0027 & $<0.002$ \\
\hline MAJOR IONS (mg/L) & - & $\cdot$ & - & . & . & $\cdot$ & • & • \\
\hline Alkalinity-HCO3 & $23 \dot{2}$ & $\cdot$ & 232 & $\cdot \dot{1}$ & $24 \dot{8}$ & $\cdot$ & 244 & • \\
\hline Alkalinity-CO3 & $<1$ &. & $<1$ &. & $<1$ &. & $<1$ & . \\
\hline Calcium & 80 & 81 & 84 & 84 & 87 & 87 & 85 & 82 \\
\hline Chloride & 9.1 & . & 8.9 &. & 8.7 & . & 10 & • \\
\hline Fluoride & $<0.1$ &. & 0.3 & . & $<0.1$ & . & $<0.1$ & • \\
\hline Magnesium & 17 & 17 & 18 & 18 & 18 & 18 & 19 & 19 \\
\hline Manganese & $<0.001$ & $<0.001$ & 0.0015 & 0.0012 & $<0.001$ & $<0.001$ & $<0.001$ & $<0.001$ \\
\hline Nitrate-N & 9.73 & . & 8.5 & $\cdot$ & 9.35 & . & 12.4 & • \\
\hline Potassium & 1.5 & 1.9 & 1.3 & 1.4 & 1.1 & 1.6 & 1.5 & 1.4 \\
\hline Sodium & 2.9 & 2.9 & 2.8 & 2.8 & 3.2 & 3.2 & 3.3 & 3.3 \\
\hline Sulfate & 5.4 & . & 7.1 & . & 6 & . & 6 & • \\
\hline
\end{tabular}

(CONTINUED) 
APPENDIX E.1

Groundwater and Surface-Water Quality Data, 1993

\begin{tabular}{|c|c|c|c|c|c|c|c|c|}
\hline \multirow{4}{*}{$\begin{array}{l}\text { Sampling Point } \\
\text { Location } \\
\text { - } \\
\text { Date Sampled }\end{array}$} & \multicolumn{8}{|c|}{ GW-364 } \\
\hline & \multicolumn{8}{|c|}{ OLF } \\
\hline & \multicolumn{2}{|c|}{$03 / 27 / 93$} & \multicolumn{2}{|c|}{$06 / 27 / 93$} & \multicolumn{2}{|c|}{$09 / 19 / 93$} & \multicolumn{2}{|c|}{$12 / 18 / 93$} \\
\hline & TOT & DIS & TOT & DIS & TOT & DIS & TOT & DIS \\
\hline METArs (mg/L) & - & - & - & - & $\cdot$ & - & • & - \\
\hline Aluminum & 0.83 & $<0.02$ & 4.6 & $<0.02$ & 12 & 0.035 & 0.69 & $<0.02$ \\
\hline Antimony & $<0.05$ & $<0.05$ & $<0.05$ & $<0.05$ & $<0.05$ & $<0.05$ & $<0.05$ & $<0.05$ \\
\hline Arsenic & $<0.05$ & $<0.05$ & $<0.05$ & $<0.05$ & $<0.05$ & $<0.05$ & $<0.05$ & $<0.05$ \\
\hline Barium & 0.089 & 0.079 & 0.15 & 0.13 & 0.24 & 0.14 & 0.096 & 0.092 \\
\hline Beryllium & $<0.0003$ & $<0.0003$ & $<0.0003$ & $<0.0003$ & 0.0013 & $<0.0003$ & $<0.0003$ & $<0.0003$ \\
\hline Boron & 0.05 & 0.057 & 0.11 & 0.098 & 0.13 & 0.12 & 0.063 & 0.067 \\
\hline Cadmium (AAS) & $<0.002$ & $<0.002$ & $<0.002$ & $<0.002$ & $<0.002$ & $<0.002$ & $<0.002$ & $<0.002$ \\
\hline Cadmium & $<0.003$ & $<0.003$ & $<0.003$ & $<0.003$ & 0.006 & $<0.003$ & $<0.003$ & $<0.003$ \\
\hline Chromium (AAS) & $<0.01$ & $<0.01$ & $<0.01$ & $<0.01$ & 0.012 & $<0.01$ & $<0.01$ & $<0.01$ \\
\hline Chromium & $<0.01$ & $<0.01$ & $<0.01$ & $<0.01$ & 0.011 & $<0.01$ & $<0.01$ & $<0.01$ \\
\hline Cobalt & $<0.005$ & $<0.005$ & $<0.005$ & $<0.005$ & 0.0072 & $<0.005$ & $<0.005$ & $<0.005$ \\
\hline Copper & 0.0045 & $<0.004$ & 0.0042 & $<0.004$ & 0.033 & 0.0041 & $<0.004$ & $<0.004$ \\
\hline Iron & 0.83 & 0.0061 & 2.8 & 0.0057 & 13 & 0.014 & 0.65 & $<0.005$ \\
\hline Lead (AAS) & $<0.004$ & $<0.004$ & $<0.004$ & $<0.004$ & 0.0084 & $<0.004$ & 0.0088 & $<0.004$ \\
\hline Mercury (CVAA) & $<0.0002$ & $<0.0002$ & $<0.0002$ & $<0.0002$ & $<0.0002$ & $<0.0002$ & $<0.0002$ & $<0.0002$ \\
\hline Molybdenum & $<0.01$ & $<0.01$ & $<0.01$ & $<0.01$ & $<0.01$ & $<0.01$ & $<0.01$ & $<0.01$ \\
\hline Nickel & $<0.01$ & $<0.01$ & $<0.01$ & $<0.01$ & 0.021 & 0.015 & $<0.01$ & $<0.01$ \\
\hline Selenium & $<0.05$ & $<0.05$ & $<0.05$ & $<0.05$ & $<0.05$ & $<0.05$ & $<0.05$ & $<0.05$ \\
\hline silver & $<0.006$ & $<0.006$ & $<0.006$ & $<0.006$ & $<0.006$ & $<0.006$ & $<0.006$ & $<0.006$ \\
\hline strontium & 0.24 & 0.24 & 0.44 & 0.44 & 0.56 & 0.53 & 0.28 & 0.28 \\
\hline Thorium & $<0.2$ & $<0.2$ & $<0.2$ & $<0.2$ & $<0.2$ & $<0.2$ & $<0.2$ & $<0.2$ \\
\hline Uranium (Fluor) & 0.001 & 0.001 & 0.003 & 0.004 & 0.008 & 0.008 & 0.003 & 0.003 \\
\hline Vanadium & $<0.005$ & $<0.005$ & $<0.005$ & $<0.005$ & 0.016 & $<0.005$ & $<0.005$ & $<0.005$ \\
\hline zinc & 0.018 & 0.015 & 0.019 & 0.012 & 0.19 & 0.069 & 0.0059 & 0.0035 \\
\hline MAJOR IONS $(\mathrm{mg} / \mathrm{L})$ & $\cdot$ & $\cdot$ & . & . & - & . & - & - \\
\hline Alkalinity-HCO3 & 169 & $\cdot$ & 237 & $\cdot$ & 226 & $\cdot$ & 193 & $\cdot$ \\
\hline Alkalinity-CO3 & $<1$ & . & $<1$ & - & $<1$ & . & $<1$ & - \\
\hline Calcium & 59 & 57 & 87 & 87 & 100 & 94 & 73 & 73 \\
\hline Chloride & 15.5 & - & 42 & . & 39 &. & 17 & - \\
\hline Fluoride & $<0.1$ & . & $<0.1$ & . & $<0.1$ & . & $<0.1$ & - \\
\hline Magnesium & 10 & 9.8 & 17 & 17 & 20 & 18 & 12 & 12 \\
\hline Mang anese & 0.049 & 0.0045 & 0.14 & 0.0073 & 0.59 & 0.018 & 0.025 & $<0.001$ \\
\hline Nitrate-N & 1.21 & . & 1.12 & $\cdot$ & 10.2 & $\cdot$ & 2.8 & - \\
\hline Potassium & $2 \cdot 3$ & 1.5 & 3.5 & 2.6 & 5.2 & 2.8 & 2.2 & 1.8 \\
\hline Sodium & 3.7 & 3.8 & 6.2 & 6.3 & 8.9 & 8.7 & 4.1 & 4.2 \\
\hline Sulfate & 17 & - & 29.6 & - & 29.5 & . & 17 & - \\
\hline
\end{tabular}

(CONTINUED) 
APPENDIX E. 1

Groundwater and Surface-Water Quality Data, 1993

\begin{tabular}{|c|c|c|c|c|c|c|c|c|}
\hline \multirow{4}{*}{$\begin{array}{l}\text { Sampling Point } \\
\text { Location } \\
\text { Date Sampled }\end{array}$} & \multicolumn{8}{|c|}{$G W-365$} \\
\hline & \multicolumn{8}{|c|}{ OLF } \\
\hline & \multicolumn{2}{|c|}{$03 / 28 / 93$} & \multicolumn{2}{|c|}{$06 / 27 / 93$} & \multicolumn{2}{|c|}{$09 / 19 / 93$} & \multicolumn{2}{|c|}{$12 / 19 / 93$} \\
\hline & TOT & Drs & TOT & DIS & TOT & DIS & TOT & DIS \\
\hline METALS (mg/L) & $\cdot$ & $\cdot$ & $\cdot$ &. & $\cdot$ & $\cdot$ & $\cdot 1$ & - \\
\hline Aluminum & $<0.02$ & $<0.02$ & $<0.02$ & $<0.02$ & 0.032 & 0.043 & $<0.02$ & $<0.02$ \\
\hline Antimony & $<0.05$ & $<0.05$ & $<0.05$ & $<0.05$ & $<0.05$ & $<0.05$ & $<0.05$ & $<0.05$ \\
\hline Arsenic & $<0.05$ & $<0.05$ & $<0.05$ & $<0.05$ & $<0.05$ & $<0.05$ & $<0.05$ & $<0.05$ \\
\hline Barium & 0.3 & 0.29 & 0.33 & 0.33 & 0.37 & 0.36 & 0.35 & 0.35 \\
\hline Beryllium & $<0.0003$ & $<0.0003$ & $<0.0003$ & $<0.0003$ & $<0.0003$ & $<0.0003$ & $<0.0003$ & $<0.0003$ \\
\hline Boron & 0.21 & 0.16 & 0.15 & 0.16 & 0.19 & 0.18 & 0.18 & 0.17 \\
\hline Cadmium (AAS) & $<0.002$ & $<0.002$ & $<0.002$ & $<0.002$ & $<0.002$ & $<0.002$ & $<0.002$ & $<0.002$ \\
\hline Cadmium & $<0.003$ & $<0.003$ & $<0.003$ & $<0.003$ & $<0.003$ & $<0.003$ & $<0.003$ & $<0.003$ \\
\hline Chromium (AAS) & $<0.01$ & $<0.01$ & $<0.01$ & $<0.01$ & $<0.01$ & $<0.01$ & $<0.01$ & $<0.01$ \\
\hline Chromium & $<0.01$ & $<0.01$ & $<0.01$ & $<0.01$ & $<0.01$ & $<0.01$ & $<0.01$ & $<0.01$ \\
\hline Cobalt & $<0.005$ & $<0.005$ & $<0.005$ & $<0.005$ & $<0.005$ & $<0.005$ & $<0.005$ & $<0.005$ \\
\hline Copper & $<0.004$ & $<0.004$ & $<0.004$ & $<0.004$ & $<0.004$ & $<0.004$ & $<0.004$ & $<0.004$ \\
\hline Iron & 1.8 & 1.4 & 0.81 & 0.89 & 0.64 & 0.73 & 1.2 & 1.1 \\
\hline Lead (AAS) & $<0.004$ & $<0.004$ & $<0.004$ & $<0.004$ & $<0.004$ & $<0.004$ & $<0.004$ & $<0.004$ \\
\hline Mercury (CVAA) & $<0.0002$ & $<0.0002$ & $<0.0002$ & $<0.0002$ & $<0.0002$ & $<0.0002$ & $<0.0002$ & $<0.0002$ \\
\hline Molybdenum & $<0.01$ & $<0.01$ & $<0.01$ & $<0.01$ & $<0.01$ & $<0.01$ & $<0.01$ & $<0.01$ \\
\hline Nickel & 0.029 & 0.037 & 0.026 & 0.026 & 0.033 & 0.033 & 0.022 & 0.023 \\
\hline Selenium & $<0.05$ & $<0.05$ & $<0.05$ & $<0.05$ & $<0.05$ & $<0.05$ & $<0.05$ & $<0.05$ \\
\hline silver & $<0.006$ & $<0.006$ & $<0.006$ & $<0.006$ & $<0.006$ & $<0.006$ & $<0.006$ & $<0.006$ \\
\hline strontium & 0.82 & 0.79 & 0.78 & 0.77 & 0.88 & 0.86 & 0.83 & 0.84 \\
\hline Thorium & $<0.2$ & $<0.2$ & $<0.2$ & $<0.2$ & $<0.2$ & $<0.2$ & $<0.2$ & $<0.2$ \\
\hline Uranium (Fluor) & 0.001 & 0.001 & 0.001 & 0.001 & 0.001 & 0.001 & 0.001 & 0.001 \\
\hline Vanadium & $<0.005$ & $<0.005$ & $<0.005$ & $<0.005$ & $<0.005$ & $<0.005$ & $<0.005$ & $<0.005$ \\
\hline zinc & 0.0054 & 0.0042 & 0.0025 & $<0.002$ & 0.009 & 0.024 & $<0.002$ & $<0.002$ \\
\hline MAJOR IONS $(\mathrm{mg} / \mathrm{L})$ & . & . & . &. & . & . & - & • \\
\hline Alkalinity-HCO3 & 334 & $\cdot$ & 348 & $\cdot$ & $344^{\circ}$ & $\cdot$ & 346 & • \\
\hline Alkalinity- $\mathrm{CO} 3$ & $<1$ &. & $<1$ &. & $<1$ & - & $<1$ & • \\
\hline Calcium & 140 & 140 & 130 & 130 & 140 & 140 & 140 & 140 \\
\hline Chloride & 73 & . & 87 & $\cdot$ & 93 & - & 86 & • \\
\hline Fluoride & $<0.1$ & $\cdot$ & $<0.1$ & $\cdot$ & $<0.1$ & . & $<0.1$ & - \\
\hline Magnesium & 26 & 26 & 25 & 25 & 27 & 26 & 27 & 27 \\
\hline Manganese & 0.057 & 0.056 & 0.035 & 0.038 & 0.035 & 0.036 & 0.035 & 0.035 \\
\hline Nitrate-N & 2.9 & . & 4.04 & $\cdot$ & 5.17 & - & 3.9 & • \\
\hline Potassium & 2.9 & 3.1 & 3.5 & 3.2 & 3.5 & 3.6 & 3.6 & 3.3 \\
\hline Sodium & 16 & 15 & 16 & 15 & 17 & 17 & 17 & 17 \\
\hline sulfate & 43.7 & $\cdot$ & 43.2 & $\cdot$ & 40 & - & 42 & • \\
\hline
\end{tabular}

(CONTINUED) 


\begin{tabular}{|c|c|c|c|c|c|c|c|c|}
\hline \multirow{4}{*}{$\begin{array}{l}\text { Sampling Point } \\
\text { Iocation } \\
\text { Date Sampled }\end{array}$} & \multicolumn{8}{|c|}{$G W-370$} \\
\hline & \multicolumn{8}{|c|}{ BG } \\
\hline & \multicolumn{2}{|c|}{$02 / 17 / 93$} & \multicolumn{2}{|c|}{$05 / 21 / 93$} & \multicolumn{2}{|c|}{$07 / 10 / 93$} & \multicolumn{2}{|c|}{$10 / 14 / 93$} \\
\hline & TOT & Drs & TOT & DIS & TOT & DIS & TOT & DIS \\
\hline METALS (mg/L) & $\cdot$ & $\cdot$ & $\cdot$ & . & . & . & . & . \\
\hline Aluminum & 0.61 & $<0.02$ & 6.5 & 0.038 & 0.69 & 0.06 & 1.6 & $<0.02$ \\
\hline Antimony & $<0.05$ & $<0.05$ & $<0.05$ & $<0.05$ & $<0.05$ & $<0.05$ & $<0.05$ & $<0.05$ \\
\hline Arsenic & $<0.05$ & $<0.05$ & $<0.05$ & $<0.05$ & $<0.05$ & $<0.05$ & $<0.05$ & $<0.05$ \\
\hline Barium & 0.068 & 0.058 & 0.15 & 0.045 & 0.064 & 0.051 & 0.081 & 0.045 \\
\hline Beryllium & 0.0012 & $<0.0003$ & 0.00061 & $<0.0003$ & $<0.0003$ & $<0.0003$ & $<0.0003$ & $<0.0003$ \\
\hline Boron & 0.015 & 0.014 & 0.049 & 0.025 & 0.01 & 0.0096 & 0.014 & 0.0071 \\
\hline Cadmium (AAS) & $<0.002$ & $<0.002$ & $<0.002$ & $<0.002$ & $<0.002$ & $<0.002$ & $<0.002$ & $<0.002$ \\
\hline Cadmium & $<0.003$ & $<0.003$ & $<0.003$ & $<0.003$ & $<0.003$ & $<0.003$ & $<0.003$ & $<0.003$ \\
\hline Chromium (AAS) & $<0.01$ & $<0.01$ & 0.012 & $<0.01$ & $<0.01$ & $<0.01$ & $<0.01$ & $<0.01$ \\
\hline Chromium & $<0.01$ & $<0.01$ & 0.012 & $<0.01$ & $<0.01$ & $<0.01$ & $<0.01$ & $<0.01$ \\
\hline Cobalt & $<0.005$ & $<0.005$ & $<0.005$ & $<0.005$ & $<0.005$ & $<0.005$ & 0.0056 & $<0.005$ \\
\hline Copper & 0.009 & 0.0068 & 0.022 & $<0.004$ & $<0.004$ & $<0.004$ & 0.0065 & $<0.004$ \\
\hline Iron & 0.48 & 0.02 & 6 & 0.01 & 0.52 & 0.043 & 1.4 & $<0.005$ \\
\hline Lead (AAS) & $<0.004$ & $<0.004$ & 0.014 & $<0.004$ & $<0.004$ & $<0.004$ & 0.0064 & $<0.004$ \\
\hline Mercury (CVAA) & $<0.0002$ & $<0.0002$ & $<0.0002$ & $<0.0002$ & $<0.0002$ & $<0.0002$ & $<0.0002$ & $<0.0002$ \\
\hline Mol ybdenum & $<0.01$ & $<0.01$ & $<0.01$ & $<0.01$ & $<0.01$ & $<0.01$ & $<0.01$ & $<0.01$ \\
\hline Nickel & $<0.01$ & $<0.01$ & $<0.01$ & 0.013 & $<0.01$ & $<0.01$ & $<0.01$ & $<0.01$ \\
\hline Selentum & $<0.05$ & $<0.05$ & $<0.05$ & $<0.05$ & $<0.05$ & $<0.05$ & $<0.05$ & $<0.05$ \\
\hline silver & $<0.006$ & $<0.006$ & $<0.006$ & $<0.006$ & $<0.006$ & $<0.006$ & $<0.006$ & $<0.006$ \\
\hline Strontium & 0.059 & 0.053 & 0.066 & 0.042 & 0.054 & 0.047 & 0.049 & 0.041 \\
\hline Thorium & $<0.2$ & $<0.2$ & $<0.2$ & $<0.2$ & $<0.2$ & $<0.2$ & $<0.2$ & $<0.2$ \\
\hline Uranium (Fluor) & 0.001 & $<0.001$ & 0.002 & $<0.001$ & 0.001 & 0.001 & 0.001 & $<0.001$ \\
\hline Vanadium & $<0.005$ & $<0.005$ & 0.0058 & $<0.005$ & $<0.005$ & $<0.005$ & $<0.005$ & $<0.005$ \\
\hline Zine & 0.018 & 0.014 & 0.029 & 0.0071 & 0.016 & 0.01 & 0.023 & 0.011 \\
\hline MAJOR IONS $(\mathrm{mg} / \mathrm{L})$ & • & • & . & • & • & • & • & • \\
\hline Alkalinity-HCO3 & 113 & • & $7 \dot{8}$ & . & $\dot{91}$ & . & $\dot{76}$ & . \\
\hline Alkalinity- $\mathrm{CO} 3$ & $<1$ & - & $<1$ & . & $<1$ & . & $<1$ & . \\
\hline Calcium & 36 & 36 & 29 & 22 & 29 & 26 & 24 & 24 \\
\hline Chloride & 1.3 & - & 1.4 & . & 1.4 & . & 1 & - \\
\hline Fluoride & 0.1 & - & 0.1 & - & 0.1 & - & 0.1 & - \\
\hline Magnesium & 3.4 & 3.3 & 4.1 & 2.4 & 3.1 & 2.8 & 2.8 & 2.5 \\
\hline Manganese & 0.012 & 0.0026 & 0.13 & 0.0058 & 0.012 & 0.0034 & 0.028 & 0.005 \\
\hline Nitrate-N & $<0.2$ & - & $<0.2$ & - & $<0.2$ & - & $<0.2$ & - \\
\hline Potassium & 0.83 & $<0.6$ & 1.9 & $<0.6$ & $<0.6$ & $<0.6$ & 0.63 & $<0.6$ \\
\hline Sodium & 10 & 10 & 11 & 9.9 & 10 & 10 & 9.9 & 9.8 \\
\hline sulfate & 11 & • & 11 & . & 12.5 & - & 10 & - \\
\hline
\end{tabular}


APPBNDIX $\mathbf{2} .1$

Groundwater and surfave-Water Quality Data, 1993

\begin{tabular}{|c|c|c|c|c|c|c|c|c|}
\hline \multirow{4}{*}{$\begin{array}{l}\text { 8ampling Point } \\
\text { Location } \\
\text { Date sampled }\end{array}$} & \multicolumn{8}{|c|}{$0 W-371$} \\
\hline & \multicolumn{8}{|c|}{ BO } \\
\hline & \multicolumn{2}{|c|}{$02 / 16 / 93$} & \multicolumn{2}{|c|}{$05 / 21 / 93$} & \multicolumn{2}{|c|}{$07 / 09 / 93$} & \multicolumn{2}{|c|}{$10 / 13 / 93$} \\
\hline & TOT & Drs & TOT & Drs & TOT & DIs & TOT & DIs \\
\hline METALS (mg/I) & $\cdot$ &. & - & $\cdot$ & $\cdot$ & $\cdot$ & $\cdot$ & . \\
\hline Aluminum & 0.023 & $<0.02$ & 0.067 & 0.079 & $<0.02$ & $<0.02$ & $<0.02$ & $<0.02$ \\
\hline Antimony & $<0.05$ & $<0.05$ & $<0.05$ & $<0.05$ & $<0.05$ & $<0.05$ & $<0.05$ & $<0.05$ \\
\hline Arsenic & $<0.05$ & $<0.05$ & $<0.05$ & $<0.05$ & $<0.05$ & $<0.05$ & $<0.05$ & $<0.05$ \\
\hline Barium & 0.13 & 0.13 & 0.15 & 0.15 & 0.13 & 0.14 & 0.14 & 0.23 \\
\hline Beryllium & $<0.0003$ & $<0.0003$ & $<0.0003$ & $<0.0003$ & $<0.0003$ & $<0.0003$ & $<0.0003$ & $<0.0003$ \\
\hline Boron & 0.044 & 0.047 & 0.059 & 0.067 & 0.038 & 0.046 & 0.042 & 0.039 \\
\hline Cadmium (AMS) & $<0.002$ & $<0.002$ & $<0.002$ & $<0.002$ & $<0.002$ & $<0.002$ & $<0.002$ & $<0.002$ \\
\hline Cadmium & $<0.003$ & $<0.003$ & $<0.003$ & $<0.003$ & $<0.003$ & $<0,003$ & $<0.003$ & $<0.003$ \\
\hline Chromium (AAS) & $<0.01$ & $<0.01$ & $<0.01$ & $<0.01$ & $<0.01$ & $<0.01$ & $<0.01$ & $<0.01$ \\
\hline Chromium & $<0.01$ & $<0.01$ & $<0.01$ & $<0.01$ & $<0.01$ & $<0.01$ & $<0.01$ & $<0.01$ \\
\hline Cobalt & $<0.005$ & $<0.005$ & $<0.005$ & $<0.005$ & $<0.005$ & $<0.005$ & $<0.005$ & $<0.005$ \\
\hline Copper & 0.0053 & $<0.004$ & $<0.004$ & $<0.004$ & $<0.004$ & $<0.004$ & $<0.004$ & $<0.004$ \\
\hline Iron & 0.52 & 0.32 & 2.7 & 0.39 & 4.9 & 0.4 & 1.2 & 0.097 \\
\hline Lead (AAS) & $<0.004$ & $<0.004$ & $<0.004$ & $<0.004$ & $<0.004$ & $<0.004$ & $<0.004$ & $<0.004$ \\
\hline Mercury (CVAA) & $<0.0002$ & $<0.0002$ & $<0.0002$ & $<0.0002$ & $<0.0002$ & 0.00026 & $<0.0002$ & $<0.0002$ \\
\hline Mol ybdenum & $<0.01$ & $<0.01$ & $<0.01$ & $<0.01$ & $<0.01$ & $<0.01$ & $<0.01$ & $<0.01$ \\
\hline Nickel & $<0.01$ & $<0.01$ & $<0.01$ & $<0.01$ & $<0.01$ & $<0.01$ & $<0.01$ & $<0.01$ \\
\hline Belentum & $<0.05$ & $<0.05$ & $<0.05$ & $<0.05$ & $<0.05$ & $<0.05$ & $<0.05$ & $<0.03$ \\
\hline sliver & $<0.006$ & $<0.006$ & $<0.006$ & 0.0077 & $<0.006$ & $<0.006$ & $<0.006$ & $<0.006$ \\
\hline strontium & 1.4 & 1.4 & 1.6 & 1.5 & 1.5 & 1.5 & 1.4 & 1.4 \\
\hline Thorium & $<0.2$ & $<0.2$ & $<0.2$ & $<0.2$ & $<0.2$ & $<0.2$ & $<0.2$ & $<0.2$ \\
\hline Uranium (Fluor) & $<0.001$ & $<0.001$ & $<0.001$ & $<0.001$ & $<0.001$ & $<0.001$ & 0.001 & $<0.001$ \\
\hline Vanadium & $<0.005$ & $<0.005$ & $<0.005$ & $<0.005$ & $<0.005$ & $<0.005$ & $<0.005$ & $<0.005$ \\
\hline zinc & 0.0029 & 0.0026 & 0.0045 & 0.0054 & 0.017 & 0.004 & $<0.002$ & 0.0026 \\
\hline MAJOR IONS $(\mathrm{mg} / \mathrm{L})$ & - & $\cdot$ &. &. & $\cdot$ & $\cdot$ & $\cdot$ & - \\
\hline Alkalinity-HCO3 & 291 & $\cdot$ & $29 \dot{7}$ & $\cdot$ & 304 & $\cdot$ & $2 \dot{9}$ & $\bullet$ \\
\hline Alkalinity-CO3 & $<1$ & $\cdot$ & $<1$ & $\cdot$ & $<1$ & • & $<1$ & 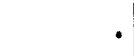 \\
\hline Calcium & 71 & 71 & 82 & 81 & 75 & 75 & 75 & 74 \\
\hline Chloride & 1.2 & $\cdot$ & 1.4 & $\cdot$ & 1.2 & . & 1 & . \\
\hline Fluoride & $<0.1$ & . & $<0.1$ & . & 0.1 & . & $<0.1$ & . \\
\hline Magnesium & 19 & 19 & 21 & 21 & 20 & 20 & 20 & 20 \\
\hline Manganese & 0.022 & 0.022 & 0.027 & 0.023 & 0.025 & 0.023 & 0.025 & 0.027 \\
\hline Nitrate-N & $<0.2$ & . & $<0.2$ & $\cdot$ & $<0.2$ & . & $<0.2$ & . \\
\hline Potassium & 3.5 & 3.1 & 3.6 & 3.4 & 3.5 & 3 & 2.9 & 3.6 \\
\hline Sodium & 17 & 17 & 18 & 18 & 17 & 18 & 17 & 17 \\
\hline sulfate & 15.6 & . & 18 & $\cdot$ & 17 & . & 17 & • \\
\hline
\end{tabular}

(CONTINUED) 
APPENDIX $\mathbf{E} .1$

Groundwater and surface-water Quality Data, 1993

\begin{tabular}{|c|c|c|c|c|c|c|c|c|}
\hline \multirow{4}{*}{$\begin{array}{l}\text { sampling Point } \\
\text { Location } \\
\text { Date sampled }\end{array}$} & \multicolumn{8}{|c|}{ ow-372 } \\
\hline & \multicolumn{8}{|c|}{ BG } \\
\hline & \multicolumn{2}{|c|}{$02 / 09 / 93$} & \multicolumn{2}{|c|}{$05 / 19 / 93$} & \multicolumn{2}{|c|}{$07 / 02 / 93$} & \multicolumn{2}{|c|}{$10 / 12 / 93$} \\
\hline & TOT & DIS & TOT & DIs & TOT & Drs & TOT & Drs \\
\hline Martals (mg/L) & $\cdot$ & $\cdot$ & $\cdot$ & - & - & $\cdot$ & . & $\cdot$ \\
\hline Aluminum & 0.2 & $<0.02$ & 0.094 & 0.053 & 0.19 & $<0.02$ & 0.22 & $<0.02$ \\
\hline Antimony & $<0.05$ & $<0.05$ & $<0.05$ & $<0.05$ & $<0.05$ & $<0.05$ & $<0.05$ & $<0.05$ \\
\hline Arsenic & $<0.05$ & $<0.05$ & 0.062 & $<0.05$ & $<0.05$ & $<0.05$ & $<0.05$ & $<0.05$ \\
\hline Barium & 0.067 & 0.064 & 0.069 & 0.065 & 0.07 & 0.067 & 0.076 & 0.07 \\
\hline Beryl11um & $<0.0003$ & $<0.0003$ & $<0.0003$ & $<0.0003$ & $<0.0003$ & $<0.0003$ & $<0.0003$ & $<0.0003$ \\
\hline Boron & 0.01 & 0.012 & 0.021 & 0.028 & 0.015 & 0.022 & 0.0089 & 0.013 \\
\hline Cadmium (MAS) & $<0.002$ & $<0.002$ & $<0.002$ & $<0.002$ & $<0.002$ & $<0.002$ & $<0.002$ & $<0.002$ \\
\hline Cadmium & $<0.003$ & $<0.003$ & $<0.003$ & $<0.003$ & $<0.003$ & $<0.003$ & $<0.003$ & $<0.003$ \\
\hline Chromium (AAS) & $<0.01$ & $<0.01$ & $<0.01$ & $<0.01$ & $<0.01$ & $<0.01$ & $<0.01$ & $<0.01$ \\
\hline Chromium & $<0.01$ & $<0.01$ & $<0.01$ & $<0.01$ & $<0.01$ & $<0.01$ & $<0.01$ & $<0.01$ \\
\hline Cobalt & $<0.005$ & $<0.005$ & $<0.005$ & $<0.005$ & $<0.005$ & $<0.005$ & $<0.005$ & $<0.005$ \\
\hline Copper & 0.0042 & $<0.004$ & $<0.004$ & $<0.004$ & $<0.004$ & $<0.004$ & 0.0081 & $<0.004$ \\
\hline Iron & 0.36 & 0.065 & 0.22 & $<0.005$ & 0.42 & 0.044 & 0.53 & 0.042 \\
\hline Lead (AAS) & $<0.004$ & $<0.004$ & $<0.004$ & $<0.004$ & $<0.004$ & $<0.004$ & $<0.004$ & $<0.004$ \\
\hline Mercury (CVAA) & $<0.0002$ & $<0.0002$ & $<0.0002$ & $<0.0002$ & $<0.0002$ & $<0.0002$ & $<0.0002$ & $<0.0002$ \\
\hline Holybdenum & $<0.01$ & $<0.01$ & $<0.01$ & $<0.01$ & $<0.01$ & $<0.01$ & $<0.01$ & $<0.01$ \\
\hline Nickel & $<0.01$ & $<0.01$ & $<0.01$ & $<0.01$ & $<0.01$ & $<0.01$ & $<0.01$ & $<0.01$ \\
\hline Selenium & $<0.05$ & $<0.05$ & $<0.05$ & $<0.05$ & $<0.05$ & $<0.05$ & $<0.05$ & $<0.05$ \\
\hline Silver & $<0.006$ & $<0.006$ & $<0.006$ & $<0.006$ & $<0.006$ & $<0.006$ & $<0.006$ & $<0.006$ \\
\hline strontium & 0.15 & 0.15 & 0.17 & 0.17 & 0.18 & 0.17 & 0.17 & 0.17 \\
\hline Thorium & $<0.2$ & $<0.2$ & $<0.2$ & $<0.2$ & $<0.2$ & $<0.2$ & $<0.2$ & $<0.2$ \\
\hline Uranium (Fluor) & $<0.001$ & $<0.001$ & $<0.001$ & $<0.001$ & 0.001 & 0.001 & $<0.001$ & 0.001 \\
\hline Vanadium & $<0.005$ & $<0.005$ & $<0.005$ & $<0.005$ & $<0.005$ & $<0.005$ & $<0.005$ & $<0.005$ \\
\hline zinc & 0.0057 & 0.0057 & 0.011 & 0.01 & 0.0075 & 0.0071 & 1.6 & 0.0064 \\
\hline MAJOR IONS $(\mathrm{mg} / \mathrm{L})$ &. & $\cdot$ &. & $\cdot$ & $\dot{.}$ & $\cdot$ & $\cdot$ & $\bullet$ \\
\hline Alkalinity-HCO3 & 184 & $\cdot$ & 180 & $\cdot$ & 182 & $\cdot$ & 184 & • \\
\hline Alkalinity-CO3 & $<1$ & . & $<1$ &. & $<1$ & . & $<1$ & $\theta^{\prime}$ \\
\hline Calcium & 60 & 59 & 59 & 57 & 59 & 58 & 63 & 62 \\
\hline Chloride & 2.7 & $\cdot$ & 2.1 & $\cdot$ & 1.9 &. & 2 & • \\
\hline Fluoride & 0.2 & $\cdot$ & 0.1 &. & 0.2 & $\cdot$ & 0.2 & $\bullet^{\circ}$ \\
\hline Magnesium & 11 & 11 & 11 & 11 & 11 & 11 & 12 & 11 \\
\hline Manganese & 0.039 & 0.028 & 0.019 & 0.012 & 0.023 & 0.019 & 0.065 & 0.026 \\
\hline Nitrate-N & $<0.2$ & $\cdot$ & $<0.2$ & $\cdot$ & $<0.2$ & $\cdot$ & $<0.2$ & $\bullet$ \\
\hline Potassium & 2 & 1.6 & 1.6 & 1.6 & 1.9 & 1.6 & 1.8 & 1.6 \\
\hline Sodium & 8.7 & 8.4 & 8.8 & 8.6 & 9.6 & 9.3 & 9.2 & 9.2 \\
\hline Sulfate & 33 & . & 31 & $\cdot$ & 31 & . & 31 & $\cdot$ \\
\hline
\end{tabular}

(CONTINUED) 
ApPINDIX $\mathbf{2 . 1}$

Oroundwater and surface-Water Quallty Data, 1993

\begin{tabular}{|c|c|c|c|c|c|c|c|c|}
\hline \multirow{4}{*}{$\begin{array}{l}\text { sampling polnt } \\
\text { Looution } \\
\text { Date sampled }\end{array}$} & \multicolumn{8}{|c|}{$0 w-373$} \\
\hline & \multicolumn{8}{|c|}{ Ba } \\
\hline & \multicolumn{2}{|c|}{$02 / 11 / 93$} & \multicolumn{2}{|c|}{$05 / 14 / 93$} & \multicolumn{2}{|c|}{$07 / 02 / 93$} & \multicolumn{2}{|c|}{$10 / 11 / 93$} \\
\hline & Tor & Drs & 20r & DIs & TOT & DIs & xor & D18 \\
\hline Murals (mg/h) & $\cdot$ & - & $\cdot$ & $\cdot$ & $\cdot$ & $\cdot$ & . & 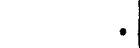 \\
\hline Aluminum & $<0.02$ & $<0.02$ & 0.051 & 0.022 & 0.84 & $<0.02$ & 0.82 & $<0.02$ \\
\hline Antimony & $<0.05$ & $<0.05$ & $<0.05$ & $<0.05$ & $<0.05$ & $<0.03$ & $<0.05$ & $<0.05$ \\
\hline Araenic & $<0.05$ & $<0.05$ & $<0.05$ & $<0.05$ & $<0.05$ & $<0.05$ & $<0.05$ & $<0.05$ \\
\hline Barlum & 0.051 & 0.042 & 0.048 & 0.026 & 0.061 & 0.036 & 0.059 & 0.039 \\
\hline Bery111um & $<0.0003$ & $<0,0003$ & $<0.0003$ & $<0,0003$ & $<0.0003$ & $<0.0003$ & $<0.0003$ & $<0.0003$ \\
\hline Boron & 0.16 & 0.16 & 0.18 & 0.15 & 0.15 & 0.17 & 0.16 & 0.15 \\
\hline Codmlum (MAs) & $<0.002$ & $<0.002$ & $<0.002$ & $<0.002$ & $<0.002$ & $<0.002$ & $<0.002$ & $<0.002$ \\
\hline Cadmi un & $<0.003$ & $<0.003$ & $<0.003$ & $<0.003$ & $<0.003$ & $<0.003$ & $<0.003$ & $<0.003$ \\
\hline Chromium (MAS) & $<0.01$ & $<0.01$ & $<0.01$ & $<0.01$ & $<0.01$ & $<0.01$ & $<0.01$ & $<0.01$ \\
\hline Chromlum & $<0.01$ & $<0.01$ & $<0.01$ & $<0.01$ & $<0.01$ & $<0.01$ & 0.01 & $<0.01$ \\
\hline Cobalt & $<0.005$ & $<0.005$ & $<0.005$ & $<0.005$ & $<0.005$ & $<0.005$ & $<0.005$ & $<0.005$ \\
\hline copper & $<0.004$ & $<0.004$ & $<0.004$ & $<0.004$ & 0.0069 & $<0.004$ & 0.013 & $<0.004$ \\
\hline Iron & 0.62 & 0.015 & 0.17 & $<0.005$ & 2.3 & 0.042 & 3.2 & 0.031 \\
\hline Lead (MAS) & $<0.004$ & $<0.004$ & $<0.004$ & $<0.004$ & 0.0099 & $<0.004$ & $<0.004$ & $<0.004$ \\
\hline mercury (CVMA) & $<0.0002$ & $<0.0002$ & $<0.0002$ & $<0.0002$ & 0.00022 & 0.0002 & $<0.0002$ & $<0.0002$ \\
\hline Nol ybdenum & $<0.01$ & $<0.01$ & $<0.01$ & $<0.01$ & $<0.01$ & $<0.01$ & $<0.01$ & $<0.01$ \\
\hline nickel & $<0.01$ & $<0.01$ & 0.023 & $<0.01$ & $<0.01$ & $<0.01$ & $<0.01$ & $<0.01$ \\
\hline selenium & $<0.05$ & $<0.05$ & $<0.05$ & $<0.05$ & $<0.05$ & $<0.05$ & $<0.05$ & $<0.05$ \\
\hline 81lvar & $<0.006$ & $<0.006$ & $<0.006$ & $<0.006$ & $<0.006$ & $<0.006$ & $<0.006$ & $<0.006$ \\
\hline Strontium & 0.059 & 0.056 & 0.062 & 0.037 & 0.064 & 0.059 & 0.061 & 0.056 \\
\hline Thorium & $<0.2$ & $<0.2$ & $<0.2$ & $<0.2$ & $<0.2$ & $<0.2$ & $<0.2$ & $<0.2$ \\
\hline Uranium (rluor) & $<0.001$ & $<0.001$ & $<0.001$ & $<0.001$ & $<0.001$ & 0.001 & $<0.001$ & $<0.001$ \\
\hline Vanadiun & $<0.005$ & $<0.005$ & $<0.005$ & $<0.005$ & $<0.005$ & $<0.005$ & $<0.00 \mathrm{~s}$ & $<0.005$ \\
\hline zinc & 0.0024 & $<0.002$ & 0.047 & 0.0059 & 0.01 & 0.0053 & 0.0058 & $<0.002$ \\
\hline MUOR IONS (mg/L) & . &. & - & . & • & $\cdot$ & . & . \\
\hline AlkallnLtky-HCO3 & $17 \dot{8}$ &. & 186 &. & $18 \dot{8}$ &. & 183 & . \\
\hline Alkalinity-CO3 & 26 &. & 24 &. & 26 &. & $<1$ & - \\
\hline Calcium & 1.4 & 1.3 & 1.3 & 1.1 & 1.5 & 1.1 & 1.4 & 1.1 \\
\hline Chloride & 2.2 &. & 1.5 & . & 1.2 & $\cdot$ & 1 & - \\
\hline Pluoride & 1.5 & .1 & 1.4 &. & 1.7 & $\cdot$ & 1.5 & . \\
\hline Magnenium & 0.14 & 0.13 & 0.14 & 0.45 & 0.38 & 0.11 & 0.38 & 0.11 \\
\hline Manganese & 0.0077 & 0.0024 & 0.0042 & 0.031 & 0.022 & 0.002 & 0.025 & 0.0025 \\
\hline Nitrate $-N$ & $<0.2$ &. & $<0.2$ &. & $<0, ?$ &. & $<0.2$ & - \\
\hline Potansium & 0.79 & 1.2 & 0.72 & 0.8 & 1.4 & 1.4 & 1.1 & 0.99 \\
\hline soditum & 100 & 100 & 110 & 110 & 110 & 110 & 110 & 210 \\
\hline sulfate & 30 & & 29 & $\cdot$ & 31 & $\cdot$ & 27 & • \\
\hline
\end{tabular}

(CONTINUED) 
APPUMDIX $\$ .1$

Oroundwater and surface-water Quallty Data, 1993

\begin{tabular}{|c|c|c|c|c|c|c|c|c|}
\hline \multirow{4}{*}{$\begin{array}{l}\text { Bampling polnt } \\
\text { Looution } \\
\text { Date sampled }\end{array}$} & \multicolumn{8}{|c|}{$a n-326$} \\
\hline & \multicolumn{8}{|c|}{83} \\
\hline & \multicolumn{2}{|c|}{$02 / 03 / 93$} & \multicolumn{2}{|c|}{$06 / 03 / 93$} & \multicolumn{2}{|c|}{$09 / 14 / 93$} & \multicolumn{2}{|c|}{$10 / 26 / 93$} \\
\hline & $\operatorname{son}$ & DIs & TOT & Drs & 20T & DIs & ror & Drs \\
\hline nurnes (mg/L) & - & $\cdot$ & $\cdot$ & . & $\cdot$ & $\cdot$ & $\cdot$ & - \\
\hline Aluminum & $<0.2$ & $<0.2$ & $<0.2$ & $<0.2$ & $<0.04$ & $<0.04$ & 0.3 & $<0.02$ \\
\hline Ant imony & $<0.5$ & $<0.5$ & $<0.3$ & $<0.5$ & $<0.1$ & $<0.1$ & $<0.03$ & $<0.08$ \\
\hline Areenlo & $<0.5$ & $<0.5$ & $<0.5$ & $<0.5$ & $<0.1$ & $<0.1$ & $<0.05$ & $<0.05$ \\
\hline Barlum & 11 & 10 & 11 & 10 & 11 & 11 & 0.35 & 0.52 \\
\hline Bery111um & $<0.003$ & $<0.003$ & $<0.003$ & $<0.003$ & $<0.0006$ & $<0.0006$ & $<0.0003$ & $<0,0003$ \\
\hline Boron & 0.18 & 0.22 & 0.19 & 0.18 & 0.21 & 0.2 & 0.018 & 0.011 \\
\hline Cadnlum (MAs) & $<0.002$ & $<0.002$ & $<0.002$ & $<0.002$ & $<0.002$ & $<0.002$ & $<0.002$ & $<0.002$ \\
\hline Cadmium & $<0.03$ & $<0.03$ & $<0.03$ & $<0.03$ & $<0.006$ & $<0.006$ & $<0.003$ & $<0.003$ \\
\hline Chromilum (MB) & $<0.01$ & $<0.01$ & $<0.01$ & $<0.01$ & $<0.01$ & $<0.01$ & $<0.01$ & $<0.01$ \\
\hline Chromiun & $<0.1$ & $<0.1$ & $<0.1$ & $<0.1$ & $<0.02$ & $<0.02$ & $<0.01$ & $<0.01$ \\
\hline Cobalt & $<0.05$ & $<0.05$ & $<0.05$ & $<0.05$ & $<0.01$ & $<0.01$ & $<0.005$ & $<0.00 s$ \\
\hline Copper & $<0.04$ & $<0.04$ & $<0.04$ & $<0.04$ & $<0.008$ & $<0.008$ & $<0.004$ & $<0.004$ \\
\hline Iron & 4.1 & $<0.05$ & 2.1 & $<0.05$ & 7 & 0.24 & 0.46 & 0.0067 \\
\hline Land (MAS) & $<0.004$ & $<0.004$ & $<0.004$ & $<0.004$ & $<0.008$ & $<0.008$ & $<0.004$ & $<0.004$ \\
\hline Maroury (CVM) & $<0.0002$ & $<0.0002$ & $<0.0002$ & $<0.0002$ & $<0.0002$ & $<0.0002$ & $<0.0002$ & $<0.0002$ \\
\hline nol ybodenum & $<0.1$ & $<0.1$ & $<0.1$ & $<0.1$ & $<0.02$ & $<0.02$ & $<0.01$ & $<0.01$ \\
\hline nlekel & $<0.1$ & $<0.1$ & $<0.1$ & $<0.1$ & 0.025 & $<0.02$ & 0.013 & 0.015 \\
\hline selenium & $<0.5$ & $<0.5$ & $<0.5$ & $<0.5$ & $<0.1$ & $<0.1$ & $<0.05$ & $<0.05$ \\
\hline 811ver & $<0.06$ & $<0.06$ & $<0.06$ & $<0.06$ & $<0.012$ & $<0.012$ & $<0.006$ & $<0.006$ \\
\hline strontium & 13 & 12 & 13 & 13 & 14 & 14 & 0.24 & 0.35 \\
\hline Thorium & $<2$ & $<2$ & $<2$ & $<2$ & $<0.4$ & $<0.4$ & $<0.2$ & $<0.2$ \\
\hline Uranium (Pluor) & $<0.001$ & $<0.001$ & $<0.001$ & $<0.001$ & 0.001 & 0.001 & $<0.001$ & 0.001 \\
\hline Vanadium & $<0.05$ & $<0.05$ & $<0.05$ & $<0.05$ & $<0.01$ & $<0.01$ & $<0.005$ & $<0.005$ \\
\hline Iine & 0.025 & $<0.02$ & 0.097 & 0.062 & 0.026 & 0.01 & 0.018 & 3.2 \\
\hline MAJOR IONS (mg/h) & . & • & - & - & - & - &. & • \\
\hline Alkalinity-HCO3 & is & . & 49 & • & $\dot{43}$ & . & ${ }_{48}^{\circ}$ & • \\
\hline Alkalindty-Co3 & $<1$ & - & 2 & . & $<1$ & - & $<1$ & • \\
\hline Calcium & 110 & 110 & 110 & 100 & 110 & 120 & 70 & 100 \\
\hline Chloride & 19 & . & 5.5 & . & 21 & - & 19 & - \\
\hline Fluoride & $<0.1$ & . & $<0.1$ & - & $<0.1$ & . & $<0.1$ & - \\
\hline Magnes Ium & 48 & 47 & 41 & 39 & 44 & 46 & 10 & 14 \\
\hline Manganese & 0.039 & 0.03 & 0.032 & 0.021 & 0.068 & 0.049 & 0.098 & 0.14 \\
\hline Nitrate-n & 826 & . & 1006 & - & 846 & - & 1171 & . \\
\hline Potass Ium & 14 & 23 & 14 & 13 & 16 & 17 & 3.8 & 4 \\
\hline sodium & 1400 & 1300 & 1500 & 1400 & 1500 & 1500 & 9.8 & 12 \\
\hline sulfate & $<10$ & . & 4.3 & . & 11 & - & 7 & . \\
\hline
\end{tabular}

(CONTINUED) 
ApPIMDIX 1.1

Croundwater and surtaoe-water guality bete, 1993

\begin{tabular}{|c|c|c|c|c|c|c|c|c|}
\hline \multirow{4}{*}{$\begin{array}{l}\text { sampling polnt } \\
\text { Loeation } \\
\text { Date sumpled }\end{array}$} & \multicolumn{8}{|c|}{$9 N-537$} \\
\hline & \multicolumn{8}{|c|}{ OLP } \\
\hline & \multicolumn{2}{|c|}{$02 / 11 / 93$} & \multicolumn{2}{|c|}{$05 / 12 / 93$} & \multicolumn{2}{|c|}{$09 / 14 / 93$} & \multicolumn{2}{|c|}{$10 / 22 / 93$} \\
\hline & $\operatorname{xos}$ & Drs & nor & D18 & Tor & DIe & ror & DIs \\
\hline nernes (mg/L) &. & $\cdot$ & . & $\cdot$ & $\cdot$ & $\cdot 1$ & $\cdot$ & \\
\hline Aluminum & $<0.2$ & $<0.2$ & 0.28 & $<0.2$ & 0.15 & $<0, i$ & 0.11 & $0.1 i$ \\
\hline Antimony & $<0.3$ & $<0.5$ & $<0.5$ & $<0,3$ & $<0.25$ & $<0.25$ & $<0.25$ & $<0.25$ \\
\hline Arsente & $<0.5$ & $<0,5$ & $<0.3$ & $<0.5$ & $<0.25$ & $<0.25$ & $<0.25$ & $<0.25$ \\
\hline Barlun & 2.3 & 2.2 & 2.5 & 2.5 & 2.3 & 2.3 & 2.4 & 2.3 \\
\hline Boryl11un & $<0.003$ & $<0.003$ & $<0.003$ & $<0.003$ & $<0.0015$ & $<0.0015$ & $<0.001 \mathrm{~s}$ & $<0.0015$ \\
\hline Boron & 0.14 & $<0.04$ & $<0.04$ & $<0.04$ & $<0.02$ & $<0.02$ & $<0.02$ & $<0.02$ \\
\hline cadniun (MS) & $<0.002$ & $<0.002$ & $<0.002$ & $<0.002$ & 40.002 & $<0.002$ & $<0.002$ & $<0.002$ \\
\hline Cadnlum & $<0.03$ & $<0.03$ & $<0.03$ & $<0.03$ & $<0.015$ & $<0.015$ & $<0.02 s$ & $<0.015$ \\
\hline Chroaiun (MAs) & $<0.01$ & $<0.01$ & $<0.01$ & $<0.01$ & $<0.01$ & $<0.01$ & $<0.01$ & $<0.01$ \\
\hline chromium & 0.15 & $<0.1$ & $<0.1$ & $<0.1$ & $<0.05$ & $<0.05$ & $<0.05$ & $<0.05$ \\
\hline Cobalt & $<0.05$ & $<0.05$ & $<0.05$ & $<0.05$ & $<0.025$ & $<0.025$ & $<0.025$ & $<0.025$ \\
\hline Copper & $<0.04$ & $<0.04$ & $<0.04$ & $<0.04$ & 0.023 & $<0.02$ & $<0.02$ & $<0.02$ \\
\hline Iron & $<0.05$ & $<0.05$ & $<0.05$ & $<0.05$ & 0.05 & $<0.025$ & 0.086 & $<0.025$ \\
\hline Lead (MAs) & $<0.004$ & $<0.004$ & $<0.004$ & $<0.004$ & $<0.004$ & $<0.004$ & $<0.004$ & $<0.004$ \\
\hline Mercury (CVMA) & $<0.0002$ & $<0.0002$ & $<0.0002$ & $<0.0002$ & $<0.0002$ & $<0.0002$ & $<0.0002$ & $<0.0002$ \\
\hline nolybdenum & $<0.1$ & $<0.1$ & $<0.1$ & $<0.1$ & $<0.05$ & $<0.05$ & $<0.05$ & $<0.05$ \\
\hline nickel & $<0.1$ & $<0.1$ & $<0.1$ & $<0.1$ & $<0.05$ & $<0.05$ & $<0.05$ & $<0.05$ \\
\hline selenium & $<0.5$ & $<0.5$ & $<0.5$ & $<0.5$ & $<0.25$ & 0.26 & $<0.25$ & $<0.25$ \\
\hline 8liver & $<0.06$ & $<0.06$ & $<0.06$ & $<0.06$ & $<0.03$ & $<0.03$ & $<0.03$ & $<0.03$ \\
\hline strontium & 2.8 & 2.5 & 3 & 3 & 2.6 & 2.6 & 2.7 & 2.7 \\
\hline Thortum & $<2$ & $<2$ & $<2$ & $<2$ & $<1$ & $<1$ & $<1$ & $<1$ \\
\hline Urantum (sluor) & 0.002 & 0.001 & 0.001 & 0.001 & 0.001 & $\cdot$ & 0.001 & 0.001 \\
\hline Vanadium & $<0.05$ & $<0.05$ & $<0.05$ & $<0.05$ & $<0.025$ & $<0.025$ & $<0.025$ & $<0.025$ \\
\hline inc & 0.034 & 0.031 & $<0.02$ & 0.023 & 0.028 & 0.028 & $<0.01$ & $<0.01$ \\
\hline MWOR IONS (mg/L) & $\cdot$ & $\cdot$ & $\cdot$ & $\cdot \cdot$ & $\cdot$ & $\cdot$ & $\cdot$ & $\cdot$ \\
\hline Alkalinlty-HCO3 & 246 & $\cdot$. & 247 &. & 252 &. & $25 \dot{2}$ & • \\
\hline Alkalinity-CO3 & $<1$ &. & $<1$ &. & $<1$ &. & $<1$ & . \\
\hline Carcium & 1100 & 1000 & 1100 & 1100 & 1100 & 1100 & 1200 & 1100 \\
\hline Chloride & 30 &. & 32 & . & 26 & $\cdot$ & 33 & • \\
\hline Pluortde & $<0.1$ &. & $<0.1$ & . & $<0.1$ &. & $<0.1$ & . \\
\hline Magnesi um & 72 & 66 & 74 & 73 & 72 & 72 & 77 & 74 \\
\hline Manganese & $<0.01$ & $<0.01$ & $<0.01$ & $<0.01$ & 0.0086 & 0.0076 & $<0.005$ & $<0.005$ \\
\hline uitrate $-N$ & 782 & . & 692 & . & 525 &. & 791.6 & . \\
\hline Potasaium & $<6$ & $<6$ & $<6$ & $<6$ & 3.9 & 3.3 & 3.1 & 4.2 \\
\hline sodium & 29 & 27 & 31 & 30 & 29 & 29 & 31 & 30 \\
\hline Sulfate & $<10$ & $\cdot$ & 2.6 & - & $<10$ & $\cdot$ & 3 & • \\
\hline
\end{tabular}

(CONTINUED) 
APPUNDIX 1.1

Oroundwater and surface-water Quallty bata, 1993

\begin{tabular}{|c|c|c|c|c|c|c|c|c|}
\hline \multirow{4}{*}{$\begin{array}{l}\text { eappilng rolnt } \\
\text { Lootion } \\
\text { Date sampled }\end{array}$} & \multicolumn{8}{|c|}{$a w-601$} \\
\hline & \multicolumn{8}{|c|}{ orf } \\
\hline & \multicolumn{2}{|c|}{$03 / 28 / 93$} & \multicolumn{2}{|c|}{$06 / 27 / 93$} & \multicolumn{2}{|c|}{$09 / 19 / 93$} & \multicolumn{2}{|c|}{$12 / 21 / 94$} \\
\hline & $\operatorname{sor}$ & Drs & $50 \pi$ & Drs & 202 & DIs & xor & D18 \\
\hline Mernes $(\mathrm{mg} / \mathrm{L})$ & - & $\cdot$ & $\cdot$ & $\cdot$ & $\cdot$ & - & - & - \\
\hline Aluninus & $<0.02$ & $<0.02$ & $<0.02$ & $<0.02$ & 0.033 & 0.026 & $<0.02$ & $<0.02$ \\
\hline Ant lmony & $<0.03$ & $<0.05$ & $<0.05$ & $<0.05$ & $<0.05$ & $<0.05$ & $<0.05$ & $<0.05$ \\
\hline Areente & $<0.03$ & $<0.05$ & $<0.08$ & $<0.05$ & $<0.05$ & $<0.05$ & $<0.0 s$ & $<0.05$ \\
\hline Dartum & 0.078 & 0.077 & 0.086 & 0.085 & 0.094 & 0.096 & 0.088 & 0.088 \\
\hline Earyl11un & $<0.0003$ & $<0.0003$ & $<0.0003$ & $<0.0003$ & $<0.0003$ & $<0.0003$ & $<0.0003$ & $<0.0003$ \\
\hline Boron & 0.089 & 0.084 & 0.08 & 0.083 & 0.12 & 0.13 & 0.11 & 0.1 \\
\hline Cadmiun (MS) & $<0.002$ & $<0.002$ & $<0.002$ & $<0.002$ & $<0.002$ & $<0.002$ & $<0.002$ & $<0.002$ \\
\hline Cadnium & $<0.003$ & $<0.003$ & $<0.003$ & $<0.003$ & 0.003 & $<0,003$ & $<0.003$ & $<0.003$ \\
\hline Chromium (MAs) & $<0.01$ & $<0.01$ & $<0.01$ & $<0.01$ & $<0.01$ & $<0.01$ & $<0.01$ & $<0.01$ \\
\hline Chronlun & $<0.01$ & $<0.01$ & $<0.01$ & $<0.01$ & $<0.01$ & $<0.01$ & $<0.01$ & $<0.01$ \\
\hline Cobalt & $<0.005$ & $<0.005$ & $<0.005$ & $<0.003$ & $<0.005$ & $<0.005$ & $<0.00 s$ & $<0.003$ \\
\hline Copper & $<0.004$ & $<0.004$ & 0.0058 & $<0.004$ & $<0.004$ & $<0.004$ & $<0.004$ & $<0.004$ \\
\hline Iron & 0.12 & $<0.003$ & 0.31 & $<0.005$ & 0.2 & 0.015 & 0.057 & $<0.00 s$ \\
\hline Lond (MMs) & $<0.004$ & $<0.004$ & $<0.004$ & $<0.004$ & $<0.004$ & $<0.004$ & $<0.004$ & $<0.004$ \\
\hline Meroury (CVM) & $<0.0002$ & $<0.0002$ & $<0.0002$ & $<0.0002$ & $<0.0002$ & $<0.0008$ & $<0.0002$ & $<0.0002$ \\
\hline Nolybdenum & $<0.01$ & $<0.01$ & $<0.01$ & $<0.01$ & $<0.01$ & $<0.01$ & $<0.01$ & $<0.01$ \\
\hline Mlokel & $<0.01$ & $<0.01$ & $<0.01$ & $<0.01$ & $<0.01$ & $<0.01$ & $<0.01$ & $<0.01$ \\
\hline Selenium & $<0,05$ & $<0.05$ & $<0.05$ & $<0.05$ & $<0.05$ & $<0.05$ & $<0.03$ & $<0.05$ \\
\hline 811ver & $<0.006$ & $<0.006$ & $<0.006$ & $<0.006$ & $<0.006$ & $<0.006$ & $<0.006$ & $<0.006$ \\
\hline strontium & 1.5 & 1.5 & 1.6 & 1.6 & 1.8 & 1.9 & 1.7 & 1.7 \\
\hline Thorium & $<0.2$ & $<0.2$ & $<0.2$ & $<0.2$ & $<0.2$ & $<0.2$ & $<0.2$ & $<0.2$ \\
\hline Urantum (Fluor) & $<0.001$ & 0.001 & 0.001 & 0.001 & $<0.001$ & $<0.001$ & $<0.001$ & 0.001 \\
\hline Vanadiun & $<0.005$ & $<0.005$ & $<0.005$ & $<0.005$ & $<0.005$ & $<0.005$ & $<0.005$ & $<0.005$ \\
\hline $\operatorname{lin} 0$ & $<0.002$ & 0.0039 & 0.013 & 0.0064 & 0.014 & 0.0056 & $<0.002$ & $<0.002$ \\
\hline MWOR IONS (mg/L) & - &. & . & . &. & . & . & • \\
\hline Alkalinity-HCO3 & 188 & . & 195 & . & 193 & . & 192 & . \\
\hline Alkalinity-CO3 & $<1$ & . & $<1$ & . & $<1$ & . & $<1$ & - \\
\hline Calcium & 76 & 76 & 83 & 82 & 89 & 89 & 82 & 82 \\
\hline Chloride & 86 & $\cdot$ & 131 & $\cdot$ & 91 & . & 101 & - \\
\hline Iuoride & 0.3 & $\cdot$ & 0.3 &. & 0.3 & . & 0.2 & - \\
\hline Magnesi um & 37 & 37 & 12 & 41 & 43 & 43 & 42 & 42 \\
\hline Manganese & 0.015 & 0.015 & 0.012 & 0.011 & 0.021 & 0.02 & 0.011 & 0.011 \\
\hline NLtrate-N & 32.1 & . & 41.6 &. & 30.5 & . & 31.9 & . \\
\hline Potessium & 2.3 & 2.5 & 2.6 & 2.1 & 2.4 & 2.3 & 2.1 & 2.4 \\
\hline sodium & 35 & 35 & 39 & 38 & 45 & 46 & 46 & 46 \\
\hline sulfate & 44.5 & . & 47.6 & . & 50 & . & 45.6 & - \\
\hline
\end{tabular}

(CONTINUED) 
APPEMDIX E.1

Oroundwater and surface-water Quality Date, 1993

\begin{tabular}{|c|c|c|c|c|c|c|c|c|}
\hline \multirow{4}{*}{$\begin{array}{l}\text { sampling point } \\
\text { Location } \\
\text { Date sumpled }\end{array}$} & \multicolumn{2}{|c|}{ on-613 } & \multicolumn{2}{|c|}{ OW-614 } & \multicolumn{4}{|c|}{$O W-621$} \\
\hline & \multicolumn{2}{|c|}{$\mathbf{8 3}$} & \multicolumn{2}{|c|}{83} & \multicolumn{4}{|c|}{80} \\
\hline & \multicolumn{2}{|c|}{$10 / 20 / 93$} & \multicolumn{2}{|c|}{$10 / 20 / 93$} & \multicolumn{2}{|c|}{$03 / 20 / 93$} & \multicolumn{2}{|c|}{$06 / 02 / 93$} \\
\hline & tor & DIs & ror & Dis & TOT & DIs & TOT & Drs \\
\hline nexhe (mg/L) & - & $\cdot$ & $\cdot$ & - & $\cdot$ & - & - & . \\
\hline A1 uminum & 0.26 & 0.026 & 0.066 & $<0.02$ & 0.6 & 0.036 & 1.4 & $0.2 \dot{1}$ \\
\hline Ant inony & $<0.03$ & $<0.05$ & $<0.05$ & $<0.03$ & $<0.05$ & $<0.05$ & $<0.05$ & $<0.05$ \\
\hline Araenio & $<0.05$ & $<0.05$ & $<0.05$ & $<0.05$ & $<0.05$ & $<0.05$ & $<0.05$ & $<0.05$ \\
\hline Barlum & 0.063 & 0.062 & 0.16 & 0.16 & 0.021 & 0.019 & 0.028 & 0.021 \\
\hline Bory 111 un & $<0.0003$ & $<0.0003$ & $<0.0003$ & $<0.0003$ & $<0.0003$ & $<0.0003$ & $<0.0003$ & $<0.0003$ \\
\hline Boron & 0.0041 & 0.0048 & 0.014 & 0.013 & 0.0088 & 0.037 & 0.043 & 0.013 \\
\hline Cadnitum (MAs) & $<0.002$ & $<0.002$ & $<0.002$ & $<0.002$ & $<0.002$ & $<0.002$ & $<0.002$ & $<0.002$ \\
\hline Cadmlum & $<0.003$ & $<0.003$ & $<0.003$ & $<0.003$ & $<0.003$ & $<0.003$ & $<0.003$ & $<0.003$ \\
\hline Chromlum (MS) & $<0.01$ & $<0.01$ & $<0.01$ & $<0.01$ & 0.063 & $<0.01$ & 0.22 & 0.036 \\
\hline Chromlum & $<0.01$ & $<0.01$ & $<0.01$ & $<0.01$ & 0.051 & $<0.01$ & 0.15 & 0.026 \\
\hline Cobalt & $<0.005$ & $<0.00 s$ & $<0.005$ & $<0.005$ & $<0.005$ & $<0.003$ & $<0.005$ & $<0.005$ \\
\hline Copper & $<0.004$ & $<0.004$ & $<0.004$ & $<0.004$ & $<0.004$ & $<0.004$ & $<0.004$ & $<0.004$ \\
\hline Iron & 0.093 & $<0.005$ & 0.1 & 0.045 & 0.85 & 0.034 & 2.2 & 0.43 \\
\hline Lead (MS) & $<0.004$ & $<0.004$ & $<0.004$ & $<0.004$ & $<0.004$ & $<0.004$ & $<0.004$ & $<0.004$ \\
\hline marousy (CVMA) & $<0.0002$ & $<0.0002$ & $<0.0002$ & $<0.0002$ & $<0.0002$ & $<0.0002$ & $<0.0002$ & $<0.0002$ \\
\hline nolybdenum & $<0.01$ & $<0.01$ & $<0.01$ & $<0.01$ & $<0.01$ & $<0.01$ & $<0.01$ & $<0.01$ \\
\hline nickel & $<0.01$ & $<0.01$ & $<0.01$ & $<0.01$ & 0.038 & 0.022 & 0.16 & 0.049 \\
\hline selentum & $<0.05$ & $<0.05$ & $<0.05$ & $<0.05$ & $<0.05$ & $<0.05$ & $<0.05$ & $<0.05$ \\
\hline silver & $<0.006$ & $<0.006$ & $<0.006$ & $<0.006$ & $<0.006$ & $<0.006$ & $<0.006$ & $<0.006$ \\
\hline stront Ium & 0.05 & 0.049 & 0.56 & 0.58 & 0.055 & 0.061 & 0.061 & 0.062 \\
\hline Thorlum & $<0.2$ & $<0.2$ & $<0.2$ & $<0.2$ & $<0.2$ & $<0.2$ & $<0.2$ & $<0.2$ \\
\hline Urantiun (rluor) & $<0.001$ & $<0.001$ & $<0.001$ & $<0.001$ & 0.003 & 0.003 & 0.004 & 0.003 \\
\hline vanediun & $<0.005$ & $<0.005$ & $<0.005$ & $<0.005$ & $<0.005$ & $<0.005$ & $<0.005$ & $<0.005$ \\
\hline zine & 0.007 & 0.0048 & 0.0025 & $<0.002$ & 0.0068 & 0.0048 & 0.025 & 0.027 \\
\hline MuOR IOAS (mg/L) & . & $\cdot \dot{ }$ & $\cdot$ & $\dot{.}$ & $\dot{\bullet}$ & $\cdot$ & $\cdot \dot{.}$ & • \\
\hline & 80 & $\cdot$ &. &. & $\cdot$ &. & 152. & - \\
\hline $\begin{array}{l}\text { Alkalinity }-\mathrm{HCO} 3 \\
\text { Alkal Inity }-\mathrm{CO} 3\end{array}$ & $<1$ & $\cdot$ & $\begin{array}{r}168 \\
<1\end{array}$ &. & $\begin{array}{r}151 \\
<1\end{array}$ &. & $\begin{array}{r}152 \\
<1\end{array}$ & $\cdot$ \\
\hline $\begin{array}{l}\text { Alkallnity }-\mathrm{CO} 3 \\
\text { Calcium }\end{array}$ & 30 & 30 & $\begin{array}{l}<1 \\
45\end{array}$ & 47 & 51 & 53 & 52 & $\dot{53}$ \\
\hline Chlorlde & 1 &. & 1 &. & 3.6 &. & 5.2 & . \\
\hline Pluoride & 0.1 & $\cdot$ & $<0.1$ &. & 0.1 &. & $<0.1$ & - \\
\hline Magnes ium & 3.1 & 3 & 9.6 & 10 & 9.3 & 9.2 & 9 & 9.1 \\
\hline Manganese & 0.0037 & $<0.001$ & 0.015 & 0.015 & 0.044 & $<0.001$ & 0.13 & 0.026 \\
\hline Hitrate-N & $<0.2$ &. & $<0.2$ &. & 4.45 & . & 6 & • \\
\hline potasesium & $<0.6$ & $<0.6$ & 1.5 & 1.6 & 1.4 & 1.3 & 1.5 & 1.4 \\
\hline sodium & 3.5 & 3.6 & 10 & 10 & 2.2 & 2.4 & 2.6 & 2.7 \\
\hline sulfate & 5 & . & 12 & . & 4.1 & $\cdot$ & 5.3 & - \\
\hline
\end{tabular}

(CONTINUED) 
APPENDIX B.1

Groundwater and surface-Water Quality Data, 1993

\begin{tabular}{|c|c|c|c|c|c|c|c|c|}
\hline \multirow{4}{*}{$\begin{array}{l}\text { 8ampling point } \\
\text { Location } \\
\text { Date sampled }\end{array}$} & \multicolumn{4}{|c|}{$0 W-621$} & \multicolumn{4}{|c|}{ OW-626 } \\
\hline & \multicolumn{4}{|c|}{ BO } & \multicolumn{4}{|c|}{$B C$} \\
\hline & \multicolumn{2}{|c|}{$09 / 20 / 93$} & \multicolumn{2}{|c|}{$12 / 20 / 93$} & \multicolumn{2}{|c|}{$03 / 10 / 93$} & \multicolumn{2}{|c|}{$04 / 26 / 93$} \\
\hline & TOT & DIs & TOT & DIs & ror & DIs & Tor & DIs \\
\hline MrTxLs (mg/L) & $\cdot$ & $\cdot$ & . & $\cdot$ & $\cdot$ & $\cdot$ & . & • \\
\hline Aluminum & 3 & $<0.02$ & 1.9 & $<0.02$ & $<0.02$ & 0.023 & $<0.02$ & $<0.02$ \\
\hline Ant inony & $<0.05$ & $<0.05$ & $<0.05$ & $<0.05$ & $<0.05$ & $<0.05$ & $<0.05$ & $<0.05$ \\
\hline Arseonic & $<0.05$ & $<0.05$ & $<0.05$ & $<0.05$ & $<0.05$ & $<0.05$ & $<0.05$ & $<0.05$ \\
\hline Barium & 0.045 & 0.023 & 0.019 & 0.019 & 0.14 & 0.14 & 0.13 & 0.13 \\
\hline Beryl11um & 0.0007 & $<0.0003$ & 0.00033 & $<0.0003$ & $<0.0003$ & 0.00038 & $<0.0003$ & $<0.0003$ \\
\hline Boron & 0.035 & 0.037 & 0.024 & 0.011 & 0.0088 & 0.0052 & $<0.004$ & 0.0045 \\
\hline Cadmium (MAS) & $<0.002$ & $<0.002$ & $<0.002$ & $<0.002$ & $<0.002$ & $<0.002$ & $<0.002$ & $<0.002$ \\
\hline Cadmitum & $<0.003$ & $<0.003$ & $<0.003$ & $<0.003$ & $<0.003$ & $<0.003$ & $<0.003$ & $<0.003$ \\
\hline Chromium (MA8) & 0.18 & $<0.01$ & 0.051 & $<0.01$ & $<0.01$ & $<0.01$ & $<0.01$ & $<0.01$ \\
\hline Chromium & 0.17 & $<0.01$ & 0.034 & $<0.01$ & $<0.01$ & $<0.01$ & $<0.01$ & $<0.01$ \\
\hline Cobalt & $<0.005$ & $<0.005$ & $<0.005$ & $<0.005$ & $<0.005$ & $<0.005$ & $<0.005$ & $<0.005$ \\
\hline Copper & 0.0055 & $<0.004$ & $<0.004$ & $<0.004$ & $<0.004$ & $<0.004$ & $<0.004$ & $<0.004$ \\
\hline Iron & 4 & $<0.005$ & 2 & $<0.005$ & 0.011 & $<0.005$ & $<0.005$ & $<0.005$ \\
\hline Lead (MAS) & $<0.004$ & $<0.004$ & $<0.004$ & $<0.004$ & $<0.004$ & $<0.004$ & $<0.004$ & $<0.004$ \\
\hline Mercury (CVMA) & $<0.0002$ & $<0.0002$ & $<0.0002$ & $<0.0002$ & $<0.0002$ & $<0.0002$ & $<0.0002$ & $<0.0002$ \\
\hline Molybdenum & $<0.01$ & $<0.01$ & $<0.01$ & $<0.01$ & $<0.01$ & $<0.01$ & $<0.01$ & $<0.01$ \\
\hline Nickel & 0.14 & 0.015 & 0.072 & 0.03 & 0.024 & 0.02 & $<0.01$ & $<0.01$ \\
\hline Selenium & $<0.05$ & $<0.05$ & $<0.05$ & $<0.05$ & $<0.05$ & $<0.05$ & $<0.05$ & $<0.05$ \\
\hline silver & $<0.006$ & $<0.006$ & $<0.006$ & $<0.006$ & $<0.006$ & $<0.006$ & $<0.006$ & 0.0065 \\
\hline Strontium & 0.07 .7 & 0.073 & 0.064 & 0.059 & 0.1 & 0.11 & 0.09 & 0.087 \\
\hline Thorlun & $<0.2$ & $<0.2$ & $<0.2$ & $<0.2$ & $<0.2$ & $<0.2$ & $<0.2$ & $<0.2$ \\
\hline Urantum (Fluor) & 0.006 & 0.006 & 0.002 & 0.002 & $<0.001$ & $<0.001$ & $<0.001$ & $<0.001$ \\
\hline Vanadium & $<0.005$ & $<0.005$ & $<0.005$ & $<0.005$ & $<0.005$ & $<0.005$ & $<0.005$ & $<0.005$ \\
\hline zine & 0.02 & 0.0076 & 0.015 & $<0.02$ & 0.0042 & 0.0044 & 0.0033 & 0.0069 \\
\hline MAJOR IONS (mg/L) & & $\cdot$ & . & $\cdot$ & $\cdot$ & . & . & • \\
\hline Alkalinlty-HCO3 & 167 &. & $157^{\circ}$ & . & 109 & . & $10 \dot{3}$ & . \\
\hline Alkalinity-CO3 & $<1$ &. & $<1$ & . & $<1$ & . & $<1$ & . \\
\hline Calcium & 64 & 61 & 56 & 53 & 41 & 42 & 36 & 36 \\
\hline Chloride & 7.8 &. & 4.8 & . & 12.6 & . & 7.5 & . \\
\hline Fluoride & $<0.1$ &. & $<0.1$ & . & $<0.1$ & . & 0.1 & . \\
\hline Magnesium & 13 & 12 & 11 & 9.8 & 2.7 & 2.8 & 2.6 & 2.6 \\
\hline Mangenese & 0.26 & 0.0067 & 0.11 & $<0.001$ & $<0.001$ & $<0.001$ & $<0.001$ & $<0.001$ \\
\hline Nitrate-N & 10.12 &. & 4 & . & $<0.2$ & . & $<0.2$ & . \\
\hline Potase ium & 1.7 & 0.94 & 1.4 & 1.6 & 1 & 1.1 & $<0.6$ & 0.86 \\
\hline Sodium & 3.6 & 3.5 & 2.3 & 2.5 & 3.9 & 4.1 & 4.1 & 3.9 \\
\hline sulfate & 8 & . & 3.1 & . & 3.9 & . & 3.5 & . \\
\hline
\end{tabular}

(CONTINUED) 
APPRNDIX I.1

Groundwater and surface-Water Quality Data, 1993

\begin{tabular}{|c|c|c|c|c|c|c|c|c|}
\hline \multirow{4}{*}{$\begin{array}{l}\text { 8ampling polnt } \\
\text { Location } \\
\text { Date sampled }\end{array}$} & \multicolumn{4}{|c|}{ OW-626 } & \multicolumn{4}{|c|}{ GW- 627} \\
\hline & \multicolumn{4}{|c|}{ BC } & \multicolumn{4}{|c|}{ Bo } \\
\hline & \multicolumn{2}{|c|}{$08 / 02 / 93$} & \multicolumn{2}{|c|}{$10 / 20 / 93$} & \multicolumn{2}{|c|}{$03 / 09 / 93$} & \multicolumn{2}{|c|}{$04 / 23 / 93$} \\
\hline & TOT & DIS & TOT & DIs & TOT & Drs & TOT & DIS \\
\hline Mrtars (mg/L) & . &. & - & - & $\cdot 1$ & $\cdot$ & • & - \\
\hline Aluminum & $<0.02$ & $<0.02$ & $<0.02$ & $<0.02$ & $<0.02$ & $<0.02$ & $<0.02$ & $<0.02$ \\
\hline Ant imony & $<0.05$ & $<0.05$ & $<0.05$ & $<0.05$ & $<0.05$ & $<0.05$ & $<0.05$ & $<0.05$ \\
\hline Arsenic & $<0.05$ & $<0.05$ & $<0.05$ & $<0.05$ & $<0.05$ & $<0.05$ & $<0.05$ & $<0.03$ \\
\hline Barium & 0.14 & 0.14 & 0.14 & 0.15 & 0.039 & 0.038 & 0.041 & 0.19 \\
\hline Bery11ium & $<0.0003$ & $<0.0003$ & $<0.0003$ & $<0.0003$ & $<0.0003$ & $<0.0003$ & $<0.0003$ & $<0.0003$ \\
\hline Boron & 0.0087 & $<0.004$ & 0.0088 & 0.0067 & 0.5 & 0.49 & 0.49 & 0.087 \\
\hline Cadmium (AAS) & $<0.002$ & $<0.002$ & $<0.002$ & $<0.002$ & $<0.002$ & $<0.002$ & $<0.002$ & $<0.002$ \\
\hline Cadnilum & $<0.003$ & $<0.003$ & $<0.003$ & $<0.003$ & $<0.003$ & $<0.003$ & $<0.003$ & $<0.003$ \\
\hline Chromium (AAS) & $<0.01$ & $<0.01$ & $<0.01$ & $<0.01$ & $<0.01$ & $<0.01$ & $<0.01$ & $<0.01$ \\
\hline Chromium & $<0.01$ & $<0.01$ & $<0.01$ & $<0.01$ & $<0.01$ & $<0.01$ & $<0.01$ & $<0.01$ \\
\hline Cobalt & $<0.005$ & $<0.005$ & $<0.005$ & $<0.005$ & $<0.005$ & $<0.005$ & $<0.005$ & $<0.005$ \\
\hline Copper & $<0.004$ & $<0.004$ & $<0.004$ & $<0.004$ & $<0.004$ & $<0.004$ & $<0.004$ & $<0.004$ \\
\hline Iron & 0.027 & 0.012 & 0.0094 & $<0.005$ & 0.17 & 0.086 & 0.15 & $<0.005$ \\
\hline Lead (AAS) & $<0.004$ & $<0.004$ & $<0.004$ & $<0.004$ & $<0.004$ & $<0.004$ & $<0.004$ & $<0.004$ \\
\hline Mercury (CVAA) & 0.00024 & $<0.0002$ & $<0.0002$ & $<0.0002$ & $<0.0002$ & $<0.0002$ & $<0.0002$ & $<0.0002$ \\
\hline Molybdenum & $<0.01$ & $<0.01$ & $<0.01$ & $<0.01$ & $<0.01$ & $<0.01$ & $<0.01$ & $<0.01$ \\
\hline Nickel & $<0.01$ & $<0.01$ & $<0.01$ & 0.012 & $<0.01$ & $<0.01$ & $<0.01$ & $<0.01$ \\
\hline Selenium & $<0.05$ & $<0.05$ & $<0.05$ & $<0.05$ & $<0.05$ & $<0.05$ & $<0.05$ & $<0.05$ \\
\hline Silvar & $<0.006$ & $<0.006$ & $<0.006$ & $<0.006$ & $<0.006$ & $<0.006$ & $<0.006$ & $<0.006$ \\
\hline strontium & 0.093 & 0.093 & 0.098 & 0.1 & 0.08 & 0.077 & 0.07 & 0.52 \\
\hline Thorium & $<0.2$ & $<0.2$ & $<0.2$ & $<0.2$ & $<0.2$ & $<0.2$ & $<0.2$ & $<0.2$ \\
\hline Uranium (Fluor) & 0.004 & 0.001 & $<0.001$ & $<0.001$ & $<0.001$ & $<0,001$ & $<0.001$ & $<0.001$ \\
\hline Vanadium & $<0.005$ & $<0.005$ & $<0.005$ & $<0.905$ & $<0.005$ & $<0.005$ & $<0.005$ & $<0.005$ \\
\hline Zinc & 0.0057 & 0.0056 & 0.0036 & 0.0068 & 0.0036 & 0.0059 & 0.0089 & 0.0026 \\
\hline MAJOR IONS $(\mathrm{mg} / \mathrm{L})$ & $\cdot$ & . & $\cdot$ & $\cdot$ & . & . & - & • \\
\hline Alkalinity-HCO3 & $10 \dot{8}$ & $\cdot$ & $11 \dot{2}$ & $\cdot$ & 469 & $\dot{\bullet}$ & 447 & $\bullet$ \\
\hline Alkalinity-CO3 & $<1$ & . & $<1$ &. & 66 & . & 80 & • \\
\hline Calcium & 38 & 38 & 43 & 45 & 0.98 & 1 & 0.96 & 53 \\
\hline Chloride & 6.1 &. & 10 &. & 22 & . & 22 & 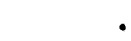 \\
\hline Fluoride & $<0.1$ & $\cdot$ & $<0.1$ & . & 4.8 & . & 5.6 & - \\
\hline Magnesium & 2.9 & 2.9 & 3.1 & 3.2 & 0.18 & 0.18 & 0.23 & 24 \\
\hline Manganese & 0.0014 & 0.0016 & $<0.001$ & 0.0012 & 0.0092 & 0.0092 & 0.0057 & 0.0011 \\
\hline Nitrate-N & $<0.2$ & . & $<0.2$ & . & $<0.2$ & . & $<0.2$ & 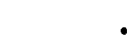 \\
\hline Potassium & 0.9 & 1.2 & 1.1 & 1.1 & 1.4 & 1.4 & 1 & 2.2 \\
\hline Sodium & 4.1 & 4.1 & 3.8 & 4 & 290 & 280 & 270 & 7.9 \\
\hline Sulfate & 3.1 & . & 3 & . & 32 & - & 31 & • \\
\hline
\end{tabular}

(CONTINUED) 
APPENDIX E.1

Groundwater and Surface-Water Quality Data, 1993

\begin{tabular}{|c|c|c|c|c|c|c|c|c|}
\hline \multirow{4}{*}{$\begin{array}{l}\text { Sampling Point } \\
\text { Location } \\
\text { Date Sampled }\end{array}$} & \multicolumn{4}{|c|}{$G W-627$} & \multicolumn{4}{|c|}{$G W-636$} \\
\hline & \multicolumn{4}{|c|}{ BG } & \multicolumn{4}{|c|}{ OLF } \\
\hline & \multicolumn{2}{|c|}{$07 / 28 / 93$} & \multicolumn{2}{|c|}{$10 / 20 / 93$} & \multicolumn{2}{|c|}{$03 / 26 / 93$} & \multicolumn{2}{|c|}{$06 / 27 / 93$} \\
\hline & TOT & DIS & TOT & DIS & TOT & DIS & TOT & DIS \\
\hline METALS (mg/L) & $\cdot$ & $\cdot$ & $\cdot$ & $\cdot$ & $\cdot$ & . & $\cdot$ & - \\
\hline Aluminum & $<0.02$ & $<0.02$ & $<0.02$ & $<0.02$ & 0.17 & $<0.02$ & $<0.02$ & $<0.02$ \\
\hline Antimony & $<0.05$ & $<0.05$ & $<0.05$ & $<0.05$ & $<0.05$ & $<0.05$ & $<0.05$ & $<0.05$ \\
\hline Arsenic & $<0.05$ & $<0.05$ & $<0.05$ & $<0.05$ & $<0.05$ & $<0.05$ & $<0.05$ & $<0.05$ \\
\hline Barium & 0.04 & 0.038 & 0.039 & 0.038 & 0.21 & 0.2 & 0.23 & 0.22 \\
\hline Beryllium & $<0.0003$ & $<0.0003$ & $<0.0003$ & $<0.0003$ & $<0.0003$ & $<0.0003$ & $<0.0003$ & $<0.0003$ \\
\hline Boron & 0.47 & 0.46 & 0.46 & 0.45 & 0.34 & 0.34 & 0.38 & 0.37 \\
\hline Cadmium (AAS) & $<0.002$ & $<0.002$ & $<0.002$ & $<0.002$ & $<0.002$ & $<0.002$ & $<0.002$ & $<0.002$ \\
\hline Cadmium & $<0.003$ & $<0.003$ & $<0.003$ & $<0.003$ & $<0.003$ & $<0.003$ & $<0.003$ & $<0.003$ \\
\hline Chromium (AAS) & $<0.01$ & $<0.01$ & $<0.01$ & $<0.01$ & $<0.01$ & $<0.01$ & $<0.01$ & $<0.01$ \\
\hline Chromium & $<0.01$ & $<0.01$ & $<0.01$ & $<0.01$ & 0.15 & $<0.01$ & $<0.01$ & $<0.01$ \\
\hline Cobalt & $<0.005$ & $<0.005$ & $<0.005$ & $<0.005$ & $<0.005$ & $<0.005$ & $<0.005$ & $<0.005$ \\
\hline Copper & $<0.004$ & $<0.004$ & $<0.004$ & $<0.004$ & $<0.004$ & $<0.004$ & $<0.004$ & $<0.004$ \\
\hline Iron & 0.62 & 0.066 & 0.35 & 0.051 & 1.4 & 0.086 & 0.69 & 0.15 \\
\hline Lead (AAS) & 0.0043 & $<0.004$ & 0.0069 & $<0.004$ & $<0.004$ & $<0.004$ & $<0.004$ & $<0.004$ \\
\hline Mercury (CVAA) & $<0.0002$ & $<0.0002$ & $<0.0002$ & $<0.0002$ & $<0.0002$ & $<0.0002$ & $<0.0002$ & $<0.0002$ \\
\hline Mol ybdenum & $<0.01$ & $<0.01$ & $<0.01$ & $<0.01$ & $<0.01$ & $<0.01$ & $<0.01$ & $<0.01$ \\
\hline Nickel & $<0.01$ & $<0.01$ & $<0.01$ & $<0.01$ & 0.15 & $<0.01$ & $<0.01$ & $<0.01$ \\
\hline Selenium & $<0.05$ & $<0.05$ & $<0.05$ & $<0.05$ & $<0.05$ & $<0.05$ & $<0.05$ & $<0.05$ \\
\hline Silver & $<0.006$ & $<0.006$ & $<0.006$ & $<0.006$ & $<0.006$ & $<0.006$ & $<0.006$ & $<0.006$ \\
\hline Strontium & 0.072 & 0.07 & 0.072 & 0.072 & 0.52 & 0.52 & 0.57 & 0.55 \\
\hline Thorium & $<0.2$ & $<0.2$ & $<0.2$ & $<0.2$ & $<0.2$ & $<0.2$ & $<0.2$ & $<0.2$ \\
\hline Uranium (Fluor) & 0.001 & $<0.001$ & $<0.001$ & 0.001 & $<0.001$ & $<0.001$ & $<0.001$ & $<0.001$ \\
\hline Vanadium & $<0.005$ & $<0.005$ & $<0.005$ & $<0.005$ & $<0.005$ & $<0.005$ & $<0.005$ & $<0.005$ \\
\hline zinc & 0.0064 & 0.0057 & 0.0094 & 0.01 & 0.0026 & 0.0047 & 0.0063 & 0.014 \\
\hline MAJOR IONS $(\mathrm{mg} / \mathrm{L})$ & $\dot{.}$ & $\cdot$ & $\bullet$ & $\cdot$ & $\dot{\bullet}$ & $\cdot$ & $\cdot$ & • \\
\hline Alkalinity-HCO3 & ${ }_{541}^{\circ}$ & $\cdot$ & 480 & $\cdot$ & 163 & $\cdot$ & 179 & - \\
\hline Alkalinity-CO3 & $<1$ & - & 62 & . & 6 & $\cdot$ & 2 & 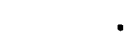 \\
\hline Calcium & 1.1 & 1 & 0.9 & 0.89 & 7.7 & 7.4 & 8.2 & 7.8 \\
\hline Chloride & 33 & . & 26 & . & 6 & $\cdot$ & 7.9 & - \\
\hline Fluoride & 6 & - & 6 & . & 0.2 & . & 0.1 & - \\
\hline Magnesium & 0.35 & 0.34 & 0.21 & 0.2 & 2.5 & 2.3 & 2.7 & 2.6 \\
\hline Manganese & 0.013 & 0.0082 & 0.011 & 0.008 & 0.018 & 0.011 & 0.018 & 0.015 \\
\hline Nitrate-N & 0.2 & - & $<0.2$ & - & $<0.2$ & - & $<0.2$ & - \\
\hline Potassium & 1.8 & 1.4 & 1.7 & 1.3 & 5.1 & 5.3 & 5 & 4.6 \\
\hline Sodium & 280 & 270 & 280 & 280 & 70 & 70 & 70 & 68 \\
\hline Sulfate & 38 & . & 30 & - & 9.3 & - & 6.7 & • \\
\hline
\end{tabular}

(CONTINUED) 
APPENDIX E.1

Groundwater and Surface-Water Quality Data, 1993

\begin{tabular}{|c|c|c|c|c|c|c|c|c|}
\hline \multirow{4}{*}{$\begin{array}{l}\text { Sampling Point } \\
\text { Location } \\
\text { Date Sampled }\end{array}$} & \multicolumn{4}{|c|}{ GW-636 } & \multicolumn{4}{|c|}{ GW-637 } \\
\hline & \multicolumn{4}{|c|}{ OLF } & \multicolumn{4}{|c|}{ orf } \\
\hline & \multicolumn{2}{|c|}{$09 / 18 / 93$} & \multicolumn{2}{|c|}{$12 / 18 / 93$} & \multicolumn{2}{|c|}{$03 / 25 / 93$} & \multicolumn{2}{|c|}{$06 / 26 / 93$} \\
\hline & TOT & Drs & TOT & DIS & TOT & DIs & TOT & DIS \\
\hline METALS (mg/L) & $\cdot$ & . & . & . &. & $\cdot 1$ & $\cdot 1$ & • \\
\hline Aluminum & $<0.02$ & $<0.02$ & $<0.02$ & $<0.02$ & 0.11 & $<0.02$ & 0.043 & 0.024 \\
\hline Antimony & $<0.05$ & $<0.05$ & $<0.05$ & $<0.05$ & $<0.05$ & $<0.05$ & $<0.05$ & $<0.05$ \\
\hline Arsenic & $<0.05$ & $<0.05$ & $<0.05$ & $<0.05$ & $<0.05$ & $<0.05$ & $<0.05$ & $<0.05$ \\
\hline Barium & 0.25 & 0.24 & 0.25 & 0.26 & 0.14 & 0.13 & 0.13 & 0.13 \\
\hline Beryllium & $<0.0003$ & $<0.0003$ & $<0.0003$ & $<0.0003$ & $<0.0003$ & $<0.0003$ & $<0.0003$ & $<0.0003$ \\
\hline Boron & 0.42 & 0.4 & 0.38 & 0.38 & 0.023 & 0.029 & 0.017 & 0.013 \\
\hline Cadmium (AAS) & $<0.002$ & $<0.002$ & $<0.002$ & $<0.002$ & $<0.002$ & $<0.002$ & $<0.002$ & $<0.002$ \\
\hline Cadmium & $<0.003$ & $<0.003$ & $<0.003$ & $<0.003$ & $<0.003$ & $<0.003$ & $<0.003$ & $<0.003$ \\
\hline Chromium (AAS) & $<0.01$ & $<0.01$ & $<0.01$ & $<0.01$ & $<0.01$ & $<0.01$ & $<0.01$ & $<0.01$ \\
\hline Chromium & $<0.01$ & $<0.01$ & $<0.01$ & $<0.01$ & $<0.01$ & $<0.01$ & $<0.01$ & $<0.01$ \\
\hline Cobalt & $<0.005$ & $<0.005$ & $<0.005$ & $<0.005$ & $<0.005$ & $<0.005$ & $<0.005$ & $<0.005$ \\
\hline Copper & $<0.004$ & $<0.004$ & $<0.004$ & $<0.004$ & $<0.004$ & $<0.004$ & $<0.004$ & $<0.004$ \\
\hline Iron & 1.1 & 0.11 & 0.92 & 0.063 & 0.14 & 0.075 & 0.047 & 0.014 \\
\hline Lead (AAS) & $<0.004$ & $<0.004$ & $<0.004$ & $<0.004$ & $<0.004$ & $<0.004$ & $<0.004$ & $<0.004$ \\
\hline Mercury (CVAA) & $<0.0002$ & $<0.0002$ & $<0.0002$ & $<0.0002$ & $<0.0002$ & $<0.0002$ & $<0.0002$ & $<0.0002$ \\
\hline Mol ybdenum & $<0.01$ & $<0.01$ & $<0.01$ & $<0.01$ & $<0.01$ & $<0.01$ & $<0.01$ & $<0.01$ \\
\hline Nickel & $<0.01$ & $<0.01$ & $<0.01$ & $<0.01$ & $<0.01$ & $<0.01$ & $<0.01$ & $<0.01$ \\
\hline Selenium & $<0.05$ & $<0.05$ & $<0.05$ & $<0.05$ & $<0.05$ & $<0.05$ & $<0.05$ & $<0.05$ \\
\hline Silver & $<0.006$ & $<0.006$ & $<0.006$ & $<0.006$ & $<0.006$ & $<0.006$ & $<0.006$ & $<0.006$ \\
\hline Strontium & 0.61 & 0.6 & 0.6 & 0.62 & 0.18 & 0.17 & 0.18 & 0.18 \\
\hline Thorium & $<0.2$ & $<0.2$ & $<0.2$ & $<0.2$ & $<0.2$ & $<0.2$ & $<0.2$ & $<0.2$ \\
\hline Uranium (Fluor) & $<0.001$ & $<0.001$ & $<0.001$ & $<0.001$ & 0.001 & 0.001 & 0.001 & 0.002 \\
\hline Vanadium & $<0.005$ & $<0.005$ & $<0.005$ & $<0.005$ & $<0.005$ & $<0.005$ & 0.0052 & $<0.005$ \\
\hline Zine & 0.0044 & 0.0089 & 0.0023 & $<0.002$ & 0.0062 & 0.0077 & 0.0063 & 0.0082 \\
\hline MAJOR IONS (mg/L) & . & $\cdot$ & . & . & . & - & - & • \\
\hline Alkalinity-HCO3 & $17 \dot{9}$ & $\cdot$ & $17 \dot{4}$ & $\cdot$ & 205 & $\cdot$ & $21 \dot{3}$ & • \\
\hline Alkalinity-CO3 & $<1$ &. & 4 & . & $<1$ & . & $<1$ & • \\
\hline Calcium & 8.1 & 7.7 & 8.8 & 9 & 73 & 72 & 75 & 76 \\
\hline Chloride & 7 & $\cdot$ & 9 & - & 2.5 & - & 2.8 & • \\
\hline Fluoride & 0.2 & $\cdot$ & $<0.1$ & - & $<0.1$ & - & $<0.1$ & • \\
\hline Magnesium & 2.6 & 2.6 & 2.8 & 2.9 & 5.5 & 5.2 & 5.6 & 5.9 \\
\hline Manganese & 0.016 & 0.012 & 0.014 & 0.0089 & 0.033 & 0.032 & 0.022 & 0.021 \\
\hline Nitrate-N & $<0.2$ &. & $<0.2$ & & $<0.2$ & - & $<0.2$ & . \\
\hline Potassium & 5.3 & 5.2 & 4.6 & 4.8 & 1.7 & 1.8 & 0.98 & 1.3 \\
\hline Sodium & 78 & 75 & 69 & 72 & 2.6 & 2.7 & 2.3 & 2.4 \\
\hline sulfate & 5 & $\cdot$ & 4 & - & 7.5 & - & 8.8 & $\bullet$ \\
\hline
\end{tabular}

(CONTINUED) 
APPENDIX E. 1

Groundwater and Surface-Water Quality Data, 1993

\begin{tabular}{|c|c|c|c|c|c|c|c|c|}
\hline \multirow{4}{*}{$\begin{array}{l}\text { Sampling Point } \\
\text { Location } \\
\text { Date Sampled }\end{array}$} & \multicolumn{4}{|c|}{$G W-637$} & \multicolumn{4}{|c|}{$G W-638$} \\
\hline & \multicolumn{4}{|c|}{ OLF } & \multicolumn{4}{|c|}{ oLf } \\
\hline & \multicolumn{2}{|c|}{$09 / 17 / 93$} & \multicolumn{2}{|c|}{$12 / 18 / 93$} & \multicolumn{2}{|c|}{$03 / 25 / 93$} & \multicolumn{2}{|c|}{$06 / 26 / 93$} \\
\hline & TOT & DIS & TOT & DIS & TOT & DIs & TOT & DIs \\
\hline METALS (mg/L) & - & $\cdot$ & $\cdot$ & $\cdot$ & $\cdot$ & $\cdot$ & $\cdot$ & - \\
\hline Al uminum & 0.036 & 0.021 & 0.024 & 0.023 & 2.6 & $<0.02$ & 28 & 0.038 \\
\hline Antimony & $<0.05$ & $<0.05$ & $<0.05$ & $<0.05$ & $<0.05$ & $<0.05$ & $<0.05$ & $<0.05$ \\
\hline Arsenic & $<0.05$ & $<0.05$ & $<0.05$ & $<0.05$ & $<0.05$ & $<0.05$ & $<0.05$ & $<0.05$ \\
\hline Barium & 0.13 & 0.14 & 0.15 & 0.16 & 0.1 & 0.062 & 0.51 & 0.1 \\
\hline Beryllium & $<0.0003$ & $<0.0003$ & $<0.0003$ & $<0.0003$ & $<0.0003$ & $<0.0003$ & 0.0019 & $<0.0003$ \\
\hline Boron & 0.023 & 0.025 & 0.025 & 0.025 & 0.028 & 0.046 & 0.061 & 0.06 \\
\hline Cadmium (AAS) & $<0.002$ & $<0.002$ & $<0.002$ & $<0.002$ & $<0.002$ & $<0.002$ & $<0.002$ & $<0.002$ \\
\hline Cadmium & $<0.003$ & $<0.003$ & $<0.003$ & $<0.003$ & $<0.003$ & $<0.003$ & 0.0094 & $<0.003$ \\
\hline Chromium (AAS) & $<0.01$ & $<0.01$ & $<0.01$ & $<0.01$ & $<0.01$ & $<0.01$ & 0.067 & $<0.01$ \\
\hline Chromium & $<0.01$ & $<0.01$ & $<0.01$ & $<0.01$ & $<0.01$ & $<0.01$ & 0.075 & $<0.01$ \\
\hline Cobalt & $<0.005$ & $<0.005$ & $<0.005$ & $<0.005$ & $<0.005$ & $<0.005$ & 0.018 & $<0.005$ \\
\hline Copper & $<0.004$ & $<0.004$ & $<0.004$ & $<0.004$ & 0.0067 & $<0.004$ & 0.054 & 0.0069 \\
\hline Iron & 0.093 & 0.048 & 0.023 & $<0.005$ & 3.7 & 0.0073 & 49 & 0.015 \\
\hline Lead (AAS) & $<0.004$ & $<0.004$ & $<0.004$ & $<0.004$ & $<0.004$ & $<0.004$ & 0.031 & $<0.004$ \\
\hline Mercury (CVAA) & $<0.0002$ & $<0.0002$ & $<0.0002$ & $<0.0002$ & $<0.0002$ & $<0.0002$ & 0.00026 & $<0.0002$ \\
\hline Molybdenum & $<0.01$ & $<0.01$ & $<0.02$ & $<0.01$ & $<0.01$ & $<0.01$ & $<0.01$ & $<0.01$ \\
\hline Nickel & $<0.01$ & $<0.01$ & $<0.01$ & $<0.01$ & $<0.01$ & $<0.01$ & 0.061 & $<0.01$ \\
\hline Selenium & $<0.05$ & $<0.05$ & $<0.05$ & $<0.05$ & $<0.05$ & $<0.05$ & $<0.05$ & $<0.05$ \\
\hline Silver & $<0.006$ & $<0.006$ & $<0.006$ & $<0.006$ & $<0.006$ & $<0.006$ & $<0.006$ & $<0.006$ \\
\hline strontium & 0.18 & 0.19 & 0.19 & 0.19 & 0.16 & 0.15 & 0.25 & 0.23 \\
\hline Thorium & $<0.2$ & $<0.2$ & $<0.2$ & $<0.2$ & $<0.2$ & $<0.2$ & $<0.2$ & $<0.2$ \\
\hline Uranium (Fluor) & $<0.001$ & $<0.001$ & 0.001 & 0.002 & 0.007 & 0.006 & 0.016 & 0.015 \\
\hline Vanadium & $<0.005$ & $<0.005$ & $<0.005$ & $<0.005$ & 0.0051 & $<0.005$ & 0.071 & $<0.005$ \\
\hline zinc & 0.0075 & 0.0068 & 0.0084 & 0.0049 & 0.033 & 0.025 & 0.1 & 0.037 \\
\hline MAJOR IONS $(\mathrm{mg} / \mathrm{L})$ & . & $\cdot$ & $\cdot$ & $\cdot$ & . & $\cdot$ & . & • \\
\hline Alkalinity- $\mathrm{HCO}$ & 216 & $\cdot$ & 212 & $\cdot$ & 164 & $\cdot$ & 202 & • \\
\hline Alkalinity-CO3 & 1 & $\cdot$ & $<1$ & $\cdot$ & $<1$ & $\cdot$ & $<1$ & • \\
\hline Calcium & 80 & 79 & 76 & 74 & 67 & 63 & 100 & 100 \\
\hline Chloride & 3 & $\cdot$ & 3 & . & 1.9 & $\cdot$ & 1.3 & - \\
\hline Fluoride & $<0.1$ & $\cdot$ & $<0.1$ & $\cdot$ & 0.3 & $\cdot$ & 0.3 & • \\
\hline Magnesium & 5.3 & 5.5 & 6.1 & 6.1 & 7.4 & 7 & 15 & 12 \\
\hline Manganese & 0.019 & 0.02 & 0.025 & 0.028 & 0.024 & 0.0017 & 0.26 & 0.013 \\
\hline Nitrate-N & $<0.2$ & $\cdot$ & $<0.2$ & $\cdot$ & 0.23 & $\cdot$ & 0.38 & - \\
\hline Potassium & 1.5 & 1.5 & 1.5 & 1.6 & 3.2 & 1.6 & 9.5 & 2.7 \\
\hline Sodium & 2.7 & 2.8 & 2.7 & 2.7 & 5.4 & 5.5 & 4.8 & 4.6 \\
\hline Sulfate & 6 & $\cdot$ & 6 & $\cdot$ & 46 & - & 64.8 & $\cdot$ \\
\hline
\end{tabular}

(CONTINUED) 
APPENDIX R.1

Groundwater and Surface-Water Quality Data, 1993

\begin{tabular}{|c|c|c|c|c|c|c|c|c|}
\hline \multirow{4}{*}{$\begin{array}{l}\text { Sampling point } \\
\text { Location } \\
\text { Date Sampled }\end{array}$} & \multicolumn{4}{|c|}{ GW-638 } & \multicolumn{4}{|c|}{ ew-639 } \\
\hline & \multicolumn{4}{|c|}{ OLF } & \multicolumn{4}{|c|}{ BG } \\
\hline & \multicolumn{2}{|c|}{$09 / 17 / 93$} & \multicolumn{2}{|c|}{$12 / 18 / 93$} & \multicolumn{2}{|c|}{$02 / 24 / 93$} & \multicolumn{2}{|c|}{$05 / 24 / 93$} \\
\hline & TOT & Drs & TOT & DIS & TOT & Drs & TOT & DIs \\
\hline $\operatorname{META} L S(\mathrm{mg} / \mathrm{L})$ & $\cdot$ & $\cdot$ & $\cdot$ & $\cdot$ & $\cdot$ & $\cdot$ & . & • \\
\hline Aluminum & 2.1 & 0.028 & 2.2 & $<0.02$ & 0.31 & $<0.02$ & 0.43 & $<0.02$ \\
\hline Antimony & $<0.05$ & $<0.05$ & $<0.05$ & $<0.05$ & $<0.05$ & $<0.05$ & $<0.05$ & $<0.05$ \\
\hline Arsenic & $<0.05$ & $<0.05$ & $<0.05$ & $<0.05$ & $<0.05$ & $<0.05$ & $<0.05$ & $<0.05$ \\
\hline Barium & 0.12 & 0.11 & 0.076 & 0.06 & 0.081 & 0.061 & 0.057 & 0.052 \\
\hline Beryllium & 0.00035 & $<0.0003$ & $<0.0003$ & $<0.0003$ & $<0.0003$ & $<0.0003$ & $<0.0003$ & $<0.0003$ \\
\hline Boron & 0.044 & 0.041 & 0.021 & 0.019 & 0.65 & 0.59 & 0.54 & 0.53 \\
\hline Cadmium (AAS) & $<0.002$ & $<0.002$ & $<0.002$ & $<0.002$ & $<0.002$ & $<0.002$ & $<0.002$ & $<0.002$ \\
\hline Cadmium & $<0.003$ & $<0.003$ & $<0.003$ & $<0.003$ & 0.0037 & $<0.003$ & $<0.003$ & $<0.003$ \\
\hline Chromium (AAS) & $<0.01$ & $<0.01$ & $<0.01$ & $<0.01$ & $<0.01$ & $<0.01$ & $<0.01$ & $<0.01$ \\
\hline Chromium & $<0.01$ & $<0.01$ & $<0.01$ & $<0.01$ & $<0.01$ & $<0.01$ & $<0.01$ & $<0.01$ \\
\hline Cobalt & $<0.005$ & $<0,005$ & $<0.005$ & $<0.005$ & $<0.005$ & $<0.005$ & $<0.005$ & $<0.005$ \\
\hline Copper & 0.085 & 0.046 & 0.011 & 0.0081 & $<0.004$ & $<0.004$ & 0.0043 & 0.0044 \\
\hline Iron & 2.4 & 0.0062 & 2.3 & $<0.005$ & 1.6 & $<0.005$ & 0.53 & 0.0054 \\
\hline Lead (AAS) & $<0.004$ & $<0.004$ & $<0.004$ & $<0.004$ & $<0.004$ & $<0.004$ & $<0.004$ & $<0.004$ \\
\hline Mercury (CVAA) & $<0.0002$ & $<0.0002$ & $<0.0002$ & $<0.0002$ & $<0.0002$ & $<0.0002$ & $<0.0002$ & $<0.0002$ \\
\hline Mol ybdenum & $<0.01$ & $<0.01$ & $<0.01$ & $<0.01$ & $<0.01$ & $<0.01$ & $<0.01$ & $<0.01$ \\
\hline Nickel & 0.011 & $<0.01$ & $<0.01$ & $<0.01$ & $<0.01$ & $<0.01$ & $<0.01$ & $<0.01$ \\
\hline Selenium & $<0.05$ & $<0.05$ & $<0.05$ & $<0.05$ & $<0.05$ & $<0.05$ & $<0.05$ & $<0.05$ \\
\hline silver & $<0.006$ & $<0.006$ & $<0.006$ & $<0.006$ & $<0.006$ & $<0.006$ & $<0.006$ & $<0.006$ \\
\hline Strontium & 0.29 & 0.28 & 0.16 & 0.16 & 0.12 & 0.094 & 0.082 & 0.08 \\
\hline Thorium & $<0.2$ & $<0.2$ & $<0.2$ & $<0.2$ & $<0.2$ & $<0.2$ & $<0.2$ & $<0.2$ \\
\hline Uranium (Fluor) & 0.018 & 0.018 & 0.015 & 0.01 & $<0.001$ & $<0.001$ & $<0.001$ & $<0.001$ \\
\hline Vanadium & $<0.005$ & $<0.005$ & 0.0053 & $<0.005$ & $<0.005$ & $<0.005$ & $<0.005$ & $<0.005$ \\
\hline zine & 0.16 & 0.11 & 0.02 & 0.0064 & 0.011 & 0.0031 & 0.0079 & 0.0069 \\
\hline MAJOR IONS $(\mathrm{mg} / \mathrm{L})$ & • & $\cdot$ &. & $\cdot$ & $\cdot$ & . & $\cdot$ & • \\
\hline Alkalinity-HCO3 & 205 & $\cdot$ & 201 & $\cdot$ & 320 & $\cdot$ & 345 & $\cdot$ \\
\hline Alkalinity- $\mathrm{CO} 3$ & $<1$ &. & $<1$ &. & 80 & . & 72 & . \\
\hline Calcium & 130 & 130 & 77 & 77 & 2.5 & 1.5 & 0.93 & 0.79 \\
\hline Chloride & $<1$ & $\cdot$ & 1 &. & 10 & - & 10 & • \\
\hline Fluoride & 0.4 & $\cdot$ & 0.2 & . & 2.3 & - & 2.4 & • \\
\hline Magnesium & 27 & 17 & 11 & 11 & 0.75 & 0.32 & 0.34 & 0.24 \\
\hline Manganese & 0.014 & 0.0029 & 0.016 & 0.0012 & 0.012 & $<0.001$ & 0.0051 & $<0.001$ \\
\hline Nitrate-N & 0.4 & . & $<0.2$ & $\cdot$ & $<0.2$ & - & $<0.2$ & . \\
\hline Potassium & 4.3 & 3.8 & 2.9 & 2.4 & 1.9 & 1.8 & 2.2 & 1.5 \\
\hline Sodium & 4.9 & 3.3 & 4.3 & 4.4 & 240 & 210 & 200 & 200 \\
\hline Sulfate & 178 & . & 44 & . & 15 & . & 29.3 & . \\
\hline
\end{tabular}

(CONTINUED) 
APPENDIX 8.1

Groundwater and Surface-Water Quality Data, 1993

\begin{tabular}{|c|c|c|c|c|c|c|c|c|}
\hline \multirow{4}{*}{$\begin{array}{l}\text { Sampling Point } \\
\text { Location } \\
\text { Date Sampled }\end{array}$} & \multicolumn{4}{|c|}{$G W-639$} & \multicolumn{4}{|c|}{$G W-640$} \\
\hline & \multicolumn{4}{|c|}{ BG } & \multicolumn{4}{|c|}{ BG } \\
\hline & \multicolumn{2}{|c|}{$07 / 11 / 93$} & \multicolumn{2}{|c|}{$10 / 15 / 93$} & \multicolumn{2}{|c|}{$02 / 22 / 93$} & \multicolumn{2}{|c|}{$05 / 24 / 93$} \\
\hline & TOT & DIS & TOT & DIS & TOT & DIS & TOT & DIS \\
\hline $\operatorname{METALS}(\mathrm{mg} / \mathrm{L})$ & $\cdot$ & $\cdot$ & $\cdot$ & $\cdot$ & $\cdot$ & - & • & $\cdot$ \\
\hline Aluminum & 0.24 & $<0.02$ & 0.43 & $<0.02$ & 0.037 & $<0.02$ & $<0.02$ & $<0.02$ \\
\hline Antimony & $<0.05$ & $<0.05$ & $<0.05$ & $<0.05$ & $<0.05$ & $<0.05$ & $<0.05$ & $<0.05$ \\
\hline Arsenic & $<0.05$ & $<0.05$ & $<0.05$ & $<0.05$ & $<0.05$ & $<0.05$ & $<0.05$ & $<0.05$ \\
\hline Barium & 0.06 & 0.054 & 0.065 & 0.059 & 0.063 & 0.061 & 0.075 & 0.074 \\
\hline Beryl11um & $<0.0003$ & $<0.0003$ & $<0.0003$ & $<0.0003$ & $<0.0003$ & $<0.0003$ & $<0.0003$ & $<0.0003$ \\
\hline Boron & 0.56 & 0.55 & 0.56 & 0.58 & 0.014 & 0.011 & 0.011 & 0.016 \\
\hline Cadmium (AAS) & $<0.002$ & $<0.002$ & $<0.002$ & $<0.002$ & $<0.002$ & $<0.002$ & $<0.002$ & $<0.002$ \\
\hline Cadmium & $<0.003$ & $<0.003$ & $<0.003$ & $<0.003$ & $<0.003$ & $<0.003$ & $<0.003$ & $<0.003$ \\
\hline Chromium (AAS) & $<0.01$ & $<0.01$ & $<0.01$ & $<0.01$ & $<0.01$ & $<0.01$ & $<0.01$ & $<0.01$ \\
\hline Chromium & $<0.01$ & $<0.01$ & $<0.01$ & $<0.01$ & $<0.01$ & $<0.01$ & $<0.01$ & $<0.01$ \\
\hline Cobalt & $<0.005$ & $<0.005$ & $<0.005$ & $<0.005$ & $<0.005$ & $<0.005$ & $<0.005$ & $<0.005$ \\
\hline Copper & 0.0052 & $<0.004$ & $<0.004$ & $<0.004$ & 0.0047 & 0.0047 & $<0.004$ & 0.018 \\
\hline Iron & 0.24 & $<0.005$ & 1 & 0.005 & 0.1 & 0.0052 & 0.061 & $<0.005$ \\
\hline Lead (AAS) & $<0.004$ & $<0.004$ & 0.004 & $<0.004$ & $<0.004$ & $<0.004$ & $<0.004$ & $<0.004$ \\
\hline Mercury (CVAA) & $<0.0002$ & $<0,0002$ & $<0.0002$ & $<0.0002$ & $<0.0002$ & $<0.0002$ & $<0.0002$ & $<0.0002$ \\
\hline Mol ybdenum & $<0.01$ & $<0.01$ & $<0.01$ & $<0.01$ & $<0.01$ & $<0.01$ & $<0.01$ & $<0.01$ \\
\hline Nickel & $<0.01$ & $<0.01$ & $<0.01$ & $<0.01$ & $<0.01$ & $<0.01$ & $<0.01$ & $<0.01$ \\
\hline Selenium & $<0.05$ & $<0.05$ & $<0.05$ & $<0.05$ & $<0.05$ & $<0.05$ & $<0.05$ & $<0.05$ \\
\hline Silver & $<0.006$ & $<0.006$ & $<0.006$ & $<0.006$ & $<0.006$ & $<0.006$ & $<0.006$ & $t<0.006$ \\
\hline Strontium & 0.083 & 0.078 & 0.091 & 0.09 & 0.043 & 0.042 & 0.056 & 0.056 \\
\hline Thorium & $<0.2$ & $<0.2$ & $<0.2$ & $<0.2$ & $<0.2$ & $<0.2$ & $<0.2$ & $<0.2$ \\
\hline Urantum (Fluor) & 0.001 & 0.001 & 0.001 & 0.001 & $<0.001$ & $<0.001$ & $<0.001$ & $<0.001$ \\
\hline Vanadium & $<0.005$ & $<0.005$ & $<0.005$ & $<0.005$ & $<0.005$ & $<0.005$ & $<0.005$ & $<0.005$ \\
\hline Zinc & 0.0097 & 0.0028 & 0.0067 & 0.0054 & 0.01 & 0.01 & 0.011 & 0.018 \\
\hline MAJOR IONS (mg/L) & $\cdot$ & . & . & . & $\cdot$ & $\cdot$ & $\cdot$ & • \\
\hline Alkalinity-HCO3 & 346 & $\cdot$ & 352 & $\cdot \dot{1}$ & $\dot{43}$ & $\cdot$ & 50 & $\cdot$ \\
\hline Alkalinity-co3 & 76 &. & 64 &. & $<1$ & . & $<1$ & 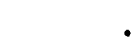 \\
\hline Calcium & 0.85 & 0.75 & 1.2 & 0.84 & 15 & 14 & 14 & 14 \\
\hline Chloride & 9.9 &. & 10 & . & 1.2 & . & 1.1 & • \\
\hline Fluoride & 3.1 & $\cdot$ & 2.6 & $\cdot$ & $<0.1$ & $\cdot$ & $<0.1$ & • \\
\hline Magnesium & 0.32 & 0.22 & 0.42 & 0.28 & 1.5 & 1.5 & 1.6 & 1.6 \\
\hline Kanganese & 0.0012 & $<0.001$ & 0.012 & $<0.001$ & 0.0093 & 0.0064 & 0.0065 & 0.0047 \\
\hline Nitrate-N & $<0.2$ & $\cdot$ & $<0.2$ & - & $<0.2$ & . & $<0.2$ & • \\
\hline Potassium & 1.3 & 1.3 & 1.9 & 1.7 & 1.4 & 1.1 & 0.85 & 0.93 \\
\hline Sodium & 210 & 200 & 210 & 220 & 4.1 & 4.1 & 3.5 & 3.7 \\
\hline Sulfate & 29.9 & . & 27 & . & 1.8 & $\cdot$ & 2.9 & $\bullet$ \\
\hline
\end{tabular}

(CONTINUED) 
APPENDIX E. 1

Groundwater and Surface-Water Quality Data, 1993

\begin{tabular}{|c|c|c|c|c|c|c|c|c|}
\hline \multirow{4}{*}{$\begin{array}{l}\text { Sampling Point } \\
\text { Location } \\
\text { Date sampled }\end{array}$} & \multicolumn{4}{|c|}{$G W-640$} & \multicolumn{4}{|c|}{ OW-641 } \\
\hline & \multicolumn{4}{|c|}{ BG } & \multicolumn{4}{|c|}{ BG } \\
\hline & \multicolumn{2}{|c|}{$07 / 10 / 93$} & \multicolumn{2}{|c|}{$10 / 14 / 93$} & \multicolumn{2}{|c|}{$02 / 22 / 93$} & \multicolumn{2}{|c|}{$05 / 24 / 93$} \\
\hline & TOT & DIS & TOT & DIS & TOT & DIS & TOT & DIS \\
\hline METALS (mg/L) & $\cdot$ & $\cdot$ & $\cdot$ & $\cdot$ & $\cdot$ & . & $\cdot$ & - \\
\hline Aluminum & 0.085 & $<0.02$ & 0.072 & $<0.02$ & 0.21 & $<0.02$ & 0.16 & $<0.02$ \\
\hline Antimony & $<0.05$ & $<0.05$ & $<0.05$ & $<0.05$ & $<0.05$ & $<0.05$ & $<0.05$ & $<0.05$ \\
\hline Arsenic & $<0.05$ & $<0.05$ & $<0.05$ & $<0.05$ & $<0.05$ & $<0.05$ & $<0.05$ & $<0.05$ \\
\hline Barium & 0.064 & 0.065 & 0.073 & 0.072 & 0.043 & 0.031 & 0.046 & 0.05 \\
\hline Beryllium & $<0.0003$ & $<0.0003$ & $<0.0003$ & $<0.0003$ & $<0.0003$ & $<0.0003$ & $<0.0003$ & $<0.0003$ \\
\hline Boron & 0.013 & 0.0062 & 0.025 & 0.011 & $<0.004$ & 0.025 & 0.013 & 0.0043 \\
\hline Cadmium (AAS) & $<0.002$ & $<0.002$ & $<0.002$ & $<0.002$ & $<0.002$ & $<0.002$ & $<0.002$ & $<0.002$ \\
\hline Cadmium & $<0.003$ & $<0.003$ & $<0.003$ & $<0.003$ & $<0.003$ & $<0.003$ & $<0.003$ & $<0.003$ \\
\hline Chromium (AAS) & $<0.01$ & $<0.01$ & $<0.01$ & $<0.01$ & $<0.01$ & $<0.01$ & $<0.01$ & $<0.01$ \\
\hline Chromium & $<0.01$ & $<0.01$ & $<0.01$ & $<0.01$ & $<0.01$ & $<0.01$ & $<0.01$ & $<0.01$ \\
\hline Cobalt & $<0.005$ & $<0.005$ & $<0.005$ & $<0.005$ & $<0.005$ & $<0.005$ & $<0.005$ & $<0.005$ \\
\hline Copper & $<0.004$ & $<0.004$ & $<0.004$ & $<0.004$ & $<0.004$ & 0.0046 & $<0.004$ & 0.016 \\
\hline Iron & 0.12 & 0.0051 & 0.077 & $<0.005$ & 0.31 & $<0.005$ & 0.37 & 0.0093 \\
\hline Lead (AAS) & $<0.004$ & $<0.004$ & $<0.004$ & $<0.004$ & $<0.004$ & $<0.004$ & $<0.004$ & $<0.004$ \\
\hline Mercury (CVAA) & $<0.0002$ & $<0.0002$ & $<0.0002$ & $<0.0002$ & $<0.0002$ & $<0.0002$ & $<0.0002$ & $<0.0002$ \\
\hline Molybdenum & $<0.01$ & $<0.01$ & $<0.01$ & $<0.01$ & $<0.01$ & $<0.01$ & $<0.01$ & $<0.01$ \\
\hline Nickel & $<0.01$ & $<0.01$ & $<0.01$ & $<0.01$ & 0.014 & 0.01 & $<0.01$ & $<0.01$ \\
\hline Selenium & $<0.05$ & $<0.05$ & $<0.05$ & $<0.05$ & $<0.05$ & $<0.05$ & $<0.05$ & $<0.05$ \\
\hline Silver & $<0.006$ & $<0.006$ & $<0.006$ & $<0.006$ & $<0.006$ & $<0.006$ & $<0.006$ & $<0.006$ \\
\hline strontium & 0.044 & 0.045 & 0.05 & 0.05 & 0.014 & 0.0095 & 0.017 & 0.017 \\
\hline Thorium & $<0.2$ & $<0.2$ & $<0.2$ & $<0.2$ & $<0.2$ & $<0.2$ & $<0.2$ & $<0.2$ \\
\hline Uranium (Fluor) & $<0.001$ & 0.001 & $<0.001$ & $<0.001$ & $<0.001$ & $<0.001$ & $<0.001$ & $<0.001$ \\
\hline Vanadium & $<0.005$ & $<0.005$ & $<0.005$ & $<0.005$ & $<0.005$ & $<0.005$ & $<0.005$ & $<0.005$ \\
\hline zinc & 0.0056 & 0.0074 & 0.0054 & 0.0074 & 0.017 & 0.021 & 0.059 & 0.12 \\
\hline MAJOR IONS $(\mathrm{mg} / \mathrm{L})$ & $\cdot$ & $\cdot$ & - & - & - & $\cdot$ & . & . \\
\hline Alkalinity-HCO3 & 49 & $\dot{.}$ & $\dot{49}$ & $\dot{\bullet}$ & $\dot{16}$ & $\cdot$ & 20 & . \\
\hline Alkalinity- $\mathrm{CO} 3$ & $<1$ &. & $<1$ & . & $<1$ &. & $<1$ & . \\
\hline Calcium & 15 & 15 & 18 & 18 & 3.8 & 2.2 & 5.6 & 4.8 \\
\hline Chloride & 1.2 & $\cdot$ & 2 & - & $<1$ &. & $<1$ & . \\
\hline Fluoride & $<0.1$ &. & $<0.1$ & - & $<0.1$ & . & $<0.1$ & . \\
\hline Magnesium & 1.5 & 1.5 & 1.7 & 1.7 & 1.2 & 0.85 & 0.95 & 0.92 \\
\hline Manganese & 0.0098 & 0.0072 & 0.0052 & 0.0048 & 0.04 & 0.013 & 0.038 & 0.008 \\
\hline Nitrate-N & $<0.2$ & $\cdot$ & $<0.2$ & - & $<0.2$ & . & $<0.2$ & ra \\
\hline Potassium & 0.64 & 0.62 & 0.77 & 0.62 & $<0.6$ & 1 & 0.8 & 0.7 \\
\hline Sodium & 3.6 & 3.7 & 3.7 & 3.7 & 2.2 & 1.8 & 2.4 & 2.5 \\
\hline Sulfate & 2.2 & . & 2 & - & $<1$ & - & 2 & re \\
\hline
\end{tabular}

(CONTINUED) 
APPENDIX $\mathbf{E} .1$

Groundwater and Surface-Water Quality Data, 1993

\begin{tabular}{|c|c|c|c|c|c|c|c|c|}
\hline \multirow{4}{*}{$\begin{array}{l}\text { Sampling Point } \\
\text { Location } \\
\text { Date Sampled }\end{array}$} & \multicolumn{4}{|c|}{$G W-641$} & \multicolumn{4}{|c|}{$6 W-642$} \\
\hline & \multicolumn{4}{|c|}{ BG } & \multicolumn{4}{|c|}{ Be } \\
\hline & \multicolumn{2}{|c|}{$07 / 10 / 93$} & \multicolumn{2}{|c|}{$10 / 24 / 93$} & \multicolumn{2}{|c|}{$02 / 08 / 93$} & \multicolumn{2}{|c|}{$05 / 11 / 93$} \\
\hline & TOT & DIs & $\operatorname{TOT}$ & DI8 & $\operatorname{Tor}$ & DIS & TOT & DI8 \\
\hline MeTaLs (mg/L) & - & - & . & - & $\cdot$ & $\cdot$ & - & • \\
\hline Aluminum & $<0.02$ & $<0.02$ & 0.31 & $<0.02$ & 0.079 & $<0.02$ & 0.3 & 0.037 \\
\hline Antimony & $<0.05$ & $<0.05$ & $<0.05$ & $<0.05$ & $<0.05$ & $<0.05$ & $<0.05$ & $<0.05$ \\
\hline Arsenic & $<0.05$ & $<0.05$ & $<0.05$ & $<0.05$ & $<0.05$ & $<0.05$ & $<0.05$ & $<0.05$ \\
\hline Barium & 0.04 & 0.039 & 0.046 & 0.04 & 0.12 & 0.12 & 0.13 & 0.12 \\
\hline Beryllium & $<0.0003$ & $<0.0003$ & 0.00037 & $<0.0003$ & $<0.0003$ & $<0.0003$ & $<0.0003$ & $<0.0003$ \\
\hline Boron & 0.0095 & $<0.004$ & 0.0056 & 0.0056 & 0.011 & 0.021 & 0.027 & 0.03 \\
\hline Cadmium (MAs) & $<0.002$ & $<0.002$ & $<0.002$ & $<0.002$ & $<0.002$ & $<0.002$ & $<0.002$ & $<0.002$ \\
\hline Cadmi um & $<0.003$ & $<0.003$ & $<0.003$ & $<0.003$ & $<0.003$ & $<0.003$ & $<0.003$ & $<0.003$ \\
\hline Chromium (MAS) & $<0.01$ & $<0.01$ & $<0.01$ & $<0.01$ & $<0.01$ & $<0.01$ & $<0.01$ & $<0.01$ \\
\hline Chromium & $<0.01$ & $<0.01$ & $<0.01$ & $<0.01$ & $<0.01$ & $<0.01$ & 0.022 & 0.013 \\
\hline Cobalt & $<0.005$ & $<0.005$ & $<0.005$ & $<0.005$ & $<0.005$ & $<0.005$ & $<0.005$ & $<0.005$ \\
\hline Copper & 0.0047 & 0.0064 & 0.0058 & $<0.004$ & $<0.004$ & $<0.004$ & 0.0069 & $<0.004$ \\
\hline Iron & 0.028 & 0.02 & 0.44 & 0.033 & 3.1 & 2.8 & 2.6 & 2.3 \\
\hline Lead (AAS) & $<0.004$ & $<0.004$ & $<0.004$ & $<0.004$ & $<0.004$ & $<0.004$ & $<0.004$ & $<0.004$ \\
\hline Mercury (CVAA) & $<0.0002$ & $<0.0002$ & $<0.0002$ & $<0.0002$ & $<0.0002$ & $<0.0002$ & $<0.0002$ & $<0.0002$ \\
\hline Mol ybdenum & $<0.01$ & $<0.01$ & $<0.01$ & $<0.01$ & $<0.01$ & $<0.01$ & $<0.01$ & $<0.01$ \\
\hline Nickel & $<0.01$ & $<0.01$ & $<0.01$ & $<0.01$ & 0.03 & 0.033 & 0.036 & 0.043 \\
\hline Selenium & $<0.05$ & $<0.05$ & $<0.05$ & $<0.05$ & $<0.05$ & $<0.05$ & $<0.05$ & $<0.05$ \\
\hline silver & $<0.006$ & $<0.006$ & $<0.006$ & $<0.006$ & $<0.006$ & $<0.006$ & $<0.006$ & $<0.006$ \\
\hline strontium & 0.014 & 0.013 & 0.016 & 0.014 & 0.021 & 0.021 & 0.027 & 0.023 \\
\hline Thorium & $<0.2$ & $<0.2$ & $<0.2$ & $<0.2$ & $<0.2$ & $<0.2$ & $<0.2$ & $<0.2$ \\
\hline Uranium (Fluor) & 0.001 & $<0.001$ & $<0.001$ & $<0.001$ & $<0.001$ & $<0.001$ & $<0.001$ & $<0.001$ \\
\hline Vanadium & $<0.005$ & $<0.005$ & $<0.005$ & $<0.005$ & $<0.005$ & $<0.005$ & $<0.005$ & $<0.005$ \\
\hline zinc & 0.038 & 0.032 & 0.044 & 0.03 & 0.0095 & 0.0087 & 0.038 & 0.026 \\
\hline MAJOR IONS $(\mathrm{mg} / \mathrm{L})$ & . & $\cdot$ & . & $\cdot$ & $\cdot$ & $\cdot$ & . & • \\
\hline Alkalinity-HCO3 & $\dot{18}$ & $\cdot$ & $\dot{18}$ &. & $\dot{60}$ & $\cdot$ & $\dot{58}$ & • \\
\hline Alkalinity- $\mathrm{CO} 3$ & $<1$ & . & $<1$ &. & $<1$ &. & $<1$ & • \\
\hline Calcium & 4.1 & 4 & 5 & 4.4 & 7.3 & 7.1 & 8.9 & 7.5 \\
\hline Chloride & $<1$ & . & 1 & .1 & $<1$ & . & 1.7 & . \\
\hline Fluoride & $<0.1$ & - & $<0.1$ &. & 0.3 &. & 0.4 & . \\
\hline Magnesiuxn & 0.87 & 0.86 & 0.89 & 0.81 & 7.6 & 7.6 & 7.6 & 7.4 \\
\hline Manganese & 0.0056 & 0.0041 & 0.033 & 0.0053 & 1.3 & 1.2 & 1.8 & 1.3 \\
\hline Nitrate-N & $<0.2$ & . & $<0.2$ & $\cdot$ & $<0.2$ & . & $<0.2$ & • \\
\hline Potassium & $<0.6$ & $<0.6$ & 0.65 & $<0.6$ & 1.8 & 1.7 & 2 & 1.8 \\
\hline Sodium & 2.2 & 2.2 & 2.3 & 2.2 & 7.4 & 7.3 & 7.7 & 7.6 \\
\hline Sulfate & $<1$ & . & $<1$ & . & 10.8 &. & 16 & • \\
\hline
\end{tabular}

(CONTINUED) 
APPENDIX 1.1

Groundwater and surface-water Quality Data, 1993

\begin{tabular}{|c|c|c|c|c|c|c|c|c|}
\hline \multirow{4}{*}{$\begin{array}{l}\text { sampling Point } \\
\text { Location } \\
\text { Date sampled }\end{array}$} & \multicolumn{4}{|c|}{$O N-642$} & \multicolumn{4}{|c|}{$a n-643$} \\
\hline & \multicolumn{4}{|c|}{80} & \multicolumn{4}{|c|}{80} \\
\hline & \multicolumn{2}{|c|}{$07 / 01 / 93$} & \multicolumn{2}{|c|}{$10 / 10 / 93$} & \multicolumn{2}{|c|}{$02 / 16 / 93$} & \multicolumn{2}{|c|}{$05 / 19 / 93$} \\
\hline & TOT & DI8 & rot & DIs & 202 & DI8 & Tor & DI8 \\
\hline NeTNLs $(\mathrm{mg} / \mathrm{L})$ & $\cdot$ & - & $\cdot$ & - & - & - & - & - \\
\hline Aluminum & 0.079 & $<0.02$ & 13 & 0.13 & 4.9 & $<0.02$ & 0.62 & $<0.02$ \\
\hline Ant imony & $<0.03$ & $<0.05$ & $<0.05$ & $<0.05$ & $<0.05$ & $<0.05$ & $<0.05$ & $<0.05$ \\
\hline Areente & $<0.05$ & $<0.05$ & $<0.05$ & $<0.05$ & $<0.05$ & $<0.05$ & $<0.05$ & $<0.08$ \\
\hline Barium & 0.12 & 0.11 & 0.96 & 0.045 & 0.081 & 0.025 & 0.051 & 0.036 \\
\hline Beryl11um & $<0.0003$ & $<0.0003$ & 0.0011 & $<0.0003$ & $<0.0003$ & $<0.0003$ & $<0.0003$ & $<0.0003$ \\
\hline Boron & 0.031 & 0.04 & 0.032 & 0.013 & 0.0064 & 0.018 & 0.016 & 0.03 \\
\hline Cadmi um (MAs) & $<0.002$ & $<0.002$ & $<0.002$ & $<0.002$ & $<0.002$ & $<0.002$ & $<0.002$ & $<0.002$ \\
\hline Cadmium & $<0.003$ & $<0.003$ & 0.0074 & $<0.003$ & $<0.003$ & $<0.003$ & $<0.003$ & $<0.003$ \\
\hline Chromiun (MAs) & $<0.01$ & $<0.01$ & 0.29 & $<0.01$ & 0.024 & $<0.01$ & $<0.01$ & $<0.01$ \\
\hline Chromiun & $<0.01$ & $<0.01$ & 0.18 & $<0.01$ & 0.02 & $<0.01$ & $<0.01$ & $<0.01$ \\
\hline Cobalt & $<0.005$ & $<0.005$ & 0.023 & $<0.005$ & $<0.005$ & $<0.005$ & $<0.005$ & $<0.005$ \\
\hline Copper & $<0.004$ & $<0.004$ & 0.049 & $<0.004$ & 0.015 & 0.0061 & $<0.004$ & $<0.004$ \\
\hline Iron & 3.1 & 2.2 & 93 & 2.1 & 3.7 & 0.013 & 0.74 & $<0.005$ \\
\hline Lead (MAS) & $<0.004$ & $<0.004$ & 0.031 & $<0.004$ & 0.0052 & $<0.004$ & $<0.004$ & $<0.004$ \\
\hline Marcury (CVMA) & $<0.0002$ & 0.00024 & $<0.0002$ & $<0.0002$ & $<0.0002$ & $<0.0002$ & $<0.0002$ & $<0.0002$ \\
\hline Molybdenum & $<0.01$ & $<0.01$ & $<0.01$ & $<0.01$ & $<0.01$ & $<0.01$ & $<0.01$ & $<0.01$ \\
\hline Nickel & 0.022 & 0.023 & 0.12 & 0.027 & 0.014 & $<0.01$ & 0.015 & 0.018 \\
\hline Selenium & $<0.05$ & $<0.05$ & $<0.05$ & $<0.05$ & $<0.05$ & $<0.05$ & $<0.05$ & $<0.05$ \\
\hline silver & $<0.006$ & $<0.006$ & $<0.006$ & $<0.006$ & $<0.006$ & $<0.006$ & $<0.006$ & $<0.006$ \\
\hline Strontiun & 0.022 & 0.022 & 0.053 & 0.023 & 0.015 & 0.0096 & 0.016 & 0.014 \\
\hline Thorium & $<0.2$ & $<0.2$ & $<0.2$ & $<0.2$ & $<0.2$ & $<0.2$ & $<0.2$ & $<0.2$ \\
\hline Uranium (Fluor) & $<0.001$ & $<0.001$ & 0.002 & 0.001 & $<0.001$ & $<0.001$ & $<0.001$ & $<0.001$ \\
\hline Vanadium & $<0.005$ & $<0.005$ & 0.058 & $<0.005$ & 0.0087 & $<0.005$ & $<0.005$ & $<0.005$ \\
\hline zinc & 0.042 & 0.015 & 0.11 & $<0.002$ & 0.022 & 0.018 & 0.033 & 0.028 \\
\hline MANOR IONS (mg/L) & . & . & . & . & . & . & . & . \\
\hline Alkalinity-HCO3 & 57 &. & $\dot{57}$ & • & $\dot{12}$ & • & ${ }_{14}$ & • \\
\hline Alkalinity-co3 & $<1$ & . & $<1$ & . & $<1$ & . & $<1$ & • \\
\hline Calcium & 7 & 6.9 & 16 & 9.2 & 4 & 2.6 & 4.5 & 3.9 \\
\hline Chloride & 1.2 & - & 1.5 & • & 1.4 & • & 1.9 & $\cdot$ \\
\hline Fluoride & 0.4 & . & 0.4 & • & $<0.1$ & - & $<0.1$ & • \\
\hline Magnesium & 7.4 & 7.5 & 12 & 7.2 & 2 & 1.1 & 1.8 & 2.5 \\
\hline Manganese & 1.4 & 1.3 & 2.4 & 1.2 & 0.087 & 0.03 & 0.03 & 0.018 \\
\hline Nitrate-N & $<0.2$ & . & $<0.2$ & • & 0.29 & - & $<0.2$ & • \\
\hline Potassium & 1.9 & 1.8 & 4.5 & 1.5 & 2.7 & 1 & 1.2 & 0.88 \\
\hline Sodium & 7.8 & 7.9 & 7.8 & 7.8 & 8.1 & 8 & 6.4 & 6 \\
\hline sulfate & 12 & - & 9.8 & - & 14.4 & . & 16 & • \\
\hline
\end{tabular}

(CONTINUED) 
APPEHDIX $\geq .1$

Oroundwater and surface-Water Quallty Data, 1993

\begin{tabular}{|c|c|c|c|c|c|c|c|c|}
\hline \multirow{4}{*}{$\begin{array}{l}\text { Sampling Polnt } \\
\text { Location } \\
\text { Date sumpled }\end{array}$} & \multicolumn{4}{|c|}{$a w-643$} & \multicolumn{4}{|c|}{$a n-644$} \\
\hline & \multicolumn{4}{|c|}{ BO } & \multicolumn{4}{|c|}{ orF } \\
\hline & \multicolumn{2}{|c|}{$07 / 08 / 93$} & \multicolumn{2}{|c|}{$10 / 12 / 93$} & \multicolumn{2}{|c|}{$03 / 25 / 93$} & \multicolumn{2}{|c|}{$06 / 25 / 93$} \\
\hline & ror & Drs & Tor & Dr8 & TOT & DIs & Tor & Dre \\
\hline Merats (mg/L) & . & $\cdot$ & - & - & $\cdot$ & $\cdot$ & $\cdot$ & . \\
\hline Aluminum & 7 & 0.024 & 1.5 & $<0.02$ & 0.051 & $<0.02$ & 1.9 & 0.025 \\
\hline Antimony & $<0.05$ & $<0.05$ & $<0.05$ & $<0.05$ & $<0.03$ & $<0.05$ & $<0.05$ & $<0.05$ \\
\hline Arsente & $<0.05$ & $<0.05$ & $<0.05$ & $<0.05$ & $<0.05$ & $<0.05$ & $<0.0 s$ & $<0.05$ \\
\hline Barium & 0.15 & 0.088 & 0.063 & 0.042 & 0.06 & 0.06 & 0.091 & 0.079 \\
\hline Bery111um & $<0.0003$ & $<0.0003$ & $<0.0003$ & $<0.0003$ & $<0.0003$ & $<0.0003$ & $<0.0003$ & $<0.0003$ \\
\hline Boron & 0.016 & 0.025 & 0.015 & 0.011 & 0.036 & 0.0089 & 0.0046 & 0.018 \\
\hline Cadmiun (MAs) & $<0.002$ & $<0.002$ & $<0.002$ & $<0.002$ & $<0.002$ & $<0.002$ & $<0.002$ & $<0.002$ \\
\hline Cadmium & 0.0036 & $<0.003$ & $<0.003$ & $<0.003$ & $<0.003$ & $<0.003$ & $<0.003$ & $<0.003$ \\
\hline Chromiun (MAs) & 0.027 & $<0.01$ & $<0.01$ & $<0.01$ & $<0.01$ & $<0.01$ & $<0.01$ & $<0.01$ \\
\hline Chromlum & 0.024 & $<0.01$ & $<0.01$ & $<0.01$ & $<0.01$ & $<0.01$ & $<0.01$ & $<0.01$ \\
\hline Cobalt & $<0.005$ & $<0.005$ & $<0.005$ & $<0.005$ & $<0.005$ & $<0.005$ & $<0.005$ & $<0.005$ \\
\hline Copper & 0.013 & 0.0044 & 0.0069 & $<0.004$ & $<0.004$ & 0.0041 & $<0.004$ & $<0.004$ \\
\hline Iron & 8 & 0.057 & 1.9 & 0.0082 & 0.22 & 0.016 & 1.9 & -0.005 \\
\hline Laad (MAs) & $<0.004$ & $<0.004$ & $<0.004$ & $<0.004$ & $<0.004$ & $<0.004$ & $<0.004$ & $<0.004$ \\
\hline Marcury (CVAA) & $<0.0002$ & 0.00025 & $<0.0002$ & $<0.0002$ & $<0.0002$ & $<0.0002$ & $<0.0002$ & $<0.0002$ \\
\hline Mol ybdenum & $<0.01$ & $<0.01$ & $<0.01$ & $<0.01$ & $<0.01$ & $<0.01$ & $<0.01$ & $<0.01$ \\
\hline Nickel & 0.027 & $<0.01$ & $<0.01$ & $<0.01$ & $<0.01$ & $<0.01$ & $<0.01$ & $<0.01$ \\
\hline seleniun & $<0.05$ & $<0.05$ & $<0.05$ & $<0.05$ & $<0.05$ & $<0.05$ & $<0.05$ & $<0.05$ \\
\hline stiver & $<0.006$ & $<0.006$ & $<0.006$ & $<0.006$ & $<0.006$ & $<0.006$ & $<0.006$ & $<0.006$ \\
\hline strontium & 0.073 & 0.061 & 0.025 & 0.018 & 0.041 & 0.041 & 0.067 & 0.067 \\
\hline Thorium & $<0.2$ & $<0.2$ & $<0.2$ & $<0.2$ & $<0.2$ & $<0.2$ & $<0.2$ & $<0.2$ \\
\hline Uranium (Fluor) & $<0.001$ & 0.001 & $<0.001$ & $<0.001$ & $<0.001$ & $<0.001$ & $<0.001$ & $<0.001$ \\
\hline Vanadium & 0.01 & $<0.005$ & $<0.005$ & $<0.005$ & $<0.005$ & $<0.005$ & $<0.005$ & $<0.005$ \\
\hline zine & 0.022 & 0.015 & 0.035 & 0.043 & 0.022 & 0.022 & 0.012 & 0.0089 \\
\hline MAJOR IONS $(\mathrm{mg} / \mathrm{L})$ &. &. & . & $\cdot$ & $\cdot$ & $\cdot$ &. & • \\
\hline & $\dot{82}$ & $\cdot$ & $\cdot$ & $\cdot$ & $\cdot$ & $\cdot$ & $\cdot$ & - \\
\hline Alkalinity-HCO3 & 82 & $\cdot$ & 14 & $\cdot$ & 58 & $\cdot$ & 108 & $\cdot$ \\
\hline Alkalinity-CO3 & $<1$ & $\cdot$ & $<1$ & $\cdot$ & $<1$ & $\cdot$ & $<1$ & • \\
\hline Calcium & 25 & 22 & 8.9 & 6.1 & 19 & 19 & 34 & 34 \\
\hline Chloride & 2.6 & . & 2 & $\cdot$ & 2.2 & . & 2.7 & • \\
\hline Fluoride & 0.2 &. & 0.1 &. & 0.1 &. & $<0.1$ & • \\
\hline Magnesium & 9.6 & 7.4 & 3.3 & 2.2 & 1.5 & 1.5 & 2.5 & 2.2 \\
\hline Manganese & 0.29 & 0.13 & 0.061 & 0.03 & 0.0037 & 0.0022 & 0.066 & 0.0029 \\
\hline Nitrate-N & $<0.2$ &. & $<0.2$ & . & $<0.2$ & . & $<0.2$ & • \\
\hline Potassium & 3.9 & 2.2 & 1.7 & 0.91 & 0.99 & 0.65 & $<0.6$ & $<0.6$ \\
\hline Sodium & 14 & 13 & 8 & 7 & 3.7 & 3.8 & 3.1 & 3.3 \\
\hline sulfate & 40.4 & $\cdot$ & 15 & . & 2.1 & . & 7.1 & • \\
\hline
\end{tabular}

(CONT INUED) 
APpundix 1.1

Groundwater and surface-Water Quallty Data, 1993

\begin{tabular}{|c|c|c|c|c|c|c|c|c|}
\hline \multirow{4}{*}{$\begin{array}{l}\text { Sampling polnt } \\
\text { Lootion } \\
\text { Date sampled }\end{array}$} & \multicolumn{4}{|c|}{ ON-644 } & \multicolumn{4}{|c|}{$a w-647$} \\
\hline & \multicolumn{4}{|c|}{ ow } & \multicolumn{4}{|c|}{ ow } \\
\hline & \multicolumn{2}{|c|}{$09 / 27 / 93$} & \multicolumn{2}{|c|}{$12 / 28 / 93$} & \multicolumn{2}{|c|}{$02 / 08 / 93$} & \multicolumn{2}{|c|}{$05 / 17 / 93$} \\
\hline & 20r & Drs & $\operatorname{sor}$ & Drs & $\operatorname{xos}$ & Drs & $x+2$ & ors \\
\hline conshs (mg/L) & $\cdot$ & $\cdot$ & $\cdot$ & $\cdot$ & $\cdot$ & $\cdot$ & $\cdot$ & • \\
\hline Aluminum & 17 & $<0.02$ & 1.2 & $<0.02$ & 0.24 & $<0.02$ & $<0.02$ & $<0.02$ \\
\hline Antiony & $<0.05$ & $<0.05$ & $<0.05$ & $<0.05$ & $<0.03$ & $<0.05$ & $<0.05$ & $<0.03$ \\
\hline Armenlo & 0.058 & $<0.05$ & $<0.05$ & $<0.05$ & $<0.05$ & $<0.05$ & $<0.05$ & $<0.05$ \\
\hline Barium & 0.17 & 0.061 & 0.086 & 0.08 & 0.033 & 0.024 & 0.025 & 0.026 \\
\hline Baryl11um & 0.0014 & $<0.0003$ & $<0.0003$ & $<0.0003$ & $<0.0003$ & $<0.0003$ & $<0.0003$ & $<0.0003$ \\
\hline Boron & 0.028 & 0.011 & 0.0078 & 0.0091 & 0.038 & 0.023 & 0.041 & 0.04 \\
\hline Cadmiun (MAB) & $<0.002$ & $<0.002$ & $<0.002$ & $<0.002$ & $<0.002$ & $<0.002$ & $<0.002$ & $<0.002$ \\
\hline Cadmitum & 0.0064 & $<0.003$ & $<0.003$ & $<0.003$ & $<0.003$ & $<0.003$ & $<0.003$ & $<0.003$ \\
\hline Chromium (Ms) & 0.046 & $<0.01$ & $<0.01$ & $<0.01$ & $<0,01$ & $<0.01$ & $<0.01$ & $<0.01$ \\
\hline Chromitum & 0.12 & $<0.01$ & $<0.01$ & $<0.01$ & $<0.01$ & $<0.01$ & $<0.02$ & $<0.01$ \\
\hline Cobalt & 0.012 & $<0.005$ & $<0.005$ & $<0.005$ & $<0.005$ & $<0.003$ & $<0.005$ & $<0.005$ \\
\hline Copper & 0.044 & $<0.004$ & $<0.004$ & $<0.004$ & $<0.004$ & $<0.004$ & $<0.004$ & $<0.004$ \\
\hline Iron & 23 & 0.0077 & 1.1 & $<0.00 s$ & 0.52 & $<0.005$ & 0.24 & 0.011 \\
\hline Lead (MAS) & 0.021 & $<0.004$ & $<0.004$ & $<0.004$ & $<0.004$ & $<0.004$ & $<0.004$ & $<0.004$ \\
\hline Mercury (CVMa) & $<0.0002$ & $<0.0002$ & $<0.0002$ & $<0.0002$ & $<0.0002$ & $<0.0002$ & $<0.0002$ & $<0.0002$ \\
\hline nolybdenum & $<0.01$ & $<0.01$ & $<0.01$ & $<0.01$ & $<0.01$ & $<0.01$ & $<0.01$ & $<0.01$ \\
\hline Nickel & 0.063 & $<0.01$ & $<0.01$ & $<0.01$ & $<0.01$ & $<0.01$ & 0.024 & 0.018 \\
\hline 8elentum & $<0.05$ & $<0.05$ & $<0.05$ & $<0.05$ & $<0.05$ & $<0.05$ & $<0.05$ & $<0.05$ \\
\hline silver & $<0.006$ & $<0.006$ & $<0.006$ & $<0.006$ & $<0.006$ & $<0.006$ & $<0.006$ & $<0.006$ \\
\hline strontlum & 0.079 & 0.054 & 0.06 & 0.061 & 1.2 & 1.1 & 1.1 & 1.2 \\
\hline Thoriun & $<0.2$ & $<0.2$ & $<0.2$ & $<0.2$ & $<0.2$ & $<0.2$ & $<0.2$ & $<0.2$ \\
\hline Uranium (Fluor) & 0.001 & $<0.001$ & $<0.001$ & $<0.001$ & $<0.001$ & $<0.001$ & $<0.001$ & $<0.001$ \\
\hline Vanadium & 0.024 & $<0.005$ & $<0.005$ & $<0.005$ & $<0.005$ & $<0.005$ & $<0.005$ & $<0.005$ \\
\hline zinc & 0.095 & 0.007 & 0.0068 & 0.0055 & 0.012 & 0.009 & 0.023 & 0.014 \\
\hline majon rons $(\mathrm{mg} / \mathrm{L})$ & $\cdot$ &. & - & $\cdot$ & • & • & - & . \\
\hline Alkalinity-HCO3 & 136 &. & $\dot{86}$ & $\cdot \dot{1}$ & 177 & . & 179 & . \\
\hline Alkalinity-CO3 & 1 & $\dot{.}$ & $<1$ & $\cdot \dot{1}$ & $<1$ & $\cdot$ & $<1$ & • \\
\hline Calcium & 37 & 26 & 29 & 30 & 63 & 63 & 60 & 60 \\
\hline Chlorlde & 3 &. & 3 &. & 1.2 & - & 1.2 & . \\
\hline Fluorida & 0.1 &. & $<0.1$ & $\cdot$ & 0.4 & . & 0.4 & . \\
\hline Magnesium & 5.4 & 1.7 & 2.2 & 2 & 32 & 33 & 30 & 31 \\
\hline Manganese & 0.34 & 0.0043 & 0.032 & $<0.001$ & 0.0094 & 0.0026 & 0.0089 & 0.0025 \\
\hline Nitrate-N & $<0.2$ &. & $<0.2$ &. & $<0.2$ & . & $<0.2$ & . \\
\hline Potaselum & 4.4 & $<0.6$ & 1.2 & $<0.6$ & 3.1 & 2.7 & 2.7 & 2.8 \\
\hline sodium & 3.8 & 3.7 & 3.5 & 3.6 & 2.4 & 1.9 & 2 & 2 \\
\hline sulfate & $<1$ & $\cdot$ & $<1$ & . & 118 & - & 105 & 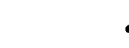 \\
\hline
\end{tabular}

(CONTINUED) 
APPIMDIX $\mathbf{2} .1$

Groundwater and Burface-Mater Quallty Data, 1993

\begin{tabular}{|c|c|c|c|c|c|c|c|c|}
\hline \multirow{4}{*}{$\begin{array}{l}\text { Sampling Polnt } \\
\text { Loeston } \\
\text { Date sampled }\end{array}$} & \multicolumn{4}{|c|}{ OW-647 } & \multicolumn{4}{|c|}{$0 N-631$} \\
\hline & \multicolumn{4}{|c|}{ owP } & \multicolumn{4}{|c|}{ so } \\
\hline & \multicolumn{2}{|c|}{$09 / 25 / 93$} & \multicolumn{2}{|c|}{$12 / 10 / 93$} & \multicolumn{2}{|c|}{$03 / 01 / 93$} & \multicolumn{2}{|c|}{$04 / 19 / 93$} \\
\hline & not & Drs & sor & Drs & xor & Drs & ror & Drs \\
\hline nernes (ng/L) & • & - & $\cdot$ & $\cdot$ & - & $\cdot$ & - & $\cdot$ \\
\hline Aluninum & 0.061 & 0.029 & 0.025 & $<0.02$ & 0.03 & 0.038 & 0.24 & $<0.02$ \\
\hline Ant Lmony & $<0.05$ & $<0.05$ & $<0.05$ & $<0.05$ & $<0.05$ & $<0.05$ & $<0.0 s$ & $<0.0 s$ \\
\hline isaento & $<0.05$ & $<0.05$ & $<0.05$ & $<0.05$ & $<0.05$ & 0.06 & $<0.03$ & $<0.05$ \\
\hline Bariun & 0.032 & 0.032 & 0.031 & 0.031 & 0.047 & 0.048 & 0.049 & 0.046 \\
\hline Deryl11un & 0.00033 & 0.00043 & $<0.0003$ & $<0.0003$ & $<0.0003$ & $<0.0003$ & $<0.0003$ & $<0.0003$ \\
\hline Boron & 0.052 & 0.047 & 0.028 & 0.026 & 0.046 & 0.057 & 0.044 & 0.035 \\
\hline Cadmi un (MAS) & $<0,002$ & $<0.002$ & 0.0074 & $<0.002$ & $<0.002$ & $<0.002$ & $<0.002$ & $<0.002$ \\
\hline Cadmium & 0.0034 & $<0.003$ & 0.0072 & $<0.003$ & $<0.003$ & $<0.003$ & $<0.003$ & $<0.003$ \\
\hline Chromium (MA8) & $<0.01$ & $<0.01$ & $<0.01$ & $<0.01$ & $<0.01$ & $<0.01$ & $<0.01$ & $<0.01$ \\
\hline Chromlun & $<0.01$ & $<0.01$ & $<0.01$ & $<0.01$ & $<0.01$ & $<0.01$ & $<0,01$ & $<0.01$ \\
\hline cobalt & $<0.005$ & $<0.005$ & $<0.005$ & $<0.005$ & $<0.003$ & $<0.005$ & $<0.005$ & $<0.00 s$ \\
\hline opper & $<0.004$ & $<0.004$ & 0.0084 & $<0.004$ & $<0.004$ & 0.078 & 0.01 & $<0.004$ \\
\hline ron & 0.16 & $<0.005$ & 0.086 & $<0.005$ & 0.045 & 0.016 & 0.3 & 0.014 \\
\hline Load (MAB) & $<0.004$ & $<0.004$ & $<0.004$ & $<0.004$ & $<0.004$ & $<0.004$ & $<0.004$ & $<0.004$ \\
\hline | Marcury (CVMa) & $<0.0002$ & $<0.0002$ & $<0.0002$ & $<0.0002$ & $<0.0002$ & $<0.0002$ & $<0.0002$ & $<0.0002$ \\
\hline Mol ybdenum & $<0.01$ & $<0.01$ & $<0.01$ & $<0.01$ & $<0.01$ & $<0.01$ & $<0.01$ & $<0.01$ \\
\hline mickel & $<0.02$ & $<0.01$ & $<0.01$ & $<0.01$ & $<0.01$ & $<0.01$ & $<0.01$ & $<0.01$ \\
\hline selendun & $<0.05$ & $<0.05$ & $<0.05$ & $<0.05$ & $<0.05$ & $<0.05$ & $<0.03$ & $<0.05$ \\
\hline silver & $<0.006$ & $<0.006$ & $<0.006$ & $<0.006$ & $<0.006$ & $<0.006$ & $<0.006$ & $<0.006$ \\
\hline stront Iun & 1.2 & 1.2 & 1.2 & 1.1 & 0.29 & 0.29 & 0.28 & 0.28 \\
\hline Thorlum & $<0.2$ & $<0.2$ & $<0.2$ & $<0.2$ & $<0.2$ & $<0.2$ & $<0.2$ & $<0.2$ \\
\hline Urantum (Fluor) & $<0.001$ & $<0.001$ & 0.001 & 0.001 & $<0.001$ & $<0.001$ & $<0.001$ & $<0.001$ \\
\hline Vanadium & $<0.005$ & $<0.005$ & $<0.005$ & $<0.005$ & $<0.005$ & $<0.005$ & $<0.005$ & $<0.005$ \\
\hline $\operatorname{lin} c$ & 0.035 & 0.021 & 0.018 & 0.0083 & $<0.002$ & 0.028 & 0.038 & 0.022 \\
\hline MWOR IONS (mg/L) & $\dot{.}$ & $\cdot$ & $\cdot \dot{\varphi}$ & $\cdot \dot{ }$ & $\cdot$ & $\cdot$ & $\cdot$ & • \\
\hline Alkalinity-HCO3 & $\begin{array}{r}\cdot \\
185\end{array}$ & $\cdot$ & $\begin{array}{r}178 \\
\end{array}$ &. & 184 & $\cdot$ & 192 & . \\
\hline Alkallnity-CO3 & $<1$ & $\cdot$ & $<1$ & $\cdot$ & $<1$ & $\cdot$ & $<1$ & - \\
\hline Calcium & 62 & 62 & 64 & 61 & 57 & 55 & 62 & 59 \\
\hline Chloride & 1.9 & $\cdot$ & 1 & $\cdot$ & 3.2 & . & 3 & - \\
\hline rluoride & 0.4 & $\cdot$ & 0.4 & . & $<0.1$ & . & $<0.1$ & - \\
\hline Magnes Lum & 33 & 33 & 33 & 32 & 9.5 & 9.2 & 9.9 & 9.7 \\
\hline Manganeme & 0.0053 & $<0.001$ & 0.0037 & $<0.001$ & 0.016 & 0.011 & 0.029 & 0.012 \\
\hline Nitrate-N & $<0.2$ & $\cdot$ & $<0.2$ &. & $<0.2$ & . & $<0.2$ & - \\
\hline Potase I um & 3 & 2.9 & 3.3 & 3.2 & 5.2 & 6.2 & 2.8 & 2.9 \\
\hline Sodiun & 2.1 & 2.1 & 2.3 & 2.2 & 2 & 2.2 & 1.5 & 1.7 \\
\hline sulfate & 103 & - & 88 & $\cdot$ & 10.2 & $\cdot$ & 10.4 & - \\
\hline
\end{tabular}

(CONTINUED) 
APpandx 1.1

Groundwater and surface-water Quallty Data, 1993

\begin{tabular}{|c|c|c|c|c|c|c|c|c|}
\hline \multirow{4}{*}{$\begin{array}{l}\text { sampling point } \\
\text { Location } \\
\text { Date sampled }\end{array}$} & \multicolumn{4}{|c|}{ CW-651 } & \multicolumn{4}{|c|}{ GN-652 } \\
\hline & \multicolumn{4}{|c|}{ so } & \multicolumn{4}{|c|}{$B 0$} \\
\hline & \multicolumn{2}{|c|}{$07 / 10 / 93$} & \multicolumn{2}{|c|}{$10 / 10 / 93$} & \multicolumn{2}{|c|}{$03 / 01 / 93$} & \multicolumn{2}{|c|}{$04 / 19 / 93$} \\
\hline & ror & DI8 & TOr & DI8 & 202 & DIs & 202 & Drs \\
\hline mornes $(m g / L)$ & $\cdot 1$ & $\cdot$ &. & $\cdot$ & $\cdot$ & $\cdot$ & $\cdot 1$ & - \\
\hline Aluminum & $<0.02$ & $<0.02$ & 8.5 & 0.044 & 2.2 & 0.03 & 1.1 & $<0.02$ \\
\hline Ant Leony & $<0.08$ & $<0.05$ & $<0.05$ & $<0.0 s$ & $<0.05$ & $<0.05$ & $<0.08$ & $<0.05$ \\
\hline Arsenic & $<0.05$ & $<0.05$ & $<0.05$ & $<0.05$ & $<0.05$ & $<0.08$ & $<0.05$ & $<0.05$ \\
\hline Bariun & 0.048 & 0.047 & 0.15 & 0.053 & 0.18 & 0.15 & 0.15 & 0.15 \\
\hline Bery111um & $<0.0003$ & $<0.0003$ & 0.00053 & 0.00043 & $<0.0003$ & $<0.0003$ & $<0.0003$ & $<0.0003$ \\
\hline Boron & 0.04 & 0.035 & 0.017 & 0.04 & 0.026 & 0.029 & 0.022 & 0.017 \\
\hline Cadmlun (MAs) & $<0.002$ & $<0.002$ & 0.0045 & $<0.002$ & $<0.002$ & $<0.002$ & $<0.002$ & $<0.002$ \\
\hline Cadmi un & $<0.003$ & $<0.003$ & 0.0064 & 0.0079 & $<0.003$ & $<0.003$ & $<0.003$ & $<0.003$ \\
\hline Chromium (MAs) & $<0.01$ & $<0.01$ & $<0.01$ & $<0.01$ & $<0.01$ & $<0.01$ & $<0.01$ & $<0.01$ \\
\hline Chromlum & $<0.01$ & $<0.01$ & $<0.01$ & $<0.01$ & $<0.01$ & $<0.01$ & $<0.01$ & $<0.01$ \\
\hline Cobalt & $<0.005$ & $<0.005$ & $<0.005$ & $<0.005$ & 0.0051 & $<0.005$ & $<0.005$ & $<0.003$ \\
\hline Copper & $<0.004$ & $<0.004$ & $<0.004$ & $<0.604$ & $<0.004$ & $<0.004$ & $<0.004$ & $<0.004$ \\
\hline Iron & 0.049 & $<0.005$ & 7 & 0.06 & 3.6 & $<0.005$ & 1.7 & $<0.005$ \\
\hline road (MAs) & $<0.004$ & $<0.004$ & $<0.004$ & $<0.004$ & $<0.004$ & $<0.004$ & $<0.004$ & $<0.004$ \\
\hline Marcury (CVM) & $<0.0002$ & $<0.0002$ & $<0.0002$ & $<0.0002$ & $<0.0002$ & $<0.0002$ & $<0.0002$ & $<0.0002$ \\
\hline Holybdenum & $<0.01$ & $<0.01$ & $<0.02$ & $<0.01$ & $<0.01$ & $<0.01$ & $<0.01$ & $<0.01$ \\
\hline nlckel & $<0.01$ & $<0.01$ & $<0.01$ & $<0.01$ & $<0.01$ & $<0.01$ & $<0.01$ & $<0.01$ \\
\hline Selentum & $<0.05$ & $<0.05$ & $<0.05$ & $<0.05$ & $<0.05$ & $<0.05$ & $<0.05$ & $<0.05$ \\
\hline 811ver & $<0.006$ & $<0.006$ & $<0.006$ & 0.0083 & $<0.006$ & $<0.006$ & $<0.006$ & $<0.006$ \\
\hline strontlum & 0.28 & 0.26 & 0.036 & 0.31 & 0.18 & 0.18 & 0.15 & 0.15 \\
\hline Thoriun & $<0.2$ & $<0.2$ & $<0.2$ & $<0.2$ & $<0.2$ & $<0.2$ & $<0.2$ & $<0.2$ \\
\hline Urantum (Fluor) & $<0.001$ & 0.001 & $<0.001$ & $<0.001$ & $<0,001$ & $<0.001$ & $<0.001$ & $<0.001$ \\
\hline Vanadium & $<0.005$ & $<0.005$ & 0.012 & $<0 ; 005$ & $<0.005$ & $<0.005$ & $<0.005$ & $<0.005$ \\
\hline ine & 0.0088 & 0.0083 & 0.027 & 0.019 & $<0.002$ & $<0.002$ & 0.011 & 0.0075 \\
\hline MuJOR IOAs $(\mathrm{mg} / \mathrm{L})$ & $\cdot$ & • &. &. & $\cdot$ & . &. & • \\
\hline Alkal inity-HCO3 & 196 & $\dot{.}$ & 203 &. & 193 & $\dot{.}$ & 187 & • \\
\hline Alkalinity- $\mathrm{CO} 3$ & $<1$ & - & $<1$ & $\cdot$ & $<1$ & - & $<1$ & • \\
\hline Calciun & 56 & 52 & 2.8 & 66 & 76 & 74 & 69 & 69 \\
\hline Chloride & 3.4 & - & 3.2 & $\cdot 1$ & 4.4 & - & 5.8 & • \\
\hline Fluoride & $<0.1$ & - & $<0.1$ & $\cdot$ & $<0.1$ & • & $<0.1$ & • \\
\hline Magnesium & 9.7 & 9 & 2.6 & 11 & 6.7 & 6.7 & 5.8 & 6 \\
\hline Manganaee & 0.011 & 0.0049 & 0.25 & 0.028 & 0.1 & 0.0027 & 0.064 & $<0.001$ \\
\hline Nitrate-N & $<0.2$ & - & $<0.2$ & $\cdot$ & $<0.2$ & - & $<0.2$ & · \\
\hline Potase Lum & 4.3 & 5.3 & 2.4 & 3.1 & 3.1 & 2.8 & 2.8 & 2.3 \\
\hline Sodium & 1.8 & 1.9 & 3 & 1.5 & 0.95 & 0.96 & 0.92 & 1 \\
\hline sulete & 9.9 & - & 9.2 & $\cdot$ & 7.5 & - & 8.3 & . \\
\hline
\end{tabular}

(CONT INUED) 
APPENDIX 2.1

Groundwater and surface-Water Quality Data, 1993

\begin{tabular}{|c|c|c|c|c|c|c|c|c|}
\hline \multirow{4}{*}{$\begin{array}{l}\text { 8ampling Polnt } \\
\text { Location } \\
\text { Date sampled }\end{array}$} & \multicolumn{4}{|c|}{ OW-652 } & \multicolumn{4}{|c|}{ aw-653 } \\
\hline & \multicolumn{4}{|c|}{ BO } & \multicolumn{4}{|c|}{ BO } \\
\hline & \multicolumn{2}{|c|}{$07 / 10 / 93$} & \multicolumn{2}{|c|}{$10 / 28 / 93$} & \multicolumn{2}{|c|}{$03 / 01 / 93$} & \multicolumn{2}{|c|}{$04 / 20 / 93$} \\
\hline & TOT & DIs & Tot & Drs & TOT & DIs & TOT & Dr8 \\
\hline Natals (mg/L) & . & - & $\cdot$ & - & . & $\cdot$ & - & • \\
\hline Aluminum & 0.63 & $<0.02$ & 0.18 & 0.042 & 5.3 & $<0.02$ & 1.5 & $<0.02$ \\
\hline Antinony & $<0.05$ & $<0.05$ & $<0.05$ & $<0.05$ & $<0.05$ & $<0.05$ & $<0.05$ & $<0.05$ \\
\hline Arsento & $<0.05$ & $<0.05$ & $<0.05$ & $<0.05$ & $<0.03$ & $<0.05$ & $<0.05$ & $<0.05$ \\
\hline Darlum & 0.16 & 0.15 & 0.16 & 0.16 & 0.12 & 0.024 & 0.049 & 0.027 \\
\hline Bary 11 ium & $<0.0003$ & $<0.0003$ & $<0.0003$ & $<0.0003$ & 0.0007 & $<0.0003$ & $<0.0003$ & $<0.0003$ \\
\hline Boron & 0.02 & 0.016 & 0.03 & 0.025 & 0.022 & 0.0069 & 0.0075 & 0.0049 \\
\hline Cadmiun (Ms) & $<0.002$ & $<0.002$ & $<0.002$ & $<0.002$ & $<0.002$ & $<0.002$ & $<0.002$ & -0.002 \\
\hline Cadnitum & $<0.003$ & $<0.003$ & $<0.003$ & $<0.003$ & $<0.003$ & $<0.003$ & $<0.003$ & $<0.003$ \\
\hline Chromi um (MA8) & $<0.01$ & $<0.01$ & $<0.01$ & $<0.01$ & 0.011 & $<0.01$ & $<0.01$ & $<0.01$ \\
\hline Chromium & $<0.01$ & $<0.01$ & $<0.01$ & $<0.01$ & $<0.01$ & $<0.01$ & $<0.01$ & $<0.01$ \\
\hline Cobalt & $<0.005$ & $<0.005$ & $<0.005$ & $<0.005$ & $<0.005$ & $<0.005$ & $<0.005$ & $<0.005$ \\
\hline Copper & 0.0049 & $<0.004$ & $<0.004$ & $<0.004$ & $<0.004$ & $<0.004$ & $<0.004$ & 0.0049 \\
\hline Iron & 0.92 & $<0.005$ & 0.23 & $<0.005$ & 3.9 & $<0.005$ & 1.3 & 0.011 \\
\hline raad (MAS) & $<0.004$ & $<0.004$ & $<0.004$ & $<0.004$ & 0.0077 & $<0.004$ & $<0.004$ & $<0.004$ \\
\hline Marcury (CVMa) & $<0.0002$ & $<0.0002$ & $<0.0002$ & $<0,0002$ & $<0.0002$ & $<0,0002$ & $<0.0002$ & $<0.0002$ \\
\hline Molybdenum & $<0.01$ & $<0.01$ & $<0.01$ & $<0.01$ & $<0.01$ & $<0.01$ & $<0.01$ & $<0.01$ \\
\hline Nickel & $<0.01$ & $<0.01$ & $<0.01$ & $<0.01$ & $<0.01$ & $<0.01$ & $<0.01$ & $<0.01$ \\
\hline Belentum & $<0.05$ & $<0.03$ & $<0.05$ & $<0.05$ & $<0.05$ & $<0.05$ & $<0.05$ & $<0.05$ \\
\hline 811ver & $<0.006$ & $<0.006$ & $<0.006$ & $<0.006$ & $<0.006$ & $<0.006$ & $<0.006$ & $<0.006$ \\
\hline Strontlum & 0.15 & 0.15 & 0.2 & 0.19 & 0.038 & 0.017 & 0.019 & 0.015 \\
\hline Thoriun & $<0.2$ & $<0.2$ & $<0.2$ & $<0.2$ & $<0.2$ & $<0.2$ & $<0.2$ & $<0.2$ \\
\hline Uranium (Fluor) & $<0.001$ & $<0.001$ & $<0.001$ & 0.001 & 0.001 & $<0.001$ & $<0.001$ & $<0.001$ \\
\hline Vanadium & $<0.005$ & $<0.005$ & $<0.005$ & $<0.005$ & $<0.005$ & $<0.005$ & $<0.005$ & $<0.005$ \\
\hline Zine & 0.011 & 0.0056 & 0.011 & 0.011 & 0.0098 & $<0.002$ & 0.012 & 0.011 \\
\hline MAJOR IONS (mg/L) &. &. &. &. &. &. & . & - \\
\hline Alkalinity-HCO3 & 208 & . & 208 & $\cdot$ & ${ }_{86}$ & $\cdot$. & 14 & • \\
\hline Alkalinity-CO3 & $<1$ &. & $<1$ & $\cdot$ & $<1$ & . & $<1$ & . \\
\hline Calcium & 76 & 73 & 78 & 77 & 3.7 & 1.9 & 2.2 & 1.9 \\
\hline Chloride & 5.7 & $\cdot$ & 5.9 & $\cdot$ & 1.1 & $\cdot$ & 1.1 & - \\
\hline Pluoride & $<0.1$ & $\cdot$ & $<0.1$ & $\cdot$ & $<0.1$ & $\cdot$ & $<0.1$ & - \\
\hline Magnesium & 6.5 & 6.3 & 8 & 7.7 & 2.3 & 1 & 1.3 & 0.95 \\
\hline Manganese & 0.04 & 0.001 & 0.0055 & $<0.001$ & 0.13 & 0.0029 & 0.049 & 0.0029 \\
\hline Hitrate-N & $<0.2$ & . & 0.45 & . & $<0.2$ & . & $<0.2$ & \\
\hline Potaes I um & 2.7 & 2.4 & 2.8 & 2.7 & 1.5 & 0.83 & $<0.6$ & $<0.6$ \\
\hline Sod1um & 0.98 & 0.87 & 1.2 & 1.2 & 3 & 2.6 & 2.5 & 2.5 \\
\hline sulfate & 8.8 & . & 7.9 &. & 2.7 & . & 2.2 & • \\
\hline
\end{tabular}

(CONTINUED) 
APPANDIX $\mathbf{E} .1$

oroundwater and surface-Water Quality Data, 1993

\begin{tabular}{|c|c|c|c|c|c|c|c|c|}
\hline \multirow{4}{*}{$\begin{array}{l}\text { Sampling point } \\
\text { zocation } \\
\text { Date sampled }\end{array}$} & \multicolumn{4}{|c|}{$a w-653$} & \multicolumn{4}{|c|}{$a W-654$} \\
\hline & \multicolumn{4}{|c|}{ BG } & \multicolumn{4}{|c|}{80} \\
\hline & \multicolumn{2}{|c|}{$07 / 10 / 93$} & \multicolumn{2}{|c|}{$10 / 10 / 93$} & \multicolumn{2}{|c|}{$02 / 24 / 93$} & \multicolumn{2}{|c|}{$04 / 16 / 93$} \\
\hline & TOT & DIs & Tot & DIs & TOT & Drs & TOT & DIS \\
\hline Marass (mg/L) & . & • & $\cdot$ & - & $\cdot 1$ & $\cdot$ & $\cdot$ & • \\
\hline Aluminum & 2.6 & $<0.02$ & $<0.02$ & $<0.02$ & 0.33 & $<0.02$ & 0.27 & 0.022 \\
\hline Antimony & $<0.05$ & $<0.05$ & $<0.05$ & $<0.03$ & $<0.05$ & $<0.08$ & $<0.05$ & $<0.05$ \\
\hline Areenic & $<0.05$ & $<0.05$ & $<0.05$ & $<0.05$ & $<0.05$ & $<0.05$ & $<0.05$ & $<0.05$ \\
\hline Barium & 0.072 & 0.026 & 0.052 & 0.027 & 0.12 & 0.091 & 0.093 & 0.087 \\
\hline Bery11ium & $<0.0003$ & $<0.0003$ & 0.00032 & $<0.0003$ & 0.0022 & $<0.0003$ & $<0.0003$ & $<0.0003$ \\
\hline Boron & 0.0057 & 0.0075 & 0.04 & 0.0068 & 0.051 & 0.05 & 0.045 & 0.045 \\
\hline Cadmium (MAs) & $<0.002$ & $<0.002$ & $<0.002$ & $<0.002$ & $<0.002$ & $<0.002$ & $<0.002$ & $<0.002$ \\
\hline Cadmium & $<0.003$ & $<0.003$ & $<0.003$ & $<0.003$ & $<0.003$ & $<0.003$ & $<0.003$ & $<0.003$ \\
\hline Chromium (Ans) & $<0.01$ & $<0.01$ & 0.018 & $<0.01$ & $<0.01$ & $<0.01$ & $<0.01$ & $<0.01$ \\
\hline Chromium & $<0.01$ & $<0.01$ & $<0.01$ & $<0.01$ & 0.011 & $<0.01$ & $<0.01$ & $<0.01$ \\
\hline Cobalt & $<0.005$ & $<0.005$ & $<0.005$ & $<0.005$ & $<0.005$ & $<0.005$ & $<0.005$ & $<0.005$ \\
\hline Copper & $<0.004$ & $<0.004$ & $<0.004$ & $<0.004$ & 0.0047 & $<0.004$ & 0.0063 & 0.0062 \\
\hline Iron & 2.2 & 0.006 & 0.018 & $<0.005$ & 1.1 & 0.07 & 0.47 & 0.094 \\
\hline Lead (MAS) & $<0.004$ & $<0.004$ & 0.0095 & $<0.004$ & $<0.004$ & $<0.004$ & $<0.004$ & $<0.004$ \\
\hline Mercury (CVMA) & $<0.0002$ & $<0.0002$ & $<0.0002$ & $<0.0002$ & $<0.0002$ & $<0.0002$ & $<0.0002$ & $<0.0002$ \\
\hline Mol ybdenum & $<0.01$ & $<0.01$ & $<0.01$ & $<0.01$ & $<0.01$ & $<0.01$ & $<0,01$ & $<0.01$ \\
\hline Nickel & $<0.01$ & $<0.01$ & $<0.01$ & $<0.01$ & 0.013 & $<0.01$ & $<0.01$ & $<0.01$ \\
\hline Selentum & $<0.05$ & $<0.05$ & $<0.05$ & $<0.05$ & $<0.05$ & $<0.05$ & $<0.05$ & $<0.05$ \\
\hline silver & $<0.006$ & $<0.006$ & $<0.006$ & $<0.006$ & $<0.006$ & $<0.006$ & $<0.006$ & $<0.006$ \\
\hline strontium & 0.024 & 0.016 & 0.31 & 0.016 & 0.19 & 0.18 & 0.18 & 0.18 \\
\hline Thorium & $<0.2$ & $<0.2$ & $<0.2$ & $<0.2$ & $<0.2$ & $<0.2$ & $<0.2$ & $<0.2$ \\
\hline Uranium (Fluor) & 0.001 & 0.001 & 0.011 & 0.005 & $<0.001$ & $<0.001$ & $<0.001$ & $<0.001$ \\
\hline Vanadium & $<0.005$ & $<0.005$ & $<0.005$ & $<0.005$ & $<0.005$ & $<0.005$ & $<0.005$ & $<0.005$ \\
\hline zine & 0.011 & 0.0079 & 0.018 & 0.0095 & 0.023 & 0.0027 & 0.02 & 0.021 \\
\hline MAJOR IONS $(\mathrm{mg} / \mathrm{L})$ & • & - & $\cdot$ & • & $\cdot$ & $\cdot$ & $\cdot$ & • \\
\hline Alkalinity-HCO3 & $\dot{14}$ & • & $\dot{14}$ & • & $10 \dot{0}$ & $\cdot$ & 108 & • \\
\hline Alkalinity-CO3 & $<1$ & . & $<1$ & . & $<1$ & . & $<1$ & . \\
\hline Calcium & 2.5 & 1.8 & 65 & 1.7 & 15 & 14 & 16 & 15 \\
\hline Chloride & 1 & - & 1.2 & - & $<1$ & . & 1.5 & . \\
\hline Fluoride & $<0.1$ & - & $<0.1$ & - & 0.3 & - & 0.4 & . \\
\hline Magnesium & 1.6 & 1 & 11 & 1 & 3.5 & 3.5 & 3.6 & 3.6 \\
\hline Manganese & 0.082 & 0.0019 & 0.02 & 0.0018 & 0.59 & 0.15 & 0.21 & 0.2 \\
\hline Nitrate-N & $<0.2$ & . & $<0.2$ & - & $<0.2$ & - & $<0.2$ & . \\
\hline Potaseilum & 0.86 & 0.74 & 3.2 & $<0.6$ & 1.4 & 1.4 & 1.4 & 1.3 \\
\hline Sodium & 2.6 & 2.5 & 1.6 & 2.5 & 29 & 29 & 31 & 30 \\
\hline sulfate & 2.1 & • & 2.9 & - & 11 & - & 16 & . \\
\hline
\end{tabular}

(CONTINUED) 
APPENDIX E.1

\begin{tabular}{|c|c|c|c|c|c|c|c|c|}
\hline \multirow{4}{*}{$\begin{array}{l}\text { Sampling Point } \\
\text { Iocation } \\
\text { - } \\
\text { Date Sampled }\end{array}$} & \multicolumn{4}{|c|}{$G W-654$} & \multicolumn{4}{|c|}{$G W-655$} \\
\hline & \multicolumn{4}{|c|}{ BG } & \multicolumn{4}{|c|}{ BG } \\
\hline & \multicolumn{2}{|c|}{$07 / 01 / 93$} & \multicolumn{2}{|c|}{$10 / 07 / 93$} & \multicolumn{2}{|c|}{$02 / 05 / 93$} & \multicolumn{2}{|c|}{$05 / 10 / 93$} \\
\hline & TOT & DIS & TOT & DIS & TOT & DIS & TOT & DIS \\
\hline METALS (mg/L) & $\cdot$ & $\cdot$ & $\cdot$ & $\cdot$ & $\cdot$ & $\cdot$ & $\cdot$ & - \\
\hline Aluminum & 0.26 & $<0.02$ & 0.1 & $<0.02$ & 1.1 & $<0.02$ & 16 & $<0.02$ \\
\hline Antimony & $<0.05$ & $<0.05$ & $<0.05$ & $<0.05$ & $<0.05$ & $<0.05$ & $<0.05$ & $<0.05$ \\
\hline Arsenic & $<0.05$ & $<0.05$ & $<0.05$ & $<0.05$ & $<0.05$ & $<0.05$ & $<0.05$ & $<0.05$ \\
\hline Barium & 0.084 & 0.078 & 0.11 & 0.11 & 0.16 & 0.14 & 0.52 & 0.13 \\
\hline Bery 111 um & $<0.0003$ & $<0.0003$ & $<0.0003$ & $<0.0003$ & $<0.0003$ & 0.00037 & 0.0015 & $<0.0003$ \\
\hline Boron & 0.067 & 0.064 & 0.068 & 0.068 & 0.02 & 0.0077 & 0.064 & 0.022 \\
\hline Cadmiun (AAS) & $<0.002$ & 0.0021 & $<0.002$ & $<0.002$ & $<0.002$ & $<0.002$ & $<0.002$ & $<0.002$ \\
\hline Cadmium & $<0.003$ & $<0.003$ & $<0.003$ & 0.0039 & $<0.003$ & $<0.003$ & $<0.003$ & $<0.003$ \\
\hline Chromium (AAS) & $<0.01$ & $<0.01$ & $<0.01$ & $<0.01$ & $<0.01$ & $<0.01$ & 0.038 & $<0.01$ \\
\hline Chromium & $<0.01$ & $<0.01$ & $<0.01$ & $<0.01$ & $<0.01$ & $<0.01$ & 0.037 & $<0.01$ \\
\hline Cobalt & $<0.005$ & $<0.005$ & $<0.005$ & $<0.005$ & $<0.005$ & $<0.005$ & 0.0092 & $<0.005$ \\
\hline Copper & $<0.004$ & $<0.004$ & $<0.004$ & $<0.004$ & 0.0058 & $<0.004$ & 0.02 & $<0.004$ \\
\hline Iron & 0.5 & 0.19 & 0.33 & 0.15 & 1.8 & 0.35 & 30 & 0.32 \\
\hline Lead (AAS) & $<0.004$ & $<0.004$ & $<0.004$ & $<0.004$ & $<0.004$ & $<0.004$ & 0.017 & $<0.004$ \\
\hline Mercury (CVAA) & $<0.0002$ & $<0.0002$ & $<0.0002$ & $<0.0002$ & $<0.0002$ & $<0.0002$ & $<0.0002$ & $<0.0002$ \\
\hline Molybdenum & $<0.01$ & $<0.01$ & $<0.01$ & $<0.01$ & $<0.01$ & $<0.01$ & $<0.01$ & $<0.01$ \\
\hline Nickel & $<0.01$ & $<0.01$ & $<0.01$ & $<0.01$ & $<0.01$ & $<0.01$ & $<0.01$ & $<0.01$ \\
\hline Selenium & $<0.05$ & $<0.05$ & $<0.05$ & $<0.05$ & $<0.05$ & $<0.05$ & $<0.05$ & $<0.05$ \\
\hline Silver & $<0.006$ & $<0.006$ & $<0.006$ & $<0.006$ & $<0.006$ & $<0.006$ & $<0.006$ & $<0.006$ \\
\hline strontium & 0.16 & 0.18 & 0.21 & 0.22 & 0.02 & 0.019 & 0.047 & 0.019 \\
\hline Thorium & $<0.2$ & $<0.2$ & $<0.2$ & $<0.2$ & $<0.2$ & $<0.2$ & $<0.2$ & $<0.2$ \\
\hline Uranium (Fluor) & $<0.001$ & $<0.001$ & $<0.001$ & $<0.001$ & $<0.001$ & $<0.001$ & 0.002 & $<0.001$ \\
\hline Vanadium & $<0.005$ & $<0.005$ & $<0.005$ & $<0,005$ & $<0.005$ & $<0.005$ & 0.04 & $<0.005$ \\
\hline zine & 0.0097 & 0.012 & 0.025 & 0.023 & 0.014 & 0.0098 & 0.063 & 0.0097 \\
\hline MAJOR IONS $(\mathrm{mg} / \mathrm{L})$ &. &. & $\cdot$ &. & $\cdot$ &. &. & - \\
\hline Alkalinity-HCO3 & 115 & $\cdot$ & 127 &. & 34 &. & 32 & • \\
\hline Alkalinity-CO3 & $<1$ &. & $<1$ &. & $<1$ &. & $<1$ & . \\
\hline Calcium & 11 & 13 & 15 & 16 & 3.2 & 3 & 5.7 & 3 \\
\hline Chloride & $<1$ & $\cdot$ & $<1$ &. & $<1$ & $\cdot$ & 1.3 & • \\
\hline Fluoride & 0.5 &. & 0.5 &. & 0.2 &. & 0.2 & - \\
\hline Magnesium & 2.8 & 3.3 & 4.1 & 4.3 & 5.6 & 5.2 & 10 & 4.9 \\
\hline Manganese & 0.094 & 0.15 & 0.25 & 0.27 & 0.034 & 0.029 & 0.15 & 0.034 \\
\hline Nitrate-N & $<0.2$ & . & $<0.2$ &. & $<0.2$ & . & $<0.2$ & • \\
\hline Potassium & 1 & 1.7 & 1.7 & 1.7 & 1.6 & 1.3 & 4.4 & 1.5 \\
\hline Sodium & 47 & 44 & 39 & 37 & 5.9 & 5.8 & 6.2 & 5.7 \\
\hline sulfate & 16 & $\cdot$ & 15 & $\cdot 1$ & 9.2 & . & 14 & . \\
\hline
\end{tabular}


APPENDIX R.1

Groundwater and Surface-Water Quality Data, 1993

\begin{tabular}{|c|c|c|c|c|c|c|c|c|}
\hline \multirow{4}{*}{$\begin{array}{l}\text { Sampling point } \\
\text { Location } \\
\text { Date sampled }\end{array}$} & \multicolumn{4}{|c|}{ GW-655 } & \multicolumn{4}{|c|}{$G W-683$} \\
\hline & \multicolumn{4}{|c|}{ BG } & \multicolumn{4}{|c|}{$\operatorname{EXP}$} \\
\hline & \multicolumn{2}{|c|}{$07 / 01 / 93$} & \multicolumn{2}{|c|}{$10 / 06 / 93$} & \multicolumn{2}{|c|}{$03 / 04 / 93$} & \multicolumn{2}{|c|}{$04 / 23 / 93$} \\
\hline & TOT & DIS & TOT & DIs & TOT & DIs & TOT & DIS \\
\hline MRTALS (mg/L) & . & - & - & $\cdot$ & - &. & - & \\
\hline Aluminum & 0.29 & $<0.02$ & 0.5 & $<0.02$ & $<0.02$ & $<0.02$ & 0.025 & $<0.02$ \\
\hline Antimony & $<0.05$ & $<0.05$ & $<0.05$ & $<0.05$ & $<0.05$ & $<0.05$ & $<0.05$ & $<0.05$ \\
\hline Arsenic & $<0.05$ & $<0.05$ & $<0.05$ & $<0.05$ & $<0.05$ & $<0.05$ & $<0.05$ & $<0.05$ \\
\hline Barium & 0.15 & 0.13 & 0.16 & 0.12 & 0.06 & 0.06 & 0.058 & 0.057 \\
\hline Beryllium & $<0.0003$ & $<0.0003$ & $<0.0003$ & $<0.0003$ & $<0.0003$ & $<0.0003$ & $<0.0003$ & $<0.0003$ \\
\hline Boron & 0.0062 & 0.0055 & 0.0075 & 0.0082 & 0.061 & 0.039 & 0.011 & 0.016 \\
\hline Cadmium (MAS) & $<0.002$ & $<0.002$ & $<0.002$ & $<0.002$ & $<0.002$ & $<0.002$ & $<0.002$ & $<0.002$ \\
\hline Cacmium & $<0.003$ & $<0.003$ & $<0.003$ & $<0.003$ & $<0.003$ & $<0.003$ & $<0,003$ & $<0.003$ \\
\hline Chromium (AAS) & $<0.01$ & $<0.01$ & $<0.01$ & $<0.01$ & $<0.01$ & $<0.01$ & $<0.01$ & $<0.01$ \\
\hline Chromium & $<0.01$ & $<0.01$ & $<0.01$ & $<0.01$ & $<0.01$ & $<0.01$ & $<0.01$ & $<0.01$ \\
\hline Cobalt & $<0.005$ & $<0.005$ & $<0.005$ & $<0.005$ & $<0.005$ & $<0.005$ & $<0.005$ & $<0.005$ \\
\hline Copper & $<0.004$ & $<0.004$ & 0.004 & $<0.004$ & $<0.004$ & $<0.004$ & $<0.004$ & $<0.004$ \\
\hline Iron & 0.75 & 0.11 & 1.5 & 0.18 & 0.0075 & $<0.005$ & 0.042 & $<0.005$ \\
\hline Lead (AAS) & $<0.004$ & $<0.004$ & $<0.004$ & $<0.004$ & $<0.004$ & $<0.004$ & $<0.004$ & $<0.004$ \\
\hline Mercury (CVAA) & $<0.0002$ & $<0.0002$ & $<0.0002$ & $<0.0002$ & $<0.00022$ & $<0.00022$ & $<0.0002$ & $<0.0002$ \\
\hline Molybdenum & $<0.01$ & $<0.01$ & $<0.01$ & $<0.01$ & $<0.01$ & $<0.01$ & $<0.01$ & $<0.01$ \\
\hline Hickel & $<0.01$ & $<0.01$ & $<0.01$ & $<0.01$ & $<0.01$ & $<0.01$ & $<0.01$ & $<0.01$ \\
\hline Selenium & $<0.05$ & $<0.05$ & $<0.05$ & $<0.05$ & $<0.05$ & $<0.05$ & $<0.05$ & $<0.05$ \\
\hline silver & $<0.006$ & $<0.006$ & $<0.006$ & $<0.006$ & $<0.006$ & $<0.006$ & $<0.006$ & $<0.006$ \\
\hline Strontium & 0.021 & 0.021 & 0.02 & 0.016 & 0.058 & 0.057 & 0.047 & 0.046 \\
\hline Thorium & $<0.2$ & $<0.2$ & $<0.2$ & $<0.2$ & $<0.2$ & $<0.2$ & $<0.2$ & $<0.2$ \\
\hline Uranium (Fluor) & $<0.001$ & $<0.001$ & $<0.001$ & $<0.001$ & 0.022 & 0.022 & 0.013 & 0.014 \\
\hline Vanadium & $<0.005$ & $<0.005$ & $<0.005$ & $<0.005$ & $<0.005$ & $<0.005$ & $<0.005$ & $<0.005$ \\
\hline zine & 0.017 & 0.021 & 0.044 & 0.013 & 0.0077 & 0.0085 & 0.012 & 0.0074 \\
\hline MAJOR IONS (mg/L) & . & . & . &. & . & . & . & . \\
\hline Alkalinity-HCO3 & 34 & • & $\dot{36}$ & $\cdot$ & $138^{\circ}$ & $\cdot$ & 132 & • \\
\hline Alkalinity- $\mathrm{CO} 3$ & $<1$ & . & $<1$ & . & $<1$ & . & $<1$ & • \\
\hline Calcium & 3.2 & 3.5 & 3.5 & 2.9 & 38 & 38 & 35 & 34 \\
\hline Chloride & $<1$ & - & $<1$ & . & 7.1 & . & 5.7 & . \\
\hline Fluoride & 0.2 & - & 0.1 & . & 0.1 & . & 0.1 & . \\
\hline Magnesium & 5.6 & 5.6 & 5.7 & 4.9 & 15 & 15 & 14 & 14 \\
\hline Manganese & 0.032 & 0.026 & 0.042 & 0.028 & $<0.001$ & $<0.001$ & 0.0015 & 0.0015 \\
\hline witrate-N & $<0.2$ & . & $<0.2$ & . & 2.7 & . & 2.3 & . \\
\hline Potassium & 1.9 & 1.9 & 2.1 & 1.1 & 1.4 & 1.3 & 0.92 & 0.96 \\
\hline Sodium & 6.5 & 6.7 & 6.2 & 5.8 & 3.6 & 3.6 & 3.4 & 3.4 \\
\hline Sulfate & 10 & . & 8 & • & 7.1 & . & 11 & • \\
\hline
\end{tabular}

(CONT INUED) 
APPENDIX E. 1

Groundwater and Surface-Water Quality Data, 1993

\begin{tabular}{|c|c|c|c|c|c|c|c|c|}
\hline \multirow{4}{*}{$\begin{array}{l}\text { Sampling point } \\
\text { Location } \\
\text { Date Sampled }\end{array}$} & \multicolumn{4}{|c|}{$G W-683$} & \multicolumn{4}{|c|}{$G W-684$} \\
\hline & \multicolumn{4}{|c|}{$\mathbf{E X P}$} & \multicolumn{4}{|c|}{ EXP } \\
\hline & \multicolumn{2}{|c|}{$07 / 12 / 93$} & \multicolumn{2}{|c|}{$10 / 12 / 93$} & \multicolumn{2}{|c|}{$03 / 03 / 93$} & \multicolumn{2}{|c|}{$04 / 22 / 93$} \\
\hline & TOT & DIS & TOT & DIS & TOT & DIS & TOT & DIS \\
\hline METALS (mg/L) & - & $\cdot$ & $\cdot$ & $\cdot$ & $\cdot$ & - & $\cdot$ & $\cdot$ \\
\hline Aluminum & $<0.02$ & $<0.02$ & 0.055 & 0.042 & 0.026 & $<0.02$ & 0.038 & $<0.02$ \\
\hline Antimony & $<0.05$ & $<0.05$ & $<0.05$ & $<0.05$ & $<0.05$ & $<0.05$ & $<0.05$ & $<0.05$ \\
\hline Arsenic & $<0.05$ & $<0.05$ & $<0.05$ & $<0.05$ & $<0.05$ & $<0.05$ & $<0.05$ & $<0.05$ \\
\hline Barium & 0.1 & 0.1 & 0.15 & 0.15 & 0.072 & 0.071 & 0.074 & 0.072 \\
\hline Beryllium & $<0.0003$ & $<0.0003$ & $<0.0003$ & $<0.0003$ & $<0.0003$ & $<0.0003$ & $<0.0003$ & $<0.0003$ \\
\hline Boron & 0.11 & 0.088 & 0.12 & 0.13 & 0.027 & 0.03 & 0.03 & 0.036 \\
\hline Cadmium (AAS) & $<0.002$ & $<0.002$ & $<0.002$ & $<0.002$ & $<0.002$ & $<0.002$ & $<0.002$ & $<0.002$ \\
\hline Cadmium & $<0.003$ & $<0.003$ & $<0.003$ & $<0.003$ & $<0.003$ & $<0.003$ & $<0.003$ & $<0.003$ \\
\hline Chromium (AAS) & $<0.01$ & $<0.01$ & $<0.01$ & $<0.01$ & $<0.01$ & $<0.01$ & $<0.01$ & $<0.01$ \\
\hline Chromium & $<0.01$ & $<0.01$ & $<0.01$ & $<0.01$ & $<0.01$ & $<0.01$ & $<0.01$ & $<0.01$ \\
\hline Cobalt & $<0.005$ & $<0.005$ & $<0.005$ & $<0.005$ & $<0.005$ & $<0.005$ & $<0.005$ & $<0.005$ \\
\hline Copper & $<0.004$ & $<0.004$ & $<0.004$ & $<0.004$ & $<0.004$ & $<0.004$ & $<0.004$ & $<0.004$ \\
\hline Iron & 0.012 & $<0.005$ & 0.18 & 0.014 & 0.02 & $<0.005$ & 0.038 & 0.016 \\
\hline Lead (AAS) & $<0.004$ & $<0.004$ & $<0.004$ & $<0.004$ & $<0.004$ & $<0.004$ & $<0.004$ & $<0.004$ \\
\hline Mercury (CVAA) & $<0.0002$ & $<0.0002$ & $<0.0002$ & $<0.0002$ & $<0.00022$ & $<0.00022$ & $<0.0002$ & $<0.0002$ \\
\hline Molybdenum & $<0.02$ & $<0.01$ & $<0.01$ & $<0.01$ & $<0.01$ & $<0.01$ & $<0.01$ & $<0.01$ \\
\hline Nickel & $<0.01$ & $<0.01$ & $<0.01$ & $<0.01$ & 0.011 & $<0.01$ & $<0.01$ & $<0.01$ \\
\hline Selenium & $<0.05$ & $<0.05$ & $<0.05$ & $<0.05$ & $<0.05$ & $<0.05$ & $<0.05$ & $<0.05$ \\
\hline Silver & $<0.006$ & $<0.006$ & $<0.006$ & $<0.006$ & $<0.006$ & $<0.006$ & $<0.006$ & $<0.006$ \\
\hline Strontium & 0.12 & 0.11 & 0.2 & 0.2 & 0.095 & 0.094 & 0.082 & 0.081 \\
\hline Thorium & $<0.2$ & $<0.2$ & $<0.2$ & $<0.2$ & $<0.2$ & $<0.2$ & $<0.2$ & $<0.2$ \\
\hline Uranium (Fluor) & 0.061 & 0.048 & 0.11 & 0.109 & 0.026 & 0.025 & 0.019 & 0.019 \\
\hline Vanadium & $<0.005$ & $<0.005$ & $<0.005$ & $<0.005$ & $<0.005$ & $<0.005$ & $<0.005$ & $<0.005$ \\
\hline zinc & 0.0078 & 0.006 & 0.012 & 0.011 & 0.0064 & 0.0062 & 0.0085 & 0.0055 \\
\hline MAJOR IONS $(\mathrm{mg} / \mathrm{L})$ & . & $\cdot$ & . & $\cdot$ & $\cdot$ & - & $\cdot$ & • \\
\hline Alkalinity $-\mathrm{HCO} 3$ & $19 \dot{1}$ & $\cdot$ & $21 \dot{4}$ & . & 129 & • & 126 & • \\
\hline Alkalinity-CO3 & $<1$ & - & $<1$ & . & $<1$ & - & $<1$ & • \\
\hline Calcium & 63 & 61 & 94 & 94 & 42 & 40 & 32 & 32 \\
\hline Chloride & 14.4 & . & 31 & . & 17 & • & 16 & • \\
\hline Fluoride & 0.2 & - & 0.2 & . & 0.1 & - & 0.2 & • \\
\hline Magnesium & 20 & 19 & 23 & 23 & 13 & 13 & 12 & 12 \\
\hline Manganese & 0.0089 & 0.0095 & 0.0055 & 0.0028 & 0.0026 & $<0.001$ & 0.0024 & 0.003 \\
\hline Nitrate-N & 10.01 & . & 32 & . & 4.3 & - & 4.1 & . \\
\hline Potassium & 2.7 & 3.6 & 2.3 & 2.3 & 15 & 15 & 20 & 20 \\
\hline Sodium & 8.6 & 8.6 & 14 & 14 & 6 & 5.7 & 6.4 & 6.3 \\
\hline Sulfate & 16.9 & . & 26 & - & 13 & - & 14 & $\cdot$ \\
\hline
\end{tabular}

(CONTINUED) 
APPENDIX E.1

Groundwater and Surface-Water Quallty Data, 1993

\begin{tabular}{|c|c|c|c|c|c|c|c|c|}
\hline \multirow{4}{*}{$\begin{array}{l}\text { Sampling Point } \\
\text { Location } \\
\text { Date Samplad }\end{array}$} & \multicolumn{4}{|c|}{$G W-684$} & \multicolumn{4}{|c|}{$G W-685$} \\
\hline & \multicolumn{4}{|c|}{$\mathbf{E x p}$} & \multicolumn{4}{|c|}{$\mathbf{E X P}$} \\
\hline & \multicolumn{2}{|c|}{$07 / 11 / 93$} & \multicolumn{2}{|c|}{$10 / 12 / 93$} & \multicolumn{2}{|c|}{$03 / 02 / 93$} & \multicolumn{2}{|c|}{$04 / 21 / 93$} \\
\hline & TOT & DIs & TOT & DIS & TOT & DIs & TOT & Drs \\
\hline MeTars (mg/L) &. & $\cdot$ & . & $\cdot$ & $\cdot$ & - & - & $\cdot$ \\
\hline Aluminum & $<0.02$ & $<0.02$ & 0.074 & $<0.02$ & 0.29 & $<0.02$ & 0.32 & $<0.02$ \\
\hline Antimony & $<0.05$ & $<0.05$ & $<0.05$ & $<0.05$ & $<0.05$ & $<0.05$ & $<0.05$ & $<0.05$ \\
\hline Armenic & $<0.05$ & $<0.05$ & $<0.05$ & $<0.05$ & $<0.05$ & $<0.05$ & $<0.05$ & $<0.05$ \\
\hline Barium & 0.099 & 0.099 & 0.13 & 0.13 & 0.12 & 0.12 & 0.11 & 0.093 \\
\hline Beryllium & $<0.0003$ & $<0.0003$ & $<0.0003$ & $<0.0003$ & $<0.0003$ & $<0.0003$ & $<0.0003$ & $<0.0003$ \\
\hline Boron & 0.098 & 0.096 & 0.093 & 0.092 & 0.04 & 0.037 & 0.029 & 0.24 \\
\hline Cadmium (AAS) & $<0.002$ & $<0.002$ & $<0.002$ & $<0.002$ & $<0.002$ & $<0.002$ & $<0.002$ & $<0.002$ \\
\hline Cadmium & $<0.003$ & $<0.003$ & $<0.003$ & $<0.003$ & $<0.003$ & $<0.003$ & $<0.003$ & $<0.003$ \\
\hline Chromium (AAS) & $<0.01$ & $<0.01$ & $<0.01$ & $<0.01$ & $<0.01$ & $<0.01$ & $<0.01$ & $<0.01$ \\
\hline Chromium & $<0.01$ & $<0.01$ & $<0.01$ & $<0.01$ & $<0.01$ & $<0.01$ & $<0.01$ & $<0.01$ \\
\hline Cobalt & $<0.005$ & $<0.005$ & $<0.005$ & $<0.005$ & $<0.005$ & $<0.005$ & $<0.005$ & $<0.005$ \\
\hline Copper & $<0.004$ & $<0.004$ & $<0.004$ & $<0.004$ & $<0.004$ & $<0.004$ & $<0.004$ & $<0.004$ \\
\hline Iron & 0.042 & 0.011 & 0.24 & $<0.005$ & 6.3 & 0.097 & 1.5 & 0.04 \\
\hline Laad (AMS) & $<0.004$ & $<0.004$ & $<0.004$ & $<0.004$ & $<0.004$ & $<0.004$ & $<0.004$ & $<0.004$ \\
\hline Mercury (CVAA) & $<0.0002$ & $<0.0002$ & $<0.0002$ & $<0.0002$ & $<0.00022$ & $<0.00022$ & $<0.0002$ & $<0.0002$ \\
\hline Mol ybdenum & $<0.01$ & $<0.01$ & $<0.01$ & $<0.01$ & $<0.01$ & $<0.01$ & $<0.01$ & $<0.01$ \\
\hline Nlckel & $<0.01$ & $<0.01$ & $<0.01$ & $<0.01$ & $<0.01$ & $<0.01$ & $<0.01$ & $<0.01$ \\
\hline Selenium & $<0.05$ & $<0.05$ & $<0.05$ & $<0.05$ & $<0.05$ & $<0.05$ & $<0.05$ & $<0.05$ \\
\hline silver & 0.015 & $<0.006$ & $<0.006$ & $<0.006$ & $<0.006$ & $<0.006$ & $<0.006$ & $<0.006$ \\
\hline strontium & 0.13 & 0.13 & 0.19 & 0.19 & 0.13 & 0.13 & 0.11 & 0.11 \\
\hline Thorium & $<0.2$ & $<0.2$ & $<0.2$ & $<0.2$ & $<0.2$ & $<0.2$ & $<0.2$ & $<0.2$ \\
\hline Uranium (Fluor) & 0.062 & 0.066 & 0.114 & 0.075 & 0.002 & 0.003 & 0.003 & 0.003 \\
\hline Vanadium & $<0.005$ & $<0.005$ & $<0.005$ & $<0.005$ & $<0.005$ & $<0.005$ & $<0.005$ & $<0.005$ \\
\hline zine & 0.0065 & 0.0051 & 0.0083 & 0.0089 & 0.0057 & $<0.002$ & 0.012 & 0.0046 \\
\hline MAJOR IONS $(\mathrm{mg} / \mathrm{L})$ & $\cdot$ & $\cdot$ & . & $\cdot$ & $\cdot$ & • &. & - \\
\hline Alkalinity-HCO3 & $17 \dot{0}$ & $\cdot$ & 174 & $\cdot$ & 250 & $\cdot$ & 245 & • \\
\hline Alkalinity-CO3 & $<1$ & $\cdot$ & $<1$ & $\cdot$ & $<1$ & $\cdot$ & $<1$ & - \\
\hline Calcium & 56 & 56 & 66 & 65 & 82 & 84 & 79 & 76 \\
\hline Chloride & 20.8 & $\cdot$ & 28 & $\cdot 1$ & 69 & $\cdot$ & 94 & • \\
\hline Fluoride & 0.3 & $\cdot$ & 0.2 & $\cdot$ & $<0.1$ & $\cdot$ & 0.1 & - \\
\hline Magnesium & 17 & 17 & 18 & 18 & 23 & 24 & 23 & 21 \\
\hline Manganese & 0.0053 & 0.0051 & 0.013 & 0.01 & 0.14 & 0.079 & 0.12 & 0.041 \\
\hline Nitrate-N & 10.3 & $\cdot$ & 18.9 & $\cdot$ & 1.7 & • & 1.63 & • \\
\hline Potassium & 17 & 16 & 14 & 14 & 1.8 & 1.7 & 1.1 & 1.3 \\
\hline sodium & 10 & 10 & 18 & 18 & 33 & 34 & 28 & 28 \\
\hline sulfate & 16.7 & $\cdot$ & $<20$ & $\cdot$ & 19 & - & 18.3 & \\
\hline
\end{tabular}

(CONTINUED) 
APPENDIX R.1

Groundwater and Surface-Water Quality Data, 1993

\begin{tabular}{|c|c|c|c|c|c|c|c|c|}
\hline \multirow{4}{*}{$\begin{array}{l}\text { Sampling Point } \\
\text { Iocation } \\
\text { Date Sampled }\end{array}$} & \multicolumn{4}{|c|}{$G W-685$} & \multicolumn{4}{|c|}{ GW-694 } \\
\hline & \multicolumn{4}{|c|}{$\operatorname{EXP}$} & \multicolumn{4}{|c|}{$\operatorname{EXP}$} \\
\hline & \multicolumn{2}{|c|}{$07 / 11 / 93$} & \multicolumn{2}{|c|}{$10 / 11 / 93$} & \multicolumn{2}{|c|}{$03 / 23 / 93$} & \multicolumn{2}{|c|}{$06 / 06 / 93$} \\
\hline & TOT & DIS & TOT & DIS & TOT & DIS & TOT & DIS \\
\hline METALS $(\mathrm{mg} / \mathrm{L})$ & - & - & $\cdot$ & $\cdot$ & $\cdot$ & $\cdot$ & $\cdot$ & . \\
\hline Aluminum & 0.051 & $<0.02$ & 0.091 & 0.029 & $<0.02$ & $<0.02$ & 0.023 & $<0.02$ \\
\hline Antimony & $<0.05$ & $<0.05$ & $<0.05$ & $<0.05$ & $<0.05$ & $<0.05$ & $<0.05$ & $<0.05$ \\
\hline Arsenic & $<0.05$ & $<0.05$ & $<0.05$ & $<0.05$ & $<0.05$ & $<0.05$ & $<0.05$ & $<0.05$ \\
\hline Barium & 0.099 & 0.098 & 0.1 & 0.1 & 0.11 & 0.1 & 0.17 & 0.16 \\
\hline Beryllium & $<0.0003$ & $<0.0003$ & $<0.0003$ & $<0.0003$ & 0.00035 & $<0.0003$ & $<0.0003$ & $<0.0003$ \\
\hline Boron & 0.03 & 0.054 & 0.057 & 0.052 & 0.1 & 0.11 & 0.17 & 0.2 \\
\hline Cadmium (AAS) & $<0.002$ & $<0.002$ & $<0.002$ & $<0.002$ & $<0.002$ & $<0.002$ & $<0.002$ & $<0.002$ \\
\hline Cadmium & $<0.003$ & $<0.003$ & 0.0034 & $<0.003$ & $<0.003$ & $<0.003$ & $<0.003$ & $<0.003$ \\
\hline Chromium (AAS) & $<0.01$ & $<0.01$ & $<0.01$ & $<0.01$ & $<0.01$ & $<0.01$ & $<0.01$ & $<0.01$ \\
\hline Chromium & $<0.01$ & $<0.01$ & $<0.01$ & $<0.01$ & $<0.01$ & $<0.01$ & $<0.01$ & $<0.01$ \\
\hline Cobalt & $<0.005$ & $<0.005$ & $<0.005$ & $<0.005$ & $<0.005$ & $<0.005$ & $<0.005$ & $<0.005$ \\
\hline Copper & $<0.004$ & $<0.004$ & $<0.004$ & $<0.004$ & $<0.004$ & $<0.004$ & $<0.004$ & $<0.004$ \\
\hline Iron & 0.16 & $<0.005$ & 2.2 & 0.44 & 0.62 & 0.045 & 1.3 & 0.58 \\
\hline Lead (AAS) & $<0.004$ & $<0.004$ & $<0.004$ & $<0.004$ & $<0.004$ & $<0.004$ & $<0.004$ & $<0.004$ \\
\hline Mercury (CVAA) & $<0.0002$ & $<0.0002$ & $<0.0002$ & $<0.0002$ & $<0.0002$ & $<0.0002$ & $<0.0002$ & $<0.0002$ \\
\hline Mol ybdenum & $<0.01$ & $<0.01$ & $<0.01$ & $<0.01$ & $<0.01$ & $<0.01$ & $<0.01$ & $<0.01$ \\
\hline Nickel & $<0.01$ & $<0.01$ & $<0.01$ & $<0.01$ & 0.017 & 0.017 & $<0.01$ & 0.018 \\
\hline Selenium & $<0.05$ & $<0.05$ & $<0.05$ & $<0.05$ & $<0.05$ & $<0.05$ & $<0.05$ & $<0.05$ \\
\hline silver & $<0.006$ & $<0.006$ & $<0.006$ & $<0.006$ & $<0.006$ & $<0.006$ & $<0.006$ & $<0.006$ \\
\hline Strontium & 0.11 & 0.11 & 0.12 & 0.12 & 0.17 & 0.17 & 0.29 & 0.28 \\
\hline Thorium & $<0.2$ & $<0.2$ & $<0.2$ & $<0.2$ & $<0.2$ & $<0.2$ & $<0.2$ & $<0.2$ \\
\hline Uranium (Fluor) & 0.003 & 0.003 & 0.005 & 0.005 & 0.115 & 0.111 & 0.121 & 0.015 \\
\hline Vanadium & $<0.005$ & $<0.005$ & $<0.005$ & $<0.005$ & $<0.005$ & $<0.005$ & $<0.005$ & $<0.005$ \\
\hline zinc & 0.014 & 0.0097 & 0.014 & 0.0071 & 0.0062 & 0.0044 & $<0.002$ & 0.002 \\
\hline MAJOR IONS (mg/L) &. &. & - & - &. &. &. & • \\
\hline Alkalinity-HCO3 & $\begin{array}{r}\cdot \\
253\end{array}$ & $\cdot$ & $25 \dot{2}$ & . & 202 & $\cdot$ & 218 & • \\
\hline Alkalinity- $\mathrm{CO} 3$ & $<1$ & . & $<1$ & - & $<1$ & . & $<1$ & • \\
\hline Calcium & 82 & 81 & 79 & 83 & 79 & 79 & 110 & 100 \\
\hline Chloride & 48.9 & $\cdot$ & 50 & - & 30 & $\cdot$ & 33 & $\cdot$ \\
\hline Fluoride & 0.1 & $\cdot$ & 0.1 & - & 0.3 & - & 0.4 & $\cdot$ \\
\hline Magnesium & 24 & 24 & 23 & 24 & 17 & 17 & 21 & 21 \\
\hline Manganese & 0.065 & 0.043 & 0.1 & 0.083 & 0.062 & 0.059 & 0.053 & 0.055 \\
\hline Nitrate-N & 2.1 & $\cdot$ & 4 & - & 10 & - & 29.25 & • \\
\hline Potassium & 1.6 & 1.3 & 1.7 & 1.7 & 2.2 & 2.1 & 3 & 3 \\
\hline Sodium & 21 & 22 & 21 & 22 & 14 & 14 & 20 & 20 \\
\hline sulfate & 19.6 & $\cdot$ & 21 & - & 21 & - & 30.2 & • \\
\hline
\end{tabular}

(CONTINUED) 
APPENDIX E. 1

Groundwater and surface-Water Quality Data, 1993

\begin{tabular}{|c|c|c|c|c|c|c|c|c|}
\hline \multirow{4}{*}{$\begin{array}{l}\text { Sampling Point } \\
\text { Location } \\
\text { - } \\
\text { Date Sampled }\end{array}$} & \multicolumn{4}{|c|}{$G W-694$} & \multicolumn{4}{|c|}{$6 w-695$} \\
\hline & \multicolumn{4}{|c|}{$\exp$} & \multicolumn{4}{|c|}{$\operatorname{Exp}$} \\
\hline & \multicolumn{2}{|c|}{$09 / 22 / 93$} & \multicolumn{2}{|c|}{$12 / 28 / 93$} & \multicolumn{2}{|c|}{$03 / 24 / 93$} & \multicolumn{2}{|c|}{$06 / 09 / 93$} \\
\hline & TOT & DIS & TOT & DIs & TOT & DIs & TOT & DIs \\
\hline MRTALS (mg/L) & $\cdot$ & $\cdot$ & $\cdot$ & . & $\cdot$ & $\cdot$ & $\cdot$ & - \\
\hline Aluminum & $<0.02$ & 0.038 & $<0.02$ & $<0.02$ & 0.057 & 0.036 & 1.6 & $<0.02$ \\
\hline Antimony & $<0.05$ & $<0.05$ & $<0.05$ & $<0.05$ & $<0.05$ & $<0.05$ & $<0.05$ & $<0.05$ \\
\hline Arsenic & $<0.05$ & $<0.05$ & $<0.05$ & $<0.05$ & $<0.05$ & $<0.05$ & $<0.05$ & $<0.05$ \\
\hline Barium & 0.18 & 0.18 & 0.16 & 0.15 & 0.025 & 0.023 & 0.028 & 0.022 \\
\hline Beryllium & $<0.0003$ & $<0.0003$ & $<0.0003$ & $<0.0003$ & $<0.0003$ & $<0.0003$ & $<0.0003$ & $<0.0003$ \\
\hline Boron & 0.22 & 0.22 & 0.14 & 0.15 & 0.049 & 0.059 & 0.069 & 0.039 \\
\hline Cadmium (AAS) & $<0.002$ & $<0.002$ & $<0.002$ & $<0.002$ & $<0.002$ & $<0.002$ & $<0.002$ & $<0.002$ \\
\hline Cadmium & $<0.003$ & $<0.003$ & $<0.003$ & $<0.003$ & $<0.003$ & $<0.003$ & $<0.003$ & $<0.003$ \\
\hline Chromium (AAS) & $<0.01$ & $<0.01$ & $<0.01$ & $<0.01$ & $<0.01$ & $<0.01$ & $<0.01$ & $<0.01$ \\
\hline Chromium & $<0.01$ & $<0.01$ & $<0.01$ & $<0.01$ & $<0.01$ & $<0.01$ & $<0.01$ & $<0.01$ \\
\hline Cobalt & $<0.005$ & $<0.005$ & $<0.005$ & $<0.005$ & $<0.005$ & $<0.005$ & $<0.005$ & $<0.005$ \\
\hline Copper & $<0.004$ & $<0.004$ & 0.0076 & $<0.004$ & $<0.004$ & $<0.004$ & 0.0065 & 0.0059 \\
\hline Iron & 1.9 & 1.8 & 0.47 & 0.29 & 0.025 & $<0.005$ & 0.74 & 0.013 \\
\hline Load (AAS) & $<0.004$ & $<0.004$ & $<0.004$ & $<0.004$ & $<0.004$ & $<0.004$ & $<0.004$ & $<0.004$ \\
\hline Marcury (CVAA) & $<0.0002$ & $<0.0002$ & $<0.0002$ & $<0.0002$ & $<0.0002$ & $<0.0002$ & $<0.0002$ & $<0.0002$ \\
\hline Molybdenum & $<0.01$ & $<0.01$ & $<0.01$ & $<0.01$ & $<0.01$ & $<0.01$ & $<0.01$ & 0.011 \\
\hline Nickel & $<0.01$ & 0.023 & $<0.01$ & $<0.01$ & $<0.01$ & $<0.01$ & $<0.01$ & $<0.02$ \\
\hline Selenium & $<0.05$ & $<0.05$ & $<0.05$ & $<0.05$ & $<0.05$ & $<0.05$ & $<0.05$ & $<0.05$ \\
\hline silver & $<0.006$ & $<0.006$ & $<0.006$ & $<0.006$ & $<0.006$ & $<0.006$ & $<0.006$ & $<0.006$ \\
\hline strontium & 0.35 & 0.35 & 0.33 & 0.32 & 0.061 & 0.059 & 0.06 & 0.05 \\
\hline Thorium & $<0.2$ & $<0.2$ & $<0.2$ & $<0.2$ & $<0.2$ & $<0.2$ & $<0.2$ & $<0.2$ \\
\hline Uranium (Fluor) & 0.198 & 0.194 & 0.3 & 0.31 & 0.002 & 0.002 & 0.003 & 0.002 \\
\hline Vanadium & $<0.005$ & $<0.005$ & $<0.005$ & $<0.005$ & $<0.005$ & $<0.005$ & $<0.005$ & $<0.005$ \\
\hline zinc & 0.0063 & 0.0081 & $<0.002$ & 0.0047 & 0.0036 & 0.0036 & 0.018 & 0.013 \\
\hline MAJOR IONS (mg/L) & - &. & . & . &. &. &. & • \\
\hline Alkalinity-HCO3 & 262 &. & 232 & $\cdot$ & 170 & $\cdot$ & 179 & . \\
\hline Alkalinity-CO3 & $<1$ &. & $<1$ &. & $<1$ &. & $<1$ & • \\
\hline Calcium & 120 & 120 & 120 & 120 & 28 & 27 & 38 & 29 \\
\hline Chloride & 40 &. & 28.5 & . & 7.3 & $\cdot 1$ & 9.2 & • \\
\hline Fluoride & 0.5 & $\cdot$ & 0.4 & . & 0.1 & . & 0.1 & • \\
\hline Magnesium & 24 & 24 & 22 & 22 & 15 & 15 & 19 & 15 \\
\hline Manganese & 0.046 & 0.045 & 0.12 & 0.12 & $<0.001$ & 0.001 & 0.0053 & 0.0012 \\
\hline Nitrate-N & 31 & $\cdot$ & 29.2 & . & 5.7 & $\cdot$ & 16 & . \\
\hline Potassium & 4.2 & 4.1 & 3.2 & 3 & 8.8 & 8.4 & 4.7 & 5.5 \\
\hline Sodium & 25 & 25 & 16 & 16 & 29 & 28 & 33 & 42 \\
\hline Sulfate & 36 & $\cdot 1$ & 40.6 & . & 12 & $\cdot$ & 12 & • \\
\hline
\end{tabular}

(CONTINUED) 
APPENDIX E.1

Groundwater and Surface-Water Quality Data, 1993

\begin{tabular}{|c|c|c|c|c|c|c|c|c|}
\hline \multirow{4}{*}{$\begin{array}{l}\text { Sampling point } \\
\text { Location } \\
\text { Date sampled }\end{array}$} & \multicolumn{4}{|c|}{$6 w-695$} & \multicolumn{4}{|c|}{ OW-703 } \\
\hline & \multicolumn{4}{|c|}{$\operatorname{Exp}$} & \multicolumn{4}{|c|}{$\mathbf{E X P}$} \\
\hline & \multicolumn{2}{|c|}{$09 / 22 / 93$} & \multicolumn{2}{|c|}{$12 / 21 / 93$} & \multicolumn{2}{|c|}{$03 / 20 / 93$} & \multicolumn{2}{|c|}{$06 / 03 / 93$} \\
\hline & TOT & DIs & TOT & DIs & TOT & DIs & TOT & DIS \\
\hline HETALS (mg/L) & $\cdot$ & $\cdot$ & $\cdot$ & $\cdot$ & $\cdot$ & $\cdot 1$ & $\cdot$ & - \\
\hline Aluminum & 2.13 & 0.023 & 2.6 & $<0.02$ & 0.036 & 0.043 & $<0.02$ & $<0.02$ \\
\hline Antimony & $<0.05$ & $<0.05$ & $<0.05$ & $<0.05$ & $<0.05$ & $<0.05$ & $<0.05$ & $<0.05$ \\
\hline Arsentc & $<0.05$ & $<0.05$ & $<0.05$ & $<0.05$ & $<0.05$ & $<0.05$ & $<0.05$ & $<0.05$ \\
\hline Barium & 0.025 & 0.026 & 0.026 & 0.02 & 0.082 & 0.081 & 0.03 & 0.21 \\
\hline Beryllium & 0.00047 & $<0.0003$ & $<0.0003$ & $<0.0003$ & $<0.0003$ & $<0.0003$ & $<0.0003$ & $<0.0003$ \\
\hline Boron & 0.043 & 0.057 & 0.045 & 0.041 & 0.12 & 0.11 & 0.069 & 0.099 \\
\hline Cadmium (AAS) & $<0.002$ & $<0.002$ & $<0.002$ & $<0.002$ & $<0.002$ & $<0.002$ & $<0.002$ & $<0.002$ \\
\hline Cadmium & $<0.003$ & $<0.003$ & $<0.003$ & $<0.003$ & $<0.003$ & $<0.003$ & $<0.003$ & $<0.003$ \\
\hline Chromium (AAS) & $<0.01$ & $<0.01$ & 0.012 & $<0.01$ & $<0.01$ & $<0.01$ & $<0.01$ & $<0.01$ \\
\hline Chromium & 0.011 & $<0.01$ & 0.013 & $<0.01$ & $<0.01$ & $<0.01$ & $<0.01$ & $<0.01$ \\
\hline Cobalt & $<0.005$ & $<0.005$ & $<0.005$ & $<0.005$ & $<0.005$ & $<0.005$ & $<0.005$ & $<0.005$ \\
\hline Copper & $<0.004$ & $<0.004$ & 0.0071 & $<0.004$ & $<0.004$ & $<0.004$ & $<0.004$ & $<0.004$ \\
\hline Iron & 1 & 0.035 & 2.5 & $<0.005$ & 0.37 & 0.017 & 4.7 & $<0.005$ \\
\hline Lead (AAS) & $<0.004$ & $<0.008$ & 0.0065 & $<0.004$ & $<0.004$ & $<0.004$ & $<0.004$ & $<0.004$ \\
\hline Mercury (CVAA) & $<0.0002$ & $<0.0002$ & $<0.0002$ & $<0.0002$ & $<0.0002$ & $<0.0002$ & $<0.0002$ & $<0.0002$ \\
\hline Mol ybdenum & $<0.01$ & $<0.01$ & $<0.01$ & $<0.01$ & $<0.01$ & $<0.01$ & $<0.01$ & $<0.01$ \\
\hline Nickel & $<0.01$ & $<0.01$ & $<0.01$ & $<0.01$ & 0.012 & 0.011 & $<0.01$ & $<0.01$ \\
\hline Selenium & $<0.05$ & $<0.05$ & $<0.05$ & $<0.05$ & $<0.05$ & $<0.05$ & $<0.05$ & 0.057 \\
\hline Silver & $<0.006$ & $<0.006$ & $<0.006$ & $<0.006$ & $<0.006$ & $<0.006$ & $<0.006$ & $<0.006$ \\
\hline strontium & 0.057 & 0.061 & 0.064 & 0.051 & 0.24 & 0.24 & 0.14 & 0.36 \\
\hline Thorium & $<0.2$ & $<0.2$ & $<0.2$ & $<0.2$ & $<0.2$ & $<0.2$ & $<0.2$ & $<0.2$ \\
\hline Oranium (Fluor) & 0.003 & 0.002 & 0.002 & 0.002 & 0.002 & 0.003 & 0.001 & 0.001 \\
\hline Vanadium & $<0.005$ & $<0.005$ & 0.0063 & $<0.005$ & $<0.005$ & $<0.005$ & $<0.005$ & $<0.005$ \\
\hline zine & 0.027 & 0.011 & 0.013 & $<0.002$ & 0.0068 & 0.0054 & 0.0052 & $<0.002$ \\
\hline MAJOR IONS $(\mathrm{mg} / \mathrm{L})$ & $\cdot$ & $\cdot$ & - & $\cdot$ & • & - & . & • \\
\hline Alkalinity-HCO3 & 175 & $\cdot$ & 183 & $\cdot$ & 201 & $\cdot$ & $17 \dot{0}$ & - \\
\hline Alkaltnity-CO3 & $<1$ & . & $<1$ & . & $<1$ & . & $<1$ & . \\
\hline Calcium & 29 & 32 & 39 & 23 & 72 & 70 & 40 & 42 \\
\hline Chloride & 6.1 & . & 9.7 &. & 22 & . & 15 & - \\
\hline Fluoride & 0.2 & $\cdot$ & 0.1 & . & 0.2 & . & 0.1 & • \\
\hline Magnesium & 16 & 18 & 16 & 13 & 32 & 32 & 28 & 29 \\
\hline Manganese & 0.008 & 0.0046 & 0.016 & $<0.001$ & 0.0033 & 0.002 & 0.1 & 0.072 \\
\hline Nitrate-N & 6.2 & $\cdot$ & 7.7 & • & 22.36 & - & 9.6 & 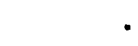 \\
\hline Potassium & 9.9 & 7.1 & 9.7 & 10 & 1.6 & 1.3 & 1.6 & 2.2 \\
\hline Sodium & 35 & 27 & 40 & 43 & 11 & 10 & 8.2 & 32 \\
\hline sulfate & 14 & $\cdot$ & 9.7 & $\cdot$ & 23.1 & . & 18 & - \\
\hline
\end{tabular}

(CONTINUED) 
APPEADIX $\mathrm{E} .1$

Oroundwater and surface-Water Quallty Data, 1993

\begin{tabular}{|c|c|c|c|c|c|c|c|c|}
\hline \multirow{4}{*}{$\begin{array}{l}\text { Sampling Polnt } \\
\text { Iooation } \\
\text { Date sampled }\end{array}$} & \multicolumn{4}{|c|}{ OW-703 } & \multicolumn{4}{|c|}{ ON-704 } \\
\hline & \multicolumn{4}{|c|}{$\operatorname{EXP}$} & \multicolumn{4}{|c|}{$\mathbf{E x p}$} \\
\hline & \multicolumn{2}{|c|}{$09 / 20 / 93$} & \multicolumn{2}{|c|}{$12 / 21 / 93$} & \multicolumn{2}{|c|}{$03 / 22 / 93$} & \multicolumn{2}{|c|}{$06 / 04 / 93$} \\
\hline & TOT & DIs & rot & Drs & TOT & DIs & $\operatorname{Tor}$ & DIS \\
\hline Merals (mg/L) & $\cdot$ & - & . & $\cdot 1$ & . & . & . & - \\
\hline Aluminum & $<0.02$ & $<0.02$ & 0.027 & $<0.02$ & 0.021 & 0.025 & 0.15 & $<0.02$ \\
\hline Ant imony & $<0.05$ & $<0.05$ & $<0.05$ & $<0.05$ & $<0.05$ & $<0.05$ & $<0.05$ & $<0.05$ \\
\hline Arsenic & 0.086 & $<0.05$ & $<0.05$ & $<0.05$ & $<0.05$ & $<0.05$ & $<0.05$ & $<0.05$ \\
\hline Barium & 0.066 & 0.058 & 0.095 & 0.093 & 0.08 & 0.077 & 0.11 & 0.068 \\
\hline Beryl11um & $<0.0003$ & $<0.0003$ & $<0.0003$ & $<0.0003$ & $<0.0003$ & $<0.0003$ & $<0.0003$ & $<0.0003$ \\
\hline Boron & 0.094 & 0.093 & 0.094 & 0.12 & 0.09 & 0.094 & 0.26 & 0.08 \\
\hline Cadmium (MAS) & $<0.002$ & $<0.002$ & $<0.002$ & $<0.002$ & $<0.002$ & $<0.002$ & $<0.002$ & $<0.002$ \\
\hline Cadmium & $<0.003$ & $<0.003$ & $<0.003$ & $<0.003$ & $<0.003$ & $<0.003$ & $<0.003$ & $<0.003$ \\
\hline Chromium (MAS) & $<0.01$ & $<0.01$ & $<0.01$ & $<0.02$ & $<0.01$ & $<0.01$ & $<0.01$ & $<0.01$ \\
\hline Chromium & $<0.01$ & $<0.01$ & $<0.01$ & $<0.01$ & $<0.01$ & $<0.01$ & $<0.01$ & $<0.01$ \\
\hline Cobalt & $<0.005$ & $<0.005$ & $<0.005$ & $<0.005$ & $<0.005$ & $<0.005$ & $<0.005$ & $<0.005$ \\
\hline Copper & $<0.004$ & $<0.004$ & $<0.004$ & $<0.004$ & $<0.004$ & $<0.004$ & $<0.004$ & $<0.004$ \\
\hline Iron & 0.68 & $<0.005$ & 0.69 & $<0.005$ & 2.3 & 0.33 & 23 & 0.045 \\
\hline Lead (MAS) & $<0.004$ & $<0.004$ & $<0.004$ & $<0.004$ & $<0.004$ & $<0.004$ & $<0.004$ & $<0.004$ \\
\hline Mercury (CVAA) & $<0.0002$ & $<0.0002$ & $<0.0002$ & $<0.0002$ & $<0.0002$ & $<0.0002$ & $<0.0002$ & $<0.0002$ \\
\hline Mol ybdenum & $<0.01$ & $<0.01$ & $<0.01$ & $<0.01$ & $<0.01$ & $<0.01$ & $<0.01$ & $<0.01$ \\
\hline NLckel & $<0.01$ & $<0.01$ & $<0.01$ & $<0.01$ & 0.01 & $<0.01$ & 0.012 & $<0.01$ \\
\hline Selenium & $<0.05$ & $<0.05$ & $<0.05$ & $<0.05$ & $<0.05$ & $<0.05$ & $<0.05$ & $<0.05$ \\
\hline silver & $<0.006$ & $<0.006$ & $<0.006$ & $<0.006$ & $<0.006$ & $<0.006$ & $<0.006$ & $<0.006$ \\
\hline strontium & 0.22 & 0.22 & 0.23 & 0.23 & 0.38 & 0.38 & 0.35 & 0.33 \\
\hline Thorium & $<0.2$ & $<0.2$ & $<0.2$ & $<0.2$ & $<0.2$ & $<0.2$ & $<0.2$ & $<0.2$ \\
\hline Uranium (Fluor) & 0.002 & 0.002 & 0.004 & 0.003 & 0.001 & 0.001 & 0.001 & 0.001 \\
\hline Vanadium & $<0.005$ & $<0.005$ & $<0.005$ & $<0.005$ & $<0.005$ & $<0.005$ & $<0.005$ & $<0.005$ \\
\hline zinc & 0.013 & 0.014 & 0.0065 & 0.0038 & 0.0064 & 0.0061 & 0.023 & 0.0074 \\
\hline MAJOR IONS (mg/L) & $\cdot$ & $\cdot$ & $\cdot$ & $\cdot$ & $\cdot$ & $\cdot$ & $\cdot$ & $\bullet$ \\
\hline Alkalintty-HCO3 & 199 & • & $21 \dot{2}$ & $\cdot$ & 185 & - & 184 & - \\
\hline Alkalinity- $\mathrm{CO} 3$ & $<1$ & . & $<1$ &. & $<1$ & . & $<1$ & . \\
\hline Calcium & 61 & 59 & 74 & 74 & 63 & 63 & 57 & 55 \\
\hline Chloride & 20.5 & . & 22.5 &. & 19.6 & . & 22 & 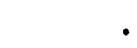 \\
\hline Fluoride & 0.2 & . & 0.1 &. & 0.2 & . & 0.2 & . \\
\hline Magnesium & 31 & 31 & 32 & 32 & 29 & 29 & 28 & 27 \\
\hline Manganese & 0.034 & 0.04 & 0.017 & 0.0028 & 0.066 & 0.062 & 0.11 & 0.043 \\
\hline Nitrate-N & 21.8 & . & 22.6 &. & 14.28 & . & 15 & 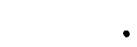 \\
\hline Potassium & 3.8 & 4.4 & 2.1 & 2 & 1.4 & 1.4 & 1.3 & 1.3 \\
\hline Sodium & 9.8 & 10 & 11 & 11 & 11 & 11 & 10 & 10 \\
\hline Sulfate & 19.1 & . & 21.9 &. & 20.7 & . & 20 & $\bullet$ \\
\hline
\end{tabular}

(CONTINUED) 
APPENDIX E.1

Groundwater and gurface-Water Quality Data, 1993

\begin{tabular}{|c|c|c|c|c|c|c|c|c|}
\hline \multirow{4}{*}{$\begin{array}{l}\text { Sampling Polnt } \\
\text { Location } \\
\text { Date sampled }\end{array}$} & \multicolumn{4}{|c|}{ 6W-704 } & \multicolumn{4}{|c|}{ GW-706 } \\
\hline & \multicolumn{4}{|c|}{$\exp$} & \multicolumn{4}{|c|}{$\mathbf{E X P}$} \\
\hline & \multicolumn{2}{|c|}{$09 / 22 / 93$} & \multicolumn{2}{|c|}{$12 / 22 / 93$} & \multicolumn{2}{|c|}{$03 / 23 / 93$} & \multicolumn{2}{|c|}{$06 / 11 / 93$} \\
\hline & Tor & Drs & TOT & Drs & TOT & Dr8 & TOT & Drs \\
\hline Matrars (mg/L) & . & $\cdot 1$ &. & $\cdot$ & $\cdot$ & $\cdot$ & - & 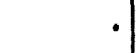 \\
\hline Aluminum & 0.06 & $<0.02$ & $<0.02$ & 0.023 & 0.04 & 0.039 & 0.024 & $<0.02$ \\
\hline Antimony & $<0.05$ & $<0.05$ & $<0.05$ & $<0.05$ & $<0.05$ & $<0.05$ & $<0.05$ & $<0.05$ \\
\hline Armenic & $<0.05$ & $<0.05$ & $<0.05$ & $<0.03$ & $<0.05$ & 0.062 & $<0.05$ & $<0.05$ \\
\hline Barlum & 0.098 & 0.082 & 0.017 & 0.015 & 0.016 & 0.014 & 0.16 & 0.16 \\
\hline Beryllium & $<0.0003$ & $<0.0003$ & $<0.0003$ & $<0.0003$ & 0.00061 & 0.00061 & $<0.0003$ & $<0.0003$ \\
\hline Boron & 0.095 & 0.088 & 0.052 & 0.091 & 0.18 & 0.17 & 0.17 & 0.16 \\
\hline Cadmium (MAS) & $<0.002$ & $<0.002$ & $<0.002$ & $<0.002$ & $<0.002$ & $<0.002$ & $<0.002$ & $<0.002$ \\
\hline Cadmium & 0.0054 & $<0.003$ & $<0.003$ & $<0.003$ & $<0.003$ & $<0.003$ & $<0.003$ & $<0.003$ \\
\hline Chromium (AAS) & $<0.01$ & $<0.01$ & $<0.01$ & $<0.01$ & $<0.01$ & $<0.01$ & $<0.01$ & $<0.01$ \\
\hline Chromium & $<0.01$ & $<0.01$ & $<0.01$ & $<0.01$ & $<0.01$ & $<0.01$ & $<0.01$ & $<0.01$ \\
\hline Cobalt & $<0.005$ & $<0.005$ & $<0.005$ & $<0.005$ & $<0.005$ & $<0.005$ & $<0.005$ & $<0.005$ \\
\hline Copper & $<0.004$ & $<0.004$ & $<0.004$ & $<0.004$ & 0.0064 & $<0.004$ & 0.0048 & $<0.004$ \\
\hline Iron & 14 & 0.26 & 9.7 & 0.063 & 3.9 & 0.016 & 0.64 & 0.5 \\
\hline Lead (AAS) & $<0.004$ & $<0.004$ & $<0.004$ & $<0.004$ & $<0.004$ & $<0.004$ & $<0.004$ & $<0.004$ \\
\hline Mercury (CVAa) & $<0.0002$ & $<0.0002$ & $<0.0002$ & $<0.0002$ & $<0.0002$ & $<0.0002$ & $<0.0002$ & $<0.0002$ \\
\hline Molybdenum & $<0.01$ & $<0.01$ & $<0.01$ & $<0.01$ & $<0.01$ & $<0.01$ & $<0.01$ & $<0.01$ \\
\hline Nickel & $<0.01$ & $<0.01$ & $<0.01$ & $<0.01$ & 0.012 & $<0.01$ & $<0.01$ & $<0.01$ \\
\hline Selenium & $<0.05$ & $<0.05$ & $<0.05$ & $<0.05$ & $<0.05$ & $<0.05$ & $<0.05$ & 0.06 \\
\hline silver & $<0.006$ & $<0.006$ & $<0.006$ & $<0.006$ & $<0.006$ & $<0.006$ & $<0.006$ & $<0.006$ \\
\hline strontium & 0.4 & 0.4 & 0.072 & 0.069 & 0.067 & 0.062 & 0.3 & 0.3 \\
\hline Thorium & $<0.2$ & $<0.2$ & $<0.2$ & $<0.2$ & $<0.2$ & $<0.2$ & $<0.2$ & $<0.2$ \\
\hline Uranium (Fluor) & 0.001 & 0.002 & $<0.001$ & $<0.001$ & 0.002 & 0.005 & 0.137 & 0.158 \\
\hline Vanadium & $<0.005$ & $<0.005$ & $<0.005$ & $<0.005$ & $<0.005$ & $<0.005$ & $<0.005$ & $<0.005$ \\
\hline Zinc & 0.013 & 0.0057 & $<0.002$ & $<0.002$ & 0.01 & 0.0055 & 0.013 & 0.0052 \\
\hline MAJOR IONS (mg/L) & . & $\cdot$ &. & $\cdot$ & $\cdot$ &. & $\cdot$ & $\cdot$ \\
\hline Alkalinity-HCO3 & $\begin{array}{r}0 \\
195\end{array}$ & $\cdot$ & 125 & $\cdot$ & 93 &. & 241 & • \\
\hline Alkalinity-CO3 & $<1$ & $\cdot$ & 2 & . & 8 & . & $<1$ & - \\
\hline Calcium & 65 & 64 & 15 & 14 & 11 & 11 & 110 & 110 \\
\hline Chloride & 21 & . & 21.7 & .1 & 31 &. & 36 & • \\
\hline Fluoride & 0.2 & . & 0.1 &. & 0.2 &. & 0.3 & - \\
\hline Magnesium & 30 & 30 & 29 & 29 & 22 & 21 & 23 & 22 \\
\hline Manganese & 0.094 & 0.045 & 0.16 & 0.088 & 0.11 & 0.05 & 0.02 & 0.018 \\
\hline Nitrate-N & 17 & $\cdot$ & 0.23 & $\cdot$ & $<0.2$ & $\cdot$ & 32 & $\theta^{\circ}$ \\
\hline Potassium & 1.5 & 1.5 & 1.3 & 1.3 & 6.6 & 6.4 & 5.3 & 5 \\
\hline Sodium & 12 & 12 & 12 & 11 & 20 & 20 & 19 & 19 \\
\hline Sulfate & 23 & $\cdot 1$ & 18.6 & $\cdot$ & 33 & $\cdot$ & 30 & • \\
\hline
\end{tabular}

(CONTINUED) 
APPEMDIX 1.1

Croundwater and Burface-Water Quallty Data, 1993

\begin{tabular}{|c|c|c|c|c|c|c|c|c|}
\hline \multirow{4}{*}{$\begin{array}{l}\text { Sampling point } \\
\text { Location } \\
\text { Date sampled }\end{array}$} & \multicolumn{4}{|c|}{ ON-706 } & \multicolumn{4}{|c|}{ OW-710 } \\
\hline & \multicolumn{4}{|c|}{$\operatorname{Exp}$} & \multicolumn{4}{|c|}{$\operatorname{Exp}$} \\
\hline & \multicolumn{2}{|c|}{$09 / 23 / 93$} & \multicolumn{2}{|c|}{$12 / 28 / 93$} & \multicolumn{2}{|c|}{$03 / 21 / 93$} & \multicolumn{2}{|c|}{$04 / 30 / 93$} \\
\hline & TOT & DI8 & TOT & DI8 & Tor & DIs & $\operatorname{son}$ & D18 \\
\hline wraxs (mg/L) & - & - & - & - & $\cdot$ & - & $\cdot$ & \\
\hline Aluminum & 0.045 & $<0.02$ & $<0.02$ & $<0.02$ & $<0.2$ & $<0.2$ & 0.22 & 0.34 \\
\hline Ant Imony & $<0.05$ & $<0.05$ & $<0.05$ & $<0.05$ & $<0.5$ & $<0.5$ & $<0.25$ & $<0.25$ \\
\hline Arsenic & $<0.05$ & $<0.05$ & $<0.05$ & $<0.05$ & $<0.5$ & $<0.3$ & $<0.25$ & $<0.25$ \\
\hline Barlum & 0.02 & 0.016 & 0.15 & 0.14 & 0.012 & $<0.01$ & 0.013 & 0.0094 \\
\hline Beryll1um & $<0.0003$ & $<0.0003$ & $<0.0003$ & $<0.0003$ & $<0.003$ & $<0.003$ & $<0.0015$ & $<0.0015$ \\
\hline Boron & 0.14 & 0.13 & 0.25 & 0.21 & 0.31 & 0.32 & 0.33 & 0.34 \\
\hline CadmLim (MAs) & $<0.002$ & $<0.002$ & $<0.002$ & $<0.002$ & $<0,002$ & $<0.002$ & $<0.002$ & $<0.002$ \\
\hline Cadmium & 0.0066 & $<0.003$ & $<0.003$ & $<0.003$ & $<0.03$ & $<0.03$ & 0.015 & $<0.015$ \\
\hline Chromium (MAS) & $<0.01$ & $<0.01$ & 0.043 & $<0.01$ & $<0.01$ & $<0.01$ & $<0.01$ & $<0.01$ \\
\hline Chromium & $<0.01$ & $<0.01$ & $<0.01$ & $<0.01$ & $<0.1$ & $<0.1$ & $<0.05$ & $<0.05$ \\
\hline Cobalt & $<0.005$ & $<0.005$ & $<0.005$ & $<0.005$ & $<0.05$ & $<0.05$ & $<0.025$ & $<0.025$ \\
\hline Copper & 0.01 & $<0.004$ & $<0.004$ & $<0.004$ & $<0.04$ & $<0.04$ & $<0.02$ & $<0.02$ \\
\hline Iron & 13 & $<0.005$ & 0.5 & 0.3 & 12 & 11 & 4.7 & 4.4 \\
\hline Lead (MAS) & $<0.004$ & $<0.004$ & $<0.004$ & $<0.004$ & $<0.004$ & $<0.004$ & $<0.004$ & $<0.004$ \\
\hline hercury (CVMA) & $<0.0002$ & $<0.0002$ & $<0.0002$ & $<0.0002$ & $<0.0002$ & $<0.0002$ & $<0.0002$ & $<0.0002$ \\
\hline Molybdenum & $<0.01$ & $<0.01$ & $<0.01$ & $<0.01$ & $<0.1$ & $<0.1$ & $<0.05$ & $<0.05$ \\
\hline Nlokel & 0.012 & $<0.01$ & $<0.01$ & $<0.01$ & $<0.1$ & $<0.1$ & $<0.05$ & $<0.05$ \\
\hline Selentum & $<0.05$ & $<0.05$ & $<0.05$ & $<0.05$ & $<0.5$ & $<0.5$ & $<0.25$ & $<0.25$ \\
\hline silver & $<0.006$ & $<0.006$ & $<0.006$ & $<0.006$ & $<0.06$ & $<0.06$ & $<0.03$ & 0.046 \\
\hline strontium & 0.062 & 0.058 & 0.32 & 0.3 & 9.7 & 9.7 & 8.4 & 8.2 \\
\hline Thorium & $<0.2$ & $<0.2$ & $<0.2$ & $<0.2$ & $<2$ & $<2$ & $<1$ & $<1$ \\
\hline Uranium (Fluor) & 0.008 & 0.006 & 0.23 & 0.27 & $<0.001$ & $<0.001$ & $<0.001$ & $<0.001$ \\
\hline Vanadium & $<0.005$ & $<0.005$ & $<0.005$ & $<0,005$ & $<0.05$ & $<0.05$ & $<0.025$ & $<0.025$ \\
\hline ainc & 0.015 & 0.0037 & 0.0049 & $<0.002$ & $<0.02$ & $<0.02$ & 0.025 & 0.02 \\
\hline MUTOR IONS $(\mathrm{mg} / \mathrm{L})$ & $\cdot$ & $\cdot$ & $\cdot$ & $\cdot$ & $\cdot$ & $\cdot \cdot$ & $\cdot$ & - \\
\hline Alkalinity-HCO3 & 101 & $\cdot$ & 243 & . & ${ }_{156}^{\circ}$ &. & ${ }_{162}$ & $\cdot$ \\
\hline Alkalinity-co3 & $<1$ & $\cdot$ & $<1$ & . & $<1$ & . & $<1$ & . \\
\hline Calciun & 13 & 13 & 120 & 120 & 630 & 640 & 570 & 550 \\
\hline Chloride & 25 & . & 26 & . & 365 & . & 349 & - \\
\hline Fluoride & 0.2 & $\cdot$ & 0.4 & . & 1.6 &. & 2.7 & . \\
\hline Magnesium & 20 & 20 & 22 & 21 & 200 & 200 & 180 & 180 \\
\hline Manganese & 0.18 & 0.08 & 0.0079 & 0.0058 & 0.22 & 0.2 & 0.081 & 0.081 \\
\hline Nitrate-N & $<0.2$ & $\cdot$ & 28.9 & . & $<11.3$ & . & 74.9 & • \\
\hline Potassium & 5.8 & 5.8 & 3.8 & 3.6 & 11 & 12 & 9.1 & 8.9 \\
\hline Sodium & 18 & 18 & 16 & 15 & 270 & 270 & 230 & 220 \\
\hline sulfate & 26 &. & 39.4 & . & 1800 &. & 2100 & 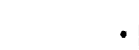 \\
\hline
\end{tabular}

(CONTINUED) 
APPINDIX $\geq .1$

Groundwater and surtace-Water Quallty Data, 1993

\begin{tabular}{|c|c|c|c|c|c|c|c|c|}
\hline \multirow{4}{*}{$\begin{array}{l}\text { sampling polnt } \\
\text { Looation } \\
\text { Date sampled }\end{array}$} & \multicolumn{4}{|c|}{ CW-710 } & \multicolumn{4}{|c|}{$0 N-711$} \\
\hline & \multicolumn{4}{|c|}{$\mathbf{E x p}$} & \multicolumn{4}{|c|}{$\mathbf{E x p}$} \\
\hline & \multicolumn{2}{|c|}{$08 / 01 / 93$} & \multicolumn{2}{|c|}{$11 / 09 / 93$} & \multicolumn{2}{|c|}{$03 / 20 / 93$} & \multicolumn{2}{|c|}{$04 / 29 / 93$} \\
\hline & TOT & Dr8 & nor & DI8 & $\operatorname{sos}$ & DIs & sor & DI8 \\
\hline Mruts (mg/L) & $\cdot 1$ & $\cdot$ & $\cdot$ & $\cdot$ & $\cdot$ &. & $\cdot$ & - \\
\hline Aluminum & $<0.04$ & 0.042 & 0.048 & $<0.04$ & 0.27 & $<0.2$ & $<0.1$ & 0.29 \\
\hline Antimony & $<0.1$ & $<0.1$ & $<0.1$ & $<0.1$ & $<0.5$ & $<0.5$ & $<0.25$ & $<0.5$ \\
\hline Arsento & $<0.1$ & $<0.1$ & $<0.1$ & $<0.1$ & $<0.3$ & $<0.5$ & $<0.23$ & $<0.3$ \\
\hline Barium & 0.007 & 0.0074 & 0.0079 & 0.0076 & 0.017 & $<0.01$ & 0.0069 & 0.027 \\
\hline Boryl11um & $<0.0006$ & $<0.0006$ & $<0.0006$ & $<0.0006$ & $<0.003$ & $<0.003$ & $<0.001 s$ & $<0.003$ \\
\hline Boron & 0.25 & 0.24 & 0.25 & 0.25 & 1.3 & 1.2 & 0.83 & 1.3 \\
\hline Cadmium (MS) & $<0.002$ & $<0.002$ & $<0.002$ & $<0.002$ & $<0,002$ & $<0,002$ & $<0.002$ & $<0.002$ \\
\hline Cadmitum & $<0.006$ & $<0.006$ & $<0.006$ & $<0.006$ & $<0.03$ & $<0.03$ & $<0.015$ & $<0.03$ \\
\hline Chromium (MAs) & $<0.01$ & $<0.01$ & $<0.01$ & $<0.01$ & $<0.01$ & $<0.01$ & $<0.01$ & $<0.01$ \\
\hline Chromiun & $<0.02$ & $<0.02$ & $<0.02$ & $<0.02$ & $<0.1$ & $<0.1$ & $<0.05$ & $<0.1$ \\
\hline cobalt & $<0.01$ & $<0.01$ & $<0.01$ & $<0.01$ & $<0.05$ & $<0.05$ & $<0.025$ & $<0.05$ \\
\hline Copper & $<0.008$ & $<0.008$ & $<0.008$ & $<0.008$ & $<0.04$ & $<0.04$ & $<0.02$ & $<0.04$ \\
\hline Iron & 11 & 9.7 & 11 & 10 & 13 & 10 & 12 & 8.3 \\
\hline Lad (MAs) & $<0.004$ & $<0.004$ & $<0.004$ & $<0.004$ & $<0.004$ & $<0.004$ & $<0.004$ & $<0.004$ \\
\hline Wreury (CVAA) & $<0.0002$ & $<0.0002$ & $<0.0002$ & $<0.0002$ & $<0.0002$ & $<0.0002$ & $<0.0002$ & $<0.0002$ \\
\hline Molybdenum & $<0.02$ & $<0.02$ & $<0.02$ & $<0.02$ & $<0.1$ & $<0.1$ & $<0.05$ & $<0.1$ \\
\hline Mlokel & $<0.02$ & $<0.02$ & $<0.02$ & $<0.02$ & $<0.1$ & $<0.1$ & $<0.05$ & 0.17 \\
\hline selentum & $<0.1$ & $<0.1$ & $<0.1$ & $<0.1$ & $<0.5$ & $<0.5$ & $<0.25$ & $<0.5$ \\
\hline stlver & $<0.012$ & $<0.012$ & $<0.012$ & $<0.012$ & $<0.06$ & $<0.06$ & $<0.03$ & $<0.06$ \\
\hline stront1um & 8.6 & 8.4 & 8.3 & 8.2 & 15 & 15 & 13 & 13 \\
\hline Thorium & $<0.4$ & $<0.4$ & $<0.4$ & $<0.4$ & $<2$ & $<2$ & $<1$ & $<2$ \\
\hline Uranium (Fluor) & $<0.001$ & 0.002 & 0.002 & 0.004 & $<0.001$ & $<0.001$ & $<0.001$ & $<0.001$ \\
\hline Vanadium & $<0.01$ & $<0.01$ & $<0.01$ & $<0.01$ & $<0.05$ & $<0.05$ & $<0.025$ & $<0.05$ \\
\hline zinc & 0.026 & 0.017 & $<0.004$ & $<0.004$ & $<0.02$ & $<0.02$ & $<0.01$ & 0.026 \\
\hline MhJOR IONS (mg/L) &. & - & - & . & - & $\dot{\bullet}$ & $\dot{\bullet}$ & • \\
\hline Alkalinity-HCO3 & 155 & $\cdot$ & $15 \dot{4}$ & $\bullet$ & $14 \dot{3}$ & • & $14 \dot{1}$ & • \\
\hline Alkalinity-CO3 & $<1$ & . & $<1$ & . & $<1$ & . & $<1$ & • \\
\hline Calcium & 580 & 580 & 590 & 580 & 610 & 610 & 550 & 530 \\
\hline Chloride & 295 & • & 339 & - & 1580 & - & 2180 & • \\
\hline Fluoride & $1 . \%$ & . & 1.8 & - & 1.5 & - & 1.6 & • \\
\hline Magnesium & 190 & 190 & 180 & 180 & 250 & 250 & 240 & 260 \\
\hline Manganase & 0.23 & 0.21 & 0.27 & 0.25 & 0.16 & 0.12 & 0.28 & 0.27 \\
\hline Nitrate-N & $<0.2$ & . & $<0.2$ & - & $<11.3$ & . & $<23$ & • \\
\hline Potasnium & 10 & 10 & 12 & 11 & 24 & 23 & 26 & 27 \\
\hline Sodium & 230 & 230 & 220 & 220 & 1300 & 1200 & 1100 & 1200 \\
\hline sulfate & 1540 & - & 1854 & - & 1790 & . & 2148 & • \\
\hline
\end{tabular}

(CONTINUED) 
ApPawdIX 1.1

Oroundwater and surface-water Ounlity Data, 1993

\begin{tabular}{|c|c|c|c|c|c|c|c|c|}
\hline \multirow{4}{*}{$\begin{array}{l}\text { Sampling point } \\
\text { Looation } \\
\text { Date sampled }\end{array}$} & \multicolumn{4}{|c|}{$0 N-711$} & \multicolumn{4}{|c|}{ ON-712 } \\
\hline & \multicolumn{4}{|c|}{$\operatorname{Exp}$} & \multicolumn{4}{|c|}{$\mathbf{E x p}$} \\
\hline & \multicolumn{2}{|c|}{$07 / 30 / 93$} & \multicolumn{2}{|c|}{$11 / 07 / 93$} & \multicolumn{2}{|c|}{$03 / 18 / 93$} & \multicolumn{2}{|c|}{$04 / 28 / 93$} \\
\hline & $\operatorname{xos}$ & DIs & $\operatorname{sot}$ & Drs & TOT & Drs & nor & Drs \\
\hline maxs $(\log / 2)$ & • & $\cdot$ & $\cdot$ & $\cdot$ & $\cdot$ & $\cdot$ & $\cdot$ & • \\
\hline Aluminum & $<0.2$ & $<0.2$ & $<0.04$ & 0.07 & 0.034 & 0.12 & $<0.02$ & $<0.02$ \\
\hline Ant 1mony & $<0.5$ & $<0.5$ & $<0.2$ & $<0.1$ & $<0.05$ & $<0.05$ & $<0.05$ & $<0.05$ \\
\hline Armente & $<0.5$ & $<0.5$ & $<0.1$ & $<0.1$ & $<0.05$ & $<0.05$ & $<0.05$ & $<0.05$ \\
\hline Barium & $<0.01$ & $<0.01$ & 0.0092 & 0.0099 & 0.064 & 0.068 & 0.064 & 0.063 \\
\hline Bery111um & $<0.003$ & $<0.003$ & $<0.0006$ & $<0.0006$ & $<0.0003$ & $<0.0003$ & $<0.0003$ & $<0.0003$ \\
\hline Boron & 1.2 & 1.2 & 1.2 & 1.2 & 0.15 & 0.15 & 0.11 & 0.1 \\
\hline Cadmlum (MAs) & $<0.002$ & $<0.002$ & $<0.002$ & $<0.002$ & $<0.002$ & $<0.002$ & $<0.002$ & $<0.002$ \\
\hline Cadmitum & $<0.03$ & $<0.03$ & $<0.006$ & $<0.006$ & $<0.003$ & $<0.003$ & $<0.003$ & $<0.003$ \\
\hline Chromium (MS) & $<0.01$ & $<0.01$ & $<0.01$ & $<0.01$ & $<0.01$ & $<0.01$ & $<0.01$ & $<0.01$ \\
\hline Chromlum & $<0.1$ & $<0.1$ & $<0.02$ & $<0.02$ & $<0.01$ & $<0.01$ & $<0.01$ & $<0.01$ \\
\hline Cobalt & $<0.05$ & $<0.05$ & $<0.01$ & $<0.01$ & $<0.005$ & $<0.005$ & $<0.005$ & $<0.005$ \\
\hline Copper & $<0.04$ & $<0.04$ & $<0.008$ & $<0.008$ & $<0.004$ & $<0.004$ & $<0.004$ & 0.011 \\
\hline Iron & 9.2 & 6.1 & 9.5 & 6.8 & 1.3 & 1.1 & 0.96 & 0.86 \\
\hline Lead (MAs) & $<0.004$ & $<0.004$ & $<0.004$ & $<0,004$ & $<0.004$ & $<0.004$ & $<0.004$ & $<0.004$ \\
\hline Meroury (CVMA) & $<0.0002$ & $<0.0002$ & $<0.0002$ & $<0.0002$ & $<0.0002$ & $<0.0002$ & $<0.0002$ & $<0.0002$ \\
\hline Molybdenum & $<0.1$ & $<0.1$ & $<0.02$ & $<0.02$ & $<0.01$ & $<0.01$ & $<0.01$ & $<0.01$ \\
\hline Nickel & $<0.1$ & $<0.1$ & $<0.02$ & $<0.02$ & $<0.01$ & $<0.01$ & $<0.01$ & $<0.01$ \\
\hline Selenium & $<0.5$ & $<0.5$ & $<0.1$ & $<0.1$ & $<0.03$ & $<0.05$ & $<0.05$ & $<0.05$ \\
\hline silver & $<0.06$ & $<0.06$ & $<0.012$ & $<0.012$ & $<0.006$ & $<0.006$ & $<0.006$ & $<0.006$ \\
\hline strontlum & 14 & 14 & 13 & 13 & 2.3 & 2.5 & 2.3 & 2.2 \\
\hline Thorlum & $<2$ & $<2$ & $<0.4$ & $<0.4$ & $<0.2$ & $<0.2$ & $<0.2$ & $<0.2$ \\
\hline Uranium (rluor) & $<0.001$ & $<0.001$ & $<0.001$ & $<0.001$ & $<0.001$ & $<0.001$ & $<0.001$ & $<0.001$ \\
\hline Vanedium & $<0.05$ & $<0.05$ & $<0.01$ & $<0.01$ & $<0.005$ & $<0.005$ & $<0.005$ & $<0.005$ \\
\hline Zinc & 0.037 & 0.028 & $<0.004$ & $<0.004$ & 0.0092 & 0.0063 & 0.0044 & 0.035 \\
\hline MuJOR IONS (mg/L) &. & $\cdot$ & $\cdot$ & $\cdot$ & $\cdot$ & $\cdot$ & . & • \\
\hline Alkalinity-HCO3 & 148 &. & 257 & $\cdot$ & 176 & $\cdot$ & 183 & • \\
\hline Alkalinity-CO3 & $<1$ & - & $<1$ & . & $<1$ & $\cdot$ & $<1$ & - \\
\hline Calcium & 590 & 590 & 550 & 530 & 93 & 99 & 89 & 86 \\
\hline Chloride & 2090 & • & 2027 & $\cdot$ & 29 & $\cdot$ & 27 & • \\
\hline Fluoride & 1.7 & . & 1.7 & $\cdot$ & 0.7 & $\cdot 1$ & 0.7 & • \\
\hline Magnealum & 240 & 240 & 220 & 220 & 40 & 43 & 42 & 40 \\
\hline Manganese & 0.2 & 0.098 & 0.086 & 0.071 & 0.015 & 0.018 & 0.014 & 0.013 \\
\hline Nitrate-N & $<2$ & . & $<22.5$ & . & $<0.2$ & . & 0.24 & 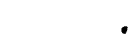 \\
\hline Potassium & 26 & 31 & 23 & 24 & 2.9 & 3.1 & 2.4 & 2.8 \\
\hline Sodium & 1200 & 1200 & 1100 & 1100 & 27 & 28 & 27 & 27 \\
\hline sulfate & 1820 & & 1580 & ${ }^{\circ}$ & 265 & $\cdot$ & 234 & • \\
\hline
\end{tabular}

( CONT INUED) 
APPENDIX 2.1

Oroundwater and surface-water Quallty Data, 1993

\begin{tabular}{|c|c|c|c|c|c|c|c|c|}
\hline \multirow{4}{*}{$\begin{array}{l}\text { 8ampling point } \\
\text { Looution } \\
\text { Date sampled }\end{array}$} & \multicolumn{4}{|c|}{ GN-712 } & \multicolumn{4}{|c|}{$0 N-713$} \\
\hline & \multicolumn{4}{|c|}{$\mathbf{E x P}$} & \multicolumn{4}{|c|}{$\mathbf{E x p}$} \\
\hline & \multicolumn{2}{|c|}{$07 / 29 / 93$} & \multicolumn{2}{|c|}{$11 / 06 / 93$} & \multicolumn{2}{|c|}{$03 / 19 / 93$} & \multicolumn{2}{|c|}{$04 / 30 / 93$} \\
\hline & $\cos$ & Dxs & xor & DI8 & Tor & Drs & $20 x$ & DI8 \\
\hline inturs (ng/L) & $\cdot$ & $\cdot$ & $\cdot$ & $\cdot$ & $\cdot$ & - & - & - \\
\hline Aluminum & $<0.02$ & $<0.02$ & $<0.02$ & $<0.02$ & $<0.02$ & $<0.02$ & $<0.02$ & 0.026 \\
\hline Ant imony & $<0.08$ & $<0.05$ & $<0.05$ & $<0.05$ & $<0.05$ & $<0.05$ & $<0.05$ & $<0.05$ \\
\hline Areenio & $<0.05$ & $<0.05$ & $<0.05$ & $<0.05$ & $<0.05$ & $<0.05$ & $<0.03$ & $<0.05$ \\
\hline Barlum & 0.06 & 0.06 & 0.056 & 0.057 & 0.051 & 0.051 & 0.04 & 0.039 \\
\hline Bery 111 um & $<0.0003$ & $<0.0003$ & $<0.0003$ & $<0.0003$ & $<0.0003$ & $<0.0003$ & $<0.0003$ & $<0.0003$ \\
\hline Boron & 0.12 & 0.11 & 0.11 & 0.11 & 0.1 & 0.11 & 0.063 & 0.069 \\
\hline Cedmitum (MAS) & $<0.002$ & $<0.002$ & $<0.002$ & $<0.002$ & $<0.002$ & $<0.002$ & $<0.002$ & $<0.002$ \\
\hline Cadmium & $<0.003$ & $<0.003$ & $<0.003$ & $<0,003$ & $<0.003$ & $<0.003$ & $<0.003$ & $<0.003$ \\
\hline Chromiun (MS) & $<0.01$ & $<0.01$ & $<0.01$ & $<0.01$ & $<0.01$ & $<0.01$ & $<0.01$ & $<0.01$ \\
\hline Chromium & $<0.01$ & $<0.01$ & $<0.01$ & $<0.01$ & $<0.01$ & $<0.01$ & $<0.01$ & $<0.01$ \\
\hline Cobalt & $<0.005$ & $<0.005$ & $<0.005$ & $<0.005$ & $<0.005$ & $<0.005$ & $<0.005$ & $<0.005$ \\
\hline Copper & $<0.004$ & $<0.004$ & $<0.004$ & $<0.004$ & $<0.004$ & $<0.004$ & $<0.004$ & $<0.004$ \\
\hline Iron & 0.99 & 0.89 & 1 & 0.9 & 2.5 & 1.5 & 2 & 1.2 \\
\hline Laad (MAs) & $<0.004$ & $<0.004$ & $<0.004$ & $<0.004$ & $<0.004$ & $<0.004$ & $<0.004$ & $<0.004$ \\
\hline Marcury (CVMA) & $<0.0002$ & $<0.0002$ & $<0.0002$ & $<0.0002$ & $<0.0002$ & $<0.0002$ & $<0.0002$ & $<0.0002$ \\
\hline Mol ybdenum & $<0.01$ & $<0.01$ & $<0.01$ & $<0.01$ & $<0.01$ & $<0.01$ & $<0.01$ & $<0.01$ \\
\hline nlckel & $<0.01$ & $<0.01$ & $<0.01$ & $<0.01$ & $<0.01$ & $<0.01$ & $<0.01$ & $<0.01$ \\
\hline 8elenium & $<0.05$ & $<0.05$ & $<0.05$ & $<0.05$ & $<0.05$ & $<0.05$ & $<0.05$ & $<0.05$ \\
\hline silver & $<0.006$ & $<0.006$ & $<0.006$ & $<0.006$ & $<0.006$ & $<0.006$ & $<0.006$ & $<0.006$ \\
\hline 8trontium & 2.3 & 2.3 & 2.1 & 2.1 & 1.8 & 1.8 & 1.3 & 1.2 \\
\hline Thorlum & $<0.2$ & $<0.2$ & $<0.2$ & $<0.2$ & $<0.2$ & $<0.2$ & $<0.2$ & $<0.2$ \\
\hline Uranium (Pluor) & 0.001 & 0.001 & $<0.001$ & $<0.001$ & $<0.001$ & 0.001 & $<0.001$ & $<0.001$ \\
\hline Vanadium & $<0.005$ & $<0.005$ & $<0.005$ & $<0,005$ & $<0.003$ & $<0.005$ & $<0.005$ & $<0.005$ \\
\hline $2 \ln 0$ & 0.01 & 0.0088 & $<0.002$ & $<0.002$ & 0.014 & 0.031 & 0.01 & 0.015 \\
\hline MAJOR IONS (mg/L) & $\cdot$ & • &. & . & $\cdot$ & $\cdot$ &. & • \\
\hline Alkalinity-HCO3 & 183 & $\cdot$ & 184 & $\cdot$ & 183 & $\cdot$ & 187 & • \\
\hline Alkalinity-CO3 & $<1$ & . & $<1$ & $\cdot$ & $<1$ & . & $<1$ & - \\
\hline Calcium & 92 & 91 & 80 & 81 & 80 & 80 & 65 & 63 \\
\hline Chloride & 21 & . & 29.9 & $\cdot$ & 1.7 &. & 29 & - \\
\hline Iluoride & 0.8 &. & 0.7 &. & 0.7 &. & 0.6 & • \\
\hline Magnesium & 40 & 40 & 37 & 37 & 36 & 36 & 30 & 30 \\
\hline Manganese & 0.013 & 0.012 & 0.027 & 0.028 & 0.029 & 0.025 & 0.043 & 0.038 \\
\hline Witrato-N & 0.2 & $\cdot$ & 0.2 & $\cdot$ & $<0.2$ & $\cdot$ & 1.4 & - \\
\hline Potaseium & 3.1 & 2.7 & 3 & 3 & 2.3 & 2.2 & 2 & 2.1 \\
\hline Sodium & 28 & 28 & 25 & 26 & 14 & 14 & 10 & 9.9 \\
\hline sulfate & 191 & . & 285 & . & 18 & . & 269 & . \\
\hline
\end{tabular}

(CONTINUED) 
APPENDIX E.1

Croundwater and surface-water Quality Data, 1993

\begin{tabular}{|c|c|c|c|c|c|c|c|c|}
\hline \multirow{4}{*}{$\begin{array}{l}\text { Bampling Polnt } \\
\text { Looation } \\
\text { - } \\
\text { Date sampled }\end{array}$} & \multicolumn{4}{|c|}{$0 W-713$} & \multicolumn{4}{|c|}{ OW-714 } \\
\hline & \multicolumn{4}{|c|}{$\mathbf{E x p}$} & \multicolumn{4}{|c|}{$\mathbf{8 x p}$} \\
\hline & \multicolumn{2}{|c|}{$07 / 31 / 93$} & \multicolumn{2}{|c|}{$11 / 09 / 93$} & \multicolumn{2}{|c|}{$03 / 21 / 93$} & \multicolumn{2}{|c|}{$04 / 29 / 93$} \\
\hline & $\operatorname{sor}$ & DIs & TOT & DI8 & $\operatorname{Tot}$ & DIs & 20T & DIs \\
\hline Mrears (mg/L) & $\cdot 1$ & $\cdot$ & . & - & - & $\cdot 1$ &. & - \\
\hline Aluminum & $<0.02$ & $<0.02$ & $<0.02$ & $<0.02$ & 0.064 & $<0.02$ & 0.2 & $<0.02$ \\
\hline Antimony & $<0.03$ & $<0.05$ & $<0.05$ & $<0.05$ & $<0.05$ & $<0.05$ & $<0.05$ & $<0.05$ \\
\hline Armentr & $<0.05$ & $<0.05$ & $<0.05$ & $<0.05$ & $<0.05$ & $<0.05$ & $<0.05$ & $<0.05$ \\
\hline Barium & 0.046 & 0.045 & 0.05 & 0.049 & 0.059 & 0.058 & 0.07 & 0.065 \\
\hline Bery111um & $<0.0003$ & $<0.0003$ & $<0.0003$ & $<0.0003$ & $<0.0003$ & $<0.0003$ & $<0.0003$ & $<0.0003$ \\
\hline Boron & 0.072 & 0.073 & 0.14 & 0.098 & 0.085 & 0.1 & 0.072 & 0.081 \\
\hline Cadmium (MS) & $<0.002$ & $<0.002$ & $<0.002$ & $<0.002$ & $<0.002$ & $<0.002$ & $<0.002$ & $<0.002$ \\
\hline Cadmium & $<0.003$ & $<0.003$ & $<0.003$ & $<0.003$ & $<0.003$ & $<0.003$ & $<0.003$ & $<0.003$ \\
\hline Chromium (aAs) & $<0.01$ & $<0.01$ & $<0.01$ & $<0.01$ & $<0.01$ & $<0.01$ & $<0.01$ & $<0.01$ \\
\hline Chromium & $<0.01$ & $<0.01$ & $<0.01$ & $<0.01$ & $<0.01$ & $<0.01$ & $<0.01$ & $<0.01$ \\
\hline Cobalt & $<0.005$ & $<0.005$ & $<0.005$ & $<0.005$ & $<0.005$ & $<0.005$ & $<0.005$ & $<0.005$ \\
\hline Copper & 0.042 & $<0.004$ & $<0.004$ & $<0.004$ & $<0.004$ & $<0.004$ & $<0.004$ & $<0.004$ \\
\hline Iron & 2.6 & 1.9 & 1.9 & 1.6 & 1.1 & 0.089 & 8.2 & 0.006 \\
\hline Lead (MAS) & $<0.004$ & $<0.004$ & $<0.004$ & $<0.004$ & $<0.004$ & $<0.004$ & $<0.004$ & $<0.004$ \\
\hline Meroury (CVMA) & $<0.0002$ & $<0.0002$ & $<0.0002$ & $<0.0002$ & $<0.0002$ & $<0.0002$ & $<0.0002$ & $<0.0002$ \\
\hline Molybdenum & $<0.01$ & $<0.01$ & $<0.01$ & $<0.01$ & $<0.01$ & $<0.01$ & $<0.01$ & $<0.01$ \\
\hline Nicke1 & 0.04 & $<0.01$ & $<0.01$ & $<0.01$ & $<0.01$ & $<0.01$ & $<0.01$ & $<0.01$ \\
\hline Selentum & $<0.05$ & $<0.05$ & $<0.05$ & $<0.05$ & $<0.05$ & $<0.05$ & $<0.05$ & $<0.05$ \\
\hline silver & $<0.006$ & $<0.006$ & $<0.006$ & $<0.006$ & $<0.006$ & $<0.006$ & $<0.006$ & $<0.006$ \\
\hline strontium & 1.8 & 1.8 & 1.9 & 1.8 & 0.2 & 0.19 & 0.19 & 0.18 \\
\hline Thorium & $<0.2$ & $<0.2$ & $<0.2$ & $<0.2$ & $<0.2$ & $<0.2$ & $<0.2$ & $<0.2$ \\
\hline Uranium (Fluor) & 0.002 & 0.002 & 0.001 & 0.002 & 0.002 & 0.002 & 0.001 & 0.002 \\
\hline Vanadium & $<0.005$ & $<0.005$ & $<0.005$ & $<0.005$ & $<0.005$ & $<0.005$ & $<0.005$ & $<0.005$ \\
\hline Zino & 0.084 & 0.0092 & 0.0098 & 0.024 & 0.0092 & 0.0082 & 0.0076 & 0.0046 \\
\hline MAJOR IONS (mg/L) &. & $\cdot$ &. & - & - & $\cdot$ & - & • \\
\hline Alkalinity-HCO3 & 184 &. & 187 & $\cdot$ & 298 &. & 191 & • \\
\hline Alkalinity-CO3 & $<1$ &. & $<1$ & . & $<1$ &. & $<1$ & • \\
\hline Calcium & 70 & 70 & 61 & 61 & 57 & 56 & 53 & 53 \\
\hline Chloride & 14.2 &. & 16 & . & 8.7 & . & 9.9 & - \\
\hline Fluoride & 0.7 &. & 0.8 & . & 0.2 &. & 0.2 & . \\
\hline Magnesium & 32 & 32 & 29 & 29 & 20 & 20 & 21 & 21 \\
\hline Manganese & 0.026 & 0.024 & 0.036 & 0.035 & 0.052 & 0.049 & 0.043 & 0.0083 \\
\hline Nitrate-N & $<0.2$ & . & $<0.2$ & . & 4.16 &. & 4.8 & . \\
\hline Potassium & 2.9 & 2.1 & 2.3 & 2.8 & 0.99 & 0.99 & 1.1 & 1.3 \\
\hline Sodium & 16 & 16 & 18 & 17 & 3.1 & 3.1 & 3.6 & 3.2 \\
\hline sulfate & 125 &. & 107 & . & 23.2 & . & 21 & . \\
\hline
\end{tabular}

(CONTINUED) 
APPENDIX E.1

Groundwater and Surface-water Quality Data, 1993

\begin{tabular}{|c|c|c|c|c|c|c|c|c|}
\hline \multirow{4}{*}{$\begin{array}{l}\text { Sampling Point } \\
\text { Location } \\
\text { Date Sampled }\end{array}$} & \multicolumn{4}{|c|}{$G W-714$} & \multicolumn{4}{|c|}{ GW-715 } \\
\hline & \multicolumn{4}{|c|}{$\operatorname{EXP}$} & \multicolumn{4}{|c|}{$\mathbf{E X P}$} \\
\hline & \multicolumn{2}{|c|}{$08 / 01 / 93$} & \multicolumn{2}{|c|}{$11 / 09 / 93$} & \multicolumn{2}{|c|}{$03 / 21 / 93$} & \multicolumn{2}{|c|}{$04 / 29 / 93$} \\
\hline & TOT & DIS & TOT & DIS & TOT & DIs & TOT & DIS \\
\hline KRTALS (mg/L) &. & • & • & • & • & • & • & $\bullet$ \\
\hline Aluminum & 0.046 & 0.022 & 0.066 & $<0.02$ & 0.027 & $<0.02$ & 0.033 & $<0.02$ \\
\hline Antimony & $<0.05$ & $<0.05$ & $<0.05$ & $<0.05$ & $<0.05$ & $<0.05$ & $<0.05$ & $<0.05$ \\
\hline Arsenic & $<0.05$ & $<0.05$ & $<0.05$ & $<0.05$ & $<0.05$ & $<0.05$ & $<0.05$ & $<0.05$ \\
\hline Barium & 0.065 & 0.064 & 0.062 & 0.057 & 0.086 & 0.088 & 0.082 & 0.082 \\
\hline Beryllium & $<0.0003$ & $<0.0003$ & $<0.0003$ & $<0.0003$ & $<0.0003$ & $<0.0003$ & $<0.0003$ & $<0.0003$ \\
\hline Boron & 0.067 & 0.063 & 0.1 & 0.077 & 0.045 & 0.055 & 0.055 & 0.045 \\
\hline Cadmium (AAS) & $<0.002$ & $<0.002$ & $<0.002$ & $<0.002$ & $<0.002$ & $<0.002$ & $<0.002$ & $<0.002$ \\
\hline Cadmium & $<0.003$ & $<0.003$ & $<0.003$ & $<0.003$ & 0.0035 & $<0.003$ & $<0.003$ & $<0.003$ \\
\hline Chromium (AAS) & $<0.01$ & $<0.01$ & $<0.01$ & $<0.01$ & $<0.01$ & $<0.01$ & $<0.01$ & $<0.01$ \\
\hline Chromium & $<0.01$ & $<0.01$ & $<0.01$ & $<0.01$ & $<0.01$ & $<0.01$ & $<0.01$ & $<0.01$ \\
\hline Cobalt & $<0.005$ & $<0.005$ & $<0.005$ & $<0.005$ & $<0.005$ & $<0.005$ & $<0.005$ & $<0.005$ \\
\hline Copper & $<0.004$ & $<0.004$ & 0.0044 & $<0.004$ & $<0.004$ & $<0.004$ & $<0.004$ & $<0.004$ \\
\hline Iron & 0.68 & 0.0052 & 9.3 & 0.58 & 0.069 & $<0.005$ & 0.35 & 0.012 \\
\hline Lead (AAS) & $<0.004$ & $<0.004$ & $<0.004$ & $<0.004$ & $<0.004$ & $<0.004$ & $<0.004$ & $<0.004$ \\
\hline Mercury (CVAA) & $<0.0002$ & $<0.0002$ & $<0.0002$ & $<0.0002$ & $<0.0002$ & $<0.0002$ & $<0.0002$ & $<0.0002$ \\
\hline Mol ybdenum & $<0.01$ & $<0.01$ & $<0.01$ & $<0.01$ & $<0.01$ & $<0.01$ & $<0.01$ & $<0.01$ \\
\hline Nickel & $<0.01$ & $<0.01$ & $<0.01$ & $<0.01$ & $<0.01$ & $<0.01$ & 0.033 & 0.032 \\
\hline Selenium & $<0.05$ & $<0.05$ & $<0.05$ & $<0.05$ & $<0.05$ & $<0.05$ & $<0.05$ & $<0.05$ \\
\hline Silver & $<0.006$ & $<0.006$ & $<0.006$ & $<0.006$ & $<0.006$ & $<0.006$ & $<0.006$ & $<0.006$ \\
\hline Strontium & 0.2 & 0.2 & 0.17 & 0.17 & 0.1 & 0.097 & 0.09 & 0.092 \\
\hline Thorium & $<0.2$ & $<0.2$ & $<0.2$ & $<0.2$ & $<0.2$ & $<0.2$ & $<0.2$ & $<0.2$ \\
\hline Uranium (Fluor) & 0.001 & 0.001 & 0.002 & 0.002 & 0.001 & 0.001 & 0.001 & 0.001 \\
\hline Vanadium & $<0.005$ & $<0.005$ & $<0.005$ & $<0.005$ & $<0.005$ & $<0.005$ & $<0.005$ & $<0.005$ \\
\hline zinc & 0.0084 & 0.0047 & 0.0066 & 0.0049 & 0.013 & 0.013 & 0.01 & 0.0081 \\
\hline MAJOR IONS $(\mathrm{mg} / \mathrm{L})$ & $\cdot$ & . & . & . & $\cdot$ & . & $\cdot$ & . \\
\hline Alkalinity-HCO3 & 195 & . & 180 & - & ${ }_{203}$ & - & ${ }_{203}$ & • \\
\hline Alkalinity- $\mathrm{CO} 3$ & $<1$ & . & $<1$ & . & $<1$ & . & $<1$ & . \\
\hline Calcium & 57 & 57 & 49 & 49 & 71 & 72 & 61 & 63 \\
\hline Chloride & 9 & - & 10 & - & 62 & . & 109 & . \\
\hline Fluoride & 0.1 & - & 0.2 & - & 0.1 & . & $<0.1$ & - \\
\hline Magnesium & 22 & 22 & 21 & 22 & 15 & 15 & 15 & 15 \\
\hline Manganese & 0.0099 & 0.0094 & 0.12 & 0.084 & 0.14 & 0.11 & 0.13 & 0.094 \\
\hline Nitrate-N & 4.03 & - & 3.2 & • & 0.6 & . & 0.82 & • \\
\hline Potassium & 0.88 & 1.3 & 0.88 & 1.5 & 2.6 & 2.6 & 4.3 & 4 \\
\hline sodium & 3.4 & 3.3 & 4.2 & 4.2 & 25 & 25 & 17 & 18 \\
\hline Sulfate & 22.5 & - & 22 & - & 7.5 & • & 6.8 & . \\
\hline
\end{tabular}

(CONTINUED) 
APPENDIX E.1

Groundwater and Surface-Water Quality Data, 1993

\begin{tabular}{|c|c|c|c|c|c|c|c|c|}
\hline \multirow{4}{*}{$\begin{array}{l}\text { Sampling Point } \\
\text { Location } \\
\text { Date Sampled }\end{array}$} & \multicolumn{4}{|c|}{ GW-715 } & \multicolumn{4}{|c|}{$G w-723$} \\
\hline & \multicolumn{4}{|c|}{$\operatorname{EXP}$} & \multicolumn{4}{|c|}{$\operatorname{EXP}$} \\
\hline & \multicolumn{2}{|c|}{$08 / 01 / 93$} & \multicolumn{2}{|c|}{$11 / 11 / 93$} & \multicolumn{2}{|c|}{$02 / 12 / 93$} & \multicolumn{2}{|c|}{$05 / 18 / 93$} \\
\hline & TOT & Drs & TOT & DIS & TOT & Drs & TOT & DIS \\
\hline METALS (mg/L) & . & $\cdot 1$ & . & $\cdot$ & $\cdot$ & $\cdot 1$ & • & • \\
\hline Aluminum & 0.34 & $<0.02$ & 0.061 & $<0.02$ & $<0.02$ & $<0.02$ & $<0.02$ & $<0.02$ \\
\hline Antimony & $<0.05$ & $<0.05$ & $<0.05$ & $<0.05$ & $<0.05$ & $<0.05$ & $<0.05$ & $<0.05$ \\
\hline Arsenic & $<0.05$ & $<0.05$ & $<0.05$ & $<0.05$ & $<0.05$ & $<0.05$ & $<0.05$ & $<0.05$ \\
\hline Barium & 0.08 & 0.075 & 0.081 & 0.081 & 0.1 & 0.062 & 0.056 & 0.054 \\
\hline Beryllium & $<0.0003$ & $<0.0003$ & $<0.0003$ & $<0.0003$ & $<0.0003$ & $<0.0003$ & $<0.0003$ & $<0.0003$ \\
\hline Boron & 0.04 & 0.036 & 0.065 & 0.031 & 0.049 & 0.046 & 0.082 & 0.081 \\
\hline Cadmium (AAS) & $<0.002$ & $<0.002$ & $<0.002$ & $<0.002$ & $<0.002$ & $<0.002$ & $<0.002$ & $<0.002$ \\
\hline Cadmium & $<0.003$ & $<0.003$ & $<0.003$ & $<0.003$ & $<0.003$ & $<0.003$ & $<0.003$ & $<0.003$ \\
\hline Chromium (AAS) & 0.064 & $<0.01$ & 0.019 & $<0.01$ & $<0.01$ & $<0.01$ & $<0.01$ & $<0.01$ \\
\hline Chromiura & 0.061 & $<0.01$ & 0.018 & $<0.01$ & $<0.01$ & $<0.01$ & $<0.01$ & $<0.01$ \\
\hline Cobalt & $<0.005$ & $<0.005$ & $<0.005$ & $<0.005$ & $<0.005$ & $<0.005$ & $<0.005$ & $<0.005$ \\
\hline Copper & 0.0063 & $<0.004$ & $<0.004$ & $<0.004$ & $<0.004$ & $<0.004$ & $<0.004$ & $<0.004$ \\
\hline Iron & 1.2 & 0.0059 & 0.32 & 0.058 & 42 & $<0.005$ & 1.8 & $<0.005$ \\
\hline Lead (AAS) & $<0.004$ & $<0.004$ & $<0.004$ & $<0.004$ & $<0.004$ & $<0.004$ & $<0.004$ & $<0.004$ \\
\hline Mercury (CVAA) & $<0.0002$ & $<0.0002$ & $<0.0002$ & $<0.0002$ & $<0.0002$ & $<0.0002$ & $<0.0002$ & $<0.0002$ \\
\hline Molybdenum & $<0.01$ & $<0.01$ & $<0.01$ & $<0.01$ & $<0.01$ & $<0.01$ & $<0.01$ & $<0.01$ \\
\hline Nickel & 0.033 & 0.021 & 0.038 & 0.053 & $<0.01$ & $<0.01$ & 0.017 & 0.011 \\
\hline Selenium & $<0.05$ & $<0.05$ & $<0.05$ & $<0.05$ & $<0.05$ & $<0.05$ & $<0.05$ & $<0.05$ \\
\hline Silver & $<0.006$ & $<0.006$ & $<0.006$ & $<0.006$ & $<0.006$ & $<0.006$ & $<0.006$ & $<0.006$ \\
\hline Strontium & 0.094 & 0.094 & 0.081 & 0.082 & 0.78 & 0.71 & 1.2 & 1.2 \\
\hline Thorium & $<0.2$ & $<0.2$ & $<0.2$ & $<0.2$ & $<0.2$ & $<0.2$ & $<0.2$ & $<0.2$ \\
\hline Uranium (Fluor) & 0.001 & 0.003 & 0.001 & 0.001 & 0.003 & 0.003 & 0.002 & 0.002 \\
\hline Vanadium & $<0.005$ & $<0.005$ & $<0.005$ & $<0.005$ & $<0.005$ & $<0.005$ & $<0.005$ & $<0.005$ \\
\hline zine & 0.012 & 0.0068 & 0.0082 & 0.058 & 0.017 & 0.0035 & 0.018 & 0.016 \\
\hline MAJOR IONS $(\mathrm{mg} / \mathrm{L})$ & $\cdot$ & $\cdot$ & $\cdot$ & $\cdot$ & $\cdot$ & $\cdot$ & $\cdot \cdot$ & $\bullet$ \\
\hline Alkalinity-HCO3 & 224 & $\cdot$ & $23 \dot{6}$ & $\cdot$ & 223 & $\cdot$ & 197 & • \\
\hline Alkalinity- $\mathrm{CO} 3$ & $<1$ & . & $<1$ &. & $<1$ &. & $<1$ & • \\
\hline Calcium & 73 & 74 & 74 & 74 & 84 & 78 & 52 & 50 \\
\hline Chloride & 51 & $\cdot$ & 45.6 & . & 11 &. & 7.5 & • \\
\hline Fluoride & $<0.1$ &. & $<0.1$ &. & 0.3 &. & 0.4 & • \\
\hline Magnesium & 17 & 17 & 18 & 18 & 27 & 26 & 26 & 26 \\
\hline Manganese & 0.33 & 0.14 & 0.11 & 0.11 & 0.38 & 0.055 & 0.02 & 0.0068 \\
\hline Nitrate-N & 1.1 &. & 0.66 & . & 20 &. & 6.39 & . \\
\hline Potassium & 2 & 2.5 & 2.2 & 1.7 & 2.9 & 2.9 & 2.4 & 2.4 \\
\hline Sodium & 17 & 17 & 19 & 19 & 7 & 6.7 & 6.3 & 6.2 \\
\hline Sulfate & 8.2 & $\cdot$ & 6.1 & . & 28 &. & 30.3 & • \\
\hline
\end{tabular}

(CONTINUED) 
APPENDIX E.1

Groundwater and Surface-Water Quality Data, 1993

\begin{tabular}{|c|c|c|c|c|c|c|c|c|}
\hline \multirow{4}{*}{$\begin{array}{l}\text { Sampling Point } \\
\text { Location } \\
\text { Date sampled }\end{array}$} & \multicolumn{4}{|c|}{$G W-723$} & \multicolumn{4}{|c|}{ GW-724 } \\
\hline & \multicolumn{4}{|c|}{$\operatorname{EXP}$} & \multicolumn{4}{|c|}{$\operatorname{EXP}$} \\
\hline & \multicolumn{2}{|c|}{$09 / 27 / 93$} & \multicolumn{2}{|c|}{$12 / 14 / 93$} & \multicolumn{2}{|c|}{$02 / 10 / 93$} & \multicolumn{2}{|c|}{$05 / 18 / 93$} \\
\hline & TOT & DIS & TOT & DIS & TOT & DIS & TOT & DIS \\
\hline METALS (mg/L) & - & - & $\cdot$ & $\cdot$ & $\cdot$ & $\cdot$ & . & - \\
\hline Aluminum & 0.037 & 0.03 & 0.035 & 0.03 & $<0.02$ & $<0.02$ & $<0.02$ & $<0.02$ \\
\hline Antimony & $<0.05$ & $<0.05$ & $<0.05$ & $<0.05$ & $<0.05$ & $<0.05$ & $<0.05$ & $<0.05$ \\
\hline Arsenic & $<0.05$ & $<0.05$ & $<0.05$ & $<0.05$ & $<0.05$ & $<0.05$ & $<0.05$ & $<0.05$ \\
\hline Barium & 0.067 & 0.058 & 0.06 & 0.056 & 0.15 & 0.15 & 0.16 & 0.16 \\
\hline Beryllium & $<0.0003$ & $<0.0003$ & $<0.0003$ & $<0.0003$ & $<0.0003$ & $<0.0003$ & $<0.0003$ & $<0.0003$ \\
\hline Boron & 0.094 & 0.094 & 0.1 & 0.08 & 0.057 & 0.072 & 0.088 & 0.082 \\
\hline Cadmium (AAS) & $<0.002$ & $<0.002$ & $<0.002$ & $<0.002$ & $<0.002$ & $<0.002$ & $<0.002$ & $<0.002$ \\
\hline Cadmium & 0.0076 & $<0.003$ & $<0.003$ & $<0.003$ & $<0.003$ & $<0.003$ & $<0.003$ & $<0.003$ \\
\hline Chromium (AAS) & $<0.01$ & $<0.01$ & $<0.01$ & $<0.01$ & $<0.01$ & $<0.01$ & $<0.01$ & $<0.01$ \\
\hline Chromium & $<0.01$ & $<0.01$ & $<0.01$ & $<0.01$ & $<0.01$ & $<0.01$ & $<0.01$ & $<0.01$ \\
\hline Cobalt & 0.0054 & $<0.005$ & $<0.005$ & $<0.005$ & $<0.005$ & $<0.005$ & $<0.005$ & $<0.005$ \\
\hline Copper & $<0.004$ & $<0.004$ & $<0.004$ & $<0.004$ & 0.0087 & $<0.004$ & $<0.004$ & $<0.004$ \\
\hline Iron & 16 & $<0.005$ & 2.6 & $<0.005$ & 0.67 & 0.45 & 0.83 & 0.52 \\
\hline Lead (AAS) & 0.0046 & $<0.004$ & $<0.004$ & $<0.004$ & $<0.004$ & $<0.004$ & $<0.004$ & $<0.004$ \\
\hline Mercury (CVAA) & $<0.0002$ & $<0.0002$ & $<0.0002$ & $<0.0002$ & $<0.0002$ & $<0.0002$ & $<0.0002$ & $<0.0002$ \\
\hline Mol ybdenum & $<0.01$ & $<0.01$ & $<0.01$ & $<0.01$ & $<0.01$ & $<0.01$ & $<0.01$ & $<0.01$ \\
\hline Hickel & $<0.01$ & $<0.01$ & $<0.01$ & $<0.01$ & $<0.01$ & $<0.01$ & 0.016 & $<0.01$ \\
\hline Selenium & $<0.05$ & $<0.05$ & $<0.05$ & $<0.05$ & $<0.05$ & $<0.05$ & $<0.05$ & $<0.05$ \\
\hline silver & $<0.006$ & $<0.006$ & $<0.006$ & $<0.006$ & $<0.006$ & $<0.006$ & $<0.006$ & $<0.006$ \\
\hline strontium & 1.6 & 1.6 & 1.5 & 1.5 & 1.2 & 1.2 & 1.2 & 1.2 \\
\hline Thorium & $<0.2$ & $<0.2$ & $<0.2$ & $<0.2$ & $<0.2$ & $<0.2$ & $<0.2$ & $<0.2$ \\
\hline Oranium (Fluor) & 0.001 & 0.001 & 0.001 & 0.001 & 0.001 & 0.001 & $<0.001$ & $<0.001$ \\
\hline Vanadium & $<ن .005$ & $<0.005$ & $<0.005$ & $<0.005$ & $<0.005$ & $<0.005$ & $<0.005$ & $<0.005$ \\
\hline zinc & 0.035 & 0.0074 & 0.004 & $<0.002$ & 0.0033 & 0.0058 & 0.03 & 0.12 \\
\hline MAJOR IONS (mg/L) & $\cdot$ &. & • & - &. &. &. & • \\
\hline Alkalinity-HCO3 & $\begin{array}{r}\circ \\
196\end{array}$ & $\cdot$ & 190 &. & 201 & $\cdot$ & 213 & • \\
\hline Alkalinity-CO3 & $<2$ &. & $<1$ &. & $<1$ &. & $<1$ & . \\
\hline Calcium & 51 & 52 & 49 & 49 & 99 & 99 & 98 & 98 \\
\hline Chloride & 9.1 & $\cdot 1$ & 10 &. & 48 &. & 48 & - \\
\hline Fluoride & 0.5 & $\cdot$ & 0.5 &. & 0.2 &. & 0.2 & • \\
\hline Magnesium & 31 & 31 & 31 & 31 & 41 & 41 & 40 & 40 \\
\hline Manganese & 0.17 & 0.018 & 0.047 & 0.01 & 0.036 & 0.037 & 0.036 & 0.035 \\
\hline Nitrate-n & 9.6 & $\cdot$ & 10.6 & . & 62 & . & 37 & - \\
\hline Potassium & 2.6 & 2.7 & 2.7 & 3.1 & 1.4 & 2 & 1.6 & 1.7 \\
\hline Sodium & 8.1 & 8.2 & 8.7 & 8.7 & 21 & 21 & 19 & 19 \\
\hline Sulfate & 33.5 & $\cdot$ & 33 & . & 26 & . & 23 & - \\
\hline
\end{tabular}

(CONTINUED) 
APPENDIX E.1

Groundwater and Surface-Water Quality Data, 1993

\begin{tabular}{|c|c|c|c|c|c|c|c|c|}
\hline \multirow{4}{*}{$\begin{array}{l}\text { Sampling Point } \\
\text { Location } \\
\text { - } \\
\text { Date Sampled }\end{array}$} & \multicolumn{4}{|c|}{ GW-724 } & \multicolumn{4}{|c|}{$G W-725$} \\
\hline & \multicolumn{4}{|c|}{$\mathbf{E X P}$} & \multicolumn{4}{|c|}{$\mathbf{E X P}$} \\
\hline & \multicolumn{2}{|c|}{$09 / 29 / 93$} & \multicolumn{2}{|c|}{$12 / 14 / 93$} & \multicolumn{2}{|c|}{$02 / 12 / 93$} & \multicolumn{2}{|c|}{$05 / 19 / 93$} \\
\hline & TOT & DIS & TOT & DIS & TOT & DIS & TOT & DIS \\
\hline HETALS (mg/L) & $\cdot$ & - & - & - & - & - & $\cdot$ & . \\
\hline Aluminum & $<0.02$ & 0.029 & $<0.02$ & 0.037 & 0.043 & 0.075 & 0.047 & 0.067 \\
\hline Antimony & $<0.05$ & $<0.05$ & $<0.05$ & $<0.05$ & $<0.05$ & $<0.05$ & $<0.05$ & $<0.05$ \\
\hline Arsenic & $<0.05$ & $<0.05$ & $<0.05$ & $<0.05$ & $<0.05$ & $<0.05$ & 0.051 & $<0.05$ \\
\hline Barium & 0.17 & 0.18 & 0.16 & 0.16 & 0.28 & 0.28 & 0.28 & 0.27 \\
\hline Beryllium & $<0.0003$ & $<0.0003$ & $<0.0003$ & $<0.0003$ & $<0.0003$ & $<0.0003$ & $<0.0003$ & $<0.0003$ \\
\hline Boron & 0.094 & 0.097 & 0.078 & 0.089 & 0.044 & 0.052 & 0.063 & 0.053 \\
\hline Cadmium (AAS) & $<0.002$ & $<0.002$ & $<0.002$ & $<0.002$ & $<0.002$ & $<0.002$ & $<0.002$ & $<0.002$ \\
\hline Cadmium & 0.0035 & $<0.003$ & $<0.003$ & $<0.003$ & $<0.003$ & $<0.003$ & $<0.003$ & $<0.003$ \\
\hline Chromium (AAS) & $<0.01$ & $<0.01$ & $<0.01$ & $<0.01$ & $<0.01$ & $<0.01$ & $<0.01$ & $<0.01$ \\
\hline Chromium & $<0.01$ & $<0.01$ & $<0.01$ & $<0.01$ & $<0.01$ & $<0.01$ & $<0.01$ & $<0.01$ \\
\hline Cobalt & $<0.005$ & $<0.005$ & $<0.005$ & $<0.005$ & $<0.005$ & $<0.005$ & $<0.005$ & $<0.005$ \\
\hline Copper & $<0.004$ & $<0.004$ & $<0.004$ & $<0.004$ & $<0.004$ & $<0.004$ & $<0.004$ & $<0.004$ \\
\hline Iron & 1 & 0.66 & 2.5 & 0.99 & 1.8 & 0.53 & 0.21 & 0.036 \\
\hline Lead (AAS) & $<0.004$ & $<0.004$ & $<0.004$ & $<0.004$ & $<0.004$ & $<0.004$ & $<0.004$ & $<0.004$ \\
\hline Mercury (CVAA) & $<0.0002$ & $<0.0002$ & $<0.0002$ & $<0.0002$ & $<0.0002$ & $<0.0002$ & $<0.0002$ & $<0.0002$ \\
\hline Mol ybdenum & $<0.01$ & $<0.01$ & $<0.01$ & $<0.01$ & $<0.01$ & $<0.01$ & $<0.01$ & $<0.01$ \\
\hline Nickel & $<0.01$ & $<0.01$ & $<0.01$ & $<0.01$ & $<0.01$ & $<0.01$ & $<0.01$ & $<0.01$ \\
\hline Selenium & $<0.05$ & $<0.05$ & $<0.05$ & $<0.05$ & $<0.05$ & $<0.05$ & $<0.05$ & $<0.05$ \\
\hline Silver & $<0.006$ & $<0.006$ & $<0.006$ & $<0.006$ & $<0.006$ & $<0.006$ & $<0.006$ & $<0.006$ \\
\hline Strontium & $1 \cdot 3$ & $1 \cdot 3$ & 1.2 & 1.2 & 0.5 & 0.51 & 0.56 & 0.55 \\
\hline Tisorium & $<0.2$ & $<0.2$ & $<0.2$ & $<0.2$ & $<0.2$ & $<0.2$ & $<0.2$ & $<0.2$ \\
\hline Uranium (Fluor) & $<0.001$ & 0.001 & 0.001 & 0.001 & 0.004 & 0.003 & 0.003 & 0.003 \\
\hline Vanadium & $<0.005$ & $<0.005$ & $<0.005$ & $<0.005$ & $<0.005$ & $<0.005$ & $<0.005$ & $<0.005$ \\
\hline zinc & 0.0066 & 0.011 & $<0.002$ & $<0.002$ & 0.0055 & 0.0059 & 0.017 & 0.019 \\
\hline MAJOR IONS $(\mathrm{mg} / \mathrm{L})$ & - & - & - & . & . & $\cdot$ & - & - \\
\hline Alkalinity-HCO3 & 203 &. & 203 & $\cdot$ & 292 & $\cdot$ & $284^{\circ}$ & • \\
\hline Alkalinity-CO3 & $<1$ & - & $<1$ &. & $<1$ & - & $<1$ & - \\
\hline Calcium & 97 & 100 & 96 & 100 & 170 & 170 & 170 & 170 \\
\hline Chloride & 54 & - & 45 & $\cdot$ & 78 & $\cdot$ & 50 & - \\
\hline Fluoride & 0.2 & - & 0.2 & . & 0.1 & $\cdot$ & $<0.1$ & • \\
\hline Magnesium & 40 & 42 & 41 & 43 & 27 & 27 & 30 & 30 \\
\hline Manganese & 0.054 & 0.061 & 0.11 & 0.11 & 0.063 & 0.055 & 0.029 & 0.029 \\
\hline Nitrate-N & 50.1 & - & 46.4 & - & 61 &. & 55 & - \\
\hline Potassium & 2.1 & 2.1 & 2.3 & 2.6 & 2.9 & 2.8 & 2.6 & 2.3 \\
\hline Sodium & 21 & 22 & 21 & 21 & 17 & 17 & 17 & 16 \\
\hline Sulfate & 23 & • & 21 & . & 59 & . & 34 & • \\
\hline
\end{tabular}

(CONTINUED) 
APPENDIX E.1

Groundwater and surface-Water Quallty Data, 1993

\begin{tabular}{|c|c|c|c|c|c|c|c|c|}
\hline \multirow{4}{*}{$\begin{array}{l}\text { Sampling Point } \\
\text { Location } \\
\text { Date Sampled }\end{array}$} & \multicolumn{4}{|c|}{ GW-725 } & \multicolumn{4}{|c|}{$G W-736$} \\
\hline & \multicolumn{4}{|c|}{$\operatorname{EXP}$} & \multicolumn{4}{|c|}{ EXP } \\
\hline & \multicolumn{2}{|c|}{$09 / 28 / 93$} & \multicolumn{2}{|c|}{$12 / 15 / 93$} & \multicolumn{2}{|c|}{$02 / 11 / 93$} & \multicolumn{2}{|c|}{$05 / 18 / 93$} \\
\hline & TOT & DIs & TOT & DIS & TOT & DIS & TOT & DIs \\
\hline METALS (mg/L) & . & $\cdot$ & $\cdot$ & $\cdot$ & $\cdot$ & $\cdot$ & $\cdot$ & • \\
\hline Aluminum & 0.054 & 0.056 & 0.029 & 0.03 & 0.066 & 0.067 & $<0.02$ & 0.046 \\
\hline Antimony & $<0.05$ & $<0.05$ & $<0.05$ & $<0.05$ & $<0.05$ & $<0.05$ & $<0.05$ & $<0.05$ \\
\hline Arsenic & $<0.05$ & $<0.05$ & $<0.05$ & $<0.05$ & $<0.05$ & $<0.05$ & $<0.05$ & $<0.05$ \\
\hline Barium & 0.25 & 0.25 & 0.26 & 0.25 & 0.22 & 0.22 & 0.21 & 0.21 \\
\hline Beryllium & $<0.0003$ & $<0.0003$ & $<0.0003$ & $<0.0003$ & $<0.0003$ & 0.00038 & $<0.0003$ & $<0.0003$ \\
\hline Boron & 0.047 & 0.051 & 0.051 & 0.04 & 0.044 & 0.041 & 0.056 & 0.043 \\
\hline Cadmium (AAS) & $<0.002$ & $<0.002$ & $<0.002$ & $<0.002$ & $<0.002$ & $<0.002$ & $<0.002$ & $<0.002$ \\
\hline Cadmium & $<0.003$ & $<0.003$ & $<0.003$ & $<0.003$ & $<0.003$ & $<0.003$ & $<0.003$ & $<0.003$ \\
\hline Chromium (AAS) & $<0.01$ & $<0.01$ & $<0.01$ & $<0.01$ & $<0.01$ & $<0.01$ & $<0.01$ & $<0.01$ \\
\hline Chromium & $<0.01$ & $<0.01$ & $<0.01$ & $<0.01$ & $<0.01$ & $<0.01$ & $<0.01$ & $<0.01$ \\
\hline Cobalt & $<0.005$ & $<0.005$ & $<0.005$ & $<0.005$ & $<0.005$ & $<0.005$ & $<0.005$ & $<0.005$ \\
\hline Copper & $<0.004$ & $<0.004$ & $<0.004$ & $<0.004$ & $<0.004$ & $<0.004$ & $<0.004$ & $<0.004$ \\
\hline Iron & 0.042 & 0.013 & 0.26 & 0.11 & 0.028 & $<0.005$ & 0.019 & 0.0069 \\
\hline Lead (AAS) & $<0.004$ & $<0.004$ & $<0.004$ & $<0.004$ & $<0.004$ & $<0.004$ & $<0.004$ & $<0.004$ \\
\hline Mercury (CVAA) & $<0.0002$ & $<0.0002$ & $<0.0002$ & $<0.0002$ & $<0.0002$ & $<0.0002$ & $<0.0002$ & $<0.0002$ \\
\hline Mol ybdenum & $<0.01$ & $<0.01$ & $<0.01$ & $<0.01$ & $<0.01$ & $<0.01$ & $<0.01$ & $<0.01$ \\
\hline Nickel & $<0.01$ & $<0.01$ & $<0.01$ & $<0.01$ & $<0.01$ & $<0.01$ & 0.01 & $<0.01$ \\
\hline Selenium & $<0.05$ & $<0.05$ & $<0.05$ & $<0.05$ & $<0.05$ & $<0.05$ & $<0.05$ & $<0.05$ \\
\hline silver & $<0.006$ & $<0.006$ & $<0.006$ & $<0.006$ & $<0.006$ & $<0.006$ & $<0.006$ & $<0.006$ \\
\hline Strontium & 0.39 & 0.4 & 0.46 & 0.45 & 0.36 & 0.36 & 0.34 & 0.35 \\
\hline Thorium & $<0.2$ & $<0.2$ & $<0.2$ & $<0.2$ & $<0.2$ & $<0.2$ & $<0.2$ & $<0.2$ \\
\hline Uranium (Fluor) & 0.006 & 0.004 & 0.006 & 0.005 & 0.007 & 0.008 & 0.003 & 0.007 \\
\hline Vanadium & $<0.005$ & $<0.005$ & $<0.005$ & $<0.005$ & $<0.005$ & $<0.005$ & $<0.005$ & $<0.005$ \\
\hline Zine & 0.0077 & 0.0091 & $<0.002$ & $<0.002$ & 0.0068 & 0.0074 & 0.01 & 0.0088 \\
\hline MAJOR IONS $(\mathrm{mg} / \mathrm{L})$ & $\cdot$ & - & - & - & - & . & • & • \\
\hline Alkalinity-HCO3 & 289 & . & 303 &. & 312 & . & 313 & • \\
\hline Alkalinity- $\mathrm{CO} 3$ & $<1$ & - & $<1$ & - & $<1$ & . & $<1$ & - \\
\hline Calcium & 170 & 170 & 180 & 170 & 180 & 180 & 170 & 180 \\
\hline Chloride & 52 & - & 52 & . & 49 & $\cdot$ & 50 & • \\
\hline Fluoride & $<0.1$ & - & $<0.1$ & - & 0.3 & - & 0.2 & - \\
\hline Magnesium & 26 & 26 & 28 & 27 & 22 & 23 & 22 & 22 \\
\hline Manganese & 0.97 & 0.96 & 0.37 & 0.36 & 0.5 & 0.52 & 0.53 & 0.54 \\
\hline Nitrate-N & 49.9 & - & 46.2 & . & 61 & - & 42 & • \\
\hline Potassium & 3.1 & 3 & 3.4 & 3.6 & 3.3 & 3.2 & 2.8 & 3 \\
\hline Sodium & 21 & 22 & 21 & 21 & 22 & 22 & 19 & 20 \\
\hline Sulfate & 38 & - & 31 & - & 40 & . & 38 & • \\
\hline
\end{tabular}

(CONTINUED) 
APPENDIX 8.1

Groundwater and Surface-Water Qual1ty Data, 1993

\begin{tabular}{|c|c|c|c|c|c|c|c|c|}
\hline \multirow{4}{*}{$\begin{array}{l}\text { Sampling point } \\
\text { Looation } \\
\text { Date sampled }\end{array}$} & \multicolumn{4}{|c|}{ GW-736 } & \multicolumn{4}{|c|}{$6 W-737$} \\
\hline & \multicolumn{4}{|c|}{$\mathbf{E X P}$} & \multicolumn{4}{|c|}{$\mathbf{E X P}$} \\
\hline & \multicolumn{2}{|c|}{$09 / 28 / 93$} & \multicolumn{2}{|c|}{$12 / 15 / 93$} & \multicolumn{2}{|c|}{$02 / 11 / 93$} & \multicolumn{2}{|c|}{$05 / 19 / 93$} \\
\hline & TOT & DIS & TOT & DIS & TOT & DIS & TOT & DIS \\
\hline METALS (mg/L) & $\cdot$ &. & $\cdot$ & $\cdot$ & $\cdot 1$ & $\cdot$ & $\cdot$ & • \\
\hline Aluminum & 0.041 & 0.044 & 0.033 & 0.032 & 0.051 & 0.055 & 0.023 & 0.054 \\
\hline Antimony & $<0.05$ & $<0.05$ & $<0.05$ & $<0.05$ & $<0.05$ & $<0.05$ & $<0.05$ & $<0.05$ \\
\hline Arsenic & $<0.05$ & $<0.05$ & $<0.05$ & $<0.05$ & $<0.05$ & $<0.05$ & $<0.05$ & $<0.05$ \\
\hline Barium & 0.28 & 0.29 & 0.22 & 0.23 & 0.25 & 0.25 & 0.23 & 0.23 \\
\hline Bery 11 ium & $<0.0003$ & $<0.0003$ & $<0.0003$ & $<0.0003$ & $<0.0003$ & $<0.0003$ & $<0.0003$ & $<0.0003$ \\
\hline Boron & 0.067 & 0.059 & 0.044 & 0.049 & 0.042 & 0.041 & 0.054 & 0.044 \\
\hline Cadmium (AAS) & $<0.002$ & $<0.002$ & $<0.002$ & $<0,002$ & $<0.002$ & $<0.002$ & $<0.002$ & $<0.002$ \\
\hline Cadmium & $<0.003$ & $<0.003$ & $<0.003$ & $<0.003$ & $<0.003$ & $<0.003$ & $<0.003$ & $<0.003$ \\
\hline Chromium (AAS) & $<0.01$ & $<0.01$ & $<0.01$ & $<0.01$ & $<0.01$ & $<0.01$ & $<0.01$ & $<0.01$ \\
\hline Chromium & $<0.01$ & $<0.01$ & $<0.01$ & $<0.01$ & $<0.01$ & 0.012 & $<0.01$ & $<0.01$ \\
\hline Cobalt & $<0.005$ & $<0.005$ & $<0.005$ & $<0.005$ & $<0.005$ & $<0.005$ & $<0.005$ & $<0.005$ \\
\hline Copper & $<0.004$ & $<0.004$ & $<0.004$ & $<0.004$ & $<0.004$ & $<0.004$ & $<0.004$ & $<0.004$ \\
\hline Iron & 0.85 & 0.7 & $<0.005$ & $<0.005$ & 0.29 & $<0.005$ & 0.023 & $<0.005$ \\
\hline Iead (AAS) & $<0.004$ & $<0.004$ & $<0.004$ & $<0.004$ & $<0.004$ & $<0.004$ & $<0.004$ & $<0.004$ \\
\hline Mercury (CVAA) & $<0.0002$ & $<0.0002$ & $<0.0002$ & $<0.0002$ & $<0.0002$ & $<0.0002$ & $<0.0002$ & $<0.0002$ \\
\hline Molybdenum & $<0.01$ & $<0.01$ & $<0.01$ & $<0.01$ & $<0.01$ & $<0.01$ & $<0.01$ & $<0.01$ \\
\hline Hickel & $<0.01$ & $<0.01$ & $<0.01$ & $<0.01$ & $<0.01$ & $<0.01$ & 0.025 & 0.015 \\
\hline Selenium & $<0.05$ & $<0.05$ & $<0.05$ & $<0.05$ & $<0.05$ & $<0.05$ & $<0.05$ & $<0.05$ \\
\hline Silver & $<0.006$ & $<0.006$ & $<0.006$ & $<0.006$ & $<0.006$ & $<0.006$ & $<0,006$ & $<0.006$ \\
\hline Strontium & 0.56 & 0.57 & 0.34 & 0.35 & 0.37 & 0.36 & 0.36 & 0.36 \\
\hline Thorium & $<0.2$ & $<0.2$ & $<0.2$ & $<0.2$ & $<0.2$ & $<0.2$ & $<0.2$ & $<0.2$ \\
\hline Uranium (Fluor) & 0.006 & 0.005 & 0.008 & 0.008 & 0.009 & 0.008 & 0.009 & 0.006 \\
\hline Vanadium & $<0.005$ & $<0.005$ & $<0.005$ & $<0.005$ & $<0.005$ & $<0.005$ & $<0.005$ & $<0.005$ \\
\hline zinc & 0.0071 & 0.0088 & $<0.002$ & $<0.002$ & 0.021 & 0.0094 & 0.011 & 0.0065 \\
\hline MANOR IONS (mg/L) &. & . & $\cdot$ &. & $\cdot$ & $\cdot$ &. & • \\
\hline Alkalinity-HCO3 & 317 &. & 316 &. & ${ }_{312}$ & $\cdot$ & 319 & . \\
\hline Alkalinity-CO3 & $<1$ &. & $<1$ &. & $<1$ &. & $<1$ & - \\
\hline Calcium & 170 & 170 & 170 & 190 & 180 & 170 & 180 & 180 \\
\hline Chloride & 65 & $\cdot$ & 55 & $\cdot$ & 52 & $\cdot$ & 51 & - \\
\hline Fluoride & 0.2 & $\cdot$ & 0.2 & $\cdot$ & 0.2 & $\cdot$ & 0.1 & - \\
\hline Magnesium & 29 & 29 & 24 & 25 & 24 & 23 & 23 & 23 \\
\hline Manganese & 0.19 & 0.19 & 0.9 & 0.97 & 0.94 & 0.77 & 0.86 & 0.82 \\
\hline Nitrate-N & 43.8 &. & 42.4 &. & 57 &. & 37 & - \\
\hline Potassium & 3.1 & 3 & 3.8 & 3.6 & 3.4 & 3.3 & 3.1 & 3 \\
\hline Sodium & 20 & 20 & 23 & 24 & 23 & 24 & 21 & 20 \\
\hline sulfate & 40 & $\cdot$ & 32 & . & 40 & . & 41 & - \\
\hline
\end{tabular}

(CONTINUED) 
APPENDIX E.1

Groundwater and Surface-Water Quality Data, 1993

\begin{tabular}{|c|c|c|c|c|c|c|c|c|}
\hline \multirow{4}{*}{$\begin{array}{l}\text { Sampling Point } \\
\text { Location } \\
\text { Date Sampled }\end{array}$} & \multicolumn{4}{|c|}{$6 w-737$} & \multicolumn{4}{|c|}{$G W-738$} \\
\hline & \multicolumn{4}{|c|}{ EXP } & \multicolumn{4}{|c|}{ EXP } \\
\hline & \multicolumn{2}{|c|}{$09 / 28 / 93$} & \multicolumn{2}{|c|}{$12 / 15 / 93$} & \multicolumn{2}{|c|}{$02 / 10 / 93$} & \multicolumn{2}{|c|}{$05 / 18 / 93$} \\
\hline & TOT & DIS & TOT & DIS & TOT & Drs & TOT & DIS \\
\hline METALS (mg/L) & $\cdot$ & $\cdot$ & $\cdot$ & $\cdot$ & - & $\cdot$ & $\cdot$ & $\cdot$ \\
\hline Aluminum & 0.056 & 0.056 & 0.025 & 0.021 & 0.19 & $<0.02$ & 0.18 & $<0.02$ \\
\hline Antimony & $<0.05$ & $<0.05$ & $<0.05$ & $<0.05$ & $<0.05$ & $<0.05$ & $<0.05$ & $<0.05$ \\
\hline Arsentc & $<0.05$ & $<0.05$ & $<0.05$ & $<0.05$ & $<0.05$ & $<0.05$ & $<0.05$ & $<0.05$ \\
\hline Barium & 0.24 & 0.23 & 0.23 & 0.24 & 0.044 & 0.043 & 0.044 & 0.043 \\
\hline Beryllium & $<0.0003$ & $<0.0003$ & $<0.0003$ & $<0.0003$ & $<0.0003$ & $<0.0003$ & $<0.0003$ & $<0.0003$ \\
\hline Boron & 0.051 & 0.05 & 0.043 & 0.038 & 0.019 & 0.022 & 0.03 & 0.027 \\
\hline Cadmium (AAS) & $<0.002$ & $<0.002$ & $<0.002$ & $<0.002$ & $<0.002$ & $<0.002$ & $<0.002$ & $<0.002$ \\
\hline Cadmium & $<0.003$ & $<0.003$ & $<0.003$ & $<0.003$ & $<0.003$ & $<0.003$ & $<0.003$ & $<0.003$ \\
\hline Chromiun (AAS) & $<0.01$ & $<0.01$ & $<0.01$ & $<0.01$ & $<0.01$ & $<0.01$ & $<0.01$ & $<0.01$ \\
\hline Chromium & $<0.01$ & $<0.01$ & $<0.01$ & $<0.01$ & $<0.01$ & $<0.01$ & $<0.01$ & $<0.01$ \\
\hline Cobalt & $<0.005$ & $<0.005$ & $<0.005$ & $<0.005$ & $<0.005$ & $<0.005$ & $<0.005$ & $<0.005$ \\
\hline Copper & $<0.004$ & $<0.004$ & $<0.004$ & $<0.004$ & 0.014 & $<0.004$ & $<0.004$ & $<0.004$ \\
\hline Iron & 0.028 & $<0.005$ & $<0.005$ & $<0.005$ & 0.21 & $<0.005$ & 0.2 & $<0.005$ \\
\hline Lead (AAS) & $<0.004$ & $<0.004$ & $<0.004$ & $<0.004$ & $<0.004$ & $<0.004$ & $<0.004$ & $<0.004$ \\
\hline Mercury (CVAA) & $<0.0002$ & $<0.0002$ & $<0.0002$ & $<0.0002$ & $<0.0002$ & $<0.0002$ & $<0.0002$ & $<0.0002$ \\
\hline Molybdenum & $<0.01$ & $<0.01$ & $<0.01$ & $<0.01$ & $<0.01$ & $<0.01$ & $<0.01$ & $<0.01$ \\
\hline Nickel & $<0.01$ & $<0.01$ & $<0.01$ & $<0.01$ & $<0.01$ & $<0.01$ & $<0.01$ & $<0.01$ \\
\hline Selenium & $<0.05$ & $<0.05$ & $<0.05$ & $<0.05$ & $<0.05$ & $<0.05$ & $<0.05$ & $<0.05$ \\
\hline Silver & $<0.006$ & $<0.006$ & $<0.006$ & $<0.006$ & $<0.006$ & $<0.006$ & $<0.006$ & $<0.006$ \\
\hline strontium & 0.39 & 0.38 & 0.36 & 0.36 & 0.1 & 0.1 & 0.1 & 0.1 \\
\hline Thorium & $<0.2$ & $<0.2$ & $<0.2$ & $<0.2$ & $<0.2$ & $<0.2$ & $<0.2$ & $<0.2$ \\
\hline Uranium (Fluor) & 0.005 & 0.006 & 0.008 & 0.008 & 0.002 & 0.002 & 0.002 & 0.002 \\
\hline Vanadium & $<0.005$ & $<0.005$ & $<0.005$ & $<0.005$ & $<0.005$ & $<0.005$ & $<0.005$ & $<0.005$ \\
\hline Zine & 0.0068 & 0.0059 & $<0.002$ & $<0.002$ & 0.016 & 0.0069 & 0.0087 & 0.0076 \\
\hline MAJOR IONS $(\mathrm{mg} / \mathrm{L})$ & $\cdot$ & $\cdot$ & $\cdot$ & $\cdot$ & $\cdot$ & $\cdot$ & $\cdot$ & - \\
\hline Alkalinity-HCO3 & 307 & $\cdot$ & 310 & $\cdot$ & $\begin{array}{r}\circ \\
279\end{array}$ & . & $\begin{array}{r}\cdot \\
283\end{array}$ & . \\
\hline Alkalinity- $\mathrm{CO} 3$ & $<1$ &. & $<1$ &. & $<1$ & . & $<1$ & . \\
\hline Calcium & 180 & 180 & 170 & 170 & 120 & 120 & 110 & 110 \\
\hline Chloride & 54 & $\cdot$ & 53 & $\cdot 1$ & 14 & $\cdot$ & 15 & • \\
\hline Fluoride & 0.1 & $\cdot$ & 0.1 & $\cdot$ & $<0.1$ & . & $<0.1$ & - \\
\hline Magnesium & 25 & 25 & 25 & 25 & 25 & 25 & 24 & 23 \\
\hline Manganese & 0.94 & 0.93 & 0.94 & 0.94 & 0.0068 & 0.0011 & 0.004 & $<0.001$ \\
\hline Nitrate-N & 49.9 & $\cdot$ & 44.3 &. & 22 & $\cdot$ & 15 & - \\
\hline Potassium & 3.3 & 3.2 & 3.5 & 3.4 & 2.8 & 2 & 1.6 & 1.7 \\
\hline Sodium & 24 & 23 & 23 & 22 & 6.7 & 6.8 & 5.6 & 5.6 \\
\hline sulfate & 38 & $\cdot$ & 30 & $\cdot$ & 30 & $\cdot$ & 27 & - \\
\hline
\end{tabular}

(CONTINUED) 
APPENDIX E.1

Groundwater and surface-Water Quality Data, 1993

\begin{tabular}{|c|c|c|c|c|c|c|c|c|}
\hline \multirow{4}{*}{$\begin{array}{l}\text { Sampling Point } \\
\text { Location } \\
\text { Date Sampled }\end{array}$} & \multicolumn{4}{|c|}{$6 w-738$} & \multicolumn{4}{|c|}{$6 w-739$} \\
\hline & \multicolumn{4}{|c|}{ EXP } & \multicolumn{4}{|c|}{$\exp$} \\
\hline & \multicolumn{2}{|c|}{$09 / 27 / 93$} & \multicolumn{2}{|c|}{$12 / 14 / 93$} & \multicolumn{2}{|c|}{$02 / 10 / 93$} & \multicolumn{2}{|c|}{$05 / 18 / 93$} \\
\hline & TOT & DIs & TOT & DIs & TOT & Dr8 & TOT & Drs \\
\hline Merars $(\mathrm{mg} / \mathrm{L})$ & - &. & $\cdot$ & $\cdot$ & $\cdot$ & $\cdot$ & $\cdot$ & - \\
\hline Aluminum & 0.5 & 0.042 & 0.27 & 0.026 & $<0.02$ & 0.02 & $<0.02$ & $<0.02$ \\
\hline Antimony & $<0.05$ & $<0.05$ & $<0.05$ & $<0.05$ & $<0.05$ & $<0.05$ & $<0.05$ & $<0.05$ \\
\hline Arsenic & $<0.05$ & $<0.05$ & $<0.05$ & $<0.05$ & $<0.05$ & $<0.05$ & $<0.05$ & $<0.05$ \\
\hline Bartum & 0.058 & 0.055 & 0.054 & 0.054 & 0.031 & 0.029 & 0.039 & 0.038 \\
\hline Beryllium & $<0.0003$ & 0.00033 & $<0.0003$ & $<0.0003$ & $<0.0003$ & $<0.0003$ & $<0.0003$ & $<0.0003$ \\
\hline Boron & 0.04 & 0.038 & 0.03 & 0.043 & 0.016 & 0.012 & 0.032 & 0.033 \\
\hline Cadmium (AAS) & $<0.002$ & $<0.002$ & $<0.002$ & $<0.002$ & $<0.002$ & $<0.002$ & $<0.002$ & $<0.002$ \\
\hline Cadmium & 0.0035 & $<0.003$ & $<0.003$ & $<0.003$ & $<0.003$ & $<0.003$ & $<0.003$ & $<0.003$ \\
\hline Chromium (AAS) & $<0.01$ & $<0.01$ & $<0.01$ & $<0.01$ & $<0.01$ & $<0.01$ & $<0.01$ & $<0.01$ \\
\hline Chromium & $<0.01$ & 0.032 & $<0.01$ & $<0.01$ & $<0.01$ & $<0.01$ & $<0.01$ & $<0.01$ \\
\hline Cobalt & $<0.005$ & $<0.005$ & $<0.005$ & $<0.005$ & $<0.005$ & $<0.005$ & $<0.005$ & $<0.005$ \\
\hline Copper & $<0.004$ & $<0.004$ & $<0.004$ & $<0.004$ & $<0.004$ & $<0.004$ & $<0.004$ & $<0.004$ \\
\hline Iron & 0.48 & 0.11 & 0.22 & $<0.005$ & 7.1 & 0.023 & 0.4 & 0.022 \\
\hline Lead (AAS) & $<0.004$ & $<0.004$ & $<0.004$ & $<0.004$ & $<0.004$ & $<0.004$ & $<0.004$ & $<0.004$ \\
\hline Mercury (CVAA) & $<0.0002$ & $<0.0002$ & $<0.0002$ & $<0.0002$ & $<0.0002$ & $<0.0002$ & $<0.0002$ & $<0.0002$ \\
\hline Mol ybdenum & $<0.01$ & $<0.01$ & $<0.01$ & $<0.01$ & $<0.01$ & $<0.01$ & $<0.01$ & $<0.01$ \\
\hline Nickel & $<0.01$ & 0.044 & $<0.01$ & $<0.01$ & $<0.01$ & $<0.01$ & 0.017 & 0.014 \\
\hline Selenium & $<0.05$ & $<0.05$ & $<0.05$ & $<0.05$ & $<0.05$ & $<0.05$ & $<0.05$ & $<0.05$ \\
\hline silver & $<0.006$ & $<0.006$ & $<0.006$ & $<0.006$ & $<0.006$ & $<0.006$ & $<0.006$ & $<0.006$ \\
\hline Strontium & 0.13 & 0.13 & 0.12 & 0.12 & 0.087 & 0.085 & 0.096 & 0.095 \\
\hline Thorium & $<0.2$ & $<0.2$ & $<0.2$ & $<0.2$ & $<0.2$ & $<0.2$ & $<0.2$ & $<0.2$ \\
\hline Uranium (Pluor) & 0.002 & 0.003 & 0.003 & 0.003 & 0.001 & 0.001 & 0.001 & $<0.001$ \\
\hline Vanadium & $<0.005$ & $<0.005$ & $<0.005$ & $<0 ; 005$ & $<0.005$ & $<0.005$ & $<0.005$ & $<0.005$ \\
\hline Zine & 0.013 & 0.0072 & $<0.002$ & 0.0024 & 0.013 & 0.0045 & 0.009 & 0.0079 \\
\hline MAJOR IONS (mg/L) & . & $\cdot$ & . & $\cdot$ & . & $\cdot$ &. & • \\
\hline Alkalinity-HCO3 & 292 &. & $28 \dot{ }$ & & 222 &. & 219 & • \\
\hline Alkalinit $y-\mathrm{CO} 3$ & $<1$ & . & $<1$ & $\cdot$ & $<1$ &. & $<1$ & - \\
\hline Calcium & 130 & 130 & 130 & 130 & 61 & 60 & 58 & 57 \\
\hline Chloride & 34 &. & 20 &. & 5.3 & $\cdot$ & 5 & - \\
\hline Fluoride & $<0.1$ &. & $<0.1$ & $\cdot$ & 0.2 & $\cdot$ & 0.2 & - \\
\hline Magnesium & 27 & 27 & 26 & 27 & 27 & 27 & 26 & 25 \\
\hline Manganese & 0.017 & 0.0081 & 0.0092 & $<0.001$ & 0.09 & 0.056 & 0.022 & 0.019 \\
\hline Nitrate-N & 40.4 & . & 24.8 &. & 7.4 &. & 4.17 & • \\
\hline Potassium & 2.1 & 2.1 & 2.8 & 2.2 & $<0.6$ & 1.3 & 1.1 & 1.2 \\
\hline Sodium & 8.3 & 8.2 & 8.7 & 8.8 & 1.7 & 1.8 & 1.9 & 1.9 \\
\hline Sulfate & 26.9 & . & 25 & . & 18 & $\cdot$ & 12.7 & - \\
\hline
\end{tabular}

(CONTINUED) 
APPENDIX 2.1

Groundwater and Surface-water Quality Data, 1993

\begin{tabular}{|c|c|c|c|c|c|c|c|c|}
\hline \multirow{4}{*}{$\begin{array}{l}\text { Sampling Point } \\
\text { Looation } \\
\text { Date Sampled }\end{array}$} & \multicolumn{4}{|c|}{ GW-739 } & \multicolumn{4}{|c|}{$6 w-740$} \\
\hline & \multicolumn{4}{|c|}{$\mathbf{E x p}$} & \multicolumn{4}{|c|}{$\mathbf{E X P}$} \\
\hline & \multicolumn{2}{|c|}{$09 / 27 / 93$} & \multicolumn{2}{|c|}{$12 / 13 / 93$} & \multicolumn{2}{|c|}{$02 / 09 / 93$} & \multicolumn{2}{|c|}{$05 / 17 / 93$} \\
\hline & TOT & DIs & TOT & DIS & TOT & Drs & TOT & DIs \\
\hline $\operatorname{METALS}(\mathrm{mg} / \mathrm{L})$ & • & $\cdot$ & $\cdot$ & $\cdot$ & - & $\cdot$ & - & - \\
\hline Aluminum & 0.039 & 0.024 & 0.032 & 0.023 & $<0.02$ & $<0.02$ & $<0.02$ & 0.02 \\
\hline Antimony & $<0.05$ & $<0.05$ & $<0.05$ & $<0.05$ & $<0.05$ & $<0.05$ & $<0.05$ & $<0.05$ \\
\hline Arsenic & $<0.05$ & $<0.05$ & $<0.05$ & $<0.05$ & $<0.05$ & $<0.05$ & $<0.05$ & $<0.05$ \\
\hline Barium & 0.044 & 0.044 & 0.038 & 0.037 & 0.072 & 0.072 & 0.079 & 0.079 \\
\hline Beryllium & 0.00043 & 0.00033 & $<0.0003$ & $<0.0003$ & $<0.0003$ & $<0.0003$ & $<0.0003$ & $<0.0003$ \\
\hline Boron & 0.033 & 0.036 & 0.035 & 0.024 & 0.015 & 0.0047 & 0.022 & 0.039 \\
\hline Cadmium (AAS) & $<0.002$ & $<0.002$ & $<0.002$ & $<0.002$ & $<0.002$ & $<0.002$ & $<0.002$ & $<0.002$ \\
\hline Cadmium & $<0.003$ & $<0.003$ & $<0.003$ & $<0.003$ & $<0.003$ & $<0.003$ & $<0.003$ & $<0.003$ \\
\hline Chromium (AAS) & $<0.01$ & $<0.01$ & $<0.01$ & $<0.01$ & $<0.01$ & $<0.01$ & $<0.01$ & $<0.01$ \\
\hline Chromium & $<0.01$ & $<0.01$ & $<0.01$ & $<0.01$ & $<0.01$ & $<0.01$ & $<0.01$ & $<0.01$ \\
\hline Cobalt & $<0.005$ & $<0.005$ & $<0.005$ & $<0.005$ & $<0.005$ & $<0.005$ & $<0.005$ & $<0,005$ \\
\hline Copper & 0.007 & $<0.004$ & $<0.004$ & $<0.004$ & $<0.004$ & $<0.004$ & $<0.004$ & $<0.004$ \\
\hline Iron & 0.95 & 0.07 & 1.6 & 0.071 & 0.35 & 0.11 & 0.21 & 0.047 \\
\hline Lead (AAS) & $<0.004$ & $<0.004$ & $<0.004$ & $<0.004$ & $<0.004$ & $<0.004$ & $<0.004$ & $<0.004$ \\
\hline Mercury (CVAA) & $<0.0002$ & $<0.0002$ & $<0.0002$ & $<0.0002$ & $<0.0002$ & $<0.0002$ & $<0.0002$ & $<0.0002$ \\
\hline Molybdenum & $<0.01$ & $<0.01$ & $<0.01$ & $<0.01$ & $<0.01$ & $<0.01$ & $<0.01$ & $<0.01$ \\
\hline Nickel & $<0.01$ & $<0.01$ & $<0.01$ & $<0.01$ & $<0.01$ & $<0.01$ & 0.012 & $<0.01$ \\
\hline Selenium & $<0.05$ & $<0.05$ & $<0.05$ & $<0.05$ & $<0.05$ & $<0.05$ & $<0.05$ & $<0.05$ \\
\hline silver & $<0.006$ & $<0.006$ & $<0.006$ & $<0.006$ & $<0.006$ & $<0.006$ & $<0.006$ & $<0.006$ \\
\hline strontium & 0.11 & 0.11 & 0.11 & 0.1 & 0.043 & 0.043 & 0.048 & 0.048 \\
\hline Thorium & $<0.2$ & $<0.2$ & $<0.2$ & $<0.2$ & $<0.2$ & $<0.2$ & $<0.2$ & $<0.2$ \\
\hline Uranium (Fluor) & 0.002 & 0.004 & $<0.001$ & 0.001 & $<0.001$ & 0.001 & $<0.001$ & $<0.001$ \\
\hline Vanadium & $<0.005$ & $<0.005$ & $<0.005$ & $<0.005$ & $<0.005$ & $<0.005$ & $<0.005$ & $<0.005$ \\
\hline zinc & 0.012 & 0.0078 & $<0.002$ & $<0.002$ & 0.0048 & 0.0034 & 0.011 & 0.01 \\
\hline MAJOR IONS $(\mathrm{mg} / \mathrm{L})$ & - &. & . & - &. &. & - & • \\
\hline Alkalinity- $\mathrm{HCO} 3$ & $235^{\circ}$ & $\cdot$ & 236 & . & 237 & $\cdot$ & $24 \dot{2}$ & • \\
\hline Alkalinity-CO3 & $<1$ &. & $<1$ & . & $<1$ &. & $<1$ & . \\
\hline Calcium & 63 & 63 & 68 & 68 & 59 & 61 & 60 & 60 \\
\hline Chloride & 6.4 &. & 7.3 & . & 5.8 &. & 6.1 & . \\
\hline Fluoride & 0.2 & . & 0.2 & . & 0.2 & $\cdot$ & 0.2 & • \\
\hline Magnesium & 29 & 29 & 30 & 30 & 29 & 29 & 28 & 28 \\
\hline Manganese & 0.049 & 0.043 & 0.087 & 0.079 & 0.015 & 0.015 & 0.0089 & 0.012 \\
\hline Nitrate-N & 8.6 &. & 8.76 & . & 3.5 &. & 3.32 & • \\
\hline Potassium & 1.3 & 1.2 & 1.5 & 1.6 & 2.4 & 0.6 & 1.3 & 1.3 \\
\hline Sodium & 2.4 & 2.5 & 2.7 & 2.6 & 1.4 & 1.4 & 1.6 & 1.7 \\
\hline Sulfate & 12.3 &. & 13.3 & - & 17 & . & 12.4 & . \\
\hline
\end{tabular}

(CONTINUED) 
APPENDIX 8.1

Groundwater and Surface-water Quality Data, 1993

\begin{tabular}{|c|c|c|c|c|c|c|c|c|}
\hline \multirow{4}{*}{$\begin{array}{l}\text { 8ampling point } \\
\text { Location } \\
\text { Date sampled }\end{array}$} & \multicolumn{4}{|c|}{$6 w-740$} & \multicolumn{4}{|c|}{$6 w-793$} \\
\hline & \multicolumn{4}{|c|}{$\mathbf{E x p}$} & \multicolumn{4}{|c|}{ AOLrs? } \\
\hline & \multicolumn{2}{|c|}{$09 / 27 / 93$} & \multicolumn{2}{|c|}{$12 / 11 / 93$} & \multicolumn{2}{|c|}{$02 / 26 / 93$} & \multicolumn{2}{|c|}{$05 / 25 / 93$} \\
\hline & TOT & DIs & TOT & DIs & TOT & DI8 & TOT & DI8 \\
\hline remas (mg/L) &. &. & . & • & . & . &. & \\
\hline Aluminum & $<0.02$ & 0.032 & 0.029 & 0.028 & $<0.02$ & $<0.02$ & 0.039 & 0.023 \\
\hline Antimony & $<0.05$ & $<0.05$ & $<0.05$ & $<0.05$ & $<0.05$ & $<0.05$ & $<0.05$ & $<0.05$ \\
\hline Arsenic & $<0.05$ & $<0.05$ & $<0.05$ & $<0.05$ & $<0.05$ & $<0.05$ & $<0.05$ & $<0.05$ \\
\hline Barium & 0.093 & 0.092 & 0.088 & 0.088 & 0.14 & 0.13 & 0.13 & 0.13 \\
\hline Beryllium & $<0.0003$ & 0.00033 & $<0.0003$ & $<0.0003$ & $<0.0003$ & $<0.0003$ & $<0.0003$ & $<0.0003$ \\
\hline Boron & 0.038 & 0.032 & 0.043 & 0.026 & 0.027 & $<0.004$ & 0.014 & 0.022 \\
\hline Cadmium (AAS) & $<0.002$ & $<0.002$ & $<0.002$ & $<0.002$ & $<0.002$ & $<0.002$ & $<0.002$ & $<0.002$ \\
\hline Cadmium & 0.0033 & $<0.003$ & $<0.003$ & $<0.003$ & $<0.003$ & $<0.003$ & $<0.003$ & $<0.003$ \\
\hline Chromium (AAS) & $<0.01$ & $<0.01$ & $<0.01$ & $<0.01$ & $<0.01$ & $<0.01$ & $<0.01$ & $<0.01$ \\
\hline Chromium & $<0.01$ & $<0.01$ & $<0.01$ & $<0.01$ & $<0.01$ & $<0.01$ & $<0.01$ & $<0.01$ \\
\hline Cobalt & $<0.005$ & $<0.005$ & $<0.005$ & $<0.005$ & $<0.005$ & $<0.005$ & $<0.005$ & $<0.005$ \\
\hline Copper & $<0.004$ & $<0.004$ & 0.0042 & $<0.004$ & 0.0064 & 0.0052 & 0.0093 & $<0.004$ \\
\hline Iron & 0.17 & 0.093 & 0.22 & 0.054 & 0.056 & $<0.005$ & 0.079 & 0.012 \\
\hline Lead (AAS) & $<0.004$ & $<0.004$ & $<0.004$ & $<0.004$ & $<0.004$ & $<0.004$ & $<0.004$ & $<0.004$ \\
\hline Mercury (CVAA) & $<0.0002$ & $<0.0002$ & $<0.0002$ & $<0.0002$ & $<0.0002$ & $<0.0002$ & $<0.0002$ & $<0.0002$ \\
\hline Mol ybdenum & $<0.01$ & $<0.01$ & $<0.01$ & $<0.01$ & $<0.01$ & $<0.01$ & $<0.01$ & $<0.01$ \\
\hline Nickel & $<0.01$ & $<0.01$ & $<0.01$ & $<0.01$ & $<0.01$ & $<0.01$ & 0.012 & $<0.01$ \\
\hline Selenium & $<0.05$ & $<0.05$ & $<0.05$ & $<0.05$ & $<0.05$ & $<0.05$ & $<0.05$ & $<0.05$ \\
\hline silver & $<0.006$ & $<0.006$ & $<0.006$ & $<0.006$ & $<0.006$ & $<0.006$ & $<0.006$ & $<0.006$ \\
\hline strontium & 0.055 & 0.055 & 0.05 & 0.05 & 0.089 & 0.089 & 0.091 & 0.085 \\
\hline Thorium & $<0.2$ & $<0.2$ & $<0.2$ & $<0.2$ & $<0.2$ & $<0.2$ & $<0.2$ & $<0.2$ \\
\hline Uranium (Fluor) & $<0.001$ & 0.001 & 0.001 & 0.001 & 0.001 & $<0.001$ & $<0.001$ & $<0.001$ \\
\hline Vanadium & $<0.005$ & $<0.005$ & $<0.005$ & $<0.005$ & $<0.005$ & $<0.005$ & $<0.005$ & $<0.005$ \\
\hline zinc & 0.012 & 0.012 & $<0.002$ & $<0.002$ & 0.0098 & 0.0087 & 0.017 & 0.012 \\
\hline MAJOR IONS (mg/L) &. &. &. & . & . &. &. & • \\
\hline Alkalinity-HCO3 & 261 &. & ${ }_{25} \dot{3}$ & - & $15 \dot{0}$ & $\cdot$ & . & 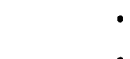 \\
\hline Alkalinity-CO3 & $<1$ &. & $<1$ &. & $\begin{array}{r}130 \\
<1\end{array}$ & $\cdot$. & $\begin{array}{r}130 \\
<1\end{array}$ & - \\
\hline Calcium & 67 & 67 & 67 & 68 & 59 & 59 & 51 & 48 \\
\hline Chloride & 7.2 &. & 8.2 & . & $<1$ &. & $<1$ & . \\
\hline Fluoride & 0.1 &. & 0.2 & . & 0.1 &. & 0.1 & . \\
\hline Magnesium & 32 & 32 & 32 & 32 & 4.7 & 4.7 & 4.3 & 4.3 \\
\hline Manganese & 0.0074 & 0.0067 & 0.0094 & 0.0081 & 0.097 & 0.092 & 0.11 & 0.11 \\
\hline Nitrate-N & 4.8 &. & 4.78 & . & $<0.2$ &. & $<0.2$ & . \\
\hline Potassium & 1.3 & 1.4 & 1.6 & 1.8 & 1.3 & $<0.6$ & 0.85 & 0.73 \\
\hline Sodium & 2.1 & 2.1 & 2.2 & 2.3 & 3.8 & 3.7 & 3.5 & 3.4 \\
\hline Sulfate & 13.3 & . & 11.9 &. & 6 &. & 4.6 & . \\
\hline
\end{tabular}

(CONTINUED) 
APPENDIX 1.1

Groundwater and surface-Water Quality Data, 1993

\begin{tabular}{|c|c|c|c|c|c|c|c|c|}
\hline \multirow{4}{*}{$\begin{array}{l}\text { sampling polnt } \\
\text { iocation } \\
\text { Date sampled }\end{array}$} & \multicolumn{4}{|c|}{ CW-793 } & \multicolumn{4}{|c|}{ 6w-794 } \\
\hline & \multicolumn{4}{|c|}{ AOLL8F } & \multicolumn{4}{|c|}{ AOLL81 } \\
\hline & \multicolumn{2}{|c|}{$07 / 11 / 93$} & \multicolumn{2}{|c|}{$10 / 18 / 93$} & \multicolumn{2}{|c|}{$03 / 01 / 93$} & \multicolumn{2}{|c|}{$05 / 25 / 93$} \\
\hline & TOT & Drs & TOT & DIs & TOT & DIs & TOT & DIs \\
\hline Merars (mg/I) & - & $\cdot$ & $\cdot$ & $\cdot$ & • & $\cdot$ & $\cdot$ & • \\
\hline Aluminum & 0.57 & $<0.02$ & 0.12 & $<0.02$ & 9.6 & 0.022 & 0.23 & $<0.02$ \\
\hline Antimony & $<0.05$ & $<0.05$ & $<0.05$ & $<0.05$ & $<0.5$ & $<0.03$ & $<0.05$ & $<0.05$ \\
\hline Armentc & $<0.05$ & $<0.05$ & $<0.05$ & $<0.05$ & $<0.5$ & $<0.05$ & $<0.05$ & $<0.05$ \\
\hline Barium & 0.14 & 0.13 & 0.15 & 0.15 & 0.19 & 0.084 & 0.091 & 0.091 \\
\hline Beryllium & $<0.0003$ & $<0.0003$ & $<0.0003$ & $<0.0003$ & $<0.003$ & $<0.0003$ & $<0.0003$ & $<0.0003$ \\
\hline Boron & 0.018 & 0.0069 & 0.01 & 0.012 & 0.13 & 0.038 & 0.052 & 0.047 \\
\hline Cadmium (MAS) & $<0.002$ & $<0.002$ & $<0.002$ & $<0.002$ & $<0.002$ & $<0.002$ & $<0.002$ & $<0.002$ \\
\hline Cadmium & $<0.003$ & $<0.003$ & $<0.003$ & $<0.003$ & $<0.03$ & $<0.003$ & $<0.003$ & $<0.003$ \\
\hline Chromium (AAS) & $<0.01$ & $<0.01$ & $<0.01$ & $<0.01$ & 0.032 & $<0.01$ & $<0.01$ & $<0.01$ \\
\hline Chromium & $<0.01$ & $<0.01$ & $<0.01$ & $<0.01$ & $<0.1$ & $<0.01$ & $<0.01$ & $<0.01$ \\
\hline Cobalt & 0.0054 & $<0.005$ & $<0.005$ & $<0.005$ & $<0.05$ & $<0.005$ & $<0.005$ & $<0.005$ \\
\hline Copper & 0.012 & $<0.004$ & 0.0041 & $<0.004$ & $<0.04$ & 0.0046 & 0.0084 & $<0.004$ \\
\hline Iron & 0.91 & $<0.005$ & 0.19 & 0.022 & 17 & 0.0093 & 0.36 & $<0.005$ \\
\hline Lead (AAS) & $<0.004$ & $<0.004$ & $<0.004$ & $<0.004$ & 0.013 & $<0.004$ & $<0.004$ & $<0.004$ \\
\hline Mercury (CVAA) & $<0.0002$ & $<0.0002$ & $<0.0002$ & $<0.0002$ & $<0.0002$ & $<0.0002$ & $<0.0002$ & $<0.0002$ \\
\hline Molybdenum & $<0.01$ & $<0.01$ & $<0.01$ & $<0.01$ & $<0.1$ & $<0.01$ & $<0.01$ & $<0.01$ \\
\hline Nickel & $<0.01$ & $<0.01$ & $<0.01$ & $<0.01$ & $<0.1$ & $<0.01$ & $<0.01$ & $<0.01$ \\
\hline Selenium & $<0.05$ & $<0.05$ & $<0.05$ & $<0.05$ & $<0.5$ & $<0.05$ & $<0.05$ & $<0.05$ \\
\hline silver & $<0.006$ & $<0.006$ & $<0.006$ & $<0.006$ & $<0.06$ & $<0.006$ & $<0.006$ & $<0.006$ \\
\hline strontium & 0.088 & 0.086 & 0.096 & 0.094 & 0.35 & 0.16 & 0.16 & 0.16 \\
\hline Thorium & $<0.2$ & $<0.2$ & $<0.2$ & $<0.2$ & $<2$ & $<0.2$ & $<0.2$ & $<0.2$ \\
\hline Uranium (Fluor) & 0.001 & $<0.001$ & $<0.001$ & $<0.001$ & 0.002 & 0.001 & 0.001 & 0.001 \\
\hline Vanadium & $<0.005$ & $<0.005$ & $<0.005$ & $<0,005$ & $<0.05$ & $<0.005$ & $<0.005$ & $<0.005$ \\
\hline Zinc & 0.011 & 0.006 & 0.0068 & 0.0062 & 0.069 & 0.0091 & 0.016 & 0.013 \\
\hline MAJOR IONS $(\mathrm{mg} / \mathrm{I})$ & - & - & - & - & - & - & • & • \\
\hline Alkalinity-HCO3 & $154^{\circ}$ & • & $164^{\circ}$ & • & 222 & $\cdot$ & $20 \dot{9}$ & $\cdot$ \\
\hline Alkalinity- $\mathrm{CO} 3$ & $<1$ & . & $<1$ & . & $<1$ & . & $<1$ & . \\
\hline Calcium & 49 & 49 & 55 & 53 & 190 & 97 & 90 & 85 \\
\hline Chloride & $<1$ & - & 1 & - & 9.5 & - & 11.5 & . \\
\hline Fluoride & 0.2 & . & 0.1 & . & 0.2 & - & $<0.1$ & . \\
\hline Magnesium & 4.5 & 4.3 & 4.7 & 4.6 & 19 & 6.5 & 6.7 & 6.6 \\
\hline Manganese & 0.16 & 0.1 & 0.15 & 0.13 & 0.58 & 0.028 & 0.031 & 0.039 \\
\hline Nitrate-N & $<0.2$ & - & $<0.2$ & . & $<0.2$ & . & $<0.2$ & . \\
\hline Potassium & $<0.6$ & 0.64 & 0.69 & 1 & 11 & 2.1 & 1.7 & 1.6 \\
\hline Sodium & 3.4 & 3.3 & 3.5 & 3.5 & 0.23 & 3.2 & 3 & 3 \\
\hline sulfate & 5.2 & . & 3.9 & . & 39.1 & - & 20.4 & . \\
\hline
\end{tabular}

(CONTINUED) 
APPENDIX $\mathbf{1} .1$

Groundwater and surface-Water Quallty Data, 1993

\begin{tabular}{|c|c|c|c|c|c|c|c|c|}
\hline \multirow{4}{*}{$\begin{array}{l}\text { Bampling Point } \\
\text { Location } \\
\text { Date sampled }\end{array}$} & \multicolumn{4}{|c|}{ 6w-794 } & \multicolumn{4}{|c|}{ OM-79s } \\
\hline & \multicolumn{4}{|c|}{ AOLL8F } & \multicolumn{4}{|c|}{ AOLLSF } \\
\hline & \multicolumn{2}{|c|}{$07 / 11 / 93$} & \multicolumn{2}{|c|}{$10 / 18 / 93$} & \multicolumn{2}{|c|}{$03 / 02 / 93$} & \multicolumn{2}{|c|}{$05 / 25 / 93$} \\
\hline & TOT & DI8 & TOT & DI8 & TOT & Dr8 & $\operatorname{TOT}$ & DI8 \\
\hline Mathrs (mg/L) & $\cdot$ & $\cdot$ & - & $\cdot$ & $\cdot$ & - & - & - \\
\hline Aluminum & 0.29 & $<0.02$ & 0.28 & $<0.02$ & 0.024 & $<0.02$ & 0.024 & 0.036 \\
\hline Antimony & $<0.05$ & $<0.05$ & $<0.05$ & $<0.05$ & $<0.05$ & $<0.05$ & $<0.05$ & $<0.05$ \\
\hline Arsente & $<0.05$ & $<0.05$ & $<0.05$ & $<0.05$ & $<0.05$ & $<0.05$ & $<0.05$ & $<0.05$ \\
\hline Barium & 0.1 & 0.12 & 0.1 & 0.1 & 0.052 & 0.051 & 0.053 & 0.053 \\
\hline Bary111um & $<0.0003$ & $<0.0003$ & $<0.0003$ & $<0.0003$ & $<0.0003$ & $<0.0003$ & $<0.0003$ & $<0.0003$ \\
\hline Boron & 0.03 & 0.04 & 0.044 & 0.041 & 0.028 & 0.026 & 0.033 & 0.031 \\
\hline Cadmium (MAs) & $<0.002$ & $<0.002$ & $<0.002$ & $<0.002$ & $<0.002$ & $<0.002$ & $<0.002$ & $<0.002$ \\
\hline Cadmium & $<0,003$ & $<0.003$ & $<0.003$ & $<0.003$ & $<0.003$ & $<0.003$ & $<0.003$ & $<0.003$ \\
\hline Chromiun (AMS) & $<0.01$ & $<0.01$ & $<0.01$ & $<0.01$ & $<0.01$ & $<0.01$ & $<0.01$ & $<0.01$ \\
\hline Chromium & $<0.01$ & $<0.01$ & $<0.01$ & $<0.01$ & $<0.01$ & $<0.01$ & $<0.01$ & $<0.01$ \\
\hline Cobalt & $<0.005$ & $<0.005$ & 0.0067 & $<0.005$ & $<0.005$ & $<0.005$ & $<0.005$ & $<0.005$ \\
\hline Copper & $<0.004$ & $<0.004$ & $<0.004$ & 0.0059 & $<0.004$ & $<0.004$ & $<0.004$ & $<0.004$ \\
\hline Iron & 0.37 & $<0.005$ & 0.53 & 0.046 & 0.021 & $<0.005$ & 0.012 & $<0.005$ \\
\hline Lead (AMS) & $<0.004$ & $<0.004$ & 0.004 & $<0.004$ & $<0.004$ & $<0.004$ & $<0.004$ & $<0.004$ \\
\hline Mercury (CVAA) & $<0.0002$ & $<0.0002$ & $<0.0002$ & $<0.0002$ & $<0.0002$ & $<0.0002$ & $<0.0002$ & $<0.0002$ \\
\hline Mol ybdenum & $<0.01$ & $<0.01$ & $<0.01$ & $<0.01$ & $<0.01$ & $<0.01$ & $<0.01$ & $<0.01$ \\
\hline Nickel & $<0.01$ & $<0.01$ & $<0.01$ & $<0.01$ & $<0.01$ & $<0.01$ & $<0.01$ & $<0.01$ \\
\hline Selenium & $<0.05$ & $<0.05$ & $<0.05$ & $<0.05$ & $<0.05$ & $<0.05$ & $<0.05$ & $<0.05$ \\
\hline silver & $<0.006$ & $<0.006$ & $<0.006$ & $<0.006$ & $<0.006$ & $<0.006$ & $<0.006$ & $<0.006$ \\
\hline strontium & 0.16 & 0.16 & 0.17 & 0.16 & 0.13 & 0.13 & 0.13 & 0.13 \\
\hline Thorium & $<0.2$ & $<0.2$ & $<0.2$ & $<0.2$ & $<0.2$ & $<0.2$ & $<0.2$ & $<0.2$ \\
\hline Uranium (Fluor) & 0.001 & 0.001 & 0.00 & 0.001 & 0.001 & 0.001 & 0.001 & 0.001 \\
\hline Vanadium & $<0.005$ & $<0.005$ & $<0.005$ & $<0.005$ & $<0.005$ & $<0.005$ & $<0.005$ & $<0.005$ \\
\hline zine & 0.011 & 0.0083 & 0.011 & 0.013 & 0.0095 & 0.013 & 0.019 & 0.022 \\
\hline MAJOR IONS $(\mathrm{mg} / \mathrm{L})$ & $\cdot$ & . & $\cdot$ & • & . & . & . & 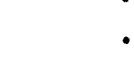 \\
\hline Alkalinity-HCO3 & 222 &. & 229 & $\cdot$ & 178 &. & 195 & $\bullet$ \\
\hline Alkalinity-CO3 & $<1$ & . & $<1$ & . & $<1$ &. & $<1$ & 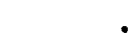 \\
\hline Calcium & 81 & 66 & 91 & 87 & 90 & 89 & 79 & 79 \\
\hline Chloride & 11.3 & $\cdot$ & 11.8 & $\cdot$ & 4.9 & $\cdot$ & 4.7 & $\cdot$ \\
\hline Fluoride & 0.1 & $\cdot$ & $<0.1$ & $\cdot$ & $<0.1$ & . & $<0.1$ & - \\
\hline Magnesium & 8.6 & 12 & 7.6 & 7.6 & 3.5 & 3.5 & 3.3 & 3.2 \\
\hline Manganese & 0.013 & 0.0037 & 0.023 & 0.0073 & 0.015 & 0.0064 & 0.022 & 0.023 \\
\hline Nitrate-N & 0.3 &. & 0.37 & . & $<0.2$ & . & $<0.2$ & 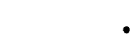 \\
\hline Potassium & 3.1 & 3 & 2.7 & 2.9 & 0.87 & 0.67 & 0.71 & 0.74 \\
\hline Sodium & 2.7 & 1.9 & 3 & 3.1 & 2.1 & 2.2 & 2.2 & 2.2 \\
\hline sulfate & 23.5 & . & 19.2 & - & 20.3 & $\cdot$ & 22.2 & • \\
\hline
\end{tabular}

(CONTINUED) 
APPENDIX $\mathbf{E} .1$

\begin{tabular}{|c|c|c|c|c|c|c|c|c|}
\hline \multirow{4}{*}{$\begin{array}{l}\text { 8ampling Point } \\
\text { Location } \\
\text { Date sampled }\end{array}$} & \multicolumn{4}{|c|}{$0 w-79 s$} & \multicolumn{4}{|c|}{ OW-800 } \\
\hline & \multicolumn{4}{|c|}{ MathsF } & \multicolumn{4}{|c|}{ orf } \\
\hline & \multicolumn{2}{|c|}{$07 / 12 / 93$} & \multicolumn{2}{|c|}{$10 / 28 / 93$} & \multicolumn{2}{|c|}{$05 / 11 / 93$} & \multicolumn{2}{|c|}{$09 / 14 / 93$} \\
\hline & TOT & DIs & TOT & DI8 & Tor & DI8 & TOT & DI8 \\
\hline $\operatorname{mrazs}(\mathrm{mg} / \mathrm{L})$ & $\cdot$ & - & - & $\cdot$ & - & $\cdot$ & $\cdot$ & $\cdot$ \\
\hline Aluminum & 0.031 & $<0.02$ & $<0.02$ & $<0.02$ & 0.065 & $<0.02$ & 2.4 & $<0.02$ \\
\hline Antimony & $<0.08$ & $<0.05$ & $<0.05$ & $<0.05$ & $<0.05$ & $<0.05$ & $<0.05$ & $<0.05$ \\
\hline Arsonic & $<0.05$ & $<0.05$ & $<0.05$ & $<0.05$ & $<0.05$ & $<0.05$ & $<0.05$ & $<0.05$ \\
\hline Barlum & 0.058 & 0.058 & 0.071 & 0.07 & 0.09 & 0.09 & 0.17 & 0.15 \\
\hline Beryl11um & $<0.0003$ & $<0.0003$ & $<0.0003$ & $<0.0003$ & $<0.0003$ & $<0.0003$ & $<0.0003$ & $<0.0003$ \\
\hline Boron & 0.026 & 0.028 & 0.029 & 0.031 & 0.033 & 0.029 & 0.037 & 0.037 \\
\hline Cadmium (AMS) & $<0.002$ & $<0.002$ & $<0.002$ & $<0.002$ & $<0.002$ & $<0.002$ & $<0.002$ & $<0.002$ \\
\hline Cadmium & $<0.003$ & $<0.003$ & $<0.003$ & $<0.003$ & $<0.003$ & $<0.003$ & $<0.003$ & $<0.003$ \\
\hline Chromium (MAS) & $<0.01$ & $<0.01$ & $<0.01$ & $<0.01$ & $<0.01$ & $<0.01$ & $<0.01$ & $<0.01$ \\
\hline Chromium & $<0.01$ & $<0.01$ & $<0.01$ & $<0.01$ & $<0.01$ & $<0.01$ & $<0.01$ & $<0.01$ \\
\hline Cobalt & $<0.005$ & $<0.005$ & $<0.005$ & $<0.005$ & $<0.005$ & $<0.005$ & $<0.005$ & $<0.005$ \\
\hline Copper & $<0.004$ & $<0.004$ & $<0.004$ & $<0.004$ & $<0.004$ & $<0.004$ & 0.011 & 0.0053 \\
\hline Iron & 0.015 & $<0.005$ & 0.023 & $<0.005$ & 0.051 & $<0.005$ & 3.5 & $<0.005$ \\
\hline Load (MAS) & $<0.004$ & $<0.004$ & 0.0043 & $<0.004$ & $<0.004$ & $<0.004$ & 0.0089 & $<0.004$ \\
\hline Mercury (CVMA) & $<0.0002$ & $<0.0002$ & $<0.0002$ & $<0.0002$ & $<0.0002$ & $<0.0002$ & $<0,0002$ & $<0.0002$ \\
\hline Molybdanum & $<0.01$ & $<0.01$ & $<0.01$ & $<0.01$ & $<0.01$ & $<0.01$ & $<0.01$ & $<0.01$ \\
\hline Nickel & $<0.01$ & $<0.01$ & $<0.01$ & $<0.01$ & $<0.01$ & 0.023 & $<0.01$ & $<0.01$ \\
\hline Selenium & $<0.05$ & $<0.05$ & $<0.05$ & $<0.05$ & $<0.05$ & $<0.05$ & $<0.05$ & $<0.05$ \\
\hline silver & $<0.006$ & $<0.006$ & $<0.006$ & $<0.006$ & $<0.006$ & $<0.006$ & $<0.006$ & $<0.006$ \\
\hline Strontium & 0.13 & 0.14 & 0.16 & 0.16 & 0.15 & 0.15 & 0.31 & 0.29 \\
\hline Thorium & $<0.2$ & $<0.2$ & $<0.2$ & $<0.2$ & $<0.2$ & $<0.2$ & $<0.2$ & $<0.2$ \\
\hline Uranium (Fluor) & 0.001 & 0.001 & 0.003 & 0.003 & 0.001 & 0.001 & 0.002 & - \\
\hline Vanadium & $<0.005$ & $<0.005$ & $<0.005$ & $<0.005$ & $<0,005$ & $<0.005$ & 0.0086 & $<0.005$ \\
\hline zinc & 0.0078 & 0.0069 & 0.011 & 0.0099 & 0.074 & 0.067 & 0.041 & 0.031 \\
\hline MAJOR IONS (mg/L) & $\cdot$ & $\cdot$ & $\cdot$ & $\cdot$ & $\cdot$ & . & . & - \\
\hline Alkalinity-HCO3 & 204 & $\cdot$ & $\begin{array}{r}\cdot \\
215\end{array}$ & $\cdot$ & 183 &. & 276 & • \\
\hline Alkalinity-CO3 & $<1$ &. & $<1$ & $\cdot$ & $<1$ & . & $<1$ & - \\
\hline Calcium & 81 & 82 & 94 & 95 & 47 & 47 & 72 & 71 \\
\hline Chloride & 4.3 & $\cdot$ & 6.7 & $\cdot$ & 1.5 & $\cdot$ & 2.4 & - \\
\hline Fluoride & $<0.1$ & $\cdot$ & $<0.1$ & $\cdot$ & $<0.1$ & $\cdot$ & 0.1 & - \\
\hline Magnesium & 3.5 & 3.5 & 4.5 & 4.5 & 17 & 17 & 34 & 31 \\
\hline Manganese & 0.052 & 0.047 & 0.018 & 0.016 & 0.0019 & 0.0033 & 0.21 & $<0.001$ \\
\hline Nitrate-N & $<0.2$ &. & 5.2 & . & $<0.2$ & . & $<0.2$ & - \\
\hline Potassium & 1.2 & 1.1 & 1.2 & 1.1 & 1.9 & 1.8 & 3.4 & 3.4 \\
\hline Sodium & 1.9 & 2 & 3 & 3 & 0.81 & 0.84 & 1.2 & 1.2 \\
\hline sulfate & 20.9 & $\cdot$ & 21 & $\cdot$ & 13 & $\cdot$ & 21 & • \\
\hline
\end{tabular}

( CONT INUED) 
Groundwater and gurfuce-Water Quality Date, 1993

\begin{tabular}{|c|c|c|}
\hline \multirow{4}{*}{$\begin{array}{l}\text { Banpling polnt } \\
\text { Iocation } \\
\text { Date samplod }\end{array}$} & \multicolumn{2}{|c|}{$O W-800$} \\
\hline & \multicolumn{2}{|c|}{ ore } \\
\hline & \multicolumn{2}{|c|}{$10 / 22 / 93$} \\
\hline & $\operatorname{sos}$ & DI8 \\
\hline nernes (mg/L) & - & • \\
\hline Mluminum & 0.42 & $<0.02$ \\
\hline Ant Imony & $<0.05$ & $<0.05$ \\
\hline Areento & $<0.05$ & $<0.05$ \\
\hline Dartum & 0.13 & 0.13 \\
\hline Bery $111 \mathrm{um}$ & $<0.0003$ & $<0.0003$ \\
\hline Boron & 0.019 & 0.019 \\
\hline Cadmium (NAS) & $<0.002$ & $<0.002$ \\
\hline Cadmium & $<0.003$ & $<0.003$ \\
\hline Chronlum (MAs) & $<0.01$ & $<0.01$ \\
\hline Chromlum & $<0.01$ & $<0.01$ \\
\hline Cobalt & $<0.005$ & $<0.005$ \\
\hline Copper & $<0.004$ & $<0.004$ \\
\hline Iron & 0.43 & 0.006 \\
\hline Iread (MAs) & $<0.004$ & $<0.004$ \\
\hline Meroury (CVAN) & $<0.0002$ & $<0.0002$ \\
\hline Molybdenum & $<0.01$ & $<0.01$ \\
\hline Nickel & $<0.01$ & $<0.01$ \\
\hline selentum & $<0.05$ & $<0.05$ \\
\hline silver & $<0.006$ & $<0.006$ \\
\hline strontium & 0.18 & 0.17 \\
\hline Thorlum & $<0.2$ & $<0.2$ \\
\hline Uranium (Fluor) & 0.001 & 0.001 \\
\hline Vanadium & $<0.005$ & $<0.005$ \\
\hline anc & 0.018 & 0.015 \\
\hline MWOR IONS (mg/L) & . & - \\
\hline Alkalinity-hCO3 & 248 & - \\
\hline Alkalindty $-\cos$ & $<1$ & - \\
\hline Calcium & 59 & 58 \\
\hline Chloride & 2 & - \\
\hline Fluoride & 0.1 & - \\
\hline Magnesium & 23 & 23 \\
\hline Manganese & 0.044 & $<0.001$ \\
\hline N1trate-N & $<0.2$ & • \\
\hline Potaseium & 2.9 & 2.6 \\
\hline sodium & 0.87 & 0.87 \\
\hline sulfate & 16 & • \\
\hline
\end{tabular}




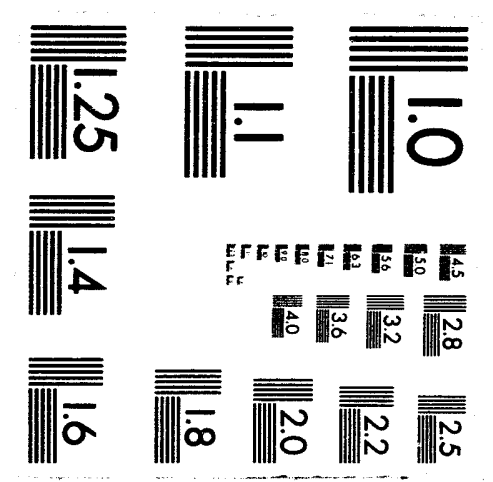



APPENDIX E.1

Groundwater and Surface-Water Quality Data, 1993

\begin{tabular}{|c|c|c|c|c|c|c|c|c|}
\hline \multirow{4}{*}{$\begin{array}{l}\text { Sampling Point } \\
\text { Location } \\
\text { Date Sampled }\end{array}$} & \multicolumn{8}{|c|}{ BCK-00.63 } \\
\hline & \multicolumn{8}{|c|}{$\operatorname{EXP}$} \\
\hline & \multicolumn{2}{|c|}{$03 / 09 / 93$} & \multicolumn{2}{|c|}{$05 / 03 / 93$} & \multicolumn{2}{|c|}{$08 / 16 / 93$} & \multicolumn{2}{|c|}{$11 / 08 / 93$} \\
\hline & TOT & DIS & TOT & DIS & TOT & DIS & TOT & Drs \\
\hline METALS (mg/L) & - &. & $\cdot$ & - & . & $\cdot$ & $\cdot$ & - \\
\hline Aluminum & 0.17 & 0.038 & 0.066 & $<0.02$ & 0.25 & $<0.02$ & 0.076 & 0.039 \\
\hline Antimony & $<0.05$ & $<0.05$ & 0.057 & $<0.05$ & $<0.05$ & $<0.05$ & $<0.05$ & $<0.05$ \\
\hline Arsenic & $<0.05$ & $<0.05$ & $<0.05$ & $<0.05$ & $<0.05$ & $<0.05$ & $<0.05$ & $<0.05$ \\
\hline Barium & 0.044 & 0.042 & 0.048 & 0.045 & 0.073 & 0.05 & 0.078 & 0.071 \\
\hline Beryllium & 0.00043 & 0.00056 & $<0.0003$ & $<0.0003$ & $<0.0003$ & $<0.0003$ & 0.0062 & $<0.0003$ \\
\hline Boron & 0.048 & 0.049 & 0.082 & 0.056 & 0.092 & 0.087 & 0.092 & 0.073 \\
\hline Cadmium (AAS) & $<0.002$ & $<0.002$ & $<0.002$ & $<0.002$ & $<0.002$ & $<0.002$ & $<0.002$ & $<0.002$ \\
\hline Cadmium & $<0.003$ & $<0.003$ & $<0.003$ & $<0.003$ & $<0.003$ & $<0.003$ & 0.0082 & $<0.003$ \\
\hline Chromium (AAS) & $<0.01$ & $<0.01$ & $<0.01$ & $<0.01$ & $<0.01$ & $<0.01$ & $<0.01$ & $<0.01$ \\
\hline Chromium & $<0.01$ & $<0.01$ & $<0.01$ & $<0.01$ & $<0.01$ & $<0.01$ & $<0.01$ & $<0.01$ \\
\hline Cobalt & $<0.005$ & $<0.005$ & $<0.005$ & $<0.005$ & $<0.005$ & $<0.005$ & 0.0064 & $<0.005$ \\
\hline Copper & $<0.004$ & $<0.004$ & $<0.004$ & $<0.004$ & $<0.004$ & $<0.004$ & 0.011 & $<0.004$ \\
\hline Iron & 0.13 & 0.02 & 0.097 & 0.019 & 0.25 & 0.014 & 0.096 & 0.033 \\
\hline Lead (AAS) & $<0.004$ & $<0.004$ & $<0.004$ & $<0.004$ & $<0.004$ & $<0.004$ & $<0.004$ & $<0.004$ \\
\hline Mercury (CVAA) & $<0.0002$ & $<0.0002$ & $<0.0002$ & $<0.0002$ & $<0.0002$ & $<0.0002$ & $<0.0002$ & $<0.0002$ \\
\hline Mol ybdenum & $<0.01$ & $<0.01$ & $<0.01$ & $<0.01$ & $<0.01$ & $<0.01$ & $<0.01$ & $<0.01$ \\
\hline Nickel & $<0.01$ & $<0.01$ & 0.01 & $<0.01$ & $<0.01$ & $<0.01$ & 0.01 & $<0.01$ \\
\hline Selenium & $<0.05$ & $<0.05$ & $<0.05$ & $<0.05$ & $<0.05$ & $<0.05$ & $<0.05$ & $<0.05$ \\
\hline Silver & $<0.006$ & $<0.006$ & $<0.006$ & $<0.006$ & $<0.006$ & $<0.006$ & $<0.006$ & $<0.006$ \\
\hline strontium & 0.056 & 0.057 & 0.055 & 0.061 & 0.092 & 0.1 & 0.13 & 0.12 \\
\hline Thorium & $<0.2$ & $<0.2$ & $<0.2$ & $<0.2$ & $<0.2$ & $<0.2$ & $<0.2$ & $<0.2$ \\
\hline Uranium (Fluor) & 0.015 & 0.019 & 0.018 & 0.017 & 0.032 & 0.033 & 0.03 & 0.03 \\
\hline Vanadium & $<0.005$ & $<0.005$ & $<0.005$ & $<0.005$ & $<0.005$ & $<0.005$ & 0.0056 & $<0.005$ \\
\hline zinc & 0.0032 & 0.0075 & 0.0079 & 0.015 & 0.0049 & $<0.002$ & 0.0032 & $<0.002$ \\
\hline MAJOR IONS $(\mathrm{mg} / \mathrm{L})$ & $\cdot$ & $\cdot$ & . & . &. & . & - & • \\
\hline Alkalinity-HCO3 & 95 & $\cdot$ & $\begin{array}{r}0 \\
111\end{array}$ & $\cdot$ & $\begin{array}{r}\bullet \\
169\end{array}$ &. & 163 & • \\
\hline Alkalinity-CO3 & $<1$ & $\cdot$ & $<1$ &. & $<1$ &. & $<1$ & - \\
\hline Calcium & 30 & 31 & 31 & 31 & 55 & 57 & 59 & 58 \\
\hline Chloride & 4.8 &. & 5.1 &. & 10.9 &. & 13 & - \\
\hline Fluoride & $<0.1$ & $\cdot$ & $<0.1$ & . & 0.1 &. & 0.2 & • \\
\hline Magnesium & 8.3 & 8.4 & 9.3 & 9.5 & 16 & 15 & 14 & 14 \\
\hline Manganese & 0.012 & 0.019 & 0.014 & 0.011 & 0.031 & 0.0095 & 0.013 & 0.0065 \\
\hline Nitrate-N & 2 & . & 2.2 & . & 5.1 & $\cdot$ & 9.9 & . \\
\hline Potassium & 0.97 & 1 & 1.2 & 1.1 & 1.8 & 1.5 & 2 & 2 \\
\hline Sodium & 3.2 & 3.3 & 3.3 & 3.2 & 6.3 & 6.6 & 8 & 8 \\
\hline Sulfate & 13 & . & 10 & . & 12.5 & $\cdot$ & 20 & . \\
\hline
\end{tabular}

(CONTINUED) 
APPENDIX E. 1

Groundwater and Surface-Water Quality Data, 1993

\begin{tabular}{|c|c|c|c|c|c|c|c|c|}
\hline \multirow{4}{*}{$\begin{array}{l}\text { Sampling Point } \\
\text { Location } \\
\text { Date Sampled }\end{array}$} & \multicolumn{8}{|c|}{ BCK-04.55 } \\
\hline & \multicolumn{8}{|c|}{ EXP } \\
\hline & \multicolumn{2}{|c|}{$03 / 09 / 93$} & \multicolumn{2}{|c|}{$05 / 03 / 93$} & \multicolumn{2}{|c|}{$08 / 16 / 93$} & \multicolumn{2}{|c|}{$11 / 08 / 93$} \\
\hline & TOT & DIS & TOT & Drs & TOT & DIS & TOT & DIS \\
\hline METALS (mg/L) &. &. & $\cdot$ &. & $\cdot$ &. & $\cdot$ & \\
\hline Aluminum & 0.091 & $<0.02$ & 0.035 & $<0.02$ & 0.087 & $<0.02$ & 0.077 & $<0.02$ \\
\hline Antimony & $<0.05$ & $<0.05$ & $<0.05$ & $<0.05$ & $<0.05$ & $<0.05$ & $<0.05$ & $<0.05$ \\
\hline Arsenic & $<0.05$ & $<0.05$ & $<0.05$ & $<0.05$ & $<0.05$ & $<0.05$ & $<0.05$ & $<0.05$ \\
\hline Barium & 0.045 & 0.044 & 0.05 & 0.047 & 0.071 & 0.073 & 0.081 & 0.073 \\
\hline Beryllium & $<0.0003$ & $<0.0003$ & $<0.0003$ & $<0.0003$ & $<0.0003$ & $<0.0003$ & 0.0072 & 0.00069 \\
\hline Boron & 0.06 & 0.046 & 0.05 & 0.06 & 0.085 & 0.082 & 0.085 & 0.13 \\
\hline Cadmium (AAS) & $<0.002$ & $<0.002$ & $<0.002$ & $<0.002$ & $<0.002$ & $<0.002$ & $<0.002$ & $<0.002$ \\
\hline Cadmium & $<0.003$ & $<0.003$ & $<0.003$ & $<0.003$ & $<0.003$ & $<0.003$ & 0.0097 & $<0.003$ \\
\hline Chromium (AAS) & $<0.01$ & $<0.01$ & $<0.01$ & $<0.01$ & $<0.01$ & $<0.01$ & $<0.01$ & $<0.01$ \\
\hline Chromium & $<0.01$ & $<0.01$ & $<0.01$ & $<0.01$ & $<0.01$ & $<0.01$ & $<0.01$ & $<0.01$ \\
\hline Cobalt & $<0.005$ & $<0.005$ & $<0.005$ & $<0.005$ & $<0.005$ & $<0.005$ & 0.0083 & $<0.005$ \\
\hline Copper & $<0.004$ & $<0.004$ & $<0.004$ & $<0.004$ & $<0.004$ & 0.005 & 0.014 & $<0.004$ \\
\hline Iron & 0.09 & 0.014 & 0.071 & 0.019 & 0.11 & $<0.005$ & 0.082 & 0.01 \\
\hline Lead (AAS) & $<0.004$ & $<0.004$ & $<0.004$ & $<0.004$ & $<0.004$ & $<0.004$ & $<0.004$ & $<0.004$ \\
\hline Mercury (CVAA) & $<0.0002$ & $<0.0002$ & $<0.0002$ & $<0.0002$ & $<0.0002$ & $<0.0002$ & $<0.0002$ & $<0.0002$ \\
\hline Mol ybdenum & $<0.01$ & $<0.01$ & $<0.01$ & $<0.01$ & $<0.01$ & $<0.01$ & $<0.01$ & $<0.01$ \\
\hline Nickel & $<0.01$ & $<0.01$ & $<0.01$ & $<0.01$ & $<0.01$ & $<0.01$ & 0.011 & $<0.01$ \\
\hline Selenium & $<0.05$ & $<0.05$ & $<0.05$ & $<0.05$ & $<0.05$ & $<0.05$ & $<0.05$ & $<0.05$ \\
\hline silver & $<0.006$ & $<0.006$ & $<0.006$ & $<0.006$ & $<0.006$ & $<0.006$ & 0.0061 & $<0.006$ \\
\hline strontium & 0.057 & 0.055 & 0.058 & 0.057 & 0.085 & 0.089 & 0.13 & 0.13 \\
\hline Thorium & $<0.2$ & $<0.2$ & $<0,2$ & $<0.2$ & $<0.2$ & $<0.2$ & $<0.2$ & $<0.2$ \\
\hline Uranium (Fluor) & 0.019 & 0.02 & 0.021 & 0.02 & 0.03 & 0.026 & 0.045 & 0.046 \\
\hline Vanadium & $<0.005$ & $<0.005$ & $<0.005$ & $<0.005$ & $<0.005$ & $<0.005$ & 0.0069 & $<0.005$ \\
\hline zinc & 0.0049 & 0.0035 & 0.017 & 0.0083 & 0.008 & 0.014 & 0.0087 & $<0.002$ \\
\hline MAJOR IONS $(\mathrm{mg} / \mathrm{L})$ & . &. &. &. &. & $\cdot$ & $\cdot$ & e \\
\hline Alkalinity-HCO3 & 102 & $\cdot$ & 118 & $\cdot$ & $\begin{array}{r}\bullet \\
165\end{array}$ & . & $16 \dot{4}$ & • \\
\hline Alkalinity-CO3 & $<1$ &. & $<1$ & . & $<1$ & . & $<1$ & $\theta^{\circ}$ \\
\hline Calcium & 32 & 31 & 33 & 32 & 52 & 53 & 62 & 58 \\
\hline Chloride & 5.1 & . & 5.2 & . & 11.9 & . & 14 & • \\
\hline Fluoride & $<0.1$ &. & $<0.1$ & . & 0.1 & . & 0.2 & 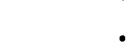 \\
\hline Magnesium & 9 & 8.9 & 10 & 9.8 & 16 & 17 & 15 & 15 \\
\hline Manganese & 0.012 & 0.014 & 0.016 & 0.011 & 0.034 & 0.022 & 0.015 & 0.0066 \\
\hline Nitrate-N & 2.3 & . & 2.6 &. & 6.3 & . & 10.3 & 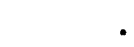 \\
\hline Potassium & 1.1 & 0.99 & 1.1 & 1.2 & 1.7 & 1.7 & 2.3 & 2 \\
\hline Sodium & 3.3 & 3.2 & 3.5 & 3.3 & 6 & 6.3 & 8.2 & 8.5 \\
\hline Sulfate & 13 & . & 11 & . & 13.1 & . & 19 & • \\
\hline
\end{tabular}

(CONTINUED) 
APPENDIX E. 1

Groundwater and Surface-Water Quality Data, 1993

\begin{tabular}{|c|c|c|c|c|c|c|c|}
\hline \multirow{4}{*}{$\begin{array}{l}\text { Sampling Point } \\
\text { Location } \\
\text { Date Sampled }\end{array}$} & \multicolumn{7}{|c|}{$\mathrm{BCK}-09.40$} \\
\hline & \multicolumn{7}{|c|}{ EXP } \\
\hline & \multicolumn{2}{|c|}{$03 / 09 / 93$} & \multicolumn{2}{|c|}{$05 / 03 / 93$} & \multirow{2}{*}{\begin{tabular}{|c|}
$08 / 16 / 93$ \\
TOT
\end{tabular}} & \multicolumn{2}{|c|}{$11 / 08 / 93$} \\
\hline & TOT & DIS & TOT & DIS & & TOT & DIS \\
\hline METALS (mg/L) & $\cdot$ & $\cdot$ & $\cdot$ & $\cdot$ & $\cdot$ & $\cdot$ & - \\
\hline Aluminum & 0.063 & 0.056 & 0.074 & 0.022 & 0.057 & 0.086 & $<0.02$ \\
\hline Antimony & $<0.05$ & $<0.05$ & $<0.05$ & $<0.05$ & $<0.05$ & $<0.05$ & $<0.05$ \\
\hline Arsenic & $<0.05$ & $<0.05$ & $<0.05$ & $<0.05$ & $<0.05$ & $<0.05$ & $<0.05$ \\
\hline Barium & 0.08 & 0.079 & 0.092 & 0.088 & 0.12 & 0.025 & 0.025 \\
\hline Beryliium & $<0.0003$ & $<0.0003$ & $<0.0003$ & $<0.0003$ & $<0.0003$ & $<0.0003$ & $<0.0003$ \\
\hline Boron & 0.15 & 0.15 & 0.16 & 0.15 & 0.43 & 0.059 & 0.065 \\
\hline Cadmium (AAS) & $<0.002$ & $<0.002$ & $<0.002$ & $<0.002$ & $<0.002$ & $<0.002$ & $<0.002$ \\
\hline Cadmium & $<0.003$ & $<0.003$ & $<0.003$ & $<0.003$ & $<0.003$ & $<0.003$ & $<0.003$ \\
\hline Chromium (AAS) & $<0.01$ & $<0.01$ & $<0.01$ & $<0.01$ & $<0.01$ & $<0.01$ & $<0.01$ \\
\hline Chromium & $<0.01$ & $<0.01$ & $<0.01$ & $<0.01$ & $<0.01$ & $<0.01$ & $<0.01$ \\
\hline Cobalt & $<0.005$ & $<0.005$ & $<0.005$ & $<0.005$ & $<0.005$ & $<0.005$ & $<0.005$ \\
\hline Copper & $<0.004$ & $<0.004$ & $<0.004$ & $<0.004$ & $<0.004$ & $<0.004$ & $<0.004$ \\
\hline Iron & 0.11 & 0.026 & 0.15 & 0.028 & 0.16 & 0.07 & $<0.005$ \\
\hline Lead (AAS) & $<0.004$ & $<0.004$ & $<0.004$ & $<0.004$ & $<0.004$ & $<0.004$ & $<0.004$ \\
\hline Mercury (CVAA) & $<0.0002$ & $<0.0002$ & $<0.0002$ & $<0.0002$ & $<0.0002$ & $<0.0002$ & $<0.0002$ \\
\hline Molybdenum & $<0.01$ & $<0.01$ & $<0.01$ & $<0.01$ & $<0.01$ & $<0.01$ & $<0.01$ \\
\hline Nickel & $<0.01$ & $<0.01$ & $<0.01$ & $<0.01$ & $<0.01$ & $<0.01$ & $<0.01$ \\
\hline Selenium & $<0.05$ & $<0.05$ & $<0.05$ & $<0.05$ & $<0.05$ & $<0.05$ & $<0.05$ \\
\hline Silver & $<0.006$ & $<0.006$ & $<0.006$ & $<0.006$ & $<0.006$ & $<0.006$ & $<0.006$ \\
\hline Strontium & 0.14 & 0.14 & 0.15 & 0.14 & 0.22 & 0.024 & 0.022 \\
\hline Thorium & $<0.2$ & $<0.2$ & $<0.2$ & $<0.2$ & $<0.2$ & $<0.2$ & $<0.2$ \\
\hline Uranium (Fluor) & 0.074 & 0.073 & 0.087 & 0.08 & 0.129 & 0.001 & 0.001 \\
\hline Vanadium & $<0.005$ & $<0.005$ & $<0.005$ & $<0.005$ & $<0.005$ & $<0.005$ & $<0.005$ \\
\hline zinc & 0.004 & 0.0037 & 0.0049 & 0.012 & 0.01 & 0.0089 & 0.014 \\
\hline MAJOR IONS (mg/L) & $\cdot$ & $\cdot$ &. &. & $\cdot$ & . & $\cdot$ \\
\hline Alkalinity-HCO3 & 122 & $\cdot$ & 146 & $\cdot$ & 187 & 130 & • \\
\hline Alkalinity- $\mathrm{CO} 3$ & $<1$ & $\cdot$ & $<1$ & $\cdot$ & $<1$ & $<1$ & - \\
\hline Calcium & 54 & 54 & 59 & 57 & 89 & 29 & 29 \\
\hline Chloride & 12 & $\cdot$ & 15 & $\cdot$ & 24.3 & 1 & - \\
\hline Fluoride & 0.2 & $\cdot$ & 0.2 & $\cdot$ & 0.3 & 0.1 & • \\
\hline Magnesium & 10 & 10 & 12 & 11 & 16 & 15 & 15 \\
\hline Manganese & 0.046 & 0.041 & 0.058 & 0.046 & 0.07 & 0.0038 & 0.0016 \\
\hline $\mathrm{Ni}$ rate- $\mathrm{N}$ & 11 & $\cdot$ & 13 & $\cdot$ & 18.8 & $<0.2$ & - \\
\hline Polassium & 1.6 & 1.6 & 1.8 & 1.8 & 3 & 0.77 & 0.72 \\
\hline Sodium & 7.8 & 7.8 & 8.9 & 8.5 & 15 & 0.75 & 0.72 \\
\hline Sulfate & 21 & $\cdot$ & 18 & . & 25.2 & 5 & - \\
\hline
\end{tabular}


APPENDIX E.1

Groundwater and Surface-Water Quality Data, 1993

\begin{tabular}{|c|c|c|c|c|c|c|c|c|}
\hline \multirow{4}{*}{$\begin{array}{l}\text { Sampling Point } \\
\text { Location } \\
\text { Date Sampled }\end{array}$} & \multicolumn{8}{|c|}{ NT-01 } \\
\hline & \multicolumn{8}{|c|}{$\operatorname{ExP}$} \\
\hline & \multicolumn{2}{|c|}{$03 / 09 / 93$} & \multicolumn{2}{|c|}{$05 / 03 / 93$} & \multicolumn{2}{|c|}{$08 / 16 / 93$} & \multicolumn{2}{|c|}{$11 / 08 / 93$} \\
\hline & TOT & DIS & TOT & DIS & TOT & DIS & TOT & DIS \\
\hline METALS (mg/L) &. & $\cdot$ &. & . & . &. & $\cdot$ & - \\
\hline Aluminum & 0.79 & 0.11 & 0.55 & 0.047 & 0.13 & 0.91 & 0.79 & 0.15 \\
\hline Antimony & $<0.05$ & $<0.05$ & $<0.05$ & $<0.05$ & $<0.05$ & $<0.05$ & $<0.05$ & $<0.05$ \\
\hline Arsenic & $<0.05$ & $<0.05$ & $<0.05$ & $<0.05$ & $<0.05$ & $<0.05$ & $<0.05$ & $<0.05$ \\
\hline Barium & 0.48 & 0.41 & 0.084 & 0.088 & 0.71 & 0.7 & 0.61 & 0.58 \\
\hline Beryllium & $<0.0003$ & 0.00059 & $<0.0003$ & $<0.0003$ & $<0.0003$ & 0.00031 & 0.00031 & $<0.0003$ \\
\hline Boron & 0.041 & 0.036 & 0.05 & 0.053 & 0.11 & 0.095 & 0.12 & 0.09 \\
\hline Cadmium (AAS) & 0.017 & 0.014 & $<0.002$ & $<0.002$ & 0.017 & 0.013 & 0.024 & 0.022 \\
\hline Cadmium & 0.016 & 0.013 & $<0.003$ & $<0.003$ & $c .016$ & 0.013 & 0.02 & 0.019 \\
\hline Chromium (AAS) & $<0.01$ & $<0.01$ & $<0.01$ & $<0.01$ & $<0.01$ & 0.019 & $<0.01$ & $<0.01$ \\
\hline Chromium & 0.012 & $<0.01$ & $<0.01$ & $<0.01$ & $<0.01$ & 0.023 & $<0.01$ & $<0.01$ \\
\hline cobalt & 0.0076 & 0.0075 & $<0.005$ & $<0.005$ & 0.0071 & 0.02 & 0.008 & 0.0083 \\
\hline Copper & $<0.004$ & $<0.004$ & $<0.004$ & $<0.004$ & 0.0042 & 0.044 & $<0.004$ & $<0.004$ \\
\hline Iron & 0.19 & $<0.005$ & 0.75 & $<0.005$ & 0.0064 & 11 & 0.19 & 0.047 \\
\hline Lead (AAS) & $<0.004$ & $<0.004$ & 0.0073 & $<0.004$ & $<0.004$ & 0.018 & $<0.004$ & $<0.004$ \\
\hline Mercury (CVAA) & $<0.0002$ & $<0.0002$ & $<0.0002$ & $<0.0002$ & $<0.0002$ & $<0.0002$ & $<0.0002$ & $<0.0002$ \\
\hline Molybdenum & $<0.01$ & $<0.01$ & $<0.01$ & $<0.01$ & $<0.01$ & $<0.01$ & $<0.01$ & $<0.01$ \\
\hline Nickel & 0.082 & 0.061 & $<0.01$ & 0.012 & 0.075 & 0.24 & 0.077 & 0.075 \\
\hline Selenium & $<0.05$ & $<0.05$ & $<0.05$ & $<0.05$ & $<0.05$ & $<0.05$ & $<0.05$ & $<0.05$ \\
\hline Silver & $<0.006$ & $<0.006$ & $<0.006$ & $<0.006$ & $<0.006$ & $<0.006$ & $<0.006$ & $<0.006$ \\
\hline Strontium & 0.48 & 0.41 & 0.42 & 0.43 & 1 & 1 & 0.92 & 0.89 \\
\hline Thorium & $<0.2$ & $<0.2$ & $<0.2$ & $<0.2$ & $<0.2$ & $<0.2$ & $<0.2$ & $<0.2$ \\
\hline Uranium (Fluor) & 0.012 & 0.013 & 0.031 & 0.035 & 0.18 & 0.113 & 0.185 & 0.207 \\
\hline Vanadium & $<0.005$ & $<0.005$ & $<0.005$ & $<0.005$ & $<0.005$ & $<0.005$ & $<0.005$ & $<0.005$ \\
\hline zinc & 0.01 & 0.0076 & 0.018 & 0.0096 & 0.013 & 0.082 & 0.021 & 0.02 \\
\hline MAJOR IONS $(\mathrm{mg} / \mathrm{L})$ & $\cdot$ & . & $\cdot$ & . & $\cdot$ & $\cdot$ & . & • \\
\hline Alkalinity-HCO3 & 100 &. & 328 & . & 205 & $\cdot$ & $245^{\circ}$ & . \\
\hline Alkalinity- $\mathrm{CO} 3$ & $<1$ & $\cdot$ & $<1$ & - & $<1$ & $\cdot$ & $<1$ & • \\
\hline Calcium & 150 & 140 & 170 & 180 & 320 & 330 & 270 & 260 \\
\hline Chloride & 9.9 & . & 44 & - & 41.6 & $\cdot$ & 29 & • \\
\hline Fluoride & 0.8 & - & 0.1 & - & 1.5 & $\cdot$ & 1.4 & • \\
\hline Magnesium & 20 & 19 & 17 & 18 & 44 & 49 & 38 & 37 \\
\hline Manganese & 3.3 & 2.8 & 0.1 & 0.011 & 3.2 & 3.7 & 3.6 & 3.5 \\
\hline Nitrate-N & 112 & . & 31 & - & 286.1 & $\cdot$ & 259 & • \\
\hline Potassium & 2.5 & 2 & 3.1 & 3.2 & 6.2 & 5.9 & 5.2 & 5 \\
\hline Sodium & 18 & 16 & 14 & 14 & 50 & 50 & 40 & 40 \\
\hline Sulfate & 23 & - & 59 & - & 66.8 &. & 74 & - \\
\hline
\end{tabular}

(CONTINUED) 
APPENDIX E.1

Groundwater and Surface-Water Quality Data, 1993

\begin{tabular}{|c|c|c|c|c|c|c|c|c|}
\hline \multirow{4}{*}{$\begin{array}{l}\text { Sampling Point } \\
\text { Location } \\
\text { - } \\
\text { Date Sampled }\end{array}$} & \multicolumn{8}{|c|}{$N T-13$} \\
\hline & \multicolumn{8}{|c|}{ EXP } \\
\hline & \multicolumn{2}{|c|}{$03 / 09 / 93$} & \multicolumn{2}{|c|}{$05 / 03 / 93$} & \multicolumn{2}{|c|}{$08 / 16 / 93$} & \multicolumn{2}{|c|}{$11 / 08 / 93$} \\
\hline & TOT & DIS & TOT & DIS & TOT & DIS & TOT & DIS \\
\hline MBTALS $(\mathrm{mg} / \mathrm{I})$ & $\cdot$ & $\cdot$ & . & - & $\cdot$ & - & - & - \\
\hline Aluminum & 0.27 & $<0.02$ & 0.37 & $<0.02$ & $<0.02$ & $<0.02$ & 5.8 & 0.1 \\
\hline Antimony & $<0.05$ & $<0.05$ & $<0.05$ & $<0.05$ & $<0.05$ & $<0.05$ & $<0.05$ & $<0.05$ \\
\hline Arsenic & $<0.05$ & $<0.05$ & $<0.05$ & $<0.05$ & $<0.05$ & $<0.05$ & $<0.05$ & $<0.05$ \\
\hline Barium & 0.035 & 0.025 & 0.043 & 0.037 & 0.13 & 0.13 & 0.17 & 0.071 \\
\hline Beryllium & $<0.0003$ & $<0.0003$ & $<0.0003$ & $<0.0003$ & $<0.0,003$ & $<0.0003$ & 0.0004 & $<0.0003$ \\
\hline Boron & 0.015 & 0.032 & 0.023 & 0.022 & 0.021 & 0.027 & 0.03 & 0.019 \\
\hline Cadmium (AAS) & $<0.002$ & $<0.002$ & $<0.002$ & $<0.002$ & $<0.002$ & $<0.002$ & $<0.002$ & $<0.002$ \\
\hline Cadmium & $<0.003$ & $<0.003$ & $<0.003$ & $<0.003$ & $<0.003$ & $<0.003$ & 0.0044 & $<0.003$ \\
\hline Chromium (AAS) & $<0.01$ & $<0.01$ & $<0.01$ & $<0.01$ & $<0.01$ & $<0.01$ & 0.02 & $<0.01$ \\
\hline Chromium & $<0.01$ & $<0.01$ & $<0.01$ & $<0.01$ & $<0.01$ & $<0.01$ & $<0.01$ & $<0.01$ \\
\hline Cobalt & $<0.005$ & $<0.005$ & $<0.005$ & $<0.005$ & $<0.005$ & $<0.005$ & 0.012 & $<0.005$ \\
\hline Copper & $<0.004$ & $<0.004$ & $<0.004$ & $<0.004$ & $<0.004$ & $<0.004$ & 0.0072 & $<0.004$ \\
\hline Iron & 0.37 & 0.073 & 0.6 & 0.11 & 1.8 & 0.12 & 10 & 0.22 \\
\hline Lead (AAS) & $<0.004$ & $<0.004$ & $<0.004$ & $<0.004$ & $<0.004$ & $<0.004$ & 0.0068 & $<0.004$ \\
\hline Mercury (CVAA) & $<0.0002$ & $<0.0002$ & $<0.0002$ & $<0.0002$ & $<0.0002$ & $<0.0002$ & $<0.0002$ & $<0.0002$ \\
\hline Mol ybdenum & $<0.01$ & $<0.01$ & $<0.01$ & $<0.01$ & $<0.01$ & $<0.01$ & $<0.01$ & $<0.01$ \\
\hline Nickel & $<0.01$ & 0.015 & $<0.01$ & $<0.01$ & $<0.01$ & $<0.01$ & $<0.01$ & $<0.01$ \\
\hline Selenium & $<0.05$ & $<0.05$ & $<0.05$ & $<0.05$ & $<0.05$ & $<0.05$ & $<0.05$ & $<0.05$ \\
\hline silver & $<0.006$ & $<0.006$ & $<0.006$ & $<0.006$ & $<0.006$ & $<0.006$ & $<0.006$ & $<0.006$ \\
\hline Strontium & 0.021 & 0.023 & 0.029 & 0.029 & 0.12 & 0.12 & 0.063 & 0.059 \\
\hline Thorium & $<0.2$ & $<0.2$ & $<0.2$ & $<0.2$ & $<0.2$ & $<0.2$ & $<0.2$ & $<0.2$ \\
\hline Uranium (Fluor) & $<0.001$ & 0.001 & $<0.001$ & $<0.001$ & $<0.001$ & $<0.001$ & 0.001 & $<0.001$ \\
\hline Vanadium & $<0.005$ & $<0.005$ & $<0.005$ & $<0.005$ & $<0.005$ & $<0.005$ & 0.01 & $<0.005$ \\
\hline zinc & 0.0042 & 0.0035 & 0.0068 & 0.0042 & $<0.002$ & $<0.002$ & 0.066 & 0.037 \\
\hline MAJOR IONS (mg/I) & - & - & . &. & . & . & . & . \\
\hline Alkalinity-HCO3 & 17 &. & 26 & $\cdot$ & 119 & . & 16 & • \\
\hline Alkalinity-CO3 & $<1$ & . & $<1$ & . & $<1$ & - & $<1$ & - \\
\hline Calcium & $5 \cdot 5$ & 6.4 & 7.5 & 7.8 & 34 & 33 & 21 & 20 \\
\hline Chloride & 1.7 & $\cdot$ & 1.6 & - & 1 & - & 2 & - \\
\hline Fluoride & $<0.1$ & . & $<0.1$ & - & $<0.1$ & - & $<0.1$ & - \\
\hline Magnesium & $1 \cdot 3$ & 1.4 & 1.6 & 1.7 & 4.4 & 4.3 & 4.2 & 3.4 \\
\hline Manganese & 0.068 & 0.059 & 0.12 & 0.11 & 1.5 & 1.5 & 2.1 & 0.19 \\
\hline Nitrate-N & $<0.2$ & . & $<0.2$ & - & $<0.2$ & - & $<0.2$ & - \\
\hline Potassium & 1.1 & 1.1 & 1 & 1.1 & 1.9 & 1.2 & 3.1 & 1.6 \\
\hline Sodium & 1.7 & 1.8 & 2.1 & 2.1 & 7.4 & 7.5 & 4.2 & 4.2 \\
\hline Sulfate & 12 & $\cdot$ & 10 & - & 4.6 & - & 57 & - \\
\hline
\end{tabular}


APPENDIX E. 1

Groundwater and Surface-Water Quality Data, 1993

\begin{tabular}{|c|c|c|c|c|c|c|c|c|}
\hline \multirow{4}{*}{$\begin{array}{l}\text { Sampling Point } \\
\text { Iocation } \\
\text { - } \\
\text { Date Sampled }\end{array}$} & \multicolumn{8}{|c|}{ ss-1 } \\
\hline & \multicolumn{8}{|c|}{ EXP } \\
\hline & \multicolumn{2}{|c|}{$03 / 09 / 93$} & \multicolumn{2}{|c|}{$05 / 03 / 93$} & \multicolumn{2}{|c|}{$08 / 16 / 93$} & \multicolumn{2}{|c|}{$11 / 08 / 93$} \\
\hline & TOT & DIS & TOT & DIS & TOT & DIS & TOT & DIS \\
\hline MBTALS $(\mathrm{mg} / \mathrm{L})$ & - & $\cdot$ & $\cdot$ & - & - & - & - & - \\
\hline Aluminum & 0.04 & $<0.02$ & 0.39 & 0.097 & 0.89 & 0.047 & 0.13 & 0.058 \\
\hline Antimony & $<0.05$ & $<0.05$ & $<0.05$ & $<0.05$ & $<0.05$ & $<0.05$ & $<0.05$ & $<0.05$ \\
\hline Arsenic & $<0.05$ & $<0.05$ & $<0.05$ & $<0.05$ & $<0.05$ & $<0.05$ & $<0.05$ & $<0.05$ \\
\hline Barium & 0.081 & 0.093 & 0.66 & 0.61 & 0.11 & 0.12 & 0.11 & 0.11 \\
\hline Beryllium & $<0.0003$ & $<0.0003$ & $<0.0003$ & $<0.0003$ & $<0.0003$ & $<0.0003$ & $<0.0003$ & $<0.0003$ \\
\hline Boron & 0.048 & 0.045 & 0.046 & 0.039 & 0.057 & 0.044 & 0.079 & 0.1 \\
\hline Cadmium (AAS) & $<0.002$ & $<0.002$ & 0.024 & 0.022 & $<0.002$ & $<0.002$ & $<0.002$ & $<0.002$ \\
\hline Cadmium & $<0.003$ & $<0.003$ & 0.017 & 0.016 & $<0.003$ & $<0.003$ & $<0.003$ & $<0.003$ \\
\hline Chromium (AAS) & $<0.01$ & $<0.01$ & $<0.01$ & $<0.01$ & $<0.01$ & $<0.01$ & $<0.01$ & $<0.01$ \\
\hline Chromium & $<0.01$ & $<0.01$ & $<0.01$ & 0.014 & $<0.01$ & $<0.01$ & $<0.01$ & $<0.01$ \\
\hline Cobalt & $<0.005$ & $<0.005$ & $<0.005$ & 0.0068 & $<0.005$ & $<0.005$ & $<0.005$ & $<0.005$ \\
\hline Copper & 0.0043 & $<0.004$ & $<0.004$ & $<0.004$ & 0.0071 & 0.0081 & $<0.004$ & $<0.004$ \\
\hline Iron & $<0.005$ & $<0.005$ & 0.23 & 0.0058 & 1.5 & 0.031 & 0.26 & $<0.005$ \\
\hline Lead (AAS) & $<0.004$ & $<0.004$ & $<0.004$ & $<0.004$ & $<0.004$ & $<0.004$ & $<0.004$ & $<0.004$ \\
\hline Mercury (CVAA) & $<0.0002$ & $<0.0002$ & $<0.0002$ & $<0.0002$ & $<0.0002$ & $<0.0002$ & $<0.0002$ & $<0.0002$ \\
\hline Molybdenum & $<0.01$ & $<0.01$ & $<0.01$ & $<0.01$ & $<0.01$ & $<0.01$ & $<0.01$ & $<0.01$ \\
\hline Nickel & $<0.01$ & $<0.01$ & 0.08 & 0.09 & $<0.01$ & 0.015 & $<0.01$ & $<0.01$ \\
\hline Selenium & $<0.05$ & $<0.05$ & $<0.05$ & $<0.05$ & $<0.05$ & $<0.05$ & $<0.05$ & $<0.05$ \\
\hline Silver & $<0.006$ & $<0.006$ & $<0.006$ & $<0.006$ & $<0.006$ & $<0.006$ & $<0.006$ & $<0.006$ \\
\hline Strontium & 0.4 & 0.39 & 0.6 & 0.58 & 0.55 & 0.53 & 0.56 & 0.57 \\
\hline Thorium & $<0.2$ & $<0.2$ & $<0.2$ & $<0.2$ & $<0.2$ & $<0.2$ & $<0.2$ & $<0.2$ \\
\hline Uranium (Fluor) & 0.031 & 0.034 & 0.016 & 0.019 & 0.045 & 0.056 & 0.04 & 0.04 \\
\hline Vanadium & $<0.005$ & $<0.005$ & $<0.005$ & $<0.005$ & $<0.005$ & $<0.005$ & $<0.005$ & $<0.005$ \\
\hline zinc & 0.007 & 0.0033 & 0.012 & 0.011 & 0.01 & 0.059 & 0.016 & 0.0021 \\
\hline MAJOR IONS (mg/L) & . & - & • & - & . & - & - & - \\
\hline Alkalinity-HCO3 & 324 & $\cdot$ & 126 & $\cdot$ & 354 & $\cdot$ & 361 & - \\
\hline Alkalinity-CO3 & $<1$ & - & $<1$ & - & $<1$ & - & $<1$ & - \\
\hline Calcium & 180 & 170 & 200 & 190 & 220 & 220 & 220 & 220 \\
\hline Chloride & 40 & - & 14 & - & 77.1 & - & 80 & - \\
\hline Fluoride & 0.2 & - & 0.8 & - & 0.2 & - & 0.3 & - \\
\hline Magnesium & 16 & 15 & 29 & 28 & 20 & 21 & 20 & 20 \\
\hline Manganese & 0.016 & 0.019 & 4 & 3.7 & 0.1 & 0.022 & 0.017 & 0.005 \\
\hline Nitrate-N & 31 & - & 142 & - & 44.2 & • & 54.9 & • \\
\hline Potassium & 3.7 & 3 & 2.9 & 3 & 4.4 & 4.1 & 4.2 & 4.3 \\
\hline Sodium & 14 & 13 & 23 & 22 & 25 & 24 & 26 & 26 \\
\hline Sulfate & 54 & - & 19 & - & 56.7 & - & 57 & $\cdot$ \\
\hline
\end{tabular}

(CONTINUED) 
APPENDIX E. 1

Groundwater and Surface-Water Quality Data, 1993

\begin{tabular}{|c|c|c|c|c|c|c|c|c|}
\hline \multirow{4}{*}{$\begin{array}{l}\text { Sampling Point } \\
\text { Location } \\
\text { Date Sampled }\end{array}$} & \multicolumn{8}{|c|}{ ss -4} \\
\hline & \multicolumn{8}{|c|}{ EXP } \\
\hline & \multicolumn{2}{|c|}{$03 / 09 / 93$} & \multicolumn{2}{|c|}{$05 / 03 / 93$} & \multicolumn{2}{|c|}{$08 / 16 / 93$} & \multicolumn{2}{|c|}{$11 / 08 / 93$} \\
\hline & TOT & DIS & TOT & DIS & TOT & DIS & TOT & DIS \\
\hline METALS (mg/L) & . & - & . & $\cdot$ & $\cdot$ & $\cdot$ & $\cdot 1$ & - \\
\hline Aluminum & 0.16 & 0.035 & 0.12 & $<0.02$ & 0.2 & $<0.02$ & 0.15 & 0.041 \\
\hline Antimony & $<0.05$ & $<0.05$ & $<0.05$ & $<0.05$ & $<0.05$ & $<0.05$ & $<0.05$ & $<0.05$ \\
\hline Arsenic & $<0.05$ & $<0,05$ & $<0.05$ & $<0.05$ & $<0.05$ & $<0.05$ & $<0.05$ & $<0.05$ \\
\hline Barium & 0.1 & 0.089 & 0.11 & 0.11 & 0.17 & 0.17 & 0.17 & 0.17 \\
\hline Beryllium & $<0.0003$ & $<0.0003$ & $<0.0003$ & $<0.0003$ & $<0.0003$ & $<0.0003$ & $<0.0003$ & $<0.0003$ \\
\hline Boron & 0.12 & 0.088 & 0.14 & 0.12 & 0.15 & 0.16 & 0.1 & 0.12 \\
\hline Cadmium (AAS) & $<0.002$ & $<0.002$ & $<0.002$ & $<0.002$ & $<0.002$ & $<0.002$ & $<0.002$ & $<0,002$ \\
\hline Cadmium & $<0.003$ & $<0.003$ & $<0.003$ & $<0.003$ & $<0.003$ & $<0.003$ & $<0.003$ & $<0.003$ \\
\hline Chromium (AAS) & $<0.01$ & $<0.01$ & $<0.01$ & $<0.01$ & $<0.01$ & $<0.01$ & $<0.01$ & $<0.01$ \\
\hline Chromium & $<0.01$ & $<0.01$ & $<0.01$ & $<0.01$ & $<0.01$ & $<0.01$ & $<0.01$ & $<0.01$ \\
\hline Cobalt & $<0.005$ & $<0.005$ & $<0.005$ & $<0.005$ & $<0.005$ & $<0.005$ & $<0.005$ & $<0.005$ \\
\hline Copper & $<0.004$ & $<0.004$ & $<0.004$ & $<0.004$ & $<0.004$ & 0.0053 & $<0.004$ & $<0.004$ \\
\hline Iron & 0.17 & $<0.005$ & 0.16 & 0.0065 & 0.22 & $<0.005$ & 0.15 & $<0.005$ \\
\hline Lead (AAS) & $<0.004$ & $<0.004$ & $<0.004$ & $<0.004$ & $<0.004$ & $<0.004$ & $<0.004$ & $<0.004$ \\
\hline Mercury (CVAA) & $<0.0002$ & $<0.0002$ & $<0.0002$ & $<0.0002$ & $<0.0002$ & $<0.0002$ & $<0.0002$ & $<0.0002$ \\
\hline Molybdenum & $<0.01$ & $<0.01$ & $<0.01$ & $<0.01$ & $<0.01$ & $<0.01$ & $<0.01$ & $<0.01$ \\
\hline Nickel & $<0.01$ & $<0.01$ & 0.022 & 0.018 & 0.012 & $<0.01$ & $<0.01$ & $<0.01$ \\
\hline Selenium & $<0.05$ & $<0.05$ & $<0.05$ & $<0.05$ & $<0.05$ & $<0.05$ & $<0.05$ & $<0.05$ \\
\hline Sllver & $<0.006$ & $<0.006$ & $<0.006$ & $<0.006$ & $<0.006$ & $<0.006$ & $<0.006$ & $<0.006$ \\
\hline Strontium & 0.15 & 0.15 & 0.16 & 0.17 & 0.33 & 0.33 & 0.36 & 0.37 \\
\hline Thorium & $<0.2$ & $<0.2$ & $<0.2$ & $<0.2$ & $<0.2$ & $<0.2$ & $<0.2$ & $<0.2$ \\
\hline Uranium (liluor) & 0.084 & 0.087 & 0.112 & 0.107 & 0.181 & 0.177 & 0.24 & 0.12 \\
\hline Vanadium & $<0.005$ & $<0.005$ & $<0.005$ & $<0.005$ & $<0.005$ & $<0.005$ & $<0.005$ & $<0.005$ \\
\hline zinc & 0.0055 & 0.0034 & 0.0069 & 0.0063 & $<0.002$ & $<0.002$ & $<0.002$ & $<0.002$ \\
\hline MAJOR IONS $(\mathrm{mg} / \mathrm{L})$ & - &. & $\cdot$ &. & $\cdot$ & $\cdot$ & $\cdot$ & • \\
\hline Alkalinity-HCO3 & ${ }_{192}^{\circ}$ &. & $\begin{array}{r}\cdot \\
203\end{array}$ &. & 227 &. & 237 & • \\
\hline Alkalinity- $\mathrm{CO} 3$ & $<1$ &. & $<1$ &. & $<1$ &. & $<1$ & . \\
\hline Calcium & 70 & 71 & 70 & 74 & 120 & 120 & 130 & 140 \\
\hline Chloride & 16 & . & 21 & . & 35.8 & . & 31 & • \\
\hline Fluoride & 0.3 &. & 0.3 &. & 0.4 & . & 0.6 & . \\
\hline Magnesium & 18 & 18 & 18 & 19 & 23 & 23 & 23 & 23 \\
\hline Manganese & 0.12 & 0.13 & 0.086 & 0.0071 & 0.11 & 0.0023 & 0.057 & 0.0013 \\
\hline Nitrate-N & 9.3 &. & 15 &. & 41.3 & . & 67.6 & . \\
\hline Potassium & 2 & 2.1 & 2.3 & 2.3 & 3.9 & 3.7 & 3.4 & 3.3 \\
\hline Sodium & 9.8 & 11 & 12 & 12 & 25 & 25 & 23 & 22 \\
\hline Sulfate & 21 & . & 23 & . & 31.2 & . & 45 & • \\
\hline
\end{tabular}

(CONTINUED) 
APPENDIX E. 1

Groundwater and Surface-Water Quality Data, 1993

\begin{tabular}{|c|c|c|c|c|c|c|c|c|}
\hline \multirow{4}{*}{$\begin{array}{l}\text { Sampling Point } \\
\text { Location } \\
\text { Date Sampled }\end{array}$} & \multicolumn{8}{|c|}{ SS-5 } \\
\hline & \multicolumn{8}{|c|}{$\operatorname{EXP}$} \\
\hline & \multicolumn{2}{|c|}{$03 / 09 / 93$} & \multicolumn{2}{|c|}{$05 / 03 / 93$} & \multicolumn{2}{|c|}{$08 / 16 / 93$} & \multicolumn{2}{|c|}{$11 / 08 / 93$} \\
\hline & TOT & DIS & TOT & DIS & TOT & DIS & TOT & DIS \\
\hline MRTALS (mg/L) & $\cdot$ & $\cdot$ &. & - & $\cdot$ & • & $\cdot$ & $\cdot$ \\
\hline Aluminum & 0.094 & 0.042 & 0.056 & $<0.02$ & 0.039 & $<0.02$ & 0.14 & $<0.02$ \\
\hline Antimony & $<0.05$ & $<0.05$ & $<0.05$ & $<0.05$ & $<0.05$ & $<0.05$ & $<0.05$ & $<0.05$ \\
\hline Arsenic & $<0.05$ & $<0.05$ & $<0.05$ & $<0.05$ & $<0.05$ & $<0.05$ & $<0.05$ & $<0.05$ \\
\hline Barium & 0.049 & 0.045 & 0.053 & 0.054 & 0.11 & 0.11 & 0.12 & 0.11 \\
\hline Beryllium & 0.0003 & 0.00043 & $<0.0003$ & $<0.0003$ & $<0.0003$ & $<0.0003$ & $<0.0003$ & $<0.0003$ \\
\hline Boron & 0.18 & 0.026 & 0.033 & 0.039 & 0.14 & 0.11 & 0.13 & 0.081 \\
\hline Cadmium (AAS) & $<0.002$ & $<0.002$ & $<0.002$ & $<0.002$ & $<0.002$ & $<0.002$ & $<0.002$ & $<0.002$ \\
\hline Cadmium & $<0.003$ & $<0.003$ & $<0.003$ & $<0.003$ & $<0.003$ & $<0.003$ & $<0.003$ & $<0.003$ \\
\hline Chromium (AAS) & $<0.01$ & $<0.01$ & $<0.01$ & $<0.01$ & $<0.01$ & $<0.01$ & $<0.01$ & $<0.01$ \\
\hline Chromium & $<0.01$ & $<0.01$ & $<0.01$ & $<0.01$ & $<0.01$ & $<0.01$ & $<0.01$ & $<0.01$ \\
\hline Cobalt & $<0.005$ & $<0.005$ & $<0.005$ & $<0.005$ & $<0.005$ & $<0.005$ & $<0.005$ & $<0.005$ \\
\hline Copper & $<0.004$ & $<0.004$ & $<0.004$ & $<0.004$ & $<0.004$ & 0.0044 & $<0.004$ & $<0.004$ \\
\hline Iron & 0.052 & $<0.005$ & 0.028 & 0.011 & 0.036 & 0.031 & 0.15 & 0.0076 \\
\hline Lead (AAS) & $<0.004$ & $<0.004$ & $<0.004$ & $<0.004$ & $<0.004$ & $<0.004$ & $<0.004$ & $<0.004$ \\
\hline Mercury (CVAA) & $<0.0002$ & $<0.0002$ & $<0.0002$ & $<0.0002$ & $<0.0002$ & $<0.0002$ & $<0.0002$ & $<0.0002$ \\
\hline Mol ybdenum & $<0.01$ & $<0.01$ & $<0.01$ & $<0.01$ & $<0.01$ & $<0.01$ & $<0.01$ & $<0.01$ \\
\hline Nickel & $<0.01$ & $<0.01$ & $<0.01$ & $<0.01$ & $<0.01$ & $<0.01$ & 0.01 & $<0.01$ \\
\hline Selenium & $<0.05$ & $<0.05$ & $<0.05$ & $<0.05$ & $<0.05$ & $<0.05$ & $<0.05$ & $<0.05$ \\
\hline silver & $<0.006$ & $<0.006$ & $<0.006$ & $<0.006$ & $<0.006$ & $<0.006$ & $<0.006$ & $<0.006$ \\
\hline strontium & 0.052 & 0.059 & 0.062 & 0.064 & 0.18 & 0.18 & 0.2 & 0.2 \\
\hline Thoriun & $<0.2$ & $<0.2$ & $<0.2$ & $<0.2$ & $<0.2$ & $<0.2$ & $<0.2$ & $<0.2$ \\
\hline Uranium (Fluor) & 0.018 & 0.019 & 0.025 & 0.026 & 0.098 & 0.078 & 0.1 & 0.11 \\
\hline Vanadium & $<0.005$ & $<0.005$ & $<0.005$ & $<0,005$ & $<0.005$ & $<0.005$ & $<0.005$ & $<0.005$ \\
\hline zinc & 0.0036 & 0.0044 & 0.0036 & 0.0036 & $<0.002$ & 0.0063 & 0.01 & $<0.002$ \\
\hline MAJOR IONS (mg/L) & $\cdot$ & $\cdot$ & $\cdot$ & $\cdot$ & $\cdot$ & $\bullet$ & • & • \\
\hline Alkalinity- $\mathrm{HCO} 3$ & 127 & $\cdot$ & 138 & $\cdot$ & 204 & . & 192 & • \\
\hline Alkalinity- $\mathrm{CO} 3$ & $<1$ & . & $<1$ & $\cdot$ & $<1$ & - & $<1$ & • \\
\hline Calcium & 36 & 39 & 39 & 40 & 84 & 81 & 86 & 86 \\
\hline Chloride & 5.4 & $\cdot$ & 6.8 & . & 23.9 & . & 22 & $\bullet$ \\
\hline Fluoride & $<0.1$ & $\cdot$ & 0.1 & $\cdot$ & 0.2 & . & 0.3 & $\theta$ \\
\hline Magnesium & 13 & 13 & 13 & 13 & 20 & 19 & 17 & 17 \\
\hline Manganese & 0.0051 & 0.0032 & 0.0055 & 0.0052 & 0.0049 & 0.027 & 0.0059 & 0.001 \\
\hline Nitrate-N & 2.5 & . & 4.5 & . & 15.2 & . & 24.8 & . \\
\hline Potassium & 0.88 & 0.97 & 1.1 & 0.99 & 1.8 & 1.8 & 2.4 & 2.5 \\
\hline Sodium & 3.4 & 3.3 & 4.1 & 4.3 & 14 & 14 & 14 & 14 \\
\hline Sulfate & 13 & . & 12 & . & 22.7 & . & 29 & $\cdot$ \\
\hline
\end{tabular}

(CONTINUED) 
APPENDIX E.1

Groundwater and Surface-Water Quality Data, 1993

\begin{tabular}{|c|c|c|c|c|c|c|c|c|}
\hline \multirow{4}{*}{$\begin{array}{l}\text { Sampling Point } \\
\text { Location } \\
\text { Date Sampled }\end{array}$} & \multicolumn{8}{|c|}{ ss-6 } \\
\hline & \multicolumn{8}{|c|}{ EXP } \\
\hline & \multicolumn{2}{|c|}{$03 / 09 / 93$} & \multicolumn{2}{|c|}{$05 / 03 / 93$} & \multicolumn{2}{|c|}{$08 / 16 / 93$} & \multicolumn{2}{|c|}{$11 / 08 / 93$} \\
\hline & TOT & DIS & TOT & DIS & TOT & DIS & TOT & Drs \\
\hline METALS $(m g / L)$ & - & - & - & - & • & $\cdot$ & $\cdot$ & 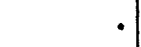 \\
\hline Aluminum & 0.042 & 0.032 & 0.039 & $<0.02$ & 0.025 & $<0.02$ & 0.069 & 0.053 \\
\hline Antimony & $<0.05$ & $<0.05$ & $<0.05$ & $<0.05$ & $<0.05$ & $<0.05$ & $<0.05$ & $<0.05$ \\
\hline Arsenic & $<0.05$ & $<0.05$ & $<0.05$ & $<0.05$ & $<0.05$ & $<0.05$ & $<0.05$ & $<0.05$ \\
\hline Barium & 0.025 & 0.037 & 0.027 & 0.027 & 0.025 & 0.027 & 0.13 & 0.13 \\
\hline Beryllium & $<0.0003$ & $<0.0003$ & $<0.0003$ & $<0.0003$ & $<0.0003$ & $<0.0003$ & $<0.0003$ & $<0.0003$ \\
\hline Boron & 0.009 & 0.015 & 0.014 & 0.0094 & 0.012 & 0.025 & 0.28 & 0.25 \\
\hline Cadmium (AAS) & $<0.002$ & $<0.002$ & $<0.002$ & $<0.002$ & $<0.002$ & $<0.002$ & $<0.002$ & $<0.002$ \\
\hline Cadmium & $<0.003$ & $<0.003$ & $<0.003$ & $<0.003$ & $<0.003$ & $<0.003$ & $<0.003$ & $<0.003$ \\
\hline Chromium (AAS) & $<0.01$ & $<0.01$ & $<0.01$ & $<0.01$ & $<0.01$ & $<0.01$ & $<0.01$ & $<0.01$ \\
\hline Chromium & $<0.01$ & $<0.01$ & $<0.01$ & $<0.01$ & $<0.01$ & $<0.01$ & $<0.01$ & $<0.01$ \\
\hline Cobalt & $<0.005$ & $<0.005$ & $<0.005$ & $<0.005$ & $<0.005$ & $<0.005$ & $<0.005$ & $<0.005$ \\
\hline Copper & $<0.004$ & $<0.004$ & $<0.004$ & $<0.004$ & $<0.004$ & $<0.004$ & $<0.004$ & $<0.004$ \\
\hline Iron & 0.038 & 0.017 & 0.051 & 0.011 & 0.031 & 0.0078 & 0.15 & 0.023 \\
\hline Lead (AAS) & $<0.004$ & $<0.004$ & $<0.004$ & $<0.004$ & $<0.004$ & $<0.004$ & $<0.004$ & $<0.004$ \\
\hline Mercury (CVAA) & $<0.0002$ & $<0.0002$ & $<0.0002$ & $<0.0002$ & $<0.0002$ & $<0.0002$ & $<0.0002$ & $<0.0002$ \\
\hline Molybdenum & $<0.01$ & $<0.01$ & $<0.01$ & $<0.01$ & $<0.01$ & $<0.01$ & $<0.01$ & $<0.01$ \\
\hline Nickel & $<0.01$ & 0.017 & $<0.01$ & $<0.01$ & $<0.01$ & $<0.01$ & $<0.01$ & 0.013 \\
\hline Selenium & $<0.05$ & $<0.05$ & $<0.05$ & $<0.05$ & $<0.05$ & $<0.05$ & $<0.05$ & $<0.05$ \\
\hline Silver & $<0.006$ & $<0.006$ & $<0.006$ & $<0.006$ & $<0.006$ & $<0.006$ & $<0.006$ & $<0.006$ \\
\hline Strontium & 0.016 & 0.025 & 0.016 & 0.016 & 0.021 & 0.023 & 0.28 & 0.28 \\
\hline Thorium & $<0.2$ & $<0.2$ & $<0.2$ & $<0.2$ & $<0.2$ & $<0.2$ & $<0.2$ & $<0.2$ \\
\hline Uranium (Fluor) & $<0.001$ & 0.001 & $<0.001$ & $<0.001$ & $<0.001$ & 0.001 & 0.15 & 0.17 \\
\hline Vanadium & $<0.005$ & $<0.005$ & $<0.005$ & $<0.005$ & $<0.005$ & $<0.005$ & $<0.005$ & $<0.005$ \\
\hline Zinc & 0.009 & 0.0059 & 0.013 & 0.012 & 0.038 & 0.029 & 0.0045 & 0.0081 \\
\hline MAJOR IONS $(\mathrm{mg} / \mathrm{L})$ &. & $\cdot$ &. & $\cdot$ & $\cdot$ & - & - & - \\
\hline Alkalinity- $\mathrm{HCO} 3$ & 90 & $\cdot$ & 109 & $\cdot$ & 150 & • & 197 & $\cdot$ \\
\hline Alkalinity-CO3 & $<1$ & $\cdot$ & $<1$ & $\cdot$ & $<1$ & - & $<1$ & - \\
\hline Calcium & 20 & 23 & 21 & 20 & 33 & 34 & 100 & 100 \\
\hline Chloride & 1.7 & $\cdot$ & 1.5 & $\cdot$ & $<1$ & - & 25 & . \\
\hline Fluoride & $<0.1$ & $\cdot$ & $<0.1$ & $\cdot$ & $<0.1$ & - & 0.4 & - \\
\hline Magnesium & 10 & 11 & 13 & 12 & 18 & 18 & 17 & 17 \\
\hline Manganese & 0.012 & 0.072 & 0.026 & 0.022 & 0.0044 & 0.0031 & 0.067 & 0.058 \\
\hline Nitrate-N & $<0.2$ & $\cdot$ & $<0.2$ & . & $<0.2$ & . & 37.8 & • \\
\hline Potassium & $<0.6$ & 0.73 & $<0.6$ & 0.62 & $<0.6$ & 0.64 & 3.1 & 3 \\
\hline Sodium & 0.45 & 0.74 & 0.47 & 0.48 & 0.56 & 0.78 & 18 & 17 \\
\hline Sulfate & 9.1 & . & 6.5 & . & 2.3 & - & 29 & • \\
\hline
\end{tabular}

(CONTINUED) 
APPENDIX B. 1

Groundwater and Surface-Water Quality Data, 1993

\begin{tabular}{|c|c|c|c|c|c|c|c|c|}
\hline \multirow{4}{*}{$\begin{array}{l}\text { Sampling Point } \\
\text { Location } \\
\text { - } \\
\text { Date Sampled }\end{array}$} & \multicolumn{8}{|c|}{ ss -8} \\
\hline & \multicolumn{8}{|c|}{ EXP } \\
\hline & \multicolumn{2}{|c|}{$03 / 09 / 93$} & \multicolumn{2}{|c|}{$05 / 03 / 93$} & \multicolumn{2}{|c|}{$08 / 16 / 93$} & \multicolumn{2}{|c|}{$11 / 08 / 93$} \\
\hline & TOT & DIS & TOT & DIS & TOT & DIS & TOT & DIS \\
\hline METALS (mg/L) & $\cdot$ & - & $\cdot$ & - & - & - & $\cdot$ & - \\
\hline Aluminum & 0.16 & $<0.02$ & 0.074 & $<0.02$ & 0.025 & $<0.02$ & 0.14 & 0.023 \\
\hline Antimony & $<0.05$ & $<0.05$ & $<0.05$ & $<0.05$ & $<0.05$ & $<0.05$ & $<0.05$ & $<0.05$ \\
\hline Arsenic & $<0.05$ & $<0.05$ & $<0.05$ & $<0.05$ & $<0.05$ & $<0.05$ & $<0.05$ & $<0.05$ \\
\hline Barium & 0.03 & 0.031 & 0.033 & 0.031 & 0.054 & 0.07 & 0.052 & 0.051 \\
\hline Bery 11 ium & $<0.0003$ & $<0.0003$ & $<0.0003$ & $<0.0003$ & $<0.0003$ & $<0.0003$ & $<0.0003$ & $<0.0003$ \\
\hline Boron & 0.018 & 0.016 & 0.031 & 0.018 & 0.023 & 0.017 & 0.036 & 0.014 \\
\hline Cadmium (AAS) & $<0.002$ & $<0.002$ & $<0.002$ & $<0.002$ & $<0.002$ & $<0.002$ & $<0.002$ & $<0.002$ \\
\hline Cadmium & $<0.003$ & $<0.003$ & $<0.003$ & $<0.003$ & $<0.003$ & $<0.003$ & $<0.003$ & $<0.003$ \\
\hline Chromium (AAS) & $<0.01$ & $<0.01$ & $<0.01$ & $<0.01$ & $<0.01$ & $<0.01$ & $<0.01$ & $<0.01$ \\
\hline Chromium & $<0.01$ & $<0.01$ & $<0.01$ & $<0.01$ & $<0.01$ & $<0.01$ & $<0.01$ & $<0.01$ \\
\hline Cobalt & $<0.005$ & $<0.005$ & $<0.005$ & $<0.005$ & $<0.005$ & $<0.005$ & $<0.005$ & $<0.005$ \\
\hline Copper & $<0.004$ & $<0.004$ & $<0.004$ & $<0.004$ & $<0.004$ & $<0.004$ & $<0.004$ & $<0.004$ \\
\hline Iron & 0.15 & $<0.005$ & 0.063 & 0.016 & 0.035 & 0.1 & 0.15 & 0.0073 \\
\hline Lead (AAS) & $<0.004$ & $<0.004$ & $<0.004$ & $<0.004$ & $<0.004$ & $<0.004$ & $<0.004$ & $<0.004$ \\
\hline Mercury (CVAA) & $<0.0002$ & $<0.0002$ & $<0.0002$ & $<0.0002$ & $<0.0002$ & $<0.0002$ & $<0.0002$ & $<0.0002$ \\
\hline Mol ybdenum & $<0.01$ & $<0.01$ & $<0.01$ & $<0.01$ & $<0.01$ & $<0.01$ & $<0.01$ & $<0.01$ \\
\hline Nickel & $<0.01$ & $<0.01$ & 0.017 & 0.011 & $<0.01$ & 0.013 & $<0.01$ & $<0.01$ \\
\hline Selenium & $<0.05$ & $<0.05$ & $<0.05$ & $<0.05$ & $<0.05$ & $<0.05$ & $<0.05$ & $<0.05$ \\
\hline silver & $<0.006$ & $<0.006$ & $<0.006$ & $<0.006$ & $<0.006$ & $<0.006$ & $<0.006$ & $<0.006$ \\
\hline strontium & 0.024 & 0.026 & 0.027 & 0.028 & 0.037 & 0.065 & 0.052 & 0.051 \\
\hline Thorium & $<0.2$ & $<0.2$ & $<0.2$ & $<0.2$ & $<0.2$ & $<0.2$ & $<0.2$ & $<0.2$ \\
\hline Uranium (Fluor) & $<0.001$ & 0.001 & $<0.001$ & 0.001 & $<0.001$ & 0.014 & 0.001 & 0.002 \\
\hline Vanadium & $<0.005$ & $<0.005$ & $<0.005$ & $<0.005$ & $<0.005$ & $<0.005$ & $<0.005$ & $<0.005$ \\
\hline zinc & 0.004 & 0.0025 & 0.01 & 0.0053 & $<0.002$ & $<0.002$ & $<0.002$ & $<0.002$ \\
\hline MAJOR IONS $(\mathrm{mg} / \mathrm{L})$ & $\cdot$ & - & $\cdot$ & - & $\cdot$ & - & - & • \\
\hline Alkalinity-HCO3 & 97 & . & 110 & • & 161 & $\cdot$ & 144 & • \\
\hline Alkalinity-CO3 & $<1$ & $\cdot$ & $<1$ & • & $<1$ & - & $<1$ & • \\
\hline Calcium & 25 & 25 & 27 & 27 & 39 & 46 & 39 & 39 \\
\hline Chloride & 3.8 & $\cdot$ & 2.9 & - & 2.9 & $\cdot$ & 6 & • \\
\hline Fluoride & $<0.1$ & $\cdot$ & $<0.1$ & - & $<0.1$ & - & 0.1 & • \\
\hline Magnesium & 8.6 & 8.8 & 9.1 & 9 & 16 & 16 & 12 & 12 \\
\hline Manganese & 0.0084 & 0.0046 & 0.0035 & 0.0029 & 0.0062 & 0.13 & 0.0016 & $<0.001$ \\
\hline Nitrate-N & 0.36 & $\cdot$ & 0.32 & - & 1.2 & • & 0.3 & $\cdot$ \\
\hline Potassium & 0.67 & $<0.6$ & 0.9 & 0.78 & 0.86 & 0.8 & 1.2 & 1.3 \\
\hline Sodium & 1.8 & 1.9 & 1.7 & 1.7 & 1.9 & 2.8 & 3.8 & 3.7 \\
\hline Sulfate & 8.3 & $\cdot$ & 6.4 & - & 2.4 & - & 7 & $\cdot$ \\
\hline
\end{tabular}



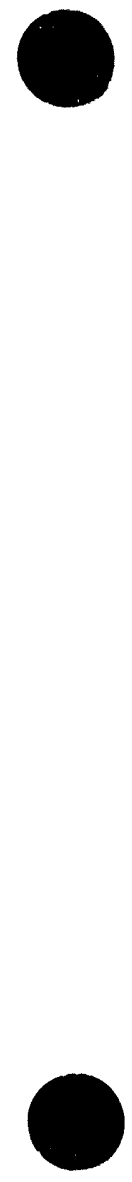

\section{APPENDIX E.2 \\ VOLATILE ORGANIC COMPOUNDS}

\section{VOLATHE ORGANIC COMPOUNDS}


APPENDLX E.2

VOC Data Summary

\begin{tabular}{|c|c|c|c|c|c|}
\hline \multirow{2}{*}{ Compound } & \multicolumn{4}{|c|}{ Number of Samples } & \multirow{2}{*}{ Total } \\
\hline & $\begin{array}{l}\text { Pust } \\
\text { Quarter }\end{array}$ & $\begin{array}{l}\text { Second } \\
\text { Qumerter }\end{array}$ & $\begin{array}{c}\text { Thind } \\
\text { Quarter }\end{array}$ & $\begin{array}{c}\text { Fourth } \\
\text { Quarter } \\
\end{array}$ & \\
\hline Total Samples & 91 & 91 & 90 & 97 & 369 \\
\hline Not Detected & 25 & 36 & 43 & 25 & 129 \\
\hline VOCs Detected & 66 & 55 & 47 & 72 & 240 \\
\hline Methylene chloride & 46 & 24 & 24 & 56 & 150 \\
\hline Trichloroethene & 24 & 24 & 26 & 24 & 98 \\
\hline 1,2-Dichloroethene & 24 & 18 & 21 & 21 & 84 \\
\hline 2-Butanone & 6 & 8 & 0 & 48 & 62 \\
\hline 4-Methyl-2-pentanone & 38 & 18 & 0 & 5 & 61 \\
\hline Acetone & 16 & 2 & 2 & 39 & 59 \\
\hline Tetrachloroethene & 13 & 12 & 10 & 14 & 49 \\
\hline Chloroform & 12 & 8 & 12 & 9 & 41 \\
\hline Carbon tetrachloride & 9 & 3 & 3 & 8 & 23 \\
\hline 1,1,1-Trichloroethane & 4 & 6 & 7 & 6 & 23 \\
\hline 1,1-Dichloroethene & 5 & 4 & 6 & 7 & 22 \\
\hline 1,1-Dichloroethane & 5 & 2 & 4 & 5 & 16 \\
\hline Vinyl chloride & 2 & 2 & 2 & 1 & 7 \\
\hline Benzene & 0 & 0 & 1 & 3 & 4 \\
\hline Bromodichloromethane & 1 & 1 & 1 & 1 & 4 \\
\hline Toluene & 0 & 0 & 1 & 2 & 3 \\
\hline Chlorobenzene & 1 & 0 & 0 & 1 & 2 \\
\hline 1,2-Dichloroethane & 0 & 1 & 0 & 1 & 2 \\
\hline Vinyl acetate & 2 & 0 & 0 & 0 & 2 \\
\hline Carbon disulfide & 1 & 0 & 0 & 0 & 1 \\
\hline Ethylbenzene & 1 & 0 & 0 & 0 & 1 \\
\hline 2-Hexanone & 1 & 0 & 0 & 0 & 1 \\
\hline Styrene & 1 & 0 & 0 & 0 & 1 \\
\hline 1,1,2-Trichloroethane & 1 & 0 & 0 & 0 & 1 \\
\hline Xylenes & 1 & 0 & 0 & 1 & 2 \\
\hline Bromoform & 0 & 0 & 0 & 0 & 0 \\
\hline Bromomethane & 0 & 0 & 0 & 0 & 0 \\
\hline Chlorodibromomethane & 0 & 0 & 0 & 0 & 0 \\
\hline Chloroethane & 0 & 0 & 0 & 0 & 0 \\
\hline Chloromethane & 0 & 0 & 0 & 0 & 0 \\
\hline 1,2-Dichloropropane & 0 & 0 & 0 & 0 & 0 \\
\hline cis-1,3-Dichloropropene & 0 & 0 & 0 & 0 & 0 \\
\hline trans-1,3-Dichloropropene & 0 & 0 & 0 & 0 & 0 \\
\hline 1,1,2,2-Tetrachloroethane & 0 & 0 & 0 & 0 & 0 \\
\hline
\end{tabular}


APPENDIX E.2

Groundwater and Surface-Water Quality Data, 1993

\begin{tabular}{|c|c|c|c|c|c|c|c|c|}
\hline \multirow{3}{*}{$\begin{array}{l}\text { Sampling Point } \\
\text { Location } \\
\text { Date Sampled }\end{array}$} & \multicolumn{4}{|c|}{ GW-013 } & \multicolumn{4}{|c|}{$G W-040$} \\
\hline & \multicolumn{4}{|c|}{ OLF } & \multicolumn{4}{|c|}{ BG } \\
\hline & $03 / 29 / 93 \mid$ & $06 / 17 / 93$ & $09 / 17 / 93 \mid$ & $12 / 19 / 93$ & $03 / 25 / 93$ & $05 / 11 / 93$ & $07 / 02 / 93$ & $10 / 09 / 93$ \\
\hline VOLATILE ORGANICS (ug/L) & $\cdot$ & $\cdot 1$ & - & $\cdot$ & $\cdot$ & $\cdot$ & $\cdot$ & - \\
\hline Acetone & 100 & 100 & 100 & 2 & 100 & 100 & 100 & 100 \\
\hline Benzene & 50 & 5u & 5u & 50 & 50 & 5u & 50 & 50 \\
\hline Bromodichloromethane & 5u & 50 & 50 & 50 & 50 & 5u & 50 & 50 \\
\hline Bromoform & 50 & 50 & 50 & 50 & su & 50 & $5 v$ & $5 \mathbf{5}$ \\
\hline Bromomethane & 100 & 100 & 100 & 100 & 100 & 100 & 100 & 100 \\
\hline 2-Butanone & 100 & 100 & 100 & 4 & 100 & 100 & 100 & 100 \\
\hline Carbon disulfide & 50 & 5u & 50 & 5u & 50 & 50 & 50 & 5u \\
\hline Carbon tetrachloride & 50 & 50 & 50 & 50 & $5 v$ & $5 v$ & su & $5 U$ \\
\hline Chlorobenzene & 50 & 5u & 50 & 50 & 50 & 50 & $5 \mathbf{v}$ & 50 \\
\hline Chlorodibromomethane & 50 & $5 u$ & 5u & 50 & 5u & $5 U$ & 5u & 50 \\
\hline Chloroethane & 100 & 100 & 100 & 100 & 100 & 100 & 100 & 100 \\
\hline Chloroform & 50 & 5u & 50 & 5u & 5u & 50 & 50 & $5 \mathbf{u}$ \\
\hline Chloromethane & 100 & 100 & 100 & 100 & 100 & 100 & 100 & 100 \\
\hline 1,1-Dichloroethane & $5 \mathbf{v}$ & 5u & 50 & 50 & $5 u$ & 50 & 50 & 50 \\
\hline 1,2-Dichloroethane & 50 & 5u & $5 \mathbf{u}$ & 50 & 50 & 5u & $5 U$ & 50 \\
\hline 1,1-Dichloroethene & 50 & $5 \mathbf{u}$ & 50 & 50 & $5 U$ & 50 & 5u & 50 \\
\hline 1,2-Dichloroethene & 5u & 50 & 5u & $5 \mathbf{u}$ & 50 & 5u & 50 & $5 \mathrm{U}$ \\
\hline 1,2-Dichloropropane & $5 v$ & 50 & 50 & 50 & 50 & 50 & 50 & 50 \\
\hline cis-1,3-Dichloropropene & 5u & 50 & 50 & $5 v$ & 5u & 50 & 50 & $5 u$ \\
\hline trans-1,3-Dichloropropene & 50 & 5u & $5 U$ & $5 U$ & su & 5u & 50 & 50 \\
\hline Ethylbenzene & 50 & 5u & $5 u$ & 50 & 50 & 5u & 50 & $5 U$ \\
\hline 2-Hexanone & 100 & 100 & 100 & 100 & 100 & 100 & 100 & 100 \\
\hline 4-Methy1-2-pentanone & 2 & 100 & 100 & 1 & 100 & 100 & 100 & 100 \\
\hline Methylene chloride & 3 & 5u & 50 & 1 & 50 & 1 & 50 & 50 \\
\hline Styrene & 50 & 50 & 50 & 50 & 50 & $5 u$ & 50 & 5u \\
\hline $1,1,2,2$-Tetrachloroethane & 5u & 50 & 50 & 50 & 50 & 50 & 50 & $5 \mathrm{U}$ \\
\hline Tetrachloroethene & 50 & 5u & $5 \mathbf{u}$ & $5 \mathbf{U}$ & $5 U$ & 5u & $5 \mathrm{U}$ & $5 \mathrm{U}$ \\
\hline Toluene & 50 & 50 & 50 & 50 & 50 & 50 & 50 & 50 \\
\hline 1,1,1-Trichloroethane & 5u & $5 U$ & 50 & 50 & 50 & 5u & 50 & $5 u$ \\
\hline $1,1,2$-Trichloroethane & 50 & 5u & 50 & 50 & su & 50 & $5 \mathbf{v}$ & 50 \\
\hline Trichloroethene & 50 & 50 & 50 & 50 & 50 & 50 & 50 & 50 \\
\hline Vinyl acetate & 100 & 100 & 100 & 100 & 100 & 100 & 100 & 100 \\
\hline vinyl chloride & 100 & 100 & 100 & 100 & 100 & 100 & 100 & 100 \\
\hline xylenes & $5 \mathrm{U}$ & 50 & 50 & 50 & 5u & $5 u$ & 50 & 50 \\
\hline
\end{tabular}

(CONTINUED) 
APPENDIX E. 2

Groundwater and Surface-Water Quality Data, 1993

\begin{tabular}{|c|c|c|c|c|c|c|c|c|}
\hline \multirow{3}{*}{$\begin{array}{l}\text { Sampling Point } \\
\text { Location } \\
\text { Date Sampled }\end{array}$} & \multirow{3}{*}{\begin{tabular}{|c|} 
GW-042 \\
BG \\
$12 / 29 / 93$
\end{tabular}} & \multicolumn{4}{|c|}{ GW-043 } & \multicolumn{3}{|c|}{ GW-044 } \\
\hline & & \multicolumn{4}{|c|}{ OLF } & \multicolumn{3}{|c|}{ OLF } \\
\hline & & $02 / 04 / 93$ & $05 / 10 / 93$ & $09 / 11 / 93$ & $10 / 19 / 93$ & $02 / 04 / 93$ & $05 / 10 / 93$ & $09 / 11 / 93$ \\
\hline VOLATILE ORGANICS (ug/L) & $\cdot$ & $\cdot$ & $\cdot$ & $\cdot$ & $\cdot$ & - & - & - \\
\hline Acetone & 100 & 100 & 100 & 100 & 2 & 100 & 200 & $10 \dot{0}$ \\
\hline Benzene & 5u & 5u & 50 & 5u & 50 & 5u & $5 \mathrm{U}$ & su \\
\hline Bromodichloromethane & 5u & $5 v$ & 50 & 50 & 50 & 50 & 50 & su \\
\hline Bromoform & su & 5u & $5 u$ & 50 & 50 & 50 & 50 & 50 \\
\hline Bromomethane & 100 & 100 & 100 & 100 & 100 & $10 \mathrm{U}$ & 100 & 100 \\
\hline 2-Butanone & 100 & 100 & 100 & 100 & 9 & 100 & 100 & 100 \\
\hline Carbon disulfide & $5 v$ & 50 & 50 & $5 \mathbf{v}$ & 5u & $5 \mathrm{U}$ & 50 & 50 \\
\hline Carbon tetrachloride & 5u & 50 & 5u & $5 \mathbf{v}$ & 5u & 50 & 50 & 5u \\
\hline Chlorobenzene & 5u & 50 & 50 & 5u & 50 & 50 & 50 & 50 \\
\hline Chlorodibromomethane & 50 & so & 50 & 50 & $5 v$ & 50 & 50 & $5 U$ \\
\hline Chloroethane & 100 & 100 & 100 & 100 & 100 & 100 & 100 & 100 \\
\hline Chloroform & 50 & 50 & su & $5 \mathbf{v}$ & 50 & 50 & 50 & 50 \\
\hline Chloromethane & 100 & 100 & 100 & 100 & 100 & 100 & 100 & 100 \\
\hline 1,1-Dichloroethane & $5 \mathbf{v}$ & 50 & 5u & su & 50 & 50 & $5 \mathrm{U}$ & 50 \\
\hline 1,2-Dichloroethane & 5v & 50 & 50 & 50 & 5v & $5 \mathrm{U}$ & $5 \mathrm{u}$ & 5u \\
\hline 1,1-Dichloroethene & so & $5 v$ & $5 \mathbf{u}$ & 50 & $5 v$ & su & $5 v$ & 50 \\
\hline 1,2-Dichloroethene & 50 & 50 & 5u & 50 & 50 & 5u & $5 \mathbf{v}$ & 5u \\
\hline 1,2-Dichloropropane & 50 & 50 & 5u & 50 & $5 \mathrm{v}$ & 50 & 50 & 50 \\
\hline Cis-1,3-Dichloropropene & $5 \mathrm{v}$ & 50 & 50 & 5u & $5 v$ & $5 \mathbf{u}$ & 5u & Su \\
\hline trans-1,3-Dichloropropene & 50 & su & 50 & 50 & 50 & $5 \mathbf{u}$ & 50 & $5 u$ \\
\hline Ethylbenzene & 50 & 50 & 50 & 50 & 50 & $5 \mathbf{v}$ & $5 \mathbf{v}$ & $5 u$ \\
\hline 2-Hexanone & 100 & 100 & 100 & 100 & 100 & 100 & 100 & 100 \\
\hline 4-Methy1-2-pentanone & 100 & 1 & 100 & 100 & 100 & 1 & 100 & 100 \\
\hline Methylene chloride & 50 & 1 & 50 & 1 & 3 & 1 & 50 & 5u \\
\hline Styrene & 50 & 50 & 50 & 50 & 50 & 5u & $5 \mathrm{U}$ & 50 \\
\hline $1,1,2,2$-Tetrachloroethane & 50 & $5 \mathrm{u}$ & 50 & 50 & 50 & $5 \mathbf{v}$ & 50 & 50 \\
\hline Tetrachloroethene & $5 u$ & 50 & $5 u$ & 50 & 50 & su & $5 v$ & 5u \\
\hline Toluene & $5 \mathrm{U}$ & 50 & 50 & $5 \mathrm{u}$ & $5 v$ & 50 & $5 \mathrm{U}$ & 50 \\
\hline 1,1,1-Trichloroethane & 5u & 50 & 5u & $5 \mathrm{U}$ & 50 & 50 & $5 v$ & 50 \\
\hline 1,1,2-Trichloroethane & 5u & 50 & 50 & 5u & 50 & 50 & 50 & 50 \\
\hline Trichloroethene & 50 & 5v & su & 50 & $5 v$ & 50 & 50 & 5u \\
\hline vinyl acetate & 100 & 100 & 100 & 100 & 100 & 100 & 100 & 100 \\
\hline vinyl chloride & 100 & 100 & 100 & 100 & 100 & 100 & 100 & 100 \\
\hline Xylenes & su & su & $5 \mathrm{U}$ & $5 U$ & 50 & 50 & $5 u$ & 50 \\
\hline
\end{tabular}

(CONTINUED) 
APPENDIX E.2

Groundwater and Surface-Water Quality Data, 1993

\begin{tabular}{|c|c|c|c|c|c|c|c|c|}
\hline \multirow{3}{*}{$\begin{array}{l}\text { Sampling Point } \\
\text { rocation } \\
\text { Date Sampled }\end{array}$} & \multirow{3}{*}{\begin{tabular}{|c|} 
GW-044 \\
OLF \\
$10 / 19 / 93$ \\
\end{tabular}} & \multicolumn{2}{|c|}{$G W-152$} & \multicolumn{4}{|c|}{ GW-056 } & \multirow{3}{*}{\begin{tabular}{|c|} 
GW-057 \\
EXP \\
$03 / 04 / 93$
\end{tabular}} \\
\hline & & \multicolumn{2}{|l|}{ BG } & \multicolumn{4}{|c|}{ BG } & \\
\hline & & $03 / 25 / 93$ & $04 / 26 / 93$ & $03 / 04 / 93$ & $04 / 22 / 93$ & $07 / 09 / 93$ & $10 / 09 / 93$ & \\
\hline VOLATILE ORGANICS (ug/L) & $\cdot$ & - & $\cdot$ & $\cdot$ & $\cdot$ & $\cdot$ & - & $\cdot$ \\
\hline Acetone & 3 & 100 & 100 & 100 & 100 & 100 & 100 & 100 \\
\hline Benzene & $5 \mathrm{u}$ & 50 & 5u & $5 u$ & 50 & 50 & $5 \mathbf{u}$ & 50 \\
\hline Bromodichloromethane & 50 & 50 & 50 & 50 & $5 \mathbf{5 u}$ & 50 & 50 & 50 \\
\hline Bromoform & 50 & 50 & 50 & 50 & 50 & 50 & 50 & 5u \\
\hline Bromomethane & 100 & 100 & 100 & 100 & 100 & 100 & 100 & 100 \\
\hline 2-Butanone & 12 & 100 & 100 & 100 & 100 & 100 & 100 & 100 \\
\hline Carbon disulfide & $5 \mathrm{v}$ & 50 & 50 & $5 u$ & su & 50 & $5 u$ & 50 \\
\hline Carbon tetrachloride & 50 & 50 & 50 & 50 & 50 & 50 & $5 u$ & 50 \\
\hline Chlorobenzene & 50 & $5 u$ & 5u & 50 & 50 & 50 & 50 & $5 U$ \\
\hline Chlorodibromomethane & 50 & $5 v$ & $5 \mathrm{U}$ & $5 U$ & $5 \mathrm{U}$ & 50 & $5 U$ & 5U \\
\hline Chloroethane & 100 & 100 & 100 & 100 & 100 & 100 & 100 & 100 \\
\hline Chloroform & $5 v$ & 50 & 50 & 50 & 50 & 50 & $5 \mathbf{U}$ & $5 U$ \\
\hline Chloromethane & 100 & 100 & 100 & 100 & 100 & 100 & 100 & 100 \\
\hline 1,1-Dichloroethane & 50 & so & 50 & $5 u$ & 50 & $5 \mathrm{v}$ & 50 & 5u \\
\hline 1,2-Dichloroethane & 50 & $5 u$ & 50 & su & $5 u$ & 50 & 50 & $5 \mathrm{U}$ \\
\hline 1,1-Dichloroethene & $5 \mathrm{u}$ & 50 & 50 & 50 & 50 & 50 & 50 & 50 \\
\hline 1,2-Dichloroethene & 50 & $5 U$ & 50 & 50 & $5 \mathrm{U}$ & 50 & su & 50 \\
\hline 1,2-Dichloropropiane & 50 & 50 & $5 \mathrm{U}$ & 50 & 50 & 50 & 50 & 5U \\
\hline cis-1,3-Dichloropropene & 5u & 5u & $5 \mathrm{U}$ & 50 & $5 \mathbf{U}$ & 50 & 50 & 50 \\
\hline trans-1,3-Dichloropropene & 50 & 50 & 50 & 50 & 5u & $5 \mathrm{u}$ & $5 U$ & 50 \\
\hline Ethylbenzene & $5 \mathrm{u}$ & 50 & 50 & 50 & $5 \mathbf{v}$ & $5 \mathrm{U}$ & $5 \mathrm{U}$ & 50 \\
\hline 2-Hexanone & 100 & 100 & 100 & 100 & $10 \mathrm{U}$ & 100 & $10 \mathrm{U}$ & 100 \\
\hline 4-Methy1-2-pentanone & 100 & 100 & 100 & 100 & 100 & 100 & 100 & 100 \\
\hline Methylene chloride & 2 & 50 & 50 & 1 & su & 50 & 5u & 50 \\
\hline Styrene & 50 & 50 & $5 \mathrm{u}$ & 50 & 50 & $5 \mathrm{u}$ & 50 & $5 U$ \\
\hline $1,1,2,2$-Tetrachloroethane & 50 & 50 & 50 & 50 & $5 \mathrm{U}$ & 50 & 50 & 5u \\
\hline Tetrachloroethene & 50 & 50 & 50 & 50 & $5 u$ & 50 & $5 u$ & 50 \\
\hline Toluene & 50 & 50 & 5u & 50 & 50 & 50 & su & 50 \\
\hline $1,1,1$-Trichloroethane & $5 \mathrm{v}$ & 50 & 50 & $5 \mathrm{U}$ & $5 u$ & $5 \mathrm{v}$ & 5u & 50 \\
\hline $1,1,2$-Trichloroethane & 50 & 50 & 50 & $5 \mathrm{U}$ & 5u & 50 & 50 & $5 U$ \\
\hline Trichloroethene & 50 & $5 v$ & 50 & $5 \mathrm{U}$ & $5 \mathrm{v}$ & $5 \mathrm{U}$ & $5 u$ & $5 \mathrm{U}$ \\
\hline Vinyl acetate & 100 & 100 & 100 & 100 & 100 & $10 \mathrm{U}$ & 100 & 100 \\
\hline vinyl chloride & 100 & 100 & 100 & 100 & 100 & 100 & 100 & 100 \\
\hline Xylenes & 50 & $5 \mathrm{U}$ & $5 \mathrm{U}$ & $5 \mathrm{U}$ & $5 u$ & 50 & $5 \mathrm{U}$ & $5 \mathrm{U}$ \\
\hline
\end{tabular}

(CONTINUED) 
APRENDIX $\mathrm{B} .2$

Groundwater and Surface-Water Quality Data, 1993

\begin{tabular}{|c|c|c|c|c|c|c|c|c|}
\hline \multirow{3}{*}{$\begin{array}{l}\text { Sampling Point } \\
\text { - } \\
\text { Location } \\
\text { - } \\
\text { Date Sampled }\end{array}$} & \multicolumn{3}{|c|}{ GW-057 } & GW-063 & \multicolumn{4}{|c|}{ GW-064 } \\
\hline & \multicolumn{3}{|c|}{$\mathbf{E X P}$} & OrF & \multicolumn{4}{|c|}{ OLF } \\
\hline & $04 / 22 / 93$ & $07 / 09 / 93$ & $10 / 09 / 93$ & $02 / 23 / 93$ & $02 / 09 / 93$ & $05 / 12 / 93$ & $09 / 11 / 93$ & $10 / 20 / 93$ \\
\hline VOLATILE ORGANICS (ug/L) & - & - & $\cdot$ & $\cdot$ & $\cdot$ & $\cdot$ & $\cdot$ & - \\
\hline Acetone & $10 \mathrm{U}$ & 100 & 100 & 5 & $10 \mathrm{U}$ & 100 & 100 & 100 \\
\hline Benzene & $5 u$ & 50 & 50 & 50 & 5u & 50 & 5u & 50 \\
\hline Bromodichloromethane & $5 \mathbf{U}$ & 5v & 5u & 5u & $5 \mathbf{u}$ & $5 \mathbf{5 u}$ & $5 \mathbf{v}$ & $5 \mathbf{v}$ \\
\hline Bromoform & 50 & 5v & 50 & 50 & 50 & $5 \mathrm{u}$ & $5 \mathbf{v}$ & 50 \\
\hline Bromomethane & 100 & 100 & 100 & 100 & 100 & 100 & 100 & 100 \\
\hline 2-Butanone & 100 & 100 & 100 & 100 & 100 & 100 & 100 & 10 \\
\hline Carbon disulfide & $5 \mathbf{v}$ & 50 & 50 & 50 & 50 & $5 U$ & 5u & 5u \\
\hline Carbon tetrachloride & 50 & $5 v$ & $5 v$ & su & 4 & 2 & 2 & 2 \\
\hline Chlorobenzene & su & 5u & 5u & 50 & 50 & $5 \mathbf{v}$ & 5u & 50 \\
\hline Chlorodibromomethane & 50 & 50 & $5 u$ & 50 & $5 \mathrm{u}$ & $5 v$ & 5u & $5 \mathbf{U}$ \\
\hline Chloroethane & 100 & 100 & 100 & $10 \mathrm{U}$ & 100 & 100 & $10 \mathrm{U}$ & 100 \\
\hline Chloroform & $5 \mathbf{U}$ & 50 & $5 \mathrm{v}$ & 50 & 3 & 2 & 2 & 2 \\
\hline Chloromethane & 100 & 100 & 100 & 100 & 100 & 100 & 100 & 200 \\
\hline 1,1-Dichloroethane & 50 & 50 & 50 & $5 U$ & 50 & $5 U$ & 50 & 50 \\
\hline 1,2-Dichloroethane & $5 v$ & 5u & 50 & 5u & 50 & $5 U$ & su & $5 \mathbf{U}$ \\
\hline 1,1-Dichloroethene & 50 & 50 & $5 \mathbf{u}$ & $5 \mathbf{v}$ & 50 & $5 \mathbf{5}$ & 5U & 5U \\
\hline 1,2-Dichloroethene & $5 \mathbf{v}$ & 5u & $5 \mathbf{u}$ & 0.9 & 9 & 6 & 7 & 6 \\
\hline 1,2-Dichloropropane & $5 v$ & 50 & $5 v$ & $5 v$ & 50 & $5 v$ & $5 \mathbf{U}$ & 5u \\
\hline cis-1,3-Dichloropropene & 50 & 50 & $5 \mathbf{u}$ & $5 \mathbf{u}$ & 50 & $5 \mathbf{v}$ & 50 & 50 \\
\hline trans-1,3-Dichloropropene & $5 U$ & $5 \mathbf{u}$ & $5 \mathbf{u}$ & $5 \mathbf{u}$ & $5 \mathbf{U}$ & $5 v$ & 5u & 50 \\
\hline Ethylbenzene & $5 \mathrm{v}$ & 5u & 50 & 50 & $5 \mathbf{v}$ & 50 & 50 & 50 \\
\hline 2-Hexanone & 100 & 100 & 100 & 100 & 100 & 100 & $10 \mathrm{v}$ & 100 \\
\hline 4-Methyl-2-pentanone & 100 & 100 & 100 & 100 & 2 & 100 & $10 \mathrm{U}$ & 100 \\
\hline Methylene chloride & 50 & 1 & $5 \mathbf{U}$ & 1 & 2 & 1 & 1 & 2 \\
\hline Styrene & 50 & 50 & 50 & 50 & 50 & 50 & 50 & 50 \\
\hline $1,1,2,2$-Tetrachloroethane & $5 \mathbf{v}$ & $5 \mathrm{u}$ & 50 & $5 \mathbf{U}$ & 50 & 50 & $5 U$ & 5u \\
\hline Tetrachloroethene & 50 & $5 \mathrm{U}$ & 5u & $5 u$ & 1 & 1 & 1 & 50 \\
\hline Toluene & $5 \mathbf{U}$ & 50 & 50 & 50 & 50 & 50 & $5 \mathrm{U}$ & 5U \\
\hline $1,1,1$-Trichloroethane & $5 \mathbf{u}$ & 50 & 50 & 50 & 1 & 0.8 & 0.9 & 50 \\
\hline $1,1,2-T r i c h l o r o e t h a n e$ & 50 & 50 & $5 v$ & $5 v$ & 50 & su & 50 & 50 \\
\hline Trichloroethene & $5 u$ & 50 & $5 U$ & 16 & 100 & 79 & 94 & 85 \\
\hline Vinyl acetate & $10 u$ & $10 \mathrm{U}$ & $10 \mathrm{U}$ & 100 & 3 & 100 & 100 & 100 \\
\hline Vinyl chloride & 100 & 100 & $10 \mathrm{U}$ & $10 U$ & 100 & 100 & $10 \mathrm{U}$ & 100 \\
\hline xylenes & $5 U$ & 50 & 5u & 50 & 50 & 50 & 50 & $5 U$ \\
\hline
\end{tabular}

(CONTINUED) 
APPENDIX E. 2

Groundwater and Surface-water Quality Data, 1993

\begin{tabular}{|c|c|c|c|c|c|c|c|c|}
\hline \multirow{3}{*}{$\begin{array}{l}\text { Sampling Point } \\
\text { Location } \\
\text { Date Sampled }\end{array}$} & \multicolumn{4}{|c|}{ GW-069 } & \multirow{3}{*}{\begin{tabular}{|c|} 
GW-079 \\
$B G$ \\
$10 / 18 / 93$
\end{tabular}} & \multirow{3}{*}{\begin{tabular}{|c|} 
GW-080 \\
BG \\
$10 / 10 / 93$
\end{tabular}} & \multirow{2}{*}{\multicolumn{2}{|c|}{$\frac{G W-082}{B G}$}} \\
\hline & \multicolumn{4}{|c|}{ BG } & & & & \\
\hline & $03 / 03 / 93$ & $04 / 21 / 93$ & $07 / 25 / 93$ & $10 / 10 / 93$ & & & $03 / 10 / 93$ & $04 / 21 / 93$ \\
\hline VOLATILE ORGANICS $(\mathrm{ug} / \mathrm{L})$ & $\cdot$ & $\cdot$ & $\cdot$ & $\cdot$ & $\cdot$ & $\cdot$ & $\cdot$ & $\cdot$ \\
\hline Acetone & 100 & 100 & 100 & 100 & 2 & 100 & 100 & 100 \\
\hline Benzene & su & 5u & 50 & 5v & 50 & 50 & 50 & 50 \\
\hline Bromodichloromethane & 50 & 5u & 50 & 50 & $5 u$ & 50 & 5u & 50 \\
\hline Bromoform & 50 & 50 & 50 & 5u & 50 & su & 50 & 5u \\
\hline Bromomethane & 100 & 100 & 100 & 100 & 100 & 100 & 100 & 100 \\
\hline 2-Butanone & 100 & 100 & 100 & 100 & 10 & 100 & 100 & 100 \\
\hline Carbon disulfide & 50 & 50 & 50 & $5 u$ & $5 v$ & 50 & $5 u$ & 50 \\
\hline Carbon tetrachloride & 50 & su & su & 5u & $5 v$ & 5u & $5 \mathrm{U}$ & 50 \\
\hline Chlorobenzene & $5 v$ & 50 & 50 & 50 & 5v & su & $5 \mathbf{v}$ & $5 U$ \\
\hline Chlorodibromomethane & 50 & 50 & 50 & 50 & $5 v$ & $5 \mathrm{U}$ & $5 \mathrm{u}$ & $5 U$ \\
\hline Chloroethane & 100 & 100 & 100 & 100 & 100 & 100 & 100 & 100 \\
\hline Chloroform & 5u & $5 u$ & $5 \mathbf{u}$ & 50 & $5 v$ & 50 & 50 & 50 \\
\hline Chloromethane & 100 & 100 & 100 & 100 & 100 & 100 & 100 & 100 \\
\hline 1,1-Dichloroethane & 50 & 50 & 50 & $5 \mathbf{u}$ & $5 v$ & 50 & $5 v$ & 50 \\
\hline 1,2-Dichloroethane & 5u & 5u & 5u & 50 & $5 u$ & $5 \mathrm{v}$ & 5u & 50 \\
\hline 1,1-Dichloroethene & $5 \mathbf{u}$ & 50 & 50 & 50 & 50 & 5u & 50 & 50 \\
\hline 1,2-Dichloroethene & 50 & 5u & 5u & $5 u$ & 50 & $5 \mathrm{u}$ & 41 & 50 \\
\hline 1,2-Dichloropropane & 50 & 50 & $5 \mathrm{U}$ & 50 & 50 & $5 v$ & 50 & su \\
\hline cis-1, 3-Dichloropropene & $5 v$ & 5u & 5u & 5u & 5u & 50 & su & 5u \\
\hline trans-1,3-Dichloropropene & 50 & 50 & $5 \mathbf{v}$ & $5 v$ & 50 & 5u & 50 & $5 \mathbf{U}$ \\
\hline Ethylbenzene & 50 & 50 & 50 & $5 \mathrm{u}$ & $5 v$ & 5u & $5 \mathrm{v}$ & $5 U$ \\
\hline 2-Hexanone & 100 & 100 & 100 & 100 & 100 & 100 & 100 & 100 \\
\hline 4-Methy1-2-pentanone & 100 & 100 & 100 & 100 & 100 & 100 & 100 & 100 \\
\hline Methylene chloride & $5 v$ & 0.8 & 1 & 50 & 2 & 50 & 5u & $5 U$ \\
\hline Styrene & $5 v$ & $5 u$ & su & 50 & 50 & 50 & 50 & $5 \mathbf{U}$ \\
\hline $1,1,2,2$-Tetrachloroethane & 50 & $5 \mathrm{v}$ & $5 \mathrm{U}$ & 50 & 50 & su & 50 & $5 \mathrm{U}$ \\
\hline Tetrachloroethene & $5 \mathrm{U}$ & 50 & 50 & $5 \mathbf{v}$ & 50 & 50 & $5 \mathbf{v}$ & 5u \\
\hline Toluene & $5 u$ & $5 v$ & $5 U$ & $5 U$ & 50 & 50 & 5u & $5 \mathrm{U}$ \\
\hline $1,1,1$-Trichloroethane & $5 U$ & $5 \mathrm{u}$ & $5 \mathrm{U}$ & 50 & 50 & 50 & $5 u$ & $5 \mathrm{U}$ \\
\hline 1,1,2-Trichloroethane & 50 & $5 u$ & 5u & 50 & 50 & 50 & $5 u$ & $5 \mathbf{U}$ \\
\hline Trichloroethene & $5 u$ & 50 & 5u & 50 & 50 & 50 & 50 & 50 \\
\hline Vinyl acetate & 100 & 100 & 100 & 100 & 100 & 100 & 100 & 100 \\
\hline Vinyl chloride & 100 & 1 & 100 & 100 & 100 & 100 & 30 & 31 \\
\hline Xylenes & 50 & 5u & 50 & 50 & 50 & su & 50 & 50 \\
\hline
\end{tabular}

(CONTINUED) 
APPENDIX E.2

Groundwater and Surface-Water Quality Data, 1993

\begin{tabular}{|c|c|c|c|c|c|c|c|c|}
\hline \multirow{3}{*}{$\begin{array}{l}\text { Sampling Point } \\
\text { Location } \\
\text { Date Sampled }\end{array}$} & \multicolumn{2}{|c|}{ GW-082 } & \multicolumn{4}{|c|}{$6 w-084$} & \multicolumn{2}{|c|}{ GW-085 } \\
\hline & \multicolumn{2}{|c|}{ BG } & \multicolumn{4}{|c|}{ OLF } & \multicolumn{2}{|c|}{ OLF } \\
\hline & $07 / 25 / 93$ & $10 / 18 / 93$ & $02 / 04 / 93$ & $05 / 10 / 93$ & $09 / 11 / 93$ & $10 / 20 / 93$ & $02 / 11 / 93$ & $05 / 12 / 93$ \\
\hline VOLATILE ORGANICS (ug/L) & $\cdot$ & $\cdot$ & - & $\cdot$ & $\cdot$ & $\cdot$ & $\cdot$ & - \\
\hline Acetone & 100 & 2 & 100 & 100 & 100 & 3 & 100 & 100 \\
\hline Benzene & su & su & 5u & 5u & $5 \mathrm{u}$ & su & 50 & 5U \\
\hline Bromodichloromethane & 50 & 5u & su & su & 50 & su & 5u & 50 \\
\hline Bromoform & su & 50 & 50 & 50 & so & su & 5u & 50 \\
\hline Bromomethane & 100 & 100 & 100 & 100 & 100 & 100 & 100 & 100 \\
\hline 2-Butanone & 100 & 10 & 100 & 100 & 100 & 8 & 100 & 100 \\
\hline Carbon disulfide & su & 5u & 5u & 5u & su & 5u & 50 & 5u \\
\hline Carbon tetrachloride & su & 50 & 50 & 5u & 50 & 5u & 5u & su \\
\hline Chlorobenzene & 50 & $5 \mathrm{U}$ & 5u & 50 & 50 & 50 & 5U & 50 \\
\hline Chlorodibromomethane & 5u & 50 & $5 u$ & 50 & su & 50 & 5u & 50 \\
\hline Chloroethane & 100 & 100 & 100 & 100 & 100 & 100 & 100 & 100 \\
\hline Chloroform & 5u & $5 \mathbf{v}$ & 50 & 50 & 50 & 5u & $5 u$ & 5u \\
\hline Chloromethane & 100 & 100 & 100 & 100 & 100 & 200 & 100 & 100 \\
\hline 1,1-Dichloroethane & 50 & 5u & 50 & $5 v$ & 50 & 5u & 5u & $5 U$ \\
\hline 1,2-Dichloroethane & 50 & 50 & 5u & 50 & 50 & su & 50 & 50 \\
\hline 1,1-Dichloroethene & 50 & 5u & 50 & su & 5u & 5u & su & 50 \\
\hline 1,2-Dichloroethene & 29 & 23 & 5u & 50 & 50 & su & 50 & su \\
\hline 1,2-Dichloropropane & 50 & 50 & 50 & 50 & 50 & 50 & 5u & 50 \\
\hline Cis-1, 3-Dichloropropene & 50 & 5u & 50 & su & 50 & 5u & 5u & 50 \\
\hline trans-1,3-Dichloropropene & 50 & 50 & 50 & su & so & so & 5u & 5u \\
\hline Ethylbenzene & 50 & 50 & $5 u$ & 50 & 5u & 50 & su & 50 \\
\hline 2-Hexanone & 100 & 100 & 100 & 100 & 100 & 100 & 100 & 100 \\
\hline 4-Methyl-2-pentanone & 100 & 100 & 100 & 100 & 100 & 100 & 100 & 100 \\
\hline Methylene chloride & 1 & 2 & 5u & 5u & 5u & 3 & 1 & 5U \\
\hline styrene & $5 v$ & 5u & 50 & $\therefore 50$ & 5u & 50 & 50 & 50 \\
\hline $1,1,2,2$-Tetrachloroethane & 5u & 5u & 50 & 50 & $5 \mathrm{u}$ & 50 & 50 & so \\
\hline Tetrachloroethene & 5u & 5u & 50 & su & su & 5u & 5u & 50 \\
\hline Toluene & 50 & 5u & 50 & 50 & 50 & 50 & su & 50 \\
\hline 1,1,1-Trichloroethane & 50 & 50 & 50 & 5u & 5u & 50 & su & 50 \\
\hline $1,1,2-\operatorname{Tr}$ ichloroethane & $5 v$ & 50 & 50 & 50 & 50 & 50 & 5u & 50 \\
\hline Trichloroethene & 50 & 5u & 50 & 50 & 5u & su & 5u & 50 \\
\hline vinyl acetate & 100 & 100 & 100 & 100 & 100 & 100 & 100 & $10 \mathrm{U}$ \\
\hline Vinyl chloride & 8 & 13 & 100 & 100 & 100 & 100 & 100 & 100 \\
\hline Xylenes & 50 & 50 & 50 & 50 & 50 & 50 & 50 & su \\
\hline
\end{tabular}

(CONTINUED) 
APPENDIX $\mathrm{E} .2$

Groundwater and Surface-Water Quality Data, 1993

\begin{tabular}{|c|c|c|c|c|c|c|c|c|}
\hline \multirow{3}{*}{$\begin{array}{l}\text { Sampling Point } \\
\text { Location } \\
\text { Date Sampled }\end{array}$} & \multicolumn{2}{|c|}{ GW-085 } & \multicolumn{4}{|c|}{$G W-086$} & \multicolumn{2}{|c|}{$G W-115$} \\
\hline & \multicolumn{2}{|c|}{ OLF } & \multicolumn{4}{|c|}{ OLF } & \multicolumn{2}{|c|}{ s3 } \\
\hline & $09 / 14 / 93$ & $10 / 22 / 93$ & $02 / 05 / 93$ & $05 / 11 / 93$ & $09 / 12 / 93$ & $10 / 21 / 93$ & $01 / 28 / 93$ & $05 / 25 / 93$ \\
\hline VOLATILE ORGANICS $(u g / L)$ & $\cdot$ & $\cdot$ & - & - & - & - & - & - \\
\hline Acetone & 100 & 100 & 100 & 100 & 100 & 100 & 100 & 100 \\
\hline Benzene & su & 50 & 5u & su & 5u & 50 & 50 & su \\
\hline Bromodichloromethane & $5 u$ & 50 & 5u & 50 & 5u & 50 & su & 5u \\
\hline Bromoform & $5 u$ & su & 5u & $5 \mathbf{U}$ & $5 u$ & 50 & su & 5u \\
\hline Bromomethane & 100 & 100 & 100 & 100 & 100 & 100 & 100 & 100 \\
\hline 2-Butanone & 100 & 100 & 100 & 100 & 100 & 100 & 100 & 100 \\
\hline Carbon disulfide & 50 & 5u & 50 & 50 & 5u & 5u & 5u & su \\
\hline Carbon tetrachloride & 50 & 5u & 5u & su & su & su & 5u & 5u \\
\hline Chlorobenzene & 50 & 5u & 5u & 5u & 5u & 50 & 50 & su \\
\hline Chlorodibromomethane & 50 & 5u & 5u & 5u & 5u & su & 50 & su \\
\hline Chloroethane & 100 & $10 \mathrm{U}$ & 100 & 100 & 100 & 100 & 100 & 100 \\
\hline Chloroform & 50 & so & 5u & su & 50 & 50 & 5u & su \\
\hline Chloromethane & 100 & 100 & 100 & 100 & 100 & 100 & 100 & 100 \\
\hline 1,1-Dichloroethane & $5 \mathbf{v}$ & 50 & su & 50 & 5u & $5 U$ & 50 & 50 \\
\hline 1,2-Dichloroethane & $5 v$ & 5u & 5u & 5u & su & 5u & su & 5u \\
\hline 1,1-Dichloroethene & 50 & 5u & 5u & 5u & 50 & $5 U$ & 50 & su \\
\hline 1,2-Dichloroethene & su & 50 & 5u & su & su & 5U & 5u & 50 \\
\hline 1,2-Dichloropropane & 5u & 5u & su & 5u & 5u & 5U & 5u & 50 \\
\hline cis-1,3-Dichloropropene & 5u & 5u & 50 & 5u & 5u & 5U & 5u & 50 \\
\hline trans-1,3-Dichloropropene & 5u & 5u & 5U & 5u & 5u & 5u & 5u & su \\
\hline Ethylbenzene & 5u & 5u & 50 & 5u & 5u & su & 5U & su \\
\hline 2-Hexanone & 100 & 100 & 100 & 100 & 100 & 100 & 200 & 100 \\
\hline 4-Methyl-2-pentanone & 100 & 100 & 100 & 1 & 100 & 100 & 2 & 100 \\
\hline Methylene chloride & 1 & $5 \mathbf{v}$ & 5u & 5u & su & 5v & 5u & 50 \\
\hline Styrene & su & 5u & 5u & 50 & 5u & $5 U$ & 50 & su \\
\hline $1,1,2,2$-Tetrachloroethane & 5u & 5u & 5u & 5u & 50 & 5u & 50 & $5 u$ \\
\hline Tetrachloroethene & $5 \mathrm{u}$ & so & 50 & 5u & 50 & 50 & su & su \\
\hline Toluene & 50 & su & 5u & 50 & 5u & 5u & $5 u$ & su \\
\hline 1,1,1-Trichloroethane & su & 5u & 50 & 50 & 50 & su & 50 & su \\
\hline 1,1,2-Trichloroethane & $5 \mathrm{U}$ & $5 \mathrm{u}$ & 50 & 5u & su & 50 & su & su \\
\hline Trichloroethene & $5 u$ & su & 50 & 50 & 50 & $5 U$ & 50 & $5 u$ \\
\hline vinyl acetate & 100 & 100 & 100 & 100 & 100 & 100 & 100 & 100 \\
\hline Vinyl chloride & 100 & 100 & 100 & 100 & 100 & 100 & 100 & $10 U$ \\
\hline xylenes & 50 & so & 50 & 50 & 50 & $5 U$ & 50 & 50 \\
\hline
\end{tabular}

(CONT INUED) 
APPENDIX E. 2

Groundwater and Surface-water Quality Data, 1993

\begin{tabular}{|c|c|c|c|c|c|c|c|c|}
\hline \multirow{3}{*}{$\begin{array}{l}\text { Sampling Point } \\
\text { Location } \\
\text { - } \\
\text { Date sampled }\end{array}$} & \multicolumn{2}{|c|}{ Gw-115 } & \multicolumn{4}{|c|}{ GW-118 } & GW-162 & $G w-286$ \\
\hline & \multicolumn{2}{|c|}{$\mathbf{8 3}$} & \multicolumn{4}{|c|}{ BO } & BG & BG \\
\hline & $09 / 11 / 93$ & $10 / 19 / 93$ & $03 / 04 / 93$ & $04 / 21 / 93$ & $07 / 25 / 93$ & $10 / 10 / 93$ & $10 / 10 / 93$ & $02 / 25 / 93$ \\
\hline VOLATILE ORCANICS (ug/L) & - & $\cdot$ & $\cdot$ & $\cdot$ & $\cdot$ & - &. & • \\
\hline Acetone & 100 & $10 \mathrm{U}$ & 100 & 100 & 100 & 7 & 100 & 200 \\
\hline Benzene & $5 \mathbf{u}$ & $5 \mathbf{v}$ & 5u & 50 & 2 & 1 & so & $5 \mathbf{5}$ \\
\hline Bromodichloromethane & $5 \mathbf{U}$ & $5 \mathbf{v}$ & su & su & su & $5 \mathbf{v}$ & 50 & $\mathbf{5 u}$ \\
\hline Bromoform & $5 v$ & 50 & 50 & su & 5u & $\mathbf{5 u}$ & su & $\mathbf{5 u}$ \\
\hline Bromomethane & 100 & 100 & 100 & 100 & 100 & 100 & 100 & 100 \\
\hline 2-Butanone & 100 & 8 & 100 & 100 & 100 & 11 & 100 & 100 \\
\hline Carbon disultide & $5 \mathbf{v}$ & $\mathbf{5 u}$ & $5 \mathbf{U}$ & 5u & 5u & $5 v$ & su & $\mathbf{5 u}$ \\
\hline Carbon tetrachloride & $5 \mathbf{u}$ & 50 & $\mathbf{5 u}$ & 5u & 50 & $5 \mathbf{v}$ & 50 & 5u \\
\hline Chlorobenzene & $5 \mathbf{v}$ & $5 \mathbf{v}$ & $5 \mathbf{v}$ & $5 \mathrm{u}$ & su & $5 \mathbf{u}$ & 50 & so \\
\hline Chlorodibromomethane & 50 & 50 & $5 \mathbf{v}$ & 50 & $5 \mathbf{u}$ & $5 \mathbf{v}$ & $5 \mathbf{v}$ & $\mathbf{5 u}$ \\
\hline Chloroethane & 100 & 100 & 100 & 100 & 100 & 100 & 100 & 100 \\
\hline Chloroform & $5 \mathbf{v}$ & $5 \mathbf{U}$ & $5 \mathbf{u}$ & $5 \mathbf{u}$ & su & $5 u$ & $5 \mathbf{U}$ & $\mathbf{5 u}$ \\
\hline Chloromethane & 100 & 100 & 100 & 100 & 100 & 100 & 100 & 100 \\
\hline 1,1-Dichloroethane & $5 \mathbf{v}$ & su & 5u & $5 \mathbf{u}$ & 5u & $5 \mathbf{u}$ & $5 \mathbf{v}$ & 50 \\
\hline 1,2-Dichloroethane & 50 & su & 5u & $5 v$ & 5u & 50 & $5 \mathbf{v}$ & $5 \mathbf{u}$ \\
\hline 1,1-Dichloroethene & $\mathbf{5 u}$ & $5 \mathbf{v}$ & $\mathbf{5 u}$ & $5 \mathbf{u}$ & su & 50 & $5 \mathbf{v}$ & $\mathbf{5 u}$ \\
\hline 1,2-Dichloroethene & $5 \mathbf{U}$ & $5 \mathbf{v}$ & $5 \mathbf{s u}$ & su & 5u & $5 \mathbf{v}$ & su & 5u \\
\hline 1,2-Dichloropropane & 5u & $5 v$ & 50 & sv & su & $5 U$ & sv & 5u \\
\hline cis-1,3-Dichloropropene & $5 \mathbf{v}$ & $5 \mathbf{v}$ & $5 \mathbf{v}$ & su & 5u & su & $5 \mathbf{v}$ & 50 \\
\hline trans-1, 3-Dichloropropene & 5u & $5 \mathbf{0}$ & $5 \mathbf{u}$ & $5 \mathbf{u}$ & 5u & $\mathbf{5 u}$ & 5v & 50 \\
\hline Bthylbenzene & $\mathbf{5 u}$ & 50 & $\mathbf{5 u}$ & $5 \mathbf{v}$ & 5u & su & $\mathbf{5 u}$ & 5u \\
\hline 2-Hexanone & 100 & 100 & 100 & $10 v$ & 100 & 100 & 100 & 100 \\
\hline 4-Methy 1-2-pentanone & 100 & 100 & 100 & 100 & 100 & 1 & 100 & 100 \\
\hline Methylene chloride & 2 & 2 & su & 50 & 5v & 6 & $\mathbf{s u}$ & 1 \\
\hline styrene & su & 50 & su & su & $5 v$ & su & 50 & 50 \\
\hline $1,1,2,2$-Tetrachloroethane & $\mathbf{5 u}$ & $5 \mathbf{v}$ & $5 \mathbf{u}$ & su & 5u & $5 \mathbf{v}$ & su & su \\
\hline Tetrachloroethene & 5u & 50 & $5 \mathbf{v}$ & $5 \mathbf{v}$ & $5 \mathbf{v}$ & $5 \mathbf{v}$ & $5 \mathbf{u}$ & 50 \\
\hline Toluene & 5u & 50 & $5 \mathbf{v}$ & $5 \mathbf{U}$ & 2 & 2 & su & su \\
\hline $1,1,1$-Trichloroethane & 50 & 50 & $5 \mathbf{u}$ & su & 5u & $5 \mathbf{u}$ & $5 \mathbf{u}$ & 50 \\
\hline $1,1,2-$ Trichloroethane & 5u & $5 \mathbf{v}$ & $5 \mathbf{v}$ & $5 U$ & 5v & su & $5 \mathbf{u}$ & su \\
\hline Trichloroethene & 50 & $5 v$ & $5 \mathbf{v}$ & su & 50 & 50 & 50 & 5u \\
\hline Vinyl acetate & 100 & 100 & 100 & 100 & $10 \mathrm{U}$ & 100 & 100 & 100 \\
\hline Vinyl chloride & 100 & 100 & 100 & 100 & 100 & 100 & 100 & 100 \\
\hline xylenes & 5u & $5 \mathbf{u}$ & su & $5 u$ & 5u & 50 & $5 u$ & 50 \\
\hline
\end{tabular}

(CONTINUED) 
APPENDIX $\mathbf{2} .2$

Groundwater and Surface-Water Quality Data, 1993

\begin{tabular}{|c|c|c|c|c|c|c|c|c|}
\hline \multirow{3}{*}{$\begin{array}{l}\text { Sampling Polnt } \\
\text { Location } \\
\text { - } \\
\text { Date sampled }\end{array}$} & \multicolumn{3}{|c|}{ CW-286 } & \multicolumn{4}{|c|}{$G W-287$} & \multirow{3}{*}{\begin{tabular}{|c|} 
Gw-311 \\
28 \\
$02 / 08 / 93$
\end{tabular}} \\
\hline & \multicolumn{3}{|c|}{ BG } & \multicolumn{4}{|c|}{$\mathbf{B Q}$} & \\
\hline & $04 / 19 / 93$ & $07 / 10 / 93$ & $10 / 07 / 93$ & $02 / 24 / 93$ & $04 / 16 / 93$ & $07 / 10 / 93$ & $10 / 07 / 93$ & \\
\hline VOLATILE ORGANICS (ug/L) & - & $\cdot$ & - & - & $\cdot$ & - & - & - \\
\hline Acetone & $10 \mathrm{u}$ & 5 & 4 & 100 & 100 & $10 \mathrm{U}$ & 3 & $10 u$ \\
\hline Benzene & 50 & 50 & 5u & $\mathbf{5 u}$ & $5 \mathbf{U}$ & 5u & su & $\mathbf{5 u}$ \\
\hline Bromodich loromethane & 50 & 5u & 5u & su & 50 & su & 5u & 5u \\
\hline Bromoform & 50 & $\mathbf{5 u}$ & $5 \mathbf{v}$ & 50 & 5u & su & $5 \mathbf{u}$ & $5 \mathbf{v}$ \\
\hline Bromomethane & 100 & 100 & 100 & 100 & 100 & 100 & 100 & 100 \\
\hline 2-Butanone & 100 & 100 & 10 & 100 & 100 & 100 & 11 & 100 \\
\hline Carbon disulfide & $\mathbf{s u}$ & $5 v$ & 5u & $5 v$ & $5 v$ & su & su & su \\
\hline Carbon tetrachloride & $5 v$ & su & 50 & 50 & su & su & 50 & 0.9 \\
\hline Chlorobenzene & 50 & su & 5u & su & 5u & $5 \mathbf{v}$ & su & $5 \mathbf{5}$ \\
\hline Chlorodibromomethane & su & 5u & su & $5 \mathbf{u}$ & su & 50 & su & $\mathbf{5 u}$ \\
\hline Chloroethane & 100 & 100 & 100 & 100 & 100 & 100 & $10 \mathrm{u}$ & 100 \\
\hline Chloroform & $5 \mathbf{v}$ & su & su & $\mathbf{5 u}$ & su & 5u & su & 1 \\
\hline Chloromethane & 100 & 100 & 100 & 100 & 100 & 100 & 100 & 100 \\
\hline 1,1-Dichloroethane & $5 \mathbf{u}$ & sv & 5v & $5 \mathbf{v}$ & 50 & 50 & su & su \\
\hline 1,2-Dichloroethane & 50 & su & 50 & su & su & $5 \mathbf{v}$ & su & 5v \\
\hline 1,1-Dichloroethene & 5u & $5 u$ & 50 & 5u & $5 \mathbf{u}$ & 5u & su & $\mathbf{5 u}$ \\
\hline 1,2-Dichloroethene & su & su & 50 & su & 50 & $5 v$ & su & 50 \\
\hline 1,2-Dichloropropane & su & 5u & $5 \mathbf{u}$ & su & $5 \mathbf{v}$ & $5 \mathbf{v}$ & su & $\mathbf{5 u}$ \\
\hline cis-1,3-Dichloropropene & su & 5u & su & 50 & su & sv & $\mathbf{s u}$ & $\mathbf{5 0}$ \\
\hline trane-1,3-Dichloropropene & su & su & su & su & su & $\mathbf{5 u}$ & $\mathbf{5 u}$ & $\mathbf{5 u}$ \\
\hline Bthylbenzene & 5u & 5u & $\mathbf{s u}$ & su & su & $5 \mathbf{v}$ & $\mathbf{s u}$ & $\mathbf{5 0}$ \\
\hline 2-Hexanone & 100 & 100 & 100 & 100 & 100 & 100 & 100 & 100 \\
\hline 4-Mathy1-2-pentanone & 100 & 100 & 100 & 100 & 100 & 100 & 100 & 100 \\
\hline Methylene chloride & $5 \mathbf{v}$ & 0.8 & 3 & 2 & $\mathbf{5 u}$ & $\mathbf{s u}$ & 3 & $\mathbf{5 u}$ \\
\hline styrene & su & 50 & su & $\therefore 50$ & 5u & su & 5u & $\mathbf{5 u}$ \\
\hline $1,1,2,2$-Tetrachloroethane & $\mathbf{5 u}$ & 5u & su & $5 \mathbf{v}$ & $\mathbf{5 u}$ & $5 \mathbf{v}$ & 5u & $\mathbf{5 u}$ \\
\hline Tetruchloroethene & 50 & 5u & su & su & 0.7 & $\mathbf{5 u}$ & 5u & $\mathbf{5 u}$ \\
\hline Toluene & $5 \mathbf{u}$ & su & su & 50 & su & su & su & $\mathbf{5 v}$ \\
\hline $1,1,1$-Trichloroethane & su & 5u & su & $\mathbf{5 u}$ & 5u & 5u & 5u & $\mathbf{s u}$ \\
\hline $1,1,2$-Trichloroethane & 5u & su & 50 & su & su & su & 5u & $\mathbf{5 0}$ \\
\hline Trichloroethene & 3 & 50 & su & su & su & $5 \mathbf{v}$ & su & 13 \\
\hline VInyl acetate & $10 \mathrm{u}$ & 100 & 100 & 100 & 100 & $10 u$ & 100 & 100 \\
\hline Vinyl chloride & 100 & 100 & 100 & 100 & 100 & 100 & $10 \mathrm{U}$ & 100 \\
\hline Xylenes & $5 \mathrm{U}$ & su & su & su & $5 \mathbf{v}$ & $5 u$ & $\mathbf{5 u}$ & su \\
\hline
\end{tabular}

(CONTINURD) 
APPENDIX $\mathbf{8 . 2}$

Groundwater and surface-Water Quality Data, 1993

\begin{tabular}{|c|c|c|c|c|c|c|c|c|}
\hline \multirow{3}{*}{$\begin{array}{l}\text { Sampling Point } \\
\text { ' } \\
\text { Location } \\
\text { Date sampled }\end{array}$} & \multicolumn{3}{|c|}{$0 \%-311$} & \multicolumn{4}{|c|}{ CW-312 } & \multirow{3}{*}{\begin{tabular}{|c|} 
ON-315 \\
$8 P 1$ \\
$02 / 03 / 93$
\end{tabular}} \\
\hline & \multicolumn{3}{|c|}{ R8 } & \multicolumn{4}{|c|}{ Rs } & \\
\hline & $05 / 11 / 93$ & $09 / 12 / 93$ & $10 / 21 / 93$ & $02 / 08 / 93$ & $05 / 11 / 93$ & $09 / 12 / 93$ & $10 / 21 / 93$ & \\
\hline VOLATILE ORCANICS (ug/L) & $\cdot$ & - & - & - & - & - & $\cdot 1$ & - \\
\hline Acetone & $10 \mathrm{U}$ & 100 & 100 & 100 & 100 & 100 & 3 & 2 \\
\hline Benzene & su & 5u & 5u & su & 5u & 5u & su & su \\
\hline Bromodlchloromethane & 5u & 5u & 50 & su & 5u & su & su & 2 \\
\hline Bromoform & $5 \mathbf{u}$ & 5u & 5u & su & su & su & su & su \\
\hline Bromomethane & 100 & 100 & 100 & 100 & $10 \mathrm{u}$ & 100 & 100 & 100 \\
\hline 2-Butanone & 100 & 100 & 100 & 100 & $10 \mathrm{v}$ & $10 v$ & 10 & 100 \\
\hline Carbon dieulfide & 5u & su & su & su & su & su & so & su \\
\hline Carbon tetrachloride & 2 & 1 & 3 & 5u & su & su & su & $\mathbf{5 u}$ \\
\hline Chlorobenzene & su & su & $5 \mathbf{u}$ & su & su & 50 & su & $\mathbf{s u}$ \\
\hline Chlorodibromomethane & $5 v$ & 50 & su & su & su & su & $\mathbf{5 u}$ & $\mathbf{s u}$ \\
\hline Chloroethane & 100 & $10 \mathrm{U}$ & $10 \mathrm{u}$ & $10 v$ & $10 \mathrm{v}$ & 100 & 100 & 100 \\
\hline Chloroform & 5u & 0.8 & 1 & 1 & 1 & su & su & 7 \\
\hline Chloromethane & 100 & 100 & 100 & 100 & 100 & 100 & 100 & 100 \\
\hline 1.1-Dichloroethane & 50 & 5u & su & $5 u$ & $5 \mathbf{v}$ & 5u & 5u & 50 \\
\hline 1,2-Dichloroethane & 5u & su & su & su & $5 u$ & 50 & su & $5 \mathbf{u}$ \\
\hline 1,1-Dichloroathene & 5u & su & su & su & su & su & su & $5 v$ \\
\hline 1,2-Dichloroethene & 50 & $5 \mathbf{u}$ & su & 5u & 5u & 5u & su & 5 \\
\hline 1,2-Dlchloropropane & 5u & su & su & su & 50 & su & su & $\mathbf{S U}$ \\
\hline c1e-1,3-Dichloropropene & 5u & 5u & Su & 5u & su & 5u & 5u & su \\
\hline trans-1,3-Dichloropropene & 5u & $5 \mathbf{v}$ & su & 5u & su & su & 5u & $\mathbf{5 0}$ \\
\hline Bthylbenzene & 5u & 5u & 5u & su & su & 5u & su & su \\
\hline 2-Hexanone & 100 & 100 & 100 & 100 & 100 & 100 & 100 & 100 \\
\hline 4-Methyl-2-pentanone & 1 & $10 \mathrm{v}$ & $10 \mathrm{u}$ & 100 & $10 \mathrm{v}$ & $10 \mathrm{U}$ & 100 & 2 \\
\hline Methylene chloride & su & 1 & 5u & sv & sv & 5u & 3 & 2 \\
\hline styrene & 5u & 5u & su & 5u & su & 5u & 5u & su \\
\hline $1,1,2,2$-Tetrachloroethane & 50 & 5u & 50 & 50 & su & $5 \mathbf{u}$ & 5u & $5 u$ \\
\hline Tetrachloroethene & su & su & 50 & 5u & su & 50 & su & 8 \\
\hline Toluene & 50 & 5u & su & su & su & 5u & $5 \mathbf{v}$ & $5 \mathbf{v}$ \\
\hline $1,1,1-T r 1$ chloroethana & 50 & 5u & 5u & 50 & su & su & 5u & $\mathbf{5 u}$ \\
\hline 1,1,2-Trlchloroethane & 5u & 5u & su & 50 & su & 5u & su & su \\
\hline Trlchloroethene & 21 & 15 & 19 & 62 & 83 & 10 & 49 & 4 \\
\hline Vinyl acetate & 100 & 100 & 100 & 200 & 100 & $10 v$ & 100 & 100 \\
\hline Vinyl chloride & 100 & 100 & 100 & 100 & 100 & $10 \mathrm{U}$ & 100 & 100 \\
\hline Xylenes & su & su & $5 u$ & $5 v$ & $\mathbf{5 u}$ & su & su & SU \\
\hline
\end{tabular}

(CONTINURD) 
APPENDIX $\$ .2$

Groundwater and Surface-Water Quality Data, 1993

\begin{tabular}{|c|c|c|c|c|c|c|c|c|}
\hline \multirow{3}{*}{$\begin{array}{l}\text { Sampling Polnt } \\
\text { Location } \\
\text { Date sampled }\end{array}$} & \multicolumn{3}{|c|}{ GW-315 } & \multicolumn{4}{|c|}{$0 *-316$} & \multirow{2}{*}{$\frac{a W-317}{\operatorname{SPI}}$} \\
\hline & \multicolumn{3}{|c|}{$\mathbf{8 P I}$} & \multicolumn{4}{|c|}{ 8PI } & \\
\hline & $06 / 03 / 93$ & $09 / 14 / 93$ & $10 / 27 / 93$ & $02 / 02 / 93$ & $06 / 01 / 93$ & $09 / 13 / 93$ & $10 / 24 / 93$ & $02 / 02 / 93$ \\
\hline VOLATILE ORGNICS (ug/L) & $\cdot$ & - & - & - & $\cdot$ & $\cdot$ & - & - \\
\hline Acetone & $10 \mathrm{v}$ & 100 & 1 & 3 & 100 & 100 & 100 & 3 \\
\hline Benzene & Su & su & $5 \mathbf{v}$ & su & su & 5u & su & 5u \\
\hline Bromodlchloromethane & 2 & 2 & 1 & 5u & su & $5 u$ & $5 \mathbf{v}$ & $\mathbf{5 v}$ \\
\hline Bromotorm & $5 \mathbf{5}$ & su & 50 & 50 & 50 & sv & 50 & 5v \\
\hline Bromomethane & 100 & 100 & $10 \mathrm{v}$ & 100 & 100 & 100 & 100 & 100 \\
\hline 2-Butanone & $10 \mathrm{v}$ & 100 & 7 & 6 & 100 & 100 & 100 & 8 \\
\hline Carbon disulfide & $5 \mathbf{v}$ & $5 \mathbf{u}$ & su & so & $5 v$ & $5 \mathbf{v}$ & $5 v$ & su \\
\hline Carbon tetrachloride & su & 50 & $5 \mathbf{v}$ & su & su & $\mathbf{5 u}$ & su & su \\
\hline Chlorobenzene & su & 5u & $5 \mathbf{5}$ & 5u & su & $5 \mathbf{5}$ & 5u & 5u \\
\hline Chlorodibromome t hane & 50 & 50 & su & 5u & $5 \mathbf{v}$ & 5u & $5 \mathbf{v}$ & 5u \\
\hline Chloroethane & 100 & 100 & $10 \mathrm{v}$ & 100 & 100 & $10 u$ & 100 & 100 \\
\hline Chloroform & 9 & 8 & 6 & $\mathbf{s u}$ & $5 \mathbf{u}$ & 5U & $5 v$ & $\mathbf{s u}$ \\
\hline Chloromethane & $10 \mathrm{v}$ & 100 & $10 \mathrm{u}$ & $10 \mathrm{v}$ & $10 \mathrm{v}$ & 100 & $10 \mathrm{v}$ & $10 \mathrm{U}$ \\
\hline 1,1-Dichloroethane & su & $5 \mathbf{v}$ & $5 \mathbf{v}$ & $5 \mathbf{v}$ & $5 u$ & $5 \mathbf{u}$ & $5 \mathbf{v}$ & 5u \\
\hline 1,2-Dichloroethane & 5u & $5 \mathbf{u}$ & 5u & 5u & Su & 50 & $5 \mathbf{u}$ & su \\
\hline 1,1-Dichloroethene & su & su & su & $5 \mathbf{v}$ & $5 \mathbf{u}$ & $5 \mathbf{v}$ & $\mathbf{s u}$ & su \\
\hline 1,2-D1chloroethene & 2 & 7 & 5 & 5u & su & 5u & $5 \mathbf{u}$ & 5u \\
\hline 1,2-Dlchloropropane & sv & 5u & su & 50 & $5 u$ & 50 & $5 v$ & 5u \\
\hline cis-1,3-Dichloropropene & 5u & $5 \mathbf{u}$ & $5 u$ & 50 & sv & 50 & su & 5u \\
\hline trans-1,3-Dichloropropene & su & $5 v$ & 5u & 50 & $5 \mathbf{v}$ & $5 \mathbf{v}$ & $\mathbf{5 u}$ & su \\
\hline Bthylbenzene & su & su & $5 \mathbf{u}$ & $5 \mathbf{v}$ & 5u & $\mathbf{5 u}$ & su & su \\
\hline 2-Hexanone & 100 & 100 & $10 \mathrm{v}$ & 100 & 100 & $10 \mathrm{v}$ & 100 & 100 \\
\hline 4-Methy1-2-pentanone & 100 & $10 u$ & 100 & 2 & 100 & 100 & 100 & 2 \\
\hline Methylene chlorlde & 1 & $5 \mathbf{v}$ & 2 & $5 \mathbf{u}$ & su & su & su & $\mathbf{5 u}$ \\
\hline styrene & su & su & 5v & 50 & $\mathbf{5 u}$ & su & $\mathbf{s u}$ & 5u \\
\hline $1,1,2,2$-Tetrachloroethane & su & su & su & $5 v$ & $5 \mathbf{u}$ & 5u & $5 \mathbf{v}$ & $\mathbf{5 u}$ \\
\hline Tetrachloroethene & 5 & 10 & 7 & $\mathbf{5 u}$ & $5 \mathbf{u}$ & $5 \mathbf{v}$ & $5 \mathbf{v}$ & su \\
\hline Toluene & su & $\mathbf{5 u}$ & su & $5 v$ & su & $5 \mathbf{v}$ & $5 v$ & 5u \\
\hline $1,1,1$-Tr1chloroethane & su & 50 & su & $5 \mathbf{v}$ & $\mathbf{5 u}$ & $5 \mathbf{u}$ & $5 \mathbf{v}$ & $5 u$ \\
\hline $1,1,2$-Trichloroethane & su & $5 \mathbf{u}$ & 5u & $5 \mathbf{v}$ & 5u & $\mathbf{5 u}$ & $5 \mathbf{5 u}$ & $5 \mathbf{u}$ \\
\hline Trichloroethene & 2 & 5 & 4 & 5u & 5u & 50 & su & su \\
\hline Vinyl acetate & $10 \mathrm{u}$ & $10 \mathrm{u}$ & $10 \mathrm{u}$ & 100 & $10 u$ & 100 & 100 & 100 \\
\hline Vinyl chloride & 100 & $10 \mathrm{U}$ & 100 & 100 & $10 \mathrm{U}$ & 100 & 100 & 100 \\
\hline Xylenes & 50 & su & 5u & 50 & $5 u$ & $5 v$ & 50 & $5 \mathbf{5}$ \\
\hline
\end{tabular}

(CONTINUED) 
APPENDIX E. 2

Groundwater and Surface-Water Quality Data, 1993

\begin{tabular}{|c|c|c|c|c|c|c|c|c|}
\hline \multirow{3}{*}{$\begin{array}{l}\text { Sampling Point } \\
\text { Location } \\
\text { Date Sampled }\end{array}$} & \multicolumn{3}{|c|}{ GW-317 } & \multicolumn{4}{|c|}{$G w-323$} & $G W-324$ \\
\hline & \multicolumn{3}{|c|}{ SPI } & \multicolumn{4}{|c|}{ SPI } & $\mathbf{8 3}$ \\
\hline & $05 / 28 / 93$ & $09 / 13 / 93$ & $10 / 24 / 93$ & $02 / 02 / 93$ & $05 / 28 / 93$ & $09 / 12 / 93$ & $10 / 23 / 93$ & $01 / 29 / 93$ \\
\hline VOLATILB OREANICS (Ug/L) & $\cdot 1$ & $\cdot$ & $\cdot$ & $\cdot$ & $\cdot$ & - & - & - \\
\hline Aoetone & 100 & 100 & 100 & 2 & 100 & 100 & 100 & 100 \\
\hline Benzene & su & su & 5u & 50 & 50 & 5u & su & 5U \\
\hline Bromodichloromethane & 50 & $\mathbf{s u}$ & 50 & 5u & su & 50 & 50 & 5u \\
\hline Bromoform & 50 & su & 5v & 50 & su & su & 5u & so \\
\hline Bromomethane & 100 & 100 & 100 & 100 & 100 & 100 & 100 & 100 \\
\hline 2-Butanone & 4 & 100 & 100 & 6 & 3 & 100 & 100 & 100 \\
\hline Carbon diaulfide & 50 & $5 \mathbf{v}$ & 50 & 50 & su & 50 & 5u & 50 \\
\hline Carbon tetrachloride & su & $5 \mathbf{v}$ & $5 \mathbf{v}$ & 50 & su & 5u & 50 & su \\
\hline Chlorobenzene & 50 & 50 & su & 50 & su & 5u & 50 & 50 \\
\hline Chlorodibromomethane & 5u & su & 5u & su & 50 & 5u & 50 & 50 \\
\hline Chloroethane & 100 & 100 & 100 & 100 & 100 & 100 & 100 & 100 \\
\hline Chloroform & 50 & 5u & 5u & 50 & 50 & $5 \mathbf{v}$ & su & 5u \\
\hline Chloromethane & 100 & 100 & 100 & 100 & 100 & 100 & 100 & 100 \\
\hline 1,1-Dichloroethane & 5u & 5u & 5u & 5u & 50 & 5u & 5u & 50 \\
\hline 1,2-Dichloroethane & 50 & 5u & 50 & 5u & 5u & 50 & 5u & su \\
\hline 1,1-Dichloroethene & so & 5u & 50 & 50 & su & $5 v$ & 5v & 50 \\
\hline 1,2-Dichloroethene & 50 & 50 & 50 & 5u & su & 5u & su & 50 \\
\hline 1,2-Dichloropropane & 50 & $5 \mathbf{v}$ & 50 & 5u & 5u & 5u & 5u & 50 \\
\hline cis-1, 3-Dichloropropene & 50 & 50 & $5 u$ & 5u & su & 50 & 5u & 5u \\
\hline trans-1,3-Dichloropropene & su & su & $5 u$ & 5u & $5 \mathbf{v}$ & 5u & 50 & 50 \\
\hline Ethylbenzene & 50 & su & 50 & 5u & 5u & 5u & $5 u$ & su \\
\hline 2-Hexanone & 100 & 100 & 100 & 100 & 100 & 100 & 100 & 100 \\
\hline 4-Methyl-2-pentanone & 100 & 100 & 100 & 2 & 100 & 100 & 100 & 100 \\
\hline Methylene chloride & 1 & su & 50 & 5u & 5u & su & 50 & 5u \\
\hline styrene & 5u & $5 u$ & 50 & 50 & su & 50 & $5 \mathbf{u}$ & su \\
\hline $1,1,2,2$-Tetrachlor oethane & 50 & $5 \mathbf{u}$ & 50 & 50 & 50 & 50 & $5 \mathbf{u}$ & 50 \\
\hline Tetrachloroethene & 5u & 5u & 50 & 50 & 5u & 50 & 50 & 50 \\
\hline Toluene & 5u & 50 & 50 & 50 & 5u & 50 & 5u & 50 \\
\hline $1,1,1$-Trichloroethane & 5u & 50 & 5u & 50 & 5u & su & 5u & su \\
\hline $1,1,2-$ Trichloroethane & 5u & 5u & 5u & 50 & $5 \mathrm{u}$ & su & su & 5u \\
\hline Trichloroethene & su & 50 & 5u & 50 & su & 50 & 5u & so \\
\hline Vinyl acetate & 100 & 100 & 100 & 100 & 100 & 100 & 100 & 100 \\
\hline vinyl chloride & 100 & 100 & 100 & 100 & 100 & 100 & 100 & 100 \\
\hline Xylenes & 50 & 50 & 50 & 50 & 50 & 50 & su & 50 \\
\hline
\end{tabular}

(CONTINUED) 
Groundwater and Surface-Water Quality Data, 1993

\begin{tabular}{|c|c|c|c|c|c|c|c|c|}
\hline \multirow{3}{*}{$\begin{array}{l}\text { Sampling Point } \\
\text { Location } \\
\text { - } \\
\text { Date Sampled }\end{array}$} & \multicolumn{3}{|c|}{ Gw-324 } & \multicolumn{4}{|c|}{$G w-325$} & GW-342 \\
\hline & \multicolumn{3}{|c|}{$\mathbf{S 3}$} & \multicolumn{4}{|c|}{83} & $\mathbf{B O}$ \\
\hline & $05 / 25 / 93$ & $09 / 11 / 93$ & $10 / 19 / 93$ & $02 / 01 / 93$ & $05 / 25 / 93$ & $09 / 11 / 93 \mid$ & $10 / 21 / 93$ & $10 / 07 / 93$ \\
\hline VOLATILB ORGANICS (ug/L) & $\cdot$ & $\cdot$ & $\cdot$ & - & - & - & $\cdot$ & $\cdot$ \\
\hline Acetone & 100 & 100 & 2 & $10 \mathrm{U}$ & 100 & 100 & 100 & 100 \\
\hline Benzene & 5u & $5 \mathbf{v}$ & $5 \mathbf{v}$ & 5u & $\mathbf{5 u}$ & $5 \mathbf{v}$ & su & 5u \\
\hline Bromodichloromethane & $5 \mathbf{u}$ & $5 \mathbf{v}$ & 50 & $\mathbf{5 0}$ & $5 \mathbf{v}$ & 5u & su & 5u \\
\hline Bromoform & $5 \mathbf{v}$ & 5u & $5 \mathbf{v}$ & $5 \mathbf{v}$ & $5 \mathbf{v}$ & 50 & su & 5u \\
\hline Bromomethane & $10 u$ & 100 & 100 & 100 & 100 & 100 & 100 & 100 \\
\hline 2-Butanone & 100 & 100 & 8 & 100 & $10 \mathrm{U}$ & 100 & 100 & $10 \mathbf{v}$ \\
\hline Carbon disulfide & $5 v$ & su & 50 & $5 \mathbf{u}$ & 5u & 50 & $\mathbf{5 u}$ & $5 \mathbf{v}$ \\
\hline Carbon tetrachloride & 50 & 5u & 5v & $5 \mathbf{v}$ & 5u & $5 \mathbf{u}$ & 5u & 5v \\
\hline Chlorobenzene & 5u & 50 & $5 \mathrm{v}$ & $5 \mathbf{v}$ & 50 & $5 \mathbf{v}$ & 0.6 & 50 \\
\hline Chlorodibromomethane & 5u & $5 \mathbf{v}$ & 50 & 50 & 5u & $5 \mathbf{u}$ & su & so \\
\hline Chloroethane & 100 & 100 & 100 & 100 & 100 & 100 & 100 & 100 \\
\hline Chloroform & $5 v$ & $5 \mathrm{v}$ & $5 v$ & $5 \mathbf{u}$ & su & $5 \mathbf{v}$ & 5u & 50 \\
\hline Chloromethane & 100 & 100 & 100 & $10 \mathrm{U}$ & $10 \mathrm{U}$ & 100 & 100 & 100 \\
\hline 1,1-Dichloroethane & 50 & 50 & $5 \mathbf{u}$ & $5 \mathbf{s u}$ & 5u & $5 \mathbf{v}$ & $5 u$ & su \\
\hline 1,2-Dichloroethane & 5u & 5u & 5v & $5 \mathbf{v}$ & 5u & 50 & $5 \mathbf{v}$ & 5u \\
\hline 1,1-Dichloroethene & 5u & 50 & 5u & $5 \mathbf{v}$ & 5u & $\mathbf{5 u}$ & $5 \mathbf{u}$ & su \\
\hline 1,2-Dichloroethene & 5u & su & 5U & $5 \mathbf{u}$ & su & $5 \mathbf{u}$ & su & $\mathbf{5 u}$ \\
\hline 1,2-Dichloropropane & $5 \mathbf{v}$ & $5 \mathbf{v}$ & $5 \mathbf{v}$ & $5 \mathbf{U}$ & 5u & 50 & $5 \mathbf{u}$ & $5 \mathbf{v}$ \\
\hline c1s-1,3-Dichloropropene & 50 & $5 \mathbf{v}$ & $5 \mathbf{v}$ & $5 \mathbf{v}$ & su & $5 \mathbf{U}$ & su & $\mathbf{5 u}$ \\
\hline trans-1, 3-Dichloropropene & $5 \mathbf{u}$ & 5u & 5u & 5u & $5 \mathrm{u}$ & $5 \mathbf{v}$ & $\mathbf{5 u}$ & $\mathbf{s u}$ \\
\hline Ethylbenzene & $5 \mathbf{v}$ & $5 u$ & $5 \mathbf{U}$ & $5 \mathbf{v}$ & $5 \mathbf{u}$ & $\mathbf{5 u}$ & 5u & $5 \mathbf{u}$ \\
\hline 2-Hexanone & 100 & 100 & 100 & 100 & 100 & 100 & 100 & 100 \\
\hline 4-Methyl-2-pentanone & 100 & 100 & 100 & 1 & 100 & 100 & $10 \mathrm{u}$ & 100 \\
\hline Methylene chloride & su & 1 & 2 & su & $5 \mathbf{u}$ & 1 & 5u & $5 \mathbf{U}$ \\
\hline styrene & 5u & $5 \mathbf{v}$ & $5 \mathbf{v}$ & 5u & $5 \mathbf{v}$ & 5u & su & 50 \\
\hline $1,1,2,2$-Tetrachloroethane & $5 \mathrm{U}$ & $5 v$ & $\mathbf{s u}$ & 5u & 50 & $5 \mathbf{v}$ & $5 \mathbf{v}$ & $5 \mathbf{v}$ \\
\hline Tetrachloroethene & 50 & $5 v$ & 50 & 5U & $5 \mathbf{v}$ & 5v & $5 \mathbf{u}$ & $\mathbf{5 u}$ \\
\hline Toluene & 5U & $5 \mathrm{U}$ & $5 U$ & $5 \mathbf{v}$ & su & $5 v$ & $5 \mathbf{u}$ & $\mathbf{5 u}$ \\
\hline $1,1,1$-Trichloroethane & $5 u$ & 50 & $5 \mathbf{U}$ & 50 & 50 & 50 & 50 & 50 \\
\hline $1,1,2$-Trichloroethane & $5 \mathrm{U}$ & 50 & $5 \mathbf{u}$ & $5 \mathbf{u}$ & $5 \mathrm{U}$ & $5 \mathbf{U}$ & 50 & 50 \\
\hline Trichloroethene & 5u & $5 U$ & 50 & $5 \mathbf{u}$ & $5 U$ & su & $5 \mathbf{U}$ & 50 \\
\hline Vinyl acetate & $10 u$ & 100 & 100 & $10 \mathrm{U}$ & $10 U$ & $10 \mathrm{U}$ & 100 & 100 \\
\hline Vinyl chloride & 100 & 100 & $10 \mathrm{U}$ & $10 \mathrm{U}$ & 100 & 100 & 100 & 100 \\
\hline Xylenes & 50 & $5 \mathrm{U}$ & $5 \mathbf{u}$ & $5 \mathbf{U}$ & $5 \mathbf{v}$ & su & $5 U$ & $5 \mathbf{U}$ \\
\hline
\end{tabular}

(CONTINUED) 
APPENDIX E. 2

Groundwater and Surface-Water Quality Data, 1993

\begin{tabular}{|c|c|c|c|c|c|c|c|c|}
\hline \multirow{3}{*}{$\begin{array}{l}\text { Sampling Point } \\
\text { Location } \\
\text { - } \\
\text { Date Sampled }\end{array}$} & \multicolumn{4}{|c|}{$G W-345$} & \multicolumn{4}{|c|}{$G W-347$} \\
\hline & \multicolumn{4}{|c|}{ s3 } & \multicolumn{4}{|c|}{ s3 } \\
\hline & $02 / 03 / 93$ & $06 / 01 / 93$ & $09 / 14 / 93$ & $10 / 25 / 93$ & $02 / 01 / 93$ & $05 / 27 / 93$ & $09 / 12 / 93$ & $10 / 22 / 93$ \\
\hline VOLATILE ORGANICS (ug/L) & $\cdot$ & - & $\cdot$ & - & - & . & $\cdot$ & - \\
\hline Acetone & 100 & 100 & 100 & 2 & 100 & 100 & 100 & 100 \\
\hline Benzene & 50 & 50 & $5 \mathbf{v}$ & 50 & 50 & 50 & 50 & 50 \\
\hline Bromodichloromethane & $5 v$ & $5 v$ & 5u & 5v & 50 & 5u & 50 & 5u \\
\hline Bromoform & 50 & $5 \mathbf{u}$ & 50 & 5u & 5u & 5u & $5 \mathrm{v}$ & 50 \\
\hline Bromomethane & 100 & 100 & 100 & 100 & 100 & 100 & 100 & 100 \\
\hline 2-Butanone & 100 & 100 & 100 & 6 & 100 & 100 & 100 & 100 \\
\hline Carbon disulfide & 50 & 50 & 50 & 50 & su & 50 & 50 & 5u \\
\hline Carbon tetrachloride & 50 & 50 & $5 v$ & 50 & $5 v$ & 5u & 50 & $5 u$ \\
\hline Chlorobenzene & 50 & 5u & $5 \mathbf{5 u}$ & 50 & 5u & 50 & 50 & 5u \\
\hline Chlorodibromomethane & 50 & 50 & su & 50 & su & 50 & 50 & 5u \\
\hline Chloroethane & 100 & 100 & 100 & 100 & 100 & 100 & 100 & 100 \\
\hline Chloroform & 50 & 50 & 2 & 50 & su & 50 & 50 & 5u \\
\hline Chloromethane & 100 & 100 & 100 & 100 & 200 & 100 & 100 & 100 \\
\hline 1,1-Dichloroethane & 50 & su & 5u & 50 & 50 & 5u & so & su \\
\hline 1,2-Dichloroethane & 50 & 5u & 5u & 50 & 5u & 50 & 5u & 50 \\
\hline 1,1-Dichloroethene & 50 & 50 & 5u & 5u & 5u & 5u & 50 & 5u \\
\hline 1,2-Dichloroethene & 50 & 5u & 5u & 5v & su & 50 & 50 & 5u \\
\hline 1,2-Dichloropropane & 5u & 50 & 50 & $5 v$ & $5 \mathbf{v}$ & 5v & 5u & 5u \\
\hline cis-1,3-Dichloropropene & 50 & su & 50 & 5u & 50 & 5v & 5o & 50 \\
\hline trans-1,3-Dichloropropene & 50 & su & 50 & 50 & 5u & 50 & 513 & 5u \\
\hline Ethylbenzene & 50 & 50 & 50 & 50 & su & 5u & 5u & 5u \\
\hline 2-Hexanone & 100 & 100 & 100 & 100 & $10 U$ & 100 & 100 & 100 \\
\hline 4-Methy1-2-pentanone & 2 & 100 & 100 & 100 & 2 & 100 & 100 & 100 \\
\hline Methylene chloride & 2 & 5u & 5 & 2 & 50 & 5u & 5u & 50 \\
\hline Styrene & 50 & $5 \mathbf{u}$ & 5u & $5 \mathbf{v}$ & 5u & 5u & 5u & 5u \\
\hline $1,1,2,2$-Tetrachloroethane & $5 v$ & 5u & 5u & 5u & 5u & 50 & 50 & 50 \\
\hline Tetrachloroethene & 50 & 5u & 50 & 50 & $5 u$ & 5u & 5u & 50 \\
\hline Toluene & 50 & 50 & $5 v$ & 50 & 50 & 50 & 50 & 5u \\
\hline $1,2,1$-Trichloroethane & 50 & 50 & 5u & $5 \mathbf{U}$ & 5u & 5u & 5u & 5u \\
\hline 1,1,2-Trichloroethane & 50 & 5u & $5 \mathrm{~J}$ & 5u & 50 & 5u & 5u & 5u \\
\hline Trichloroethene & 50 & su & 50 & 50 & 50 & 5u & 50 & 5u \\
\hline Vinyl acetate & 100 & 100 & 100 & 100 & 100 & 100 & 100 & 100 \\
\hline Vinyl chloride & 100 & 100 & 100 & 100 & 100 & 100 & 100 & 100 \\
\hline Xylenes & 50 & 50 & 5u & $5 \mathrm{U}$ & 50 & 50 & 50 & 50 \\
\hline
\end{tabular}

(CONTINUED) 
APPENDIX E.2

Groundwater and Surface-Water Quality Data, 1993

\begin{tabular}{|c|c|c|c|c|c|c|c|c|}
\hline \multirow{3}{*}{$\begin{array}{l}\text { Sampling Point } \\
\text { Location } \\
\text { Date Sampled }\end{array}$} & \multicolumn{4}{|c|}{$G W-348$} & \multicolumn{4}{|c|}{$G W-364$} \\
\hline & \multicolumn{4}{|c|}{ s3 } & \multicolumn{4}{|c|}{ OLF } \\
\hline & $02 / 01 / 93$ & $05 / 27 / 93$ & $09 / 12 / 93$ & $10 / 22 / 93$ & $03 / 27 / 93$ & $06 / 27 / 93$ & $09 / 19 / 93$ & $12 / 18 / 93$ \\
\hline VOLATILE ORGANICS (Ug/L) &. & . & . & $\cdot$ & - & - & $\cdot$ & • \\
\hline Acetone & 100 & 100 & 100 & 2 & 100 & 100 & 100 & $\dot{5}$ \\
\hline Benzene & 50 & 50 & 50 & 50 & 50 & 50 & 5u & 50 \\
\hline Bromodichloromethane & 50 & 50 & 5u & $5 v$ & su & 50 & 5u & 50 \\
\hline Bromoform & $5 u$ & 50 & 5u & 50 & 50 & 5v & 50 & 50 \\
\hline Bromomethane & 100 & 100 & 100 & 100 & 100 & 100 & 100 & 100 \\
\hline 2-Butanone & 100 & 100 & 100 & 7 & 200 & 100 & 100 & 9 \\
\hline Carbon disulfide & 50 & 50 & 50 & 50 & su & 50 & $5 \mathbf{v}$ & 50 \\
\hline Carbon tetrachloride & $5 U$ & 50 & 50 & 50 & SU & 50 & 50 & 50 \\
\hline Chlorobenzene & 50 & 5u & 5u & 50 & 5u & 5u & $5 \mathrm{v}$ & 50 \\
\hline Chlorodibromomethane & 5u & 5u & 50 & 50 & 50 & su & 50 & 50 \\
\hline Chloroethane & 100 & 100 & 100 & 100 & 100 & 100 & 100 & 100 \\
\hline Chloroform & 50 & 50 & 50 & 50 & su & 50 & 50 & 50 \\
\hline Chloromethane & 100 & 100 & 100 & 100 & 100 & 100 & 100 & 100 \\
\hline 1,1-Dichloroethane & $5 \mathbf{v}$ & 50 & 50 & 50 & 2 & 50 & 3 & 2 \\
\hline 1,2-Dichloroethane & 5u & 50 & 5u & 50 & 5u & 50 & $5 \mathbf{u}$ & 50 \\
\hline 1,1-Dichloroethene & 5u & 50 & 50 & 50 & 50 & $5 u$ & 5 & 2 \\
\hline 1,2-Dichloroethene & 5u & 50 & 5u & 50 & su & 6 & 7 & 4 \\
\hline 1,2-Dichloropropane & 5u & 50 & 50 & 50 & su & 50 & 50 & 50 \\
\hline Cis-1,3-Dichloropropene & 5u & 50 & 50 & 50 & 5U & 50 & $5 \mathbf{v}$ & 50 \\
\hline trans-1,3-Dichloropropene & 50 & 5u & 50 & $5 \mathbf{v}$ & su & su & $5 \mathbf{v}$ & su \\
\hline Ethylbenzene & 5u & 50 & 50 & su & 50 & 5u & su & so \\
\hline 2-Hexanone & 100 & 100 & 100 & 100 & 100 & 100 & 100 & 100 \\
\hline 4-Methy1-2-pentanone & 2 & 100 & 100 & 100 & 100 & 1 & 100 & 100 \\
\hline Methylene chloride & $5 \mathrm{v}$ & 50 & 50 & 2 & su & so & 1 & 0.6 \\
\hline Styrene & 50 & 50 & 5u & 50 & su & 50 & 50 & 50 \\
\hline $1,1,2,2$-Tetrachloroethane & 50 & 50 & $5 u$ & 50 & 5U & 50 & 5u & 50 \\
\hline Tetrachloroethene & 50 & 50 & 5u & $5 u$ & su & 50 & 5u & 0.3 \\
\hline Toluene & 50 & $5 \mathrm{u}$ & 50 & $5 v$ & $5 \mathrm{U}$ & 50 & 5u & 50 \\
\hline $1,1,1$-Trichloroethane & 50 & 50 & 50 & 50 & 5u & 50 & 2 & 1 \\
\hline 1,1,2-Trichloroethane & 50 & 50 & $5 \mathrm{U}$ & 50 & 50 & 50 & 50 & 50 \\
\hline Trichloroethene & 50 & 50 & 50 & 50 & 6 & 16 & 15 & $\varepsilon$ \\
\hline Vinyl acetate & 100 & 100 & 100 & 100 & 100 & 100 & 100 & 100 \\
\hline Vinyl chloride & 100 & 100 & 100 & 100 & 100 & 100 & 100 & 100 \\
\hline xylenes & 50 & $5 \mathrm{U}$ & $5 U$ & $5 U$ & 5u & 50 & $5 \mathrm{U}$ & 50 \\
\hline
\end{tabular}

(CONTINUED) 
APPENDIX E. 2

Groundwater and Surface-Water Quality Data, 1993

\begin{tabular}{|c|c|c|c|c|c|c|c|c|}
\hline \multirow{3}{*}{$\begin{array}{l}\text { Sampling Point } \\
\text { Location } \\
\text { Date Sampled }\end{array}$} & \multicolumn{4}{|c|}{$G W-365$} & \multicolumn{4}{|c|}{ GW-370 } \\
\hline & \multicolumn{4}{|c|}{ OLF } & \multicolumn{4}{|c|}{ BG } \\
\hline & $03 / 28 / 93$ & $06 / 27 / 93$ & $09 / 19 / 93$ & $12 / 19 / 93$ & $02 / 17 / 93$ & $05 / 21 / 93$ & $07 / 10 / 93$ & $10 / 14 / 93$ \\
\hline VOLATILE ORGANICS (ug/L) & $\cdot$ & . & $\cdot$ & - & $\cdot$ & $\cdot$ & - & • \\
\hline Acetone & 100 & 100 & 100 & 100 & 4 & 100 & 100 & 3 \\
\hline Benzene & 50 & 50 & 50 & 50 & 5u & 50 & 50 & 50 \\
\hline Bromodichloromethane & 5u & 5u & 5u & 5u & so & 5u & 5u & 50 \\
\hline Bromoform & 50 & 50 & 50 & 5u & 50 & 50 & 5u & 50 \\
\hline Bromomethane & 100 & 100 & 100 & 100 & 100 & 100 & 100 & 100 \\
\hline 2-Butanone & 100 & 100 & 100 & 9 & 100 & 100 & 100 & 9 \\
\hline Carbon disulfide & 50 & 5u & 50 & 5u & 50 & 50 & $5 \mathbf{v}$ & 50 \\
\hline Carbon tetrachloride & 5u & 50 & su & 5u & su & 5u & 5u & 5u \\
\hline Chlorobenzene & 50 & su & 50 & 5u & 5u & 5u & 50 & su \\
\hline Chlorodibromomethane & 50 & 50 & $5 \mathrm{v}$ & 50 & $5 v$ & 5u & 50 & 50 \\
\hline Chloroethane & 100 & 100 & 100 & 100 & 100 & 100 & 200 & 100 \\
\hline Chloroform & 1 & $5 u$ & $5 u$ & 5u & 50 & 5u & 50 & 50 \\
\hline Chloromethane & 100 & 100 & 100 & 100 & 100 & 100 & 100 & 100 \\
\hline 1,1-Dichloroethane & 5 & 50 & 4 & 4 & 5u & 50 & 5v & 50 \\
\hline 1,2-Dichloroethane & 50 & 50 & 5u & 50 & $5 \mathrm{u}$ & 50 & 50 & 5u \\
\hline 1,1-Dichloroethene & 21 & 20 & 14 & 13 & 50 & 5u & 50 & 50 \\
\hline 1,2-Dichloroethene & 23 & 19 & 14 & 12 & su & 5u & 50 & 50 \\
\hline 1,2-Dichloropropane & 50 & 50 & 5u & 50 & su & 5u & 50 & 50 \\
\hline Cis-1,3-Dichloropropene & 5u & 50 & 50 & 5v & su & 50 & $5 \mathbf{v}$ & 50 \\
\hline trans-1,3-Dichloropropene & $5 v$ & 50 & 5u & su & 50 & 50 & 5u & so \\
\hline Ethylbenzene & 50 & 50 & 50 & 50 & su & 5u & 50 & 50 \\
\hline 2-Hexanone & 100 & 100 & 100 & 100 & 100 & 100 & 100 & 100 \\
\hline 4-Nethy1-2-pentanone & 2 & 100 & 100 & 100 & 3 & 100 & 100 & 100 \\
\hline Methylene chloride & 3 & su & 50 & 0.6 & 2 & 50 & 5u & 3 \\
\hline Styrene & 50 & 50 & 50 & $5 u$ & su & su & 5v & 50 \\
\hline $1,1,2,2$-Tetrachloroethane & 50 & 50 & 50 & 50 & 50 & 5u & $5 \mathrm{v}$ & su \\
\hline Tetrachloroethene & 2 & su & 1 & 1 & 50 & 5u & $5 v$ & su \\
\hline Toluene & 50 & 50 & 50 & $5 U$ & 5u & $5 \mathrm{u}$ & $5 \mathrm{v}$ & 50 \\
\hline $1,1,1$-Trichloroethane & 11 & 5 & 2 & 2 & su & 5u & 50 & 50 \\
\hline 1,1,2-Trichloroethane & 50 & 50 & 50 & 5u & 50 & 50 & $5 u$ & 50 \\
\hline Trichloroethene & 89 & 77 & 46 & 39 & 50 & 50 & 50 & 50 \\
\hline vinyl acetate & 100 & 100 & 100 & 100 & 100 & 100 & 100 & 100 \\
\hline Vinyl chloride & 100 & 100 & 100 & 100 & 100 & 100 & 100 & 100 \\
\hline Xylenes & 50 & 50 & 50 & 50 & 50 & 50 & 50 & 50 \\
\hline
\end{tabular}

(CONTINUED) 
APPENDIX $\mathrm{E}, 2$

Groundwater and Surface-Water Quality Data, 1993

\begin{tabular}{|c|c|c|c|c|c|c|c|c|}
\hline \multirow{3}{*}{$\begin{array}{l}\text { Sampling Point } \\
\text { - } \\
\text { Location } \\
\text { Date Sampled }\end{array}$} & \multicolumn{4}{|c|}{ Gw-371 } & \multicolumn{4}{|c|}{ GW-372 } \\
\hline & \multicolumn{4}{|c|}{ BG } & \multicolumn{4}{|c|}{ BG } \\
\hline & $02 / 16 / 93$ & $05 / 21 / 93$ & $07 / 09 / 93$ & $10 / 13 / 93$ & $02 / 09 / 93$ & $05 / 19 / 93$ & $07 / 02 / 93$ & $10 / 12 / 93$ \\
\hline VOLATILE ORGANICS (ug/L) & - & $\cdot$ & $\cdot$ & - & - & • & $\cdot$ & - \\
\hline Acetone & 100 & 100 & 5 & 100 & 1 & 100 & 100 & 100 \\
\hline Benzene & $5 u$ & $5 \mathbf{v}$ & 5u & 5U & $5 v$ & $5 v$ & 50 & $5 \mathbf{v}$ \\
\hline Bromodichloromethane & $5 v$ & $5 v$ & 5v & $5 \mathbf{v}$ & 5v & $5 \mathbf{u}$ & 50 & 5u \\
\hline Bromoform & 50 & $5 \mathrm{v}$ & $5 U$ & 50 & 50 & 5u & 50 & $5 \mathbf{v}$ \\
\hline Bromomethane & 100 & $10 \mathrm{v}$ & 100 & 100 & 100 & 100 & 100 & 100 \\
\hline 2-Butanone & 100 & 100 & 100 & 100 & 100 & 100 & 100 & 100 \\
\hline Carbon disulfide & $5 \mathbf{v}$ & 5u & su & 5u & $5 U$ & $5 \mathbf{U}$ & 5u & 50 \\
\hline Carbon tetrachloride & $5 v$ & 50 & 50 & 50 & $5 v$ & $5 \mathbf{v}$ & 5u & $5 \mathbf{5 u}$ \\
\hline Chlorobenzene & $5 \mathbf{v}$ & $5 \mathrm{u}$ & 50 & $5 \mathbf{v}$ & $5 v$ & $5 \mathbf{U}$ & 50 & $5 \mathbf{v}$ \\
\hline Chlorodibromomethane & $5 u$ & 50 & $5 v$ & 50 & su & $5 \mathbf{U}$ & 50 & 5u \\
\hline Chloroethane & $10 \mathrm{U}$ & 100 & 100 & 100 & 100 & $10 \mathrm{U}$ & 100 & 100 \\
\hline Chloroform & $5 \mathbf{u}$ & $5 \mathbf{s}$ & 0.6 & 50 & $5 \mathbf{u}$ & $5 \mathbf{v}$ & $5 U$ & 50 \\
\hline Chloromethane & 100 & 100 & 100 & 100 & 100 & $10 \mathrm{U}$ & 100 & 100 \\
\hline 1,1-Dichloroethane & $5 u$ & 50 & $5 \mathrm{v}$ & 50 & $5 v$ & $5 \mathbf{u}$ & 5u & 50 \\
\hline 1,2-Dichloroethane & 5u & 50 & $5 v$ & 50 & 50 & 50 & 50 & $5 \mathbf{v}$ \\
\hline 1,1-Dichloroethene & $5 \mathrm{u}$ & 50 & 5u & 50 & 50 & $5 \mathrm{U}$ & $5 \mathbf{U}$ & $5 \mathbf{v}$ \\
\hline 1,2-Dichloroethene & $5 v$ & $5 \mathrm{U}$ & 5v & 50 & $5 \mathbf{v}$ & $5 u$ & 5u & $5 \mathbf{u}$ \\
\hline 1,2-Dichloropropane & 5u & 50 & 5v & 50 & 50 & $5 v$ & $5 \mathbf{v}$ & $5 \mathbf{v}$ \\
\hline Cis-1,3-Dichloropropene & 50 & $5 \mathrm{v}$ & $5 v$ & $5 \mathbf{v}$ & $5 \mathbf{u}$ & $5 \mathbf{U}$ & 5u & 5v \\
\hline trans-1,3-Dichloropropene & 5u & su & 50 & $5 \mathbf{v}$ & $5 \mathbf{U}$ & $5 \mathbf{v}$ & $5 \mathbf{v}$ & $5 \mathbf{v}$ \\
\hline Ethylbenzene & $5 U$ & 50 & $5 \mathrm{U}$ & $5 v$ & $5 \mathbf{v}$ & 50 & $5 \mathbf{v}$ & $5 v$ \\
\hline 2-Hexanone & $10 \mathrm{U}$ & 100 & 100 & 100 & $10 U$ & 100 & 100 & $10 \mathrm{U}$ \\
\hline 4-Methyl-2-pentanone & $10 \mathrm{U}$ & 100 & 100 & 100 & 3 & 100 & 100 & 100 \\
\hline Methylene chloride & $5 u$ & $5 v$ & 1 & $5 v$ & 2 & $5 \mathbf{v}$ & 50 & 0.7 \\
\hline Styrene & $5 \mathbf{U}$ & $5 U$ & 50 & $5 \mathbf{v}$ & $5 \mathbf{U}$ & $5 U$ & $5 \mathrm{U}$ & $5 \mathbf{U}$ \\
\hline $1,1,2,2$-Tetrachloroethane & $5 \mathbf{u}$ & $5 \mathrm{U}$ & $5 v$ & $5 \mathbf{v}$ & $5 \mathbf{U}$ & $5 \mathbf{U}$ & $5 \mathrm{v}$ & $5 U$ \\
\hline Tetrachloroethene & $5 \mathbf{U}$ & $5 \mathrm{u}$ & $5 \mathrm{U}$ & $5 \mathbf{U}$ & 50 & $5 \mathbf{u}$ & $5 U$ & $5 \mathbf{U}$ \\
\hline Toluene & $5 U$ & $5 \mathrm{U}$ & $5 \mathbf{U}$ & $5 \mathbf{U}$ & $5 \mathrm{U}$ & 50 & 50 & 50 \\
\hline 1,1,1-Trichloroethane & 50 & $5 v$ & 50 & $5 U$ & $5 \mathbf{S}$ & 50 & $5 \mathbf{U}$ & $5 \mathbf{v}$ \\
\hline $1,1,2$-Trichloroethane & 5u & 50 & $5 \mathbf{v}$ & $\mathbf{5 U}$ & $5 \mathbf{U}$ & 50 & su & 50 \\
\hline Trichloroethene & 50 & 50 & 50 & $5 \mathbf{U}$ & $5 U$ & 50 & $5 \mathbf{U}$ & $5 \mathbf{u}$ \\
\hline vinyl acetate & 100 & 100 & 100 & 100 & 100 & 100 & 100 & 100 \\
\hline Vinyl chloride & 100 & 100 & 100 & 100 & 100 & 100 & 100 & 100 \\
\hline xylenes & $5 v$ & $5 v$ & 50 & 50 & $5 v$ & 50 & $5 \mathrm{U}$ & 50 \\
\hline
\end{tabular}

(CONTINUED) 
APPENDIX 8.2

Groundwater and Surface-Water Quality Data, 1993

\begin{tabular}{|c|c|c|c|c|c|c|c|c|}
\hline \multirow{3}{*}{$\begin{array}{l}\text { Sampling Point } \\
\text { Location } \\
\text { Date Sampled }\end{array}$} & \multicolumn{4}{|c|}{$G W-373$} & \multicolumn{4}{|c|}{$G W-526$} \\
\hline & \multicolumn{4}{|c|}{ BG } & \multicolumn{4}{|c|}{$\mathbf{s 3}$} \\
\hline & $02 / 11 / 93$ & $05 / 14 / 93$ & $07 / 02 / 93$ & $10 / 11 / 93$ & $02 / 03 / 93$ & $06 / 03 / 93$ & $09 / 14 / 93$ & $10 / 26 / 93$ \\
\hline VOLATILE ORGANICS (ug/L) & - & - & . & - & . & - & - & - \\
\hline Acetone & 2 & 100 & 100 & 3 & 4 & 200 & 100 & 2 \\
\hline Benzene & 5u & 5u & 5v & 50 & su & 5u & 5u & 50 \\
\hline Bromodichloromethane & 50 & $5 v$ & 50 & 5U & su & su & 5u & 5u \\
\hline Bromoform & 5u & su & 5u & 50 & 5u & su & 5u & 50 \\
\hline Bromomethane & 100 & 100 & 100 & 100 & 100 & 100 & 100 & 100 \\
\hline 2-Butanone & 100 & 100 & 100 & 9 & 100 & $10 u$ & 100 & 8 \\
\hline Carbon disulfide & 50 & 5v & 5u & 50 & 5u & 50 & 50 & 50 \\
\hline Carbon tetrachloride & $5 U$ & $5 U$ & 50 & 5u & $5 v$ & 5v & 5u & 5u \\
\hline Chlorobenzene & 5u & 5u & 50 & 5v & 5u & su & 5u & 50 \\
\hline Chlorodibromomethane & 5u & 50 & 50 & su & 50 & 5u & 5u & 50 \\
\hline Chloroethane & 100 & 100 & 100 & 100 & 100 & 100 & 100 & 100 \\
\hline Chloroform & 5u & 5U & 50 & 50 & su & 50 & 5u & 2 \\
\hline Chloromethane & 100 & 100 & 100 & 100 & 100 & 100 & 100 & 100 \\
\hline 1,1-Dichloroethane & 5v & 50 & 50 & 5u & 50 & su & 50 & 5U \\
\hline 1,2-Dichloroethane & 50 & su & 50 & su & 5u & 5v & su & 50 \\
\hline 1,1-Dichloroethene & 50 & su & 50 & 50 & 50 & su & 5u & 5u \\
\hline 1,2-Dichloroethene & 50 & 50 & 50 & su & su & su & 50 & $5 U$ \\
\hline 1,2-Dichloropropane & su & su & 5u & su & 5u & su & su & 50 \\
\hline cis-1,3-Dichloropropene & 50 & 50 & 5u & 5u & su & 50 & 5u & 50 \\
\hline trans-1, 3-Dichloropropene & 50 & 5u & 5u & su & 5u & 50 & 5u & 50 \\
\hline Ethylbenzene & 50 & 50 & 50 & su & 5u & $5 \mathbf{u}$ & 5u & su \\
\hline 2-Hexanone & 100 & 100 & 100 & 100 & 100 & 100 & 100 & 100 \\
\hline 4-Methy 1-2-pentanone & 1 & 2 & 100 & 100 & 2 & 100 & 100 & 100 \\
\hline Methylene chloride & 2 & 2 & su & 3 & 2 & su & 5u & 5 \\
\hline Styrene & 5u & 5u & 5u & $\because 50$ & 5u & 50 & 5u & $5 u$ \\
\hline $1,1,2,2$-Tetrachloroethane & 5u & 50 & su & $5 \mathbf{v}$ & 5u & 50 & 5u & 50 \\
\hline Tetrachloroethene & 50 & $5 u$ & 50 & 50 & 5u & su & 50 & 50 \\
\hline Toluene & 5U & 50 & 5u & 50 & $5 u$ & su & su & $5 u$ \\
\hline 1,1,1-Trichloroethane & 50 & 5U & 50 & su & $5 v$ & 50 & 5u & 5u \\
\hline 1,1,2-Trichloroethane & 50 & 50 & 50 & 50 & $5 u$ & 50 & $5 U$ & 50 \\
\hline Trichloroethene & $5 \mathrm{u}$ & 5u & 50 & 5u & 50 & 50 & 50 & 5u \\
\hline vinyl acetate & 100 & 100 & 100 & 100 & 100 & 100 & 100 & 100 \\
\hline Vinyl chloride & 100 & 100 & 100 & 100 & 100 & 100 & 100 & 100 \\
\hline Xylenes & 5u & 50 & $5 U$ & 50 & 50 & 50 & 50 & 50 \\
\hline
\end{tabular}

(CONTINUED) 
APPENDIX 8.2

Groundwater and Surface-water Quality Data, 1993

\begin{tabular}{|c|c|c|c|c|c|c|c|c|}
\hline \multirow{3}{*}{$\begin{array}{l}\text { Sampling Point } \\
\text { Location } \\
\text { Date Sampled }\end{array}$} & \multicolumn{4}{|c|}{ GW-537 } & \multicolumn{4}{|c|}{ GW-601 } \\
\hline & \multicolumn{4}{|c|}{ orf } & \multicolumn{4}{|c|}{ orf } \\
\hline & $02 / 11 / 93$ & $05 / 12 / 93$ & $09 / 14 / 93$ & $10 / 22 / 93$ & $03 / 28 / 93$ & $06 / 27 / 93$ & $09 / 19 / 93$ & $12 / 21 / 94$ \\
\hline VOLATILE ORGANICS (ug/L) & $\cdot$ & $\cdot$ & $\cdot$ & $\cdot$ & - & $\cdot$ & $\cdot$ & - \\
\hline Acetone & 100 & 100 & 100 & 100 & 100 & 100 & 100 & 100 \\
\hline Benzene & 50 & 5u & su & 50 & $5 v$ & 5u & su & su \\
\hline Bromodichloromethane & 50 & 5u & 50 & $5 \mathbf{u}$ & 5v & 5u & 5u & su \\
\hline Bromoform & so & su & su & su & $5 u$ & su & 5u & su \\
\hline Bromomethane & 100 & 100 & 100 & 100 & 100 & 100 & 100 & 100 \\
\hline 2-Butanone & 100 & 100 & 100 & 100 & 100 & 100 & 100 & 5 \\
\hline Carbon disulfide & su & su & 50 & 5u & 50 & $5 v$ & su & $5 U$ \\
\hline Carbon tetrachloride & su & $5 u$ & 5u & su & 4 & $5 \mathbf{u}^{\prime}$ & 2 & 3 \\
\hline Chlorobenzene & 50 & 5u & 5u & 50 & 50 & 50 & su & 5u \\
\hline Chlorodibromomethane & su & 50 & 5v & su & 5u & 50 & 5u & 5u \\
\hline Chloroethane & 100 & 100 & 100 & 100 & 200 & 100 & 100 & 100 \\
\hline Chloroform & 0.8 & 0.8 & 0.9 & 50 & 1 & 50 & 0.6 & 0.7 \\
\hline Chloromethane & 100 & 100 & 100 & 100 & 100 & 100 & 100 & 100 \\
\hline 1,1-Dichloroethane & $5 \mathbf{v}$ & su & 50 & 50 & su & $5 \mathbf{v}$ & 5u & 50 \\
\hline 1,2-Dichloroethane & 50 & su & su & 50 & 5u & 5u & $5 \mathbf{v}$ & su \\
\hline 1,1-Dichloroethene & 50 & 5u & 50 & 5u & 5u & 5u & 0.7 & 0.8 \\
\hline 1,2-Dichloroethene & $5 v$ & 50 & 5u & 50 & 2 & su & 5u & 1 \\
\hline 1,2-Dichloropropane & 50 & 5u & 5u & 50 & 50 & 5u & 50 & 50 \\
\hline cis-1,3-Dichloropropene & 5v & 50 & 5u & 5u & su & $5 v$ & 50 & su \\
\hline trans-1, 3-Dichloropropene & su & 50 & 5u & 5u & 50 & su & $5 \mathrm{U}$ & 50 \\
\hline Ethylbenzene & 50 & 50 & 5u & 5u & $5 u$ & 5u & 5u & $5 u$ \\
\hline 2-Hexanone & 100 & 100 & 100 & 100 & 100 & 100 & 100 & 100 \\
\hline 4-Methy1-2-pentanone & 100 & 100 & 100 & 100 & 2 & 3 & 100 & 100 \\
\hline Methylene chloride & $5 \mathbf{v}$ & 50 & 1 & 50 & 3 & 5u & su & 0.6 \\
\hline Styrene & 50 & 50 & 5u & 50 & 50 & $5 \mathbf{u}$ & $5 \mathbf{v}$ & 50 \\
\hline $1,1,2,2$-Tetrachloroethane & su & su & $5 v$ & 5u & $5 u$ & 5u & su & $5 \mathbf{u}$ \\
\hline Tetrachloroethene & 50 & 5u & 50 & 5u & 1 & $5 \mathbf{u}$ & 1 & 1 \\
\hline Toluene & $5 v$ & $5 v$ & 50 & 50 & 50 & 5u & $5 \mathbf{v}$ & $5 U$ \\
\hline $1,1,1$-Trichloroethane & 5u & 50 & 50 & 5u & 50 & su & 0.5 & 0.5 \\
\hline 1,1,2-Trichloroethane & $5 \mathbf{u}$ & 5u & $5 v$ & 50 & 50 & 50 & $5 \mathbf{5 u}$ & su \\
\hline Trichloroethene & su & $5 u$ & 50 & 50 & 140 & 140 & 120 & 120 \\
\hline Vinyl acetate & 100 & 100 & 100 & 100 & 100 & 100 & 100 & 100 \\
\hline Vinyl chloride & 100 & 100 & 100 & 100 & 100 & 100 & 100 & 100 \\
\hline xylenes & 50 & su & 50 & $5 \mathrm{U}$ & su & 5u & 5u & su \\
\hline
\end{tabular}

(CONTINUED) 
APPENDIX $\mathrm{E} .2$

Groundwater and surface-water Quality Data, 1993

\begin{tabular}{|c|c|c|c|c|c|c|c|c|}
\hline \multirow{3}{*}{$\begin{array}{l}\text { Sampling Point } \\
\text { Location } \\
\text { Date Sampled }\end{array}$} & \multirow{3}{*}{\begin{tabular}{|c|}
$6 N-613$ \\
83 \\
$10 / 20 / 93$ \\
\end{tabular}} & \multirow{3}{*}{\begin{tabular}{|c|} 
OW-614 \\
83 \\
$10 / 20 / 93$ \\
\end{tabular}} & \multicolumn{4}{|c|}{ aw-621 } & \multirow{2}{*}{\multicolumn{2}{|c|}{$\frac{G W-626}{B Q}$}} \\
\hline & & & \multicolumn{4}{|c|}{$\mathbf{B Q}$} & & \\
\hline & & & $03 / 20 / 93$ & $06 / 02 / 93$ & $09 / 20 / 93$ & $12 / 20 / 93$ & $03 / 10 / 93$ & $04 / 26 / 93$ \\
\hline VOLATILS ORGANICS $\left(\mathrm{ug} / \mathrm{L}_{1}\right)$ & $\cdot 1$ & $\cdot 1$ & $\cdot 1$ & $\cdot 1$ & $\cdot$ & $\cdot$ & $\cdot$ & • \\
\hline Acetone & 2 & 1 & 100 & 100 & 100 & 100 & 1000 & 200 \\
\hline Benzene & 50 & 5u & su & so & so & 0.3 & 500 & 100 \\
\hline Bromodichloromethane & 5u & su & su & 50 & su & so & 500 & 100 \\
\hline Bromoform & su & 5u & $\mathbf{s u}$ & su & su & su & 500 & 100 \\
\hline Bromomethane & 100 & 100 & 100 & 100 & 100 & 100 & 1000 & 200 \\
\hline 2-Butanone & 7 & 9 & 100 & 100 & 100 & 10 & 1000 & 200 \\
\hline Carbon dieulfida & su & 50 & su & su & su & su & 500 & 100 \\
\hline Carbon tetrachloride & 5u & su & so & su & su & 2 & 500 & 100 \\
\hline Chlorobenzene & 50 & su & 5u & 5u & so & 5u & 500 & 100 \\
\hline Chlorodibromomethane & su & su & su & su & su & 50 & 500 & 100 \\
\hline Chloroethane & 100 & 100 & 100 & 100 & 100 & 100 & 1000 & 200 \\
\hline Chlorotorm & 5u & sv & 5u & su & su & 50 & 500 & 100 \\
\hline Chloromethane & 100 & 100 & 100 & 100 & 100 & 100 & 1000 & 200 \\
\hline 1,1-Dichloroethane & su & su & 5u & su & su & 5u & 51 & 18 \\
\hline 1,2-Dichloroethane & 5u & 5u & so & su & su & 5u & 500 & 4 \\
\hline 1,1-Dichloroethene & 5u & 5u & su & 50 & su & 5u & 28 & 6 \\
\hline 1,2-Dichloroethene & su & 5u & 5u & su & su & 5u & 1200 & 400 \\
\hline 1,2-Dichloropropane & 5u & su & su & su & su & 50 & 500 & 100 \\
\hline cis-1,3-Dichloropropene & 5u & $5 \mathbf{u}$ & 5u & su & su & 50 & 500 & 100 \\
\hline trans-1,3-Dichloropropene & 5u & su & so & su & su & 50 & 500 & 100 \\
\hline Rthylbenzune & su & 5u & 5u & 50 & su & su & 500 & 100 \\
\hline 2-Hexanone & 100 & 100 & 100 & 100 & 100 & 100 & 1000 & 200 \\
\hline 4-Methy1-2-pentanone & 100 & 100 & 3 & 100 & 100 & 100 & 1000 & 200 \\
\hline Methylene chloride & 3 & 2 & 2 & 0.9 & 5u & 5u & $50 u$ & 3 \\
\hline Styrene & 5u & 5u & 5u & 5u & su & 50 & 500 & 100 \\
\hline $1,1,2,2$-Tetrachloroethane & 5u & $3 v$ & so & 50 & su & 50 & 500 & 100 \\
\hline Tetrachloroethene & 5u & 5u & 5u & 5u & su & 50 & 140 & 29 \\
\hline Toluene & 50 & su & 50 & su & 5u & 5u & 500 & 100 \\
\hline $1,1,1$-Trichloroethane & 5u & 50 & 5u & su & su & 5u & 17 & 4 \\
\hline $1,1,2$-Trichloroethane & 5u & 50 & 50 & 50 & su & 50 & 500 & 100 \\
\hline Trichloroethene & su & su & sv & 50 & 2 & su & 110 & 34 \\
\hline Vinyl acetate & 100 & 100 & 100 & 100 & 100 & 100 & $100 u$ & 200 \\
\hline Vinyl chloride & 100 & 100 & 100 & 100 & 100 & 100 & 72 & 200 \\
\hline Xylenes & 50 & $5 u$ & 5u & su & 50 & su & 500 & 100 \\
\hline
\end{tabular}

(CONTINURD) 
APPENDIX $\mathbf{2 . 2}$

Groundwater and surtace-Water Quality Data, 1993

\begin{tabular}{|c|c|c|c|c|c|c|c|c|}
\hline \multirow{3}{*}{$\begin{array}{l}\text { 8ampling Point } \\
\text { Location } \\
\text { Date sampled }\end{array}$} & \multicolumn{2}{|c|}{ OW- 626} & \multicolumn{4}{|c|}{ ew-627 } & \multicolumn{2}{|c|}{ OW-636 } \\
\hline & \multicolumn{2}{|l|}{$\mathbf{B O}$} & \multicolumn{4}{|c|}{$B O$} & \multicolumn{2}{|c|}{ orf } \\
\hline & $08 / 02 / 93$ & $10 / 20 / 93$ & $03 / 09 / 93$ & $04 / 23 / 93 \mid$ & $07 / 28 / 93$ & $10 / 20 / 93$ & $03 / 26 / 93$ & $06 / 27 / 93$ \\
\hline VOLATILE ORGANICS (ug/L) & - & $\cdot$ & - & $\cdot$ & $\cdot$ & $\cdot$ & - & $\cdot$ \\
\hline Acetone & 200 & 3 & 100 & 100 & 100 & 3 & 100 & 100 \\
\hline Banzene & 100 & 100 & su & su & $\mathbf{5 u}$ & su & su & su \\
\hline Bromodichloromathane & 100 & 100 & su & su & su & su & su & su \\
\hline Bromotorm & 100 & 100 & su & su & su & su & su & so \\
\hline Bromomethane & 200 & 200 & 100 & 100 & 100 & 100 & 100 & 200 \\
\hline 2-Butanona & 200 & 23 & 100 & 100 & 100 & 9 & 100 & 100 \\
\hline Carbon disulfide & 100 & 100 & su & su & su & su & su & $\mathbf{s u}$ \\
\hline Carbon tetrachloride & 100 & 100 & so & su & su & su & su & 50 \\
\hline Chlorobenzene & 100 & 100 & so & 5u & su & su & su & su \\
\hline Chlorodibromomathane & 100 & 100 & su & su & su & su & su & 5u \\
\hline Chloroethane & 200 & 200 & 100 & 100 & 100 & 100 & 100 & 100 \\
\hline Chloroform & 100 & 100 & su & 5u & su & su & su & su \\
\hline Chloromethane & 200 & 200 & 100 & 100 & 100 & 100 & 100 & 100 \\
\hline 1,1-Dichloroethane & 19 & 19 & 3 & 5 & 6 & 3 & su & su \\
\hline 1,2-Dichloroethane & 100 & 3 & su & su & su & su & su & 5v \\
\hline 1,1-Dichloroethene & 9 & 12 & su & su & 1 & 5u & su & 5u \\
\hline 1,2-Dichloroethene & 250 & 330 & su & su & 5u & 5u & su & 5u \\
\hline 1,2-Dichloropropane & 100 & 100 & so & so & su & 5u & 5u & so \\
\hline cis-1,3-Dichloropropen & 100 & 100 & su & su & su & 5u & 5u & su \\
\hline trans-1,3-Diohloropropene & 100 & 100 & su & su & su & su & su & 5u \\
\hline zthylbenzene & 100 & 100 & 5u & su & su & so & su & su \\
\hline 2-Hexanone & 200 & 200 & 100 & 100 & 100 & 100 & 100 & 100 \\
\hline 4-Methy1-2-pentanone & 200 & 200 & 2 & 100 & 100 & 100 & 100 & 100 \\
\hline Methylene chloride & 100 & 7 & 5u & su & 1 & 3 & su & su \\
\hline styrene & 100 & 100 & su & $\therefore 50$ & su & su & su & su \\
\hline $1,1,2,2$-Tetrachloroethane & 100 & 100 & su & su & 5u & 5u & su & 5u \\
\hline Tetrachloroethene & 29 & 32 & 39 & 51 & 74 & 37 & 5u & su \\
\hline Toluene & 100 & 100 & su & su & 5u & 5u & su & su \\
\hline 1,1,1-Trichloroethane & 6 & 6 & su & 5u & 50 & 5u & so & 5u \\
\hline $1,1,2-\operatorname{Tr}$ lchloroethane & 100 & 100 & 50 & so & 5u & su & 50 & so \\
\hline Trichloroethene & 35 & 36 & 6 & 7 & 10 & 5 & so & su \\
\hline Vinyl acetate & 200 & 200 & 100 & 100 & 100 & 100 & 200 & 100 \\
\hline vinyl chloride & 200 & 200 & 100 & 100 & 0.8 & 100 & 100 & 100 \\
\hline xylenes & 100 & 100 & 50 & 50 & su & 5u & su & su \\
\hline
\end{tabular}

(CONTINUED) 
APPINDIX 2.2

Oroundwater and surface-Water Quallty Data, 1993

\begin{tabular}{|c|c|c|c|c|c|c|c|c|}
\hline \multirow{3}{*}{$\begin{array}{l}\text { Sampling polnt } \\
\text { Location } \\
\text { Date sampled }\end{array}$} & \multicolumn{2}{|c|}{ GW-636 } & \multicolumn{4}{|c|}{ ON-637 } & \multicolumn{2}{|c|}{$a w-638$} \\
\hline & \multicolumn{2}{|c|}{ OLF } & \multicolumn{4}{|c|}{ ow } & \multicolumn{2}{|c|}{ or? } \\
\hline & $09 / 18 / 93 \mid$ & $12 / 18 / 93$ & $03 / 25 / 93$ & $06 / 26 / 93$ & $09 / 17 / 93$ & $12 / 18 / 93$ & $03 / 25 / 93$ & $0126 / 93$ \\
\hline VOLATIL ORCARICs (ug/L) & $\cdot$ & - & $\cdot 1$ & $\cdot$ & $\cdot$ & • & $\cdot 1$ & - \\
\hline Acetone & 100 & 3 & 100 & 100 & 100 & 100 & 100 & 100 \\
\hline Benzene & su & su & so & su & so & su & su & so \\
\hline Bromodlehloromethane & su & su & su & su & su & su & su & su \\
\hline Bronotorm & su & su & 50 & su) & su & su & su & so \\
\hline Bromomethane & 100 & 100 & 100 & 100 & 100 & 100 & 100 & 100 \\
\hline 2-Butanone & 100 & 9) & 100 & 100 & 100 & 11 & 100 & 100 \\
\hline Carbon dieullida & su & su & so & su & su & su & su & su \\
\hline Carbon tetrachloride & su & su & su & su & su & su & so & su \\
\hline Chlorobenzene & su & su & su & su & so & su & so & so \\
\hline Chlorodlbromomethane & su & so & su & su & su & su & su & so \\
\hline Chloroethane & 100 & 100 & 100 & 100 & 100 & 100 & 100 & 100 \\
\hline Chlorotorm & su & so & so & su & su & so & su & su \\
\hline Chloromethane & 100 & 100 & 100 & 100 & 100 & 100 & 100 & 100 \\
\hline 1,1-Dichloroethane & su & su & su & su & su & so & su) & so \\
\hline 1,2-Dichloroethane & su & su & su & su & su & su & su) & so \\
\hline 1,1-Dichloroethene & su| & so & su & su & su & su & su & su \\
\hline 1,2-Dichloroethene & su & 50 & su & su & su & su & su & su \\
\hline 1,2-Dichloropropane & su & so & su & su & so & $\mathbf{s u}$ & su & so \\
\hline C1e-1,3-Dichloropropen & su & su & su & su & su & su & su & so \\
\hline trann-1, 3-Dich loropropane & su & su & su & su & su & su & su & su \\
\hline zthylbenzene & su & su & su & su & su & so & su & 50 \\
\hline 2-Hexanone & 100 & 100 & 100 & 100 & 100 & 100 & 100 & 100 \\
\hline 1-Mathyl-2-pentanone & 100 & 100 & 200 & 100 & 200 & 100 & 100 & 100 \\
\hline Mathylene ohloride & 0.9 & 0.6 & su & su & sv & 0.7 & su & su \\
\hline styrene & su & su & su & su & su & su & su & su \\
\hline $1,1,2,2$-Tetrachloroethane & su & su & su & su & su & su & so & su \\
\hline Tetrachloroethene & su & 5u & su & su & su & su & su & su \\
\hline Toluene & su & su & su & su & su & su & su & su \\
\hline 1,1,1-Triohloroathane & su & su & su & su & su & su & su & su \\
\hline 1,2,2-Trichloroethane & 5u & su & so & su & so & su & su & su \\
\hline Trichlorouthene & su & su & su & su & su & su & su & su \\
\hline VInyl acetate & 100 & 200 & 100 & 100 & 100 & 200 & 200 & 100 \\
\hline vinyl chloride & 100 & 100 & 100 & 100 & 100 & 100 & 100 & 100 \\
\hline xylenes & su & su & su & su & su & su & su & 5u \\
\hline
\end{tabular}

(CONTINUED) 
APPLHDIX $\mathbf{2 . 2}$

Oroundwater and surface-water Quality Data, 1993

\begin{tabular}{|c|c|c|c|c|c|c|c|c|}
\hline \multirow{3}{*}{$\begin{array}{l}\text { Sampling polnt } \\
\text { Location } \\
\text { Date sampled }\end{array}$} & \multicolumn{2}{|c|}{$0 w-638$} & \multicolumn{4}{|c|}{ an-639 } & \multicolumn{2}{|c|}{ OW-640 } \\
\hline & \multicolumn{2}{|c|}{ ory } & \multicolumn{4}{|c|}{80} & \multicolumn{2}{|c|}{20} \\
\hline & $09 / 17 / 93$ & $12 / 18 / 930$ & $02 / 24 / 93$ & $05 / 24 / 93$ & $07 / 12 / 93$ & $10 / 23 / 93$ & $02 / 22 / 93$ & $05 / 24 / 93$ \\
\hline Vothats onenurcs (ug/L) & $\cdot$ & . & $\cdot$ & - & $\cdot$ & $\cdot$ & $\cdot 1$ & - \\
\hline nootone & 100 & 100 & 100 & 200 & 100 & 100 & 100 & $10 \dot{0}$ \\
\hline Bensene & su & su & su & su & su & su & su & so \\
\hline Dromodiohloromethane & su & su & su & so & su & su & su & su \\
\hline Eromosorm & su & su & so & su & su & su & so & su \\
\hline Bromomethane & 100 & 100 & 100 & 100 & 100 & 100 & 100 & 100 \\
\hline 2-Butanone & 100 & 11 & 100 & 4 & 100 & 100 & 100 & 4 \\
\hline Carbon diaulelde & su & su & so & su & su & so & su & su \\
\hline Carbon tetrachloride & su & so & su & su & su & so & su & so \\
\hline Chlorobanaene & su & su & su & su & su & su & so & 50 \\
\hline Chlorodlbromonethane & su & so & so & su & su & sv & su & so \\
\hline Chloroethane & 100 & 100 & 100 & 100 & 100 & 100 & 100 & 100 \\
\hline Chlorotorm & su & sv & so & su & su & so & su & so \\
\hline Chloromathane & 100 & 100 & 100 & 100 & 100 & 100 & 200 & 100 \\
\hline 1,1-DLchloroethane & so & su & so & su & su & so & su & so \\
\hline 1,2-D1chloroethane & su & so & su & su & su & su & su & so \\
\hline 1,1-Dichloroethene & so & su & su & su & su & 30 & su & so \\
\hline 1,2-Dichloroethene & so & su & su & su & su & so & su & so \\
\hline 1,2-Dlehioropropane & su & so & su & so & so & so & su & su \\
\hline ole-1,3-DLChLloropropene & su & so & su & so & so & so & su & su \\
\hline trana-1, 3-Dlehloropropane & so & su & so & su & su & so & so & so \\
\hline Ithylbenzene & so & so & so & su & su & su & so & so \\
\hline 2-Hexanone & 100 & 100 & 100 & 100 & 100 & 100 & 100 & 100 \\
\hline 4-Methy1-2-pentanone & 100 & 100 & 100 & 1 & 100 & 100 & 100 & 100 \\
\hline Methylene ohloride & su & su & 1 & su & su & su & 1 & so \\
\hline styrene & so & su & 5u & su & su & so & su & su \\
\hline $1,1,2,2$-Tetrachloroethane & su & su & su & su & so & su & su & su \\
\hline Tetrachloroethene & su & su & su & su & su & su & su & su \\
\hline Toluene & su & su & 5u & su & su & su & su & su \\
\hline 1,1,1-Trichloroethane & su & su & su & su & su & so & su & su \\
\hline 1,1,2-Trichloroethane & su & 5u & su & su & su & su & su & so \\
\hline Trichloroethene & su & su & su & su & 5u & su & su & so \\
\hline Vinyl acetate & 100 & 100 & 100 & 100 & 100 & 100 & 100 & 100 \\
\hline Vinyl chloride & 100 & 100 & 100 & 100 & 100 & 100 & 100 & 100 \\
\hline xylenes & $5 \mathbf{v}$ & su & su & su & su & 50 & su & so \\
\hline
\end{tabular}

(CONTrkU:D) 
Appand IX 1.2

Groundwater and surfaee-water Quallty Data, 1993

\begin{tabular}{|c|c|c|c|c|c|c|c|c|}
\hline \multirow{3}{*}{$\begin{array}{l}\text { Bempling rolat } \\
\text { Location } \\
\text { Date sumpled }\end{array}$} & \multicolumn{2}{|c|}{$0 n-640$} & \multicolumn{4}{|c|}{$0 w-641$} & \multicolumn{2}{|c|}{$m-642$} \\
\hline & \multicolumn{2}{|c|}{$\mathbf{8 0}$} & \multicolumn{4}{|c|}{$\mathbf{s 0}$} & \multicolumn{2}{|c|}{80} \\
\hline & $|07 / 10 / 93|$ & $10 / 14 / 93$ & $02 / 22 / 93$ & $\mid 05 / 24 / 93$ & $07 / 10 / 93$ & $10 / 14 / 93$ & $02 / 08 / 93 \mid$ & $\mid 05 / 11 / 93$ \\
\hline vouarres onenurcs (ug/L) & $\cdot$ & $\cdot$ & $\cdot$ & $\cdot$ & $\cdot$ & $\cdot$ & - & $\cdot$ \\
\hline ncotone & 100 & 3 & 100 & 100 & 100 & $\dot{2}$ & $10 \dot{0}$ & $10 \dot{0}$ \\
\hline Bensene & su & so & su & su & 30 & su & so & su \\
\hline Bromodlehloromathane & su & so & su & su & so & so & so & so \\
\hline Dromotorm & so & so & so & su & so & so & so & so \\
\hline Eromomethane & 100 & 100 & 100 & 100 & 200 & 200 & 100 & 100 \\
\hline 2-Butanone & 100 & 6 & 100 & 3 & 200 & e) & 200 & 100 \\
\hline Carbon dleulfide & so & so & su & su & so & so & so & su \\
\hline carbon tetrachloride & so & so & su & so & so & so & so & so \\
\hline Ch1orobensene & so & so & so & sv & su & su & so & so \\
\hline Chlorodibronomathane & su & so & su & sv & so & su & so & su \\
\hline Chloroethane & 100 & 100 & 100 & 100 & 100 & 100 & 200 & 100 \\
\hline Chlorolorm & su & so & su & so & su & so & so & su \\
\hline Chloromethane & 100 & 100 & 100 & 100 & 100 & 100 & 100 & 100 \\
\hline 1,1-Diohloroethane & so & so & so & so & so & su & so & su \\
\hline 1,2-Diohloroethune & so & so & su & su & so & su & so & su \\
\hline 1,1-Diohlorouthene & su & so & so & su & so & so & sv & su \\
\hline 1,2-D1chloroothene & so & so & so & so & su & su & so & su \\
\hline 1,2-Diohloropropane & su & so & su & su & su & so & 50 & su \\
\hline ols-1,3-Diohloropropene & so & so & so & so & so & su & so & su \\
\hline trane-1,3-Diehloropropene & so & so & so & su & so & su & so & su \\
\hline Ethylbenzene & su & so & su & su & su & so & so & su \\
\hline 2-Hexanone & 100 & 100 & 100 & 100 & 100 & 100 & 100 & 100 \\
\hline 4-kethy1-2-pentunone & 100 & 100 & 100 & 100 & 100 & 100 & 100 & 100 \\
\hline Mathylene chlorida & su & 2 & 1 & su & su & 2 & so & 1 \\
\hline styrone & su & so & su & so & su & su & 50 & su \\
\hline $1,1,2,2$-Tetrachloroethane & so & so & so & so & so & su & so & su \\
\hline Tetruchloroethene & su & so & su & so & su & su & so & su \\
\hline 2oluene & su & su & su & su & so & su & so & su \\
\hline 1,1,1-Triohloroethane & so & so & so & so & su & su & 50 & su \\
\hline 1,1,2-Trichloroethane & so & su & so & su & su & su & su & su \\
\hline Triehloroethene & so & so & su & su & su & su & so & su \\
\hline Vinyl acotate & 100 & 100 & 100 & 100 & 100 & 100 & 100 & 200 \\
\hline vinyl ohloride & 100 & 100 & 200 & 100 & 100 & 100 & 100 & 100 \\
\hline xylenes: & su & su & su & so & so & su & so & su \\
\hline
\end{tabular}

(CONTrmusd) 
APPEHDIX 1.2

Oroundwater and surface-Water Quallty Data, 1993

\begin{tabular}{|c|c|c|c|c|c|c|c|c|}
\hline \multirow{3}{*}{$\begin{array}{l}\text { sanpling polnt } \\
\text { Location } \\
\text { Date sampled }\end{array}$} & \multirow{2}{*}{\multicolumn{2}{|c|}{$\frac{0 N-642}{80}$}} & \multicolumn{4}{|c|}{$a N-643$} & \multicolumn{2}{|c|}{ ON-644 } \\
\hline & & & \multicolumn{4}{|c|}{$\mathbf{B O}$} & \multicolumn{2}{|c|}{ ozr } \\
\hline & $07 / 01 / 93$ & $10 / 10 / 93$ & $02 / 26 / 93$ & $05 / 29 / 93$ & $07 / 08 / 93$ & $10 / 12 / 93$ & $03 / 25 / 93$ & $06 / 25 / 93$ \\
\hline vowarrus oneanics $(4 \mathrm{~g} / \mathrm{L})$ & $\cdot$ & $\cdot 1$ & $\cdot$ & $\cdot$ & . & $\cdot$ & $\cdot$ & . \\
\hline Noetone & 100 & 2 & 100 & $10 \dot{0}$ & 100 & 200 & 100 & 100 \\
\hline Benzene & su & su & so & su & su & su & sv & su \\
\hline Bromodichloromethane & so & so & so & su & su & su & su & su \\
\hline Bromotorm & su & so & $\mathbf{s u}$ & su & su & su & su & su \\
\hline Bromomathane & 200 & 100 & 100 & 100 & 100 & 100 & 100 & 100 \\
\hline 2-Butanone & 100 & 8 & 100 & 100 & 100 & 100 & 100 & 100 \\
\hline Carbon disulelde & su & so & $\mathbf{s u}$ & su & su & su & su & su \\
\hline Carbon tetraohloride & su & so & so & su & su & su & so & 50 \\
\hline Chlorobansene & so & su & so & su & su & su & su & su \\
\hline Chlorodlbromomothane & su & su & su & su & su & su & su & so \\
\hline Chloroothane & 100 & 100 & 100 & 100 & 100 & 100 & 100 & 100 \\
\hline Chloroform & su & su & su & so & su & su & $\mathbf{s u}$ & su \\
\hline Chloromothane & 100 & 100 & 100 & 100 & 100 & 100 & 100 & 100 \\
\hline 1,1-Dichloroethane & so & su & so & su & su & so & su & 50 \\
\hline 1,2-Dichloroethane & su & su & so & su & su & so & su & su \\
\hline 1,1-Diohloroethene & su & su & so & su & su & su & su & so \\
\hline 1,2-Dichloroethene & so & su & so & su & so & sv & su & su \\
\hline 1,2-DLehloropropane & su & su & 50 & 5v & su & 50 & su & so \\
\hline C1a-1,3-DLchloropropene & so & su & su & su & so & su & su & su \\
\hline trana-1, 3-DLohloropropene & su & su & so & su & su & su & 5u & su \\
\hline Ithylbenzene & su & su & su & su & su & su & su & su \\
\hline 2-Hexanone & 100 & 100 & 100 & 100 & 100 & 100 & 100 & 100 \\
\hline 4-Methy1-2-pentanone & 100 & 100 & 100 & 100 & 100 & 100 & 100 & 100 \\
\hline Mathylene chloride & so & 3 & 1 & su & su & su & su & 5u \\
\hline 8tyrane & su & su & su & su & su & su & su & 5u \\
\hline $1,1,2,2$-Tetrachloronthane & su & su & 50 & su & su & su & su & su \\
\hline Tetrachloroethene & so & su & su & su & 5u & su & su & 5u \\
\hline Toluene & su & so & sv & su & $5 v$ & 5u & su & su \\
\hline 1,1,1-Trichloroethane & su & su & 5u & su & su & 5v & su & su \\
\hline 1,1,2-Trichloroethane & su & su & su & su & so & su & su & su \\
\hline Trichloroethene & su & su & su & su & su & 5u & su & su \\
\hline VInyl acetate & 100 & 100 & 100 & 100 & 100 & 100 & 100 & 100 \\
\hline vinyl chlorlde & 100 & 100 & 100 & 100 & 100 & 100 & 100 & 100 \\
\hline xylenea & 5u & su & 50 & su & 5u & 2 & 5u & su \\
\hline
\end{tabular}

(CONTINURD) 
APPINDIX 2.2

Groundwater and surface-Water Quality Data, 1993

\begin{tabular}{|c|c|c|c|c|c|c|c|c|}
\hline \multirow{3}{*}{$\begin{array}{l}\text { 8ampling polnt } \\
\text { Location } \\
\text { Date sampled }\end{array}$} & \multicolumn{2}{|c|}{ OW-644 } & \multicolumn{4}{|c|}{ OW-647 } & \multicolumn{2}{|c|}{$6 w-651$} \\
\hline & \multicolumn{2}{|c|}{ ow } & \multicolumn{4}{|c|}{ orf } & \multicolumn{2}{|c|}{$B O$} \\
\hline & $09 / 17 / 93$ & $12 / 18 / 93$ & $02 / 08 / 93$ & $05 / 17 / 93$ & $09 / 25 / 93$ & $12 / 10 / 93$ & $03 / 01 / 93$ & $04 / 19 / 93$ \\
\hline VOLATIL ORCNHICs (ug/L) &. & $\cdot$ & . & $\cdot$ & $\cdot$ & $\cdot 1$ & $\cdot$ & • \\
\hline Acetone & 100 & 100 & 100 & 100 & 100 & 100 & 100 & 100 \\
\hline Benzene & 5v & su & 5u & so & su & su & so & su \\
\hline Bromodichloromethane & su & su & su & so & su & 50 & so & 5u \\
\hline Bromotorm & su & su & su & su & su & 50 & su & su \\
\hline Bromomethane & 100 & 100 & 100 & 100 & 100 & 100 & 100 & 100 \\
\hline 2-Butanone & 100 & 100 & 100 & 100 & 100 & 100 & 100 & 100 \\
\hline Carbon disulfide & 50 & su & su & so & su & so & $\mathbf{s u}$ & su \\
\hline Carbon tetrachloride & so & su & su & so & so & 50 & su & 50 \\
\hline Chlorobenzene & su & su & su & 5u & su & su & su & so \\
\hline Chlorodibromomethane & sv & su & su & su & su & su & su & 5u \\
\hline Chloroethane & 100 & 100 & 100 & 100 & 100 & 100 & 100 & 100 \\
\hline Chlorotorm & su & su & 50 & so & su & 50 & so & so \\
\hline Chloromethane & 100 & 100 & 100 & 100 & 100 & 100 & 100 & 100 \\
\hline 1,1-Dichloroethane & so & 5u & 5u & so & su & 5u & su & 50 \\
\hline 1,2-Dichloroethane & su & su & 5v & su & su & 50 & su & 50 \\
\hline 1,1-Dichloroethene & su & 5u & 50 & 5u & su & 5u & su & 50 \\
\hline 1,2-Dichloroethene & 50 & su & su & so & so & 5u & 50 & su \\
\hline 1,2-Dichloropropane & 50 & 5u & 5u & 50 & su & 5u & 5u & 50 \\
\hline Cis-1,3-Dichloropropene & 5u & su & 5u & 50 & su & 50 & su & 5u \\
\hline tranu-1,3-Dichloropropene & 5u & 5u & 5u & su & su & 5u & su & 5u \\
\hline Ethylbenzene & su & 5u & 50 & 5u & su & 50 & su & so \\
\hline 2-Hexanone & 100 & 100 & 100 & 100 & 100 & 100 & 100 & 100 \\
\hline 4-Methy 1-2-pentanone & 100 & 100 & 100 & 100 & 100 & 100 & 100 & 100 \\
\hline Mathylene ohloride & su & su & su & su & su & 1 & su & su \\
\hline styrene & 50 & 5u & 5u & su & 5u & 5u & su & 50 \\
\hline $1,1,2,2$-Tetrachloroethane & $5 u$ & su & 50 & 50 & 50 & su & 5u & 50 \\
\hline Tetrachloroethene & su & 5u & 5u & 5u & 5u & 5u & su & 5u \\
\hline Toluene & 50 & 5u & 5u & 5v & su & 5u & su & 5u \\
\hline $1,1,1-\operatorname{Tr}$ ichloroethane & 50 & su & 5u & 50 & su & 5u & su & su \\
\hline 1,1,2-Trichloroethane & 5u & 5u & 50 & 5u & su & su & su & 50 \\
\hline Trichloroethene & $5 v$ & sv & 5u & 50 & su & 5u & 5u & su \\
\hline Vinyl acetate & 100 & 100 & 100 & 100 & 100 & 100 & 100 & 100 \\
\hline Vinyl ohloride & 100 & 100 & 100 & 100 & 100 & 100 & 100 & 100 \\
\hline Xylenes & 50 & 50 & 50 & 50 & su & 50 & 5u & 50 \\
\hline
\end{tabular}

(CONTINUED) 
APPENDIX 8.2

Groundwater and Surface-Water Quality Data, 1993

\begin{tabular}{|c|c|c|c|c|c|c|c|c|}
\hline \multirow{3}{*}{$\begin{array}{l}\text { Sampling Point } \\
\text { Location } \\
\text { Date Sampled }\end{array}$} & \multicolumn{2}{|c|}{ Qw-651 } & \multicolumn{4}{|c|}{$0 w-652$} & \multicolumn{2}{|c|}{ aw-653 } \\
\hline & \multicolumn{2}{|c|}{ BG } & \multicolumn{4}{|c|}{ BO } & \multicolumn{2}{|c|}{$\mathbf{B O}$} \\
\hline & $07 / 10 / 93$ & $10 / 10 / 93$ & $03 / 01 / 93$ & $04 / 19 / 93$ & $07 / 10 / 93$ & $10 / 18 / 93$ & $03 / 01 / 93$ & $04 / 20 / 93$ \\
\hline VOLATIIS ORGaNICS (ug/L) & $\cdot$ & - & $\cdot$ & $\cdot$ & $\cdot$ & $\cdot$ & $\cdot$ & $\cdot$ \\
\hline Acetone & 100 & 100 & 100 & 100 & 100 & 100 & 100 & 100 \\
\hline Benzene & su & su & 5u & su & su & su & so & $\mathbf{s u}$ \\
\hline Bromodichloromethane & su & 5u & 5v & su & su & su & su & su \\
\hline Bromoform & su & 5u & su & su & su & su & su & so \\
\hline Bromomethane & 100 & 100 & 100 & 100 & 100 & 100 & 100 & 100 \\
\hline 2-Butanone & 100 & 100 & 100 & 100 & 100 & 100 & 100 & 100 \\
\hline Carbon disulfide & 50 & su & 50 & so & su & su & su & su \\
\hline Carbon tetrachloride & 50 & 50 & 5u & 50 & 50 & su & su & su \\
\hline Chlorobenzene & su & su & 50) & su & 5u & su & su & su \\
\hline Chlorodibromomethane & 5u & su & 5u & su & 50 & su & 5u & su \\
\hline Chloroethane & 100 & 100 & 100 & 100 & 100 & 100 & 100 & 100 \\
\hline Chlorotorm & 5u & 5u & su & 50 & $5 \mathbf{u}$ & 5u & su & su \\
\hline Chloromethane & 100 & 100 & 100 & 100 & 100 & 100 & 100 & 100 \\
\hline 1,1-Dichloroethane & su & su & 50 & 5u & su & su & $5 u$ & su \\
\hline 1,2-Dichloroethane & 5u & 5u & su & 5u & 5u & su & 5u & su \\
\hline 1,1-Dichloroethene & 5u & su & so & 5u & su & 5u & so & su \\
\hline 1,2-Dichloroethene & 5u & 5u & 5v & 50 & so & su & 7 & su \\
\hline 1,2-Dichloropropane & su & 5u & 50 & sv & $5 v$ & su & 50 & su \\
\hline cis-1,3-Dichloropropene & $5 u$ & su & 5u & 5u & su & su & 5u & su \\
\hline trans-1,3-Dichloropropene & 5u & 5u & 5u & 50 & 5u & su & 5u & 50 \\
\hline Ethylbenzene & 5u & 5u & 5u & 50 & 50 & su & 50 & su \\
\hline 2-Hexanone & 100 & 100 & 100 & 100 & 100 & 100 & 100 & 100 \\
\hline 4-Methy1-2-pentanone & 100 & 100 & 100 & 100 & 100 & 100 & 100 & 1 \\
\hline Methylene chloride & su & 50 & 5u & 50 & 0.8 & 5u & 50 & 5u \\
\hline styrene & 50 & su & 50 & 50 & su & su & 5u & 5u \\
\hline $1,1,2,2$-Tetrachloroethane & 50 & 5u & su & 50 & su & su & 5u & 50 \\
\hline Tetrachloroethene & $5 \mathbf{5 u}$ & 5u & 5u & su & su & su & 2 & 2 \\
\hline Toluene & 5u & so & 50 & 50 & su & 5u & su & 5u \\
\hline $1,1,1-\operatorname{Tr}$ ichloroethane & 5u & su & su & 50 & 5u & su & 50 & su \\
\hline 1.1,2-Trichloroothane & 5u & 5u & su & 5u & su & 5u & 50 & su \\
\hline ichloroethene & 50 & 50 & $5 U$ & 9 & su & 50 & su & 5u \\
\hline nyl acetate & 100 & 100 & 100 & $10 \mathrm{U}$ & 100 & 100 & 100 & 100 \\
\hline Vinyl chloride & 100 & 100 & 100 & 100 & 100 & 100 & 100 & 100 \\
\hline xylenes & 50 & su & 50 & 5u & 50 & 5u & 50 & 5u \\
\hline
\end{tabular}

(CONTINUED) 
APPENDIX B.2

Groundwater and Surface-water Quality Data, 1993

\begin{tabular}{|c|c|c|c|c|c|c|c|c|}
\hline \multirow{3}{*}{$\begin{array}{l}\text { Sampling Point } \\
\text { Location } \\
\text { Date Sampled }\end{array}$} & \multicolumn{2}{|c|}{$G W-653$} & \multicolumn{4}{|c|}{ GW-654 } & \multicolumn{2}{|c|}{ GW-655 } \\
\hline & \multicolumn{2}{|c|}{ BG } & \multicolumn{4}{|c|}{ BG } & \multicolumn{2}{|c|}{$\mathbf{B G}$} \\
\hline & $07 / 10 / 93$ & $10 / 10 / 93$ & $02 / 24 / 93$ & $04 / 16 / 93$ & $07 / 01 / 93$ & $10 / 07 / 93$ & $02 / 05 / 93$ & $05 / 10 / 93$ \\
\hline VOLATILE ORGANICS (ug/L) & $\cdot$ & - & $\cdot$ & $\cdot$ & $\cdot$ & $\cdot$ & $\cdot 1$ & - \\
\hline Acetone & 100 & 100 & 100 & 100 & 100 & 4 & 100 & 100 \\
\hline Benzene & 50 & 50 & $5 \mathbf{u}$ & 5u & 50 & 5u & 50 & 5u \\
\hline Bromodichloromethane & 50 & 50 & 50 & 50 & 50 & 50 & 5v & 5v \\
\hline Bromoform & 5u & 5v & $5 \mathbf{u}$ & $5 u$ & $5 \mathrm{v}$ & 5u & $5 \mathbf{v}$ & 5u \\
\hline Bromomethane & 100 & 100 & 200 & 100 & 100 & 100 & 200 & 100 \\
\hline 2-Butanone & 100 & 100 & 100 & 100 & 100 & 11 & 11 & 100 \\
\hline Carbon disulfide & $5 \mathbf{u}$ & 50 & 5u & $5 u$ & 50 & 50 & 5u & 5u \\
\hline Carbon tetrachloride & 50 & 50 & 50 & 50 & 50 & 5u & $5 v$ & 50 \\
\hline Chlorobenzene & 50 & $5 \mathbf{u}$ & 5u & 50 & 5u & 5u & $5 u$ & 50 \\
\hline Chlorodibromomethane & 50 & 50 & 50 & 5u & 50 & 5u & $5 v$ & 50 \\
\hline Chloroethane & $10 v$ & 100 & 100 & 100 & 100 & 100 & 100 & 100 \\
\hline Chloroform & 50 & 50 & 50 & 50 & 50 & 5U & 50 & 50 \\
\hline Chloromethane & 100 & 100 & 100 & $10 \mathrm{U}$ & 100 & 100 & 100 & 100 \\
\hline 1,1-Dichloroethane & 5u & $5 u$ & $5 v$ & 5u & $5 U$ & 50 & 50 & 50 \\
\hline 1,2-Dichloroethane & 50 & 50 & 50 & 50 & $5 \mathbf{u}$ & 50 & 5u & 50 \\
\hline 1,1-Dichloroethene & 50 & 50 & $5 v$ & $5 v$ & $5 v$ & 50 & $5 v$ & 5u \\
\hline 1,2-Dichloroethene & 3 & 7 & 5u & 50 & 5v & 50 & $5 v$ & 5u \\
\hline 1,2-Dichloropropane & 50 & 50 & $5 v$ & 50 & 50 & 50 & 50 & 50 \\
\hline cis-1,3-Dichloropropene & 50 & 5u & 50 & 50 & 50 & 50 & 50 & $5 \mathbf{v}$ \\
\hline trans-1,3-Dichloropropene & 50 & $5 u$ & $5 v$ & 50 & su & 50 & 50 & 50 \\
\hline Ethylbenzene & 50 & su & 5u & 50 & 5u & 5U & 5u & 5u \\
\hline 2-Hexanone & 100 & 100 & 100 & 100 & 100 & 100 & 100 & 100 \\
\hline 4-Methyl-2-pentanone & 100 & 100 & 100 & 100 & 100 & 100 & 100 & 100 \\
\hline Methylene chloride & $5 \mathbf{v}$ & 50 & 1 & 50 & 50 & 3 & 5u & 50 \\
\hline Styrene & 50 & 50 & 50 & 50 & 50 & 50 & 50 & 50 \\
\hline $1,1,2,2$-Tetrachloroethane & 50 & 50 & 50 & 5u & 5u & 5u & 50 & 50 \\
\hline Tetrachloroethene & 50 & 2 & $5 \mathrm{u}$ & 50 & 50 & 50 & $5 \mathbf{U}$ & $5 v$ \\
\hline Toluene & 5u & 5u & $5 \mathbf{u}$ & $5 \mathrm{v}$ & 5u & 50 & 50 & 50 \\
\hline $1,1,1$-Trichloroethane & $5 v$ & $5 u$ & 50 & 50 & 50 & 50 & $5 U$ & 50 \\
\hline 1,1,2-Trichloroethane & 50 & 50 & 50 & 50 & 50 & 50 & 50 & 50 \\
\hline Trichloroethene & su & 5u & $5 v$ & 50 & su & 50 & 50 & 50 \\
\hline Vinyl acetate & 100 & 100 & $10 \mathrm{U}$ & 100 & 100 & 100 & 100 & 100 \\
\hline Vinyl chloride & 100 & 100 & 100 & 100 & 100 & 100 & 100 & 100 \\
\hline Xylenes & 50 & 50 & 50 & 50 & 50 & 50 & 5U & 50 \\
\hline
\end{tabular}

(CONTINUED) 
APPENDIX E. 2

Groundwater and Surface-water Quality Data, 1993

\begin{tabular}{|c|c|c|c|c|c|c|c|c|}
\hline \multirow{3}{*}{$\begin{array}{l}\text { Sampling Point } \\
\text { Location } \\
\text { - } \\
\text { Date Sampled }\end{array}$} & \multicolumn{2}{|c|}{$G W-655$} & \multicolumn{4}{|c|}{$G W-683$} & \multicolumn{2}{|c|}{$G W-684$} \\
\hline & \multicolumn{2}{|c|}{ BG } & \multicolumn{4}{|c|}{$\mathbf{B X P}$} & \multicolumn{2}{|c|}{$\mathbf{E X P}$} \\
\hline & $07 / 01 / 93$ & $10 / 06 / 93$ & $03 / 04 / 93$ & $04 / 23 / 93$ & $07 / 12 / 93$ & $10 / 12 / 93$ & $03 / 03 / 93$ & $04 / 22 / 93$ \\
\hline VOLATILE ORGANICS (ug/L) & $\cdot$ & $\cdot$ & - & $\cdot$ & $\cdot$ & $\cdot$ & $\cdot$ & $\cdot$ \\
\hline Acetone & $10 \mathrm{v}$ & 100 & 100 & 100 & 100 & 2 & 100 & 100 \\
\hline Benzene & 50 & su & $5 \mathbf{u}$ & 5u & 50 & $5 \mathbf{u}$ & 50 & $5 \boldsymbol{v}$ \\
\hline Bromodichloromethane & $5 \mathbf{v}$ & 50 & su & 50 & 50 & $5 \mathbf{u}$ & $5 v$ & $5 \mathbf{v}$ \\
\hline Bromoform & 50 & 50 & 50 & 50 & $5 v$ & $5 u$ & 50 & $5 \mathbf{v}$ \\
\hline Bromomethane & 100 & 100 & $10 \mathrm{U}$ & 100 & 100 & $10 \mathrm{U}$ & $10 \mathrm{v}$ & 100 \\
\hline 2-Butanone & 100 & 100 & 100 & 100 & 100 & 10 & 100 & 100 \\
\hline Carbon disulfide & 50 & 50 & $5 \mathrm{v}$ & 5u & 50 & 50 & $5 \mathbf{u}$ & $5 \mathbf{v}$ \\
\hline Carbon tetrachloride & 50 & $5 u$ & 5u & 50 & $5 \mathbf{v}$ & $5 U$ & $5 \mathbf{u}$ & $5 \mathbf{v}$ \\
\hline Chlorobenzene & $5 \mathbf{v}$ & 50 & 50 & 5u & 5U & $5 \mathbf{u}$ & $5 v$ & $5 \mathbf{v}$ \\
\hline Chlorodibromomethane & $5 \mathbf{5 u}$ & 5u & 5U & 5u & 50 & $5 \mathbf{s u}$ & $5 u$ & $5 \mathbf{v}$ \\
\hline Chloroethane & $10 \mathrm{U}$ & 100 & 100 & 100 & 100 & 100 & 100 & 100 \\
\hline Chloroform & $5 \mathbf{v}$ & 50 & $5 \mathbf{v}$ & 5u & 50 & $5 U$ & $5 u$ & 50 \\
\hline Chloromethane & 100 & 100 & $10 \mathrm{u}$ & 100 & $10 u$ & 100 & 100 & 100 \\
\hline 1,1-Dichloroethane & $5 \mathbf{v}$ & 50 & 50 & $5 \mathbf{u}$ & 5U & 5u & 50 & $5 \mathbf{U}$ \\
\hline 1,2-Dichloroethane & 5u & $5 \mathrm{U}$ & $5 \mathbf{u}$ & $5 \mathbf{u}$ & 5u & $5 u$ & $5 v$ & $5 \boldsymbol{v}$ \\
\hline 1,1-Dichloroethene & 5u & 5u & 5u & su & 5u & su & 5u & $5 \mathbf{U}$ \\
\hline 1,2-Dichloroethene & 50 & $5 \mathbf{u}$ & $5 \mathbf{v}$ & 5u & 5u & 5v & 50 & $5 \mathbf{v}$ \\
\hline 1,2-Dichloropropane & 50 & 50 & 50 & 50 & $5 \mathbf{v}$ & 5u & 5v & $5 \mathbf{v}$ \\
\hline cis-1,3-Dichloropropene & 50 & 50 & 5u & 5u & 5u & 50 & su & $5 \mathbf{v}$ \\
\hline trans-1,3-Dichloropropene & 50 & 50 & $5 \mathrm{u}$ & 5u & 50 & 50 & su & 50 \\
\hline Ethylbenzene & 5v & 50 & 5u & 5u & 5u & 50 & su & $5 \mathbf{v}$ \\
\hline 2-Hexanone & 100 & 100 & $10 \mathrm{U}$ & 100 & 100 & $10 \mathrm{U}$ & 100 & 100 \\
\hline 4-Methy1-2-pentanone & 100 & 100 & $10 \mathrm{U}$ & 100 & 100 & $10 \mathrm{U}$ & 100 & 100 \\
\hline Methylene chloride & 50 & $5 u$ & $5 \mathbf{v}$ & 5u & 5u & 3 & $5 \mathrm{U}$ & 1 \\
\hline Styrene & 50 & 50 & $5 \mathbf{v}$ & 5u & 50 & 5v & su & $\mathbf{5 U}$ \\
\hline $1,1,2,2$-Tetrachloroethane & $5 \mathbf{u}$ & 50 & $5 \mathbf{v}$ & $5 U$ & 50 & 5u & 50 & 50 \\
\hline Tetrachloroethene & 50 & $5 \mathrm{U}$ & $5 \mathbf{v}$ & $5 v$ & $5 \mathbf{U}$ & 50 & 50 & 50 \\
\hline Toluene & $5 U$ & $5 \mathbf{U}$ & $5 u$ & 50 & 50 & 50 & 50 & $5 \mathbf{v}$ \\
\hline $1,1,1$-Trichloroethane & 5u & $5 U$ & 50 & 50 & 50 & 5u & 5u & $5 \mathbf{U}$ \\
\hline $1,1,2$-Trichloroethane & $5 \mathbf{U}$ & $5 v$ & $5 v$ & 50 & 5u & $5 \mathbf{v}$ & 5u & $\mathbf{5 u}$ \\
\hline Trichloroethene & 50 & 50 & $5 v$ & 50 & so & $5 \mathbf{v}$ & 5u & 50 \\
\hline Vinyl acetate & 100 & 100 & 100 & 100 & 100 & 100 & $10 \mathrm{U}$ & 100 \\
\hline vinyl chloride & 100 & 100 & 100 & 100 & 100 & 100 & 100 & 100 \\
\hline xylenes & 50 & $5 U$ & $5 U$ & 50 & $5 U$ & 50 & $5 U$ & 50 \\
\hline
\end{tabular}

(CONTINUED) 
APPENDIX E.2

Groundwater and Surface-Water Quality Data, 1993

\begin{tabular}{|c|c|c|c|c|c|c|c|c|}
\hline \multirow{3}{*}{$\begin{array}{l}\text { Sampling Point } \\
\text { Location } \\
\text { Date Sampled }\end{array}$} & \multicolumn{2}{|c|}{$G W-684$} & \multicolumn{4}{|c|}{ GW-685 } & \multicolumn{2}{|c|}{$G W-694$} \\
\hline & \multicolumn{2}{|c|}{$\mathbf{E x P}$} & \multicolumn{4}{|c|}{$\mathbf{E X P}$} & \multicolumn{2}{|c|}{$\mathbf{E X P}$} \\
\hline & $07 / 11 / 93$ & $10 / 12 / 93$ & $03 / 02 / 93$ & $04 / 21 / 93$ & $07 / 11 / 93$ & $10 / 11 / 93$ & $03 / 23 / 93$ & $06 / 06 / 93$ \\
\hline VOLATILE ORGANICS (ug/L) & $\cdot$ & $\cdot$ & $\cdot$ & $\cdot$ & $\cdot$ & $\cdot$ & $\cdot$ & - \\
\hline Acetone & 100 & 2 & 100 & 100 & 100 & 3 & $10 \mathrm{U}$ & 100 \\
\hline Benzene & 50 & 5v & 50 & 50 & 5u & 50 & 5u & 5u \\
\hline Bromodichloromethane & su & $5 v$ & 50 & 5u & $5 U$ & 5u & su & 50 \\
\hline Bromoform & 50 & su & 5u & 50 & 5u & 50 & 5u & su \\
\hline Bromomethane & 100 & 100 & 100 & 200 & 100 & 100 & 100 & 100 \\
\hline 2-Butanone & 100 & 9 & 100 & 100 & 100 & 11 & 100 & 100 \\
\hline Carbon disulfide & 5u & su & $5 v$ & 5u & 5u & 50 & 5u & 50 \\
\hline Carbon tetrachloride & 5u & 50 & 50 & 5u & 50 & 50 & 5u & 50 \\
\hline Chlorobenzene & 50 & su & 5u & $5 U$ & 50 & 5v & 5u & 50 \\
\hline Chlorodibromomethane & $5 \mathbf{u}$ & 50 & 5u & 50 & 50 & 50 & 5u & 5u \\
\hline Chloroethane & 100 & 100 & 100 & 100 & 100 & 100 & 100 & 100 \\
\hline Chloroform & 50 & 50 & $5 \mathrm{U}$ & su & 50 & 50 & 50 & 50 \\
\hline Chloromethane & 100 & 100 & 100 & 100 & 100 & 100 & 100 & 100 \\
\hline 1,1-Dichloroethane & 5u & 5u & 50 & 50 & $5 u$ & 50 & 5u & 50 \\
\hline 1,2-Dichloroethane & 5u & 5u & 50 & 50 & 5u & 50 & $5 \mathbf{u}$ & 50 \\
\hline 1,1-Dichloroethene & 50 & 50 & $5 v$ & 5u & 50 & 50 & 5u & 50 \\
\hline 1,2-Dichloroethene & su & $5 u$ & 2 & 2 & 2 & 50 & 9 & 11 \\
\hline 1,2-Dichloropropane & 5u & su & 50 & 50 & $5 u$ & 50 & 5u & 5u \\
\hline cis-1, 3-Dichloropropene & 50 & 50 & 50 & 50 & so & 50 & 5u & 5u \\
\hline trans-1,3-Dichloropropene & 50 & 50 & su & 5u & 5u & 50 & 5u & 5u \\
\hline Ethylbenzene & 50 & su & 50 & 5u. & su & 50 & $5 \mathbf{u}$ & 50 \\
\hline 2-Hexanone & 100 & 100 & 100 & 100 & $10 \mathrm{u}$ & 100 & 100 & 100 \\
\hline 4-Methy1-2-pentanone & 100 & 100 & 100 & 100 & 100 & 100 & 2 & 100 \\
\hline Methylene chloride & 0.8 & 3 & su & 5u & 50 & 3 & 2 & 1 \\
\hline styrene & su & 50 & 50 & $\therefore 50$ & 5u & 50 & $5 u$ & 5u \\
\hline $1,1,2,2$-Tetrachloroethane & 50 & su & 5u & 5u & 5u & su & 50 & 50 \\
\hline Tetrachloroethene & 50 & 50 & su & 50 & 50 & 50 & $5 u$ & 50 \\
\hline Toluene & 50 & 50 & 50 & 5u & $5 u$ & 50 & 5u & 5v \\
\hline $1,1,1$-Trich1oroethane & $5 u$ & 50 & 5v & 50 & 5u & 50 & 5v & 0.5 \\
\hline 1,1,2-Trichloroethane & 50 & 50 & 5u & 5u & 50 & 50 & 5u & 50 \\
\hline Trichloroethene & 50 & 50 & 50 & 5v & 50 & 50 & 14 & 14 \\
\hline vinyl acetate & 100 & 100 & 100 & 100 & 100 & 100 & 100 & 100 \\
\hline vinyl chloride & 100 & 100 & 100 & 100 & 100 & 100 & 100 & 100 \\
\hline xylenes & $5 u$ & 50 & 50 & 5u & 50 & 50 & 50 & 50 \\
\hline
\end{tabular}

(CONTINUED) 
APPENDIX $\mathbf{2 . 2}$

Groundwater and Surface-Water Quality Data, 1993

\begin{tabular}{|c|c|c|c|c|c|c|c|c|}
\hline \multirow{3}{*}{$\begin{array}{l}\text { Sampling Point } \\
\text { Location } \\
\text { Date Sampled }\end{array}$} & \multicolumn{2}{|c|}{$G w-694$} & \multicolumn{4}{|c|}{ GW-695 } & \multicolumn{2}{|c|}{ GW-703 } \\
\hline & \multicolumn{2}{|c|}{ EXP } & \multicolumn{4}{|c|}{ EXP } & \multicolumn{2}{|c|}{$\mathbf{E X P}$} \\
\hline & $09 / 22 / 93$ & $12 / 28 / 93$ & $03 / 24 / 93$ & $06 / 09 / 93$ & $09 / 22 / 93$ & $12 / 21 / 93$ & $03 / 20 / 93$ & $06 / 03 / 93$ \\
\hline VOLATILE ORGANICS (ug/L) & $\cdot$ &. & . & $\cdot$ & - & . & $\cdot$ & • \\
\hline Acetone & 100 & 4 & 100 & 100 & 100 & 100 & 2 & 100 \\
\hline Benzene & 50 & 5u & su & 50 & 5u & 50 & 5u & su \\
\hline Bromodichloromethane & 5u & 50 & 5u & 50 & 5u & 50 & 5u & 5u \\
\hline Bromoform & 5u & 50 & 50 & su & 50 & 5v & su & 5u \\
\hline Bromomethane & 100 & 100 & 100 & 100 & 100 & 100 & 100 & 100 \\
\hline 2-Butanone & 100 & 7 & 100 & 100 & 100 & 6 & 100 & 100 \\
\hline Carbon disulfide & su & 50 & 5v & 50 & 5u & 5v & 5u & 5u \\
\hline Carbon tetrachloride & 5u & 50 & 50 & 50 & $5 U$ & $5 v$ & $5 \mathrm{U}$ & su \\
\hline Chlorobenzene & su & 5u & 5u & 50 & 5u & 5u & su & 50 \\
\hline Chlorodibromomethane & 50 & 50 & 50 & 50 & 5u & 50 & 50 & $5 U$ \\
\hline Chloroethane & 100 & 100 & 100 & 100 & 100 & 100 & 100 & $10 U$ \\
\hline Chloroform & 5u & $5 \mathrm{U}$ & 5u & 50 & $5 U$ & su & 50 & 5u \\
\hline Chloromethane & 100 & 100 & 100 & 100 & 100 & 100 & 100 & 100 \\
\hline 1,1-Dichloroethane & 5u & 5u & 50 & 5u & $5 \mathbf{U}$ & $5 \mathbf{v}$ & 5u & 5u \\
\hline 1,2-Dichloroethane & su & $5 \mathrm{u}$ & 5u & 5u & 50 & 50 & su & 50 \\
\hline 1,1-Dichloroethene & $5 u$ & $5 v$ & 5u & so & 5u & 0.5 & 2 & 1 \\
\hline 1,2-Dichloroethene & 11 & 13 & 2 & $5 \mathbf{v}$ & 50 & 2 & 8 & 7 \\
\hline 1,2-Dichloropropane & su & $5 v$ & 50 & $5 v$ & su & 5u & su & 5u \\
\hline cis-1,3-Dichloropropene & 50 & $5 u$ & 50 & 50 & 5u & 5u & 5u & 50 \\
\hline trans-1,3-Dichloropropene & $5 \mathbf{u}$ & $5 v$ & 5u & $5 \mathrm{v}$ & su & 50 & su & 5u \\
\hline Ethylbenzene & 50 & 50 & 50 & $5 v$ & su & $5 v$ & 5u & su \\
\hline 2-Hexanone & 100 & 100 & 100 & 200 & 100 & 100 & 100 & 100 \\
\hline 4-Methy1-2-pentanone & 100 & 100 & 100 & 100 & 100 & 100 & 2 & 100 \\
\hline Methylene chloride & 50 & 1 & 5U & 50 & 50 & 0.8 & 2 & 50 \\
\hline Styrene & 5u & 5u & 5u & 50 & su & 50 & 5u & 50 \\
\hline $1,1,2,2$-Tetrachloroethane & 50 & 5u & $5 U$ & 50 & 5u & $5 u$ & 5u & 50 \\
\hline Tetrachloroethene & 50 & 50 & $5 U$ & 50 & $5 U$ & $5 \mathrm{u}$ & 50 & 50 \\
\hline Toluene & $5 v$ & 5v & $5 U$ & 50 & 50 & $5 \mathbf{v}$ & 5u & so \\
\hline $1,1,1$-Trichloroethane & $5 v$ & 50 & 50 & 50 & 50 & 50 & 5u & 50 \\
\hline 1,1,2-Trichloroethane & 50 & so & 50 & 5u & 5u & su & 50 & 50 \\
\hline Trichloroethene & 11 & 15 & 4 & 50 & 4 & 4 & 28 & 23 \\
\hline Vinyl acetate & 100 & 100 & 100 & 100 & 100 & 100 & $10 u$ & 100 \\
\hline Vinyl chloride & 100 & 100 & 100 & 100 & 100 & 100 & 100 & 100 \\
\hline xylenes & 50 & 50 & 50 & su & 50 & su & 50 & so \\
\hline
\end{tabular}

(CONTINUED) 
APPENDIX E.2

Groundwater and Surface-water Quality Data, 1993

\begin{tabular}{|c|c|c|c|c|c|c|c|c|}
\hline \multirow{3}{*}{$\begin{array}{l}\text { Sampling Point } \\
\text { Location } \\
\text { Date Sampled }\end{array}$} & \multicolumn{2}{|c|}{ GW-703 } & \multicolumn{4}{|c|}{ GW-704 } & \multirow{2}{*}{\multicolumn{2}{|c|}{$\frac{\text { GW-706 }}{\text { EXP }}$}} \\
\hline & \multicolumn{2}{|c|}{$\mathbf{E x p}$} & \multicolumn{4}{|c|}{$\mathbf{E X P}$} & & \\
\hline & $09 / 20 / 93$ & $12 / 21 / 93$ & $03 / 22 / 93$ & $06 / 04 / 93$ & $09 / 22 / 93$ & $12 / 22 / 93$ & $03 / 23 / 93$ & $06 / 11 / 93$ \\
\hline VOLATILE ORGANICS (ug/L) & - & $\cdot$ & $\cdot$ & $\cdot 1$ & $\cdot$ & . & $\cdot$ & . \\
\hline Acetone & 100 & 100 & 100 & 100 & 100 & 2 & 100 & 100 \\
\hline Benzene & 50 & 50 & su & $5 v$ & $5 v$ & 5u & su & 5u \\
\hline Bromodichloromethane & 5u & 5u & su & 50 & su & $5 \mathbf{v}$ & 5u & 5u \\
\hline Bromoform & 50 & 5u & 5u & su & $5 v$ & 50 & 5u & 5u \\
\hline Bromomethane & 100 & 100 & 100 & 100 & 100 & 100 & 100 & 100 \\
\hline 2-Butanone & 100 & 7 & 100 & 100 & 100 & 3 & 100 & 100 \\
\hline Carbon disulfide & su & 5u & 5u & 5u & 50 & su & 5u & 50 \\
\hline Carbon tetrachloride & 50 & 0.4 & 2 & 1 & 5u & su & 50 & 50 \\
\hline Chlorobenzene & su & 5u & 5u & 50 & su & su & 5u & 5u \\
\hline Chlorodibromomethane & su & su & 5u & su & su & su & 5u & su \\
\hline Chloroethane & 100 & 200 & 100 & 100 & 100 & 100 & 100 & 100 \\
\hline Chloroform & 0.5 & 0.5 & 5u & 0.4 & 5u & su & 5u & 50 \\
\hline Chloromethane & 100 & 100 & 100 & 100 & 100 & 100 & 100 & 100 \\
\hline 1,1-Dichloroethane & 5u & 0.5 & 1 & 5u & 5u & su & 50 & 5u \\
\hline 1,2-Dichloroethane & 5u & $5 u$ & 5u & 5u & 5u & su & 50 & 5u \\
\hline 1,1-Dichloroethene & 5u & 2 & 6 & 6 & 6 & 4 & 5u & 5u \\
\hline 1,2-Dichloroethene & 6 & 13 & 3 & 3 & 4 & 2 & 7 & 5 \\
\hline 1,2-Dichloropropane & 50 & 5v & 50 & 5v & su & 5u & 5u & 50 \\
\hline cis-1,3-Dichloropropene & 50 & 5u & 50 & 50 & 5u & 50 & 5u & su \\
\hline trans-1, 3-Dichloropropene & 50 & 50 & 50 & 5u & 5u & 5u & 5u & 50 \\
\hline Ethylbenzene & 50 & 5u & 5u & 50 & su & 50 & 50 & 50 \\
\hline 2-Hexanone & 100 & 100 & 100 & 100 & 100 & 100 & 100 & 100 \\
\hline 4-Methyl-2-pentanone & 100 & 100 & 6 & 100 & 100 & 100 & 4 & 100 \\
\hline Nethylene chloride & 5u & 0.6 & 1 & 1 & 50 & su & 2 & 5U \\
\hline Styrene & 5u & su & su & 5u & 5u & su & 5u & 5u \\
\hline $1,1,2,2$-Tetrachloroethane & 5u & su & 50 & 5u & 5u & 5u & 5u & 5u \\
\hline Tetrachloroethene & 50 & 5u & 50 & 0.5 & 50 & su & 50 & 50 \\
\hline Toluene & $5 u$ & 50 & 50 & $5 v$ & su & su & 5v & 50 \\
\hline $1,1,1$-Trichloroethane & 0.5 & 0.9 & 2 & 2 & 2 & Su & 5u & 5u \\
\hline 1,1,2-Trichloroethane & 50 & 50 & 5u & 50 & 5u & su & 5u & 50 \\
\hline Trichloroethene & 21 & 22 & 120 & 92 & 86 & 55 & 9 & 6 \\
\hline Vinyl acetate & 100 & 100 & 100 & 100 & 100 & 100 & 100 & 100 \\
\hline vinyl chloride & 100 & 100 & 100 & 100 & 100 & 100 & 100 & 100 \\
\hline xylenes & su & 50 & 50 & su & su & su & 5u & su \\
\hline
\end{tabular}

(CONTINUED) 
APPENDIX E. 2

\begin{tabular}{|c|c|c|c|c|c|c|c|c|}
\hline \multirow{3}{*}{$\begin{array}{l}\text { Sampling Point } \\
\text { Location } \\
\text { Date Sampled }\end{array}$} & \multicolumn{2}{|c|}{ GW-706 } & \multicolumn{4}{|c|}{$G W-710$} & \multicolumn{2}{|c|}{ GW-711 } \\
\hline & \multicolumn{2}{|c|}{$\mathbf{E x p}$} & \multicolumn{4}{|c|}{$\mathbf{E X P}$} & \multicolumn{2}{|c|}{$\mathbf{m x P}$} \\
\hline & $09 / 23 / 93$ & $12 / 28 / 93$ & $03 / 21 / 93$ & $04 / 30 / 93$ & $08 / 01 / 93$ & $11 / 09 / 93$ & $03 / 20 / 93$ & $04 / 29 / 93$ \\
\hline VOLATILE ORGANICS (ug/L) & $\cdot$ & $\cdot$ & $\cdot$ & $\cdot$ & $\cdot$ & $\cdot$ & $\cdot$ & $\cdot$ \\
\hline Acetone & 100 & 2 & 100 & 100 & 100 & 100 & 100 & $10 \dot{0}$ \\
\hline Benzene & $5 \mathrm{u}$ & $5 u$ & su & 50 & 50 & 5u & 5u & 5u \\
\hline Bromodiehloromethane & su & su & 5u & 5u & su & su & su & su \\
\hline Bromoform & 5u & su & 5u & su & so & 5u & 5u & su \\
\hline Bromomethane & 100 & 100 & 100 & 100 & 100 & 100 & 100 & 100 \\
\hline 2-Butanone & 100 & 6 & 100 & 100 & 100 & 100 & 100 & 100 \\
\hline Carbon disulfide & 50 & 50 & $5 \mathbf{v}$ & 5u & 50 & su & 5u & 5u \\
\hline Carbon tetrachloride & 5u & 5u & su & 5u & 5u & 5u & su & 5u \\
\hline Chlorobenzene & 50 & 5u & 5u & 5u & su & 5u & so & 50 \\
\hline Chlorodibromomethane & su & 50 & 50 & su & su & 50 & 5u & 5u \\
\hline Chloroethane & 100 & 100 & 100 & 100 & 100 & 100 & 100 & 100 \\
\hline Chloroform & $5 \mathbf{u}$ & 50 & 5u & $5 u$ & 50 & su & su & su \\
\hline Chloromethane & 100 & 100 & 100 & 100 & 100 & 100 & 100 & 100 \\
\hline 1,1-Dichloroethane & 50 & su & 5u & $5 u$ & su & su & su & 5u \\
\hline 1,2-Dichloroethane & 5u & su & su & 50 & 50 & 5u & 5u & su \\
\hline 1,1-Dichloroethene & 5u & 5u & 50 & 50 & $5 u$ & 5u & 50 & su \\
\hline 1,2-Dichloroethene & 5 & su & su & so & 50 & 5u & 50 & 5u \\
\hline 1,2-Dichloropropane & 5u & $5 \mathbf{v}$ & $5 \mathbf{u}$ & 50 & su & 5u & 5u & su \\
\hline cis-1,3-Dichloropropene & 5u & su & 50 & 5u & 5u & 50 & su & su \\
\hline trans-1,3-Dichloropropene & 5u & 5u & $5 \mathbf{v}$ & 5u & 5u & 5u & 5u & 5u \\
\hline Ethylbenzene & $5 \mathrm{U}$ & $5 \mathbf{u}$ & 50 & 50 & 5u & 5u & 50 & su \\
\hline 2-Hexanone & 100 & 100 & 100 & 100 & 100 & 100 & 100 & 100 \\
\hline 4-Methyl-2-pentanone & 100 & 100 & 100 & 100 & 100 & 100 & 100 & 100 \\
\hline Methylene chloride & 5u & 1 & su & 2 & 50 & 5u & su & 50 \\
\hline styrene & 5u & 5u & su & $\therefore 50$ & 50 & 50 & 50 & su \\
\hline $1,1,2,2$-Tetrachloroethane & 50 & 5u & 50 & 50 & 50 & 5v & 5u & su \\
\hline Tetrachloroethene & 50 & 50 & 5u & 5u & 5u & 5u & 50 & 5u \\
\hline Toluene & 5u & $5 u$ & $5 v$ & 50 & 50 & 50 & 50 & 5u \\
\hline $1,1,1$-Trichloroethane & 5u & $5 \mathrm{u}$ & 50 & $5 \mathrm{u}$ & 50 & 50 & 5u & $5 v$ \\
\hline $1,1,2$-Trichloroethane & so & 50 & su & 50 & 5u & 5v & so & 50 \\
\hline Trichloroethene & 5 & $5 u$ & $5 v$ & 50 & 5u & 50 & 50 & $5 u$ \\
\hline Vinyl acetate & 100 & 100 & 100 & 100 & 100 & 100 & 100 & 100 \\
\hline vinyl chloride & 100 & 100 & 100 & 100 & 100 & 100 & 100 & 100 \\
\hline xylenes & su & 50 & 5u & 50 & 50 & 5u & su & 50 \\
\hline
\end{tabular}

(CONTINUED) 


\begin{tabular}{|c|c|c|c|c|c|c|c|c|}
\hline \multirow{3}{*}{$\begin{array}{l}\text { 8ampling Point } \\
\text { Iocation } \\
\text { Date sampled }\end{array}$} & \multicolumn{2}{|c|}{ OW-711 } & \multicolumn{4}{|c|}{ ON-712 } & \multicolumn{2}{|c|}{ OW-713 } \\
\hline & \multicolumn{2}{|c|}{$\mathbf{E X P}$} & \multicolumn{4}{|c|}{$\mathbf{E x P}$} & \multicolumn{2}{|c|}{$\mathbf{m x p}$} \\
\hline & $07 / 30 / 93$ & $11 / 07 / 93$ & $03 / 18 / 93$ & $04 / 28 / 93$ & $07 / 29 / 93$ & $11 / 06 / 93$ & $03 / 19 / 93$ & $04 / 30 / 93$ \\
\hline VOLATILE ORGANICS (ug/L) & $\cdot$ & $\cdot$ & $\cdot$ & $\cdot$ & • & $\cdot$ & $\cdot$ & $\cdot$ \\
\hline Noetone & 100 & 100 & 100 & 100 & 100 & 100 & 7 & 100 \\
\hline Benzene & 50 & su & 50 & so & so & su & su & su \\
\hline Bromodichloromethane & 5u & su & 50 & su & su & 5u| & su & su \\
\hline Bromoform & 50 & su & 50 & 5v & 5u & 50 & $\mathbf{5 u}$ & so \\
\hline Bromomathane & 100 & $10 u$ & 100 & 100 & 100 & 100 & 100 & 100 \\
\hline 2-Butanone & 100 & 100 & 100 & 100 & 100 & 100 & 100 & 2 \\
\hline Carbon diauleide & 5v & su & 5u & 50 & 5u & 5u & su & 5u \\
\hline Carbon tetrachloride & 5u & 5u & 50 & 5u & su & su & su & so \\
\hline Chlorobenzene & 5u & su & so & 50 & su & 5u & su & su \\
\hline Chlorodibromomethane & su & 5u & so & 50 & su & 5u & 5u & so \\
\hline Chloroethane & 100 & 100 & 100 & 100 & 100 & 100 & 100 & 100 \\
\hline Chlorotorm & 5u & su & 5u & 5v & 5u & 5u & su & 50 \\
\hline Chloromethane & 100 & 100 & 100 & 100 & 100 & 100 & 100 & 100 \\
\hline 1,1-Dichloroethane & su & 5u & $5 u$ & su & su & so & su & su \\
\hline 1,2-Dichloroethane & 50 & 5u & 5u & 50 & su & su & su & su \\
\hline 1,1-Dichloroethene & su & su & su & su & su & 5u & su & so \\
\hline 1,2-Dichloroethene & su & 5u & so & 5u & su & 5u & su & so \\
\hline 1,2-Dichloropropane & 50 & so & 5u & su & su & 5u & su & so \\
\hline cis-1,3-Dichloropropen & 50 & 50 & su & su & su & 50 & su & 50 \\
\hline trans-1, 3-Dich loropropene & su & 5u & 5u & su & su & 5u & su & su \\
\hline Bthylbenzene & 5u & 5u & 5u & su & su & su & su & so \\
\hline 2-Hexanone & 100 & 100 & 100 & 100 & 100 & 100 & 100 & 100 \\
\hline 4-Methy1-2-pentanone & 100 & 100 & 100 & 100 & 100 & 100 & 2 & 100 \\
\hline Methylene chloride & 1 & 1 & 5u & 5u & 1 & su & 2 & su \\
\hline styrene & su & 5u & 50 & su & 5u & 5u & su & su \\
\hline $1,1,2,2$-Tetrachloroethane & 50 & 5u & so & $5 u$ & su & 5u & 5u & su \\
\hline Tetrachloroethene & su & su & su & $5 v$ & so & 50 & su & so \\
\hline Toluene & su & 5u & 5u & 5u & su & 5u & su & $\rightarrow 0$ \\
\hline 1,1,1-Trichloroethane & 50 & 50 & 50 & 5v & su & 5u & $5 u$ & 1 \\
\hline 1,1,2-Trichloroethane & 5u & 50 & 5u & 50 & su & so & 50 & 50 \\
\hline Trichloroethene & 5u & su & 5u & 5v & su & su & su & su \\
\hline Vinyl acetate & 100 & 100 & 100 & 100 & 100 & 100 & 100 & 100 \\
\hline Vinyl chloride & 100 & 100 & 100 & 100 & 100 & 100 & 100 & 100 \\
\hline xylenes & su & $5 v$ & 5u & 50 & su & Su & 50 & su \\
\hline
\end{tabular}

(CONTINUED) 
APPENDIX 1.2

Croundwater and surface-Water Quality Data, 1993

\begin{tabular}{|c|c|c|c|c|c|c|c|c|}
\hline \multirow{3}{*}{$\begin{array}{l}\text { sampling polnt } \\
\text { Location } \\
\text { Date sampled }\end{array}$} & \multicolumn{2}{|c|}{ ow-713 } & \multicolumn{4}{|c|}{ aW-714 } & \multicolumn{2}{|c|}{ an-71s } \\
\hline & \multicolumn{2}{|c|}{$\mathbf{E x p}$} & \multicolumn{4}{|c|}{$\mathbf{E x p}$} & \multicolumn{2}{|c|}{$\mathbf{m x P}$} \\
\hline & $07 / 31 / 93$ & $11 / 09 / 93$ & $03 / 21 / 93$ & $04 / 29 / 93$ & $08 / 01,93$ & $11 / 09 / 93$ & $03 / 21 / 93$ & $04 / 29 / 93$ \\
\hline VOLATIL OReNAICs (ug/L) & $\cdot$ & $\cdot$ & $\cdot$ & $\cdot 1$ & $\cdot$ & $\cdot$ & $\cdot$ & - \\
\hline Aontone & 100 & 100 & $10 \dot{0}$ & 100 & $10 \dot{0}$ & 100 & $10 \dot{u}$ & $10 \dot{v}$ \\
\hline Benzene & su & su & so & so & su & so & su & so \\
\hline Bromodiohloromethane & su & su & su & so & su & so & su & su \\
\hline Bromotorm & su & su & su & so & su & su & su & su \\
\hline Bromomethane & 100 & 100 & 100 & 200 & 100 & 100 & 100 & 100 \\
\hline 2-Butanone & 100 & 100 & 100 & 200 & 100 & 100 & 100 & 100 \\
\hline Carbon disulfide & so & su & su & su & su & su & su & su \\
\hline Carbon tetrachloride & su & su & 5u & su & so & su & su & su \\
\hline Chlorobenzene & su & so & su & su & su & so & su & sv \\
\hline Chlorodibromomethane & su & su & su & su & su & so & su & so \\
\hline Chloroethane & 100 & 100 & 100 & 100 & 100 & 100 & 100 & 100 \\
\hline Chloroform & su & su & su & so & su & su & su & so \\
\hline Chloromethane & 100 & 100 & 100 & 100 & 100 & 100 & 100 & 100 \\
\hline 1,1-Dichloroethane & su & su & so & su & su & su & su & so \\
\hline 1,2-Dichloroethane & su & 5u & su & su & $\mathbf{s u}$ & su & so & so \\
\hline 1,1-Dichloroethene & su & su & so & su & su & su & su & so \\
\hline 1,2-Dichloroethene & su & su & su & so & su & su & su & su \\
\hline 1,2-Dichloropropane & su & 5u & su & su & su & su & su & su \\
\hline cis-1,3-Dichloropropene & su & su & su & su & su & so & su & su \\
\hline trane-1,3-Dichloropropene & su & so & so & 5v & su & su & su & so \\
\hline Ethylbenzene & su & su & su & su & su & su & su & su \\
\hline 2-Hexanone & 100 & 100 & 100 & 100 & 100 & 100 & 100 & 100 \\
\hline 4-Mathy1-2-pentanone & 100 & 100 & 100 & 100 & 100 & 100 & 100 & 100 \\
\hline Mathylene chlorlde & 5u & 1 & 1 & 2 & su & 1 & 0.9 & su \\
\hline styrane & su & su & su & 5u & su & so & su & su \\
\hline 1,1,2,2-Tetrachloroethane & su & su & 5u & 5u & su & su & su & su \\
\hline Tatrachloroethene & su & su & 50 & so & su & su & su & su \\
\hline Toluene & su & su & 5u & so & su & su & su & su \\
\hline $1,1,1$-Trichloroethane & 5u & 50 & 5u & so & su & su & su & su \\
\hline $1,1,2$-Trichloroethane & su & su & su & 5u & su & su & su & su \\
\hline Trichloroethene & su & 50 & su & su & su & 5u & su & 5U \\
\hline Vinyl acetate & 100 & 100 & 100 & 100 & 100 & 100 & 100 & 100 \\
\hline vinyl chloride & 100 & 100 & 100 & 100 & 100 & 100 & 100 & 100 \\
\hline xylenes & su & su & 5u & 5u & 5u & su & su & su \\
\hline
\end{tabular}

(CONTINUBD) 
Oroundwater and surface-Wator Quality Data, 1993

\begin{tabular}{|c|c|c|c|c|c|c|c|c|}
\hline \multirow{3}{*}{$\begin{array}{l}\text { gampling point } \\
\text { Locetion } \\
\text { Date suapled }\end{array}$} & \multicolumn{2}{|c|}{$0 w-713$} & \multicolumn{4}{|c|}{$0 w-723$} & \multicolumn{2}{|c|}{ ON-724 } \\
\hline & \multicolumn{2}{|c|}{$\mathbf{E x p}$} & \multicolumn{4}{|c|}{ exp } & \multicolumn{2}{|c|}{$\exp$} \\
\hline & $08 / 01 / 93$ & $11 / 12 / 93$ & $02 / 12 / 93$ & $05 / 18 / 93$ & $09 / 27 / 93$ & $12 / 24 / 93$ & $02 / 10 / 93 \mid$ & $09 / 18 / 93$ \\
\hline voratris onenurcs (ug/L) & $\cdot$ & $\cdot$ & $\cdot$ & $\cdot$ & $\cdot$ & $\cdot$ & - & - \\
\hline Acetone & 100 & 100 & 100 & 100 & 200 & 100 & 100 & $10 \dot{0}$ \\
\hline Bensene & su & so & so & sv & su & su & so & su \\
\hline Bromodichloromethane & su & su & su & su & su & su & su & so \\
\hline Bromotorm & su & su & su & su & su & su & so & su \\
\hline Bromomothane & 100 & 100 & 200 & 200 & 100 & 100 & 100 & 100 \\
\hline 2-Butanone & 100 & 100 & 2 & 100 & 100 & 100 & 100 & 100 \\
\hline Carbon dieuzlide & su & so & su & so & su & su & so & su \\
\hline Carbon tetrachloride & su & so & 1 & su & so & su & su & su \\
\hline Ch1orobenzene & so & so & su & su & su & su & su & su \\
\hline ChlorodLbyomomethane & su & so & su & so & so & su & 50 & su \\
\hline Chloroethane & 100 & 100 & 100 & 100 & 100 & 100 & 100 & 100 \\
\hline Chloroform & so & su & su & so & su & su & su & su \\
\hline Chloromethane & 100 & 100 & 100 & 100 & 100 & 100 & 100 & 100 \\
\hline 1,1-Dichloroethane & so & su & su & su & su & su & su & su \\
\hline 1,2-Dichlorouthane & su & su & su & so & su & su & so & su \\
\hline 1,1-Dichloroethene & so & su & su & so & su & so & su & su \\
\hline 1,2-Diehloroethene & so & su & 2 & su & su & su & 2 & su \\
\hline 1,2-Dlohloropropane & su & so & su & so & su & so & su & so \\
\hline C1e-1,3-Dichloropropane & su & su & su & su & su & so & su & su \\
\hline trane-1, 3-Dlohloropropane & sul & su & su & so & su & su & so & su \\
\hline Ethylbonzene & su & su & su & so & su & so & su & su \\
\hline 2-Hexanone & 100 & 100 & 100 & 100 & 100 & 100 & 100 & 100 \\
\hline 1-Mothy1-2-pentanone & 100 & 100 & 1 & 100 & 100 & 100 & 100 & 100 \\
\hline nethylene chloride & so & 1 & 3 & sv & su & su & 1 & su \\
\hline Styrene & so & su & su & su & su & so & su & su \\
\hline 1,1,2,2-Tetrachloroethane & so & su & su & su & su & so & so & su \\
\hline Tetrachloroethene & so & su & su & su & su & su & 2 & su \\
\hline Toluene & so & su & su & so & su & so & so & so \\
\hline $1,1,1-\operatorname{Tr} 1$ chlorouthane & so & su & su & so & su & su & su & su \\
\hline 1,1,2-Trichloroethane & su & su & su & su & su & so & su & su \\
\hline Trlehloroethene & su & so & 21 & 3 & 4 & 5 & 93 & 46 \\
\hline Vinyl acetate & 100 & 100 & 100 & 100 & 100 & 100 & 100 & 100 \\
\hline Vinyl chloride & 100 & 100 & 100 & 100 & 100 & 100 & 100 & 100 \\
\hline xylenes & so & su & su & so & su & su & su & su \\
\hline
\end{tabular}

(CONTINUED) 
APrudux 8.2

Groundwater and surface-water Quality Date, 1983

\begin{tabular}{|c|c|c|c|c|c|c|c|c|}
\hline \multirow{3}{*}{$\begin{array}{l}\text { Sampling polnt } \\
\text { Loontion } \\
\text { Date sanpled }\end{array}$} & \multicolumn{2}{|c|}{ ON-724 } & \multicolumn{4}{|c|}{ ow-72s } & \multicolumn{2}{|c|}{$0 N-736$} \\
\hline & \multicolumn{2}{|c|}{$\mathbf{s x p}$} & \multicolumn{4}{|c|}{$\mathbf{m e P}$} & \multicolumn{2}{|c|}{$\mathbf{z x p}$} \\
\hline & $|09 / 29 / 83|$ & $12 / 14 / 93$ & $02 / 12 / 93$ & $05 / 10 / 93$ & $09 / 28 / 93$ & $12 / 15 / 93$ & $|02 / 11 / 93|$ & $05 / 21 / 93$ \\
\hline votarses onenurcs (ug/L) & $\cdot 1$ & $\cdot$ & $\cdot 1$ & $\cdot 1$ & $\cdot 1$ &. & $\cdot$ & - \\
\hline Acetone & 100 & 100 & $i$ & 100 & 100 & $\dot{4}$ & 100 & 100 \\
\hline Eensene & su & su & su & su & su & 1 & so & su \\
\hline Eromodlahloromethane & so & sv & su & su & so & su & su & so \\
\hline Eromotorm & su & $\mathbf{s u}$ & su & su & so & su & su & su \\
\hline Bromomethane & 100 & 100 & 100 & 100 & 100 & 100 & 100 & 200 \\
\hline 2-Dutanone & 100 & 100 & 2 & 120 & 100 & 7 & 100 & 100 \\
\hline Carbon dieullide & so & su & su & so & so & su & 2 & su \\
\hline Carbon tetraohlorida & so & 2 & so & su & su & su & 2 & so \\
\hline Chlorobensene & so & su & su & su & so & su & 1 & su \\
\hline Chlorodibromonethane & su & su & su & su & so & su & su & su \\
\hline Chloroethane & 100 & 100 & 100 & 100 & 100 & 100 & 100 & 100 \\
\hline Chlorolorm & 7 & so & so & su & so & so & 2 & su \\
\hline Chloromethane & 100 & 100 & 100 & 100 & 100 & 100 & 100 & 100 \\
\hline 1,1-DLohlorouthane & su & su & so & su & so & su & su & so \\
\hline 1,2-Dlchloroethane & su & su & so & su & su & su & su & su \\
\hline 1,1-Dlohloroethene & su & su & su & su & su & su & 2 & su \\
\hline 1,2-DLChloroethene & su & 3 & 2 & 3 & 2 & 3 & 7 & 5 \\
\hline 1,2-Dlehloropropane & so & so & su & so & so & su & so & su \\
\hline Cle-1,3-DLChLoropropene & so & su & su & su & su & su & su & su \\
\hline trans-1,3-Diehloropropane & su & su & su & su & su & su & su & su \\
\hline Ethylbenzene & su & so & su & su & su & su & 2 & su \\
\hline 2-Hexanone & 100 & 100 & 100 & 100 & 100 & 200 & 100 & 100 \\
\hline 1-Methy 1-2-pantanone & 100 & 100 & 1 & 100 & 100 & 1 & 1 & 100 \\
\hline Methylene chloride & su & su & 2 & su & so & 2 & 2 & su \\
\hline styrone & so & su & so & su & su & su & 1 & su \\
\hline $1,1,2,2$-Tetrachloroethane & su & so & su & su & su & su & su & su \\
\hline Tetrachloroethene & su & 3 & su & so & 0.8 & 1 & 7 & 3 \\
\hline Toluene & su & su & su & su & su & 2 & su & 51 \\
\hline 1,1,1-Trichloroethane & su & su & su & su & so & su & su & su \\
\hline 1,1,2-Trlohloroethane & so & 5u & so & su & so & 5u & 1 & so \\
\hline Trlehloroethene & 3 & 140 & 19 & 53 & 47 & 53 & 12 & 12 \\
\hline Vinyl acetate & 100 & 100 & 100 & 100 & 100 & 100 & 100 & 100 \\
\hline Vinyl chloride & 100 & 100 & 100 & 100 & 100 & 100 & 100 & 100 \\
\hline xylenes & su & su & su & su & su & $5 \mathbf{s u}$ & 5 & so \\
\hline
\end{tabular}

(CONTINUED) 
Appmatx 1.2

Oroundwater and surfacomater Quadlty Data, 1993

\begin{tabular}{|c|c|c|c|c|c|c|c|c|}
\hline \multirow{3}{*}{$\begin{array}{l}\text { ienpling polnt } \\
\text { Loention } \\
\text { Date sempled }\end{array}$} & \multicolumn{2}{|c|}{$0 w-736$} & \multicolumn{4}{|c|}{$0 N-737$} & \multicolumn{2}{|c|}{$0 N-730$} \\
\hline & \multicolumn{2}{|c|}{$\mathbf{E x p}$} & \multicolumn{4}{|c|}{$\operatorname{Ixp}$} & \multicolumn{2}{|c|}{$\operatorname{mxp}$} \\
\hline & $09 / 20 / 93 \mid$ & $12 / 13 / 93$ & $02 / 21 / 93$ & $05 / 19 / 93$ & $\mid 0 s / 28 / 93$ & $12 / 15 / 93$ & $02 / 10 / 93$ & $05 / 18 / 93$ \\
\hline vouarise onenurcs (ug/t) & $\cdot$ & - & $\cdot$ & - & $\cdot$ & $\cdot$ & $\cdot$ & - \\
\hline Naetone & 100 & $\dot{4}$ & $\dot{2}$ & 100 & 100 & $\dot{4}$ & $10 \dot{0}$ & 100 \\
\hline Bensene & su & so & su & su & su & sv & so & su \\
\hline Eromod lehlorowethane & su & so & su & su & su & su & so & so \\
\hline Bronolorm & su & so & su & so & su & su & so & so \\
\hline Bromomothane & 100 & 100 & 100 & 100 & 100 & 100 & 100 & 100 \\
\hline 2-Dutanone & 100 & 7 & 100 & 100 & 100 & - & 100 & 100 \\
\hline Carbon diusteldo & so & so & so & so & so & su & so & so \\
\hline Carbon tetrachloride & su & so) & 1 & so & $\mathbf{s u}$ & su & 1 & so \\
\hline Chlorobenzene & so & so & su & su & su & su & so & su \\
\hline Chlorodlbromomethane & so & su & su & su & $\mathbf{s 0}$ & so & su & su \\
\hline Chloroethane & 100 & 100 & 100 & 100 & 100 & 100 & 100 & 100 \\
\hline Chlorotorm & so & so & 2 & 2 & 1 & su & 2 & 2 \\
\hline Chloromethane & 100 & 200 & 100 & 100 & 100 & 100 & 100 & 100 \\
\hline 1,1-DLohloroethane & so & su & su & su & su & su & so & so \\
\hline 1,2-DLohloronthane & so & su & su & su & so & su & su & su \\
\hline 1,1-Dichloroethene & su & su & su & su & su & su & su & su \\
\hline 1,2-Dichloronthene & 3 & s) & 4 & 4 & 3 & 4 & 4 & 3 \\
\hline 1,2-DLehloropropane & su & so & so & so & su & su & so & su \\
\hline ole-1, 3-Dichloropropene & su & so & su & su & so & su & su & su \\
\hline trana-1, 3-Dlohloropropene & su & so & su & su & so & su & su & su \\
\hline athylbenzone & su & so & su & su & su & su & so & su \\
\hline 2-Hexanone & 100 & 100 & 100 & 100 & 100 & 100 & 100 & 100 \\
\hline 4-Methy1-2-pentanone & 100 & 1 & 1 & 100 & 100 & 1 & 100 & 10u \\
\hline Methylene chloride & su & 2 & 2 & su & su & 2 & 1 & su \\
\hline Styrane & su & so & su & so & so & su & so & sv \\
\hline $1,1,2,2$-Tetraohloroethane & su & so & su & su & su & so & so & su \\
\hline ratrachloroethene & 1 & 3 & 4 & 3 & 3 & 3 & su & so \\
\hline Toluene & so & so & su & su & so & su & su & su \\
\hline 1,1,1-Trichloroethane & su & su & su & su & su & so & su & su \\
\hline 1,2,2-Trlenloroethane & su & su & su & su & so & su & su & so \\
\hline Trlehloroethene & 9 & 13 & 9 & 9 & 9 & 11 & 55 & 51 \\
\hline Vinyl acetate & 100 & 100 & 100 & 100 & 100 & 100 & 100 & 100 \\
\hline Vinyl ohloride & 100 & 100 & 100 & 100 & 100 & 100 & 100 & 100 \\
\hline xylenes & so & su & su & so & so & su & su & su \\
\hline
\end{tabular}

(CONTINUED) 


\begin{tabular}{|c|c|c|c|c|c|c|c|c|}
\hline \multirow{3}{*}{$\begin{array}{l}\text { sampling polnt } \\
\text { Location } \\
\text { Date sampled }\end{array}$} & \multirow{2}{*}{\multicolumn{2}{|c|}{$\frac{0 N-738}{\operatorname{2xp}}$}} & \multicolumn{4}{|c|}{$0 N-739$} & \multicolumn{2}{|c|}{$0 N-740$} \\
\hline & & & \multicolumn{4}{|c|}{$\mathbf{s x p}$} & \multicolumn{2}{|c|}{$\mathbf{E x p}$} \\
\hline & $09 / 27 / 93$ & $12 / 24 / 93$ & $02 / 10 / 93$ & $05 / 18 / 93$ & $09 / 27 / 93$ & $12 / 13 / 93$ & $02 / 09 / 93 \mid$ & $05 / 17 / 93$ \\
\hline voraris onenurcs (ug/h) & $\cdot$ & $\cdot$ & - & $\cdot$ & $\cdot 1$ & $\cdot$ & $\cdot$ & - \\
\hline Acetone & 100 & 100 & 200 & 100 & 100 & 100 & 100 & $10 \dot{0}$ \\
\hline Densene & so & so & so & so & su & su & so & so \\
\hline Bromodichloromothane & su & su & so & so & su & su & su & su \\
\hline Bromotorm & so & so & su & so & su & su & su & so \\
\hline Dromomethane & 100 & 100 & 100 & 100 & 100 & 100 & 100 & 100 \\
\hline 2-Butanona & 100 & 100 & 100 & 100 & 100 & 100 & 100 & 100 \\
\hline Carbon dieulelde & su & su & su & so & su & so & su & so \\
\hline Carbon tetraohloride & su & 1 & so & su & su & 1 & 1 & su \\
\hline Chlorobensen & su & so) & so & su & su & so & su & su \\
\hline Chlorodibramomethane & su & su & so & so & su & su & su & so \\
\hline Chloroethane & 100 & 100 & 100 & 100 & 100 & 100 & 100 & 100 \\
\hline Chlorotorm & 1 & 2 & so & so & su & 1 & 2 & 2 \\
\hline Chloromethane & 100 & 100 & 200 & 200 & 100 & 100 & 100 & 100 \\
\hline 1,1-Dlehloroethane & so & su & so) & su & su & so & su & 50 \\
\hline 1,2-Dichloroothane & su & su & so & su & su & so & 5u & su \\
\hline 1,1-Dlchloroethane & so & su & so & su & su & su & su & so \\
\hline 1,2-Diehloroethene & 3 & 3 & 2 & su & 2 & 2 & 4 & 2 \\
\hline 1,2-Dichloropropane & su & so & 50 & su & so & so & su & 50 \\
\hline ole-1,3-Dichloropropene & so & su & so & su & su & su & su & so \\
\hline trans-1,3-Diohloropropene & su & su & su & su & su & so & su & so \\
\hline Ethylbonzene & so & so & su & su & su & su & su & 5u \\
\hline 2-Hexanone & 100 & 100 & 2 & 100 & 100 & 100 & 100 & 100 \\
\hline 4-Mothyl-2-pentanone & 100 & 100 & 2 & 100 & 100 & 100 & 2 & 100 \\
\hline methylene chloride & so & su & 2 & su & su & so & 2 & su \\
\hline styrene & su & su & su & su & su & su & su & su \\
\hline $1,1,2,2$-Tetrachloroethane & 5u & su & su & su & so & su & su & 5u \\
\hline Tetrachloroethene & su & su & su & su & su & su & su & su \\
\hline Toluene & 5v & su & su & su & su & su & su & su \\
\hline 1,1,1-Trichloroethane & su & su & su & su & su & 0.7 & su & su \\
\hline 1,1,2-Trichloroethane & su & su & su & su & 5u & su & su & su \\
\hline Trich soothene & 44) & so & 32 & 33 & 39 & 42 & 50 & 38 \\
\hline VInyl wetate & 100 & 100 & 1 & 100 & 100 & 100 & 100 & 100 \\
\hline Vinyl chloride & 100 & 100 & 100 & 100 & 100 & 100 & 100 & 100 \\
\hline xylenes & su & su & su & su & so & su & su & su \\
\hline
\end{tabular}

(Contruned) 
APPENDIX 1.2

Groundwater and surface-water Quallty Data, 1993

\begin{tabular}{|c|c|c|c|c|c|c|c|c|}
\hline \multirow{3}{*}{$\begin{array}{l}\text { Eampling point } \\
\text { Location } \\
\text { Date sampled }\end{array}$} & \multicolumn{2}{|c|}{$a n-740$} & \multicolumn{4}{|c|}{ ON-793 } & \multicolumn{2}{|c|}{ aw-794 } \\
\hline & \multicolumn{2}{|c|}{$\mathbf{E x p}$} & \multicolumn{4}{|c|}{ MOLIsF } & \multicolumn{2}{|c|}{ AOLLSF } \\
\hline & $09 / 27 / 93$ & $12 / 11 / 93$ & $02 / 26 / 93$ & $05 / 25 / 93$ & $07 / 11 / 93$ & $10 / 18 / 93$ & $03 / 01 / 93$ & $05 / 25 / 93$ \\
\hline VOHATIL ORCNAICS (ug/L) & • & $\cdot 1$ & $\cdot$ & $\cdot 1$ & $\cdot 1$ & • & $\cdot$ & $\bullet^{\circ}$ \\
\hline Acetone & 100 & 100 & 200 & 100 & 100 & 2 & 100 & $10 \dot{U}$ \\
\hline Benzene & su & su & su & so & su & su & su & su \\
\hline Bromodiohloromathane & su & 5u & su & so & su & su & so & su \\
\hline Bromotorm & su & su & su & 5v & su & su & su & su \\
\hline Bromomothane & 100 & $10 u$ & 100 & 100 & 100 & 100 & 100 & 100 \\
\hline 2-Butanone & 100 & 100 & 100 & 3 & 100 & 9 & 100 & 5 \\
\hline Carbon disulfide & su & su & so & so & su & su & su & su \\
\hline Carbon tetrachloride & su & su & su & 5u & su & su & 5u & su \\
\hline Chlorobanzene & so & su & su & su & so & su & su & su \\
\hline Chlorodibromomethane & so & su & su & so & su & su & su & su \\
\hline Chloronthane & 100 & 100 & 100 & 100 & 100 & 100 & 100 & 100 \\
\hline Chlorotorm & 1 & 1 & 5u & 50 & su & 5u & 5u & su \\
\hline Chloromethane & 100 & 100 & 100 & 100 & 100 & 100 & 100 & 100 \\
\hline 1,1-Diohloroethane & su & su & su & su & su & 5u & su & su \\
\hline 1,2-Dichloroethane & su & su & su & 5u & su & 50 & su & su \\
\hline 1,1-Dichloroethene & su & 5u & su & so & su & 5v & su & 50 \\
\hline 1,2-Dichloroethene & 2 & 2 & 5u & su & su & 5u & 5u & su \\
\hline 1,2-Dichloropropane & so & su & so & 50 & su & 5u & 5v & su \\
\hline ole-1,3-Dichloropropene & 50 & su & 5u & so & 5u & 5u & su & so \\
\hline trans-1,3-Dichloropropene & so & 5u & su & su & su & 50 & 5u & su \\
\hline Bthylbenzene & su & su & su & so & su & su & 5u & 50 \\
\hline 2-Hexanone & 100 & 100 & 100 & 100 & 100 & 100 & 100 & 100 \\
\hline 4-Mothyl-2-pentanone & 100 & 100 & 100 & 2 & 100 & 100 & 100 & 1 \\
\hline Methylene chloride & su & 1 & 5u & su & su & 2 & su & 50 \\
\hline styrene & 50 & 5u & su & su & su & so & 50 & su \\
\hline $1,1,2,2$-Tetrachloroethane & su & su & su & 5u & su & 5u & su & 5u \\
\hline Tetrachlorouthane & 5u & su & su & su & su & so & su & 50 \\
\hline Toluene & 5u & 5u & 5u & so & so & 50 & 50 & 50 \\
\hline $1,1,1$-Trichloroethane & su & 5u & 5u & 5u & su & 5u & 50 & 5u \\
\hline $1,1,2-$ Trichloroethane & su & su & su & 5u & su & 5v & su & 5u \\
\hline Trichloroethene & 33 & 35 & 5u & su & su & 5u & su & 5u \\
\hline Vinyl acetate & 100 & 100 & 100 & 100 & 100 & 100 & 100 & 200 \\
\hline Vinyl chloride & 100 & 100 & 100 & 100 & 100 & 100 & 100 & 100 \\
\hline Xylenes & su & su & 5u & su & su & 50 & 5u & su \\
\hline
\end{tabular}

(CONTrRUED) 
APPENDIX 2.2

\begin{tabular}{|c|c|c|c|c|c|c|c|c|}
\hline \multirow{3}{*}{$\begin{array}{l}\text { 8ampling Point } \\
\text { Looation } \\
\text { Date 8ampled }\end{array}$} & \multicolumn{2}{|c|}{ ow-794 } & \multicolumn{4}{|c|}{ 6w-795 } & \multirow{2}{*}{\multicolumn{2}{|c|}{$\frac{0 w-800}{\text { ox }}$}} \\
\hline & \multicolumn{2}{|c|}{ AOLLSF } & \multicolumn{4}{|c|}{ AOLLSF } & & \\
\hline & $07 / 11 / 93$ & $10 / 18 / 93$ & $03 / 02 / 93$ & $05 / 25 / 93$ & $07 / 12 / 93$ & $10 / 18 / 93$ & $05 / 11 / 93$ & $09 / 14 / 93$ \\
\hline VOLATILE ORCANICS (ug/L) & $\cdot$ & $\cdot$ & $\cdot$ & $\cdot$ & $\cdot$ & $\cdot$ & $\cdot$ & - \\
\hline Aoetone & 100 & 2 & 1 & 100 & 100 & 1 & 100 & 100 \\
\hline Benzene & su & su & so & su & 5u & so & so & su \\
\hline Bromodichloromethane & su & su & sv & su & 50 & sv & so & su \\
\hline Bromoform & su & su & su & su & so & su & so & su \\
\hline Bromomethane & 100 & 100 & 100 & 100 & 100 & 100 & 100 & 100 \\
\hline 2-Butanone & 100 & 10 & 100 & 100 & 100 & 8 & 100 & 100 \\
\hline Carbon dieulfide & 5v & su & 5u & su & su & $5 \mathbf{v}$ & su & su \\
\hline Carbon tetrachloride & su & 5u & su & so & 5u & 5u & su & su \\
\hline Chlorobenzene & 5v & su & su & 5u & so & su & su & su \\
\hline Chlorodibromomethane & su & sv & 5u & su & su & su & su & su \\
\hline Chloroethane & 100 & 100 & 100 & 100 & 100 & 100 & 100 & 100 \\
\hline Chloroform & 5u & su & $5 \mathbf{5 u}$ & $5 v$ & 50 & $5 \mathbf{v}$ & su & su \\
\hline Chloromethane & 100 & 100 & 100 & 100 & 100 & 100 & 100 & 100 \\
\hline 1,1-Dichloroethane & 5v & 50 & 50 & su & 50 & su & su & su \\
\hline 1,2-Dichloroethane & 5u & su & 50 & su & su & su & su & su \\
\hline 1,1-Dichloroethene & su & su & su & 5u & $5 \mathbf{v}$ & su & su & su \\
\hline 1,2-Dichloroethene & su & 50 & su & su & 5u & su & su & su \\
\hline 1,2-Dichloropropane & 5u & su & sv & $5 v$ & 50 & su & su & su \\
\hline cis-1,3-Dichloropropene & 50 & 50 & 50 & 50 & $5 \mathbf{v}$ & su & su & su \\
\hline trans-1,3-Dichloropropene & su & su & su & $5 \mathbf{v}$ & su & 5u & su & su \\
\hline Bthylbenzene & 5u & su & su & 5u & 5u & su & 5u & su \\
\hline 2-Hexanone & 100 & 100 & 100 & 100 & 100 & 100 & 100 & 100 \\
\hline A-Methy 1-2-pentanone & 100 & 100 & 2 & 100 & 100 & 100 & 100 & 100 \\
\hline Methylene chloride & 5u & 3 & 1 & 5u & 5u & 2 & 5u & su \\
\hline Styrene & su & 5u & 5u & 5u & 50 & $5 \mathbf{v}$ & 5u & 5u \\
\hline $1,1,2,2$-Tetrachloroethane & 50 & 5u & su & 50 & su & $5 \mathbf{u}$ & su & 5u \\
\hline Tetrachloroethene & su & 5u & su & 5u & 50 & su & su & su \\
\hline Toluene & 50 & su & 5u & 5u & 50 & 5u & 50 & 5u \\
\hline $1,1,1$-Trichloroethane & su & su & $5 v$ & su & su & 50 & 5u & su \\
\hline 1,1,2-Trichloroethane & su & 5u & su & 50 & su & 50 & su & su \\
\hline Trichloroathene & 50 & su & $5 \mathbf{v}$ & 50 & 50 & su & 50 & 50 \\
\hline vinyl acetate & 100 & 100 & 100 & 100 & 100 & 100 & 100 & 100 \\
\hline Vinyl chloride & 100 & 100 & 100 & 100 & 100 & 100 & 100 & 100 \\
\hline Xylenes & 50 & 5u & 5u & 50 & $5 \mathbf{u}$ & 5u & su & 5U \\
\hline
\end{tabular}

(CONTINUED) 
Groundwater and Surface-Water Quality Data, 1993

\begin{tabular}{|c|c|}
\hline \multirow{3}{*}{$\begin{array}{l}\text { Sampling Point } \\
\text { Location } \\
\text { - } \\
\text { Date Sampled }\end{array}$} & GW-800 \\
\hline & OLF \\
\hline & $10 / 22 / 93$ \\
\hline VOLATILE ORGANICS (ug/L) & - \\
\hline Acetone & 100 \\
\hline Benzene & $5 \mathbf{U}$ \\
\hline Bromodichloromethane & $5 v$ \\
\hline Bromoform & 50 \\
\hline Bromomethane & 100 \\
\hline 2-Butanone & 100 \\
\hline Carbon disulfide & $\mathbf{5 u}$ \\
\hline Carbon tetrachloride & $5 \mathbf{v}$ \\
\hline Chlorobenzene & $\mathbf{5 u}$ \\
\hline Chlorodibromomethane & $5 \mathbf{v}$ \\
\hline Chloroethane & 100 \\
\hline Chloroform & 50 \\
\hline Chloromethane & 100 \\
\hline 1,1-Dichloroethane & 5u \\
\hline 1,2-Dichloroethane & $\mathbf{5 u}$ \\
\hline 1,1-Dichloroethene & $5 \mathbf{5}$ \\
\hline 1,2-Dichloroethene & $\mathbf{5 v}$ \\
\hline 1,2-Dichloropropane & 50 \\
\hline cis-1,3-Dichloropropene & 50 \\
\hline trans-1,3-Dichloropropene & $5 \boldsymbol{U}$ \\
\hline Ethylbenzene & $5 \mathbf{v}$ \\
\hline 2-Hexanone & 100 \\
\hline 4-Methyl-2-pentanone & 100 \\
\hline Methylene chloride & 50 \\
\hline Styrene & 50 \\
\hline $1,1,2,2$-Tetrachloroethane & 50 \\
\hline Tetrachloroethene & $5 \mathbf{5}$ \\
\hline Toluene & $5 \mathbf{U}$ \\
\hline 1,1,1-Trichloroethane & $5 \mathbf{U}$ \\
\hline 1,1,2-Trichloroethane & 5u \\
\hline Trichloroethene & 5u \\
\hline Vinyl acetate & 100 \\
\hline Vinyl chloride & 100 \\
\hline Xylenes & 50 \\
\hline
\end{tabular}


APPENDIX $\mathrm{E} .2$

Groundwater and Surface-Water Quality Data, 1993

\begin{tabular}{|c|c|c|c|c|c|c|c|c|}
\hline \multirow{3}{*}{$\begin{array}{l}\text { Sampling Point } \\
\text { - } \\
\text { Iocation } \\
\text { Date Sampled }\end{array}$} & \multicolumn{4}{|c|}{$\mathrm{BCK}-00.63$} & \multicolumn{4}{|c|}{ BCK-04.55 } \\
\hline & \multicolumn{4}{|c|}{$\mathbf{E X P}$} & \multicolumn{4}{|c|}{$\mathbf{E X P}$} \\
\hline & $03 / 09 / 93$ & $05 / 03 / 93$ & $08 / 16 / 93$ & $11 / 08 / 93$ & $03 / 09 / 93$ & $05 / 03 / 93$ & $08 / 16 / 93$ & $11 / 08 / 93$ \\
\hline VOLATIIE ORGANICS $(\mathrm{ug} / \mathrm{I})$ & $\cdot$ & $\cdot$ & - & $\cdot$ & $\cdot$ & - & - & - \\
\hline Acetone & 100 & $10 \mathrm{U}$ & 100 & 100 & 1 & 100 & 100 & 100 \\
\hline Benzene & $5 \mathbf{v}$ & 5u & $5 \mathbf{u}$ & 5U & 50 & $5 \mathbf{v}$ & $\mathbf{5 0}$ & $5 v$ \\
\hline Bromodichloromethane & 50 & 50 & $5 u$ & 50 & $5 \mathbf{u}$ & 50 & $5 \mathbf{v}$ & $5 u$ \\
\hline Bromoform & $5 \mathbf{v}$ & $5 \mathbf{u}$ & $5 \mathbf{u}$ & 50 & $5 v$ & $5 \mathbf{u}$ & $5 \mathbf{v}$ & $5 \mathbf{U}$ \\
\hline Bromomethane & 100 & 100 & 100 & 100 & 100 & 100 & 100 & 100 \\
\hline 2-Butanone & 100 & 100 & 100 & 100 & 100 & 100 & 100 & 100 \\
\hline Carbon disulfide & $5 \mathbf{v}$ & $5 \mathbf{v}$ & 5u & 5U & $5 \mathbf{U}$ & $5 v$ & 50 & $5 v$ \\
\hline Carbon tetrachloride & $5 \mathbf{v}$ & $5 v$ & $5 v$ & 50 & 5v & $5 \mathbf{v}$ & $5 \mathbf{U}$ & 50 \\
\hline Chlorobenzene & $5 \mathbf{v}$ & 5u & $5 \mathbf{v}$ & 50 & 50 & $5 \mathbf{v}$ & 50 & 50 \\
\hline Chlorodibromomethane & $\mathbf{5 u}$ & $5 \mathbf{v}$ & 50 & 50 & $5 \mathbf{u}$ & $5 \mathbf{v}$ & $5 \mathbf{v}$ & $5 \mathbf{v}$ \\
\hline Chloroethane & 100 & $10 \mathrm{U}$ & $10 \mathrm{v}$ & 100 & 100 & 100 & 100 & 100 \\
\hline Chloroform & $5 \mathbf{v}$ & $5 \mathbf{u}$ & $5 U$ & $5 \mathbf{v}$ & 50 & $5 \mathbf{v}$ & 50 & $5 v$ \\
\hline Chloromethane & 100 & 100 & 100 & 100 & 100 & 100 & 100 & 100 \\
\hline 1,1-Dichloroethane & $5 \mathbf{v}$ & 50 & $5 \mathbf{v}$ & 5u & 5u & $\mathbf{5 v}$ & $5 \mathbf{u}$ & $5 \mathbf{v}$ \\
\hline 1,2-Dichloroethane & $5 \mathbf{u}$ & 5u & $5 \mathbf{u}$ & $5 \mathbf{v}$ & $5 \mathbf{5}$ & $5 \mathbf{v}$ & $\mathbf{5 u}$ & $5 \mathbf{U}$ \\
\hline 1,1-Dichloroethene & $5 \mathbf{v}$ & 50 & $5 \mathbf{v}$ & $5 \mathbf{v}$ & 50 & 50 & $5 \mathbf{v}$ & $5 \mathbf{U}$ \\
\hline 1,2-Dichloroethene & $5 \mathbf{v}$ & 50 & $5 \mathbf{v}$ & $5 v$ & 50 & $5 \mathbf{v}$ & $5 \mathbf{v}$ & $5 \mathbf{5}$ \\
\hline 1,2-Dichloropropane & 50 & 50 & 50 & $5 v$ & 50 & $5 \mathbf{v}$ & $5 \mathbf{v}$ & $5 \mathbf{v}$ \\
\hline cis-1, 3-Dichloropropene & $5 \mathbf{v}$ & 50 & $5 \mathbf{v}$ & 5u & 5u & 50 & $5 \mathbf{v}$ & 5u \\
\hline $\operatorname{trans-1,3-Dichloropropene~}$ & $5 v$ & $5 \mathrm{v}$ & $5 \mathbf{v}$ & 50 & 50 & $\mathbf{5 u}$ & 50 & $5 \mathbf{v}$ \\
\hline Ethylbenzene & 5v & 5v & $5 \mathbf{u}$ & $5 \mathbf{v}$ & $5 \mathbf{v}$ & $5 \mathbf{v}$ & 5u & $5 \mathbf{v}$ \\
\hline 2-Hexanone & 100 & 100 & 100 & 100 & 100 & 100 & $10 U$ & 100 \\
\hline 4-Methy1-2-pentanone & 2 & 1 & 100 & 100 & 2 & 1 & 100 & 100 \\
\hline Methylene chloride & 2 & 2 & $5 \mathbf{v}$ & 1 & 2 & 2 & $\mathbf{5 u}$ & 1 \\
\hline Styrene & $5 \mathbf{u}$ & 50 & $5 \mathbf{u}$ & $5 \mathbf{v}$ & $5 \mathbf{u}$ & 50 & $\mathbf{5 u}$ & 50 \\
\hline $1,1,2,2$-Tetrachloroethane & 5u & 50 & $5 \mathbf{u}$ & $\mathbf{5 U}$ & 5u & $5 \mathbf{v}$ & $5 \mathbf{v}$ & $\mathbf{5 u}$ \\
\hline Tetrachloroethene & 50 & 50 & $5 \mathbf{v}$ & $5 \mathbf{U}$ & 5u & $5 \mathbf{v}$ & $\mathbf{5 u}$ & 50 \\
\hline Toluene & $5 \mathbf{u}$ & 5U & $5 \mathbf{v}$ & $5 \mathbf{u}$ & 5u & 50 & $5 v$ & $5 \mathbf{u}$ \\
\hline 1,1,1-Trichloroethane & 50 & $5 v$ & $5 \mathbf{v}$ & 50 & $5 v$ & $5 \mathbf{v}$ & $5 \mathbf{v}$ & $5 \mathbf{u}$ \\
\hline $1,1,2$-Trichloroethane & $5 \mathbf{v}$ & su & $5 \mathbf{U}$ & $5 \mathbf{U}$ & $5 \mathbf{v}$ & $5 \mathbf{U}$ & $5 \mathbf{U}$ & $5 \mathbf{5}$ \\
\hline Trichloroethene & $5 \mathbf{v}$ & 50 & $\mathbf{5 u}$ & $5 \mathbf{U}$ & $5 \mathbf{u}$ & $5 \mathbf{v}$ & $5 \mathbf{5}$ & $5 \mathbf{U}$ \\
\hline Vinyl acetate & 100 & 100 & 100 & $10 U$ & 100 & 100 & 100 & 100 \\
\hline Vinyl chloride & 100 & 100 & 100 & 100 & 100 & 100 & 100 & 100 \\
\hline Xylenes & 50 & $5 v$ & $5 v$ & $5 \mathbf{v}$ & $5 \mathrm{U}$ & $5 v$ & $5 \mathbf{U}$ & 50 \\
\hline
\end{tabular}

(CONTINUED) 
APPEADIX E.2

Groundwater and Surface-Water Quality Data, 1993

\begin{tabular}{|c|c|c|c|c|c|c|c|c|}
\hline \multirow{3}{*}{$\begin{array}{l}\text { Sampling Point } \\
\text { Location } \\
\text { Date sampled }\end{array}$} & \multicolumn{4}{|c|}{ BCK-09.40 } & \multicolumn{4}{|c|}{ NT-01 } \\
\hline & \multicolumn{4}{|c|}{$\mathbf{E X P}$} & \multicolumn{4}{|c|}{$\mathbf{E X P}$} \\
\hline & $03 / 09 / 93$ & $05 / 03 / 93$ & $08 / 16 / 93$ & $11 / 08 / 93$ & $03 / 09 / 93$ & $05 / 03 / 93$ & $08 / 16 / 93$ & $11 / 08 / 93$ \\
\hline VOLATILE ORGANICS (ug/L) & $\cdot$ & $\cdot$ & $\cdot$ & $\cdot$ & - & - & $\cdot$ & - \\
\hline Acetone & 100 & 100 & 100 & 100 & 100 & $\dot{3}$ & 100 & 100 \\
\hline Benzene & 50 & 50 & 50 & 50 & 5u & su & 5v & su \\
\hline Bromodichloromethane & $5 u$ & 5u & 50 & 5u & 5u & 5u & 5u & 5u \\
\hline Bromoform & su & 50 & 5u & 5u & su & 50 & 50 & 5u \\
\hline Bromomethane & 100 & 100 & 100 & 100 & 100 & 100 & 100 & 100 \\
\hline 2-Butanone & 100 & 100 & 100 & 100 & 100 & 100 & 100 & 100 \\
\hline Carbon disulfide & 50 & 5u & 50 & 5U & 50 & 50 & 5u & 50 \\
\hline Carbon tetrachloride & 50 & 50 & 5u & 5u & su & 50 & su & 50 \\
\hline Chlorobenzene & su & 50 & 50 & 5u & 5u & 50 & $5 \mathbf{u}$ & 50 \\
\hline Chlorodibromomethane & su & 5u & 5u & 50 & 5u & 5u & su & 5u \\
\hline Chloroethane & 100 & 100 & 100 & 100 & 100 & 100 & 100 & 100 \\
\hline Chloroform & 5u & 5u & 50 & 5u & 50 & 50 & su & 50 \\
\hline Chloromethane & 100 & 100 & 100 & 100 & 100 & 100 & 100 & 100 \\
\hline 1,1-Dichloroethane & 50 & 5u & 5u & su & 5u & 50 & 5u & 5u \\
\hline 1,2-Dichloroethane & 50 & 50 & 5u & 50 & 5u & 5u & 5u & 50 \\
\hline 1,1-Dichloroethene & 5v & su & 5u & 50 & 5v & 5v & 5u & 5U \\
\hline 1,2-Dichloroethene & 8 & 9 & 1 & 5u & 5u & 5u & 5u & 50 \\
\hline 1,2-Dichloropropane & su & 50 & 5v & 5v & 5v & 5u & su & $5 \mathbf{v}$ \\
\hline Cis-1,3-Dichloropropene & 50 & 50 & 5u & 5v & 50 & 50 & 5u & 50 \\
\hline trans-1,3-Dichloropropene & 50 & 50 & 5u & 50 & 50 & 50 & su & 5u \\
\hline Ethylbenzene & $5 \mathbf{u}$ & 50 & 5u & su & 5u & 5u & su & 5u \\
\hline 2-Hexanone & 100 & 100 & 100 & 100 & 100 & 100 & 100 & 100 \\
\hline 4-Methy 1-2-pentanone & 100 & 1 & 100 & 100 & 100 & 2 & 100 & 100 \\
\hline Methylene chloride & 5u & 2 & 1 & su & 1 & 2 & 1 & su \\
\hline Styrene & 5u & 5u & 50 & 50 & 50 & 5u & 50 & 50 \\
\hline $1,1,2,2$-Tetrachloroethane & 50 & 5u & 5u & 5u & 50 & 5u & 5u & 50 \\
\hline Tetrachloroethene & 1 & 1 & 5u & 5u & 4 & 1 & 2 & 3 \\
\hline Toluene & su & 50 & 50 & 50 & 5u & su & $5 v$ & SU \\
\hline $1,1,1$-Trichloroethane & 50 & 5v & 5u & 5u & 50 & 5u & 50 & 5u \\
\hline $1,1,2-\operatorname{Tr} 1 \mathrm{ch}$ loroethane & 5u & 5u & 5u & $5 \mathbf{u}$ & 50 & 50 & 50 & 50 \\
\hline Trichloroethene & 5u & 5u & 0.7 & su & 50 & 5u & $5 \mathbf{v}$ & 5u \\
\hline vinyl acetate & 100 & 100 & 100 & 100 & 100 & 100 & 100 & 100 \\
\hline vinyl chloride & 100 & 100 & 100 & 100 & 100 & 100 & 100 & 100 \\
\hline xylenes & $5 u$ & 50 & 5u & 5u & 5u & su & 5u & 50 \\
\hline
\end{tabular}

(CONTINUED) 
APPENDIX E.2

Groundwater and Surface-Water Quality Data, 1993

\begin{tabular}{|c|c|c|c|c|c|c|c|c|}
\hline \multirow{3}{*}{$\begin{array}{l}\text { Sampling Point } \\
\text { Location } \\
\text { Date Sampled }\end{array}$} & \multicolumn{4}{|c|}{$N T-13$} & \multicolumn{4}{|c|}{ ss-1 } \\
\hline & \multicolumn{4}{|c|}{$\mathbf{E X P}$} & \multicolumn{4}{|c|}{$\mathbf{E x P}$} \\
\hline & $03 / 09 / 93$ & $05 / 03 / 93$ & $08 / 16 / 93$ & $11 / 08 / 93$ & $03 / 09 / 93$ & $05 / 03 / 93$ & $08 / 16 / 93$ & $11 / 08 / 93$ \\
\hline VOLATITE ORGANICS (Ug/L) & $\cdot$ & $\cdot$ & $\cdot$ & $\cdot$ & . & $\cdot$ & . & - \\
\hline Acetons & 1 & 100 & 100 & 100 & 100 & 100 & 100 & $100^{\circ}$ \\
\hline Benzene & 50 & 5u & 50 & 50 & $5 v$ & $\mathbf{5 u}$ & 50 & su \\
\hline Bromodichloromethane & 5u & 50 & 5u & 5u & $5 u$ & 5u & 50 & 5u \\
\hline Bromoform & 5u & 50 & su & $5 u$ & $5 u$ & 5u & 5v & 5u \\
\hline Bromomethane & 100 & 100 & 100 & 100 & 100 & 100 & 100 & $10 \mathrm{U}$ \\
\hline 2-Butanone & 100 & 100 & 100 & 100 & 100 & 100 & 100 & 100 \\
\hline Carbon disulfide & 50 & 50 & 50 & 50 & 50 & su & 50 & 50 \\
\hline Carbon tetrachloride & 5u & 5v & 5u & 5u & 5v & su & 50 & su \\
\hline Chlorobenzene & 50 & 50 & 50 & $5 U$ & 50 & 50 & 50 & $5 \mathbf{v}$ \\
\hline Chlorodibromomethane & 50 & 50 & 5u & 5u & 5u & 50 & 50 & 50 \\
\hline Chloroethane & 100 & 100 & 100 & 100 & 100 & 100 & 100 & 100 \\
\hline Chloroform & 50 & 50 & 50 & $5 u$ & 0.7 & 5u & 5u & $5 \mathbf{U}$ \\
\hline Chloromethane & 100 & 100 & 100 & 100 & 100 & 100 & 100 & 100 \\
\hline 1,1-Dichloroethane & 50 & $5 \mathbf{u}$ & 5u & 50 & 50 & 50 & 50 & $5 \mathbf{v}$ \\
\hline 1,2-Dichloroethane & 5u & 50 & 5u & 50 & 50 & 50 & 50 & 50 \\
\hline 1,1-Dichloroethene & 5u & $5 v$ & 50 & 50 & 50 & 50 & 5u & $5 \mathbf{u}$ \\
\hline 1,2-Dichloroethene & 50 & su & 5u & 50 & 50 & 50 & 5u & 50 \\
\hline 1,2-Dichloropropane & 50 & su & 5u & 50 & su & 5u & 5u & 50 \\
\hline Cis-1,3-Dichloropropene & 50 & 5u & $5 \mathbf{v}$ & 50 & 50 & 5u & 50 & 50 \\
\hline $\operatorname{trans-1}, 3-$ Dichloropropene & su & su & 5u & 50 & 5u & 5v & 5u & 5u \\
\hline Ethylbenzene & 5u & su & 5u & 5u & su & 5u & 50 & 50 \\
\hline 2-Hexanone & 100 & 100 & 100 & 100 & 100 & 100 & 100 & 100 \\
\hline 4-Methy1-2-pentanone & 2 & 1 & 100 & 100 & 100 & 1 & 100 & 100 \\
\hline Methylene chloride & 2 & 2 & 5u & 1 & 0.9 & 2 & 50 & $5 u$ \\
\hline Styrene & 5u & 50 & 50 & $\therefore 50$ & 5u & 5u & 5U & 50 \\
\hline $1,1,2,2$-Tetrachloroethane & 50 & $5 v$ & 50 & 5u & 50 & 50 & 5u & 50 \\
\hline Tetrachloroethene & 50 & 5u & 5u & 50 & 2 & 3 & $5 U$ & 1 \\
\hline Toluene & 5u & 5u & 50 & 5u & 5u & 5u & 50 & 50 \\
\hline 1,1,1-Trichloroethane & 5u & 50 & 5u & 50 & $5 \mathbf{v}$ & 5u & 50 & 50 \\
\hline 1,1,2-Trichloroethane & 5u & 5u & 5u & 5u & 5u & 5u & $5 U$ & 50 \\
\hline Trichloroethene & 50 & 50 & 50 & 50 & $5 v$ & 50 & 5U & 50 \\
\hline vinyl acetate & 100 & 100 & 100 & 100 & 100 & 100 & 100 & 100 \\
\hline Vinyl chloride & 100 & 100 & 100 & 100 & 100 & 100 & 100 & 100 \\
\hline Xylenes & $5 \mathrm{U}$ & $5 \mathbf{v u}$ & $5 u$ & 5u & 50 & $5 u$ & 50 & 50 \\
\hline
\end{tabular}

(CONT INUED) 
APPENDIX E.2

Groundwater and Surface-Water Quallty Data, 1993

\begin{tabular}{|c|c|c|c|c|c|c|c|c|}
\hline \multirow{3}{*}{$\begin{array}{l}\text { Sampling Point } \\
\text { Location } \\
\text { Date sampled }\end{array}$} & \multicolumn{4}{|c|}{ ss-4 } & \multicolumn{4}{|c|}{ ss-5 } \\
\hline & \multicolumn{4}{|c|}{$\mathbf{E X P}$} & \multicolumn{4}{|c|}{ EXP } \\
\hline & $03 / 09 / 93$ & $05 / 03 / 93$ & $08 / 16 / 93$ & $11 / 08 / 93$ & $03 / 09 / 93$ & $05 / 03 / 93$ & $08 / 16 / 93$ & $11 / 08 / 93$ \\
\hline VOWATIIE ORGANICS (ug/L) & - & $\cdot$ & $\cdot$ & $\cdot$ & - & - & - & - \\
\hline Acetone & 100 & 100 & 100 & 100 & 100 & 2 & 100 & $100^{\circ}$ \\
\hline Benzene & $\mathbf{s u}$ & 5u & 5u & 5u & su & 50 & 50 & 5u \\
\hline Bromodichloromethane & 50 & 5u & 5u & 50 & 5u & 50 & $5 u$ & 50 \\
\hline Bromoform & 50 & $5 \mathbf{v}$ & 50 & 50 & 5u & 50 & 5u & 5u \\
\hline Bromomethane & 100 & 100 & 100 & 100 & 100 & 100 & 100 & 100 \\
\hline 2-Butanone & 100 & 100 & 100 & 100 & 100 & 100 & 100 & 100 \\
\hline Carbon disulfide & su & 5v & 5u & $5 \mathbf{v}$ & 5u & 5u & su & 50 \\
\hline Carbon tetrachloride & 5u & 5u & 50 & $5 \mathbf{v}$ & 5u & 50 & 5u & su \\
\hline Chlorobenzene & 5u & 50 & 50 & su & 5u & 5v & $5 u$ & 5v \\
\hline Chlorodibromomethane & 5u & so & 50 & su & 5u & 50 & 5u & 50 \\
\hline Chloroethane & 100 & 100 & 100 & 100 & 100 & 100 & 100 & 100 \\
\hline Chloroform & 50 & 5u & 5u & 50 & 5u & 50 & $5 u$ & 5u \\
\hline Chloromethane & 100 & 100 & 100 & 100 & 100 & 100 & 100 & 100 \\
\hline 1,1-Dichloroethane & $5 \mathbf{u}$ & 50 & 5u & su & 5u & $5 v$ & 50 & 5u \\
\hline 1,2-Dichloroethane & su & 5u & $5 \mathbf{v}$ & 50 & su & 5u & 50 & 50 \\
\hline 1,1-Dichloroethene & 50 & 50 & 50 & 50 & $5 v$ & $5 v$ & 50 & 5u \\
\hline 1,2-Dichloroethene & 2 & 4 & 7 & 3 & 5u & $5 \mathrm{v}$ & 2 & 50 \\
\hline 1,2-Dichloropropane & 50 & $5 v$ & 50 & $5 v$ & su & $5 \mathrm{v}$ & 5u & $5 \mathbf{v}$ \\
\hline Cis-1,3-Dichloropropene & 5u & $5 v$ & su & $5 \mathbf{v}$ & 50 & 50 & 5u & 5u \\
\hline trans-1,3-Dichloropropene & su & 50 & $5 \mathbf{v}$ & $5 \mathbf{v}$ & su & $5 \mathbf{v}$ & 50 & 50 \\
\hline Ethylbenzene & 5u & 50 & 50 & 5u & 5u & 50 & 50 & 5u \\
\hline 2-Hexanone & 100 & 100 & 100 & 100 & 100 & 100 & 100 & 100 \\
\hline 4-Methy1-2-pentanone & 100 & 1 & 100 & 100 & 100 & 1 & 100 & 100 \\
\hline Methylene chloride & $5 \mathbf{v}$ & 2 & 1 & 50 & 50 & 2 & $5 \mathbf{u}$ & 5u \\
\hline styrene & 5u & 50 & 50 & 50 & 5u & 50 & su & Su \\
\hline $1,1,2,2$-Tetrachloroethane & su & 50 & 50 & 5u & $5 v$ & $5 \mathbf{v}$ & 5u & 5u \\
\hline Tetrachloroethene & 5u & 5u & $5 \mathrm{v}$ & 50 & 50 & 50 & 50 & 50 \\
\hline Toluene & 50 & 50 & $5 \mathbf{v}$ & 50 & 5v & 50 & 50 & $5 \mathrm{U}$ \\
\hline $1,1,1$-Trichloroethane & $5 v$ & 50 & 50 & 5u & 50 & $5 v$ & 5u & $5 \mathbf{v}$ \\
\hline $1,1,2$-Trichloroethane & 5u & 50 & 50 & 50 & 50 & $5 v$ & su & $5 \mathbf{v}$ \\
\hline Trichloroethene & 4 & 4 & 4 & 4 & 50 & 50 & 2 & 1 \\
\hline Vinyl acetate & 100 & 100 & 100 & 100 & 100 & 100 & 100 & 100 \\
\hline Vinyl chloride & 100 & 100 & 100 & 100 & 100 & 100 & 100 & 100 \\
\hline Xylenes & 50 & 50 & $5 v$ & $5 u$ & 5u & $5 \mathbf{v}$ & $5 v$ & 50 \\
\hline
\end{tabular}

(CONTINUED) 
APPENDIX 8.2

Groundwater and Surface-Water Quality Data, 1993

\begin{tabular}{|c|c|c|c|c|c|c|c|c|}
\hline \multirow{3}{*}{$\begin{array}{l}\text { Sampling Point } \\
\text { Location } \\
\text { Date Sampled }\end{array}$} & \multicolumn{4}{|c|}{$88-6$} & \multicolumn{4}{|c|}{ 8s-8 } \\
\hline & \multicolumn{4}{|c|}{ EXP } & \multicolumn{4}{|c|}{$\mathbf{E X P}$} \\
\hline & $03 / 09 / 93$ & $05 / 03 / 93$ & $08 / 16 / 93$ & $11 / 08 / 93$ & $03 / 09 / 93$ & $05 / 03 / 93$ & $08 / 16 / 93$ & $11 / 08 / 93$ \\
\hline VOLATILE ORGANICS (ug/L) & $\cdot$ & $\cdot$ & $\cdot$ & $\cdot$ & $\cdot$ & $\cdot$ & $\cdot$ & - \\
\hline Acetone & 100 & 100 & 100 & 100 & $10 \mathrm{u}$ & 100 & 100 & 100 \\
\hline Benzene & su & su & $5 v$ & su & 50 & 5u & 5u & su \\
\hline Bromodichloromethane & su & 5u & su & su & 50 & su & 50 & 5u \\
\hline Bromoform & 201 & 5v & 5u & 5u & 5v & 50 & 50 & su \\
\hline Bromomethane & 100 & 100 & 100 & 100 & 100 & 100 & 100 & 100 \\
\hline 2-Butanone & 100 & 100 & 100 & 100 & 100 & 100 & 100 & 100 \\
\hline Carbon disulfide & 50 & 5u & 5u & $5 \mathbf{v}$ & 50 & 50 & 50 & su \\
\hline Carbon tetrachloride & $5 \mathbf{u}$ & su & 5u & 50 & su & 5u & 5u & 5u \\
\hline Chlorobenzene & 50 & 5v & 50 & su & 50 & 50 & 50 & 5u \\
\hline Chlorodibromomethane & 50 & su & 5v & 50 & 50 & 5u & 5u & 5u \\
\hline Chloroethane & 100 & 100 & 100 & 100 & 100 & 100 & 100 & 100 \\
\hline Chloroform & 50 & $5 u$ & 5u & 5u & 5u & 5u & su & so \\
\hline Chloromethane & 100 & 100 & 100 & 100 & 100 & 100 & 100 & 100 \\
\hline 1,1-Dichloroethane & 50 & 5u & 50 & $5 v$ & $5 v$ & 50 & 50 & 5u \\
\hline 1,2-Dichloroethane & su & 5u & 5u & 5v & $5 \mathbf{5 u}$ & 5u & 5u & 50 \\
\hline 1,1-Dichloroethene & 50 & $5 \mathbf{v}$ & 5u & 5u & 5u & 5u & su & 5u \\
\hline 1,2-Dichloroethene & 50 & 5u & 5u & 7 & 50 & 50 & 5u & 5u \\
\hline 1,2-Dichloropropane & 50 & $5 u$ & 5u & su & 50 & 5u & su & $5 \mathbf{U}$ \\
\hline Cis-1,3-Dichloropropene & 5u & 50 & 50 & 5u & 50 & 50 & $5 U$ & 5u \\
\hline trans-1,3-Dichloropropene & 50 & 5u & 5u & 5u & 50 & 5u & 50 & 5u \\
\hline Ethylbenzene & 50 & 5u & $5 u$ & 50 & 50 & 5u & 5u & 50 \\
\hline 2-Hexanone & 100 & 100 & 100 & 100 & 100 & 100 & 100 & 100 \\
\hline 4-Methy1-2-pentanone & 2 & 100 & 100 & 100 & 3 & 1 & 100 & 100 \\
\hline Methylene chloride & 2 & 2 & 50 & 5u & 2 & 2 & 5u & 1 \\
\hline Styrene & 5v & 50 & su & su & 50 & 50 & 5u & 50 \\
\hline $1,1,2,2$-Tetrachloroethane & 50 & 50 & 50 & 50 & 5u & 50 & 50 & 50 \\
\hline Tetrachloroethene & $5 v$ & $5 u$ & 5u & 1 & 50 & 50 & 50 & $5 v$ \\
\hline Toluene & 50 & 50 & 50 & 5u & 50 & su & 50 & 50 \\
\hline $1,1,1$-Trichloroethane & 50 & 50 & 5u & 5u & 50 & $5 u$ & 50 & $5 U$ \\
\hline 1,1,2-Trichloroethane & 50 & 50 & 50 & 5u & 5u & 50 & 5u & 5u \\
\hline Trichloroethene & 50 & 5u & 5u & 1 & 5u & 5u & 5u & 50 \\
\hline Vinyl acetate & 100 & 100 & $10 \mathrm{U}$ & 100 & 100 & 100 & 100 & 100 \\
\hline Vinyl chloride & 100 & 100 & 100 & 100 & 100 & 100 & 100 & 100 \\
\hline xylenes & 5u & 50 & 50 & 50 & 50 & 50 & 50 & 50 \\
\hline
\end{tabular}


APPENDIX E.3

FIELD, MISCELLANEOUS, AND

GROSS ALPHA AND GROSS BETA ACTIVITIES 


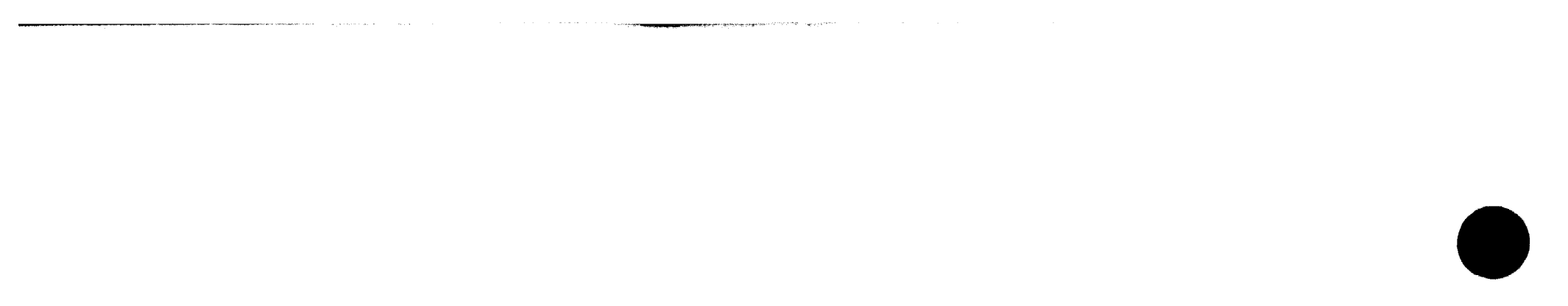

•

-

. 
APpundix 8.3

Oroundwater and surface-water Quallty Data, 1993

\begin{tabular}{|c|c|c|c|c|c|c|c|c|}
\hline \multirow{3}{*}{$\begin{array}{l}\text { sampling point } \\
\text { Loeation } \\
\text { Date sampled }\end{array}$} & \multicolumn{4}{|c|}{ On-013 } & \multicolumn{4}{|c|}{ ON-040 } \\
\hline & \multicolumn{4}{|c|}{ OLF } & \multicolumn{4}{|c|}{$\mathbf{B O}$} \\
\hline & $03 / 29 / 93$ & $06 / 17 / 93$ & $09 / 17 / 93$ & $12 / 19 / 93$ & $03 / 25 / 93$ & $\mid 05 / 11 / 93$ & $07 / 02 / 93$ & $10 / 09 / 93$ \\
\hline TIELD MEASUREMENTS & &. & - & $\cdot$ & - & $\cdot$ & $\cdot$ & • \\
\hline Dapth to Water (ft) & 2.33 & 5.4 & 6.5 & 3.3 & 11.55 & 18.93 & 21.2 & 21.2 \\
\hline Water remp (degreas C) & 12.5 & 16.8 & 20.1 & 14 & 16.2 & 17.1 & 18.1 & 16.8 \\
\hline pH (pH unite) & 6.6 & 6.3 & 6.5 & 6.6 & 6.9 & 5.8 & 6.4 & 5.5 \\
\hline sp. Cond. (unho/om) & 382 & 498 & 590 & 510 & 148 & 133 & 142 & 156 \\
\hline Dissolved Oxygen (ppm) & 1.9 & 1.7 & 5.9 & 4.3 & 6.3 & 4.4 & 4.5 & 1.7 \\
\hline Oxidation/Reduction (mV) & 40 & 45 & 206 & 44 & 205 & 204 & 36 & 142 \\
\hline MISCELLAMEOUS PARAMTERS & - & . & • &. & . & . & $\cdot$ & • \\
\hline pH (pH unites) & $6 . \dot{9}$ & 7.4 & $\dot{7}$ & $\dot{7}$ & 6.3 & $6 . \overline{7}$ & $6 . \overline{7}$ & 6.4 \\
\hline Sp. Cond. (wmho/cm) & 585 & 554 & 576 & 567 & 250 & 248 & 151 & 158 \\
\hline TDS (mg/h) & 354 & 336 & 338 & 334 & 216 & 344 & 232 & 228 \\
\hline TsS (mg/L) & 4 & 2 & 4 & 6 & 726 & 422 & 159 & 78 \\
\hline Turbidity (ATU) & 12 & 4.8 & 11 & 4.1 & 1000 & 300 & 180 & 120 \\
\hline RADIOCHEMICAL PARAKTIERS & . & . & . &. & - & . & . & • \\
\hline Gross Alpha (pC1/L) & 2.17 & -3.47 & -0.01 & -0.37 & 9.99 & -1.19 & 5.67 & -1.96 \\
\hline Grose Alphe $(\mathrm{CE}+/-)$ & $1.7 n$ & 1200.00 & 3.00 & 1.00 & 4.80 & 1.80 & 5.50 & 1.80 \\
\hline Grose Beta $(\mathrm{pCl} / \mathrm{L})$ & 1.76 & -12.50 & 2.65 & -2.53 & 22.70 & -3.19 & -8.38 & 2.97 \\
\hline Grose Beta $(\mathrm{CE}+/-)$ & & 2400.00 & 4.80 & 2.90 & 6.30 & 6.00 & 12.00 & 2.80 \\
\hline
\end{tabular}

(CONTINUED) 
AppundX 1.3

Oroundwater and surfece-water Quality Date, 1993

\begin{tabular}{|c|c|c|c|c|c|c|c|c|}
\hline \multirow{3}{*}{$\begin{array}{l}\text { Inpling polnt } \\
\text { tocation } \\
\text { Date sampled }\end{array}$} & \multirow{3}{*}{\begin{tabular}{|c|}
$0 w-042$ \\
00 \\
$12 / 29 / 93$ \\
\end{tabular}} & \multicolumn{4}{|c|}{$a N-043$} & \multicolumn{3}{|c|}{ OW-044 } \\
\hline & & \multicolumn{4}{|c|}{ ors } & \multicolumn{3}{|c|}{ ors } \\
\hline & & $02 / 04 / 93$ & $05 / 10 / 93$ & $09 / 11 / 93$ & $10 / 19 / 93$ & $02 / 04 / 93$ & $05 / 10 / 93$ & $09 / 12 / 83$ \\
\hline Fred masursunrs & $\cdot$ & $\cdot$ & $\cdot$ & $\cdot$ & $\cdot \mid$ & $\cdot$ & $\cdot$ & $\bullet$ \\
\hline Dapth to Mater (st) & 20.1 & 24.21 & 17.23 & 20.92 & 32 & 23.77 & 16.31 & 30.75 \\
\hline water Temp (degrees C) & 15.3 & 14.1 & 15.3 & 15 & 16.2 & 13.0 & 15 & 14 \\
\hline pH (pH undte) & 6.2 & 7 & 6.5 & 6.1 & 7.2 & 7.4 & 7.3 & 7.1 \\
\hline ap. Cond. (umho/on) & 138 & 305 & 123 & 137 & 171 & 287 & 227 & 253 \\
\hline Diasolved Oxygen (ppm) & 1.1 & 4.1 & 4.4 & 3.5 & 7.3 & 4.1 & 4.6 & 3.3 \\
\hline Oxldation/Reduction (mV) & 202 & 138 & 228 & 204 & 170 & 180 & 219 & 180 \\
\hline MIscenLATous parnitizes & $\cdot$ & $\cdot$ & $\cdot$ & & $\cdot$ & $\cdot$ & $\cdot \dot{1}$ & - \\
\hline pH (pH unite) & 6.4 & 7.7 & 7 & 6.7 & 6.0 & 0.2 & $\dot{8}$ & 7.6 \\
\hline 8p. Cond. (umho/cm) & 38 & 184 & 225 & 138 & 144 & 300 & 268 & 268 \\
\hline Tos $(\mathrm{mg} / \mathrm{L})$ & 36 & 132 & 206 & 220 & 132 & 212 & 178 & 188 \\
\hline $288(\mathrm{mg} / \mathrm{r})$ & $<1$ & 3 & 3 & s & 17 & 2 & $<1$ & $<1$ \\
\hline Turbidity (nTv) & 1.4 & 6 & 8.6 & 15 & 20 & 1.2 & 3.2 & 1.2 \\
\hline RADIOCHEMTCAL PARAMLERS & & - & - & - & . & - & . & • \\
\hline nrose alpha (pCl/L) & -2.48 & 2.01 & $1.6 \dot{0}$ & -2.48 & -0.62 & 1.29 & 2.13 & -1.95 \\
\hline :oes Alpha $(C x+1-1)$ & 1.90 & 1.30 & 0.80 & 2.30 & 2.10 & 1.30 & 0.78 & 2.60 \\
\hline Grose Beta $(\mathrm{pCi} / \mathrm{L})$ & -4.77 & 2.81 & 0.22 & -13.90 & -7.90 & 1.82 & 4.49 & -9.66 \\
\hline Grose Beta $(\mathrm{Cs}+1-1$ & 3.20 & 2.60 & 1.40 & 5.80 & 5.80 & 2.60 & 1.60 & 5.70 \\
\hline
\end{tabular}

(CONTINUED) 
Appenotx 8.3

Oroundwater and surfsee-water Quallty Data, 1993

\begin{tabular}{|c|c|c|c|c|c|c|c|c|}
\hline \multirow{3}{*}{$\begin{array}{l}\text { Sampling point } \\
\text { Lootion } \\
\text { Dete sampled }\end{array}$} & \multirow{3}{*}{\begin{tabular}{|c|}
$0 \mathrm{~N}-044$ \\
025 \\
$10 / 28 / 93$ \\
\end{tabular}} & \multirow{2}{*}{\multicolumn{2}{|c|}{$\frac{a n-052}{10}$}} & \multicolumn{4}{|c|}{ ow-0s6 } & \multirow{2}{*}{$\frac{0 w-037}{\exp }$} \\
\hline & & & & \multicolumn{4}{|c|}{$\mathbf{s 0}$} & \\
\hline & & $03 / 23 / 93$ & $04 / 26 / 93$ & $03 / 04 / 93$ & $04 / 22 / 93 \mid$ & $07 / 09 / 93$ & $10 / 09 / 93$ & $03 / 04 / 93$ \\
\hline 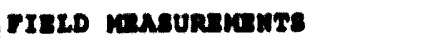 & & - & - & $\cdot$ & . & $\cdot$ & $\cdot$ & • \\
\hline Dopth to vater $(2 t)$ & 32.9 & 3.75 & 7.22 & 5.9 & 6.41 & 8.85 & 8.2 & 3.9 \\
\hline Water Tamp (degrees C) & 14.0 & 9.5 & 12 & 13.3 & 13.7 & 16.5 & 15.3 & 13.5 \\
\hline pH (pH undte) & 7.2 & 6.8 & 7.4 & 7.6 & 7.4 & 7 & 7.3 & 7.2 \\
\hline Ip. Cond. (umho/em) & 293 & 370 & 341 & 713 & 1191 & 978 & 866 & 1560 \\
\hline Diseolved Oxygen (ppm) & 7.1 & 7.9 & 3.5 & 7 & s) & 4.4 & 3.4 & 6.1 \\
\hline Oxddation/Raduotion (mV) & 255 & 206 & 164 & 62 & 141 & 38 & 34 & 169 \\
\hline MIsctLLAMOUS PARAMTIRS & . & $\cdot$ & $\cdot$ & $\cdot$ & $\cdot$ & $\cdot$ & $\cdot$ & - \\
\hline pH (pH unite) & 7.5 & 7.1 & 7.1 & $\dot{\theta}$ & $7 . \dot{0}$ & 7.7 & 7.3 & $7 . \dot{9}$ \\
\hline sp. Cond, (umho/cm) & 270 & 376 & 370 & 1080 & 1410 & 1050 & 969 & 1530 \\
\hline 208 (mg/L) & 210 & 216 & 286 & 584 & 746 & 598 & 630 & 734 \\
\hline 288 (mg/L) & $<1$ & 17 & 133 & 27 & 19 & 13 & 29 & 3 \\
\hline Turbldity (Nru) & 1.5 & 0.5 & 105 & 11 & 18 & 14 & 15 & 4.9 \\
\hline RADIOCKEMICAL PARANTERS & $\cdot$ & $\cdot$ & $\cdot$ & $\cdot$ & $\cdot$ & $\cdot$ & $\cdot$ & - \\
\hline Orose Alpha (pCi/L) & 0.61 & 20.00 & 32.70 & 3.82 & 2.70 & 4.54 & 1.19 & 6.76 \\
\hline Oross Alpha $(\mathrm{Cz}+1-)$ & 2.50 & 4.10 & 7.00 & 2.00 & 2.30 & 2.90 & 2.50 & 2.80 \\
\hline Orowe Beta $(\mathrm{PC} 1 / L)$ & -8.81 & 30.30 & 44.40 & 7.04 & 4.33 & 6.70 & 4.71 & 8.14 \\
\hline Orose Beta $(\mathrm{Cz}+1-1)$ & 5.60 & 4.10 & 7.50 & 3.30 & 2.90 & 3.10 & 3.20 & 3.60 \\
\hline
\end{tabular}

(CONTINUED) 
APREndX 2.3

Oroundwater and sureace-water Quality Data, 1993

\begin{tabular}{|c|c|c|c|c|c|c|c|c|}
\hline \multirow{3}{*}{$\begin{array}{l}\text { osmiling polnt } \\
\text { Locetion } \\
\text { Date sampled }\end{array}$} & \multicolumn{3}{|c|}{ ON-0S7 } & an-063 & \multicolumn{4}{|c|}{ ON-064 } \\
\hline & \multicolumn{3}{|c|}{$\mathbf{E x p}$} & ow & \multicolumn{4}{|c|}{ ors } \\
\hline & $|04 / 22 / 93|$ & $07 / 09 / 93$ & $10 / 09 / 93$ & $02 / 23 / 93$ & $02 / 09 / 93$ & $05 / 12 / 93$ & $09 / 11 / 93$ & $10 / 20 / 93$ \\
\hline FreLd masunenenrs & $\cdot$ & $\cdot$ & $\cdot$ & - & - & - & $\cdot$ & - \\
\hline Dopth to Water $(\mathrm{ft})$ & 5.16 & 7.15 & 6.98 & 23.83 & 25.8 & 26.51 & 28.3 & 27.68 \\
\hline Water ramp (degreas C) & 13 & 16.9 & 17.7 & 12.6 & 13.4 & 19.6 & 14.9 & 15.2 \\
\hline pH (pH units) & 7.2 & 6.0 & 6.9 & 7.1 & 7.1 & 7.3 & 7.5 & 7.1 \\
\hline sp. cond. (umho/cm) & 780 & 1039 & 940 & 452 & 635 & 359 & 571 & 669 \\
\hline Diseolved oxygen (ppm) & 4.3 & 4.9 & 6 & 9 & 2.4 & 2.5 & 2.8 & 7.8 \\
\hline Oxidation/Roduction (mV) & 134 & 68 & 143 & 204 & 203 & 155 & 114 & 222 \\
\hline MISCELIAYMOUS PARAMTIRRS & & & $\cdot$ &. &. & $\cdot$ & $\cdot$ & - \\
\hline pH (pH unite) & $7 . \dot{7}$ & $7 . \dot{3}$ & 7.1 & $7 . \dot{2}$ & $7 . \dot{2}$ & $7 . \dot{8}$ & $7 . \dot{6}$ & 7.3 \\
\hline 8p. Cond. (unho/om) & 920 & 1090 & 1050 & 174 & 203 & 636 & 660 & 658 \\
\hline $\operatorname{TDS}(m g / L)$ & 532 & 616 & 686 & 258 & 430 & 372 & 428 & 428 \\
\hline $288(m g / L)$ & 2 & 2 & $<1$ & 20 & 3 & 5 & 2 & 9 \\
\hline Turbidlty (mTU) & 2.5 & 2.6 & 1.1 & 16 & 16 & 9.5 & 5.8 & 13 \\
\hline RADTOCHAMTCAY PARNATERS & & $\cdot$ & . &. & $\cdot$ & $\cdot$ & $\cdot$ & - \\
\hline Oross Alpha (pCi/L) & 1.60 & 1.98 & 1.93 & 1.53 & 0.67 & -2.70 & -1.87 & -0.20 \\
\hline Orons Aphe $(\mathrm{Cz}+1-1$ & 3.80 & 2.70 & 2.80 & 2.10 & 2.90 & 4.20 & 3.10 & 1.30 \\
\hline arose Bata (pCi/L) & 6.43 & 4.92 & 3.23 & 5.84 & 11.90 & 15.40 & 4.71 & 13.80 \\
\hline Orone Beta $(C z+/-)$ & 3.20 & 3.10 & 3.10 & 3.00 & 3.60 & 6.50 & 6.20 & 3.40 \\
\hline
\end{tabular}

(CONrimued) 
APPIHDIX $\mathrm{R.3}$

Groundwater and surface-water Quallty Data, 1993

\begin{tabular}{|c|c|c|c|c|c|c|c|c|}
\hline \multirow{3}{*}{$\begin{array}{l}\text { 8ampling point } \\
\text { Lootion } \\
\text { Date sampled }\end{array}$} & \multicolumn{4}{|c|}{ ow-069 } & \multirow{3}{*}{\begin{tabular}{|c|} 
OW-079 \\
80 \\
$10 / 18 / 93$ \\
\end{tabular}} & \multirow{3}{*}{\begin{tabular}{|c|}
$0 N-080$ \\
80 \\
$10 / 10 / 93$ \\
\end{tabular}} & \multirow{2}{*}{\multicolumn{2}{|c|}{$\frac{a w-082}{80}$}} \\
\hline & \multicolumn{4}{|c|}{80} & & & & \\
\hline & $03 / 03 / 93$ & $04 / 21 / 93$ & $07 / 25 / 93$ & $10 / 10 / 93$ & & & $03 / 10 / 93$ & $04 / 21 / 93$ \\
\hline FI2W masuramars & $\cdot$ & - & - & - & - & $\cdot$ & - & - \\
\hline Dopth to Water (tt) & 8.13 & 7.8 & 10.98 & 11.15 & 25.65 & 25.85 & 20.11 & 19.65 \\
\hline Water remp (degrees $c$ ) & 14.3 & 14 & 16.2 & 14.6 & 15.7 & 13.9 & 15.3 & 13.6 \\
\hline pH (pH unite) & 9.2 & 8.8 & 8.7 & 0.4 & 8.1 & 6.4 & 7.5 & 7.6 \\
\hline 8p. Cond. (umho/cm) & 358 & 330 & 408 & 389 & 235 & 133 & 327 & 316 \\
\hline Dissolved Oxygen (ppm) & 2.4 & 3 & 1.5 & 0.7 & 1.3 & 0.4 & 3.5 & 2.3 \\
\hline Oxidation/Raduction (mV) & -112 & -47 & -155 & -233 & 70 & -18 & 178 & 48 \\
\hline MISCELIAMOUS PARMATERS & $\cdot$ & - & - & - & $\cdot$ & $\cdot$ & - & - \\
\hline pH (pH unite) & 8.2 & 8.8 & $8 . \dot{6}$ & $8 . \dot{6}$ & $8 . \dot{1}$ & 6.9 & $7 . \dot{5}$ & $7 . \dot{8}$ \\
\hline 8p. Cond. (unho/om) & 380 & 375 & 400 & 396 & 210 & 132 & 396 & 359 \\
\hline 208 (mg/L) & 236 & 204 & 232 & 292 & 130 & 180 & 248 & 208 \\
\hline $288(\mathrm{mg} / \mathrm{L})$ & $<1$ & $<1$ & $<1$ & $<1$ & $<1$ & 129 & 8 & $<1$ \\
\hline Turbidity (Arro) & 0.5 & 1 & 1.2 & 0.4 & 1.2 & 85 & 2.1 & 2.4 \\
\hline RADIOCKRMICAX PARAYTTRRS &. & - & . & - & . & $\cdot$ & - & • \\
\hline Gross Alpha (pCi/L) & 3.45 & 0.17 & -4.90 & -0.32 & -0.93 & 2.06 & 1.57 & 0.00 \\
\hline Crose Alpha $(\mathrm{Cz}+/-)$ & 1.70 & 1.60 & 2.50 & 1.80 & 2.00 & 2.30 & 3.90 & 1.70 \\
\hline Grose Beta $(\mathrm{pCl} / \mathrm{L})$ & 7.78 & 1.75 & 0.00 & 3.72 & -3.07 & 0.90 & 3.32 & -2.40 \\
\hline erone Beta $(\mathrm{CE}+/-)$ & 3.40 & 2.90 & 3.00 & 3.00 & 6.00 & 2.60 & 5.40 & 2.70 \\
\hline
\end{tabular}

(COATINUED) 
APPENDIX $\mathrm{E} .3$

Groundwater and surtace-water Quality Data, 1993

\begin{tabular}{|c|c|c|c|c|c|c|c|c|}
\hline \multirow{3}{*}{$\begin{array}{l}\text { Sampling Point } \\
\text { Iocation } \\
\text { Date sampled }\end{array}$} & \multicolumn{2}{|c|}{ OW-082 } & \multicolumn{4}{|c|}{ CW-084 } & \multicolumn{2}{|c|}{ CW-085 } \\
\hline & \multicolumn{2}{|c|}{ BO } & \multicolumn{4}{|c|}{ ow } & \multicolumn{2}{|c|}{ our } \\
\hline & $07 / 25 / 93$ & $10 / 18 / 93$ & $02 / 04 / 93$ & $05 / 10 / 93$ & $09 / 11 / 93$ & $10 / 20 / 93$ & $02 / 11 / 93$ & $05 / 12 / 93$ \\
\hline TIELD MBABUREMEKTS & $\cdot$ & $\cdot$ & $\cdot$ & $\cdot$ & $\cdot$ & $\cdot$ & $\cdot$ & • \\
\hline Depth to Water (ft) & 20.85 & 20.95 & 15 & 14.91 & 17.3 & 17 & 13.84 & 13.51 \\
\hline Water Temp (degrees C) & 15.7 & 14.8 & 15.3 & 16.2 & 13.8 & 16.1 & 14.7 & 17.9 \\
\hline pH (pH unita) & 7.9 & 7.4 & 7.8 & 7.7 & 7.7 & 7.6 & 7.2 & 7.1 \\
\hline sp. Cond. (umho/cm) & 361 & 341 & 279 & 256 & 277 & 306 & 1908 & 2090 \\
\hline Diseolved Oxygen (ppm) & 2.1 & 2.1 & 2.8 & 1.2 & 2 & 8 & 1.7 & 1.2 \\
\hline Oxidation/Reduction (mV) & 14 & 121 & 141 & 170 & 157 & 195 & 184 & 157 \\
\hline MISCELLANEOUS PARAMETERS & $\cdot$ & $\cdot$ & • & $\cdot$ &. & . & - & • \\
\hline pH (pH units) & 7.7 & 7.7 & 8.3 & 8.3 & 7.4 & 7.8 & $7 . \dot{2}$ & 7.8 \\
\hline sp. Cond. (unho/cm) & 404 & 370 & 317 & 304 & 302 & 295 & 2130 & 2330 \\
\hline $\operatorname{TDS}(\mathrm{mg} / \mathrm{L})$ & 270 & 210 & 218 & 208 & 206 & 216 & 1500 & 1768 \\
\hline Tss (mg/L) & 5 & $<1$ & 47 & 43 & 30 & 17 & $\mathbf{s}$ & 8 \\
\hline Turbidity (NTU) & 10 & 0.8 & 22 & 21 & 12 & 11 & 2.2 & 3 \\
\hline RADIOCHEMICAL PARAMETERS & $\cdot$ & . & - & $\cdot$ & . & • & - & - \\
\hline Orose Alpha (pCi/L) & -3.42 & -1.30 & 3.57 & 0.20 & -0.66 & 0.77 & 17.30 & -2.24 \\
\hline Grose Alpha $(\mathrm{CE}+/-1)$ & 2.50 & 2.40 & 1.80 & 0.74 & 2.90 & 1.40 & 4.10 & 7.20 \\
\hline Gross Beta $(\mathrm{pCi} / \mathrm{L})$ & -1.66 & -7.74 & 7.67 & -1.12 & -7.51 & 5.16 & 120.00 & 144.00 \\
\hline Gross Beta $(\mathrm{CE}+/-)$ & 2.90 & 5.60 & 3.00 & 1.30 & 5.80 & 2.70 & 7.00 & 12.00 \\
\hline
\end{tabular}

(CONTINUED) 
APPENDIX E. 3

Groundwater and Surface-water Quality Data, 1993

\begin{tabular}{|c|c|c|c|c|c|c|c|c|}
\hline \multirow{3}{*}{$\begin{array}{l}\text { Sampling Point } \\
\text { Location } \\
\text { Date Sampled }\end{array}$} & \multicolumn{2}{|c|}{ GW-085 } & \multicolumn{4}{|c|}{ GW-086 } & \multicolumn{2}{|c|}{ GW-115 } \\
\hline & \multicolumn{2}{|c|}{ orf } & \multicolumn{4}{|c|}{ OLF } & \multicolumn{2}{|c|}{$\mathbf{s 3}$} \\
\hline & $09 / 14 / 93$ & $10 / 22 / 93$ & $02 / 05 / 93$ & $05 / 11 / 93$ & $09 / 12 / 93$ & $10 / 21 / 93$ & $01 / 28 / 93$ & $05 / 25 / 93$ \\
\hline FIBLD MEASUREMENTS & - & - & - & - & - & $\cdot$ & $\cdot$ & - \\
\hline Depth to Water (ft) & 15.35 & 14.7 & 13.52 & 13.72 & 15.4 & 14.88 & 8.2 & 10.3 \\
\hline Water Temp (degrees $C$ ) & 18.7 & 11.2 & 15.6 & 18.7 & 16.3 & 17.5 & 16 & 16.7 \\
\hline pH (pH units) & 7.2 & 7.2 & 6.7 & 6.3 & 6.1 & 6 & 7.5 & 7.4 \\
\hline Sp. Cond. (umho/cm) & 2400 & 2410 & 252 & 215 & 245 & 253 & 414 & 361 \\
\hline Dissolved Oxygen (ppm) & 4.5 & 6 & 9.6 & 2.5 & 1.9 & 6.7 & 2 & 1.5 \\
\hline Oxidation/Reduction (mv) & 274 & 250 & 292 & 209 & 239 & 256 & -99 & -99 \\
\hline MISCELLANEOUS PARAMETERS & $\cdot$ & • & • & • & • & $\cdot \dot{ }$ & $\dot{\bullet}$ & $\cdot$ \\
\hline pH (pH units) & 7.5 & 7.2 & 6.3 & 6.7 & 6.4 & 6.5 & $7 . \dot{9}$ & $7 . \dot{6}$ \\
\hline Sp. Cond. (umho/cm) & 2650 & 2490 & 267 & 245 & 238 & 259 & 435 & 463 \\
\hline $\operatorname{TDS}(\mathrm{mg} / \mathrm{L})$ & 1950 & 1872 & 268 & 280 & 208 & 252 & 276 & 292 \\
\hline TSS (mg/L) & 1 & 3 & 511 & 1050 & 812 & 1318 & $<1$ & 1 \\
\hline Turbidity (NTU) & 1.8 & 0.7 & 320 & 480 & 360 & 620 & 1.9 & 4.1 \\
\hline RADIOCHEMICAI PARAMETERS & • & • & • & • & • & $\cdot$ & • & • \\
\hline Gross Alpha (pCi/L) & -3.08 & 0.44 & 5.10 & 9.27 & 8.75 & 7.92 & 2.35 & -1.99 \\
\hline Gross Alphe $(\mathrm{CE}+/-)$ & 3.30 & 2.10 & 2.30 & 2.30 & 5.20 & 6.40 & 1.80 & 3.30 \\
\hline Gross Beta (pCi/L) & 116.00 & 77.60 & 8.10 & 24.70 & 16.10 & 7.98 & 5.65 & -3.68 \\
\hline Gross Beta $(\mathrm{CE}+/-)$ & 16.00 & 9.60 & 4.30 & 3.40 & 7.00 & 10.00 & 3.00 & 5.40 \\
\hline
\end{tabular}

(CONTINUED) 
APPENDIX 8.3

Groundwater and Surface-Water Quality Data, 1993

\begin{tabular}{|c|c|c|c|c|c|c|c|c|}
\hline \multirow{3}{*}{$\begin{array}{l}\text { Sampling Point } \\
\text { Location } \\
\text { Date Sampled }\end{array}$} & \multicolumn{2}{|c|}{ GW-115 } & \multicolumn{4}{|c|}{$G W-118$} & $O W-162$ & $G W-286$ \\
\hline & \multicolumn{2}{|c|}{ s3 } & \multicolumn{4}{|c|}{ BG } & BG & $\mathbf{B G}$ \\
\hline & $09 / 11 / 93$ & $10 / 19 / 93$ & $03 / 04 / 93$ & $04 / 21 / 93$ & $07 / 25 / 93$ & $10 / 10 / 93$ & $10 / 10 / 93$ & $02 / 25 / 93$ \\
\hline FIEID MEASUREMENTS & $\cdot$ & $\cdot$ & • & $\cdot$ & - & $\cdot$ & $\cdot 1$ & $\bullet$ \\
\hline Depth to Water (ft) & 10.4 & 14.2 & 9.21 & 7.2 & 4.25 & 3.55 & 22.45 & 9.2 \\
\hline Water Temp (degrees C) & 16.2 & 17.4 & 13.6 & 13.1 & 17.3 & 16 & 14.2 & 7.1 \\
\hline $\mathrm{pH}$ (pH units) & 7.4 & 7.6 & 10.5 & 10 & 10.4 & 10 & 8.3 & 7.6 \\
\hline Sp. Cond. (umho/cm) & 413 & 439 & 8530 & 8080 & 9170 & 8930 & 224 & 279 \\
\hline Dissolved Oxygen (ppm) & 1.6 & 0.9 & 1.3 & 0.9 & 0.9 & 1.1 & 1.1 & 6.8 \\
\hline Oxidation/Reduction (mV) & -34 & 18 & 60 & 2 & -75 & -147 & $12: 3$ & 187 \\
\hline MISCELLAKEOUS PARAMETERS & $\cdot$ & $\cdot$ & - & $\cdot$ & $\cdot$ & - &. & • \\
\hline pH (pH units) & $7 . \dot{8}$ & $7 . \dot{7}$ & $9 . \dot{2}$ & $9 . \dot{8}$ & $10 . \dot{2}$ & $10 . \dot{1}$ & 8.1 & 7.9 \\
\hline Sp. Cond. (umho/cm) & 465 & 462 & 9440 & 9120 & 9210 & 9020 & 243 & 312 \\
\hline TDS (mg/L) & 298 & 310 & 5054 & 5016 & 5064 & 5116 & 236 & 202 \\
\hline TSS (mg/I) & $<1$ & $<1$ & 24 & 54 & 28 & 8 & 6 & 42 \\
\hline Turbidity (RTU) & 4.9 & 2.7 & 10 & 6 & 17 & 1 & 20 & 16 \\
\hline RADIOCHEMICAI PARAMETERS &. & . & - & - & - & - & $\cdot$ & - \\
\hline Gross Alpha (pci/L) & 0.18 & 1.43 & 6.60 & -2.52 & -52.30 & 9.63 & -0.31 & -0.33 \\
\hline Gross Alpha $(\mathrm{CE}+/-)$ & 1.10 & 1.60 & 3.90 & 11.00 & 29.00 & 22.00 & 2.10 & 1.50 \\
\hline Gross Beta $(\mathrm{pci} / \mathrm{L})$ & -0.64 & 4.06 & 5.51 & -1.49 & $-21 \cdot 30$ & 14.90 & -0.33 & -0.70 \\
\hline Gross Beta $(\mathrm{CE}+/-)$ & 2.60 & 2.60 & 6.60 & 12.00 & 29.00 & 54.00 & 2.60 & 2.60 \\
\hline
\end{tabular}

(CONTINURD) 
APPENDIX Z.3

Groundwater and Surface-Water Quallty Data, 1993

\begin{tabular}{|c|c|c|c|c|c|c|c|c|}
\hline \multirow{3}{*}{$\begin{array}{l}\text { Sampling point } \\
\text { Location } \\
\text { Date Sampled }\end{array}$} & \multicolumn{3}{|c|}{ GW-286 } & \multicolumn{4}{|c|}{$G W-287$} & $6 w-311$ \\
\hline & \multicolumn{3}{|c|}{ BG } & \multicolumn{4}{|c|}{ BG } & Rs \\
\hline & $04 / 19 / 93$ & $07 / 10 / 93$ & $10 / 07 / 93$ & $02 / 24 / 93$ & $04 / 16 / 93$ & $07 / 10 / 93$ & $10 / 07 / 93$ & $02 / 08 / 93$ \\
\hline FIELD MEASUREMENTS & - & - & . & - & $\cdot 1$ & - & - & - \\
\hline Depth to Water (ft) & 9.25 & 9.2 & 9.98 & 9 & 8.9 & 9.8 & 9.15 & 36.25 \\
\hline Water Temp (degrees C) & 16.2 & 16.7 & 16.9 & 4.5 & 11 & 18.7 & 18.1 & 14.1 \\
\hline pH (pH units) & 7.7 & 7.5 & 7.8 & 6.9 & 7.2 & 6.9 & 7 & 7.5 \\
\hline Sp. Cond. (umho/cm) & 272 & 243 & 271 & 229 & 252 & 218 & 241 & 403 \\
\hline Dissolved Oxygen (ppm) & 2.5 & 4 & 5.2 & 8.2 & 5.1 & 7.4 & 5.8 & 5.3 \\
\hline Oxidation/Reduction (mv) & 140 & 99 & 211 & 246 & 171 & 202 & 209 & 200 \\
\hline MISCELLANEOUS PARANETERS & - & . & . & . & $\cdot$ & . & $\cdot$ & . \\
\hline pH (pH units) & & $8 . \dot{1}$ & $7 . \dot{9}$ & $7 . \dot{5}$ & $7 . \dot{2}$ & $7 . \dot{7}$ & $7 . \dot{6}$ & 7.8 \\
\hline Sp. Cond. (umho/cm) & 281 & 277 & 288 & 255 & 261 & 272 & 288 & 453 \\
\hline $\operatorname{TDS}(\mathrm{mg} / \mathrm{L})$ & 192 & 278 & 248 & 156 & 142 & 166 & 254 & 288 \\
\hline TsS (mg/L) & 7 & $<1$ & 2 & 7 & 3 & 3 & 11 & 51 \\
\hline Turbidity (NTU) & 80 & 14 & 4.2 & 22 & 9 & 9 & 15 & 54 \\
\hline RADIOCHEMICAL PARAMETERS & $\bullet$ & . & . & . &. & . & $\cdot$ & • \\
\hline Gross Alpha (pCi/L) & 0.87 & 1.64 & 4.59 & -0.48 & 5.38 & 1.48 & 0.00 & -0.74 \\
\hline Gross Alpha $(\mathrm{CE}+/-)$ & 1.70 & 1.70 & 2.50 & 1.40 & 1.20 & 1.70 & 1.70 & 2.20 \\
\hline Gross Beta $(\mathrm{pCi} / \mathrm{L})$ & -1.66 & -0.08 & 7.39 & 0.17 & 7.54 & 0.33 & 2.22 & 10.10 \\
\hline Gross Beta $(\mathrm{CE}+/-)$ & 2.70 & 2.60 & 3.20 & 2.60 & 1.40 & 2.60 & 2.90 & 3.40 \\
\hline
\end{tabular}

(CONT INUED) 
APPENDIX E.3

Groundwater and Surface-Water Quality Data, 1993

\begin{tabular}{|c|c|c|c|c|c|c|c|c|}
\hline \multirow{3}{*}{$\begin{array}{l}\text { Sampling Point } \\
\text { L } \\
\text { Location } \\
\text { Date Sampled }\end{array}$} & \multicolumn{3}{|c|}{$G W-311$} & \multicolumn{4}{|c|}{$G W-312$} & $G W-315$ \\
\hline & \multicolumn{3}{|c|}{ RS } & \multicolumn{4}{|c|}{ RS } & SPI \\
\hline & $05 / 11 / 93$ & $09 / 12 / 93$ & $10 / 21 / 93$ & $02 / 08 / 93$ & $05 / 11 / 93$ & $09 / 12 / 93$ & $10 / 21 / 93$ & $02 / 03 / 93$ \\
\hline FIELD MEASUREMENTS & $\cdot$ &. & - & $\cdot$ & $\cdot$ & -1 & $\cdot$ & - \\
\hline Depth to Water (ft) & 37.02 & 41.35 & 40 & 34.65 & 35.21 & 39.65 & 38 & 53.35 \\
\hline Water Temp (degrees C) & 18.6 & 17.3 & 15.9 & 14.2 & 16.9 & 17.2 & 15.5 & 14.8 \\
\hline $\mathrm{pH}$ (pH units) & 7.5 & 7.7 & 7.5 & 12.2 & 12.1 & 12.1 & 12.1 & 7.1 \\
\hline Sp. Cond. (umho/cm) & 359 & 385 & 428 & 1556 & 1333 & 2350 & 2630 & 510 \\
\hline Dissolved Oxygen (ppm) & 6.6 & 5.1 & 7 & 3.4 & 1.4 & 2.9 & 6 & 6.5 \\
\hline Oxidation/Reduction (mV) & 181 & 182 & 266 & -62 & -78 & -10 & -28 & 179 \\
\hline MISCELLANEOUS PARAMETERS & . & $\cdot$ & . &. & . &. &. & - \\
\hline pH (pH units) & 7.9 & 7.9 & 7.4 & 11.7 & 12 & 12 & 12 & 7.1 \\
\hline Sp. Cond. (umho/cm) & 426 & 399 & 417 & 1110 & 1980 & 1920 & 2350 & 517 \\
\hline TDS (mg/L) & 242 & 260 & 264 & 396 & 470 & 522 & 682 & 346 \\
\hline TSS (mg/L) & $<1$ & 7 & 40 & 81 & 4 & 4 & 25 & 4 \\
\hline Turbidity (NTU) & 2.5 & 3.3 & 68 & 16 & 3.6 & 1.2 & 3.5 & 0.5 \\
\hline RADIOCKEMICAL PARAMETERS & - &. & . &. & . & . &. & - \\
\hline Gross Alpha (pci/L) & -0.00 & -2.75 & 1.00 & -0.55 & -0.35 & -1.88 & -0.20 & 5.35 \\
\hline Gross Alpha $(\mathrm{CE}+/-)$ & 0.56 & 2.60 & 1.50 & 3.30 & 0.55 & 3.10 & 1.40 & 3.40 \\
\hline Gross Beta $(\mathrm{pCi} / \mathrm{L})$ & -1.65 & -7.51 & -0.41 & 7.36 & 1.43 & -5.53 & 7.27 & 12.90 \\
\hline Gross Beta $(\mathrm{CE}+/-)$ & 1.40 & 5.70 & 2.40 & 3.40 & 1.40 & 5.70 & 2.90 & 3.40 \\
\hline
\end{tabular}

(CONTINUED) 
APPENDIX $\mathbf{8} \cdot 3$

Groundwater and Surface-Water Quality Data, 1993

\begin{tabular}{|c|c|c|c|c|c|c|c|c|}
\hline \multirow{3}{*}{$\begin{array}{l}\text { Sampling Point } \\
\text { Iocation } \\
\text { - } \\
\text { Date Sampled }\end{array}$} & \multicolumn{3}{|c|}{ GW-315 } & \multicolumn{4}{|c|}{$G W-316$} & \multirow{2}{*}{$\frac{\text { GW-317 }}{\text { SPI }}$} \\
\hline & \multicolumn{3}{|c|}{ SPI } & \multicolumn{4}{|c|}{ SPI } & \\
\hline & $06 / 03 / 93$ & $09 / 14 / 93$ & $10 / 27 / 93$ & $02 / 02 / 93$ & $06 / 01 / 93$ & $09 / 13 / 93$ & $10 / 24 / 93$ & $02 / 02 / 93$ \\
\hline FIELD MEASUREMENTS & $\cdot$ & $\cdot$ & • & $\cdot$ & - & - & $\cdot$ & - \\
\hline Depth to Water ( $f t)$ & 58.92 & 62.33 & 61.15 & 52.9 & 58.52 & 59.57 & 60.7 & 69.28 \\
\hline Water Temp (degrees C) & 16.9 & 16.7 & 14.5 & 12.7 & 18 & 18.5 & 12 & 11.7 \\
\hline pH (pH units) & 7.2 & 6.9 & 6.5 & 7.4 & 8.1 & 7.7 & 7.8 & 7.5 \\
\hline Sp. Cond. (umho/cm) & 457 & 582 & 569 & 335 & 368 & 381 & 354 & 355 \\
\hline Dissolved Oxygen (ppm) & 6.4 & 2.5 & 2.5 & 7.8 & 7.2 & 6.8 & 8.4 & 8.8 \\
\hline Oxidation/Reduction (mv) & 203 & 161 & 212 & 157 & 113 & 205 & 230 & 207 \\
\hline MISCELLANEOUS PARAMETERS & • & & - & • & $\cdot$ & • & . & - \\
\hline pH (pH units) & 7.8 & 7.4 & 7.2 & 7.9 & 8 & 8 & 8 & 7.9 \\
\hline Sp. Cond. (umho/cm) & 530 & 599 & 628 & 389 & 364 & 390 & 406 & 419 \\
\hline TDS (mg/L) & 322 & 380 & 412 & 208 & 204 & 208 & 226 & 222 \\
\hline TSS (mg/L) & $<1$ & $<1$ & $<1$ & $<1$ & $<1$ & 1 & $<1$ & $<1$ \\
\hline Turbidity (NTU) & 1 & 0.9 & 0.5 & 0.9 & 2.7 & 8 & 2.4 & 1.5 \\
\hline RADIOCHEMICAL PARAMETERS & - & $\cdot$ & - & - & - & - & $\cdot$ & • \\
\hline Gross Alphe (pCi/I) & -2.46 & 0.33 & 2.54 & 2.21 & 1.75 & -0.01 & -0.45 & 1.55 \\
\hline Gross Alpha $(\mathrm{CE}+/-)$ & 4.60 & 1.50 & 2.60 & 1.50 & 3.60 & 0.95 & 2.10 & 1.40 \\
\hline Gross Beta (pCi/L) & 15.70 & 31.30 & 53.80 & 2.16 & 5.42 & -0.97 & 2.64 & 3.58 \\
\hline Gross Beta $(\mathrm{CE}+/-)$ & 7.10 & 5.00 & 4.90 & 2.60 & 5.00 & 2.60 & 2.70 & 2.70 \\
\hline
\end{tabular}

(CONTINUED) 
APPENDIX $\mathrm{E} \cdot 3$

Groundwater and Surface-Water Quality Data, 1993

\begin{tabular}{|c|c|c|c|c|c|c|c|c|}
\hline \multirow{3}{*}{$\begin{array}{l}\text { Sampling Point } \\
\text { Location } \\
\text { Date Sampled }\end{array}$} & \multicolumn{3}{|c|}{$G W-317$} & \multicolumn{4}{|c|}{$G W-323$} & GW-324 \\
\hline & \multicolumn{3}{|c|}{ SPI } & \multicolumn{4}{|c|}{ SPI } & s3 \\
\hline & $05 / 28 / 93$ & $09 / 13 / 93$ & $10 / 24 / 93$ & $02 / 02 / 93$ & $05 / 28 / 93$ & $09 / 12 / 93$ & $10 / 23 / 93$ & $01 / 29 / 93$ \\
\hline FIEID MEASURBNERTS & - & • & • & $\cdot$ & - & • & $\cdot$ & • \\
\hline Depth to Water (ft) & 74.83 & 78.64 & 77.51 & 86 & 86 & 97.9 & 86.21 & 9.35 \\
\hline Water Temp (degrees C) & 17.3 & 15 & 11.8 & 12 & 15.3 & 15.2 & 11.9 & 13.8 \\
\hline pH (pH units) & 7.8 & 7.7 & 7.9 & 7.5 & 7.6 & 7.4 & 7.5 & 7.9 \\
\hline Sp. Cond. (umho/cm) & 346 & 380 & 323 & 365 & 339 & 366 & 325 & 206 \\
\hline Dissolved Oxygen (ppm) & 7.7 & 6.9 & 8.9 & 8.4 & 8.3 & 7.6 & 8.8 & 0.6 \\
\hline Oxidation/Reduction (mv) & 149 & 14 & 227 & 204 & 193 & 163 & 233 & -87 \\
\hline MISCELLANEOUS PARAMBTERS & . & . & . & . & . & $\cdot$ & $\cdot$ & • \\
\hline pH (pH units) & $\dot{8}$ & $\dot{8}$ & $7 . \dot{9}$ & $7 . \dot{8}$ & $7 . \dot{9}$ & $\dot{8}$ & $7 . \dot{9}$ & $8 . \dot{2}$ \\
\hline Sp. Cond. (umho/cm) & 374 & 419 & 360 & 419 & 377 & 375 & 409 & 202 \\
\hline TDS (mg/L) & 178 & 222 & 226 & 216 & 186 & 208 & 228 & 158 \\
\hline TSS (mg/L) & $<1$ & 3 & $<1$ & $<1$ & $<1$ & $<1$ & $<1$ & $<1$ \\
\hline Turbidity (NTU) & 1 & 2.4 & 2.3 & 0.4 & 2.5 & 0.9 & 0.7 & 1 \\
\hline RADIOCKRMICAL PARAMETERS & . & . & . & . & . & . & . & • \\
\hline Gross Alpha (pCi/L) & 2.94 & 0.16 & -1.64 & 2.20 & 2.08 & 0.68 & 0.75 & 1.59 \\
\hline Gross Alphe $(\mathrm{CE}+/-)$ & 3.80 & 1.40 & 1.90 & 1.50 & 3.70 & 1.20 & 2.30 & 1.30 \\
\hline Gross Beta $(\mathrm{pCi} / \mathrm{L})$ & 7.40 & -1.43 & 1.98 & 2.16 & 6.92 & -1.21 & 1.81 & 0.75 \\
\hline Gross Beta $(\mathrm{CE}+/-)$ & 5.10 & 2.30 & 2.70 & 2.60 & 5.10 & 2.60 & 2.60 & 2.60 \\
\hline
\end{tabular}

(CONTINUED) 
APPENDIX 8.3

Groundwater and Surface-Water Quality Data, 1993

\begin{tabular}{|c|c|c|c|c|c|c|c|c|}
\hline \multirow{3}{*}{$\begin{array}{l}\text { Sampling point } \\
\text { Location } \\
\text { Date Sampled }\end{array}$} & \multicolumn{3}{|c|}{$G w-324$} & \multicolumn{4}{|c|}{ GW-325 } & $6 w-342$ \\
\hline & \multicolumn{3}{|c|}{ s3 } & \multicolumn{4}{|c|}{$\mathbf{8 3}$} & BC \\
\hline & $05 / 25 / 93$ & $09 / 11 / 93$ & $10 / 19 / 93$ & $02 / 01 / 93$ & $05 / 25 / 93$ & $09 / 11 / 93$ & $10 / 21 / 93$ & $10 / 07 / 93$ \\
\hline FIELD MEASUREMENTS & - & - & - & - & - & - & - & • \\
\hline Depth to Water (ft) & 15.15 & 17.9 & 17.85 & 12.1 & 16.05 & 17.9 & 17.9 & 13.74 \\
\hline Water Temp (degrees $C$ ) & 14.9 & 14.5 & 15.2 & 13.5 & 14.5 & 18.9 & 15.7 & 17.7 \\
\hline pH (pH units) & 7.8 & 7.9 & 7.5 & 5.2 & 5.3 & 5.3 & 5.9 & 6 \\
\hline Sp. Cond. (umho/cm) & 168 & 193 & 207 & 49 & 35 & 42 & 50 & 94 \\
\hline Dissolved Oxygen (ppm) & 0.3 & 0.5 & 0.5 & 6 & 4.5 & 6.7 & 3 & 5.7 \\
\hline Oxidation/Reduction (mv) & -105 & 14 & -8 & -232 & 226 & 175 & 196 & 8 \\
\hline MISCELIAMIEOUS PARAMETERS & . & • & - & . & . & . & - & . \\
\hline pH (pH units) & $8 . \dot{1}$ & $8 . \dot{2}$ & i & $5 . \dot{7}$ & $5 . \dot{8}$ & $6 . \dot{1}$ & $5 . \dot{9}$ & 7.7 \\
\hline Sp. Cond. (umho/cm) & 212 & 210 & 210 & 46 & 37 & 36 & 41 & 129 \\
\hline $\operatorname{TDS}(\mathrm{mg} / \mathrm{L})$ & 128 & 148 & 150 & 56 & 50 & 74 & 88 & 152 \\
\hline TSS $(\mathrm{mg} / \mathrm{L})$ & 1 & $<1$ & $<1$ & 2 & 6 & 194 & 57 & 11 \\
\hline Turbidity (NTU) & 0.7 & 0.9 & 0.9 & 11 & 27 & 50 & 800 & 7.5 \\
\hline RADIOCHEMICAL PARAMETERS & . & . & . & . & . & . & . & • \\
\hline Gross Alpha (pCi/L) & 0.00 & 0.33 & $-0.3 \dot{2}$ & $1.1 \dot{2}$ & 0.56 & 0.29 & 10.80 & 0.00 \\
\hline Gross Alphe $(\mathrm{CB}+/-)$ & 3.10 & 1.10 & 2.20 & 1.60 & 3.20 & 0.94 & 3.20 & 2.00 \\
\hline Gross Beta $(\mathrm{pCi} / \mathrm{L})$ & -3.77 & -2.66 & -7.50 & 4.41 & -1.63 & -0.10 & 15.50 & 1.97 \\
\hline Gross Beta $(\mathrm{CE}+/-)$ & 5.20 & 2.50 & 5.70 & 2.70 & 5.30 & 2.90 & 3.50 & 2.70 \\
\hline
\end{tabular}

(CONTINUED) 
APRENDIX E.3

Groundwater and Surface-Water Quallty Data, 1993

\begin{tabular}{|c|c|c|c|c|c|c|c|c|}
\hline \multirow{3}{*}{$\begin{array}{l}\text { Sampling Point } \\
\text { Location } \\
\text { Date sampled }\end{array}$} & \multicolumn{4}{|c|}{ Ow-345 } & \multicolumn{4}{|c|}{$a w-347$} \\
\hline & \multicolumn{4}{|c|}{83} & \multicolumn{4}{|c|}{83} \\
\hline & $02 / 03 / 93$ & $06 / 01 / 93$ & $09 / 14 / 93$ & $10 / 26 / 93$ & $02 / 01 / 93$ & $05 / 27 / 93$ & $09 / 12 / 93$ & $10 / 22 / 93$ \\
\hline FIELd Mrasurements & $\cdot$ & $\cdot$ & $\cdot$ & - & $\cdot$ & $\cdot 1$ & $\cdot$ & - \\
\hline Depth to water $(\mathrm{ft})$ & 18.6 & 18.1 & 19.6 & 20.52 & 17.4 & 18.65 & 19.55 & 19.55 \\
\hline Water Temp (dagreas C) & 12 & 19.7 & $15 \cdot 3$ & 14.8 & 13.5 & 15.2 & 14.2 & 13 \\
\hline pH (pH units) & 5.2 & 5.9 & 5 & 4.6 & 6.6 & 7.2 & 6.9 & 7.4 \\
\hline Sp. Cond. (umho/cm) & 188 & 105 & 134 & 152 & 133 & 151 & 286 & 229 \\
\hline Dissolved Oxygen (ppm) & 2.4 & 5.8 & 2.6 & 3.2 & 8.6 & 7.6 & 6.7 & 8.6 \\
\hline oxidation/Reduction (mv) & 249 & 195 & 239 & 234 & 198 & 153 & 200 & 201 \\
\hline MISCELLANBOUS PARAMETERS & . &. & . & . & . & . & . & - \\
\hline pH (pH units) & 5.6 & $6 . \dot{2}$ & $5 . \dot{9}$ & 6 & $7 . \dot{3}$ & 7.6 & 7.8 & 7.6 \\
\hline sp. Cond. (umho/cm) & 114 & 121 & 246 & 686 & 156 & 168 & 295 & 214 \\
\hline TDS $(m g / L)$ & 98 & 84 & 216 & 514 & 116 & 92 & 178 & 132 \\
\hline TSS (mg/L) & 2 & 4 & 4 & 12 & 24 & 4 & 15 & 39 \\
\hline Turbidity (NTU) & 11 & 3.9 & 4.1 & 7.2 & 34 & 4 & 12 & 64 \\
\hline RADIOCKEMICAL PARANETERS & • & $\cdot$ & - & • & - & . & - & - \\
\hline Gross Alpha (pC1/L) & 3.33 & -1.70 & 0.47 & -14.00 & 1.81 & 0.85 & 2.50 & 3.64 \\
\hline Gross Alpha $(\mathrm{CE}+/-)$ & 2.00 & 2.90 & 1.40 & 27.00 & 1.90 & 3.40 & 1.60 & 2.70 \\
\hline Gross Beta $(\mathrm{pCl} / \mathrm{L})$ & 25.70 & 8.99 & 15.10 & 13.70 & 4.71 & 5.24 & 5.53 & 11.10 \\
\hline Gross Beta $(\mathrm{CE}+/-)$ & 3.90 & 5.20 & 3.60 & 27.00 & 2.70 & 4.90 & 3.10 & 3.20 \\
\hline
\end{tabular}

(CONTINURD) 
APPENDIX $\mathbf{8 . 3}$

Groundwater and surface-Water Qunllty Data, 1993

\begin{tabular}{|c|c|c|c|c|c|c|c|c|}
\hline \multirow{3}{*}{$\begin{array}{l}\text { Sampling Point } \\
\text { Location } \\
\text { Date sampled }\end{array}$} & \multicolumn{4}{|c|}{$O N-348$} & \multicolumn{4}{|c|}{ ON-364 } \\
\hline & \multicolumn{4}{|c|}{83} & \multicolumn{4}{|c|}{ ove } \\
\hline & $02 / 01 / 93$ & $05 / 27 / 93$ & $09 / 22 / 93$ & $10 / 22 / 93$ & $03 / 27 / 93$ & $06 / 27 / 93$ & $09 / 19 / 93$ & $12 / 18 / 93$ \\
\hline FIRLD MEASUREMEMT8 & $\cdot 1$ & • & $\cdot$ & $\cdot$ & $\cdot$ & • & . & • \\
\hline Dapth to Water (ft) & 21.95 & 2.3 .9 & 25.7 & 24.8 & 5.47 & 23.05 & 21.31 & 15.05 \\
\hline Water Temp (degreos C) & 14.6 & 16 & 14.8 & 14 & 12.4 & 19.4 & 14 & 11.3 \\
\hline pH (pH units) & 7.5 & 7.4 & 7.1 & 7 & 7.6 & 7.3 & 7.6 & $\mathbf{8}$ \\
\hline sp. Cond. (umho/cm) & 546 & 497 & 525 & 556 & 403 & 563 & 616 & 477 \\
\hline Dissolved Oxygen (ppm) & 2.1 & 4.7 & 1.3 & 1.2 & 3.7 & 2.2 & 5.1 & 4.6 \\
\hline Oxidation/Reduction (mV) & 148 & 94 & 163 & 206 & 158 & 185 & 237 & 185 \\
\hline MISCELLAKEOUS PARAMETERS &. & . & . & . & . &. &. & • \\
\hline pH (pH units) & $7 . \dot{5}$ & 7.7 & 7.7 & $7 . \dot{6}$ & $7 . \dot{8}$ & 7.7 & 7.5 & 7.7 \\
\hline 8p. Cond. (umho/om) & 576 & 544 & 563 & 586 & 427 & 602 & 647 & 462 \\
\hline $\operatorname{TDS}(\mathrm{mg} / \mathrm{x})$ & 336 & 314 & 338 & 344 & 260 & 408 & 468 & 284 \\
\hline TsS (mg/L) & $<1$ & $<1$ & $<1$ & $<1$ & 8 & 34 & 241 & 6 \\
\hline Turbidity (NTU) & 1.2 & 1 & 1 & 2.7 & 29 & 250 & 320 & 21 \\
\hline RADTOCHEMICAI PARNAETERS & $\cdot$ & - & . & - & - & . & $\cdot$ & - \\
\hline Grose Alpha (pC1/L) & 2.42 & 0.00 & $1.4 \dot{2}$ & 0.31 & 1.65 & -1.34 & 2.77 & 3.73 \\
\hline Gross Alphe $(\mathrm{CB}+/-)$ & 2.50 & 3.40 & 1.40 & 2.30 & 2.10 & 3.00 & 3.60 & 2.00 \\
\hline Grose Beta $(p C i / L)$ & 37.30 & 31.60 & 18.40 & 29.80 & 5.26 & 1.25 & 22.30 & 4.16 \\
\hline Grose Bata $(\mathrm{CB}+/-)$ & 4.40 & 6.50 & 4.00 & 4.00 & 2.90 & 5.00 & 6.30 & 3.20 \\
\hline
\end{tabular}

(CONTINUED) 
APPEHDIX $\mathrm{R.3}$

Oroundwater and surface-water Quality Data, 1993

\begin{tabular}{|c|c|c|c|c|c|c|c|c|}
\hline \multirow{3}{*}{$\begin{array}{l}\text { 8ampling Point } \\
\text { Looation } \\
\text { Date sampled }\end{array}$} & \multicolumn{4}{|c|}{ on-365 } & \multicolumn{4}{|c|}{$0 x-370$} \\
\hline & \multicolumn{4}{|c|}{ orf } & \multicolumn{4}{|c|}{ BO } \\
\hline & $03 / 28 / 93$ & $06 / 27 / 93$ & $09 / 19 / 93$ & $12 / 19 / 93$ & $02 / 17 / 93$ & $05 / 21 / 93$ & $07 / 10 / 93$ & $10 / 14 / 93$ \\
\hline TIRLD MasURAMHTs & $\cdot$ & $\cdot$ & $\cdot$ & $\cdot$ & $\cdot$ & - & $\cdot$ & • \\
\hline Dopth to Water (ft) & 3.1 & 14.1 & 15.37 & $8 . \dot{8}$ & 13 & 13.75 & 14.9 & 16.1 \\
\hline Water Temp (degreen C) & 13 & 24.9 & 15.8 & 11.9 & 14.2 & 14.2 & 18.5 & 17.2 \\
\hline pH (pH unita) & 7 & 7 & 7.1 & 7.2 & 6.8 & 7.2 & 6.6 & 6.1 \\
\hline sp. Cond. (umho/cm) & 911 & 929 & 956 & 919 & 200 & 170 & 192 & 169 \\
\hline Diseolved Oxygen (ppm) & 0.9 & 1 & 1.3 & 0.4 & 3.9 & 6.9 & 2.8 & 3.1 \\
\hline Oxidation/Reduction (mv) & -38 & -30 & 82 & -80 & 171 & 116 & 175 & 183 \\
\hline MISCELLASEOUS PARAMETERS & & $\cdot$ &. & $\cdot$ & $\cdot$ & . & $\dot{.}$ & $\bullet$ \\
\hline pH (pH unite) & 7.9 & 7.4 & $7 . \dot{1}$ & $7 . \dot{2}$ & $7 . \dot{4}$ & $\dot{7}$ & 7.4 & 6.9 \\
\hline 8p. Cond. (unho/cm) & 890 & 929 & 943 & 928 & 263 & 190 & 202 & 173 \\
\hline $\operatorname{TDS}(\mathrm{mg} / \mathrm{L})$ & 572 & 586 & 584 & 558 & 170 & 200 & 180 & 212 \\
\hline $288(m g / L)$ & s & 2 & 1 & 1 & 18 & 307 & 53 & 135 \\
\hline Turbidity (NISU) & 9.5 & 7 & 2 & 5 & 7.8 & 5.5 & 14 & 38 \\
\hline RADTOCHEMICAL PARAMETERS & $\cdot$ & . & - & - & $\dot{\bullet}$ & • & $\dot{.}$ & • \\
\hline Orose Alpha $(\mathrm{pCi} / \mathrm{L})$ & $4.2 \dot{9}$ & -2.17 & -2.10 & -0.47 & 0.20 & 4.67 & $4.8 \dot{8}$ & 4.93 \\
\hline Grose Alpha $(\mathrm{Cz}+/-)$ & 3.90 & 3.10 & 2.40 & 1.30 & 2.80 & 2.70 & 3.30 & 2.10 \\
\hline Grose Beta $(\mathrm{PCi} / \mathrm{L})$ & 4.41 & 1.40 & 4.40 & 0.89 & 2.99 & 5.12 & -8.69 & 3.08 \\
\hline Crose Bete $(\mathrm{CE}+/-)$ & 3.10 & 4.90 & 4.90 & 3.10 & 3.30 & 3.10 & 5.60 & 2.90 \\
\hline
\end{tabular}

(CONTINUED) 
APPIADIX 1.3

Groundwater and surfaon-water Quality Data, 1993

\begin{tabular}{|c|c|c|c|c|c|c|c|c|}
\hline \multirow{3}{*}{$\begin{array}{l}\text { Sampling polnt } \\
\text { Location } \\
\text { Date sampled }\end{array}$} & \multicolumn{4}{|c|}{$0 x-371$} & \multicolumn{4}{|c|}{ ON-372 } \\
\hline & \multicolumn{4}{|c|}{80} & \multicolumn{4}{|c|}{ DO } \\
\hline & $02 / 16 / 93$ & $05 / 21 / 93$ & $07 / 09 / 93$ & $10 / 13 / 93$ & $02 / 09 / 93$ & $05 / 29 / 93$ & $07 / 02 / 93$ & $10 / 22 / 93$ \\
\hline TIELD MEABURERETS & - & • & $\cdot$ & • & $\cdot$ & - & . & $\cdot$ \\
\hline Dopth to Water (tt) & 16.1 & 16.22 & 17.65 & 18.05 & 20.75 & 19.3 & 23.55 & 27.54 \\
\hline Water Temp (degrees C) & 13.2 & 14.3 & 19.3 & 15.6 & 16 & 15.3 & 18.3 & 11.8 \\
\hline pH (pH unites) & 7.3 & 6.4 & 7.3 & 6.7 & 7.4 & 7.6 & 7.7 & 7.3 \\
\hline 8p. Cond. (unho/cm) & 481 & 397 & 451 & 532 & 425 & 333 & 373 & 397 \\
\hline Dissolved Oxygan (ppa) & 8.1 & 3.2 & 0.7 & 1.9 & 9.5 & 6.6 & 7.7 & 3.6 \\
\hline Oxidation/Raduction (mv) & -3 & 152 & -58 & -9 & 160 & 192 & 91 & 142 \\
\hline MI8CLLLAROUS PARMETERS & . &. &. &. & $\cdot$ & $\cdot$ & $\cdot$ & . \\
\hline pH (pH unite) & 7.4 & 7.4 & 7.6 & 7.6 & 7.6 & $\dot{0}$ & 7.8 & 7.7 \\
\hline 8p. Cond. (unho/cm) & 570 & 590 & 366 & 569 & 430 & 403 & 401 & 420 \\
\hline $\operatorname{TDS}(m g / L)$ & 326 & 328 & 348 & 372 & 202 & 244 & 266 & 328 \\
\hline$T 88(m g / L)$ & 9 & 4 & 13 & $<1$ & 4 & 5 & 3 & 4 \\
\hline Turbldity (ATU) & 4.9 & 120 & 2.7 & 4.7 & 2.5 & 12 & 4.4 & 16 \\
\hline PADIOCFEMICAL PARAMETERS &. &. & $\cdot$ & $\cdot$ & $\cdot$ & $\cdot$ & $\cdot$ & $\cdot$ \\
\hline Orose Alpha $(\mathrm{pC1} / \mathrm{L})$ & -1.32 & -3.06 & 0.38 & 2.80 & -1.05 & 1.42 & 0.70 & -0.01 \\
\hline Grose Alpha $(\mathrm{CB}+/-)$ & 2.10 & 2.10 & 1.30 & 1.90 & 2.00 & 3.60 & 1.30 & 2.40 \\
\hline Gross Beta (pCl/L) & 0.92 & -1.35 & -3.87 & 4.86 & -0.25 & -5.73 & -4.29 & -4.94 \\
\hline Grose Beta $(\mathrm{CB}+/-)$ & 3.20 & 2.80 & 2.70 & 3.10 & 2.90 & 5.60 & 2.70 & 5.60 \\
\hline
\end{tabular}

(CONTINURD) 
APPENDIX 8.3

Groundwater and surface-Mater Quallty Data, 1993

\begin{tabular}{|c|c|c|c|c|c|c|c|c|}
\hline \multirow{3}{*}{$\begin{array}{l}\text { Sampling polnt } \\
\text { tooation } \\
\text { Date sanpled }\end{array}$} & \multicolumn{4}{|c|}{ ow-373 } & \multicolumn{4}{|c|}{$0 N-526$} \\
\hline & \multicolumn{4}{|c|}{ so } & \multicolumn{4}{|c|}{$\mathbf{8 3}$} \\
\hline & $02 / 11 / 93$ & $05 / 14 / 93$ & $07 / 02 / 93$ & $10 / 11 / 93$ & $02 / 03 / 93$ & $06 / 03 / 93$ & $09 / 14 / 93$ & $10 / 26 / 93$ \\
\hline 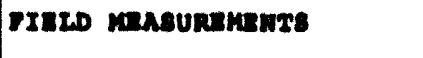 & • & - & - & $\cdot$ & • & $\cdot$ & $\cdot$ & • \\
\hline Dopth to Water (ft) & 18.25 & 16.74 & 18.8 & 20.83 & 11.97 & 12 & 13.08 & 13.35 \\
\hline Water Temp (degrees C) & 14.3 & 14.7 & 17.6 & 14.4 & 14.5 & 15.1 & 17.6 & 15.5 \\
\hline ph (pH unites) & 9.2 & 8.1 & 8.8 & 9.1 & 8.2 & 7.7 & 8.2 & 8.4 \\
\hline 8p. Cond. (umho/cm) & 476 & 441 & 418 & 462 & 7270 & 5870 & 7500 & 7100 \\
\hline DLesolved Oxygen (ppm) & 8.4 & 4.5 & 12 & 4.8 & 5.3 & $\mathbf{s . 3}$ & 0.5 & 1.2 \\
\hline Oxidation/Raduction (mV) & 158 & 172 & 157 & 123 & 118 & 168 & 101 & 189 \\
\hline MISCELFNTEOUS PARANTERS & - & - & - & - & - & - & - & - \\
\hline pH (ph units) & 9.1 & 9 & 9.1 & 9.1 & 8.1 & 8.2 & 8.1 & 8.1 \\
\hline 8p. Cond. (waho/om) & 484 & 494 & 468 & 480 & 7580 & 6940 & 7470 & 7620 \\
\hline $\operatorname{TDs}(\mathrm{mg} / \mathrm{L})$ & 290 & 306 & 306 & 358 & 6214 & 6028 & 6246 & 6320 \\
\hline T88 (mg/tu) & 3 & 15 & 12 & 10 & 14 & 9 & 8 & 28 \\
\hline Turbldity (MTU) & 3.5 & 5.1 & 6.5 & 17 & 6.3 & 7.4 & 12 & 26 \\
\hline RADIOCEIMICAL PARALTERS & . & - & . & . & . & . & - & - \\
\hline Gross Alpha (pCl/L) & 0.76 & -1.57 & 0.18 & -1.92 & 2.04 & 121.00 & -14.20 & 0.79 \\
\hline Grose Npha $(\mathrm{Cz}+/-1$ & 1.70 & 4.00 & 1.20 & 2.10 & 1.40 & 80.00 & 24.00 & 2.40 \\
\hline Grose Beta $(\mathrm{pCl} / \mathrm{L})$ & 0.79 & -2.33 & -4.66 & 0.08 & 3.53 & 287.00 & -22.10 & 44.70 \\
\hline Grone Beta $(\mathrm{CX}+1-)$ & 2.40 & 5.50 & 2.70 & 2.70 & 2.70 & 120.00 & 46.00 & 4.60 \\
\hline
\end{tabular}

( Consraved 
APPLNDIX 8.3

Groundwater and surface-Water Quallty Data, 1993

\begin{tabular}{|c|c|c|c|c|c|c|c|c|}
\hline \multirow{3}{*}{$\begin{array}{l}\text { sumpling polnt } \\
\text { Location } \\
\text { Date sampled }\end{array}$} & \multicolumn{4}{|c|}{ ou-537 } & \multicolumn{4}{|c|}{ O11-601 } \\
\hline & \multicolumn{4}{|c|}{ ow } & \multicolumn{4}{|c|}{ OLP } \\
\hline & $02 / 11 / 93$ & $05 / 12 / 93 \mid$ & $09 / 14 / 93$ & $10 / 22 / 93$ & $03 / 28 / 93$ & $06 / 27 / 93$ & $09 / 19 / 93$ & $12 / 21 / 94$ \\
\hline TIELd Magunaverse & - & . & $\cdot$ & $\cdot 1$ &. & $\cdot 1$ & . & 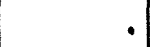 \\
\hline Dopth to Water $(f t)$ & 6.8 & 6.45 & 8.1 & 7.47 & 59.6 & 71.33 & 71.27 & 67.35 \\
\hline Water Teap (degrees C) & 14.7 & 16.2 & 17.1 & 15.6 & 14 & 16.1 & 14.6 & 11.7 \\
\hline pH (pH units) & 6.6 & 6.8 & 6.9 & 6.9 & 7.6 & 7.3 & 7.7 & 7.9 \\
\hline 8p. Cond. (umho/om) & 5430 & 4890 & 5180 & 5400 & 894 & 896 & 912 & 924 \\
\hline Diesolved Oxygen (ppen) & 1.1 & 1.1 & 3.6 & 4 & 4.2 & 4.2 & 1.3 & 1.4 \\
\hline Oxldation/Raduction (mv) & 108 & 178 & 264 & 220 & 180 & 173 & 140 & 162 \\
\hline MISCELLAMROUS PARMATTERS &. &. & $\cdot$ & . & $\cdot$ & $\cdot$ & $\cdot$ & . \\
\hline pH (pH unita) & 6.8 & 7.6 & 7.1 & 6.9 & 8.1 & 7.9 & 7.7 & 7.6 \\
\hline 8p. Cond. (uwho/am) & 6080 & 5660 & 5480 & 5710 & 904 & 948 & 980 & 1010 \\
\hline TDS $(m g / L)$ & 4700 & 4682 & 4438 & 4478 & $\mathbf{5 4 0}$ & 542 & 584 & 560 \\
\hline Ts8 (mg/L) & 4 & 5 & 4 & 5 & 1 & 13 & $<1$ & $<1$ \\
\hline Turbldity (NTU) & 1.9 & 1.1 & 1.6 & 1.9 & 0.8 & 0.5 & 0.5 & 0.7 \\
\hline RADIOCHEMICAL PARAMTERS &. &. &. & . &. & & . & . \\
\hline Grose Alpha $(\mathrm{pCl} / \mathrm{L})$ & 39.80 & 0.00 & 16.60 & 19.70 & 1.48 & -2.15 & 0.33 & 2.08 \\
\hline Orose Alpha $(\mathrm{Cz}+/-)$ & 14.00 & 20.00 & 33.00 & 12.00 & 2.70 & 3.00 & 3.10 & 1.80 \\
\hline Crose Beta $(\mathrm{pCl} / \mathrm{L})$ & 287.00 & 373.00 & 126.00 & 432.00 & 15.50 & 18.70 & 23.40 & 21.80 \\
\hline Grose Bata $(\mathrm{Cs}+1-)$ & 23.00 & 42.00 & 80.00 & 27.00 & 3.50 & 6.20 & 6.40 & 4.20 \\
\hline
\end{tabular}

(CONTIMURD) 
APPENDIX $\mathrm{B} .3$

Groundwater and Surface-Water Quallty Data, 1993

\begin{tabular}{|c|c|c|c|c|c|c|c|c|}
\hline \multirow{3}{*}{$\begin{array}{l}\text { Sampling Point } \\
\text { Location } \\
\text { Date sampled }\end{array}$} & \multirow{3}{*}{\begin{tabular}{|c|} 
ON-613 \\
83 \\
$10 / 20 / 93$ \\
\end{tabular}} & \multirow{3}{*}{\begin{tabular}{|c|}
$6 W-614$ \\
83 \\
$10 / 20 / 93$ \\
\end{tabular}} & \multicolumn{4}{|c|}{ ON-621 } & \multicolumn{2}{|c|}{ ON-626 } \\
\hline & & & \multicolumn{4}{|c|}{ BO } & \multicolumn{2}{|c|}{$\mathbf{B O}$} \\
\hline & & & $03 / 20 / 93$ & $06 / 02 / 93$ & $09 / 20 / 93$ & $12 / 20 / 93$ & $03 / 10 / 93$ & $04 / 26 / 93$ \\
\hline FIELd maguRERENTs & - & $\cdot 1$ & $\cdot$ & • & - & $\cdot$ & $\cdot$ & • \\
\hline Depth to Water (ft) & 16.25 & 10.1 & 10.35 & 13.66 & 19.35 & 12.58 & 23.72 & 21.8 \\
\hline Water Temp (degrees C) & 14.8 & 14.8 & 12.6 & 14.2 & 15 & 12.6 & 14.8 & 14.1 \\
\hline ph (pH unite) & 6.8 & 6.9 & 7.3 & 7.5 & 7.6 & 7.7 & 8.1 & 8.2 \\
\hline sp. Cond. (umho/am) & 176 & 327 & 302 & 309 & 360 & 316 & 246 & 194 \\
\hline Diesolved Oxygen (ppm) & 2.2 & 0.3 & 7.6 & 4.2 & 5.3 & 5.3 & 2.9 & 3.8 \\
\hline Oxidation/Reduction (mv) & 235 & 123 & 195 & 190 & 190 & 180 & 65 & 171 \\
\hline MISCELLANEOUS PARAMETERS & $\cdot$ & - & - & - & - & - & - & • \\
\hline ph (ph unite) & 7.3 & 7.8 & $7 . \dot{7}$ & $8 . \dot{1}$ & $7 . \overline{7}$ & 7.9 & $\dot{8}$ & $\dot{8}$ \\
\hline sp. Cond. (umho/cm) & 180 & 329 & 359 & 347 & 408 & 358 & 267 & 225 \\
\hline $\operatorname{TDS}(\mathrm{mg} / \mathrm{L})$ & 144 & 224 & 222 & 232 & 272 & 198 & 166 & 162 \\
\hline $288(\mathrm{mg} / \mathrm{L})$ & 8 & s & 5 & 23 & 37 & 34 & 1 & 1 \\
\hline Turbidity (RTU) & 3.1 & 2 & 14 & 34 & 35 & 26 & 0.5 & 0.8 \\
\hline RADTOCKEMICAI PARAYETERS & $\cdot$ & - & - & - & - & $\cdot$ & - & • \\
\hline Gross Alpha (pCl/L) & 1.79 & 1.23 & 0.00 & 1.47 & 3.51 & 2.41 & 2.38 & 0.16 \\
\hline Grose Alpha $(\mathrm{Cz}+/-)$ & 1.60 & 1.50 & 1.80 & 4.90 & 4.50 & 1.70 & 3.90 & 1.40 \\
\hline Orose Beta $(\mathrm{pCl} / \mathrm{L})$ & 2.82 & 2.92 & 5.13 & 7.89 & 24.10 & 5.79 & 2.97 & 0.26 \\
\hline Grose Beta $(\mathrm{CB}+/-)$ & 2.50 & 2.60 & 2.80 & 6.60 & 6.30 & 3.30 & 5.30 & 2.60 \\
\hline
\end{tabular}

(CONTIRURD) 
APPENDIX E.3

Groundwater and Surface-Water Quality Data, 1993

\begin{tabular}{|c|c|c|c|c|c|c|c|c|}
\hline \multirow{3}{*}{$\begin{array}{l}\text { Sampling Point } \\
\text { - } \\
\text { Iocation } \\
\text { - } \\
\text { Date Sampled }\end{array}$} & \multicolumn{2}{|c|}{$G W-626$} & \multicolumn{4}{|c|}{$G W-627$} & \multicolumn{2}{|c|}{$G W-636$} \\
\hline & \multicolumn{2}{|c|}{ BG } & \multicolumn{4}{|c|}{ BG } & \multicolumn{2}{|c|}{ OLF } \\
\hline & $08 / 02 / 93$ & $10 / 20 / 93$ & $03 / 09 / 93$ & $04 / 23 / 93$ & $07 / 28 / 93$ & $10 / 20 / 93$ & $03 / 26 / 93$ & $06 / 27 / 93$ \\
\hline FIELD MEASUREMENTS & $\cdot$ & $\cdot$ & $\cdot$ & $\cdot$ & $\cdot$ & - & $\cdot$ & $\bullet$ \\
\hline Depth to Water (ft) & 26.55 & 28.45 & 22.43 & 22.4 & 24.58 & 25.1 & 1.65 & 8.25 \\
\hline Water Temp (degrees C) & 16.2 & 15.8 & $15 \cdot 3$ & 16 & 18.4 & 16 & 13.8 & 14.5 \\
\hline pH (pH units) & 8 & 8 & 9.2 & 8.9 & 9.1 & 8.6 & 8.4 & 8.1 \\
\hline Sp. Cond. (umho/cm) & 250 & 242 & 1183 & 1053 & 1204 & 1208 & 304 & 315 \\
\hline Dissolved Oxygen (ppm) & $3 \cdot 3$ & 2.7 & 0.4 & 1.1 & 1.1 & 0.6 & 4.1 & 0.4 \\
\hline Oxidation/Reduction (mv) & 196 & 107 & -105 & -18 & -115 & -62 & 141 & 152 \\
\hline MISCELIANEOUS PARAMETERS & $\cdot$ & - & . & . & $\cdot$ & $\cdot$ & . & • \\
\hline pH (pH units) & 7.9 & 8 & 9.1 & 9 & 9.1 & 9.2 & 8.5 & 8.5 \\
\hline Sp. Cond. (umho/cm) & 234 & 247 & 1190 & 1130 & 1180 & 1120 & 365 & 359 \\
\hline $\operatorname{TDS}(\mathrm{mg} / \mathrm{L})$ & 146 & 182 & 704 & 732 & 728 & 738 & 226 & 230 \\
\hline TsS $(m g / L)$ & $<1$ & $<1$ & $<1$ & $<1$ & $<1$ & 2 & 7 & $<1$ \\
\hline Turbidity (NTU) & 0.8 & 0.3 & 1 & 1 & 2.7 & 0.9 & 5.5 & 2 \\
\hline RADIOCHEMICAL PARAMBTERS & $\cdot$ & - & $\cdot$ & - & - & $\cdot$ & - & - \\
\hline Gross Alpha (pci/L) & $-22 \cdot 30$ & -1.59 & 1.25 & -0.38 & -6.24 & -0.82 & 0.00 & -2.87 \\
\hline Gross Alpha $(\mathrm{CE}+/-)$ & 33.00 & 2.30 & 1.30 & 3.10 & 3.60 & 3.20 & 1.60 & 2.50 \\
\hline Gross Beta (pCi/L) & 17.90 & $-8 \cdot 32$ & 5.84 & 1.70 & -0.96 & -6.67 & 6.37 & -1.61 \\
\hline Gross Beta $(\mathrm{CE}+/-)$ & 52.00 & 5.70 & 3.40 & 2.90 & 3.00 & 5.50 & 2.90 & 4.80 \\
\hline
\end{tabular}

(CONTINUED) 
APPENDIX $\mathrm{E} .3$

Groundwater and Surface-Water Quality Data, 1993

\begin{tabular}{|c|c|c|c|c|c|c|c|c|}
\hline \multirow{3}{*}{$\begin{array}{l}\text { Sampling Point } \\
\text { - } \\
\text { Location } \\
\text { Date Sampled }\end{array}$} & \multicolumn{2}{|c|}{$G N-636$} & \multicolumn{4}{|c|}{$G W-637$} & \multicolumn{2}{|c|}{$G w-638$} \\
\hline & \multicolumn{2}{|c|}{ OLF } & \multicolumn{4}{|c|}{ OLF } & \multicolumn{2}{|c|}{ Ore } \\
\hline & $09 / 18 / 93$ & $12 / 18 / 93$ & $03 / 25 / 93$ & $06 / 26 / 93$ & $09 / 17 / 93$ & $12 / 18 / 93$ & $03 / 25 / 93$ & $06 / 26 / 93$ \\
\hline FIELD MEASUREMENTS & - & - & - & $\cdot$ & $\cdot$ & - & - & - \\
\hline Depth to Water (ft) & 10.15 & 3.99 & 5.9 & 12.92 & 14.45 & 7.6 & 5.6 & 11 \\
\hline Water Temp (degrees C) & 14.5 & 12.8 & 13.7 & 14.6 & 16.5 & $12 \cdot 1$ & 13.3 & 19.4 \\
\hline pH (pH units) & $8 \cdot 4$ & 8.2 & 7.5 & $7 \cdot 3$ & $7 \cdot 2$ & $7 \cdot 3$ & 7 & 6.9 \\
\hline Sp. Cond. (umho/cm) & 360 & 364 & 389 & 377 & 430 & 413 & 387 & 503 \\
\hline Dissolved Oxygen (ppm) & 3.1 & 0.3 & 2.9 & 4.3 & 4.4 & 3.5 & 6.2 & 7.1 \\
\hline Oxidation/Reduction (mV) & 169 & 69 & 111 & 164 & 173 & 119 & 176 & 231 \\
\hline MISCELIAMEOUS PARAMETERS & $\cdot$ & $\cdot$ & - & $\cdot$ & $\cdot$ & $\cdot$ & $\cdot$ & - \\
\hline pH (pH units) & B. 5 & 8.5 & 7.6 & 7.9 & 7.7 & 7.7 & 7.4 & 7.5 \\
\hline Sp. Cond. (umho/cm) & 364 & 358 & 409 & 414 & 433 & 410 & 419 & 499 \\
\hline TDS (mg/L) & 240 & 236 & 252 & 254 & 258 & 262 & 308 & 550 \\
\hline TSS (mg/L) & 1 & 14 & 4 & 2 & $<1$ & 15 & 48 & 5405 \\
\hline Turbidity (NTU) & 1.5 & 0.9 & 6 & 1.5 & 5.7 & 1.8 & 54 & 3100 \\
\hline RADTOCHEMICAL PARAMETERS & $\cdot$ & - & $\cdot$ & $\cdot$ & $\cdot$ & - & $\cdot$ & - \\
\hline Gross Alpha (pcl/I) & -0.72 & -0.60 & -0.19 & $-2 \cdot 42$ & -0.72 & 1.39 & 5.01 & 5.67 \\
\hline Gross Alpha $(\mathrm{CB}+/-)$ & 2.80 & 1.00 & 1.80 & 2.60 & 2.80 & 1.50 & 4.20 & 4.30 \\
\hline Gross Beta (pCi/L) & 3.44 & -1.66 & 2.89 & -2.73 & 0.46 & -2.56 & 15.60 & 8.17 \\
\hline Gross Beta $(\mathrm{CE}+/-)$ & 4.80 & 2.90 & 2.70 & 4.80 & 4.60 & 2.90 & 5.90 & 5.50 \\
\hline
\end{tabular}

(CONTINUED) 
APPENDIX E.3

Groundwater and Surface-Water Quality Data, 1993

\begin{tabular}{|c|c|c|c|c|c|c|c|c|}
\hline \multirow{3}{*}{$\begin{array}{l}\text { Sampling Point } \\
\text { - } \\
\text { Location } \\
\text { Date sampled }\end{array}$} & \multicolumn{2}{|c|}{$G W-638$} & \multicolumn{4}{|c|}{$G w-639$} & \multicolumn{2}{|c|}{ Gw-640 } \\
\hline & \multicolumn{2}{|c|}{ OLF } & \multicolumn{4}{|c|}{ BG } & \multicolumn{2}{|c|}{ BG } \\
\hline & $09 / 17 / 93$ & $12 / 18 / 93$ & $02 / 24 / 93$ & $05 / 24 / 93$ & $07 / 11 / 93$ & $10 / 15 / 93$ & $02 / 22 / 93$ & $05 / 24 / 93$ \\
\hline FIELD MEASUREKENTS & $\cdot$ & $\cdot$ & $\cdot 1$ & - & $\cdot 1$ & - & $\cdot$ & $\bullet$ \\
\hline Depth to Water $(f t)$ & 5.9 & 5.99 & 10.11 & 11.4 & 12.85 & 13.25 & 15.7 & 18 \\
\hline Water Temp (degrees C) & 20.3 & 9.8 & 12.7 & 15.7 & 18.1 & 14.5 & 13.7 & 15 \\
\hline pH (pH units) & 7 & 7.1 & 9.2 & 9 & 9.3 & 9 & 6.2 & 6.1 \\
\hline Sp. Cond. (umho/cm) & 657 & 449 & 768 & 757 & 775 & 859 & 97 & 86 \\
\hline Dissolved Oxygen (ppm) & 6.2 & 10.01 & 1.5 & 1.6 & 2.3 & 4.1 & 3.7 & 3.5 \\
\hline Oxidation/Reduction (mV) & 314 & 211 & 99 & 152 & 6 & 88 & 172 & 273 \\
\hline MISCELLANEOUS PARAMETERS & $\cdot$ & . & $\cdot$ & . & $\cdot$ & . &. & - \\
\hline pH (pH units) & 7.6 & 7.5 & 9.2 & 9.3 & 9.2 & 9.2 & 6.8 & 6.7 \\
\hline Sp. Cond. (umho/cm) & 697 & 454 & 868 & 870 & 868 & 860 & 100 & 102 \\
\hline TDS $(m g / L)$ & 516 & 312 & 542 & 562 & 546 & 564 & 70 & 76 \\
\hline TsS $(m g / L)$ & 91 & 31 & 21 & 24 & 6 & 12 & 7 & 4 \\
\hline Turbidity (NTU) & 370 & 37 & 14 & 60 & 6.9 & 80 & 2.4 & 3.1 \\
\hline RADIOCKEMICAI PARAMETERS & $\cdot$ & $\cdot$ & $\cdot$ & . &. & . & $\cdot$ & - \\
\hline Gross Alpha (pCi/L) & 8.70 & 9.13 & 0.46 & 0.00 & 0.43 & -0.77 & 0.59 & -0.56 \\
\hline Gross Alpha $(\mathrm{CE}+/-)$ & 4.40 & 3.00 & 2.20 & 3.80 & 1.50 & 3.00 & 1.50 & 3.00 \\
\hline Gross Beta ( $\mathrm{pCi} / \mathrm{L})$ & 13.50 & 2.14 & 0.72 & 0.17 & -4.58 & -7.74 & 7.60 & -1.47 \\
\hline Gross Beta $(\mathrm{CE}+/-)$ & 5.70 & 3.10 & 2.80 & 5.60 & 2.80 & 5.40 & 3.00 & 5.40 \\
\hline
\end{tabular}

(CONTIMUED) 
APPENDIX E.3

Groundwater and Surface-Water Quality Data, 1993

\begin{tabular}{|c|c|c|c|c|c|c|c|c|}
\hline \multirow{3}{*}{$\begin{array}{l}\text { Sampling Point } \\
\text { Location } \\
\text { Date Sampled }\end{array}$} & \multicolumn{2}{|c|}{$G W-640$} & \multicolumn{4}{|c|}{$G W-641$} & \multicolumn{2}{|c|}{$G W-642$} \\
\hline & \multicolumn{2}{|c|}{ BG } & \multicolumn{4}{|c|}{ BG } & \multicolumn{2}{|c|}{ BG } \\
\hline & $07 / 10 / 93$ & $10 / 14 / 93$ & $02 / 22 / 93$ & $05 / 24 / 93$ & $07 / 10 / 93$ & $10 / 14 / 93$ & $02 / 08 / 93$ & $05 / 11 / 93$ \\
\hline FIELD MEASUREMENTS & - & $\cdot$ & - & $\cdot$ & - & . & - & $\bullet$ \\
\hline Depth to Water $(f t)$ & 20.22 & 20.67 & 16.9 & 18.95 & 21.08 & 22 & 19.66 & 19.83 \\
\hline Water Temp (degrees C) & 15.8 & 15.3 & 13.7 & 15.2 & 19.6 & 16 & 14.5 & 17.8 \\
\hline pH (pH units) & 6.3 & 5.6 & 5.7 & 5.4 & 5.7 & 4.7 & 6.6 & 5.8 \\
\hline sp. Cond. (umho/cm) & 93 & 107 & 56 & 33 & 44 & 46 & 168 & 125 \\
\hline Dissolved Oxygen (ppm) & 3.1 & 3.3 & 5.7 & 5.6 & 4.5 & 5.2 & 9.5 & 5.4 \\
\hline Oxidation/Reduction (mV) & 186 & 251 & 202 & 265 & 163 & 255 & -8 & 94 \\
\hline MISCELLANEOUS PARAMETERS & . &. & . & . & . &. & . & • \\
\hline pH (pH units) & 7.3 & $6 . \dot{7}$ & $6 . \dot{3}$ & $6 . \dot{1}$ & $6 . \dot{4}$ & $\dot{6}$ & $6 . \dot{9}$ & $6 . \dot{7}$ \\
\hline Sp. Cond. (umho/cm) & 102 & 112 & 37 & 46 & 43 & 45 & 162 & 145 \\
\hline $\operatorname{TDS}(\mathrm{mg} / \mathrm{L})$ & 78 & 130 & 42 & 48 & 36 & 110 & 136 & 110 \\
\hline TSS $(\mathrm{mg} / \mathrm{L})$ & 5 & 5 & 83 & 3 & 3 & 9 & 9 & 5 \\
\hline Turbidity (NTU) & 3.1 & 2.2 & 7 & 4.5 & 0.9 & 39 & 19 & 5.4 \\
\hline RADIOCHEMICAI PARAMETERS & . & $\cdot$ & . & . &. &. &. & . \\
\hline Gross Alpha (pCi/L) & -0.16 & 0.44 & .00 & -0.84 & 0.15 & 1.27 & -1.00 & -0.62 \\
\hline Gross Alpha (CE $+/-$ ) & 0.93 & 1.30 & 1.50 & 2.90 & 0.99 & 2.10 & 1.60 & 0.43 \\
\hline Gross Beta $(\mathrm{pCi} / \mathrm{L})$ & -4.22 & 0.26 & 1.11 & -2.28 & -4.96 & -1.31 & 0.08 & -2.64 \\
\hline Gross Beta $(\mathrm{CB}+/-)$ & 2.90 & 2.80 & 2.70 & 5.30 & 3.00 & 2.70 & 2.80 & 1.40 \\
\hline
\end{tabular}

(CONTINUED) 
APPEWDIX $\mathrm{E} \cdot 3$

Groundwater and Surface-Water Quality Data, 1993

\begin{tabular}{|c|c|c|c|c|c|c|c|c|}
\hline \multirow{3}{*}{$\begin{array}{l}\text { Sampling Point } \\
\text { Location } \\
\text { Date Sampled }\end{array}$} & \multicolumn{2}{|c|}{$G W-642$} & \multicolumn{4}{|c|}{$G w-643$} & \multicolumn{2}{|c|}{$G W-644$} \\
\hline & \multicolumn{2}{|c|}{ BG } & \multicolumn{4}{|c|}{ BG } & \multicolumn{2}{|c|}{ orf } \\
\hline & $07 / 01 / 93$ & $10 / 10 / 93$ & $02 / 16 / 93$ & $05 / 19 / 93$ & $07 / 08 / 93$ & $10 / 12 / 93$ & $03 / 25 / 93$ & $06 / 25 / 93$ \\
\hline FIELD MRASUREMRNTS & $\cdot$ & - & - & - & $\cdot$ & $\cdot$ & - & - \\
\hline Depth to Water (ft) & 22.45 & 23.25 & 15.65 & 16.27 & 21.1 & 23.8 & 7.1 & 11.16 \\
\hline Water Temp (degrees C) & 18.6 & 15 & 14.2 & 15.2 & 15.8 & 13.4 & 13.5 & 14.5 \\
\hline pH (pH units) & $6 \cdot 4$ & 6 & 5.7 & 6.1 & 6 & 4.9 & 6.2 & $6 \cdot 3$ \\
\hline Sp. Cond. (umho/cm) & 125 & 138 & 104 & 67 & 227 & 67 & 125 & 156 \\
\hline Dissolved Oxygen (ppm) & 2.8 & 0.9 & 10.1 & $4 \cdot 4$ & 1.5 & 5.8 & 4.1 & 4.5 \\
\hline Oxidation/Reduction (mv) & -9 & -10 & 225 & 244 & 250 & 246 & 188 & 208 \\
\hline MISCELIANEOUS RARAMTTERS & - & - & $\cdot$ & - & - & - & - & - \\
\hline pH (pH units) & 6.8 & 7.1 & 6 & 6.4 & 7.2 & 6.1 & 6.4 & 7.1 \\
\hline sp. Cond. (umho/cm) & 136 & 137 & 65 & 74 & 273 & 73 & 123 & 203 \\
\hline $\operatorname{TDS}(\mathrm{mg} / \mathrm{L})$ & 106 & 186 & 82 & 86 & 168 & 188 & 106 & 152 \\
\hline TSS (mg/L) & 3 & 540 & 135 & 51 & 35 & 50 & 2 & 49 \\
\hline Turbidity (NTU) & 12 & 60 & 56 & 25 & 11 & 27 & 2.9 & 19 \\
\hline RADIOCHEMICAT PARAMETERS & - & - & - & - & $\cdot$ & • & . & • \\
\hline Gross Alpha (pcI/L) & -2.38 & 2.10 & 2.73 & -0.14 & 0.82 & 2.61 & -0.29 & 0.95 \\
\hline Gross Alpha $(\mathrm{CE}+/-)$ & 2.40 & 2.40 & 3.60 & 2.00 & 1.30 & 1.50 & 1.40 & 2.60 \\
\hline Gross Beta (pCL/L) & 0.64 & 3.30 & 1.57 & 1.72 & -3.49 & 1.28 & 0.66 & -6.95 \\
\hline Grose Beta $(\mathrm{CE}+/-)$ & 5.10 & 2.80 & 3.30 & 2.90 & 2.80 & 2.80 & 2.50 & 5.60 \\
\hline
\end{tabular}

(CONTINUED) 
Groundwater and Surface-Water Quality Data, 1993

\begin{tabular}{|c|c|c|c|c|c|c|c|c|}
\hline \multirow{3}{*}{$\begin{array}{l}\text { Sampling Point } \\
\text { Location } \\
\text { Date Sampled }\end{array}$} & \multicolumn{2}{|c|}{$G W-644$} & \multicolumn{4}{|c|}{$G W-647$} & \multicolumn{2}{|c|}{$G w-651$} \\
\hline & \multicolumn{2}{|c|}{ OLF } & \multicolumn{4}{|c|}{ OLF } & \multicolumn{2}{|c|}{ BG } \\
\hline & $09 / 17 / 93$ & $12 / 18 / 93$ & $02 / 08 / 93$ & $05 / 17 / 93$ & $09 / 25 / 93$ & $12 / 10 / 93$ & $03 / 01 / 93$ & $04 / 19 / 93$ \\
\hline FIELD MEASUREMERTS & - & - & - & $\cdot$ & - & $\cdot$ & - & • \\
\hline Depth to Water (ft) & 11.55 & 9 & 69.08 & 68.9 & 69 & 69 & 5.1 & 5.43 \\
\hline Water Termp (degrees C) & 16.2 & 14.2 & 14.7 & 15.4 & 15 & 13 & 9.9 & 15.5 \\
\hline pH (pH units) & 6.8 & 6 & 7.8 & 7.5 & 7 & 7.4 & 7.6 & 7.6 \\
\hline Sp. Cond. (umho/cm) & 316 & 175 & 566 & 448 & 520 & 506 & 404 & 392 \\
\hline Dissolved Oxygen (ppm) & 3.5 & 0.8 & 2.1 & 0.9 & 3.1 & 2.8 & 7.4 & 6.3 \\
\hline Oxidation/Reduction (mv) & 223 & 216 & 181 & 198 & 128 & 193 & 216 & 158 \\
\hline MISCELIANEOUS PARAMETERS & - & - & •. & - & - & $\cdot$ & - & - \\
\hline pH (pH units) & 7.1 & 6.8 & 7.9 & $7 . \dot{7}$ & $7 . \dot{8}$ & $7 . \overline{7}$ & $7 . \dot{7}$ & $\dot{8}$ \\
\hline Sp. Cond. (umho/cm) & 248 & 169 & 573 & 570 & 545 & 500 & 405 & 400 \\
\hline TDS $(m g / I)$ & 168 & 126 & 406 & 366 & 340 & 372 & 248 & 236 \\
\hline TSS (mg/L) & 53 & 27 & 16 & $<1$ & 1 & 1 & 2 & 85 \\
\hline Turbidity (NTU) & 19 & 14 & 9.3 & 1 & 5 & 1.6 & 2.4 & 1.1 \\
\hline RADTOCHEMICAL PARAMETERS & - & - & - & - & - & $\cdot$ & - & • \\
\hline Gross Alpha $(\mathrm{pCl} / \mathrm{L})$ & 0.69 & -0.01 & -1.70 & 3.74 & 2.11 & 0.34 & -0.37 & 1.71 \\
\hline Gross Alphe $(\mathrm{CE}+/-)$ & 3.10 & 1.10 & 1.80 & 3.90 & 4.00 & 2.50 & 2.30 & 2.00 \\
\hline Gross Beta $(\mathrm{pCl} / \mathrm{L})$ & 5.01 & -2.98 & 1.01 & -4.59 & 9.41 & 3.31 & 2.98 & 2.03 \\
\hline Gross Beta $(\mathrm{CE}+/-)$ & 5.00 & 2.90 & 2.90 & 5.60 & 5.50 & 3.10 & 3.60 & 3.00 \\
\hline
\end{tabular}

( CONTINUED) 
APPBNDIX R.3

Groundwater and Surface-Water Quality Data, 1993

\begin{tabular}{|c|c|c|c|c|c|c|c|c|}
\hline \multirow{3}{*}{$\begin{array}{l}\text { Sampling Point } \\
\text { Location } \\
\text { Date Sampled }\end{array}$} & \multicolumn{2}{|c|}{$G W-651$} & \multicolumn{4}{|c|}{ GW-652 } & \multicolumn{2}{|c|}{ GW-653 } \\
\hline & \multicolumn{2}{|c|}{ BQ } & \multicolumn{4}{|c|}{ BG } & \multicolumn{2}{|c|}{$\mathbf{B O}$} \\
\hline & $07 / 10 / 93$ & $10 / 10 / 93$ & $03 / 01 / 93$ & $04 / 19 / 93$ & $07 / 10 / 93$ & $10 / 18 / 93$ & $03 / 01 / 93$ & $04 / 20 / 93$ \\
\hline FIELD MEASUREMENTS & - & • & $\cdot$ & - & $\cdot$ & - & - & • \\
\hline Depth to Water (ft) & 8.8 & 10.8 & 9.25 & 9.49 & 12.32 & 11.3 & 20.5 & 19.95 \\
\hline Water Temp (degrees C) & 17.6 & 12.6 & 12.6 & 13.7 & 14.1 & 15.5 & 14.5 & 14.3 \\
\hline pH (pH units) & 7.5 & 7.7 & 7.3 & 7.3 & 7.1 & 7.5 & 5.6 & 5.3 \\
\hline sp. Cond. (umho/cm) & 338 & 369 & 407 & 394 & 352 & 433 & 34 & 31 \\
\hline Dissolved Oxygen (ppm) & 8 & 6.2 & 5.3 & 3.5 & 5.2 & 5.7 & 7.7 & 7.3 \\
\hline Oxidation/Reduction (mv) & 181 & 188 & 210 & 177 & 176 & 170 & 309 & 299 \\
\hline MISCELLANEOUS PARAMETERS & $\cdot$ & - & - & . & - & • & - & • \\
\hline pH (pH units) & $8 . \dot{1}$ & $7 . \dot{9}$ & $7 . \dot{6}$ & $7 . \dot{8}$ & $7 . \dot{9}$ & $7 . \dot{6}$ & $5 . \dot{7}$ & 5.8 \\
\hline Sp. Cond. (umho/cm) & 372 & 401 & 407 & 408 & 406 & 406 & 34 & 33 \\
\hline TDS (mg/L) & 234 & 326 & 284 & 266 & 250 & 282 & 70 & 56 \\
\hline TSS (mg/L) & $<1$ & 4 & 17 & 12 & 5 & $<1$ & 314 & 82 \\
\hline Turbidity (NTU) & 2.4 & 5.6 & 44 & 1.9 & 11 & 28 & 70 & 29 \\
\hline RADIOCKEMICAI PARAMETERS & $\cdot$ & . & . & . & . & . & . & . \\
\hline Gross Alpha $(\mathrm{pci} / L)$ & 1.21 & 0.30 & 0.22 & 1.67 & 0.47 & 0.31 & 12.90 & 3.93 \\
\hline Gross Alpha $(\mathrm{CE}+/-)$ & 1.70 & 1.80 & 2.80 & 2.20 & 1.60 & 2.80 & 5.60 & 3.50 \\
\hline Gross Beta $(\mathrm{pC} i / L)$ & 4.87 & 0.94 & 9.19 & 0.89 & 0.66 & 0.30 & 11.60 & -3.09 \\
\hline Gross Beta $(\mathrm{CE}+/-)$ & 2.90 & 2.80 & 3.90 & 2.90 & 2.60 & 6.00 & 7.20 & 5.30 \\
\hline
\end{tabular}

(CONTINUED) 
Groundwater and surface-Water Quality Data, 1993

\begin{tabular}{|c|c|c|c|c|c|c|c|c|}
\hline \multirow{3}{*}{$\begin{array}{l}\text { Sampling Point } \\
\text { Location } \\
\text { Date Sampled }\end{array}$} & \multicolumn{2}{|c|}{$6 w-653$} & \multicolumn{4}{|c|}{ Gw-654 } & \multicolumn{2}{|c|}{$G W-655$} \\
\hline & \multicolumn{2}{|c|}{ BO } & \multicolumn{4}{|c|}{ BG } & \multicolumn{2}{|c|}{ BG } \\
\hline & $07 / 10 / 93$ & $10 / 10 / 93$ & $02 / 24 / 93$ & $04 / 16 / 93$ & $07 / 01 / 93$ & $10 / 07 / 93$ & $02 / 05 / 93$ & $05 / 10 / 93$ \\
\hline FIELD MEASUREREKTS & $\cdot$ & $\cdot$ & $\cdot$ & - &. & • & $\cdot \mid$ & \\
\hline Depth to Water (ft) & 23.73 & 24.63 & 6.4 & 6.1 & 7.95 & 8.7 & 28.2 & 23.69 \\
\hline Water Temp (degrees $C$ ) & 15.1 & 13.3 & 8.3 & 11.3 & 17.8 & 17 & 14.3 & 16.3 \\
\hline pH (pH units) & 5.1 & 5.6 & 7 & 7.3 & 7 & 7.2 & 6.5 & 6 \\
\hline Sp. Cond. (umho/cm) & 28 & 32 & 239 & 244 & 221 & 256 & 99 & 78 \\
\hline Dissolved Oxygen (ppm) & 7.4 & 7.4 & 6.8 & 5.7 & 3.3 & 5.8 & 5.2 & 12.2 \\
\hline Oxidation/Reduction (mV) & 286 & 284 & 107 & 10 & -4 & 163 & 92 & 144 \\
\hline MISCRLLAMEOUS PARAMETERS & • & $\cdot$ & $\cdot$ & • & $\cdot$ & • & $\cdot$ & \\
\hline pH (pH units) & $6 . \dot{6}$ & $6 . \dot{1}$ & 7.7 & 7.4 & 7.6 & $7 . \dot{4}$ & 6.5 & 6.8 \\
\hline sp. Cond. (umho/cm) & 32 & 32 & 233 & 248 & 262 & 284 & 96 & 95 \\
\hline TDS $(\mathrm{mg} / \mathrm{L})$ & 46 & 142 & 154 & 154 & 182 & 258 & 92 & 150 \\
\hline TsS (mg/L) & $<1$ & 158 & 4 & 5 & $<1$ & $<1$ & 22 & 624 \\
\hline Turbidity (NTU) & 12 & 42 & 18 & 13 & 2.4 & 3.5 & 22 & 180 \\
\hline RADIOCHEMICAL PARAMETERS & $\cdot$ & • & $\cdot$ & $\dot{\bullet}$ & $\cdot$ & • & $\cdot$ & \\
\hline Gross Aipha (pCi/L) & 6.36 & $8.5 \dot{6}$ & 0.31 & $3.5 \dot{2}$ & -2.58 & 3.44 & $2.1 \dot{8}$ & 14.10 \\
\hline Gross Alphe $(\mathrm{CB}+/-)$ & 3.80 & 8.00 & 1.50 & 1.00 & 2.60 & 2.30 & 1.60 & 3.90 \\
\hline Gross Beta (pCi/L) & 6.73 & 16.00 & 1.89 & 1.31 & -3.20 & 4.14 & -4.35 & 13.70 \\
\hline Gross Beta $(\mathrm{CE}+/-)$ & 5.60 & 12.00 & 2.70 & 1.30 & 4.70 & 3.00 & 3.70 & 5.90 \\
\hline
\end{tabular}

(CONTINUED) 
APPENDIX 8.3

Groundwater and surface-Water Quality Data, 1993

\begin{tabular}{|c|c|c|c|c|c|c|c|c|}
\hline \multirow{3}{*}{$\begin{array}{l}\text { Sampling Point } \\
\text { Looation } \\
\text { Date sampled }\end{array}$} & \multicolumn{2}{|c|}{ GW-655 } & \multicolumn{4}{|c|}{ GW-683 } & \multicolumn{2}{|c|}{$G W-684$} \\
\hline & \multicolumn{2}{|c|}{ BG } & \multicolumn{4}{|c|}{$\operatorname{ExP}$} & \multicolumn{2}{|c|}{$\mathbf{E x P}$} \\
\hline & $07 / 01 / 93$ & $10 / 06 / 93$ & $03 / 04 / 93$ & $04 / 23 / 93$ & $07 / 12 / 93$ & $10 / 12 / 93$ & $03 / 03 / 93$ & $04 / 22 / 93$ \\
\hline FIELD MEASUREHENTS & $\cdot$ & $\cdot$ & $\cdot$ & $\cdot$ & $\cdot$ & $\cdot$ & $\cdot$ & - \\
\hline Depth to Watar $(f t)$ & 30.25 & 35.95 & 87.5 & 87.99 & 88.92 & 88.8 & 15.15 & 14.85 \\
\hline Water Tamp (degrees C) & 18.6 & 16.5 & 11.9 & 13.4 & 16 & 13.3 & 13.1 & 12.5 \\
\hline pH (pH units) & 6.1 & 6.2 & 7.1 & 7.3 & 7.3 & 7.3 & 7.5 & 7.9 \\
\hline Sp. Cond. (umho/cm) & 87 & 92 & 417 & 267 & 484 & 602 & 453 & 310 \\
\hline Dissolved Oxygen (ppm) & 2.5 & 4.6 & 9.2 & 6.9 & 6.9 & 7.8 & 6 & 5.5 \\
\hline Oxidation/Reduction (mv) & 25 & 151 & 283 & 216 & 143 & 233 & 196 & 177 \\
\hline MISCELIANEOUS PARAMETERS & $\cdot$ & $\cdot$ & • & . & $\cdot$ & $\cdot$ &. & • \\
\hline pH (pH units) & 6.9 & $6 . \overline{7}$ & 7.9 & 7.3 & $7 . \dot{5}$ & 7.4 & 7.8 & 8.1 \\
\hline Sp. Cond. (umho/cm) & 93 & 93 & 335 & 305 & 515 & 710 & 350 & 371 \\
\hline TDS (mg/L) & 74 & 64 & 178 & 160 & 308 & 504 & 226 & 222 \\
\hline TSS (mg/L) & 13 & 54 & 2 & 1 & $<1$ & 2 & $<1$ & $<1$ \\
\hline Turbidity (NTU) & 11 & 16 & 2 & 1.3 & 0.9 & 2.5 & 3.9 & 1.5 \\
\hline RADIOCHEMICAL PARAMETERS & . & $\cdot$ & - & . & . &. & . & - \\
\hline Gross Alpha (pCi/L) & -2.03 & 0.43 & 7.31 & 5.33 & 22.50 & 35.80 & 9.05 & 5.89 \\
\hline Gross Alpha $(\mathrm{CE}+/-)$ & 2.40 & 1.70 & 2.30 & 2.30 & 5.50 & 5.10 & 2.50 & 2.50 \\
\hline Gross Beta $(\mathrm{pCi} / \mathrm{L})$ & -1.65 & 2.21 & 15.80 & 10.60 & 26.20 & 64.40 & 20.90 & 27.50 \\
\hline Gross Beta $(\mathrm{CE}+/-)$ & 5.00 & 2.70 & 3.80 & 3.20 & 7.70 & 5.50 & 4.00 & 4.00 \\
\hline
\end{tabular}

(CONTINUED) 
APPENDIX $\mathrm{E} .3$

Groundwater and surface-Water Quallty Data, 1993

\begin{tabular}{|c|c|c|c|c|c|c|c|c|}
\hline \multirow{3}{*}{$\begin{array}{l}\text { sampling point } \\
\text { Location } \\
\text { Date sampled }\end{array}$} & \multicolumn{2}{|c|}{$G W-684$} & \multicolumn{4}{|c|}{$G W-683$} & \multicolumn{2}{|c|}{$6 w-694$} \\
\hline & \multicolumn{2}{|c|}{$\mathbf{E X P}$} & \multicolumn{4}{|c|}{$\exp$} & \multicolumn{2}{|c|}{$\mathbf{E X P}$} \\
\hline & $07 / 11 / 93$ & $10 / 12 / 93$ & $03 / 02 / 93$ & $04 / 21 / 93$ & $07 / 11 / 93$ & $10 / 11 / 93$ & $03 / 23 / 93$ & $06 / 06 / 93$ \\
\hline FIELd MEASUREMENTS & $\cdot$ & - & $\cdot$ & - & $\cdot$ & $\cdot$ & • & - \\
\hline Depth to Water (ft) & 15.61 & 15.55 & 7.28 & 7.35 & 8.7 & 8.14 & 25.44 & 29.14 \\
\hline Water Temp (degrees C) & 15.6 & 13.2 & 12.8 & 13.3 & 18.5 & 14.3 & 13.6 & 14.6 \\
\hline pH (pH unit») & 7.3 & 7.8 & 7.2 & 7.2 & 7.3 & 7.5 & 6.9 & 6.7 \\
\hline Sp. Cond. (umho/cm) & 459 & 494 & 707 & 620 & 590 & 634 & 603 & 748 \\
\hline Diesolved Oxygen (ppm) & 7.6 & 7 & 10.6 & 7.3 & 10.3 & 5.4 & 3.6 & 1.1 \\
\hline Oxidation/Reduction (mV) & 103 & 180 & 80 & 25 & 67 & 16 & 46 & -37 \\
\hline MISCELLANEOUS PARAMETERS & - & - & . & • & . &. & - & • \\
\hline pH (pH units) & $7 . \dot{9}$ & $7 . \dot{7}$ & $7 . \dot{6}$ & $7 . \dot{8}$ & $7 . \overline{7}$ & 7.7 & $7 . \dot{2}$ & 7.8 \\
\hline Sp. Cond. (umho/cm) & 510 & 591 & 723 & 724 & 665 & 680 & 620 & 822 \\
\hline $\operatorname{TDS}(\mathrm{mg} / \mathrm{L}$ ) & 322 & 432 & 412 & 422 & 388 & 456 & 360 & 514 \\
\hline Tss (mg/L) & $<1$ & 3 & 12 & 10 & 2 & 5 & 3 & 3 \\
\hline Turblaity (NTU) & 1 & 4.5 & 52 & 12 & 2.9 & 18 & 2.7 & 5.4 \\
\hline RADIOCHEMICAL PARAMETERS & . & . & - & . & - & . & - & - \\
\hline Gross Alpha (pCi/L) & 21.20 & 38.20 & 0.75 & 1.93 & 3.21 & 4.48 & 31.80 & 56.90 \\
\hline Gross Alpha $(\mathrm{CB}+/-)$ & 4.00 & 5.20 & 3.30 & 3.30 & 2.30 & 2.20 & 5.10 & 9.20 \\
\hline Gross Beta $(\mathrm{pCl} / \mathrm{L})$ & 46.60 & 63.60 & 3.16 & 5.21 & 7.10 & 10.90 & 34.60 & 88.40 \\
\hline Gross Beta $(\mathrm{CE}+/-)$ & 4.70 & 5.50 & 3.70 & 3.30 & 3.10 & 3.40 & 4.20 & 9.40 \\
\hline
\end{tabular}

(CONTINUED) 
APRENDIX $\mathrm{B}, 3$

Groundwater and surface-water Qual1ty Data, $199 \mathrm{~J}$

\begin{tabular}{|c|c|c|c|c|c|c|c|c|}
\hline \multirow{3}{*}{$\begin{array}{l}\text { 8ampling Polnt } \\
\text { Location } \\
\text { Date sampled }\end{array}$} & \multicolumn{2}{|c|}{ an-694 } & \multicolumn{4}{|c|}{$0 w-695$} & \multicolumn{2}{|c|}{ aH-703 } \\
\hline & \multicolumn{2}{|c|}{$\mathbf{E x P}$} & \multicolumn{4}{|c|}{$\mathbf{E x p}$} & \multicolumn{2}{|c|}{$\mathbf{E x P}$} \\
\hline & $09 / 22 / 93$ & $12 / 28 / 93$ & $03 / 24 / 93$ & $06 / 09 / 93$ & $09 / 22 / 93$ & $12 / 21 / 93$ & $03 / 20 / 93$ & $06 / 03 / 93$ \\
\hline FIELD MaAOREMNTS & $\cdot$ & - & - & - & - & - & - & - \\
\hline Depth to Water (ft) & 30.9 & 28.3 & 23.85 & 29.65 & 31 & 26.52 & 39 & 42.8 \\
\hline Water Tenp (degrees C) & 15.3 & 12.7 & 14.6 & 18.8 & 16.4 & 9.3 & 10 & 25.3 \\
\hline ph (pH unite) & 6.7 & 6.7 & 8.4 & 7.9 & 8.3 & 8.3 & 7.7 & 8 \\
\hline Sp. Cond. (umho/cm) & 930 & 758 & 389 & 438 & 417 & 373 & 608 & 427 \\
\hline Diasolved Oxygen (ppm) & 0.7 & 5.3 & 7.2 & 7.2 & 11 & 7.5 & 8.4 & 1.5 \\
\hline Oxidation/Reduction (mv) & 2 & 70 & 146 & 162 & 98 & 207 & 174 & 175 \\
\hline MISCELTANEOUS PARAMTERS & $\cdot$ & - & $\cdot$ & - & $\cdot$ & - & - & - \\
\hline ph (pH unite) & 7.1 & 7.2 & 8.3 & 8.3 & 8.3 & 8.2 & 7.8 & 8.4 \\
\hline 8p. Cond. (umho/cm) & 918 & 815 & 407 & 438 & 416 & 441 & 708 & 475 \\
\hline TDS (mg/x) & 568 & 488 & 252 & 280 & 272 & 286 & 400 & 284 \\
\hline TsS $(\mathrm{mg} / \mathrm{L})$ & 1 & 2 & 12 & 149 & 83 & 325 & $<1$ & 8 \\
\hline Turbldity (NTU) & 2 & 2.7 & 3.1 & 40 & 20 & 200 & 2.6 & 8.7 \\
\hline RADIOCHEMICAL PARAYETERS & - & - & . & . & . & - & : & • \\
\hline Grose Alpha (pCi/L) & 50.90 & 108.00 & 3.49 & 4.46 & 5.77 & 10.90 & 0.19 & -2.64 \\
\hline Gross Alpha $(\mathrm{CE}+/-)$ & 13.00 & 8.90 & 2.30 & 4.20 & 5.00 & 3.40 & 2.30 & 4.30 \\
\hline Gross Beta (pCi/L) & 112.00 & 161.00 & 15.70 & 14.50 & 30.90 & 16.90 & 6.39 & -3.95 \\
\hline Gross Beta $(\mathrm{CE}+/-)$ & 17.00 & 8.00 & 3.40 & 6.10 & 6.70 & 4.10 & 2.90 & 6.00 \\
\hline
\end{tabular}

(CONTINUED) 
APPENDIX 8.3

Groundwater and surface-Water Quality Data, 1993

\begin{tabular}{|c|c|c|c|c|c|c|c|c|}
\hline \multirow{3}{*}{$\begin{array}{l}\text { 8ampling Polnt } \\
\text { Location } \\
\text { Date sampled }\end{array}$} & \multicolumn{2}{|c|}{ Ow-703 } & \multicolumn{4}{|c|}{ cW-704 } & \multicolumn{2}{|c|}{ aw-706 } \\
\hline & \multicolumn{2}{|c|}{$\mathbf{E x p}$} & \multicolumn{4}{|c|}{$\mathbf{E x p}$} & \multicolumn{2}{|c|}{$\mathbf{E x p}$} \\
\hline & $09 / 20 / 93$ & $12 / 21 / 93$ & $03 / 22 / 93$ & $06 / 04 / 93$ & $09 / 22 / 93$ & $12 / 22 / 93$ & $03 / 23 / 93$ & $06 / 11 / 93$ \\
\hline FIELD MEASUREIUNTS & $\cdot$ & - & $\cdot$ & • & $\cdot$ & • & $\cdot$ & • \\
\hline Depth to Water (ft) & 45.45 & 41.45 & 28.3 & 33.82 & 36.5 & 32 & 12.57 & 17.46 \\
\hline Water Temp (dagreas C) & 15 & 11.6 & 13.4 & 18.2 & 14.4 & 11 & 14 & 17.7 \\
\hline pH (pH unita) & & 8 & 7.5 & 7.5 & 7.7 & 8.4 & 5.2 & 7.2 \\
\hline 8p. Cond. (unho/cm) & 538 & 552 & 578 & 527 & 394 & 333 & 680 & 745 \\
\hline Dlesolved Oxygen (ppm) & 5.5 & 5.1 & s) & 1.5 & 2.4 & 1.6 & 4.5 & 1 \\
\hline Oxidation/Reduction (mv) & 108 & 196 & 40 & 85 & -60 & 25 & -16 & -61 \\
\hline MISCELLANEOUS PARAMETERS & $\cdot$ & $\cdot$ & $\cdot$ & $\cdot$ & $\cdot$ & $\cdot$ & . & - \\
\hline pH (pH unites) & & 7.9 & 8.1 & 8 & 7.8 & 8.3 & $8 . \dot{6}$ & 7.4 \\
\hline Sp. Cond. (unho/cm) & 5.83 & 675 & 606 & 588 & 612 & 350 & 363 & 845 \\
\hline $\operatorname{TDS}(\mathrm{mg} / \mathrm{I})$ & 354 & 396 & 348 & 258 & 344 & 180 & 190 & 544 \\
\hline TSS (mg/L) & 2 & 2 & 3 & 52 & 24 & 21 & 7 & 1 \\
\hline Turbidity (NTU) & 8.2 & 2.5 & 15 & 125 & 38 & 17 & 15 & 2 \\
\hline RADTOCHEMICAL PARAMEIERS & - & $\cdot$ & $\cdot$ & $\cdot$ & $\cdot$ & $\cdot$ & . & 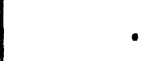 \\
\hline Gross Npha (pCl/L) & -0.66 & 2.91 & -1.48 & 0.92 & 1.30 & 0.82 & 0.00 & 54.40 \\
\hline Gross Alpha $(\mathrm{CB}+/-)$ & 5.00 & 1.80 & 3.30 & 3.80 & 4.20 & 1.20 & 1.50 & 9.00 \\
\hline Grose Beta $(\mathrm{PCI} / \mathrm{L})$ & 28.60 & 42.70 & 4.94 & 9.93 & 13.90 & 3.64 & 7.42 & 97.90 \\
\hline Grose Beta $(\mathrm{Cz}+/-)$ & 7.10 & 6.10 & 5.20 & 5.90 & 5.80 & 2.70 & 2.90 & 9.80 \\
\hline
\end{tabular}

(CONTINURD) 
APREndX 8.3

Groundwater and surface-water Quality Data, 1993

\begin{tabular}{|c|c|c|c|c|c|c|c|c|}
\hline \multirow{3}{*}{$\begin{array}{l}\text { 8ampling point } \\
\text { Location } \\
\text { Date sampled }\end{array}$} & \multicolumn{2}{|c|}{ 0u-706 } & \multicolumn{4}{|c|}{$0 w-710$} & \multicolumn{2}{|c|}{ ON-711 } \\
\hline & \multicolumn{2}{|c|}{$\mathbf{A x p}$} & \multicolumn{4}{|c|}{$\mathbf{2 x p}$} & \multicolumn{2}{|c|}{$\mathbf{E x p}$} \\
\hline & $09 / 23 / 93$ & $12 / 28 / 93$ & $03 / 21 / 93$ & $04 / 30 / 93$ & $08 / 01 / 93$ & $11 / 09 / 93$ & $03 / 20 / 93$ & $04 / 29 / 93$ \\
\hline FIELD MASUREMANS & & $\cdot$ & - & - & - & - & $\cdot$ & • \\
\hline Depth to Water (ft) & 18.55 & 15.6 & 66.85 & 64.59 & 67.93 & 68.7 & 61.1 & 59.8 \\
\hline Water Tamp (degreos C) & 16.1 & 12.9 & 12.2 & 16 & 15.6 & 13 & 12.2 & 13.3 \\
\hline pH (pH units) & & 7.2 & 7.6 & 7.3 & 7.5 & 7.5 & 7.7 & 7.5 \\
\hline 8p. Cond. (umho/cm) & 852 & 783 & 3850 & 3590 & 3600 & 3520 & 6680 & 7410 \\
\hline Diseolved oxygen (ppm) & 2 & 1.9 & 0.5 & 0.5 & 2 & 9.5 & 1.5 & 2.5 \\
\hline Oxidation/Reduction (mv) & 22 & 60 & -120 & -111 & -119 & -53 & -107 & -101 \\
\hline MISCELLAKLOUS PARAMETERS & 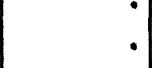 & $\cdot$ & $\dot{\bullet}$ & • & • & • & $\cdot$ & $\bullet$ \\
\hline pH (pH units) & $8 . \dot{4}$ & $7 . \dot{5}$ & i & 6.5 & $7 . \dot{6}$ & 7.4 & $7 . \dot{1}$ & $7 . \dot{6}$ \\
\hline sp. Cond. (umho/cm) & 333 & 835 & 4190 & 4050 & 3990 & 4060 & 8610 & 8660 \\
\hline $\operatorname{TDS}(m g / x)$ & 168 & 508 & 3862 & 3782 & 3758 & 3738 & 6526 & 6344 \\
\hline T8s (mg/L) & 27 & $<1$ & 12 & 2 & 7 & 9 & 23 & 23 \\
\hline Turbldity (NTU) & 120 & 3.9 & 20 & 27 & 11 & 5 & 21 & 38 \\
\hline RADIOCHEMICAL PARAMETERS & & . & . & - & - & - & • & - \\
\hline Grose Alpha $(\mathrm{pCl} / \mathrm{L})$ & 11.70 & 99.20 & 1.00 & -4.53 & -6.39 & -6.99 & $1.4 \dot{5}$ & 3.24 \\
\hline Gross Alphe $(\mathrm{Cz}+/-)$ & 7.40 & 8.50 & 4.20 & 3.10 & 41.00 & 22.00 & 4.60 & 3.80 \\
\hline Gross Bata $(\mathrm{PC} 1 / \mathrm{L})$ & 0.00 & 150.00 & 7.84 & -11.80 & 15.00 & -20.10 & 16.80 & 8.84 \\
\hline Oross Beta $(\mathrm{CE}+/-)$ & & 7.70 & 3.20 & 2.90 & 53.00 & 28.00 & 3.70 & 3.40 \\
\hline
\end{tabular}

(CONTINURD) 
APPENDIX 8.3

Groundwater and surface-water Quality Data, 1993

\begin{tabular}{|c|c|c|c|c|c|c|c|c|}
\hline \multirow{3}{*}{$\begin{array}{l}\text { 8ampling point } \\
\text { Location } \\
\text { Date sumpled }\end{array}$} & \multicolumn{2}{|c|}{$0 N-711$} & \multicolumn{4}{|c|}{ ON-712 } & \multicolumn{2}{|c|}{$0 N-713$} \\
\hline & \multicolumn{2}{|c|}{$\exp$} & \multicolumn{4}{|c|}{$\mathbf{E x p}$} & \multicolumn{2}{|c|}{$\mathbf{m x p}$} \\
\hline & $07 / 30 / 93$ & $11 / 07 / 93$ & $03 / 28 / 93$ & $04 / 28 / 93$ & $07 / 29 / 93$ & $11 / 06 / 93$ & $03 / 19 / 93$ & $04 / 30 / 93$ \\
\hline TIELD MAABURMERTS & - & $\cdot$ & $\cdot$ & - & - & - & - & • \\
\hline Depth to Water (ft) & 51.21 & 66 & 32 & 31.2 & 35.49 & 34.35 & 36.25 & 35.01 \\
\hline Water Temp (degrees C) & 14.7 & 12.3 & 11.2 & 15 & 25.1 & 12.2 & 11.4 & 13.7 \\
\hline pH (pH unite) & 7.2 & 7.4 & 7.6 & 7.3 & 7.6 & 7.5 & 7.6 & 7.5 \\
\hline sp. Cond. (unho/cm) & 7240 & 7640 & 809 & 753 & 833 & 749 & 685 & 511 \\
\hline Dineolved Oxygen (ppm) & 1.1 & 0.4 & 0.9 & 4.2 & 0.7 & 0.5 & 1.5 & 0.3 \\
\hline Oxidation/Reduction (mv) & -103 & -85 & -8 & 70 & -133 & 62 & -32 & -83 \\
\hline MISCELTAKOUS PARANETERS & - & $\cdot$ & $\cdot$ & $\cdot$ & - & - & $\cdot$ & - \\
\hline pH (pH unite) & 7.4 & 7.4 & $\dot{8}$ & 0.1 & 7.8 & $7 . \dot{7}$ & $7 . \dot{5}$ & $7 . \dot{8}$ \\
\hline sp. Cond. (umho/cm) & 8560 & 8340 & 853 & 832 & 836 & 828 & 732 & 628 \\
\hline $\operatorname{TDS}(\mathrm{mg} / \mathrm{L})$ & 6132 & 6164 & 642 & 596 & 652 & 568 & 502 & 424 \\
\hline Ts8 (mg/L) & 15 & 14 & 4 & 2 & $<1$ & 1 & 4 & 4 \\
\hline Turbldity (NTu) & 15 & 32 & 11 & 7 & 4.3 & 12 & 7.7 & 18 \\
\hline RADTOCHEMICAI PARAHLTERS & - & $\cdot$ & - & . & - & - & - & • \\
\hline Grose Alpha (pC1/L) & 3.52 & -6.49 & 1.71 & 5.37 & -2.80 & -0.19 & -0.53 & -1.16 \\
\hline Grose Alpha $(\mathrm{Cz}+/-)$ & 47.00 & 28.00 & 1.80 & 2.50 & 37.00 & 2.50 & 2.00 & 2.00 \\
\hline Grose Beta $(\mathrm{pCl} / \mathrm{L})$ & 18.60 & 3.59 & 3.37 & -8.64 & 31.00 & 1.15 & 3.30 & -8.13 \\
\hline Grose Beta $(\mathrm{CE}+/-)$ & 54.00 & 31.00 & 2.90 & 2.70 & 53.00 & 3.10 & 2.70 & 2.80 \\
\hline
\end{tabular}

(CONT IRUED) 


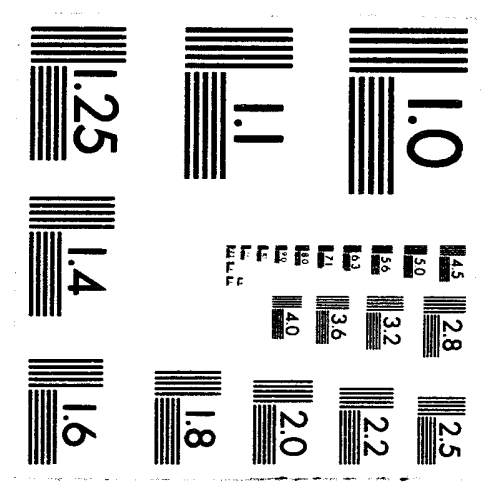



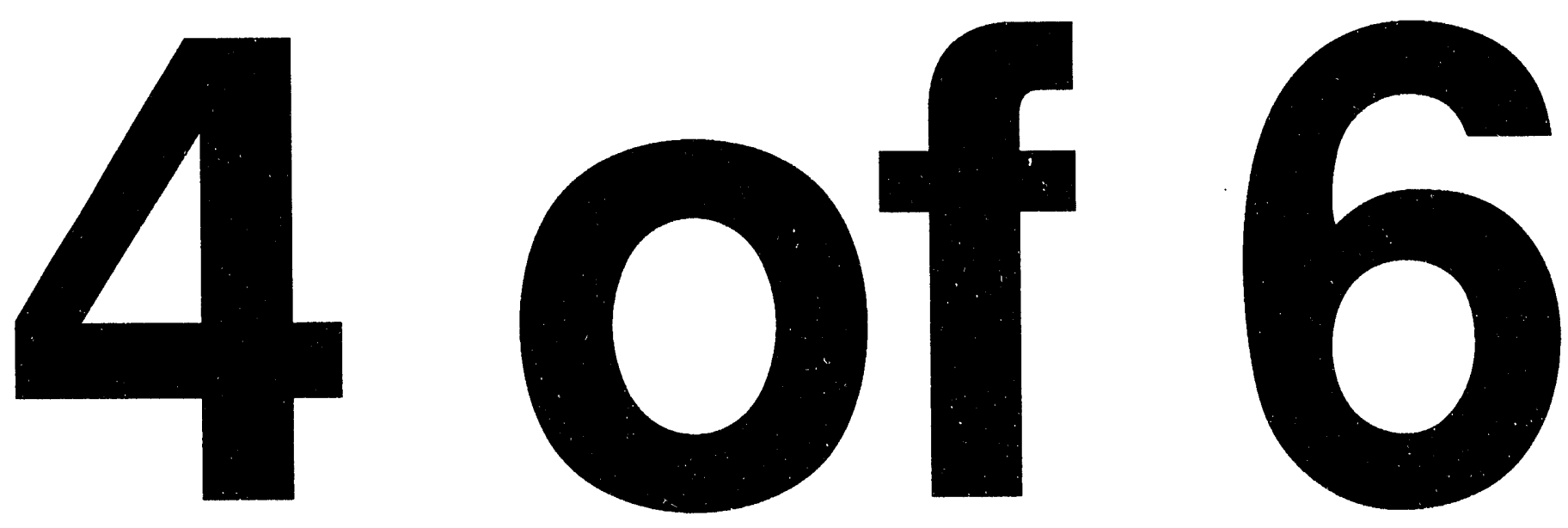
APPENDIX E. 3

Groundwater and Surface-Water Quality Data, 1993

\begin{tabular}{|c|c|c|c|c|c|c|c|c|}
\hline \multirow{3}{*}{$\begin{array}{l}\text { Sampling Point } \\
\text { - } \\
\text { Location } \\
\text { - } \\
\text { Date Sampled }\end{array}$} & \multicolumn{2}{|c|}{ GW-713 } & \multicolumn{4}{|c|}{ GW-714 } & \multicolumn{2}{|c|}{ GW-715 } \\
\hline & \multicolumn{2}{|c|}{ EXP } & \multicolumn{4}{|c|}{ EXP } & \multicolumn{2}{|c|}{$\mathbf{E X P}$} \\
\hline & $07 / 31 / 93$ & $11 / 09 / 93$ & $03 / 21 / 93$ & $04 / 29 / 93$ & $08 / 01 / 93$ & $11 / 09 / 93$ & $03 / 21 / 93$ & $04 / 29 / 93$ \\
\hline FIELD MEASUREMENTS & $\cdot 1$ & $\cdot$ & - & $\cdot$ & - & - & $\cdot$ & - \\
\hline Depth to water (ft) & 41.6 & 38.25 & 23.22 & 25.89 & 31.7 & 31.12 & 22.44 & 23.75 \\
\hline water Temp (degrees C) & 15.5 & 12.2 & 12.2 & 16.8 & 18 & 12.6 & 13.2 & 15.5 \\
\hline pH (pH units) & 7.8 & 7.4 & 7.6 & 7.6 & 7.9 & 7.6 & 7.1 & 7.2 \\
\hline Sp. Cond. (unho/cm) & 683 & 562 & 509 & 436 & 489 & 392 & 586 & 496 \\
\hline Dissolved Oxygen (ppm) & 1.1 & 0.3 & 2 & 9 & 6 & 1.3 & 6.6 & 0.9 \\
\hline Oxidation/Reduction (mv) & -136 & -10 & 81 & 148 & 85 & -69 & 118 & 99 \\
\hline MISCELIAANEOUS PARAMETERS & - & - & - & $\cdot$ & - & $\cdot$ & $\cdot$ & - \\
\hline $\mathrm{pH}$ (pH units) & 7.7 & 7.8 & 7.6 & 8.2 & 8.1 & 7.9 & 7.2 & 8 \\
\hline Sp. Cond. (umho/cm) & 668 & 633 & 473 & 466 & 454 & 432 & 638 & 555 \\
\hline $\operatorname{TDS}(\mathrm{mg} / \mathrm{L})$ & 476 & 438 & 276 & 264 & 274 & 256 & 376 & 298 \\
\hline TSS (mg/L) & 5 & 4 & 6 & 20 & $<1$ & 12 & 1 & 6 \\
\hline Turbidity (NTU) & 24 & 15 & 3.8 & 54 & 3.8 & 18 & 3 & 3 \\
\hline RADIOCHEMICAL PARAMETERS & $\cdot$ & - & - & $\cdot$ & - & $\cdot$ & - & - \\
\hline Gross Alpha (pci/L) & $-22 \cdot 30$ & -0.17 & 2.23 & -3.05 & -2.80 & 0.93 & -0.35 & -3.15 \\
\hline Gross Alpha $(\mathrm{CE}+f-)$ & 33.00 & 2.20 & 2.40 & 1.60 & 37.00 & 2.20 & 2.10 & 1.80 \\
\hline Gross Beta $(\mathrm{pCi} / \mathrm{L})$ & -17.90 & 1.13 & 2.94 & -3.17 & $-22 \cdot 80$ & 6.28 & 5.66 & -4.22 \\
\hline Gross Beta $(\mathrm{CE}+/-)$ & 50.00 & 3.00 & 2.70 & 3.00 & 49.00 & 3.30 & 2.90 & 2.90 \\
\hline
\end{tabular}

(CONTINUED) 


\begin{tabular}{|c|c|c|c|c|c|c|c|c|}
\hline \multirow{3}{*}{$\begin{array}{l}\text { Sampling Point } \\
\text { Location } \\
\text { - } \\
\text { Date Sampled }\end{array}$} & \multicolumn{2}{|c|}{ GW-715 } & \multicolumn{4}{|c|}{ GW-723 } & \multicolumn{2}{|c|}{ GW-724 } \\
\hline & \multicolumn{2}{|c|}{ EXP } & \multicolumn{4}{|c|}{$\mathbf{E X P}$} & \multicolumn{2}{|c|}{$\mathbf{E X P}$} \\
\hline & $08 / 01 / 93$ & $11 / 11 / 93$ & $02 / 12 / 93$ & $05 / 18 / 93$ & $09 / 27 / 93$ & $12 / 14 / 93$ & $02 / 10 / 93$ & $05 / 18 / 93$ \\
\hline FIELD MEASUREMENTS & $\cdot$ & - & - & - & - & - & - & • \\
\hline Depth to Water (ft) & 30.1 & 29.15 & 70.5 & 69.97 & 76.45 & 71.55 & 29.65 & 31.35 \\
\hline Water Temp (degrees $C$ ) & 14.5 & 12.8 & 14.4 & 15.8 & 14.6 & 12.2 & 13.6 & 15.8 \\
\hline pH (pH units) & 7.1 & 7 & 7.4 & 7.9 & 7.8 & 8.4 & 7.1 & 7.3 \\
\hline Sp. Cond. (umho/cm) & 552 & 454 & 652 & 509 & 493 & 512 & 899 & 762 \\
\hline Dissolved Oxygen (ppm) & 1.1 & 1.9 & 9.1 & 8.4 & 7.9 & 11.2 & 2.4 & $3 \cdot 4$ \\
\hline Oxidation/Reduction (mv) & 119 & 182 & 138 & 184 & 71 & 179 & 22 & -45 \\
\hline MISCELIAANEOUS PARAMETERS & - & - & - & - & $\cdot$ & - & - & - \\
\hline pH (pH units) & 7.8 & 7.1 & 7.7 & 7.9 & $7 \cdot 4$ & 7.9 & 7.5 & 7.5 \\
\hline Sp. Cond. (umho/cm) & 566 & 552 & 649 & 516 & 507 & 497 & 990 & 934 \\
\hline $\operatorname{TDS}(\mathrm{mg} / \mathrm{L})$ & 334 & 354 & 358 & 298 & 280 & 304 & 546 & 604 \\
\hline TSS (mg/L) & 6 & 1 & 89 & 9 & $<1$ & 6 & 3 & 2 \\
\hline Turbidity (NTU) & 9.9 & 54 & 80 & 14 & 36 & 9.2 & 7.8 & 9.1 \\
\hline RADIOCHEMICAL PARAMETERS & $\cdot$ & - & - & - & $\cdot$ & - & - & - \\
\hline Gross Alpha (pCi/L) & -33.80 & 0.17 & 0.54 & 3.24 & -1.51 & 0.64 & 4.97 & 2.08 \\
\hline Gross Alpha $(\mathrm{CE}+/-)$ & 32.00 & 1.30 & 7.80 & 4.00 & 3.50 & 2.40 & 2.80 & 3.80 \\
\hline Gross Beta (pCi/L) & -11.40 & 2.65 & 4.96 & 10.70 & 9.41 & 9.78 & 11.10 & 40.40 \\
\hline Gross Beta $(\mathrm{CE}+/-)$ & 50.00 & 2.50 & 6.90 & 6.50 & 5.50 & 3.40 & 3.50 & 7.90 \\
\hline
\end{tabular}

(CONTINUED) 
APPENDIX E. 3

Groundwater and Surface-Water Quality Data, 1993

\begin{tabular}{|c|c|c|c|c|c|c|c|c|}
\hline \multirow{3}{*}{$\begin{array}{l}\text { Sampling Point } \\
\text { Location } \\
\text { - } \\
\text { Date Sampled }\end{array}$} & \multicolumn{2}{|c|}{ GW-724 } & \multicolumn{4}{|c|}{ GW-725 } & \multicolumn{2}{|c|}{$\mathrm{GW}-736$} \\
\hline & \multicolumn{2}{|c|}{$\mathbf{E X P}$} & \multicolumn{4}{|c|}{ EXP } & \multicolumn{2}{|c|}{ EXP } \\
\hline & $09 / 29 / 93$ & $12 / 14 / 93$ & $02 / 12 / 93$ & $05 / 19 / 93$ & $09 / 28 / 93$ & $12 / 15 / 93$ & $02 / 11 / 93$ & $05 / 18 / 93$ \\
\hline FIELD MEASUREMENTS & - & - & - & - & - & - & - & • \\
\hline Depth to water (ft) & 34.15 & 28.77 & 11.02 & 13.25 & 13.65 & 10.65 & 9.95 & 10.9 \\
\hline Water Temp (degrees $C$ ) & 15.2 & 10.8 & 15 & 15.5 & 15.1 & 13.3 & 13.2 & 16 \\
\hline pH (pH units) & 7.5 & 7.3 & 7 & 7.1 & 6.8 & 7 & 6.8 & 6.8 \\
\hline Sp. Cond. (umho/cm) & 1262 & 814 & 1182 & 1069 & 1077 & 740 & 1080 & 915 \\
\hline Dissolved Oxygen (ppm) & 7.3 & 1 & 2.2 & 1.7 & 0.4 & 3.1 & 2.2 & 1.3 \\
\hline Oxidation/Reduction (mV) & 33 & -60 & -1 & 105 & 20 & 98 & 182 & 175 \\
\hline MISCELILANEOUS PARAMETERS & - & . & . & . & . & • & • & • \\
\hline pH (pH units) & 7.7 & 7.6 & 7.2 & 7.3 & 7.2 & 7.3 & 6.9 & 7 \\
\hline Sp. Cond. (umho/cm) & 3.2 & 930 & 1180 & 1180 & 1130 & 1140 & 1180 & 1100 \\
\hline $\operatorname{TDS}(\mathrm{mg} / \mathrm{L})$ & 494 & 556 & 722 & 760 & 576 & 764 & 710 & 700 \\
\hline TSS (mg/L) & $<1$ & 3 & 4 & 1 & $<1$ & 1 & 1 & $<1$ \\
\hline Turbidity (NTU) & 7 & 16 & 10 & 2 & 0.8 & 1.5 & 0.7 & 1.4 \\
\hline RADIOCHEMICAL PARAMETERS & - & - & - & - & - & - & - & • \\
\hline Gross Alpha (pCi/L) & 0.92 & -0.36 & 0.26 & 2.06 & 0.63 & 4.61 & 7.80 & 2.04 \\
\hline Gross Alpha $(\mathrm{CE}+/-)$ & 3.40 & 2.60 & 3.70 & 3.80 & 3.80 & 3.50 & 4.50 & 3.70 \\
\hline Gross Beta (pCi/L) & 41.80 & 32.00 & 24.20 & 41.60 & 46.50 & 53.70 & 22.90 & 45.20 \\
\hline Gross Beta $(\mathrm{CE}+/-)$ & 4.90 & 4.40 & 4.40 & 7.90 & 5.20 & 5.30 & 3.90 & 8.00 \\
\hline
\end{tabular}

(CONTINUED) 
Groundwater and Surface-Water Quality Data, 1993

\begin{tabular}{|c|c|c|c|c|c|c|c|c|}
\hline \multirow{3}{*}{$\begin{array}{l}\text { Sampling Point } \\
\text { Location } \\
\text { - } \\
\text { Date Sampled }\end{array}$} & \multicolumn{2}{|c|}{ GW-736 } & \multicolumn{4}{|c|}{ GW-737 } & \multicolumn{2}{|c|}{ GW-738 } \\
\hline & \multicolumn{2}{|c|}{ EXP } & \multicolumn{4}{|c|}{ EXP } & \multicolumn{2}{|c|}{ EXP } \\
\hline & $09 / 28 / 93$ & $12 / 15 / 93$ & $02 / 11 / 93$ & $05 / 19 / 93$ & $09 / 28 / 93$ & $12 / 15 / 93$ & $02 / 10 / 93$ & $05 / 18 / 93$ \\
\hline FIELD MEASUREKENTS & $\cdot$ & $\cdot$ & - & $\cdot$ & $\cdot$ & $\cdot$ & - & - \\
\hline Depth to water (ft) & 13.07 & 11.12 & 12.65 & 11.2 & 13.6 & 12 & 28.97 & 28.75 \\
\hline Water Temp (degrees $C$ ) & 15.3 & 13.3 & 13.6 & 14.7 & $16 \cdot 5$ & 12.8 & 14 & 15.9 \\
\hline pH (pH units) & 6.8 & 7 & 6.9 & 6.7 & 7.1 & 7 & 6.2 & 7.1 \\
\hline Sp. Cond. (umho/cm) & 1166 & 1050 & 1122 & 878 & 1197 & 1050 & 745 & 595 \\
\hline Dissolved Oxygen (ppm) & 0.8 & 3 & 4.1 & 1.1 & 0.7 & 4.2 & 1.2 & 2.1 \\
\hline Oxidation/Reduction (mv) & 178 & 157 & 183 & 197 & 155 & 167 & 186 & 167 \\
\hline MISCELLANEOUS PARAMETERS & - & - & $\cdot$ & - & . & - & $\cdot$ & - \\
\hline pH (pH units) & 7.4 & 7.2 & - & 7.2 & 7.4 & 7.3 & $7 \cdot 3$ & 7 \\
\hline sp. Cond. (umho/cm) & 1120 & 1120 & 1220 & 1120 & 1120 & 1110 & 804 & 727 \\
\hline $\operatorname{TDS}(\mathrm{mg} / \mathrm{L}$ ) & 696 & 740 & 712 & 760 & 694 & 726 & 492 & 440 \\
\hline TSS (mg/L) & $<1$ & $<1$ & 1 & $<1$ & $<1$ & $<1$ & 3 & 3 \\
\hline Turbidity (NTU) & 0.9 & 0.6 & 1 & 1.1 & 1.4 & 0.8 & 3.9 & 4.9 \\
\hline RADIOCHEMICAL PARAMETERS & $\cdot$ & - & $\cdot$ & $\cdot$ & $\cdot$ & $\cdot$ & - & • \\
\hline Gross Alpha (pCi/L) & 3.51 & 4.12 & 9.66 & 0.95 & 0.00 & 5.85 & 7.81 & 0.59 \\
\hline Gross Alpha $(\mathrm{CE}+/-)$ & 4.00 & 3.40 & 4.80 & 3.50 & 3.70 & 3.60 & 3.30 & $\therefore 50$ \\
\hline Gross Beta (pCi/L) & 57.90 & 52.00 & 23.60 & 42.00 & 52.80 & 60.80 & $41 \cdot 40$ & 30.20 \\
\hline Gross Beta $(\mathrm{CE}+/-)$ & 5.50 & 5.20 & 3.90 & 4.90 & 5.40 & 5.50 & 4.80 & 7.40 \\
\hline
\end{tabular}

(CONTINUED) 
APPENDIX E.3

Groundwater and Surface-Water Quality Data, 1993

\begin{tabular}{|c|c|c|c|c|c|c|c|c|}
\hline \multirow{3}{*}{$\begin{array}{l}\text { Sampling Point } \\
\text { Location } \\
\text { - } \\
\text { Date Sampled }\end{array}$} & \multicolumn{2}{|c|}{ GW-738 } & \multicolumn{4}{|c|}{ GW-739 } & \multicolumn{2}{|c|}{ GW-740 } \\
\hline & \multicolumn{2}{|c|}{$\mathbf{E X P}$} & \multicolumn{4}{|c|}{ EXP } & \multicolumn{2}{|c|}{$\mathbf{E X P}$} \\
\hline & $09 / 27 / 93$ & $12 / 14 / 93$ & $02 / 10 / 93$ & $05 / 18 / 93$ & $09 / 27 / 93$ & $12 / 13 / 93$ & $02 / 09 / 93$ & $05 / 17 / 93$ \\
\hline FIELD MEASUREMENTS & - & - & $\cdot$ & $\cdot$ & - & - & - & - \\
\hline Depth to Water (ft) & 30.1 & 27.78 & 75 & 74.95 & 77.45 & 71.95 & 69.45 & 70.86 \\
\hline Water Temp (degrees $C$ ) & 14.7 & 13.3 & 13 & 15 & 14 & 13 & 14.5 & 15.3 \\
\hline pH (pH units) & 7.3 & 6.9 & 7.5 & 7.4 & 7.3 & 7.7 & 7.3 & 7.3 \\
\hline Sp. Cond. (umho/cm) & 786 & 772 & 502 & 426 & 491 & 487 & 529 & 428 \\
\hline Dissolved Oxygen (ppm) & 1.4 & 3.2 & 2.7 & 3.3 & 2.3 & 5.8 & 3.5 & 3.3 \\
\hline Oxidation/Reduction (mV) & 54 & 161 & 143 & 193 & 82 & 129 & 55 & 102 \\
\hline MISCELLANEOUS PARAMETERS & - & $\cdot$ & . & . & . & . & . & . \\
\hline $\mathrm{pH}$ (pH units) & $7 . \dot{4}$ & $7 . \dot{3}$ & $7 . \dot{6}$ & $7 . \dot{7}$ & $7 . \dot{6}$ & 7.7 & $7 . \dot{5}$ & $7 . \dot{6}$ \\
\hline Sp. Cond. (umho/cm) & 824 & 848 & 530 & 496 & 535 & 522 & 529 & 527 \\
\hline $\operatorname{TDS}(\mathrm{mg} / \mathrm{L})$ & 478 & 496 & 312 & 292 & 280 & 314 & 306 & 292 \\
\hline TSS $(\mathrm{mg} / \mathrm{L})$ & $<1$ & 4 & 16 & 3 & 1 & 5 & 3 & $<1$ \\
\hline Turbidity (NTU) & 7.9 & 3.2 & 22 & 3 & 3.7 & 6.7 & 3.4 & 2.3 \\
\hline RADIOCHEMICAL PARAMETERS & • & $\cdot$ & $\cdot$ & . & . & . & $\dot{\bullet}$ & . \\
\hline Gross Alpha $(\mathrm{pC} i / L)$ & 0.33 & 0.18 & 1.57 & 2.33 & -1.22 & -0.96 & 0.17 & 0.28 \\
\hline Gross Alpha $(\mathrm{CE}+/-)$ & 4.10 & 2.60 & 1.50 & 3.80 & 3.60 & 2,20 & 1.30 & 3.40 \\
\hline Gross Beta $(\mathrm{pCi} / \mathrm{L})$ & 47.50 & 60.10 & 1.08 & 7.72 & 14.20 & 9.51 & 0.17 & 0.33 \\
\hline Gross Beta $(\mathrm{CE}+1-)$ & 7.50 & 5.40 & 2.80 & 6.30 & 5.80 & 3.40 & 2.80 & 5.90 \\
\hline
\end{tabular}

(CONTINUED) 
APPENDIX E.3

Groundwater and Surface-Water Quality Data, 1993

\begin{tabular}{|c|c|c|c|c|c|c|c|c|}
\hline \multirow{3}{*}{$\begin{array}{l}\text { Sampling Point } \\
\text { Location } \\
\text { Date Sampled }\end{array}$} & \multicolumn{2}{|c|}{ GW-740 } & \multicolumn{4}{|c|}{ GW-793 } & \multicolumn{2}{|c|}{ GW-794 } \\
\hline & \multicolumn{2}{|c|}{ EXP } & \multicolumn{4}{|c|}{ AGLLSF } & \multicolumn{2}{|c|}{ AGLLSF } \\
\hline & $09 / 27 / 93$ & $12 / 11 / 93$ & $02 / 26 / 93$ & $05 / 25 / 93$ & $07 / 11 / 93$ & $10 / 18 / 93$ & $03 / 01 / 93$ & $05 / 25 / 93$ \\
\hline FIELD MEASUREMENTS & - & - & - & - & $\cdot 1$ & $\cdot$ & - & - \\
\hline Depth to Water $(\mathrm{ft})$ & 72.65 & 67.8 & 7.15 & 8.5 & 10.05 & 10.07 & 9 & 16.1 \\
\hline Water Temp (degrees C) & 14.3 & 12.1 & 13.3 & 14.5 & 16.5 & 16.3 & 12.3 & 13.6 \\
\hline pH (pH units) & 7.7 & 7.5 & 7.1 & 6.8 & 7.2 & 6.8 & 7.4 & 7.4 \\
\hline sp. Cond. (umho/cm) & 538 & 488 & 282 & 240 & 252 & 265 & 441 & 403 \\
\hline Dissolved Oxygen (ppm) & 3 & 2.7 & 0.5 & 0.6 & 0.4 & 0.6 & 8.5 & 5.4 \\
\hline Oxidation/Reduction (mV) & -35 & 18 & 140 & 170 & 106 & 157 & 109 & 177 \\
\hline MISCELIANEOUS PARAMETERS & $\cdot$ & • & • & • & $\cdot$ & $\cdot$ & • & • \\
\hline pH (pH units) & 7.7 & 7.6 & 7.5 & 7.3 & $7 . \dot{6}$ & 7.6 & 7.6 & 7.7 \\
\hline sp. Cond. (umho/cm) & 559 & 537 & 295 & 296 & 292 & 308 & 523 & 483 \\
\hline TDS (mg/L) & 302 & 324 & 198 & 188 & 186 & 192 & 372 & 296 \\
\hline TSS (mg/L) & $<1$ & $<1$ & 5 & 1 & 16 & 5 & 224 & 15 \\
\hline Turbidity (NTU) & 2.3 & 1.2 & 2.8 & 1.1 & 7 & 2.5 & 110 & 16 \\
\hline RADIOCHEMICAL PARAMETERS & - & - & - & - & . & . & • & • \\
\hline Gross Alpha (pCi/L) & 1.85 & 0.65 & 0.49 & -5.55 & -4.03 & -0.15 & 2.55 & -5.92 \\
\hline Gross Alpha $(\mathrm{CE}+/-)$ & 4.10 & 2.40 & 1.40 & 4.40 & 7.90 & 2.20 & 2.00 & 4.70 \\
\hline Gross Beta $(\mathrm{pCi} / \mathrm{L})$ & 16.90 & 8.40 & 0.95 & 3.98 & -0.98 & 3.21 & 4.57 & 1.00 \\
\hline Gross Beta $(\mathrm{CE}+/-)$ & 5.90 & 3.30 & 2.70 & 5.50 & 11.00 & 3.10 & 2.90 & 5.40 \\
\hline
\end{tabular}

(CONTINUED) 
APPENDIX E.3

Groundwater and Surface-water Quality Data, 1993

\begin{tabular}{|c|c|c|c|c|c|c|c|c|}
\hline \multirow{3}{*}{$\begin{array}{l}\text { Sampling Point } \\
\text { - } \\
\text { Location } \\
\text { Date Sampled }\end{array}$} & \multicolumn{2}{|c|}{ GW-794 } & \multicolumn{4}{|c|}{ GW-795 } & \multicolumn{2}{|c|}{ GW-800 } \\
\hline & \multicolumn{2}{|c|}{ AGLLSF } & \multicolumn{4}{|c|}{ AGLISF } & \multicolumn{2}{|c|}{ OLF } \\
\hline & $07 / 11 / 93$ & $10 / 18 / 93$ & $03 / 02 / 93$ & $05 / 25 / 93$ & $07 / 12 / 93$ & $10 / 18 / 93$ & $05 / 11 / 93$ & $09 / 14 / 93$ \\
\hline FIELD MEASUREMENTS & . & $\cdot$ & - & $\cdot$ & $\cdot$ & $\cdot$ & $\cdot$ & - \\
\hline Depth to water (ft) & 20.8 & 20.25 & 3.65 & 6.3 & 10.25 & 10.46 & 18.76 & 21.93 \\
\hline Water Temp (degrees C) & 17.8 & 15.8 & 11.7 & 13.3 & 15.7 & 17.2 & 15.7 & 15.7 \\
\hline pH (pH units) & 7.7 & 7.2 & $7 \cdot 1$ & 7 & 7.2 & 6.7 & $7 \cdot 4$ & 7.2 \\
\hline Sp. Cond. (umho/cm) & 436 & 432 & 396 & 344 & 370 & 434 & 326 & 505 \\
\hline Dissolved Oxygen (ppm) & 9 & 8.2 & 2.8 & 0.6 & 0.6 & 0.6 & 5.8 & 6.7 \\
\hline Oxidation/Reduction (mv) & 89 & 187 & 181 & 260 & 170 & 164 & 154 & 254 \\
\hline MISCELLANEOUS PARAMETERS & & & . & . & - & $\cdot$ & $\cdot$ & - \\
\hline pH (pH units) & 7.8 & 7.8 & 7.3 & 7.4 & 7.3 & 7.5 & 7.7 & 7.4 \\
\hline Sp. Cond. (umho/cm) & 479 & 482 & 480 & 428 & 426 & 484 & 369 & 547 \\
\hline TDS (mg/I) & 298 & 300 & 266 & 262 & 268 & 302 & 180 & 334 \\
\hline TSS (mg/L) & 11 & 10 & $<1$ & $<1$ & $<1$ & $<1$ & $<1$ & 66 \\
\hline Turbidity (NTU) & 15 & 13 & 1.1 & 1.1 & 1.8 & 1 & 1.1 & 66 \\
\hline RADIOCHEMICAI PARAMETERS & $\cdot$ & • & $\cdot$ & $\cdot$ & $\cdot$ & $\cdot$ & • & • \\
\hline Grose Alpha (pci/L) & -4.51 & 2.20 & 0.35 & -6.19 & 0.00 & 3.24 & -0.18 & -2.26 \\
\hline Gross Alpha $(\mathrm{CE}+/-)$ & 7.60 & 2.60 & 1.50 & 4.30 & 8.30 & 2.70 & 0.57 & 3.00 \\
\hline Gross Beta (pCi/L) & -3.27 & 7.15 & 2.28 & 2.32 & -8.51 & 10.00 & 0.31 & -6.00 \\
\hline Gross Beta $(\mathrm{CE}+/-)$ & 11.00 & 3.40 & 2.80 & 5.40 & 11.00 & 3.50 & 1.40 & 5.70 \\
\hline
\end{tabular}

(CONTINUED) 


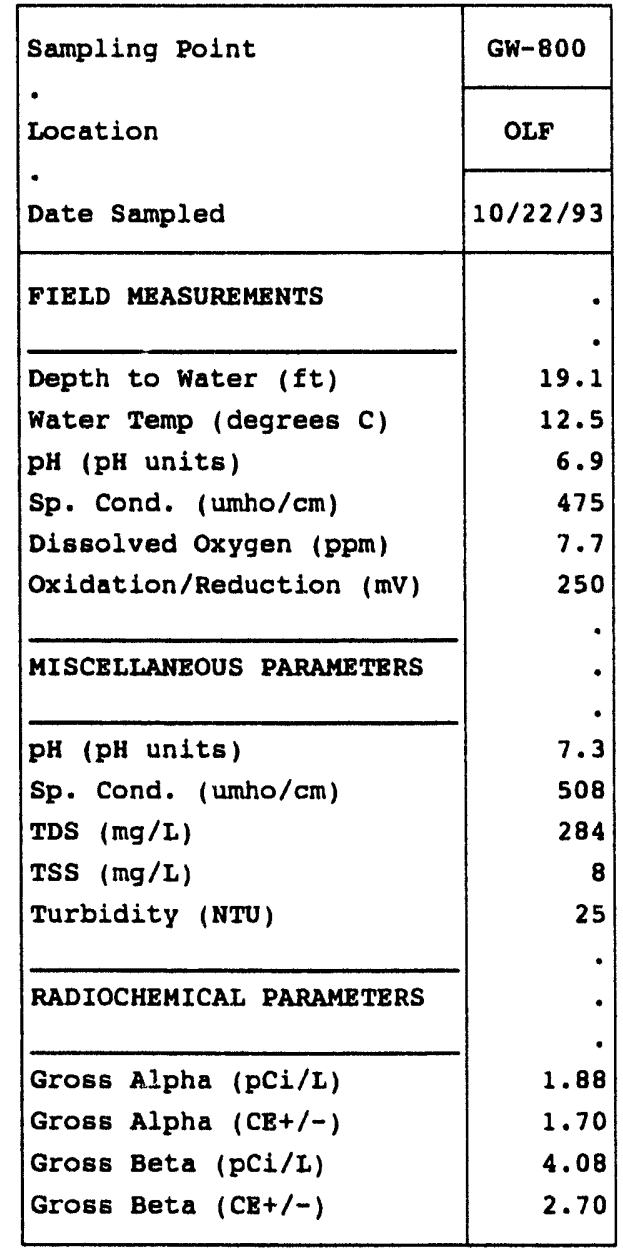


APPENDIX E. 3

Groundwater and Surface-Water Quality Data, 1993

\begin{tabular}{|c|c|c|c|c|c|c|c|c|}
\hline \multirow{3}{*}{$\begin{array}{l}\text { Sampling Point } \\
\text { Location } \\
\text { Date Sampled }\end{array}$} & \multicolumn{4}{|c|}{$B C K=00.63$} & \multicolumn{4}{|c|}{ BCK-04.55 } \\
\hline & \multicolumn{4}{|c|}{$\mathbf{E X P}$} & \multicolumn{4}{|c|}{$\mathbf{E X P}$} \\
\hline & $03 / 09 / 93$ & $05 / 03 / 93$ & $08 / 16 / 93$ & $11 / 08 / 93$ & $03 / 09 / 93$ & $05 / 03 / 93$ & $08 / 16 / 93$ & $11 / 08 / 93$ \\
\hline FIELD MRASUREMENTS & - & - & - & - & - & - & - & - \\
\hline Depth to Water ( $f t)$ & $\cdot$ & . & . & • & . & . &. & • \\
\hline Water Temp (degrees $c$ ) & 7 & 14 & 17.7 & 5.7 & 8 & 14 & 16.8 & 6.5 \\
\hline pH (pH units) & 8 & 8.1 & 7.6 & 7.9 & 7.7 & 7.9 & 7.5 & 7.5 \\
\hline Sp. Cond. (umho/cm) & 162 & 200 & 360 & 290 & 170 & 222 & 350 & 307 \\
\hline Dissolved Oxygen (ppm) & 7.8 & 9.2 & 8.5 & 10.8 & 10.7 & 8.8 & 7.4 & 9.8 \\
\hline Oxidation/Reduction (mv) & $\cdot$ & - & - & - & - & - & - & - \\
\hline MISCELIANEOUS PARAMETERS & . & - & - & . & . & . & . & . \\
\hline pH (pH units) & 7.7 & 7.9 & 8.1 & 8.1 & 7.8 & 7.9 & 7.9 & 8 \\
\hline Sp. Cond. (unho/cm) & 241 & 278 & 412 & 447 & 258 & 289 & 410 & 457 \\
\hline TDS (mg/L) & 130 & 176 & 258 & 384 & 136 & 162 & 264 & 376 \\
\hline TSS (mg/L) & 2 & 2 & 4 & $<1$ & 2 & 1 & 1 & $<1$ \\
\hline Turbidity (NTU) & 4 & 3.2 & 2.5 & 3 & 3.4 & 3 & 2.3 & 2.9 \\
\hline RADIOCHEMICAL PARAMETERS & $\cdot$ & - & - & - & . & - & - & - \\
\hline Gross Alpha (pCi/L) & 6.99 & 5.06 & 18.30 & 13.80 & 7.94 & 5.81 & 11.70 & 12.10 \\
\hline Gross Alpha $(\mathrm{CE}+/-)$ & 2.70 & 2.40 & 8.70 & 3.60 & 2.90 & 2.60 & 7.80 & 3.40 \\
\hline Gross Beta $(\mathrm{pCi} / \mathrm{L})$ & 10.50 & 10.20 & 21.50 & 32.40 & 13.90 & 8.17 & 21.80 & 30.50 \\
\hline Gross Beta $(\mathrm{CE}+/-)$ & 3.30 & 3.10 & 12.00 & 5.30 & 3.40 & 3.00 & 12.00 & 5.10 \\
\hline
\end{tabular}

(CONTINUED) 
APPENDIX E. 3

Groundwater and Surface-Water Quality Data, 1993

\begin{tabular}{|c|c|c|c|c|c|c|c|c|}
\hline \multirow{3}{*}{$\begin{array}{l}\text { Sampling Point } \\
\text { Location } \\
\text { Date Sampled }\end{array}$} & \multicolumn{4}{|c|}{ BCK-09.40 } & \multicolumn{4}{|c|}{ NT-01 } \\
\hline & \multicolumn{4}{|c|}{ EXP } & \multicolumn{4}{|c|}{ EXP } \\
\hline & $03 / 09 / 93$ & $05 / 03 / 93$ & $08 / 16 / 93$ & $11 / 08 / 93$ & $03 / 09 / 93$ & $05 / 03 / 93$ & $08 / 16 / 93$ & $11 / 08 / 93$ \\
\hline FIELD MEASUREMENTS & $\cdot$ & - & - & - & • & $\cdot$ & - & • \\
\hline Depth to Water $(f t)$ &. & . & . & . & . &. & . & . \\
\hline Water Temp (degrees C) & 7 & 15 & - & 5.8 & 9 & 15 & 25.6 & 6.7 \\
\hline pH (pH units) & 7.7 & 8.2 & - & 7.7 & 7 & 7.4 & 7 & 7.1 \\
\hline sp. Cond. (umho/cm) & 230 & 350 & - & 480 & 690 & 820 & 2330 & 1110 \\
\hline Dissolved Oxygen (ppm) & 11.7 & 8.4 & - & 10.6 & 8.1 & 6.6 & 6.8 & 9.9 \\
\hline Oxidation/Reduction (mV) & $\cdot$ & - & - & - & $\cdot$ & $\cdot$ & • & $\cdot$ \\
\hline MISCELLANEOUS PARAMETERS & $\cdot$ & • & - & - & - & - & • & • \\
\hline pH (pH units) & 8.1 & 8.1 & 7.8 & 8.1 & 7 & 7.6 & 7.5 & 7.7 \\
\hline Sp. Cond. (unho/cm) & 418 & 478 & 645 & 250 & 1170 & 1110 & 2280 & 1760 \\
\hline TDS (mg/L) & 230 & 288 & 424 & 254 & 772 & 772 & 1746 & 1382 \\
\hline TSS $(\mathrm{mg} / \mathrm{L})$ & 2 & 1 & 2 & 4 & 5 & 7 & 4 & 8 \\
\hline Turbidity (NTU) & 2.8 & 3 & 2.6 & 4.1 & 7.1 & 1.3 & 2.2 & 3.3 \\
\hline RADIOCHEMICAL PARAMETERS & . & • & . & . & - & - & • & • \\
\hline Gross Alpha $(\mathrm{pCi} / \mathrm{L})$ & 26.80 & 19.10 & 41.00 & -0.01 & 8.78 & 16.30 & 45.50 & 72.80 \\
\hline Gross Alpha $(\mathrm{CE}+/-)$ & 4.80 & 4.40 & 12.00 & 1.00 & 5.10 & 7.10 & 7.20 & 8.70 \\
\hline Gross Beta $(\mathrm{pCi} / \mathrm{L})$ & 41.30 & 43.80 & 95.50 & -1.47 & 63.60 & 64.40 & 384.00 & 318.00 \\
\hline Gross Beta $(\mathrm{CE}+/-)$ & 4.60 & 4.70 & 16.00 & 2.70 & 6.00 & 8.60 & 12.00 & 11.00 \\
\hline
\end{tabular}

(CONTINURD) 
APPENDIX 8.3

Groundwater and Surface-Water Quality Data, 1993

\begin{tabular}{|c|c|c|c|c|c|c|c|c|}
\hline \multirow{3}{*}{$\begin{array}{l}\text { Sampling Point } \\
\text { Location } \\
\text { Date Sampled }\end{array}$} & \multicolumn{4}{|c|}{$N T-13$} & \multicolumn{4}{|c|}{ SS-1 } \\
\hline & \multicolumn{4}{|c|}{ EXP } & \multicolumn{4}{|c|}{ EXP } \\
\hline & $03 / 09 / 93$ & $05 / 03 / 93$ & $08 / 16 / 93$ & $11 / 08 / 93$ & $03 / 09 / 93$ & $05 / 03 / 93$ & $08 / 16 / 93$ & $11 / 08 / 93$ \\
\hline FIELD MEASUREMENTS & $\cdot$ & • & $\cdot$ & - & - & $\cdot$ & - & - \\
\hline Depth to Water (ft) &. & . &. & . & - &. & . & . \\
\hline Water Temp (degrees C) & 6 & 13 & 21.3 & 8.3 & 8 & 18 & 20.6 & 12.6 \\
\hline ph (pH units) & 7.3 & 8.2 & 7.5 & 5.8 & 6.8 & 6.8 & 7.5 & 7.3 \\
\hline Sp. Cond. (umho/om) & 30 & 60 & 230 & 140 & 760 & 1220 & 705 & 1000 \\
\hline Dissolved Oxygen (ppm) & 11.1 & 9.4 & 7 & 8.4 & 10.1 & 7.6 & 7.1 & 6.9 \\
\hline Oxidation/Reduction (mV) & $\cdot$ & - & $\cdot$ & - & - & $\cdot$ & - & • \\
\hline MISCELLANEOUS PARAMETERS & & . &. & . & . & $\cdot$ & . & • \\
\hline pH (pH units) & 7.1 & 7.5 & $7 . \dot{6}$ & $6 . \dot{9}$ & 7.6 & $7 . \dot{2}$ & $7 . \dot{9}$ & $7 . \dot{8}$ \\
\hline Sp. Cond. (umho/cm) & 57 & 72 & 239 & 170 & 1100 & 1540 & 1330 & 1290 \\
\hline TDS $(m g / L)$ & 40 & 52 & 166 & 230 & 670 & 1052 & 942 & 1070 \\
\hline TSS (mg/L) & 4 & 3 & 1 & 38 & $<1$ & 9 & 5 & 10 \\
\hline Turbidity (NTU) & 6.9 & 8.4 & 6.7 & 44 & 0.8 & 3 & 1.3 & 1.6 \\
\hline RADIOCHEMICAL PARAMETERS &. & . & . & . & . & . & . & • \\
\hline Gross Alpha (pCi/L) & 0.28 & 0.00 & 1.73 & 0.81 & $12.90^{\circ}$ & 15.10 & 18.90 & 18.20 \\
\hline Gross Alpha $(\mathrm{CE}+/-)$ & 1.60 & 1.40 & 6.00 & 1.30 & 3.50 & 3.60 & 4.10 & 4.50 \\
\hline Gross Beta $(\mathrm{pCi} / \mathrm{L})$ & 1.55 & 2.46 & -2.04 & 0.23 & 27.10 & 178.00 & 52.60 & 63.60 \\
\hline Gross Beta $(\mathrm{CE}+/-)$ & 2.70 & 2.60 & 11.00 & 2.80 & 4.50 & 8.30 & 5.00 & 5.40 \\
\hline
\end{tabular}

(CONTINUED) 
APPENDIX E. 3

Groundwater and Surface-water Quality Data, 1993

\begin{tabular}{|c|c|c|c|c|c|c|c|c|}
\hline \multirow{3}{*}{$\begin{array}{l}\text { Sampling Point } \\
\text { Location } \\
\text { Date Sampled }\end{array}$} & \multicolumn{4}{|c|}{ ss-4 } & \multicolumn{4}{|c|}{ Ss-5 } \\
\hline & \multicolumn{4}{|c|}{$\mathbf{E X P}$} & \multicolumn{4}{|c|}{$\mathbf{E X P}$} \\
\hline & $03 / 09 / 93$ & $05 / 03 / 93$ & $08 / 16 / 93$ & $11 / 08 / 93$ & $03 / 09 / 93$ & $05 / 03 / 93$ & $08 / 16 / 93$ & $11 / 08 / 93$ \\
\hline FIELD MEASUREMENTS & $\cdot 1$ & - & & . & . & $\cdot$ &. & - \\
\hline Depth to Water $(f t)$ & $\cdot$ & - & $\cdot$ & $\cdot$ & - & - & - & - \\
\hline Water Temp (degrees C) & 10 & 15 & 16.9 & 10.3 & 12 & 12 & 13.9 & 12.4 \\
\hline pH (ph units) & 7.3 & 7.6 & 7.8 & 7.6 & 7 & 7.6 & 7.8 & 7 \\
\hline Sp. Cond. (umho/ckit) & 381 & 475 & 780 & 700 & 220 & 260 & 500 & 495 \\
\hline Dissolved Oxygen (ppin) & 8.7 & 8.8 & 8.4 & 9.2 & 7.8 & 6.6 & 5.4 & 5.6 \\
\hline Oxidation/Reduction (mv) & $\cdot$ & $\cdot$ & - & • & - & - & $\cdot$ & - \\
\hline MISCELIANEOUS PARAMETERS & $\cdot$ & • & $\cdot$ & • & - & • & $\cdot$ & - \\
\hline pH (pH units) & 7.8 & 7.8 & $8 . \dot{1}$ & $\dot{8}$ & $7 . \dot{3}$ & 7.5 & 7.5 & 7.4 \\
\hline Sp. Cond. (umho/cm) & 550 & 625 & 920 & 959 & 309 & 353 & 633 & 629 \\
\hline $\operatorname{TDS}(\mathrm{mg} / \mathrm{L})$ & 306 & 334 & 616 & 788 & 166 & 194 & 380 & 494 \\
\hline TsS (mg/L) & 10 & 6 & 10 & 41 & 3 & $<1$ & $<1$ & $\mathbf{5}$ \\
\hline Turbidity (NTU) & 2.5 & 2.4 & 2.7 & 2 & 3 & 2.1 & 2.2 & 4 \\
\hline RADIOCHEMICAI PARAMETERS & . & . & $\cdot$ & $\cdot$ & • & • & . & • \\
\hline Gross Alpha (pCi/L) & 25.80 & 29.60 & 57.00 & 55.10 & 8.69 & 6.11 & 30.60 & 32.80 \\
\hline Gross Alpha $(\mathrm{CE}+/-)$ & 5.00 & 6.20 & 14.00 & 9.10 & 3.00 & 2.70 & 11.00 & 6.10 \\
\hline Gross Beta $(\mathrm{pC} i / L)$ & 37.10 & 44.30 & 118.00 & 143.00 & 12.50 & 15.70 & 65.20 & 73.30 \\
\hline Gross Beta $(\mathrm{CE}+/-)$ & 4.50 & 4.80 & 17.00 & 16.00 & 3.40 & 3.50 & 15.00 & 9.20 \\
\hline
\end{tabular}

(CONTINUED) 
APPENDIX $\mathrm{E} .3$

Groundwater and Surface-Water Quality Data, 1993

\begin{tabular}{|c|c|c|c|c|c|c|c|c|}
\hline \multirow{3}{*}{$\begin{array}{l}\text { Sampling Point } \\
\text { Location } \\
\text { Date Sampled }\end{array}$} & \multicolumn{4}{|c|}{ SS-6 } & \multicolumn{4}{|c|}{ Ss-8 } \\
\hline & \multicolumn{4}{|c|}{ ExP } & \multicolumn{4}{|c|}{$\mathbf{E X P}$} \\
\hline & $03 / 09 / 93$ & $05 / 03 / 93$ & $08 / 16 / 93$ & $11 / 08 / 93$ & $03 / 09 / 93$ & $05 / 03 / 93$ & $08 / 26 / 93$ & $11 / 08 / 93$ \\
\hline FIELD MEASUREMENTS &. & . & . & . & . & . & . & • \\
\hline Depth to Water $(f t)$ & . &. & . & . &. & . & . & . \\
\hline Water Temp (degrees C) & 8 & 13 & 16 & 9.8 & 11 & 12 & 13.2 & 12.3 \\
\hline pH (pH units) & 7.6 & 8.4 & 7.7 & 7.6 & 7.5 & 7.9 & 7.5 & 7.1 \\
\hline Sp. Cond. (umho/cm) & 132 & 170 & 238 & 182 & 155 & 172 & 248 & 230 \\
\hline Dissolved Oxygen (ppm) & 10.2 & 9.3 & 7.9 & 10.3 & 9 & 9.4 & 6.5 & 7.2 \\
\hline Oxidation/Reduction (mV) & . & $\cdot$ & - & - & . & - & - & • \\
\hline MISCELLANEOUS PARAMETERS & . & $\cdot$ & - & . & . & - & . & • \\
\hline pH (pH units) & 8 & 8.1 & 7.9 & 8 & 7.3 & 7.5 & 7.6 & 7.6 \\
\hline Sp. Cond. (umho/cm) & 189 & 219 & 288 & 728 & 213 & 232 & 317 & 297 \\
\hline TDS (mg/L) & 94 & 104 & 172 & 580 & 104 & 120 & 184 & 280 \\
\hline TSS (mg/L) & $<1$ & 3 & 2 & 3 & 1 & 3 & 4 & $<1$ \\
\hline Turbidity (NTU) & 1.9 & 2 & 2.3 & 2.4 & 2.1 & 2.2 & 2.4 & 3.5 \\
\hline RADIOCHEMICAL PARAMETERS & . & . & - & . & . & . & . & • \\
\hline Gross Alphe (PCi/L) & -0.15 & -0.90 & 2.29 & 54.30 & 0.00 & 0.48 & 6.91 & -0.66 \\
\hline Gross Alpha $(\mathrm{CB}+/-)$ & 1.60 & 1.30 & 6.10 & 8.70 & 1.70 & 1.70 & 6.90 & 0.81 \\
\hline Gross Beta (pCi/L) & 0.82 & 0.34 & -1.70 & 126.00 & 1.65 & -1.38 & 6.47 & -1.70 \\
\hline Gross Beta $(\mathrm{CE}+/-)$ & 2.70 & 2.50 & 11.00 & 14.00 & 2.80 & 2.40 & 11.00 & 2.70 \\
\hline
\end{tabular}




\section{APPENDIX E.4}

\section{RADIONUCLIDES}


APPENDIX E. 4

Groundwater and Surface-Water Quality Data, 1993

\begin{tabular}{|c|c|c|c|c|c|c|c|c|}
\hline & GW-040 & $G W-042$ & GW-043 & GW-044 & GW-079 & GW-080 & GW-084 & GW-115 \\
\hline Location & BG & BG & OLF & OLF & BG & BG & OLF & s3 \\
\hline Date Sampled & $10 / 09 / 93$ & $12 / 29 / 93$ & $10 / 19 / 93$ & $10 / 19 / 93$ & $10 / 18 / 93$ & $10 / 10 / 93$ & $10 / 20 / 93$ & $10 / 19 / 93$ \\
\hline Gross Alpha (pci/L) & -1.96 & $-2 \cdot 48$ & -0.62 & 0.61 & -0.93 & 2.06 & 0.77 & 1.43 \\
\hline Gross Alpha $(\mathrm{CE}+/-)$ & 1.80 & 1.90 & 2.10 & 2.50 & 2.00 & 2.30 & 1.40 & 1.60 \\
\hline Gross Beta (pci/L) & 2.97 & -4.77 & -7.90 & -8.81 & -3.07 & 0.90 & 5.16 & 4.06 \\
\hline Gross Beta $(\mathrm{CB}+/-)$ & 2.80 & 3.20 & 5.80 & 5.60 & 6.00 & 2.60 & 2.70 & 2.60 \\
\hline RADIONUCLIDES & $\cdot$ & • &. & $\cdot$ & . & $\cdot$ & $\cdot$ & . \\
\hline Americium $241(\mathrm{pCi} / \mathrm{L})$ & 0.00 & 0.89 & 0.00 & -3.54 & -1.46 & -1.63 & -1.15 & 0.68 \\
\hline Americium 241 (CE+/-) & 8.20 & 1.00 & 6.30 & 7.90 & 5.00 & 7.30 & 2.60 & 3.00 \\
\hline Cesium $137(\mathrm{pci} / 1)$ & - & 0.08 & 13.10 & 18.60 & 4.51 & - & - & - \\
\hline Cesium $137(\mathrm{CE}+/-)$ & . & 0.06 & 35.20 & 42.00 & 35.30 & $\cdot$ & - & - \\
\hline Iodine $129(\mathrm{pCi} / \mathrm{L})$ & 0.01 & 0.10 & 0.10 & 0.10 & 0.10 & 0.10 & 0.02 & 0.10 \\
\hline Iodine $129(\mathrm{CB}+/-)$ & 0.75 & 1.00 & 3.30 & 3.50 & -0.05 & 1.50 & 0.32 & 2.90 \\
\hline Iodine 131 (pci/L) & -0.02 & 0.05 & -0.06 & 0.04 & 1.70 & -0.02 & 0.10 & 0.01 \\
\hline Iodine $131(\mathrm{CE}+/-)$ & 0.10 & 0.16 & 0.34 & 0.22 & 0.29 & 0.19 & 2.20 & 0.34 \\
\hline Neptunium 237 (pCi/L) & -0.55 & 0.00 & 17.80 & -5.47 & -5.51 & 1.10 & 0.00 & 0.00 \\
\hline Neptunium $237(\mathrm{CE}+/-)$ & 2.20 & 0.49 & 31.00 & 19.00 & 19.00 & 2.20 & 1.80 & 1.80 \\
\hline Potassium 40 (pci/L) & - & - & $\cdot$ & - & $\cdot$ & - & . & - \\
\hline Potassium $40(\mathrm{CE}+/-)$ & - & - & $\cdot$ & . & - & • & - & - \\
\hline Plutonium 238 (pCi/L) & -0.69 & 0.22 & 0.00 & 13.60 & 6.87 & 0.00 & 0.00 & 0.00 \\
\hline Plutonium $238(\mathrm{CE}+/-)$ & 3.10 & 0.75 & 42.00 & 43.00 & 41.00 & 3.40 & 2.20 & 2.20 \\
\hline Plutonium $239(\mathrm{pci} / \mathrm{L})$ & 0.00 & 0.00 & 14.80 & -6.82 & 0.00 & 0.00 & 0.00 & 0.64 \\
\hline Plutonium $239(\mathrm{CE}+/-)$ & 2.40 & 0.75 & 30.00 & 27.00 & 19.00 & 2.40 & 2.20 & 1.30 \\
\hline Protactinium (pCi/L) & - & $\cdot$ & 2050.00 & 1960.00 & 2360.00 & - & - & - \\
\hline Protactinium $(\mathrm{CE}+/-)$ & - & - & 6440.00 & 7260.00 & 6040.00 & - & - & - \\
\hline Radium (Bq/L) & 0.06 & 0.24 & 0.00 & 0.01 & 0.02 & 0.12 & 0.03 & 0.02 \\
\hline Radium $(\mathrm{CE}+/-)$ & 0.05 & 0.08 & 0.03 & 0.03 & 0.03 & 0.04 & 0.04 & 0.03 \\
\hline Ruthenium $106(\mathrm{pCi} / \mathrm{L})$ & - & 0.38 & $\cdot$ & - & - & - & - & - \\
\hline Ruthenium $106(\mathrm{CE}+/-)$ & - & 0.80 & $\cdot$ & - & - & - & - & - \\
\hline Strontium (pCi/I) & 38.10 & 34.80 & 35.70 & 57.90 & 57.90 & 60.50 & 51.20 & 33.60 \\
\hline strontium $(\mathrm{CE}+/-)$ & 22.00 & 25.00 & 23.00 & 25.00 & 25.00 & 24.00 & 25.00 & 23.00 \\
\hline Technetium $99(\mathrm{pCi} / \mathrm{L})$ & 38.20 & 109.00 & 53.00 & 18.00 & 53.80 & 47.50 & $25 \cdot 40$ & 37.10 \\
\hline Technetium $99(\mathrm{CE}+/-)$ & 59.00 & 62.00 & 60.20 & 59.10 & 60.30 & 61.00 & 59.00 & 60.00 \\
\hline Thorium $234(\mathrm{pCi} / 1)$ & . & 7.90 & -207.00 & 998.00 & 327.00 & - & • & - \\
\hline Thorium $234(\mathrm{CE}+/-)$ & - & 1.90 & 575.00 & 346.00 & 608.00 & - & - & - \\
\hline Thorium $228(\mathrm{pCi} / 1)$ & . & 7.43 & - & - & 1390.00 & - & - & - \\
\hline Thorium $228(C E+/-)$ & . & 8.10 & - & - & 1930.00 & - & - & - \\
\hline Tritium (Bq/L) & 25.00 & 8.00 & 12.00 & -5.00 & 26.00 & 9.00 & 1.00 & 11.00 \\
\hline Tritium $(\mathrm{CE}+/-)$ & 26.00 & 22.00 & 24.00 & 23.00 & 24.00 & 25.00 & 24.00 & 26.00 \\
\hline Uranium 234 (pCi/L) & 0.86 & 1.86 & 9.14 & -7.23 & 0.00 & 0.90 & 4.15 & 7.30 \\
\hline Uranium $234(\mathrm{CE}+/-)$ & 1.20 & 1.20 & 32.00 & 29.00 & 21.00 & $1 \cdot 30$ & 3.70 & 4.90 \\
\hline Uranium 235 (pCi/L) & $-6 \cdot 59$ & 0.03 & -1.85 & 43.40 & $-42 \cdot 80$ & 10.50 & 16.90 & 121.00 \\
\hline Uranium $235(\mathrm{CE}+/-)$ & 59.00 & 0.36 & 68.10 & 90.40 & 68.50 & 70.00 & 58.00 & 170.00 \\
\hline Uranium 238 (pCi/L) & 0.00 & -0.19 & 9.14 & 22.70 & 7.30 & 0.00 & 5.81 & 0.00 \\
\hline Uranium $238(\mathrm{CE}+/-)$ & 1.50 & 0.74 & 18.00 & 25.00 & 15.00 & 1.60 & 5.00 & 2.30 \\
\hline
\end{tabular}

(CONTINUED) 
APPENDIX E.4

Groundwater and Surface-water Quality Data, 1993

\begin{tabular}{|c|c|c|c|c|c|c|c|c|}
\hline \multirow{3}{*}{$\begin{array}{l}\text { Sampling Point } \\
\text { - } \\
\text { Location } \\
\text { - } \\
\text { Date Sampled }\end{array}$} & $G w-162$ & $G W-324$ & $G W-325$ & $G W-342$ & GW-372 & GW-373 & \multicolumn{2}{|c|}{ Gw-537 } \\
\hline & BG & s3 & s3 & BG & BG & BG & \multicolumn{2}{|c|}{ OLF } \\
\hline & $10 / 10 / 93$ & $10 / 19 / 93$ & $10 / 21 / 93$ & $10 / 07 / 93$ & $10 / 12 / 93$ & $10 / 11 / 93$ & $02 / 11 / 93$ & $05 / 12 / 93$ \\
\hline Gross Alpha (pCi/L) & -0.31 & -0.32 & 10.80 & 0.00 & -0.01 & -1.92 & 39.80 & 0.00 \\
\hline Gross Alpha $(\mathrm{CE}+/-)$ & $2 \cdot 10$ & 2.20 & 3.20 & 2.00 & 2.40 & 2.10 & 14.00 & 20.00 \\
\hline Gross Beta (pci/L) & -0.33 & -7.50 & 15.50 & 1.97 & -4.94 & 0.08 & 287.00 & 373.00 \\
\hline Gross Beta $(\mathrm{CE}+/-)$ & 2.60 & 5.70 & 3.50 & 2.70 & 5.60 & 2.70 & 23.00 & 42.00 \\
\hline RADIONUCLIDES & - & $\cdot$ & . & $\cdot$ & $\cdot$ & $\cdot$ & $\cdot$ & $\cdot$ \\
\hline Americium 241 (pCi/L) & -5.22 & 10.40 & $\begin{array}{r}\cdot \\
1.23\end{array}$ & -4.82 & 0.00 & 3.92 & • & • \\
\hline Americium $241(\mathrm{CE}+/-)$ & 8.50 & 13.00 & 3.00 & 7.90 & 6.80 & 11.00 & - & - \\
\hline Cesium $137(\mathrm{pci} / 1)$ & - & -7.53 & - & - & 28.50 & - & - & - \\
\hline Cesium 137 (CE $+/-$ ) & - & 84.80 & $\cdot$ & $\cdot$ & 26.00 & $\cdot$ & - & - \\
\hline Iodine 129 (pCi/L) & -0.42 & 0.10 & -0.04 & 0.10 & 0.01 & 0.10 & $\cdot$ & - \\
\hline Iodine $129(\mathrm{CE}+/-)$ & 0.98 & 2.10 & 0.24 & 1.00 & 0.77 & 2.30 & - & - \\
\hline Iodine 131 (pCi/L) & -0.07 & 0.02 & 0.10 & 0.01 & -0.02 & 0.06 & $\cdot$ & - \\
\hline Iodine $131(\mathrm{CE}+/-)$ & 0.19 & 0.24 & 2.10 & 0.15 & 0.10 & 0.23 & - & - \\
\hline Neptunium 237 (pci/L) & 0.55 & 0.00 & 0.00 & 0.59 & $-11 \cdot 90$ & 0.00 & - & - \\
\hline Neptunium $237(\mathrm{CB}+/-)$ & 1.90 & 22.00 & 1.80 & 2.10 & 27.00 & 1.50 & - & - \\
\hline Potassium $40(\mathrm{pCi} / \mathrm{L})$ & $\cdot$ & $\cdot$ & $\cdot$ & $\cdot$ & $\cdot$ & $\cdot$ & - & - \\
\hline Potsssium $40(\mathrm{CE}+/-)$ & $\cdot$ & $\cdot$ & $\cdot$ & $\cdot$ & $\cdot$ & $\cdot$ & $\cdot$ & - \\
\hline Plutonium 238 (pCi/L) & 0.68 & $-27 \cdot 50$ & 0.00 & $-1 \cdot 48$ & 0.00 & -0.68 & - & - \\
\hline Plutonium $238(\mathrm{CB}+/-)$ & 3.60 & 36.00 & 2.30 & 3.00 & 42.00 & 3.00 & • & - \\
\hline Plutonium 239 (pci/L) & 0.00 & 13.70 & 0.00 & 0.74 & $-7 \cdot 38$ & 0.68 & $\cdot$ & - \\
\hline Plutonium $239(\mathrm{CB}+/-)$ & 2.40 & 27.00 & 2.30 & 1.50 & 30.00 & 1.40 & - & - \\
\hline Protactinium (pci/L) & . & 579.00 & $\cdot$ & - & -781.00 & $\cdot$ & - & - \\
\hline Protactinium $(\mathrm{CE}+/-)$ & $\cdot$ & 13700.00 & - & - & 4620.00 & $\cdot$ & - & - \\
\hline Radium $(\mathrm{Bq} / \mathrm{L})$ & 0.07 & 0.00 & 0.05 & 0.06 & 0.08 & 0.04 & - & - \\
\hline Radium $(\mathrm{CE}+/-)$ & 0.04 & 0.03 & 0.05 & 0.05 & 0.06 & 0.05 & - & - \\
\hline Ruthenium 106 (pCi/L) & 363.00 & $\cdot$ & - & $\cdot$ & $\cdot$ & $\cdot$ & $\cdot$ & - \\
\hline Ruthenium $106(\mathrm{CE}+/-)$ & 350.00 & $\cdot$ & - & - & $\cdot$ & $\cdot$ & $\cdot$ & - \\
\hline Strontium (pCi/L) & 24.80 & 60.60 & 0.13 & $51 \cdot 40$ & $14 \cdot 70$ & $49 \cdot 40$ & 0.35 & -3.71 \\
\hline Strontium $(\mathrm{CE}+/-)$ & 20.00 & 25.00 & 19.00 & 23.00 & 21.00 & 23.00 & 21.00 & 18.00 \\
\hline Technetium 99 (pCi/L) & 20.20 & 103.00 & 99.10 & 55.20 & 78.00 & 11.00 & 1260.00 & 1300.00 \\
\hline Cechnetium $99(\mathrm{CE}+1-)$ & 60.00 & 61.80 & 61.00 & 61.00 & 61.00 & 58.00 & 1400.00 & 2700.00 \\
\hline Thorium $234(\mathrm{pci} / 1)$ & $\cdot$ & -219.00 & $\cdot$ & - & 186.00 & $\cdot$ & $\cdot$ & - \\
\hline Thorium 234 (CE $+/-$ ) & $\cdot$ & 3050.00 & $\cdot$ & $\cdot$ & 488.00 & $\cdot$ & $\cdot$ & - \\
\hline Thorium $228(\mathrm{pCi} / \mathrm{l})$ & $\cdot$ & $\cdot$ & $\cdot$ & $\cdot$ & $\cdot$ & - & $\cdot$ & - \\
\hline Thorium $228(\mathrm{CE}+/-)$ & $\cdot$ & $\cdot$ & $\cdot$ & - & $\cdot$ & $\cdot$ & $\cdot$ & - \\
\hline Tritium (Bg/L) & 17.00 & -2.00 & -7.00 & 0.00 & 6.00 & -1.00 & -3.00 & 16.00 \\
\hline Tritium $(\mathrm{CE}+/-)$ & 25.00 & 23.00 & 23.00 & 22.00 & 24.00 & 23.00 & 24.00 & 34.00 \\
\hline Uranium 234 (pCi/L) & 0.00 & 0.00 & 3.08 & 1.77 & 8.27 & 5.52 & $\cdot$ & - \\
\hline Uranium $234(\mathrm{CE}+/-)$ & 1.90 & 21.00 & 3.10 & 1.80 & 29.00 & 4.20 & $\cdot$ & - \\
\hline Uranium 235 (pCi/L) & 0.22 & 94.90 & 3.49 & 17.60 & $18 \cdot 10$ & 200.00 & - & - \\
\hline Uranium $235(\mathrm{CE}+/-)$ & 68.00 & 167.00 & 70.00 & 170.00 & 58.90 & 160.00 & $\cdot$ & - \\
\hline Uranium $238(\mathrm{pCi} / \mathrm{L})$ & 2.78 & 7.55 & 2.31 & 3.10 & $16 \cdot 50$ & 0.79 & - & - \\
\hline Uranium $238(\mathrm{CB}+/-)$ & 2.50 & 15.00 & 3.40 & 2.30 & 23.00 & 1.60 & - & - \\
\hline
\end{tabular}


APPENDIX $\mathrm{E}, 4$

Groundwater and Surface-water Quality Data, 1993

\begin{tabular}{|c|c|c|c|c|c|c|c|c|}
\hline \multirow{3}{*}{$\begin{array}{l}\text { Sampling Point } \\
\text { Location } \\
\text { Date Sampled }\end{array}$} & \multicolumn{2}{|c|}{$G w-537$} & $G W-613$ & $G W-614$ & $G W-642$ & \multicolumn{3}{|c|}{$G W-694$} \\
\hline & \multicolumn{2}{|c|}{ OLF } & s3 & \$3 & BG & \multicolumn{3}{|c|}{$\mathbf{E X P}$} \\
\hline & $09 / 14 / 93$ & $10 / 22 / 93$ & $10 / 20 / 93$ & $10 / 20 / 93$ & $10 / 10 / 93$ & $03 / 23 / 93$ & $06 / 06 / 93$ & $09 / 22 / 93$ \\
\hline Gross Alpha (pCi/L) & 16.60 & 19.70 & 1.79 & 1.23 & 2.10 & 31.80 & 56.90 & 50.90 \\
\hline Gross Alpha $(\mathrm{CE}+/-)$ & 33.00 & 12.00 & 1.60 & 1.50 & 2.40 & 5.10 & 9.20 & 13.00 \\
\hline Gross Beta (pci/L) & 426.00 & 432.00 & 2.82 & 2.92 & 3.30 & 34.60 & $88 \cdot 40$ & 112.00 \\
\hline Gross Beta $(\mathrm{CE}+/-)$ & 80.00 & 27.00 & 2.50 & 2.60 & 2.80 & 4.20 & 9.40 & 17.00 \\
\hline RADIONUCLIDES & $\cdot$ & $\cdot$ & $\cdot$ & $\cdot$ & $\cdot$ & • & $\cdot$ & $\cdot$ \\
\hline$\overline{\text { Americium } 241(\mathrm{pCi} / \mathrm{L})}$ & $\cdot$ & . & 0.00 & 10.40 & $\begin{array}{r}\cdot \\
-3.88\end{array}$ & - & . & $\cdot$ \\
\hline Americium $241(\mathrm{CE}+/-)$ & $\cdot$ & - & 2.30 & 6.90 & 7.80 & - & . & - \\
\hline Cesium 137 (pci/l) & . & . & . & . & - & - & $\cdot$ & - \\
\hline Cesium 137 (CE +/-) & . & - & $\cdot$ & - & - & - & - & - \\
\hline Iodine $129(\mathrm{pci} / \mathrm{L})$ & . & . & 0.10 & 0.10 & 0.10 & - & - & - \\
\hline Iodine $129(\mathrm{CE}+/-)$ & $\cdot$ & $\cdot$ & 2.30 & 2.20 & 1.00 & - & - & $\cdot$ \\
\hline Iodine 131 (pCi/L) & . & - & -0.03 & -0.05 & 0.01 & - & $\cdot$ & - \\
\hline Iodine $131(\mathrm{CE}+/-)$ & - & - & 0.36 & 0.29 & 0.13 & - & $\cdot$ & $\cdot$ \\
\hline Neptunium 237 (pCi/L) & $\cdot$ & - & 0.00 & 0.00 & 0.59 & - & - & - \\
\hline Neptunium 237 (CE+/-) & $\cdot$ & $\cdot$ & 1.70 & 1.80 & 2.10 & $\cdot$ & - & - \\
\hline Potassium $40(\mathrm{pCi} / \mathrm{L})$ & $\cdot$ & $\cdot$ & - & $\cdot$ & - & $\cdot$ & - & - \\
\hline Potassium $40(\mathrm{CE}+/-)$ & - & $\cdot$ & $\cdot$ & $\cdot$ & - & $\cdot$ & $\cdot$ & • \\
\hline Plutonium $238(\mathrm{pCi} / \mathrm{L})$ & $\cdot$ & $\cdot$ & 0.00 & 0.00 & 0.00 & $\cdot$ & $\cdot$ & $\cdot$ \\
\hline Plutonium $238(\mathrm{CE}+/-)$ & $\cdot$ & . & 2.20 & 2.30 & 3.60 & $\cdot$ & . & - \\
\hline Plutonium 239 (pCi/L) & $\cdot$ & $\cdot$ & 0.63 & 0.00 & 0.74 & $\cdot$ & $\cdot$ & $\cdot$ \\
\hline Plutonium $239(\mathrm{CE}+1-)$ & - & . & 1.30 & 2.30 & 1.50 & . & $\cdot$ & - \\
\hline Protactinium ( $\mathrm{pCi} / \mathrm{L})$ & - & - & $\cdot$ & $\cdot$ & $\cdot$ & $\cdot$ & $\cdot$ & - \\
\hline Protactinium $(\mathrm{CE}+/-)$ & $\cdot$ & $\cdot$ & $\cdot$ & $\cdot$ & $\cdot$ & $\cdot$ & $\cdot$ & - \\
\hline Radium $(\mathrm{Bq} / \mathrm{L})$ & - & - & 0.04 & 0.03 & 0.05 & $\cdot$ & - & - \\
\hline Radium $(\mathrm{CE}+/-)$ & $\cdot$ & . & 0.05 & 0.04 & 0.04 & $\cdot$ & $\cdot$ & $\cdot$ \\
\hline Ruthenium $106(\mathrm{pCi} / \mathrm{L})$ & - & $\cdot$ & $\cdot$ & $\cdot$ & - & $\cdot$ & $\cdot$ & - \\
\hline Ruthenium $106(\mathrm{CE}+/-)$ & $\cdot$ & $\cdot$ & $\cdot$ & $\cdot$ & $\cdot$ & $\cdot$ & $\cdot$ & - \\
\hline strontium (pCi/I) & 22.90 & 36.90 & 41.50 & 0.07 & 5.51 & $-12 \cdot 70$ & 4.17 & 17.20 \\
\hline Strontium (CE+/-) & 23.00 & 22.00 & 24.00 & 19.00 & 18.00 & 17.00 & 20.00 & 22.00 \\
\hline Technetium 99 (pci/L) & 1190.00 & 1040.00 & 55.10 & 68.10 & 46.50 & 4290.00 & -2430.00 & 280.00 \\
\hline Technetium $99(\mathrm{CE}+/-)$ & 89.00 & 87.00 & 60.00 & 61.00 & 61.00 & 3500.00 & 3300.00 & 66.00 \\
\hline Thorium $234(\mathrm{pCi} / 1)$ & - & $\cdot$ & - & . & - & $\cdot$ & - & - \\
\hline Thorium 234 (CE $+/-)$ & - & $\cdot$ & . & . & - & $\cdot$ & $\cdot$ & - \\
\hline Thorium $228(\mathrm{pCi} / 1)$ & - & - & - & $\cdot$ & - & $\cdot$ & - & - \\
\hline Thorium $228(\mathrm{CE}+/-)$ & $\cdot$ & $\cdot$ & - & $\cdot$ & $\cdot$ & $\cdot$ & - & - \\
\hline Tritium (Bq/L) & 120.00 & 13.00 & - & 0.00 & 5.00 & 26.00 & 7.00 & 12.00 \\
\hline Tritium $(\mathrm{CE}+/-)$ & $\cdot$ & 28.00 & $\cdot$ & 23.00 & 23.00 & 31.00 & 41.00 & $\cdot$ \\
\hline Uranium $234(\mathrm{pCi} / \mathrm{L})$ & $\cdot$ & $\cdot$ & 5.46 & 5.89 & 2.19 & $\cdot$ & $\cdot$ & $\cdot$ \\
\hline Uranium $234(\mathrm{CE}+/-)$ & $\cdot$ & - & 4.90 & 4.50 & 2.50 & . & $\cdot$ & - \\
\hline Uranium $235(\mathrm{pCi} / \mathrm{L})$ & - & - & 5.27 & -13.80 & 13.60 & $\cdot$ & $\cdot$ & $\cdot$ \\
\hline Uranium $235(\mathrm{CE}+/-)$ & - & - & 170.00 & 68.00 & 90.00 & $\cdot$ & - & - \\
\hline uranium 238 (pCi/L) & - & $\cdot$ & 1.09 & 0.00 & 1.46 & $\cdot$ & $\cdot$ & - \\
\hline Uranium $238(\mathrm{CE}+/-)$ & - & - & 3.80 & 2.40 & 2.10 & - & - & - \\
\hline
\end{tabular}

(CONTINUED) 
APPENDIX $\mathrm{E} .4$

Groundwater and Surface-Water Quality Data, 1993

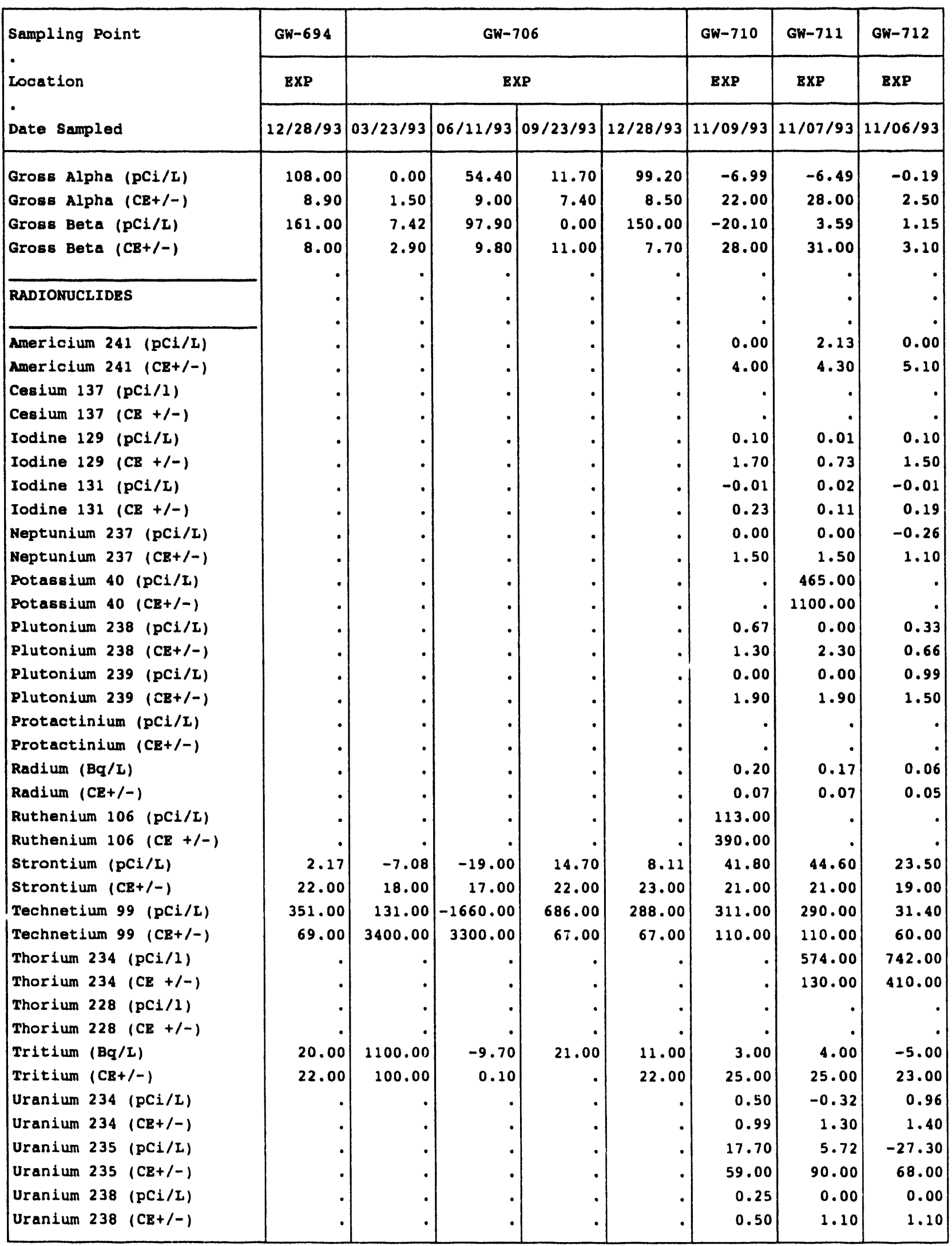


APPENDIX E. 4

Groundwater and Surface-Water Quality Data, 1993

\begin{tabular}{|c|c|c|c|c|c|c|c|c|}
\hline \multirow{3}{*}{$\begin{array}{l}\text { Sampling Point } \\
\text { Location } \\
\text { - } \\
\text { Date Sampled }\end{array}$} & \multirow{3}{*}{\begin{tabular}{|c|}
$6 w-713$ \\
$\operatorname{Exp}$ \\
$11 / 09 / 93$ \\
\end{tabular}} & \multirow{3}{*}{\begin{tabular}{|c|} 
GW-714 \\
EXP \\
$11 / 09 / 93$ \\
\end{tabular}} & \multirow{3}{*}{\begin{tabular}{|c|} 
GW- 715 \\
EXP \\
$11 / 11 / 93$ \\
\end{tabular}} & \multicolumn{4}{|c|}{ GW-793 } & \multirow{3}{*}{\begin{tabular}{|c|} 
GW-794 \\
AGLLSF \\
$03 / 01 / 93$
\end{tabular}} \\
\hline & & & & \multicolumn{4}{|c|}{ AGLLSF } & \\
\hline & & & & $02 / 26 / 93$ & $05 / 25 / 93$ & $07 / 11 / 93$ & $10 / 18 / 93$ & \\
\hline Grose Alpha (pCi/I) & -0.17 & 0.93 & 0.17 & 0.49 & -5.55 & -4.03 & -0.15 & 2.55 \\
\hline Gross Alpha $(\mathrm{CE}+/-)$ & 2.20 & 2.20 & 1.30 & 1.40 & 4.40 & 7.90 & 2.20 & 2.00 \\
\hline Gross Beta (pCi/L) & 1.13 & 6.28 & 2.65 & 0.95 & 3.98 & -0.98 & 3.21 & 4.57 \\
\hline Gross Beta $(\mathrm{CE}+/-)$ & 3.00 & 3.30 & 2.50 & 2.70 & 5.50 & 11.00 & 3.10 & 2.90 \\
\hline RADIONUCLIDES & • & • & • & $\cdot$ &. & $\cdot$ & $\cdot$ & • \\
\hline Americium 241 (pCi/L) & $\begin{array}{r}. \\
1.28\end{array}$ & 2.05 & 22.60 &. & . & . & • & • \\
\hline Americlum $241(\mathrm{CE}+/-)$ & 2.60 & 4.10 & 11.00 & - &. & . & - & - \\
\hline Cesium $137(\mathrm{pc} 1 / 1)$ & - & - & 34.80 & - & . & . & - & - \\
\hline Cesium 137 (CE $+/-$ ) & - & - & 36.00 & $\cdot$ & $\cdot$ & . & - & - \\
\hline Iodine $129(\mathrm{pCl} / \mathrm{L})$ & 0.10 & 0.10 & 0.10 & $\cdot$ & . & $\cdot$ & $\cdot$ & - \\
\hline Iodine $129(\mathrm{CE}+/-)$ & 1.40 & 1.70 & 1.60 & . & - & - & - & - \\
\hline Iodine $131(\mathrm{pCi} / \mathrm{L})$ & -0.07 & 0.05 & -0.01 & - & - & . & $\cdot$ & - \\
\hline Iodine $131(\mathrm{CE}+/-)$ & 0.26 & 0.24 & 0.23 & - & $\cdot$ & - & - & - \\
\hline Neptunium $237(\mathrm{pCi} / \mathrm{L})$ & 0.00 & -0.27 & 1.71 & - & - & $\cdot$ & . & - \\
\hline Neptunium $237(\mathrm{CB}+/-)$ & 0.73 & 1.10 & 3.40 & - & - & $\cdot$ & - & - \\
\hline Potassium 40 (pCi/L) & - & 353.00 & 332.00 & - & - & 558.00 & $\cdot$ & - \\
\hline Potassium $40(\mathrm{CE}+/-)$ & - & 950.00 & 950.00 & - & - & 1100.00 & - & - \\
\hline Plutonium $230(\mathrm{pCl} / \mathrm{L})$ & 0.00 & 0.34 & -12.80 & - & $\cdot$ & $\cdot$ & - & - \\
\hline Plutonium $238(\mathrm{CE}+/-)$ & 1.10 & 0.68 & 13.00 & - & . & - & - & - \\
\hline Plutonium 239 (pCi/L) & -0.32 & -0.34 & -4.27 & - & - & . & $\cdot$ & - \\
\hline Plutonium $239(\mathrm{Cz}+/-)$ & 1.30 & 1.40 & 9.50 & - & - & - & $\cdot$ & • \\
\hline Protactinium (pCi/L) & - & - & $\cdot$ & . & . & - & $\cdot$ & - \\
\hline Protactinium $(\mathrm{CE}+/-)$ & & - & $\cdot$ & - & - & - & $\cdot$ & - \\
\hline Radium (Bq/L) & 0.03 & 0.10 & 0.04 & • & - & - & - & - \\
\hline Radium $(\mathrm{CE}+/-)$ & 0.04 & 0.05 & 0.04 & $\cdot$ & - & - & - & - \\
\hline Ruthenium $106(\mathrm{pCi} / \mathrm{L})$ & - & 182.00 & 214.00 & - & - & - & . & - \\
\hline Ruthenium $106(\mathrm{CE}+/-)$ & - & 350.00 & 360.00 & - & $\cdot$ & $\cdot$ & - & - \\
\hline strontium $(\mathrm{pCi} / L)$ & 34.10 & 50.20 & 18.30 & - & - & - & - & - \\
\hline Strontium $(\mathrm{CE}+/-)$ & 20.00 & 22.00 & 26.00 & - & • & - & - & • \\
\hline Technetium 99 (pCi/L) & 301.00 & 152.00 & 7.60 & - & - & $\cdot$ & - & - \\
\hline Technetium $99(\mathrm{CB}+/-)$ & 68.00 & 63.00 & 59.00 & - & - &. & $\cdot$ & • \\
\hline Thorium 234 (pCi/l) & $\cdot$ & - & - & - & - & - & $\cdot$ & • \\
\hline Thorium $234(\mathrm{CE}+/-)$ & - & - & - & - & - & - & - & • \\
\hline Thorium $228(\mathrm{pCi} / 1)$ & - & - & - & - & - & - & $\cdot$ & • \\
\hline Thorium $228(\mathrm{CE}+/-)$ & - & - & - & - & - & - & . & • \\
\hline Tritium (Bq/L) & 50.00 & -2.00 & 42.00 & - & - & - & . & $\cdot$ \\
\hline Tritium $(\mathrm{CE}+/-)$ & 27.00 & 23.00 & 26.00 & - & - & - & - & • \\
\hline Uranium $234(\mathrm{pCi} / \mathrm{L})$ & 0.38 & 1.84 & 0.45 & 0.00 & 6.65 & 13.20 & -10.80 & 8.34 \\
\hline Uranium $234(\mathrm{CE}+/-)$ & 1.30 & 1.90 & 0.90 & 16.00 & 13.00 & 19.00 & 49.00 & 12.00 \\
\hline Uranium 235 (pCi/L) & 10.70 & 11.90 & 15.90 & 50.50 & 12.80 & 24.70 & 21.80 & -23.60 \\
\hline Uranium $235(\mathrm{CE}+/-)$ & 59.00 & 68.00 & 69.00 & 81.00 & 90.00 & 90.00 & 59.00 & 70.00 \\
\hline Uranium $238(\mathrm{pCl} / \mathrm{L})$ & 0.38 & 0.73 & -0.22 & 25.60 & 6.65 & 6.59 & 0.00 & $-17 \cdot 50$ \\
\hline Uranium $238(\mathrm{CE}+/-)$ & 0.75 & 1.00 & 0.90 & 36.00 & 13.00 & 35.00 & 38.00 & 60.00 \\
\hline
\end{tabular}

(CONTINUED) 
APPENDIX $\mathrm{B}, 4$

Groundwater and Surface-Water Quality Data, 1993

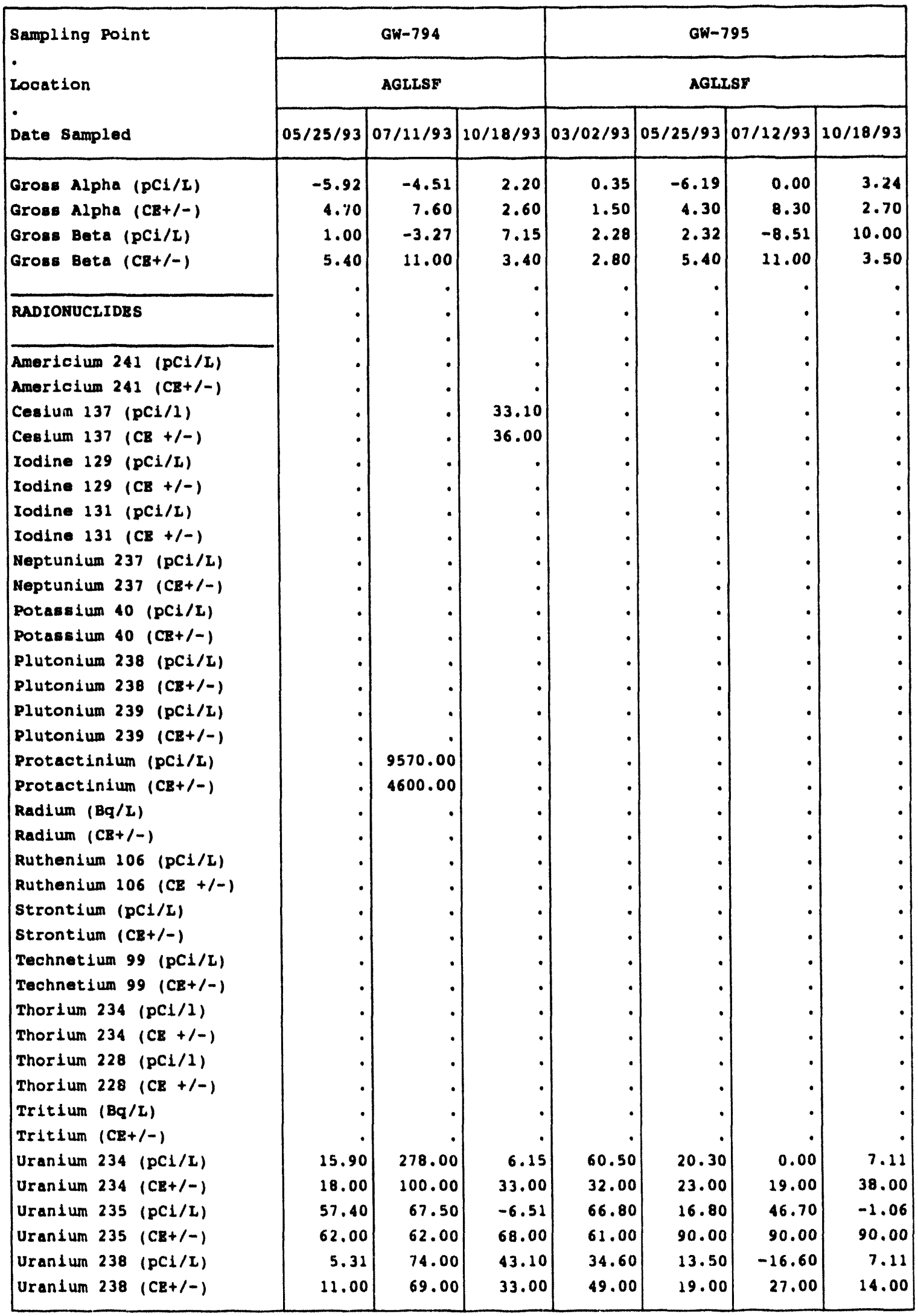


APPENDIX 8.4

Groundwater and Surface-water Quality Data, 1993

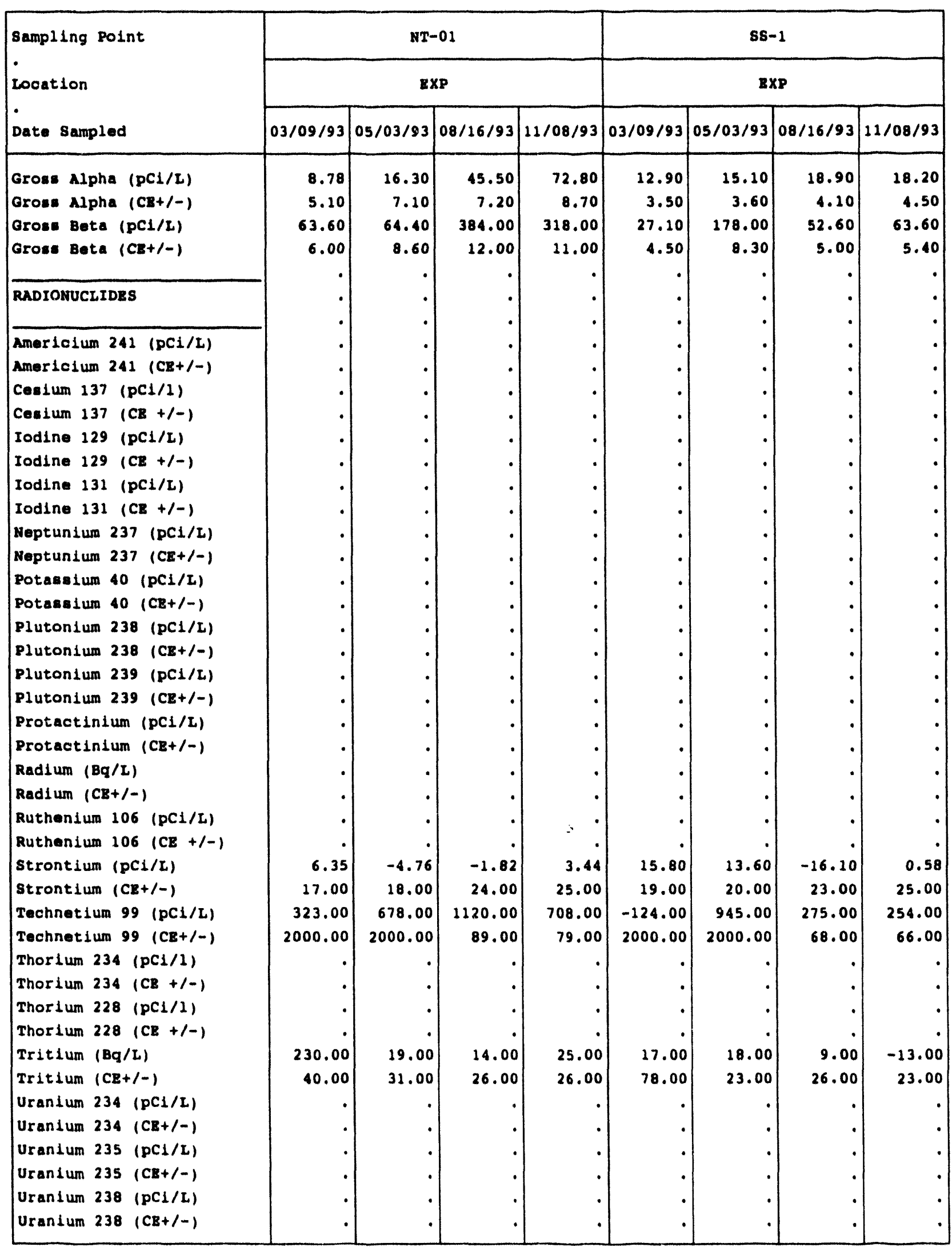



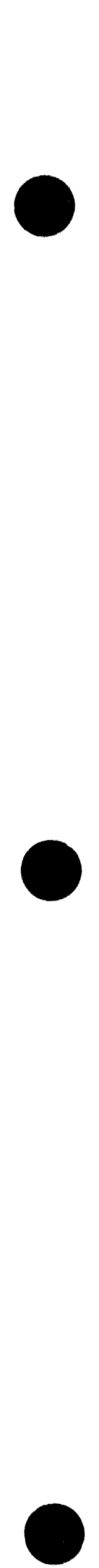
APPENDIX F

FIELD DUPLICATE DATA 


\title{
EXPLANATION
}

SAMPLING POINT:

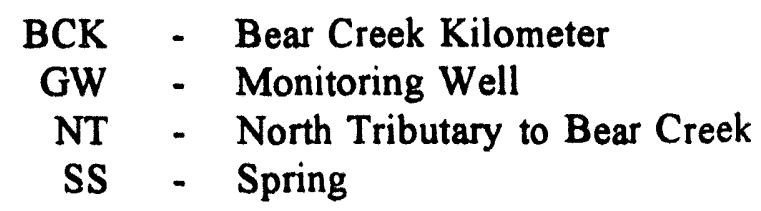

\section{LOCATION:}

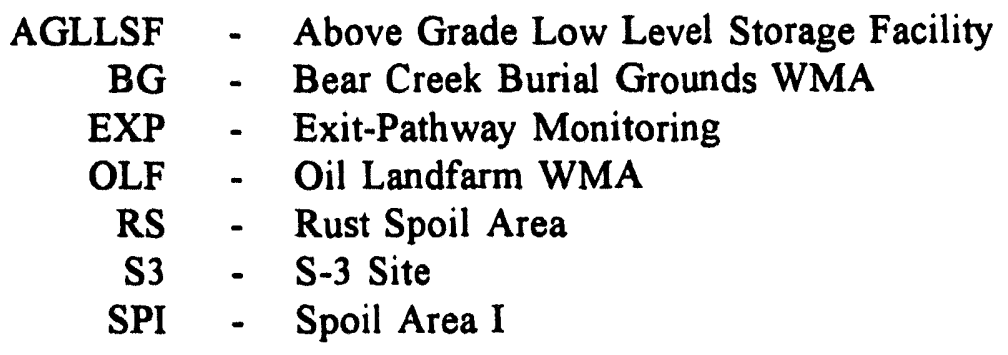

\begin{abstract}
ALL DATA:
- Not Analyzed
\end{abstract}

\section{VOLATILE ORGANIC COMPOUNDS:}

All results in micrograms per Liter (ug/L).

$\mathrm{U}$ - Compound not detected at the reported minimum attainable detection limit.

METALS, MAJOR IONS, MISCELLANEOUS, AND RADIOCHEMICAL PARAMETERS:

All analyses are by Inductively Coupled Plasma (ICP) Spectroscopy unless otherwise noted.

Bicarbonate and carbonate alkalinity reported as $\mathrm{CaCO}_{3}$.

$<\quad-\quad$ Compound analyzed but not detected at the reported minimum attainable detection limit

$\mathrm{mg} / \mathrm{L}$ - milligrams per Liter

AAS - Atomic Absorption Spectroscopy

CVAA - Cold Vapor Atomic Absorption

DIS - Dissolved Concentration (Filtered Sample)

TOT - Total Concentration (Unfiltered Sample) 
Fluor - Fluorometric

TDS - Total Dissolved Solids

TSS - Total Suspended Solids

Sp. Cond. - Specific Conductance

ppm - parts per million

umho/cm - micromhos per centimeter

NTU - Nephelometric Turbidity Unit

$\mathrm{mV}$ - millivolts

$\mathrm{pCi} / \mathrm{L}$ - picoCuries per liter

$\mathrm{Bq} / \mathrm{L}$ - Becquerels per liter

$\mathrm{CE}+\% \quad$ - Counting Error (two standard deviations) 
APPENDIX F.1

TRACE METALS AND MAJOR IONS 
APPENDIX F.1

Field Dupllcate Groundwater Quality Data, 1993

\begin{tabular}{|c|c|c|c|c|c|c|c|c|}
\hline \multirow{4}{*}{$\begin{array}{l}\text { Sampling Point } \\
\text { Location } \\
\text { - } \\
\text { Date Sampled }\end{array}$} & \multicolumn{2}{|c|}{$G W-044$} & \multicolumn{2}{|c|}{$G W-069$} & \multicolumn{4}{|c|}{ GW-115 } \\
\hline & \multicolumn{2}{|c|}{ OLF } & \multicolumn{2}{|c|}{ BG } & \multicolumn{4}{|c|}{ S3 } \\
\hline & \multicolumn{2}{|c|}{$09 / 11 / 93$} & \multicolumn{2}{|c|}{$10 / 10 / 93$} & \multicolumn{2}{|c|}{$05 / 25 / 93$} & \multicolumn{2}{|c|}{$09 / 11 / 93$} \\
\hline & TOT & DIS & $\operatorname{TOT}$ & DIS & TOT & DIS & TOT & DIS \\
\hline METALS (mg/I) & - & - & - & - & - & $\cdot$ & - & - \\
\hline Aluminum & 0.022 & $<0.02$ & $<0.02$ & 0.022 & 0.028 & 0.024 & $<0.02$ & $<0.02$ \\
\hline Antimony & $<0.05$ & $<0.05$ & $<0.05$ & $<0.05$ & $<0.05$ & $<0.05$ & $<0.05$ & $<0.05$ \\
\hline Arbenic & $<0.05$ & $<0.05$ & $<0.05$ & $<0.05$ & $<0.05$ & $<0.05$ & $<0.05$ & $<0.05$ \\
\hline Barium & 0.23 & 0.23 & 0.41 & 0.42 & 0.23 & 0.23 & 0.24 & 0.24 \\
\hline Beryllium & $<0.0003$ & $<0.0003$ & $<0.0003$ & $<0.0003$ & $<0.0003$ & $<0.0003$ & $<0.0003$ & $<0.0003$ \\
\hline Boron & 0.0076 & 0.029 & 0.11 & 0.12 & 0.023 & 0.036 & 0.01 & 0.013 \\
\hline Cadmium (AAS) & $<0.002$ & $<0.002$ & $<0.002$ & $<0.002$ & $<0.002$ & $<0.002$ & $<0.002$ & $<0.002$ \\
\hline Cadmium & $<0.003$ & $<0.003$ & $<0.003$ & $<0.003$ & $<0.003$ & $<0.003$ & $<0.003$ & $<0.003$ \\
\hline Chromium (AAS) & $<0.01$ & $<0.01$ & $<0.01$ & $<0.01$ & $<0.01$ & $<0.01$ & $<0.01$ & $<0.01$ \\
\hline Chromium & $<0.01$ & $<0.01$ & $<0.01$ & $<0.01$ & $<0.01$ & $<0.01$ & $<0.01$ & $<0.01$ \\
\hline Cobalt & $<0.005$ & $<0.005$ & $<0.005$ & $<0.005$ & $<0.005$ & $<0.005$ & $<0.005$ & $<0.005$ \\
\hline Copper & 0.01 & 0.0046 & $<0.004$ & $<0.004$ & $<0.004$ & $<0.004$ & 0.0042 & $<0.004$ \\
\hline Iron & 0.044 & $<0.005$ & 0.018 & 0.0096 & 0.42 & 0.42 & 0.4 & 0.31 \\
\hline Lead (AAS) & 0.0089 & $<0.004$ & $<0.004$ & $<0.004$ & $<0.004$ & $<0.004$ & $<0.004$ & $<0.004$ \\
\hline Mercury (CVAA) & $<0.0002$ & $<0.0002$ & $<0.0002$ & $<0.0002$ & $<0.0002$ & $<0.0002$ & $<0.0002$ & $<0.0002$ \\
\hline Molybdenum & $<0.01$ & $<0.01$ & $<0.01$ & $<0.01$ & $<0.01$ & $<0.01$ & $<0.01$ & $<0.01$ \\
\hline Nickel & $<0.01$ & $<0.01$ & $<0.01$ & $<0.01$ & $<0.01$ & $<0.01$ & $<0.01$ & $<0.01$ \\
\hline Selenium & $<0.05$ & $<0.05$ & $<0.05$ & $<0.05$ & $<0.05$ & $<0.05$ & $<0.05$ & $<0.05$ \\
\hline silver & $<0.006$ & $<0.006$ & $<0.006$ & $<0.006$ & $<0.006$ & $<0.006$ & $<0.006$ & $<0.006$ \\
\hline strontium & 0.077 & 0.076 & 0.91 & 0.95 & 0.11 & 0.11 & 0.12 & 0.12 \\
\hline Thorium & $<0.2$ & $<0.2$ & $<0.2$ & $<0.2$ & $<0.2$ & $<0.2$ & $<0.2$ & $<0.2$ \\
\hline Uranium (Fluor) & $<0.001$ & $<0.001$ & $<0.001$ & $<0.001$ & $<0.001$ & $<0.001$ & 0.003 & $<0.001$ \\
\hline Vanadium & $<0.005$ & $<0.005$ & $<0.005$ & $<0.005$ & $<0.005$ & $<0.005$ & $<0.005$ & $<0.005$ \\
\hline zinc & 0.025 & 0.03 & 0.0043 & 0.0042 & 0.0035 & 0.011 & 0.0063 & 0.0029 \\
\hline MAJOR IONS $(\mathrm{mg} / \mathrm{L})$ & $\cdot$ & $\cdot$ & . & $\cdot$ & $\cdot$ & $\cdot$ & $\cdot$ & - \\
\hline Alkalinity-HCO3 & 132 & $\cdot$ & 133 & $\cdot$ & 226 & $\cdot$ & 235 & • \\
\hline Alkalinity-CO3 & $<1$ & . & 6 &. & $<1$ & . & $<1$ & - \\
\hline Calcium & 51 & 50 & 11 & 12 & 67 & 68 & 73 & 71 \\
\hline Chloride & 1 & - & 53.6 & . & 4 & . & 3.8 & - \\
\hline Fluoride & 0.1 & . & $<0.1$ & . & 0.1 & - & $<0.1$ & - \\
\hline Magnesium & 3.3 & 3.2 & 6.1 & 6.5 & 11 & 11 & 11 & 11 \\
\hline Manganese & 0.024 & $<0.001$ & 0.0028 & 0.0037 & 0.3 & 0.3 & 0.31 & 0.27 \\
\hline Nitrate-N & $<0.2$ & . & 0.26 & . & $<0.2$ & - & $<0.2$ & - \\
\hline Potassium & $<0.6$ & $<0.6$ & 4.4 & 4.7 & 2 & 1.7 & 1.7 & 3 \\
\hline sodium & 4.7 & 4.7 & 64 & 66 & 9.5 & 9.5 & 10 & 10 \\
\hline sulfate & 6.7 & - & 1.8 & . & 17.5 & . & 15.8 & - \\
\hline
\end{tabular}

(CONTINUED) 
APPENDIX F.1

Field Duplicate Groundwater Quality Data, 1993

\begin{tabular}{|c|c|c|c|c|c|c|c|c|}
\hline \multirow{4}{*}{$\begin{array}{l}\text { Sampling Point } \\
\text { Location } \\
\text { Date Sampled }\end{array}$} & \multicolumn{2}{|c|}{$G W-315$} & \multicolumn{2}{|c|}{$G W-324$} & \multicolumn{2}{|c|}{ GW-371 } & \multicolumn{2}{|c|}{ GW-373 } \\
\hline & \multicolumn{2}{|c|}{ SPI } & \multicolumn{2}{|c|}{ s3 } & \multicolumn{2}{|c|}{ BG } & \multicolumn{2}{|c|}{ BG } \\
\hline & \multicolumn{2}{|c|}{$10 / 27 / 93$} & \multicolumn{2}{|c|}{$01 / 29 / 93$} & \multicolumn{2}{|c|}{$10 / 13 / 93$} & \multicolumn{2}{|c|}{$02 / 11 / 93$} \\
\hline & TOT & DIS & TOT & DIS & TOT & DIs & TOT & DIs \\
\hline $\operatorname{matars}(\mathrm{mg} / \mathrm{L})$ & $\cdot$ & $\cdot$ & $\cdot$ & $\cdot$ & - & $\cdot$ & $\cdot$ & - \\
\hline Aluminum & $<0.02$ & $<0.02$ & $<0.02$ & $<0.02$ & $<0.02$ & $<0.02$ & 0.031 & $<0.02$ \\
\hline Antimony & $<0.05$ & $<0.05$ & $<0.05$ & $<0.05$ & $<0.05$ & $<0.05$ & $<0.05$ & $<0.05$ \\
\hline Arsenic & $<0.05$ & $<0.05$ & $<0.05$ & $<0.05$ & $<0.05$ & $<0.05$ & $<0.05$ & $<0.05$ \\
\hline Barium & 0.06 & 0.056 & 0.2 & 0.2 & 0.14 & 0.14 & 0.052 & 0.042 \\
\hline Beryllium & $<0.0003$ & $<0.0003$ & $<0.0003$ & $<0.0003$ & $<0.0003$ & $<0.0003$ & $<0.0003$ & $<0.0003$ \\
\hline Boron & 0.019 & 0.015 & 0.012 & 0.0084 & 0.04 & 0.042 & 0.16 & 0.16 \\
\hline Cadmium (AAS) & $<0.002$ & $<0.002$ & $<0.002$ & $<0.002$ & $<0.002$ & $<0.002$ & $<0.002$ & $<0.002$ \\
\hline Cadmitum & $<0.003$ & $<0.003$ & $<0.003$ & $<0.003$ & $<0.003$ & $<0.003$ & $<0.003$ & $<0.003$ \\
\hline Chromium (AAS) & $<0.01$ & $<0.01$ & $<0.01$ & $<0.01$ & $<0.01$ & $<0.01$ & $<0.01$ & $<0.01$ \\
\hline Chromium & $<0.01$ & $<0.01$ & $<0.01$ & $<0.01$ & $<0.01$ & $<0.01$ & $<0.01$ & $<0.01$ \\
\hline Cobalt & $<0.005$ & $<0.005$ & $<0.005$ & $<0.005$ & $<0.005$ & $<0.005$ & $<0.005$ & $<0.005$ \\
\hline Copper & $<0.004$ & $<0.004$ & $<0.004$ & $<0.004$ & $<0.004$ & $<0.004$ & $<0.004$ & $<0.004$ \\
\hline Iron & 0.022 & $<0.005$ & 0.086 & 0.032 & 1.4 & 0.44 & 0.63 & 0.022 \\
\hline Lead (AAS) & $<0.004$ & $<0.004$ & $<0.004$ & $<0.004$ & $<0.004$ & $<0.004$ & $<0.004$ & $<0.004$ \\
\hline Mercury (CVAA) & $<0.0002$ & $<0.0002$ & $<0.0002$ & $<0.0002$ & $<0.0002$ & $<0.0002$ & $<0.0002$ & $<0.0002$ \\
\hline Mol ybdenum & $<0.01$ & $<0.01$ & $<0.01$ & $<0.01$ & $<0.01$ & $<0.01$ & $<0.01$ & $<0.01$ \\
\hline Nickel & $<0.01$ & $<0.01$ & $<0.01$ & $<0.01$ & $<0.01$ & $<0.01$ & $<0.01$ & $<0.01$ \\
\hline Selenium & $<0.05$ & $<0.05$ & $<0.05$ & $<0.05$ & $<0.05$ & $<0.05$ & $<0.05$ & $<0.05$ \\
\hline silver & $<0.006$ & $<0.006$ & $<0.006$ & $<0.006$ & $<0.006$ & $<0.006$ & $<0.006$ & $<0.006$ \\
\hline Strontium & 0.17 & 0.17 & 0.22 & 0.21 & 1.5 & 1.5 & 0.06 & 0.056 \\
\hline Thorium & $<0.2$ & $<0.2$ & $<0.2$ & $<0.2$ & $<0.2$ & $<0.2$ & $<0.2$ & $<0.2$ \\
\hline Uranium (Fluor) & 0.002 & 0.002 & $<0.001$ & $<0.001$ & 0.001 & $<0.001$ & $<0.001$ & $<0.001$ \\
\hline Vanadium & $<0.005$ & $<0.005$ & $<0.005$ & $<0.005$ & $<0.005$ & $<0.005$ & $<0.005$ & $<0.005$ \\
\hline Zinc & 0.02 & 0.011 & 0.0096 & 0.0071 & 0.002 & 0.0071 & 0.0028 & 0.002 \\
\hline MAJOR IONS $(\mathrm{mg} / \mathrm{L})$ & $\cdot$ & . & - & $\cdot$ & - & - & • & - \\
\hline Alkalinity- $\mathrm{HCO} 3$ & 221 & $\cdot$ & 100 & $\cdot$ & 299 & $\cdot$ & $18 \dot{\circ}$ & • \\
\hline Alkalinity-CO3 & $<1$ &. & $<1$ &. & $<1$ & . & 24 & . \\
\hline Calcium & 100 & 100 & 28 & 28 & 75 & 78 & 1.5 & 1.2 \\
\hline Chloride & 13 & $\cdot$ & 1 & . & 1 & . & 2.2 & - \\
\hline Fluoride & $<0.1$ & $\cdot$ & 0.1 & . & $<0.1$ & - & 1.5 & - \\
\hline Magnesium & 10 & 10 & 5.2 & 5.2 & 20 & 21 & 0.15 & 0.13 \\
\hline Manganese & 0.0014 & $<0.001$ & 0.015 & 0.015 & 0.027 & 0.031 & 0.0086 & 0.002 \\
\hline Nitrate-N & 20.5 & . & $<0.2$ &. & $<0.2$ & . & $<0.2$ & . \\
\hline Potassium & 3.6 & 3.3 & $<0.6$ & 0.78 & 3.1 & 3.3 & 0.75 & $<0.6$ \\
\hline Sodium & 7 & 7 & 5 & 5 & 18 & 18 & 110 & 100 \\
\hline Sulfate & 19 &. & 8.9 & . & 15 & . & 30 & • \\
\hline
\end{tabular}

(CONTINUED) 
APPENDIX F.1

Fleld Duplicate Groundwater Quality Data, 1993

\begin{tabular}{|c|c|c|c|c|c|c|c|c|}
\hline \multirow{4}{*}{$\begin{array}{l}\text { Sampling Point } \\
\text { Iocation } \\
\text { - } \\
\text { Date Sampled }\end{array}$} & \multicolumn{4}{|c|}{$G W-537$} & \multicolumn{4}{|c|}{$G w-626$} \\
\hline & \multicolumn{4}{|c|}{ OLF } & \multicolumn{4}{|c|}{ BG } \\
\hline & \multicolumn{2}{|c|}{$02 / 11 / 93$} & \multicolumn{2}{|c|}{$05 / 12 / 93$} & \multicolumn{2}{|c|}{$03 / 10 / 93$} & \multicolumn{2}{|c|}{$08 / 02 / 93$} \\
\hline & TOT & DIS & TOT & DIS & TOT & DIS & TOT & DIS \\
\hline METALS (mg/L) & $\cdot$ & - & - & - & - & - & - & - \\
\hline Aluminum & $<0.2$ & $<0.2$ & 0.29 & 0.63 & $<0.02$ & 0.036 & $<0.02$ & $<0.02$ \\
\hline Antimony & $<0.5$ & $<0.5$ & $<0.5$ & $<0.5$ & $<0.05$ & $<0.05$ & $<0.05$ & $<0.05$ \\
\hline Argenic & $<0.5$ & $<0.5$ & $<0.5$ & $<0.5$ & $<0.05$ & $<0.05$ & $<0.05$ & $<0.05$ \\
\hline Barium & 2.8 & 2.1 & 2.4 & 2.5 & 0.13 & 0.14 & 0.14 & 0.14 \\
\hline Bery1lium & $<0.003$ & $<0.003$ & $<0.003$ & $<0.003$ & $<0.0003$ & 0.0005 & $<0.0003$ & $<0.0003$ \\
\hline Boron & $<0.04$ & $<0.04$ & $<0.04$ & $<0.04$ & 0.0067 & 0.035 & $<0.004$ & $<0.004$ \\
\hline Cadmium (AAS) & $<0.002$ & $<0.002$ & $<0.002$ & $<0.002$ & $<0.002$ & $<0.002$ & $<0.002$ & $<0.002$ \\
\hline Cadmium & $<0.03$ & $<0.03$ & $<0.03$ & $<0.03$ & $<0.003$ & $<0.003$ & $<0.003$ & $<0.003$ \\
\hline Chromium (AAS) & $<0.01$ & $<0.01$ & $<0.01$ & $<0.01$ & $<0.01$ & $<0.01$ & $<0.01$ & $<0.01$ \\
\hline Chromium & 0.23 & 0.9 & $<0.1$ & $<0.1$ & $<0.01$ & $<0.01$ & $<0.01$ & $<0.01$ \\
\hline Cobalt & $<0.05$ & 0.053 & $<0.05$ & $<0.05$ & $<0.005$ & $<0.005$ & $<0.005$ & $<0.005$ \\
\hline Copper & $<0.04$ & $<0.04$ & $<0.04$ & $<0.04$ & $<0.004$ & $<0.004$ & $<0.004$ & $<0.004$ \\
\hline Iron & 1 & 3.8 & $<0.05$ & $<0.05$ & 0.0077 & 0.0056 & 0.016 & 0.0089 \\
\hline Lead (AAS) & $<0.004$ & $<0,004$ & $<0.004$ & $<0.004$ & $<0.004$ & $<0.004$ & $<0.004$ & $<0.004$ \\
\hline Mercury (CVAA) & $<0.0002$ & $<0.0002$ & $<0.0002$ & $<0.0002$ & $<0.0002$ & $<0.0002$ & $<0.0002$ & $<0.0002$ \\
\hline Mol ybdenum & $<0.1$ & $<0.1$ & $<0.1$ & $<0.1$ & $<0.01$ & $<0.01$ & $<0.01$ & $<0.01$ \\
\hline Nickel & 0.56 & 1.2 & $<0.1$ & $<0.1$ & $<0.01$ & 0.023 & $<0.01$ & $<0.01$ \\
\hline Selenium & $<0.5$ & $<0.5$ & $<0.5$ & $<0.5$ & $<0.05$ & $<0.05$ & $<0.05$ & $<0.05$ \\
\hline Silver & $<0.06$ & $<0.06$ & $<0.06$ & $<0.06$ & $<0.006$ & $<0.006$ & $<0.006$ & $<0.006$ \\
\hline Strontium & 3.2 & $2 \cdot 4$ & 2.9 & 3 & 0.096 & 0.1 & 0.094 & 0.093 \\
\hline Thorium & $<2$ & $<2$ & $<2$ & $<2$ & $<0.2$ & $<0.2$ & $<0.2$ & $<0.2$ \\
\hline Uranium (Fluor) & 0.002 & 0.002 & 0.001 & 0.001 & $<0.001$ & $<0.001$ & 0.001 & 0.001 \\
\hline Vanadium & $<0.05$ & $<0.05$ & $<0.05$ & $<0.05$ & $<0.005$ & $<0.005$ & $<0.005$ & $<0.005$ \\
\hline Zinc & 0.053 & 0.049 & $<0.02$ & $<0.02$ & 0.0057 & 0.0058 & 0.0047 & 0.0061 \\
\hline MAJOR IONS (mg/L) & - & $\cdot$ & . & - & - & $\cdot$ & $\cdot$ & - \\
\hline Alkalinity-HCO3 & 233 & . & 246 & $\cdot$ & 107 & . & 109 & • \\
\hline Alkalinity-CO3 & $<1$ & - & $<1$ & - & $<1$ & . & $<1$ & - \\
\hline Calcium & 1300 & 1000 & 1100 & 1100 & 38 & 41 & 38 & 38 \\
\hline Chloride & 28 & - & 33 & - & 14.5 & - & 6 & - \\
\hline Eluoride & $<0.1$ & - & $<0.1$ & $\cdot$ & $<0.1$ & - & $<0.1$ & - \\
\hline Magnesium & 84 & 66 & 73 & 74 & 2.5 & 2.7 & 2.9 & $2 \cdot 9$ \\
\hline Manganese & 0.05 & 0.14 & $<0.01$ & $<0.01$ & $<0.001$ & $<0.001$ & 0.0013 & 0.0015 \\
\hline Nitrate-N & 677 & - & 769 & - & $<0.2$ & - & $<0.2$ & - \\
\hline Potassium & $<6$ & 10 & $<6$ & $<6$ & 1 & 1.1 & 1.1 & 0.93 \\
\hline Sodium & 35 & 27 & 30 & 30 & 3.8 & 4.5 & 4.1 & 4.1 \\
\hline Sulfate & $<10$ & . & 2 & - & 3.9 & • & 2.8 & - \\
\hline
\end{tabular}

(CONTINUED) 
APRENDIX F.1

Fleld Duplicate Groundwater Quality Data, 1993

\begin{tabular}{|c|c|c|c|c|c|c|c|c|}
\hline \multirow{4}{*}{$\begin{array}{l}\text { Sampling Point } \\
\text { Location } \\
\text { Date Sampled }\end{array}$} & \multicolumn{2}{|c|}{$G W-627$} & \multicolumn{6}{|c|}{ GW-636 } \\
\hline & \multicolumn{2}{|c|}{ BG } & \multicolumn{6}{|c|}{ OLF } \\
\hline & \multicolumn{2}{|c|}{$04 / 23 / 93$} & \multicolumn{2}{|c|}{$03 / 26 / 93$} & \multicolumn{2}{|c|}{$06 / 27 / 93$} & \multicolumn{2}{|c|}{$09 / 18 / 93$} \\
\hline & TOT & DIs & TOT & DIS & TOT & DIS & TOT & DIs \\
\hline METALS $(\mathrm{mg} / \mathrm{L})$ & $\cdot$ & $\cdot$ & $\cdot$ & $\cdot$ & - & $\cdot$ & $\cdot$ & • \\
\hline Aluminum & $<0.02$ & $<0.02$ & $<0.02$ & $<0.02$ & $<0.02$ & $<0.02$ & $<0.02$ & $<0.02$ \\
\hline Ant imony & $<0.05$ & $<0.05$ & $<0.05$ & $<0.05$ & $<0.05$ & $<0.05$ & $<0.05$ & $<0.05$ \\
\hline Arsenic & $<0.05$ & $<0.05$ & $<0.05$ & $<0.05$ & $<0.05$ & $<0.05$ & $<0.05$ & $<0.05$ \\
\hline Barium & 0.041 & 0.041 & 0.21 & 0.2 & 0.23 & 0.21 & 0.24 & 0.24 \\
\hline Beryllium & $<0.0003$ & $<0.0003$ & $<0.0003$ & $<0.0003$ & $<0.0003$ & $<0.0003$ & $<0.0003$ & $<0.0003$ \\
\hline Boron & 0.46 & 0.48 & 0.2 & 0.33 & 0.38 & 0.31 & 0.39 & 0.39 \\
\hline Cadmium (AAS) & $<0.002$ & $<0.002$ & $<0.002$ & $<0.002$ & $<0.002$ & $<0 ., 502$ & $<0.002$ & $<0.002$ \\
\hline Cadmium & $<0.003$ & $<0.003$ & $<0.003$ & $<0.003$ & $<0.003$ & $<0.003$ & $<0.003$ & $<0.003$ \\
\hline Chromium (AAS) & $<0.01$ & $<0.01$ & $<0.01$ & $<0.01$ & $<0.01$ & $: 0.01$ & $<0.01$ & $<0.01$ \\
\hline Chromium & $<0.01$ & $<0.01$ & $<0.01$ & $<0.01$ & $<0.01$ & $<0.01$ & $<0.01$ & $<0.01$ \\
\hline Cobalt & $<0.005$ & $<0.005$ & $<0.005$ & $<0.005$ & $<0.005$ & $<0.005$ & $<0.005$ & $<0.005$ \\
\hline Copper & $<0.004$ & $<0.004$ & $<0.004$ & $<0.004$ & $<0.004$ & $<0.004$ & $<0.004$ & $<0.004$ \\
\hline Iron & 0.19 & 0.04 & 1.4 & 0.098 & 0.71 & 0.22 & 1.3 & 0.13 \\
\hline Lead (AAS) & $<0.004$ & $<0.004$ & $<0.004$ & $<0.004$ & $<0.004$ & $<0.004$ & $<0.004$ & $<0.004$ \\
\hline Mercury (CVAA) & $<0.0002$ & $<0.0002$ & $<0.0002$ & $<0.0002$ & $<0.0002$ & $<0.0002$ & $<0.0002$ & $<0.0002$ \\
\hline Molybdenum & $<0.01$ & $<0.01$ & $<0.01$ & $<0.01$ & $<0.01$ & $<0.01$ & $<0.01$ & $<0.01$ \\
\hline Nickel & $<0.01$ & $<0.01$ & $<0.01$ & $<0.01$ & $<0.01$ & $<0.01$ & $<0.01$ & $<0.01$ \\
\hline Selenium & $<0.05$ & $<0.05$ & $<0.05$ & $<0.05$ & $<0.05$ & $<0.05$ & $<0.05$ & $<0.05$ \\
\hline silver & $<0.006$ & $<0.006$ & $<0.006$ & $<0.006$ & $<0.006$ & $<0.006$ & $<0.006$ & $<0.006$ \\
\hline Strontium & 0.07 & 0.07 & 0.51 & 0.52 & 0.57 & 0.55 & 0.6 & 0.61 \\
\hline Thorium & $<0.2$ & $<0.2$ & $<0.2$ & $<0.2$ & $<0.2$ & $<0.2$ & $<0.2$ & $<0.2$ \\
\hline Uranium (Fluor) & $<0.001$ & 0.001 & $<0.001$ & $<0.001$ & $<0.001$ & $<0.001$ & $<0.001$ & $<0.001$ \\
\hline Vanadium & $<0.005$ & $<0.005$ & $<0.005$ & $<0.005$ & $<0.005$ & $<0.005$ & $<0.005$ & $<0.005$ \\
\hline zinc & 0.0049 & 0.0066 & 0.003 & 0.004 & 0.0042 & $<0.002$ & 0.0077 & 0.027 \\
\hline MAJOR IONS $(\mathrm{mg} / \mathrm{L})$ & $\cdot$ & $\cdot$ & $\cdot$ & $\cdot$ & . & $\cdot$ & $\cdot$ & - \\
\hline Alkalinity-HCO3 & 443 &. & $164^{\circ}$ &. & 179 & $\cdot$ & 177 & • \\
\hline Alkalinity- $\mathrm{CO} 3$ & 82 & $\cdot$ & 6 & $\cdot$ & 2 & $\cdot$ & $<1$ & - \\
\hline Calcium & 0.96 & 0.9 & 7.4 & 7.4 & 8.1 & 7.4 & 7.6 & 7.7 \\
\hline Chloride & 22 & $\cdot$ & 6.2 & $\cdot$ & 8 & $\cdot$ & 7 & • \\
\hline Fluoride & 5.6 & $\cdot$ & 0.2 & $\cdot$ & 0.1 & $\cdot$ & 0.2 & • \\
\hline Magnesium & 0.23 & 0.2 & 2.4 & 2.4 & 2.7 & 2.5 & 2.6 & 2.6 \\
\hline Manganese & 0.0063 & 0.0045 & 0.019 & 0.01 & 0.018 & 0.015 & 0.021 & 0.012 \\
\hline Nitrate-N & $<0.2$ &. & $<0.2$ &. & $<0.2$ & $\cdot$ & $<0.2$ & • \\
\hline Potassium & 1.5 & 1.1 & 5.5 & 5 & 5.1 & 5.4 & 5.1 & 5.4 \\
\hline Sodium & 270 & 270 & 69 & 70 & 70 & 67 & 74 & 76 \\
\hline Sulfate & 31 & $\cdot$ & 9.3 & $\cdot$ & 5.2 & $\cdot$ & 4 & $\bullet$ \\
\hline
\end{tabular}

(CONTINUED) 
Field Duplicate Groundwater Quallty Data, 1993

\begin{tabular}{|c|c|c|c|c|c|c|c|c|}
\hline \multirow{4}{*}{$\begin{array}{l}\text { Sampling Point } \\
\text { Location } \\
\text { - } \\
\text { Date sampled }\end{array}$} & \multicolumn{2}{|c|}{$e w-636$} & \multicolumn{6}{|c|}{$G w-639$} \\
\hline & \multicolumn{2}{|c|}{ OLF } & \multicolumn{6}{|c|}{ BO } \\
\hline & \multicolumn{2}{|c|}{$12 / 18 / 93$} & \multicolumn{2}{|c|}{$02 / 24 / 93$} & \multicolumn{2}{|c|}{$05 / 24 / 93$} & \multicolumn{2}{|c|}{$07 / 11 / 93$} \\
\hline & TOT & Drs & TOT & DIs & TOT & Drs & TOT & DI8 \\
\hline MBTALS (mg/L) & . & - & $\cdot$ & $\cdot$ & $\cdot$ & $\cdot$ & $\cdot 1$ & \\
\hline Aluminum & $<0.02$ & $<0.02$ & 0.7 & $<0.02$ & 0.48 & $<0.02$ & 0.27 & $<0.02$ \\
\hline Antimony & $<0.05$ & $<0.05$ & $<0.05$ & $<0.05$ & $<0.05$ & $<0.05$ & $<0.05$ & $<0.05$ \\
\hline Arsenic & $<0.05$ & $<0.05$ & $<0.05$ & $<0.05$ & $<0.05$ & $<0.05$ & $<0.05$ & $<0.05$ \\
\hline Barium & 0.25 & 0.25 & 0.078 & 0.06 & 0.056 & 0.058 & 0.058 & 0.056 \\
\hline Beryllium & $<0.0003$ & $<0.0003$ & 0.00053 & $<0.0003$ & $<0.0003$ & $<0.0003$ & $<0.0003$ & $<0.0003$ \\
\hline Boron & 0.38 & 0.38 & 0.59 & 0.59 & 0.52 & 0.6 & 0.57 & 0.57 \\
\hline Cadmium (AAS) & $<0.002$ & $<0.002$ & $<0.002$ & $<0.002$ & $<0.002$ & $<0.002$ & $<0.002$ & $<0.002$ \\
\hline Cadmi um & $<0.003$ & $<0.003$ & 0.0032 & $<0.003$ & $<0.003$ & $<0.003$ & $<0,003$ & $<0.003$ \\
\hline Chromium (MAs) & $<0.01$ & $<0.01$ & $<0.01$ & $<0.01$ & $<0.01$ & $<0.01$ & $<0.01$ & $<0.01$ \\
\hline Chromium & $<0.01$ & $<0.01$ & $<0.01$ & $<0.01$ & $<0.01$ & 0.01 & $<0.01$ & $<0.01$ \\
\hline Cobalt & $<0.005$ & $<0.005$ & $<0.005$ & $<0.005$ & $<0.005$ & $<0.005$ & $<0.005$ & $<0.005$ \\
\hline Coppex & $<0.004$ & $<0.004$ & 0.0064 & $<0.004$ & 0.0044 & $<0.004$ & $<0.004$ & $<0.004$ \\
\hline Iron & 0.68 & 0.058 & 1.9 & 0.0092 & 0.6 & 0.014 & 0.21 & 0.0083 \\
\hline Lead (AAS) & $<0.004$ & $<0.004$ & $<0.004$ & $<0.004$ & $<0.004$ & $<0.004$ & $<0.004$ & $<0.004$ \\
\hline Mercury (CVMA) & $<0.0002$ & $<0.0002$ & $<0.0002$ & $<0.0002$ & $<0.0002$ & $<0.0002$ & $<0.0002$ & $<0.0002$ \\
\hline Mol ybdenum & $<0.01$ & $<0.01$ & $<0.01$ & $<0.01$ & $<0.01$ & $<0.01$ & $<0.01$ & $<0.01$ \\
\hline Nickel & $<0.01$ & $<0.01$ & $<0.01$ & $<0.01$ & $<0.01$ & 0.016 & $<0.01$ & $<0.01$ \\
\hline Selentum & $<0.05$ & $<0.05$ & $<0.05$ & $<0.05$ & $<0.05$ & $<0.05$ & $<0.05$ & $<0.05$ \\
\hline Silver & $<0.006$ & $<0.006$ & $<0.006$ & $<0.006$ & $<0.006$ & $<0.006$ & $<0.006$ & $<0.006$ \\
\hline strontium & 0.61 & 0.61 & 0.1 & 0.092 & 0.079 & 0.086 & 0.082 & 0.081 \\
\hline Thorium & $<0.2$ & $<0.2$ & $<0.2$ & $<0.2$ & $<0.2$ & $<0.2$ & $<0.2$ & $<0.2$ \\
\hline Urantum (Fluor) & $<0.001$ & $<0.001$ & $<0.001$ & $<0.001$ & $<0.001$ & $<0.001$ & 0.001 & 0.001 \\
\hline Vanadium & $<0.005$ & $<0.005$ & $<0.005$ & $<0.005$ & $<0.005$ & $<0.005$ & $<0.005$ & $<0.005$ \\
\hline zinc & 0.0023 & $<0.002$ & 0.013 & 0.0045 & 0.008 & 0.0062 & 0.0052 & 0.0061 \\
\hline MAJOR IONS $(\mathrm{mg} / \mathrm{L})$ & . &. & $\cdot$ & . & $\cdot$ & $\cdot$ &. & • \\
\hline Alkalinity-HCO3 & 173 & $\cdot$ & 336 & $\cdot$ & 342 & $\cdot$ & 354 & • \\
\hline Alkalinity-CO3 & 4 & . & 68 &. & 78 & . & 72 & 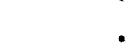 \\
\hline Calcium & 8.8 & 8.7 & 1.6 & 1.2 & 0.89 & 0.84 & 0.79 & 0.75 \\
\hline Chloride & 9 & $\cdot$ & 10 & $\cdot$ & 9.9 & . & 9.9 & $\cdot$ \\
\hline Fluoride & $<0.1$ & . & 2.3 &. & 2.4 & . & 2.8 & 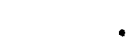 \\
\hline Magnesium & 2.8 & 2.8 & 0.52 & 0.3 & 0.34 & 0.23 & 0.31 & 0.23 \\
\hline Manganese & 0.013 & 0.0085 & 0.019 & $<0.001$ & 0.0057 & $<0.001$ & 0.0013 & $<0.001$ \\
\hline Nitrate-N & $<0.2$ & . & $<0.2$ & . & $<0.2$ & . & $<0.2$ & 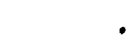 \\
\hline Potassium & 4.9 & 4.9 & 1.9 & 1.3 & 2.2 & 1.7 & 1.3 & 1.3 \\
\hline Sodium & 70 & 69 & 220 & 210 & 190 & 210 & 200 & 200 \\
\hline sulfate & 4 & . & 15 & . & 27.4 & . & 29.4 & 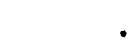 \\
\hline
\end{tabular}

(CONTINUED) 
APPEHDIX T.1

Field Dupllcate Groundwater Quallty Data, 1993

\begin{tabular}{|c|c|c|c|c|c|c|c|c|}
\hline \multirow{4}{*}{$\begin{array}{l}\text { sampling point } \\
\text { Location } \\
\text { Date sumpled }\end{array}$} & \multicolumn{4}{|c|}{$O W-684$} & \multicolumn{4}{|c|}{$6 W-685$} \\
\hline & \multicolumn{4}{|c|}{$\mathbf{E x p}$} & \multicolumn{4}{|c|}{$\mathbf{E x p}$} \\
\hline & \multicolumn{2}{|c|}{$07 / 11 / 93$} & \multicolumn{2}{|c|}{$10 / 12 / 93$} & \multicolumn{2}{|c|}{$03 / 02 / 93$} & \multicolumn{2}{|c|}{$04 / 21 / 93$} \\
\hline & TOT & DIs & TOT & DI8 & TOT & DIs & Tor & DIS \\
\hline MeTALs (mg/L) &. & $\cdot$ & $\cdot$ & $\cdot$ & $\cdot$ & $\cdot$ & - & 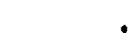 \\
\hline Aluminum & 0.028 & 0.025 & 0.091 & $<0.02$ & 0.26 & $<0.02$ & 0.27 & $<0.02$ \\
\hline Ant imony & $<0.05$ & $<0.05$ & $<0.03$ & $<0.05$ & $<0.05$ & $<0.05$ & $<0.05$ & $<0.05$ \\
\hline Arsenio & $<0.05$ & $<0.05$ & $<0.05$ & $<0.05$ & $<0.05$ & $<0.05$ & $<0.05$ & $<0.05$ \\
\hline Barium & 0.1 & 0.1 & 0.13 & 0.13 & 0.12 & 0.11 & 0.1 & 0.097 \\
\hline Beryll lum & $<0.0003$ & $<0.0003$ & $<0.0003$ & 0.00033 & $<0.0003$ & $<0.0003$ & $<0.0003$ & $<0.0003$ \\
\hline Boron & 0.14 & 0.12 & 0.088 & 0.085 & 0.041 & 0.045 & 0.14 & 0.1 \\
\hline Cadmi un (AMS) & $<0.002$ & $<0.002$ & $<0.002$ & $<0.002$ & $<0.002$ & $<0.002$ & $<0.002$ & $<0.002$ \\
\hline Cadnium & $<0.003$ & $<0.003$ & $<0.003$ & $<0.003$ & $<0.003$ & $<0.003$ & $<0.003$ & $<0.003$ \\
\hline Chromium (MAs) & $<0.01$ & $<0.01$ & $<0.01$ & $<0.01$ & $<0.01$ & $<0.01$ & $<0.01$ & $<0.01$ \\
\hline Chromium & $<0.01$ & $<0.01$ & $<0.01$ & $<0.01$ & $<0.01$ & $<0.01$ & $<0.01$ & $<0.01$ \\
\hline Cobalt & $<0.005$ & $<0.005$ & $<0.005$ & $<0.005$ & $<0,005$ & $<0.005$ & $<0.005$ & $<0.005$ \\
\hline Copper & $<0.004$ & $<0.004$ & $<0.004$ & $<0.004$ & $<0.004$ & $<0.004$ & $<0.004$ & $<0.004$ \\
\hline Iron & 0.027 & 0.012 & 0.24 & $<0.005$ & 5.3 & 0.1 & 1.5 & 0.042 \\
\hline Lead (MAS) & $<0.004$ & $<0.004$ & $<0.004$ & $<0.004$ & $<0.004$ & $<0.004$ & $<0.004$ & $<0.004$ \\
\hline Marcury (CVAN) & $<0.0002$ & $<0.0002$ & $<0.0002$ & $<0.0002$ & $<0.00022$ & $<0.00022$ & $<0.0002$ & $<0.0002$ \\
\hline Molybdenum & $<0.01$ & $<0.01$ & $<0.01$ & $<0.01$ & $<0.01$ & $<0.01$ & $<0.01$ & $<0.01$ \\
\hline Nickel & $<0.01$ & $<0.01$ & $<0.01$ & $<0.01$ & 0.02 & 0.017 & $<0.01$ & $<0.01$ \\
\hline Selenium & $<0.05$ & $<0.05$ & $<0.05$ & $<0.05$ & $<0.05$ & $<0.05$ & $<0.05$ & $<0.05$ \\
\hline silver & $<0.006$ & $<0.006$ & $<0.006$ & $<0.006$ & $<0.006$ & $<0.006$ & $<0.006$ & $<0.006$ \\
\hline strontium & 0.14 & 0.13 & 0.19 & 0.19 & 0.13 & 0.12 & 0.11 & 0.11 \\
\hline Thorlum & $<0.2$ & $<0.2$ & $<0.2$ & $<0.2$ & $<0.2$ & $<0.2$ & $<0.2$ & $<0.2$ \\
\hline Uranium (Fluor) & 0.071 & 0.072 & 0.067 & 0.082 & 0.002 & 0.002 & 0.002 & 0.003 \\
\hline Vanadium & $<0.005$ & $<0.005$ & $<0.005$ & $<0.005$ & $<0.005$ & $<0.005$ & $<0.005$ & $<0.005$ \\
\hline $2 \ln c$ & 0.0052 & 0.0047 & 0.01 & 0.025 & 0.015 & $<0.002$ & 0.012 & 0.0054 \\
\hline MAJOR IONS (mg/L) & . & $\cdot$ & . &. &. & . & $\cdot$ & $\bullet^{\circ}$ \\
\hline Alkallnity-HCO3 & 169 & $\cdot$ & 173 & $\cdot$ & 251 &. & $250^{\circ}$ & . \\
\hline Alkalinity-CO3 & $<1$ &. & $<1$ &. & $<1$ & . & $<1$ & • \\
\hline Calcium & 56 & 58 & 64 & 64 & 82 & 81 & 76 & 81 \\
\hline Chlorlde & 21.3 & $\cdot$ & 28 & . & 71 & . & 71 & - \\
\hline Fluoride & 0.3 & . & 0.2 & $\cdot$ & 0.1 & . & 0.1 & $\bullet$ \\
\hline Magnesium & 17 & 18 & 18 & 17 & 23 & 23 & 21 & 23 \\
\hline Manganese & 0.0052 & 0.0049 & 0.014 & 0.01 & 0.14 & 0.076 & 0.12 & 0.043 \\
\hline Nitrate-N & 10.4 & $\cdot$ & 19.6 & . & 1.8 & . & 1.9 & $\bullet^{\circ}$ \\
\hline Potassium & 16 & 16 & 14 & 14 & 1.7 & 1.7 & 2.4 & 1.6 \\
\hline Sodium & 10 & 10 & 17 & 17 & 33 & 33 & 27 & 29 \\
\hline Sulfate & 18 & . & $<20$ & . & 20 & . & 21 & • \\
\hline
\end{tabular}

(CONTINUED) 
APPENDIX F.1

Field Duplleate Oroundwater Quality Data, 1993

\begin{tabular}{|c|c|c|c|c|c|c|c|c|}
\hline \multirow{4}{*}{$\begin{array}{l}\text { Sampling Point } \\
\text { Locution } \\
\text { Date sanpled }\end{array}$} & \multicolumn{8}{|c|}{$0 w-704$} \\
\hline & \multicolumn{8}{|c|}{$\mathbf{E x p}$} \\
\hline & \multicolumn{2}{|c|}{$03 / 22 / 93$} & \multicolumn{2}{|c|}{$06 / 04 / 93$} & \multicolumn{2}{|c|}{$09 / 22 / 93$} & \multicolumn{2}{|c|}{$12 / 22 / 93$} \\
\hline & TOT & D18 & ror & DI8 & $\operatorname{tor}$ & DI8 & $\operatorname{ros}$ & DIs \\
\hline $\operatorname{mrnhs}(\mathrm{mg} / \mathrm{x})$ & $\cdot$ & $\cdot$ & $\cdot$ & $\cdot$ & $\cdot$ & $\cdot$ & $\cdot$ & - \\
\hline Aluminum & 0.036 & 0.043 & 0.18 & 0.032 & 0.04 & $<0.02$ & $<0.02$ & $<0.02$ \\
\hline Ant imony & $<0.05$ & $<0.05$ & $<0.05$ & $<0.05$ & $<0.05$ & $<0.05$ & $<0.05$ & $<0.05$ \\
\hline Arsento & $<0.05$ & $<0.05$ & $<0.05$ & $<0.05$ & $<0.05$ & $<0.05$ & $<0.08$ & $<0.08$ \\
\hline Bartum & 0.082 & 0.078 & 0.11 & 0.071 & 0.098 & 0.084 & 0.017 & 0.014 \\
\hline Beryl11 um & $<0.0003$ & $<0.0003$ & $<0.0003$ & $<0.0003$ & $<0.0003$ & $<0.0003$ & $<0.0003$ & $<0.0003$ \\
\hline Boron & 0.078 & 0.087 & 0.089 & 0.083 & 0.086 & 0.083 & 0.097 & 0.14 \\
\hline Cadni un (MAs) & $<0.002$ & $<0.002$ & $<0.002$ & $<0.002$ & $<0.002$ & $<0.002$ & $<0.002$ & $<0.002$ \\
\hline Cadnitun & $<0.003$ & $<0.003$ & $<0.003$ & $<0.003$ & 0.0035 & $<0.003$ & $<0.003$ & $<0.003$ \\
\hline Chromiun (MAs) & $<0.01$ & $<0.01$ & $<0.01$ & $<0.01$ & $<0.01$ & $<0.01$ & $<0.01$ & $<0.01$ \\
\hline Chromium & $<0.02$ & $<0.01$ & $<0.01$ & $<0.01$ & $<0.01$ & $<0.01$ & $<0.01$ & $<0.01$ \\
\hline Cobalt & $<0.005$ & $<0.005$ & $<0.005$ & $<0.005$ & $<0.005$ & $<0.005$ & $<0.005$ & $<0.003$ \\
\hline Coppar & 0.0052 & $<0.004$ & 0.0041 & $<0.004$ & $<0.004$ & $<0.004$ & $<0.004$ & 0.0093 \\
\hline Iron & 2.7 & 0.34 & 23 & 0.039 & 14 & 0.25 & 8.8 & 0.063 \\
\hline Lead (MAS) & $<0.004$ & $<0.004$ & $<0.004$ & $<0.004$ & $<0.004$ & $<0.004$ & $<0.004$ & $<0.004$ \\
\hline Mercury (CVMA) & $<0.0002$ & $<0.0002$ & $<0.0002$ & $<0.0002$ & $<0.0002$ & $<0.0002$ & $<0.0002$ & $<0.0002$ \\
\hline Mol ybdenum & $<0.01$ & $<0.01$ & $<0.01$ & $<0.01$ & $<0.01$ & $<0.01$ & $<0.01$ & $<0.01$ \\
\hline Nicke1 & $<0.01$ & $<0.01$ & 0.015 & $<0.01$ & $<0.01$ & $<0.01$ & $<0.01$ & $<0.01$ \\
\hline selentum & $<0.05$ & $<0.05$ & $<0.05$ & $<0.05$ & $<0,05$ & $<0.05$ & $<0.05$ & $<0.05$ \\
\hline silver & $<0.006$ & $<0.006$ & $<0.006$ & $<0.006$ & $<0.006$ & $<0.006$ & $<0.006$ & $<0.006$ \\
\hline strontium & 0.39 & 0.39 & 0.35 & 0.34 & 0.4 & 0.41 & 0.07 & 0.08 \\
\hline Thorlum & $<0.2$ & $<0.2$ & $<0.2$ & $<0.2$ & $<0.2$ & $<0.2$ & $<0.2$ & $<0.2$ \\
\hline Urantum (Fluor) & 0.001 & 0.001 & 0.001 & 0.001 & 0.002 & 0.001 & $<0.001$ & $<0.001$ \\
\hline Vanadium & $<0.005$ & $<0.005$ & $<0.005$ & $<0.005$ & $<0.005$ & $<0.005$ & $<0.005$ & $<0.005$ \\
\hline zine & 0.013 & 0.0056 & 0.021 & 0.0055 & 0.014 & 0.0037 & $<0.002$ & $<0.002$ \\
\hline MhJOR IOHS $(\mathrm{mg} / \mathrm{L})$ & $\cdot$ & $\cdot$ & $\cdot$ & . &. & . & . & - \\
\hline Alkalinity-HCO3 & 187 & $\cdot$ & 185 & $\cdot$ & $194^{\circ}$ & $\cdot$ & 123 & . \\
\hline Alkalinity- $\mathrm{CO} 3$ & $<1$ & $\cdot$ & $<1$ & . & $<1$ & $\cdot$ & 2 & . \\
\hline Calcium & 63 & 64 & 58 & 56 & 65 & 66 & 15 & 15 \\
\hline Chloride & 19.2 & $\cdot$ & 23 & $\cdot$ & 22 & $\cdot$ & 22.6 & - \\
\hline Fluoride & 0.2 & $\cdot$ & 0.2 & . & 0.2 & $\cdot$ & 0.1 & - \\
\hline Magnesium & 29 & 29 & 28 & 27 & 30 & 30 & 28 & 30 \\
\hline Manganese & 0.07 & 0.663 & 0.11 & 0.042 & 0.095 & 0.045 & 0.15 & 0.091 \\
\hline Nitrate-N & 14.01 & $\cdot$ & 17 & $\cdot$ & 17 & . & 0.23 & • \\
\hline Potaseium & 1.5 & 1.5 & 1.2 & 1.2 & 1.6 & 1.5 & 1.4 & 1.6 \\
\hline Sodium & 11 & 11 & 10 & 10 & 12 & 12 & 11 & 12 \\
\hline sulfare & 20.3 &. & 20 & . & 23 &. & 18.9 & • \\
\hline
\end{tabular}

(CONTINUED) 
APPLHDIX F.1

Field Duplleate Groundwater Quallty Data, 1993

\begin{tabular}{|c|c|c|c|c|c|c|c|c|}
\hline \multirow{4}{*}{$\begin{array}{l}\text { sempling polnt } \\
\text { Location } \\
\text { Date sanpled }\end{array}$} & \multicolumn{4}{|c|}{ on-712 } & \multicolumn{2}{|c|}{$04-713$} & \multicolumn{2}{|c|}{ On-714 } \\
\hline & \multicolumn{4}{|c|}{$\mathbf{8 x p}$} & \multirow{2}{*}{\multicolumn{2}{|c|}{$\frac{\text { Exp }}{07 / 31 / 93}$}} & \multirow{2}{*}{\multicolumn{2}{|c|}{$\frac{\operatorname{sxp}}{11 / 09 / 93}$}} \\
\hline & \multicolumn{2}{|c|}{$03 / 18 / 93$} & \multicolumn{2}{|c|}{$04 / 28 / 93$} & & & & \\
\hline & ror & DIs & Tor & urs & TOT & DI8 & xor & D18 \\
\hline $\operatorname{marazs}(\mathrm{mg} / \mathrm{h})$ & $\cdot$ & $\cdot$ &. & - & $\cdot$ & - & $\cdot$ & $\cdot$ \\
\hline Al uninum & 0.094 & 0.12 & $<0.02$ & $<0.02$ & $<0.02$ & $<0.02$ & 0.068 & $<0.02$ \\
\hline Antimony & $<0.05$ & $<0.05$ & $<0.05$ & $<0.03$ & $<0.05$ & $<0.05$ & $<0.05$ & $<0.05$ \\
\hline Arsento & $<0.05$ & $<0.05$ & $<0.05$ & $<0.05$ & $<0.05$ & $<0.08$ & $<0.08$ & $<0.05$ \\
\hline Derlun & 0.067 & 0.068 & 0.063 & 0.063 & 0.045 & 0.045 & 0.059 & 0.057 \\
\hline Bery111um & $<0.0003$ & $<0.0003$ & $<0.0003$ & $<0.0003$ & $<0.0003$ & $<0.0003$ & $<0.0003$ & $<0.0003$ \\
\hline Doron & 0.15 & 0.14 & 0.11 & 0.11 & 0.075 & 0.073 & 0.099 & 0.11 \\
\hline Cadalun (MAs) & $<0.002$ & $<0.002$ & $<0.002$ & $<0.002$ & $<0.002$ & $<0.002$ & $<0.002$ & $<0.002$ \\
\hline Cadnitun & $<0.003$ & $<0,003$ & $<0.003$ & $<0.003$ & $<0.003$ & $<0.003$ & $<0.003$ & $<0.003$ \\
\hline Chromium (MAs) & $<0.01$ & $<0.01$ & $<0.01$ & $<0.01$ & $<0.01$ & $<0.01$ & $<0.01$ & $<0.01$ \\
\hline Chromiun & $<0.01$ & $<0.01$ & $<0.01$ & $<0.01$ & $<0.01$ & $<0.01$ & $<0.01$ & $<0.01$ \\
\hline Cobalt & $<0.005$ & $<0.00 s$ & $<0.005$ & $<0.005$ & $<0.005$ & $<0.005$ & $<0.005$ & $<0.005$ \\
\hline Copper & 0.016 & $<0.004$ & $<0.004$ & $<0.004$ & 0.0051 & $<0.004$ & $<0.004$ & $<0.004$ \\
\hline Iron & 1.3 & 1.1 & 0.98 & 0.89 & 2.3 & 2.9 & 6 & 0.59 \\
\hline Lead (MS) & $<0.004$ & $<0.004$ & $<0.004$ & $<0.004$ & $<0.004$ & $<0.004$ & $<0.004$ & $<0.004$ \\
\hline Mercury (CVMA) & 0.00032 & $<0.0002$ & $<0.0002$ & $<0.0002$ & $<0.0002$ & $<0.0002$ & $<0.0002$ & $<0.0002$ \\
\hline Nolybdenum & $<0.01$ & $<0.01$ & $<0.01$ & $<0.01$ & $<0.01$ & $<0.01$ & $<0.01$ & $<0.01$ \\
\hline mlokel & $<0.01$ & $<0.01$ & $<0.01$ & $<0.01$ & $<0.01$ & $<0.01$ & $<0.01$ & $<0.01$ \\
\hline selentum & $<0.05$ & $<0.05$ & $<0.05$ & $<0.05$ & $<0.05$ & $<0.05$ & $<0.08$ & $<0.0 s$ \\
\hline S11ver & $<0.006$ & $<0.006$ & $<0.006$ & $<0.006$ & $<0.006$ & $<0.006$ & $<0.006$ & $<0.006$ \\
\hline strontium & 2.4 & 2.3 & 2.2 & 2.2 & 1.7 & 1.7 & 0.17 & 0.17 \\
\hline Thorlum & $<0.2$ & $<0.2$ & $<0,2$ & $<0.2$ & $<0.2$ & $<0.2$ & $<0.2$ & $<0.2$ \\
\hline Uranium (Fluor) & $<0.001$ & 0.001 & $<0.001$ & $<0.001$ & $<0.001$ & $<0.001$ & 0.002 & 0.003 \\
\hline Vanadium & $<0.003$ & $<0.00 s$ & $<0.005$ & $<0.005$ & $<0.005$ & $<0.005$ & $<0.005$ & $<0.005$ \\
\hline Zine & 0.012 & 0.0066 & 0.0088 & 0.0073 & 0.012 & 0.0087 & 0.0068 & 0.0038 \\
\hline MUJOR IONS (mg/L) & $\cdot$ & $\cdot$ & . & $\cdot$ & $\cdot$ & $\cdot$ & . & - \\
\hline Alkalinity-HCO3 & $17 \dot{9}$ & . & 176 & $\cdot$ & 185 & $\cdot$ & 188 & . \\
\hline Alkalinity- $\mathrm{CO}$ & $<1$ & . & $<1$ &. & $<1$ & $\cdot$ & $<1$ & . \\
\hline Calcium & 96 & 99 & 89 & 88 & 69 & 70 & 49 & 49 \\
\hline Chloride & 34 & • & 28 & $\cdot$ & 14.1 & $\cdot$ & 10 & . \\
\hline Fluoride & 0.7 & • & 0.7 & $\cdot$ & 0.7 &. & 0.2 & . \\
\hline Magnesium & 42 & 43 & 41 & 41 & 32 & 32 & 22 & 22 \\
\hline Manganese & 0.017 & 0.017 & 0.011 & 0.014 & 0.024 & 0.023 & 0.11 & 0.084 \\
\hline Nitrate-N & $<0.2$ & • & 0.24 & . & $<0.2$ & . & 3.2 & - \\
\hline Potanes i um & 2.9 & 3 & 3.5 & 2.9 & 2.6 & 2.4 & 1.1 & 1.4 \\
\hline sodlum & 28 & 29 & 27 & 27 & 16 & 16 & 4.1 & 1.2 \\
\hline sulfate & 308 & . & 247 & . & 124 & . & 22 & - \\
\hline
\end{tabular}

(CONTINURD) 
APpEHDIX $\mathrm{r}, 1$

rleld Duplleate Groundwater Quallty Date, 1993

\begin{tabular}{|c|c|c|c|c|c|c|c|c|}
\hline \multirow{4}{*}{$\begin{array}{l}\text { Bempling polnt } \\
\text { Loostion } \\
\text { Dete sampled }\end{array}$} & \multicolumn{4}{|c|}{ OW-725 } & \multicolumn{2}{|c|}{ ON-736 } & \multicolumn{2}{|c|}{ Cw-740 } \\
\hline & \multicolumn{4}{|c|}{ exp } & \multirow{2}{*}{\multicolumn{2}{|c|}{$\frac{\exp }{12 / 15 / 93}$}} & \multirow{2}{*}{\multicolumn{2}{|c|}{$\frac{\text { Exp }}{09 / 27 / 93}$}} \\
\hline & \multicolumn{2}{|c|}{$02 / 12 / 93$} & \multicolumn{2}{|c|}{$05 / 19 / 93$} & & & & \\
\hline & $\operatorname{sor}$ & DI8 & Tor & DIs & 20T & Drs & TOT & DIs \\
\hline narnse $(\mathrm{mg} / \mathrm{L})$ & $\cdot$ & - & $\cdot$ & - & - & - & $\cdot$ & - \\
\hline Aluminum & 0.059 & $<0.02$ & 0.058 & 0.053 & 0.029 & 0.022 & 0.027 & $<0.02$ \\
\hline Ant imony & $<0.05$ & $<0.05$ & $<0.05$ & $<0.05$ & $<0.05$ & $<0.03$ & $<0.08$ & $<0.05$ \\
\hline Armenic & $<0.05$ & $<0.05$ & $<0.05$ & $<0.05$ & $<0.05$ & $<0.05$ & $<0.05$ & $<0.05$ \\
\hline Bartum & 0.29 & 0.29 & 0.29 & 0.28 & 0.22 & 0.23 & 0.092 & 0.091 \\
\hline Beryl11um & $<0.0003$ & $<0.0003$ & $<0.0003$ & $<0.0003$ & $<0.0003$ & $<0.0003$ & 0.00033 & 0.00032 \\
\hline Boron & 0.045 & 0.061 & 0.051 & 0.072 & 0.037 & 0.048 & 0.033 & 0.033 \\
\hline Cadmi un (MS) & $<0.002$ & $<0.002$ & $<0.002$ & $<0.002$ & $<0.002$ & $<0.002$ & $<0.002$ & $<0.002$ \\
\hline Cadmlun & $<0.003$ & $<0.003$ & $<0.003$ & $<0.003$ & $<0.003$ & $<0.003$ & $<0.003$ & $<0.003$ \\
\hline Chrondum (MAs) & $<0.01$ & $<0.01$ & $<0.01$ & $<0.01$ & $<0.01$ & $<0.01$ & $<0.01$ & $<0.01$ \\
\hline Chronitum & $<0.01$ & $<0.01$ & $<0.01$ & $<0.01$ & $<0.01$ & $<0.01$ & $<0.01$ & $<0.01$ \\
\hline Cobalt & $<0.005$ & $<0.005$ & $<0.005$ & $<0.005$ & $<0.005$ & $<0.005$ & $<0.005$ & $<0.005$ \\
\hline Copper & $<0.004$ & $<0.004$ & $<0.004$ & $<0.004$ & $<0.004$ & $<0.004$ & $<0.004$ & $<0.004$ \\
\hline Iron & 1.7 & 0.51 & 0.091 & 0.041 & $<0.005$ & $<0.00 s$ & 0.19 & 0.092 \\
\hline Lead (MAs) & $<0.004$ & $<0.004$ & $<0.004$ & $<0.004$ & $<0.004$ & $<0.004$ & $<0.004$ & $<0.004$ \\
\hline (nercury (CVMA) & $<0.0002$ & $<0.0002$ & $<0.0002$ & $<0.0002$ & $<0.0002$ & $<0.0002$ & $<0.0002$ & $<0.0002$ \\
\hline Mol ybdenum & $<0.01$ & $<0.01$ & $<0.01$ & $<0.01$ & $<0.01$ & $<0.01$ & $<0.01$ & $<0.01$ \\
\hline Nickel & $<0.01$ & $<0.01$ & $<0.01$ & $<0.01$ & $<0.01$ & $<0.01$ & $<0.01$ & $<0.01$ \\
\hline Selenium & $<0.05$ & $<0.05$ & $<0.05$ & $<0.05$ & $<0.05$ & $<0.05$ & $<0.05$ & $<0.05$ \\
\hline 811ver & $<0.006$ & $<0.006$ & $<0.006$ & $<0.006$ & $<0.006$ & $<0.006$ & $<0.006$ & $<0.006$ \\
\hline strontium & 0.51 & 0.52 & 0.58 & 0.57 & 0.34 & 0.35 & 0.055 & 0.054 \\
\hline Thor ium & $<0.2$ & $<0.2$ & $<0.2$ & $<0.2$ & $<0,2$ & $<0.2$ & $<0.2$ & $<0.2$ \\
\hline Uranium (rluor) & 0.004 & 0.004 & 0.004 & 0.003 & 0.008 & 0.01 & $<0.001$ & $<0.001$ \\
\hline Vanadiun & $<0.005$ & $<0.005$ & $<0.005$ & $<0.005$ & $<0.005$ & $<0.005$ & $<0.005$ & $<0.005$ \\
\hline inc & 0.011 & 0.0025 & 0.017 & 0.0066 & $<0.002$ & $<0.002$ & 0.0074 & 0.0081 \\
\hline MuJOR IONS $(\mathrm{mg} / \mathrm{L})$ & $\cdot$ & $\cdot$ & . & . &. & $\cdot$ & - & - \\
\hline Alkadinity-HCO3 & 290 &. & 285 &. & 314 & $\cdot$ & 259 & . \\
\hline Alkalinity-CO3 & $<1$ & $\cdot$ & $<1$ & . & $<1$ & . & $<1$ & - \\
\hline Calcium & 170 & 170 & 180 & 170 & 170 & 170 & 66 & 66 \\
\hline Chloride & 74 & $\cdot$ & 31 & $\cdot$ & 54 & $\cdot$ & 7.1 & - \\
\hline Pluoride & 0.1 & $\cdot$ & 0.1 & $\cdot$ & 0.2 & $\cdot$ & 0.1 & - \\
\hline Magnasiun & 27 & 27 & 31 & 31 & 24 & 24 & 32 & 31 \\
\hline Manganese & 0.062 & 0.055 & 0.03 & 0.03 & 0.94 & 0.92 & 0.006 & 0.006 \\
\hline Nitrate-n & 58 & $\cdot$ & 62 & . & 42.3 & $\cdot$ & 4.9 & - \\
\hline Potaseivm & 3.1 & 2.7 & 2.7 & 2.5 & 3.7 & 3.1 & 1.4 & 1.4 \\
\hline Sodium & 18 & 17 & 17 & 17 & 23 & 24 & 2.1 & 2.1 \\
\hline sulfate & 61 & $\cdot$ & 36 & $\cdot$ & 31 & $\cdot$ & 11.9 & - \\
\hline
\end{tabular}

(CONT INUED) 


\begin{tabular}{|c|c|c|}
\hline \multirow{4}{*}{$\begin{array}{l}\text { sampling point } \\
\text { Looation } \\
\text { Date sampled }\end{array}$} & \multicolumn{2}{|c|}{$9 n-800$} \\
\hline & \multicolumn{2}{|c|}{ orf } \\
\hline & \multicolumn{2}{|c|}{$10 / 22 / 93$} \\
\hline & nor & Drs \\
\hline MrnLs (mg/L) & $\cdot$ & - \\
\hline Aluminum & 0.33 & $<0.02$ \\
\hline Antimony & $<0.05$ & $<0.05$ \\
\hline Araenic & $<0.08$ & $<0.05$ \\
\hline Barlum & 0.13 & 0.13 \\
\hline Dory111um & $<0.0003$ & $<0.0003$ \\
\hline Doron & 0.021 & 0.02 \\
\hline Cadmium (MA8) & $<0.002$ & $<0.002$ \\
\hline Cadmium & $<0.003$ & $<0.003$ \\
\hline Chromdun (AAs) & $<0.01$ & $<0.01$ \\
\hline Chromium & $<0.01$ & $<0.01$ \\
\hline Cobalt & $<0.005$ & $<0.005$ \\
\hline Copper & $<0.004$ & $<0.004$ \\
\hline Iron & 0.46 & $<0.005$ \\
\hline Lead (MAs) & $<0.004$ & $<0.004$ \\
\hline Mercury (CVMA) & $<0.0002$ & $<0.0002$ \\
\hline Molybdanum & $<0.01$ & $<0.01$ \\
\hline Hlokel & $<0.01$ & $<0.01$ \\
\hline selentum & $<0.05$ & $<0.05$ \\
\hline 811ver & $<0.006$ & $<0.006$ \\
\hline strontium & 0.18 & 0.17 \\
\hline Thorium & $<0.2$ & $<0.2$ \\
\hline Uranium (Fluor) & 0.001 & 0.001 \\
\hline Vanadium & $<0.005$ & $<0.005$ \\
\hline 21 nc & 0.022 & 0.017 \\
\hline MWOR IONS (mg/L) & $\cdot$ & - \\
\hline Alkalinity-HCO3 & 237 & 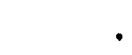 \\
\hline Alkalinity-CO3 & $<1$ & - \\
\hline Calcium & 61 & 57 \\
\hline Chloride & 2 & $\theta^{\circ}$ \\
\hline Pluoride & 0.1 & $\bullet$ \\
\hline Magnesium & 24 & 23 \\
\hline Manganese & 0.047 & $<0.001$ \\
\hline Nitrate-n & $<0.2$ & $\bullet$ \\
\hline Potaseium & 2.3 & 2.6 \\
\hline Sodium & 0.9 & 0.88 \\
\hline sulfate & 16 & $\bullet$ \\
\hline
\end{tabular}


APPENDIX F.2

VOLATILE ORGANIC COMPOUNDS 
APPENDIX F. 2

Field Duplicate Data, 1993

\begin{tabular}{|c|c|c|c|c|c|c|c|c|}
\hline \multirow{3}{*}{$\begin{array}{l}\text { Sampling Point } \\
\text { Location } \\
\text { - } \\
\text { Date Sampled }\end{array}$} & \multirow{3}{*}{\begin{tabular}{|c|} 
GW-044 \\
OLF \\
$09 / 11 / 93$ \\
\end{tabular}} & \multirow{3}{*}{\begin{tabular}{c|}
$G W-069$ \\
$B G$ \\
$10 / 10 / 93$ \\
\end{tabular}} & \multicolumn{2}{|c|}{ GW-115 } & \multirow{3}{*}{\begin{tabular}{|c|} 
GW-315 \\
SPI \\
$10 / 27 / 93$ \\
\end{tabular}} & \multirow{3}{*}{\begin{tabular}{|c|} 
GW-324 \\
s3 \\
$02 / 29 / 93$ \\
\end{tabular}} & \multirow{3}{*}{\begin{tabular}{|c|} 
GW-371 \\
$B G$ \\
$10 / 13 / 93$ \\
\end{tabular}} & \multirow{3}{*}{$\begin{array}{c}\text { GW-373 } \\
B G \\
02 / 11 / 93\end{array}$} \\
\hline & & & \multicolumn{2}{|c|}{ s3 } & & & & \\
\hline & & & $05 / 25 / 93$ & $09 / 11 / 93$ & & & & \\
\hline VOLATILE ORGANICS (ug/I) & - & - & $\cdot$ & $\cdot$ & - & • & - & - \\
\hline Acetone & 100 & $10 \mathrm{U}$ & 100 & 100 & 2 & 100 & 100 & 100 \\
\hline Benzene & 50 & $5 \mathbf{u}$ & 5u & 50 & 50 & $5 u$ & $5 v$ & 50 \\
\hline Bromodichloromethane & 50 & $5 v$ & 5u & 5u & 1 & 50 & $5 \mathbf{v}$ & 50 \\
\hline Bromoform & 5v & $5 \mathbf{v}$ & $5 \mathbf{v}$ & 50 & $5 \mathbf{0}$ & 50 & $5 \mathbf{U}$ & 50 \\
\hline Bromomethane & 100 & 100 & 100 & 100 & $10 \mathrm{U}$ & 100 & 100 & 100 \\
\hline 2-Butanone & 100 & 100 & 100 & 100 & 6 & 100 & 100 & 100 \\
\hline Carbon disulfide & $5 \mathbf{u}$ & $5 \mathbf{u}$ & $5 \mathbf{v}$ & 50 & 50 & 50 & $5 \mathbf{v}$ & 50 \\
\hline Carbon tetrachloride & 50 & $5 v$ & $5 \mathbf{5 u}$ & 50 & 50 & $5 \mathbf{v}$ & $5 \mathbf{u}$ & 5u \\
\hline Chlorobenzene & 50 & $5 u$ & $5 \mathbf{v}$ & 50 & 5u & 5u & $5 \mathbf{u}$ & su \\
\hline Chlorodibromomethane & 50 & $5 \mathbf{u}$ & $5 \mathbf{v}$ & $5 \mathbf{u}$ & 5u & $5 \mathbf{U}$ & $5 \mathbf{v}$ & 5u \\
\hline Chloroethane & 100 & 100 & 100 & 100 & $10 \mathrm{v}$ & 100 & 100 & 100 \\
\hline Chloroform & 50 & $5 \mathbf{u}$ & $5 \mathbf{v}$ & $5 \mathbf{u}$ & 6 & 50 & $5 \mathbf{v}$ & 50 \\
\hline Chloromethane & 100 & 100 & 100 & 100 & 200 & 100 & 100 & 100 \\
\hline 1,1-Dichloroethane & 5u & $5 U$ & 5v & $5 \mathbf{v}$ & 5u & $5 \mathbf{v}$ & $5 \mathbf{u}$ & su \\
\hline 1,2-Dichloroethane & 50 & 5u & 5v & 50 & 50 & 50 & $5 \mathbf{u}$ & 50 \\
\hline 1,1-Dichloroethene & $5 v$ & $5 \mathbf{v}$ & 5U & 50 & $5 \mathbf{v}$ & 50 & $5 \mathbf{u}$ & 50 \\
\hline 1,2-Dichloroethene & $5 \mathbf{u}$ & su & 5v & 50 & 5 & 50 & 50 & 50 \\
\hline 1,2-Dichloropropane & $5 u$ & $5 v$ & 50 & $5 \mathbf{v}$ & 50 & $5 \mathbf{v}$ & $5 \mathbf{u}$ & 5U \\
\hline cis-1,3-Dichloropropene & $5 \mathbf{u}$ & 50 & 5u & 5u & 50 & $5 v$ & $5 \mathbf{u}$ & 50 \\
\hline trans-1,3-Dichloropropene & 50 & 50 & 5v & 50 & 50 & 50 & $5 \mathbf{v}$ & $\mathbf{5 0}$ \\
\hline Ethylbenzene & 50 & $5 \mathbf{u}$ & 50 & 5u & 5u & 50 & 5U & 50 \\
\hline 2-Hexanone & 100 & 100 & 100 & 100 & 100 & 100 & 100 & 100 \\
\hline 4-Methy1-2-pentanone & 100 & 100 & 100 & 100 & 100 & 100 & $10 \mathrm{U}$ & 2 \\
\hline Methylene chloride & 1 & $5 \mathbf{u}$ & 50 & 1 & 2 & $\mathbf{5 u}$ & $5 U$ & 2 \\
\hline styrene & 5u & 50 & 5u & $\therefore 50$ & $5 \mathbf{u}$ & $5 \mathbf{v}$ & $5 \mathbf{u}$ & $5 \mathbf{v}$ \\
\hline $1,1,2,2$-Tetrachloroethane & $5 \mathbf{v}$ & 5v & $5 \mathbf{u}$ & $5 \mathbf{v}$ & $5 \mathbf{v}$ & 50 & $5 \mathbf{u}$ & $5 \mathbf{U}$ \\
\hline Tetrachloroethene & 50 & 50 & $5 U$ & 50 & 8 & $5 \mathbf{U}$ & $5 v$ & $5 \mathbf{v}$ \\
\hline Toluene & 50 & $5 \mathrm{U}$ & 50 & 50 & 50 & $5 \mathbf{v}$ & $5 \mathbf{u}$ & 50 \\
\hline 1,1,1-Trichloroethane & $5 \mathrm{v}$ & $5 \mathbf{U}$ & 50 & 50 & $5 \mathbf{u}$ & 50 & 50 & $5 \mathbf{U}$ \\
\hline 1,1,2-Trichloroethane & 50 & $5 u$ & 50 & 50 & $5 \mathrm{U}$ & 5u & $5 \mathbf{u}$ & $5 \mathbf{U}$ \\
\hline Trichloroethene & 50 & $5 u$ & 50 & $5 \mathbf{0}$ & 4 & $5 \mathbf{U}$ & 5u & $5 \mathbf{U}$ \\
\hline Vinyl acetate & $10 \mathrm{U}$ & $10 \mathrm{U}$ & 100 & 100 & 100 & 100 & 100 & 100 \\
\hline Vinyl chloride & 100 & $10 \mathrm{U}$ & 100 & 100 & 100 & 100 & 100 & 200 \\
\hline xylenes & 50 & $5 u$ & $5 v$ & $5 v$ & 50 & 50 & 5u & Su \\
\hline
\end{tabular}

(CONTINUED) 
APPENDIX F. 2

Field Duplicate Data, 1993

\begin{tabular}{|c|c|c|c|c|c|c|c|c|}
\hline \multirow{3}{*}{$\begin{array}{l}\text { Sampling Point } \\
\text { Location } \\
\text { Date Sampled }\end{array}$} & \multicolumn{2}{|c|}{ GW-537 } & \multicolumn{2}{|c|}{$G W-626$} & \multirow{3}{*}{\begin{tabular}{|c|} 
GW-627 \\
BG \\
$04 / 23 / 93$
\end{tabular}} & \multicolumn{3}{|c|}{ GW-636 } \\
\hline & \multicolumn{2}{|c|}{ OLF } & \multicolumn{2}{|c|}{ BG } & & \multicolumn{3}{|c|}{ OLF } \\
\hline & $02 / 11 / 93$ & $05 / 12 / 93$ & $03 / 10 / 93$ & $08 / 02 / 93$ & & $03 / 26 / 93$ & $06 / 27 / 93$ & $09 / 18 / 93$ \\
\hline VOLATILE ORGANICS (ug/L) & $\cdot$ & $\cdot$ & $\cdot$ & $\cdot$ & $\cdot$ & . & $\cdot$ & $\cdot$ \\
\hline Acetone & 100 & 100 & 1000 & 200 & 100 & 100 & 100 & 100 \\
\hline Benzene & 5u & 50 & 500 & 100 & $5 u$ & 50 & $5 u$ & 5u \\
\hline Bromodichloromethane & 5u & su & 500 & 100 & 50 & 50 & 5u & 50 \\
\hline Bromoform & 50 & 50 & 500 & 100 & 5u & 50 & $5 u$ & 5u \\
\hline Bromomethane & 100 & 100 & 1000 & 200 & 100 & 100 & 100 & 100 \\
\hline 2-Butanone & 100 & 100 & 1000 & 200 & 100 & 100 & 100 & 100 \\
\hline Carbon disulfide & 50 & $5 \mathrm{u}$ & 500 & 100 & 5u & 50 & 5u & 5u \\
\hline Carbon tetrachloride & 50 & $5 u$ & 500 & 100 & 50 & 5u & 50 & 5u \\
\hline Chlorobenzene & 50 & 50 & 500 & 100 & 50 & 5v & 50 & 5u \\
\hline Chlorodibromomethane & su & 50 & 500 & 100 & 5u & 50 & su & 50 \\
\hline Chloroethane & 100 & 100 & 1000 & 200 & 100 & 100 & 100 & 100 \\
\hline Chloroform & 1 & 0.8 & 500 & 100 & 50 & 5u & $5 \mathbf{u}$ & 50 \\
\hline Chloromethane & 100 & 100 & 1000 & 200 & 100 & 100 & 100 & 100 \\
\hline 1,1-Dichloroethane & 50 & 50 & 51 & 19 & 5 & 5u & $5 \mathbf{U}$ & 5U \\
\hline 1,2-Dichloroethane & 50 & $5 \mathbf{v}$ & 500 & 100 & $5 u$ & $5 \mathbf{U}$ & $5 u$ & su \\
\hline 1,1-Dichloroethene & 50 & 50 & 28 & 8 & 5u & 5U & 5u & 5U \\
\hline 1,2-Dichloroethene & 50 & 50 & 1300 & 240 & 5u & 5u & 5u & 5u \\
\hline 1,2-Dichloropropane & 50 & 50 & 500 & 100 & 5v & 5u & 5u & 50 \\
\hline cis-1,3-Dichloropropene & 50 & $5 v$ & 500 & 100 & 5u & 50 & su & $5 U$ \\
\hline trans-1,3-Dichloropropene & 50 & 50 & 500 & 100 & 5u & 5u & 5u & 5U \\
\hline Ethylbenzene & 50 & 50 & 500 & 100 & 50 & $5 \mathbf{v}$ & 50 & $5 \mathbf{U}$ \\
\hline 2-Hexanone & 100 & 100 & 1000 & 200 & 100 & 100 & 100 & 100 \\
\hline 4-Methy1-2-pentanone & 100 & 100 & 1000 & 200 & 100 & $10 U$ & $10 u$ & 100 \\
\hline Methylene chloride & 2 & 1 & 14 & 10.0 & 50 & 50 & 50 & 0.8 \\
\hline Styrene & 50 & 50 & $50 u$ & $10 \mathrm{U}$ & 5u & $5 U$ & 5u & $5 \mathbf{v}$ \\
\hline $1,1,2,2$-Tetrachloroethane & 50 & 50 & 500 & 100 & 50 & $5 U$ & $5 \mathrm{u}$ & $5 U$ \\
\hline Tetrachloroethene & 50 & 5u & 130 & 28 & 52 & 5u & 50 & $5 U$ \\
\hline Toluene & $5 \mathrm{v}$ & $5 \mathrm{U}$ & $50 U$ & 100 & 5u & $5 U$ & 50 & 50 \\
\hline 1,1,1-Trichloroethane & $5 \mathrm{U}$ & 50 & 17 & 6 & 50 & 50 & $5 \mathrm{U}$ & $5 U$ \\
\hline 1,1,2-Trichloroethane & 50 & 50 & 500 & 100 & $5 v$ & $5 U$ & 50 & 50 \\
\hline Trichloroethene & 50 & 50 & 120 & 33 & 7 & $5 U$ & 50 & 50 \\
\hline Vinyl acetate & 100 & 100 & 1000 & 200 & 100 & 100 & 100 & 100 \\
\hline Vinyl chloride & 100 & 100 & 71 & 200 & 100 & 100 & 100 & 100 \\
\hline xylenes & 50 & $5 \mathrm{U}$ & 500 & 100 & $5 U$ & 50 & $5 U$ & 50 \\
\hline
\end{tabular}

(CONTINUED) 
APPENDIX F.2

Field Duplicate Data, 1993

\begin{tabular}{|c|c|c|c|c|c|c|c|c|}
\hline \multirow{3}{*}{$\begin{array}{l}\text { Sampling Point } \\
\text { Location } \\
\text { Date Sampled }\end{array}$} & \multirow{3}{*}{\begin{tabular}{|c|} 
GW-636 \\
OLF \\
$12 / 18 / 93$ \\
\end{tabular}} & \multicolumn{3}{|c|}{ GW-639 } & \multicolumn{2}{|c|}{$G W-684$} & \multicolumn{2}{|c|}{ GW-685 } \\
\hline & & \multicolumn{3}{|c|}{ BG } & \multicolumn{2}{|c|}{$\operatorname{Exp}$} & \multicolumn{2}{|c|}{$\mathbf{E X P}$} \\
\hline & & $02 / 24 / 93$ & $05 / 24 / 93$ & $07 / 11 / 93$ & $07 / 11 / 93$ & $10 / 12 / 93$ & $03 / 02 / 93$ & $04 / 21 / 93$ \\
\hline VOLATILE ORGANICS (ug/L) & $\cdot$ & $\cdot$ & $\cdot$ & $\cdot$ & $\cdot$ & $\cdot$ & $\cdot$ & $\cdot$ \\
\hline Acetone & 100 & 100 & 100 & 100 & 100 & 3 & 100 & $10 \dot{0}$ \\
\hline Benzene & $5 v$ & 50 & 50 & 50 & 50 & $5 v$ & su & su \\
\hline Bromodichloromethane & $5 u$ & 5u & 5u & $5 v$ & 50 & 50 & 50 & $5 v$ \\
\hline Bromoform & 50 & 50 & 50 & 50 & 5u & 50 & 5u & 50 \\
\hline Bromomethane & 100 & 100 & 100 & 100 & 100 & 100 & 100 & 100 \\
\hline 2-Butanone & 8 & 100 & 5 & 100 & 100 & 9 & 100 & 100 \\
\hline Carbon disulfide & 50 & 5u & 5u & 5u & 5u & 50 & su & su \\
\hline Carbon tetrachloride & 5u & 50 & 50 & 50 & 50 & 50 & 5u & $5 U$ \\
\hline Chlorobenzene & 5u & 50 & 5u & 5u & 5u & $5 u$ & 5u & 50 \\
\hline Chlorodibromomethane & 50 & 50 & 50 & $5 u$ & 5u & 50 & 5u & $5 v$ \\
\hline Chloroethane & 100 & 100 & 100 & 100 & 100 & 100 & 100 & 100 \\
\hline Chloroform & 50 & 5u & $5 u$ & 5u & 50 & 50 & 50 & 5u \\
\hline Chloromethane & 100 & 100 & 100 & 100 & 100 & 100 & 100 & 100 \\
\hline 1,1-Dichloroethane & 50 & 5u & 50 & su & 50 & 5u & su & 50 \\
\hline 1,2-Dichloroethane & 50 & $5 u$ & 5u & 50 & 50 & 50 & 5u & 50 \\
\hline 1,1-Dichloroethene & su & su & su & $5 v$ & 50 & 5u & 5u & 5u \\
\hline 1,2-Dichloroethene & su & su & 50 & 5u & 50 & 5u & 2 & 1 \\
\hline 1,2-Dichloropropane & 5u & 5u & su & 5u & 5u & $5 u$ & $5 u$ & 5u \\
\hline cis-1,3-Dichloropropene & 50 & 5u & su & 50 & $5 \mathrm{u}$ & 50 & $5 u$ & Su \\
\hline trans-1,3-Dichloropropene & 50 & 50 & su & 5u & 50 & 50 & 50 & su \\
\hline Ethylbenzene & 5u & 50 & $5 \mathrm{v}$ & 5u & 50 & $5 \mathbf{u}$ & 50 & 5u \\
\hline 2-Hexanone & 100 & 100 & 100 & 100 & 100 & 100 & 100 & 100 \\
\hline 4-Methy1-2-pentanone & 100 & 100 & 1 & 100 & 100 & 100 & 100 & 100 \\
\hline Methylene chloride & 0.6 & 2 & 5v & $5 u$ & 0.8 & 3 & 5u & 50 \\
\hline Styrene & 50 & $5 u$ & 50 & 5u & 50 & 50 & 5u & 50 \\
\hline $1,1,2,2$-Tetrachloroethane & 50 & 50 & 5u & 5u & 5u & 5u & 50 & 50 \\
\hline Tetrachloroethene & 5u & 50 & 50 & 50 & 50 & $5 v$ & su & 5u \\
\hline Toluene & 50 & su & $5 \mathrm{u}$ & 5u & 5u & 5u & 50 & $5 u$ \\
\hline $1,1,1$-Trichloroethane & $5 U$ & su & 50 & 5u & $5 v$ & 50 & 5u & 5u \\
\hline 1,1,2-Trichloroethane & 50 & su & 50 & 5u & 50 & 5u & 5u & 5u \\
\hline Trichloroethene & 50 & so & su & 5u & 50 & 5u & 5u & 50 \\
\hline Vinyl acetate & 100 & 100 & 100 & 100 & 100 & 100 & 100 & 100 \\
\hline vinyl chloride & 100 & 100 & 100 & 100 & 100 & 100 & 100 & 100 \\
\hline Xylenes & $5 \mathrm{v}$ & su & 50 & 50 & $5 \mathrm{u}$ & 50 & su & $5 U$ \\
\hline
\end{tabular}

(CONTINUED) 
APPENDIX $\$ .2$

Field Duplicate Data, 1993

\begin{tabular}{|c|c|c|c|c|c|c|c|c|}
\hline \multirow{3}{*}{$\begin{array}{l}\text { Sampling point } \\
\text { - } \\
\text { Location } \\
\text { Date Sampled }\end{array}$} & \multicolumn{4}{|c|}{ GW-704 } & \multicolumn{2}{|c|}{$G W-712$} & \multirow{3}{*}{\begin{tabular}{|c|} 
GW-713 \\
BXP \\
$07 / 31 / 93$ \\
\end{tabular}} & \multirow{3}{*}{\begin{tabular}{|c|} 
GW-714 \\
EXP \\
$11 / 09 / 93$ \\
\end{tabular}} \\
\hline & \multicolumn{4}{|c|}{ EXP } & \multicolumn{2}{|c|}{ Exp } & & \\
\hline & $03 / 22 / 93$ & $06 / 04 / 93$ & $09 / 22 / 93$ & $12 / 22 / 93$ & $03 / 18 / 93$ & $04 / 28 / 93$ & & \\
\hline VOLATILE ORGANICS (ug/L) & - & - & - & - & - & - & $\cdot$ & - \\
\hline Acetone & 100 & 100 & 100 & 4 & 100 & $10 U$ & 100 & $10 \mathrm{U}$ \\
\hline Benzene & $5 \mathbf{u}$ & 50 & 50 & 5u & $5 \mathbf{u}$ & $5 \mathbf{v}$ & $5 \mathbf{v u}$ & $\mathbf{5 u}$ \\
\hline Bromodichloromethane & $5 \mathbf{v}$ & $5 v$ & $5 \mathbf{v}$ & su & $5 \mathbf{v}$ & $5 \mathbf{v}$ & $\mathbf{5 u}$ & 5u \\
\hline Bromoform & 5u & 50 & $5 \mathbf{v}$ & 5v & $5 u$ & $5 v$ & $5 \mathbf{v}$ & $5 U$ \\
\hline Bromomethane & 100 & 100 & 100 & $10 \mathrm{U}$ & 100 & 100 & 100 & 100 \\
\hline 2-Butanone & 100 & $10 \mathrm{U}$ & 100 & 3 & 100 & 100 & 100 & 100 \\
\hline Carbon disulfide & 50 & 50 & 50 & Su & $\mathbf{5 u}$ & $5 \mathbf{u}$ & $5 \mathbf{u}$ & 5u \\
\hline Carbon tetrachloride & 2 & 2 & 50 & 50 & $5 \mathbf{v}$ & $5 u$ & su & 5u \\
\hline Chlorobenzene & $5 \mathbf{u}$ & $5 v$ & $5 v$ & $5 \mathbf{u}$ & $5 u$ & $5 \mathbf{u}$ & $5 \mathbf{U}$ & $5 \mathbf{v}$ \\
\hline Chlorodibromomethane & 50 & $5 \mathbf{u}$ & 5u & $5 v$ & $5 \mathbf{u}$ & 50 & $\mathbf{5 u}$ & 50 \\
\hline Chloroethane & 100 & 100 & 100 & 100 & 100 & 100 & 100 & 100 \\
\hline Chloroform & $\mathbf{5 u}$ & 0.5 & $5 v$ & $5 \mathbf{v}$ & su & $5 \mathbf{v}$ & $5 \mathbf{v}$ & 5u \\
\hline Chloromethane & 100 & $10 \mathrm{U}$ & 100 & 100 & $10 \mathrm{U}$ & 100 & 100 & 100 \\
\hline 1,1-Dichloroethane & 1 & $5 \mathrm{U}$ & $5 \mathbf{U}$ & $\mathbf{s u}$ & $5 v$ & 5u & su & $\mathbf{5 u}$ \\
\hline 1,2-Dichloroethane & $5 \mathbf{u}$ & $5 \mathbf{v}$ & $5 \mathbf{v}$ & $5 \mathbf{v}$ & $5 \mathbf{v}$ & $5 \mathbf{v}$ & 50 & $5 v$ \\
\hline 1,1-Dichloroethene & 6 & 7 & 6 & 6 & $5 \mathbf{v}$ & $5 \mathbf{v}$ & $5 \mathbf{u}$ & $\mathbf{5 U}$ \\
\hline 1,2-Dichloroethene & 3 & 3 & 4 & 2 & su & $\mathbf{5 U}$ & $5 \mathbf{U}$ & 50 \\
\hline 1,2-Dichloropropane & 50 & $5 v$ & 50 & $5 \mathbf{v}$ & $5 \mathbf{v}$ & $5 \mathbf{v}$ & $5 \mathbf{u}$ & $5 \mathbf{U}$ \\
\hline Cis-1,3-Dichloropropene & 50 & 50 & 50 & $\mathbf{5 u}$ & $5 \mathbf{U}$ & $5 \mathbf{U}$ & 5u & 5u \\
\hline trans-1,3-Dichloropropene & $5 \mathbf{v}$ & $5 v$ & $5 \mathrm{U}$ & $5 \mathbf{v}$ & $5 v$ & $5 \mathbf{v}$ & 5u & 50 \\
\hline Ethylbenzene & $5 \mathbf{5}$ & 50 & 50 & $5 \mathbf{v}$ & $5 \mathbf{u}$ & $5 \mathbf{U}$ & 5u & 5U \\
\hline 2-Hexanone & 100 & 100 & 100 & 100 & 100 & 100 & 100 & 100 \\
\hline 4-Methy 1-2-pentanone & 5 & 100 & $10 \mathrm{U}$ & 100 & 100 & 100 & 100 & 100 \\
\hline Methylene chloride & 1 & 1 & su & $5 \mathbf{v}$ & $5 v$ & $5 \mathbf{u}$ & $5 \mathbf{u}$ & 1 \\
\hline styrene & $5 \mathbf{U}$ & $5 U$ & su & $5 \mathbf{v}$ & 5u & $5 \mathbf{5}$ & $5 v$ & 50 \\
\hline $1,1,2,2$-Tetrachloroethane & $5 U$ & su & 5u & $5 \mathbf{v}$ & $5 v$ & 5u & $5 \mathbf{v}$ & 50 \\
\hline Tetrachloroethene & $5 \mathbf{u}$ & 0.5 & 5u & 1 & 5u & $5 U$ & $5 \mathbf{u}$ & $5 \mathbf{v}$ \\
\hline Toluene & $5 \mathbf{U}$ & $5 \mathbf{v}$ & 50 & su & $5 \mathbf{v}$ & $5 \mathbf{v}$ & $5 v$ & 50 \\
\hline $1,1,1$-Trichloroethane & 2 & 2 & 2 & $5 \mathbf{u}$ & 50 & $5 \mathbf{v}$ & 5U & 50 \\
\hline $1,1,2$-Trichloroethane & $5 U$ & 50 & $5 \mathrm{v}$ & $5 v$ & $5 v$ & $5 v$ & 5v & 50 \\
\hline Trichloroethene & 120 & 100 & 86 & 56 & 5u & $5 \boldsymbol{v}$ & $5 v$ & 5u \\
\hline Vinyl acetate & 100 & $10 \mathrm{U}$ & 100 & 100 & 100 & 100 & 100 & 100 \\
\hline Vinyl chloride & 100 & 100 & 100 & 100 & 100 & 100 & 100 & 100 \\
\hline Xylenes & 50 & $5 v$ & 50 & $5 u$ & su & 50 & $5 v$ & 50 \\
\hline
\end{tabular}

(CONTTNUED) 
APPENDIX 5.2

Field Duplicate Data, 1993

\begin{tabular}{|c|c|c|c|c|c|}
\hline \multirow{3}{*}{$\begin{array}{l}\text { Sampling Point } \\
\text { - } \\
\text { Location } \\
\text { Date Sampled }\end{array}$} & \multicolumn{2}{|c|}{ OW-725 } & $G W-736$ & $G W-740$ & $G W-800$ \\
\hline & \multicolumn{2}{|c|}{$\operatorname{EXP}$} & $\mathbf{E X P}$ & EXP & OLF \\
\hline & $02 / 12 / 93$ & $05 / 19 / 93$ & $12 / 15 / 93$ & $09 / 27 / 93$ & $10 / 22 / 93$ \\
\hline VOLATILE ORGANICS (ug/L) & $\cdot$ & $\cdot$ & $\cdot$ & . & - \\
\hline Acetone & 100 & 100 & 3 & 100 & 100 \\
\hline Benzene & 50 & 50 & su & 50 & su \\
\hline Bromodichloromethane & 50 & $5 \mathbf{u}$ & 5u & 5u & 50 \\
\hline Bromoform & 50 & $5 v$ & su & 5u & su \\
\hline Bromomethane & 100 & 100 & 100 & 100 & 100 \\
\hline 2-Butanone & 100 & 100 & 8 & $10 u$ & 100 \\
\hline Carbon disulfide & 50 & 5u & 50 & su & su \\
\hline Carbon tetrachloride & 5u & 5u & 5u & 50 & 5u \\
\hline Chlorobenzene & 5u & 5u & 50 & 50 & su \\
\hline Chlorodibromomethane & $5 \mathbf{u}$ & 50 & 50 & su & 5u \\
\hline Chloroethane & 100 & 100 & 100 & $10 u$ & 100 \\
\hline Chloroform & 50 & 5u & $5 v$ & 1 & $5 v$ \\
\hline Chloromethane & 100 & 100 & 100 & 100 & 100 \\
\hline 1,1-Dichloroethane & 5u & 5u & 50 & 50 & 5U \\
\hline 1,2-Dichloroethane & 50 & 50 & 50 & 5u & 5u \\
\hline 1,1-Dichloroethene & su & 50 & 50 & 50 & 5u \\
\hline 1,2-Dichloroethene & 2 & 3 & 5 & 2 & 50 \\
\hline 1,2-Dichloropropane & $5 v$ & 50 & su & 5u & 5u \\
\hline cis-1,3-Dichloropropene & 50 & 5u & $5 v$ & 50 & 50 \\
\hline trans-1,3-Dichloropropene & 5u & 5u & 50 & 5u & 5u \\
\hline Ethylbenzene & 50 & 5u & 50 & su & 5U \\
\hline 2-Hexanone & 100 & 100 & 100 & 100 & 100 \\
\hline 4-Methy1-2-pentanone & 2 & 100 & 1 & 100 & 100 \\
\hline Methylene chloride & 2 & 50 & 1 & 50 & 5u \\
\hline Styrene & 50 & 50 & 5u & 50 & 50 \\
\hline $1,1,2,2$-Tetrachloroethane & 50 & 50 & 50 & su & 50 \\
\hline Tetrachloroethene & 50 & 50 & 3 & $5 u$ & 50 \\
\hline Toluene & 50 & su & 5u & 50 & 5u \\
\hline 1,1,1-Trichloroethane & 5u & so & 50 & $5 u$ & su \\
\hline $1,1,2$-Trichloroethane & 5u & 50 & 50 & $5 u$ & 5u \\
\hline Trichloroethene & 19 & 53 & 13 & 32 & su \\
\hline Vinyl acetate & 100 & 100 & 100 & 100 & 100 \\
\hline Vinyl chloride & 100 & 100 & 100 & 100 & 100 \\
\hline xylenes & $5 v$ & 50 & 50 & 50 & 50 \\
\hline
\end{tabular}




\section{APPENDIX F.3}

FIELD, MISCELLANEOUS, AND GROSS ALPHA AND GROSS BETA ACTIVITIES 
APPERDIX $\mathbf{7 . 3}$

Fleld Dupllcate Groundwater Data, 1993

\begin{tabular}{|c|c|c|c|c|c|c|c|c|}
\hline \multirow{3}{*}{$\begin{array}{l}\text { 8ampling Polnt } \\
\text { Location } \\
\text { Date sampled }\end{array}$} & OW-044 & OW-069 & \multicolumn{2}{|c|}{ on-115 } & an-31s & $6 x-324$ & ON-371 & $O W-373$ \\
\hline & oxf & BO & \multicolumn{2}{|c|}{83} & SPI & 83 & Be & $B 0$ \\
\hline & $09 / 11 / 93$ & $10 / 10 / 93$ & $05 / 25 / 93$ & $09 / 11 / 93$ & $10 / 27 / 93$ & $01 / 29 / 93$ & $10 / 13 / 93$ & $02 / 11 / 93$ \\
\hline FIELD RARBURMENTS & $\cdot$ & - & $\cdot$ & - & - & - & - & $\cdot$ \\
\hline Depth to Water (ft) & & &. & . & . & . & . & • \\
\hline Water Tamp (degraes C) & 14.2 & 14.5 & 16.7 & 16.6 & 14.5 & 14 & 15.2 & 14.1 \\
\hline pH (pH unite) & 7.2 & 8.5 & 7.4 & 7.3 & 6.5 & 7.9 & 6.7 & 9.1 \\
\hline 8p. Cond. (unho/cm) & 252 & 386 & 360 & 414 & 569 & 205 & 521 & 471 \\
\hline Diesolved oxygen (ppra) & 3.7 & 0.5 & 1.4 & 2.2 & 2.5 & 0.5 & 1.2 & 7.5 \\
\hline Oxidation/Reduction (mV) & 180 & -137 & -104 & -35 & 213 & -85 & -5 & 161 \\
\hline MISCELIANEOUS PARAMETERS &. & . & . & . & . & . & . & $\bullet$ \\
\hline pH (pH unite) & 7.6 & 8.5 & 7.6 & 7.0 & 7.2 & $8 . \dot{2}$ & 7.6 & 9.1 \\
\hline 8p. Cond. (unho/cm) & 267 & 396 & 464 & 465 & 640 & 202 & 571 & 484 \\
\hline TDS (mg/L) & 188 & 290 & 286 & 298 & 402 & 148 & 368 & 288 \\
\hline T88 (mg/L) & $<1$ & $<1$ & 2 & $<1$ & $<1$ & $<1$ & $<1$ & 4 \\
\hline Turbidity (NTU) & 1.1 & 0.3 & 4.3 & 3.8 & 0.6 & 1 & 4.5 & 3.4 \\
\hline RADIOCHEMICAI PARAMETERS & $\cdot 1$ & - & . & . & . & . & - & • \\
\hline Grose Alpha (pCi/L) & -1.62 & 1.75 & 0.00 & 0.18 & 4.33 & 1.23 & 0.66 & 2.24 \\
\hline Grous Alpha $(\mathrm{CE}+/-)$ & 2.70 & 2.10 & 3.50 & 1.10 & 2.90 & 1.20 & 1.50 & 1.90 \\
\hline Gross Bata (PC1/L) & -6.59 & 5.44 & -1.17 & -1.82 & 54.80 & 1.99 & 0.35 & 2.97 \\
\hline Grose Beta $(\mathrm{CB}+1-)$ & 5.90 & 3.10 & 5.50 & 2.50 & 5.00 & 2.70 & 2.80 & 2.50 \\
\hline
\end{tabular}

(CONTINUED) 
APPandix $\mathbf{P . 3}$

Fleld Duplloate Groundwater Data, 1993

\begin{tabular}{|c|c|c|c|c|c|c|c|c|}
\hline \multirow{3}{*}{$\begin{array}{l}\text { sampling polnt } \\
\text { Location } \\
\text { Date sampled }\end{array}$} & \multicolumn{2}{|c|}{ OW-537 } & \multicolumn{2}{|c|}{$O N-626$} & \multirow{2}{*}{$\frac{01-627}{80}$} & \multicolumn{3}{|c|}{$0 N-636$} \\
\hline & \multicolumn{2}{|c|}{ oLP } & \multicolumn{2}{|c|}{$B O$} & & \multicolumn{3}{|c|}{ ote } \\
\hline & $02 / 21 / 93$ & $05 / 22 / 93$ & $03 / 10 / 93$ & $08 / 02 / 93$ & $04 / 23 / 93$ & $03 / 26 / 93$ & $06 / 27 / 93$ & $09 / 18 / 93$ \\
\hline FIELD MEAUREMENTS & & - & - & - & 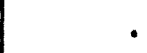 & $\cdot$ & - & $\cdot$ \\
\hline Dopth to Mater (ft) & & & • & & • & . &. & 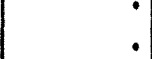 \\
\hline water Temp (degrees C) & 14.7 & 16.3 & 14.8 & 16.4 & 15.8 & 13.8 & 14.3 & 14.4 \\
\hline ph (ph unlte) & 6.6 & 6.8 & 0.2 & 8 & 9 & 0.4 & 7.9 & 0.3 \\
\hline sp. Cond. (unho/cm) & 3480 & 4960 & 247 & 250 & 2017 & 303 & 310 & 349 \\
\hline Diesolved Oxygen (ppm) & 1.3 & 1.1 & 2.7 & 3.3 & 0.6 & 4.7 & 0.5 & 3.9 \\
\hline Oxidation/Roduction (mV) & 120 & 172 & 74 & 157 & -14 & 139 & 152 & 171 \\
\hline MI8CELLAMEOUS PARANTTERS & . & . & - & & . &. & . & - \\
\hline$\therefore$ (ph unita) & 6.8 & 7.6 & $\dot{8}$ & 8.1 & $\dot{9}$ & 8.5 & 8.5 & 8.5 \\
\hline p. Cond. (umho/om) & 5920 & 5670 & 268 & 235 & 1240 & 364 & 361 & 362 \\
\hline TDS (mg/L) & 4578 & 4612 & 154 & 144 & 730 & 232 & 244 & 241 \\
\hline Tss (mg/L) & s & 3 & $<1$ & $<1$ & $<1$ & 6 & 1 & 3 \\
\hline Turbidity (ArU) & 2 & 1 & 0.7 & 0.6 & 1.4 & 5.5 & 2.5 & 1.5 \\
\hline RADIOCHEMICAL PARAMETERS & - & - & - & - & - & . & . & - \\
\hline Grome Alphe (pCi/L) & 38.40 & 0.00 & -0.59 & -13.90 & 3.90 & 0.50 & -3.04 & -2.27 \\
\hline Gross Alpha $(\mathrm{CE}+/-1$ & 14.00 & 20.00 & 3.40 & 35.00 & 3.80 & 1.70 & 2.60 & 2.40 \\
\hline Grone Bata (pCi/t) & 262.00 & 458.00 & -0.16 & 19.60 & -0.09 & 3.27 & 5.22 & 3.83 \\
\hline Grose Beta $(\mathrm{CE}+1-)$ & 22.00 & 46.00 & 5.10 & 52.00 & 2.80 & 2.70 & 5.20 & 5.00 \\
\hline
\end{tabular}

(CONTIMUED) 
Appundix 7.3

Fleld Duplleate Oroundwater Data, 1993

\begin{tabular}{|c|c|c|c|c|c|c|c|c|}
\hline \multirow{3}{*}{$\begin{array}{l}\text { Bampling polnt } \\
\text { Loention } \\
\text { Date sampled }\end{array}$} & \multirow{3}{*}{\begin{tabular}{|c|} 
on-636 \\
orf \\
$12 / 18 / 93$ \\
\end{tabular}} & \multicolumn{3}{|c|}{ ON-639 } & \multicolumn{2}{|c|}{$0 w-684$} & \multicolumn{2}{|c|}{ ON-6es } \\
\hline & & \multicolumn{3}{|c|}{ so } & \multicolumn{2}{|c|}{$\mathbf{E x p}$} & \multicolumn{2}{|c|}{$\mathbf{I x P}$} \\
\hline & & $02 / 24 / 93$ & $05 / 24 / 93$ & $07 / 11 / 93$ & $07 / 11 / 93$ & $10 / 12 / 93$ & $03 / 02 / 53$ & $04 / 21 / 93$ \\
\hline 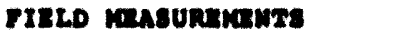 & - & $\cdot$ & $\cdot$ & $\cdot$ & $\cdot$ & $\cdot$ & - & - \\
\hline Dapth to Water $(t t)$ & . & . & . &. &. & . & . & • \\
\hline Water Tanp (degrees $C$ ) & 13 & 12.9 & 15.6 & 16.7 & 15.2 & 13.2 & 13.3 & 13.6 \\
\hline pH (pH units) & 6.2 & 9.3 & 9.1 & 9.3 & 7.4 & 7.7 & 7.1 & 7.3 \\
\hline 8p. Cond. (unho/cm) & 374 & 778 & 722 & 760 & 433 & 308 & 736 & 6.36 \\
\hline Diweolved Oxygen (ppm) & 0.4 & 1.4 & 1.3 & 0.7 & 7.6 & 7.1 & 9.3 & 8.8 \\
\hline Oxldation/Raduction (nv) & 68 & 100 & 154 & -4 & 114 & 184 & 104 & 43 \\
\hline MISCELLNIEOUS PARAMTERS & . & . & . & . &. &. & . & . \\
\hline pH (pH unIte) & 0.5 & 9.2 & 9.3 & 9.3 & 7.9 & 7.7 & 7.6 & 7.0 \\
\hline sp. Cond. (unho/cm) & 356 & 871 & 872 & 970 & 510 & 594 & 722 & 726 \\
\hline $\operatorname{TDS}(\mathrm{mg} / \mathrm{L})$ & 240 & 538 & 562 & ss4 & 322 & 142 & 414 & 414 \\
\hline$T 88(m g / L)$ & 4 & 13 & 30 & 6 & $<1$ & 2 & 13 & 8 \\
\hline Turbldity (NTU) & 0.8 & 12 & 30 & 6.3 & 1 & 3.8 & 36 & 11 \\
\hline RADIOCKaMTCAL PARANTERS & - & . & . & . &. & . & . & 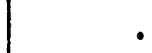 \\
\hline Orous Alphe $(\mathrm{pCl} / \mathrm{L})$ & -0.01 & -0.45 & -1.10 & -0.44 & 22.60 & 29.80 & -0.77 & -0.28 \\
\hline Orose Alpha $(C 2+1-)$ & 1.20 & 2.00 & 3.80 & 1.20 & 4.20 & 4.60 & 3.10 & 2.40 \\
\hline Grose Bete $(\mathrm{pCl} / \mathrm{L})$ & -1.19 & 1.44 & -3.05 & -3.87 & 46.20 & 57.60 & 3.35 & 2.30 \\
\hline Orons Bete $(\mathrm{Cs}+/-)$ & 2.90 & 2.80 & 5.30 & 2.80 & 4.70 & 5.30 & 3.70 & 3.10 \\
\hline
\end{tabular}

(CONT InURD) 
APpundx 7.3

Field Duplloate Oroundwater Data, 1993

\begin{tabular}{|c|c|c|c|c|c|c|c|c|}
\hline \multirow{3}{*}{$\begin{array}{l}\text { Iapiling polnt } \\
\text { Lootion } \\
\text { Date sempled }\end{array}$} & \multicolumn{4}{|c|}{ ON-704 } & \multicolumn{2}{|c|}{$a N-712$} & $0 N-713$ & $9 w-714$ \\
\hline & \multicolumn{4}{|c|}{$\mathbf{x e}$} & \multicolumn{2}{|c|}{$\mathbf{E x p}$} & $\mathbf{E x p}$ & $\mathbf{E x p}$ \\
\hline & $03 / 22 / 93$ & $06 / 04 / 93$ & $09 / 22 / 33$ & $12 / 22 / 03$ & $03 / 28 / 93$ & $04 / 28 / 93$ & $07 / 31 / 93$ & $12 / 09 / 93$ \\
\hline preto masuranures & $\cdot 1$ & $\cdot$ & $\cdot 1$ & $\cdot 1$ & $\cdot$ & $\cdot 1$ & $\cdot$ & $\bullet$ \\
\hline Dopth to Water (ft) & & &. &. &. &. &. & - \\
\hline Mater Tamp (dogreas $c$ ) & 13.3 & 13.7 & 14.3 & 12,1 & 11.3 & 14.9 & 15 & 12.6 \\
\hline pH (pH undte) & 7.4 & 7.4 & 7.7 & 0.5 & 7.6 & 7.4 & 7.8 & 7.6 \\
\hline ip. Cond. (unho/cm) & 378 & 308 & 398 & 327 & 867 & 738 & 641 & 402 \\
\hline Diesolved Oxygen (ppon) & 3.5 & 1.6 & 2.3 & 1 & 1.1 & 3.5 & 0.7 & 1.3 \\
\hline Oxldation/Raduction (nV) & 42 & 63 & -72 & 19 & -25 & ss & -137 & -67 \\
\hline MISCELLNDOUS PARNATERS & . & $\cdot$ &. &. &. &. &. & - \\
\hline ph (ph undte) & $0 . \dot{1}$ & $\dot{8}$ & 7.8 & 8. & $\dot{1}$ & $\dot{0}$ & $7 . \dot{7}$ & $1 . \dot{8}$ \\
\hline 8p. Cond, (unho/om) & 609 & 384 & 611 & 348 & 896 & 846 & 666 & 432 \\
\hline TDS $(m g / L)$ & 344 & 254 & 342 & 174 & 636 & 592 & 482 & 260 \\
\hline $\operatorname{Tas}(\mathrm{mg} / \mathrm{L})$ & 4 & 48 & 21 & 19 & 2 & 1 & s & 13 \\
\hline Turbldity (mru) & 16 & 130 & 39 & 17 & 24 & 3.9 & 22 & 18 \\
\hline RADIOCKRMICAL PARNATIERS & $\cdot 1$ & $\cdot$ & $\cdot$ &. & $\cdot$ &. & $\cdot$ & - \\
\hline arose Alphe $(\mathrm{pCi} / \mathrm{L})$ & -1.93 & 0.00 & 3.12 & 0.49 & 2.63 & -1.53 & -27.90 & 2.14 \\
\hline Orose Alphe $(C x+/-)$ & 3.50 & 3.60 & 4.30 & 1.10 & 1.90 & 1.90 & 33.00 & 2.30 \\
\hline Orose Beta (pCl/h) & 3.83 & 6.27 & 17.10 & 1.02 & 2.92 & -6.84 & -3.26 & 3.61 \\
\hline Oross beta $(\mathrm{Cx}+1-)$ & 5.20 & 5.60 & 5.90 & 2.60 & 2.90 & 2.80 & 51.00 & 3.10 \\
\hline
\end{tabular}

(Contrinuso) 
APpewdX $\mathbf{1 . 3}$

Nield Duplloate Groundwater Data, 1993

\begin{tabular}{|c|c|c|c|c|c|}
\hline \multirow{3}{*}{$\begin{array}{l}\text { Sanpling polnt } \\
\text { tooation } \\
\text { Date sampled }\end{array}$} & \multicolumn{2}{|c|}{$0 N-725$} & ON-736 & ON-740 & ON-800 \\
\hline & \multicolumn{2}{|c|}{$\exp$} & $\operatorname{mxp}$ & $\mathbf{e x p}$ & ors \\
\hline & $02 / 12 / 93$ & $05 / 19 / 93$ & $12 / 15 / 93$ & $09 / 27 / 93$ & $10 / 22 / 93$ \\
\hline FIERd MaguRELants & $\cdot$ & - & $\cdot$ & $\cdot$ & • \\
\hline Depth to Mater $(t t)$ &. & - & . &. & . \\
\hline Water Temp (degrees C) & 15 & 13.6 & 23.4 & 14.2 & 14.1 \\
\hline ph (pH unite) & 6.9 & 7.1 & 7 & 7.3 & 6.9 \\
\hline 8p. Cond. (unho/cm) & 1240 & 1039 & 1070 & 497 & 444 \\
\hline Diesolved Oxygen (ppa) & 2.3 & 1.8 & 3.4 & 3.2 & 7.8 \\
\hline Oxldation/Raduction (mV) & -4 & 98 & 156 & -36 & 231 \\
\hline MISCRLHNROUS PARANTERS & - & - & . & . & • \\
\hline PH (PH undte) & 7.2 & 7.3 & 7.2 & 7.6 & 7.2 \\
\hline 8p. Cond, (unho/cm) & 1180 & 1190 & 1120 & 563 & 501 \\
\hline Tos $(n g / 2)$ & 714 & 758 & 752 & 306 & 286 \\
\hline $288(\mathrm{mg} / \mathrm{L})$ & 4 & 1 & $<1$ & $<1$ & 43 \\
\hline Turbldity (WTU) & 10 & 2.1 & 0.5 & 2.3 & 140 \\
\hline RADTOCHEMICAL PARNETRRB & . & - & . & . & • \\
\hline Orose Alphe $(p C 1 / L)$ & 3.78 & 1.74 & 4.92 & 4.98 & 0.67 \\
\hline Gross Alpha $(\mathrm{Cs}+/-)$ & 3.50 & 3.70 & 3.50 & 4.60 & 1.50 \\
\hline Orose Bata (pC1/L) & 39.90 & 45.80 & 61.80 & 13.10 & 2.91 \\
\hline Orose Bata $(\mathrm{Cs}+1-)$ & 4.90 & 8.10 & 5.50 & 5.70 & 2.60 \\
\hline
\end{tabular}




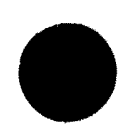

-

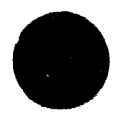


II 
APPENDIX F.4

Field Duplicate Groundwater Quality Data, 1993

\begin{tabular}{|c|c|c|c|}
\hline \multirow{3}{*}{$\begin{array}{l}\text { Sampling Point } \\
\text { Location } \\
\text { Date Sampled }\end{array}$} & \multicolumn{2}{|c|}{ GW-537 } & GW-714 \\
\hline & \multicolumn{2}{|c|}{ OLF } & EXP \\
\hline & $02 / 11 / 93$ & $05 / 12 / 93$ & $11 / 09 / 93$ \\
\hline Grose Alpha (pCi/L) & 38.40 & 0.00 & 2.14 \\
\hline Gross Alpha $(\mathrm{CE}+/-)$ & 14.00 & 20.00 & 2.30 \\
\hline Gross Beta $(\mathrm{pCi} / \mathrm{L})$ & 262.00 & 458.00 & 3.61 \\
\hline Gross Beta $(\mathrm{CE}+/-)$ & 22.00 & 46.00 & 3.10 \\
\hline RADIONUCLIDES & $\cdot$ & • & - \\
\hline Americium $241(\mathrm{pCi} / \mathrm{L})$ & $\cdot$ & • & 5.51 \\
\hline Americium $241(\mathrm{CE}+/-)$ & $\cdot$ & - & 5.50 \\
\hline Iodine $129(\mathrm{pCi} / \mathrm{L})$ & $\cdot$ & - & 0.10 \\
\hline Iodine $129(\mathrm{CE}+/-)$ & $\cdot$ & - & 1.10 \\
\hline Iodine $131(\mathrm{pCi} / \mathrm{L})$ & $\cdot$ & $\cdot$ & 0.04 \\
\hline Iodine $131(\mathrm{CE}+/-)$ & $\cdot$ & - & 0.15 \\
\hline Neptunium 237 (pCi/L) & $\cdot$ & $\cdot$ & 0.54 \\
\hline Neptunium $237(\mathrm{CE}+/-)$ & $\cdot$ & $\cdot$ & 1.10 \\
\hline Plutonium 238 (pCi/L) & $\cdot$ & $\cdot$ & 0.34 \\
\hline Plutonium $238(\mathrm{CE}+/-)$ & $\cdot 1$ & $\cdot$ & 0.67 \\
\hline Plutonium 239 (pCi/L) & $\cdot$ & $\cdot$ & 0.00 \\
\hline Plutonium $239(\mathrm{CB}+/-)$ & $\cdot$ & $\cdot$ & 0.95 \\
\hline Radium $(B q / L)$ & $\cdot$ & $\cdot$ & 0.03 \\
\hline Radium $(\mathrm{CE}+/-)$ & $\cdot$ & - & 0.03 \\
\hline Strontiun (pCi/L) & -12.50 & -4.85 & 90.80 \\
\hline strontium $(\mathrm{CE}+/-)$ & 19.00 & 18.00 & 26.00 \\
\hline Technetium 99 (pCi/L) & 1810.00 & 920.00 & 138.00 \\
\hline Technetium $99(\mathrm{CE}+/-)$ & 1400.00 & 2700.00 & 63.00 \\
\hline Tritium (Bq/L) & -2.00 & 15.00 & 88.00 \\
\hline Tritium $(\mathrm{Cz}+/-)$ & 24.00 & 23.00 & 29.00 \\
\hline Uranium $234(\mathrm{pCi} / \mathrm{L})$ & $\cdot$ & $\cdot$ & -0.27 \\
\hline Uranium $234 \quad(\mathrm{CE}+/-)$ & $\cdot$ & $\cdot$ & 1.10 \\
\hline Uranium 235 (pCi/L) & $\cdot$ & $\cdot$ & 34.60 \\
\hline Uranium $235 \quad(\mathrm{CE}+/-)$ & $\cdot$ & $\cdot$ & 90.00 \\
\hline Uranium 238 (pCi/L) & $\cdot$ & $\cdot$ & 0.00 \\
\hline Uranium $238(C E+/-)$ & $\cdot$ & $\cdot$ & 0.93 \\
\hline
\end{tabular}




\section{APPENDIX G}

ION-CHARGE BALANCE CALCULATIONS 


\section{EXPLANATION}

\section{SAMPLING POINT:}

$$
\begin{array}{rll}
\text { BCK } & \text { - } & \text { Bear Creek Kilometer } \\
\text { GW } & - \text { Monitoring Well } \\
\text { NT } & - & \text { North Tributary to Bear Creek } \\
\text { SS } & - \text { Spring }
\end{array}
$$

\section{LOCATION:}

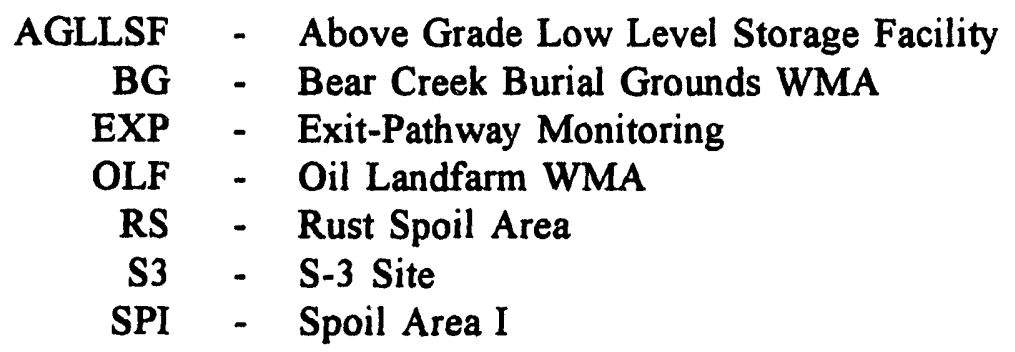

NOTES:

$$
\begin{array}{rll}
\mathrm{mg} / \mathrm{L} & - & \text { milligrams per Liter } \\
\mathrm{meq} / \mathrm{L} & - & \text { milliequivalents per Liter } \\
\text { TDS } & - & \text { Total Dissolved Solids } \\
\text { TSS } & - & \text { Total Suspended Solids } \\
\text { NTU } & - & \text { Nephelometric Turbidity Unit }
\end{array}
$$

Bicarbonate and carbonate alkalinities (reported as $\mathrm{CaCO}_{3}$ ) were converted to $\mathrm{HCO}_{3}$ and $\mathrm{CO}_{3}$ as described in Mercier and Turner (1990).

Charge balances for each sample were evaluated by computing a relative percent difference (RPD) in the summed milliequivalent concentrations of the principal cations and anions using the following formula:

$$
\text { RPD }(\%)=\left(\frac{\sum \text { cations }-\sum \text { anions }}{\sum \text { cations }+\sum \text { anions }}\right) \times 100
$$




\section{EXPLANATION}

Using this formula, the RPD calculated for the following samples exceeded $10 \%$ :

\begin{tabular}{|c|c|c|c|}
\hline Monitoring Well & Location & Sampling Date & RPD \\
\hline GW-042 & BG & $12 / 29 / 93$ & -17.8 \\
\hline GW- 057 & EXP & $3 / 4 / 93$ & -12.8 \\
\hline GW-064 & OLF & $9 / 11 / 93$ & 19.9 \\
\hline GW-085 & OLF & $2 / 11 / 93$ & -77.8 \\
\hline GW-118 & BG & $7 / 25 / 93$ & -15.9 \\
\hline GW-312 & RS & $2 / 8 / 93$ & 13.9 \\
\hline GW-312 & $\mathbf{R S}$ & $5 / 11 / 93$ & -19.6 \\
\hline GW-312 & RS & $10 / 21 / 93$ & -14.8 \\
\hline GW-345 & S3 & $2 / 3 / 93$ & -28.3 \\
\hline GW-345 & S3 & $10 / 26 / 93$ & 87.2 \\
\hline GW-347 & S3 & $10 / 22 / 93$ & -15.9 \\
\hline GW-526 & S3 & $10 / 26 / 93$ & -85.3 \\
\hline GW-537 & OLF & $9 / 14 / 93$ & 17.9 \\
\hline GW-601 & OLF & $6 / 27 / 93$ & -11.6 \\
\hline GW-627 & BG & $4 / 23 / 93$ & -41.4 \\
\hline GW-643 & BG & $10 / 12 / 93$ & 10.9 \\
\hline GW-644 & OLF & $9 / 17 / 93$ & -27.8 \\
\hline GW-653 & BG & $3 / 1 / 93$ & -70.4 \\
\hline GW-653 & BG & $4 / 20 / 93$ & -11.5 \\
\hline GW-653 & BG & $10 / 10 / 93$ & -15 \\
\hline GW-710 & EXP & $8 / 1 / 93$ & 11.4 \\
\hline GW-711 & EXP & $3 / 20 / 93$ & 10.1 \\
\hline GW-712 & EXP & $11 / 6 / 93$ & -11.8 \\
\hline GW-713 & EXP & $3 / 19 / 93$ & 29.8 \\
\hline GW-713 & EXP & $4 / 30 / 93$ & -25.6 \\
\hline$G W-715$ & EXP & $4 / 29 / 93$ & -16.4 \\
\hline NT-01 & EXP & $11 / 8 / 93$ & -18.2 \\
\hline
\end{tabular}


APPENDIX $G$

Ion-Charge Balance Calculations, 1993

\begin{tabular}{|c|c|c|c|c|c|c|c|c|c|c|c|c|c|c|}
\hline \multirow{3}{*}{$\begin{array}{l}\text { Sampling Point } \\
\text { Location } \\
\text { Date Sampled }\end{array}$} & \multicolumn{4}{|c|}{ GW-013 } & \multicolumn{4}{|c|}{ GW-040 } & GW-042 & \multicolumn{4}{|c|}{$G W-043$} & \multirow{2}{*}{\begin{tabular}{|c|} 
GN-044 \\
OrF
\end{tabular}} \\
\hline & \multicolumn{4}{|c|}{ OLF } & \multicolumn{4}{|c|}{ BG } & BG & \multicolumn{4}{|c|}{ OLF } & \\
\hline & $03 / 29 / 93$ & $06 / 17 / 93$ & $09 / 17 / 93$ & $12 / 19 / 93$ & $03 / 25 / 93$ & $05 / 11 / 93$ & $07 / 02 / 93 \mid$ & $10 / 09 / 93$ & $12 / 29 / 93$ & $02 / 04 / 93$ & $05 / 10 / 93$ & $09 / 11 / 93 \mid$ & $10 / 19 / 93$ & $02 / 04 / 93$ \\
\hline CATIONS (meq/L) & $\cdot$ & $\cdot$ & $\cdot$ & $\cdot$ & $\cdot$ & $\cdot$ & $\cdot$ & $\cdot$ & $\cdot 1$ & $\cdot$ & $\cdot$ & $\cdot$ & $\cdot$ & • \\
\hline Calcium & 4.54 & 4.79 & 5.49 & 4.99 & 0.33 & 0.29 & 0.28 & 0.38 & 0.12 & 1.05 & 0.70 & 0.75 & 0.90 & 2.40 \\
\hline Magnesium & 0.90 & 0.99 & 1.07 & 0.99 & 0.50 & 0.51 & 0.55 & 0.59 & 0.23 & 0.30 & 0.20 & 0.24 & 0.27 & 0.26 \\
\hline Potassium & 0.05 & 0.03 & 0.04 & 0.04 & 0.04 & 0.04 & 0.05 & 0.05 & 0.00 & 0.02 & 0.02 & 0.02 & 0.02 & 0.02 \\
\hline Sodium & 0.16 & 0.16 & 0.20 & 0.18 & 0.48 & 0.48 & 0.52 & 0.52 & 0.10 & 0.29 & 0.31 & 0.29 & 0.31 & 0.20 \\
\hline Hydrogen Ion & 0.00 & 0.00 & 0.00 & 0.00 & 0.00 & 0.00 & 0.00 & 0.00 & 0.00 & 0.00 & 0.00 & 0.00 & 0.00 & 0.00 \\
\hline$\cdot$ & & & & & $\dot{1}$ & 33 & . & 1.54 & 0.45 & 1.66 & 1.23 & 1.30 & 1.50 & 2.88 \\
\hline Total Cations & 5.65 & 5.96 & 6.80 & 6.20 & 1.35 & 1.33 &. &. &. & & &. & & \\
\hline ANIONS (meq/L) & $\cdot$ & $\cdot$ & $\cdot$ & $\cdot$ & $\cdot$ & $\cdot$ & $\cdot$ & $\cdot$ & $\cdot$ & $\cdot$ & $\cdot$ & $\cdot$ & $\cdot$ & $\bullet$ \\
\hline Alkalinity- $\mathrm{CO} 3$ & 0.00 & 0.00 & 0.00 & 0.00 & 0.00 & 0.00 & 0.00 & 0.00 & 0.00 & 0.00 & 0.00 & 0.00 & 0.00 & 0.00 \\
\hline Alkalinity-HCO3 & 5.98 & 5.84 & 5.68 & 6.19 & 0.76 & 0.74 & 0.82 & 0.76 & 0.50 & 1.50 & 1.04 & 1.24 & 1.24 & 2.86 \\
\hline Chloride & 0.12 & 0.12 & 0.11 & 0.14 & 0.08 & 0.06 & 0.05 & 0.06 & 0.04 & 0.03 & 0.05 & 0.03 & 0.03 & 0.03 \\
\hline Fluoride & 0.00 & 0.00 & 0.00 & 0.00 & 0.02 & 0.02 & 0.02 & 0.02 & 0.01 & 0.01 & 0.00 & 0.00 & 0.00 & 0.01 \\
\hline Nitrate Nitrogen & 0.00 & 0.00 & 0.00 & 0.00 & 0.00 & 0.00 & 0.00 & 0.00 & 0.00 & 0.00 & 0.00 & 0.00 & 0.00 & 0.00 \\
\hline Sulfate & 0.08 & 0.12 & 0.08 & 0.06 & 0.62 & 0.62 & 0.65 & 0.60 & 0.10 & 0.23 & 0.16 & 0.26 & 0.19 & 0.19 \\
\hline Hydroxyl Ion & 0.00 & 0.00 & 0.00 & 0.00 & 0.00 & 0.00 & 0.00 & 0.00 & 0.00 & 0.00 & 0.00 & 0.00 & 0.00 & 0.00 \\
\hline Total Anions & 6.18 & 6.08 & 5.87 & 6.40 & 1.48 & 1.44 & 1.53 & 1.44 & 0.65 & 1.76 & 1.25 & 1.53 & 1.45 & 3.08 \\
\hline & & $\cdot$ & $\cdot$ & $:$ & 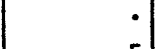 & $\cdot$ & $\cdot$ & $:$ & -18 & $\dot{-3}$ & $\dot{-1}$ & $\dot{-8}$ & $\dot{1}$ & -3 \\
\hline CHARGE BALANCE (8) & -4 & -1 & 7 & -2 & -5 & -4 & -4 & 4 & -18 & -3 & -1 &. &. & -3 \\
\hline $\operatorname{TDS}(\mathrm{mg} / \mathrm{I})$ & 354 & 336 & 338 & 334 & 216 & 344 & 232 & 228 & 56 & 132 & 106 & 120 & 132 & 212 \\
\hline TSS (mg/L) & 4 & 2 & 4 & 6 & 726 & 422 & 159 & 78 & $<1$ & 3 & 3 & 5 & 17 & 1 \\
\hline Turbidity (NTU) & 12 & 4.8 & 11 & 4.1 & 1000 & 300 & 180 & 120 & 1.4 & 6 & 8.6 & 15 & 20 & 1.2 \\
\hline
\end{tabular}

(CONTINUED) 
APPENDIX G

Ion-Charge Balance Calculations, 1993

\begin{tabular}{|c|c|c|c|c|c|c|c|c|c|c|c|c|c|c|}
\hline \multirow{3}{*}{$\begin{array}{l}\text { Sampling Point } \\
\text { Location } \\
\text { Date Sampled }\end{array}$} & \multicolumn{3}{|c|}{ GW-044 } & \multicolumn{2}{|c|}{ GW-052 } & \multicolumn{4}{|c|}{ GW-056 } & \multicolumn{4}{|c|}{ GW-057 } & \multirow{2}{*}{$\frac{\text { Gu-063 }}{\text { OrF }}$} \\
\hline & \multicolumn{3}{|c|}{ OLF } & \multicolumn{2}{|c|}{ BG } & \multicolumn{4}{|c|}{ BG } & \multicolumn{4}{|c|}{$\operatorname{Exp}$} & \\
\hline & $05 / 10 / 93$ & $09 / 11 / 93$ & $10 / 19 / 93$ & $03 / 25 / 93$ & $04 / 26 / 93$ & $03 / 04 / 93$ & $04 / 22 / 93$ & $07 / 09 / 93$ & $10 / 09 / 93$ & $03 / 04 / 93$ & $04 / 22 / 93$ & $07 / 09 / 93$ & $10 / 09 / 93$ & $02 / 23 / 93$ \\
\hline CATIONS (meq/L) & - & - & $\cdot 1$ & $\cdot$ & $\cdot$ & . & - & $\cdot$ & - & $\cdot$ & - & $\cdot$ & - & 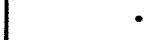 \\
\hline Calcium & 2.25 & 2.45 & 2.45 & 2.35 & 2.35 & 4.59 & 4.84 & 5.49 & 5.49 & 4.39 & 4.39 & 5.49 & 5.99 & 2.99 \\
\hline Magnesium & 0.24 & 0.26 & 0.24 & 0.57 & 0.63 & 1.73 & 1.65 & 1.81 & 1.73 & 1.40 & 1.32 & 1.97 & 1.81 & 1.48 \\
\hline Potassium & 0.02 & 0.00 & 0.02 & 0.04 & 0.04 & 0.08 & 0.05 & 0.05 & 0.05 & 0.04 & 0.04 & 0.06 & 0.05 & 0.05 \\
\hline Sodium & 0.21 & 0.20 & 0.20 & 0.43 & 0.40 & 4.31 & 6.09 & 3.18 & 2.26 & 5.22 & 2.61 & 3.31 & 3.13 & 0.13 \\
\hline Hydrogen Ion & 0.00 & 0.00 & 0.00 & 0.00 & 0.00 & 0.00 & 0.00 & 0.00 & 0.00 & 0.00 & 0.00 & 0.00 & 0.00 & 0.00 \\
\hline$\cdot$ & & & & & & & & & - & -1 & $\because$ & $\cdot$ & & \\
\hline Total Cations & 2.71 & 2.91 & 2.90 & 3.38 & 3.43 & 10.70 & 12.62 & 10.52 & 9.53 & 11.05 & 8.35 & 10.83 & 10.98 & 4.66 \\
\hline ANIONS (meq/L) & $\cdot$ & $\cdot$ & $\cdot$ & $\cdot$ & $\cdot$ & $\cdot$ & $\cdot$ & $\cdot$ & $\cdot$ & $\cdot$ & $\cdot$ & $\cdot$ & $\bullet$ & - \\
\hline Alkalinity-CO3 & 0.00 & 0.00 & 0.00 & 0.00 & 0.00 & 0.00 & 0.00 & 0.00 & 0.00 & 0.00 & 0.00 & 0.00 & 0.00 & 0.00 \\
\hline Alkalinity- $\mathrm{HCO} 3$ & 2.56 & 2.70 & 2.82 & 2.10 & 2.30 & 4.34 & 4.40 & 5.50 & 6.27 & 4.16 & 4.36 & 5.32 & 6.04 & 4.36 \\
\hline Chloride & 0.03 & 0.03 & 0.03 & 0.51 & 0.52 & 6.46 & 8.49 & 5.28 & 3.05 & 9.62 & 3.67 & 5.22 & 3.75 & 0.13 \\
\hline Fluoride & 0.00 & 0.01 & 0.00 & 0.02 & 0.02 & 0.00 & 0.00 & 0.01 & 0.00 & 0.00 & 0.01 & 0.01 & 0.00 & 0.01 \\
\hline Nitrate Nitrogen & 0.00 & 0.00 & 0.00 & 0.45 & 0.86 & 0.00 & 0.02 & 0.00 & 0.00 & 0.02 & 0.02 & 0.00 & 0.02 & 0.13 \\
\hline Sulfate & 0.18 & 0.14 & 0.17 & 0.42 & 0.39 & 0.54 & 0.54 & 0.54 & 0.75 & 0.50 & 0.54 & 0.60 & 0.87 & 0.19 \\
\hline Hydroxyl Ion & 0.00 & 0.00 & 0.00 & 0.00 & 0.00 & 0.00 & 0.00 & 0.00 & 0.00 & 0.00 & 0.00 & 0.00 & 0.00 & 0.00 \\
\hline$\cdot$ & & & & & & & & & & & & $\cdot$ & & \\
\hline Total Anions & 2.77 & 2.87 & 3.01 & 3.49 & 4.09 & 11.34 & 13.45 & 11.32 & 10.07 & 14.30 & 8.59 & 11.14 & 10.68 & 4.81 \\
\hline CHARGE BALANCE (8) & -1 & 1 & -2 & -2 & -9 & -3 & -3 & -4 & -3 & -13 & -1 & -1 & 1 & -2 \\
\hline & . & $\begin{array}{r}\cdot \\
188\end{array}$ & 210 & 216 & 286 & 584 & $74 \dot{6}$ & 598 & 630 & 734 & 532 & 616 & $68 \dot{8}$ & 258 \\
\hline $\begin{array}{l}\text { TDS }(m g / L) \\
\text { TSS }(m g / L)\end{array}$ & $\begin{array}{r}178 \\
<1\end{array}$ & $\begin{array}{r}188 \\
<1\end{array}$ & $\begin{array}{r}210 \\
<1\end{array}$ & $\begin{array}{r}216 \\
17\end{array}$ & 153 & 27 & 19 & 13 & 29 & 3 & 2 & 2 & $<1$ & 20 \\
\hline Turbidity (NTU) & 3.2 & 1.2 & 1.5 & 8.5 & 105 & 11 & 18 & 14 & 15 & 4.9 & 2.5 & 2.6 & 1.1 & 16 \\
\hline
\end{tabular}

(CONTINUED) 
APPENDIX $G$

Ion-Charge Balance Calculations, 1993

\begin{tabular}{|c|c|c|c|c|c|c|c|c|c|c|c|c|c|c|}
\hline \multirow{3}{*}{$\begin{array}{l}\text { Sampling Point } \\
\text { Location } \\
\text { Date Sampled }\end{array}$} & \multicolumn{4}{|c|}{ GW-064 } & \multicolumn{4}{|c|}{ GW-069 } & \multirow{3}{*}{\begin{tabular}{|c|} 
GW-079 \\
BG \\
$10 / 28 / 93$ \\
\end{tabular}} & \multirow{3}{*}{\begin{tabular}{|c|}
$G W-080$ \\
$B G$ \\
$10 / 10 / 93$ \\
\end{tabular}} & \multicolumn{4}{|c|}{ Gw-082 } \\
\hline & \multicolumn{4}{|c|}{ OLF } & \multicolumn{4}{|c|}{ BG } & & & \multicolumn{4}{|c|}{ BG } \\
\hline & $02 / 09 / 93$ & $05 / 12 / 93$ & $09 / 11 / 93$ & $10 / 20 / 93$ & $03 / 03 / 93$ & $04 / 21 / 93$ & $07 / 25 / 93$ & $10 / 10 / 93$ & & & $03 / 10 / 93$ & $04 / 21 / 93$ & $07 / 25 / 93$ & $10 / 18 / 93$ \\
\hline CATIONS (meq/L) & $\cdot$ & $\cdot$ & $\cdot$ & $\cdot$ & - & $\cdot$ & $\cdot$ & $\cdot$ & - & - & $\cdot 1$ & $\cdot$ & $\cdot 1$ & \\
\hline Calcium & 4.99 & 5.49 & 5.49 & 5.99 & 0.37 & 0.43 & 0.55 & 0.60 & 1.20 & 0.22 & 3.29 & 2.84 & 3.14 & 2.99 \\
\hline Magnesium & 1.32 & 1.40 & 1.32 & 1.32 & 0.46 & 0.44 & 0.49 & 0.51 & 0.37 & 0.40 & 0.53 & 0.53 & 0.61 & 0.58 \\
\hline Potassium & 0.05 & 0.05 & 0.13 & 0.05 & 0.11 & 0.12 & 0.12 & 0.11 & 0.07 & 0.07 & 0.03 & 0.04 & 0.04 & 0.05 \\
\hline Sodium & 0.20 & 0.20 & 0.21 & 0.22 & 2.57 & 2.48 & 2.74 & 2.78 & 0.23 & 0.61 & 0.20 & 0.19 & 0.20 & 0.20 \\
\hline Hydrogen Ion & 0.00 & 0.00 & 0.00 & 0.00 & 0.00 & 0.00 & 0.00 & 0.00 & 0.00 & 0.00 & 0.00 & 0.00 & 0.00 & 0.00 \\
\hline Total Cations & 6.56 & 1.14 & 7,14 & 7.57 & 3.51 & 3.47 & 3.91 & 4.01 & 1.87 & 1.30 & 4.05 & 3.60 & 4.00 & 3.82 \\
\hline & & & & & & & & & & &. & $\cdot$ & $\cdot 1$ & \\
\hline ANIONS (meg/L) & $\cdot$ & $\cdot$ & $\cdot$ & $\cdot$ & - & $\cdot$ & $\cdot$ & $\cdot$ & - & $\cdot$ & - & $\bullet$ & $\cdot 1$ & - \\
\hline Alkalinity- $\mathrm{CO} 3$ & 0.00 & 0.00 & 0.00 & 0.00 & 0.40 & 0.20 & 0.12 & 0.16 & 0.00 & 0.00 & 0.00 & 0.00 & 0.00 & 0.00 \\
\hline Alkalinity-HCO3 & 5.72 & 5.70 & 3.42 & 5.86 & 2.22 & 2.22 & 2.62 & 2.64 & 2.06 & 0.86 & 3.62 & 3.20 & 3.52 & 3.46 \\
\hline Chloride & 0.31 & 0.34 & 0.32 & 0.42 & 1.10 & 1.02 & 1.10 & 1.38 & 0.03 & 0.06 & 0.37 & 0.21 & 0.48 & 0.50 \\
\hline Fluoride & 0.00 & 0.00 & 0.00 & 0.00 & 0.01 & 0.01 & 0.00 & 0.01 & 0.01 & 0.01 & 0.01 & 0.01 & 0.01 & 0.01 \\
\hline Nitrate Nitrogen & 0.59 & 0.34 & 0.55 & 0.64 & 0.00 & 0.00 & 0.00 & 0.02 & 0.00 & 0.00 & 0.00 & 0.00 & 0.00 & 0.00 \\
\hline Sulfate & 0.52 & 0.35 & 0.48 & 0.52 & 0.04 & 0.16 & 0.08 & 0.05 & 0.14 & 0.44 & 0.15 & 0.27 & 0.15 & 0.14 \\
\hline Hydroxyl Ion & 0.00 & 0.00 & 0.00 & 0.00 & 0.02 & 0.01 & 0.00 & 0.00 & 0.00 & 0.00 & 0.00 & 0.00 & 0.00 & 0.00 \\
\hline - & & & & & & & & & & & & & & \\
\hline Total Anions & 7.14 & 6.72 & 4.77 & 7.44 & 3.78 & 3.61 & 3.92 & 4.25 & 2.24 & 1.36 & 4.15 & 3.69 & 4.16 & 4.11 \\
\hline CHARGE BALANCE (8) & -4 & 3 & 20 & 1 & -4 & -2 & -0 & -3 & -9 & -2 & -1 & -1 & -2 & -4 \\
\hline & $\cdot$ & - & $\cdot$ & - & - & - & $\cdot$ & $\cdot$ & - & $\cdot$ & - & $\cdot$ & - & 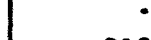 \\
\hline $\operatorname{TDS}(\mathrm{mg} / \mathrm{L})$ & 430 & 372 & 428 & 428 & 236 & 204 & 232 & 292 & 130 & 180 & 248 & 208 & 270 & 210 \\
\hline TSS (mg/L) & 3 & 5 & 2 & 9 & $<1$ & $<1$ & $<1$ & $<1$ & $<1$ & 129 & 8 & $<1$ & 5 & $<1$ \\
\hline Turbidity (NTU) & 16 & 9.5 & 5.8 & 13 & 0.5 & 1 & 1.2 & 0.4 & 1.2 & 85 & 2.1 & 2.4 & 10 & 0.8 \\
\hline
\end{tabular}

(CONTINUED) 
APPENDIX G

Ion-Charge Balance Calculations, 1993

\begin{tabular}{|c|c|c|c|c|c|c|c|c|c|c|c|c|c|c|}
\hline \multirow{3}{*}{$\begin{array}{l}\text { Sampling Point } \\
\text { Location } \\
\text { Date Sampled }\end{array}$} & \multicolumn{4}{|c|}{$G w-084$} & \multicolumn{4}{|c|}{ GW-085 } & \multicolumn{4}{|c|}{ Gw-086 } & \multicolumn{2}{|c|}{ Ga-115 } \\
\hline & \multicolumn{4}{|c|}{ OLF } & \multicolumn{4}{|c|}{ OLF } & \multicolumn{4}{|c|}{ orf } & \multicolumn{2}{|c|}{ s3 } \\
\hline & $02 / 04 / 93$ & $05 / 10 / 93$ & $09 / 11 / 93$ & $10 / 20 / 93$ & $02 / 11 / 93$ & $05 / 12 / 93$ & $09 / 14 / 93$ & $10 / 22 / 93$ & $02 / 05 / 93$ & $05 / 11 / 93$ & $09 / 12 / 93$ & $10 / 21 / 93$ & $01 / 28 / 93$ & $05 / 25 / 93$ \\
\hline CATIONS (meq/L) & - & • & - & - & - & - & $\cdot$ & - & - & $\cdot$ & $\cdot$ & $\bullet$ & - & \\
\hline Calcium & 2.50 & 2.64 & 2.79 & 2.74 & 16.97 & 20.46 & 21.96 & 20.46 & 1.95 & 2.05 & 2.10 & 1.85 & 3.44 & 3.44 \\
\hline Magnesium & 0.30 & 0.30 & 0.31 & 0.29 & 1.81 & 2.06 & 2.30 & 2.22 & 0.24 & 0.24 & 0.26 & 0.24 & 0.78 & 0.90 \\
\hline Potassium & 0.04 & 0.02 & 0.00 & 0.02 & 0.04 & 0.05 & 0.07 & 0.06 & 0.03 & 0.03 & 0.02 & 0.03 & 0.05 & 0.05 \\
\hline Sodium & 0.23 & 0.23 & 0.23 & 0.23 & 0.61 & 0.52 & 0.57 & 0.61 & 0.22 & 0.22 & 0.23 & 0.22 & 0.38 & 0.43 \\
\hline Hydrogen Ion & 0.00 & 0.00 & 0.00 & 0.00 & 0.00 & 0.00 & 0.00 & 0.00 & 0.00 & 0.00 & 0.00 & 0.00 & 0.00 & 0.00 \\
\hline - & 3.07 & 3.18 & 3.34 & 3.29 & 19.43 & 23.09 & 24.89 & 23.35 & 2.44 & 2.53 & 2.60 & 2.34 & 4.66 & 4.83 \\
\hline ANIONS (meq/L) & & $\cdot$ & $\bullet$ & $\cdot$ &. & &. & - & • & - & & - & & $\bullet$ \\
\hline Alkalinity-CO3 & 0.00 & 0.00 & 0.00 & 0.00 & 0.00 & $0 . \dot{00}$ & 0.00 & 0.00 & 0.00 & 0.00 & 0.00 & 0.00 & 0.00 & 0.00 \\
\hline Alkalinity-HCO3 & 2.98 & 2.98 & 3.06 & 3.06 & 2.46 & 2.48 & 2.56 & 2.42 & 1.92 & 1.90 & 1.82 & 1.84 & 4.24 & 4.48 \\
\hline Chloride & 0.00 & 0.00 & 0.00 & 0.03 & 0.45 & 0.45 & 0.39 & 0.42 & 0.04 & 0.05 & 0.04 & 0.06 & 0.10 & 0.12 \\
\hline Fluoride & 0.01 & 0.00 & 0.01 & 0.01 & 0.01 & 0.00 & 0.00 & 0.01 & 0.00 & 0.00 & 0.00 & 0.00 & 0.01 & 0.01 \\
\hline Nitrate Nitrogen & 0.00 & 0.00 & 0.00 & 0.00 & 152.73 & 17.14 & 21.71 & 22.32 & 0.32 & 0.22 & 0.35 & 0.40 & 0.00 & 0.00 \\
\hline sulfate & 0.19 & 0.17 & 0.16 & 0.15 & 0.23 & 0.16 & 0.23 & 0.00 & 0.29 & 0.31 & 0.23 & 0.19 & 0.37 & 0.37 \\
\hline Hydroxyl Ion & 0.00 & 0.00 & 0.00 & 0.00 & 0.00 & 0.00 & 0.00 & 0.00 & 0.00 & 0.00 & 0.00 & 0.00 & 0.00 & 0.00 \\
\hline & & & & & & & & & 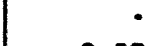 & & & & & \\
\hline Total Anions & 3.17 & 3.15 & 3.23 & 3.24 & 155.88 & 20.23 & 24.89 & 25.17 & 2.57 & 2.48 & 2.44 & 2.48 & 4.72 & 4.97 \\
\hline$\overline{\text { CHARGE BALANCE (8) }}$ & -2 & 1 & 2 & 1 & -78 & 7 & 0 & -4 & -3 & 1 & 3 & -3 & -1 & -1 \\
\hline & 218 & 208 & 206 & 216 & 1500 & 1768 & 1950 & $1872^{\circ}$ & 268 & $28 \dot{0}$ & 208 & $252^{\circ}$ & 276 & 292 \\
\hline $\begin{array}{l}\text { TDS (mg/L) } \\
\text { ISS (mg/L) }\end{array}$ & $\begin{array}{r}218 \\
47\end{array}$ & $\begin{array}{r}200 \\
43\end{array}$ & 30 & 17 & 5 & 8 & 1 & 3 & 511 & 1050 & 812 & 1318 & $<1$ & 1 \\
\hline Turbidity (NTU) & 22 & 21 & 12 & 11 & 2.2 & 3 & 1.8 & 0.7 & 320 & 480 & 360 & 620 & 1.9 & 4.1 \\
\hline
\end{tabular}

(CONTINUED) 
APPEIDIX 6

Ion-Charge Balance Calculations, 1993

\begin{tabular}{|c|c|c|c|c|c|c|c|c|c|c|c|c|c|c|}
\hline \multirow{3}{*}{$\begin{array}{l}\text { Sampling Point } \\
\text { Location } \\
\text { Date Sampled }\end{array}$} & \multicolumn{2}{|c|}{ GN-115 } & \multicolumn{4}{|c|}{$6 w-118$} & GW-162 & \multicolumn{4}{|c|}{ Ga-286 } & \multicolumn{3}{|c|}{ Gw-287 } \\
\hline & \multicolumn{2}{|c|}{ s3 } & \multicolumn{4}{|c|}{ BG } & BG & \multicolumn{4}{|c|}{ BG } & \multicolumn{3}{|c|}{ B6 } \\
\hline & $09 / 11 / 93$ & $10 / 19 / 93$ & $03 / 04 / 93$ & $\mid 04 / 21 / 93$ & $07 / 25 / 93$ & $10 / 10 / 93$ & $10 / 10 / 93$ & $02 / 25 / 93$ & $04 / 19 / 93$ & $07 / 10 / 93$ & $10 / 07 / 93$ & $02 / 24 / 93$ & $04 / 16 / 93$ & $07 / 10 / 93$ \\
\hline CATIONS (meg/I) & $\cdot 1$ & $\cdot$ & $\cdot$ & $\cdot$ & - & $\cdot$ & $\cdot$ & - & $\cdot$ & $\cdot$ & $\cdot$ & $\cdot$ & $\cdot$ & - \\
\hline Calcium & 3.59 & 3.49 & 0.12 & 0.15 & 0.06 & 0.05 & 1.35 & 2.45 & 2.20 & 2.35 & 2.35 & 1.90 & 1.80 & 1.70 \\
\hline Magnesium & 0.90 & 0.99 & 0.15 & 0.38 & 0.05 & 0.00 & 0.36 & 0.38 & 0.34 & 0.38 & 0.37 & 0.47 & 0.44 & 0.44 \\
\hline Potassium & 0.07 & 0.05 & 0.61 & 0.56 & 0.87 & 0.66 & 0.08 & 0.02 & 0.02 & 0.02 & 0.02 & 0.03 & 0.02 & 0.03 \\
\hline Sodium & 0.43 & 0.43 & 87.00 & 78.30 & 82.65 & 82.65 & 0.65 & 0.22 & 0.20 & 0.20 & 0.22 & 0.17 & 0.16 & 0.15 \\
\hline Hydrogen Ion & 0.00 & 0.00 & 0.00 & 0.00 & 0.00 & 0.00 & 0.00 & 0.00 & 0.00 & 0.00 & 0.00 & 0.00 & 0.00 & 0.00 \\
\hline Total Cations & 5.00 & 4.95 & 87.88 & 79.39 & 83.63 & 83.36 & 2.44 & 3.06 & 2.75 & 2.95 & 2.94 & 2.57 & 2.42 & 2.32 \\
\hline ANIOAs (meq $/ L$ ) & & & & $\cdot$ & & $\cdot$ & & $\cdot$ & & & $\cdot$ & & $\cdot$ & • \\
\hline Alkalinity-CO3 & 0.00 & 0.00 & 3.04 & 3.52 & 3.88 & 3.84 & 0.00 & 0.00 & 0.00 & 0.00 & 0.00 & 0.00 & 0.00 & 0.00 \\
\hline Alkalinity-HCO3 & 4.70 & 4.70 & 1.98 & 1.42 & 0.86 & 0.98 & 2.30 & 2.80 & 2.70 & 2.82 & 2.84 & 2.40 & 2.30 & 2.50 \\
\hline Chloride & 0.11 & 0.11 & 90.84 & 86.32 & 110.30 & 89.14 & 0.08 & 0.03 & 0.04 & 0.04 & 0.06 & 0.07 & 0.08 & 0.07 \\
\hline Fluoride & 0.00 & 0.00 & 0.09 & 0.09 & 0.08 & 0.06 & 0.01 & 0.01 & 0.01 & 0.01 & 0.01 & 0.01 & 0.01 & 0.01 \\
\hline Nitrate Mitrogen & 0.00 & 0.00 & 0.00 & 0.00 & 0.00 & 0.00 & 0.00 & 0.00 & 0.00 & 0.00 & 0.00 & 0.00 & 0.00 & 0.00 \\
\hline Sulfate & 0.33 & 0.31 & 0.00 & 0.00 & 0.00 & 0.00 & 0.18 & 0.13 & 0.13 & 0.15 & 0.12 & 0.17 & 0.23 & 0.13 \\
\hline Hydroxyl Ion & 0.00 & 0.00 & 0.02 & 0.06 & 0.16 & 0.13 & 0.00 & 0.00 & 0.00 & 0.00 & 0.00 & 0.00 & 0.00 & 0.00 \\
\hline - & 5.14 & 5.12 & 95.96 & 91.41 & 115.27 & 94.15 & 2.57 & 2.96 & 2.87 & 3.02 & 3.03 & 2.65 & 2.61 & 2.72 \\
\hline 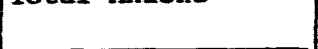 & & & & & & & & . & & . & . & . & - & • \\
\hline CHARGE BALANCE (8) & -1 & -2 & -4 & -7 & -16 & -6 & -3 & 2 & -2 & -1 & -1 & -2 & -4 & -8 \\
\hline $\operatorname{TDS}(m \mathrm{~m} / L)$ & $\begin{array}{r}\cdot \\
298\end{array}$ & 310 & 5054 & 5016 & $5064^{\circ}$ & 5116 & 236 & 202 & 192 & 178 & $24 \dot{8}$ & 156 & $14 \dot{2}$ & 166 \\
\hline TSS (mg/L) & $<1$ & $<1$ & 24 & 54 & 28 & 8 & 6 & 42 & 7 & $<1$ & 2 & 7 & 3 & 3 \\
\hline Turbidity (MTU) & 4.9 & 2.7 & 10 & 6 & 17 & 1 & 20 & 16 & 80 & 24 & 4.2 & 22 & 9 & 9 \\
\hline
\end{tabular}

(COMrrmued) 
APPSEDIX G

Ion-Charge Balance Calculations, 1993

\begin{tabular}{|c|c|c|c|c|c|c|c|c|c|c|c|c|c|c|}
\hline \multirow{3}{*}{$\begin{array}{l}\text { Sampling Point } \\
\text { Location } \\
\text { Date Sampled }\end{array}$} & \multirow{3}{*}{\begin{tabular}{|c|} 
GW-287 \\
$B G$ \\
$10 / 07 / 93$ \\
\end{tabular}} & \multicolumn{4}{|c|}{$6 n-311$} & \multicolumn{4}{|c|}{$601-312$} & \multicolumn{4}{|c|}{ con-315 } & \multirow{2}{*}{$\frac{6-316}{\operatorname{sPI}}$} \\
\hline & & \multicolumn{4}{|c|}{ RS } & \multicolumn{4}{|c|}{ RS } & \multicolumn{4}{|c|}{ SPI } & \\
\hline & & 02/08/93 & $05 / 11 / 93$ & $09 / 12 / 93$ & $10 / 21 / 93$ & $|02 / 08 / 93|$ & $05 / 11 / 93$ & $09 / 12 / 93$ & $10 / 21 / 93$ & $02 / 03 / 93$ & $06 / 03 / 93$ & $09 / 14 / 93$ & $10 / 27 / 93$ & $02 / 02 / 93$ \\
\hline CATIONS (meq/L) & $\cdot$ & $\cdot$ & - & $\cdot$ & $\cdot$ & $\cdot 1$ & $\cdot$ & $\cdot$ & • & - & - & - & $\cdot$ & $\cdot$ \\
\hline Calcium & 2.00 & 4.39 & 3.89 & 4.09 & 3.79 & 7.49 & 6.99 & 9.98 & 7.49 & 4.29 & 4.39 & 4.99 & 4.99 & 2.05 \\
\hline Magnesium & 0.44 & 0.44 & 0.42 & 0.39 & 0.39 & 0.02 & 0.00 & 0.00 & 0.00 & 0.65 & 0.76 & 0.90 & 0.82 & 1.73 \\
\hline Potassium & 0.03 & 0.04 & 0.04 & 0.02 & 0.03 & 0.26 & 0.22 & 0.23 & 0.25 & 0.09 & 0.07 & 0.08 & 0.09 & 0.06 \\
\hline Sodium & 0.20 & 0.09 & 0.08 & 0.07 & 0.07 & 0.41 & 0.41 & 0.70 & 0.57 & 0.28 & 0.22 & 0.25 & 0.30 & 0.10 \\
\hline Hydrogen Ion & 0.00 & 0.00 & 0.00 & 0.00 & 0.00 & 0.00 & 0.00 & 0.00 & 0.00 & 0.00 & 0.00 & 0.00 & 0.00 & 0.00 \\
\hline - & & & & & & $\cdot$ & & 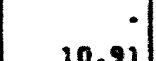 & $8 \dot{0}$ & 5.31 & 5.4 & 6.22 & 6.20 & 3.93 \\
\hline Total Cations & 2.66 & 4.96 & $\begin{array}{r}4.43 \\
.\end{array}$ & $\begin{array}{r}4.57 \\
.1\end{array}$ & $\begin{array}{r}4.27 \\
.\end{array}$ & $\begin{array}{r}8.17 \\
.1\end{array}$ & $\begin{array}{r}7.62 \\
.\end{array}$ & $\begin{array}{r}10.91 \\
.\end{array}$ & $\begin{array}{r}8.30 \\
.\end{array}$ & $\begin{array}{r}3.31 \\
.1\end{array}$ & &. & 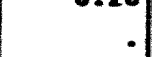 & \\
\hline ANIONS (meq/I) & $\cdot$ & $\cdot$ & • & $\cdot$ & $\cdot$ & $\cdot$ & $\cdot$ & $\cdot$ & $\cdot$ & $\cdot$ & $\cdot$ & $\cdot$ & - & - \\
\hline Alkalinity-CO3 & 0.00 & 0.00 & 0.00 & 0.00 & 0.00 & 1.00 & 1.04 & 0.96 & 1.00 & 0.00 & 0.00 & 0.00 & 0.00 & 0.00 \\
\hline Alkalinity-nCO3 & 2.74 & 4.52 & 4.28 & 4.20 & 4.18 & 0.00 & 0.00 & 0.00 & 0.00 & 3.58 & 4.32 & 4.58 & 4.36 & 3.82 \\
\hline Chloride & 0.08 & 0.06 & 0.07 & 0.08 & 0.08 & 0.04 & 0.05 & 0.04 & c.03 & 0.31 & 0.24 & 0.23 & 0.37 & 0.05 \\
\hline Fluoride & 0.01 & 0.00 & 0.00 & 0.00 & 0.00 & 0.01 & 0.02 & 0.01 & 0.01 & 0.01 & 0.00 & 0.00 & 0.00 & 0.01 \\
\hline Nitrate Nitrogen & 0.00 & 0.03 & 0.04 & 0.04 & 0.04 & 0.00 & 0.02 & 0.02 & 0.01 & 0.56 & 0.64 & 1.07 & 1.41 & 0.03 \\
\hline Sulfate & 0.15 & 0.09 & 0.20 & 0.08 & 0.06 & 0.12 & 0.21 & 0.08 & 0.12 & 0.79 & 0.56 & 0.46 & 0.42 & 0.08 \\
\hline Hydroxyl Ion & 0.00 & 0.00 & 0.00 & 0.00 & 0.00 & 5.01 & 10.00 & 10.00 & 10.00 & 0.00 & 0.00 & 0.00 & 0.00 & 0.00 \\
\hline - & & & & & & & & & & & & & & \\
\hline Total Anions & 2.98 & 4.69 & 4.59 & 4.39 & 4.36 & 6.18 & 11.33 & 11.11 & 11.18 & 5.24 & 5.75 & 6.34 & 6.55 & 3.98 \\
\hline CHARGE BALAMCE (*) & -6 & 3 & -2 & 2 & -1 & 14 & -20 & -1 & -15 & 2 & -3 & -1 & -3 & -1 \\
\hline $\operatorname{TDS}(m g / L)$ & 254 & 288 & $24 \dot{2}$ & 260 & 264 & 396 & 470 & 522 & 682 & 346 & 322 & 300 & 112 & 200 \\
\hline TSS (mg/L) & 11 & 51 & $<1$ & 7 & 40 & 81 & 4 & 4 & 25 & 4 & $<1$ & $<1$ & $<1$ & $<1$ \\
\hline Turbidity (Mro) & 15 & 54 & 2.5 & 3.3 & 68 & 16 & 3.6 & 1.2 & 3.5 & 0.5 & 1 & 0.9 & 0.5 & 0.9 \\
\hline
\end{tabular}

(COMTIMUED) 
APPEDIX 6

Ion-Charge Belance Calculations, 1993

\begin{tabular}{|c|c|c|c|c|c|c|c|c|c|c|c|c|c|c|}
\hline \multirow{3}{*}{$\begin{array}{l}\text { Sampling Point } \\
\text { Location } \\
\text { Date Sampled }\end{array}$} & \multicolumn{3}{|c|}{ an-316 } & \multicolumn{4}{|c|}{ Gn-317 } & \multicolumn{4}{|c|}{$60-323$} & \multicolumn{3}{|c|}{$c-324$} \\
\hline & \multicolumn{3}{|c|}{ SPI } & \multicolumn{4}{|c|}{ SPI } & \multicolumn{4}{|c|}{$\operatorname{sex}$} & \multicolumn{3}{|c|}{$\mathbf{8 3}$} \\
\hline & $06 / 01 / 93$ & $09 / 13 / 93$ & $10 / 24 / 93$ & $02 / 02 / 93$ & $05 / 28 / 93$ & $09 / 13 / 93$ & $10 / 24 / 93$ & $02 / 02 / 93$ & $05 / 28 / 93$ & $|00 / 22 / 93|$ & $10 / 23 / 93$ & $01 / 29 / 93$ & $05 / 25 / 93$ & $09 / 12 / 93$ \\
\hline CArIons (meg/L) & $\cdot$ & $\cdot$ & $\cdot$ & $\cdot$ & - & $\cdot$ & $\cdot$ & $\cdot$ & $\cdot$ & $\cdot$ & $\cdot$ & $\cdot$ & $\cdot$ & " \\
\hline Calcium & 2.25 & 2.25 & 2.05 & 2.30 & 2.25 & 2.50 & 2.10 & 2.30 & 2.20 & 2.10 & 2.10 & 1.40 & 1.45 & 1.55 \\
\hline Magnesium & 1.97 & 1.89 & 1.89 & 1.65 & 1.65 & 1.97 & 1.73 & 2.06 & 2.14 & 2.06 & 2.22 & 0.43 & 0.45 & 0.47 \\
\hline Potassium & 0.05 & 0.05 & 0.06 & 0.04 & 0.02 & 0.03 & 0.02 & 0.04 & 0.03 & 0.03 & 0.03 & 0.03 & 0.00 & 0.02 \\
\hline Sodium & 0.06 & 0.11 & 0.06 & 0.15 & 0.19 & 0.08 & 0.30 & 0.04 & 0.07 & 0.06 & 0.08 & 0.22 & 0.21 & 0.23 \\
\hline Hydrogen Ion & 0.00 & 0.00 & 0.00 & 0.00 & 0.00 & 0.00 & 0.00 & 0.00 & 0.00 & 0.00 & 0.00 & 0.00 & 0.00 & 0.00 \\
\hline - rotal Cations & 4.33 & 4.29 & 4.05 & 4.12 & 4.10 & 4.58 & 4.15 & 4.23 & 4.43 & 4.24 & 4.43 & 2.07 & 2.11 & 2.26 \\
\hline & & & & & & & & $\cdot$ & $\bullet$ & $\cdot$ & $\bullet$ & $\cdot 1$ & $\bullet$ & \\
\hline AnIOMs (meg/L) & $\cdot$ & $\cdot$ & $\cdot$ & $\cdot$ & $\cdot$ & $\cdot$ & $\cdot$ & $\cdot$ & - & $\cdot$ & $\cdot$ & $\cdot$ & $\cdot$ & $\bullet$ \\
\hline Alkalinity-CO3 & 0.00 & $0 . \dot{00}$ & $0.0 \mathrm{c}$ & 0.00 & 0.00 & 0.00 & 0.00 & 0.00 & 0.00 & 0.00 & 0.00 & 0.00 & 0.00 & 0.00 \\
\hline Alkalinity-HCO3 & 3.94 & 4.18 & 3.96 & 4.12 & 3.94 & 4.42 & 3.94 & 4.24 & 4.14 & 4.10 & 4.30 & 2.00 & 2.04 & 2.00 \\
\hline Chloride & 0.04 & 0.04 & 0.06 & 0.00 & 0.00 & 0.00 & 0.03 & 0.00 & 0.03 & 0.03 & 0.03 & 0.04 & 0.03 & 0.00 \\
\hline Fluoride & 0.00 & 0.00 & 0.00 & 0.01 & 0.00 & 0.00 & 0.00 & 0.00 & 0.00 & 0.00 & 0.00 & 0.01 & 0.01 & 0.00 \\
\hline Mitrate Nitrogen & 0.02 & 0.02 & 0.02 & 0.02 & 0.00 & 0.00 & 0.01 & 0.00 & 0.00 & 0.00 & 0.00 & 0.00 & 0.00 & 0.00 \\
\hline sulfate & 0.04 & 0.08 & 0.08 & 0.11 & 0.12 & 0.11 & 0.08 & 0.04 & 0.05 & 0.05 & 0.04 & 0.19 & 0.19 & 0.25 \\
\hline Bydroxyl Ion & 0.00 & 0.00 & 0.00 & 0.00 & 0.00 & 0.00 & 0.00 & 0.00 & 0.00 & 0.00 & 0.00 & 0.00 & 0.00 & 0.00 \\
\hline rotal Anions & 4.04 & 4.32 & $\begin{array}{r}. \\
4.12\end{array}$ & 4.25 & 4.05 & 4.53 & 4.06 & 4.28 & 4.21 & 4.18 & 4.37 & 2.24 & 2.27 & 2.23 \\
\hline$\overline{\text { Charge Balance (8) }}$ & $\dot{3}$ & $-\dot{0}$ & $\dot{-1}$ & -1 & $\dot{1}$ & $\dot{1}$ & $\dot{1}$ & -1 & 3 & $\dot{1}$ & $\dot{1}$ & -4 & $-\dot{4}$ & $\dot{1}$ \\
\hline (1) & & • & . &. &. &. & . &. & - &. & - & . & - & • \\
\hline $\operatorname{TDS}(m g / L)$ & 204 & 208 & 226 & 222 & 178 & 222 & 226 & 216 & 186 & 200 & 228 & 158 & 228 & 148 \\
\hline ISS $(\mathrm{mg} / \mathrm{L})$ & $<1$ & 1 & $<1$ & $<1$ & $<1$ & 3) & $<1$ & $<1$ & $<1$ & $<1$ & $<1$ & $<1$ & 1 & $<1$ \\
\hline Turbidity (2mo) & 2.7 & 8 & 2.4 & 1.5 & 1 & 2.4 & 2.3 & 0.4 & 2.5 & 0.9 & 0.7 & 1 & 0.7 & 0.9 \\
\hline
\end{tabular}

(CONTIMUED) 
APPETDIX 6

Ion-Charge Balance Calculations, 1993

\begin{tabular}{|c|c|c|c|c|c|c|c|c|c|c|c|c|c|c|}
\hline \multirow{3}{*}{$\begin{array}{l}\text { Sampling point } \\
\text { Location } \\
\text { Date Sampled }\end{array}$} & \multirow{3}{*}{\begin{tabular}{|c|}
$60-324$ \\
53 \\
$10 / 19 / 93$ \\
\end{tabular}} & \multicolumn{4}{|c|}{ GW-325 } & $c a-342$ & \multicolumn{4}{|c|}{$6-345$} & \multicolumn{4}{|c|}{$006-347$} \\
\hline & & \multicolumn{4}{|c|}{ s3 } & BE & \multicolumn{4}{|c|}{ s3 } & \multicolumn{4}{|c|}{$\mathbf{s 3}$} \\
\hline & & $02 / 01 / 93$ & $05 / 25 / 93$ & $09 / 11 / 93$ & $10 / 21 / 93$ & $10 / 07 / 93$ & $02 / 03 / 93$ & $06 / 01 / 93$ & $\mid 09 / 24 / 93$ & $10 / 26 / 93$ & $02 / 01 / 93$ & $05 / 27 / 93$ & $09 / 12 / 93$ & $10 / 22 / 93$ \\
\hline Carrous $($ neq $/ L)$ & $\cdot$ & $\cdot$ & $\cdot$ & - & - & $\cdot 1$ & $\cdot$ & $\cdot$ & - & - & - & - & - & - \\
\hline Calcium & 1.50 & 0.13 & 0.12 & 0.17 & 0.16 & 0.18 & 0.24 & 0.55 & 1.65 & 6.49 & 1.20 & 1.40 & 2.89 & 1.15 \\
\hline Magnesium & 0.49 & 0.12 & 0.10 & 0.09 & 0.10 & 0.12 & 0.14 & 0.21 & 0.46 & 4.28 & 0.16 & 0.17 & 0.42 & 0.15 \\
\hline Potassium & 0.02 & 0.03 & 0.00 & 0.02 & 0.02 & 0.06 & 0.05 & 0.08 & 0.17 & 0.43 & 0.09 & 0.08 & 0.10 & 0.07 \\
\hline Sodium & 0.23 & 0.16 & 0.07 & 0.06 & 0.07 & 0.24 & 0.30 & 0.30 & 0.34 & 69.60 & 0.04 & 0.04 & 0.05 & 0.03 \\
\hline Eydrogen ion & 0.00 & 0.00 & 0.00 & 0.00 & 0.00 & 0.00 & 0.00 & 0.00 & 0.00 & 0.00 & 0.00 & 0.00 & 0.00 & 0.00 \\
\hline Total Cations & 2.24 & 0.44 & 0.29 & 0.35 & 0.34 & 0.90 & 0.75 & 1.15 & 2.62 & 80.80 & 1.48 & 1.69 & 3.46 & 1.40 \\
\hline ANIOAs (meq/L) & & & - & & $\cdot$ & $\cdot$ & $\cdot$ & $\cdot$ & - & - & - & - & - & - \\
\hline Alkalinity-CO3 & 0.00 & 0.00 & 0.00 & 0.00 & 0.00 & & 0.00 & 0.00 & 0.00 & 0.00 & 0.00 & 0.00 & 0.00 & 0.00 \\
\hline Alkalinity-HCO3 & 2.06 & 0.18 & 0.16 & 0.24 & 0.18 & 0.76 & 0.40 & 0.48 & 0.54 & 0.44 & 1.48 & 1.66 & 3.12 & 1.86 \\
\hline Chloride & 0.03 & 0.05 & 0.05 & 0.05 & 0.06 & 0.00 & 0.13 & 0.14 & 0.15 & 0.20 & 0.00 & 0.00 & 0.00 & 0.00 \\
\hline Fluoride & 0.00 & 0.00 & 0.00 & 0.00 & 0.00 & 0.01 & $0.00 i$ & 0.00 & 0.00 & 0.00 & 0.00 & 0.00 & 0.01 & 0.01 \\
\hline Witrate Mitrogen & 0.00 & 0.00 & 0.00 & 0.00 & 0.00 & 0.00 & 0.55 & 0.24 & 1.43 & 4.71 & 0.00 & 0.00 & 0.00 & 0.00 \\
\hline Sulfate & 0.15 & 0.16 & 0.11 & 0.06 & 0.06 & 0.12 & 0.25 & 0.18 & 0.29 & 0.19 & 0.02 & 0.03 & 0.13 & 0.06 \\
\hline Hydroxyl Ion & 0.00 & 0.00 & 0.00 & 0.00 & 0.00 & 0.00 & 0.00 & 0.00 & 0.00 & 0.00 & 0.00 & 0.00 & 0.00 & 0.00 \\
\hline$\cdot$ & & & & & & & & & & & & & & ר \\
\hline Total Anions & 2.23 & 0.38 & 0.31 & 0.35 & 0.30 & 0.90 & 1.33 & 1.03 & 2.41 & 5.54 & 1.50 & 1.69 & 3.25 & 1.93 \\
\hline CaARGE BALAMCE (8) & $\dot{0}$ & $\dot{\theta}$ & -4 & -0 & 7 & 0 & -28 & 5 & 4 & 97 & -1 & $\dot{0}$ & 3 & -16 \\
\hline TDS (mg/I) & $\begin{array}{r}. \\
150\end{array}$ & 56 & 50 & 74 & $\dot{88}$ & 152 & 98 & 84 & 216 & 514 & 116 & $\dot{22}$ & 178 & 132 \\
\hline TDS (mg/L) & $<1$ & 2 & 6 & 194 & 57 & 11 & 2 & 4 & 4 & 12 & 24 & 4 & 15 & 39 \\
\hline Turbidity (Mru) & 0.9 & 11 & 27 & 50 & 800 & 7.5 & 11 & 3.9 & 4.1 & 7.2 & 34 & 4 & 12 & 64 \\
\hline
\end{tabular}

(COMTIMUED) 
APPEADIX $G$

Ion-Charge Balance Calculations, 1993

\begin{tabular}{|c|c|c|c|c|c|c|c|c|c|c|c|c|c|c|}
\hline \multirow{3}{*}{$\begin{array}{l}\text { Sampling Point } \\
\text { Location } \\
\text { Date Sampled }\end{array}$} & \multicolumn{4}{|c|}{$G W-348$} & \multicolumn{4}{|c|}{ Gu-364 } & \multicolumn{4}{|c|}{ Gu-365 } & \multicolumn{2}{|c|}{$60-370$} \\
\hline & \multicolumn{4}{|c|}{ s3 } & \multicolumn{4}{|c|}{ otr } & \multicolumn{4}{|c|}{ OLP } & \multicolumn{2}{|c|}{86} \\
\hline & $02 / 01 / 93$ & $05 / 27 / 93$ & 09/12/93 & $10 / 22 / 93$ & $03 / 27 / 93$ & $06 / 27 / 93$ & $09 / 19 / 93$ & $12 / 18 / 93$ & $03 / 28 / 93$ & $06 / 27 / 93$ & $09 / 19 / 93$ & $12 / 19 / 93$ & $02 / 17 / 93$ & $05 / 21 / 93$ \\
\hline CATrons (meq/L) & - & $\cdot$ & $\cdot$ & $\cdot$ & $\cdot$ & $\cdot$ & $\cdot$ & $\cdot$ & - & $\cdot$ & $\cdot$ & $\bullet$ & $\cdot$ & 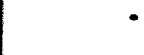 \\
\hline Calcium & 4.04 & 4.19 & 4.34 & 4.09 & 2.84 & 4.34 & 4.69 & 3.64 & 6.99 & 6.49 & 6.99 & 6.99 & 1.80 & 1.10 \\
\hline Magnesium & 1.40 & 1.48 & 1.48 & 1.56 & 0.81 & 1.40 & 1.48 & 0.99 & 2.14 & 2.06 & 2.14 & 2.22 & 0.27 & 0.20 \\
\hline Potassium & 0.05 & 0.04 & 0.04 & 0.04 & 0.04 & 0.07 & 0.07 & 0.05 & 0.08 & 0.08 & 0.09 & 0.08 & 0.00 & 0.00 \\
\hline Sodiun & 0.13 & 0.12 & 0.14 & 0.14 & 0.17 & 0.27 & 0.38 & 0.18 & 0.65 & 0.65 & 0.74 & 0.74 & 0.43 & 0.43 \\
\hline Hydrogen Ion & 0.00 & 0.00 & 0.00 & 0.00 & 0.00 & 0.00 & 0.00 & 0.00 & 0.00 & 0.00 & 0.00 & 0.00 & 0.00 & 0.00 \\
\hline - & $\cdot$ & & & & 2. & 6 & 6.62 & .86 &, $8 \dot{8}$ & 9.28 & 9.96 & 10.03 & 2.50 & 1.73 \\
\hline Total Cations & $\begin{array}{r}5.62 \\
.1\end{array}$ & 5.83 & 6.00 & $\begin{array}{r}3.83 \\
.\end{array}$ & .00 & $\begin{array}{r}0.00 \\
.1\end{array}$ & 0.02 & & & & . & 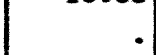 &. & \\
\hline AwIONS (meq/L) & $\cdot$ & $\cdot$ & $\cdot$ & $\cdot$ & $\cdot$ & $\cdot$ & $\cdot$ & $\cdot$ & $\cdot$ & $\bullet$ & $\cdot$ & $\cdot$ & $\cdot$ & $\bullet$ \\
\hline Alkalinity $-\mathrm{CO} 3$ & 0.00 & 0.00 & 0.00 & 0.00 & 0.00 & 0.00 & 0.00 & 0.00 & 0.00 & 0.00 & 0.00 & 0.00 & 0.00 & 0.00 \\
\hline Alkalinity-HCO3 & 4.64 & 4.64 & 4.96 & 4.88 & 3.38 & 4.74 & 4.52 & 3.86 & 6.67 & 6.95 & 6.87 & 6.91 & 2.26 & 1.56 \\
\hline Chloride & 0.26 & 0.25 & 0.25 & 0.28 & 0.44 & 1.18 & 1.10 & 0.48 & 2.06 & 2.45 & 2.62 & 2.43 & 0.04 & 0.04 \\
\hline Fluoride & 0.00 & 0.02 & 0.00 & 0.00 & 0.00 & 0.00 & 0.00 & 0.00 & 0.00 & 0.00 & 0.00 & 0.00 & 0.01 & 0.01 \\
\hline Nitrate Nitrogen & 0.69 & 0.61 & 0.67 & 0.81 & 0.09 & 0.08 & 0.73 & 0.20 & 0.21 & 0.29 & 0.37 & 0.28 & 0.00 & 0.00 \\
\hline Sulfate & 0.11 & 0.15 & 0.12 & 0.12 & 0.35 & 0.62 & 0.61 & 0.35 & 0.91 & 0.90 & 0.83 & 0.87 & 0.23 & 0.23 \\
\hline Hydroxyl Ion & 0.00 & 0.00 & 0.00 & 0.00 & 0.00 & 0.00 & 0.00 & 0.00 & 0.00 & 0.00 & 0.00 & 0.00 & 0.00 & 0.00 \\
\hline - & $\cdot 1$ & $\cdot$ & $=0$ & $\dot{0}$ & . & 6.62 & 6.96 & 4.89 & 9.85 & 10.60 & 10.70 & 10.49 & 2.53 & 1.83 \\
\hline Total Anlons & 3.10 & 3.00 & 3.00 & 0.10 & 8.00 &. & & 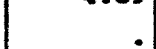 & 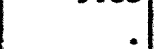 & & . & . &. & \\
\hline CHARGE BATANCE (8) & -1 & 1 & 0 & -2 & -5 & -4 & -2 & -0 & 0) & -7 & -4 & -2 & -1 & -3 \\
\hline$\overline{\operatorname{TDS}(\mathrm{mg} / L)}$ & 336 & 314 & 338 & 344 & 260 & 408 & 468 & 284 & 572 & 586 & 584 & 558 & 170 & 200 \\
\hline ISS $(\mathrm{mg} / \mathrm{L})$ & $<1$ & $<1$ & $<1$ & $<1$ & 8 & 34 & 241 & 6 & 5 & 2 & 1 & 1 & 18 & 307 \\
\hline Turbidity (NTO) & 1.2 & 1 & 1 & 2.7 & 29 & 250 & 320 & 21 & 9.5 & 7 & 2 & 5 & 7.8 & 5.5 \\
\hline
\end{tabular}

(COMTIMUED) 
APPESTIX G

Ion-Charge Balance Calculations, 1993

\begin{tabular}{|c|c|c|c|c|c|c|c|c|c|c|c|c|c|c|}
\hline \multirow{3}{*}{$\begin{array}{l}\text { Sampling Point } \\
\text { Location } \\
\text { Date Sampled }\end{array}$} & \multicolumn{2}{|c|}{$G W-370$} & \multicolumn{4}{|c|}{ Gw-371 } & \multicolumn{4}{|c|}{ Gw-372 } & \multicolumn{4}{|c|}{$60-373$} \\
\hline & \multicolumn{2}{|c|}{ BG } & \multicolumn{4}{|c|}{ BG } & \multicolumn{4}{|c|}{ BG } & \multicolumn{4}{|c|}{ BG } \\
\hline & $07 / 10 / 93$ & $10 / 14 / 93$ & $02 / 16 / 93$ & $05 / 21 / 93$ & 07/09/93 & $10 / 13 / 93$ & $02 / 09 / 93$ & 05/19/93 & $07 / 02 / 93$ & $10 / 12 / 93$ & $02 / 11 / 93$ & $|05 / 14 / 93|$ & $|07 / 02 / 93|$ & $10 / 11 / 93$ \\
\hline CATIONs (meq/L) & $\cdot$ & $\cdot$ & $\cdot$ & $\cdot$ & $\cdot$ & $\cdot$ & $\cdot$ & • & $\bullet$ & $\cdot$ & $\cdot$ & $\cdot$ & $\cdot$ & 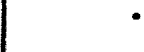 \\
\hline Calcium & 1.30 & 1.20 & 3.54 & 4.04 & 3.74 & 3.69 & 2.94 & 2.84 & 2.89 & 3.09 & 0.06 & 0.05 & 0.05 & 0.05 \\
\hline Magnesium & 0.23 & 0.21 & 1.56 & 1.73 & 1.65 & 1.65 & 0.90 & 0.90 & 0.90 & 0.90 & 0.01 & 0.04 & 0.01 & 0.01 \\
\hline Potassium & 0.00 & 0.00 & 0.08 & 0.09 & 0.08 & 0.09 & 0.04 & 0.04 & 0.04 & 0.04 & 0.03 & 0.02 & 0.04 & 0.03 \\
\hline Sodium & 0.43 & 0.43 & 0.74 & 0.78 & 0.78 & 0.74 & 0.37 & 0.37 & 0.40 & 0.40 & 4.35 & 4.78 & 4.78 & 4.78 \\
\hline Hydrogen Ion & 0.00 & 0.00 & 0.00 & 0.00 & 0.00 & 0.00 & 0.00 & 0.00 & 0.00 & 0.00 & 0.00 & 0.00 & 0.00 & 0.00 \\
\hline Total Cations & 1.96 & 1.83 & 5.92 & 6.64 & 6.25 & 6.17 & 4.26 & 4.16 & 4.24 & 4.44 & 4.46 & 4.90 & 4.88 & 4.87 \\
\hline ANIONS (meg/L) & $\cdot$ & . & $\cdot$ & $\cdot$ &. & $\cdot$ & . & $\cdot$ & - & $\cdot$ & $\cdot$ & $\cdot$ & $\cdot$ & - \\
\hline Alkalinity-CO3 & 0.00 & 0.00 & 0.00 & 0.00 & 0.00 & 0.00 & 0.00 & 0.00 & 0.00 & 0.00 & 0.52 & 0.48 & 0.52 & 0.00 \\
\hline Alkalinity-HCO3 & 1.82 & 1.52 & 5.82 & 5.94 & 6.08 & 5.98 & 3.68 & $3.60 !$ & 3.64 & 3.68 & 3.56 & 3.72 & 3.76 & 3.66 \\
\hline Chloride & 0.04 & 0.03 & 0.03 & 0.04 & 0.03 & 0.03 & 0.08 & 0.06 & 0.05 & 0.06 & 0.06 & 0.04 & 0.03 & 0.03 \\
\hline Fluoride & 0.01 & 0.01 & 0.00 & 0.00 & 0.01 & 0.00 & 0.01 & 0.01 & 0.01 & 0.01 & 0.08 & 0.07 & 0.09 & 0.08 \\
\hline Nitrate Nitrogen & 0.00 & 0.00 & 0.00 & 0.00 & 0.00 & 0.00 & 0.00 & 0.00 & 0.00 & 0.00 & 0.00 & 0.00 & 0.00 & 0.00 \\
\hline Sulfate & 0.26 & 0.21 & 0.32 & 0.37 & 0.35 & 0.35 & 0.69 & 0.65 & 0.65 & 0.65 & 0.62 & 0.60 & 0.65 & 0.56 \\
\hline Hydroxy 1 Ion & 0.00 & 0.00 & 0.00 & 0.00 & 0.00 & 0.00 & 0.00 & 0.00 & 0.00 & 0.00 & 0.01 & 0.01 & 0.01 & 0.01 \\
\hline$\cdot$ & & & & & & & & & & & & & & \\
\hline Total Anions & 2.12 & 1.76 & 6.17 & 6.35 & 6.47 & 6.36 & 4.45 & 4.31 & 4.35 & 4.39 & 4.86 & 4.93 & 5.06 & 4.34 \\
\hline CHARGE BALANCE (8) & -4 & 2 & $-\bar{z}$ & 2 & -2 & -2 & -2 & -2 & -1 & 1 & -4 & -0 & -2 & 6 \\
\hline $\operatorname{TDS}(m g / L)$ & 180 & 212 & 326 & 328 & 348 & 372 & 282 & 244 & 266 & 328 & 290 & 306 & 306 & 358 \\
\hline TsS $(m g / L)$ & 53 & 135 & 9 & 4 & 13 & $<1$ & 4 & 5 & 3 & 4 & 3 & 15 & 12 & 10 \\
\hline Turbidity (NTU) & 14 & 38 & 4.9 & 120 & 2.7 & 4.7 & 2.5 & 12 & 4.4 & 16 & 3.5 & 5.1 & 6.5 & 17 \\
\hline
\end{tabular}

(CONTINUED) 
APPENDIX G

Ion-Charge Balance Calculations, 1993

\begin{tabular}{|c|c|c|c|c|c|c|c|c|c|c|c|c|c|c|}
\hline \multirow{3}{*}{$\begin{array}{l}\text { Sampling Point } \\
\text { Location } \\
\text { Date Sampled }\end{array}$} & \multicolumn{4}{|c|}{$G W-526$} & \multicolumn{4}{|c|}{ GW-537 } & \multicolumn{4}{|c|}{$G W-601$} & \multirow{3}{*}{\begin{tabular}{|c|}
$G W-613$ \\
$S 3$ \\
$10 / 20 / 93$ \\
\end{tabular}} & \multirow{3}{*}{\begin{tabular}{|c|}
$G W-614$ \\
53 \\
$10 / 20 / 93$
\end{tabular}} \\
\hline & \multicolumn{4}{|c|}{ s3 } & \multicolumn{4}{|c|}{ OLF } & \multicolumn{4}{|c|}{ OLF } & & \\
\hline & $02 / 03 / 93$ & $06 / 03 / 93$ & $09 / 14 / 93$ & $10 / 26 / 93$ & $02 / 11 / 93$ & $05 / 12 / 93$ & $09 / 14 / 93$ & $10 / 22 / 93$ & $03 / 28 / 93$ & $06 / 27 / 93$ & $09 / 19 / 93$ & $12 / 21 / 94$ & & \\
\hline CATIONS (meg/L) & & & & & & & $\cdot$ & & & $\cdot 1$ & $\cdot$ & $\cdot 1$ & $\cdot$ & $\cdot$ \\
\hline Calcium & 5.49 & 4.99 & 5.99 & 4.99 & 49.90 & 54.89 & 54.89 & 54.89 & 3.79 & 4.09 & 4.44 & 4.09 & 1.50 & 2.35 \\
\hline Magnesium & 3.87 & 3.21 & 3.78 & 1.15 & 5.43 & 6.00 & 5.92 & 6.09 & 3.04 & 3.37 & 3.54 & 3.45 & 0.25 & 0.82 \\
\hline Potassium & 0.59 & 0.33 & 0.43 & 0.10 & 0.00 & 0.00 & 0.08 & 0.11 & 0.06 & 0.05 & 0.06 & 0.06 & 0.00 & 0.04 \\
\hline Sodium & 56.55 & 60.90 & 65.25 & 0.52 & 1.17 & 1.30 & 1.26 & 1.30 & 1.52 & 1.65 & 2.00 & 2.00 & 0.16 & 0.43 \\
\hline Hydrogen Ion & 0.00 & 0.00 & 0.00 & 0.00 & 0.00 & 0.00 & 0.00 & 0.00 & 0.00 & 0.00 & 0.00 & 0.00 & 0.00 & 0.00 \\
\hline Total Cations & 66.49 & 69.43 & 75.46 & 6.77 & 56.50 & 62.20 & 62.16 & 62.39 & 8.42 & 9.17 & 10.04 & 9.61 & 1.90 & 3.64 \\
\hline ANIONS (meg/L) & $\cdot 1$ & $\cdot$ &. & & & $\cdot$ &. & &. &. &. &. &. & $\cdot$ \\
\hline Alkalinity-CO3 & 0.00 & 0.04 & 0.00 & 0.00 & 0.00 & 0.00 & 0.00 & 0.00 & 0.00 & 0.00 & 0.00 & 0.00 & 0.00 & 0.00 \\
\hline Alkalinity-HCO3 & 1.00 & 0.98 & 0.86 & 0.96 & 4.92 & 4.94 & 5.04 & 5.04 & 3.76 & 3.90 & 3.86 & 3.84 & 1.76 & 3.36 \\
\hline Chloride & 0.54 & 0.16 & 0.59 & 0.54 & 0.85 & 0.90 & 0.73 & 0.93 & 2.43 & 3.70 & 2.57 & 2.85 & 0.03 & 0.03 \\
\hline Fluoride & 0.00 & 0.00 & 0.00 & 0.00 & 0.00 & 0.00 & 0.00 & 0.00 & 0.02 & 0.02 & 0.02 & 0.01 & 0.01 & 0.00 \\
\hline Nitrate Nitrogen & 58.98 & 71.83 & 60.41 & 83.61 & 55.84 & 49.41 & 37.49 & 56.52 & 2.29 & 2.97 & 2.18 & 2.28 & 0.00 & 0.00 \\
\hline Sulfate & 0.00 & 0.09 & 0.23 & 0.15 & 0.00 & 0.05 & 0.00 & 0.06 & 0.93 & 0.99 & 1.04 & 0.95 & 0.10 & 0.25 \\
\hline Hydroxyl Ion & 0.00 & 0.00 & 0.00 & 0.00 & 0.00 & 0.00 & 0.00 & 0.00 & 0.00 & 0.00 & 0.00 & 0.00 & 0.00 & 0.00 \\
\hline$\dot{T}$ Total Anions & 60.52 & 73.10 & 62.09 & 85.26 & 61.60 & 55.31 & 43.26 & 62.55 & 9.42 & 11.57 & 9.66 & 9.92 & 1.90 & 3.64 \\
\hline & & $\cdot$ &. & $\begin{array}{r}\cdot \\
-85\end{array}$ & -4 & $\dot{6}$ & $\begin{array}{r}\cdot \\
18\end{array}$ & $\dot{0}$ & -6 & $\begin{array}{r}\cdot \\
-12\end{array}$ & - & $\dot{-2}$ & $\dot{0}$ & $\dot{0}$ \\
\hline CHARGE BALANCE (\&) & & $\begin{array}{r}-3 \\
.\end{array}$ &. &. & & & & &. & & $\cdot$ & $\cdot 1$ & $\cdot 1$ & $\cdot$ \\
\hline TDS (mg/L) & 6214 & 6028 & 6246 & 6320 & 4700 & 4682 & 4438 & 4478 & 540 & 542 & 584 & 560 & 144 & 224 \\
\hline TSS (mg/L) & 14 & 9 & 8 & 28 & 4 & 5 & 4 & 5 & 1 & 13 & $<1$ & $<1$ & 8 & 5 \\
\hline Turbidity (NTU) & 6.3 & 7.4 & 12 & 26 & 1.9 & 1.1 & 1.6 & 1.9 & 0.8 & 0.5 & 0.5 & 0.7 & 3.1 & 2 \\
\hline
\end{tabular}

(CONTINUED) 
APPBNDIX 6

Ion-Charge be Calculations, 1993

\begin{tabular}{|c|c|c|c|c|c|c|c|c|c|c|c|c|c|c|}
\hline \multirow{3}{*}{$\begin{array}{l}\text { Sampling Point } \\
\text { Location } \\
\text { Date Sampled }\end{array}$} & \multicolumn{4}{|c|}{ GW-621 } & \multicolumn{4}{|c|}{ GW-626 } & \multicolumn{4}{|c|}{$G W-627$} & \multicolumn{2}{|c|}{$G W-636$} \\
\hline & \multicolumn{4}{|c|}{ BG } & \multicolumn{4}{|c|}{ BG } & \multicolumn{4}{|c|}{ BG } & \multicolumn{2}{|c|}{ OLF } \\
\hline & $03 / 20 / 93$ & $06 / 02 / 93$ & $09 / 20 / 93$ & $12 / 20 / 93$ & $03 / 10 / 93$ & $04 / 26 / 93$ & $08 / 02 / 93$ & $10 / 20 / 93$ & $03 / 09 / 93$ & $04 / 23 / 93$ & $07 / 28 / 93$ & $10 / 20 / 93$ & $03 / 26 / 93$ & $06 / 27 / 93$ \\
\hline CATIONS (meq/L) & - & $\cdot$ & $\cdot$ & $\cdot$ & $\cdot$ & • & $\cdot$ & $\cdot$ & $\cdot$ & $\cdot$ & $\cdot$ & $\cdot$ & $\bullet$ & • \\
\hline Calcium & 2.64 & 2.64 & 3.04 & 2.64 & 2.10 & 1.80 & 1.90 & 2.25 & 0.05 & 2.64 & 0.05 & 0.04 & 0.37 & 0.39 \\
\hline Magnesium & 0.76 & 0.75 & 0.99 & 0.81 & 0.23 & 0.21 & 0.24 & 0.26 & 0.01 & 1.97 & 0.03 & 0.02 & 0.19 & 0.21 \\
\hline Potassium & 0.03 & 0.04 & 0.02 & 0.04 & 0.03 & 0.02 & 0.03 & 0.03 & 0.04 & 0.06 & 0.04 & 0.03 & 0.14 & 0.12 \\
\hline sodium & 0.10 & 0.12 & 0.15 & 0.11 & 0.18 & 0.17 & 0.18 & 0.17 & 12.18 & 0.34 & 11.75 & 12.18 & 3.04 & 2.96 \\
\hline Hydrogen Ion & 0.00 & 0.00 & 0.00 & 0.00 & 0.00 & 0.00 & 0.00 & 0.00 & 0.00 & 0.00 & 0.00 & 0.00 & 0.00 & 0.00 \\
\hline Total Cations & 3.54 & 3.55 & 4.21 & 3.60 & 2.53 & 2.20 & 2.34 & 2.71 & 12.28 & 5.02 & 11.86 & 12.27 & 3.74 & 3.68 \\
\hline ANIONS (meq/L) & & $\cdot$ & $\cdot$ & & - & $\cdot$ & $\cdot$ & $\cdot$ & $\cdot$ & $\cdot$ & $\cdot$ & $\cdot$ & $\cdot$ & • \\
\hline Alkalinity-CO3 & 0.00 & 0.00 & 0.00 & 0.00 & 0.00 & 0.00 & 0.00 & 0.00 & 1.32 & 1.60 & 0.00 & 1.24 & 0.12 & 0.04 \\
\hline Alkalinity-HCO3 & 3.02 & 3.04 & 3.34 & 3.14 & 2.18 & 2.06 & 2.16 & 2.24 & 9.37 & 8.93 & 10.81 & 9.59 & 3.26 & 3.58 \\
\hline Chloride & 0.10 & 0.15 & 0.22 & 0.14 & 0.36 & 0.21 & 0.17 & 0.28 & 0.62 & 0.62 & 0.93 & 0.73 & 0.17 & 0.22 \\
\hline Fluoride & 0.01 & 0.00 & 0.00 & 0.00 & 0.00 & 0.01 & 0.00 & 0.00 & 0.25 & 0.29 & 0.32 & 0.32 & 0.01 & 0.01 \\
\hline Nitrate Nitrogen & 0.32 & 0.43 & 0.72 & 0.29 & 0.00 & 0.00 & 0.00 & 0.00 & 0.00 & 0.00 & 0.01 & 0.00 & 0.00 & 0.00 \\
\hline Sulfate & 0.09 & 0.11 & 0.17 & 0.06 & 0.08 & 0.07 & 0.06 & 0.06 & 0.67 & 0.65 & 0.79 & 0.62 & 0.19 & 0.14 \\
\hline Hydroxyl Ion & 0.00 & 0.00 & 0.00 & 0.00 & 0.00 & 0.00 & 0.00 & 0.00 & 0.01 & 0.01 & 0.01 & 0.02 & 0.00 & 0.00 \\
\hline & & & & & & & & & & & & & & \\
\hline Total Anions & 3.53 & 3.72 & 4.45 & 3.62 & 2.62 & 2.35 & 2.40 & 2.58 & 12.24 & 12.10 & 12.88 & 12.52 & 3.75 & 3.99 \\
\hline CHARGE BALANCE (8) & 0 & -2 & -3 & -0 & -2 & -3 & -1 & 2 & 0 & -41 & -4 & -1 & -0 & -4 \\
\hline TDS $(\mathrm{mg} / \mathrm{L})$ & 222 & 232 & $27 \dot{2}$ & 198 & $16 \dot{6}$ & 162 & 146 & 182 & 704 & 732 & $728^{\circ}$ & 738 & 226 & 230 \\
\hline 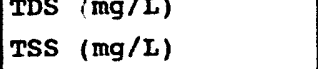 & 5 & 23 & 37 & 34 & 1 & 1 & $<1$ & $<1$ & $<1$ & $<1$ & $<1$ & 2 & 7 & $<1$ \\
\hline Turbidity (NTU) & 14 & 34 & 35 & 26 & 0.5 & 0.8 & 0.8 & 0.3 & 1 & 1 & 2.7 & 0.9 & 5.5 & 2 \\
\hline
\end{tabular}

(CONTINUED) 
Ion-Charge Balance Calculations, 1993

\begin{tabular}{|c|c|c|c|c|c|c|c|c|c|c|c|c|c|c|}
\hline \multirow{3}{*}{$\begin{array}{l}\text { Sampling Point } \\
\text { Location } \\
\text { Date Sampled }\end{array}$} & \multicolumn{2}{|c|}{ GW-636 } & \multicolumn{4}{|c|}{ GW-637 } & \multicolumn{4}{|c|}{$G W-638$} & \multicolumn{4}{|c|}{ GW-639 } \\
\hline & \multicolumn{2}{|c|}{ OLF } & \multicolumn{4}{|c|}{ OLF } & \multicolumn{4}{|c|}{ OLF } & \multicolumn{4}{|c|}{ BG } \\
\hline & $09 / 18 / 93$ & $12 / 18 / 93$ & $03 / 25 / 93$ & $06 / 26 / 93$ & $|09 / 17 / 93|$ & $12 / 18 / 93$ & $03 / 25 / 93$ & $06 / 26 / 93$ & $09 / 17 / 93$ & $12 / 18 / 93$ & $02 / 24 / 93 \mid$ & $05 / 24 / 93$ & $07 / 11 / 93$ & $10 / 15 / 93$ \\
\hline CATIONS (meq/L) & & $\cdot$ & $\cdot$ & $\cdot$ & $\cdot$ & $\cdot$ & $\cdot$ & $\cdot$ & • & $\cdot$ & $\cdot$ & $\cdot$ & $\cdot$ & \\
\hline Calcium & 0.38 & 0.45 & 3.59 & 3.79 & 3.94 & 3.69 & 3.14 & 4.99 & 6.49 & 3.84 & 0.07 & 0.04 & 0.04 & 0.04 \\
\hline Magnesium & 0.21 & 0.24 & 0.43 & 0.49 & 0.45 & 0.50 & 0.58 & 0.99 & 1.40 & 0.90 & 0.03 & 0.02 & 0.02 & 0.02 \\
\hline Potassium & 0.13 & 0.12 & 0.05 & 0.03 & 0.04 & 0.04 & 0.04 & 0.07 & 0.10 & 0.06 & 0.05 & 0.04 & 0.03 & 0.04 \\
\hline Sodium & 3.26 & 3.13 & 0.12 & 0.10 & 0.12 & 0.12 & 0.24 & 0.20 & 0.14 & 0.19 & 9.14 & 8.70 & 8.70 & 9.57 \\
\hline Hydrogen Ion & 0.00 & 0.00 & 0.00 & 0.00 & 0.00 & 0.00 & 0.00 & 0.00 & 0.00 & 0.00 & 0.00 & 0.00 & 0.00 & 0.00 \\
\hline Total Cations & 3.99 & 3.94 & 4.18 & 4.42 & 4.55 & 4.35 & 4.00 & 6.25 & 8.13 & 5.00 & 9.28 & 8.80 & 8.79 & 9.68 \\
\hline ANIONS (meq/L) & &. & & & & $\cdot$ & & & & . & $\cdot$ &. & & $\cdot$ \\
\hline Alkalinity- $\mathrm{CO} 3$ & 0.00 & 0.08 & 0.00 & 0.00 & 0.02 & 0.00 & 0.00 & 0.00 & 0.00 & $0.0 \dot{0}$ & 1.60 & 1.44 & 1.52 & 1.28 \\
\hline Alkalinity-HCO3 & 3.58 & 3.48 & 4.10 & 4.26 & 4.32 & 4.24 & 3.28 & 4.04 & 4.10 & 4.02 & 6.39 & 6.89 & 6.91 & 7.03 \\
\hline Chloride & 0.20 & 0.25 & 0.07 & 0.08 & 0.08 & 0.08 & 0.05 & 0.04 & 0.00 & 0.03 & 0.28 & 0.28 & 0.28 & 0.28 \\
\hline Fluoride & 0.01 & 0.00 & 0.00 & 0.00 & 0.00 & 0.00 & 0.02 & 0.02 & 0.02 & 0.01 & 0.12 & 0.13 & 0.16 & 0.14 \\
\hline Nitrate Nitrogen & 0.00 & 0.00 & 0.00 & 0.00 & 0.00 & 0.00 & 0.02 & 0.03 & 0.03 & 0.00 & 0.00 & 0.00 & 0.00 & 0.00 \\
\hline Sulfate & 0.10 & 0.08 & 0.16 & 0.18 & 0.12 & 0.12 & 0.96 & 1.35 & 3.71 & 0.92 & 0.31 & 0.61 & 0.62 & 0.56 \\
\hline Hydroxyl Ion & 0.00 & 0.00 & 0.00 & 0.00 & 0.00 & 0.00 & 0.00 & 0.00 & 0.00 & 0.00 & 0.02 & 0.02 & 0.02 & 0.02 \\
\hline Total Anions & 3.89 & 3.90 & 4.32 & 4.52 & 4.55 & 4.45 & 4.32 & 5.47 & 7.85 & 4.97 & 8.72 & 9.37 & 9.51 & 9.31 \\
\hline CHARGE BALANCE (₹) & $\dot{1}$ & $\dot{1}$ & $\dot{-2}$ & $\dot{-1}$ & $\dot{0}$ & $\dot{-1}$ & -4 & $\dot{7}$ & $\dot{2}$ & $\dot{0}$ & $\dot{3}$ & -3 & -4 & $\dot{2}$ \\
\hline &. & $\cdot 1$ & $\cdot$ & $\cdot$ & $\cdot$ & & $\cdot$ & $\cdot$ & • & $\cdot$ & $\cdot$ & $\cdot$ & $\cdot$ & $\cdot$ \\
\hline $\operatorname{TDS}(\mathrm{mg} / \mathrm{L})$ & 240 & 236 & 252 & 254 & 258 & 262 & 308 & 550 & 516 & 312 & 542 & 562 & 546 & 564 \\
\hline TSS (mg/L) & 1 & 14 & 4 & 2 & $<1$ & 15 & 48 & 5405 & 91 & 31 & 21 & 24 & 6 & 12 \\
\hline Turbidity (NTU) & 1.5 & 0.9 & 6 & 1.5 & 5.7 & 1.8 & 54 & 3100 & 370 & 37 & 14 & 60 & 6.9 & 80 \\
\hline
\end{tabular}

(CONTINUED) 
APPENDIX G

Ion-Charge Balance Calculations, 1993

\begin{tabular}{|c|c|c|c|c|c|c|c|c|c|c|c|c|c|c|}
\hline \multirow{3}{*}{$\begin{array}{l}\text { Sampling Point } \\
\text { Location } \\
\text { Date Sampled }\end{array}$} & \multicolumn{4}{|c|}{ GW-640 } & \multicolumn{4}{|c|}{$G W-641$} & \multicolumn{4}{|c|}{$G W-642$} & \multicolumn{2}{|c|}{$G W-643$} \\
\hline & \multicolumn{4}{|c|}{ BG } & \multicolumn{4}{|c|}{ BG } & \multicolumn{4}{|c|}{ BG } & \multicolumn{2}{|l|}{ BG } \\
\hline & $02 / 22 / 93$ & $05 / 24 / 93$ & $07 / 10 / 93$ & $10 / 14 / 93$ & $02 / 22 / 93$ & $05 / 24 / 93$ & $07 / 10 / 93$ & $10 / 14 / 93$ & $02 / 08 / 93$ & $05 / 11 / 93$ & $07 / 01 / 93$ & $10 / 10 / 93$ & $02 / 16 / 930$ & $05 / 19 / 93$ \\
\hline CATrons (meg/L) & $\cdot$ & $\cdot$ & $\cdot$ & $\cdot$ & $\cdot$ & $\cdot$ & $\cdot$ & $\cdot$ & $\cdot$ & $\cdot$ & $\cdot$ & $\cdot$ & $\cdot$ & \\
\hline Calcium & 0.70 & 0.70 & 0.75 & 0.90 & 0.11 & 0.24 & 0.20 & 0.22 & 0.35 & 0.37 & 0.34 & 0.46 & 0.13 & 0.19 \\
\hline Magnesium & 0.12 & 0.13 & 0.12 & 0.14 & 0.07 & 0.08 & 0.07 & 0.07 & 0.63 & 0.61 & 0.62 & 0.59 & 0.09 & 0.12 \\
\hline Potassium & 0.03 & 0.02 & 0.02 & 0.02 & 0.03 & 0.02 & 0.00 & 0.00 & 0.04 & 0.05 & 0.05 & 0.04 & 0.03 & 0.02 \\
\hline Sodium & 0.18 & 0.16 & 0.16 & 0.16 & 0.08 & 0.11 & 0.10 & 0.10 & 0.32 & 0.33 & 0.34 & 0.34 & 0.35 & 0.26 \\
\hline Hydrogen Ion & 0.00 & 0.00 & 0.00 & 0.00 & 0.00 & 0.00 & 0.00 & 0.00 & 0.00 & 0.00 & 0.00 & 0.00 & 0.00 & 0.00 \\
\hline - & 1.03 & 1.02 & 1.05 & 1.22 & 0.28 & 0.44 & 0.37 & 0.38 & 1.34 & 1.36 & 1.35 & 1.43 & 0.59 & 0.60 \\
\hline ANIONS (meq/L) & & & $\cdot$ & . & $\cdot$ & $\cdot$ & $\cdot$ & $\cdot$ &. & $\cdot$ & $\cdot$ & $\cdot$ & $\cdot$ & $\bullet$ \\
\hline Alkalinity- $\mathrm{CO} 3$ & 0.00 & 0.00 & 0.00 & 0.00 & 0.00 & 0.00 & 0.00 & 0.00 & 0.00 & 0.00 & 0.00 & 0.00 & 0.00 & 0.00 \\
\hline Alkalinity-HCO3 & 0.86 & 1.00 & 0.98 & 0.98 & 0.32 & 0.40 & 0.36 & 0.36 & 1.20 & 1.16 & 1.14 & 1.14 & 0.24 & 0.28 \\
\hline Chloride & 0.03 & 0.03 & 0.03 & 0.06 & 0.00 & 0.00 & 0.00 & 0.03 & 0.00 & 0.05 & 0.03 & 0.04 & 0.04 & 0.05 \\
\hline Fluoride & 0.00 & 0.00 & 0.00 & $0: 00$ & 0.00 & 0.00 & 0.00 & 0.00 & 0.02 & 0.02 & 0.02 & 0.02 & 0.00 & 0.00 \\
\hline Nitrate Nitrogen & 0.00 & 0.00 & 0.00 & 0.00 & 0.00 & 0.00 & 0.00 & 0.00 & 0.00 & 0.00 & 0.00 & 0.00 & 0.02 & 0.00 \\
\hline Sulfate & 0.04 & 0.06 & 0.05 & 0.04 & 0.00 & 0.04 & 0.00 & 0.00 & 0.22 & 0.33 & 0.25 & 0.20 & 0.30 & 0.33 \\
\hline Hydroxyl Ion & 0.00 & 0.00 & 0.00 & 0.00 & 0.00 & 0.00 & 0.00 & 0.00 & 0.00 & 0.00 & 0.00 & 0.00 & 0.00 & 0.00 \\
\hline - & 0.93 & 1.09 & 1.06 & 1.08 & 0.32 & 0.44 & 0.36 & 0.39 & 1.44 & 1.56 & 1.44 & 1.41 & 0.60 & 0.67 \\
\hline & & &. & & &. & & &. & $\cdot 1$ & $\cdot 1$ & $\cdot$ &. & . \\
\hline CHARGE BALANCE (₹) & 5 & -4 & -0 & 6 & -6 & 0 & 1 & -1 & -4 & -7 & -3 & 1 & -0 & -5 \\
\hline TDS (mg/L) & 70 & 76 & 78 & 130 & 42 & 48 & 36 & 110 & 136 & 110 & 106 & 186 & 82 & 86 \\
\hline TSS (mg/L) & 7 & 4 & 5 & 5 & 83 & 3 & 3 & 9 & 9 & 5 & 3 & 540 & 135 & 51 \\
\hline Turbidity (NTU) & 2.4 & 3.1 & 3.1 & 2.2 & 7 & 4.5 & 0.9 & 39 & 19 & 5.4 & 12 & 60 & 56 & 25 \\
\hline
\end{tabular}

(CONTINUED) 
Ion-Charge Balance Calculations, 1993

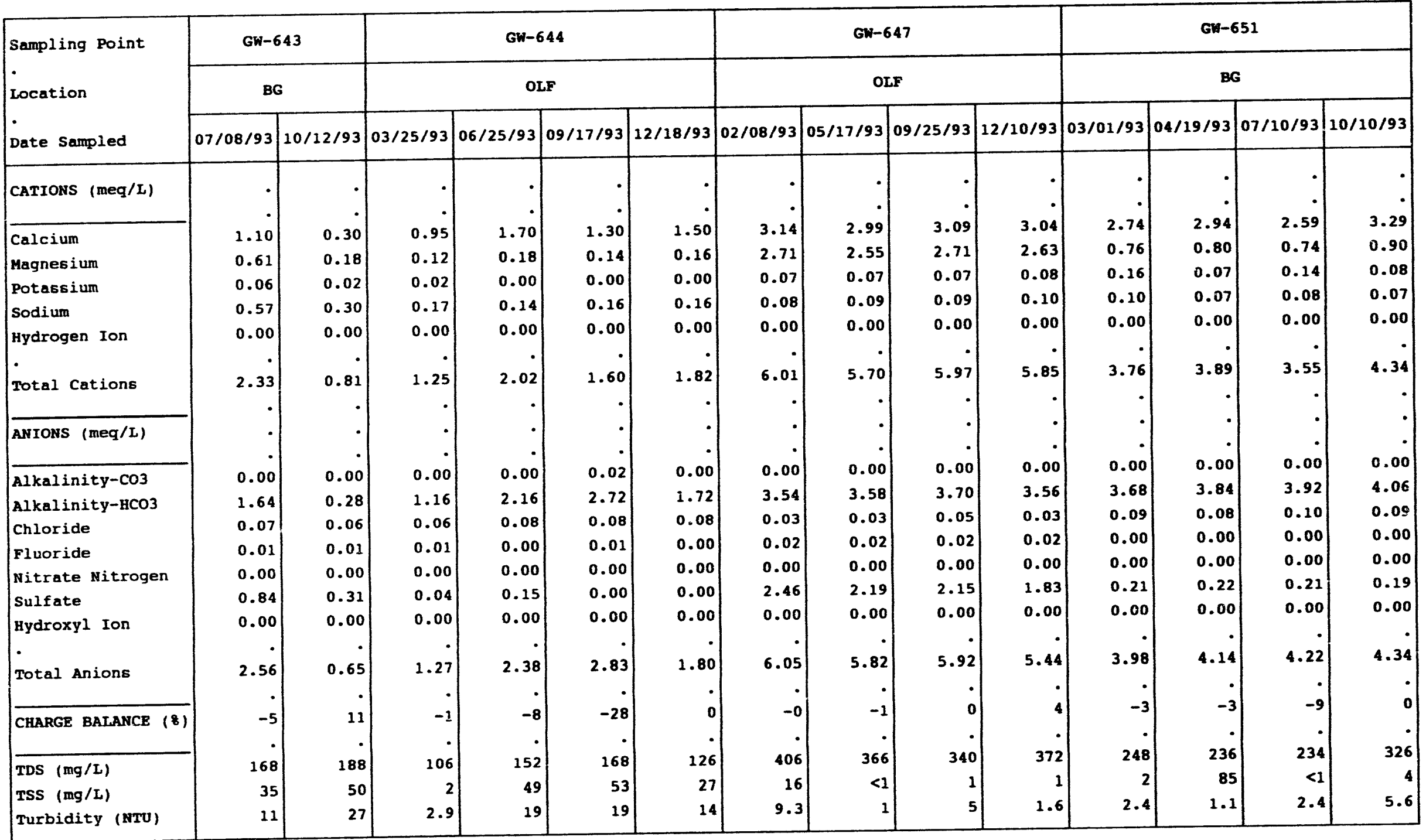

(CONTINUED) 
APPENDIX $G$

Ion-Charge Balance Calculations, 1993

\begin{tabular}{|c|c|c|c|c|c|c|c|c|c|c|c|c|c|c|}
\hline \multirow{3}{*}{$\begin{array}{l}\text { Sampling Point } \\
\text { Location } \\
\text { Date Sampled }\end{array}$} & \multicolumn{4}{|c|}{ GW-652 } & \multicolumn{4}{|c|}{$G W-653$} & \multicolumn{4}{|c|}{$G W-654$} & \multicolumn{2}{|c|}{ GW-655 } \\
\hline & \multicolumn{4}{|c|}{ BG } & \multicolumn{4}{|c|}{ BG } & \multicolumn{4}{|c|}{ BG } & \multicolumn{2}{|c|}{ BG } \\
\hline & $03 / 01 / 93$ & $04 / 19 / 93$ & $07 / 10 / 93$ & $10 / 18 / 93$ & $03 / 01 / 93$ & $04 / 20 / 93$ & $07 / 10 / 93$ & $10 / 10 / 93$ & $02 / 24 / 93$ & $04 / 16 / 93$ & $07 / 01 / 93$ & $10 / 07 / 93$ & $02 / 05 / 93$ & $05 / 10 / 93$ \\
\hline CATIONS (meq/L) & - & - & - & - & - & $\cdot$ & $\cdot$ & - & - & $\cdot$ & - & - & • & - \\
\hline Calcium & 3.69 & 3.44 & 3.64 & 3.84 & 0.09 & 0.09 & 0.09 & 0.08 & 0.70 & 0.75 & 0.65 & 0.80 & 0.15 & 0.15 \\
\hline Magnesium & 0.55 & 0.49 & 0.52 & 0.63 & 0.08 & 0.08 & 0.08 & 0.08 & 0.29 & 0.30 & 0.27 & 0.35 & 0.43 & 0.40 \\
\hline Potassium & 0.07 & 0.06 & 0.06 & 0.07 & 0.02 & 0.00 & 0.02 & 0.00 & 0.04 & 0.03 & 0.04 & 0.04 & 0.03 & 0.04 \\
\hline Sodium & 0.04 & 0.04 & 0.04 & 0.05 & 0.11 & 0.11 & 0.11 & 0.11 & 1.26 & 1.30 & 1.91 & 1.61 & 0.25 & 0.25 \\
\hline Hydrogen Ion & 0.00 & 0.00 & 0.00 & 0.00 & 0.00 & 0.00 & 0.00 & 0.00 & 0.00 & 0.00 & 0.00 & 0.00 & 0.00 & 0.00 \\
\hline - & & & & & $\cdot$ & & & & : & & & 8 & 0 & 0 \\
\hline Total Cations & 4.36 & 4.04 & 4.26 & 4.60 & 0.31 & 0.28 & 0.30 & 0.28 & 2.28 & 2.38 & 2.88 & 2.81 & 0.86 & 0.84 \\
\hline ANIONS (meq/L) & $\cdot$ & - & $\cdot$ & - & $\cdot$ &. & $\cdot$ & $\cdot$ & - & . & . & - & - & • \\
\hline Alkalinity-CO3 & 0.00 & 0.00 & 0.00 & 0.00 & 0.00 & 0.00 & 0.00 & 0.00 & 0.00 & 0.00 & 0.00 & 0.00 & 0.00 & 0.00 \\
\hline Alkalinity-HCO3 & 3.86 & 3.74 & 4.16 & 4.16 & 1.72 & 0.28 & 0.28 & 0.28 & 2.00 & 2.16 & 2.30 & 2.54 & 0.68 & 0.64 \\
\hline Chloride & 0.12 & 0.16 & 0.16 & 0.17 & 0.03 & 0.03 & 0.03 & 0.03 & 0.00 & 0.04 & 0.00 & 0.00 & 0.00 & 0.04 \\
\hline Fluoride & 0.00 & 0.00 & 0.00 & 0.00 & 0.00 & 0.00 & 0.00 & 0.00 & 0.02 & 0.02 & 0.03 & 0.03 & 0.01 & 0.01 \\
\hline Nitrate Nitrogen & 0.00 & 0.00 & 0.00 & 0.03 & 0.00 & 0.00 & 0.00 & 0.00 & 0.00 & 0.00 & 0.00 & 0.00 & 0.00 & 0.00 \\
\hline Sulfate & 0.16 & 0.17 & 0.18 & 0.16 & 0.06 & 0.05 & 0.04 & 0.06 & 0.23 & 0.33 & 0.33 & 0.31 & 0.19 & 0.29 \\
\hline Hydroxyl Ion & 0.00 & 0.00 & 0.00 & 0.00 & 0.00 & 0.00 & 0.00 & 0.00 & 0.00 & 0.00 & 0.00 & 0.00 & 0.00 & 0.00 \\
\hline . & & & & & $\cdot$ & & & & - & $\cdot$ & & - & $\cdot$ & \\
\hline Total Anions & 4.14 & 4.07 & 4.50 & 4.52 & 1.81 & 0.36 & 0.35 & 0.37 & 2.24 & 2.56 & 2.66 & 2.88 & 0.88 & 0.98 \\
\hline CHARGE BALANCE (8) & 3 & -0 & -3 & 1 & -70 & -11 & -8 & -15 & 1 & -3 & 4 & -1 & -1 & -8 \\
\hline & $\cdot$ & - & $\cdot$ & - & $\cdot$ & $\cdot$ & $\cdot$ & $\cdot$ & - & • & - & • & $\cdot$ & - \\
\hline $\operatorname{TDS}(\mathrm{mg} / L)$ & 284 & 266 & 250 & 282 & 70 & 56 & 46 & 142 & 154 & 154 & 182 & 258 & 92 & 150 \\
\hline TSS (mg/L) & 17 & 12 & 5 & $<1$ & 314 & 82 & $<1$ & 158 & 4 & 5 & $<1$ & $<1$ & 22 & 624 \\
\hline Turbidity (NTU) & 44 & 1.9 & 11 & 28 & 70 & 29 & 12 & 42 & 18 & 13 & 2.4 & 3.5 & 22 & 180 \\
\hline
\end{tabular}

(CONTINUED) 
Ion-Charge Balance Calculations, 1993

\begin{tabular}{|c|c|c|c|c|c|c|c|c|c|c|c|c|c|c|}
\hline \multirow{3}{*}{$\begin{array}{l}\text { Sampling Point } \\
\text { Location } \\
\text { - } \\
\text { Date Sampled }\end{array}$} & \multicolumn{2}{|c|}{ GW-655 } & \multicolumn{4}{|c|}{$G W-683$} & \multicolumn{4}{|c|}{$G W-684$} & \multicolumn{4}{|c|}{$G w-685$} \\
\hline & \multicolumn{2}{|c|}{ BG } & \multicolumn{4}{|c|}{$\mathbf{E X P}$} & \multicolumn{4}{|c|}{$\mathbf{E x P}$} & \multicolumn{4}{|c|}{ EXP } \\
\hline & $07 / 01 / 93$ & $10 / 06 / 93$ & $03 / 04 / 93$ & $04 / 23 / 93$ & $07 / 12 / 93$ & $10 / 12 / 93$ & $03 / 03 / 93$ & $04 / 22 / 93$ & $07 / 11 / 93$ & $10 / 12 / 93$ & $03 / 02 / 93$ & $04 / 21 / 93$ & $07 / 11 / 93$ & $10 / 11 / 93$ \\
\hline CATIONS (meg/L) & & & $\cdot$ & & &. & &. & &. & & &. & $\cdot$ \\
\hline Calcium & 0.17 & 0.14 & 1.90 & 1.70 & 3.04 & 4.69 & 2.00 & 1.60 & 2.79 & 3.24 & 4.19 & 3.79 & 4.04 & 4.14 \\
\hline Magnesium & 0.46 & 0.40 & 1.23 & 1.15 & 1.56 & 1.89 & 1.07 & 0.99 & 1.40 & 1.48 & 1.97 & 1.73 & 1.97 & 1.97 \\
\hline Potassium & 0.05 & 0.03 & 0.03 & 0.02 & 0.09 & 0.06 & 0.38 & 0.51 & 0.41 & 0.36 & 0.04 & 0.03 & 0.03 & 0.04 \\
\hline Sodium & 0.29 & 0.25 & 0.16 & 0.15 & 0.37 & 0.61 & 0.25 & 0.27 & 0.43 & 0.78 & 1.48 & 1.22 & 0.96 & 0.96 \\
\hline Hydrogen Ion & 0.00 & 0.00 & 0.00 & 0.00 & 0.00 & 0.00 & 0.00 & 0.00 & 0.00 & 0.00 & 0.00 & 0.00 & 0.00 & 0.00 \\
\hline Total Cations & 0.98 & 0.83 & 3.32 & 3.02 & 5.07 & 7.25 & 3.70 & 3.37 & 5.04 & 5.87 & 7.69 & 6.77 & 7.01 & 7.12 \\
\hline ANIONS (meq/L) & & & & & & $\cdot$ & & $\cdot$ & $\cdot$ & & $\cdot$ & $\cdot$ &. & $\cdot$ \\
\hline Alkalinity-CO3 & 0.00 & 0.00 & 0.00 & 0.00 & 0.00 & 0.00 & 0.00 & 0.00 & 0.00 & 0.00 & 0.00 & 0.00 & 0.00 & 0.00 \\
\hline Alkalinity-HCO3 & 0.68 & 0.72 & 2.76 & 2.64 & 3.82 & 4.28 & 2.58 & 2.52 & 3.40 & 3.48 & 5.00 & 4.90 & 5.06 & 5.04 \\
\hline Chloride & 0.00 & 0.00 & 0.20 & 0.16 & 0.41 & 0.87 & 0.48 & 0.45 & 0.59 & 0.79 & 1.95 & 2.65 & 1.38 & 1.41 \\
\hline Fluoride & 0.01 & 0.01 & 0.01 & 0.01 & 0.01 & 0.01 & 0.01 & 0.01 & 0.02 & 0.01 & 0.00 & 0.01 & 0.01 & 0.01 \\
\hline Nitrate Nitrogen & 0.00 & 0.00 & 0.19 & 0.16 & 0.71 & 2.28 & 0.31 & 0.29 & 0.74 & 1.35 & 0.12 & 0.12 & 0.15 & 0.29 \\
\hline Sulfate & 0.21 & 0.17 & 0.15 & 0.23 & 0.35 & 0.54 & 0.27 & 0.29 & 0.35 & 0.00 & 0.40 & 0.38 & 0.41 & 0.44 \\
\hline Hydroxyl Ion & 0.00 & 0.00 & 0.00 & 0.00 & 0.00 & 0.00 & 0.00 & 0.00 & 0.00 & 0.00 & 0.00 & 0.00 & 0.00 & 0.00 \\
\hline Total Anions & 0.90 & 0.89 & 3.30 & 3.20 & 5.30 & 7.99 & 3.64 & 3.57 & 5.08 & 5.63 & 7.46 & 8.05 & 7.00 & 7.18 \\
\hline CHARGE BALANCE (8) & 4 & -4 & 0 & -3 & -2 & -5 & 1 & -3 & -0 & 2 & 2 & -9 & 0 & $-\dot{0}$ \\
\hline TDS $(\mathrm{mg} / \mathrm{L})$ & 74 & 64 & 178 & 160 & 308 & 504 & 226 & 222 & 322 & 432 & 412 & 422 & 388 & 456 \\
\hline TSS (mg/L) & 13 & 54 & 2 & 1 & $<1$ & 2 & $<1$ & $<1$ & $<1$ & 3 & 12 & 10 & 2 & 5 \\
\hline Turbidity (NTU) & 11 & 16 & 2 & 1.3 & 0.9 & 2.5 & 3.9 & 1.5 & 1 & 4.5 & 52 & 12 & 2.9 & 18 \\
\hline
\end{tabular}

(CONTINUED) 
APPENDIX G

Ion-Charge Balance Calculations, 1993

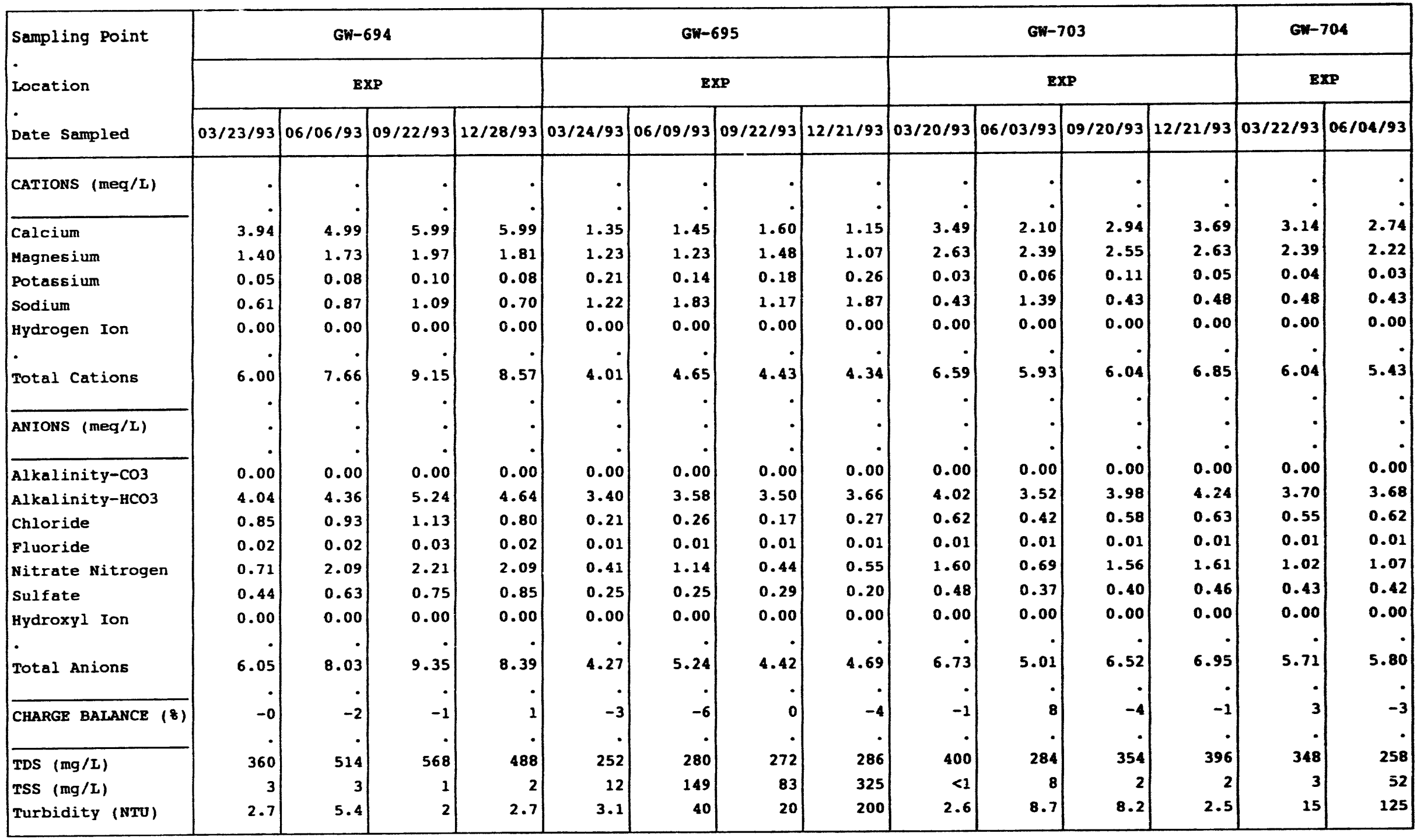

(CONTINUED) 
APPENDIX 6

Ion-Charge Balance Calculations, 1993

\begin{tabular}{|c|c|c|c|c|c|c|c|c|c|c|c|c|c|c|}
\hline \multirow{3}{*}{$\begin{array}{l}\text { Sampling point } \\
\text { Location } \\
\text { Date Sampled }\end{array}$} & \multicolumn{2}{|c|}{ GW-704 } & \multicolumn{4}{|c|}{ GW-706 } & \multicolumn{4}{|c|}{$G W-710$} & \multicolumn{4}{|c|}{$6 w-711$} \\
\hline & \multicolumn{2}{|c|}{$\mathbf{E X P}$} & \multicolumn{4}{|c|}{ EXP } & \multicolumn{4}{|c|}{$\mathbf{E X P}$} & \multicolumn{4}{|c|}{$\mathbf{E X P}$} \\
\hline & $09 / 22 / 93$ & $12 / 22 / 93$ & $03 / 23 / 93$ & $06 / 11 / 93$ & $09 / 23 / 93$ & $12 / 28 / 93$ & $03 / 21 / 93$ & $04 / 30 / 93$ & $08 / 01 / 93$ & $11 / 09 / 93$ & $03 / 20 / 93$ & $04 / 29 / 93$ & $07 / 30 / 93$ & $11 / 07 / 93$ \\
\hline CATIONS (meg/L) & $\cdot$ & - & $\cdot$ & $\cdot$ & - & - & - & ' & - & $\cdot$ & - & $\cdot 1$ & $\bullet$ & - \\
\hline Calcium & 3.19 & 0.70 & 0.55 & 5.49 & 0.65 & 5.99 & 31.94 & 27.44 & 28.94 & 28.94 & 30.44 & 26.45 & 29.44 & 26.45 \\
\hline Magnesium & 2.47 & 2.39 & 1.73 & 1.81 & 1.65 & 1.73 & 16.45 & 14.81 & 15.63 & 14.81 & 20.57 & 21.39 & 19.74 & 18.10 \\
\hline Potassium & 0.04 & 0.05 & 0.16 & 0.13 & 0.15 & 0.09 & 0.31 & 0.23 & 0.26 & 0.28 & 0.59 & 0.69 & 0.79 & 0.61 \\
\hline Sodium & 0.52 & 0.48 & 0.87 & 0.83 & 0.78 & 0.65 & 11.75 & 9.57 & 10.00 & 9.57 & 52.20 & 52.20 & 52.20 & 47.85 \\
\hline Hydrogen Ion & 0.00 & 0.00 & 0.00 & 0.00 & 0.00 & 0.00 & 0.00 & 0.00 & 0.00 & 0.00 & 0.00 & 0.00 & 0.00 & 0.00 \\
\hline - & & & & & & & & & - & & & & $\cdot$ & \\
\hline Total Cations & 6.22 & 3.61 & 3.31 & 8.25 & 3.23 & 8.46 & 60.44 & 52.05 & 54.83 & 53.60 & 103.79 & 100.73 & 102.18 & 93.01 \\
\hline ANIONS (meq/L) & & & $\cdot$ &. & - & $\cdot$ & $\cdot$ & . & - & . & - & - & - & • \\
\hline Alkalinity-CO3 & 0.00 & & 0.16 & & & & $0 . \dot{00}$ & 0.00 & 0.00 & 0.00 & 0.00 & 0.00 & 0.00 & 0.00 \\
\hline $\begin{array}{l}\text { Alkalinity }-\mathrm{CO} 3 \\
\text { Alkalinity-HCO3 }\end{array}$ & $\begin{array}{l}0.00 \\
3.90\end{array}$ & $\begin{array}{l}0.04 \\
2.50\end{array}$ & $\begin{array}{l}0.16 \\
1.86\end{array}$ & $\begin{array}{l}0.00 \\
4.82\end{array}$ & $\begin{array}{l}0.00 \\
2.02\end{array}$ & 4.86 & 3.12 & 3.24 & 3.10 & 3.08 & 2.86 & 2.82 & 2.96 & 3.14 \\
\hline chloride & 0.59 & 0.61 & 0.87 & 1.02 & 0.71 & 0.73 & 10.30 & 9.85 & 8.32 & 9.56 & 44.57 & 61.50 & 58.96 & 57.18 \\
\hline Fluoride & 0.01 & 0.01 & 0.01 & 0.02 & 0.01 & 0.02 & 0.08 & 0.09 & 0.09 & 0.09 & 0.08 & 0.08 & 0.09 & 0.09 \\
\hline Nitrate Nitrogen & 1.21 & 0.02 & 0.00 & 2.28 & 0.00 & 2.06 & 0.00 & 5.35 & 0.00 & 0.00 & 0.00 & 0.00 & 0.00 & 0.00 \\
\hline Sulfate & 0.48 & 0.39 & 0.69 & 0.62 & 0.54 & 0.82 & 37.49 & 43.74 & 32.08 & 38.62 & 37.29 & 44.74 & 37.91 & 32.91 \\
\hline Hydroxyl Ion & 0.00 & 0.00 & 0.00 & 0.00 & 0.00 & 0.00 & 0.00 & 0.00 & 0.00 & 0.00 & 0.00 & 0.00 & 0.00 & 0.00 \\
\hline - & & & & & & & & & & & & & & \\
\hline Total Anions & 6.19 & 3.56 & 3.59 & 8.76 & 3.28 & 8.50 & 50.99 & 62.26 & 43.59 & 51.35 & 84.79 & 109.14 & 99.92 & 93.32 \\
\hline CHARGE BAIANCE (8) & : & $\dot{1}$ & -4 & $\begin{array}{r}-3 \\
-3\end{array}$ & $\dot{-1}$ & $-\dot{0}$ & $\dot{8}$ & $-\dot{9}$ & $\dot{11}$ & $\dot{2}$ & 10 & -4 & $\dot{1}$ & -0 \\
\hline &. & & & & . & - & & & & & - & - & $\cdot$ & - \\
\hline $\operatorname{TDS}(\mathrm{mg} / \mathrm{L})$ & 344 & 180 & 190 & 544 & 168 & 508 & 3862 & 3782 & 3758 & 3738 & 6526 & 6344 & 6132 & 6164 \\
\hline TSS (mg/L) & 24 & 21 & 7 & 1 & 27 & $<1$ & 12 & 2 & 7 & 9 & 23 & 23 & 15 & 14 \\
\hline Turbidity (NTO) & 38 & 17 & 15 & 2 & 120 & 3.9 & 20 & 27 & 11 & 5 & 21 & 38 & 15 & 32 \\
\hline
\end{tabular}

(CONTINUED) 
APPEMDIX G

Ion-Charge Balance Calculations, 1993

\begin{tabular}{|c|c|c|c|c|c|c|c|c|c|c|c|c|c|c|}
\hline \multirow{3}{*}{$\begin{array}{l}\text { Sampling Point } \\
\text { Location } \\
\text { Date Sampled }\end{array}$} & \multicolumn{4}{|c|}{ GW-712 } & \multicolumn{4}{|c|}{ GN-713 } & \multicolumn{4}{|c|}{$64-714$} & \multicolumn{2}{|c|}{$0 *-715$} \\
\hline & \multicolumn{4}{|c|}{ EXP } & \multicolumn{4}{|c|}{ EXP } & \multicolumn{4}{|c|}{$\mathbf{E x P}$} & \multicolumn{2}{|c|}{$\mathbf{E x P}$} \\
\hline & $03 / 18 / 93$ & $04 / 28 / 93$ & $07 / 29 / 93$ & $11 / 06 / 93$ & $03 / 19 / 93$ & $04 / 30 / 93$ & $07 / 31 / 93$ & $11 / 09 / 93$ & $03 / 21 / 93$ & $04 / 29 / 93$ & $08 / 01 / 93$ & $11 / 09 / 93$ & $03 / 21 / 93$ & $04 / 29 / 93$ \\
\hline CATIONS (meq/L) & - & - & $\cdot$ & - & - & $\cdot$ & - & $\cdot 1$ & - & $\cdot$ & - & $\cdot$ & $\cdot$ & - \\
\hline Calcium & 4.94 & 4.29 & 4.54 & 4.04 & 3.99 & 3.14 & 3.49 & 3.04 & 2.79 & 2.64 & 2.84 & 2.45 & 3.59 & 3.14 \\
\hline Magnesium & 3.54 & 3.29 & 3.29 & 3.04 & 2.96 & 2.47 & 2.63 & 2.39 & 1.65 & 1.73 & 1.81 & 1.81 & 1.23 & 1.23 \\
\hline Potassium & 0.08 & 0.07 & 0.07 & 0.08 & 0.06 & 0.05 & 0.05 & 0.07 & 0.03 & 0.03 & 0.03 & 0.04 & 0.07 & 0.10 \\
\hline Sodium & 1.22 & 1.17 & 1.22 & 1.13 & 0.61 & 0.43 & 0.70 & 0.74 & 0.13 & 0.14 & 0.14 & 0.28 & 1.09 & 0.78 \\
\hline Hydrogen Ion & 0.00 & 0.00 & 0.00 & 0.00 & 0.00 & 0.00 & 0.00 & 0.00 & 0.00 & 0.00 & 0.00 & 0.00 & 0.00 & 0.00 \\
\hline - & & & & & - & & - & $\cdot$ & - & $\cdot$ & - & $\cdot$ & $\bullet$ & - \\
\hline Total Cations & 9.77 & 8.83 & 9.12 & 8.29 & 7.62 & 6.10 & 6.88 & 6.24 & 4.60 & 4.54 & 4.83 & 4.48 & 5.98 & 5.26 \\
\hline ANIONS (meq/L) & $\cdot$ & $\cdot$ & $\cdot$ & - & - & $\cdot$ & - & $\cdot 1$ & $\cdot$ & $\cdot$ & - & $\cdot$ & $\cdot$ & - \\
\hline Alkalinity- $\mathrm{CO} 3$ & 0.00 & 0.00 & 0.00 & 0.00 & 0.00 & 0.00 & 0.00 & 0.00 & 0.00 & 0.00 & 0.00 & 0.00 & 0.00 & 0.00 \\
\hline Alkalinity-HCO3 & 3.52 & 3.66 & 3.66 & 3.68 & 3.66 & 3.74 & 3.68 & 3.74 & 3.96 & 3.82 & 3.90 & 3.60 & 4.06 & 4.06 \\
\hline Chloride & 0.82 & 0.76 & 0.59 & 0.84 & 0.05 & 0.82 & 0.40 & 0.45 & 0.25 & 0.28 & 0.25 & 0.28 & 1.75 & 3.07 \\
\hline Fluoride & 0.04 & 0.04 & 0.04 & 0.04 & 0.04 & 0.03 & 0.04 & 0.04 & 0.01 & 0.01 & 0.01 & 0.01 & 0.01 & 0.00 \\
\hline Nitrate Nitrogen & 0.00 & 0.02 & 0.01 & 0.01 & 0.00 & 0.10 & 0.00 & 0.00 & 0.30 & 0.34 & 0.29 & 0.23 & 0.04 & 0.06 \\
\hline Sulfate & 5.52 & 4.87 & 3.98 & 5.94 & 0.37 & 5.60 & 2.60 & 2.23 & 0.48 & 0.44 & 0.47 & 0.46 & 0.16 & 0.24 \\
\hline Hydroxyl Ion & 0.00 & 0.00 & 0.00 & 0.00 & 0.00 & 0.00 & 0.00 & 0.00 & 0.00 & 0.00 & 0.00 & 0.00 & 0.00 & 0.00 \\
\hline . & & & & & & & & & & & - & & & - \\
\hline Total Anions & 9.89 & 9.35 & 8.28 & 10.51 & 4.12 & 10.29 & 6.72 & 6.46 & 4.99 & 4.89 & 4.91 & 4.58 & 6.01 & 7.33 \\
\hline CHARGE BALANCE (\&) & $\begin{array}{r}\cdot \\
-1\end{array}$ & -3 & $\cdot \dot{5}$ & -12 & 30 & -26 & $\dot{1}$ & $-\dot{1}$ & -4 & -4 & $-\dot{1}$ & -1 & $\begin{array}{r}-0 \\
-0\end{array}$ & -26 \\
\hline &. &. &. & & & & . &. & . & .1 & . &. &. & \\
\hline TDS (mg/L) & 642 & 596 & 652 & 568 & 502 & 424 & 476 & 438 & 276 & 264 & 274 & 256 & 376 & 298 \\
\hline TSS (mg/i) & 4 & 2 & $<1$ & 1 & 4 & 4 & 5 & 4 & 6 & 20 & $<1$ & 12 & 1 & 6 \\
\hline Turbidity (NTU) & 11 & 7 & 4.3 & 12 & 7.7 & 18 & 24 & 15 & 3.8 & 54 & 3.8 & 18 & 3 & 3 \\
\hline
\end{tabular}

(CONTINUED) 
APPEADIX $G$

Ion-Charge Belance Calculations, 1993

\begin{tabular}{|c|c|c|c|c|c|c|c|c|c|c|c|c|c|c|}
\hline \multirow{3}{*}{$\begin{array}{l}\text { Sampling Point } \\
\text { L } \\
\text { Location } \\
\text { Date Sampled }\end{array}$} & \multicolumn{2}{|c|}{$6 w-715$} & \multicolumn{4}{|c|}{ Gw-723 } & \multicolumn{4}{|c|}{$6 w-724$} & \multicolumn{4}{|c|}{$60-725$} \\
\hline & \multicolumn{2}{|c|}{ Exp } & \multicolumn{4}{|c|}{ EXP } & \multicolumn{4}{|c|}{ Exp } & \multicolumn{4}{|c|}{$\mathbf{E x P}$} \\
\hline & $08 / 01 / 93$ & $11 / 11 / 93$ & $02 / 12 / 93$ & $05 / 18 / 93$ & $09 / 27 / 93$ & $12 / 14 / 93$ & $02 / 10 / 93$ & $05 / 18 / 93$ & $09 / 29 / 93$ & $12 / 14 / 93$ & $02 / 12 / 93$ & $\mid 05 / 19 / 93$ & $09 / 28 / 93$ & $12 / 15 / 93$ \\
\hline CATIONS (meq/L) & $\cdot$ & $\cdot$ & $\cdot$ & $\cdot$ & $\cdot$ & $\cdot$ & $\cdot$ & $\cdot$ & $\cdot$ & • & • & $\cdot$ & $\cdot$ & - \\
\hline Calcium & 3.69 & 3.69 & 3.89 & 2.50 & 2.59 & 2.45 & 4.94 & 4.89 & 4.99 & 4.99 & 8.48 & 8.48 & 8.48 & 8.40 \\
\hline Magnesium & 1.40 & 1.48 & 2.14 & 2.14 & 2.55 & 2.55 & 3.37 & 3.29 & 3.45 & 3.54 & 2.22 & 2.47 & 2.24 & 2.22 \\
\hline Potassium & 0.06 & 0.04 & 0.07 & 0.06 & 0.07 & 0.08 & 0.05 & 0.04 & 0.05 & 0.07 & 0.07 & 0.06 & 0.08 & 0.09 \\
\hline Sodium & 0.74 & 0.83 & 0.29 & 0.27 & 0.36 & 0.38 & 0.91 & 0.83 & 0.96 & 0.91 & 0.74 & 0.70 & 0.96 & 0.91 \\
\hline Hydrogen Ion & 0.00 & 0.00 & 0.00 & 0.00 & 0.00 & 0.00 & 0.00 & 0.00 & 0.00 & 0.00 & 0.00 & 0.00 & 0.00 & 0.00 \\
\hline - Total Cations & 5.89 & 6.04 & 6.40 & 4.96 & $\begin{array}{r}\cdot 1 \\
5.57\end{array}$ & 5.45 & 9.28 & 9.05 & 9.46 & 9.51 & $\begin{array}{r}11.52 \\
\end{array}$ & 11.71 & 11.66 & 11.71 \\
\hline & | & . & &. & & & . & & & & &. & & - \\
\hline ARIONS (meq/I) & $\cdot$ & $\cdot$ & $\cdot$ & $\cdot$ & $\cdot$ & $\cdot$ & $\cdot$ & - & $\cdot$ & - & $\cdot$ & $\cdot$ & $\cdot 1$ & - \\
\hline Alkalinity-CO3 & 0.00 & 0.00 & 0.00 & 0.00 & 0.00 & 0.00 & 0.00 & 0.00 & 0.00 & 0.00 & 0.00 & 0.00 & 0.00 & 0.00 \\
\hline Alkalinity- $\mathrm{BCO} 3$ & 4.48 & 4.72 & 4.46 & 3.94 & 3.92 & 3.80 & 4.02 & 4.26 & 4.06 & 4.06 & 5.84 & 5.68 & 5.78 & 6.06 \\
\hline Chloride & 1.44 & 1.29 & 0.31 & 0.21 & 0.26 & 0.28 & 1.35 & 1.35 & 1.52 & 1.27 & 2.20 & 1.41 & 1.47 & 1.47 \\
\hline Fluoride & 0.00 & 0.00 & 0.02 & 0.02 & 0.03 & 0.03 & 0.01 & 0.01 & 0.01 & 0.01 & 0.01 & 0.00 & 0.00 & 0.00 \\
\hline Mitrate Mitrogen & 0.08 & 0.05 & 1.43 & 0.46 & 0.69 & 0.76 & 4.43 & 2.64 & 3.58 & 3.31 & 4.36 & 3.93 & 3.56 & 3.30 \\
\hline Sulfate & 0.17 & 0.13 & 0.58 & 0.63 & 0.70 & 0.69 & 0.54 & 0.48 & 0.48 & 0.44 & 1.23 & 0.71 & 0.79 & 0.65 \\
\hline Hydroxyl Ion & 0.00 & 0.00 & 0.00 & 0.00 & 0.00 & 0.00 & 0.00 & 0.00 & 0.00 & 0.00 & 0.00 & 0.00 & 0.00 & 0.00 \\
\hline • & & & & & & & & & & & & & & \\
\hline Total Anions & 6.17 & 6.18 & 6.79 & 5.26 & 5.58 & 5.55 & 10.35 & 8.74 & 9.65 & 9.09 & 13.63 & 11.72 & 11.60 & 11.47 \\
\hline CHARGE BALAMCE (8) & $-\dot{2}$ & -1 & -3 & $-\dot{3}$ & $\begin{array}{r}-0 \\
-0\end{array}$ & -1 & -5 & $\dot{2}$ & $\begin{array}{r}1 \\
-1\end{array}$ & $\dot{2}$ & $-\dot{8}$ & -0 & $\dot{0}$ & $\dot{1}$ \\
\hline 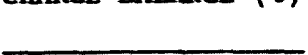 & & &. &. & & &. & &. &. &. &. &. & - \\
\hline $\operatorname{TDS}(m g / L)$ & 334 & 354 & 358 & 298 & 280 & 304 & 546 & 604 & 494 & 556 & 722 & 760 & 576 & 764 \\
\hline TSS $(m g / L)$ & 6 & 1 & 89 & 9 & $<1$ & 6 & 3 & 2 & $<1$ & 3 & 4 & 1 & $<1$ & 1 \\
\hline Turbidity (MTU) & 9.9 & 54 & 80 & 14 & 36 & 9.2 & 7.8 & 9.1 & 7 & 16 & 10. & 2 & 0.8 & 1.5 \\
\hline
\end{tabular}

(CONTIMUED) 
APPESTIX 6

Ion-Charge Balance Calculations, 1993

\begin{tabular}{|c|c|c|c|c|c|c|c|c|c|c|c|c|c|c|}
\hline \multirow{3}{*}{$\begin{array}{l}\text { Sampling Point } \\
\text { Locetion } \\
\text { Date Sampled }\end{array}$} & \multicolumn{4}{|c|}{ Gw-736 } & \multicolumn{4}{|c|}{ 6-737 } & \multicolumn{4}{|c|}{$6-730$} & \multicolumn{2}{|c|}{$6-739$} \\
\hline & \multicolumn{4}{|c|}{$\mathbf{E x p}$} & \multicolumn{4}{|c|}{$\exp$} & \multicolumn{4}{|c|}{ 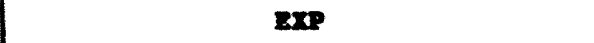 } & \multicolumn{2}{|c|}{$\mathbf{E x P}$} \\
\hline & $02 / 11 / 93$ & $05 / 18 / 93$ & $09 / 28 / 93$ & $12 / 15 / 93$ & $02 / 11 / 93$ & $05 / 19 / 93$ & $09 / 28 / 93$ & $12 / 15 / 93$ & $02 / 10 / 93$ & $05 / 18 / 93$ & $09 / 27 / 93$ & $12 / 14 / 93$ & $02 / 10 / 93$ & $05 / 18 / 93$ \\
\hline CATIONS (meq $/ \mathrm{L}$ ) & $\cdot$ & $\cdot$ & $\cdot$ & $\cdot$ & - & - & $\cdot$ & - & $\cdot$ & - & $\cdot$ & - & $\cdot$ & • \\
\hline Calcium & 8.98 & 8.98 & 8.48 & 9.48 & 8.48 & 6.98 & 8.98 & 8.48 & 5.99 & 5.49 & 6.49 & 6.49 & 2.99 & 2.84 \\
\hline Magnesium & 1.89 & 1.81 & 2.39 & 2.06 & 1.89 & 1.89 & 2.06 & 2.06 & 2.06 & 1.89 & 2.22 & 2.22 & 2.22 & 2.06 \\
\hline Potassium & 0.08 & 0.08 & 0.08 & 0.09 & 0.08 & 0.08 & 0.08 & 0.09 & 0.05 & 0.04 & 0.05 & 0.06 & 0.03 & 0.03 \\
\hline Sodium & 0.96 & 0.87 & 0.87 & 1.04 & 1.04 & 0.87 & 1.00 & 0.96 & 0.30 & 0.24 & 0.36 & 0.38 & 0.08 & 0.00 \\
\hline Hydrogen Ion & 0.00 & 0.00 & 0.00 & 0.00 & 0.00 & 0.00 & 0.00 & 0.00 & 0.00 & 0.00 & 0.00 & 0.00 & 0.00 & 0.00 \\
\hline 1. & & & & & & & & & & & $\cdot$ & & & \\
\hline Total Cations & 11.91 & 11.74 & 11.82 & 12.67 & 11.50 & 11.82 & 12.12 & 11.58 & 8.39 & 7.67 & 9.12 & 9.15 & 5.33 & 5.01 \\
\hline ArIONS (meq/L) & $\cdot 1$ & $\cdot$ & $\cdot$ & $\cdot$ & - & - & $\cdot$ & - &. & - & $\cdot$ & - & $\cdot$ & • \\
\hline Alkalinity-co3 & 0.00 & 0.00 & 0.00 & 0.00 & 0.00 & 0.00 & 0.00 & 0.00 & 0.00 & 0.00 & 0.00 & 0.00 & 0.00 & 0.00 \\
\hline Alkalinity-HCO3 & 6.23 & 6.25 & 6.33 & 6.31 & 6.23 & 6.37 & 6.13 & 6.19 & 5.58 & 5.66 & 5.84 & 5.72 & 4.44 & 4.38 \\
\hline Chloride & 1.38 & 1.41 & 1.83 & 1.55 & 1.47 & 1.44 & 1.52 & 1.50 & 0.39 & 0.42 & 0.96 & 0.56 & 0.15 & 0.14 \\
\hline Fluoride & 0.02 & 0.01 & 0.01 & 0.01 & 0.01 & 0.01 & 0.01 & 0.01 & 0.00 & 0.00 & 0.00 & 0.00 & 0.01 & 0.01 \\
\hline witrate Nitrogen & 4.36 & 3.00 & 3.13 & 3.03 & 4.07 & 2.64 & 3.56 & 3.16 & 1.57 & 1.07 & 2.80 & 1.77 & 0.53 & 0.30 \\
\hline Sulfate & 0.83 & 0.79 & 0.83 & 0.67 & 0.83 & 0.85 & 0.79 & 0.62 & 0.62 & 0.56 & 0.36 & 0.52 & 0.37 & 0.26 \\
\hline Hydroxyl Ion & 0.00 & 0.00 & 0.00 & 0.00 & 0.00 & 0.00 & 0.00 & 0.00 & 0.00 & 0.00 & 0.00 & 0.00 & 0.00 & 0.00 \\
\hline - & & & & & & & & & & & & & & \\
\hline Total Anions & 12.82 & 11.47 & 12.14 & 11.57 & 12.62 & 11.31 & 12.02 & 11.48 & 8.17 & 7.71 & 10.24 & 8.57 & 5.50 & 5.09 \\
\hline CHARGE BALANCE (8) & $\begin{array}{r}-4 \\
-4\end{array}$ & $\dot{1}$ & $\begin{array}{r}-1 \\
-1\end{array}$ & $\dot{5}$ & $\begin{array}{r}-5 \\
-5\end{array}$ & $\dot{2}$ & $\dot{0}$ & : & $i$ & $-\dot{0}$ & $\begin{array}{r}-6 \\
-6\end{array}$ & $\dot{3}$ & $\begin{array}{r}-2 \\
-2\end{array}$ & -1 \\
\hline & $\cdot$ & $\cdot$ & $\cdot$ & $\cdot$ & - & $\cdot$ & $\cdot$ & $\cdot$ & $\cdot$ & $\cdot$ & $\cdot$ & - & $\cdot 1$ & 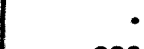 \\
\hline $\operatorname{TDS}(\mathrm{mg} / L)$ & 710 & 700 & 696 & 740 & 712 & 760 & 694 & 726 & 492 & 440 & 478 & 496 & 312 & 292 \\
\hline TSS (mg/L) & 1 & $<1$ & $<1$ & $<1$ & 1 & $<1$ & $<1$ & $<1$ & 3 & 3 & $<1$ & 4 & 16 & 3 \\
\hline Turbidity (NTU) & 0.7 & 1.4 & 0.9 & 0.6 & 1 & 1.1 & 1.4 & 0.8 & 3.9 & 4.9 & 7.9 & 3.2 & 22 & 3 \\
\hline
\end{tabular}

(CONTIMUED) 
APPEIDIX G

Ion-Charge Balance Calculations, 1993

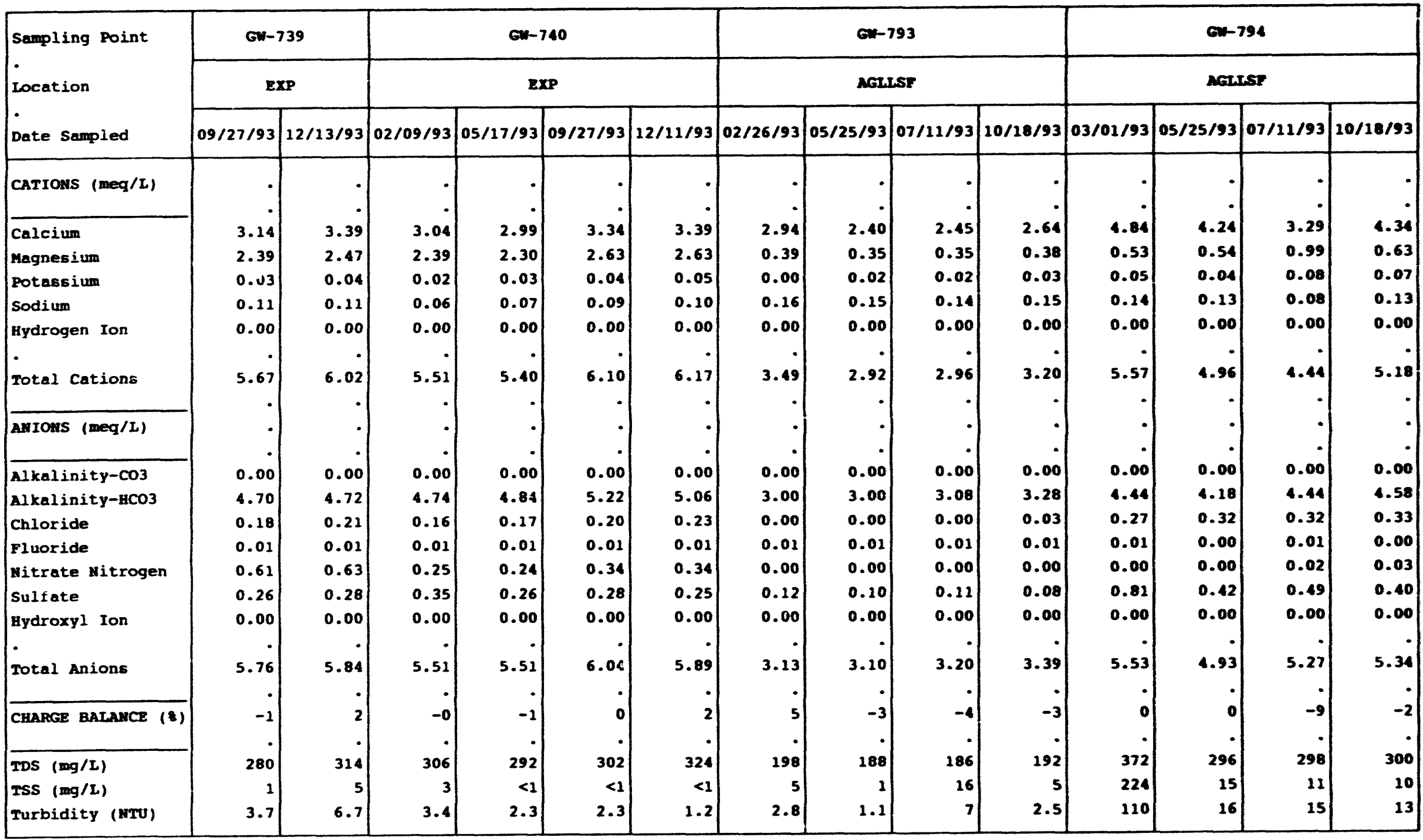

(CONTINUED) 
APPEADIX $G$

Ion-Charge Balance Calculations, 1993

\begin{tabular}{|c|c|c|c|c|c|c|c|}
\hline \multirow{3}{*}{$\begin{array}{l}\text { Sampling Point } \\
\text { Location } \\
\text { Date Sampled }\end{array}$} & \multicolumn{4}{|c|}{ Gw-795 } & \multicolumn{3}{|c|}{$6 \%-800$} \\
\hline & \multicolumn{4}{|c|}{ AGTSF } & \multicolumn{3}{|c|}{ orf } \\
\hline & $|03 / 02 / 93|$ & $05 / 25 / 93$ & $\mid 07 / 12 / 93$ & $10 / 18 / 93$ & $05 / 11 / 93$ & $09 / 14 / 93$ & $10 / 22 / 93$ \\
\hline CATIOAs (meq/L) & - & - & - & $\cdot 1$ & $\cdot$ & $\cdot$ & - \\
\hline Calcium & 4.44 & 3.94 & 4.09 & 4.74 & 2.35 & 3.54 & 2.89 \\
\hline Magnesium & 0.29 & 0.26 & 0.29 & 0.37 & 1.40 & 2.55 & 1.89 \\
\hline Potassium & 0.02 & 0.02 & 0.03 & 0.03 & 0.05 & 0.09 & 0.07 \\
\hline Sodium & 0.10 & 0.10 & 0.09 & 0.13 & 0.04 & 0.05 & 0.04 \\
\hline Hydrogen Ion & 0.00 & 0.00 & 0.00 & 0.00 & 0.00 & 0.00 & 0.00 \\
\hline rotal Cations & $\begin{array}{r}\cdot \\
4.84\end{array}$ & 4.32 & 4.49 & 5.27 & 3.83 & 6.23 & 4.89 \\
\hline AMIONS (meg/L) & & $\cdot$ & & $\cdot$ &. & . & • \\
\hline Alkalinity-CO3 & 0.00 & 0.00 & 0.00 & 0.00 & 0.00 & 0.00 & 0.00 \\
\hline Alkalinity-HCO3 & 3.56 & 3.90 & 4.08 & 4.30 & 3.66 & 5.52 & 4.96 \\
\hline Chloride & 0.14 & 0.13 & 0.12 & 0.19 & 0.04 & 0.07 & 0.06 \\
\hline Fluoride & 0.00 & 0.00 & 0.00 & 0.00 & 0.00 & 0.01 & 0.01 \\
\hline Witrate Mitrogen & 0.00 & 0.00 & 0.00 & 0.37 & 0.00 & 0.00 & 0.00 \\
\hline Sulfate & 0.42 & 0.46 & 0.44 & 0.44 & 0.27 & 0.44 & 0,33 \\
\hline Hydroxyl Ion & 0.00 & 0.00 & 0.00 & 0.00 & 0.00 & 0.00 & 0.00 \\
\hline - & & & & & & & \\
\hline Total Anions & 4.12 & 4.49 & 4.63 & 5.29 & 3.97 & 6.03 & 5.35 \\
\hline$\overline{\text { Charge Batance (*) }}$ & 8 & -2 & -2 & -0 & -2 & 2 & -4 \\
\hline $\operatorname{TDS}(\mathrm{mg} / \mathrm{L})$ & 266 & $26 \dot{2}$ & $26 \dot{0}$ & 302 & 180 & 334 & 284 \\
\hline $\begin{array}{l}\text { TDS (mg/L) } \\
\text { TSS (mg/L) }\end{array}$ & $<1$ & $<1$ & $<1$ & $<1$ & $<1$ & 66 & 8 \\
\hline Turbidity (MTU) & 1.1 & 1.1 & 1.8 & 1 & 1.1 & 66 & 25 \\
\hline
\end{tabular}


APPENDIX $G$

Ion-Charge Balance Calculations, 1993

\begin{tabular}{|c|c|c|c|c|c|c|c|c|c|c|c|c|c|c|}
\hline \multirow{3}{*}{$\begin{array}{l}\text { Sampling Point } \\
\text { Location } \\
\text { Date Sampled }\end{array}$} & \multicolumn{4}{|c|}{ BCK-00.63 } & \multicolumn{4}{|c|}{ BCK-04.55 } & \multicolumn{3}{|c|}{ BCK-09.40 } & \multicolumn{3}{|c|}{ NT-01 } \\
\hline & \multicolumn{4}{|c|}{ EXP } & \multicolumn{4}{|c|}{$\operatorname{EXP}$} & \multicolumn{3}{|c|}{ EXP } & \multicolumn{3}{|c|}{$\operatorname{EXP}$} \\
\hline & $03 / 09 / 93$ & $05 / 03 / 93$ & $08 / 16 / 93$ & $11 / 08 / 93$ & $03 / 09 / 93$ & $05 / 03 / 93$ & $08 / 16 / 93$ & $11 / 08 / 93$ & $03 / 09 / 93$ & $05 / 03 / 93$ & $11 / 08 / 93$ & $03 / 09 / 93$ & $05 / 03 / 93$ & $08 / 16 / 93$ \\
\hline CATroNs (meq/L) & & - & $\cdot$ & - & $\cdot$ & $\cdot$ & • & - & $\cdot$ & $\cdot$ & $\cdot$ & $\cdot$ & $\cdot$ & $\bullet$ \\
\hline Calcium & 1.55 & 1.55 & 2.84 & 2.89 & 1.55 & 1.60 & 2.64 & 2.89 & 2.69 & 2.84 & 1.45 & 6.99 & 8.98 & 16.47 \\
\hline Magnesium & 0.69 & 0.78 & 1.23 & 1.15 & 0.73 & 0.81 & 1.40 & 1.23 & 0.82 & 0.90 & 1.23 & 1.56 & 1.48 & 4.03 \\
\hline Potassium & 0.03 & 0.03 & 0.04 & 0.05 & 0.03 & 0.03 & 0.04 & 0.05 & 0.04 & 0.05 & 0.02 & 0.05 & 0.08 & 0.15 \\
\hline Sodium & 0.14 & 0.14 & 0.29 & 0.35 & 0.14 & 0.14 & 0.27 & 0.37 & 0.34 & 0.37 & 0.03 & 0.70 & 0.61 & 2.17 \\
\hline Hydrogen Ion & 0.00 & 0.00 & 0.00 & 0.00 & 0.00 & 0.00 & 0.00 & 0.00 & 0.00 & 0.00 & 0.00 & 0.00 & 0.00 & 0.00 \\
\hline Total Cations & 2.41 & 2.50 & 4.40 & 4.44 & 2.44 & 2.58 & 4.36 & 4.55 & 3.90 & 4.16 & 2.73 & 9.30 & 11.15 & 22.82 \\
\hline ANIONS (meq/L) & $\cdot$ & - & $\cdot$ &. & $\cdot$ & $\cdot$ & . & $\cdot$ & $\cdot$ & $\cdot$ & $\cdot$ & $\cdot$ &. & 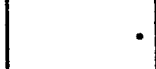 \\
\hline Alkalinity- $\mathrm{CO} 3$ & 0.00 & 0.00 & 0.00 & 0.00 & 0.00 & 0.00 & 0.00 & 0.00 & 0.00 & 0.00 & 0.00 & 0.00 & 0.00 & 0.00 \\
\hline Alkalinity-HCO3 & 1.90 & 2.22 & 3.38 & 3.26 & 2.04 & 2.36 & 3.30 & 3.28 & 2.44 & 2.92 & 2.60 & 2.00 & 6.55 & 4.10 \\
\hline Chloride & 0.14 & 0.14 & 0.31 & 0.37 & 0.14 & 0.15 & 0.34 & 0.39 & 0.34 & 0.42 & 0.03 & 0.28 & 1.24 & 1.17 \\
\hline Fluoride & 0.00 & 0.00 & 0.01 & 0201 & 0.00 & 0.00 & 0.01 & 0.01 & 0.01 & 0.01 & 0.01 & 0.04 & 0.01 & 0.08 \\
\hline Nitrate Nitrogen & 0.14 & 0.16 & 0.36 & 0.71 & 0.16 & 0.19 & 0.45 & 0.74 & 0.79 & 0.93 & 0.00 & 8.00 & 2.21 & 20.43 \\
\hline Sulfate & 0.27 & 0.21 & 0.26 & 0.42 & 0.27 & 0.23 & 0.27 & 0.40 & 0.44 & 0.37 & 0.10 & 0.48 & 1.23 & 1.39 \\
\hline Hydroxyl Ion & 0.00 & 0.00 & 0.00 & 0.00 & 0.00 & 0.00 & 0.00 & 0.00 & 0.00 & 0.00 & 0.00 & 0.00 & 0.00 & 0.00 \\
\hline Total Anions & 2.45 & 2.73 & 4.32 & 4.76 & 2.62 & 2.92 & 4.36 & 4.82 & 4.01 & 4.66 & 2.74 & 10.80 & 11.24 & 27.17 \\
\hline & & • & $\cdot$ & $\cdot$ & $\cdot$ & $\cdot$ & $\cdot$ & $\dot{2}$ & $\cdot$ & $\cdot \dot{0}$ & $-\dot{0}$ & $\dot{-7}$ & -0 & $\dot{-9}$ \\
\hline CHARGE BALANCE (8) & -1 & -4 & 1 & -3 & -3 & -6 & -0 & -3 & $\begin{array}{r}-1 \\
.\end{array}$ & $\begin{array}{r}-6 \\
.\end{array}$ & $\begin{array}{r}-0 \\
.\end{array}$ & $\begin{array}{r}-7 \\
.\end{array}$ & -0 & -9 \\
\hline TDS (mg/L) & 130 & 176 & 258 & 384 & 136 & 162 & 264 & 376 & 230 & 288 & 254 & 772 & 772 & 1746 \\
\hline TSS (mg/L) & 2 & 2 & 4 & $<1$ & 2 & 1 & 1 & $<1$ & 2 & 1 & 4 & 5 & 7 & 4 \\
\hline Turbidity (NTU) & 4 & 3.2 & 2.5 & 3 & 3.4 & 3 & 2.3 & 2.9 & 2.8 & 3 & 4.1 & 7.1 & 1.3 & 2.2 \\
\hline
\end{tabular}

(CONTINUED) 
Al: $: * 2 y=6$

Ion-Charge Balance Cat_-iations, 1993

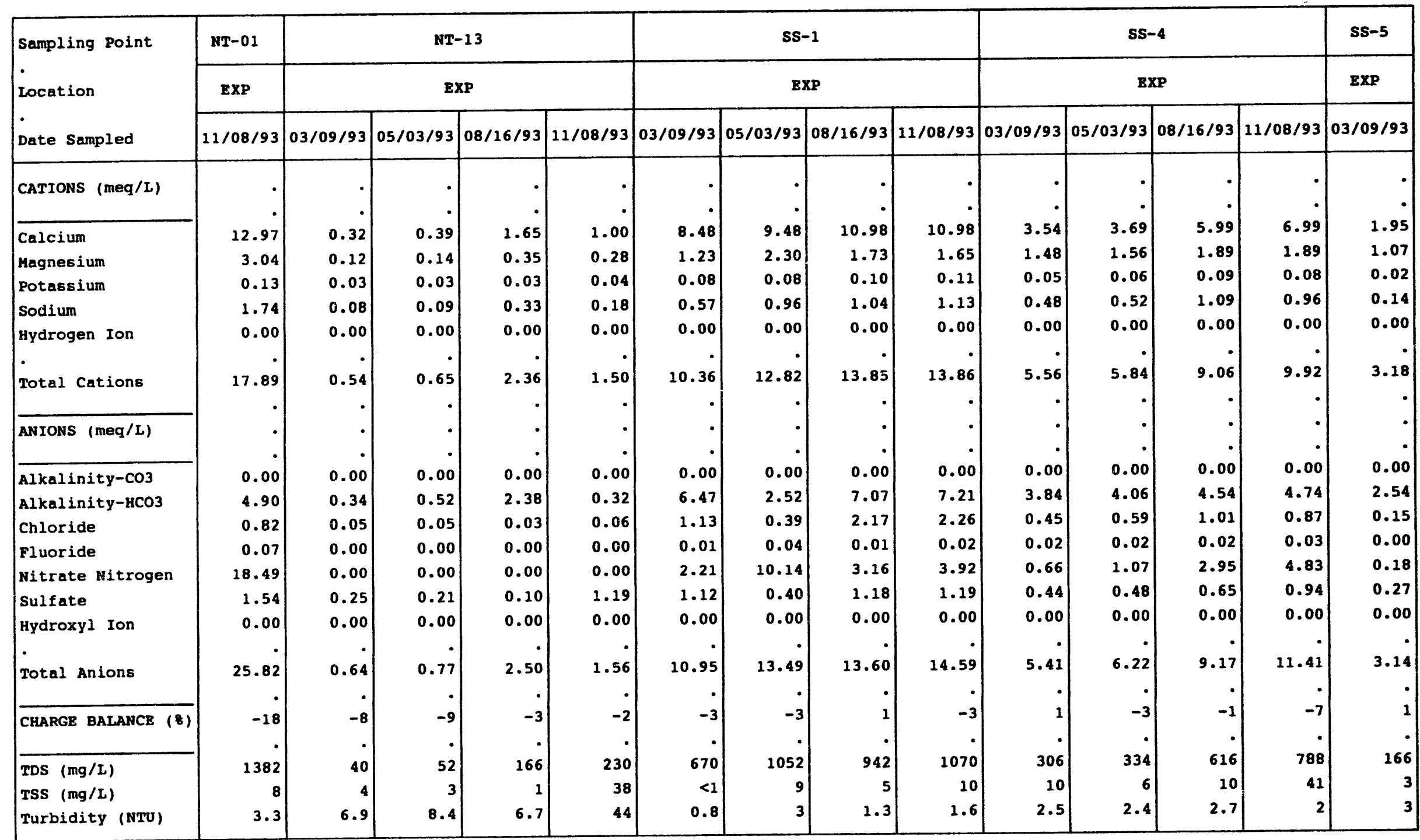

(CONTINUED) 
Ion-Charge Balance Calculations, 1993

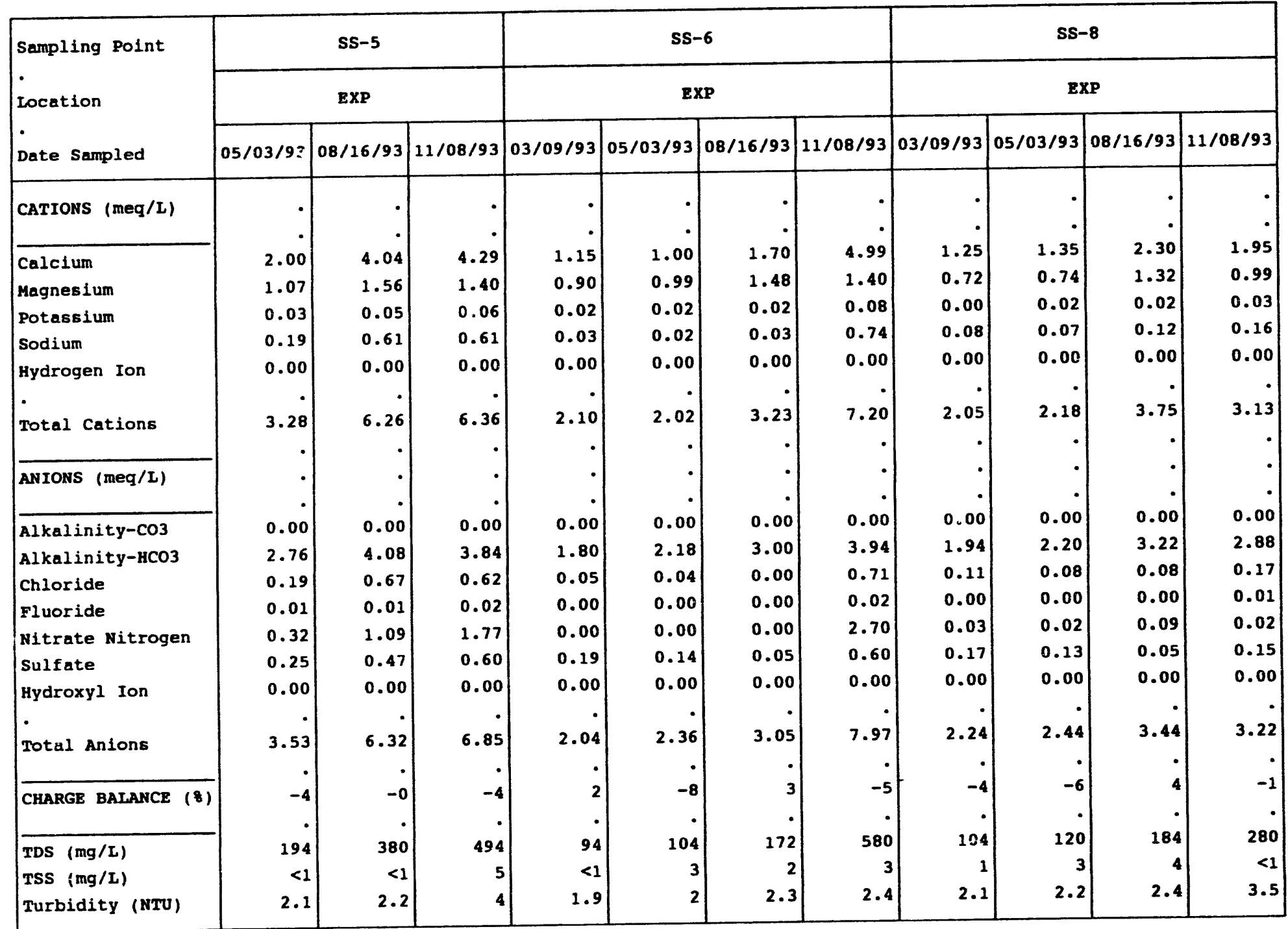




\section{APPENDIX H}

TRACE METAL DATA SUMMARY 
SAMPLING POINT:

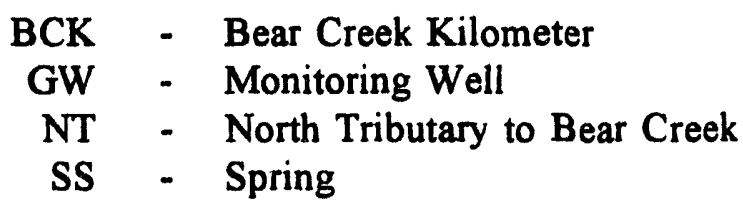

\section{LOCATION:}

$$
\begin{array}{rll}
\text { AGLLSF } & \text { - } & \text { Above Grade Low Level Storage Facility } \\
\text { BG } & - \text { Bear Creek Burial Grounds WMA } \\
\text { EXP } & - \text { Exit-Pathway Monitoring } \\
\text { OLF } & - \text { Oil Landfarm WMA } \\
\text { RS } & - \text { Rust Spoil Area } \\
\text { S3 } & - \text { S-3 Site } \\
\text { SPI } & - \text { Spoil Area I }
\end{array}
$$

NOTES:
$\mathrm{mg} / \mathrm{L}$ - milligrams per Liter
- Concentration less than the Maximum Contaminant Level (MCL)
TOT - Total Concentration (Unfiltered Sample)
DIS - Dissolved Concentration (Filtered Sample)
TSS - Total Suspended Solids

\section{MCLs:}

$\begin{array}{lc}\text { Metal } & \text { Maximum Co } \\ \text { Barium (ICP) } & 2.0 \\ \text { Cadmium (AAS) } & 0.005 \\ \text { Chromium (AAS) } & 0.10 \\ \text { Lead (AAS) } & 0.05 \\ \text { Mercury (CVAA) } & 0.002\end{array}$

Because of inherent analytical interferences, ICP results for arsenic, selenium, and silver were not evaluated with respect to MCLs. Historical AAS results for these metals have shown only isolated and sporadic occurrences where concentrations exceeded MCLs. 
SCREENING LEVELS:

\section{Metal (ICP)}

Aluminum

Beryllium

Boron

Cobalt

Copper

Iron

Manganese

Molybdenum

Nickel

Strontium

Thorium

Uranium

Vanadium

Zinc

\section{Screening Level (mg/L)}

8.3

0.0066

1.1

0.012

0.44

13.91

2.4

0.023

0.18

0.88

$<0.2$

0.01

0.064

0.14

Values represent the maximum total concentration (in $\mathrm{mg} / \mathrm{L}$ ) that would be expected in uncontaminated, unfiltered groundwater samples collected from monitoring wells located upgradient from waste-management sites at the Y-12 Plant. 


\section{APPENDIX H.1}

TRACE METAL CONCENTRATIONS THAT EXCEED MAXIMUM CONTAMINANT LEVELS 
Trace Metal Concentrations That Exceed Maximum Contaminant Levels, 1993

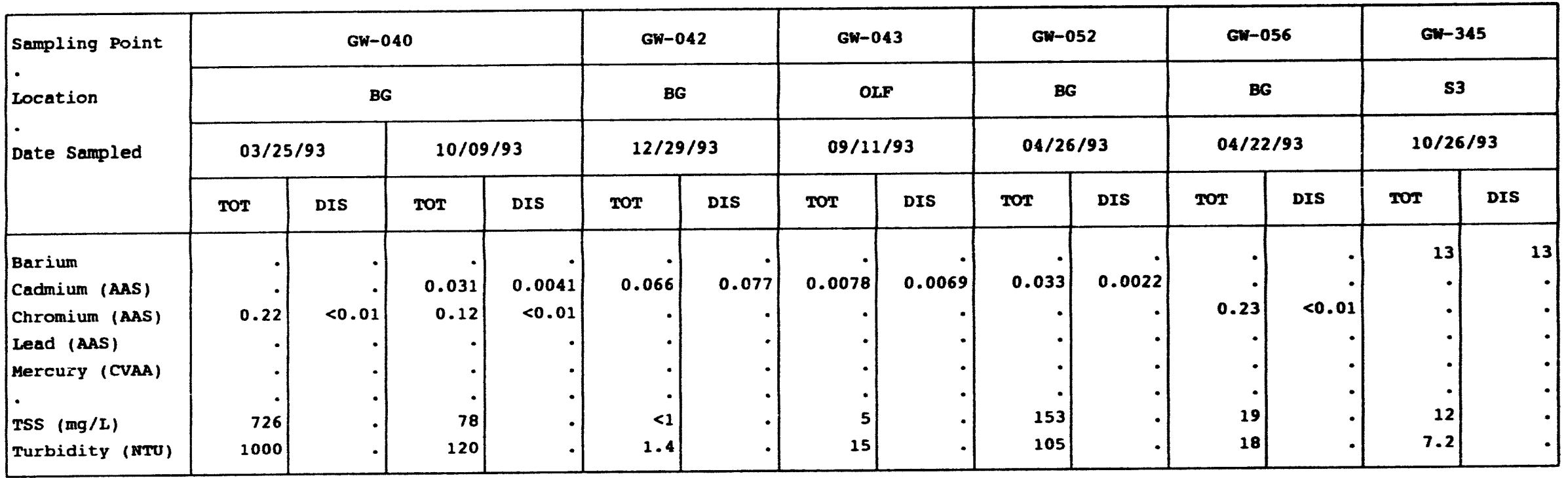

(CONTINUED)

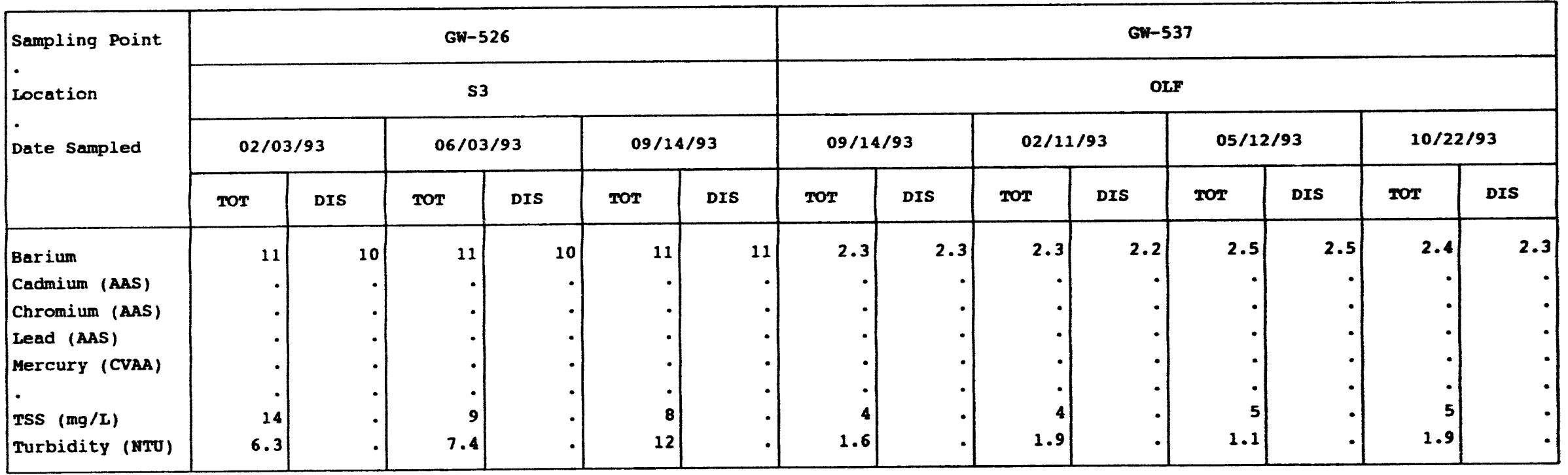

(CONTINUED) 
APPENDIX H.1

Trace Metal Concentrations That Exceed Maximum Contaminant Levels, 1993

\begin{tabular}{|c|c|c|c|c|c|c|c|c|c|c|c|c|c|c|}
\hline \multirow{4}{*}{$\begin{array}{l}\text { Sampling Point } \\
\text { Location } \\
\text { - } \\
\text { Date Sampled }\end{array}$} & \multicolumn{4}{|c|}{$G W-621$} & \multicolumn{2}{|c|}{ GW-642 } & \multicolumn{2}{|c|}{$G w-647$} & \multicolumn{2}{|c|}{ ss-1 } & \multicolumn{4}{|c|}{$m-01$} \\
\hline & \multicolumn{4}{|c|}{ BG } & \multirow{2}{*}{\multicolumn{2}{|c|}{$\frac{\text { BG }}{10 / 10 / 93}$}} & \multirow{2}{*}{\multicolumn{2}{|c|}{$\frac{\text { OLF }}{12 / 10 / 93}$}} & \multirow{2}{*}{\multicolumn{2}{|c|}{$\begin{array}{c}\text { EXP } \\
05 / 03 / 93\end{array}$}} & \multicolumn{4}{|c|}{$\operatorname{ExP}$} \\
\hline & \multicolumn{2}{|c|}{$06 / 02 / 93$} & \multicolumn{2}{|c|}{$09 / 20 / 93$} & & & & & & & \multicolumn{2}{|c|}{$03 / 09 / 93$} & \multicolumn{2}{|c|}{$08 / 16 / 93$} \\
\hline & TOT & DIS & TOT & DIS & TOT & DIS & TOT & DIS & TOT & DIS & TOT & DIS & TOT & DIS \\
\hline $\begin{array}{l}\text { Barium } \\
\text { Cadmium (AAS) } \\
\text { Chromium (AAS) } \\
\text { Lead (AAS) } \\
\text { Mercury (CVAA) } \\
\text { TSS (mg/L) } \\
\text { Turbidity (NTU) }\end{array}$ & $\begin{array}{r}. \\
0.22 \\
. \\
. \\
23 \\
34\end{array}$ & $\begin{array}{r}. \\
. \\
0.036 \\
. \\
. \\
. \\
.\end{array}$ & $\begin{array}{r}\cdot \\
0.18 \\
. \\
. \\
37 \\
35\end{array}$ & $\begin{array}{r}. \\
<0.01 \\
. \\
. \\
. \\
. \\
.\end{array}$ & $\begin{array}{r}\cdot \\
. \\
0.29 \\
. \\
. \\
. \\
540 \\
60\end{array}$ & $\begin{array}{r}. \\
. \\
.01 \\
. \\
. \\
. \\
.\end{array}$ & $\begin{array}{r}0.0074 \\
. \\
. \\
. \\
\dot{1} \\
1.6\end{array}$ & $\begin{array}{r}<0.002 \\
. \\
. \\
. \\
.\end{array}$ & $\begin{array}{r}. \\
0.024 \\
. \\
. \\
. \\
9 \\
3\end{array}$ & $\begin{array}{r}0.022 \\
. \\
. \\
. \\
. \\
.\end{array}$ & $\begin{array}{r}. \\
0.017 \\
. \\
. \\
. \\
5 \\
7.1\end{array}$ & $\begin{array}{r}0.014 \\
\cdot \\
\cdot \\
\cdot \\
\cdot \\
\cdot\end{array}$ & $\begin{array}{r}. \\
0.017 \\
. \\
. \\
. \\
. \\
4 \\
2.2\end{array}$ & $\begin{array}{r}0.013 \\
. \\
. \\
. \\
. \\
.\end{array}$ \\
\hline
\end{tabular}

(CONTINUED)

\begin{tabular}{|c|c|c|}
\hline \multirow{4}{*}{$\begin{array}{l}\text { Sampling Point } \\
\text { Location } \\
\text { Date Sampled }\end{array}$} & \multicolumn{2}{|c|}{ MT-01 } \\
\hline & \multicolumn{2}{|c|}{$\operatorname{ExP}$} \\
\hline & \multicolumn{2}{|c|}{$11 / 08 / 93$} \\
\hline & TOT & DIS \\
\hline Barium & - & \\
\hline Cadmium (AAS) & 0.024 & 0.022 \\
\hline Chromium (AAS) & - & • \\
\hline Lead (AAS) & - & - \\
\hline Mercury (CVAA) & - & - \\
\hline - & - & - \\
\hline TSS (mg/I) & 8 & - \\
\hline Turbidity (NTU) & 3.3 & . \\
\hline
\end{tabular}


APPENDIX $\mathrm{H.2}$

TRACE METAL CONCENTRATIONS THAT EXCEED SCREENING LEVELS 
APPENDIX H.2

Trace Metal Concentrations That Exceed

Screening Levels, 1993

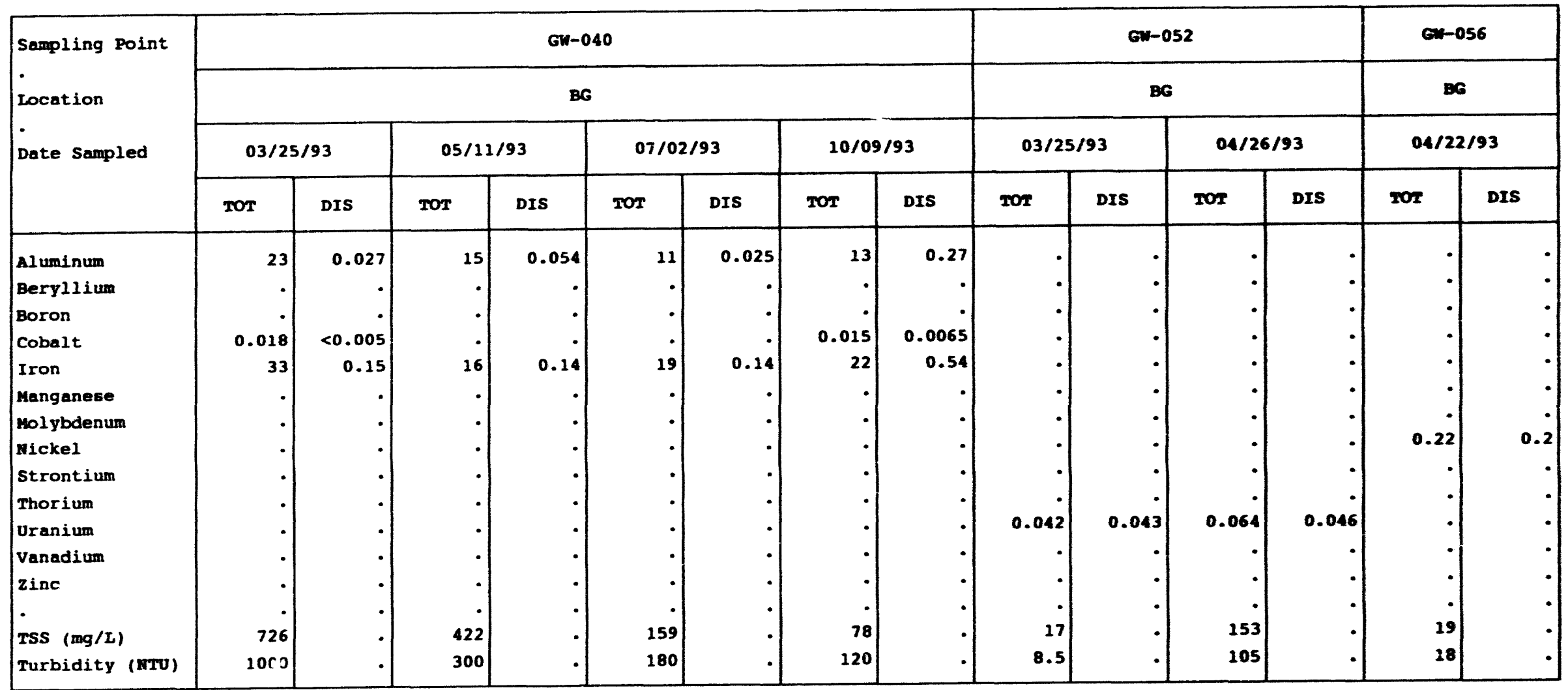

(CONTINUED) 
APPERDIX 11.2

Trace Metal Concentrations That Exceed

Screening Levels, 1993

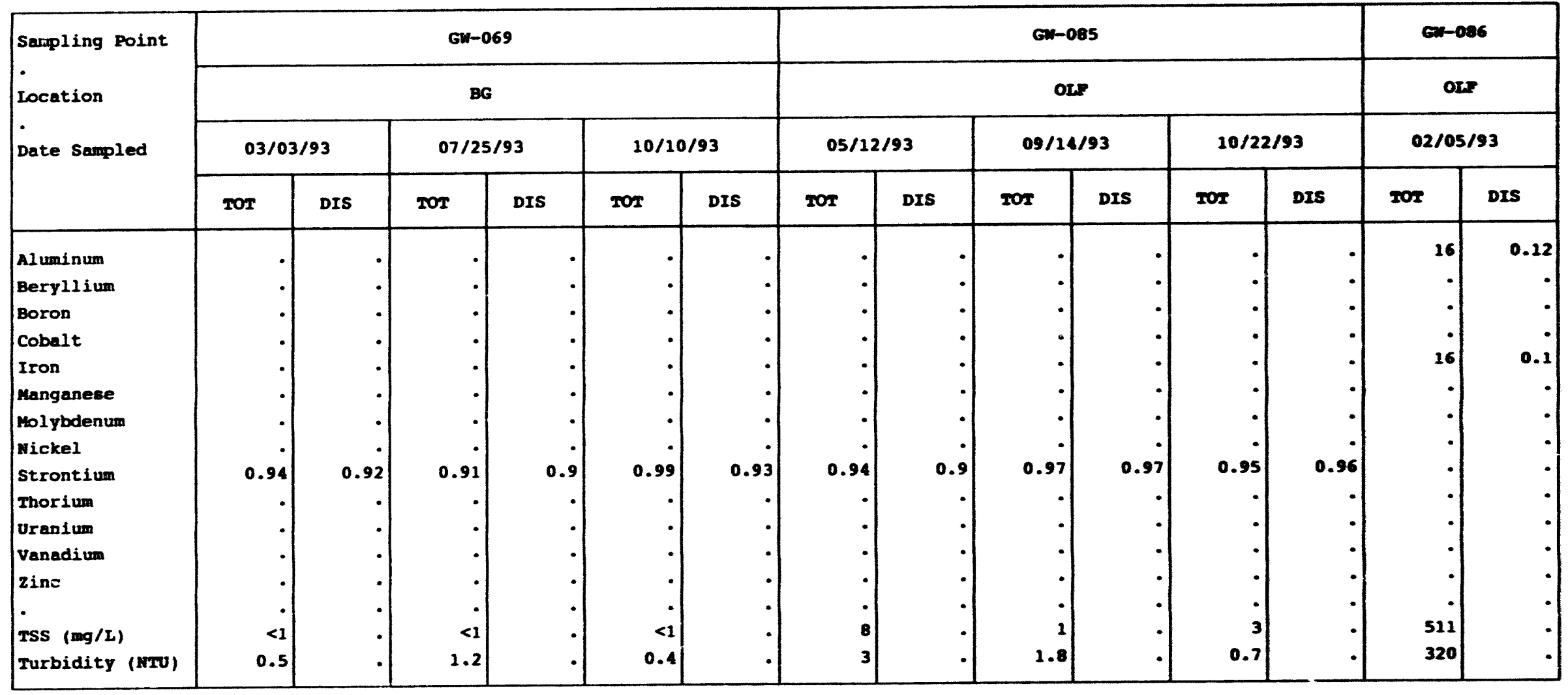

(CONTIMUED) 
APPEADIX H.2

Trace metal concentrations That Exceed

screening Levels, 1993

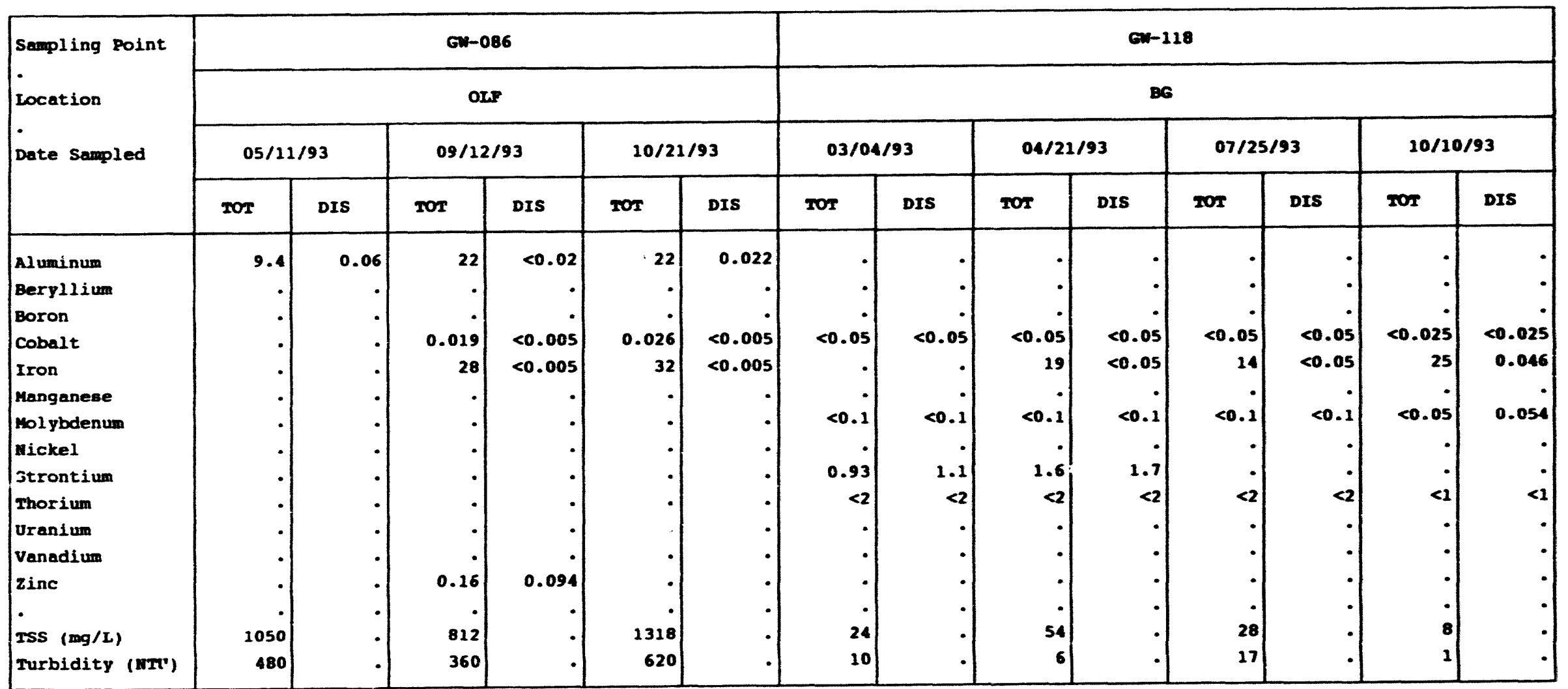

(CONTINUED) 


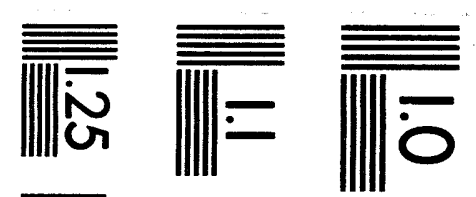

$$
\begin{aligned}
& \text { 莓|| }
\end{aligned}
$$

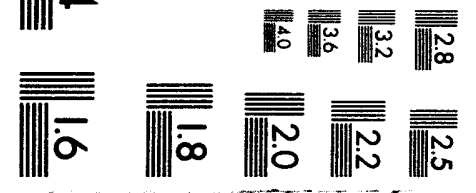



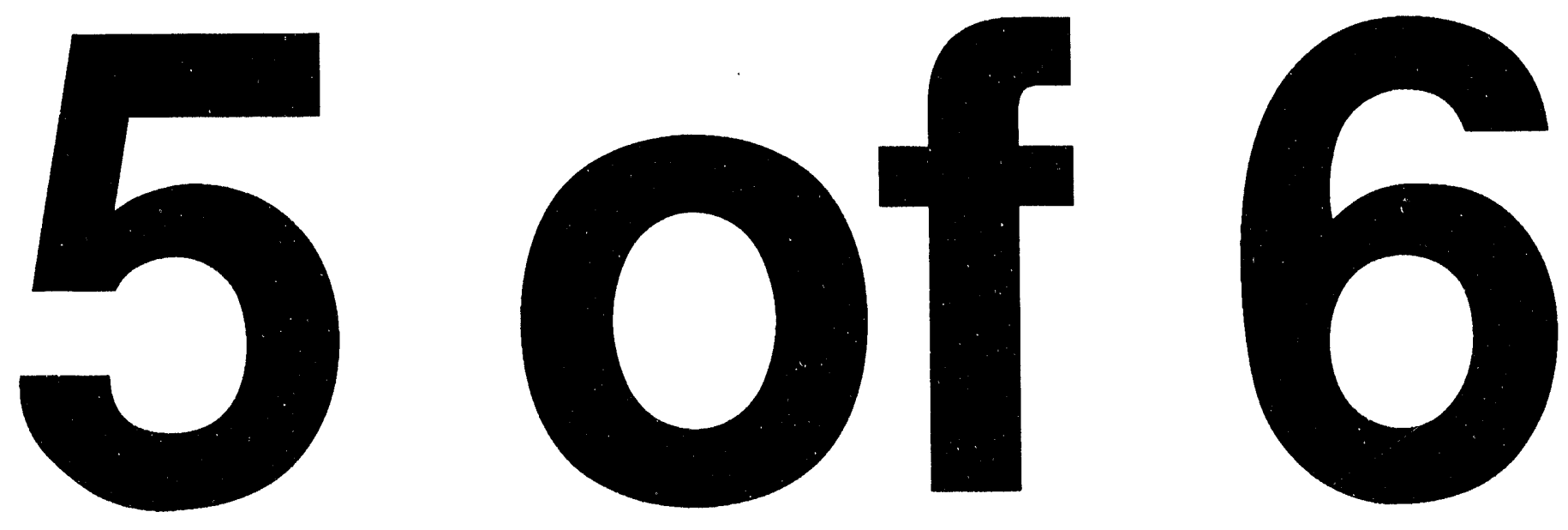
APPENDIX H. 2

Trace Metal Concentrations That Exceed

Screening Levels, 1993

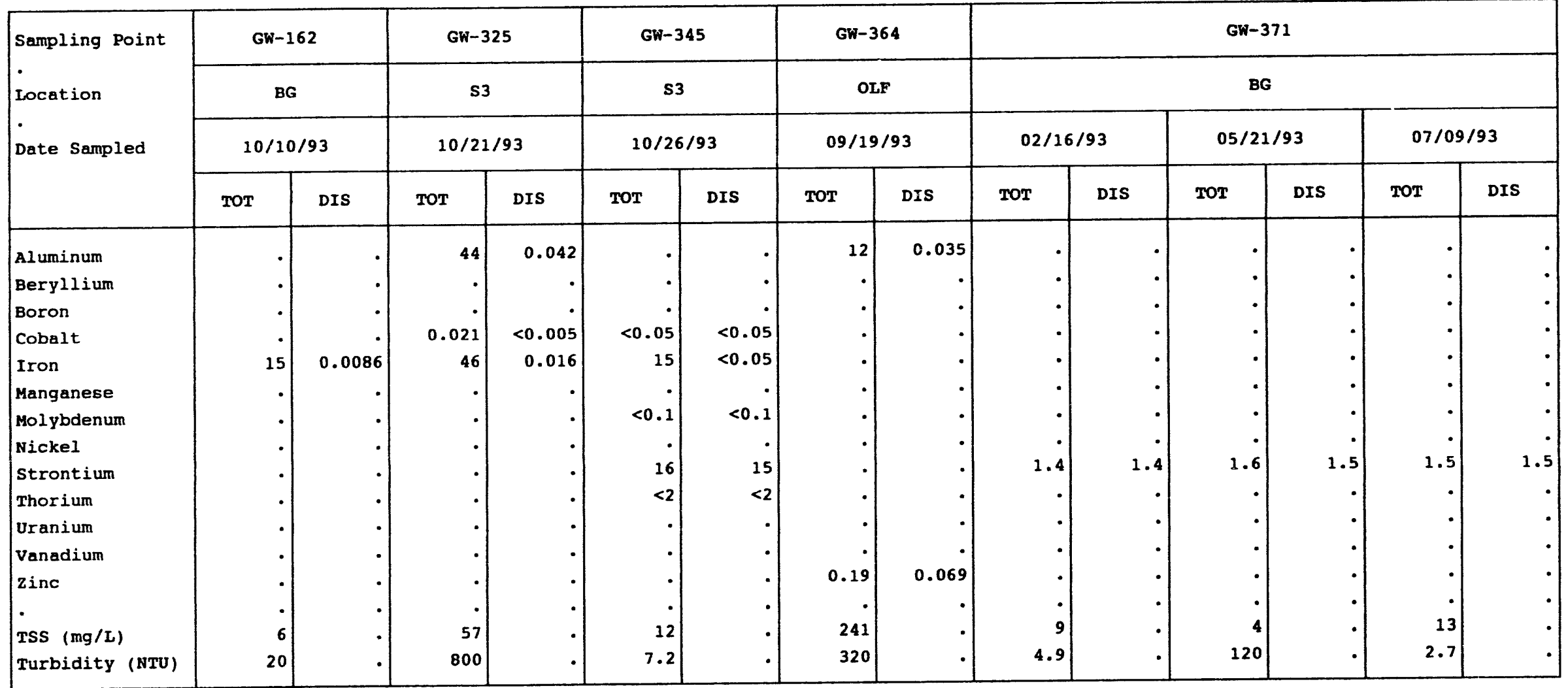

(CONTINUED) 
APPENDIX H.2

Trace Metal Concentrations That Exceed

Screening Levels, 1993

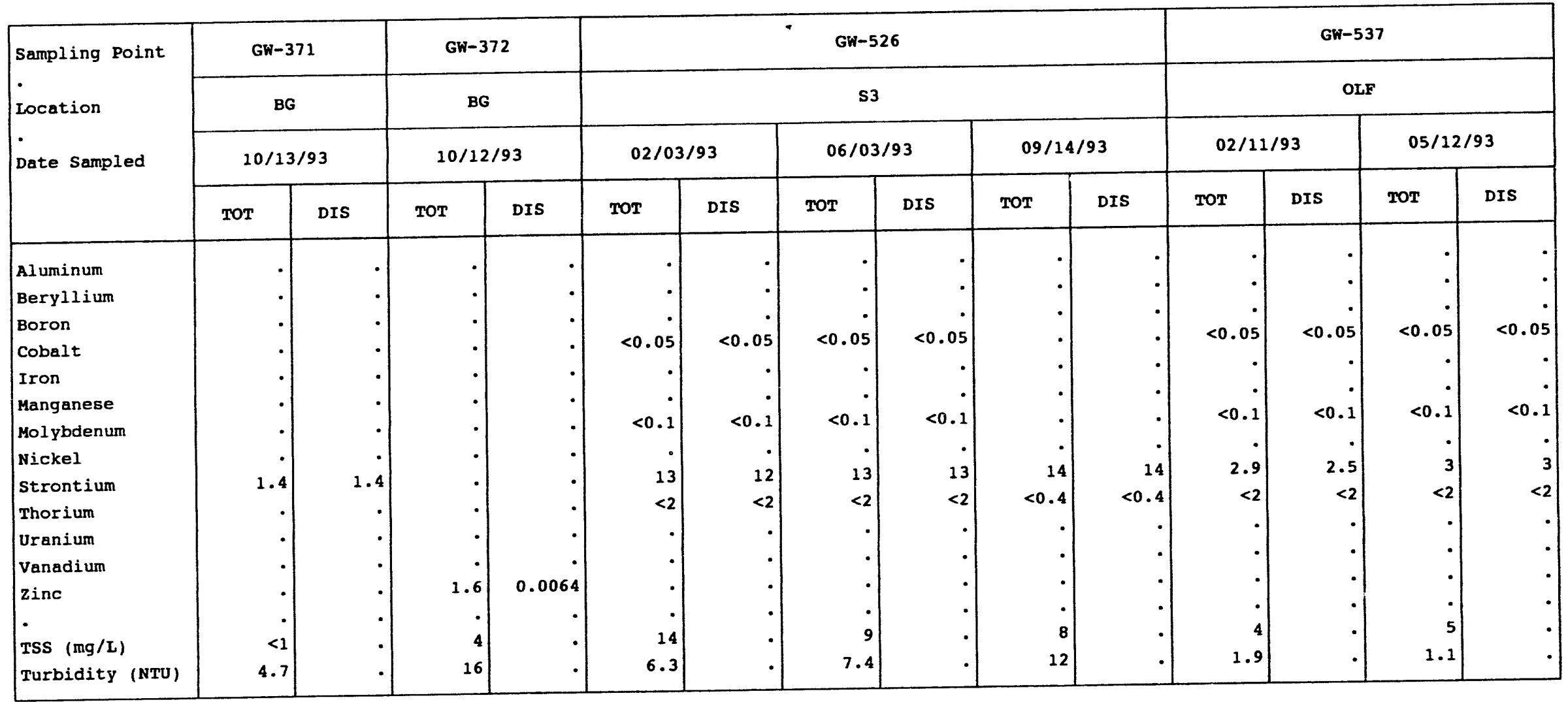

(CONTINUED) 
APPENDIX $\mathrm{H} .2$

Trace Metal Concentrations That Exceed

Screening Levels, 1993

\begin{tabular}{|c|c|c|c|c|c|c|c|c|c|c|c|c|c|c|}
\hline \multirow{4}{*}{$\begin{array}{l}\text { Sampling Point } \\
\text { Location } \\
\text { Date Sampled }\end{array}$} & \multicolumn{4}{|c|}{ GW-537 } & \multicolumn{8}{|c|}{$G W-601$} & \multicolumn{2}{|c|}{ GW-638 } \\
\hline & \multicolumn{4}{|c|}{ OLF } & \multicolumn{8}{|c|}{ OLF } & \multirow{2}{*}{\multicolumn{2}{|c|}{$\frac{\text { OLF }}{06 / 26 / 93}$}} \\
\hline & \multicolumn{2}{|c|}{$09 / 11 / 93$} & \multicolumn{2}{|c|}{$10 / 22 / 93$} & \multicolumn{2}{|c|}{$03 / 28 / 93$} & \multicolumn{2}{|c|}{$06 / 27 / 93$} & \multicolumn{2}{|c|}{$09 / 19 / 93$} & \multicolumn{2}{|c|}{$12 / 21 / 94$} & & \\
\hline & TOT & DIS & TOT & DIS & TOT & DIS & TOT & DIS & TOT & DIS & TOT & DIS & TOT & DIS \\
\hline Aluminum & . & . & - & . & . & . & . & . & . & . & . & . & 28 & 0.038 \\
\hline Beryllium & - & . & - & . & . & . & . & . & . & . & . &. & . & • \\
\hline Boron & - & - & - & - & . & . & . & . & . & . & . & . & . & . \\
\hline Cobalt & $<0.025$ & $<0.025$ & $<0.025$ & $<0.025$ & . & . & . & . & . & . & . & . & 0.018 & $<0.005$ \\
\hline Iron & . & . & . & . & . & . & . & . & . & . & . & . & 49 & 0.015 \\
\hline Manganese & & . & . & . & . & . & . & . & - & . & . & . &. & . \\
\hline Molybdenum & $<0.05$ & $<0.05$ & $<0.05$ & $<0.05$ & . & . & . & . & . & . & . & . & . & . \\
\hline Nickel & . & . & . & . & . & . & . & . & . & . & . & . & . & . \\
\hline Strontium & 2.6 & 2.6 & 2.7 & 2.7 & 1.5 & 1.5 & 1.6 & 1.6 & 1.8 & 1.9 & 1.7 & 1.7 & . & - \\
\hline Thorium & $<1$ & $<1$ & $<1$ & $<1$ &. & . & . & . & . &. &. & . & . & . \\
\hline Uranium & - & - & . & $\cdot$ &. & . & . & - & . & . & . & . & 0.016 & 0.015 \\
\hline Vanadium & . & . & . &. &. & . & . & . & . & . &. & . & 0.071 & $<0.005$ \\
\hline zine & . & . & . &. &. & . & . & . & . &. &. & . & . & . \\
\hline . & . & . & . &. &. & . & . & . & . &. &. & . &. & . \\
\hline TSS (mg/L) & 4 & . & 5 &. & 1 & . & 13 & . & $<1$ &. & $<1$ &. & 5405 & . \\
\hline Turbidity (NTU) & 1.6 & . & 1.9 &. & 0.8 & . & 0.5 & . & 0.5 &. & 0.7 & . & 3100 & . \\
\hline
\end{tabular}

(CONTINUED) 
APPENDIX Н.2

Trace Metal Concentrations That Exceed Screening Lavels, 1993

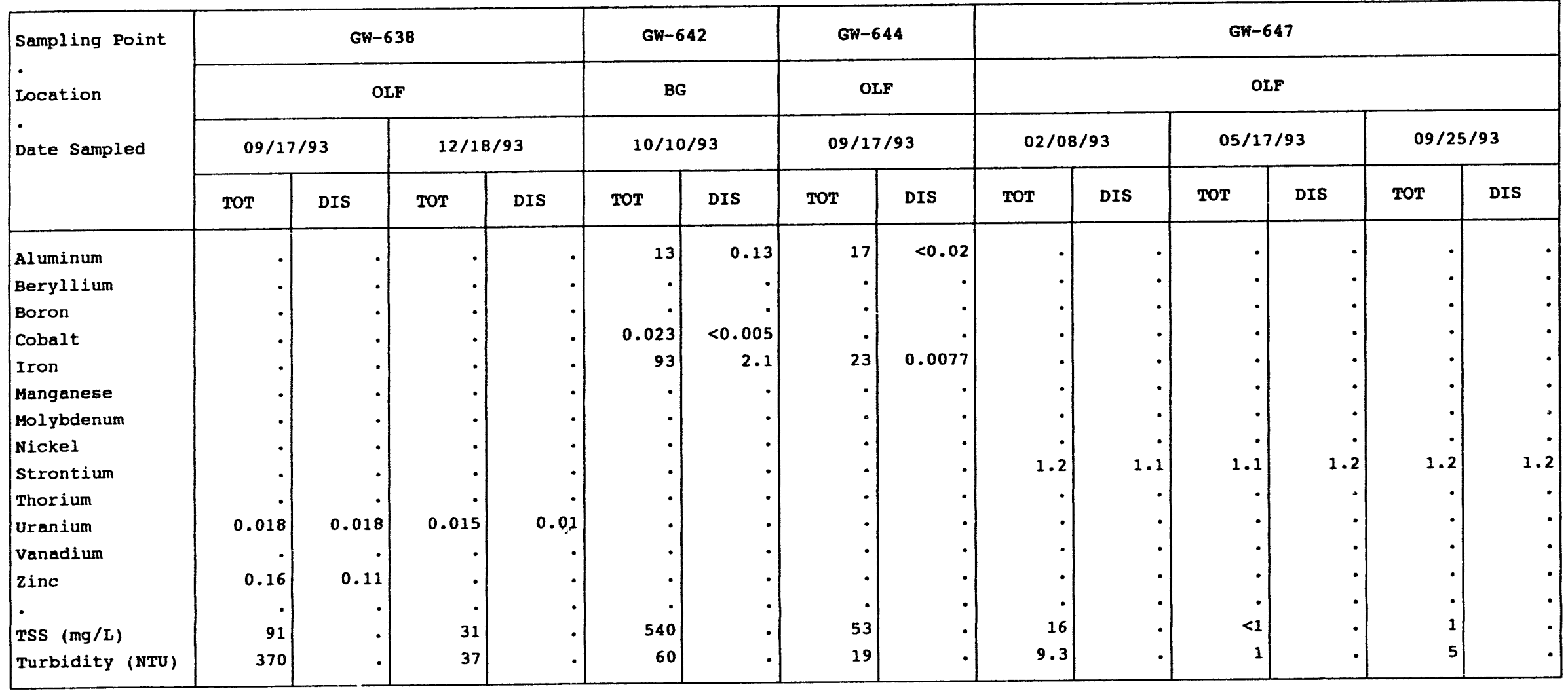

(CONTINUED) 
APPENDIX H. 2

Trace Metal Concentrations That Exceed

Screening Levels, 1993

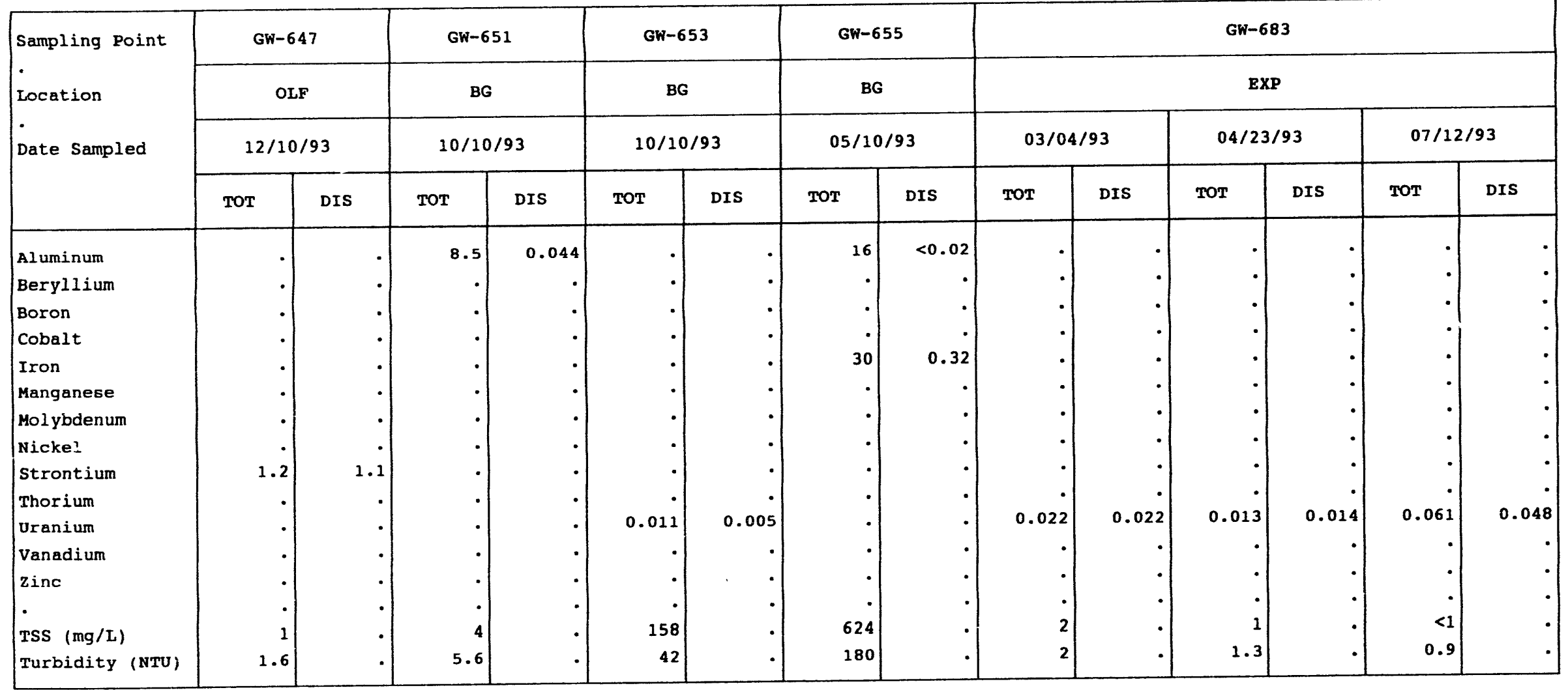

(CONTINUED) 
APPENDIX H. 2

Trace Metal Concentrations That Exceed

Screening Levels, 1993

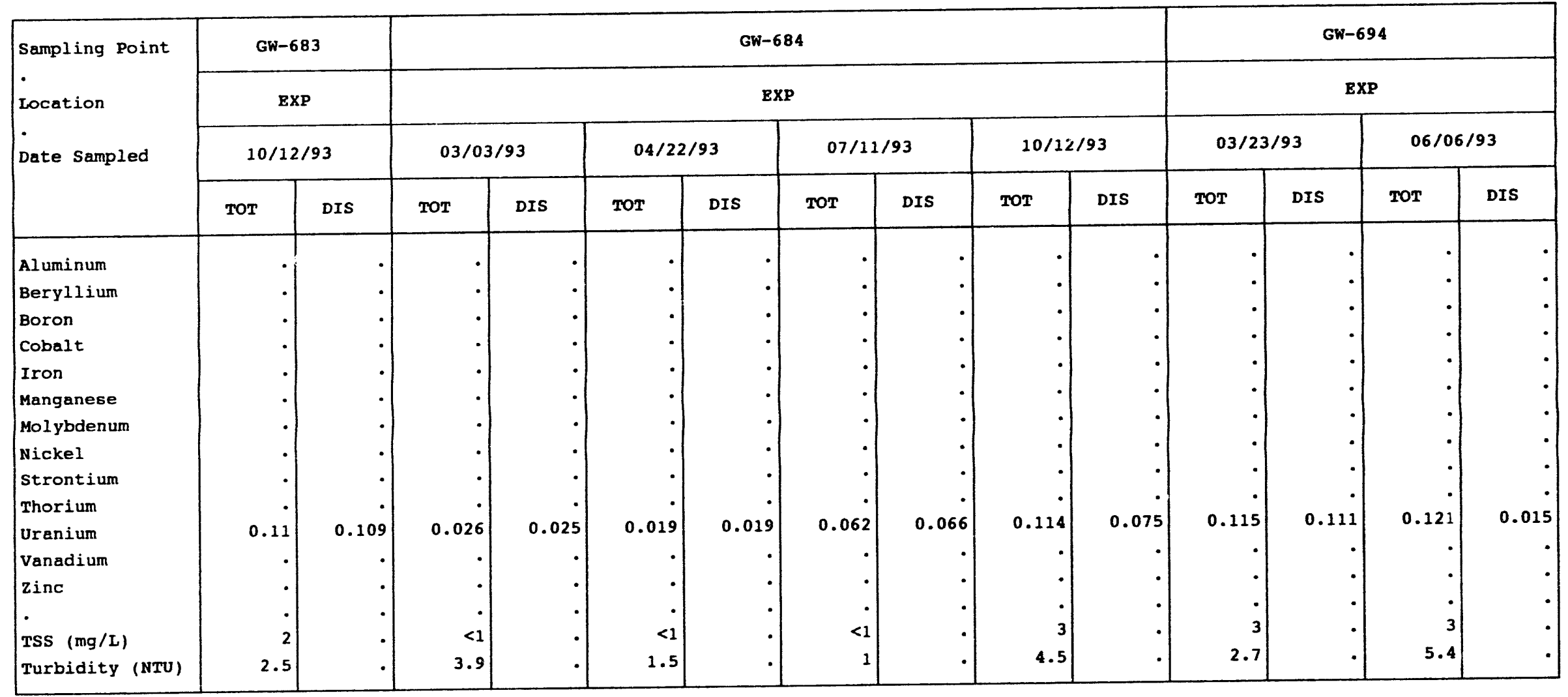

(CONTINUED) 
APPENDIX H. 2

Trace Metal Concentrations That Exceed

Screening Levels, 1993

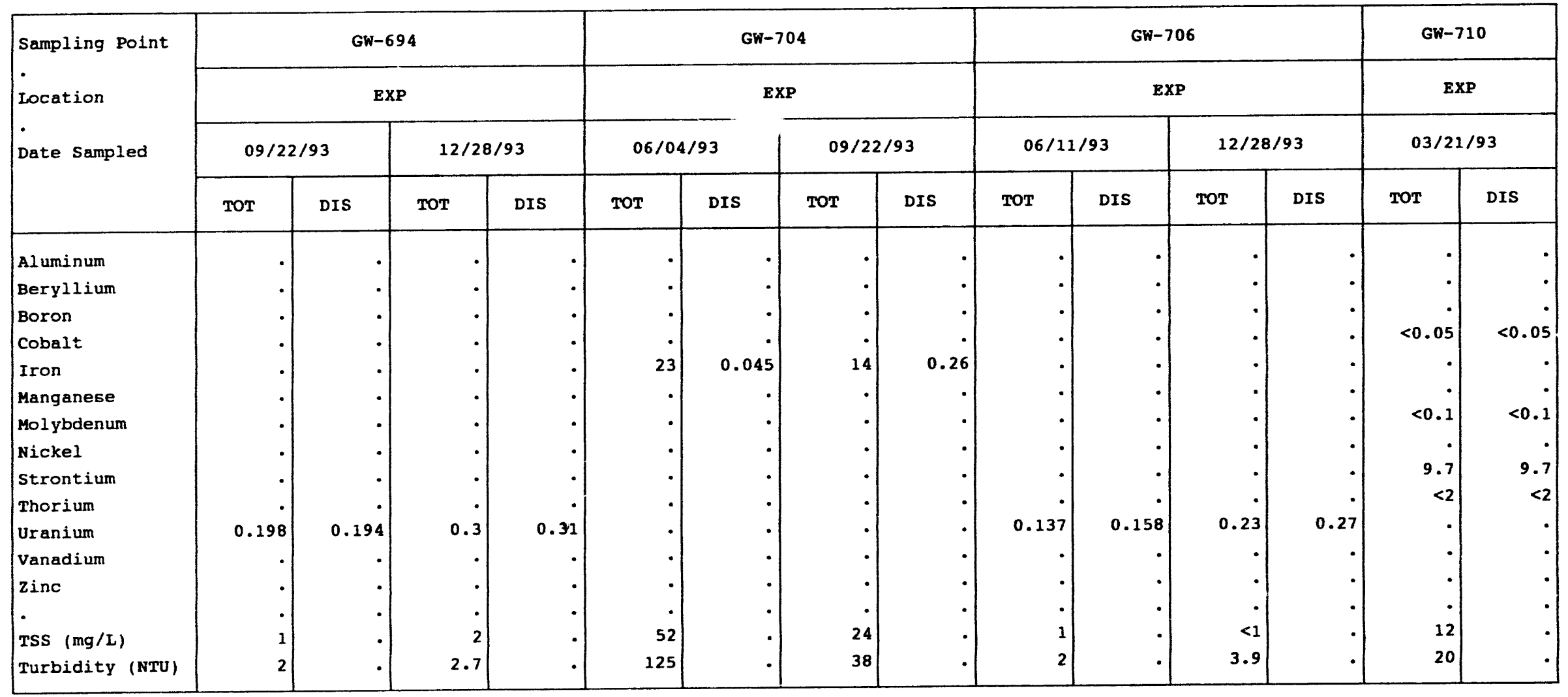

(CONTINUED) 
APPBNDIX H. 2

Trace Metal Concentrations That Exceed

Screening Levels, 1993

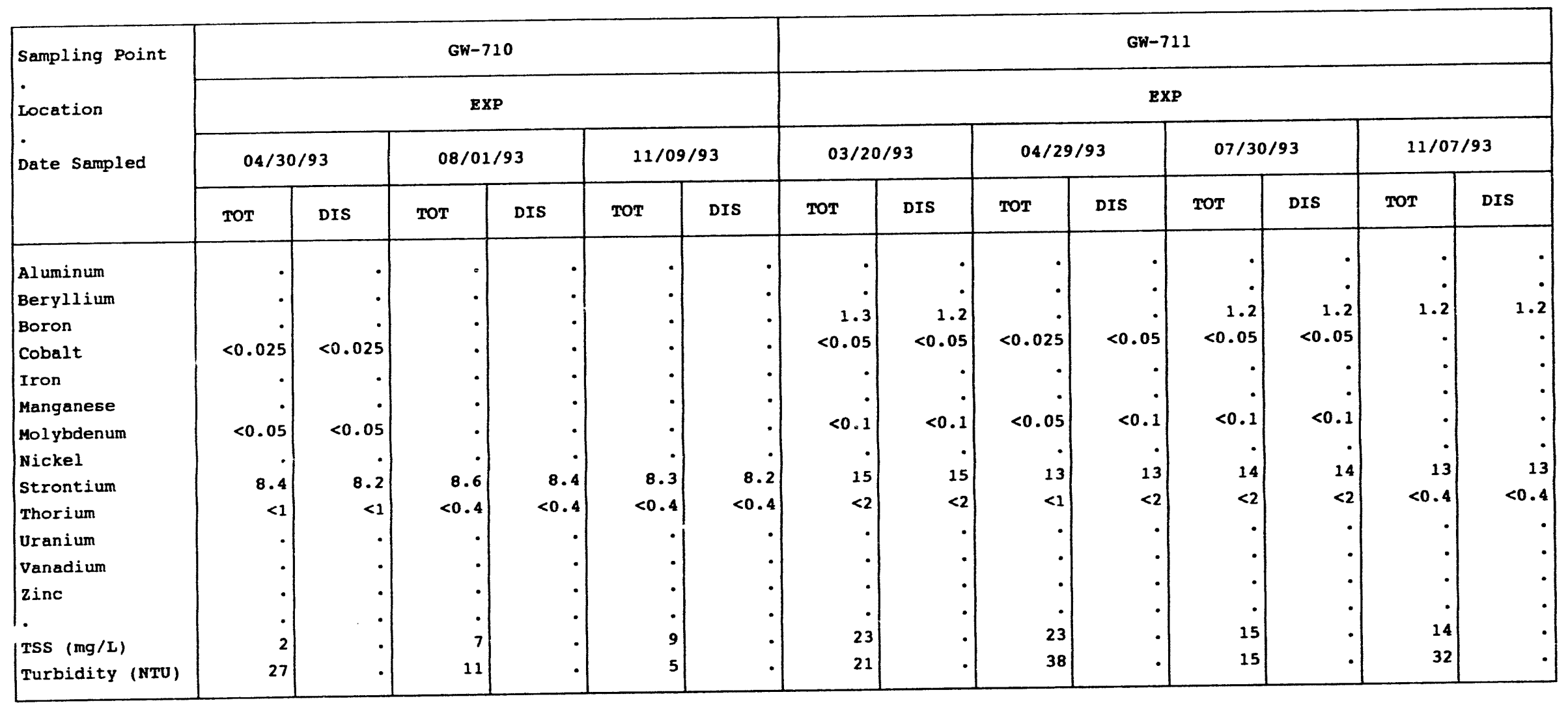

(CONTINUED) 
APPENDIX H.2

Trace Metal Concentrations That Exceed

Screening Levels, 1993

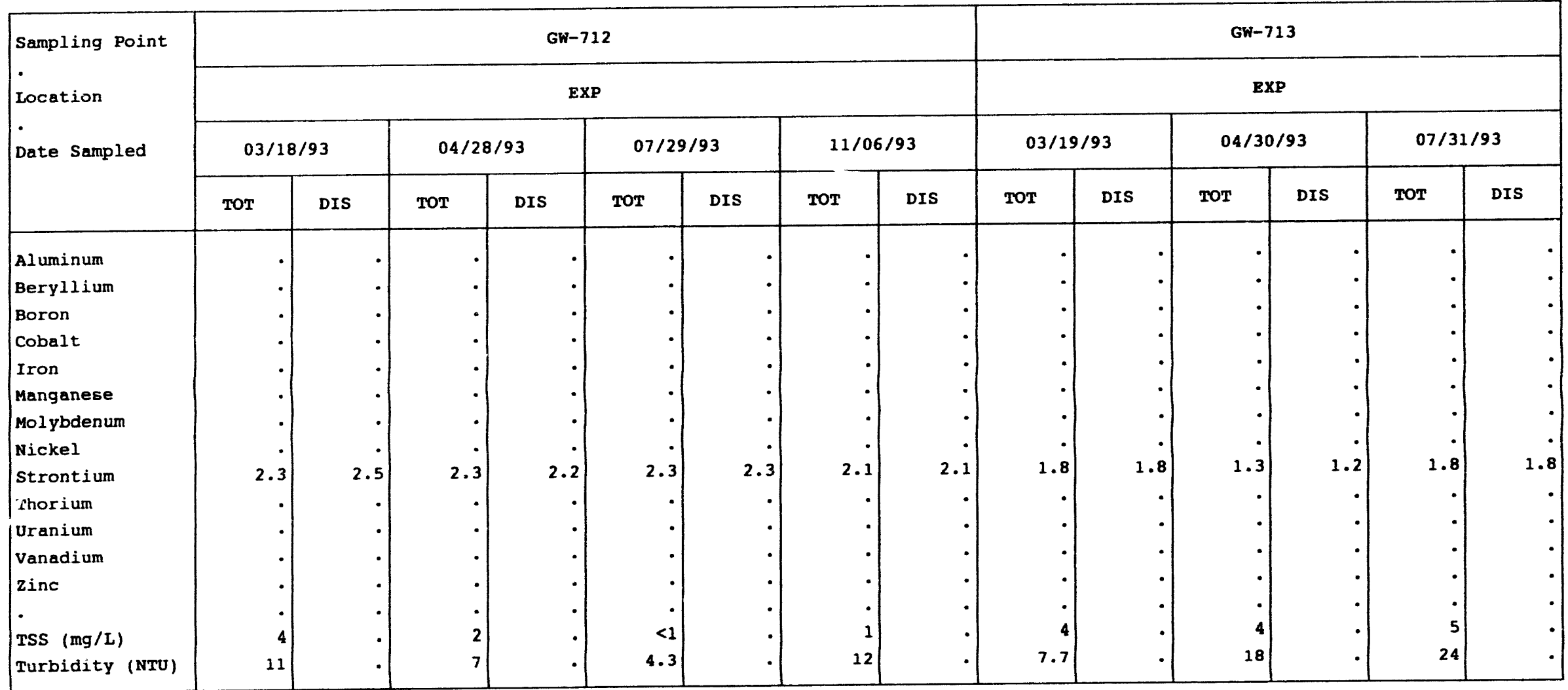

(CONTINUED) 
APPENDIX Н.2

Trace Metal Concentrations That Exceed

Screening Levels, 1993

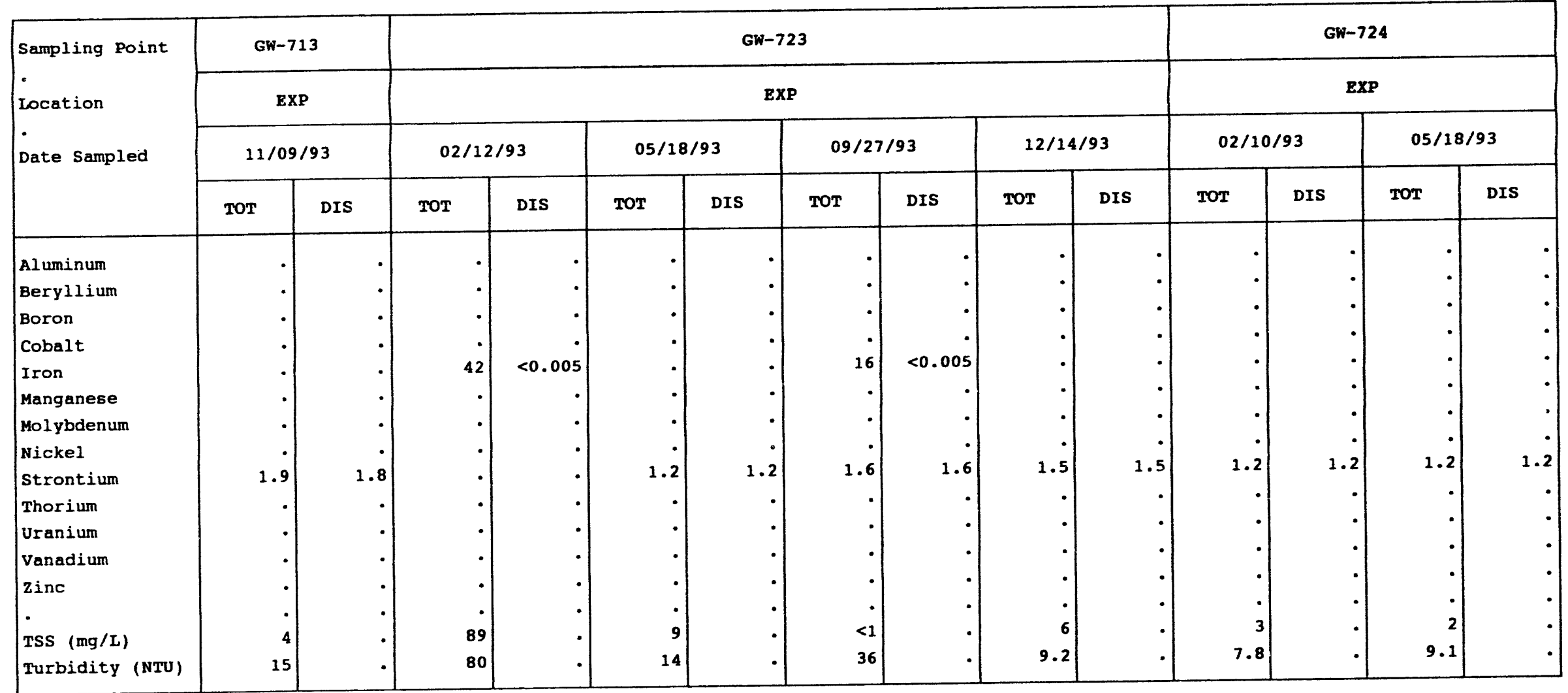

(CONTINUED) 
APPENDIX $\mathrm{H.2}$

Trace Metal Concentrations That Exceed

Screening Levels, 1993

\begin{tabular}{|c|c|c|c|c|c|c|}
\hline \multirow{4}{*}{$\begin{array}{l}\text { Sampling Point } \\
\text { Location } \\
\text { Date Sampled }\end{array}$} & \multicolumn{4}{|c|}{ GW-724 } & \multicolumn{2}{|c|}{ GW-794 } \\
\hline & \multicolumn{4}{|c|}{ EXP } & \multirow{2}{*}{\multicolumn{2}{|c|}{$\frac{\text { AGLLSF }}{03 / 01 / 93}$}} \\
\hline & \multicolumn{2}{|c|}{$09 / 29 / 93$} & \multicolumn{2}{|c|}{$12 / 14 / 93$} & & \\
\hline & TOT & DIS & TOT & DIS & TOT & DIS \\
\hline Aluminum &. & . & - & - & 9.6 & 0.022 \\
\hline Beryllium & $\cdot$ & - & - & - & - & . \\
\hline Boron & - & - & . & - & - & . \\
\hline Cobalt &. & . & . & . & $<0.05$ & $<0.005$ \\
\hline Iron & . & . & - & - & 17 & 0.0093 \\
\hline Manganese & $\cdot$ & $\cdot$ & - & - & - & • \\
\hline Mol ybdenum & $\cdot$ & • & - & - & $<0.1$ & $<0.01$ \\
\hline Nickel & $\cdot$ & - & - & - & - & - \\
\hline Strontium & 1.3 & 1.3 & 1.2 & 1.2 & - & • \\
\hline Thorium & . & . & . & . & $<2$ & $<0.2$ \\
\hline Uranium & . & . & . & - & - & • \\
\hline Vanadium &. & . & . & . & . & . \\
\hline zine & . & . & . & . & - & - \\
\hline . & $\cdot$ & . & - & - & - & - \\
\hline TSS (mg/L) & $<1$ & . & 3 & - & 224 & • \\
\hline Turbidity (NTU) & 7 &. & 16 & . & 110 & . \\
\hline
\end{tabular}


APPENDIX H.2

Trace Metal Concentrations That Bxceed

Screening Levels, 1993

\begin{tabular}{|c|c|c|c|c|c|c|c|c|c|c|c|c|c|c|}
\hline \multirow{4}{*}{$\begin{array}{l}\text { Sampling Point } \\
\text { Location } \\
\text { - } \\
\text { Date Sampled }\end{array}$} & \multicolumn{8}{|c|}{ BCK-00.63 } & \multicolumn{6}{|c|}{$B C K-04.55$} \\
\hline & \multicolumn{8}{|c|}{ EXP } & \multicolumn{6}{|c|}{ EXP } \\
\hline & \multicolumn{2}{|c|}{$03 / 09 / 93$} & \multicolumn{2}{|c|}{$05 / 03 / 93$} & \multicolumn{2}{|c|}{$08 / 16 / 93$} & \multicolumn{2}{|c|}{$11 / 08 / 93$} & \multicolumn{2}{|c|}{$03 / 09 / 93$} & \multicolumn{2}{|c|}{$05 / 03 / 93$} & \multicolumn{2}{|c|}{$08 / 16 / 93$} \\
\hline & TOT & DIS & тот & DIS & TOT & DIS & тот & DIS & TOT & DIS & TOT & DIS & TOT & DIS \\
\hline $\begin{array}{l}\text { Beryllium } \\
\text { Strontium } \\
\text { Uranium } \\
\text { TSS (mg/L) } \\
\text { Turbidity (NTU) }\end{array}$ & $\begin{array}{r}\cdot \\
. \\
0.015 \\
. \\
2 \\
4\end{array}$ & $\begin{array}{r}. \\
. \\
0.019 \\
. \\
. \\
.\end{array}$ & \begin{tabular}{r|}
$\cdot$ \\
0.018 \\
. \\
2 \\
3.2
\end{tabular} & $\begin{array}{r}. \\
. \\
0.017 \\
. \\
.\end{array}$ & $\begin{array}{r}. \\
0.032 \\
. \\
4 \\
2.5\end{array}$ & $\begin{array}{r}\cdot \\
. \\
0.033 \\
. \\
. \\
.\end{array}$ & $\begin{array}{r}\cdot \\
0.03 \\
. \\
<1 \\
3\end{array}$ & $\begin{array}{r}. \\
0.03 \\
. \\
.\end{array}$ & $\begin{array}{r}. \\
0.019 \\
. \\
2 \\
3.4\end{array}$ & $\begin{array}{r}\cdot \\
. \\
0.02 \\
. \\
.\end{array}$ & $\begin{array}{r}. \\
0.021 \\
\dot{1} \\
3\end{array}$ & $\begin{array}{r}\cdot \\
0.02 \\
. \\
. \\
.\end{array}$ & $\begin{array}{r}\cdot \\
0.03 \\
. \\
1 \\
2.3\end{array}$ & $\begin{array}{r}. \\
0.026 \\
. \\
.\end{array}$ \\
\hline
\end{tabular}

(CONTINUED)

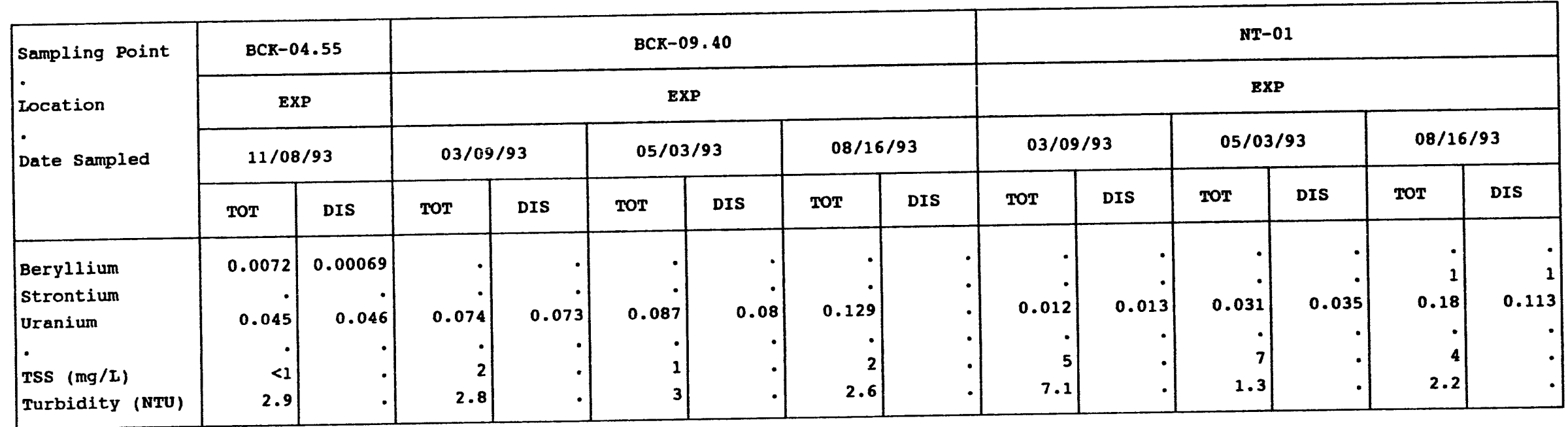

(CONTINUED) 
APPENDIX H.2

Trace Metal Concentrations That Exceed

Screening Levels, 1993

\begin{tabular}{|c|c|c|c|c|c|c|c|c|c|c|c|c|c|c|}
\hline \multirow{4}{*}{$\begin{array}{l}\text { Sampling Point } \\
\text { Location } \\
\text { Date Sampled }\end{array}$} & \multicolumn{2}{|c|}{ NT-01 } & \multicolumn{8}{|c|}{ SS-1 } & \multicolumn{4}{|c|}{ ss-4 } \\
\hline & \multicolumn{2}{|c|}{ EXP } & \multicolumn{8}{|c|}{$\operatorname{EXP}$} & \multicolumn{4}{|c|}{$\mathbf{E X P}$} \\
\hline & \multicolumn{2}{|c|}{$11 / 08 / 93$} & \multicolumn{2}{|c|}{$03 / 09 / 93$} & \multicolumn{2}{|c|}{$05 / 03 / 93$} & \multicolumn{2}{|c|}{$08 / 16 / 93$} & \multicolumn{2}{|c|}{$11 / 08 / 93$} & \multicolumn{2}{|c|}{$03 / 09 / 93$} & \multicolumn{2}{|c|}{$05 / 03 / 93$} \\
\hline & TOT & DIS & TOT & DIS & TOT & DIS & TOT & DIS & TOT & DIS & TOT & DIS & TOT & DIS \\
\hline Beryliium & • & & $\cdot$ & - & $\cdot 1$ & $\cdot$ & $\cdot 1$ & $\cdot$ & $\cdot$ & $\cdot 1$ & $\cdot 1$ & $\cdot$ & $\cdot$ & - \\
\hline Strontium & 0.92 & 0.89 & $\cdot$ & • & $\cdot$ & $\cdot$ & $\cdot$ & $\cdot$ & $\cdot$ & $\cdot$ & $\cdot 1$ & $\cdot$ & $\cdot$ & - \\
\hline Uranium & 0.185 & 0.207 & 0.031 & 0.034 & 0.016 & 0.019 & 0.045 & 0.056 & 0.04 & 0.04 & 0.084 & 0.087 & 0.112 & 0.107 \\
\hline - & - & • & $\cdot$ & • & $\cdot$ & $\cdot$ & $\cdot$ & $\cdot$ & $\cdot$ & $\cdot 1$ & $\cdot 1$ & - & $\cdot$ & - \\
\hline TSS (mg/L) & 8 & - & $<1$ & - & 9 & $\cdot$ & 5 & $\cdot$ & 10 & $\cdot$ & 10 & $\cdot$ & 6 & - \\
\hline Turbidity (NTU) & 3.3 & - & 0.8 & - & 3 & $\cdot$ & 1.3 & $\cdot$ & 1.6 &. & 2.5 & $\cdot$ & 2.4 & . \\
\hline
\end{tabular}

(CONTINUED)

\begin{tabular}{|c|c|c|c|c|c|c|c|c|c|c|c|c|c|c|}
\hline \multirow{4}{*}{$\begin{array}{l}\text { Sampling Point } \\
\text { Location } \\
\text { Date Sampled }\end{array}$} & \multicolumn{4}{|c|}{ ss-4 } & \multicolumn{8}{|c|}{ ss-5 } & \multicolumn{2}{|c|}{ ss-6 } \\
\hline & \multicolumn{4}{|c|}{ EXP } & \multicolumn{8}{|c|}{ EXP } & \multirow{2}{*}{\multicolumn{2}{|c|}{$\frac{\operatorname{EXP}}{11 / 08 / 93}$}} \\
\hline & \multicolumn{2}{|c|}{$08 / 16 / 93$} & \multicolumn{2}{|c|}{$11 / 08 / 93$} & \multicolumn{2}{|c|}{$03 / 09 / 93$} & \multicolumn{2}{|c|}{$05 / 03 / 93$} & \multicolumn{2}{|c|}{$08 / 16 / 93$} & \multicolumn{2}{|c|}{$11 / 08 / 93$} & & \\
\hline & TOT & DIS & TOT & DIS & TOT & DIS & TOT & DIS & TOT & DIS & TOT & DIS & TOT & DIS \\
\hline Beryllium & . & - & $\cdot 1$ & - & - & - & - & - & - & - &. & • &. & • \\
\hline Strontium & - & - & $\cdot$ & - & . & - & - & - & - & - & $\cdot 1$ & - & $\cdot$ & - \\
\hline Uranium & 0.181 & 0.177 & 0.24 & 0.12 & 0.018 & 0.019 & 0.025 & 0.026 & 0.098 & 0.078 & 0.1 & 0.11 & 0.15 & 0.17 \\
\hline . & . & - & $\cdot$ & - & - & - & • & - & • & - & $\cdot 1$ & • & - & $\cdot$ \\
\hline TSS $(\mathrm{mg} / \mathrm{L})$ & 10 & - & 41 & - & 3 & - & $<1$ & - & $<1$ & - & 5 & - & 3 & • \\
\hline Turbidity (NTU) & 2.7 & - & 2 & . & 3 & . & 2.1 & . & 2.2 & • & 4 & . & 2.4 & . \\
\hline
\end{tabular}

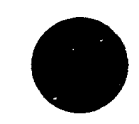




\section{APPENDIX I}

QA/QC DATA SUMMARIES 
SAMPLING POINT:

$\begin{aligned} \text { BCK } & \text { - Bear Creek Kilometer } \\ \text { GW } & - \text { Monitoring Well } \\ \text { NT } & - \text { North Tributary to Bear Creek } \\ \text { SS } & - \text { Spring }\end{aligned}$

\section{LOCATION:}

$$
\begin{aligned}
\text { AGLLSF } & \text { - } \\
\text { BG } & \text { - Beare Grade Low Level Storage Facility } \\
\text { EXP } & - \text { Exit-Pathway Monitoring } \\
\text { OLF } & - \text { Oil Landfarm WMA } \\
\text { RS } & - \text { Rust Spoil Area } \\
\text { S3 } & - \text { S-3 Site } \\
\text { SPI } & - \text { Spoil Area I }
\end{aligned}
$$

\section{NOTES:}

- Not analyzed

U - Compound not detected at the reported minimum attainable detection limit.

B - Well was bailed

W - Well Wizard (Dedicated sampling equipment)

NA - Not Applicable

\section{UNITS:}

$\mu \mathrm{g} / \mathrm{L} \quad-\quad$ micrograms per liter

$\mathrm{mg} / \mathrm{L}$ - milligrams per liter

$\mathrm{pCi} / \mathrm{L}$ - picoCuries per liter

$\mathrm{Bq} / \mathrm{L} \quad-\quad$ Becquerels per liter

CE +/- - Counting Error (two standard deviations) 
APPENDIX 1.1

CORRELATION OF GROUNDWATER AND SURFACE-WATER SAMPLES TO ASSOCIATED BLANKS 
APPENDIX I. 1

Correlation of samples to Associated Blanks

\begin{tabular}{|c|c|c|c|c|c|c|}
\hline $\begin{array}{l}\text { sampling } \\
\text { pt. }\end{array}$ & Location & $\begin{array}{l}\text { Pump } \\
\text { No. }\end{array}$ & Date & $\begin{array}{c}\text { Sample } \\
\text { No. }\end{array}$ & $\begin{array}{c}\text { Trip Blank } \\
\text { No. }\end{array}$ & $\begin{array}{c}\text { Lab Blank } \\
\text { No. }\end{array}$ \\
\hline \multicolumn{7}{|c|}{ First quarter 1993} \\
\hline$G W-013$ & OLF & $\mathbf{w}$ & $03 / 29 / 93$ & $930329-084$ & $930329-086$ & $930330-003$ \\
\hline$G W-040$ & BG & B & $03 / 25 / 93$ & $930326-017$ & $9303215-021$ & $930331-039$ \\
\hline GW-043 & OLF & $\mathbf{w}$ & $02 / 04 / 93$ & $930204-293$ & $930204-297$ & $930205-035$ \\
\hline$G W-044$ & OLF & $\mathbf{W}$ & $02 / 04 / 93$ & $930204-291$ & $930204-297$ & $930205-035$ \\
\hline GW-052 & $B G$ & B & $03 / 25 / 93$ & $930326-019$ & $930326-021$ & $930331-039$ \\
\hline GW-056 & BG & $\mathbf{w}$ & $03 / 04 / 93$ & $930305-005$ & $930305-011$ & $930308-001$ \\
\hline GW -057 & EXP & w & $03 / 04 / 93$ & $930305-006$ & $930305-011$ & $930308-001$ \\
\hline GW-063 & OLF & $\mathbf{B}$ & $02 / 23 / 93$ & $930223-085$ & $930223-087$ & $930225-001$ \\
\hline$G W-064$ & OLF & $w$ & $02 / 09 / 93$ & $930209-064$ & $930209-066$ & $930210-022$ \\
\hline GW-069 & BG & $\mathbf{w}$ & $03 / 03 / 93$ & $930303-124$ & $930303-127$ & $930304-076$ \\
\hline GW-082 & BG & $w$ & $03 / 10 / 93$ & $.930311-076$ & $930311-082$ & $930311-022$ \\
\hline$G W-084$ & OLF & $w$ & $02 / 04 / 93$ & $930204-295$ & $930204-297$ & $930209-001$ \\
\hline GW-085 & OLF & 8 & $02 / 11 / 93$ & $930211-337$ & $930211-343$ & $930216-001$ \\
\hline$G W-086$ & OLF & 8 & $02 / 05 / 93$ & $930205-072$ & $930205-074$ & $930208-001$ \\
\hline GW-115 & s3 & $w$ & $01 / 28 / 93$ & $930128-114$ & $930128-047$ & $930129-016$ \\
\hline GW-118 & BG & B & $03 / 04 / 93$ & $930304-236$ & $930304-238$ & $930305-002$ \\
\hline$G W-286$ & BG & 10 & $02 / 25 / 93$ & $930226-306$ & $930226-308$ & $930301-001$ \\
\hline$G W-287$ & BG & 10 & $02 / 24 / 93$ & $930225-007$ & $930225-015$ & $930225-030$ \\
\hline GW-311 & RS & 8 & $02 / 08 / 93$ & $930208-159$ & $930208-161$ & $930210-021$ \\
\hline GW-312 & RS & B & $02 / 08 / 93$ & $930208-157$ & $930208-161$ & $930210-021$ \\
\hline$G W-315$ & SPI & 10 & $02 / 03 / 93$ & $930204-003$ & $930204-007$ & $930204-034$ \\
\hline GW-316 & SPI & 10 & $02 / 02 / 93$ & $930203-002$ & $930203-008$ & $930203-057$ \\
\hline GW -317 & SPI & 10 & $02 / 02 / 93$ & $930203-004$ & $930203-008$ & $930203-057$ \\
\hline$G W-323$ & SPI & 10 & $02 / 02 / 93$ & $930203-006$ & $930203-008$ & $930203-057$ \\
\hline$G W-324$ & s3 & 10 & $01 / 29 / 93$ & $930129-099$ & $930129-101$ & $930201-001$ \\
\hline$G W-325$ & s3 & 10 & $02 / 01 / 93$ & $930201-132$ & $930201-134$ & $930202-004$ \\
\hline$G W-345$ & s3 & 10 & $02 / 03 / 93$ & $930204-005$ & $930204-007$ & $930204-034$ \\
\hline GW -347 & s3 & 10 & $02 / 01 / 93$ & $930201-128$ & $930201-134$ & $930202-004$ \\
\hline$G W-348$ & s3 & 10 & $02 / 01 / 93$ & $930201-130$ & $930201-134$ & $930202-004$ \\
\hline$G W-364$ & OLF & 8 & $03 / 27 / 93$ & $930329-011$ & $930329-013$ & $930331-039$ \\
\hline$G W-365$ & OLF & 8 & $03 / 28 / 93$ & $930329-021$ & $930329-023$ & $930330-003$ \\
\hline GW-370 & BG & 6 & $02 / 17 / 93$ & $930217-070$ & $930217-072$ & $930218-002$ \\
\hline GW-371 & BG & 6 & $02 / 16 / 93$ & $930216-140$ & $930216-142$ & $930217-004$ \\
\hline$G W-372$ & BG & 6 & $02 / 09 / 93$ & $930209-088$ & $930209-090$ & $930210-022$ \\
\hline GW-373 & BG & 6 & $02 / 11 / 93$ & $930211-332$ & $930211-336$ & $930212-068$ \\
\hline GW-526 & s3 & 10 & $02 / 03 / 93$ & $930204-001$ & $930204-007$ & $930204-034$ \\
\hline GW-537 & OLF & 8 & $02 / 11 / 93$ & $930211-338$ & $930211-343$ & $930216-001$ \\
\hline$G W-601$ & OLF & 6 & $03 / 28 / 93$ & $930329-029$ & $930329-031$ & $930330-003$ \\
\hline$G W-621$ & BG & 10 & $03 / 20 / 93$ & $930322-010$ & $930322-014$ & $930323-042$ \\
\hline GW-626 & BG & 6 & $03 / 10 / 93$ & $930311-078$ & $930311-082$ & $930311-022$ \\
\hline$G W-627$ & BG & 6 & $03 / 09 / 93$ & $930309-123$ & $930309-125$ & $930310-001$ \\
\hline GW-636 & OLF & 8 & $03 / 26 / 93$ & $930329-006$ & $930329-010$ & $930401-019$ \\
\hline GW -637 & OLF & 8 & $03 / 25 / 93$ & $930325-117$ & $930325-117$ & $930326-001$ \\
\hline$G W-638$ & OLF & 8 & $03 / 25 / 93$ & $930325-119$ & $930325-121$ & $930326-001$ \\
\hline GW-639 & BG & 6 & $02 / 24 / 93$ & $930225-003$ & $930225-011$ & $930225-030$ \\
\hline
\end{tabular}


APPENDIX I. 1

Correlation of Samples to Associated Blanks

\begin{tabular}{|c|c|c|c|c|c|c|}
\hline $\begin{array}{l}\text { sampling } \\
\text { pt. }\end{array}$ & Location & $\begin{array}{l}\text { Pump } \\
\text { No. }\end{array}$ & Date & $\begin{array}{c}\text { Sample } \\
\text { No. }\end{array}$ & $\begin{array}{c}\text { Trip Blank } \\
\text { No. }\end{array}$ & $\begin{array}{c}\text { Lab Blank } \\
\text { No. }\end{array}$ \\
\hline$G W-640$ & BG & 6 & $02 / 22 / 93$ & $930222-104$ & $930222-108$ & $930223-001$ \\
\hline$G W-641$ & BG & 6 & $02 / 22 / 93$ & $930222-105$ & $930222-108$ & $930223-001$ \\
\hline$G W-642$ & BG & 6 & $02 / 08 / 93$ & $930208-162$ & $930208-164$ & $930210-021$ \\
\hline$G W-643$ & BG & 6 & $02 / 16 / 93$ & $930216-138$ & $930216-142$ & $930217-004$ \\
\hline$G W-644$ & OLE & 8 & $03 / 25 / 93$ & $930325-115$ & $930325-121$ & $930326-001$ \\
\hline GW -647 & OLF & 7 & $02 / 08 / 93$ & $930209-002$ & $930209-004$ & $930210-021$ \\
\hline$G W-651$ & BG & 10 & $03 / 01 / 93$ & $930302-009$ & $930302-015$ & $930302-060$ \\
\hline$G W-652$ & BG & 10 & $03 / 01 / 93$ & $930302-010$ & $930302-015$ & $930302-060$ \\
\hline$G W-653$ & BG & 10 & $03 / 01 / 93$ & $930302-011$ & $930302-015$ & $930302-060$ \\
\hline$G W-654$ & BG & 10 & $02 / 24 / 93$ & $930225-008$ & $930225-015$ & $930225-030$ \\
\hline GW-655 & BG & 6 & $02 / 05 / 93$ & $930205-069$ & $930205-071$ & $930208-001$ \\
\hline$G W-683$ & EXP & 10 & $03 / 04 / 93$ & $930305-007$ & $930305-011$ & $930308-001$ \\
\hline$G W-684$ & EXP & 10 & $03 / 03 / 93$ & $930303-105$ & $930303-107$ & $930304-069$ \\
\hline$G W-685$ & EXP & 10 & $03 / 02 / 93$ & $930302-081$ & $930302-085$ & $930303-001$ \\
\hline$G W-694$ & EXP & 8 & $03 / 23 / 93$ & $930323-069$ & $930323-071$ & $930325-011$ \\
\hline GW-695 & EXP & 8 & $03 / 24 / 93$ & $930324-097$ & $930324-099$ & $930325-001$ \\
\hline GW-703 & EXP & 10 & $03 / 20 / 93$ & $930322-012$ & $930322-014$ & $930322-005$ \\
\hline GW-704 & EXP & 10 & $03 / 22 / 93$ & $930322-111$ & $930322-115$ & $930323-042$ \\
\hline GW-706 & EXP & 10 & $03 / 23 / 93$ & $930323-056$ & $930323-058$ & $930325-011$ \\
\hline GW-710 & EXP & 2 & $03 / 21 / 93$ & $930322-006$ & $930322-009$ & $930323-002$ \\
\hline GW-711 & EXP & 8 & $03 / 20 / 93$ & $930322-037$ & $930,322-043$ & $930323-002$ \\
\hline GW-712 & EXP & 4 & $03 / 18 / 93$ & $930319-002$ & $930319-006$ & $930322-002$ \\
\hline GW -713 & EXP & 4 & $03 / 19 / 93$ & $930319-114$ & $930319-116$ & $930322-005$ \\
\hline GW -714 & EXP & 8 & $03 / 21 / 93$ & $930322-041$ & $930322-044$ & $930323-002$ \\
\hline GW-715 & EXP & 8 & $03 / 21 / 93$ & $930322-039$ & $930322-044$ & $930323-002$ \\
\hline$G W-723$ & EXP & 2 & $02 / 12 / 93$ & $930212-105$ & $930212-111$ & $930216-089$ \\
\hline$G W-724$ & EXP & 10 & $02 / 10 / 93$ & $930210-183$ & $930210-187$ & $930211-001$ \\
\hline GW -725 & EXP & 10 & $02 / 12 / 93$ & $930212-106$ & $930212-111$ & $930216-089$ \\
\hline GW-736 & EXP & 7 & $02 / 11 / 93$ & $930211-327$ & $930211-331$ & $930212-068$ \\
\hline GW-737 & EXP & 7 & $02 / 11 / 93$ & $930211-328$ & $930211-331$ & $930212-068$ \\
\hline GW-738 & EXP & 10 & $02 / 10 / 93$ & $930210-185$ & $930210-187$ & $930211-001$ \\
\hline GW-739 & EXP & 7 & $02 / 10 / 93$ & $930210-026$ & $930210-029$ & $930211-011$ \\
\hline$G W-740$ & EXP & 7 & $02 / 09 / 93$ & $930210-024$ & $930210-028$ & $930211-011$ \\
\hline GW-793 & AGLLSF & 6 & $02 / 26 / 93$ & $930226-313$ & $930226-315$ & $930226-343$ \\
\hline GW-794 & AGLLSF & 6 & $03 / 01 / 93$ & $930302-005$ & $930302-008$ & $930302-060$ \\
\hline GW-795 & AGLLSF & 6 & $03 / 02 / 93$ & $930302-069$ & $930302-071$ & $930303-002$ \\
\hline ss-1 & EXP & NA & $03 / 09 / 93$ & $930309-107$ & $930309-109$ & $930310-002$ \\
\hline ss -4 & EXP & NA & $03 / 09 / 93$ & $930309-103$ & $930309-109$ & $930310-002$ \\
\hline ss -5 & EXP & NA & $03 / 09 / 93$ & $930309-101$ & $930309-109$ & $930310-002$ \\
\hline ss-6 & EXP & NA & $03 / 09 / 93$ & $930309-097$ & $930309-109$ & $930310-001$ \\
\hline ss-8 & EXP & NA & $03 / 09 / 93$ & $930309-093$ & $930309-109$ & $930310-001$ \\
\hline $\mathrm{BCK}-00.63$ & EXP & NA & $03 / 09 / 93$ & $930309-089$ & $930309-109$ & $930310-001$ \\
\hline BCK-04.55 & EXP & NA & $03 / 09 / 93$ & $930309-091$ & $930309-109$ & $930310-001$ \\
\hline $\mathrm{BCK}-09.40$ & EXP & NA & $03 / 09 / 93$ & $930309-099$ & $930309-109$ & $930310-002$ \\
\hline NT-01 & EXP & NA & $03 / 09 / 93$ & $930309-105$ & $930309-109$ & $930310-002$ \\
\hline$N T-13$ & EXP & NA & $03 / 09 / 93$ & $930309-095$ & $930309-109$ & $930310-001$ \\
\hline
\end{tabular}


APPENDIX I.I

Correlation of samples to Associated Blanks

\begin{tabular}{|c|c|c|c|c|c|c|}
\hline $\begin{array}{l}\text { sampling } \\
\text { Pt. }\end{array}$ & Iocation & $\begin{array}{l}\text { Pump } \\
\text { No. }\end{array}$ & Date & $\begin{array}{c}\text { sample } \\
\text { No. }\end{array}$ & $\begin{array}{c}\text { Trip Blank } \\
\text { No. }\end{array}$ & $\begin{array}{c}\text { Lab Blank } \\
\text { No. }\end{array}$ \\
\hline
\end{tabular}

Second Quarter 1993

\begin{tabular}{|c|c|c|c|c|c|c|}
\hline GW-013 & OLF & $w$ & $06 / 17 / 93$ & $930617-164$ & $930617-166$ & $930619-007$ \\
\hline$G W-040$ & BG & B & $05 / 11 / 93$ & $930511-097$ & $930511-101$ & $930513-045$ \\
\hline$G W-043$ & OLF & $\mathbf{W}$ & $05 / 10 / 93$ & $930510-150$ & $930510-156$ & $930512-001$ \\
\hline$G W-044$ & OLF & W & $05 / 10 / 93$ & $930510-151$ & $930510-156$ & $930512-001$ \\
\hline GW-052 & BG & B & $04 / 26 / 93$ & $930427-004$ & $930427-008$ & $930428-001$ \\
\hline$G W-056$ & BG & W & $04 / 22 / 93$ & $930423-013$ & $930423-019$ & $930423-001$ \\
\hline GW-057 & EXP & W & $04 / 22 / 93$ & $930423-015$ & $930423-019$ & $930423-001$ \\
\hline$G W-064$ & OLF & $\mathbf{W}$ & $05 / 12 / 93$ & $930513-009$ & $930513-017$ & $930514-001$ \\
\hline GW-069 & BG & $w$ & $04 / 21 / 93$ & $930422-029$ & $930422-033$ & $930422-001$ \\
\hline GW-082 & BG & $\mathbf{w}$ & $04 / 21 / 93$ & $930422-031$ & $930422-033$ & $930422-001$ \\
\hline$G W-084$ & OLF & $\mathbf{W}$ & $05 / 10 / 93$ & $930510-152$ & $930510-156$ & $930512-001$ \\
\hline GW-085 & OLF & 5 & $05 / 12 / 93$ & $930513-011$ & $930513-017$ & $930514-001$ \\
\hline$G W-086$ & OLF & 5 & $05 / 11 / 93$ & $930512-019$ & $930512-027$ & $930513-045$ \\
\hline$G W-115$ & s3 & W & $05 / 25 / 93$ & $930526-002$ & $930526-010$ & $930528-043$ \\
\hline$G W-118$ & BG & B & $04 / 21 / 93$ & $930422-027$ & $930422-027$ & $930422-001$ \\
\hline$G W-286$ & BG & 7 & $04 / 19 / 93$ & $930419-083$ & $930419-089$ & $930421-001$ \\
\hline GW-287 & BG & 7 & $04 / 16 / 93$ & $930416-078$ & $930416-080$ & $930419-001$ \\
\hline$G W-311$ & RS & 5 & $05 / 11 / 93$ & $930512-021$ & $930512-027$ & $930513-045$ \\
\hline GW-312 & RS & 5 & $05 / 11 / 93$ & $930512-023$ & $930512-027$ & $930513-045$ \\
\hline GW-315 & SPI & 8 & $06 / 03 / 93$ & $930603-171$ & $930603-175$ & $930606-001$ \\
\hline$G W-316$ & SPI & 8 & $06 / 01 / 93$ & $930602-004$ & $930602-006$ & $930605-001$ \\
\hline$G W-317$ & SPI & 8 & $05 / 28 / 93$ & $930528-145$ & $930528-149$ & $930604-019$ \\
\hline$G W-323$ & SPI & 8 & $05 / 28 / 93$ & $930528-146$ & $930528-149$ & $930604-019$ \\
\hline$G W-324$ & s3 & 8 & $05 / 25 / 93$ & $930526-006$ & $930526-010$ & $930528-043$ \\
\hline$G W-325$ & s3 & 8 & $05 / 25 / 93$ & $930526-008$ & $930526-010$ & $930529-016$ \\
\hline$G W-345$ & s3 & 8 & $06 / 01 / 93$ & $930602-002$ & $930602-006$ & $930605-001$ \\
\hline$G W-347$ & s3 & 8 & $05 / 27 / 93$ & $930527-332$ & $930527-334$ & $930530-001$ \\
\hline$G W-348$ & s3 & 8 & $05 / 27 / 93$ & $930527-330$ & $930527-334$ & $930530-001$ \\
\hline$G W-364$ & OLF & 5 & $06 / 27 / 93$ & $930628-014$ & $930628-020$ & $930630-009$ \\
\hline$G W-365$ & OLF & 4 & $06 / 27 / 93$ & $930628-082$ & $930628-084$ & $930630-009$ \\
\hline GW-370 & BG & 4 & $05 / 21 / 93$ & $930521-106$ & $930521-110$ & $930522-012$ \\
\hline GW-371 & BG & 4 & $05 / 21 / 93$ & $930521-108$ & $930521-110$ & $930522-012$ \\
\hline GW-372 & BG & 4 & $05 / 19 / 93$ & $930520-002$ & $930520-006$ & $930522-012$ \\
\hline GW -373 & BG & 7 & $05 / 14 / 93$ & $930514-075$ & $930514-077$ & $930518-127$ \\
\hline GW-526 & s3 & 8 & $06 / 03 / 93$ & $930603-172$ & $930603-175$ & $930606-001$ \\
\hline GW-537 & OLF & 5 & $05 / 12 / 93$ & $930513-013$ & $930513-017$ & $930514-001$ \\
\hline$G W-601$ & OLF & 10 & $06 / 27 / 93$ & $930628-010$ & $930628-012$ & $930630-009$ \\
\hline$G W-621$ & BG & 7 & $06 / 02 / 93$ & $930603-009$ & $930603-011$ & $930605-001$ \\
\hline$G W-626$ & BG & 6 & $04 / 26 / 93$ & $930427-006$ & $930427-008$ & $930429-001$ \\
\hline GW-627 & BG & 6 & $04 / 23 / 93$ & $930423-106$ & $930423-110$ & $930426-001$ \\
\hline$G W-636$ & OLF & 5 & $06 / 27 / 93$ & $330628-016$ & $930628-020$ & $930630-001$ \\
\hline GW-637 & OLF & 5 & $06 / 26 / 93$ & $930628-005$ & $930628-009$ & $930630-001$ \\
\hline$G W-638$ & OLF & 5 & $06 / 26 / 93$ & $930628-007$ & $930628-009$ & $930630-001$ \\
\hline GW-639 & BG & 4 & $05 / 24 / 93$ & $930525-002$ & $930525-010$ & $930526-019$ \\
\hline
\end{tabular}


APPENDIX I. 1

Correlation of Samples to Associated Blanks

\begin{tabular}{|c|c|c|c|c|c|c|}
\hline $\begin{array}{l}\text { sampling } \\
\text { pt. }\end{array}$ & Location & $\begin{array}{l}\text { Pump } \\
\text { No. }\end{array}$ & Date & $\begin{array}{c}\text { sample } \\
\text { No. }\end{array}$ & $\begin{array}{c}\text { Trip Blank } \\
\text { No. }\end{array}$ & $\begin{array}{c}\text { Lab Blank } \\
\text { No. }\end{array}$ \\
\hline$G W-640$ & BG & 4 & $05 / 24 / 93$ & $930525-006$ & $930525-010$ & $930526-019$ \\
\hline$G W-641$ & BG & 4 & $05 / 24 / 93$ & $930525-008$ & $930525-010$ & $930526-019$ \\
\hline$G W-642$ & BG & 7 & $05 / 11 / 93$ & $930511-099$ & $930511-101$ & $930514-001$ \\
\hline$G W-643$ & BG & 4 & $05 / 19 / 93$ & $930520-004$ & $930520-006$ & $930522-012$ \\
\hline$G W-644$ & OLF & 10 & $06 / 25 / 93$ & $930625-292$ & $930625-294$ & $930628-002$ \\
\hline$G W-647$ & OLF & 10 & $05 / 17 / 93$ & $930518-020$ & $930518-022$ & $930520-057$ \\
\hline$G W-651$ & BG & 7 & $04 / 19 / 93$ & $930419-087$ & $930419-089$ & $930420-001$ \\
\hline$G W-652$ & BG & 7 & $04 / 19 / 93$ & $930419-085$ & $930419-089$ & $930421-001$ \\
\hline$G W-653$ & BG & 7 & $04 / 20 / 93$ & $930421-014$ & $930421-016$ & $930421-113$ \\
\hline$G W-654$ & BG & 7 & $04 / 16 / 93$ & $930416-076$ & $930416-080$ & $930419-001$ \\
\hline$G W-655$ & BG & 7 & $05 / 10 / 93$ & $930510-160$ & $930510-162$ & $930512-001$ \\
\hline$G W-683$ & EXP & 7 & $04 / 23 / 93$ & $930423-079$ & $930423-081$ & $930426-001$ \\
\hline$G W-684$ & EXP & 7 & $04 / 22 / 93$ & $930423-017$ & $930423-019$ & $930423-001$ \\
\hline$G W-685$ & EXP & 7 & $04 / 21 / 93$ & $930421-120$ & $930421-122$ & $930422-001$ \\
\hline$G W-694$ & EXP & 7 & $06 / 06 / 93$ & $930607-017$ & $930607-019$ & $930610-001$ \\
\hline$G W-695$ & EXP & 7 & $06 / 09 / 93$ & $930609-056$ & $930609-058$ & $930611-083$ \\
\hline GW-703 & EXP & 7 & $06 / 03 / 93$ & $930604-006$ & $930604-008$ & $930607-002$ \\
\hline$G W-704$ & EXP & 7 & $06 / 04 / 93$ & $930604-102$ & $930604-106$ & $930607-002$ \\
\hline GW-706 & EXP & 7 & $06 / 11 / 93$ & $930611-136$ & $930611-138$ & $930614-047$ \\
\hline GW-710 & ExP & 2 & $04 / 30 / 93$ & $930430-075$ & $930430-077$ & $930504-053$ \\
\hline GW-711 & EXP & 8 & $04 / 29 / 93$ & $930430-012$ & $930430-014$ & $930430-007$ \\
\hline GW-712 & EXP & 10 & $04 / 28 / 93$ & $930428-110$ & $930428-114$ & $930429-001$ \\
\hline$G W-713$ & EXP & 10 & $04 / 30 / 93$ & $930430-047$ & $930430-049$ & $930427-131$ \\
\hline GW-714 & EXP & 8 & $04 / 29 / 93$ & $930430-015$ & $930430-014$ & $930430-007$ \\
\hline$G W-715$ & EXP & 8 & $04 / 29 / 93$ & $930430-017$ & $930430-014$ & $930430-007$ \\
\hline$G W-723$ & EXP & 2 & $05 / 18 / 93$ & $930518-162$ & $930518-164$ & $930520-057$ \\
\hline$G W-724$ & EXP & 10 & $05 / 18 / 93$ & $930519-004$ & $930519-010$ & $930520-057$ \\
\hline$G W-725$ & EXP & 6 & $05 / 19 / 93$ & $930519-210$ & $930519-214$ & $930521-067$ \\
\hline GW -736 & EXP & 10 & $05 / 18 / 93$ & $930519-006$ & $930519-010$ & $930520-057$ \\
\hline GW -737 & EXP & 10 & $05 / 19 / 93$ & $930519-194$ & $930519-196$ & $930520-057$ \\
\hline$G W-738$ & EXP & 10 & $05 / 18 / 93$ & $930519-008$ & $930519-010$ & $930520-057$ \\
\hline GW-739 & EXP & 6 & $05 / 18 / 93$ & $930518-160$ & $930518-164$ & $930520-057$ \\
\hline$G W-740$ & EXP & 10 & $05 / 17 / 93$ & $930518-023$ & $930518-025$ & $930520-057$ \\
\hline GW-793 & AGLLSF & 4 & $05 / 25 / 93$ & $930525-084$ & $930525-090$ & $930527-064$ \\
\hline$G W-794$ & AGLISF & 4 & $05 / 25 / 93$ & $930525-085$ & $930525-090$ & $930529-016$ \\
\hline GW-795 & AGLLSF & 4 & $05 / 25 / 93$ & $930525-086$ & $930525-090$ & $930528-043$ \\
\hline$G W-800$ & OLF & 5 & $05 / 11 / 93$ & $930512-025$ & $930512-027$ & $930514-001$ \\
\hline ss-1 & EXP & NA & $05 / 03 / 93$ & $930503-093$ & $930503-095$ & $930505-041$ \\
\hline$s s-4$ & EXP & NA & $05 / 03 / 93$ & $930503-089$ & $930503-095$ & $930505-041$ \\
\hline$s s-5$ & EXP & NA & $05 / 03 / 93$ & $930503-085$ & $930503-095$ & $930505-041$ \\
\hline ss -6 & EXP & NA & $05 / 03 / 93$ & $930503-087$ & $930503-095$ & $930505-041$ \\
\hline ss - 8 & EXP & NA & $05 / 03 / 93$ & $930503-079$ & $930503-095$ & $930504-053$ \\
\hline $\mathrm{BCK}-00.63$ & EXP & NA & $05 / 03 / 93$ & $930503-075$ & $930503-095$ & $930505-041$ \\
\hline $\mathrm{BCK}-04.55$ & EXP & NA & $05 / 03 / 93$ & $930503-077$ & $930503-095$ & $930504-053$ \\
\hline $\mathrm{BCK}-09.40$ & EXP & NA & $05 / 03 / 93$ & $930503-083$ & $930503-095$ & $930505-041$ \\
\hline NT-01 & EXP & NA & $05 / 03 / 93$ & $930503-091$ & $930503-095$ & $930505-041$ \\
\hline$N T-13$ & EXP & NA & $05 / 03 / 93$ & $930503-081$ & $930503-095$ & $930504-053$ \\
\hline
\end{tabular}


APPENDIX I. 1

Correlation of samples to Associated Blanks

\begin{tabular}{|c|c|c|c|c|c|c|}
\hline $\begin{array}{c}\text { sampling } \\
\text { pt. }\end{array}$ & Location & $\begin{array}{l}\text { Pump } \\
\text { Na. }\end{array}$ & Date & $\begin{array}{c}\text { sample } \\
\text { No. }\end{array}$ & $\begin{array}{c}\text { Trip Blank } \\
\text { No. }\end{array}$ & $\begin{array}{c}\text { Lab Blank } \\
\text { No. }\end{array}$ \\
\hline
\end{tabular}

Third Quarter 1993

\begin{tabular}{|c|c|c|c|c|c|c|}
\hline GW-013 & OLF & w & $09 / 17 / 93$ & $930918-017$ & $930918-012$ & $930920-116$ \\
\hline GW-040 & BG & B & $07 / 02 / 93$ & $930702-104$ & $930702-110$ & $930707-090$ \\
\hline GW-043 & OLF & $\mathbf{w}$ & $09 / 11 / 93$ & $930913-025$ & $930913-037$ & $930913-064$ \\
\hline GW-044 & OLF & $\mathbf{w}$ & $09 / 11 / 93$ & $930913-027$ & $930913-037$ & $930913-064$ \\
\hline GW-056 & BG & w & $07 / 09 / 93$ & $930709-065$ & $930709-069$ & $930712-037$ \\
\hline GW-057 & $\operatorname{EXP}$ & w & $07 / 09 / 93$ & $930709-067$ & $930709-069$ & $930712-037$ \\
\hline$G W-064$ & OLF & w & $09 / 11 / 93$ & $930913-031$ & $930913-037$ & $930914-009$ \\
\hline$G W-069$ & BG & $\boldsymbol{w}$ & $07 / 25 / 93$ & $930726-003$ & $930726-009$ & $930728-001$ \\
\hline$G W-082$ & BG & w & $07 / 25 / 93$ & $930726-005$ & $930726-009$ & $930728-001$ \\
\hline$G W-084$ & OLF & w & $09 / 11 / 93$ & $930913-033$ & $930913-037$ & $930914-009$ \\
\hline$G W-085$ & OLF & 7 & $09 / 14 / 93$ & $930915-020$ & $930915-017$ & $930916-020$ \\
\hline$G W-086$ & OLF & 7 & $09 / 12 / 93$ & $930913-066$ & $930913-068$ & $930914-009$ \\
\hline GW-115 & s3 & w & $09 / 11 / 93$ & $930913-137$ & $930913-139$ & $930915-110$ \\
\hline$G W-118$ & BG & B & $07 / 25 / 93$ & $930726-007$ & $930726-009$ & $930728-001$ \\
\hline$G W-286$ & BG & 4 & $07 / 10 / 93$ & $930712-029$ & $930712-031$ & $930713-001$ \\
\hline$G W-287$ & BG & 4 & $07 / 10 / 93$ & $930712-023$ & $930712-031$ & $930712-080$ \\
\hline GW-311 & $\mathbf{R S}$ & 7 & $09 / 12 / 93$ & $930913-071$ & $930913-068$ & $930914-009$ \\
\hline$G W-312$ & RS & 7 & $09 / 12 / 93$ & $930913-069$ & $930913-068$ & $930914-009$ \\
\hline GW-315 & SPI & 6 & $09 / 14 / 93$ & $930915-013$ & $930915-026$ & $930916-031$ \\
\hline GW-316 & SPI & 6 & $09 / 13 / 93$ & $930913-244$ & $930913-248$ & $930916-020$ \\
\hline$G W-317$ & SPI & 6 & $09 / 13 / 93$ & $930913-246$ & $930913-248$ & $930915-107$ \\
\hline$G W-323$ & SPI & 6 & $09 / 12 / 93$ & $930913-151$ & $930913-148$ & $930915-107$ \\
\hline GW-324 & s3 & 6 & $09 / 11 / 93$ & $930913-142$ & $930913-139$ & $930915-110$ \\
\hline$G W-325$ & s3 & 6 & $09 / 11 / 93$ & $930913-144$ & $930913-139$ & $930915-110$ \\
\hline$G W-345$ & s3 & 6 & $09 / 14 / 93$ & $930915-009$ & $930915-026$ & $930916-031$ \\
\hline$G W-347$ & s3 & 6 & $09 / 12 / 93$ & $930913-146$ & $930913-148$ & $930915-107$ \\
\hline GW-348 & s3 & 6 & $09 / 12 / 93$ & $930913-149$ & $930913-148$ & $930915-107$ \\
\hline$G W-364$ & OLF & 3 & $09 / 19 / 93$ & $930920-021$ & $930920-025$ & $930920-030$ \\
\hline$G W-365$ & OLF & 3 & $09 / 19 / 93$ & $930920-023$ & $930920-025$ & $930920-030$ \\
\hline$G W-370$ & BG & 5 & $07 / 10 / 93$ & $930712-002$ & $930712-008$ & $930712-080$ \\
\hline GW-371 & BG & 5 & $07 / 09 / 93$ & $930709-057$ & $930709-059$ & $930712-037$ \\
\hline GW-372 & BG & 5 & $07 / 02 / 93$ & $930702-106$ & $930702-110$ & $930707-090$ \\
\hline GW -373 & BG & 5 & $07 / 02 / 93$ & $930702-108$ & $930702-110$ & $930707-090$ \\
\hline$G W-526$ & s3 & 6 & $09 / 14 / 93$ & $930915-011$ & $930915-026$ & $930916-031$ \\
\hline GW-537 & OLF & 7 & $09 / 14 / 93$ & $930915-018$ & $930915-017$ & $930916-020$ \\
\hline GW-601 & OLF & 7 & $09 / 19 / 93$ & $930920-027$ & $930920-048$ & $930920-030$ \\
\hline GW-621 & BG & 7 & $09 / 20 / 93$ & $930921-001$ & $930921-005$ & $930921-025$ \\
\hline$G W-626$ & BG & 8 & $08 / 02 / 93$ & $930802-115$ & $930802-119$ & $930803-040$ \\
\hline GW-627 & BG & 8 & $07 / 28 / 93$ & $930728-103$ & $930728-105$ & $930729-001$ \\
\hline GW-636 & OLF & 3 & $09 / 18 / 93$ & $930920-016$ & $930920-020$ & $930920-030$ \\
\hline GW-637 & OLF & 3 & $09 / 17 / 93$ & $930918-019$ & $930918-012$ & $930921-039$ \\
\hline GW -638 & OLF & 3 & $09 / 17 / 93$ & $930918-015$ & $930918-012$ & $930920-116$ \\
\hline GW-639 & BG & 5 & $07 / 11 / 93$ & $930712-009$ & $930712-017$ & $930713-014$ \\
\hline$G W-640$ & BG & 5 & $07 / 10 / 93$ & $930712-004$ & $930712-008$ & $930712-080$ \\
\hline
\end{tabular}


APPENDIX I. 1

Correlation of Samples to Associated BlankB

\begin{tabular}{|c|c|c|c|c|c|c|}
\hline $\begin{array}{l}\text { Sampling } \\
\text { pt. }\end{array}$ & Location & $\begin{array}{l}\text { Pump } \\
\text { No. }\end{array}$ & Date & $\begin{array}{c}\text { Sample } \\
\text { No. }\end{array}$ & $\begin{array}{c}\text { Trip Blank } \\
\text { No. }\end{array}$ & $\begin{array}{c}\text { Lab Blank } \\
\text { No. }\end{array}$ \\
\hline$G W-641$ & BG & 5 & $07 / 10 / 93$ & $930712-006$ & $930712-008$ & $930712-080$ \\
\hline$G W-642$ & BG & 5 & $07 / 01 / 93$ & $930702-014$ & $930702-018$ & $930707-032$ \\
\hline$G W-643$ & BG & 5 & $07 / 08 / 93$ & $930708-048$ & $930708-050$ & $930709-031$ \\
\hline$G W-644$ & OLF & 3 & $09 / 17 / 93$ & $930918-013$ & $930918-012$ & $930920-116$ \\
\hline$G W-647$ & OLF & 10 & $09 / 25 / 93$ & $930927-011$ & $930927-013$ & $930928-089$ \\
\hline$G W-651$ & BG & 4 & $07 / 10 / 93$ & $930712-018$ & $930712-022$ & $930712-080$ \\
\hline$G W-652$ & BG & 4 & $07 / 10 / 93$ & $930712-027$ & $930712-031$ & $930713-001$ \\
\hline$G W-653$ & BG & 4 & $07 / 10 / 93$ & $930712-020$ & $930712-022$ & $930713-014$ \\
\hline$G W-654$ & BG & 4 & $07 / 01 / 93$ & $930702-024$ & $930702-026$ & $930707-090$ \\
\hline$G W-655$ & $\mathbf{B G}$ & 5 & $07 / 01 / 93$ & $930702-016$ & $930702-018$ & $930707-032$ \\
\hline GW-683 & EXP & 4 & $07 / 12 / 93$ & $930712-110$ & $930712-112$ & $930714-024$ \\
\hline$G W-684$ & EXP & 4 & $07 / 11 / 93$ & $930712-032$ & $930712-036$ & $930713-001$ \\
\hline GW-685 & EXP & 4 & $07 / 11 / 93$ & $930712-025$ & $930712-036$ & $930713-001$ \\
\hline GW-694 & EXP & 5 & $09 / 22 / 93$ & $930922-062$ & $930922-064$ & $930923-093$ \\
\hline GW-695 & EXP & 7 & $09 / 22 / 93$ & $930923-004$ & $930923-010$ & $930923-093$ \\
\hline GW-703 & EXP & 7 & $09 / 20 / 93$ & $930921-003$ & $930921-005$ & $930921-025$ \\
\hline GW-704 & EXP & 7 & $09 / 22 / 93$ & $930923-006$ & $930923-010$ & $930923-093$ \\
\hline GW-706 & EXP & 7 & $09 / 23 / 93$ & $930924-007$ & $930924-009$ & $930927-018$ \\
\hline GW -710 & EXP & 2 & $08 / 01 / 93$ & $930802-039$ & $930802-043$ & $930802-075$ \\
\hline GW -711 & $\mathbf{E X P}$ & 7 & $07 / 30 / 93$ & $930730-105$ & $930730-107$ & $930802-008$ \\
\hline GW-712 & EXP & 10 & $07 / 29 / 93$ & $930729-374$ & $930729-376$ & $930802-008$ \\
\hline GW -713 & EXP & 10 & $07 / 31 / 93$ & $930802-032$ & $930802-036$ & $930802-075$ \\
\hline GW -714 & EXP & 10 & $08 / 01 / 93$ & $930802-037$ & $930802-043$ & $930803-040$ \\
\hline GW-715 & EXP & 10 & $08 / 01 / 93$ & $930802-041$ & $930802-043$ & $930803-040$ \\
\hline GW -723 & EXP & 2 & $09 / 27 / 93$ & $930928-059$ & $930928-056$ & $930929-174$ \\
\hline GW-724 & $\mathbf{E X P}$ & 6 & $09 / 29 / 93$ & $930929-187$ & $930929-189$ & $930930-079$ \\
\hline GW-725 & EXP & 10 & $09 / 28 / 93$ & $930928-117$ & $930928-119$ & $930930-001$ \\
\hline$G W-736$ & EXP & 5 & $09 / 28 / 93$ & $930928-129$ & $930928-131$ & $930930-001$ \\
\hline GW -737 & EXP & 5 & $09 / 28 / 93$ & $930928-132$ & $930928-131$ & $930930-001$ \\
\hline GW-738 & EXP & 5 & $09 / 27 / 93$ & $930928-061$ & $930928-056$ & $930929-174$ \\
\hline GW-739 & EXP & 10 & $09 / 27 / 93$ & $930927-237$ & $930927-239$ & $930929-174$ \\
\hline GW-740 & EXP & 5 & $09 / 27 / 93$ & $930928-054$ & $930928-056$ & $930929-174$ \\
\hline GW-793 & AGLISF & 5 & $07 / 11 / 93$ & $930712-013$ & $930712-017$ & $930713-014$ \\
\hline GW-794 & AGLLSF & 5 & $07 / 11 / 93$ & $930712-015$ & $930712-017$ & $930712-080$ \\
\hline GW-795 & AGLISF & 5 & $07 / 12 / 93$ & $930712-076$ & $930712-078$ & $930713-001$ \\
\hline$G W-800$ & OLF & 7 & $09 / 14 / 93$ & $930915-015$ & $930915-017$ & $930916-020$ \\
\hline ss-1 & EXP & NA & $08 / 16 / 93$ & $930816-143$ & $930816-147$ & $930818-001$ \\
\hline ss -4 & EXP & NA & $08 / 16 / 93$ & $930816-141$ & $930816-147$ & $930818-001$ \\
\hline ss -5 & EXP & NA & $08 / 16 / 93$ & $930816-137$ & $930816-147$ & $930817-086$ \\
\hline ss-6 & $\operatorname{EXP}$ & NA & $08 / 16 / 93$ & $930816-139$ & $930816-147$ & $930817-086$ \\
\hline ss -8 & EXP & NA & $08 / 16 / 93$ & $930816-131$ & $930816-147$ & $930817-086$ \\
\hline $\mathrm{BCK}-00.63$ & EXP & NA & $08 / 16 / 93$ & $930816-127$ & $930816-147$ & $930817-086$ \\
\hline BCK-04.55 & EXP & NA & $08 / 16 / 93$ & $930816-129$ & $930816-147$ & $930817-086$ \\
\hline BCK-09.40 & EXP & NA & $08 / 16 / 93$ & $930816-136$ & $930816-147$ & $930818-001$ \\
\hline$N T-01$ & EXP & NA & $08 / 16 / 93$ & $930816-145$ & $930816-147$ & $930818-001$ \\
\hline$N T-13$ & EXP & NA & $08 / 16 / 93$ & $930816-133$ & $930816-147$ & $930817-086$ \\
\hline
\end{tabular}


APPENDIX I.1

correlation of Samples to Associated Blanks

\begin{tabular}{|c|c|c|c|c|c|c|}
\hline $\begin{array}{c}\text { sampling } \\
\text { pt. }\end{array}$ & Location & $\begin{array}{l}\text { Pump } \\
\text { No. }\end{array}$ & Date & $\begin{array}{c}\text { Sample } \\
\text { No. }\end{array}$ & $\begin{array}{c}\text { Trip Blank } \\
\text { No. }\end{array}$ & $\begin{array}{c}\text { Lab Blank } \\
\text { No. }\end{array}$ \\
\hline
\end{tabular}

Fourth Quarter 1993

\begin{tabular}{|c|c|c|c|c|c|c|}
\hline$G W-013$ & OLF & W & $12 / 19 / 93$ & $931219-041$ & $931219-043$ & $931220-103$ \\
\hline GW -040 & BG & 10 & $10 / 09 / 93$ & $931011-005$ & $931011-007$ & $931011-055$ \\
\hline GW-042 & BG & $\boldsymbol{W}$ & $12 / 29 / 93$ & $931229-046$ & $931229-048$ & $940103-032$ \\
\hline$G W-043$ & OLF & $\boldsymbol{w}$ & $10 / 19 / 93$ & $931019-052$ & $931019-054$ & $931021-024$ \\
\hline$G W-044$ & OLF & W & $10 / 19 / 93$ & $931019-050$ & $931019-054$ & $931021-024$ \\
\hline$G W-056$ & BG & w & $10 / 09 / 93$ & $931011-010$ & $931011-012$ & $931011-055$ \\
\hline GW-057 & EXP & w & $10 / 09 / 93$ & $931011-008$ & $931011-012$ & $931011-055$ \\
\hline$G W-064$ & OLF & W & $10 / 20 / 93$ & $931020-072$ & $931020-076$ & $931025-022$ \\
\hline$G W-069$ & BG & W & $10 / 10 / 93$ & $931011-034$ & $931011-032$ & $931012-055$ \\
\hline GW-079 & BG & 8 & $10 / 18 / 93$ & $931018-207$ & $931018-209$ & $931020-001$ \\
\hline GW -080 & BG & $\mathbf{W}$ & $10 / 10 / 93$ & $931011-038$ & $931011-032$ & $931012-055$ \\
\hline GW-082 & BG & $\boldsymbol{W}$ & $10 / 18 / 93$ & $931018-205$ & $931018-204$ & $931020-001$ \\
\hline$G W-084$ & OLF & W & $10 / 20 / 93$ & $931020-074$ & $931020-076$ & $931025-022$ \\
\hline$G W-085$ & OLF & 6 & $10 / 22 / 93$ & $931022-088$ & $931022-094$ & $931026-013$ \\
\hline$G W-086$ & OLF & 6 & $10 / 21 / 93$ & $931021-103$ & $931021-105$ & $931023-006$ \\
\hline GW-115 & s3 & $\mathbf{W}$ & $10 / 19 / 93$ & $931019-057$ & $931019-059$ & $931021-024$ \\
\hline GW-118 & BG & B & $10 / 10 / 93$ & $931011-040$ & $931011-032$ & $931014-001$ \\
\hline GW-162 & BG & 10 & $10 / 10 / 93$ & $931011-013$ & $931011-017$ & $931011-055$ \\
\hline$G W-286$ & BG & 6 & $10 / 07 / 93$ & $931008-066$ & $931008-072$ & $931011-042$ \\
\hline GW-287 & BG & 6 & $10 / 07 / 93$ & $931008-070$ & $931008-072$ & $931012-038$ \\
\hline$G W-311$ & $\mathbf{R S}$ & 6 & $10 / 21 / 93$ & $931021-099$ & $931021-105$ & $931023-006$ \\
\hline$G W-312$ & $\mathbf{R S}$ & 6 & $10 / 21 / 93$ & $931021-101$ & $931021-105$ & $931025-031$ \\
\hline$G W-315$ & SPI & 10 & $10 / 27 / 93$ & $931027-029$ & $931027-033$ & $931028-007$ \\
\hline GW-316 & SPI & 10 & $10 / 24 / 93$ & $931025-014$ & $931025-016$ & $931027-002$ \\
\hline GW-317 & SPI & 10 & $10 / 24 / 93$ & $931025-012$ & $931025-016$ & $931027-002$ \\
\hline$G W-323$ & SPI & 10 & $10 / 23 / 93$ & $931025-009$ & $931025-011$ & $931027-001$ \\
\hline$G W-324$ & s3 & 10 & $10 / 19 / 93$ & $931019-055$ & $931019-059$ & $931021-024$ \\
\hline$G W-325$ & s3 & 10 & $10 / 21 / 93$ & $931022-037$ & $931022-039$ & $931023-006$ \\
\hline$G W-342$ & BG & 10 & $10 / 07 / 93$ & $931008-077$ & $931008-079$ & $931008-118$ \\
\hline GW-345 & s3 & 10 & $10 / 26 / 93$ & $931026-042$ & $931026-044$ & $931028-007$ \\
\hline$G W-348$ & $\mathbf{s} 3$ & 10 & $10 / 22 / 93$ & $931022-135$ & $931022-139$ & $931025-031$ \\
\hline$G W-364$ & OLF & 11 & $12 / 18 / 93$ & $931218-053$ & $931218-059$ & $931221-083$ \\
\hline$G W-365$ & OLF & 11 & $12 / 19 / 93$ & $931219-050$ & $931219-052$ & $931222-020$ \\
\hline GW-370 & BG & 10 & $10 / 14 / 93$ & $931014-063$ & $931014-065$ & $931018-003$ \\
\hline GW-371 & BG & 10 & $10 / 13 / 93$ & $931014-004$ & $931014-008$ & $931014-010$ \\
\hline GW-372 & BG & 10 & $10 / 12 / 93$ & $931012-151$ & $931012-153$ & $931015-141$ \\
\hline GW-373 & BG & 10 & $10 / 11 / 93$ & $931011-127$ & $931011-129$ & $931014-001$ \\
\hline GW-526 & s3 & 10 & $10 / 26 / 93$ & $931026-040$ & $931026-044$ & $931028-007$ \\
\hline$G W-537$ & OLF & 6 & $10 / 22 / 93$ & $931022-086$ & $931022-094$ & $931026-013$ \\
\hline$G W-601$ & OLF & 11 & $12 / 21 / 94$ & $931222-001$ & $931222-003$ & $931223-001$ \\
\hline GW-613 & s3 & 10 & $10 / 20 / 93$ & $931020-079$ & $931020-083$ & $931021-024$ \\
\hline$G W-614$ & s3 & 10 & $10 / 20 / 93$ & $931020-081$ & $931020-083$ & $931021-024$ \\
\hline$G W-621$ & BG & 6 & $12 / 20 / 93$ & $931220-117$ & $931220-119$ & $931222-020$ \\
\hline
\end{tabular}


APPENDIX I. 1

Correlation of samples to Associated Blanks

\begin{tabular}{|c|c|c|c|c|c|c|}
\hline $\begin{array}{c}\text { sampling } \\
\text { Pt. }\end{array}$ & Iocation & $\begin{array}{l}\text { Pump } \\
\text { No. }\end{array}$ & Date & $\begin{array}{c}\text { Sample } \\
\text { No. }\end{array}$ & $\begin{array}{c}\text { Trip Blank } \\
\text { No. }\end{array}$ & $\begin{array}{c}\text { Lab Blank } \\
\text { No. }\end{array}$ \\
\hline$G W-626$ & BG & 8 & $10 / 20 / 93$ & $931021-001$ & $931021-005$ & $931025-022$ \\
\hline$G W-627$ & BG & 8 & $10 / 20 / 93$ & $931021-003$ & $931021-005$ & $931025-022$ \\
\hline$G W-636$ & OLF & 6 & $12 / 18 / 93$ & $931218-048$ & $931218-052$ & $931221-083$ \\
\hline$G W-637$ & OLF & 6 & $12 / 18 / 93$ & $931218-046$ & $931218-052$ & $931221-083$ \\
\hline$G W-638$ & OLF & 11 & $12 / 18 / 93$ & $931218-057$ & $931218-059$ & $931222-020$ \\
\hline GW-639 & BG & 10 & $10 / 15 / 93$ & $931015-229$ & $931015-231$ & $931019-038$ \\
\hline$G W-640$ & BG & 10 & $10 / 14 / 93$ & $931014-066$ & $931014-065$ & $931018-003$ \\
\hline$G W-641$ & BG & 10 & $10 / 14 / 93$ & $931014-068$ & $931014-065$ & $931018-003$ \\
\hline$G W-642$ & BG & 10 & $10 / 10 / 93$ & $931011-015$ & $931011-017$ & $931012-038$ \\
\hline$G W-643$ & BG & 10 & $10 / 12 / 93$ & $931012-149$ & $931012-153$ & $931015-141$ \\
\hline$G W-644$ & OLF & 11 & $12 / 18 / 93$ & $931218-055$ & $931218-059$ & $931222-020$ \\
\hline$G W-647$ & OLF & 8 & $12 / 10 / 93$ & $931210-052$ & $931210-054$ & $931213-006$ \\
\hline$G W-651$ & $\mathbf{B G}$ & 6 & $10 / 10 / 93$ & $931011-018$ & $931011-022$ & $931012-055$ \\
\hline$G W-652$ & BG & 6 & $10 / 18 / 93$ & $931018-198$ & $931018-200$ & $931019-038$ \\
\hline$G W-653$ & BG & 6 & $10 / 10 / 93$ & $931011-020$ & $931011-022$ & $931012-055$ \\
\hline$G W-654$ & BG & 6 & $10 / 07 / 93$ & $931008-068$ & $931008-072$ & $931012-038$ \\
\hline$G W-655$ & $\mathbf{B G}$ & 10 & $10 / 06 / 93$ & $931007-002$ & $931007-004$ & $931008-005$ \\
\hline$G W-683$ & EXP & 6 & $10 / 12 / 93$ & $931013-001$ & $931013-007$ & $931014-001$ \\
\hline$G W-684$ & EXP & 6 & $10 / 12 / 93$ & $931013-003$ & $931013-007$ & $931014-001$ \\
\hline$G W-685$ & $\mathbf{E X P}$ & 6 & $10 / 11 / 93$ & $931011-124$ & $931011-126$ & $931014-001$ \\
\hline$G W-694$ & EXP & 6 & $12 / 28 / 93$ & $931228-136$ & $931228-143$ & $931229-014$ \\
\hline GW-695 & EXP & 8 & $12 / 21 / 93$ & $931221-132$ & $931221-136$ & $931223-001$ \\
\hline GW -703 & EXP & 8 & $12 / 21 / 93$ & $931221-134$ & $931221-136$ & $931223-001$ \\
\hline GW -704 & EXP & 6 & $12 / 22 / 93$ & $931222-059$ & $931222-063$ & $931227-026$ \\
\hline GW-706 & EXP & 8 & $12 / 28 / 93$ & $931229-007$ & $931229-010$ & $931229-014$ \\
\hline GW-710 & EXP & 2 & $11 / 09 / 93$ & $931109-175$ & $931109-177$ & $931111-002$ \\
\hline GW-711 & $\mathbf{E X P}$ & 10 & $11 / 07 / 93$ & $931108-004$ & $931108-006$ & $931110-008$ \\
\hline GW -712 & EXP & 8 & $11 / 06 / 93$ & $931108-001$ & $931108-003$ & $931110-008$ \\
\hline GW-713 & EXP & 8 & $11 / 09 / 93$ & $931109-166$ & $931109-168$ & $931111-002$ \\
\hline$G W-714$ & EXP & 10 & $11 / 09 / 93$ & $931109-170$ & $931109-174$ & $931111-002$ \\
\hline GW-715 & EXP & 10 & $11 / 11 / 93$ & $931111-112$ & $931111-114$ & $931116-002$ \\
\hline$G W-723$ & EXP & 2 & $12 / 14 / 93$ & $931214-033$ & $931214-035$ & $931215-020$ \\
\hline GW-724 & EXP & 8 & $12 / 14 / 93$ & $931215-003$ & $931215-021$ & $931215-020$ \\
\hline GW-725 & EXP & 11 & $12 / 15 / 93$ & $931216-009$ & $931216-012$ & $931216-050$ \\
\hline GW -736 & EXP & 11 & $12 / 15 / 93$ & $931216-003$ & $931216-012$ & $931216-050$ \\
\hline GW -737 & EXP & 11 & $12 / 15 / 93$ & $931216-007$ & $931216-012$ & $931216-050$ \\
\hline GW-738 & EXP & 11 & $12 / 14 / 93$ & $931215-001$ & $931215-005$ & $931215-020$ \\
\hline$G W-739$ & $\mathbf{E X P}$ & 10 & $12 / 13 / 93$ & $931213-122$ & $931213-124$ & $931214-002$ \\
\hline$G W-740$ & $\operatorname{EXP}$ & 8 & $12 / 11 / 93$ & $931213-007$ & $931213-009$ & $931213-006$ \\
\hline GW-793 & AGLLSF & 10 & $10 / 18 / 93$ & $931019-001$ & $931019-007$ & $931020-001$ \\
\hline$G W-794$ & AGLLSF & 10 & $10 / 18 / 93$ & $931019-003$ & $931019-007$ & $931020-001$ \\
\hline GW-795 & AGLLSF & 10 & $10 / 18 / 93$ & $931019-005$ & $931019-007$ & $931020-001$ \\
\hline GW -800 & OLF & 6 & $10 / 22 / 93$ & $931022-090$ & $931022-094$ & $931026-013$ \\
\hline ss-1 & EXP & NA & $11 / 08 / 93$ & $931108-114$ & $931108-118$ & $931110-011$ \\
\hline ss-4 & EXP & NA & $11 / 08 / 93$ & $931108-112$ & $931108-118$ & $931110-011$ \\
\hline ss -5 & EXP & NA & $11 / 08 / 93$ & $931108-108$ & $931108-118$ & $931110-011$ \\
\hline ss -6 & EXP & NA & $11 / 08 / 93$ & $931108-106$ & $931108-118$ & $931110-011$ \\
\hline
\end{tabular}


APPENDIX I. 1

Correlation of Samples to Associated Blanks

\begin{tabular}{lcclccc}
\hline $\begin{array}{c}\text { Sampling } \\
\text { Pt. }\end{array}$ & Location & $\begin{array}{c}\text { Pump } \\
\text { No, }\end{array}$ & Date & $\begin{array}{c}\text { Sample } \\
\text { No. }\end{array}$ & $\begin{array}{c}\text { Trip Blank } \\
\text { No. }\end{array}$ & $\begin{array}{c}\text { Lab Blank } \\
\text { No. }\end{array}$ \\
\hline & EXP & NA & $11 / 08 / 93$ & $931108-102$ & $931108-118$ & $931110-008$ \\
BCK-00.63 & EXP & NA & $11 / 08 / 93$ & $931108-098$ & $931108-118$ & $931110-008$ \\
BCK-04.55 & EXP & NA & $11 / 08 / 93$ & $931108-100$ & $931108-118$ & $931110-008$ \\
BCK-09.40 & EXP & NA & $11 / 08 / 93$ & $931108-110$ & $931108-118$ & $931110-011$ \\
NT-01 & EXP & NA & $11 / 08 / 93$ & $931108-116$ & $931108-118$ & $931110-011$ \\
NT-13 & EXP & NA & $11 / 08 / 93$ & $931108-104$ & $931108-118$ & $931110-008$
\end{tabular}




\section{APPENDEX $\mathbf{L}$.2}

TRIP BLANK DATA SUMMARY 
APPENDDX 12

1993 Trip Blank Data Summary

\begin{tabular}{|c|c|c|c|c|c|c|c|c|c|c|}
\hline \multirow{2}{*}{ Compound } & \multicolumn{2}{|c|}{ First } & \multicolumn{2}{|c|}{ Second } & \multicolumn{2}{|c|}{ Thind } & \multicolumn{2}{|c|}{ Fourth } & \multicolumn{2}{|c|}{ Total } \\
\hline & No. & $\%$ & No. & $\%$ & No. & $\%$ & No. & $\%$ & No. & $\%$ \\
\hline Total Samples & 54 & & 47 & & 41 & & 55 & & 197 & \\
\hline Not Detected & 22 & 43 & 23 & 50 & 25 & 62 & 17 & 31 & 87 & 44 \\
\hline VOCs Detected & 32 & 57 & 24 & 50 & 16 & 38 & 38 & 69 & 110 & 56 \\
\hline Methylene chloride & 26 & 48 & 22 & 47 & 15 & 37 & 36 & 65 & 99 & 50 \\
\hline Acetone & 17 & 31 & 2 & 4 & 3 & 7 & 28 & 51 & 50 & 25 \\
\hline 2-Butanone & 3 & 6 & 2 & 4 & & & 26 & 47 & 31 & 16 \\
\hline 4-Methyl-2-pentanone & 16 & 30 & 6 & 13 & & & 1 & 2 & 23 & 12 \\
\hline Tetrachloroethane & & & & & & & 1 & 2 & 1 & 1 \\
\hline 1,1,1-Trichloroethane & & & 1 & 2 & & & & & 1 & 1 \\
\hline Xylenes & & & & & & & 1 & 2 & 1 & 1 \\
\hline
\end{tabular}


Trip Blank Data Summary, First Quarter 1993

\begin{tabular}{|c|c|c|c|c|c|c|c|c|}
\hline Sample No. & $\begin{array}{c}930128- \\
047\end{array}$ & $\begin{array}{l}930129- \\
101\end{array}$ & $\begin{array}{c}930201- \\
134\end{array}$ & $\begin{array}{c}930203- \\
008\end{array}$ & $\begin{array}{l}930204- \\
007\end{array}$ & $\begin{array}{l}930204- \\
297\end{array}$ & $\begin{array}{l}930205- \\
071\end{array}$ & $\begin{array}{c}930205- \\
074\end{array}$ \\
\hline Acetone & 100 & 100 & 6 & 3 & 1 & 100 & 100 & 100 \\
\hline 2-Butanone & 100 & 100 & 6 & 5 & 100 & 100 & 100 & 100 \\
\hline 4-Methy 1-2-pent anone & 1 & $10 U$ & 2 & 2 & 2 & 100 & 100 & 100 \\
\hline Methylene chloride & $5 \mathbf{v}$ & $5 \mathbf{v}$ & $5 t$ & $5 \mathbf{v}$ & 2 & $5 \mathbf{u}$ & $5 \mathbf{U}$ & $5 \mathbf{v}$ \\
\hline
\end{tabular}

(CONTTNUED)

\begin{tabular}{|c|c|c|c|c|c|c|c|c|}
\hline Sample No. & $\begin{array}{c}930208- \\
161\end{array}$ & $\begin{array}{c}930208- \\
164\end{array}$ & $\begin{array}{l}930209- \\
004\end{array}$ & $\begin{array}{c}930209- \\
066\end{array}$ & $\begin{array}{c}930209- \\
090\end{array}$ & $\begin{array}{c}930210- \\
028\end{array}$ & $\begin{array}{l}930210- \\
029\end{array}$ & $\begin{array}{c}930210- \\
187\end{array}$ \\
\hline Acetone & 100 & 100 & 100 & 100 & 2 & 100 & 100 & 100 \\
\hline 2-Butanone & 100 & 100 & 100 & 100 & 100 & 100 & 100 & 100 \\
\hline 4-Methyl-2-pentanone & $10 \mathrm{U}$ & 100 & 100 & 100 & 2 & 100 & 100 & 100 \\
\hline Methylene chloride & 0.9 & 1 & 50 & 0.7 & 2 & 2 & 2 & 1 \\
\hline
\end{tabular}

(CONTINUED)

\begin{tabular}{|c|c|c|c|c|c|c|c|c|}
\hline Sample No. & $\begin{array}{c}930211- \\
331\end{array}$ & $\begin{array}{c}930211- \\
336\end{array}$ & $\begin{array}{c}930211- \\
343\end{array}$ & $\begin{array}{l}930212- \\
111\end{array}$ & $\begin{array}{c}930216- \\
142\end{array}$ & $\begin{array}{c}930217- \\
072\end{array}$ & $\begin{array}{c}930222- \\
108\end{array}$ & $\begin{array}{l}930223- \\
087\end{array}$ \\
\hline Acetone & 1 & 100 & 100 & 2 & 100 & 3 & 100 & 100 \\
\hline 2-Butanone & 100 & $10 \mathrm{u}$ & 100 & 1 & 100 & 100 & 100 & 100 \\
\hline 4-Methyl-2-pentanone & 1 & 100 & 100 & 100 & 100 & 2 & 100 & 100 \\
\hline Methylene chloride & 2 & 2 & 50 & 3 & 1 & 2 & $5 u$ & 1 \\
\hline
\end{tabular}

(CONTINUED)

\begin{tabular}{|c|c|c|c|c|c|c|c|c|}
\hline Sample No. & $\begin{array}{c}930225- \\
011\end{array}$ & $\begin{array}{c}930225- \\
015\end{array}$ & $\begin{array}{c}930226- \\
308\end{array}$ & $\begin{array}{c}930226- \\
315\end{array}$ & $\begin{array}{c}930302- \\
008\end{array}$ & $\begin{array}{c}930302- \\
015\end{array}$ & $\begin{array}{c}930302- \\
071\end{array}$ & $\begin{array}{c}930302- \\
085\end{array}$ \\
\hline Acetone & 100 & 100 & 100 & 100 & 100 & 100 & 100 & 100 \\
\hline 2-Butanone & 100 & 100 & 100 & 100 & 100 & 100 & 100 & 100 \\
\hline 4-Methy1-2-pentanone & 100 & 100 & 100 & 100 & 100 & 100 & 100 & 100 \\
\hline Methylene chloride & $5 \mathbf{v}$ & 50 & 1 & $5 \mathrm{u}$ & 50 & 5u & 2 & 5u \\
\hline
\end{tabular}

(CONTINUED) 
Trip Blank Data Summary, First Quarter 1993

\begin{tabular}{|c|c|c|c|c|c|c|c|c|}
\hline Sample No. & $\begin{array}{c}930303- \\
107\end{array}$ & $\begin{array}{c}930303- \\
127\end{array}$ & $\begin{array}{c}930304- \\
238\end{array}$ & $\begin{array}{c}930305- \\
011\end{array}$ & $\begin{array}{c}930311- \\
082\end{array}$ & $\begin{array}{c}930319- \\
006\end{array}$ & $\begin{array}{c}930319- \\
116\end{array}$ & $\begin{array}{c}930322- \\
009\end{array}$ \\
\hline Acetone & 100 & 100 & 13 & 100 & 100 & 87 & 4 & 100 \\
\hline 2-Butanone & 100 & 100 & 100 & 100 & 100 & 100 & 100 & 100 \\
\hline 4-Methyl-2-pentanone & 100 & $10 \mathrm{U}$ & 100 & 100 & 100 & 100 & 2 & 100 \\
\hline Methylene chloride & 50 & $\mathbf{5 u}$ & $\mathbf{5 u}$ & $5 \mathbf{u}$ & 50 & $5 \mathbf{U}$ & 2 & $5 \mathbf{v}$ \\
\hline
\end{tabular}

(CONTINUED)

\begin{tabular}{|c|c|c|c|c|c|c|c|c|}
\hline Sample No. & $\begin{array}{c}930322- \\
014\end{array}$ & $\begin{array}{c}930322- \\
043\end{array}$ & $\begin{array}{c}930322- \\
044\end{array}$ & $\begin{array}{c}930322- \\
115\end{array}$ & $\begin{array}{c}930323- \\
058\end{array}$ & $\begin{array}{c}930323- \\
071\end{array}$ & $\begin{array}{c}930324- \\
099\end{array}$ & $\begin{array}{c}930325- \\
121\end{array}$ \\
\hline Acetone & 3 & 81 & 7 & 9 & 7 & 100 & 100 & $10 \mathrm{U}$ \\
\hline 2-Butanone & 100 & 100 & 100 & $10 \mathrm{u}$ & $10 \mathrm{U}$ & 100 & 100 & 100 \\
\hline 4-Methy1-2-pentanone & 1 & 100 & 3 & 5 & 5 & 4 & 100 & 100 \\
\hline Methylene chloride & 2 & 50 & 2 & 2 & 3 & 3 & 3 & $5 \mathbf{v}$ \\
\hline
\end{tabular}

(CONTINUED)

\begin{tabular}{|c|c|c|c|c|c|c|}
\hline Sample No. & $\begin{array}{c}930326- \\
021\end{array}$ & $\begin{array}{c}930329- \\
010\end{array}$ & $\begin{array}{c}930329- \\
013\end{array}$ & $\begin{array}{c}930329- \\
023\end{array}$ & $\begin{array}{c}930329- \\
031\end{array}$ & $\begin{array}{c}930329- \\
086\end{array}$ \\
\hline Acetone & $10 U$ & 100 & 100 & 100 & 2 & 4 \\
\hline 2-Butanone & $10 u$ & 100 & 100 & 100 & 100 & 100 \\
\hline 4-Methyl-2-pentanone & 100 & 100 & 100 & 1 & 1 & 2 \\
\hline Methylene chloride & 50 & $5 \mathbf{u}$ & 50 & 3 & 3 & 2 \\
\hline
\end{tabular}


APPENDIX I. 2

Trip Blank Data Summary, Second Quarter 1993

\begin{tabular}{|c|c|c|c|c|c|c|c|c|}
\hline Sample No. & $\begin{array}{c}930416- \\
080\end{array}$ & $\begin{array}{c}930419- \\
089\end{array}$ & $\begin{array}{c}930421- \\
016\end{array}$ & $\begin{array}{c}930421- \\
122\end{array}$ & $\begin{array}{c}930422- \\
033\end{array}$ & $\begin{array}{c}930423- \\
019\end{array}$ & $\begin{array}{c}930423- \\
081\end{array}$ & $\begin{array}{c}930423- \\
110\end{array}$ \\
\hline Acetone & $10 \mathrm{U}$ & 100 & 100 & 100 & 100 & 100 & 100 & 100 \\
\hline 2-Butanone & 100 & 100 & 100 & 100 & $10 \mathrm{U}$ & 200 & 100 & 100 \\
\hline 4-Methy 1-2-pentanone & $10 \mathrm{U}$ & 100 & 1 & 100 & 100 & 100 & 100 & 100 \\
\hline Methylene chloride & 5u & 5u & 1 & su & $5 \mathbf{v}$ & 50 & 2 & 2 \\
\hline 1,1,1-Trichloroethane & 50 & $5 \mathbf{u}$ & 5u & su & 5u & 5U & $\mathbf{5 u}$ & $5 \mathbf{U}$ \\
\hline
\end{tabular}

(CONTINUED)

\begin{tabular}{|c|c|c|c|c|c|c|c|c|}
\hline Sample No. & $\begin{array}{c}930427- \\
008\end{array}$ & $\begin{array}{c}930428- \\
114\end{array}$ & $\begin{array}{c}930430- \\
014\end{array}$ & $\begin{array}{c}930430- \\
049\end{array}$ & $\begin{array}{c}930430- \\
077\end{array}$ & $\begin{array}{c}930503- \\
095\end{array}$ & $\begin{array}{c}930510- \\
156\end{array}$ & $\begin{array}{c}930510- \\
162\end{array}$ \\
\hline Acetone & $10 \mathrm{U}$ & $10 \mathrm{U}$ & 100 & 3 & 100 & 100 & $10 v$ & 100 \\
\hline 2-Butanone & 100 & 100 & 100 & 2 & $10 v$ & 100 & 100 & 100 \\
\hline 4-Methyl-2-pentanone & 100 & $10 \mathrm{U}$ & $10 \mathrm{U}$ & 1 & 100 & 1 & 100 & 100 \\
\hline Methylene chloride & 1 & $5 \mathbf{u}$ & 1 & 1 & 2 & 2 & 1 & 2 \\
\hline 1,1,1-Trlchloroethane & 50 & $5 \mathbf{U}$ & su & 1 & 5u & 5u & 5u & $\mathbf{5 u}$ \\
\hline
\end{tabular}

(CONTINUED)

\begin{tabular}{|c|c|c|c|c|c|c|c|c|}
\hline Sample No. & $\begin{array}{c}930511- \\
101\end{array}$ & $\begin{array}{c}930512- \\
027\end{array}$ & $\begin{array}{c}930513- \\
017\end{array}$ & $\begin{array}{c}930514- \\
077\end{array}$ & $\begin{array}{c}930518- \\
022\end{array}$ & $\begin{array}{c}930518- \\
025\end{array}$ & $\begin{array}{c}930518- \\
164\end{array}$ & $\begin{array}{l}930519- \\
010\end{array}$ \\
\hline Acetone & 100 & 100 & $10 \mathrm{u}$ & 100 & 100 & 100 & 100 & 100 \\
\hline 2-Butanone & 100 & 100 & 100 & 100 & 100 & 100 & 100 & 100 \\
\hline 4-Methy1-2-pentanone & $10 \mathrm{U}$ & $10 \mathrm{U}$ & 100 & 1 & 100 & 100 & $10 u$ & 100 \\
\hline Methylene chloride & 2 & 2 & 50 & 2 & $5 \mathbf{U}$ & 50 & $5 \mathbf{v}$ & 50 \\
\hline $1,1,1$-Trichloroethane & $5 u$ & $5 \mathrm{v}$ & $5 \mathbf{v}$ & $5 v$ & $5 v$ & $5 v$ & $5 \mathbf{s u}$ & $5 \mathbf{v}$ \\
\hline
\end{tabular}

(CONTINUED)

\begin{tabular}{|c|c|c|c|c|c|c|c|c|}
\hline Sample No. & $\begin{array}{c}930519- \\
196\end{array}$ & $\begin{array}{c}930519- \\
214\end{array}$ & $\begin{array}{c}930520- \\
006\end{array}$ & $\begin{array}{c}930521- \\
110\end{array}$ & $\begin{array}{c}930525- \\
010\end{array}$ & $\begin{array}{c}930525- \\
090\end{array}$ & $\begin{array}{c}930526- \\
010\end{array}$ & $\begin{array}{c}930527- \\
334\end{array}$ \\
\hline Acetone & 100 & 100 & 100 & 100 & 100 & 100 & 100 & 100 \\
\hline 2-Butanone & 100 & 100 & 100 & 100 & 2 & 100 & 100 & 100 \\
\hline 4-Methyl-2-pentanone & $10 \mathrm{U}$ & 100 & 100 & 100 & 1 & 100 & 100 & 100 \\
\hline Methylene chloride & 50 & $5 U$ & $5 U$ & 50 & $5 v$ & 1 & $5 v$ & 1 \\
\hline 1,1,1-Trichloroethane & $5 U$ & $5 \mathbf{u}$ & $5 u$ & $5 U$ & $5 U$ & $5 \mathbf{U}$ & $5 \mathbf{U}$ & 50 \\
\hline
\end{tabular}

(CONTINUED) 
APPENDIX I. 2

Trip Blank Data Sumnary, Second Quarter 1993

\begin{tabular}{|c|c|c|c|c|c|c|c|c|}
\hline Sample No. & $\begin{array}{c}930528- \\
149\end{array}$ & $\begin{array}{c}930602- \\
006\end{array}$ & $\begin{array}{c}930603- \\
011\end{array}$ & $\begin{array}{c}930603- \\
175\end{array}$ & $\begin{array}{c}930604- \\
008\end{array}$ & $\begin{array}{c}930604- \\
106\end{array}$ & $\begin{array}{c}930607- \\
019\end{array}$ & $\begin{array}{c}930609- \\
058\end{array}$ \\
\hline Acetone & 17 & 100 & 100 & 100 & 100 & 100 & 100 & 100 \\
\hline 2-Butanone & 100 & 100 & 100 & 100 & 100 & 100 & 100 & 100 \\
\hline 4-Methyl-2-pentanone & 100 & 100 & 100 & 100 & 100 & 100 & 100 & 100 \\
\hline Methylene chloride & 1 & 1 & 5u & 2 & 1 & 1 & 1 & 5u \\
\hline $1,1,1$-Trichloroethane & 5u & 50 & $5 u$ & 5u & 50 & su & so & 5u \\
\hline
\end{tabular}

(CONTINUED)

\begin{tabular}{|c|c|c|c|c|c|c|c|}
\hline Sample No. & $\begin{array}{c}930611- \\
138\end{array}$ & $\begin{array}{c}930617- \\
166\end{array}$ & $\begin{array}{c}930625- \\
294\end{array}$ & $\begin{array}{c}930628- \\
009\end{array}$ & $\begin{array}{c}930628- \\
012\end{array}$ & $\begin{array}{c}930628- \\
020\end{array}$ & $\begin{array}{c}930628- \\
084\end{array}$ \\
\hline Acetone & 100 & 100 & 100 & 100 & 100 & 100 & 100 \\
\hline 2-Butanone & 100 & 100 & 100 & 100 & 100 & 100 & 100 \\
\hline 4-Methy1-2-pentanone & 100 & 100 & 100 & 100 & 1 & 100 & 100 \\
\hline Methylene chloride & 50 & 50 & 50 & 50 & 5u & 5u & 1 \\
\hline 1,1,1-Trichloroethane & 5u & 50 & 50 & 50 & su & 50 & 5u \\
\hline
\end{tabular}


APPENDIX 1.2

Trip Blank Data Summary, Third Quarter 1993

\begin{tabular}{|c|c|c|c|c|c|c|c|c|}
\hline Sample No. & $\begin{array}{c}930702- \\
018\end{array}$ & $\begin{array}{c}930702- \\
026\end{array}$ & $\begin{array}{l}930702- \\
110\end{array}$ & $\begin{array}{l}930708- \\
050\end{array}$ & $\begin{array}{l}930709- \\
059\end{array}$ & $\begin{array}{c}930709- \\
069\end{array}$ & $\begin{array}{l}930712- \\
008\end{array}$ & $\begin{array}{l}930712- \\
017\end{array}$ \\
\hline $\begin{array}{l}\text { Acetone } \\
\text { Methylene chloride }\end{array}$ & $\begin{array}{r}100 \\
50\end{array}$ & $\begin{array}{r}100 \\
50\end{array}$ & $\begin{array}{r}100 \\
50\end{array}$ & $\begin{array}{r}100 \\
50\end{array}$ & $\begin{array}{r}100 \\
1\end{array}$ & $\begin{array}{r}100 \\
1\end{array}$ & $\begin{array}{r}10 v \\
5 v\end{array}$ & $\begin{array}{r}100 \\
1\end{array}$ \\
\hline
\end{tabular}

(CONTINUED)

\begin{tabular}{|c|c|c|c|c|c|c|c|c|}
\hline Sample No. & $\begin{array}{c}930712- \\
022\end{array}$ & $\begin{array}{c}930712- \\
031\end{array}$ & $\begin{array}{c}930712- \\
036\end{array}$ & $\begin{array}{l}930712- \\
078\end{array}$ & $\begin{array}{c}930712- \\
112\end{array}$ & $\begin{array}{c}930726- \\
009\end{array}$ & $\begin{array}{l}930728- \\
105\end{array}$ & $\begin{array}{c}930729- \\
376\end{array}$ \\
\hline Acetone & $10 \mathrm{u}$ & 7 & 100 & 100 & 100 & 100 & 100 & 12 \\
\hline Methylene chloride & 50 & $\mathbf{5 u}$ & 1 & 1 & 50 & 1 & 1 & 1 \\
\hline
\end{tabular}

(CONTINUER)

\begin{tabular}{|c|c|c|c|c|c|c|c|c|}
\hline Sample No. & $\begin{array}{l}930730- \\
107\end{array}$ & $\begin{array}{c}930802- \\
036\end{array}$ & $\begin{array}{c}930802- \\
043\end{array}$ & $\begin{array}{c}930802- \\
119\end{array}$ & $\begin{array}{c}930913- \\
037\end{array}$ & $\begin{array}{c}930913- \\
068\end{array}$ & $\begin{array}{c}930913- \\
139\end{array}$ & $\begin{array}{c}930913- \\
148\end{array}$ \\
\hline Acetone & 11 & 100 & 100 & 100 & 100 & 100 & 100 & 100 \\
\hline Methylene chloride & 1 & su & 1 & su & 1 & $5 \mathrm{v}$ & $\mathbf{5 v}$ & 50 \\
\hline
\end{tabular}

(CONTINURD)

\begin{tabular}{|c|c|c|c|c|c|c|c|c|}
\hline Sample No. & $\begin{array}{c}930913- \\
248\end{array}$ & $\begin{array}{l}930915- \\
017\end{array}$ & $\begin{array}{l}930915- \\
026\end{array}$ & $\begin{array}{l}930918- \\
012\end{array}$ & $\begin{array}{c}930920- \\
020\end{array}$ & $\begin{array}{l}930920- \\
025\end{array}$ & $\begin{array}{c}930920- \\
048\end{array}$ & $\begin{array}{l}930921- \\
005\end{array}$ \\
\hline $\begin{array}{l}\text { Acetone } \\
\text { Methylene chloride }\end{array}$ & $\begin{array}{r}10 v \\
5 v\end{array}$ & $\begin{array}{r}100 \\
1\end{array}$ & $\begin{array}{r}100 \\
5 U\end{array}$ & $\begin{array}{r}100 \\
50\end{array}$ & $\begin{array}{r}100 \\
1\end{array}$ & $\begin{array}{l}100 \\
0.9\end{array}$ & $\begin{array}{l}100 \\
0.9\end{array}$ & $\begin{array}{r}100 \\
50\end{array}$ \\
\hline
\end{tabular}

(CONTINUED)

\begin{tabular}{|c|c|c|c|c|c|c|c|c|}
\hline Sample No. & $\begin{array}{c}930922- \\
064\end{array}$ & $\begin{array}{l}930923- \\
010\end{array}$ & $\begin{array}{c}930924- \\
009\end{array}$ & $\begin{array}{c}930927- \\
013\end{array}$ & $\begin{array}{c}930927- \\
239\end{array}$ & $\begin{array}{l}930928- \\
056\end{array}$ & $\begin{array}{c}930928- \\
119\end{array}$ & $\begin{array}{c}930928- \\
131\end{array}$ \\
\hline $\begin{array}{l}\text { Acetone } \\
\text { Methylene chloride }\end{array}$ & $\begin{array}{r}100 \\
5 U\end{array}$ & $\begin{array}{r}100 \\
50\end{array}$ & $\begin{array}{r}10 U \\
5 U\end{array}$ & $\begin{array}{r}100 \\
50\end{array}$ & $\begin{array}{r}10 U \\
5 U\end{array}$ & $\begin{array}{r}100 \\
5 U\end{array}$ & $\begin{array}{r}100 \\
50\end{array}$ & $\begin{array}{r}10 U \\
5 U\end{array}$ \\
\hline
\end{tabular}

(CONTINUED) 
Trip Blank Data Sumnary, Th1rd Quarter 1993

\begin{tabular}{|l|r|}
\hline Sample No. & $\begin{array}{c}930929- \\
189\end{array}$ \\
\hline $\begin{array}{l}\text { Acetone } \\
\text { Methylene chloride }\end{array}$ & $\begin{array}{r}\text { 5U } \\
\hline\end{array}$
\end{tabular}


APPEHDIX 1.2

Trip Blank Data Summary, Fourth Quarter 1993

\begin{tabular}{|c|c|c|c|c|c|c|c|c|}
\hline Sample No. & $\begin{array}{c}931007- \\
004\end{array}$ & $\begin{array}{l}931008- \\
072\end{array}$ & $\begin{array}{l}931008- \\
079\end{array}$ & $\begin{array}{c}931011- \\
007\end{array}$ & $\begin{array}{l}931011- \\
012\end{array}$ & $\begin{array}{l}931011- \\
017\end{array}$ & $\begin{array}{l}931011- \\
022\end{array}$ & $\begin{array}{c}931011- \\
126\end{array}$ \\
\hline Acetone & $10 u$ & 4 & 100 & 100 & 100 & 100 & 200 & 3 \\
\hline 2-Butanone & 100 & 9 & 100 & 100 & 100 & 100 & 100 & 8 \\
\hline 4-Mothy 1-2-pentanone & 100 & 100 & 100 & 100 & 100 & 100 & 100 & 100 \\
\hline Mothylene chloride & 5u & 3 & su & su & $\mathbf{s u}$ & $\mathbf{5 v}$ & $\mathbf{5 u}$ & 3 \\
\hline Tetrachloroethene & su & su & su & su & $\mathbf{s u}$ & so & su & su \\
\hline Xylenes & su & $\mathbf{5 u}$ & su & $5 v$ & $\mathbf{5 u}$ & $\mathbf{5 0}$ & $5 \mathbf{v}$ & $\mathbf{s u}$ \\
\hline
\end{tabular}

(CONTINUED)

\begin{tabular}{|c|c|c|c|c|c|c|c|c|}
\hline Sample Ho. & $\begin{array}{c}931011- \\
129\end{array}$ & $\begin{array}{c}931012- \\
153\end{array}$ & $\begin{array}{c}931013- \\
007\end{array}$ & $\begin{array}{l}931014- \\
008\end{array}$ & $\begin{array}{c}931014- \\
065\end{array}$ & $\begin{array}{c}931015- \\
231\end{array}$ & $\begin{array}{c}931018- \\
200\end{array}$ & $\begin{array}{c}931018- \\
204\end{array}$ \\
\hline Acetone & 3 & 100 & 100 & 100 & 3 & 100 & $10 u$ & 3 \\
\hline 2-Butanone & 10 & 100 & 100 & 100 & 7 & 100 & $10 u$ & 11 \\
\hline 4-Methyl-2-pentanone & 100 & $10 u$ & 100 & 100 & 100 & 100 & 100 & 100 \\
\hline Methylene chlorlde & 3 & 0.7 & su & su & 3 & 50 & $\mathbf{5 u}$ & 2 \\
\hline Tetrachloroethene & 50 & $5 \mathbf{v}$ & $5 \mathbf{v}$ & 50 & su & 50 & $5 \mathbf{u}$ & su \\
\hline Xylenes & su & $5 \mathbf{u}$ & $5 \mathbf{v}$ & 50 & 50 & su & $\mathbf{5 u}$ & su \\
\hline
\end{tabular}

(CONTINURD)

\begin{tabular}{|c|c|c|c|c|c|c|c|c|}
\hline Sample No. & $\begin{array}{c}931019- \\
007\end{array}$ & $\begin{array}{c}931019- \\
054\end{array}$ & $\begin{array}{l}931019- \\
059\end{array}$ & $\begin{array}{l}931020- \\
076\end{array}$ & $\begin{array}{c}931020- \\
083\end{array}$ & $\begin{array}{c}931021- \\
005\end{array}$ & $\begin{array}{c}931021- \\
105\end{array}$ & $\begin{array}{c}931022- \\
039\end{array}$ \\
\hline Acetone & 2 & 3 & 3 & 3 & 2 & 3 & 100 & 100 \\
\hline 2-Butanona & 7 & 9 & 9 & 9 & 9 & 9 & 9 & 100 \\
\hline 4-Mathy 1-2-pentanone & 100 & $10 u$ & 100 & 100 & 100 & 100 & 100 & 100 \\
\hline Methylene chloride & 3 & 3 & 2 & 3 & 3 & 3 & su & su \\
\hline Tetrachloroethene & $5 \mathbf{v}$ & $5 \mathbf{U}$ & 5u & 50 & 50 & su & $5 \mathbf{u}$ & 50 \\
\hline Xylenes & $5 \mathbf{v}$ & 50 & 5U & su & 50 & $\mathbf{s u}$ & 5u & su \\
\hline
\end{tabular}

(CONTINUED) 
APPENDIX 1.2

Trip Blank Data Summary, Fourth Quarter 1993

\begin{tabular}{|c|c|c|c|c|c|c|c|c|}
\hline sample No. & $\begin{array}{c}931022- \\
094\end{array}$ & $\begin{array}{c}931022- \\
139\end{array}$ & $\begin{array}{c}931025- \\
011\end{array}$ & $\begin{array}{c}931025- \\
016\end{array}$ & $\begin{array}{l}931026- \\
044\end{array}$ & $\begin{array}{l}931027- \\
033\end{array}$ & $\begin{array}{l}931108- \\
003\end{array}$ & $\begin{array}{l}931108- \\
006\end{array}$ \\
\hline Acetone & 100 & 2 & 9 & 8 & 3 & 2 & 9 & 28 \\
\hline 2-Butanono & 100 & 8 & 100 & 100 & 7 & 7 & 100 & 100 \\
\hline 4-Methy 1-2-pantanone & 100 & 100 & 100 & 100 & 100 & 100 & 100 & 100 \\
\hline methylene ohlorlde & su & 3 & 1 & 1 & 2 & 3 & 1 & 1 \\
\hline Tetrachloroethene & su & su & su & su & su & su & sv & su \\
\hline Xylenes & su & $\mathbf{s u}$ & su & so & $\mathbf{s u}$ & su & $\mathbf{s u}$ & 50 \\
\hline
\end{tabular}

(COATINURD)

\begin{tabular}{|c|c|c|c|c|c|c|c|c|}
\hline Bample No. & $\begin{array}{c}931108- \\
118\end{array}$ & $\begin{array}{c}931109- \\
168\end{array}$ & $\begin{array}{c}931109- \\
174\end{array}$ & $\begin{array}{c}931109- \\
177\end{array}$ & $\begin{array}{c}931111- \\
114\end{array}$ & $\begin{array}{c}931210- \\
054\end{array}$ & $\begin{array}{c}931213- \\
009\end{array}$ & $\begin{array}{c}931213- \\
124\end{array}$ \\
\hline Acetone & 100 & 100 & 100 & 100 & 100 & $10 v$ & 100 & 100 \\
\hline 2-Butanone & 100 & 100 & 100 & 100 & 100 & 100 & 200 & 100 \\
\hline 4-Methy1-2-pentanone & 100 & 100 & 100 & 100 & 100 & 100 & 100 & 100 \\
\hline Metinglene chloride & su & 1 & 1 & su & 2 & 1 & 1 & 1 \\
\hline Totrashloroethene & 50 & su & su & su & su & su & su & $5 \mathbf{v}$ \\
\hline Xylenes & su & su & su & $\mathbf{s u}$ & su & su & su & su \\
\hline
\end{tabular}

(CONTIMUED)

\begin{tabular}{|c|c|c|c|c|c|c|c|c|}
\hline Sample No. & $\begin{array}{l}931214- \\
035\end{array}$ & $\begin{array}{l}931215- \\
005\end{array}$ & $\begin{array}{c}931215- \\
021\end{array}$ & $\begin{array}{c}931216- \\
012\end{array}$ & $\begin{array}{c}931218- \\
052\end{array}$ & $\begin{array}{c}931218- \\
059\end{array}$ & $\begin{array}{c}931219- \\
043\end{array}$ & $\begin{array}{l}931219- \\
052\end{array}$ \\
\hline Acetone & 100 & 100 & 100 & 4 & 7 & 8 & 5 & 6 \\
\hline 2-Butanone & 100 & 100 & 100 & 7 & 10 & 9 & 8 & 11 \\
\hline 4-Methy 1-2-pentanone & 100 & 100 & 100 & 1 & 100 & 100 & 100 & 200 \\
\hline Methylene chloride & $\mathbf{s u}$ & su & 1 & 2 & su & 0.8 & 2 & 0.8 \\
\hline Tetrachloroethene & su & $5 U$ & su & su & su & su & 5u & su \\
\hline Xylenes & $5 u$ & $5 u$ & su & su & $\mathbf{s u}$ & 50 & 50 & su \\
\hline
\end{tabular}

(CONTINUzD) 
APPENDIX 1.2

Trip Blank Data summary, rourth Quarter 1993

\begin{tabular}{|c|c|c|c|c|c|c|c|}
\hline Sample No. & $\begin{array}{c}931220- \\
119\end{array}$ & $\begin{array}{c}931221- \\
136\end{array}$ & $\begin{array}{c}931222- \\
003\end{array}$ & $\begin{array}{c}931222- \\
063\end{array}$ & $\begin{array}{c}931228- \\
143\end{array}$ & $\begin{array}{c}931229- \\
010\end{array}$ & $\begin{array}{c}931229- \\
048\end{array}$ \\
\hline Aoetone & 11 & 8 & 100 & 4 & 4 & 3 & 100 \\
\hline 2-Butanone & 9 & 8 & 9 & 4 & 6 & 4 & 100 \\
\hline 1-Mathyl-2-pentanone & 100 & 100 & 100 & 100 & 1011 & 100 & 100 \\
\hline Methylene ohloride & 2 & 0.7 & 0.8 & 1 & 1 & 1 & su \\
\hline Tetrachloroethene & su & su & su & 1 & su & su & su \\
\hline Xylenes & su & su & so & 1 & su & so & su \\
\hline
\end{tabular}


APPENDIX 1.3

LABORATORY BLANK DATA SUMMARY 
APPDNDIX 13

1993 Laborntory Blank Data Summary

\begin{tabular}{|c|c|c|c|c|c|c|c|c|c|c|}
\hline \multirow{2}{*}{ Compound } & \multicolumn{2}{|c|}{ Fint } & \multicolumn{2}{|c|}{ Second } & \multicolumn{2}{|c|}{ Thind } & \multicolumn{2}{|c|}{ Fourth } & \multicolumn{2}{|c|}{ Total } \\
\hline & No. & $\%$ & No. & $\%$ & No. & $\%$ & No. & $\%$ & No. & $\%$ \\
\hline Total Samples & 42 & & 35 & & 31 & & 35 & & 144 & \\
\hline Not Detected & 14 & 33 & 16 & 47 & 20 & 65 & 11 & 31 & 62 & 43 \\
\hline VOCs Detected & 28 & 67 & 19 & 53 & 11 & 35 & 24 & 69 & 82 & 57 \\
\hline Methylene chloride & 25 & 60 & 11 & 31 & 10 & 32 & 22 & 63 & 68 & 47 \\
\hline 4-Methyl-2-pentanone & 16 & 38 & 9 & 26 & 2 & 7 & 6 & 17 & 33 & 23 \\
\hline Acetone & 8 & 19 & & & & & 16 & 46 & 24 & 17 \\
\hline 2-Butanone & 2 & 5 & 4 & 11 & & & 16 & 46 & 22 & 15 \\
\hline 2-Hexanone & 1 & 2 & 8 & 23 & 1 & 3 & 3 & 9 & 13 & 9 \\
\hline Vinyl acetate & & & 3 & 9 & & & & & 3 & 2 \\
\hline Styrene & & & & & & & 1 & 3 & 1 & 1 \\
\hline 1,1,1-Trichloroethane & & & 1 & 3 & & & & & 1 & 1 \\
\hline 1,1,2-Trichloroethane & & & 1 & 3 & & & & & 1 & 1 \\
\hline
\end{tabular}


APPENDIX 1.3

Voce Detected in Laboratory Blank Bamplea, Firet Quarter 1993

\begin{tabular}{|c|c|c|c|c|c|c|c|c|}
\hline Sample No. & $\begin{array}{c}930129- \\
016\end{array}$ & $\begin{array}{c}930201- \\
001\end{array}$ & $\begin{array}{c}930202- \\
004\end{array}$ & $\begin{array}{c}930203- \\
057\end{array}$ & $\begin{array}{l}930204- \\
034\end{array}$ & $\begin{array}{l}930205- \\
035\end{array}$ & $\begin{array}{l}930208- \\
001\end{array}$ & $\begin{array}{l}930209- \\
001\end{array}$ \\
\hline Acetone & 1 & 100 & 1 & 2 & 100 & 100 & 100 & 100 \\
\hline 2-Butanona & 100 & 100 & 100 & 5 & 100 & 100 & 100 & 100 \\
\hline 2-kexanone & 100 & 100 & 100 & 100 & $10 u$ & 100 & 100 & 100 \\
\hline 4-Mathy 1-2-pentanone & 2 & 100 & 2 & 2 & 2 & 2 & 100 & 100 \\
\hline Mathylene ohlorlde & $\mathbf{5 u}$ & 50 & 50 & su & 1 & 1 & su & su \\
\hline
\end{tabular}

(CON:INUSD)

\begin{tabular}{|c|c|c|c|c|c|c|c|c|}
\hline Sample No. & $\begin{array}{l}930210- \\
021\end{array}$ & $\begin{array}{l}930210- \\
022\end{array}$ & $\begin{array}{c}930211- \\
001\end{array}$ & $\begin{array}{c}930211- \\
011\end{array}$ & $\begin{array}{c}930212- \\
068\end{array}$ & $\begin{array}{l}930216- \\
001\end{array}$ & $\begin{array}{c}930216- \\
089\end{array}$ & $\begin{array}{l}930217- \\
004\end{array}$ \\
\hline Acetone & 100 & 2 & 100 & 100 & 1 & 100 & 1 & 100 \\
\hline 2-Butanone & 100 & $10 u$ & 100 & 100 & 100 & 100 & 2 & 100 \\
\hline 2-Hexanone & 100 & 100 & 100 & 1 & 100 & $10 u$ & 100 & 100 \\
\hline 4-Mathy 1-2-pentanone & 100 & 1 & 100 & 2 & 1 & 100 & 1 & 100 \\
\hline Methylene chlorlde & 50 & 2 & 2 & 3 & 2 & 1 & 3 & 5u \\
\hline
\end{tabular}

(CONTINUED)

\begin{tabular}{|c|c|c|c|c|c|c|c|c|}
\hline Sample No. & $\begin{array}{c}930218- \\
002\end{array}$ & $\begin{array}{c}930223- \\
001\end{array}$ & $\begin{array}{c}930225- \\
001\end{array}$ & $\begin{array}{c}930225- \\
030\end{array}$ & $\begin{array}{c}930226- \\
343\end{array}$ & $\begin{array}{c}930301- \\
001\end{array}$ & $\begin{array}{c}930302- \\
060\end{array}$ & $\begin{array}{c}930303- \\
001\end{array}$ \\
\hline Acetone & 2 & 100 & 100 & $10 \mathrm{v}$ & 100 & 100 & 100 & 100 \\
\hline 2-Butanone & 100 & 100 & 100 & 100 & $10 u$ & 100 & 100 & 100 \\
\hline 2-Hexanone & 100 & 100 & 100 & 100 & $10 U$ & 100 & 100 & 100 \\
\hline 4-Methy 1-2-pentanone & 2 & 100 & 100 & 100 & 100 & 100 & 100 & 100 \\
\hline Methylene chloride & 2 & 1 & 50 & 1 & $5 U$ & 1 & $5 u$ & 50 \\
\hline
\end{tabular}

(CONTINURD)

\begin{tabular}{|c|c|c|c|c|c|c|c|c|}
\hline Sample No. & $\begin{array}{c}930303- \\
002\end{array}$ & $\begin{array}{c}930304- \\
069\end{array}$ & $\begin{array}{c}930304- \\
076\end{array}$ & $\begin{array}{c}930305- \\
002\end{array}$ & $\begin{array}{c}930308- \\
001\end{array}$ & $\begin{array}{c}930310- \\
001\end{array}$ & $\begin{array}{c}930310- \\
002\end{array}$ & $\begin{array}{c}930311- \\
022\end{array}$ \\
\hline Acetone & 100 & 100 & 100 & 100 & 100 & 100 & $10 u$ & 100 \\
\hline 2-Butanone & 100 & $10 \mathrm{U}$ & $10 \mathrm{v}$ & $10 \mathrm{U}$ & $10 \mathrm{v}$ & 100 & 100 & 100 \\
\hline 2-Hexanone & 100 & 100 & $10 \mathrm{U}$ & 100 & 100 & $10 \mathrm{U}$ & 100 & 100 \\
\hline 4-Methy 1-2-pentanone & 2 & 100 & 100 & 100 & 100 & 3 & 100 & 100 \\
\hline Methylene chloride & 2 & 1 & $5 U$ & 1 & 1 & 2 & 1 & 5u \\
\hline
\end{tabular}

(CONTINUED) 
APPENDIX 1.3

VOCs Detected in Laboratory Blank Samples, First Quarter 1993

\begin{tabular}{|c|c|c|c|c|c|c|c|c|}
\hline Sample No. & $\begin{array}{c}930322- \\
002\end{array}$ & $\begin{array}{c}930322- \\
005\end{array}$ & $\begin{array}{c}930323- \\
002\end{array}$ & $\begin{array}{c}930323- \\
042\end{array}$ & $\begin{array}{c}930325- \\
001\end{array}$ & $\begin{array}{c}930325- \\
011\end{array}$ & $\begin{array}{c}930326- \\
001\end{array}$ & $\begin{array}{c}930330- \\
003\end{array}$ \\
\hline Acetone & 100 & 4 & 100 & 100 & 100 & 100 & 100 & 100 \\
\hline 2-Butanone & 100 & 100 & 100 & 100 & 100 & 100 & 100 & 100 \\
\hline 2-Hexanone & 100 & 100 & 100 & 100 & $10 \mathrm{U}$ & 100 & 100 & 100 \\
\hline 4-Methyl-2-pentanone & 100 & 2 & 100 & 3 & 100 & 4 & 100 & 2 \\
\hline Methylene chloride & $5 \mathbf{u}$ & 2 & 2 & 2 & $5 \mathbf{v}$ & 2 & 2 & 2 \\
\hline
\end{tabular}

(CONTINUED)

\begin{tabular}{|l|r|r|}
\hline Sample No. & $\begin{array}{r}930331- \\
039\end{array}$ & $\begin{array}{c}930401- \\
019\end{array}$ \\
\hline Acetone & $10 \mathrm{U}$ & $10 \mathrm{U}$ \\
2-Butanone & $10 \mathrm{U}$ & $10 \mathrm{U}$ \\
2-Hexanone & $10 \mathrm{U}$ & $10 \mathrm{U}$ \\
4-Methyl-2-pentanone & $10 \mathrm{U}$ & $10 \mathrm{U}$ \\
Methylene chloride & $5 \mathrm{U}$ & 2 \\
\hline
\end{tabular}


APPENDIX 1.3

vocs Detected in Laboratory Blank Samples, Second Quarter 1993

\begin{tabular}{|c|c|c|c|c|c|c|c|c|}
\hline Sample No. & $\begin{array}{c}930419- \\
001\end{array}$ & $\begin{array}{c}930420- \\
001\end{array}$ & $\begin{array}{c}930421- \\
001\end{array}$ & $\begin{array}{c}930421- \\
113\end{array}$ & $\begin{array}{c}930422- \\
001\end{array}$ & $\begin{array}{c}930423- \\
001\end{array}$ & $\begin{array}{c}930426- \\
001\end{array}$ & $\begin{array}{c}930427- \\
131\end{array}$ \\
\hline 2-Butanone & 100 & 100 & $10 \mathrm{u}$ & 100 & 100 & 100 & 100 & 3 \\
\hline 2-Hexanone & 100 & $10 \mathrm{v}$ & 100 & 5 & 100 & 100 & 100 & 2 \\
\hline 4-Methyl-2-pentanone & 100 & $10 \mathrm{u}$ & 100 & 3 & 100 & 100 & 100 & 2 \\
\hline Methylene chloride & 50 & 1 & 1 & 50 & $5 \mathbf{v}$ & $\mathbf{5 u}$ & $\mathbf{5 0}$ & 50 \\
\hline 1,1,1-Trichloroethane & 50 & $5 \mathbf{u}$ & 5u & 5u & 50 & 50 & $5 \mathbf{v}$ & 1 \\
\hline 1,1,2-Trichloroethane & 5u & $5 \mathbf{v}$ & 5v & 5v & 50 & $5 \mathbf{v}$ & $5 \mathbf{v}$ & 5u \\
\hline Vinyl acetate & 100 & 100 & 100 & 1 & 100 & 100 & 100 & 100 \\
\hline
\end{tabular}

(CONTINUED)

\begin{tabular}{|c|c|c|c|c|c|c|c|c|}
\hline Sample No. & $\begin{array}{c}930428- \\
001\end{array}$ & $\begin{array}{c}930429- \\
001\end{array}$ & $\begin{array}{c}930430- \\
007\end{array}$ & $\begin{array}{c}930504- \\
053\end{array}$ & $\begin{array}{c}930505- \\
041\end{array}$ & $\begin{array}{c}930512- \\
001\end{array}$ & $\begin{array}{c}930513- \\
045\end{array}$ & $\begin{array}{l}930514- \\
001\end{array}$ \\
\hline 2-Butanone & $10 \mathrm{U}$ & $10 \mathrm{U}$ & 100 & 100 & 100 & 100 & 100 & $10 \mathrm{U}$ \\
\hline 2-Hexanone & 100 & $10 \mathrm{v}$ & 100 & 2 & 2 & 100 & 5 & 100 \\
\hline 4-Methyl-2-pentanone & 100 & 100 & 100 & 2 & 2 & 100 & 5 & 100 \\
\hline Methylene chloride & 1 & 50 & $5 u$ & 3 & 2 & 1 & $5 U$ & $5 \mathbf{U}$ \\
\hline $1,1,1$-Trichloroethane & $5 U$ & 50 & $5 \mathbf{v}$ & $5 \mathbf{U}$ & $5 \mathbf{U}$ & $5 \mathbf{U}$ & $\mathbf{5 U}$ & $5 \mathbf{v}$ \\
\hline $1,1,2$-Trichloroethane & 50 & 50 & $5 \mathbf{v}$ & $5 \mathbf{v}$ & 50 & $5 \mathbf{v}$ & $5 \mathbf{U}$ & 50 \\
\hline Vinyl acetate & 100 & 100 & $10 u$ & 100 & 100 & 100 & 100 & 100 \\
\hline
\end{tabular}

(CONTINUED)

\begin{tabular}{|c|c|c|c|c|c|c|c|c|}
\hline Sample No. & $\begin{array}{c}930518- \\
127\end{array}$ & $\begin{array}{c}930520- \\
057\end{array}$ & $\begin{array}{c}930521- \\
067\end{array}$ & $\begin{array}{c}930522- \\
012\end{array}$ & $\begin{array}{c}930526- \\
019\end{array}$ & $\begin{array}{c}930527- \\
064\end{array}$ & $\begin{array}{c}930528- \\
043\end{array}$ & $\begin{array}{c}930529- \\
016\end{array}$ \\
\hline 2-Butanone & 100 & 100 & 100 & 100 & 5 & 4 & 100 & 5 \\
\hline 2-Hexanone & 100 & 100 & 100 & 100 & 1 & 2 & 100 & 2 \\
\hline 4-Methyl-2-pentanone & 100 & $10 \mathrm{U}$ & 100 & 100 & 2 & 2 & 100 & 3 \\
\hline Methylene chloride & 50 & $5 U$ & 50 & 50 & 50 & $5 \mathbf{U}$ & 50 & $5 v$ \\
\hline 1,1,1-Trichloroethane & 5u & 50 & $5 U$ & $5 U$ & $5 \mathbf{U}$ & $5 U$ & $5 U$ & 50 \\
\hline $1,1,2$-Trichloroethane & 1 & $5 \mathrm{U}$ & 50 & $5 U$ & 5u & $5 U$ & 50 & 50 \\
\hline vinyl acetate & 5 & 100 & 100 & 100 & 100 & 100 & 100 & 100 \\
\hline
\end{tabular}

(CONTINUED) 
APPENDIX I. 3

VOCs Detected in Iaboratory Blank Samples, Second Quarter 1993

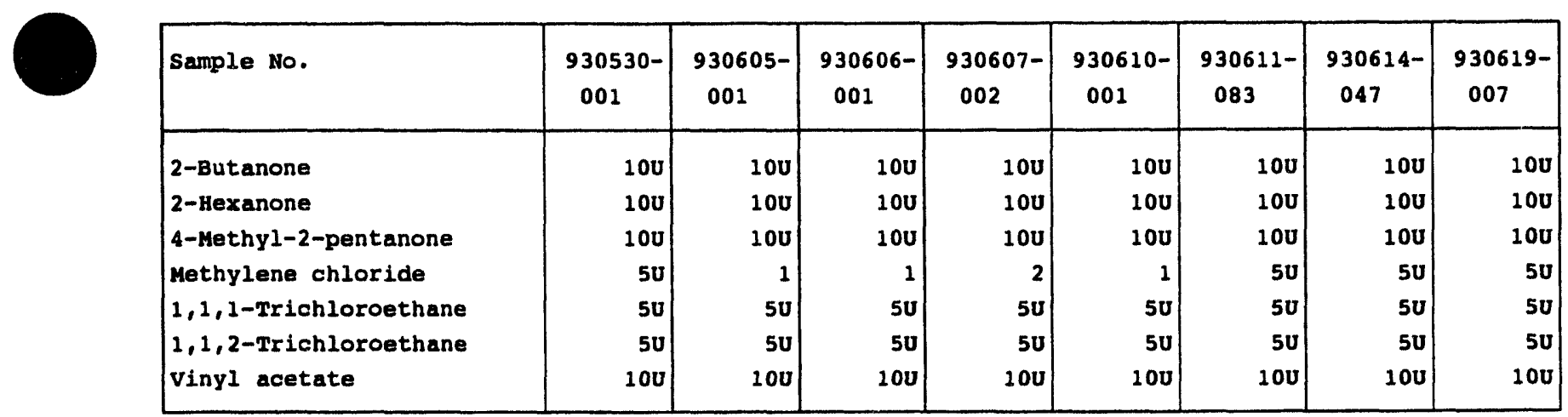

(CONTINUED)

\begin{tabular}{|c|c|c|c|}
\hline Sample No. & $\begin{array}{c}930628- \\
002\end{array}$ & $\begin{array}{c}930630- \\
001\end{array}$ & $\begin{array}{c}930630- \\
009\end{array}$ \\
\hline 2-Butanone & 100 & 100 & 100 \\
\hline 2-Hexanone & 100 & 100 & 100 \\
\hline 4-Methy1-2-pentanone & 100 & 100 & 2 \\
\hline Methylene chloride & 1 & $5 \mathbf{v}$ & 50 \\
\hline 1,1,1-Trichloroethane & $5 u$ & 50 & 50 \\
\hline $1,1,2$-Trichloroethane & 5u & 50 & 5u \\
\hline vinyl acetate & 100 & 100 & 1 \\
\hline
\end{tabular}


APPENDIX I.3

vocs Detected in Iaboratory Blank Samples, Third Quarter 1993

\begin{tabular}{|c|c|c|c|c|c|c|c|c|}
\hline Sample No. & $\begin{array}{c}930707- \\
032\end{array}$ & $\begin{array}{c}930707- \\
090\end{array}$ & $\begin{array}{c}930709- \\
031\end{array}$ & $\begin{array}{c}930712- \\
037\end{array}$ & $\begin{array}{c}930712- \\
080\end{array}$ & $\begin{array}{c}930713- \\
001\end{array}$ & $\begin{array}{c}930713- \\
014\end{array}$ & $\begin{array}{c}930714- \\
024\end{array}$ \\
\hline 2-Hexanone & 100 & 3 & 100 & $10 \mathrm{U}$ & 100 & 100 & 100 & 100 \\
\hline 4-Methyl-2-pentanone & 100 & 3 & 100 & 100 & $10 u$ & 100 & 100 & 100 \\
\hline Methylene chloride & $5 \mathbf{v}$ & 3 & 50 & 2 & $5 \mathbf{v}$ & 0.9 & $5 \mathbf{v}$ & 50 \\
\hline
\end{tabular}

(CONTINUED)

\begin{tabular}{|c|c|c|c|c|c|c|c|c|}
\hline Sample No. & $\begin{array}{c}930728- \\
001\end{array}$ & $\begin{array}{c}930729- \\
001\end{array}$ & $\begin{array}{c}930802- \\
008\end{array}$ & $\begin{array}{c}930802- \\
075\end{array}$ & $\begin{array}{c}930803- \\
040\end{array}$ & $\begin{array}{c}930817- \\
086\end{array}$ & $\begin{array}{c}930818- \\
001\end{array}$ & $\begin{array}{c}930913- \\
064\end{array}$ \\
\hline 2-Hexanone & 100 & 100 & 100 & 100 & 100 & 100 & 100 & 100 \\
\hline 4-Methy1-2-pentanone & 100 & 100 & 100 & 2 & 100 & 100 & 100 & 100 \\
\hline Methylene chloride & 1 & 1 & 1 & 50 & 50 & 50 & 1 & 5U \\
\hline
\end{tabular}

(CONTINUED)

\begin{tabular}{|c|c|c|c|c|c|c|c|c|}
\hline Sample No. & $\begin{array}{c}930914- \\
009\end{array}$ & $\begin{array}{c}930915- \\
107\end{array}$ & $\begin{array}{c}930915- \\
110\end{array}$ & $\begin{array}{l}930916- \\
020\end{array}$ & $\begin{array}{c}930916- \\
031\end{array}$ & $\begin{array}{c}930920- \\
030\end{array}$ & $\begin{array}{c}930920- \\
116\end{array}$ & $\begin{array}{c}930921- \\
025\end{array}$ \\
\hline 2-Hexanone & 100 & 100 & 100 & 100 & 100 & 100 & 100 & 100 \\
\hline 4-Methyl-2-pentanone & 100 & 100 & 100 & 100 & 100 & 100 & 100 & 100 \\
\hline Methylene chloride & 1 & 50 & $5 U$ & 2 & 50 & 50 & 5u & 50 \\
\hline
\end{tabular}

(CONTINUED)

\begin{tabular}{|c|c|c|c|c|c|c|c|}
\hline Sample No. & $\begin{array}{c}930921- \\
039\end{array}$ & $\begin{array}{c}930923- \\
093\end{array}$ & $\begin{array}{c}930927- \\
018\end{array}$ & $\begin{array}{c}930928- \\
089\end{array}$ & $\begin{array}{c}930929- \\
174\end{array}$ & $\begin{array}{c}930930- \\
001\end{array}$ & $\begin{array}{c}930930- \\
079\end{array}$ \\
\hline 2-Hexanone & 100 & 100 & 100 & 100 & 100 & 100 & $10 U$ \\
\hline 4-Methy1-2-pentanone & 100 & $10 \mathrm{U}$ & 100 & 100 & 100 & 100 & 100 \\
\hline Methylene chloride & 5u & 5u & 5u & $5 U$ & 50 & 1 & 5u \\
\hline
\end{tabular}


APPENDIX I.3

VOCs Detected in Laboratory Blank Samples, Fourth Quarter 1993

\begin{tabular}{|c|c|c|c|c|c|c|c|c|}
\hline Sample No. & $\begin{array}{c}931008- \\
005\end{array}$ & $\begin{array}{c}931008- \\
118\end{array}$ & $\begin{array}{c}931011- \\
042\end{array}$ & $\begin{array}{c}931011- \\
055\end{array}$ & $\begin{array}{c}931012- \\
038\end{array}$ & $\begin{array}{c}931012- \\
055\end{array}$ & $\begin{array}{c}931014- \\
001\end{array}$ & $\begin{array}{c}931014- \\
010\end{array}$ \\
\hline Acetone & 100 & 100 & 4 & 100 & 3 & 100 & 3 & 100 \\
\hline 2-Butanone & 7 & 100 & 11 & 100 & 10 & 100 & 11 & 100 \\
\hline 2-Hexanone & $10 \mathrm{U}$ & 100 & 100 & 100 & 100 & 100 & $10 \mathrm{U}$ & 100 \\
\hline 4-Methy1-2-pentanone & 100 & 100 & 100 & 100 & 100 & 100 & 100 & 100 \\
\hline Methylene chloride & 50 & 5u & 3 & $5 v$ & 3 & 50 & 3 & 5v \\
\hline styrene & 0.5 & $5 \mathbf{v}$ & $5 \mathbf{v}$ & 50 & 5u & 50 & 5v & 5u \\
\hline
\end{tabular}

(CONTINUED)

\begin{tabular}{|c|c|c|c|c|c|c|c|c|}
\hline Sample No. & $\begin{array}{c}931015- \\
141\end{array}$ & $\begin{array}{c}931018 \\
003\end{array}$ & $\begin{array}{c}931019- \\
038\end{array}$ & $\begin{array}{c}931020- \\
001\end{array}$ & $\begin{array}{c}931021- \\
024\end{array}$ & $\begin{array}{c}931023- \\
006\end{array}$ & $\begin{array}{c}931025- \\
022\end{array}$ & $\begin{array}{c}931025- \\
031\end{array}$ \\
\hline Acetone & 100 & 4 & 100 & 3 & 3 & 100 & 3 & 3 \\
\hline 2-Butanone & 100 & 10 & 100 & 10 & 11 & 100 & 9 & 9 \\
\hline 2-Hexanone & 100 & 100 & 100 & 100 & 100 & 100 & 100 & 100 \\
\hline 4-Methy 1-2-pentanone & 100 & 1 & $10 \mathrm{U}$ & 1 & 1 & $10 \mathrm{U}$ & 100 & 100 \\
\hline Methylene chloride & $5 \mathrm{U}$ & 3 & 50 & 3 & 2 & 0.6 & 2 & 2 \\
\hline Styrene & 50 & 5u & 50 & $5 \mathbf{v}$ & 50 & 50 & $5 \mathbf{U}$ & 50 \\
\hline
\end{tabular}

(CONTINUED)

\begin{tabular}{|c|c|c|c|c|c|c|c|c|}
\hline Sample No. & $\begin{array}{c}931026- \\
013\end{array}$ & $\begin{array}{c}931027- \\
001\end{array}$ & $\begin{array}{c}931027- \\
002\end{array}$ & $\begin{array}{c}931028- \\
007\end{array}$ & $\begin{array}{c}931110- \\
008\end{array}$ & $\begin{array}{c}931110- \\
011\end{array}$ & $\begin{array}{c}931111- \\
002\end{array}$ & $\begin{array}{c}931116- \\
002\end{array}$ \\
\hline Acetone & 100 & 8 & 100 & 3 & 100 & 100 & 100 & 100 \\
\hline 2-Butanone & 100 & 100 & 100 & 15 & 100 & 100 & 100 & 100 \\
\hline 2-Hexanone & 100 & $10 \mathrm{U}$ & 100 & 100 & 100 & 100 & 100 & 100 \\
\hline 4-Methyl-2-pentanone & 100 & 100 & 100 & $10 u$ & 100 & 100 & 100 & 100 \\
\hline Methylene chloride & $5 U$ & 1 & $5 U$ & 3 & 2 & $5 U$ & 2 & 1 \\
\hline styrene & $5 \mathbf{u}$ & 50 & 50 & $5 \mathrm{U}$ & 50 & 50 & $5 \mathbf{u}$ & 50 \\
\hline
\end{tabular}

(CONTINUED) 
APPENDIX 1.3

Voce Detected In I aboratory Blank Samples, Fourth Quarter 1993

\begin{tabular}{|c|c|c|c|c|c|c|c|c|}
\hline Sample No. & $\begin{array}{c}931213- \\
006\end{array}$ & $\begin{array}{c}931214- \\
002\end{array}$ & $\begin{array}{l}931215- \\
020\end{array}$ & $\begin{array}{c}931216- \\
050\end{array}$ & $\begin{array}{c}931220- \\
103\end{array}$ & $\begin{array}{c}931221- \\
083\end{array}$ & $\begin{array}{c}931222- \\
020\end{array}$ & $\begin{array}{c}931223- \\
001\end{array}$ \\
\hline Acetone & 100 & 100 & 100 & 4 & 3 & $\mathbf{5}$ & 4 & 100 \\
\hline 2-Butanone & 100 & 100 & 100 & 9 & 8 & 8 & 7 & 100 \\
\hline 2-Hexanone & 100 & 100 & 100 & 100 & $10 u$ & 1 & 1 & 100 \\
\hline 4-Methyl-2-pentanone & 100 & 100 & 100 & 1 & 1 & 100 & 100 & 100 \\
\hline Methylene chloride & 1 & 1 & su & 2 & 1 & 0.6 & 0.5 & 0.6 \\
\hline styrene & su & $5 U$ & $5 \mathbf{v}$ & $5 v$ & $5 \mathbf{u}$ & $5 \mathbf{U}$ & 50 & 5u \\
\hline
\end{tabular}

(CONTINUED)

\begin{tabular}{|l|r|r|}
\hline Sample No. & $\begin{array}{c}931227- \\
026\end{array}$ & $\begin{array}{c}931229- \\
\end{array}$ \\
\hline Acetone & 2 & 5 \\
2-Butanone & 4 & 9 \\
2-Hexanone & $10 U$ & 1 \\
4-Methyl-2-pentanone & $10 U$ & 2 \\
Methylene chloride & $5 U$ & 2 \\
Styrene & $5 U$ & $5 U$ \\
\hline
\end{tabular}


KYYWWAS YLVA GLVSNI LNGWdI IOA bT XIangddV 

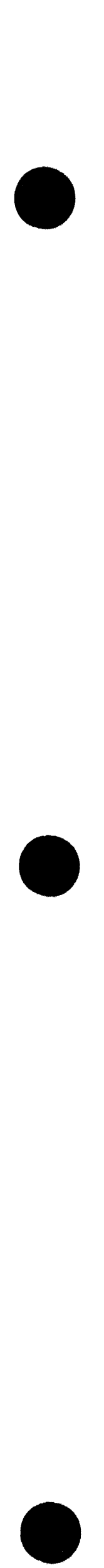
APPENDIX 1.4

Equipment RIneate Data sumary, Firet Quarter 1993

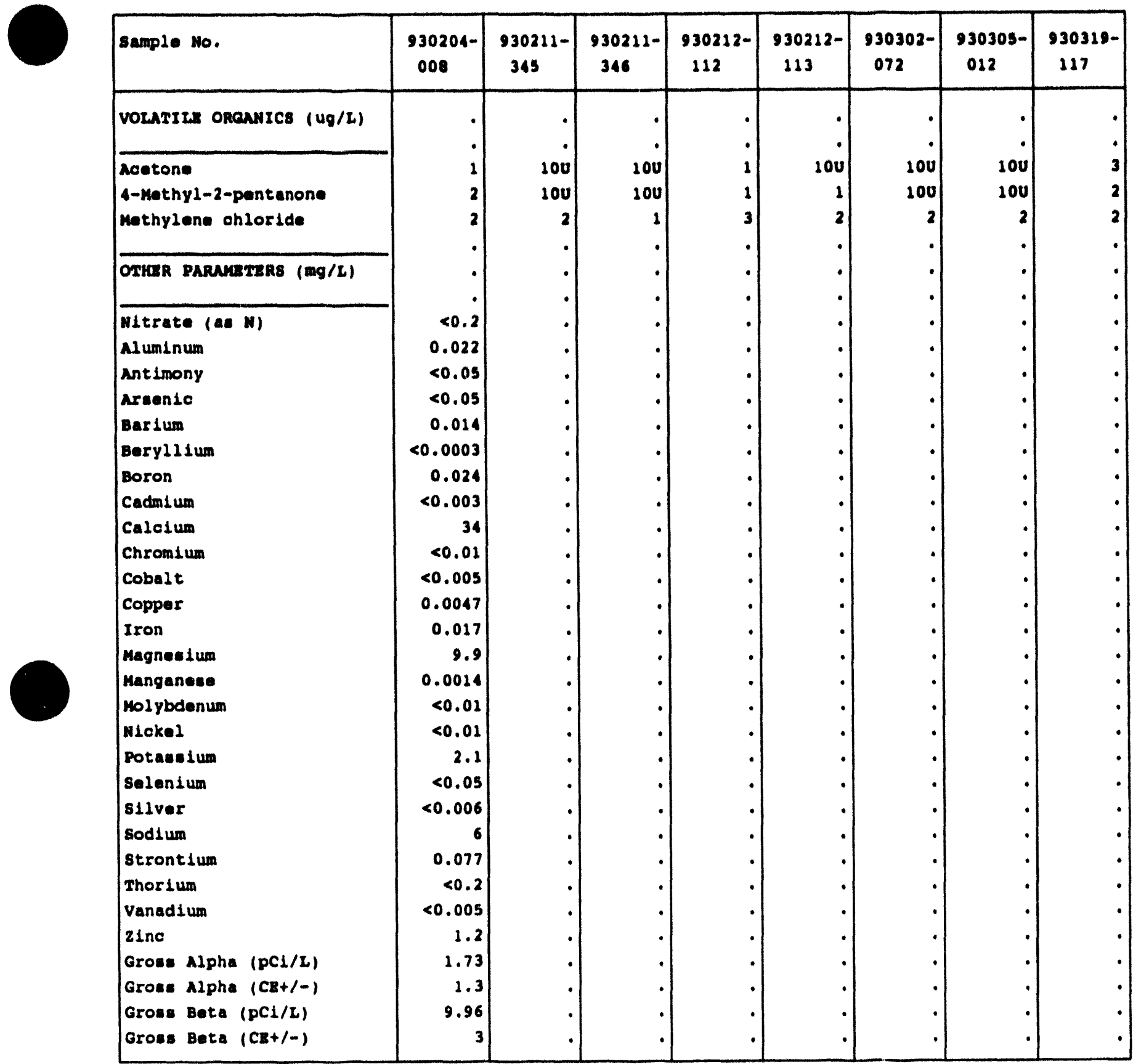

(CONTINURD) 
ApPEnDTX 1.4

equipment Rineate Dete Bumary, Fret querter 1993

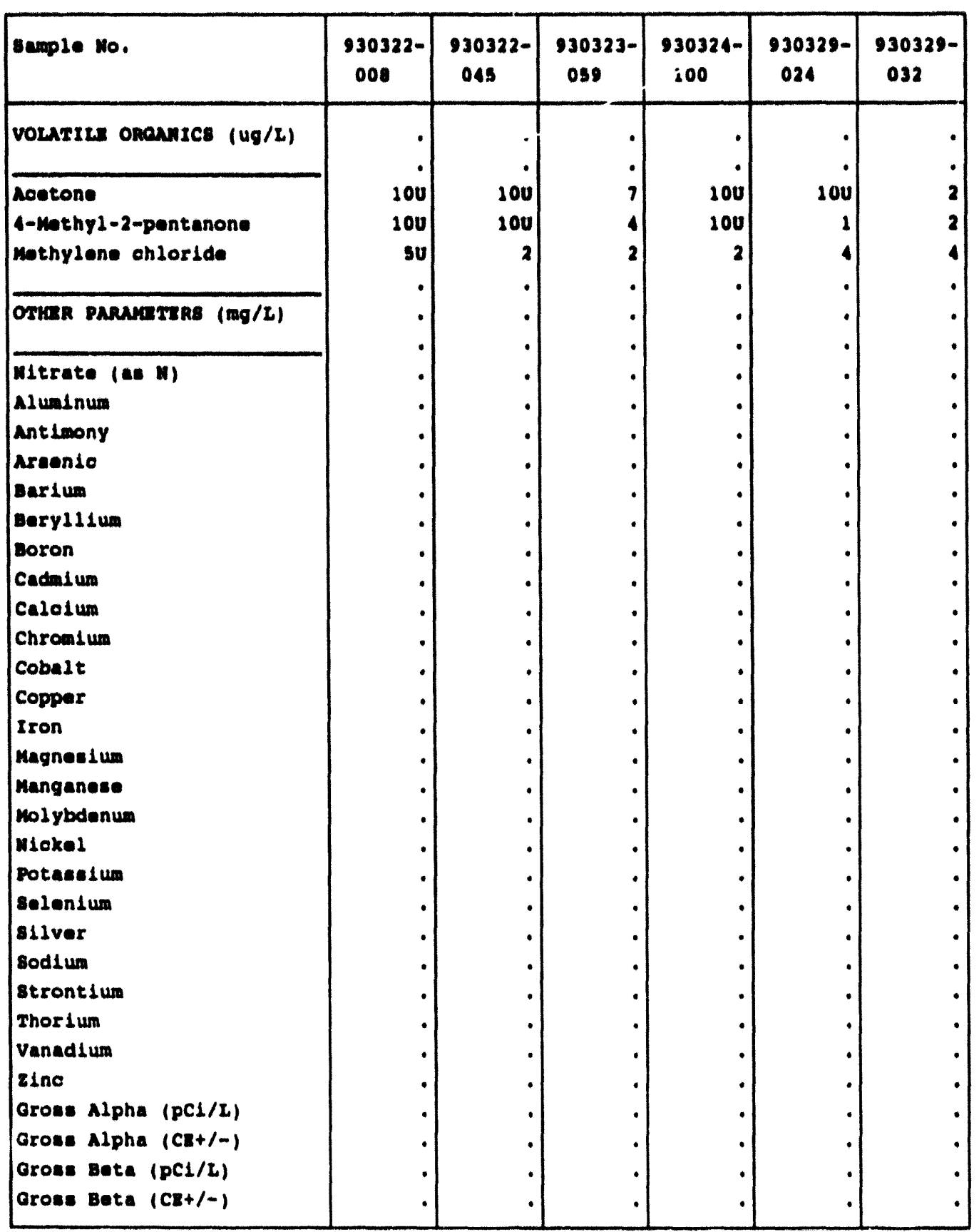


APPEnDIX 1.4

IquLpent RLneate Data sumary, second Quarter 1993

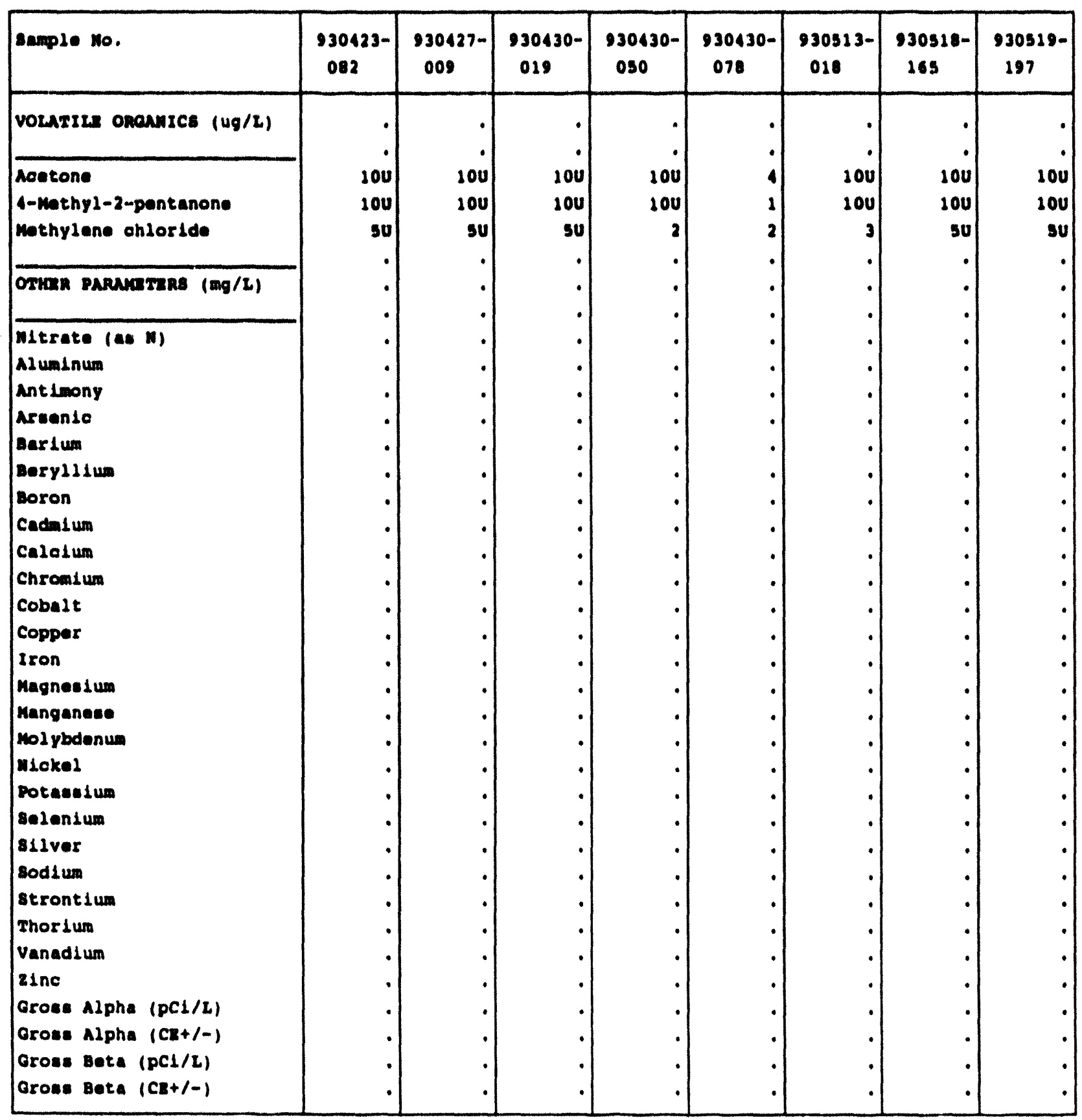

(CONTINUED) 
APRHDXX I.4

Equdpment Rlneate Data sumary, seoond Quarter 1993

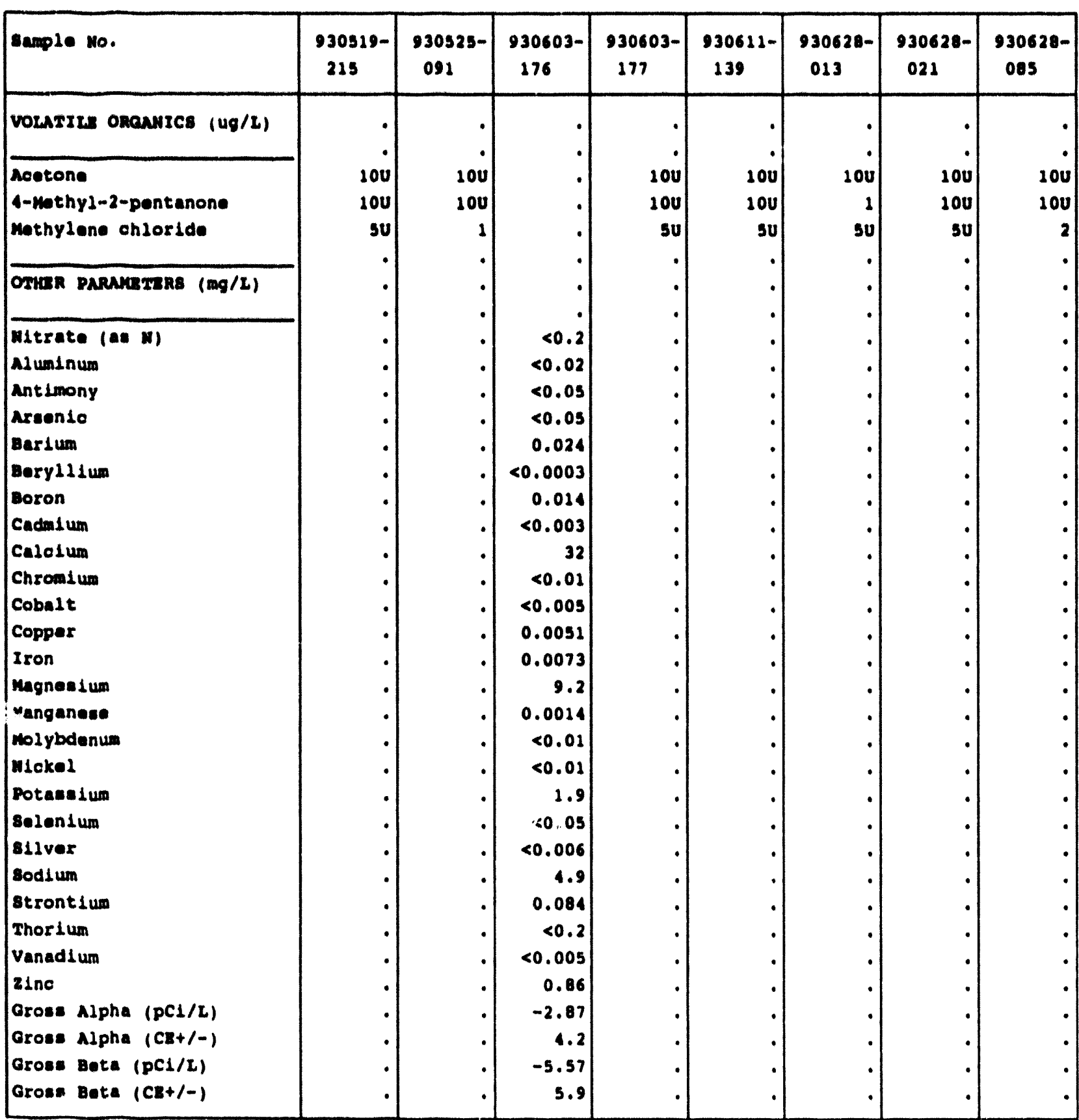


APPENDIX 1.4

Equipment RInete Data Summary, Third Quarter 1993

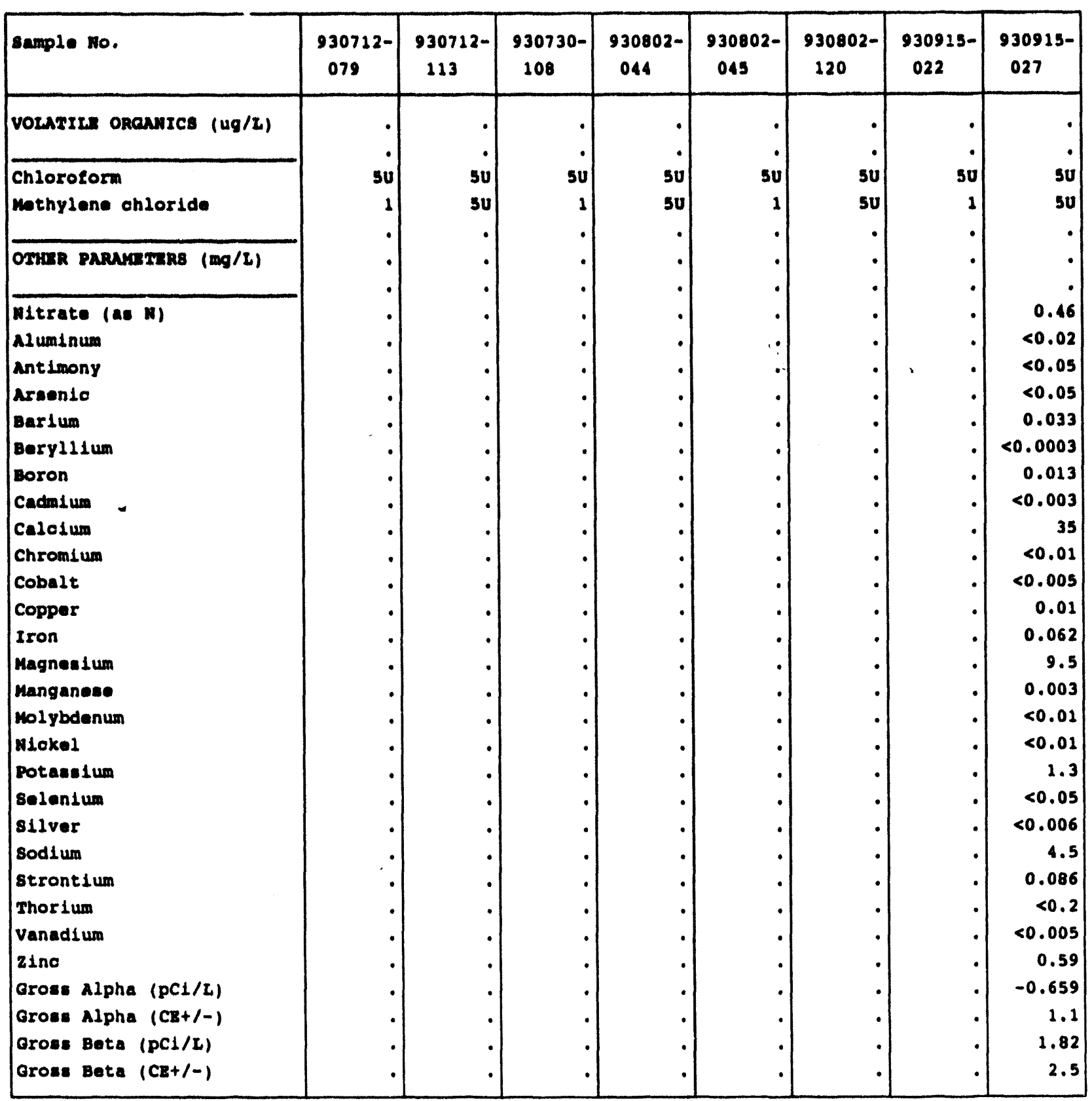

(CONTINUED) 
APPEADIX 1.4

Bquipment Rineate Data Bummary, Th1rd Quarter 1993

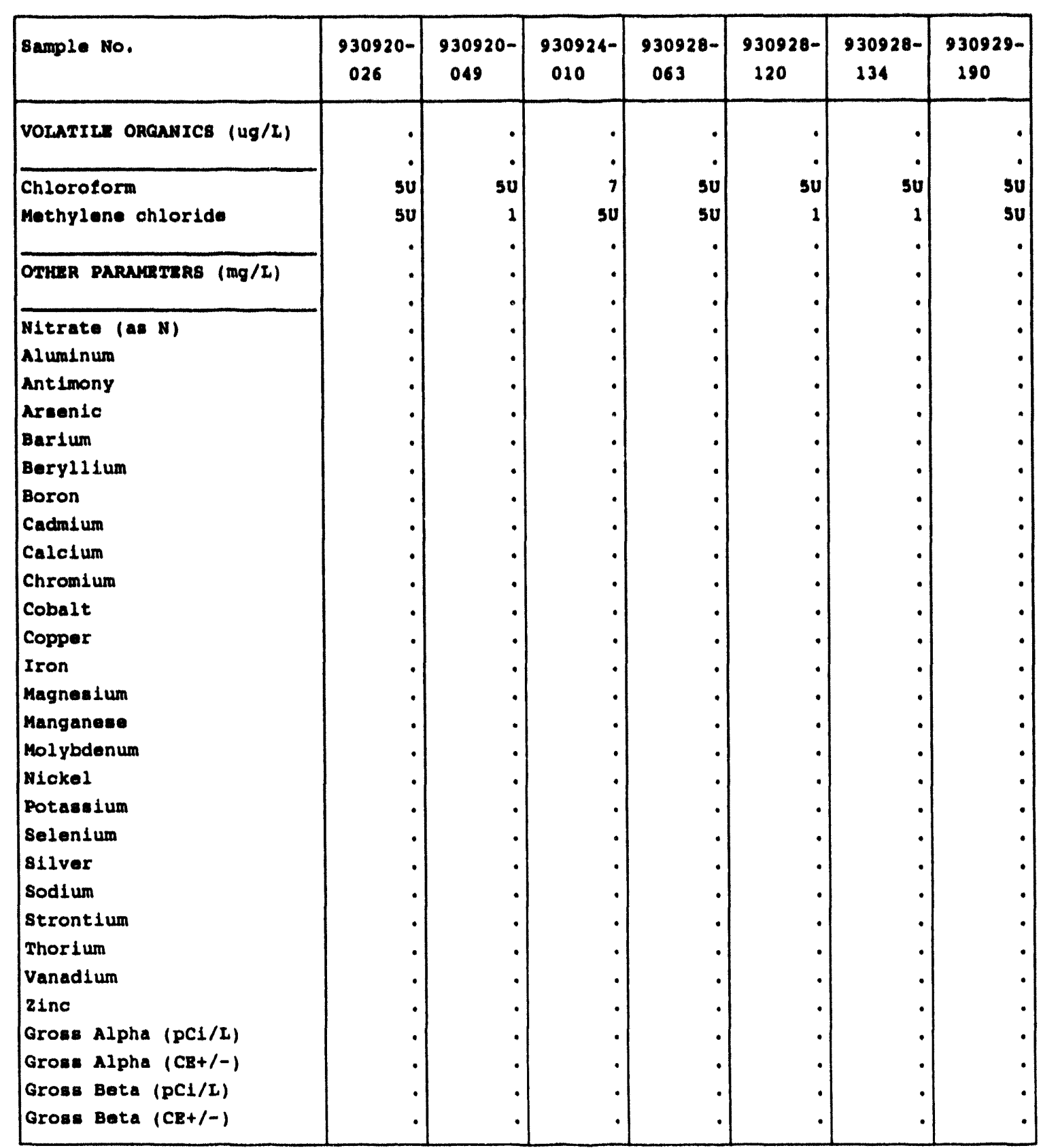


APPENDIX 1.4

Equipment Rinsate Data Summary, Fourth Quarter 1993

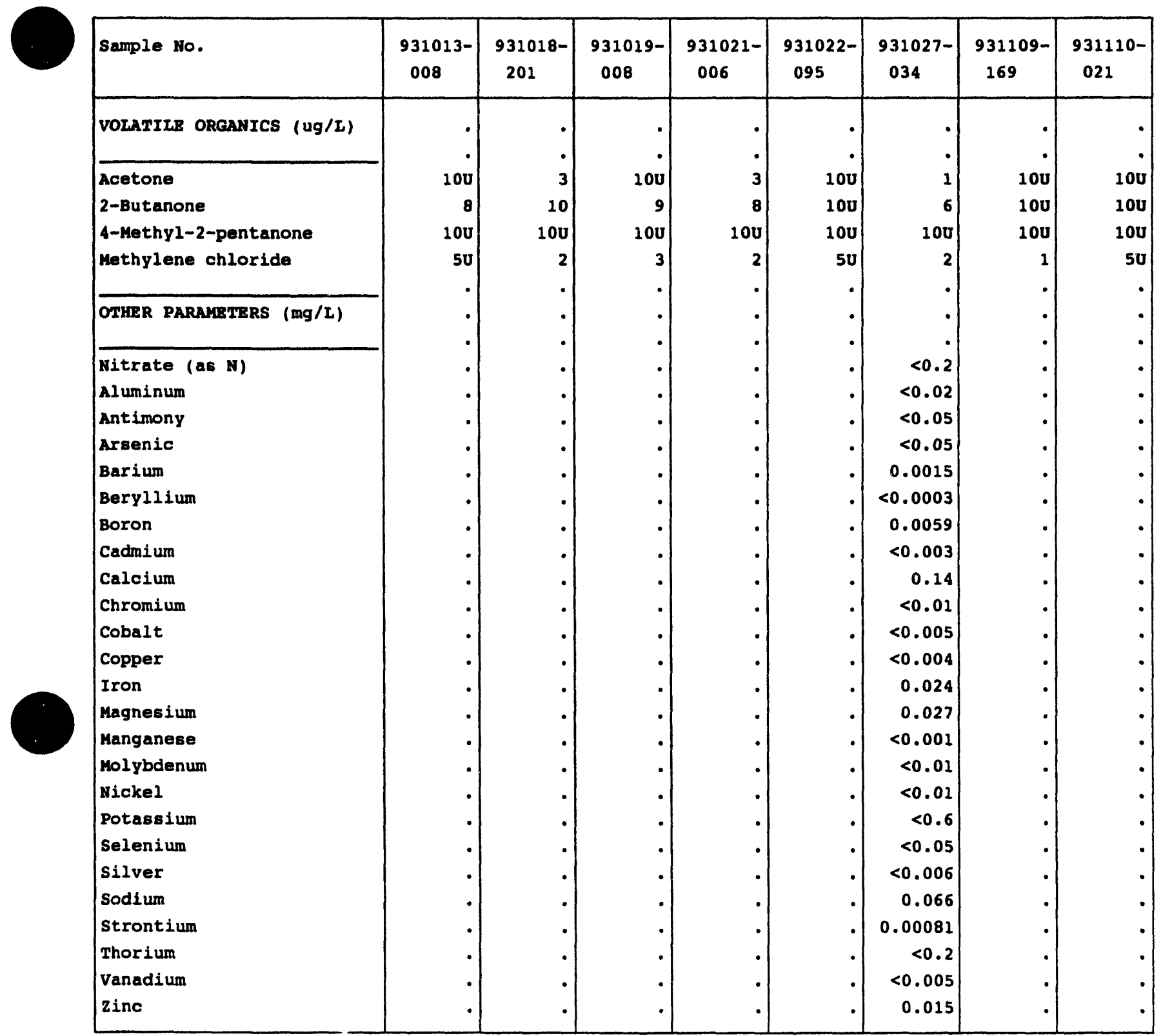

(CONTINUED) 
APPENDIX I.4

Equipment Rinsate Data Summary, Fourth Quarter 1993

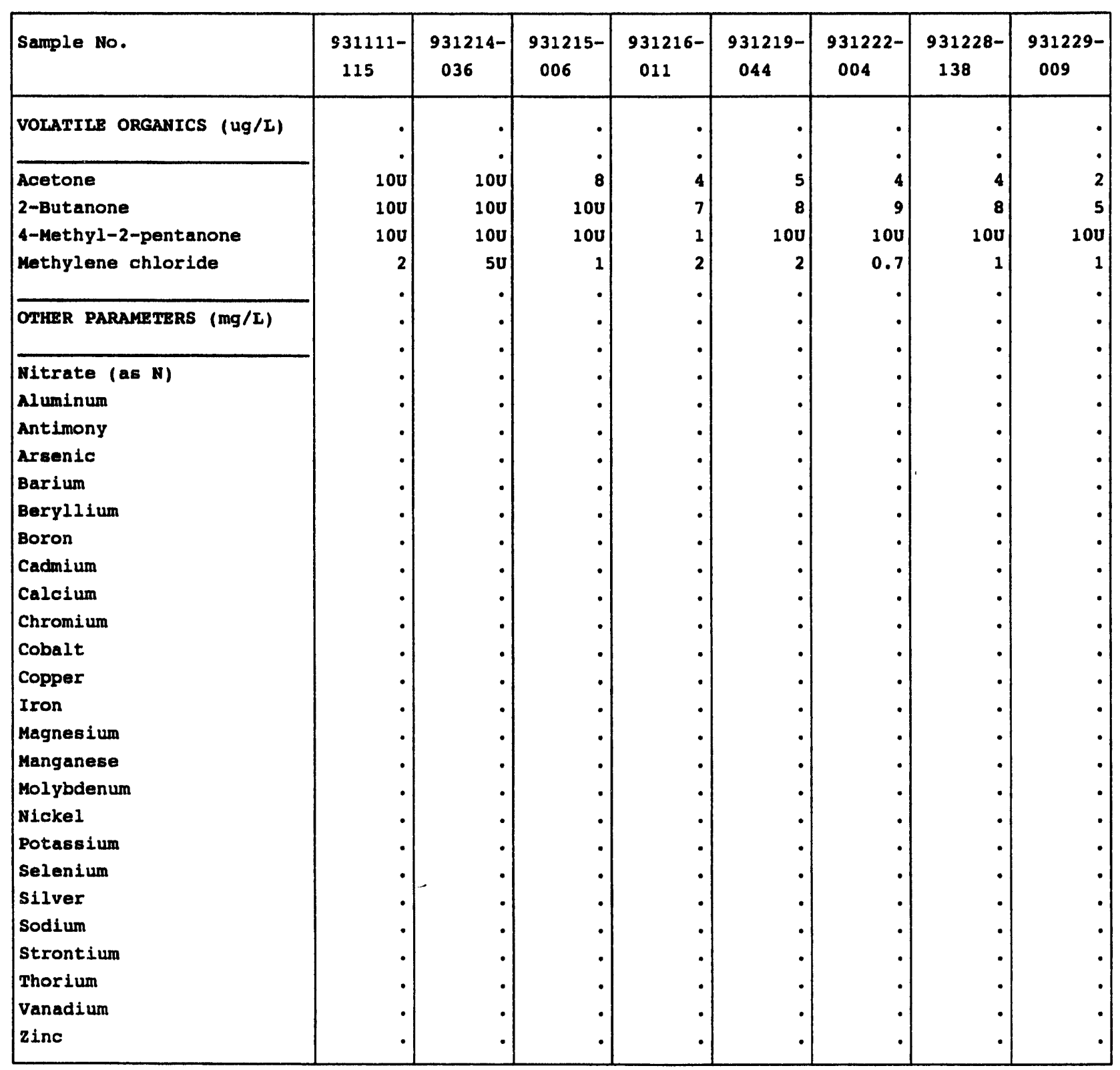


APPENDIX 1.4

Equipment Rinsate Radiochemical Data Summary, Eourth Quarter 1993

\begin{tabular}{|c|c|c|c|c|c|c|c|c|}
\hline Sample No. & $\begin{array}{c}931013- \\
008\end{array}$ & $\begin{array}{c}931018- \\
201\end{array}$ & $\begin{array}{c}931019- \\
008\end{array}$ & $\begin{array}{c}931021- \\
006\end{array}$ & $\begin{array}{c}931022- \\
095\end{array}$ & $\begin{array}{c}931027- \\
034\end{array}$ & $\begin{array}{c}931109- \\
169\end{array}$ & $\begin{array}{c}931110- \\
021\end{array}$ \\
\hline Grose Alpha $(\mathrm{pCl} / \mathrm{L})$ & $\cdot$ & $\cdot$ & $\cdot$ & $\cdot$ & $\cdot$ & 1.53 & $\cdot$ & . \\
\hline Gross Alpha $(\mathrm{Cz}+/-)$ & $\cdot$ & $\cdot$ & $\cdot$ & $\cdot$ & $\cdot$ & 1.6 & $\cdot$ & • \\
\hline Gross Beta (pCi/L) &. & $\cdot$ & $\cdot$ & $\cdot$ & $\cdot$ & 0.508 & $\cdot$ & • \\
\hline Gross Beta $(\mathrm{Cz}+1-)$ & $\cdot$ & $\cdot$ & $\cdot$ & $\cdot$ & $\cdot$ & 2.6 & - & - \\
\hline Americium 241 (pCl/L) & $\cdot$ & $\cdot$ & 0 & 5.14 & 6 & -0.662 & 8.03 & 4.21 \\
\hline Americium $241(\mathrm{CB}+/-)$ & $\cdot$ & $\cdot$ & 6.2 & 7.3 & 6.9 & 2.3 & 16 & 4.9 \\
\hline Neptunium 237 (pci/L) & $\cdot$ & $\cdot$ & 0 & 0.551 & 2.74 & 0 & 7.08 & 3.35 \\
\hline Neptunium 237 (CE+/-) & $\cdot$ &. & 2.1 & 1.1 & 2.4 & 1.8 & 7.1 & 4.7 \\
\hline Radium, total (Bq/L) & $\cdot 1$ & $\cdot$ & $\cdot$ & $\cdot$ & $\cdot$ & 0.029 & 0.077 & 0.047 \\
\hline Radium $(\mathrm{CE}+/-)$ & $\cdot$ & $\cdot$ & $\cdot$ & $\cdot$ &. & 0.048 & 0.048 & 0.039 \\
\hline Strontium, total $(\mathrm{pC} i / L)$ & $\cdot$ &. & 23.2 & 54.9 & 19.6 & 20 & 96.2 & 115 \\
\hline Strontium $(\mathrm{CE}+/-)$ & $\cdot$ & $\cdot$ & 21 & 24 & 20 & 21 & 28 & 29 \\
\hline Technetium 99 (pCi/L) & $\cdot 1$ & $\cdot$ & 187 & 121 & 189 & $-79 \cdot 3$ & 201 & 158 \\
\hline Technetium $99(\mathrm{CE}+/-)$ & $\cdot$ & $\cdot$ & 65 & 63 & 65 & 56 & 65 & 64 \\
\hline Thorium $234(\mathrm{pCi} / 1)$ & $\cdot$ & $\cdot$ & 914 & - & 213 & $\cdot$ & $\cdot$ & - \\
\hline Thorium $234(\mathrm{CE}+/-)$ & $\cdot$ & $\cdot$ & 410 & $\cdot$ & 520 & $\cdot$ & - & - \\
\hline Tritium (Bq/L) & $\cdot$ & $\cdot$ & $\cdot$ & . & 33 & -4 & 12 & -13 \\
\hline Tritium (CE+/-) & $\cdot$ & $\cdot$ & $\cdot$ & . & 29 & 23 & 25 & 22 \\
\hline Oranium $234(\mathrm{pCi} / \mathrm{L})$ & $\cdot$ & $\cdot$ & 7.11 & 0 & -7.06 & 5.46 & 4.47 & -0.345 \\
\hline Uranium $234(\mathrm{CB}+/-)$ & $\cdot$ & $\cdot$ & 38 & 28 & 32 & 4.9 & 2.3 & 0.77 \\
\hline Uranium 235 (pCi/L) & $\cdot$ & $\cdot$ & 3.8 & 61.3 & 49.6 & -6.59 & -26.4 & 29.7 \\
\hline Uranium $235(\mathrm{CE}+/-)$ & $\cdot$ & $\cdot$ & 68 & 90 & 27 & 59 & 68 & 90 \\
\hline Uranium $238(\mathrm{pCi} / \mathrm{L})$ & $\cdot$ & $\cdot$ & 7.11 & 5.64 & 14.1 & 5.46 & 3.98 & -0.517 \\
\hline Uranium $238(\mathrm{CE}+/-)$ & $\cdot$ & . & 14 & 11 & 20 & 5.8 & 2.5 & 0.91 \\
\hline
\end{tabular}

(CONTINUED) 
APPENDIX 1.4

Equipment Rinsate Radiochemical Data Summary, Fourth Quarter 1993

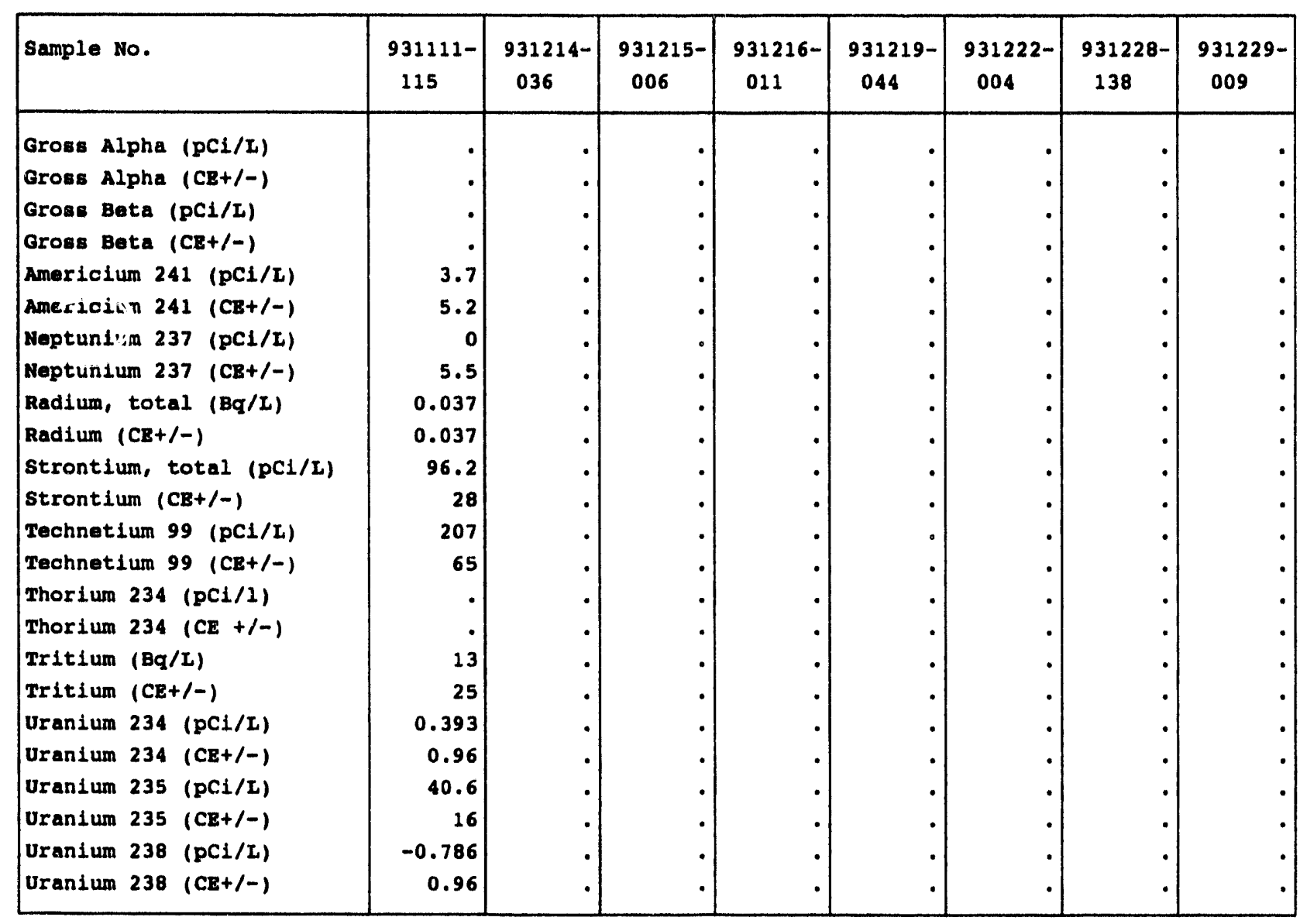


APPENDIX J

VALIDATED RESULTS OF VOLATIL ORGANIC COMPOUND ANALYSES 


\section{EXPLANATION}

\section{SAMPLING POINT:}

$\begin{array}{rll}\text { BCK } & - \text { Bear Creek Kilometer } \\ \text { GW } & - \text { Monitoring Well } \\ \text { NT } & - \text { North Tributary to Bear Creek } \\ \text { SS } & - \text { Spring }\end{array}$

\section{LOCATION/STTE:}

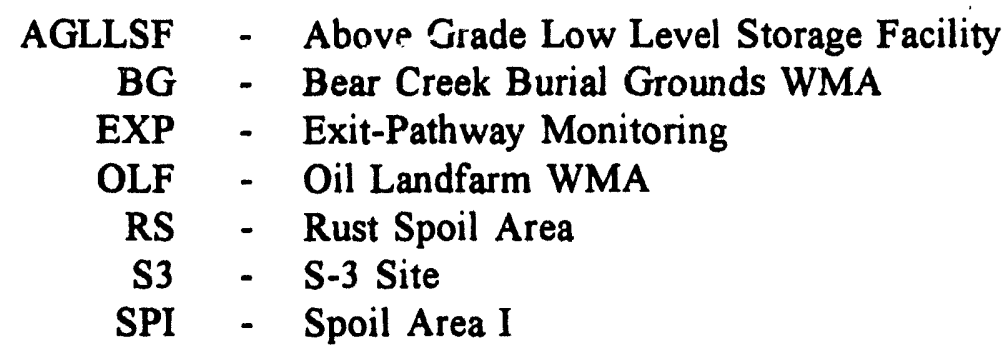

\section{NOTES:}

All results in micrograms per Liter (ug/L).
FP1 - False Positive result, screened by laboratory blank
FP2 - False Positive result, screened by trip blank
FP3 - Anomalous Result
U - Compound not detected at the reported minimum attainable detection limit.

Results of VOC analyses reported for each groundwater sample were evaluated using results for each associated laboratory and trip blank sample to identify false positives (i.e., results that are probable artifacts of the groundwater sampling or analysis procedures). To identify the false positives, the maximum concentration of each VOC detected in either blank sample (Appendix I) is multiplied by a qualification factor to determine the blank qualification result (BQR) for the compound. A factor of 5 is used for most compounds and a factor of 10 is used for the common laboratory reagents methylene chloride, acetone, 2-butanone, and toluene (U.S. Environmental Protection Agency 1988b). If a compound detected in the blank samples is also detected in an associated groundwater sample, the concentration reported for the groundwater sample (Appendix E) is compared to the corresponding $\mathrm{BQR}$. If the concentration of the compound in the groundwater sample is at or below the $B Q R$, the result is considered a false positive and is excluded from the summed VOC concentration determined for the sample.

If a compound was detected in only one of at least four consecutive quarterly samples collected at a sampling point, the result was assumed to be anomalous. Anomalous VOC results were not identified in samples for which elevated analytical detection limits were reported for the compound during any other consecutive sampling event. 
Validated voc Results, 1993

\begin{tabular}{|c|c|c|c|c|c|c|c|c|c|c|c|c|c|}
\hline \multirow{4}{*}{$\begin{array}{l}\text { Sampling Point } \\
\text { Location } \\
\text { - } \\
\text { Date Sampled }\end{array}$} & \multicolumn{8}{|c|}{ GW-013 } & \multicolumn{5}{|c|}{ GW-040 } \\
\hline & \multicolumn{8}{|c|}{ OLF } & \multicolumn{5}{|c|}{ BG } \\
\hline & \multicolumn{2}{|c|}{$03 / 29 / 93$} & \multicolumn{2}{|c|}{$06 / 17 / 93$} & \multicolumn{2}{|c|}{$09 / 17 / 93$} & \multicolumn{2}{|c|}{$12 / 19 / 93$} & \multicolumn{2}{|c|}{$03 / 25 / 93$} & \multicolumn{3}{|c|}{$05 / 11 / 93$} \\
\hline & $u g / L$ & 8 sum & ug/L & 8 sum & $\mathrm{ug} / \mathrm{L}$ & 8 sum & $u g / L$ & 8 sum & $u g / L$ & sum & $\mathrm{ug} / \mathrm{L}$ & sum & \\
\hline Acetone & 100 & $\cdot$ & & $\cdot$ & 100 & $\cdot$ & FP1 & - & 100 & $\cdot 1$ & 100 & & $\bullet$ \\
\hline Benzene & 50 & $\cdot$ & 50 & $\cdot$ & $\mathbf{5 0}$ & $\cdot$ & 5v & $\cdot$ & 5v & $\cdot$ & 50 & & $\cdot$ \\
\hline Bromodichloromethane & su & $\cdot$ & 50 & $\cdot$ & 5u & $\cdot$ & 50 & $\cdot$ & 50 & $\cdot$ & 50 & & $\cdot$ \\
\hline 2-Butanone & 100 & $\cdot$ & 100 & $\cdot 1$ & 100 & $\cdot$ & FP1 & $\cdot$ & 100 & $\cdot 1$ & 100 & & $\cdot$ \\
\hline Carbon disulfide & 50 & $\cdot$ & 50 & $\cdot$ & 50 & $\cdot$ & 50 & $\bullet$ & $\begin{array}{l}50 \\
50\end{array}$ & $\cdot$ & 50 & &. \\
\hline Carbon tetrachloride & 50 & $\cdot$ & 50 &. & $\begin{array}{l}50 \\
50\end{array}$ &. & 50 &. & 50 &. & sv & & - \\
\hline Chlorobenzene & $\begin{array}{l}50 \\
50\end{array}$ &. & 50 &. & 50 & $\cdot$ & 5v & - & 5v & $\cdot$ & 50 & & - \\
\hline $\begin{array}{l}\text { Chloroform } \\
1,1 \text {-Dichloroethane }\end{array}$ & 50 &. & 5v &. & su & .1 & 5v & - & 50 & $\cdot$ & 50 & & - \\
\hline $\begin{array}{l}\text { 1,1-Dichloroethane } \\
\text { 1,2-Dichloroethane }\end{array}$ & 50 & $\cdot$ & 50 &. & 50 & $\cdot$ & $5 \mathbf{v}$ & $\cdot$ & 50 &. & 50 & & $\cdot$ \\
\hline & 50 & $\cdot$ & 50 & $\cdot$ & 50 &. & 50 & $\cdot$ & 50 & $\cdot$ & 5v & & $\cdot$ \\
\hline & 50 & $\cdot$ & 50 & $\cdot$ & 50 & $\cdot 1$ & 50 & $\cdot$ & 50 & $\cdot$ & 50 & & • \\
\hline Ethylbenzene & 50 & $\cdot$ & 5u & $\cdot$ & 50 & $\cdot$ & 5u & $\cdot$ & 5u & $\cdot$ & 50 & & - \\
\hline 2-Hexanone & 100 & $\cdot$ & 100 & $\cdot$ & 100 & $\cdot 1$ & 100 & - & 100 & $\cdot$ & 100 & & • \\
\hline 4-Methy1-2-pentancne & FP1 & $\cdot$ & 100 & $\cdot$ & 100 & $\cdot$ & FP1 & $\cdot$ & 100 & $\cdot$ & 100 & & - \\
\hline Methylene chloride & FP1 & $\cdot$ & 50 & $\cdot$ & 50 & $\cdot$ & FP1 & $\bullet$ & 50 & $\cdot$ & FP2 & & - \\
\hline Styrene & $\begin{array}{l}50 \\
50\end{array}$ & $\cdot$ & $\begin{array}{l}50 \\
50\end{array}$ & $\cdot$ & $\begin{array}{l}50 \\
50\end{array}$ & $\cdot$ & 50 &. & $\begin{array}{l}50 \\
50\end{array}$ & $\cdot$ & 50 & & • \\
\hline Tetrachloroethene & $\begin{array}{l}30 \\
50\end{array}$ & $\cdot$ & 50 & $\bullet$ & 50 &. & 5v &. & so &. & 50 & & $\cdot$ \\
\hline $\begin{array}{l}\text { Toluene } \\
1,1,1-T r i c h l o r o e t h e n e\end{array}$ & 50 &. & 50 & $\dot{\bullet}$ & 50 & $\cdot$ & so &. & 5u & $\cdot$ & 50 & & - \\
\hline $\begin{array}{l}\text { 1,1,1-Trichloroethane } \\
\text { 1,1,2-Trichloroethane }\end{array}$ & 50 & . & 5u & $\cdot$ & 50 & $\cdot$ & 5u & $\cdot$ & 50 & $\cdot$ & 50 & & - \\
\hline $\begin{array}{l}1,1,2 \text {-Trichloroethane } \\
\text { Trichloroethene }\end{array}$ & $5 v$ & $\cdot$ & 50 & $\cdot$ & 50 & $\cdot$ & 50 & $\cdot$ & 50 & $\cdot$ & 50 & & - \\
\hline vinyl acetate & 100 & $\cdot$ & 100 & $\cdot$ & 100 & $\cdot$ & 100 & $\cdot$ & 100 & $\cdot$ & 100 & & - \\
\hline vinyl chloride & 100 & $\cdot$ & 100 & $\cdot$ & 100 & $\cdot$ & 100 & $\cdot$ & 100 & $\cdot$ & $\begin{array}{r}100 \\
50\end{array}$ & & - \\
\hline Xylenes & 50 & $\cdot$ & . & $\cdot$ &. & $\cdot$ &. & $\cdot$ & $\cdot$ & . &. & & . \\
\hline Summed & 0 & • & 0 &. & 0 & $\cdot$ & o) & $\cdot$ & 0 & $\cdot$ & 0 & & \\
\hline
\end{tabular}

(CONTINUED) 
APPENDIX J

Validated voc Results, 1993

\begin{tabular}{|c|c|c|c|c|c|c|c|c|c|c|c|c|c|}
\hline \multirow{4}{*}{$\begin{array}{l}\text { Sampling Point } \\
\text { Location } \\
\text { Date Sampled }\end{array}$} & \multicolumn{4}{|c|}{ GW-040 } & \multicolumn{9}{|c|}{ GW-043 } \\
\hline & \multicolumn{4}{|c|}{ BG } & \multicolumn{9}{|c|}{ OLF } \\
\hline & \multicolumn{2}{|c|}{$07 / 02 / 93$} & \multicolumn{2}{|c|}{$10 / 09 / 93$} & \multicolumn{2}{|c|}{$02 / 04 / 93$} & \multicolumn{2}{|c|}{$05 / 10 / 93$} & \multicolumn{2}{|c|}{$09 / 11 / 93$} & \multicolumn{3}{|c|}{$10 / 19 / 93$} \\
\hline & ug/L & 8 sum & ug/L & 8 sum & ug/L & \& sum & ug/L & sum & $u g / L$ & E sum & $\mathbf{u g} / \mathbf{L}$ & s sum & \\
\hline Acetone & 100 & $\cdot$ & 100 & $\cdot$ & 100 & $\cdot$ & 100 & $\cdot$ & 100 & $\cdot 1$ & FP1 & & $\cdot$ \\
\hline Benzene & 50 & $\cdot$ & 50 & $\cdot$ & 50 & $\cdot$ & 50 & $\cdot$ & 50 & $\cdot 1$ & so & & $\cdot$ \\
\hline Bromodichloromethane & 50 & $\cdot$ & 50 & $\cdot$ & 5u & $\cdot$ & 50 & $\cdot$ & 50 & $\cdot$ & 50 & & $\cdot$ \\
\hline 2-Butanone & 100 & $\cdot$ & 100 & $\cdot$ & 100 & $\cdot$ & 100 & $\cdot$ & 100 & $\cdot$ & FP1 & & $\cdot$ \\
\hline Carbon disulfide & 50 & $\cdot$ & 50 & $\cdot$ & 50 & $\cdot$ & 50 & $\cdot$ & so & $\cdot$ & so & & $\cdot$ \\
\hline Carbon tetrachloride & 50 & $\cdot$ & 50 & $\cdot$ & 50 & $\cdot$ & so & $\cdot$ & 50 & $\cdot$ & 50 & & $\cdot$ \\
\hline Chlorobenzene & 50 & $\cdot$ & 50 & $\cdot$ & 50 & $\cdot$ & 50 & $\cdot$ & 50 & $\cdot$ & 50 & & $\cdot$ \\
\hline Chloroform & 50 & $\cdot$ & 50 & $\cdot$ & 50 & $\cdot$ & 50 & $\cdot$ & 50 & $\cdot$ & 50 & & $\cdot$ \\
\hline 1,1-Dichlcroethane & 50 & $\cdot$ & 50 & $\cdot$ & su & $\cdot$ & 50 & $\cdot$ & 50 & $\cdot$ & 5v & & $\cdot$ \\
\hline 1,2-Dichloroethene & 50 & $\cdot$ & 50 & $\cdot$ & $5 v$ & $\cdot$ & 50 & $\cdot$ & 50 & $\cdot$ & 50 & & $\cdot$ \\
\hline 1,1-Dichloroethene & 50 & $\cdot$ & 50 & $\cdot$ & 5v & $\cdot$ & 50 & $\cdot$ & 50 & $\cdot$ & 50 & & $\cdot$ \\
\hline 1,2-Dichloroethene & 50 & $\cdot$ & 50 & $\cdot$ & su & $\cdot$ & 50 & $\cdot$ & 50 & $\cdot$ & 50 & & $\cdot$ \\
\hline Ethylbenzene & su & $\cdot$ & 50 & $\cdot$ & $5 \mathbf{0}$ & $\cdot$ & 50 & $\cdot$ & 5v & $\cdot$ & 50 & & $\cdot$ \\
\hline 2-Hexanone & 100 & $\cdot$ & 100 & $\cdot$ & 100 & $\cdot$ & 100 & $\cdot$ & 100 & $\cdot$ & 100 & & $\cdot$ \\
\hline 4-Methy1-2-pentanone & 100 & $\cdot$ & 100 & $\cdot$ & FP1 & $\cdot$ & 100 & $\cdot$ & 100 & $\cdot$ & 100 & & $\cdot$ \\
\hline Methylene chloride & 50 & $\cdot$ & 50 & $\cdot$ & FP1 & $\cdot$ & 50 & $\cdot$ & $\mathbf{F P 3}$ & $\cdot$ & FP1 & & $\cdot$ \\
\hline styrene & 50 & $\cdot$ & 5u & $\cdot$ & 50 & $\cdot$ & 50 & $\cdot$ & 50 & $\cdot$ & 50 & & $\cdot$ \\
\hline Tetrachloroethene & 5u & $\cdot$ & 50 & $\cdot$ & 50 & • & 50 & $\cdot$ & 50 & $\cdot$ & 50 & & $\cdot$ \\
\hline Toluene & 5u & $\cdot$ & 50 & $\cdot$ & 50 & $\cdot$ & 50 & $\cdot$ & 50 & $\cdot$ & 50 & & $\cdot$ \\
\hline 1,1,1-Trichloroethane & 50 & $\cdot$ & 5v & $\cdot$ & 50 & $\cdot$ & 50 & $\cdot$ & 50 & $\cdot$ & 50 & & $\cdot$ \\
\hline 1,1,2-Trichloroethane & 50 & $\cdot$ & 50 & $\cdot$ & 50 & $\cdot$ & 50 & $\cdot$ & 50 & $\cdot$ & 50 & & $\cdot$ \\
\hline Trichloroethene & 50 & $\cdot$ & 50 & $\cdot$ & 50 & $\cdot$ & 50 & $\cdot$ & 50 & $\cdot$ & 50 & & $\cdot$ \\
\hline Vinyl acetate & 100 & $\cdot$ & 100 & $\cdot$ & 100 & $\cdot$ & 100 & $\cdot$ & 100 & $\cdot 1$ & 100 & & $\cdot$ \\
\hline Vinyl chloride & 100 & $\cdot$ & 100 & $\cdot$ & 100 & $\cdot$ & 100 & $\cdot$ & 100 & $\cdot$ & 100 & & $\cdot$ \\
\hline Xylenes & 5u & - & 50 & $\cdot$ & 50 & $\cdot$ & 50 & $\cdot$ & 50 & $\cdot$ & 50 & & $\cdot$ \\
\hline Summed vocs & 0 & - & 0 & - & 0 & $\cdot$ & 0 & $\cdot$ & 0 & $\cdot$ & o) & & - \\
\hline
\end{tabular}

(CONTINUED) 
APPESWIIX $J$

Validated voc Results, 1993

\begin{tabular}{|c|c|c|c|c|c|c|c|c|c|c|c|c|}
\hline \multirow{4}{*}{$\begin{array}{l}\text { Sampling Point } \\
\text { Location } \\
\text { Date Sampled }\end{array}$} & \multicolumn{8}{|c|}{ GN-044 } & \multicolumn{4}{|c|}{$G w-056$} \\
\hline & \multicolumn{8}{|c|}{ OLF } & \multicolumn{4}{|c|}{ BG } \\
\hline & \multicolumn{2}{|c|}{$02 / 04 / 93$} & \multicolumn{2}{|c|}{$05 / 10 / 93$} & \multicolumn{2}{|c|}{$09 / 11 / 93$} & \multicolumn{2}{|c|}{$10 / 19 / 93$} & \multicolumn{2}{|c|}{$03 / 04 / 93$} & \multicolumn{2}{|c|}{$04 / 22 / 93$} \\
\hline & $u g / L$ & sum & ug/L & 8 sum & $\operatorname{ug} / \mathrm{x}$ & - sum & $u g / L$ & 8 sum & $u g / x$ & $8 \mathrm{sem}$ & ug/I & s sum \\
\hline Acetone & 100 & • & 100 & . & 100 & . & $\mathbf{F P 1}$ & • & 100 & - & 100 & • \\
\hline Benzene & 50 & • & 50 & $\cdot$ & 50 &. & su & - & 50 & - & 50 & • \\
\hline Bromodichloromethane & 50 & - & 50 & - & 50 & $\cdot$ & so & - & su & - & 50 & • \\
\hline 2-Butanone & 100 & . & 100 & - & 100 & $\cdot$ & $\mathbf{F P 1}$ & - & 100 & - & 100 & • \\
\hline Carbon disulfide & 5v & - & 50 & . & 5v &. & 50 & - & 50 & - & so & • \\
\hline Carbon tetrachloride & 50 & - & 50 & $\cdot$ & 5v & $\cdot$ & 50 & • & 50 & - & 50 & • \\
\hline Chlorobenzene & 50 & - & 50 & - & 50 & . & 50 & - & 50 & - & 50 & • \\
\hline Chloroform & 50 & - & 50 & $\cdot$ & $5 v$ & $\cdot$ & 50 & $\cdot$ & 50 & $\cdot$ & so & • \\
\hline 1,1-Dichloroethane & 5v & - & 50) & - & 50 & $\cdot$ & so) & - & so & - & 50) & • \\
\hline 1,2-Dichloroethane & 50 & $\cdot$ & so & - & so &. & 50 & $\cdot$ & 50 & $\cdot$ & 50 & • \\
\hline 1,1-Dichloroethene & 5v & . & 50 & - & so & $\cdot$ & so & .1 & so & - & 50 & . \\
\hline 1,2-Dichloroethene & 50 & - & 50 & - & 50 & $\cdot$ & 5v & $\cdot$ & 50 & - & 50 & • \\
\hline Ethylbenzene & so & - & 50 & . & 50 & $\cdot$ & so & $\cdot$ & 5v & - & 50 & . \\
\hline 2-Hexanone & 100 & . & 100 & - & 100 & - & 100 & $\cdot$ & 100 & - & 100 & . \\
\hline 4-Methy1-2-pentanone & FP1 & - & 100 & $\cdot$ & 100 & $\cdot$ & 100 & $\cdot$ & 100 & - & 100 & • \\
\hline Methylene chloride & FP1 & - & so & - & so & $\cdot$ & FP1 & $\cdot$ & FP3 & - & 50 & • \\
\hline Styrene & 50 & - & 50 & . & 50 & $\cdot$ & so & $\cdot$ & so! & - & 50 & • \\
\hline Tetrachloroethene & 50 & - & 50 & • & su & $\cdot$ & 5v & $\cdot$ & 50 & - & 5v & . \\
\hline Toluene & 50 & - & sv & - & 50 & $\cdot$ & 50 & $\cdot$ & 50) & - & 50 & . \\
\hline 1,1,1-Trichloroethane & 50 & - & so & $\cdot$ & sv & $\cdot$ & 50 & $\cdot$ & 50 & - & 50) & . \\
\hline 1,1,2-Trichloroethane & 50 & - & 50 & $\cdot$ & 50 & $\cdot$ & 50 & $\cdot$ & 50 & - & 50 & . \\
\hline Trichloroethene & 5v & - & so & - & 5v & $\cdot$ & 50 & $\cdot$ & so & - & 50 & . \\
\hline vinyl acetate & 100 & - & 100 & - & 100 & - & 100 &. & 100 & - & 100 & . \\
\hline Vinyl chloride & 100 & • & 100 & * & 100 & - & 100 &. & 100 & - & 100 & . \\
\hline xylenes & 50 & - & 5v & $\cdot$ & 50 & $\cdot$ & so & $\cdot$ & so & - & 50 & . \\
\hline Summed voC & 0 & - & 0 & - & 0 & - & 0 & - & 0 & - & 0 & . \\
\hline
\end{tabular}

(CONTINUED) 
APPEWDIX $J$

validated voc Results, 1993

\begin{tabular}{|c|c|c|c|c|c|c|c|c|c|c|c|c|}
\hline \multirow{4}{*}{$\begin{array}{l}\text { Sampling Point } \\
\text { Location } \\
\text { Date Sampled }\end{array}$} & \multicolumn{4}{|c|}{ Gw-056 } & \multicolumn{8}{|c|}{$62-057$} \\
\hline & \multicolumn{4}{|c|}{ BG } & \multicolumn{8}{|c|}{$\mathbf{E x P}$} \\
\hline & \multicolumn{2}{|c|}{$07 / 09 / 93$} & \multicolumn{2}{|c|}{$10 / 09 / 93$} & \multicolumn{2}{|c|}{$03 / 04 / 93$} & \multicolumn{2}{|c|}{$04 / 22 / 93$} & \multicolumn{2}{|c|}{$07 / 09 / 93$} & \multicolumn{2}{|c|}{$10 / 09 / 93$} \\
\hline & $\operatorname{ug} / 2$ & s sum & $u g / x$ & 8 sum & $u g / L$ & en & $\operatorname{ug} / 2$ & 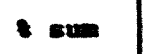 & $\log / 2$ & $2=$ & $\log / 2$ & ex \\
\hline Acetone & 100 & - & 200 & - & 100 & - & 100 & .1 & 100 & - & 100 & . \\
\hline Benzene & sv & - & 50 & - & 50 & - & 50 & $\cdot$ & 50) & - & 50 & 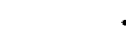 \\
\hline Bromodichloromethane & 5v & - & 5v & - & 50 & - & 5v & $\cdot$ & 5v & - & 50 & r \\
\hline 2-Butanone & 100 & - & 100 & - & 100 & - & 100 & $\cdot$ & 100 & - & 100 & 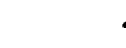 \\
\hline Carbon disulfide & 50 & - & 5v & - & 5v & - & 50 & $\cdot$ & so & $\cdot$ & 50 & 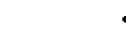 \\
\hline Carbon tetrachloride & 50 & - & 50 & - & 5v & - & 50 & $\cdot$ & 50 & $\cdot$ & 50 & 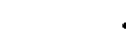 \\
\hline Chlorobenzene & 50 & - & 50 & - & 50 & - & 50 & $\cdot$ & so & $\cdot$ & 50 & 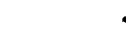 \\
\hline Chloroform & 50 & - & 5v & - & 50 & $\cdot$ & 50 & $\cdot$ & 50 & $\cdot$ & so & 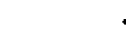 \\
\hline 1,1-Dichloroethane & su & - & 50 & - & 50 & $\cdot$ & 50 & $\cdot$ & so & $\cdot$ & 50 & 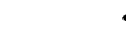 \\
\hline 1,2-Dichloroethane & 5v & - & 50 & $\cdot$ & 50 & • & 50 & $\cdot$ & 50 & $\cdot$ & so & 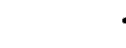 \\
\hline 1,1-Dichloroethene & 50 & $\cdot$ & 50 & $\cdot$ & 50 & $\cdot$ & 50 & $\cdot$ & 50 & $\cdot$ & 50 & 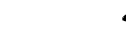 \\
\hline 1,2-Dichloroethene & 50 & - & 50 & $\cdot$ & 50 & - & 50 & $\cdot$ & 50 & $\cdot$ & 50 & 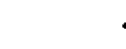 \\
\hline Ethylbenzene & so & - & 50 & - & so & $\cdot$ & so & $\cdot$ & 50 & $\cdot$ & so & 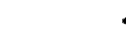 \\
\hline 2-Hexanone & 100 & $\cdot$ & 100 & $\cdot$ & 100 & $\cdot$ & 200 & $\cdot$ & 100 & $\cdot$ & 100 & 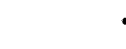 \\
\hline 4-methy 1-2-pentanone & 100 & - & 100 & - & 100 & - & 100 & $\cdot$ & 100 & - & 100 & . \\
\hline nethylene chloride & 5v & $\cdot$ & 50 & - & 5v & $\cdot$ & 50 & $\cdot$ & $\mathbf{F P 1}$ & $\cdot$ & so| & 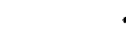 \\
\hline Styrene & $5 v$ & - & so & - & 5v & $\cdot$ & 50 & $\cdot 1$ & 50 & - & so! & . \\
\hline Tetrachloroethene & so & - & 50 & - & 50 & $\cdot$ & so & $\cdot$ & so & $\cdot$ & 50) & rata \\
\hline Toluene & 50 & - & 50 & $\cdot$ & 50 & - & 50 & $\cdot$ & so & $\cdot$ & 50) & 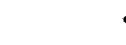 \\
\hline 1,1,1-Trichloroethane & 50 & - & so & - & 50 & - & so & $\cdot$ & so & - & 50 & . \\
\hline 1,1,2-Trichloroetinane & 5v & $\cdot$ & 50 & - & 50 & $\cdot$ & sv & .1 & so & $\cdot$ & 50 & 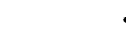 \\
\hline Trichloroethene & 5v & - & so & - & 50 & - & 50 & .1 & so & - & 50 & r \\
\hline Vinyl acetate & 100 & $\cdot$ & 100 & $\cdot$ & 100 & - & 100 & $\cdot$ & 100 & $\cdot$ & 100 & . \\
\hline vinyl chloride & 100 & - & 100 & - & 100 & - & 100 & $\cdot$ & 100 & $\cdot$ & 100 & sina \\
\hline xylenes & 50 & $\cdot$ & 50 & - & 50 & - & 50 & $\cdot$ & so & - & 5v & . \\
\hline Sumed VOCs & 0 & - & 0 & - & -) & $\cdot$ & o) & $\cdot$ & - & - & of & se \\
\hline
\end{tabular}

(COmTrmued) 
ApPSuWIX J

Velldated voc Results, 1993

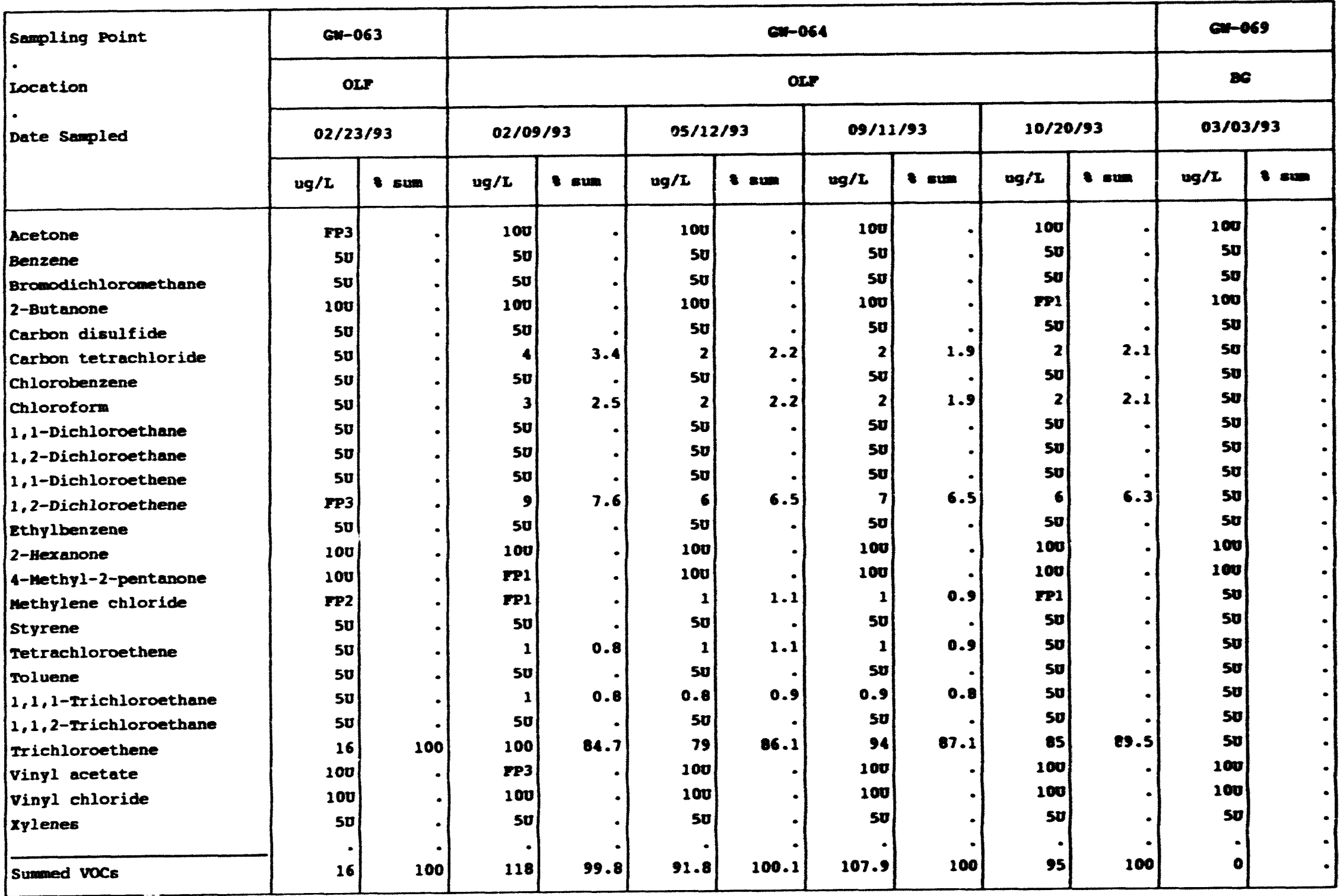

(Corrtrued) 
APREADIX J

Validated voc peaults, 1993

\begin{tabular}{|c|c|c|c|c|c|c|c|c|c|c|c|c|}
\hline \multirow{4}{*}{$\begin{array}{l}\text { Sampling Point } \\
\text { Location } \\
\text { Date Sampled }\end{array}$} & \multicolumn{6}{|c|}{ Qw-069 } & \multicolumn{2}{|c|}{0079} & \multicolumn{4}{|c|}{ Gim-eaz } \\
\hline & \multicolumn{6}{|c|}{$\mathbf{B C}$} & \multicolumn{2}{|c|}{-6} & \multicolumn{4}{|c|}{86} \\
\hline & \multicolumn{2}{|c|}{$04 / 21 / 93$} & \multicolumn{2}{|c|}{$07 / 25 / 93$} & \multicolumn{2}{|c|}{$10 / 10 / 93$} & \multicolumn{2}{|c|}{$10 / 18 / 93$} & \multicolumn{2}{|c|}{$03 / 10 / 93$} & \multicolumn{2}{|c|}{$04 / 21 / 93$} \\
\hline & ug/L & sum & ug/L & sum & $u g / x$ & $8 \mathrm{sen}$ & $u g / L$ & t som & $\operatorname{ug} / 2$ & se & $\log / x$ & - su \\
\hline Acetone & 100 & . & 100 & • & 100 & - & $\mathbf{P P 1}$ & • & 100 & - & 100 & $\cdot$ \\
\hline Benzene & 50 &. & 50) & $\cdot$ & so & - & so & - & 50 & $\cdot$ & 50 & • \\
\hline Bromodichloronethane & so & . & 50 & $\cdot$ & so & - & 50 & - & 50 & - & 50 & • \\
\hline 2-Butinnoie & 100 & $\cdot$ & 100 & $\cdot$ & 100 & - & $\mathbf{P P 1}$ & $\cdot$ & 100 & $\cdot$ & 100 & • \\
\hline Carbon disulfide & so & . & 50 &. & 50 & - & so & . & 50 & - & so & • \\
\hline Carbon tetrachloride & so &. & 50 &. & 50 & - & 50 & . & 50 & $\cdot$ & 50 & • \\
\hline Chlorobenzene & so & . & so &. & so & - & so & - & so & $\cdot$ & so & • \\
\hline Chloroform & 50 & $\cdot$ & so & $\cdot$ & 50 & - & so & $\cdot$ & 50 & - & 50 & $\cdot$ \\
\hline 1,1-Dichloroethane & so &. & 50 &. & 50 & - & so & - & 50 & $\cdot$ & so & • \\
\hline 1,2-Dichloroethane & 50 & $\cdot 1$ & so & $\cdot$ & so) & - & so & - & so & $\cdot$ & so & $\cdot$ \\
\hline 1,1-Dichloroethene & 50 & $\cdot$ & so &. & 50 & - & 50 & $\cdot$ & 50 & $\cdot$ & so & • \\
\hline 1,2-Dichloroethene & so & $\cdot 1$ & so & $\cdot$ & 50) & - & 50 & - & 41 & 57.7 & so & 61.7 \\
\hline Ethylbenzene & so & $\cdot$ & so & $\cdot$ & 50 & - & so & $\cdot$ & so & $\cdot$ & 50 & $\cdot$ \\
\hline 2-Hexanone & 100 & $\cdot$ & 100 & $\cdot$ & 200 & - & 100 & - & 200 & $\cdot$ & 100 & • \\
\hline 4-Hethy1-2-pentanone & 100 &. & $\because 100$ & $\cdot$ & 100 & - & 100 & . & 100 & - & 100 & • \\
\hline Methylene chloride & 0.8 & 100 & 1 & 100 & so & - & $\mathbf{F P 1}$ & $\cdot$ & so & $\cdot$ & so & • \\
\hline Styrene & so & $\cdot 1$ & so & $\cdot$ & so & - & sv & . & so & - & so & • \\
\hline Tetrachloroethene & so & $\cdot$ & so & $\cdot$ & so & - & so & $\cdot$ & 50 & $\cdot$ & 50 & • \\
\hline Toluene & 5v &. & 50 &. & sv) & - & 50 & - & so & - & so & - \\
\hline 1,1,1-Trichloroethane & so & $\cdot 1$ & so &. & so & - & 50 & $\cdot$ & 50 & $\cdot$ & so & • \\
\hline 1,1,2-Irichloroethane & 50 & $\cdot$ & so & $\cdot$ & sv) & - & so & - & 50 & $\cdot$ & so & • \\
\hline Irichloroethene & 5v &. & 50 &. & so & - & so & - & so &. & so & • \\
\hline Vinyl scetate & 100 & $\cdot$ & 200 &. & 100 & • & 100 & $\cdot$ & 100 & $\cdot$ & 100 & - \\
\hline vinyl chloride & FP3 & $\cdot \mid$ & 100 &. & 100 & - & 100 & - & 30 & 42.3 & 31 & 38.3 \\
\hline xylenes & 50 & $\cdot$ & 50 & $\cdot$ & so & - & 50 & - & 50 & $\cdot$ & so & • \\
\hline Summed vocs & 0.8 & 100 & 1 & 100 & 0 & - & i & $\cdot$ & 71 & 200 & e1 & 200 \\
\hline
\end{tabular}

(COmrrmued) 
validated voc Reanlte, 1993

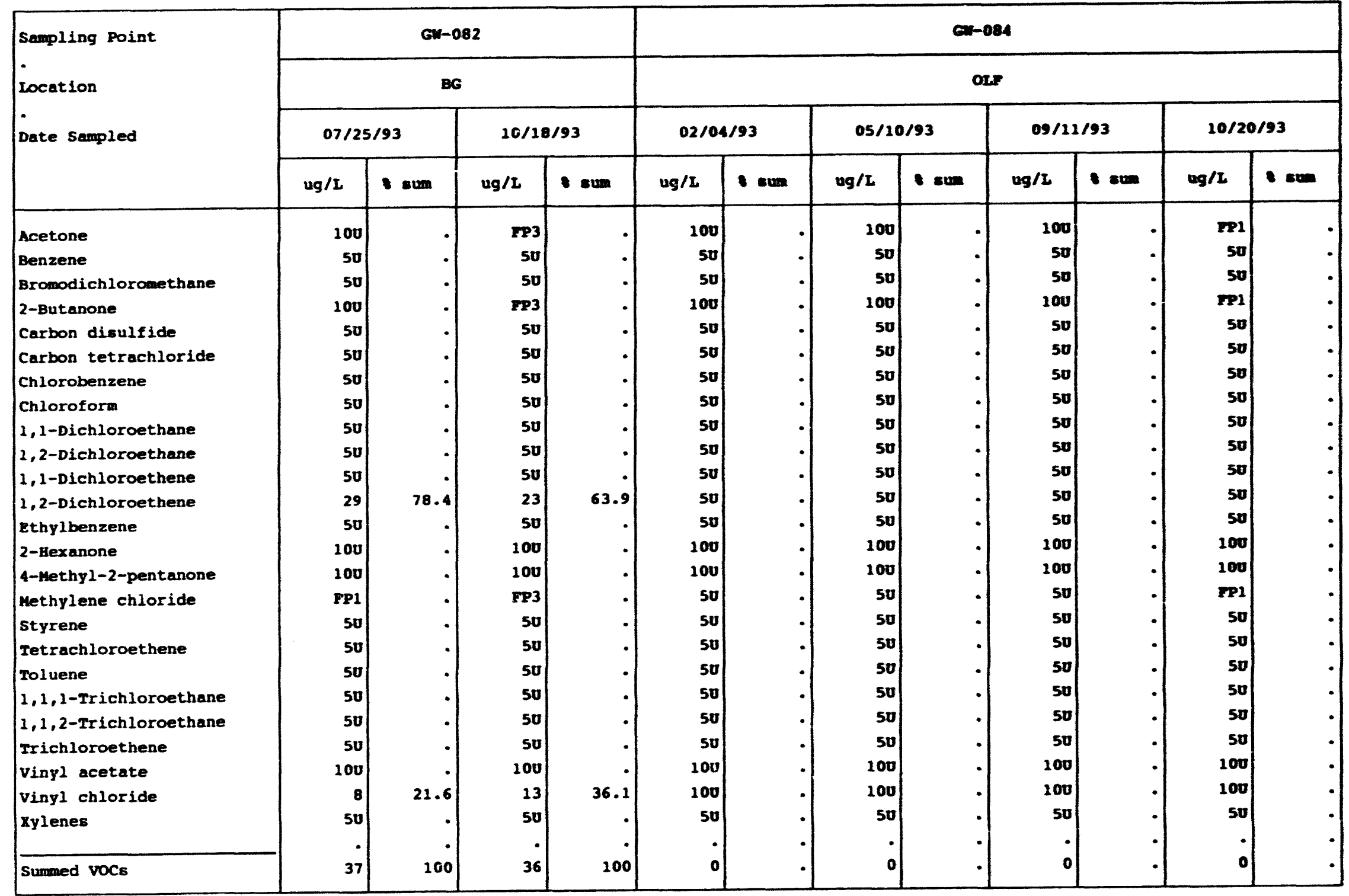

(CONTIMUED) 
APPEADIX $J$

Validated VOC Results, 1993

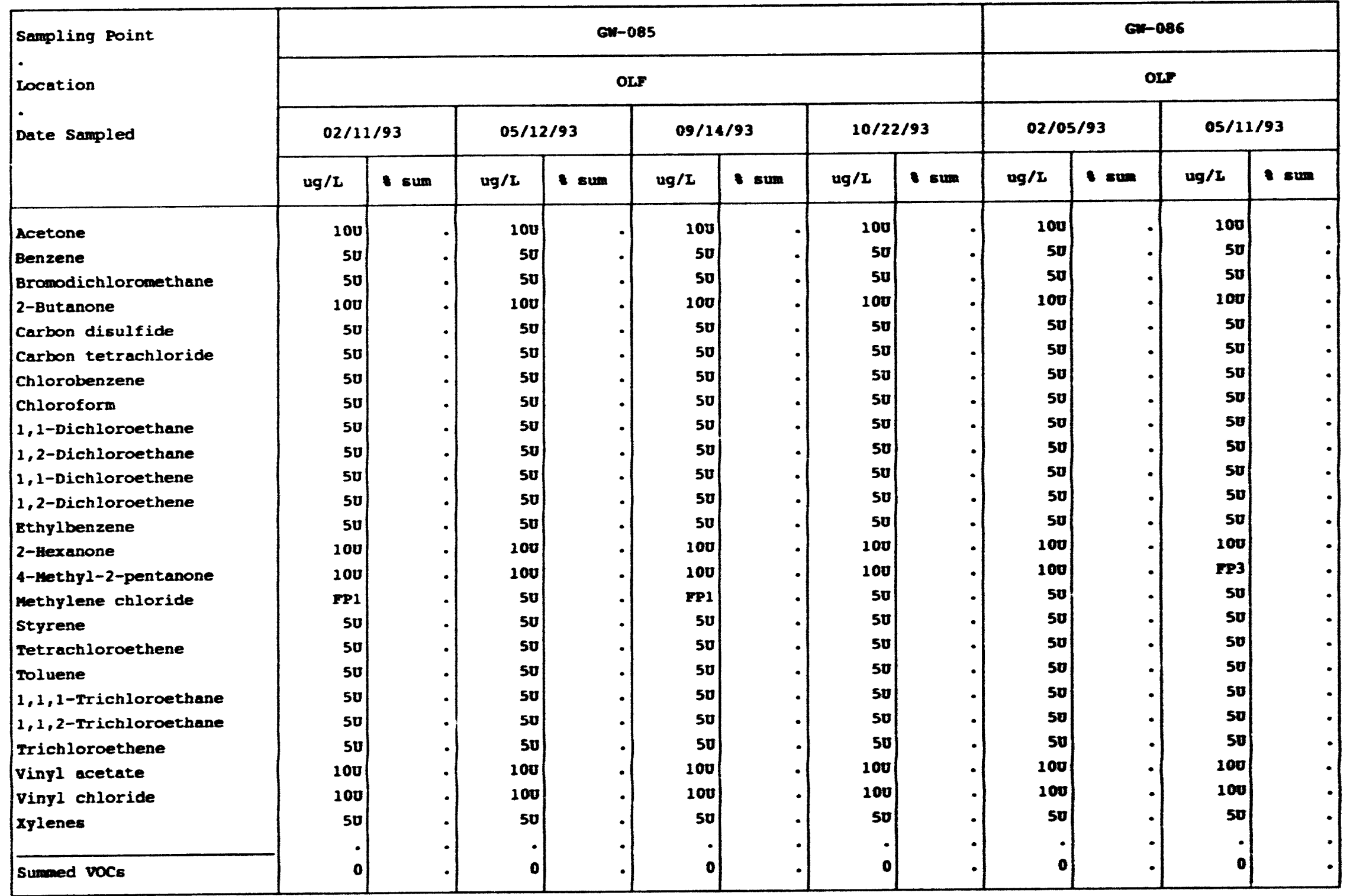

(CONTINUED) 
Validated voc Results, 1993

\begin{tabular}{|c|c|c|c|c|c|c|c|c|c|c|c|c|}
\hline \multirow{4}{*}{$\begin{array}{l}\text { Sampling Point } \\
\text { Location } \\
\text { Date Sampled }\end{array}$} & \multicolumn{4}{|c|}{$G w-086$} & \multicolumn{8}{|c|}{$G W-115$} \\
\hline & \multicolumn{4}{|c|}{ OLF } & \multicolumn{8}{|c|}{ s3 } \\
\hline & \multicolumn{2}{|c|}{$09 / 12 / 93$} & \multicolumn{2}{|c|}{$10 / 21 / 93$} & \multicolumn{2}{|c|}{$01 / 28 / 93$} & \multicolumn{2}{|c|}{$05 / 25 / 93$} & \multicolumn{2}{|c|}{$09 / 11 / 93$} & \multicolumn{2}{|c|}{$10 / 19 / 93$} \\
\hline & ug/L & 8 sum & $\mathrm{ug} / \mathrm{L}$ & 8 sum & $\mathrm{ug} / \mathrm{L}$ & 8 sum & $u g / L$ & 8 sum & $\mathrm{ug} / \mathrm{L}$ & sum & $u g / L$ & sum \\
\hline Acetone & 100 & - & 100 & - & 100 & - & 100 & - & 100 & - & 100 & 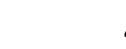 \\
\hline Benzene & 50 & - & 5u & - & 50 & . & 50 & - & 5u & - & $5 \mathbf{v}$ & ra \\
\hline Bromodichloromethane & $5 v$ & - & su & - & 50 & - & 5u & - & 5u & - & 5u & ra \\
\hline 2-Butanone & 100 & • & 100 & - & 100 & . & 100 & - & 100 & - & FP1 & ra \\
\hline Carbon disulfide & 5u & - & 50 & - & 50 & - & 50 & - & 50 & - & 50 & ra \\
\hline Carbon tetrachloride & 50 & - & 50 & - & 50 & - & 5U & - & 5v & - & $5 v$ & ra \\
\hline Chlorobenzene & $5 \mathbf{u}$ & - & 50 & - & 50 & - & 50 & - & 5u & - & 50 & ra \\
\hline Chloroform & 5u & - & 50 & - & $5 \mathrm{v}$ & - & su & - & 50 & - & 50 & ra \\
\hline 1,1-Dichloroethane & 50 & - & 5u & - & 5u & . & 50 & - & 50 & - & 50 & ra \\
\hline 1,2-Dichloroethane & 50 & - & su & - & 5v & - & 50 & - & 5u & . & 50 & ra \\
\hline 1,1-Dichloroethene & $5 u$ & - & 50 & - & 5u & - & 50 & - & 5u & - & 50 & ra \\
\hline 1,2-Dichloroethene & 5u & . & $5 v$ & . & $5 \mathrm{v}$ & - & 50 & - & 50 & - & 50 & ra \\
\hline Ethylbenzene & $5 \mathbf{u}$ & - & 5u & - & 50 & - & 5u & - & 50 & - & 50 & ra \\
\hline 2-Hexanone & 100 & - & 100 & - & 100 & - & 100 & - & 100 & - & 100 & ra \\
\hline 4-Methy1-2-pentanone & 100 & - & 100 & - & FP1 & . & 100 & - & 100 & - & 100 & ra \\
\hline Methylene chloride & 50 & - & 5u & - & 5u & . & 50 & - & FP3 & - & FP1 & ra \\
\hline Styrene & $5 v$ & - & 5u & - & 5v & - & so & - & 5v & - & 50 & ra \\
\hline Tetrachloroethene & 50 & - & 50 & - & 50 & - & 50 & - & 50 & - & 50 & ra \\
\hline Toluene & $5 v$ & - & 50 & - & 50 & - & 50 & - & 50 & - & 50 & ra \\
\hline $1,1,1-\operatorname{Trich}$ loroethane & 50 & - & 5u & - & 5u & - & 50 & - & 50 & - & 50 & ra \\
\hline 1,1,2-Trichloroethane & 50 & - & 50 & - & 50 & - & 50 & - & 50 & - & 50 & ra \\
\hline Trichloroethene & 50 & - & su & - & 5v & $\cdot$ & 50 & - & 50 & - & 50 & ra \\
\hline Vinyl acetate & 100 & . & 100 & - & 100 & . & 100 & - & 100 & - & 100 & ra \\
\hline vinyl chloride & 100 & - & 100 & - & 100 & - & 100 & - & 100 & - & 100 & ra \\
\hline Xylenes & 50 & . & 50 & - & 50 & - & 50 & - & 50 & - & 50 & ra \\
\hline Summed vocs & $\dot{0}$ & • & $\dot{0}$ & • & 0 & $\cdot$ & $\dot{0}$ & $\cdot$ & $\dot{0}$ & $\cdot$ & $\dot{0}$ & 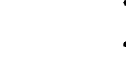 \\
\hline
\end{tabular}

(CONTINUED) 
APPENDIX $J$

Validated VOC Results, 1993

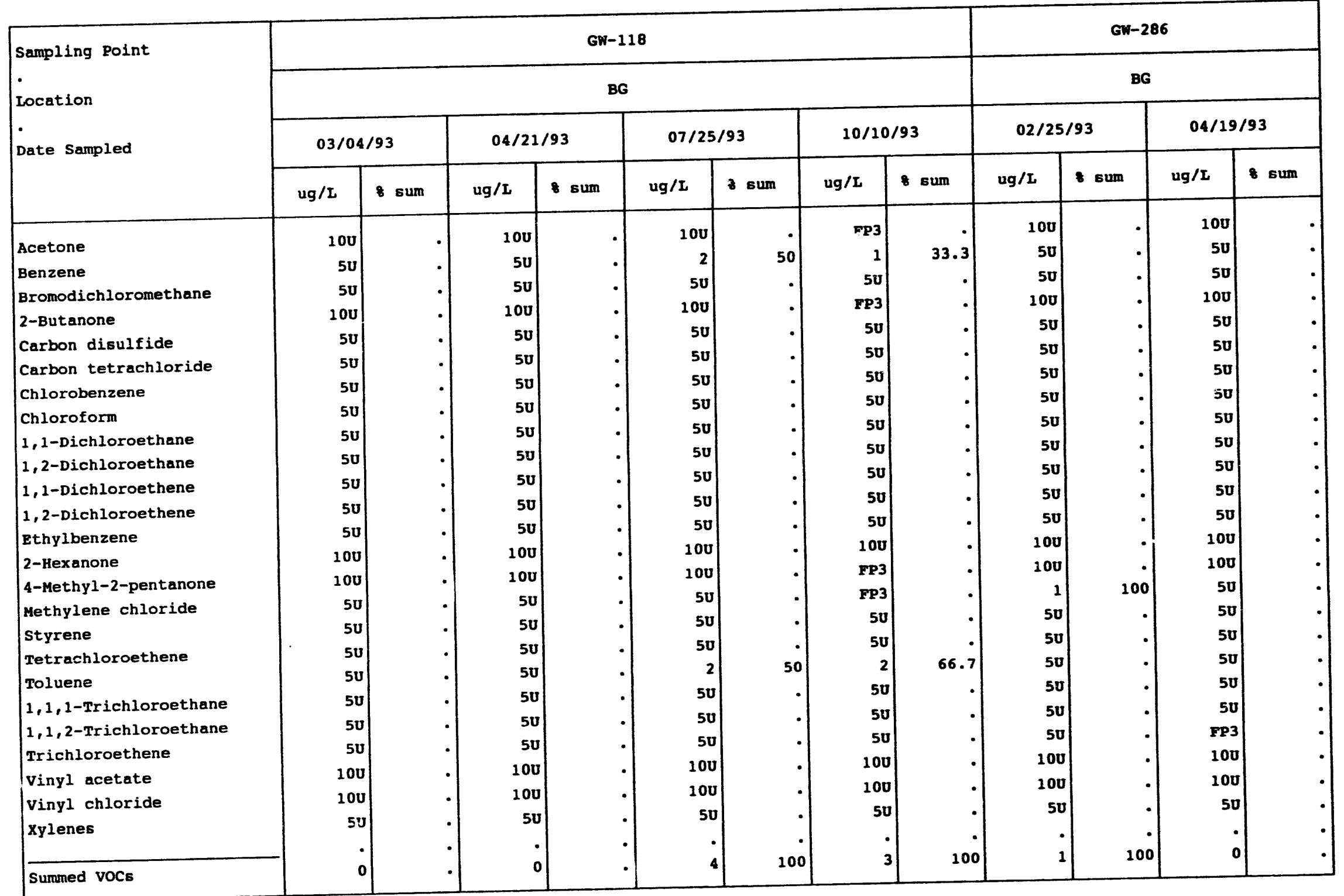

(CONTINUED) 
validated VOC ResultB, 1993

\begin{tabular}{|c|c|c|c|c|c|c|c|c|c|c|c|c|c|}
\hline \multirow{4}{*}{$\begin{array}{l}\text { Sampling Point } \\
\text { Location } \\
\text { Date Sampled }\end{array}$} & \multicolumn{4}{|c|}{$G W-286$} & \multicolumn{9}{|c|}{$G W-287$} \\
\hline & \multicolumn{4}{|c|}{ BG } & \multicolumn{9}{|c|}{ BG } \\
\hline & \multicolumn{2}{|c|}{$07 / 10 / 93$} & \multicolumn{2}{|c|}{$10 / 07 / 93$} & \multicolumn{2}{|c|}{$02 / 24 / 93$} & \multicolumn{2}{|c|}{$04 / 16 / 93$} & \multicolumn{2}{|c|}{$07 / 10 / 93$} & \multicolumn{3}{|c|}{$10 / 07 / 93$} \\
\hline & $u g / L$ & 8 sum & ug/L & sum & ug/L & 8 sum & $\mathbf{u g} / \mathbf{L}$ & 8 sum & ug/x & 8 sum & $\operatorname{ug} / \mathrm{L}$ & sum & \\
\hline Acetone & FP2 & - & FP3 & - & 100 & $\cdot$ & 100 & $\cdot 1$ & 100 & $\bullet$ & FP1 & & $\bullet$ \\
\hline Benzene & so & $\cdot$ & 50 & - & 50 & $\cdot$ & 5u & $\cdot$ & 50 & $\cdot$ & 50 & & $\cdot$ \\
\hline Bromodichloromethane & $5 U$ & $\cdot$ & 50 & • & 50 & $\cdot$ & 50 & $\cdot$ & 50 & $\cdot$ & 50 & & $\cdot$ \\
\hline 2-Butanone & 100 & $\cdot$ & FP3 & $\cdot$ & 100 & $\cdot$ & 100 & $\cdot$ & 100 & $\cdot$ & FP1 & & $\cdot$ \\
\hline Carbon disulfide & 50 & $\cdot$ & 50 & $\cdot$ & 50 & $\cdot$ & 50 & $\cdot$ & 50 & $\cdot$ & 50 & & $\bullet$ \\
\hline Carbon tetrachloride & 50 & $\cdot$ & 50 & $\cdot$ & 50 & $\cdot$ & $\begin{array}{l}50 \\
50\end{array}$ & 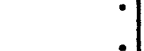 & 50 &. & 50 & &. \\
\hline Chlorobenzene & 50 & $\cdot$ & $\begin{array}{l}50 \\
50\end{array}$ &. & 50 &. & 50 &. & $5 v$ & $\cdot$ & 50 & & . \\
\hline $\begin{array}{l}\text { Chloroform } \\
\text { 1,1-Dichloroethane }\end{array}$ & 50 & $\cdot$ & $\begin{array}{l}50 \\
50\end{array}$ & $\cdot$ & 5u & $\cdot$ & 50 & $\cdot 1$ & $5 v$ & $\cdot$ & 50 & & $\cdot$ \\
\hline $\begin{array}{l}\text { 1,1-Dichloroethane } \\
\text { 1,2-Dichloroethane }\end{array}$ & $5 \mathrm{U}$ &. & 5v &. & 50 & $\cdot$ & 50 & $\cdot$ & 50 & $\cdot 1$ & 50 & & - \\
\hline $\begin{array}{l}\text { 1,2-Dichloroethane } \\
\text { 1,1-Dichloroethene }\end{array}$ & 5u & $\cdot$ & 50 & $\cdot$ & 50 & $\cdot$ & 50 & $\cdot$ & 50 & $\cdot$ & 50 & & - \\
\hline 1,2-Dichloroethene & 50 & $\cdot$ & 5u & $\cdot$ & 50 & $\cdot$ & 5u & $\cdot$ & 50 & $\cdot$ & 50 & & $\bullet^{\circ}$ \\
\hline Ethylbenzene & $5 u$ & $\cdot$ & 50 & $\cdot$ & 50 & $\cdot$ & so & $\cdot$ & 5u & $\cdot$ & 50 & & • \\
\hline 2-Hexanone & 100 & $\cdot$ & 100 & $\cdot$ & 100 & $\cdot$ & 100 & $\cdot$ & 100 & $\cdot$ & 100 & & - \\
\hline 4-Methyl-2-pentanone & 100 & $\cdot$ & 100 &. & 100 & $\cdot$ & 100 & $\cdot$ & $\begin{array}{r}100 \\
50\end{array}$ & $\cdot$ & $\begin{array}{l}100 \\
\text { FP1 }\end{array}$ & & : \\
\hline Methylene chloride & FP1 & $\cdot$ & $\begin{array}{r}3 \\
50\end{array}$ & 100 & $\begin{array}{r}\text { FP3 } \\
5 U\end{array}$ &. & 50 & $\cdot$ & 50 &. & 50 & & - \\
\hline Styrene & $\begin{array}{l}50 \\
5 v\end{array}$ & $\cdot$ & $\begin{array}{l}50 \\
50\end{array}$ & $\cdot$ & 5u &. & FP3 & $\cdot$ & 50 & $\cdot 1$ & 50 & & • \\
\hline $\begin{array}{l}\text { Tetrachloroethene } \\
\text { Toluene }\end{array}$ & 50 &. & 5u & $\cdot$ & 50 & $\cdot$ & 50 & $\cdot$ & 50 & $\cdot 1$ & so & & $\bullet$ \\
\hline & 50 & $\cdot$ & 5o & $\cdot$ & 50 & $\cdot$ & 50 & $\cdot$ & 50 & $\cdot$ & 50 & & $\bullet$ \\
\hline 1,1,2-Trichloroethane & 50 & $\cdot$ & 5u & $\cdot$ & 50 & $\cdot$ & 50 & $\cdot$ & 50 & $\cdot$ & 50 & & • \\
\hline Trichloroethene & $5 U$ & $\cdot$ & 50 & $\cdot$ & 50 & $\cdot$ & 5u & $\cdot$ & 50 & $\cdot 1$ & 50 & & $\bullet$ \\
\hline Vinyl acetate & 100 & $\cdot$ & 100 & $\cdot$ & 100 & $\cdot$ & 100 & $\cdot$ & 100 & $\cdot$ & 100 & & $\cdot$ \\
\hline Vinyl chloride & 100 & $\cdot$ & 100 & $\cdot$ & 100 & $\cdot$ & 100 & $\cdot$ & 100 & $\cdot$ & $\begin{array}{r}100 \\
5 \pi\end{array}$ & & - \\
\hline Xylenes & $\begin{array}{r}50 \\
.\end{array}$ &. & 50 & $\cdot$ &. &. & 50 & $\cdot$ &. & $\cdot$ &. & & • \\
\hline Summed VOCs & 0 &. & 3 & 100 & 0 & $\cdot$ & 0 & $\cdot$ & 0 & $\cdot 1$ & 0 & & • \\
\hline
\end{tabular}

(CONTINUED) 
APPENDIX J

Validated VOC Results, 1993

\begin{tabular}{|c|c|c|c|c|c|c|c|c|c|c|c|c|}
\hline \multirow{4}{*}{$\begin{array}{l}\text { Sampling Point } \\
\text { Location } \\
\text { Date Sampled }\end{array}$} & \multicolumn{8}{|c|}{$G W-311$} & \multicolumn{4}{|c|}{$G N-312$} \\
\hline & \multicolumn{8}{|c|}{ RS } & \multicolumn{4}{|c|}{ RS } \\
\hline & \multicolumn{2}{|c|}{$02 / 08 / 93$} & \multicolumn{2}{|c|}{$05 / 11 / 93$} & \multicolumn{2}{|c|}{$09 / 12 / 93$} & \multicolumn{2}{|c|}{$10 / 21 / 93$} & \multicolumn{2}{|c|}{$02 / 08 / 93$} & \multicolumn{2}{|c|}{$05 / 11 / 93$} \\
\hline & $\mathrm{ug} / \mathrm{x}$ & 8 sum & ug/L & 8 sum & ug/L & 8 sum & ug/t & 8 sum & $\mathrm{ug} / \mathrm{L}$ & 8 sum & $u g / x$ & 8 sum \\
\hline Acetone & 100 & - & 100 & $\cdot$ & 100 & $\cdot$ & 100 & $\cdot$ & 100 & $\cdot$ & 100 & - \\
\hline Benzene & 50 & $\cdot$ & 50 & $\cdot$ & 50 & $\cdot$ & 50 & $\cdot$ & 50 & $\cdot$ & 50 & • \\
\hline Bromodichloromethane & 50 & • & 50 & $\cdot$ & 50 & $\cdot$ & $5 \mathbf{u}$ & $\cdot$ & 50 & $\cdot$ & 5u & • \\
\hline 2-Butanone & 100 & $\cdot$ & 100 & $\cdot$ & 100 & $\cdot$ & 100 & $\cdot$ & 100 & $\cdot$ & 100 & • \\
\hline Carbon disulfide & 50 & - & 50 & $\cdot$ & 50 & $\cdot$ & 50 & $\cdot$ & 50 & $\cdot 1$ & 50 & • \\
\hline Carbon tetrachloride & 0.9 & 6 & 2 & 8.7 & 1 & 6 & 3 & 13 & 5v & $\cdot 1$ & 50 & • \\
\hline Chlorobenzene & 50 & $\cdot$ & 50 & $\cdot$ & 50 & $\cdot$ & 50 & $\cdot$ & 5v & $\cdot$ & 5u & • \\
\hline Chloroform & 1 & 6.7 & $5 \mathbf{v}$ & $\cdot$ & 0.8 & 4.8 & 1 & 4.3 & 1 & 1.6 & 1 & 1.2 \\
\hline 1,1-Dichloroethane & 50 & $\cdot$ & 50 & $\cdot$ & 50 & $\cdot$ & 50 & $\cdot$ & 50 & $\cdot$ & 50 & • \\
\hline 1,2-Dichloroethane & 50 & • & 5v & $\cdot$ & 50 & $\cdot$ & 50 & $\cdot$ & 50 & $\cdot$ & 50 & • \\
\hline 1,1-Dichloroethene & $5 \mathrm{U}$ & . & 50 & $\cdot$ & 50 & $\cdot$ & 50 & $\cdot$ & 50 & $\cdot$ & $5 v$ & • \\
\hline 1,2-Dichloroethene & $5 \mathbf{v}$ & $\cdot$ & 5u & $\cdot$ & 50 & $\cdot$ & 5u & $\cdot$ & 50 & $\cdot$ & 50 & • \\
\hline Ethylbenzene & 50 & - & 50 & $\cdot$ & 50 & $\cdot$ & 50 & $\cdot$ & 50 & $\cdot$ & 50 & • \\
\hline 2-Hexanone & 100 & $\cdot$ & 100 & $\cdot$ & 100 & $\cdot$ & 100 & $\cdot$ & 100 & $\cdot$ & 100 & • \\
\hline 4-Methy1-2-pentanone & 100 & $\cdot$ & FP1 & $\cdot$ & 100 & $\cdot$ & 100 & $\cdot$ & 100 & $\cdot$ & 100 & • \\
\hline Methylene chloride & $5 \mathrm{U}$ & . & 50 & $\cdot$ & FP1 & $\cdot$ & 50 & $\cdot$ & 5u & $\cdot$ & 50 & - \\
\hline Styrene & 50 & $\cdot$ & 50 & $\cdot$ & 50 & $\cdot$ & 50 & $\cdot$ & 50 & $\cdot$ & 50 & • \\
\hline Tetrachloroethene & 5v & - & 50 & $\cdot$ & 50 & $\cdot$ & $5 v$ & $\cdot 1$ & 50 & $\cdot$ & 50 & • \\
\hline Toluene & 50 & $\cdot$ & $5 v$ &. & $5 \mathbf{u}$ & $\cdot$ & 50 & $\cdot$ & 50 & $\cdot$ & 50 & • \\
\hline 1,1,1-Trichloroethane & 50 & $\cdot$ & $5 v$ & $\cdot$ & 5u & $\cdot$ & 50 & $\cdot$ & 50 & $\cdot$ & 50 & • \\
\hline 1,1,2-Trichloroethane & $5 v$ & - & 50 & $\cdot$ & 50 & $\cdot$ & 50 & $\cdot$ & 50 & $\cdot$ & 50 & • \\
\hline Trichloroethene & 23 & 87.2 & 21 & 91.3 & 15 & 89.3 & 19 & 82.6 & 62 & 98.4 & 83 & 98.8 \\
\hline Vinyl acetate & 100 & - & 100 & $\cdot$ & 100 & $\cdot$ & 100 & $\cdot$ & 100 & $\cdot$ & 100 & • \\
\hline Vinyl chloride & 100 & $\cdot 1$ & 100 & $\cdot$ & 100 & $\cdot$ & 100 & $\cdot$ & 100 & $\cdot$ & 100 & • \\
\hline Xylenes & 5u & $\cdot$ & 50 &. & 50 & $\cdot$ & 50 & $\cdot$ & $5 u$ & $\cdot$ & 50 & • \\
\hline Summed VOCs & 14.9 & 99.9 & 23 & 100 & 16.8 & 100.1 & 23 & 99.9 & 63 & 100 & 84 & 100 \\
\hline
\end{tabular}

(CONTINUED) 
Validated VOC Results, 1993

\begin{tabular}{|c|c|c|c|c|c|c|c|c|c|c|c|c|}
\hline \multirow{4}{*}{$\begin{array}{l}\text { Sampling Point } \\
\text { Location } \\
\text { Date Sampled }\end{array}$} & \multicolumn{4}{|c|}{ GW-312 } & \multicolumn{8}{|c|}{ GW-315 } \\
\hline & \multicolumn{4}{|c|}{ RS } & \multicolumn{8}{|c|}{ SPI } \\
\hline & \multicolumn{2}{|c|}{$09 / 12 / 93$} & \multicolumn{2}{|c|}{$10 / 21 / 93$} & \multicolumn{2}{|c|}{$02 / 03 / 93$} & \multicolumn{2}{|c|}{$06 / 03 / 93$} & \multicolumn{2}{|c|}{$09 / 14 / 93$} & \multicolumn{2}{|c|}{$10 / 27 / 93$} \\
\hline & $u g / L$ & 8 sum & $\mathrm{ug} / \mathrm{I}$ & 8 sum & $\mathrm{ug} / \mathrm{L}$ & 8 sum & $\operatorname{ug} / \mathrm{L}$ & 8 sum & ug/L & 8 sum & ug $/ 2$ & 8 sum \\
\hline Acetone & 100 & $\cdot 1$ & FP3 & $\cdot 1$ & FP2 & - & 100 & $\cdot$ & 100 & $\cdot 1$ & FP1 & - \\
\hline Benzene & 50 & $\cdot$ & $5 U$ & $\cdot$ & 50 & $\cdot$ & su & $\cdot$ & 5v & $\cdot$ & 50 & $\cdot$ \\
\hline Bromodichloromethane & 50 & $\cdot$ & 50 & $\cdot$ & 2 & 7.7 & 2 & 10 & 2 & 6.3 & 1 & 4.3 \\
\hline 2-Butanone & 100 & $\cdot$ & FP3 & $\cdot$ & 100 & $\cdot$ & 100 & $\cdot$ & 100 & $\cdot$ & FP1 & $\cdot$ \\
\hline Carbon disulfide & 50 & $\cdot$ & 50 & $\cdot$ & 50 & $\cdot$ & 50 & $\cdot$ & 50 & $\cdot$ & 50 & $\cdot$ \\
\hline Carbon tetrachloride & $5 \mathrm{u}$ & $\cdot$ & 50 & $\cdot$ & 5u & $\cdot$ & 50 & $\cdot$ & 50 & $\cdot$ & 50 & $\cdot$ \\
\hline Chlorobenzene & 50 & $\cdot$ & 50 & $\cdot$ & 5u & $\cdot$ & 5u & $\cdot$ & 50 & $\cdot$ & 50 & $\dot{.}$ \\
\hline Chloroform & su & $\cdot$ & 50 & $\cdot$ & 7 & 26.9 & 9 & 45 & 8 & 25 & 6 & 26.1 \\
\hline 1,1-Dichloroethane & 50 & $\cdot$ & 5U & $\cdot$ & 50 & $\cdot 1$ & 50 & $\cdot$ & 50 & $\cdot$ & 50 & $\cdot$ \\
\hline 1,2-Dichloroethane & 50 & $\cdot$ & 5u & $\cdot$ & su & $\cdot$ & 50 & $\cdot$ & 50 & $\cdot$ & 50 & $\cdot$ \\
\hline 1,1-Dichloroethene & 50 & $\cdot$ & 50 & $\cdot$ & 50 & $\cdot$ & 50 & $\cdot$ & 5u & $\cdot$ & 50 & $\cdot$ \\
\hline 1,2-Dichloroethene & 50 & $\cdot$ & 50 & $\cdot$ & 5 & 19.2 & 2 & 10 & 7 & 21.9 & 5 & 21.7 \\
\hline Ethylbenzene & 50 & $\cdot$ & 50 & $\cdot 1$ & 5u & $\cdot$ & 5u & $\cdot$ & 50 & $\cdot$ & 5u & $\cdot$ \\
\hline 2-Hexanone & 100 & $\cdot$ & 100 & $\cdot$ & 100 & $\cdot$ & 100 & $\cdot$ & 100 & $\cdot$ & 100 & $\bullet$ \\
\hline 4-Methy1-2-pentanone & 100 & $\cdot$ & 100 & $\cdot$ & FP1 & $\cdot$ & 100 & $\cdot$ & 100 & $\cdot$ & 100 & $\cdot$ \\
\hline Methylene chloride & 50 & $\cdot$ & FP3 & $\cdot$ & FP1 & $\cdot$ & FP1 & $\cdot$ & 5u & $\cdot$ & FP1 & $\cdot$ \\
\hline styrene & 50 & $\cdot$ & 50 & $\cdot$ & su & $\cdot$ & 50 & $\cdot$ & 5u & $\bullet$ & 50 & $\dot{0}$ \\
\hline Tetrachloroethene & 50 & $\cdot$ & 50 & $\cdot$ & 8 & 30.8 & 5 & 25 & 10 & 31.3 & 7 & 30.4 \\
\hline Toluene & 50 & $\cdot$ & 50 & $\cdot$ & 5u & $\cdot$ & 50 & $\cdot$ & 50 & $\cdot$ & 50 & - \\
\hline 1,1,1-Trichloroethane & 50 & $\cdot$ & 50 & $\cdot$ & 5u & $\cdot 1$ & 50 & $\cdot$ & 50 & $\cdot$ & 5u & $\bullet$ \\
\hline 1,1,2-Trichloroethane & 50 & $\cdot$ & 50 & $\cdot$ & 5u & $\cdot$ & 5u & $\cdot$ & 5u & $\because$ & 50 & $\bullet$ \\
\hline Trichloroethene & 10 & 100 & 49 & 100 & 4 & 15.4 & 2 & 10 & 5 & 15.6 & 4 & 17.4 \\
\hline Vinyl acetate & 100 & $\cdot$ & 100 & $\cdot$ & 100 & $\cdot$ & 100 & $\cdot$ & 100 & $\cdot$ & 100 & - \\
\hline vinyl chloride & 100 & $\cdot$ & 100 & $\cdot$ & 100 & $\cdot$ & 100 & $\cdot 1$ & 100 & - & 100 & - \\
\hline xylenes & 50 & $\cdot$ & 5u & $\cdot$ & 50 & $\cdot$ & 50 & $\cdot$ & 5u & $\cdot$ & 50 & - \\
\hline Summed & 10 & 100 & 49 & 100 & 26 & 100 & 20 & 100 & 32 & 100.1 & 23 & 99.9 \\
\hline
\end{tabular}

(CONTINUED) 
APPENDIX $J$

Validated VOC Results, 1993

\begin{tabular}{|c|c|c|c|c|c|c|c|c|c|c|c|c|c|}
\hline \multirow{4}{*}{$\begin{array}{l}\text { Sampling Point } \\
\text { Location } \\
\text { Date Sampled }\end{array}$} & \multicolumn{8}{|c|}{ GW-316 } & \multicolumn{5}{|c|}{ GW-317 } \\
\hline & \multicolumn{8}{|c|}{ SPI } & \multicolumn{5}{|c|}{ SPI } \\
\hline & \multicolumn{2}{|c|}{$02 / 02 / 93$} & \multicolumn{2}{|c|}{$06 / 01 / 93$} & \multicolumn{2}{|c|}{$09 / 13 / 93$} & \multicolumn{2}{|c|}{$10 / 24 / 93$} & \multicolumn{2}{|c|}{$02 / 02 / 93$} & \multicolumn{3}{|c|}{$05 / 28 / 93$} \\
\hline & $\mathrm{ug} / \mathrm{L}$ & 8 sum & ug/L & 8 sum & $u g / L$ & 8 sum & ug/L & 8 sum & $u g / L$ & 8 sum & $u g / L$ & 8 sum & \\
\hline Acetone & FP3 & $\cdot$ & 100 & - & 100 & $\cdot$ & 100 & - & FP1 & $\cdot$ & 100 & & - \\
\hline Benzene & 50 & $\cdot$ & 50 & $\cdot$ & 50 & $\cdot$ & 5u & $\cdot$ & 50 & $\cdot$ & 50 & & $\bullet$ \\
\hline Bromodichloromethane & 50 & $\cdot$ & 50 & $\cdot$ & 50 & $\cdot$ & 50 & $\cdot$ & 50 & $\cdot$ & so & & - \\
\hline 2-Butanone & FP3 & $\cdot$ & 100 & $\cdot$ & 100 & $\cdot$ & 100 & $\cdot$ & FP1 & $\cdot$ & FP3 & & - \\
\hline Carbon disulfide & 50 & $\cdot$ & 50 & $\cdot$ & $\begin{array}{l}5 v \\
5 v\end{array}$ & $\cdot$ & $\begin{array}{l}50 \\
50\end{array}$ & $\cdot$ & $\begin{array}{l}50 \\
50\end{array}$ & $\cdot$ & $\begin{array}{l}50 \\
50\end{array}$ & & • \\
\hline Carbon tetrachloride & $\begin{array}{l}50 \\
50\end{array}$ & $\cdot$ & $\begin{array}{l}50 \\
50\end{array}$ & $\cdot$ & 50 & $\cdot$ & 50 &. & 50 &. & 50 & & . \\
\hline $\begin{array}{l}\text { Chlorobenzene } \\
\text { Chloroform }\end{array}$ & so &. & 50 &. & 50 &. & 50 & $\cdot$ & 50 & $\cdot$ & 50 & & - \\
\hline 1,1-Dichloroethane & 5v & $\cdot$ & 50 & $\cdot$ & 50 & $\cdot$ & 50 & $\cdot$ & 50 & $\cdot$ & 5v & & - \\
\hline 1,2-Dichloroethane & 50 & $\cdot$ & 50 & $\cdot$ & 50 & $\cdot$ & 5v & $\cdot$ & 50 & $\cdot$ & 5v & & $\cdot$ \\
\hline 1,1-Dichloroethene & 50 & $\cdot$ & 5u & $\cdot$ & 50 & $\cdot$ & $5 \mathbf{u}$ & $\cdot$ & 50 & $\cdot$ & 50 & & $\cdot$ \\
\hline 1,2-Dichloroethene & 50 & $\cdot$ & 50 & $\cdot$ & 50 & $\cdot$ & $5 \mathbf{5}$ & $\cdot$ & 50 & $\cdot$ & 50 & & $\cdot$ \\
\hline Ethylbenzene & 50 & $\cdot$ & 50 & $\cdot$ & 50 & $\cdot$ & 5u & $\cdot$ & 5u & $\cdot$ & 50 & & $\cdot$ \\
\hline 2-Hexanone & 100 & $\cdot$ & 100 & $\cdot 1$ & 100 & $\cdot$ & 100 & $\cdot$ & 100 & $\cdot 1$ & 100 & & $\cdot$ \\
\hline 4-Methyl-2-pentanone & FP3 & $\cdot$ & 100 & $\cdot$ & 100 & $\cdot$ & 100 & $\cdot$ & FP1 & $\bullet$ & 100 & & $\cdot 1$ \\
\hline Methylene chloride & 50 & $\cdot$ & 50 & $\cdot$ & 50 & $\cdot$ & 50 & $\cdot$ & $\begin{array}{l}50 \\
5 u\end{array}$ & $\cdot$ & $\begin{array}{r}\text { FP2 } \\
50\end{array}$ & &. \\
\hline Styrene & 50 & $\cdot$ & $\begin{array}{l}50 \\
50\end{array}$ & $\cdot$ & $\begin{array}{l}50 \\
50\end{array}$ & $\cdot$ & $\begin{array}{l}50 \\
50\end{array}$ & $\dot{.}$ & 50 & $\cdot$ & 50 & &. \\
\hline $\begin{array}{l}\text { Tetrachloroethene } \\
\text { Toluene }\end{array}$ & $\begin{array}{l}50 \\
5 v\end{array}$ & $\cdot$ & 50 & $\cdot$ & 50 & $\cdot$ & 50 &. & 50 &. & 50 & &. \\
\hline & 50 &. & 50 &. & 50 &. & 50 & $\cdot$ & 5u & $\cdot$ & so & &. \\
\hline $\begin{array}{l}\text { 1,1,1-Trichloroethane } \\
1,1,2 \text {-Trichloroethane }\end{array}$ & 50 & $\cdot$ & su & . & 5u & . & 5u & $\cdot$ & 50 & $\cdot$ & 50 & & $\cdot$ \\
\hline Trichloroethene & 50 & $\cdot$ & 50 & $\cdot$ & su & $\cdot$ & 50 & $\cdot$ & 50 & $\cdot$ & 50 & & $\cdot$ \\
\hline Vinyl acetate & 100 & $\cdot 1$ & 100 & $\cdot$ & 100 & $\cdot$ & 100 & $\cdot$ & 100 & $\cdot 1$ & 100 & & - \\
\hline Vinyl chloride & 100 & $\cdot$ & 100 &. & 100 & $\cdot$ & 100 & $\cdot$ & 100 & $\cdot$ & 100 & & - \\
\hline xylenes & 50 & $\cdot$ & 50 & $\cdot$ & 5u & $\cdot$ & 50 & $\cdot$ & 50 & $\cdot$ & 50 & & $\bullet$ \\
\hline Summed VOCs & 0 & $\cdot$ & 0 & $\cdot$ & 0 & $\cdot$ & 0 & $\cdot$ & 0 & $\cdot$ & 0 & & - \\
\hline
\end{tabular}

(CONTINUED) 
Validated vOC Results, 1993

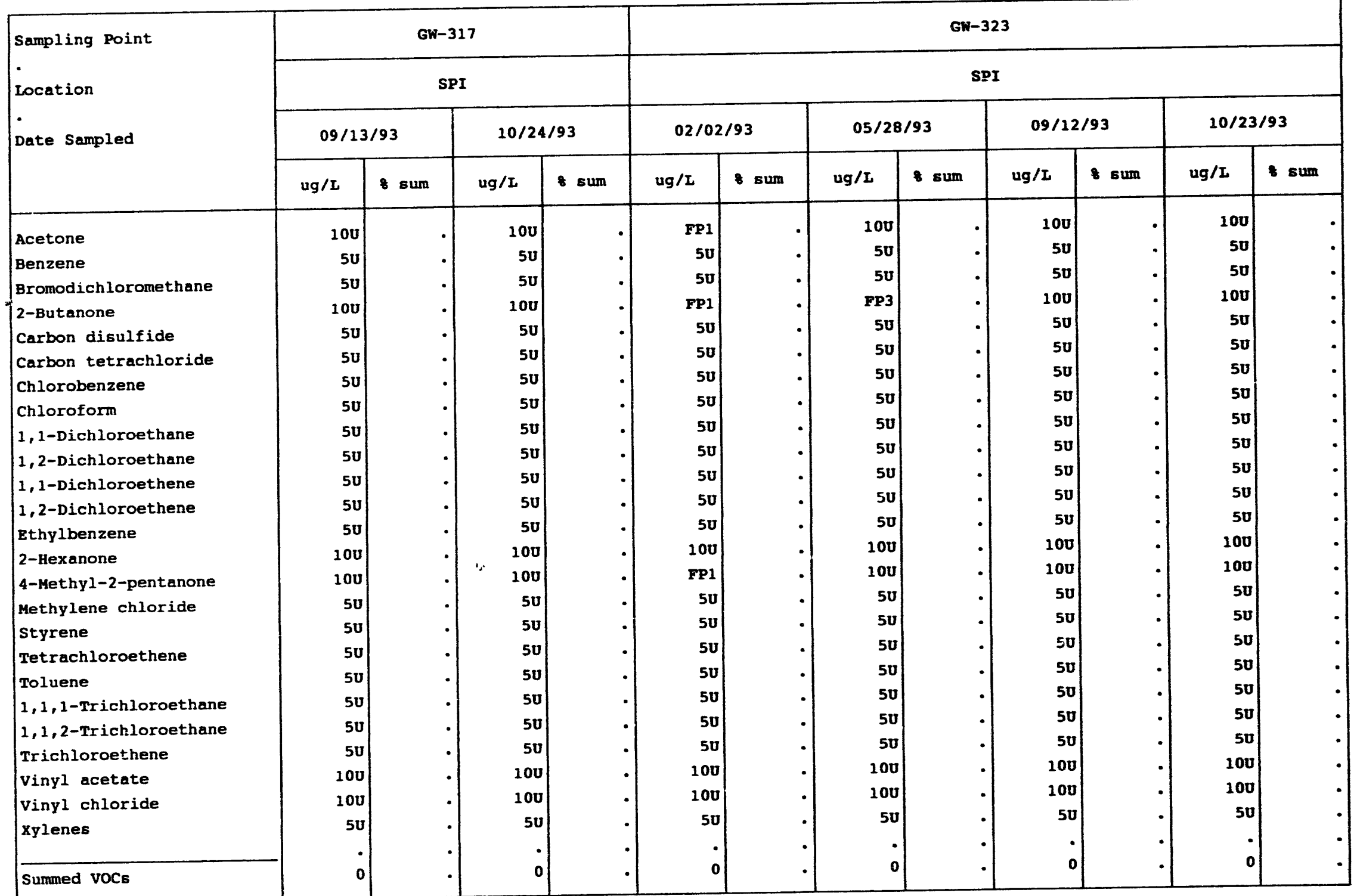

(CONTINUED) 
APPBNDIX $J$

Validated vOC Results, 1993

\begin{tabular}{|c|c|c|c|c|c|c|c|c|c|c|c|c|}
\hline \multirow{4}{*}{$\begin{array}{l}\text { Sampling Point } \\
\text { Location } \\
\text { Date Sampled }\end{array}$} & \multicolumn{8}{|c|}{$G W-324$} & \multicolumn{4}{|c|}{ GW-325 } \\
\hline & \multicolumn{8}{|c|}{ s3 } & \multicolumn{4}{|c|}{ s3 } \\
\hline & \multicolumn{2}{|c|}{$01 / 29 / 93$} & \multicolumn{2}{|c|}{$05 / 25 / 93$} & \multicolumn{2}{|c|}{$09 / 11 / 93$} & \multicolumn{2}{|c|}{$10 / 19 / 93$} & \multicolumn{2}{|c|}{$02 / 01 / 93$} & \multicolumn{2}{|c|}{$05 / 25 / 93$} \\
\hline & $\mathrm{ug} / \mathrm{L}$ & 8 sum & $\mathrm{ug} / \mathrm{L}$ & 8 sum & $\mathrm{ug} / \mathrm{L}$ & \& sum & $\mathrm{ug} / \mathrm{L}$ & 8 sum & ug/L & 8 sum & ugit & s sum \\
\hline Acetone & 100 & - & 100 & - & 100 & - & FP1 & - & 100 &. & 100 & \\
\hline Benzene & 5u & - & 50 & . & 5u & - & $5 v$ & - & 50 & $\cdot$ & 5v & . \\
\hline Bromodichloromethane & 50 & - & 50 & • & 50 & - & 50 & - & 50 & $\cdot$ & 50 & \\
\hline 2-Butanone & 100 & - & 100 & - & 100 & - & FP1 & - & 100 & $\cdot$ & 100 & . \\
\hline Carbon disulfide & 50 & - & 50 & - & 50 & - & 50 & - & 50 & $\cdot$ & 50 & . \\
\hline Carbon tetrachloride & 5u & - & 50 & • & 50 & - & 50 & - & 50 & $\cdot$ & 50 & . \\
\hline Chlorobenzene & 50 & - & 50 & . & 50 & - & 50 & - & 50 & $\cdot 1$ & 50 & . \\
\hline Chloroform & so & - & 5u & $\cdot$ & 50 & - & 5u & - & 5u & $\cdot$ & 50 & 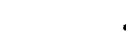 \\
\hline 1,1-Dichloroethane & 50 & - & 50 & $\cdot$ & 50 & - & 50 & - & 50 & $\cdot$ & 5v & . \\
\hline 1,2-Dichloroethane & 50 & - & 50 & $\cdot$ & 50 & - & 50 & • & 50 & $\cdot$ & 50 & . \\
\hline 1,1-Dichloroethene & 50 & - & 50 & - & 50 & - & 50 & - & 50 & $\cdot$ & 50 & . \\
\hline 1,2-Dichloroethene & 5u & - & 50 & . & 5u & - & 50 & - & so & $\cdot$ & su & . \\
\hline Ethylbenzene & 50 & • & 50 & $\cdot$ & 50 & - & 50 & - & 50 & $\cdot$ & 50 & 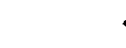 \\
\hline 2-Hexanone & 100 & - & 100 & $\cdot$ & 100 & - & 100 & - & 100 &. & 100 & . \\
\hline 4-Methyl-2-pentanone & 100 & • & 100 & $\cdot$ & 100 & • & 100 & • & FP1 & $\cdot$ & 100 & . \\
\hline Methylene chloride & 5u & - & 5u & • & FP3 & $\cdot$ & FP1 & - & 50 & $\cdot 1$ & 50) & . \\
\hline Styrene & so & $\cdot$ & 50 & $\cdot$ & 50 & • & 50 & - & su & $\cdot$ & 50 & . \\
\hline Tetrachloroethene & 50 & - & 5u & $\cdot$ & su & - & 50 & - & 50 & $\cdot$ & 50 & \\
\hline Toluene & 50 & $\cdot$ & 50 & $\cdot$ & 50 & - & 50 & $\cdot$ & 50 & $\cdot$ & 5v & . \\
\hline 1,1,1-Trichloroethane & 50 & $\cdot$ & 50 & $\cdot$ & 50 & $\cdot$ & 50 & - & 50 & $\cdot 1$ & 5u & . \\
\hline 1,1,2-Trichloroethane & 50 & $\cdot$ & 50 & $\cdot$ & 50 & $\cdot$ & 50 & - & 50 &. & 50 & 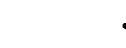 \\
\hline Trichloroethene & 50 & $\cdot$ & su & $\cdot$ & 50 & • & 50 & - & 50 & $\cdot$ & 50 & . \\
\hline Vinyl acetate & 100 & $\cdot$ & 100 & $\cdot$ & 100 & $\cdot$ & 100 & - & 100 &. & 100 & . \\
\hline Vinyl chloride & 100 & $\cdot$ & 100 & $\cdot$ & 100 & $\cdot$ & 100 & - & 100 & $\cdot$ & 100 & . \\
\hline Xylenes & 5u & $\cdot$ & 50 & $\cdot$ & 50 & - & 50 & - & 50 & $\cdot$ & 50 & . \\
\hline Sumned vOCs & & . & 0 & . & 0 & $\cdot$ & 0 & - & 0 &. & 0 & . \\
\hline
\end{tabular}

(CONTINUED) 

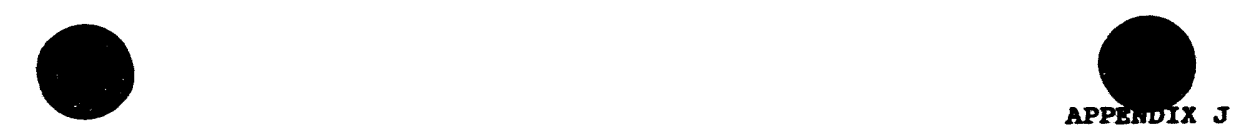

Validated VOC Results, 1993

\begin{tabular}{|c|c|c|c|c|c|c|c|c|c|c|c|c|}
\hline \multirow{4}{*}{$\begin{array}{l}\text { Sampling Point } \\
\text { Location } \\
\text { Date Sampled }\end{array}$} & \multicolumn{4}{|c|}{ GW-325 } & \multicolumn{8}{|c|}{$G N-345$} \\
\hline & \multicolumn{4}{|c|}{ s3 } & \multicolumn{8}{|c|}{ s3 } \\
\hline & \multicolumn{2}{|c|}{$09 / 11 / 93$} & \multicolumn{2}{|c|}{$10 / 21 / 93$} & \multicolumn{2}{|c|}{$02 / 03 / 93$} & \multicolumn{2}{|c|}{$06 / 01 / 93$} & \multicolumn{2}{|c|}{$09 / 14 / 93$} & \multicolumn{2}{|c|}{$10 / 26 / 93$} \\
\hline & ug/L & s sum & $\mathrm{ug} / \mathrm{L}$ & sum & $u g / I$ & 8 sum & $\lg / L$ & s sum & $u g / x$ & s sum & us/L & s sum \\
\hline Acetone & 100 & $\cdot$ & 100 & - & 100 & $\cdot$ & 100 & - & 100 & $\cdot 1$ & FP1 & r \\
\hline Benzene & 5u & - & 5v & - & 50 & $\cdot$ & 50 & $\cdot$ & 50 & $\cdot$ & 50 & • \\
\hline Bromodichloromethene & 50 & $\cdot$ & 50 & • & 5u & $\cdot$ & 50 & $\cdot$ & 50 & $\cdot$ & 50 & - \\
\hline 2-Butanone & 100 & - & 100 & • & 100 & $\cdot$ & 100 & $\cdot$ & 100 & $\cdot$ & FP1 & - \\
\hline Carbon disulfide & 50 & • & 50 & • & 5u & $\cdot$ & 50 & $\cdot$ & 50 & $\cdot$ & $\mathbf{5 0}$ & • \\
\hline Carbon tetrachloride & $5 u$ & - & 50 & • & 50 & $\cdot$ & 50 & $\cdot$ & 50 & $\cdot$ & 50 & - \\
\hline Chlorobenzene & 50 & $\cdot$ & FP3 & - & 5u & $\cdot$ & 5v & $\cdot$ & 50 & $\cdot$ & 50 & - \\
\hline Chloroform & 50 & $\cdot$ & 50 & • & 50 & $\cdot$ & 50 & $\cdot$ & FP3 & $\cdot$ & 50 & - \\
\hline 1,1-Dichloroethane & $5 \mathbf{5}$ & $\cdot$ & 50 & • & 50 & $\cdot$ & 50 & $\cdot$ & 5u & $\cdot$ & 50 & - \\
\hline 1,2-Dichloroethane & 50 & $\cdot$ & so & • & 50 & $\cdot$ & 50 & $\cdot$ & so & $\cdot$ & 50 & - \\
\hline 1,1-Dichloroethene & 50 & $\cdot$ & 50 & • & 50) & $\cdot$ & 50 & $\cdot$ & so & $\cdot$ & 50 & • \\
\hline 1,2-Dichloroethene & 50 & - & 50 & • & 50 & $\cdot$ & 50 & - & 50 & $\cdot$ & 50 & . \\
\hline Ethylbenzene & 50 & $\cdot$ & 50 & • & 50 & $\cdot$ & 50 & $\cdot$ & 50 & $\cdot$ & 50 & - \\
\hline 2-Hexanone & 100 & - & 100 & • & 100 & $\cdot$ & 100 & $\cdot$ & 100 & $\cdot$ & 100 & - \\
\hline 4-Methy1-2-pentanone & 100 & - & 100 & • & FP1 & $\cdot$ & 100 & $\cdot$ & 100 & $\cdot$ & 100 & - \\
\hline Methylene chloride & FP3 & $\cdot$ & 50 & • & FP1 & $\cdot$ & 5u & $\cdot$ & FP3 & $\cdot$ & FP1 & - \\
\hline Styrene & $\mathbf{5 u}$ & • & 50 & • & 50 & $\cdot$ & 5u & $\cdot$ & 50 & $\cdot$ & 50 & - \\
\hline Tetrachloroethene & 50 & $\cdot$ & 5u & • & 50 & $\cdot$ & 50 & $\cdot$ & 50 & $\cdot$ & 50 & r \\
\hline Toluene & 5u & $\cdot$ & 50 & • & 50 & $\cdot$ & 50 & - & 50 & $\cdot$ & 50 & - \\
\hline $1,1,1$-Trichloroethane & 50 & $\cdot$ & so & • & 50 & $\cdot$ & 50 & - & 50 & $\cdot$ & 50 & 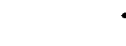 \\
\hline 1,1,2-Trichloroethane & 50 & $\cdot$ & 5u & • & 5v & $\cdot$ & 50 & $\cdot$ & 50 & $\cdot$ & 5u & 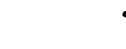 \\
\hline Trichloroethene & 5u & $\cdot$ & 50 & • & 50 & $\cdot$ & 50 & $\cdot$ & 50 & $\cdot$ & 50 & . \\
\hline Vinyl acetate & 100 & $\cdot$ & 100 & $\cdot$ & 100 & $\cdot$ & 100 & $\cdot$ & 100 & $\cdot$ & 100 & " \\
\hline Vinyl chloride & 100 & - & 100 & • & 100 & $\cdot$ & 100 & $\cdot$ & 100 & $\cdot$ & 100 & 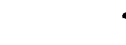 \\
\hline xylenes & 50 & $\cdot$ & 50 & • & 5v & $\cdot$ & 50 & $\cdot$ & 50 & $\cdot$ & 50 & 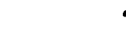 \\
\hline Sumned voCs & 0 & - & 0) & - & o) & $\cdot$ & o & - & 0 & $\cdot$ & - & 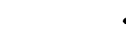 \\
\hline
\end{tabular}

(CONT INUED) 
APPEIDIX J

valideted VOC Results, 1993

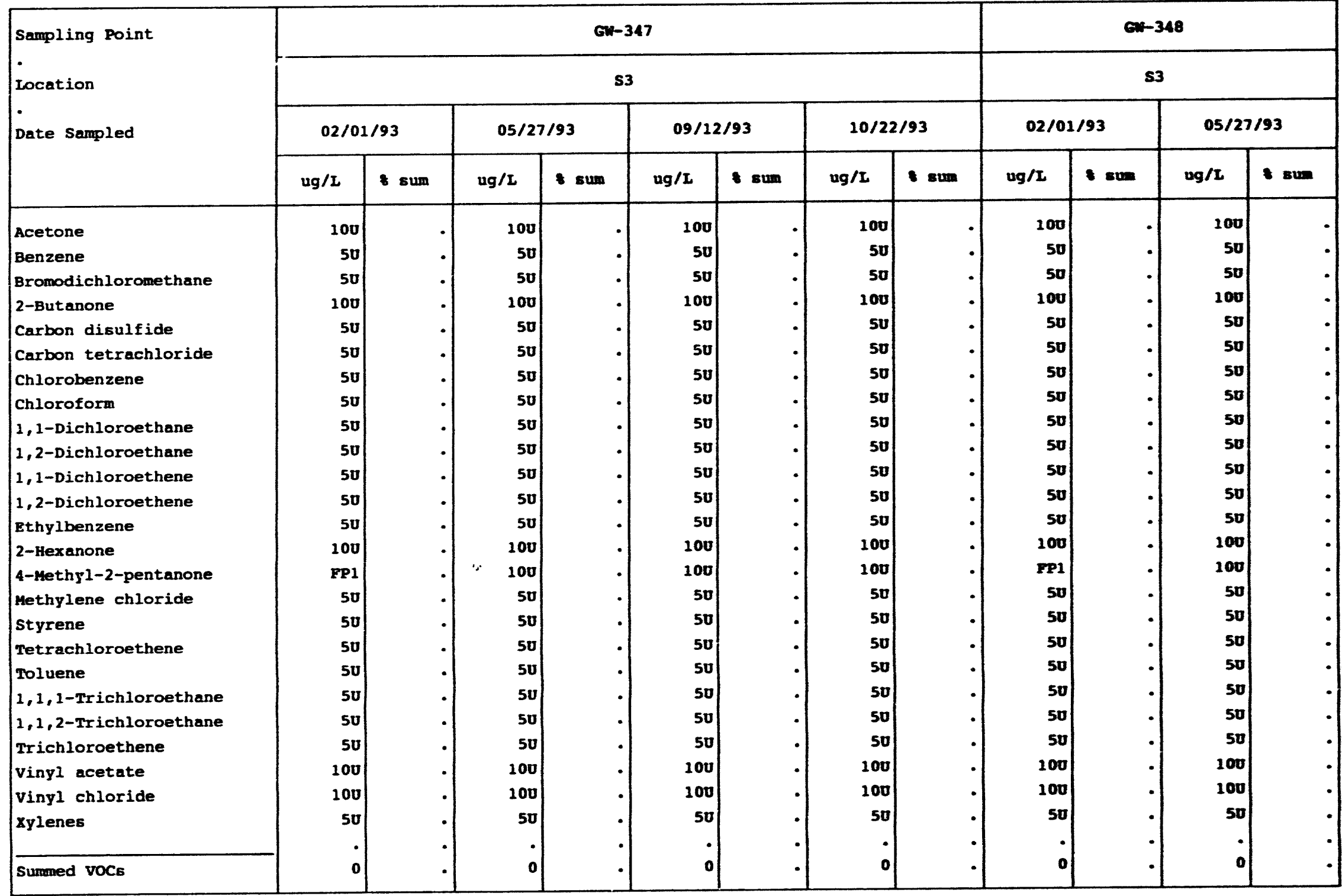

(CONTINUED) 
Validated voc Results, 1993

\begin{tabular}{|c|c|c|c|c|c|c|c|c|c|c|c|c|}
\hline \multirow{4}{*}{$\begin{array}{l}\text { Sampling Point } \\
\text { Location } \\
\text { Date Sampled }\end{array}$} & \multicolumn{4}{|c|}{$G W-348$} & \multicolumn{8}{|c|}{$6 w-364$} \\
\hline & \multicolumn{4}{|c|}{53} & \multicolumn{8}{|c|}{ OLF } \\
\hline & \multicolumn{2}{|c|}{$09 / 12 / 93$} & \multicolumn{2}{|c|}{$10 / 22 / 93$} & \multicolumn{2}{|c|}{$03 / 27 / 93$} & \multicolumn{2}{|c|}{$06 / 27 / 93$} & \multicolumn{2}{|c|}{$09 / 19 / 93$} & \multicolumn{2}{|c|}{$12 / 18 / 93$} \\
\hline & $u g / t$ & sum & $\mathrm{ug} / \mathrm{L}$ & sum & $u g / L$ & $8 \operatorname{sun}$ & $u g / L$ & $\sin$ & $\operatorname{ug} / \mathbf{L}$ & $c \operatorname{com}$ & $u g / 2$ & en \\
\hline Acetone & 100 & - & FP1 & - & 100 &. & 100 & $\cdot 1$ & 100 & $\cdot 1$ & FP1 & - \\
\hline Benzene & so & - & 50 & - & sv & $\cdot$ & 50 & $\cdot$ & $\mathbf{5 0}$ & $\cdot$ & 50 & - \\
\hline Bromodichloromethane & 50 & - & su & - & 50 & $\cdot$ & 50 & $\cdot 1$ & 50 & $\cdot$ & 50 & • \\
\hline 2-Butanone & 100 & - & $\mathbf{F P 1}$ & - & 100 & $\cdot$ & 100 & $\cdot$ & 100 &. & FP1 & • \\
\hline Carbon disulfide & 50 & - & 50 & - & so & $\cdot$ & 50 & $\cdot$ & 50 & $\cdot$ & 50 & • \\
\hline Carbon tetrachloride & 5v & . & 50 & • & 50 & $\cdot$ & 50 & $\cdot$ & 50 & $\cdot$ & so & - \\
\hline Chlorobenzene & 50 & . & 50 & • & 50 & $\cdot 1$ & so & $\cdot$ & 50 & $\cdot$ & 50 & - \\
\hline Chloroform & 50 & - & 50 & - & 50 & $\cdot$ & so) & $\cdot$ & 50 & $\cdot$ & 50 & • \\
\hline 1,1-Dichloroethane & 50 & - & 5u & - & 2 & 25 & 50 & $\cdot$ & 3 & 9.4 & 2 & 12.3 \\
\hline 1,2-Dichloroethane & 50 & - & so & - & so & $\cdot$ & 50) & $\cdot$ & 50 & $\cdot 1$ & 50 & - \\
\hline 1,1-Dichloroethene & 5u & - & 5v & - & so & $\cdot$ & so & $\cdot$ & 5 & 15.6 & 2 & 12.3 \\
\hline 1,2-Dichloroethene & 50 & - & 50 & - & 50 & $\cdot$ & 6) & 27.3 & 7 & 21.9 & 4 & 24.5 \\
\hline Ethylbenzene & 50 & - & 5v & - & so &. & 50 & $\cdot$ & so & $\cdot$ & 50 & - \\
\hline 2-Hexanone & 100 & - & 100 & - & 100 & $\cdot 1$ & 100 & $\cdot$ & 100 & $\cdot$ & 100 & - \\
\hline 4-Methy1-2-pentanone & 100 & - & 100 & - & 100 & $\cdot$ & $\mathbf{F P 1}$ & $\cdot$ & 100 & $\cdot$ & 100 & - \\
\hline Hethylene chloride & 50 & - & $\mathbf{F P 1}$ & - & 50 &. & 50 & $\cdot$ & FP3 & $\cdot 1$ & FP1 & - \\
\hline Styrene & so & - & so & - & 50 &. & 50 & $\cdot$ & 50 & $\cdot$ & 50 & - \\
\hline Tetrachloroethene & 5u & - & so & - & so & $\cdot$ & 50 & $\cdot$ & 50 & $\cdot$ & 0.3 & 1.8 \\
\hline Toluene & 50 & - & 50 & - & 50 & $\cdot$ & 50 & $\cdot$ & so & $\cdot$ & so & - \\
\hline 1,1,1-Trichloroethane & so & - & 50 & - & 50) & $\cdot 1$ & 50 & $\cdot$ & 2 & 6.3 & FP3 & - \\
\hline 1,1,2-Trichloroethane & sv & - & so & - & 50 & $\cdot$ & 50 & $\cdot$ & 5v & $\cdot$ & 50 & • \\
\hline Trichloroethene & 50 & - & so & - & 6 & 75 & 16) & 72.7 & 15 & 46.9 & 8 & 49.1 \\
\hline Vinyl acetate & 100 & - & 100 & - & 100 & $\cdot$ & 100 & $\cdot$ & 100 & $\cdot$ & 100 & - \\
\hline vinyl chloride & 100 & - & 100 & - & 100 & $\cdot$ & 100 & $\cdot$ & 100 & $\cdot$ & 100 & - \\
\hline xylenes & 50 & - & 50 & - & 50) &. & so & $\cdot$ & so & $\cdot$ & so & - \\
\hline Summed VOCs & $\dot{0}$ & • & $\dot{0}$ & • & $\dot{8}$ & 100 & 22 & 100 & 32 & 200.1 & 16.3 & 100 \\
\hline
\end{tabular}

(CONTINUED) 
APRADIX $\mathrm{J}$

Valldated voC Reaults, 1993

\begin{tabular}{|c|c|c|c|c|c|c|c|c|c|c|c|c|}
\hline \multirow{4}{*}{$\begin{array}{l}\text { Sampling Point } \\
\text { Location } \\
\text { Date Sampled }\end{array}$} & \multicolumn{8}{|c|}{ Gu-365 } & \multicolumn{4}{|c|}{$6-370$} \\
\hline & \multicolumn{8}{|c|}{ orf } & \multicolumn{4}{|c|}{ BG } \\
\hline & \multicolumn{2}{|c|}{$03 / 28 / 93$} & \multicolumn{2}{|c|}{$06 / 27 / 93$} & \multicolumn{2}{|c|}{$09 / 19 / 93$} & \multicolumn{2}{|c|}{$12 / 19 / 93$} & \multicolumn{2}{|c|}{$02 / 17 / 93$} & \multicolumn{2}{|c|}{$05 / 22 / 93$} \\
\hline & $u g / L$ & sum & $u g / L$ & t sum & $u g / L$ & sum & $\operatorname{ug} / x$ & sum & $\mathrm{ug} / \mathrm{x}$ & $\operatorname{sen}$ & $u g / 2$ & - In \\
\hline Acetone & 100 & $\cdot$ & 100 & . & 100 & - & 100 &. & FP1 & - & 100 & . \\
\hline Benzene & so & $\cdot 1$ & 50 & $\cdot$ & so & - & so &. & $\mathbf{5 0}$ & - & so & . \\
\hline Bromodichloromethane & 50 & $\cdot$ & 50) & - & 50 & - & 50 & $\cdot$ & so & - & so & . \\
\hline 2-Butanone & 100 & $\cdot$ & 100 & - & 100 & • & $\mathbf{P P 1}$ & $\cdot$ & 100 & - & 100 & . \\
\hline Carbon disulfide & 50 & $\cdot$ & 50 & - & 50 & - & so & $\cdot$ & 50 & - & 50 & • \\
\hline Carbon tetrachloride & 50 & $\cdot$ & 5v & - & so & - & 50 & $\cdot$ & so & - & 50 & • \\
\hline Chlorobenzene & so & $\cdot$ & 50 & - & 5v & - & 50 & $\cdot$ & 50 & - & 50 & • \\
\hline Chloroform & FP3 & $\cdot$ & 50 & - & 50 & - & 50 & $\cdot 1$ & so & - & 50 & . \\
\hline 1,1-Dichloroethane & 5 & 3.3 & 50 & $\cdot$ & 4 & 4.9 & 4 & 5.6 & 50 & - & 50 & • \\
\hline 1,2-Dichloroethane & so &. & 50 & - & 50 & - & so &. & 50 & - & 50 & • \\
\hline 1,1-Dichloroethene & 21 & 13.9 & 20 & 16.5 & 24 & 17.3 & 13 & 18.3 & 50 & • & so & • \\
\hline 1,2-Dichloroethene & 23 & 15.2 & 19 & 15.7 & 14 & 17.3 & 12 & 16.9 & 50 & $\cdot$ & 50 & • \\
\hline Ethylbenzene & 50 & $\cdot$ & so & - & so & - & 50 & $\cdot 1$ & sv & - & so & • \\
\hline 2-Hexanone & 100 & $\cdot 1$ & 100 & - & 100 & - & 100 &. & 100 & - & 100 & - \\
\hline 4-Methyl-2-pentanone & $\mathbf{F P 1}$ & $\cdot$ & 100 & - & 100 & - & 100 &. & $\mathbf{F P 1}$ & - & 100 & • \\
\hline Methylene chloride & $\mathbf{F P 1}$ & $\cdot$ & 5u & $\cdot$ & 50 & - & FP1 & $\cdot$ & $\mathbf{F P 1}$ & $\cdot$ & 50. & • \\
\hline styrene & sv & $\cdot$ & 50 & $\cdot$ & 50 & - & so & $\cdot$ & so & - & 50 & • \\
\hline Tetrachloroethene & 2 & 1.3 & 50 & - & 1 & 1.2 & 1 & 1.4 & so & $\cdot$ & so & • \\
\hline Toluene & 50 & $\cdot$ & 5v & $\cdot$ & 50) & - & 50 & $\cdot$ & so & $\cdot$ & 50! & • \\
\hline 1,1,1-Trichloroethane & 11 & 7.3 & 5 & 4.1 & 2 & 2.5 & 2 & 2.8 & so & $\cdot$ & 50 & • \\
\hline 1,1,2-Trichloroethane & 50 &. & so & - & 50 & - & sv &. & 50 & - & so & • \\
\hline Irichloroethene & 89 & 58.9 & 77 & 63.6 & 46 & 56.8 & 39 & 54.9 & so & - & 50 & • \\
\hline Vinyl acetate & 100 &. & 100 & . & 100 & - & 100 &. & 100 & - & 100 & - \\
\hline Vingl chloride & 100 & $\cdot$ & 100 & $\cdot$ & 100 & - & 100 & $\cdot$ & 100 & - & 200 & • \\
\hline xylenes & so & $\cdot$ & 5v & $\cdot$ & so & - & 50 & $\cdot$ & 50 & - & 50 & • \\
\hline Summed VOCs & 151 & 99.9 & 121 & 99.9 & 81 & 100 & 71 & 99.9 & - & . & 0 & . \\
\hline
\end{tabular}

(CONTIMUED) 
validated voc mesulte, 1993

\begin{tabular}{|c|c|c|c|c|c|c|c|c|c|c|c|c|c|}
\hline \multirow{4}{*}{$\begin{array}{l}\text { Sampling Point } \\
\text { rocation } \\
\text { Date Sampled }\end{array}$} & \multicolumn{4}{|c|}{ GN-370 } & \multicolumn{9}{|c|}{$60-371$} \\
\hline & \multicolumn{4}{|c|}{ BG } & \multicolumn{9}{|c|}{ BG } \\
\hline & \multicolumn{2}{|c|}{$07 / 10 / 93$} & \multicolumn{2}{|c|}{$10 / 14 / 93$} & \multicolumn{2}{|c|}{$02 / 16 / 93$} & \multicolumn{2}{|c|}{$05 / 21 / 93$} & \multicolumn{2}{|c|}{$07 / 09 / 93$} & \multicolumn{3}{|c|}{$10 / 13 / 93$} \\
\hline & ug/L & s sum & $u g / L$ & - sum & ug/L & $8 \mathrm{sum}$ & $u g / \mathbf{L}$ & sun & $\operatorname{ug} / x$ & son & $\operatorname{leg} / \mathrm{L}$ & con & \\
\hline Acetone & 100 & - & $\mathbf{F P 1}$ & - & 100 & $\cdot$ & 100 & - & FP3 & $\cdot$ & 100 & & • \\
\hline Benzene & su & - & 50 & - & 50 & - & 50 & • & 50 & $\cdot$ & so & & $\cdot$ \\
\hline Bromodichloromethane & 5u & $\cdot$ & so & - & 50 & - & 50) & - & so & - & 50 & & $\cdot$ \\
\hline 2-Butanone & 100 & - & FP1 & - & 100 & $\cdot$ & 100 & • & 100 & - & 100 & & $\cdot$ \\
\hline Carbon disulfide & so & - & 50 & - & 50 & $\cdot$ & so & • & 50 & $\cdot$ & so & & $\cdot$ \\
\hline Carbon tetrachloride & 50 & - & 50 & - & 50 & $\cdot$ & so) & $\cdot$ & so & $\cdot$ & 50 & & $\cdot$ \\
\hline Chlorobenzene & 50 & - & so & - & so & $\cdot$ & 50) & - & 50 & $\cdot$ & 50 & & - \\
\hline Chloroform & 5u & - & so & - & so & - & 50 & - & $\mathbf{r P 3}$ & - & so & & $\cdot$ \\
\hline 1,1-Dichloroethane & so & - & 50 & - & so & $\cdot$ & so & - & so & $\cdot$ & 50 & & $\cdot$ \\
\hline 1,2-Dichloroethane & 50 & - & 50 & - & 50 & $\cdot$ & so & $\cdot$ & so & - & 50 & & $\cdot$ \\
\hline 1,1-Dichloroethene & so & . & so & - & 50 & $\cdot$ & so & - & so & - & so & & $\cdot$ \\
\hline 1,2-Dichloroethene & 5v & - & 50 & - & 50 & $\cdot$ & 50 & • & 50 & $\cdot$ & so & & $\cdot$ \\
\hline Ethylbenzene & 50 & - & 50 & - & 50 & $\cdot$ & 50 & $\cdot$ & 50 & $\cdot$ & so & & $\cdot$ \\
\hline 2-Hexanone & 100 & - & 100 & - & 100 & $\cdot$ & 100 & - & 100 & - & 100 & & $\cdot$ \\
\hline 4-Methy1-2-pentanone & 100 & - & 100 & - & 100 & $\cdot$ & 200 & - & 200 & - & 100 & & $\cdot$ \\
\hline Methylene chloride & so & - & $\mathbf{F P 1}$ & - & so &. & so & . & EP1 & - & so & & - \\
\hline styrene & so & - & 50 & - & 50 & $\cdot$ & 50 & - & 58) & $\cdot$ & 50) & & $\cdot$ \\
\hline Tetrachloroethene & 50 & - & 50 & - & 50 & $\cdot$ & 50) & - & 50 & - & 50) & & $\cdot$ \\
\hline Tolvene & so & $\cdot$ & so & - & 50 & $\cdot$ & so & - & 50 & - & 50 & & $\cdot$ \\
\hline 1,1,1-Trichloroethane & so & $\cdot$ & so & - & 50 & $\cdot$ & 50 & $\cdot$ & 50 & $\cdot$ & 50 & & $\cdot$ \\
\hline 1,1,2-Trichloroethane & so & $\cdot$ & so & - & so & $\cdot$ & 50 & - & so & - & 50 & & $\cdot$ \\
\hline Trichloroethene & 50 & $\cdot$ & so & - & 50 & $\cdot$ & 50 & - & so & $\cdot$ & 5v & & $\cdot$ \\
\hline Vinyl acetate & 100 & $\cdot$ & 100 & - & 100 & - & 100 & - & 100 & - & 100 & & - \\
\hline vinyl chloride & 100 & $\cdot$ & 200 & - & 100 & $\cdot$ & 100 & • & 100 & - & 100 & & - \\
\hline xylenes & so &. & 50 & - & 50 & $\cdot$ & 50 & - & 50 & - & 50 & & $\cdot$ \\
\hline Summed vocs & 0 & . & 0 & - & 0 & - & o) & - & - & - & 이 & & $\cdot$ \\
\hline
\end{tabular}

(CONTInUED) 
APPENDIX $\mathrm{J}$

Validated VOC Results, 1993

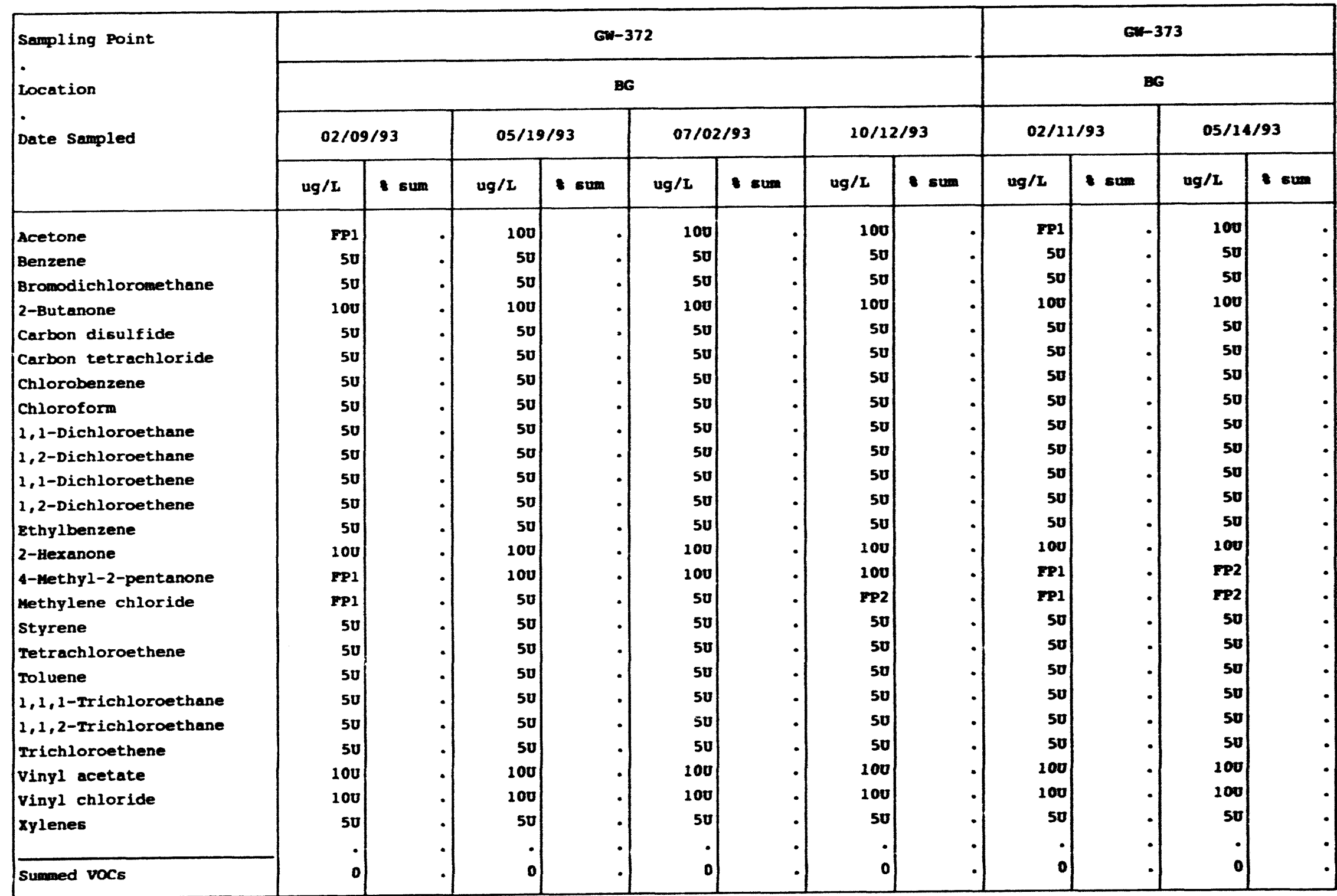

(CONTIMUED) 
Validated voC Results, 1993

\begin{tabular}{|c|c|c|c|c|c|c|c|c|c|c|c|c|}
\hline \multirow{4}{*}{$\begin{array}{l}\text { Sampling Point } \\
\text { Location } \\
\text { Date Sampled }\end{array}$} & \multicolumn{4}{|c|}{$G W-373$} & \multicolumn{8}{|c|}{$G W-526$} \\
\hline & \multicolumn{4}{|c|}{ BG } & \multicolumn{8}{|c|}{ s3 } \\
\hline & \multicolumn{2}{|c|}{$07 / 02 / 93$} & \multicolumn{2}{|c|}{$10 / 11 / 93$} & \multicolumn{2}{|c|}{$02 / 03 / 93$} & \multicolumn{2}{|c|}{$06 / 03 / 93$} & \multicolumn{2}{|c|}{$09 / 14 / 93$} & \multicolumn{2}{|c|}{$10 / 26 / 93$} \\
\hline & $\mathrm{ug} / \mathrm{L}$ & 8 sum & $\mathrm{ug} / \mathrm{L}$ & 8 sum & ug/L & 8 sum & ug/I & sum & ug/L & 8 sum & ug/t & 8 sum \\
\hline Acetone & 100 & $\cdot$ & FP1 & - & FP3 & $\cdot$ & 100 & $\cdot$ & 100 & . & FP1 & \\
\hline Benzene & su & - & 50 & - & 50 & $\cdot$ & 50 & $\cdot$ & 50 & - & 5u & \\
\hline Bromodichloromethane & su & - & 50 & $\cdot$ & 50 & $\cdot$ & 50 & $\cdot$ & 50 & $\cdot$ & 50 & \\
\hline 2-Butanone & 100 & $\bullet$ & FP1 & - & 100 & $\cdot$ & 100 & $\cdot$ & 100 & $\cdot$ & FP1 & \\
\hline Carbon disulfide & 50 & $\cdot$ & 50 & $\cdot$ & 5u & $\cdot$ & su & $\cdot$ & su & $\cdot$ & 50 & \\
\hline Carbon tetrachloride & 5u & $\cdot$ & 50 & $\cdot$ & 50 & $\cdot$ & 50 & $\cdot$ & $\begin{array}{l}50 \\
50\end{array}$ & $\cdot$ & 50 & \\
\hline Chlorobenzene & 50 & $\cdot$ & 50 & $\cdot$ & 50 & $\cdot$ & $\begin{array}{l}50 \\
50\end{array}$ &. & 50 &. & FP3 & \\
\hline Chloroform & 50 & $\cdot$ & 50 & $\cdot$ & 50 &. & 50 &. & 5u & - & $5 v$ & \\
\hline 1,1-Dichloroethane & 50 & $\cdot$ & 50 & $\cdot$ & $\begin{array}{l}50 \\
5 U\end{array}$ &. & 50 &. & 50 &. & 50 & \\
\hline $\begin{array}{l}\text { 1,2-Dichloroethane } \\
\text { 1,1-Dichloroethene }\end{array}$ & 50 & $\cdot$ & $\begin{array}{l}50 \\
50\end{array}$ & $\dot{0}$ & 50 &. & 50 &. & 50 & $\cdot$ & su & \\
\hline $\begin{array}{l}\text { 1,1-Dichloroethene } \\
\text { 1,2-Dichloroethene }\end{array}$ & $\begin{array}{l}5 U \\
5 U\end{array}$ & $\cdot$ & $\begin{array}{l}5 \mathrm{U} \\
5 \mathrm{U}\end{array}$ & . & 50 &. & 50 & $\cdot$ & 50 &. & 50 & \\
\hline $\begin{array}{l}\text { 1,2-Dichloroethene } \\
\text { Ethylbenzene }\end{array}$ & $\begin{array}{l}50 \\
50\end{array}$ & $\cdot$ & $\begin{array}{l}50 \\
50\end{array}$ & - & 50 & $\cdot 1$ & 50 & $\cdot$ & 50 & . & 50 & \\
\hline $\begin{array}{l}\text { Ethylbenzene } \\
\text { 2-Hexanone }\end{array}$ & $\begin{array}{r}50 \\
100\end{array}$ & $\cdot$ & 100 & - & 100 & $\cdot 1$ & 100 & $\cdot$ & 100 & $\cdot$ & 100 & \\
\hline & 100 & . & 100 & - & FP3 & $\cdot$ & 100 & $\cdot$ & 100 & $\cdot$ & 100 & \\
\hline & 50 & - & FP1 & - & FP3 &. & 5u & $\cdot$ & 50 &. & FP1 & \\
\hline $\begin{array}{l}\text { Methylene chloride } \\
\text { styrene }\end{array}$ & 50 & - & 50 & - & 50 &. & $5 \mathrm{u}$ & $\cdot$ & 50 & $\cdot$ & 5u & \\
\hline $\begin{array}{l}\text { Styrene } \\
\text { Tetrachloroethene }\end{array}$ & 50 & - & 50 & . & 5v & $\cdot$ & 50 & $\cdot 1$ & 50 &. & 50 & \\
\hline $\begin{array}{l}\text { Tetrachloroethene } \\
\text { Toluene }\end{array}$ & $5 \mathrm{U}$ & - & 50 & - & 5u &. & 50 & $\cdot$ & 50 &. & 50 & \\
\hline & 50 & . & 50 & - & 50 &. & $5 \mathrm{v}$ &. & 50 & . & 5v & \\
\hline & 5v & - & 50 & - & 50 &. & 50 & $\cdot$ & 50 & . & su & \\
\hline & 50 & - & 50 & - & 50 & .1 & 50 & $\cdot 1$ & 50 &. & 50 & \\
\hline & 100 & - & 100 & - & 100 &. & 100 &. & 100 &. & 100 & \\
\hline $\begin{array}{l}\text { vinyl acetate } \\
\text { vinyl chloride }\end{array}$ & 100 & - & 100 & . & 100 &. & 100 & $\cdot$ & 100 &. & 100 & \\
\hline $\begin{array}{l}\text { vinyl chloride } \\
\text { xylenes }\end{array}$ & 50 & . & 5u & . & 50 &. & 50 & . & 50 & $\cdot$ & 50 & \\
\hline & $\cdot$ & - & $\cdot$ & - & $\cdot$ & $\cdot$ & $\cdot$ & $\cdot$ & $\cdot$ & $\cdot$ & $\cdot$ & \\
\hline Summed vOCs & 0 & . & o) & • & 0 & $\cdot$ & 0 & $\cdot$ & 이 & $\cdot$ & of & \\
\hline
\end{tabular}

(CONTINUED) 
AF: 2 DIX I

Validated voc keswits, 1993

\begin{tabular}{|c|c|c|c|c|c|c|c|c|c|c|c|c|}
\hline \multirow{4}{*}{$\begin{array}{l}\text { Sampling Point } \\
\text { Location } \\
\text { Date Sampled }\end{array}$} & \multicolumn{8}{|c|}{ GN-537 } & \multicolumn{4}{|c|}{ GW-601 } \\
\hline & \multicolumn{8}{|c|}{ OLF } & \multicolumn{4}{|c|}{ OLF } \\
\hline & \multicolumn{2}{|c|}{$02 / 11 / 93$} & \multicolumn{2}{|c|}{$05 / 12 / 93$} & \multicolumn{2}{|c|}{$09 / 14 / 93$} & \multicolumn{2}{|c|}{$10 / 22 / 93$} & \multicolumn{2}{|c|}{$03 / 28 / 93$} & \multicolumn{2}{|c|}{$06 / 27 / 93$} \\
\hline & ug/L & sum & $u g / L$ & sum & ug $/ \mathrm{L}$ & sum & ug/L & 8 sum & ug/I & 8 sum & ug/t & sum \\
\hline Acetone & & $\cdot$ & 100 & $\cdot$ & 100 & - & 100 & $\cdot$ & 100 & $\cdot$ & 100 & $\theta^{\circ}$ \\
\hline Benzene & 50 & $\cdot$ & 50 & $\cdot$ & 50 & $\cdot$ & 50 & $\cdot$ & 50 & $\cdot$ & 5u & - \\
\hline Bromodichloromethane & 50 & $\cdot$ & 50 & $\cdot$ & 5u & $\cdot$ & 50 & $\cdot$ & $\begin{array}{r}50 \\
100\end{array}$ &. & $\begin{array}{r}50 \\
100\end{array}$ & . \\
\hline 2-Butanone & 100 & $\cdot$ & 100 & $\cdot$ & $\begin{array}{r}100 \\
50\end{array}$ & $\cdot$ & $\begin{array}{r}100 \\
50\end{array}$ & $\dot{.}$ & $\begin{array}{r}100 \\
50\end{array}$ &. & so & . \\
\hline $\begin{array}{l}\text { Carbon disulfide } \\
\text { Carbon tetrachlor:de }\end{array}$ & $\begin{array}{l}50 \\
50\end{array}$ & $\cdot$ & $\begin{array}{l}50 \\
50\end{array}$ & $\cdot$ & 50 & $\cdot$ & so & $\cdot$ & 4 & 2.6 & 50 & - \\
\hline $\begin{array}{l}\text { Carbon tetrachlor:de } \\
\text { Chlorobenzene }\end{array}$ & 50 & . & 5v & $\cdot$ & su & $\cdot$ & 50 & $\cdot$ & 50 & $\cdot$ & 50 & • \\
\hline Chloroform & 0.8 & 100 & 0.8 & 100 & 0.9 & 100 & 50 & $\cdot$ & 1 & 0.7 & 50 & - \\
\hline 1,1-Dichloroethane & 50 & $\cdot$ & 50 & $\cdot$ & 50 & $\cdot$ & 50 & $\cdot$ & 50 & $\cdot$ & 50 & - \\
\hline 1,2-Dichloroethane & 50 & $\cdot$ & 50 & $\cdot$ & 50 & $\cdot$ & 50 & $\cdot$ & 5u & $\cdot$ & 50 & • \\
\hline 1,1-Dichloroethene & 50 & $\cdot$ & 5u & $\cdot$ & 50 & $\cdot$ & 50 & $\cdot$ & so & 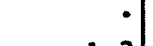 & $5 \mathbf{0}$ & - \\
\hline 1,2-Dichloroethene & 50 & $\cdot$ & 50 & $\cdot$ & 50 & $\cdot$ & 50 & $\cdot$ & 2 & 1.3 & 50 & - \\
\hline Ethylbenzene & $\mathbf{5 u}$ & $\cdot$ & 50 & $\cdot$ & 50 & $\cdot$ & 50 & $\cdot$ & $\begin{array}{r}50 \\
100\end{array}$ &. & $\begin{array}{r}50 \\
100\end{array}$ & • \\
\hline 2-Hexanone & 100 & $\cdot$ & 100 & $\bullet$ & 100 & 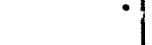 & 100 & $\cdot$ & 2 & 1.3 & 3 & 2.1 \\
\hline 4-Methy1-2-pentanone & 100 & $\cdot$ & 100 & $\cdot$ & 100 & $\cdot$ & 50 & . & 3 & 2 & so & • \\
\hline Hethylene chloride & 50 & $\cdot$ & 50 & $\cdot$ & $\begin{array}{r}501 \\
50\end{array}$ & $\cdot$ & 50 & . & 50 &. & 50 & • \\
\hline Styrene & $\begin{array}{l}50 \\
50\end{array}$ &. & su & $\cdot$ & 50 &. & 5u & $\cdot$ & 1 & 0.7 & 50 & • \\
\hline $\begin{array}{l}\text { Tetrachloroethene } \\
\text { Toluene }\end{array}$ & su & $\cdot$ & 50 &. & 50 & $\cdot$ & 50 & $\cdot$ & 50 & $\cdot$ & 5u & - \\
\hline $\begin{array}{l}\text { Toluene } \\
1,1,1-\text { Trichloroethane }\end{array}$ & 5u & $\cdot$ & 5u & $\cdot$ & su & $\cdot$ & 50 & $\cdot$ & 5u & $\cdot$ & 50 & - \\
\hline 1,1,2-Trichloroethane & 50 & $\cdot$ & 5v & $\cdot$ & 50 & $\cdot$ & 50 & $\cdot$ & 50 & $\cdot$ & 5u & • \\
\hline Trichloroethene & 5u & $\cdot$ & 50 & $\cdot$ & 50 & $\cdot$ & 50 & $\cdot$ & 140 & 91.5 & 140 & 97.9 \\
\hline Vinyl acetate & 100 & $\cdot$ & 100 & $\cdot$ & 100 & $\cdot 1$ & 100 & $\cdot$ & 100 & $\cdot$ & 100 & - \\
\hline vinyl chloride & 100 & $\cdot$ & 100 & $\cdot$ & 100 & $\cdot$ & 100 & $\cdot$ & 100 & $\cdot$ & 100 & - \\
\hline xylenes & 50 & $\cdot$ & 50 & $\cdot$ & 50 & $\cdot$ & 50 & $\bullet$ & 50 & $\cdot 1$ & 50 & - \\
\hline Summed vocs & 0.8 & 100 & 0.8 & 100 & 0.9 & 100 & 0 & $\cdot$ & 153 & 100.1 & 143 & 100 \\
\hline
\end{tabular}

(CONTINUED) 
Validated voC Resulte, 1993

\begin{tabular}{|c|c|c|c|c|c|c|c|c|c|c|c|c|}
\hline \multirow{4}{*}{$\begin{array}{l}\text { Sampling Point } \\
\text { Location } \\
\text { Date Sampled }\end{array}$} & \multicolumn{4}{|c|}{ GN-601 } & \multicolumn{2}{|c|}{ GW-613 } & \multicolumn{2}{|c|}{ GW-614 } & \multicolumn{4}{|c|}{$G W-621$} \\
\hline & \multicolumn{4}{|c|}{ OLF } & \multirow{2}{*}{\multicolumn{2}{|c|}{$\frac{53}{10 / 20 / 93}$}} & \multirow{2}{*}{\multicolumn{2}{|c|}{$\frac{53}{10 / 20 / 93}$}} & \multicolumn{4}{|c|}{ BG } \\
\hline & \multicolumn{2}{|c|}{$09 / 19 / 93$} & \multicolumn{2}{|c|}{$12 / 21 / 94$} & & & & & \multicolumn{2}{|c|}{$03 / 20 / 93$} & \multicolumn{2}{|c|}{$06 / 02 / 93$} \\
\hline & $\mathrm{ug} / \mathrm{L}$ & sum & ug/I & 8 sum & $u g / L$ & 8 sum & ug/L & sum & ug/L & sum & ug/L & - sum \\
\hline Acetone & 100 & $\cdot$ & 100 & $\cdot 1$ & FP1 & - & FP1 & - & 100 & $\cdot 1$ & 100 & • \\
\hline Benzene & 5u & $\cdot$ & 5u & $\cdot$ & 50 & $\cdot$ & 5v & - & 50 & $\cdot$ & 50 & - \\
\hline Bromodichloromethane & 5u & $\cdot$ & 50 & $\cdot$ & 50 & • & 50 & - & 50 & $\cdot$ & 50 & - \\
\hline 2-Butanone & 100 & $\cdot$ & FP3 & $\cdot$ & FP1 & - & FP1 & - & 100 & $\cdot$ & 100 & • \\
\hline Carbon disulfide & 50 & $\cdot$ & 50 & : & 50 & $\cdot$ & 50 & - & 50 & $\cdot$ & $\begin{array}{l}50 \\
50\end{array}$ & - \\
\hline Carbon tetrachloride & 2 & 1.6 & 3 & 2.4 & $\begin{array}{l}50 \\
50\end{array}$ & $\dot{\bullet}$ & $\begin{array}{ll}50 \\
50\end{array}$ & $\cdot$ & $\begin{array}{l}50 \\
5 u\end{array}$ &. & 50 & • \\
\hline Chlorobenzene & 50 & 0.5 & $\begin{array}{r}50 \\
0.7\end{array}$ & $0 . \dot{5}$ & 50 & . & 50 & • & 50 & $\cdot$ & 5u & - \\
\hline $\begin{array}{l}\text { Chloroform } \\
1,1 \text {-Dichloroethene }\end{array}$ & $\begin{array}{r}0.6 \\
5 \mathrm{U}\end{array}$ &. & $\begin{array}{r}0.7 \\
50\end{array}$ & $\cdot$ & 5u & - & 50 & - & 50 & $\cdot$ & so & - \\
\hline $\begin{array}{l}\text { 1,1-Dichloroethane } \\
\text { 1,2-Dichloroethane }\end{array}$ & 50 & & su & . & 5u & - & 50 & - & 50 & $\cdot$ & 50 & - \\
\hline 1,1-Dichloroethene & 0.7 & 0.6 & 0.8 & 0.6 & 5u & - & 50 & - & 50 & $\cdot$ & 50 & • \\
\hline 1,2-Dichloroethene & 5u & $\cdot$ & 1 & 0.8 & 5u & - & 50 & - & 50 & $\cdot$ & 50 & - \\
\hline Ethylbenzene & 50 & $\cdot$ & 50 & $\cdot$ & 50 & • & 50 & $\cdot$ & $\begin{array}{r}50 \\
100\end{array}$ &. & $\begin{array}{r}50 \\
100\end{array}$ & - \\
\hline 2-Hexanone & 100 & $\cdot$ & 100 &. & $\begin{array}{l}100 \\
100\end{array}$ & • & $\begin{array}{l}100 \\
100\end{array}$ & $\bullet$ & FP3 & $\cdot$ & 100 & • \\
\hline 4-Methy1-2-pentanone & $\begin{array}{r}100 \\
50\end{array}$ & $\cdot$ & $\begin{array}{l}100 \\
0.6\end{array}$ & $0 . \dot{5}$ & FP1 & • & FP1 & - & 2 & 100 & 0.9 & 100 \\
\hline $\begin{array}{l}\text { Methylene chloride } \\
\text { Styrene }\end{array}$ & 50 & $\cdot$ & $5 v$ & $\cdot$ & su & - & 5v & - & 50 & $\cdot$ & 50 & - \\
\hline $\begin{array}{l}\text { Styrene } \\
\text { Tetrachloroethene }\end{array}$ & 1 & 0.8 & 1 & 0.8 & 50 & - & 5u & - & 50 & $\cdot$ & 5u & $\cdot$ \\
\hline Toluene & su & $\cdot$ & $5 v$ & $\cdot$ & 50 & - & 5v & • & 50 & $\cdot$ & 5u & • \\
\hline $1,1,1$-Trichloroethane & 0.5 & 0.4 & 0.5 & 0.4 & 5u & - & 5v & • & 50 & $\cdot$ & 50 & • \\
\hline 1,1,2-Trichloroethane & 50 & $\cdot$ & su & $\cdot$ & 50 & - & 50 & $\cdot$ & 50 & $\cdot$ & 50 & $\cdot$ \\
\hline Trichloroethene & 120 & 96.2 & 120 & 94 & 50 & • & 50 & - & 50 & $\cdot$ & 50 & - \\
\hline vinyl acetate & 100 & $\cdot$ & 100 & $\cdot$ & 100 & $\cdot$ & $\begin{array}{l}100 \\
100\end{array}$ & $\cdot$ & $\begin{array}{l}100 \\
100\end{array}$ &. & $\begin{array}{l}100 \\
100\end{array}$ & • \\
\hline vinyl chloride & $\begin{array}{r}100 \\
5 v\end{array}$ & & $\begin{array}{r}100 \\
50\end{array}$ &. & $\begin{array}{r}100 \\
50\end{array}$ & • & 50 & . & 50 &. & 5v & . \\
\hline Xylenes &. & & . &. & . & . & $\cdot$ & • & $\cdot$ &. & $\cdot$ & • \\
\hline Summed vOCs & 124.8 & 100.1 & 127.6 & 100 & 0 & - & 0 & • & 2 & 100 & 0.9 & 100 \\
\hline
\end{tabular}

(CONTINUED) 
APPENDIX $J$

Velidated voc Results, 1993

\begin{tabular}{|c|c|c|c|c|c|c|c|c|c|c|c|c|}
\hline \multirow{4}{*}{$\begin{array}{l}\text { Sampling Point } \\
\text { Location } \\
\text { Date Sampled }\end{array}$} & \multicolumn{4}{|c|}{ GW-621 } & \multicolumn{8}{|c|}{$G W-626$} \\
\hline & \multicolumn{4}{|c|}{ BG } & \multicolumn{8}{|c|}{ BG } \\
\hline & \multicolumn{2}{|c|}{$09 / 20 / 93$} & \multicolumn{2}{|c|}{$12 / 20 / 93$} & \multicolumn{2}{|c|}{$03 / 10 / 93$} & \multicolumn{2}{|c|}{$04 / 26 / 93$} & \multicolumn{2}{|c|}{$08 / 02 / 93$} & \multicolumn{2}{|c|}{$10 / 20 / 93$} \\
\hline & ug/I & $\&$ sum & ug/L & 8 sum & $\mathrm{ug} / \mathrm{L}$ & $8 \mathrm{sum}$ & ugil & s sum & ug/L & 8 sum & ug/L & sum \\
\hline Acetone & 100 & - & 100 & $\cdot$ & 1000 & $\cdot$ & 200 & $\cdot$ & 200 & - & 5 & 1.1 \\
\hline Benzene & 50 & • & FP3 & $\cdot$ & 500 & $\cdot$ & 100 & $\cdot$ & 100 & • & 100 & $\cdot$ \\
\hline Bromodich loromethane & 5u & • & 50 & $\cdot$ & 500 & $\cdot$ & 100 & $\cdot$ & 100 & $\cdot$ & 100 & $\cdot$ \\
\hline 2-Butanone & 100 & - & FP1 & $\cdot$ & 1000 & $\cdot$ & 200 & $\cdot$ & 200 & $\cdot$ & 23 & 4.9 \\
\hline Carbon disulfide & 50 & . & 50 & $\cdot$ & 500 & $\cdot$ & 100 & $\cdot$ & 100 & $\cdot$ & 100 & $\cdot$ \\
\hline Carbon tetrachloride & 50 & $\cdot$ & FP3 & $\cdot$ & 500 & $\cdot$ & 100 & $\cdot$ & 100 & $\cdot$ & 100 & $\cdot$ \\
\hline Chlorobenzene & 5u & - & 50 & • & 500 & $\cdot$ & 100 & $\cdot$ & 100 & $\cdot$ & 100 & $\cdot$ \\
\hline Chloroform & 50 & $\cdot$ & 50 & • & 500 & $\cdot$ & 100 &. & 100 & $\cdot$ & 100 & $\cdot$ \\
\hline 1,1-Dichloroethane & 50 & - & $5 U$ & $\cdot$ & 51 & 3.2 & 18 & 3.6 & 19 & 5.5 & 19 & 4 \\
\hline 1,2-Dichloroethane & 5u & $\cdot$ & 50 & $\cdot$ & 500 & $\cdot$ & 4 & 0.8 & 100 & $\cdot$ & 3 & 0.6 \\
\hline 1,1-Dichloroethene & 5v & $\cdot$ & 5u & $\cdot$ & 28 & 1.7 & 6 & 1.2 & 9 & 2.6 & 12 & 2.5 \\
\hline 1,2-Dichloroethene & 50 & $\cdot$ & su & $\cdot$ & 1200 & 74.2 & 400 & 80.3 & 250 & 71.8 & 330 & 69.8 \\
\hline Ethylbenzene & 50 & $\cdot$ & 50 & $\cdot$ & 500 & $\cdot$ & 100 & $\cdot$ & 100 & $\cdot$ & 100 & - \\
\hline 2-Hexanone & 100 & $\cdot$ & 100 & $\cdot$ & 1000 & $\cdot$ & 200 & $\cdot$ & 200 & $\cdot$ & 200 & • \\
\hline 4-Methyl-2-pentanone & 100 & $\cdot$ & 100 & $\cdot$ & 1000 & $\cdot$ & 200 & $\cdot$ & 200 & $\cdot$ & 200 & • \\
\hline Methylene chloride & 5u & $\cdot$ & 50 & • & 500 & $\cdot$ & 3 & 0.6 & 100 & $\cdot$ & 7 & 1.5 \\
\hline Styrene & 50 & $\cdot$ & su & $\cdot$ & 500 & $\cdot$ & 100 & $\cdot$ & 100 & $\cdot$ & 100 & $\cdot$ \\
\hline Tetrachloroethene & 50 & $\cdot$ & 50 & $\cdot$ & 140 & 8.7 & 29 & 5.8 & 29 & 8.3 & 32 & 6.8 \\
\hline Toluene & 50 & $\cdot$ & 50 & $\cdot$ & 500 & $\cdot$ & 100 & $\cdot$ & 100 & $\cdot$ & 100 & - \\
\hline 1,1,1-Trichloroethane & 50 & $\cdot$ & su & $\cdot$ & 17 & 1.1 & 4 & 0.8 & 6 & 1.7 & 6 & 1.3 \\
\hline 1,1,2-Trichloroethane & 5u & - & 50 & $\cdot$ & 500 & $\cdot$ & 100 & $\cdot$ & 100 & $\cdot$ & 100 & • \\
\hline Trichloroethene & FP3 & $\cdot$ & 50 & $\cdot$ & 110 & 6.8 & 34 & 6.8 & 35 & 10.1 & 36 & 7.6 \\
\hline Vinyl acetate & 100 & - & 100 & - & 1000 & $\cdot$ & 200 & $\cdot$ & 200 & $\cdot$ & 200 & $\bullet$ \\
\hline vinyl chloride & 100 & - & 100 & $\cdot$ & 72 & 4.4 & 200 & $\cdot$ & 200 & $\cdot$ & 200 & • \\
\hline Xylenes & 50 & - & $5 v$ & $\cdot$ & 500 & $\cdot$ & 100 & $\cdot$ & 100 & $\cdot$ & 100 & - \\
\hline Summed & 0 & . & 0 & - & 1618 & 100.1 & 498 & 99.9 & 348 & 100 & 473 & 100.1 \\
\hline
\end{tabular}

(CONTINUED) 
APPELUIX $J$

Valideted voC Results, 1993

\begin{tabular}{|c|c|c|c|c|c|c|c|c|c|c|c|c|c|}
\hline \multirow{4}{*}{$\begin{array}{l}\text { Sampling Point } \\
\text { Location } \\
\text { Date Sampled }\end{array}$} & \multicolumn{8}{|c|}{ GW-627 } & \multicolumn{5}{|c|}{$G w-636$} \\
\hline & \multicolumn{8}{|c|}{ BG } & \multicolumn{5}{|c|}{ OLF } \\
\hline & \multicolumn{2}{|c|}{$03 / 09 / 93$} & \multicolumn{2}{|c|}{$04 / 23 / 93$} & \multicolumn{2}{|c|}{$07 / 28 / 93$} & \multicolumn{2}{|c|}{$10 / 20 / 93$} & \multicolumn{2}{|c|}{$03 / 26 / 93$} & \multicolumn{3}{|c|}{$06 / 27 / 93$} \\
\hline & $u g / L$ & 8 sum & ug/I & 8 sum & $\mathrm{ug} / \mathrm{L}$ & 8 sum & ug/L & sum & $u g / L$ & 8 sum & ug/L & 8 sum & \\
\hline Acetone & 100 & - & 100 & $\cdot$ & 100 & $\cdot$ & FP1 & $\cdot$ & 100 & $\cdot$ & 100 & & $\cdot$ \\
\hline Benzene & 50 & - & 50 & $\cdot$ & 50 & $\cdot$ & su & $\cdot$ & 50 & $\cdot$ & 5u & & $\cdot$ \\
\hline Bromodichloromethane & 52 & - & su & $\cdot$ & 50 & $\cdot$ & 50 & $\cdot$ & 5u & $\cdot$ & 5u & & $\cdot$ \\
\hline 2-Butanone & 100 & - & 100 & $\cdot$ & 100 & $\cdot$ & FP1 & $\cdot$ & 100 & $\cdot$ & 100 & & $\cdot$ \\
\hline Carbon disulfide & su & - & 50 & $\cdot$ & so & $\cdot$ & 50 & $\cdot$ & 50 & $\cdot$ & 50 & & $\cdot$ \\
\hline Carbon tetrachloride & 50 & - & 5v & $\cdot$ & 50 & $\cdot$ & 50 & $\cdot$ & 50 & $\cdot$ & 50 & & $\cdot$ \\
\hline Chlorobenzene & 50 & - & 50 & $\cdot$ & 50 & $\cdot 1$ & 50 & $\cdot$ & 50 & $\cdot$ & 50 & & $\cdot$ \\
\hline Chloroform & su & - & 50 & $\cdot$ & 50 & $\cdot$ & 50 & $\cdot$ & 50 & $\cdot$ & 50 & & $\cdot$ \\
\hline 1,1-Dichloroethane & 3 & 6.3 & 5 & 7.9 & 6 & 6.7 & 3 & 6.7 & 50 & $\cdot$ & 50 & & $\cdot$ \\
\hline 1,2-Dichloroethane & su & $\cdot$ & $5 v$ & $\cdot$ & 50 & $\cdot$ & 50 & $\cdot$ & 50 & $\cdot$ & 50 & & $\cdot$ \\
\hline 1,1-Dichloroethene & 50 & $\cdot$ & 50 & $\cdot$ & FP3 & $\cdot 1$ & 5u & $\cdot$ & 50 & $\cdot$ & 50 & & $\cdot$ \\
\hline 1,2-Dichloroethene & 50 & $\cdot$ & 5v & $\cdot$ & 50 & $\cdot$ & 50 & $\cdot$ & 50 & $\cdot$ & 50 & & $\cdot$ \\
\hline Ethylbenzene & 50 & $\cdot$ & 50 & $\cdot$ & 5u & $\cdot$ & su & $\cdot$ & 50 & $\cdot$ & so & & $\cdot$ \\
\hline 2-Hexanone & 100 & - & 100 & $\cdot$ & 100 & $\cdot$ & 100 & $\cdot$ & 100 & $\cdot$ & 100 & & $\cdot$ \\
\hline 4-Methyl-2-pentanone & FP1 & $\cdot$ & 100 & $\cdot 1$ & 100 & $\cdot$ & 100 & $\cdot$ & 100 & $\cdot$ & 100 & & $\cdot$ \\
\hline Methylene chloride & 5u & $\cdot$ & 50 & $\cdot$ & FP1 & $\cdot$ & FP1 & $\cdot$ & 50 & $\cdot$ & 50 & & $\cdot$ \\
\hline Styrene & 5u & - & $5 \mathrm{v}$ & $\cdot$ & 50 & $\cdot$ & 50 & $\cdot$ & 5u & $\cdot$ & 5v & & $\cdot$ \\
\hline Tetrachloroethene & 39 & 81.3 & 51 & 81 & 74 & 82.2 & 37 & 82.2 & 50 & $\cdot$ & 50 & & $\cdot$ \\
\hline Toluene & 5u & • & $5 v$ & $\cdot$ & 5u & $\cdot$ & 50 & $\cdot$ & 50 & $\cdot$ & 50 & & $\cdot$ \\
\hline $1,1,1$-Trichloroethane & 50 & $\cdot$ & $5 v$ & $\cdot$ & 50 & $\cdot$ & 5u & $\cdot$ & 50 & $\cdot$ & 5u & & $\cdot$ \\
\hline 1,1,2-Trichloroethane & 50 & $\cdot$ & 50 & $\cdot$ & $5 v$ & $\cdot 1$ & 50 & $\cdot$ & 50 & $\cdot$ & $5 v$ & & $\cdot$ \\
\hline Trichloroethene & 6 & 12.5 & 7 & 11.1 & 10 & 11.1 & 5 & 11.1 & 50 & $\cdot$ & 5u & & $\cdot$ \\
\hline vinyl acetate & 100 & - & 100 & $\cdot 1$ & 100 & $\cdot$ & 100 & $\cdot$ & 100 & • & 100 & & $\cdot$ \\
\hline vinyl chloride & 100 & . & 100 & $\cdot 1$ & FP3 & $\cdot$ & 200 & $\cdot$ & 100 & • & 100 & & - \\
\hline Xylenes & $5 \mathrm{U}$ & . & 50 & $\cdot$ & 50 & $\cdot$ & 50 & $\cdot$ & 50 & • & 50 & & - \\
\hline Summed VOCs & 48 & 100.1 & 63 & 100 & 90 & 100 & 45 & 100 & 0 & • & 0 & & - \\
\hline
\end{tabular}

(CONTINUED) 
Validated vOC Results, 1993

\begin{tabular}{|c|c|c|c|c|c|c|c|c|c|c|c|c|c|}
\hline \multirow{4}{*}{$\begin{array}{l}\text { Sampling Point } \\
\text { Location } \\
\text { Date Sampled }\end{array}$} & \multicolumn{4}{|c|}{$G W-636$} & \multicolumn{9}{|c|}{$G w-637$} \\
\hline & \multicolumn{4}{|c|}{ OLF } & \multicolumn{9}{|c|}{ OLF } \\
\hline & \multicolumn{2}{|c|}{$09 / 18 / 93$} & \multicolumn{2}{|c|}{$12 / 18 / 93$} & \multicolumn{2}{|c|}{$03 / 25 / 93$} & \multicolumn{2}{|c|}{$06 / 26 / 93$} & \multicolumn{2}{|c|}{$09 / 17 / 93$} & \multicolumn{3}{|c|}{$12 / 18 / 93$} \\
\hline & $\mathrm{ug} / \mathrm{L}$ & 8 sum & ug/t & 8 sum & ug $/ \mathrm{L}$ & sum & ug $/ \mathbf{L}$ & 8 sum & $u g / L$ & 8 sum & $u g / x$ & s sum & \\
\hline Acetone & 100 & - & FP1 & . & 100 & $\cdot$ & 100 & $\cdot$ & 100 & $\cdot$ & 200 & & $\cdot$ \\
\hline Benzene & 50 & • & 50 & - & 50 & $\cdot$ & 50 & $\cdot$ & 50 & $\cdot$ & 50 & & $\cdot$ \\
\hline Bromodichloromethane & 50 & - & 5u & $\cdot$ & 50 & $\cdot$ & 50 & $\cdot$ & 50 & $\cdot$ & $5 v$ & & $\cdot$ \\
\hline 2-Butanone & 100 & - & FP1 & - & 100 & $\cdot 1$ & 100 & $\cdot$ & 100 & $\cdot$ & FP1 & & $\cdot$ \\
\hline Carbon disulfide & 50 & - & $5 v$ & - & $5 v$ & $\cdot$ & 50 & $\cdot$ & 50 & $\cdot$ & 50 & & $\cdot$ \\
\hline Carbon tetrachloride & 50 & - & 50 & - & su & $\cdot$ & 50 & $\cdot$ & 50 & $\cdot$ & 50 & & $\cdot$ \\
\hline Chiorobenzene & 50 & • & 5u & - & 5u & $\cdot$ & 5u & $\cdot$ & 50 & $\cdot$ & 50 & & $\cdot$ \\
\hline Chloroform & 50 & - & $5 u$ & - & 50 & $\cdot$ & 5u & $\cdot$ & 50 & $\cdot$ & 50 & & $\cdot$ \\
\hline 1,1-Dichloroethane & 50 & • & 50 & - & 50 & $\cdot$ & 50 & $\cdot$ & 50 & $\cdot$ & 50 & & $\cdot$ \\
\hline 1,2-Dichloroethane & 50 & - & 50 & - & 50 & $\cdot$ & 50 & $\cdot$ & 50 & $\cdot$ & 50 & & $\cdot$ \\
\hline 1,1-Dichloroethene & 50 & - & 50 & - & 50 & $\cdot$ & 5u & $\cdot$ & 50 & $\cdot$ & 50 & & $\cdot$ \\
\hline 1,2-Dichloroethene & su & - & 50 & - & 50 & $\cdot$ & 50 & $\cdot$ & 50 & $\cdot$ & 50 & & $\cdot$ \\
\hline Ethylbenzene & su & • & 50 & - & 50 & $\cdot$ & 50 & $\cdot$ & 5u & $\cdot$ & 50 & & $\cdot$ \\
\hline 2-Hexanone & 100 & - & 100 & - & 100 & $\cdot$ & 100 & $\cdot$ & 100 & $\cdot$ & 100 & & $\cdot$ \\
\hline 4-Methy1-2-pentanone & 100 & • & 100 & - & 100 & $\cdot$ & 100 & $\cdot$ & 100 & $\cdot$ & 100 & & - \\
\hline Methylene chloride & FP3 & - & FP1 & - & sv & $\cdot$ & 50 & $\cdot$ & 50 & $\cdot$ & FP1 & & $\cdot$ \\
\hline Styrene & 50 & - & $5 \mathbf{U}$ & - & 50 & $\cdot$ & 50 & $\cdot$ & 50 & $\cdot$ & 50 & & $\cdot$ \\
\hline Tetrachloroethene & 5u & • & 5u & - & 5u & $\cdot$ & 50 & $\cdot$ & 50 & $\cdot$ & 50 & & $\cdot$ \\
\hline Toluene & 50 & • & 5u & • & 50 & $\cdot$ & 50 & $\cdot$ & 50 & $\cdot$ & 50 & & - \\
\hline 1,1,1-Trichloroethane & 5u & - & 5u & - & 5u & $\cdot$ & 50 & $\cdot$ & 5u & $\cdot$ & 50 & & $\cdot$ \\
\hline 1,1,2-Trichloroethane & 50 & - & 5u & • & su & $\cdot$ & 50 & $\cdot$ & 50 & $\cdot$ & 50 & & - \\
\hline Trichloroethene & $5 u$ & - & 50 & $\cdot$ & 5u & $\cdot$ & 50 & $\cdot$ & 50 & $\cdot$ & 50 & & $\cdot$ \\
\hline Vinyl acetate & 100 & • & 100 & - & 100 & $\cdot$ & 100 & $\cdot$ & 100 & $\cdot$ & 100 & & $\cdot$ \\
\hline vinyl chloride & 100 & • & 100 & - & 100 & $\cdot$ & 100 & $\cdot$ & 100 & $\cdot$ & 100 & & $\cdot$ \\
\hline xylenes & 50 & - & $5 v$ & - & 5u & $\cdot$ & 50 & $\cdot$ & 50 & - & 50 & & $\cdot$ \\
\hline Summed vOCs & 0 & - & 0 & . & 0 & $\cdot$ & 0 & $\cdot$ & 0 & - & 0 & & $\cdot$ \\
\hline
\end{tabular}

(CONTINued) 
validated VOC Results, 1993

\begin{tabular}{|c|c|c|c|c|c|c|c|c|c|c|c|c|c|}
\hline \multirow{4}{*}{$\begin{array}{l}\text { Sampling point } \\
\text { Location } \\
\text { Date Sampled }\end{array}$} & \multicolumn{8}{|c|}{$G W-638$} & \multicolumn{5}{|c|}{ GW-639 } \\
\hline & \multicolumn{8}{|c|}{ OLF } & \multicolumn{5}{|c|}{ BG } \\
\hline & \multicolumn{2}{|c|}{$03 / 25 / 93$} & \multicolumn{2}{|c|}{$06 / 26 / 93$} & \multicolumn{2}{|c|}{$09 / 17 / 93$} & \multicolumn{2}{|c|}{$12 / 18 / 93$} & \multicolumn{2}{|c|}{$02 / 24 / 93$} & \multicolumn{3}{|c|}{$05 / 24 / 93$} \\
\hline & $\mathrm{ug} / \mathrm{L}$ & 8 sum & $u g / I$ & s sum & ug/I & 8 sum & $\mathrm{ug} / \mathrm{I}$ & 8 sum & ug/I & 8 sum & $u g / L$ & 850 & \\
\hline Acetone & 100 & - & 100 & - & 100 & - & 100 & - & 100 & - & 100 & & - \\
\hline Benzene & 5u & $\cdot$ & 50 & - & 50 & - & 5v & - & 50 & - & $5 \mathbf{u}$ & & $\cdot$ \\
\hline Bromodichloromethane & 50 & $\cdot$ & 50 & $\cdot$ & 50 & $\cdot$ & 50 & - & 50 & - & 50 & & $\cdot$ \\
\hline 2-Butanone & 100 & $\cdot$ & 100 & • & 100 & $\cdot$ & FP1 & - & 100 & • & FP3 & & $\cdot$ \\
\hline Carbon disulfide & 50 & - & 50 & $\cdot$ & 50 & $\cdot$ & 50 & - & 5u & - & 50 & & $\cdot$ \\
\hline Carbon tetrachloride & 50 & $\cdot$ & 50 & $\cdot$ & 50 & $\cdot$ & 50 & $\cdot$ & 50 & - & 50 & & $\cdot$ \\
\hline Chlorobenzene & 50 & $\cdot$ & 50 & $\cdot$ & 50 & - & $5 \mathbf{v}$ & - & $\mathbf{5 u}$ & - & 50 & & $\cdot$ \\
\hline Chloroform & 50 & $\cdot$ & 50 & $\cdot$ & 50 & $\cdot$ & 5u & • & 5u & - & 50 & & - \\
\hline 1,1-Dichloroethane & 50 & $\cdot$ & 50 & - & 50 & $\cdot$ & su & • & 50 & - & $\mathbf{5 u}$ & & $\bullet$ \\
\hline 1,2-Dichloroethane & 50 & $\cdot$ & 50 & $\cdot$ & 50 & $\cdot$ & 50 & • & 50 & - & 50 & & $\cdot$ \\
\hline 1,1-Dichloroethene & 50 & $\cdot$ & 50 & $\cdot$ & 50 & $\cdot$ & 50 & - & 50 & - & 50 & & $\cdot$ \\
\hline 1,2-Dichloroethene & 50 & $\cdot$ & 50 & $\cdot$ & 50 & $\cdot$ & 50 & $\cdot$ & 50 & $\bullet$ & 50 & & $\cdot$ \\
\hline Ethylbenzene & 5u & $\cdot$ & 50 & - & 5v & $\cdot$ & 50 & $\cdot$ & 50 & $\cdot$ & 50 & & - \\
\hline 2-Hexanone & 100 & $\cdot$ & 100 & - & 100 & - & 100 & - & 100 & - & 100 & & $\cdot$ \\
\hline 4-Methy 1-2-pentanone & 100 & $\cdot$ & 100 & - & 100 & $\cdot$ & 100 & $\cdot$ & 100 & - & FP3 & & - \\
\hline Methylene chloride & 50 & $\cdot$ & 50 & - & so & - & 50 & - & FP3 & - & 50 & & • \\
\hline Styrene & 50 & $\cdot$ & 50 & - & $5 v$ & $\cdot$ & 50 & $\cdot$ & 50 & - & 50 & & $\cdot$ \\
\hline Tetrachloroethene & 50 & . & 50 & - & 50 & - & 50 & - & 50 & - & 50 & & $\cdot$ \\
\hline Toluene & 5u & $\cdot$ & 5u & - & 50 & $\cdot$ & $5 \mathbf{v}$ & $\cdot$ & 50 & - & 50 & & $\cdot$ \\
\hline 1,1,1-Trichloroethane & 50 & - & 50 & - & 50 & - & 50 & $\cdot$ & 50 & $\cdot$ & 50 & & $\cdot$ \\
\hline $1,1,2-\operatorname{Tr}$ ichloroethane & 50 & $\cdot$ & 50 & - & 50 & - & so & • & 50 & • & 50 & & $\cdot$ \\
\hline Trichloroethene & 50 & $\cdot$ & 50 & - & 50 & - & 5u & - & 5u & - & 50 & & $\cdot$ \\
\hline Vinyl acetate & 100 & $\cdot$ & 100 & - & 100 & - & 100 & • & 100 & $\cdot$ & 100 & & $\cdot$ \\
\hline Vinyl chloride & 100 & . & 100 & - & 100 & - & 100 & • & 100 & • & 100 & & - \\
\hline xylenes & 50 & - & 50 & - & 50 & - & 50 & - & 50 & • & 50 & & - \\
\hline Surmed VOCs & 0 & - & 0 & - & o & - & 0 & • & 0 & - & 0 & & - \\
\hline
\end{tabular}

(CONTINUED) 
APPENDIX J

Validated VOC Results, 1993

\begin{tabular}{|c|c|c|c|c|c|c|c|c|c|c|c|c|}
\hline \multirow{4}{*}{$\begin{array}{l}\text { Sampling Point } \\
\text { Location } \\
\text { Date Sampled }\end{array}$} & \multicolumn{4}{|c|}{ GW-639 } & \multicolumn{8}{|c|}{ GW-640 } \\
\hline & \multicolumn{4}{|c|}{ BG } & \multicolumn{8}{|c|}{ BG } \\
\hline & \multicolumn{2}{|c|}{$07 / 11 / 93$} & \multicolumn{2}{|c|}{$10 / 15 / 93$} & \multicolumn{2}{|c|}{$02 / 22 / 93$} & \multicolumn{2}{|c|}{$05 / 24 / 93$} & \multicolumn{2}{|c|}{$07 / 10 / 93$} & \multicolumn{2}{|c|}{$10 / 14 / 93$} \\
\hline & $\mathrm{ug} / \mathrm{L}$ & 8 sum & $\mathrm{ug} / \mathrm{L}$ & 8 sum & $\mathrm{ug} / \mathrm{L}$ & 8 sum & $\mathrm{ug} / \mathrm{L}$ & 8 sum & $\mathbf{u g} / \mathbf{L}$ & ร sum & $\operatorname{ug} / \mathrm{L}$ & sum \\
\hline Benzene & 50 &. & 5u & • & 50 & $\cdot$ & 50 & $\cdot$ & 50 & $\cdot$ & 5v & • \\
\hline Bromodichloromethane & 50 & $\cdot$ & 50 & - & 50 & $\cdot$ & 50 & $\cdot$ & 5v & $\cdot$ & 50 & • \\
\hline 2-Butanone & 100 & $\cdot$ & 100 & - & 100 & $\cdot$ & FP1 & $\cdot$ & 100 & $\cdot$ & FP1 & • \\
\hline Carbon disulfide & su & $\cdot$ & 50 & - & su & $\cdot$ & su & $\cdot$ & 5v & $\cdot$ & 50 & • \\
\hline Carbon tetrachloride & 50 & $\cdot 1$ & 50 & - & 5v & $\cdot$ & 50 & $\cdot 1$ & 50 & $\cdot 1$ & 50 & • \\
\hline Chlorobenzene & 50 & $\cdot$ & 50 & - & so & $\cdot$ & 50 & $\cdot$ & 50 & $\cdot$ & 50 & - \\
\hline Chloroform & 50 & $\cdot 1$ & 5u & . & 50 & $\cdot$ & 50 & $\cdot$ & so & $\cdot$ & 50 & • \\
\hline 1,1-Dichloroethane & 5u & $\cdot$ & 50 & • & 50 & $\cdot$ & 50 & $\cdot$ & 50 & $\cdot$ & so & • \\
\hline 1,1-Dichloroethene & 5u & $\cdot 1$ & 50 & - & 5v & $\cdot$ & 50 & $\cdot$ & 5u & $\cdot$ & 50 & • \\
\hline 1,2-Dichloroethene & 5u & $\cdot$ & 5u & - & 50 & $\cdot$ & 5u & $\cdot$ & 50 & $\cdot$ & 50 & • \\
\hline Ethylbenzene & 50 & $\cdot$ & 50 & - & 50 & $\cdot$ & $5 v$ & $\cdot$ & 50 & $\cdot$ & 50 & $\cdot$ \\
\hline 2-Hexanone & 100 &. & 100 & • & 100 & $\cdot$ & 100 & $\cdot$ & 100 & $\cdot$ & 100 & • \\
\hline 4-Methyl-2-pentanone & 100 &. & 100 & - & 100 &. & 100 & $\cdot$ & 100 & $\cdot$ & 100 & • \\
\hline Methylene chloride & so & $\cdot$ & 50 & - & FP1 & $\cdot$ & 5u & $\cdot 1$ & su & $\cdot$ & FP1 & • \\
\hline Styrene & 50 & $\cdot$ & 50 & - & 50 & $\cdot$ & 50 & $\cdot$ & so & $\cdot$ & 50 & • \\
\hline Tetrachloroethene & 50 & $\cdot$ & 50 & - & 50 & $\cdot$ & 50 & $\cdot$ & 5u & $\cdot 1$ & 50 & • \\
\hline Toluene & 50 & $\cdot$ & 5u & - & so & $\cdot$ & 50 & $\cdot$ & 50 & $\cdot$ & 50 & • \\
\hline $1,1,1$-Trichloroethane & 5v & $\cdot$ & su & - & 50 & $\cdot$ & 50 & $\cdot$ & 50 & $\cdot$ & 50 & • \\
\hline 1,1,2-Trichloroethane & 50 & $\cdot$ & 50 & - & 50 & $\cdot$ & 5u & $\cdot$ & 50 & $\cdot$ & so & • \\
\hline Trichloroethene & 50 & $\cdot$ & 50 & - & 5v & $\cdot 1$ & 5u & $\cdot$ & $5 \mathbf{v}$ & $\cdot$ & 50 & • \\
\hline vinyl acetate & 100 &. & 100 & - & 100 & $\cdot$ & 100 & $\cdot$ & 100 & .1 & 100 & • \\
\hline Vinyl chloride & 100 & $\cdot$ & 100 & - & 100 & $\cdot$ & 100 & $\cdot$ & 100 & $\cdot$ & 100 & • \\
\hline xylenes & 50 & $\cdot$ & 50 & - & 50 & $\cdot$ & 50 & $\cdot$ & so & $\cdot$ & 50 & • \\
\hline Summed vOCs & $\dot{0}$ &. & 0 & . & 0 &. & 0 &. & 0 &. & 0 & • \\
\hline
\end{tabular}

(CONTINUED) 
Validated VOC Results, 1993

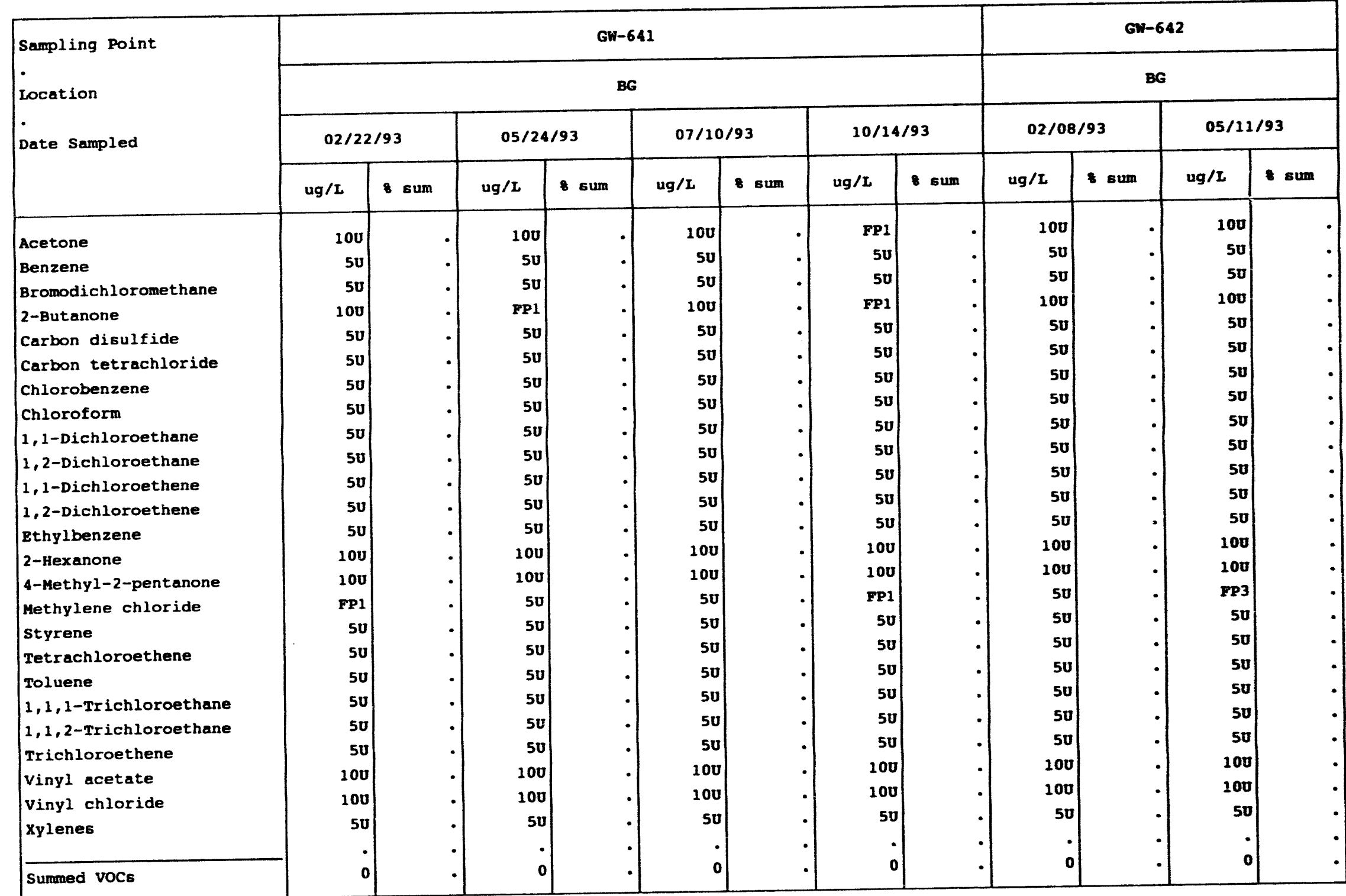

(CONTINUED) 
Validated VOC Results, 1993

\begin{tabular}{|c|c|c|c|c|c|c|c|c|c|c|c|c|c|}
\hline \multirow{4}{*}{$\begin{array}{l}\text { Sampling Point } \\
\text { Location } \\
\text { Date Sampled }\end{array}$} & \multicolumn{4}{|c|}{$G W-642$} & \multicolumn{9}{|c|}{ GW-643 } \\
\hline & \multicolumn{4}{|c|}{ BG } & \multicolumn{9}{|c|}{ BG } \\
\hline & \multicolumn{2}{|c|}{$07 / 01 / 93$} & \multicolumn{2}{|c|}{$20 / 10 / 93$} & \multicolumn{2}{|c|}{$02 / 16 / 93$} & \multicolumn{2}{|c|}{$05 / 19 / 93$} & \multicolumn{2}{|c|}{$07 / 08 / 93$} & \multicolumn{3}{|c|}{$10 / 12 / 93$} \\
\hline & $u g / r$ & 8 sum & $\mathrm{ug} / \mathrm{L}$ & 8 sum & $\operatorname{ug} / 2$ & sum & $u g / L$ & sum & $\operatorname{ug} / \mathrm{L}$ & sum & $u g / L$ & $8 s=$ & \\
\hline Acetone & 100 & • & FP1 & - & 100 & $\cdot 1$ & 100 & - & 100 & $\cdot$ & 100 & & $\bullet$ \\
\hline Benzene & 50 & - & 5u & - & 50 & $\cdot$ & 5u & - & 50 & $\cdot$ & 50 & & $\cdot$ \\
\hline Bromodichloromethane & su & - & 50 & $\cdot$ & 5u & $\cdot 1$ & 5v & $\cdot$ & 50 & $\cdot$ & 50 & & $\cdot$ \\
\hline 2-Butanone & 100 & - & FP1 & - & 100 & $\cdot 1$ & 100 & - & 100 & $\cdot 1$ & 100 & & $\cdot$ \\
\hline Carbon disulfide & 50 & - & so & - & 50 & $\cdot$ & 50 & - & 5v & $\cdot$ & 50 & & $\cdot$ \\
\hline Carbon tetrachloride & 50 & - & 50 & - & su & $\cdot$ & 5v & - & 50 & $\cdot$ & 501 & & $\cdot$ \\
\hline Chlorobenzene & 5u & - & so & - & 5v & $\cdot$ & 50 & $\cdot$ & 5v & $\cdot$ & 50 & & $\cdot$ \\
\hline Chloroform & 5u & - & 50 & • & 5v & $\cdot$ & 5u & - & 50 & $\cdot$ & 50 & & $\cdot$ \\
\hline 1,1-Dichloroethane & 50 & - & 50 & - & 50 & $\cdot$ & 50 & - & 50 & $\cdot 1$ & 50 & & $\cdot$ \\
\hline 1,2-Dichloroethane & 50 & - & 50 & - & 5v & $\cdot$ & 50 & - & 50 & $\cdot$ & so & & - \\
\hline 1,1-Dichloroethene & 50 & - & 50 & • & 50 & $\cdot$ & 50 & • & 50 & $\cdot$ & 50| & & $\cdot$ \\
\hline 1,2-Dichloroethene & 50 & - & 50 & - & 50 & $\cdot$ & 50 & • & 50 & $\cdot$ & 50| & & - \\
\hline Ethylbenzene & 50 & - & 50 & $\cdot$ & 50 & $\cdot$ & 50 & $\bullet$ & 50 & $\cdot 1$ & 50 & & $\cdot$ \\
\hline 2-Hexanone & 100 & - & 100 & - & 100 & $\cdot 1$ & 100 & - & 100 & $\cdot$ & 100 & & - \\
\hline 4-Methyl-2-pentanone & 100 & - & 100 & - & 100 & $\cdot$ & 100 & - & 100 & $\cdot$ & 100 & & $\cdot$ \\
\hline Methylene chloride & 5u & - & FP1 & • & FP2 & $\cdot$ & 50 & $\cdot$ & so & $\cdot$ & 50 & & $\cdot$ \\
\hline styrene & 50 & - & 5u & - & 50 & $\cdot$ & 50 & - & 50 & $\cdot$ & 50 & & $\cdot$ \\
\hline Tetrachloroethene & 50 & - & 50 & - & 50 & $\cdot$ & 50 & - & 50 & $\cdot$ & 50 & & - \\
\hline Toluene & so & - & 50 & - & 5u & $\cdot$ & 50 & - & 50 & $\cdot 1$ & 50 & & $\cdot$ \\
\hline 1,1,1-Trichloroethane & 50 & - & 5u & $\cdot$ & 50 & $\cdot$ & 50 & - & 50 & $\cdot$ & 50 & & - \\
\hline 1,1,2-Trichloroethane & 50 & - & so & - & 50 & $\cdot$ & 50 & - & 50 & $\cdot 1$ & so & & - \\
\hline Trichloroethene & 50 & - & 50 & $\cdot$ & 50 & $\cdot$ & 50 & - & 50 & $\cdot$ & 50 & & $\cdot$ \\
\hline Vinyl acetate & 100 & - & 100 & $\cdot$ & 100 & $\cdot 1$ & 100 & - & 100 & $\cdot 1$ & 100 & & $\cdot$ \\
\hline vinyl chloride & 100 & - & 100 & . & 100 &. & 100 & - & 100 & $\cdot 1$ & 100 & & - \\
\hline xylenes & 50 & - & 50 & - & 50 & $\cdot$ & 50 & - & 50 & $\cdot 1$ & FP3 & & $\cdot 1$ \\
\hline Summed VOCs & 0 & . & o) & . & o &. & o & - & o & - & o) & & $\bullet$ \\
\hline
\end{tabular}

(CONTINUED) 
Validated VOC Results, 1993

\begin{tabular}{|c|c|c|c|c|c|c|c|c|c|c|c|c|c|}
\hline \multirow{4}{*}{$\begin{array}{l}\text { Sampling Point } \\
\text { Location } \\
\text { Date Sampled }\end{array}$} & \multicolumn{8}{|c|}{$G w-647$} & \multicolumn{5}{|c|}{$6 w-652$} \\
\hline & \multicolumn{8}{|c|}{ OLF } & \multicolumn{5}{|c|}{ B6 } \\
\hline & \multicolumn{2}{|c|}{$02 / 08 / 93$} & \multicolumn{2}{|c|}{$05 / 17 / 93$} & \multicolumn{2}{|c|}{$09 / 25 / 93$} & \multicolumn{2}{|c|}{$12 / 10 / 93$} & \multicolumn{2}{|c|}{$03 / 01 / 93$} & \multicolumn{3}{|c|}{$04 / 19 / 93$} \\
\hline & $u g / L$ & $8 \mathrm{sum}$ & ug/L & sum & ug/I & $8 \mathrm{sum}$ & ug/t & sum & $u g / L$ & sum & ug/L & s sum & \\
\hline Acetone & 100 & - & 100 & - & 100 & - & 100 & - & 100 & - & 100 & & $\cdot$ \\
\hline Benzene & 50 & - & 5u & $\cdot$ & 50 & $\cdot$ & 50 & $\cdot$ & 50 & - & 50 & & $\cdot$ \\
\hline Bromodichloromethane & 50 & - & 50 & $\cdot$ & so & $\cdot$ & 50 & $\cdot$ & 50 & $\cdot$ & 50 & & $\cdot$ \\
\hline 2-Butanone & 100 & - & 100 & $\cdot$ & 100 & $\cdot$ & 100 & - & 100 & $\cdot$ & 100 & & $\cdot$ \\
\hline Carbon disulfide & 50 & - & 50 & $\cdot$ & 50 & $\cdot$ & 50 & $\cdot$ & 50 & $\cdot$ & 50 & & $\cdot$ \\
\hline Carbon tetrachloride & 5u & - & 50 & $\cdot$ & 50 & $\cdot$ & 50 & $\cdot$ & 50 & $\bullet$ & 50 & & $\cdot$ \\
\hline Chlorobenzene & 50 & • & 50 & $\cdot$ & 50 & $\cdot$ & 50 & $\cdot$ & 50 & $\cdot$ & 50 & & $\bullet$ \\
\hline Chloroform & 50 & - & 50 & $\cdot$ & 50 & $\cdot$ & 50 & $\cdot$ & 50 & $\bullet$ & 50 & & $\bullet$ \\
\hline 1,1-Dichloroethane & 5u & • & 50 & $\cdot$ & 50 & $\cdot$ & 50 & $\cdot$ & 50 & $\bullet$ & $\begin{array}{c}50 \\
50\end{array}$ & & $\bullet$ \\
\hline 1,2-Dichloroethane & 50 & - & 50 & $\cdot$ & 50 & $\cdot$ & 50 & $\cdot$ & 50 & $\bullet$ & 30 & & $\bullet$ \\
\hline 1,1-Dichloroethene & 50 & $\cdot$ & 50 & $\cdot$ & 50 & $\cdot$ & 50 & $\cdot$ & $\begin{array}{l}20 \\
50\end{array}$ & $\bullet$ & 30 & &. \\
\hline 1,2-Dichloroethene & 50 & $\cdot$ & 50 & $\cdot$ & $\begin{array}{l}50 \\
50\end{array}$ &. & 50 &. & 50 &. & 50 & & . \\
\hline $\begin{array}{l}\text { Bthylbenzene } \\
\text { 2-Hexanone }\end{array}$ & 100 & . & 100 &. & 100 &. & 100 &. & 100 & - & 100 & & . \\
\hline 4-Methyl-2-pentanone & 100 & - & 100 &. & 100 &. & 100 & $\cdot$ & 100 & - & 100 & & $\cdot$ \\
\hline Hethylene chloride & 50 & - & 50 & $\cdot 1$ & 5u & $\cdot$ & FP1 & $\cdot$ & 50 & - & 50 & & $\cdot$ \\
\hline styrene & 50 & - & 5o & $\cdot$ & 5v & $\cdot$ & 50 & $\cdot$ & 50 & - & 50 & & $\cdot$ \\
\hline Tetrachloroethene & 50 & - & 50 & $\cdot$ & 50 & $\cdot$ & 50 & - & 50 & - & 50 & & $\cdot$ \\
\hline Toluene & 5u & - & 50 & $\cdot$ & 50 & $\cdot$ & 50 & $\cdot$ & 50 & - & 50 & & $\cdot$ \\
\hline $1,1,1$-Trichloroethane & 50 & - & su & $\cdot$ & 50 & $\cdot$ & 50 & $\cdot$ & 50 & - & 50 & & $\cdot$ \\
\hline 1,1,2-Trichloroethane & 5u & - & 50 & $\cdot$ & 50 & $\cdot$ & 50 & $\cdot$ & 50 & • & 50 & & - \\
\hline Trichlcroethene & 50 & - & 50 & $\cdot$ & 50 & $\cdot$ & so & $\cdot$ & 50 & $\cdot$ & FP3 & & • \\
\hline vinyl acetate & 100 & - & 100 & $\cdot$ & 100 & $\cdot 1$ & 100 & $\cdot$ & 100 & - & 100 & & - \\
\hline vinyl chloride & 100 & - & 100 & $\cdot$ & 100 & $\cdot$ & 100 & $\cdot$ & 100 & - & 100 & & $\cdot$ \\
\hline xylenes & 50 & - & 50 & $\cdot$ & 50 & $\cdot$ & 50 & $\cdot$ & 50 & - & 50 & & - \\
\hline Surmed vocs & $\mathbf{0}$ & - & o & $\cdot 1$ & 0 & $\cdot$ & 0 & $\bullet$ & $\mathbf{0}$ & - & 0 & & - \\
\hline
\end{tabular}

(CONTINUED) 
APPEADIX $J$

Validated VOC Results, 1993

\begin{tabular}{|c|c|c|c|c|c|c|c|c|c|c|c|c|}
\hline \multirow{4}{*}{$\begin{array}{l}\text { Sampling Point } \\
\text { Location } \\
\text { Date Sampled }\end{array}$} & \multicolumn{4}{|c|}{$6 w-652$} & \multicolumn{8}{|c|}{ Git-653 } \\
\hline & \multicolumn{4}{|c|}{ BG } & \multicolumn{8}{|c|}{ BG } \\
\hline & \multicolumn{2}{|c|}{$07 / 10 / 93$} & \multicolumn{2}{|c|}{$10 / 18 / 93$} & \multicolumn{2}{|c|}{$03 / 01 / 93$} & \multicolumn{2}{|c|}{$04 / 20 / 93$} & \multicolumn{2}{|c|}{$07 / 10 / 93$} & \multicolumn{2}{|c|}{$10 / 10 / 93$} \\
\hline & ug/L & sum & ug/I & sum & ug/L & 8 sen & $u g / L$ & s sum & $u g / x$ & sm & $\operatorname{ug} / 2$ & $\operatorname{sun}$ \\
\hline scetone & 100 & - & 100 & - & 100 & $\cdot 1$ & 100 & $\cdot 1$ & 100 & - & 100 & $\bullet$ \\
\hline Benzene & 50 & - & 50 & $\cdot$ & 5v & $\cdot 1$ & 50 & $\cdot$ & 50 & $\cdot$ & 50 & $\cdot$ \\
\hline Bromodichloromethane & 50 & - & 50 & $\cdot$ & so & $\cdot$ & sv & $\cdot$ & 50 & - & 50 & • \\
\hline 2-Butanone & 100 & - & 100 & • & 100 & $\cdot$ & 100 & $\cdot$ & 100 & - & 100 & • \\
\hline Carbon disulfide & su & - & 5v & - & 50 & $\cdot$ & 50 & $\cdot$ & 50 & - & 50) & $\cdot$ \\
\hline Carbon tetrachloride & 50 & - & 5v & $\cdot$ & so & $\cdot$ & sv & $\cdot$ & 50 & $\cdot$ & 50 & • \\
\hline Chlorobenzene & 50 & - & su & • & 50 & $\cdot$ & 5v & $\cdot$ & so & $\cdot$ & 50) & $\cdot$ \\
\hline Chloroform & So & - & 5v & • & 50 & $\cdot$ & 5v & $\cdot$ & 5v & - & 50 & $\cdot$ \\
\hline 1,1-Dichloroethane & 50 & $\cdot$ & 5v & $\cdot$ & 50 & $\cdot$ & 50 & $\cdot 1$ & 5v & $\cdot$ & 50 & $\cdot$ \\
\hline 1,2-Dichloroethane & 50 & - & 50 & $\cdot$ & 50 & $\cdot$ & so & $\cdot$ & 50 & $\cdot$ & 50 & • \\
\hline 1,1-Dichloroethene & 50 & - & 50 & $\cdot$ & 50 & $\cdot$ & 58 & $\cdot$ & 50 & $\cdot$ & 50 & $\bullet$ \\
\hline 1,2-Dichloroethene & 50 & - & $\therefore$ & $\cdot$ & 7 & 77.8 & 50 & $\cdot$ & 3 & 100 & 7 & 77.8 \\
\hline Ethylbenzene & 50 & - & 50 & $\cdot$ & so & $\cdot$ & so & $\cdot$ & 5o & $\cdot$ & 50 & $\bullet$ \\
\hline 2-Hexanone & 100 & - & 100 & . & 100 & $\cdot 1$ & 100 & $\cdot$ & 100 & - & 100 & $\cdot$ \\
\hline 4-Methy1-2-pentanone & 100 & - & 100 & $\cdot$ & 100 & $\cdot$ & FP3 & $\cdot$ & 200 & - & 100 & $\cdot$ \\
\hline Hethylene chloride & FP1 & - & 50 & $\cdot$ & 50 & $\cdot$ & 50 & $\cdot$ & 5v & $\cdot$ & so & • \\
\hline Styrene & 50 & - & 50 & $\cdot$ & 50 & $\cdot$ & 50) & $\cdot$ & 5v & - & so & - \\
\hline Tetrachloroethene & 50 & $\cdot$ & so & $\cdot$ & 2 & 22.2 & 2 & 100 & 50 & $\cdot$ & 2 & 22.2 \\
\hline Toluene & 50 & - & 50 & $\cdot$ & 50 & $\cdot$ & 50 & $\cdot$ & 50 & $\cdot$ & 50 & $\cdot$ \\
\hline $1,1,1$-Trichloroethane & 50 & • & 50 & $\cdot$ & 5u & $\cdot$ & 50 & $\cdot$ & so & $\cdot$ & 50 & $\cdot$ \\
\hline 1,1,2-Trichloroethane & 50 & - & so & $\cdot$ & 50 & $\cdot$ & 50| & $\cdot$ & 50 & $\cdot$ & 50 & $\bullet$ \\
\hline Irichloroethene & so & - & so & $\cdot$ & 5o & $\cdot$ & 50 & $\cdot$ & 50 & $\cdot$ & so & $\cdot$ \\
\hline Vinyl acetate & 100 & $\cdot$ & 100 & $\cdot$ & 100 & $\cdot$ & 100 & $\cdot$ & 100 & $\cdot$ & 100 & 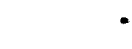 \\
\hline vinyl chloride & 100 & - & 100 & $\cdot$ & 100 & $\cdot$ & 100 & $\cdot$ & 100 & - & 100 & $\cdot$ \\
\hline xylenes & 50 & $\cdot$ & 50 & $\cdot$ & 50 & $\cdot$ & 50 & $\cdot$ & 50 & $\cdot$ & 50 & $\bullet$ \\
\hline Summed vOCs & & - & 0 & . & 9 & 100 & 2 & 100 & 3 & 100 & 9 & 100 \\
\hline
\end{tabular}

(CONTINUED) 
Validated voC Results, 1993

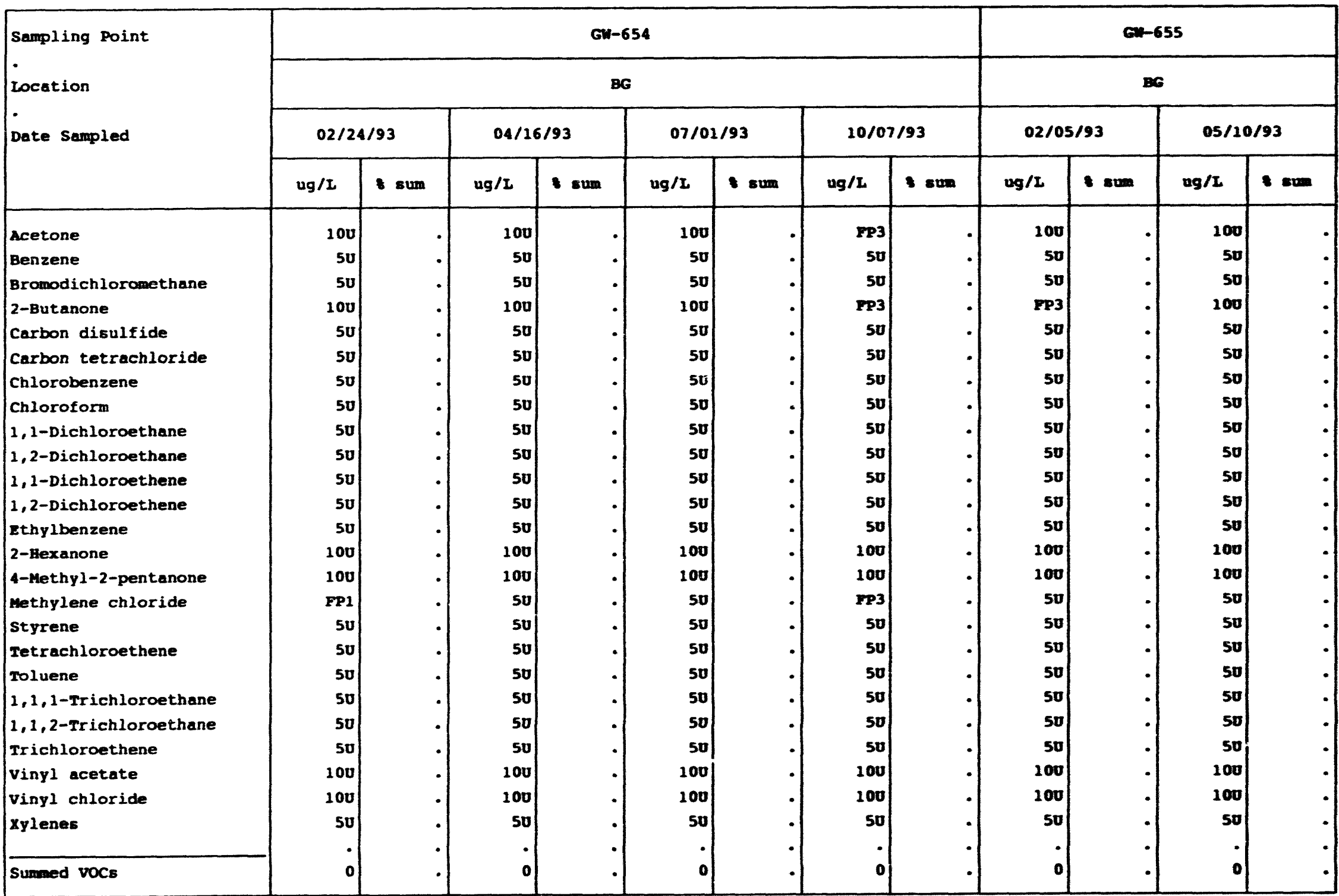

(COntrimued) 
APPEDDIX $J$

validated voc Results, 1993

\begin{tabular}{|c|c|c|c|c|c|c|c|c|c|c|c|c|}
\hline \multirow{4}{*}{$\begin{array}{l}\text { Sampling Point } \\
\text { location } \\
\text { Date Sompled }\end{array}$} & \multicolumn{4}{|c|}{ Gw-655 } & \multicolumn{8}{|c|}{ Gn-683 } \\
\hline & \multicolumn{4}{|c|}{ BG } & \multicolumn{8}{|c|}{$\mathbf{E x P}$} \\
\hline & \multicolumn{2}{|c|}{$07 / 01 / 93$} & \multicolumn{2}{|c|}{$10 / 06 / 93$} & \multicolumn{2}{|c|}{$03 / 04 / 93$} & \multicolumn{2}{|c|}{$04 / 23 / 93$} & \multicolumn{2}{|c|}{$07 / 12 / 93$} & \multicolumn{2}{|c|}{$10 / 12 / 93$} \\
\hline & ug/I & sum & $u g / z$ & sum & $\mathbf{u g} / \mathbf{I}$ & $\bullet \operatorname{cum}$ & $u g / L$ & 8 sm & $\operatorname{ug} / \mathbf{L}$ & $\operatorname{sen}$ & $\operatorname{ug} / \mathbf{L}$ & tsan \\
\hline Acetone & & $\cdot$ & 100 & - & 100 & $\cdot$ & 100 & $\cdot$ & 100 & $\cdot$ & FP3 & 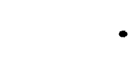 \\
\hline Benzene & 50 & $\cdot 1$ & su & $\cdot$ & $5 n$ & $\cdot$ & 50 & $\cdot$ & 50 & $\cdot 1$ & 50 & $\cdot$ \\
\hline Bromodichloromethane & so & $\cdot$ & 50 & - & 50 & $\cdot$ & 50 & $\cdot$ & 50 & $\cdot$ & 50 & - \\
\hline 2-Butanone & 100 & $\cdot$ & 100 & $\cdot$ & 100 & $\cdot$ & 100 & $\cdot$ & 100 & $\cdot$ & FP3 & - \\
\hline Carbon disulfide & 50 & $\cdot$ & so & - & 50) & $\cdot$ & 50 & $\cdot$ & 50 & $\cdot$ & 50 & • \\
\hline Carbon tetrachlcride & 50 & $\cdot$ & so & $\cdot$ & 50 & $\cdot$ & 50 & $\cdot$ & 50 & $\cdot$ & 50 & - \\
\hline Chlorobenzene & 50 & $\cdot$ & 50 & $\cdot$ & 50 & $\cdot$ & 50 & $\cdot$ & 50 & $\cdot$ & 50 & $\bullet$ \\
\hline Chloroform & 50 & $\cdot$ & 50 & $\cdot$ & 50 & $\cdot$ & 50 & $\cdot$ & 50 & $\cdot$ & 50 & $\bullet$ \\
\hline 1,1-Dichloroethane & 50 & $\cdot$ & 50 & $\bullet$ & 50 & $\bullet$ & 50 & $\cdot$ & 50 & $\cdot$ & 50 & $\bullet$ \\
\hline 1,2-Dichloroethane & 50 & $\cdot$ & 50 & $\bullet$ & 50 & $\cdot$ & su & $\bullet$ & 50 & $\bullet$ & 50 & $\bullet$ \\
\hline 1,1-Dichloroethene & $\begin{array}{l}50 \\
50\end{array}$ & $\cdot$ & $\begin{array}{l}50 \\
50\end{array}$ & $\cdot$ & 50 & 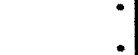 & 50 &. & 50 &. & so & - \\
\hline $\begin{array}{l}\text { 1,2-Dichloroetnene } \\
\text { Ethylbenzene }\end{array}$ & 50 &. & so & $\bullet$ & 50 & - & sv & $\cdot$ & so & $\cdot$ & 50 & • \\
\hline 2-Hexanone & 100 & $\cdot$ & 100 & - & 100 & - & 100 & $\cdot$ & 100 & $\cdot$ & 100 & - \\
\hline 4-Hethy1-2-pentanone & 100 & $\cdot$ & 100 & $\cdot$ & 100 & $\cdot$ & 100 & $\cdot$ & 100 & $\cdot$ & 100 & - \\
\hline Hethylene chloride & 50) & $\cdot$ & 50 & - & 50 & $\cdot$ & 50 & $\cdot$ & 50 & $\cdot$ & FP3 & $\cdot$ \\
\hline styrene & 50 & $\cdot$ & 50 & $\cdot$ & so & $\cdot$ & so & - & su & $\cdot$ & so & • \\
\hline Tetrachloroethene & 50 & $\cdot$ & 50 & $\cdot$ & 50 & $\cdot$ & 50 & $\cdot$ & so & $\cdot$ & so & • \\
\hline Toluene & 50! & $\cdot$ & so & $\cdot$ & 50 & $\cdot$ & 50 & $\cdot$ & 50 & $\cdot$ & 50 & • \\
\hline 1,1,1-Irichloroethane & 50) & $\cdot$ & 50 & $\cdot$ & 5v & $\cdot$ & 50 & $\cdot$ & 50 & $\cdot$ & 50 & $\cdot$ \\
\hline 1,1,2-Trichloroethane & 50 & $\cdot$ & 50 & $\cdot$ & 50) & $\cdot$ & 50 & $\cdot$ & so & $\cdot$ & 50 & - \\
\hline Trichloroethene & 50) & $\cdot$ & 5v & $\cdot$ & 50 & $\cdot$ & 50 & $\cdot$ & 50| & $\cdot$ & 50 & $\bullet$ \\
\hline Vinyl acetate & 100 & $\cdot$ & 100 & $\cdot$ & 100 & $\cdot$ & 100 & $\cdot$ & 100 & $\cdot$ & 100 & - \\
\hline vinyl chloride & 100 & $\cdot$ & 100 & - & 100 & $\cdot$ & 100 & $\cdot$ & 100 & $\cdot$ & 100 & - \\
\hline Xylenes & 50 & $\cdot$ & 50 & $\cdot$ & 50 & $\cdot$ & su & $\cdot$ & 50 & $\cdot$ & 50 & $\bullet$ \\
\hline Summed VOCs & 0) & - & $\mathbf{0}$ & - & 0 & - & 0 & - & o) & $\cdot$ & - & - \\
\hline
\end{tabular}

(CONTINOED) 


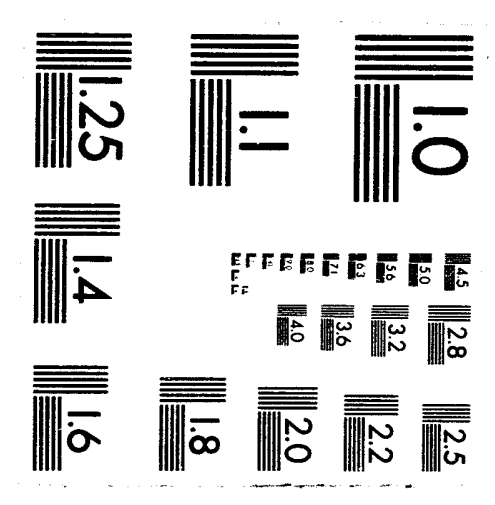



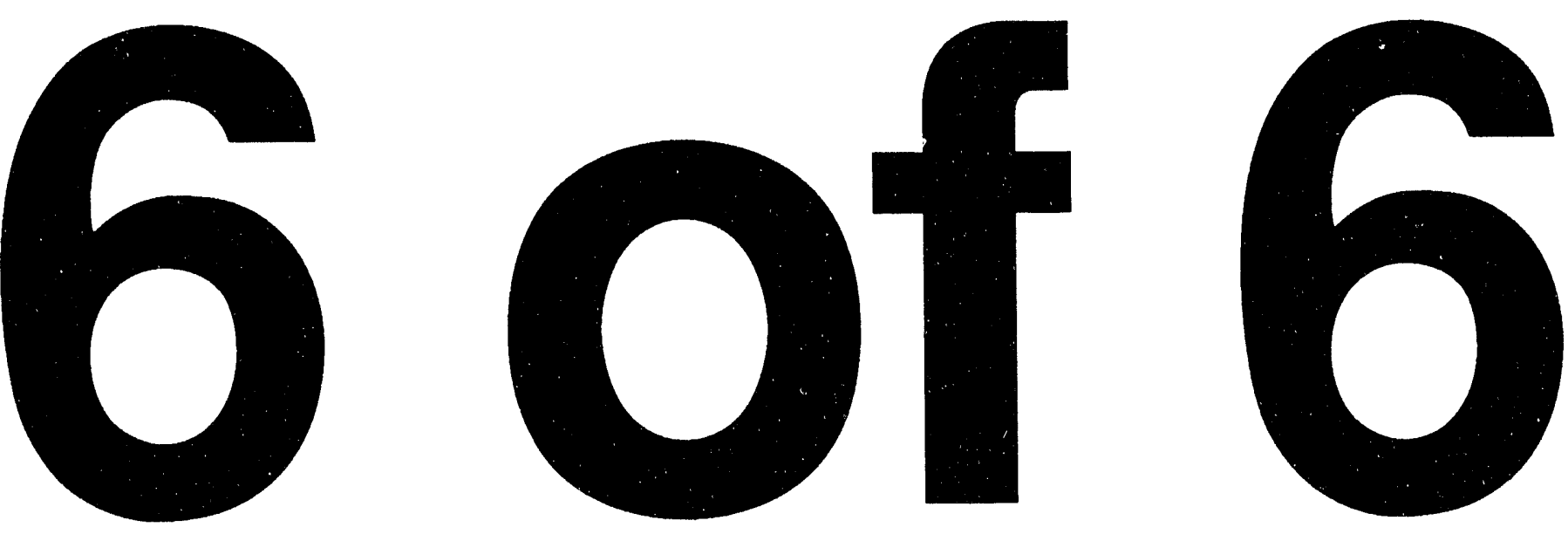
Validated vOC Results, 1993

\begin{tabular}{|c|c|c|c|c|c|c|c|c|c|c|c|c|c|}
\hline \multirow{4}{*}{$\begin{array}{l}\text { Sampling Point } \\
\text { Location } \\
\text { Date Sampled }\end{array}$} & \multicolumn{8}{|c|}{$G W-684$} & \multicolumn{5}{|c|}{$G W-685$} \\
\hline & \multicolumn{8}{|c|}{ EXP } & \multicolumn{5}{|c|}{ EXP } \\
\hline & \multicolumn{2}{|c|}{$03 / 03 / 93$} & \multicolumn{2}{|c|}{$04 / 22 / 93$} & \multicolumn{2}{|c|}{$07 / 11 / 93$} & \multicolumn{2}{|c|}{$10 / 12 / 93$} & \multicolumn{2}{|c|}{$03 / 02 / 93$} & \multicolumn{3}{|c|}{$04 / 21 / 93$} \\
\hline & $\mathrm{ug} / \mathrm{L}$ & 8 sum & $u g / L$ & 8 sum & $\mathrm{ug} / \mathrm{I}$ & 8 sum & ug/I & \& sum & ug/L & 8 sum & $\mathrm{ug} / \mathrm{L}$ & $\& 5$ & sum \\
\hline Acetone & 100 & $\cdot$ & 100 & $\cdot$ & 100 & $\cdot 1$ & FP1 & - & 100 & $\cdot$ & 100 & & - \\
\hline Benzene & 50 & $\cdot$ & 50 & $\cdot$ & $5 \mathrm{v}$ & $\cdot$ & 50 & $\cdot$ & 50 & $\cdot$ & 5u & & $\cdot$ \\
\hline Bromodichloromethane & 50 & $\cdot$ & $5 \mathbf{v}$ & $\cdot$ & 50 & $\cdot$ & $5 \mathbf{u}$ & $\cdot$ & 50 & $\cdot$ & $\begin{array}{r}50 \\
100\end{array}$ & & $\cdot$ \\
\hline 2-Butanone & 100 & $\cdot$ & 100 & $\cdot$ & 100 & $\cdot$ & $\begin{array}{r}\text { FP1 } \\
50\end{array}$ & $\cdot$ & $\begin{array}{r}100 \\
5 U\end{array}$ &. & $\begin{array}{r}100 \\
50\end{array}$ & &. \\
\hline Carbon disulfide & $\begin{array}{l}5 \mathrm{U} \\
5 \mathrm{U}\end{array}$ &. & $\begin{array}{l}50 \\
50\end{array}$ &. & $\begin{array}{l}5 \mathrm{U} \\
5 \mathrm{U}\end{array}$ & $\cdot$ & $\begin{array}{l}50 \\
50\end{array}$ & $\cdot$ & 50 &. & 50 & & . \\
\hline $\begin{array}{l}\text { Carbon tetrachloride } \\
\text { Chlorobenzene }\end{array}$ & 50 &. & $5 \mathrm{U}$ &. & $5 \mathrm{u}$ &. & 5u &. & 50 &. & 50 & & . \\
\hline Chloroform & $5 \mathrm{U}$ & $\cdot$ & 50 & $\cdot$ & 5u & $\cdot$ & 50 &. & 50 & $\cdot$ & 50 & & - \\
\hline 1,1-Dichloroethane & 50 & $\cdot$ & 50 & $\cdot$ & 50 & $\cdot$ & 50 & $\cdot$ & 50 &. & 50 & & • \\
\hline 1,2-Dichloroethane & 50 & $\cdot$ & 5u & $\cdot$ & 50 & $\cdot$ & 50 & $\cdot$ & 50 & $\cdot$ & 50 & & - \\
\hline 1,1-Dichloroethene & 50 & $\cdot$ & su & $\cdot$ & 50 & $\cdot$ & 50 & $\cdot$ & $5 \mathrm{U}$ & $\cdot$ & 50 & & - \\
\hline 1,2-Dichloroethene & 50 & $\cdot$ & 50 & $\cdot$ & $5 \mathrm{u}$ & $\cdot$ & 50 & $\cdot$ & 2 & 100 & 2 & & 100 \\
\hline Ethylbenzene & 50 & $\cdot$ & $5 \mathbf{u}$ & $\cdot$ & 50 & $\cdot$ & 50 & $\cdot$ & 50 & $\cdot$ & 50 & & - \\
\hline 2-Hexanone & 100 & $\cdot$ & 100 & $\cdot$ & 100 & $\cdot$ & 100 & $\cdot$ & 100 & $\cdot$ & 100 & & - \\
\hline 4-Methyl-2-pentanone & 100 & $\cdot$ & 100 & $\cdot$ & 100 & $\cdot$ & 100 & $\cdot$ & 100 & $\cdot$ & 100 & & - \\
\hline Methylene chloride & 50 & $\cdot$ & FP3 & $\cdot$ & FP1 & $\cdot$ & FP1 & $\cdot$ & 50 & $\cdot$ & 50 & & - \\
\hline Styrene & 50 & $\cdot$ & 50 & $\cdot$ & 50 & $\cdot$ & 50 & $\cdot$ & 50 & $\cdot$ & 50 & & - \\
\hline Tetrachloroethene & $5 v$ & $\cdot$ & 50 & $\cdot$ & 50 & - & su & $\cdot$ & 50 & $\cdot 1$ & $5 U$ & & - \\
\hline Toluene & $5 U$ & $\cdot$ & su & $\cdot$ & 50 & $\cdot$ & 50 & $\cdot$ & $5 \mathrm{U}$ & $\cdot$ & 50 & & - \\
\hline $1,1,1$-Trichloroethane & 5U & $\cdot$ & 50 & $\cdot$ & 5u & $\cdot$ & 50 & $\cdot$ & 50 & $\cdot$ & 50 & & • \\
\hline 1,1,2-Trichloroethane & 50 & $\cdot$ & 50 & $\cdot$ & 50 & $\cdot$ & 50 & $\cdot$ & 50 & $\cdot$ & $5 U$ & & - \\
\hline Trichloroethene & 50 & $\cdot 1$ & 5u & $\cdot$ & 50 & $\cdot$ & 50 & $\cdot$ & 50 & $\cdot$ & 50 & & - \\
\hline vinyl acetate & 100 & $\cdot$ & 100 & $\cdot$ & 100 & - & 100 & $\cdot$ & 100 & $\cdot$ & 100 & & - \\
\hline vinyl chloride & 100 & $\cdot 1$ & 100 & $\cdot$ & 100 & $\cdot$ & 100 & $\cdot$ & 100 & $\cdot 1$ & 100 & & - \\
\hline xylenes & $5 \mathrm{v}$ & $\cdot$ & 50 & $\cdot$ & 50 & $\cdot$ & 50 & $\cdot$ & 50 & $\cdot$ & 50 & & - \\
\hline Summed vOCs & 0 & $\cdot 1$ & 0 & - & 0 & $\cdot$ & 0 &. & 2 & 100 & 2 & & 100 \\
\hline
\end{tabular}

(CONTINUED) 
APPENDIX $J$

Validated voC Results, 1993

\begin{tabular}{|c|c|c|c|c|c|c|c|c|c|c|c|c|}
\hline \multirow{4}{*}{$\begin{array}{l}\text { Sampling Point } \\
\text { Location } \\
\text { Date Sampled }\end{array}$} & \multicolumn{4}{|c|}{ GW-685 } & \multicolumn{8}{|c|}{ GW-694 } \\
\hline & \multicolumn{4}{|c|}{ EXP } & \multicolumn{8}{|c|}{$\operatorname{EXP}$} \\
\hline & \multicolumn{2}{|c|}{$07 / 11 / 93$} & \multicolumn{2}{|c|}{$10 / 11 / 93$} & \multicolumn{2}{|c|}{$03 / 23 / 93$} & \multicolumn{2}{|c|}{$06 / 06 / 93$} & \multicolumn{2}{|c|}{$09 / 22 / 93$} & \multicolumn{2}{|c|}{$12 / 28 / 93$} \\
\hline & $u g / I$ & 8 sum & ug $/ \mathrm{L}$ & 8 sum & $u g / L$ & 8 sum & $\mathrm{ug} / \mathrm{L}$ & 8 sum & ug/L & 8 sum & $u g / L$ & 8 sum \\
\hline Acetone & 100 & $\cdot$ & FP1 & - & 100 &. & 100 &. & 100 &. & FP1 & • \\
\hline Benzene & 50 & $\cdot$ & 50 & • & $5 \mathrm{U}$ & $\cdot$ & 5v & $\cdot$ & 50 & $\cdot$ & 50 & • \\
\hline Bromodichloromethane & $5 v$ &. & 50 & . & 50 & . & 50 & . & 5u & $\cdot$ & $5 v$ & • \\
\hline 2-Butanone & 100 & $\cdot$ & FP1 & - & 100 & $\cdot$ & 100 &. & 100 & $\cdot$ & FP1 & - \\
\hline Carbon disulfide & $5 \mathrm{U}$ &. & 50 & - & $5 \mathrm{U}$ & $\cdot$ & 50 & - & su & $\cdot$ & 50 & • \\
\hline Carbon tetrachloride & $5 \mathrm{U}$ &. & 50 & - & 50 & $\cdot$ & 50 &. & 50 & $\cdot$ & 50 & - \\
\hline Chlorobenzene & 50 &. & 50 & . & 50 &. & 50 &. & 50 &. & 50 & • \\
\hline Chloroform & 50 & $\cdot$ & 50 & - & 50 & . & 50 & $\cdot$ & 50 & $\cdot$ & $5 \mathrm{U}$ & • \\
\hline 1,1-Dichloroethane & 50 &. & $5 u$ & - & 50 &. & 50 &. & 50 &. & 50 & • \\
\hline 1,2-Dichloroethane & 50 &. & 5u & - & $5 v$ & $\cdot$ & $5 v$ &. & 50 & $\cdot$ & 50 & • \\
\hline 1,1-Dichloroethene & 50 &. & 50 & - & 50 &. & 50 &. & 50 & .1 & 50 & • \\
\hline 1,2-Dichloroethene & 2 & 100 & 50 & - & 9 & 39.1 & 11 & 44 & 11 & 50 & 13 & 46.4 \\
\hline Ethylbenzene & 50 &. & $5 \mathbf{v}$ & . & $5 v$ &. & 50 &. & $5 U$ &. & 50 & . \\
\hline 2-Hexanone & 100 &. & 100 & - & 100 &. & 100 &. & 100 &. & 100 & . \\
\hline 4-Methy1-2-pentanone & 100 & $\cdot$ & 100 & . & FP1 &. & 100 &. & 100 &. & 100 & . \\
\hline Methylene chloride & $5 U$ & $\cdot$ & FP1 & - & FP1 & $\cdot$ & FP3 & $\cdot$ & 50 & $\cdot$ & FP1 & - \\
\hline styrene & $5 \mathrm{U}$ &. & 50 & . & 50 &. & 50 &. & 50 &. & $5 \mathrm{v}$ & . \\
\hline Tetrachloroethene & $5 \mathrm{v}$ & $\cdot$ & 50 & - & 5u & $\cdot$ & $5 \mathrm{U}$ & . & 50 & $\cdot$ & 50 & - \\
\hline Toluene & $5 \mathrm{U}$ &. & $5 u$ & - & 50 &. & 50 & - & $5 U$ &. & 50 & - \\
\hline 1,1,1-Trichloroethane & 50 &. & $5 v$ & - & $5 \mathrm{v}$ & $\cdot 1$ & FP3 & . & 50 & $\cdot$ & $5 \mathrm{u}$ & - \\
\hline 1,1,2-Trichloroethane & $5 \mathrm{U}$ &. & su & - & $5 v$ &. & su &. & 50 &. & su & - \\
\hline Trichloroethene & $5 U$ & $\cdot$ & $5 v$ & - & 14 & 60.9 & 14 & 56 & 11 & 50 & 15 & 53.6 \\
\hline Vinyl acetate & 100 &. & 100 & . & 100 &. & 100 & - & 100 &. & 100 & . \\
\hline Vinyl chloride & 100 &. & 100 & - & 100 & $\cdot$ & 100 & - & 100 & . & 100 & - \\
\hline Xylenes & 50 &. & $5 v$ & . & 50 &. & 50 & . & 50 &. & 5u & . \\
\hline Summed VOCs & 2 & 100 & 0 & . & 23 & 100 & 25 & 100 & 22 & 100 & 28 & 100 \\
\hline
\end{tabular}

(CONTINUED) 
Validated VOC Results, 1993

\begin{tabular}{|c|c|c|c|c|c|c|c|c|c|c|c|c|}
\hline \multirow{4}{*}{$\begin{array}{l}\text { Sampling Point } \\
\text { Location } \\
\text { Date Sampled }\end{array}$} & \multicolumn{8}{|c|}{ GW-695 } & \multicolumn{4}{|c|}{ GW-703 } \\
\hline & \multicolumn{8}{|c|}{ EXP } & \multicolumn{4}{|c|}{ EXP } \\
\hline & \multicolumn{2}{|c|}{$03 / 24 / 93$} & \multicolumn{2}{|c|}{$06 / 09 / 93$} & \multicolumn{2}{|c|}{$09 / 22 / 93$} & \multicolumn{2}{|c|}{$12 / 21 / 93$} & \multicolumn{2}{|c|}{$03 / 20 / 93$} & \multicolumn{2}{|c|}{$06 / 03 / 93$} \\
\hline & $\mathrm{ug} / \mathrm{L}$ & 8 sum & $\mathrm{ug} / \mathrm{I}$ & 8 sum & ug $/ \mathrm{L}$ & 8 sum & $\mathrm{ug} / \mathrm{L}$ & s sum & $\mathrm{ug} / \mathrm{L}$ & 8 sum & $\mathrm{ug} / \mathrm{L}$ & 8 sum \\
\hline Acetone & 100 & $\cdot$ & 100 &. & 100 & . & 100 & $\cdot$ & FP3 & . & 100 & , \\
\hline Benzene & 50 &. & 50 &. & 50 & . & 50 & . & 50 & $\cdot$ & 50 & . \\
\hline Bromodichloromethane & 50 &. & 50 &. & $5 v$ & . & 50 & $\cdot$ & 5u & $\cdot$ & 50 & . \\
\hline 2-Butanone & 100 & . & 100 &. & 100 & - & FP2 & $\cdot$ & 100 & $\cdot$ & 100 & . \\
\hline Carbon disulfide & 50 & $\cdot$ & 50 & $\cdot$ & 50 & $\cdot$ & 5u & $\cdot$ & 50 & $\cdot$ & $5 U$ & r \\
\hline Carbon tetrachloride & 50 & $\cdot$ & 50 &. & 50 & - & 50 & $\cdot$ & 50 & - & 50 & . \\
\hline Chlorobenzene & 50 & $\cdot$ & 50 & $\cdot$ & 50 & - & 50 & $\cdot$ & 50 & - & 50 & r \\
\hline chloroform & 50 & $\cdot$ & 50 &. & 50 & - & 50 & $\cdot$ & 50 & - & 50 & . \\
\hline 1,1-Dichloroethane & 50 & $\cdot$ & 50 & $\cdot$ & 50 & . & 50 & $\cdot$ & 50 & - & 50 & . \\
\hline 1,2-Dichloroethane & 50 &. & 50 &. & 50 & - & 5u & . & 50 & - & 5u & . \\
\hline 1,1-Dichloroethene & 50 &. & 50 & $\cdot$ & 50 & $\cdot$ & FP3 & $\cdot$ & 2 & 5.3 & 1 & 3.2 \\
\hline 1,2-Dichloroethene & 2 & 33.3 & $5 \mathrm{u}$ & $\cdot$ & 50 & . & 2 & 33.3 & 8 & 21.1 & 7 & 22.6 \\
\hline Ethylbenzene & 50 & $\cdot$ & 50 & $\cdot$ & 50 & $\cdot$ & 50 & $\cdot$ & 50 & $\cdot$ & 5u & 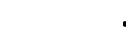 \\
\hline 2-Hexanone & 100 &. & 100 &. & 100 & - & 100 & $\cdot$ & 100 & - & 100 & . \\
\hline 4-Methyl-2-pentanone & 100 & $\cdot$ & 100 & $\cdot$ & 100 & $\cdot$ & 100 & $\cdot$ & FP3 & $\cdot$ & 100 & r \\
\hline Methylene chloride & $5 v$ & $\cdot$ & $5 v$ & $\cdot$ & $5 \mathrm{u}$ & . & FP1 & $\cdot$ & FP3 & $\cdot$ & 50 & r \\
\hline Styrene & 50 & $\cdot$ & 50 & $\cdot$ & 50 & $\cdot$ & 50 & $\cdot$ & 50 & - & 50 & . \\
\hline Tetrachloroethene & 50 &. & $5 \mathrm{u}$ & $\cdot$ & 50 & $\cdot$ & 50 & - & 50 & • & 50 & ra \\
\hline Toluene & 50 & $\cdot$ & 50 & $\cdot$ & 50 & $\cdot$ & 50 & $\cdot$ & 50 & $\cdot$ & 50 & . \\
\hline $1,1,1-$ Trichloroethane & 5u & $\cdot$ & 50 & $\cdot$ & 50 & $\cdot$ & 50 & $\cdot$ & 50 & $\cdot$ & 50 & ra \\
\hline $1,1,2$-Trichloroethane & 5u &. & 50 & $\cdot$ & 50 & - & 5u & $\cdot$ & 50 & - & 50 & ra \\
\hline Trichloroethene & 4 & 66.7 & 50 &. & 4 & 100 & 4 & 66.7 & 28 & 73.7 & 23 & 74.2 \\
\hline Vinyl acetate & 100 & $\cdot$ & 100 & $\cdot$ & 100 & . & 100 & $\cdot$ & 100 & - & 100 & ra \\
\hline vinyl chloride & 100 & $\cdot$ & 100 & $\cdot$ & 100 & . & 100 & $\cdot$ & 100 & • & 100 & . \\
\hline xylenes & 5u &. & $5 v$ &. & 50 & . & 5u & - & 50 & • & $5 U$ & ra \\
\hline Summed VOCE & 6 & 100 & 0 &. & 4 & 100 & 6 & 100 & 38 & 100.1 & 31 & 100 \\
\hline
\end{tabular}

(CONTINUED) 
APPENDIX $J$

Validated Voc Results, 1993

\begin{tabular}{|c|c|c|c|c|c|c|c|c|c|c|c|c|}
\hline \multirow{4}{*}{$\begin{array}{l}\text { Sampling Point } \\
\text { Location } \\
\text { - } \\
\text { Date Sampled }\end{array}$} & \multicolumn{4}{|c|}{ GW-703 } & \multicolumn{8}{|c|}{ GW-704 } \\
\hline & \multicolumn{4}{|c|}{ EXP } & \multicolumn{8}{|c|}{ EXP } \\
\hline & \multicolumn{2}{|c|}{$09 / 20 / 93$} & \multicolumn{2}{|c|}{$12 / 21 / 93$} & \multicolumn{2}{|c|}{$03 / 22 / 93$} & \multicolumn{2}{|c|}{$06 / 04 / 93$} & \multicolumn{2}{|c|}{$09 / 22 / 93$} & \multicolumn{2}{|c|}{$12 / 22 / 93$} \\
\hline & ug/L & 8 sum & $\mathrm{ug} / \mathrm{L}$ & 8 sum & ug/L & 8 sum & $u g / L$ & 8 sum & ug/I & 8 sum & ug/L & 8 sum \\
\hline Acetone & 100 &. & 100 &. & 100 & - & 100 & - & 100 & - & FP1 & $\bullet$ \\
\hline Benzene & $5 \mathrm{v}$ & $\cdot$ & 50 & $\cdot$ & 50 & - & 50 & - & 50 & - & 50 & • \\
\hline Bromodichloromethane & 50 & . & 50 & . & $5 \mathrm{U}$ & . & 50 & - & 50 & - & 50 & - \\
\hline 2-Butanone & 100 &. & FP2 & $\cdot$ & 100 & - & 100 & - & 100 & . & FP1 & . \\
\hline Carbon disulfide & 50 & $\cdot$ & 50 & - & 50 & - & 50 & - & 50 & - & 50 & - \\
\hline Carbon tetrachloride & 50 & $\cdot$ & FP3 & - & 2 & 1.5 & 1 & 1 & 5u & - & 5u & - \\
\hline Chlorobenzene & 50 & $\cdot 1$ & 50 & . & 50 & - & 50 & - & 5u & - & 50 & - \\
\hline Chloroform & 0.5 & 1.8 & 0.5 & 1.3 & 50 & . & FP3 & . & 50 & . & 50 & - \\
\hline 1,1-Dichloroethane & 50 & $\cdot$ & FP3 & $\cdot$ & FP3 & - & 50 & - & 50 & - & 50 & • \\
\hline 1,2-Dichloroethane & 5u &. & 50 & $\cdot$ & 50 & - & 50 & - & $5 \mathrm{u}$ & $\cdot$ & 50 & • \\
\hline 1,1-Dichloroethene & 50 & $\cdot$ & 2 & 5.2 & 6 & 4.5 & 6 & 5.8 & 6 & 6.1 & 4 & 6.6 \\
\hline 1,2-Dichloroethene & 6 & 21.4 & 13 & 33.9 & 3 & 2.3 & 3 & 2.9 & 4 & 4.1 & 2 & 3.3 \\
\hline Ethylbenzene & 50 &. & 50 & . & 50 & . & $5 \mathrm{U}$ & - & 50 & $\cdot$ & $5 \mathrm{U}$ & - \\
\hline 2-Hexanone & 100 &. & 100 & $\cdot$ & 100 & $\cdot$ & 100 & - & 100 & $\cdot$ & 100 & • \\
\hline 4-Methyl-2-pentanone & 100 &. & 100 &. & FP1 & - & 100 & - & 100 & $\cdot$ & 100 & • \\
\hline Methylene chloride & 50 &. & FP1 & $\cdot$ & FP1 & • & FP1 & - & 50 & . & 50 & - \\
\hline Styrene & 50 & $\cdot 1$ & $5 U$ & $\cdot$ & 50 & $\cdot$ & $5 v$ & - & 50 & $\cdot$ & $5 u$ & • \\
\hline Tetrachloroethene & 50 & $\cdot$ & $5 \mathrm{U}$ & $\cdot$ & 50 & . & FP3 & . & 50 & . & 50 & . \\
\hline Toluene & 50 & $\cdot$ & 50 & $\cdot$ & 50 & $\cdot$ & 50 & - & 50 & $\cdot$ & $5 u$ & • \\
\hline 1,1,1-Trichloroethane & 0.5 & 1.8 & 0.9 & 2.3 & 2 & 1.5 & 2 & 1.9 & 2 & 2 & 50 & - \\
\hline 1,1,2-Trichloroethane & 50 & $\cdot$ & 50 &. & 50 & - & 50 & - & 50 & $\cdot$ & 50 & • \\
\hline Trichloroethene & 21 & 75 & 22 & 57.3 & 120 & 90.2 & 92 & 88.5 & 86 & 87.8 & 55 & 90.2 \\
\hline vinyl acetate & 100 &. & 100 & $\cdot$ & 100 & • & 100 & • & 100 & $\cdot$ & 100 & $\bullet$ \\
\hline vinyl chloride & 100 &. & 100 & $\cdot$ & 100 & - & 100 & - & 100 & $\cdot$ & 100 & • \\
\hline Xylenes & 50 &. & 50 & $\cdot$ & 50 & . & 50 & - & 50 & - & 50 & - \\
\hline Summed VOCs & 28 & 100 & 38.4 & 100 & 133 & 100 & 104 & 100.1 & 98 & 100 & 61 & 100.1 \\
\hline
\end{tabular}

(CONTINUED) 
Validated vOC Results, 1993

\begin{tabular}{|c|c|c|c|c|c|c|c|c|c|c|c|c|}
\hline \multirow{4}{*}{$\begin{array}{l}\text { Sampling Point } \\
\text { Location } \\
\text { Date Sampled }\end{array}$} & \multicolumn{8}{|c|}{$G W-706$} & \multicolumn{4}{|c|}{$G W-710$} \\
\hline & \multicolumn{8}{|c|}{ EXP } & \multicolumn{4}{|c|}{ EXP } \\
\hline & \multicolumn{2}{|c|}{$03 / 23 / 93$} & \multicolumn{2}{|c|}{$06 / 11 / 93$} & \multicolumn{2}{|c|}{$09 / 23 / 93$} & \multicolumn{2}{|c|}{$12 / 28 / 93$} & \multicolumn{2}{|c|}{$03 / 21 / 93$} & \multicolumn{2}{|c|}{$04 / 30 / 93$} \\
\hline & $\operatorname{ug} / \mathrm{L}$ & 8 sum & $u g / L$ & 8 sum & ug/L & 8 sum & $\mathrm{ug} / \mathrm{L}$ & 8 sum & $\mathrm{ug} / \mathrm{L}$ & 8 sum & $\mathrm{ug} / \mathrm{L}$ & 8 sum \\
\hline Acetone & 100 & $\cdot$ & 100 & $\cdot$ & 100 & - & FP3 & $\cdot$ & 100 & - & 100 & \\
\hline Benzene & $5 \mathrm{v}$ & $\cdot$ & 50 & $\cdot$ & 5v & $\cdot$ & su & $\cdot$ & 50 & $\cdot$ & 50 & \\
\hline Bromodichloromethane & $5 v$ & $\cdot$ & 50 & $\cdot$ & 50 & $\cdot$ & 50 & $\cdot$ & 50 & $\cdot$ & 50 & \\
\hline 2-Butanone & 100 & $\cdot 1$ & 100 & $\cdot$ & 100 & $\cdot$ & FP3 & $\cdot$ & 100 & $\cdot$ & 100 & \\
\hline Carbon disulfide & $5 u$ & $\cdot$ & 50 & $\cdot$ & 50 & $\cdot$ & $5 \mathbf{U}$ & $\cdot$ & 50 & $\cdot$ & 5u & \\
\hline Carbon tetrachloride & 50 & $\cdot$ & 50 & $\cdot$ & 50 & $\cdot$ & $5 v$ & $\cdot$ & $5 U$ & $\cdot$ & 50 & \\
\hline Chlorobenzene & 50 & $\cdot$ & $5 u$ & $\cdot$ & 50 & $\cdot$ & 50 & $\cdot$ & 50 & $\cdot$ & 5u & \\
\hline Chloroform & 50 & $\cdot$ & 50 & $\cdot$ & $5 U$ & $\cdot$ & $5 u$ & $\cdot$ & 50 & $\cdot$ & $5 \mathrm{U}$ & \\
\hline 1,1-Dichloroethane & $5 u$ & $\cdot$ & $5 U$ & $\cdot$ & 50 & $\cdot$ & 50 & • & 50 & $\cdot$ & $5 U$ & \\
\hline 1,2-Dichloroethane & 50 & $\cdot$ & 50 & $\cdot$ & 50 & $\cdot$ & 50 & $\cdot$ & 50 & $\cdot$ & 50 & \\
\hline 1,1-Dichloroethene & 50 & $\cdot$ & su & $\cdot$ & 5u & $\cdot$ & 50 & $\cdot$ & 50 & $\cdot$ & 50 & \\
\hline 1.2-Dichloroethene & 7 & 43.8 & 5 & 45.5 & 5 & 50 & 50 & $\cdot$ & 50 & $\cdot$ & 50 & \\
\hline Ethylbenzene & $5 U$ & $\cdot$ & 50 & $\cdot$ & $5 u$ & $\cdot$ & su & $\cdot$ & $5 U$ & $\cdot$ & 50 & \\
\hline 2-Hexanone & 100 & $\cdot$ & 100 & $\cdot$ & 100 & $\cdot$ & 100 & $\cdot$ & 100 & $\cdot$ & 100 & \\
\hline 4-Methyl-2-pentanone & FP1 & $\cdot$ & $\because 100$ &. & 100 & $\cdot$ & 100 & $\cdot$ & 100 & $\cdot$ & 100 & \\
\hline Methylene chloride & FP1 & $\cdot$ & 50 & $\cdot$ & 50 & $\cdot$ & FP3 & $\cdot$ & $5 U$ & $\cdot$ & FP1 & \\
\hline Styrene & 50 & $\cdot$ & 5u & $\cdot$ & 50 & $\cdot$ & $5 U$ & $\cdot$ & 50 & $\cdot$ & 50 & \\
\hline Tetrachloroethene & 50 & $\cdot$ & 50 & $\cdot$ & 50 & $\cdot$ & 50 & $\cdot$ & 50 & $\cdot$ & 50 & \\
\hline Toluene & 50 & $\cdot$ & 5u & $\cdot$ & 50 & $\cdot$ & $5 U$ & $\cdot$ & 50 & $\cdot 1$ & 5u & \\
\hline $1,1,1$-Trichloroethane & 50 & $\cdot$ & $5 v$ & $\cdot$ & 50 & $\cdot$ & 50 & $\cdot$ & 50 & $\cdot$ & 50 & \\
\hline 1,1,2-Trichloroethane & $5 v$ & $\cdot$ & 50 & $\cdot$ & 50 & $\cdot$ & 50 & $\cdot$ & 50 & $\cdot$ & 50 & \\
\hline Trichloroethene & 9 & 56.3 & 6 & 54.5 & 5 & 50 & 50 & $\cdot$ & 50 & $\cdot$ & 50 & \\
\hline Vinyl acetate & 100 & $\cdot$ & 100 & $\cdot$ & 100 & $\cdot$ & 100 & $\cdot$ & 10 & $\cdot$ & 100 & \\
\hline vinyl chloride & 100 & $\cdot$ & 100 & $\cdot$ & 100 & $\cdot$ & 100 & $\cdot$ & 100 & $\cdot$ & 100 & \\
\hline xylenes & $5 \mathrm{U}$ & $\cdot$ & $5 U$ & $\cdot$ & 5u & $\cdot$ & 50 & $\cdot$ & 50 & $\cdot$ & 50 & \\
\hline Summed VOCs & 16 & 100.1 & 11 & 100 & 10 & 100 & 0 & . & 0 & . & 0 & \\
\hline
\end{tabular}

(CONTINUED) 
APPENDIX J

Validated vOC Results, 1993

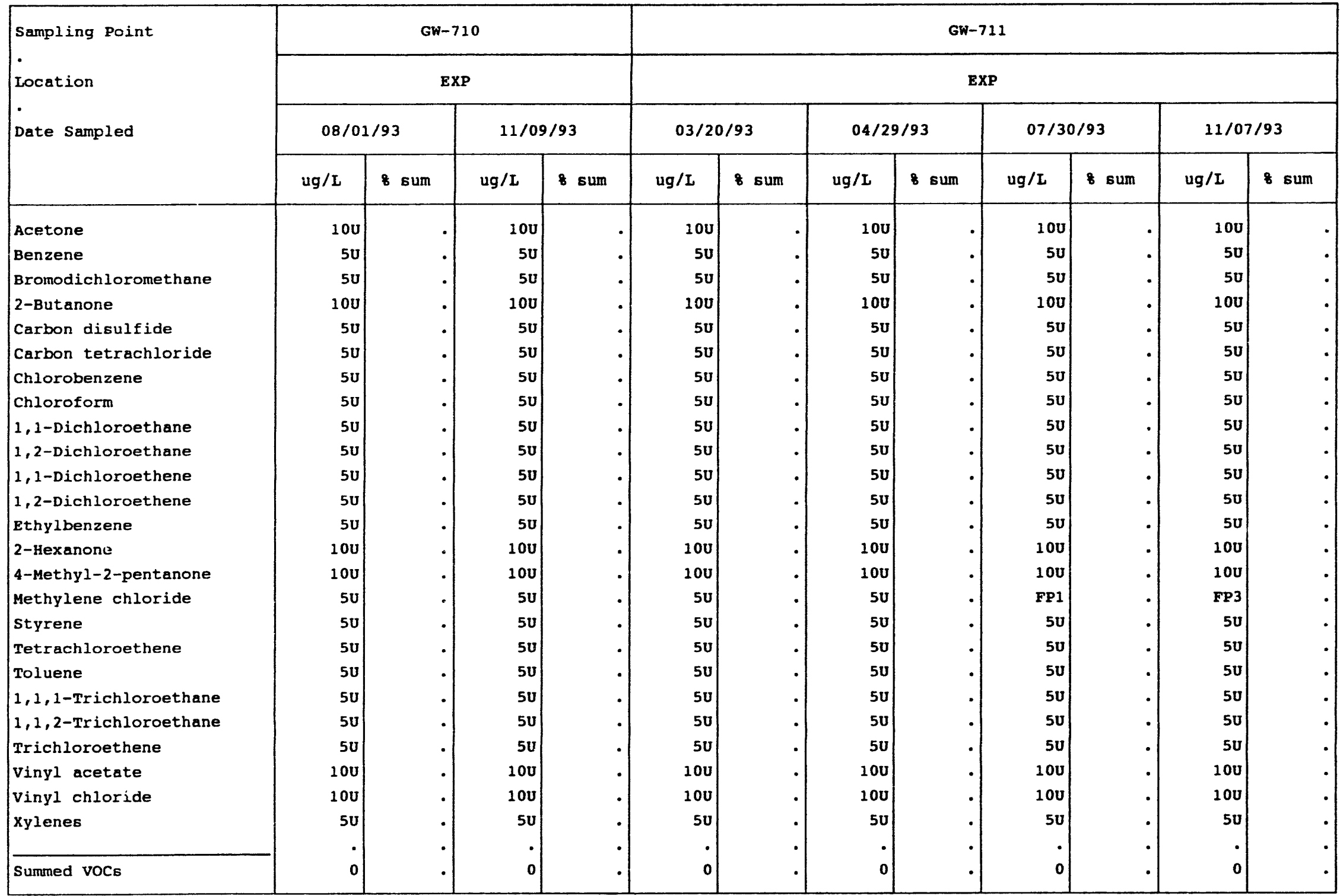

(CONTINUED) 
Validated VOC Results, 1993

\begin{tabular}{|c|c|c|c|c|c|c|c|c|c|c|c|c|c|}
\hline \multirow{4}{*}{$\begin{array}{l}\text { Sampling Point } \\
\text { Location } \\
\text { Date Sampled }\end{array}$} & \multicolumn{8}{|c|}{ GW-712 } & \multicolumn{5}{|c|}{ GW-713 } \\
\hline & \multicolumn{8}{|c|}{ EXP } & \multicolumn{5}{|c|}{$\mathbf{E X P}$} \\
\hline & \multicolumn{2}{|c|}{$03 / 18 / 93$} & \multicolumn{2}{|c|}{$04 / 28 / 93$} & \multicolumn{2}{|c|}{$07 / 29 / 93$} & \multicolumn{2}{|c|}{$11 / 06 / 93$} & \multicolumn{2}{|c|}{$03 / 19 / 93$} & \multicolumn{3}{|c|}{$04 / 30 / 93$} \\
\hline & $\mathrm{ug} / \mathrm{L}$ & 8 sum & $\mathrm{ug} / \mathrm{L}$ & 8 sum & $u g / x$ & 8 sum & ug $/ \mathrm{L}$ & 8 sum & $u g / L$ & 8 sum & $\mathrm{ug} / \mathrm{L}$ & 8 sun & \\
\hline Acetone & 100 & • & 100 & $\cdot$ & 100 & - & 100 & - & FP1 & - & 100 & & ${ }^{\circ}$ \\
\hline Benzene & 5u & - & $5 \mathrm{U}$ & • & 50 & - & 50 & - & $5 \mathbf{U}$ & $\cdot$ & 50 & & $\cdot$ \\
\hline Bromodichloromethane & 50 & - & su & - & 50 & - & 50 & - & $5 U$ & $\cdot$ & 50 & & $\cdot$ \\
\hline 2-Butanone & 100 & - & 100 & • & 100 & - & 100 & - & 100 & $\cdot$ & FP1 & & $\cdot$ \\
\hline Carbon disulfide & $5 u$ & - & $5 v$ & - & 50 & - & 50 & - & 5u & $\cdot$ & 50 & & $\cdot$ \\
\hline Carbon tetrachloride & 50 & - & $5 U$ & $\cdot$ & $5 \mathrm{U}$ & - & 50 & - & 50 & $\cdot$ & 50 & & $\cdot$ \\
\hline Chlorobenzene & 50 & - & $5 U$ & • & $5 \mathrm{u}$ & - & 50 & - & 50 & $\cdot$ & 50 & & $\cdot$ \\
\hline Chloroform & 50 & - & 50 & $\cdot$ & 50 & - & $5 \mathrm{U}$ & - & $5 U$ & $\cdot$ & 50 & & $\cdot$ \\
\hline 1,1-Dichloroethane & $5 U$ & - & 50 & • & $5 U$ & - & 50 & - & 50 & $\cdot$ & 50 & & - \\
\hline 1,2-Dichloroethane & $5 v$ & - & 50 & $\bullet$ & 50 & • & 5u & - & 50 & $\cdot$ & 50 & & $\cdot$ \\
\hline 1,1-Dichloroethene & $5 v$ & - & 50 & • & $5 u$ & - & 5u & - & $5 U$ & $\cdot$ & 50 & & $\cdot$ \\
\hline 1,2-Dichloroethene & 50 & • & 50 & $\cdot$ & $5 U$ & $\cdot$ & 50 & - & 50 & $\cdot$ & 50 & & $\cdot$ \\
\hline Ethylbenzene & 50 & • & 50 & • & $5 u$ & - & 50 & - & $5 \mathrm{U}$ & $\cdot$ & 50 & & $\cdot$ \\
\hline 2-Hexanone & 100 & • & 100 & $\cdot$ & 100 & - & 100 & - & $10 \mathrm{U}$ & $\cdot$ & 100 & & $\cdot$ \\
\hline 4-Methy1-2-pentanone & 100 & - & 100 & • & 100 & - & 100 & - & FF1 & $\cdot$ & 100 & & $\cdot$ \\
\hline Methylene chloride & 50 & • & 50 & • & FP1 & - & 50 & - & FP1 & $\cdot$ & 50 & & $\cdot$ \\
\hline styrene & 50 & - & 50 & • & 50 & $\cdot$ & su & - & $5 U$ & $\cdot$ & 5U & & $\cdot$ \\
\hline Tetrachloroethene & 50 & - & 50 & $\cdot$ & $5 \mathrm{U}$ & - & 50 & - & $5 U$ & $\cdot$ & 50 & & - \\
\hline Toluene & 50 & - & 50 & $\cdot$ & 50 & • & 50 & - & 50 & $\cdot$ & 50 & & • \\
\hline 1,1,1-Trichloroethane & $5 U$ & - & 50 & • & su & - & 5u & - & 50 & $\cdot$ & FP1 & & $\cdot$ \\
\hline $1,1,2$-Trichloroethane & 50 & - & 50 & • & 50 & - & 50 & - & SU & $\cdot$ & 50 & & $\cdot$ \\
\hline Trichloroethene & 50 & - & 50 & • & 50 & - & 50 & . & 50 & $\cdot$ & 50 & & $\cdot$ \\
\hline Vinyl acetate & 100 & - & 100 & • & 100 & - & 100 & - & 100 & $\cdot$ & 100 & & $\cdot$ \\
\hline vinyl chloride & 100 & - & 100 & - & 100 & - & 100 & - & 100 & - & 100 & & - \\
\hline xylenes & 50 & - & 50 & • & $5 \mathrm{U}$ & - & 50 & - & $5 \mathbf{u}$ & $\cdot$ & $5 \mathbf{v}$ & & $\cdot$ \\
\hline Summed VOCs & 0 & • & 0 & - & 0 & - & 0 & - & $\mathbf{0}$ & - & $\mathbf{0}$ & & $\cdot$ \\
\hline
\end{tabular}

(CONTINUED) 
APPENDIX J

Validated VOC Results, 1993

\begin{tabular}{|c|c|c|c|c|c|c|c|c|c|c|c|c|}
\hline \multirow{4}{*}{$\begin{array}{l}\text { Sampling Point } \\
\text { Location } \\
\text { Date Sampled }\end{array}$} & \multicolumn{4}{|c|}{ GW-713 } & \multicolumn{8}{|c|}{ GW-714 } \\
\hline & \multicolumn{4}{|c|}{$\operatorname{EXP}$} & \multicolumn{8}{|c|}{ EXP } \\
\hline & \multicolumn{2}{|c|}{$07 / 31 / 93$} & \multicolumn{2}{|c|}{$11 / 09 / 93$} & \multicolumn{2}{|c|}{$03 / 21 / 93$} & \multicolumn{2}{|c|}{$04 / 29 / 93$} & \multicolumn{2}{|c|}{$08 / 01 / 93$} & \multicolumn{2}{|c|}{$11 / 09 / 93$} \\
\hline & $\mathrm{ug} / \mathrm{L}$ & 8 sum & ug/L & 8 sum & $\mathrm{ug} / \mathrm{L}$ & 8 sum & $u g / L$ & 8 sum & $\mathrm{ug} / \mathrm{L}$ & 8 sum & ug/L & 8 sum \\
\hline Acetone & 100 & . & 100 & • & 100 & - & 100 & $\cdot$ & 100 & $\cdot$ & 100 & 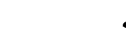 \\
\hline Benzene & $5 \mathrm{v}$ & . & $5 \mathrm{U}$ & • & 50 & - & 50 & $\cdot$ & 50 & $\cdot$ & $5 U$ & . \\
\hline Bromodichloromethane & 50 & $\cdot$ & 50 & - & 50 & - & 50 & $\cdot$ & $5 U$ & $\cdot$ & 50 & . \\
\hline 2-Butanone & 100 & $\cdot$ & 100 & - & 100 & • & 100 & $\cdot$ & 100 & $\cdot 1$ & 100 & . \\
\hline Carbon disulfide & su & $\cdot$ & 50 & - & 50 & - & 50 & $\cdot$ & 50 & $\cdot$ & $5 U$ & . \\
\hline Carbon tetrachloride & 50 & $\cdot$ & 50 & - & 50 & • & 50 & $\cdot$ & 50 & $\cdot$ & 50 & . \\
\hline Chlorobenzene & 50 & $\cdot$ & 50 & - & 50 & - & 50 & $\cdot$ & 5u & $\cdot$ & 50 & . \\
\hline Chloroform & 50 & $\cdot$ & 50 & - & 50 & - & 50 & $\cdot$ & 50 & $\cdot$ & 50 & . \\
\hline 1,1-Dichloroethane & 5u & $\cdot$ & 50 & - & 50 & - & $5 U$ &. & 50 & $\cdot$ & 50 & . \\
\hline 1,2-Dichloroethane & 50 & $\cdot$ & 50 & • & $5 v$ & - & 50 & $\cdot$ & 50 & $\cdot$ & 50 & . \\
\hline 1,1-Dichloroethene & 5u & $\cdot$ & 50 & - & 50 & • & $5 U$ & $\cdot$ & 50 & $\cdot$ & 50 & . \\
\hline 1,2-Dichloroethene & 5u & $\cdot$ & 50 & - & 50 & - & 50 & $\cdot$ & 50 & $\cdot$ & 50 & . \\
\hline Ethylbenzene & $5 v$ & $\cdot$ & 50 & - & 50 & - & 50 & $\cdot$ & 5u & $\cdot$ & 5u & . \\
\hline 2-Hexanone & 100 & $\cdot$ & $\because \quad 100$ & - & 100 & - & 100 & $\cdot$ & 100 & $\cdot$ & 100 & . \\
\hline 4-Methyl-2-pentanone & 100 & $\cdot$ & 100 & - & 100 & - & 100 & $\cdot$ & 100 & $\cdot$ & 100 & . \\
\hline Methylene chloride & 50 & $\cdot$ & FP1 & - & FP1 & - & FP2 & $\cdot$ & 50 & $\cdot$ & FP1 & . \\
\hline Styrene & 5u & $\cdot$ & 50 & - & $5 v$ & - & 50 & $\cdot$ & 50 & $\cdot$ & 50 & . \\
\hline Tetrachloroethene & 50 & $\cdot$ & 50 & - & 50 & • & $5 v$ & $\cdot$ & 50 & $\cdot$ & 50 & . \\
\hline Toluene & $5 v$ & $\cdot$ & 50 & - & 50 & - & 50 & $\cdot$ & 50 & $\cdot$ & 50 & . \\
\hline 1,1,1-Trichloroethane & 50 & $\cdot$ & 50 & - & 50 & • & 50 & $\cdot$ & 50 & $\cdot$ & 50 & . \\
\hline 1,1,2-Trichloroethane & 50 & $\cdot$ & 50 & - & 50 & - & 50 & $\cdot$ & 50 & $\cdot$ & $5 v$ & \\
\hline Trichloroethene & $5 v$ & $\cdot$ & 50 & - & 50 & - & 50 & $\cdot$ & 50 & $\cdot$ & 50 & r \\
\hline Vinyl acetate & 100 & $\cdot$ & 100 & - & 100 & - & 100 & • & 100 & $\cdot$ & 100 & . \\
\hline vinyl chloride & 100 & $\cdot$ & 100 & - & 100 & - & 100 & $\cdot$ & 100 & $\cdot$ & 100 & 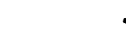 \\
\hline xylenes & 50 & $\cdot$ & 50 & - & $5 v$ & - & 50 & $\cdot$ & 50 & $\cdot$ & $5 \mathrm{U}$ & . \\
\hline Summed vocs & & $\cdot$ & 0 & - & 0 & - & 0 & $\cdot$ & 0 & $\cdot$ & 0 & . \\
\hline
\end{tabular}

(CONTINUED) 
Validated VOC Results, 1993

\begin{tabular}{|c|c|c|c|c|c|c|c|c|c|c|c|c|}
\hline \multirow{4}{*}{$\begin{array}{l}\text { Sampling Point } \\
\text { Location } \\
\text { Date Sampled }\end{array}$} & \multicolumn{8}{|c|}{ GW-715 } & \multicolumn{4}{|c|}{ GW-723 } \\
\hline & \multicolumn{8}{|c|}{$\operatorname{EXP}$} & \multicolumn{4}{|c|}{ EXP } \\
\hline & \multicolumn{2}{|c|}{$03 / 21 / 93$} & \multicolumn{2}{|c|}{$04 / 29 / 93$} & \multicolumn{2}{|c|}{$08 / 01 / 93$} & \multicolumn{2}{|c|}{$11 / 11 / 93$} & \multicolumn{2}{|c|}{$02 / 12 / 93$} & \multicolumn{2}{|c|}{$05 / 18 / 93$} \\
\hline & ug $/ \mathrm{L}$ & 8 sum & $u g / L$ & 8 sum & ug $/ \mathrm{L}$ & 8 sum & $\mathrm{ug} / \mathrm{L}$ & 8 sum & $\mathrm{ug} / \mathrm{L}$ & 8 sum & $\mathrm{ug} / \mathrm{L}$ & 8 sum \\
\hline Acetone & 100 & . & 100 & $\cdot 1$ & 100 & - & 100 & - & 100 & - & 100 & - \\
\hline Benzene & $5 u$ & . & 50 &. & 50 & . & 50 & - & 50 & - & 50 & . \\
\hline Bromodichloromethane & 50 & . & 50 &. & su & - & 5u & - & 5u & - & 50 & . \\
\hline 2-Butanone & 100 & . & 100 & $\cdot$ & 100 & . & 100 & - & FP1 & - & 100 & - \\
\hline Carbon disulfide & 50 & . & su & $\cdot$ & 50 & - & 50 & - & 50 & - & 50 & • \\
\hline Carbon tetrachloride & 50 & • & 50 & $\cdot$ & 50 & - & 50 & - & FP3 & - & 50 & • \\
\hline Chlorobenzene & $5 \mathrm{U}$ & • & 50 & $\cdot$ & 50 & - & 50 & - & 50 & $\cdot$ & 50 & $\cdot$ \\
\hline Chloroform & 50 & • & 50 & $\cdot$ & 50 & - & 50 & - & 5u & - & 50 & - \\
\hline 1,1-Dichloroethane & 50 & • & 50 & $\cdot$ & 50 & - & 50 & - & 50 & • & 50 & $\cdot$ \\
\hline 1,2-Dichloroethane & 50 & • & 50 & $\cdot$ & 50 & - & 50 & - & 50 & - & 50 & . \\
\hline 1,1-Dichloroethene & 50 & . & 50 & $\cdot$ & 50 & - & 5u & - & 50 & - & 50 & • \\
\hline 1,2-Dichloroethene & 50 & . & 50 & $\cdot$ & 50 & . & 50 & . & FP3 & - & $5 \mathbf{v}$ & - \\
\hline Ethylbenzene & 50 & - & 50 & $\cdot$ & 5u & - & so & - & 50 & - & 50 & . \\
\hline 2-Hexanone & 100 & • & 100 & $\cdot$ & 100 & - & 100 & * & 100 & - & 100 & . \\
\hline 4-Methy1-2-pentanone & 100 & . & 100 & $\cdot 1$ & 100 & - & 100 & - & FP1 & - & 100 & • \\
\hline Methylene chloride & FP3 & . & 50 & $\cdot$ & su & - & FP1 & - & FP1 & $\cdot$ & 50 & $\cdot$ \\
\hline Styrene & 50 & . & 50 & $\cdot$ & 50 & - & 50 & - & 50 & - & 50 & . \\
\hline Tetrachloroethene & 50 & - & 50 & $\cdot$ & $5 \mathrm{U}$ & - & 50 & - & 50 & $\cdot$ & 50 & . \\
\hline Toluene & 50 & - & 50 & $\cdot 1$ & 50 & - & 50 & - & 50 & - & 50 & . \\
\hline 1,1,1-Trichloroethane & 50 & - & 50 & $\cdot$ & 5u & - & 50 & - & 50 & - & 50 & • \\
\hline 1,1,2-Trichloroethane & $5 \mathrm{u}$ & • & 5u & $\cdot$ & su & - & 50 & - & 50 & - & 50 & . \\
\hline Trichloroethene & 50 & - & 5u & $\cdot 1$ & $5 U$ & - & 50 & - & 21 & 100 & 3 & 100 \\
\hline Vinyl acetate & 100 & • & 100 & $\cdot$ & 100 & - & 100 & - & 100 & . & 100 & • \\
\hline Vinyl chloride & 100 & - & 100 & $\cdot$ & 100 & . & 100 & - & 100 & - & 100 & . \\
\hline Xylenes & 50 & . & 50 &. & 50 & - & 50 & - & 50 & - & 50 & • \\
\hline Summed VOCs & 0 & • & 0 & $\cdot$ & $\mathbf{0}$ & . & o & - & 21 & 100 & 3 & 100 \\
\hline
\end{tabular}


APPENDIX $\mathrm{J}$

Validated VOC Results, 1993

\begin{tabular}{|c|c|c|c|c|c|c|c|c|c|c|c|c|}
\hline \multirow{4}{*}{$\begin{array}{l}\text { Sampling Point } \\
\text { Location } \\
\text { Date Sampled }\end{array}$} & \multicolumn{4}{|c|}{ GW-723 } & \multicolumn{8}{|c|}{$G W-724$} \\
\hline & \multicolumn{4}{|c|}{ EXP } & \multicolumn{8}{|c|}{ EXP } \\
\hline & \multicolumn{2}{|c|}{$09 / 27 / 93$} & \multicolumn{2}{|c|}{$12 / 14 / 93$} & \multicolumn{2}{|c|}{$02 / 10 / 93$} & \multicolumn{2}{|c|}{$05 / 18 / 93$} & \multicolumn{2}{|c|}{$09 / 29 / 93$} & \multicolumn{2}{|c|}{$12 / 14 / 93$} \\
\hline & $u g / L$ & 8 sum & $\mathrm{ug} / \mathrm{I}$ & 8 sum & $u g / L$ & 8 sum & $\mathrm{ug} / \mathrm{I}$ & sum & ug $/ \mathrm{L}$ & 8 sum & $u g / I$ & 8 sum \\
\hline Acetone & 100 & - & 100 & - & 100 & - & 100 & $\cdot$ & 100 & $\cdot$ & 100 & - \\
\hline Benzene & $5 v$ & - & $5 v$ & - & $5 v$ & - & 50 & $\cdot$ & 50 & $\cdot$ & 50 & $\cdot$ \\
\hline Bromodichloromethane & $5 v$ & - & 50 & - & $5 \mathbf{v}$ & • & 50 & $\cdot$ & 50 & $\cdot$ & 5u & . \\
\hline 2-Butanone & 100 & - & 100 & - & 100 & - & 100 & $\cdot$ & 100 & $\cdot$ & 100 & $\cdot$ \\
\hline Carbon disulfide & 50 & • & 50 & - & 50 & - & 5u & $\cdot$ & $5 v$ & $\cdot$ & 5u & • \\
\hline Carbon tetrachloride & $5 \mathrm{v}$ & • & 5u & - & so & - & 50 & $\cdot$ & $5 \mathbf{5 u}$ & $\cdot$ & FP3 & $\cdot$ \\
\hline Chlorcbenzene & 50 & - & 50 & - & $5 v$ & - & 50 & $\cdot$ & 50 & $\cdot$ & 50 & • \\
\hline Chloroform & 5v & - & su & - & 50 & - & 5u & $\cdot$ & FP3 & $\cdot$ & 50 & • \\
\hline 1,1-Dichloroethane & $5 v$ & - & $5 u$ & - & $5 v$ & - & 50 & $\cdot$ & $5 U$ & $\cdot$ & $5 u$ & • \\
\hline 1,2-Dichloroethane & 50 & - & 50 & - & $5 v$ & - & 50 & $\cdot$ & $5 v$ & $\cdot$ & 50 & $\cdot$ \\
\hline 1,1-Dichloroethene & su & - & 50 & - & 5u & - & su & $\cdot$ & 50 & $\cdot$ & $5 v$ & • \\
\hline 1,2-Dichloroethene & 50 & - & $5 v$ & $\cdot$ & 2 & 2 & 5u & $\cdot$ & 50 & $\cdot$ & 3 & 2.1 \\
\hline Ethylbenzene & 50 & - & 50 & $\cdot$ & 50 & - & 5u & $\cdot$ & 50 & $\cdot$ & 50 & • \\
\hline 2-Hexanone & 100 & , & 100 & $\cdot$ & 100 & - & 100 & $\cdot 1$ & 100 & $\cdot$ & 100 & • \\
\hline 4-Methy1-2-pentanone & 100 & - & 100 & $\cdot$ & 100 & $\cdot$ & 100 & $\cdot 1$ & 100 & $\cdot$ & 100 & $\cdot$ \\
\hline Methylene chloride & 5u & - & 50 & $\cdot$ & FP1 & - & 50 & $\cdot$ & 50 & $\cdot$ & 50 & • \\
\hline styrene & 50 & - & 50 & $\cdot$ & 50 & $\cdot$ & $5 v$ & $\cdot$ & 50 & $\cdot$ & 50 & $\cdot$ \\
\hline Tetrachloroethene & su & - & 5u & . & 2 & 2 & $5 v$ & $\cdot$ & $5 v$ & $\cdot$ & 3 & 2.1 \\
\hline Toluene & 5u & - & su & $\cdot$ & 50 & - & 50 & $\cdot$ & 50 & $\cdot$ & $5 v$ & $\cdot$ \\
\hline $1,1,1$-Trichloroethane & su & - & 50 & $\cdot$ & su & $\cdot$ & 50 & $\cdot$ & 5u & $\cdot$ & su & • \\
\hline $1,1,2$-Trichloroethane & $5 \mathrm{v}$ & - & 50 & • & 5u & $\cdot$ & 50 & $\cdot$ & 50 & $\cdot$ & 50 & $\cdot$ \\
\hline Trichloroethene & 4 & 100 & 5 & 100 & 95 & 96 & 46 & 100 & 3 & 100 & 140 & 95.9 \\
\hline vinyl acetate & 100 & - & 100 & • & 100 & $\cdot$ & 100 & $\cdot$ & 100 & $\cdot$ & 100 & $\cdot$ \\
\hline vinyl chloride & 100 & • & 100 & • & 100 & $\cdot$ & 100 & $\cdot$ & 100 & $\cdot$ & 100 & - \\
\hline Xylenes & 50 & $\cdot$ & 50 & - & 50 & $\cdot$ & $5 v$ & $\cdot$ & $5 v$ & $\cdot$ & 50 & $\cdot$ \\
\hline Summed vocs & 4 & 100 & 5 & 100 & 99 & 100 & 46 & 100 & 3 & 100 & 146 & 100.1 \\
\hline
\end{tabular}

(CONTINUED) 
APPEANIX J

Validated VoC Results, 1993

\begin{tabular}{|c|c|c|c|c|c|c|c|c|c|c|c|c|}
\hline \multirow{4}{*}{$\begin{array}{l}\text { Sampling Point } \\
\text { Location } \\
\text { - } \\
\text { Date Sampled }\end{array}$} & \multicolumn{8}{|c|}{ GW-725 } & \multicolumn{4}{|c|}{ GW-736 } \\
\hline & \multicolumn{8}{|c|}{$\operatorname{EXP}$} & \multicolumn{4}{|c|}{ EXP } \\
\hline & \multicolumn{2}{|c|}{$02 / 12 / 93$} & \multicolumn{2}{|c|}{$05 / 19 / 93$} & \multicolumn{2}{|c|}{$09 / 28 / 93$} & \multicolumn{2}{|c|}{$12 / 15 / 93$} & \multicolumn{2}{|c|}{$02 / 11 / 93$} & \multicolumn{2}{|c|}{$05 / 18 / 93$} \\
\hline & $\mathrm{ug} / \mathrm{L}$ & 8 sum & ug/L & 8 sum & ug $/ \mathrm{L}$ & 8 sum & $\mathrm{ug} / \mathrm{L}$ & 8 sum & ug/L & 8 sum & ug $/ \mathrm{L}$ & 8 sum \\
\hline Acetone & FP1 & $\cdot 1$ & 100 & - & 100 & $\cdot$ & FP1 & $\cdot$ & 100 & $\cdot$ & 100 & 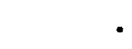 \\
\hline Benzene & $5 v$ & $\cdot$ & 50 & $\cdot$ & 50 & $\cdot$ & FP3 & $\cdot$ & 50 & $\cdot$ & 50 & - \\
\hline Bromodichloromethane & $5 v$ & $\cdot$ & su & $\cdot$ & 50 & $\cdot$ & 50 & $\cdot$ & 50 & $\cdot$ & 50 & - \\
\hline 2-Butanone & FP1 & $\cdot$ & 100 & $\cdot$ & 100 & $\cdot$ & FP1 & $\cdot$ & 100 & $\cdot$ & 100 & - \\
\hline Carbon disulfide & 50 & $\cdot$ & 50 & $\cdot$ & $5 v$ & $\cdot$ & 50 & $\cdot$ & FP3 & $\cdot$ & 50 & - \\
\hline Carbon tetrachloride & 50 & $\cdot$ & 50 & $\cdot$ & 50 & $\cdot$ & 50 & $\cdot$ & FP3 & $\cdot$ & 50 & - \\
\hline Chlorobenzene & 50 & $\cdot$ & 50 & $\cdot$ & 50 & $\cdot$ & 50 & $\cdot$ & FP3 & $\cdot$ & 50 & - \\
\hline Chloroform & 5u & $\cdot$ & 50 & $\cdot$ & $5 U$ & $\cdot$ & 50 & $\cdot$ & FP3 & $\cdot$ & 5u & 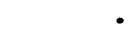 \\
\hline 1,1-Dichloroethane & 5u & $\cdot$ & 50 & $\cdot$ & 50 & $\cdot$ & 50 & $\cdot$ & 50 & $\cdot$ & 50 & . \\
\hline 1,2-Dichloroethane & 50 & $\cdot$ & 50 & $\cdot$ & $5 U$ & $\cdot$ & su & $\cdot$ & 50 & $\cdot$ & 50 & . \\
\hline 1,1-Dichloroethene & 50 & $\cdot$ & 50 & $\cdot$ & 50 & $\cdot$ & $5 u$ & $\cdot$ & FP3 & $\cdot$ & 50 & - \\
\hline 1,2-Dichloroethene & 2 & 9.5 & 3 & 5.4 & 2 & 4 & 3 & 5.1 & 7 & 26.9 & 5 & 25 \\
\hline Ethylbenzene & 50 & $\cdot$ & 50 & $\cdot$ & 50 & $\cdot$ & 50 & $\cdot$ & FP3 & $\cdot$ & 50 & - \\
\hline 2-Hexanone & 100 & $\cdot$ & 100 & $\cdot$ & 100 & $\cdot$ & 100 & $\cdot$ & 100 & $\cdot$ & 100 & - \\
\hline 4-Methyl-2-pentanone & FP1 & $\cdot$ & 100 & $\cdot$ & 100 & $\cdot$ & FP1 & $\cdot$ & FP1 & $\cdot$ & 100 & . \\
\hline Methylene chloride & FP1 & $\cdot$ & 50 & $\cdot$ & 50 & $\cdot$ & FP1 &. & FP1 & $\cdot$ & 50 & - \\
\hline Styrene & 50 & $\cdot$ & 50 & $\cdot$ & 50 & $\cdot$ & 50 & $\cdot 1$ & FP3 & $\cdot$ & 50 & - \\
\hline Tetrachloroethene & 5u & $\cdot$ & 5u & $\cdot$ & 0.8 & 1.6 & 1 & 1.7 & 7 & 26.9 & 3 & 15 \\
\hline Toluene & 50 & $\cdot$ & 50 & $\cdot$ & $5 v$ & $\cdot$ & 2 & 3.4 & su & $\cdot$ & 5u & • \\
\hline 1,1,1-Trichloroethane & 50 & $\cdot$ & 50 & $\cdot$ & 50 & $\cdot$ & 50 & $\cdot$ & 50 & $\cdot$ & su & . \\
\hline 1,1,2-Trichloroethane & 50 &. & 50 & $\cdot$ & 50 & $\cdot$ & 50 & $\cdot$ & FP3 & $\cdot$ & 50 & 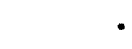 \\
\hline Trichloroethene & 19 & 90.5 & 53 & 94.6 & 47 & 94.4 & 53 & 89.8 & 12 & 46.2 & 12 & 60 \\
\hline Vinyl acetate & 100 & $\cdot$ & 100 & $\cdot$ & 100 & $\cdot$ & 100 &. & 100 & $\cdot$ & 100 & - \\
\hline vinyl chloride & 100 & $\cdot$ & 100 & $\cdot$ & 100 & $\cdot$ & 100 & $\cdot$ & 100 & $\cdot$ & 100 & - \\
\hline xylenes & 50 & $\cdot$ & 50 & $\cdot$ & 50 & $\cdot$ & 50 & $\cdot$ & FP3 & $\cdot$ & 50 & - \\
\hline Summed VOCs & 21 & 100 & 56 & 100 & 49.8 & 100 & 59 & 100 & 26 & 100 & 20 & 100 \\
\hline
\end{tabular}

(CONTINUED) 
APPENDIX J

validated vOC Results, 1993

\begin{tabular}{|c|c|c|c|c|c|c|c|c|c|c|c|c|}
\hline \multirow{4}{*}{$\begin{array}{l}\text { Sampling Point } \\
\text { Location } \\
\text { Date Sampled }\end{array}$} & \multicolumn{4}{|c|}{ GW-736 } & \multicolumn{8}{|c|}{ GW-737 } \\
\hline & \multicolumn{4}{|c|}{$\mathbf{E X P}$} & \multicolumn{8}{|c|}{$\mathbf{E X P}$} \\
\hline & \multicolumn{2}{|c|}{$09 / 28 / 93$} & \multicolumn{2}{|c|}{$12 / 15 / 93$} & \multicolumn{2}{|c|}{$02 / 11 / 93$} & \multicolumn{2}{|c|}{$05 / 19 / 93$} & \multicolumn{2}{|c|}{$09 / 28 / 93$} & \multicolumn{2}{|c|}{$12 / 15 / 93$} \\
\hline & ug/L & \& sum & ug/L & 8 sum & ug/L & 8 sum & ug/L & 8 sum & ug/L & 8 sum & $\mathrm{ug} / \mathrm{L}$ & 8 sum \\
\hline Acetone & 100 & $\cdot$ & FP3 &. & FP1 & - & 100 & $\cdot$ & 100 & $\cdot$ & FP1 & - \\
\hline Benzene & $5 \mathrm{u}$ & $\cdot$ & 50 & $\cdot$ & 50 & - & 50 & $\cdot$ & 50 & $\cdot$ & 50 & $\bullet$ \\
\hline Bromodichloromethane & 50 & $\cdot$ & 50 & $\cdot$ & 50 & • & $5 \mathrm{~J}$ & $\cdot$ & 50 & $\cdot$ & 50 & $\bullet$ \\
\hline 2-Butanone & 100 & $\cdot$ & FP3 & $\cdot 1$ & 100 & - & 100 & $\cdot$ & 100 & $\cdot$ & FP1 & • \\
\hline Carbon disulfide & 50 & $\cdot$ & 50 & $\cdot$ & $5 v$ & - & 5u & $\cdot$ & 50 & $\cdot 1$ & 50 & • \\
\hline Carbon tetrachloride & 50 & $\cdot$ & 50 & $\cdot$ & FP3 & - & 50 & $\cdot$ & 50 & $\cdot$ & 50 & • \\
\hline Chlorobenzene & $5 v$ & $\cdot$ & 50 & $\cdot$ & 50 & - & 50 & $\cdot$ & 50 & $\cdot$ & 50 & • \\
\hline Chloroform & 50 & $\cdot$ & 50 & $\cdot$ & 2 & 10.5 & 2 & 11.1 & 1 & 6.3 & 50 & $\cdot$ \\
\hline 1,1-Dichloroethane & 50 & $\cdot$ & 50 & $\cdot$ & 50 & - & 50 & $\cdot$ & 50 & $\cdot$ & 50 & $\cdot$ \\
\hline 1,2-Dichloroethane & 50 & $\cdot$ & 50 & $\cdot$ & 50 & - & 50 & $\cdot$ & 5u & $\cdot$ & 50 & • \\
\hline 1,1-Dichloroethene & $5 u$ & $\cdot$ & 50 & $\cdot$ & 50 & - & 50 & $\cdot$ & 50 & $\cdot$ & 50 & • \\
\hline 1,2-Dichloroethene & 3 & 23.1 & 5 & 23.8 & 4 & 21.1 & 4 & 22.2 & 3 & 18.8 & 4 & 22.2 \\
\hline Ethylbenzene & su & $\cdot$ & 50 & $\cdot$ & 50 & - & 50 &. & 50 & $\cdot$ & 50 & • \\
\hline 2-Hexanone & 100 & $\cdot$ & 100 & $\cdot$ & 100 & - & 100 & $\cdot$ & 100 & $\cdot$ & 100 & • \\
\hline 4-Methy1-2-pentanone & 100 & $\cdot$ & FP3 & $\cdot$ & FP1 & - & 100 & $\cdot$ & 100 & $\cdot$ & FP1 & $\cdot$ \\
\hline Methylene chloride & 50 & $\cdot$ & FP3 & $\cdot$ & FP1 & - & 50 & $\cdot$ & 50 & $\cdot$ & FP1 & • \\
\hline Styrene & 50 & $\cdot$ & $5 U$ & $\cdot$ & 50 & - & 50 & $\cdot$ & 50 & $\cdot$ & $5 \mathbf{v}$ & • \\
\hline Tetrachloroethene & 1 & 7.7 & 3 & 14.3 & 4 & 21.1 & 3 & 16.7 & 3 & 18.8 & 3 & 16.7 \\
\hline Toluene & 50 & - & $5 \mathrm{v}$ & $\cdot$ & 50 & - & 50 & $\cdot$ & 50 & $\cdot$ & 50 & $\cdot$ \\
\hline 1,1,1-Trichloroethane & 50 & $\cdot$ & $5 v$ & $\cdot$ & 50 & - & 50 & $\cdot$ & 50 & $\cdot$ & 50 & • \\
\hline $1,1,2$-Trichloroethane & $5 v$ & $\cdot$ & 50 & $\cdot$ & 50 & - & 50 & $\cdot$ & 50 & $\cdot$ & 50 & • \\
\hline Trichloroethene & 9 & 69.2 & 13 & 61.9 & 9 & 47.4 & 9 & 50 & 9 & 56.3 & 11 & 61.1 \\
\hline Vinyl acetate & 100 & . & 100 & $\cdot$ & 100 & • & 100 & $\cdot$ & 100 & $\cdot$ & 100 & • \\
\hline vinyl chloride & 100 & $\cdot$ & 100 & $\cdot 1$ & 100 & - & 100 & $\cdot$ & 100 & $\cdot$ & 100 & • \\
\hline xylenes & 50 & $\cdot$ & 50 & $\cdot$ & 5u & - & $5 v$ & $\cdot$ & 50 & $\cdot$ & 50 & $\cdot$ \\
\hline summed vocs & 13 & 100 & $\dot{21}$ & 100 & $\dot{19}$ & 100.1 & 18 & 100 & 16 & 100.2 & 18 & 100 \\
\hline
\end{tabular}

(CONTINUED) 


\begin{tabular}{|c|c|c|c|c|c|c|c|c|c|c|c|c|}
\hline \multirow{4}{*}{$\begin{array}{l}\text { Sampling Point } \\
\text { Location } \\
\text { Date Sampled }\end{array}$} & \multicolumn{8}{|c|}{ GW-738 } & \multicolumn{4}{|c|}{ GW-739 } \\
\hline & \multicolumn{8}{|c|}{ EXP } & \multicolumn{4}{|c|}{$\mathbf{E X P}$} \\
\hline & \multicolumn{2}{|c|}{$02 / 10 / 93$} & \multicolumn{2}{|c|}{$05 / 18 / 93$} & \multicolumn{2}{|c|}{$09 / 27 / 93$} & \multicolumn{2}{|c|}{$12 / 14 / 93$} & \multicolumn{2}{|c|}{$02 / 10 / 93$} & \multicolumn{2}{|c|}{$05 / 18 / 93$} \\
\hline & $u g / L$ & 8 sum & ug $/ \mathbf{L}$ & 8 sum & $u g / L$ & 8 sum & ug/L & 8 sum & ug/L & sum & ug/L & s sum \\
\hline Acetone & 100 & $\cdot$ & 100 & $\cdot$ & 100 & $\cdot$ & 100 & $\cdot$ & 100 & $\cdot$ & 100 & $\cdot$ \\
\hline Benzene & 50 & $\cdot$ & $5 \mathbf{5}$ & $\cdot$ & 50 & $\cdot$ & 50 & $\cdot$ & 50 & $\cdot$ & 50 & - \\
\hline Bromodichloromethane & 50 & $\cdot$ & su & $\cdot$ & 50 & $\cdot$ & 50 & $\cdot$ & 50 & $\cdot$ & $\begin{array}{r}50 \\
100\end{array}$ & - \\
\hline 2-Butanone & 100 & $\cdot$ & 100 & $\cdot$ & 100 & $\cdot$ & $\begin{array}{r}100 \\
5 n\end{array}$ & $\cdot$ & $\begin{array}{r}100 \\
50\end{array}$ & $\cdot$ & $\begin{array}{r}100 \\
5 \mathrm{U}\end{array}$ & • \\
\hline Carbon disulfide & $\begin{array}{r}5 \mathrm{U} \\
1\end{array}$ & $\begin{array}{r}. \dot{6} \\
1.6\end{array}$ & $\begin{array}{l}50 \\
50\end{array}$ & $\cdot \dot{1}$ & $\begin{array}{l}50 \\
50\end{array}$ & $\cdot$ & $\begin{array}{r}30 \\
1\end{array}$ & 1.8 & 50 &. & 50 & • \\
\hline $\begin{array}{l}\text { Carbon tetrachloride } \\
\text { Chlorobenzene }\end{array}$ & $\begin{array}{r}1 \\
5 v\end{array}$ &. & 50 &. & $5 \mathrm{u}$ &. & 50 & $\cdot$ & $5 \mathrm{v}$ &. & 50 & • \\
\hline Chloroform & 2 & 3.2 & 2 & 3.6 & 1 & 2.1 & 2 & 3.6 & 50 & $\cdot$ & 50 & • \\
\hline 1,1-Dichloroethane & $5 \mathrm{v}$ & $\cdot$ & 50 & $\cdot 1$ & 50 & $\cdot$ & 50 & $\cdot$ & 50 & $\cdot$ & 50 & $\cdot$ \\
\hline 1,2-Dichloroethane & 50 & $\cdot$ & 50 & $\cdot$ & 50 & $\cdot$ & 50 & $\cdot$ & 5u & $\cdot$ & 50 & $\cdot$ \\
\hline 1,1-Dichloroethene & 50 & $\cdot$ & 50 & $\cdot$ & $5 \mathbf{v}$ & $\cdot$ & 50 & $\cdot$ & 50 & $\cdot$ & so & $\cdot$ \\
\hline 1,2-Dichloroethene & 4 & 6.5 & 3 & 5.4 & 3 & 6.3 & 3 & 5.4 & 2 & 5.9 & 50 & • \\
\hline Ethylbenzene & 50 & $\cdot$ & $5 \mathbf{u}$ & $\cdot$ & 50 & $\cdot$ & 50 & $\cdot$ & 50 & $\cdot$ & 50 & • \\
\hline 2-Hexanone & 100 & $\cdot$ & 100 & $\cdot$ & 100 & $\cdot$ & 100 & $\cdot$ & FP1 & - & 100 & . \\
\hline 4-Methyl-2-pentanone & 100 & $\cdot$ & 100 & $\cdot$ & 100 & $\cdot$ & 100 & $\cdot 1$ & FP1 & $\cdot$ & 100 & $\bullet$ \\
\hline Methylene chloride & FP1 & $\cdot$ & 50 & $\cdot$ & 50 & $\cdot$ & 50 & $\cdot$ & FP1 & $\cdot$ & 50 & $\cdot$ \\
\hline Styrene & 5u & $\cdot$ & 50) & $\cdot$ & 50 & $\cdot$ & 5u & $\cdot$ & 50 & $\cdot$ & 50 & $\cdot$ \\
\hline Tetrachloroethene & 50 & $\cdot$ & 50 & $\cdot$ & 50 & $\cdot$ & 50 & $\cdot$ & 50 & $\cdot$ & 50 & $\cdot$ \\
\hline Toluene & 50 & $\cdot$ & 5u & $\cdot$ & 5u & $\cdot$ & 50 & $\cdot$ & 50 & $\cdot$ & 50 & $\cdot$ \\
\hline $1,1,1$-Trichloroethane & 5u & $\cdot$ & su & $\cdot$ & 50 & $\cdot$ & 50 & $\cdot$ & 5u & $\bullet$ & 50 & - \\
\hline 1,1,2-Trichloroethane & 50 & & 5U & & 5u & & 50 & 80 & $\begin{array}{l}5 \mathrm{v} \\
32\end{array}$ & 94.1 & $\begin{array}{l}50 \\
33\end{array}$ & 100 \\
\hline Trichloroethene & 55 & 88.7 & $\begin{array}{r}51 \\
100\end{array}$ & 91.1 & $\begin{array}{r}44 \\
100\end{array}$ & $\begin{array}{r}91.7 \\
.1\end{array}$ & $\begin{array}{r}50 \\
100\end{array}$ & $\begin{array}{r}89.3 \\
.\end{array}$ & $\begin{array}{r}32 \\
\text { FP3 }\end{array}$ & $\begin{array}{r}94.1 \\
.\end{array}$ & 100 & . \\
\hline vinyl acetate & $\begin{array}{l}10 \mathrm{U} \\
10 \mathrm{U}\end{array}$ & & $\begin{array}{l}100 \\
100\end{array}$ & $\cdot \dot{.}$ & 100 &. & 100 &. & 100 &. & 100 & . \\
\hline $\begin{array}{l}\text { Vinyl chloride } \\
\text { xylenes }\end{array}$ & $\begin{array}{r}100 \\
50\end{array}$ & $\cdot$ & 50 & $\cdot$ & 50 &. & su &. & 50 & . & 50 & • \\
\hline & $\cdot$ & $\cdot$ & $\cdot$ & & $\cdot$ & & $\cdot$ & & $\cdot$ & $\cdot$ & $\cdot$ & $\dot{0}$ \\
\hline Summed voCs & 62 & 100 & 56 & 100.1 & 48 & 100.1 & 56 & 100.1 & 34 & 100 & 33 & 100 \\
\hline
\end{tabular}

(CONTINUED) 
Validated VOC Results, 1993

\begin{tabular}{|c|c|c|c|c|c|c|c|c|c|c|c|c|}
\hline \multirow{4}{*}{$\begin{array}{l}\text { Sampling Point } \\
\text { Location } \\
\text { Date Sampled }\end{array}$} & \multicolumn{4}{|c|}{ GW-739 } & \multicolumn{8}{|c|}{$G W-740$} \\
\hline & \multicolumn{4}{|c|}{ EXP } & \multicolumn{8}{|c|}{ EXP } \\
\hline & \multicolumn{2}{|c|}{$09 / 27 / 93$} & \multicolumn{2}{|c|}{$12 / 13 / 93$} & \multicolumn{2}{|c|}{$02 / 09 / 93$} & \multicolumn{2}{|c|}{$05 / 17 / 93$} & \multicolumn{2}{|c|}{$09 / 27 / 93$} & \multicolumn{2}{|c|}{$12 / 11 / 93$} \\
\hline & ug/L & 8 sum & $u g / L$ & 8 sum & $u g / L$ & 8 sum & ug/t & 8 sum & ug $/ \mathrm{L}$ & 8 sum & $\mathrm{ug} / \mathrm{L}$ & 8 sum \\
\hline Acetone & 100 & $\cdot$ & 100 & - & 100 & $\cdot$ & 100 & $\cdot$ & 100 & $\cdot$ & 100 & • \\
\hline Benzene & 50 & $\cdot$ & 50 & $\cdot$ & 50 & $\cdot$ & 50 & $\cdot$ & 50 & $\cdot$ & 5u & $\cdot$ \\
\hline Bromodichloromethane & $5 U$ & $\cdot$ & 5u & $\cdot$ & 50 & $\cdot$ & 50 & $\cdot$ & 50 & $\cdot$ & $5 U$ & $\cdot$ \\
\hline 2-Butanone & 100 & $\cdot$ & 100 & $\cdot$ & 100 & $\cdot$ & 100 & $\cdot$ & 100 & $\cdot$ & 100 & $\cdot$ \\
\hline Carbon disulfide & 50 & $\cdot$ & 50 & • & 50 & $\cdot$ & $5 v$ & $\cdot$ & $5 v$ & $\cdot 1$ & 50 & • \\
\hline Carbon tetrachloride & 5u & $\cdot$ & FP3 & $\cdot$ & FP3 & $\cdot$ & 50 & $\cdot 1$ & 50 & $\cdot 1$ & $5 U$ & $\cdot$ \\
\hline Chlorobenzene & 50 & . & 50 & • & 5u & $\cdot$ & 50 & $\cdot$ & 5v & $\cdot 1$ & 50 & • \\
\hline Chloroform & 50 & $\cdot$ & FP3 & $\cdot$ & 2 & 3.4 & 2 & 4.8 & 1 & 2.8 & 1 & 2.6 \\
\hline 1,1-Dichloroethane & 50 & $\cdot$ & 50 & • & 50 & $\cdot$ & 50 & $\cdot$ & 50 & $\cdot$ & 50 & • \\
\hline 1,2-Dichloroethane & 50 & $\cdot$ & 50 & $\cdot$ & 50 & $\cdot$ & $5 \mathbf{u}$ & $\cdot$ & 5v & $\cdot$ & 50 & $\cdot$ \\
\hline 1,1-Dichloroethene & 50 & $\cdot$ & su & $\cdot$ & 50 & $\cdot$ & $5 v$ & $\cdot$ & so & $\cdot$ & $5 \mathbf{U}$ & $\cdot$ \\
\hline 1,2-Dichloroethene & 2 & 4.9 & 2 & 4.5 & 4 & 6.9 & 2 & 4.8 & 2 & 5.6 & 2 & 5.1 \\
\hline Ethylbenzene & 50 & $\cdot$ & 50 & $\cdot$ & 50 & $\cdot$ & $5 v$ & $\cdot$ & 5u & $\cdot$ & 50 & $\cdot$ \\
\hline 2-Hexanone & 100 & $\cdot$ & 100 & $\cdot$ & 200 & $\cdot$ & 100 & $\cdot$ & 100 & $\cdot$ & 100 & $\cdot$ \\
\hline 4-Methyl-2-pentanone & 100 & . & 100 & $\cdot$ & FP3 & $\cdot$ & 100 & $\cdot$ & 100 & $\cdot$ & 100 & • \\
\hline Methylene chloride & 50 & $\cdot$ & 50 & $\cdot$ & 2 & 3.4 & $5 v$ & $\cdot$ & 50 & $\cdot$ & 1 & 2.6 \\
\hline styrene & 50 & $\cdot$ & 50 & $\cdot$ & 5v & $\cdot$ & 50 & $\cdot$ & 50 & $\cdot$ & 50 & $\cdot$ \\
\hline Tetrachloroethene & 50 & $\cdot$ & 50 & $\cdot$ & 50 & $\cdot$ & $5 v$ & $\cdot$ & 50 & $\cdot$ & 50 & $\cdot$ \\
\hline Toluene & 50 & $\cdot$ & 50 & $\cdot$ & 50 & $\cdot$ & 50 & $\cdot 1$ & 50 & $\cdot$ & 50 & $\cdot$ \\
\hline 1,1,1-Trichloroethane & 50 & $\cdot$ & FP3 & $\cdot$ & 5u & $\cdot$ & 50 & $\cdot$ & 50 & $\cdot$ & 50 & $\cdot$ \\
\hline 1,1,2-Trichloroethane & 50 & $\cdot$ & 50 & $\cdot$ & 50 & $\cdot$ & 50 & $\cdot$ & su & $\cdot$ & 50 & $\cdot$ \\
\hline Trichloroethene & 39 & 95.1 & 42 & 95.5 & 50 & 86.2 & 38 & 90.5 & 33 & 91.7 & 35 & 89.7 \\
\hline vinyl acetate & $10 \mathrm{U}$ & $\cdot$ & 100 & $\cdot$ & 100 & $\cdot$ & 100 & $\cdot$ & $10 u$ & $\cdot$ & 100 & $\cdot$ \\
\hline vinyl chloride & 100 & . & 100 & - & 100 &. & 100 & $\cdot$ & 100 & $\cdot$ & 100 & • \\
\hline xylenes & 50 & $\cdot$ & 50 & $\cdot$ & 50 & $\cdot$ & 50 & $\cdot$ & 50 & $\cdot$ & 50 & • \\
\hline Summed vocs & 41 & 100 & 44 & 100 & 58 & 99.9 & 42 & 100.1 & 36 & 100.1 & 39 & 100 \\
\hline
\end{tabular}

(CONTINUED) 
Validated voc Results, 1993

\begin{tabular}{|c|c|c|c|c|c|c|c|c|c|c|c|c|c|}
\hline \multirow{4}{*}{$\begin{array}{l}\text { Sampling Point } \\
\text { Location } \\
\text { Date Sampled }\end{array}$} & \multicolumn{8}{|c|}{ GW-793 } & \multicolumn{5}{|c|}{ GW-794 } \\
\hline & \multicolumn{8}{|c|}{ AGLLSF } & \multicolumn{5}{|c|}{ AGLLSF } \\
\hline & \multicolumn{2}{|c|}{$02 / 26 / 93$} & \multicolumn{2}{|c|}{$05 / 25 / 93$} & \multicolumn{2}{|c|}{$07 / 11 / 93$} & \multicolumn{2}{|c|}{$10 / 18 / 93$} & \multicolumn{2}{|c|}{$03 / 01 / 93$} & \multicolumn{3}{|c|}{$05 / 25 / 93$} \\
\hline & ug/I & 8 sum & ug $/ \mathrm{L}$ & 8 sum & $u g / L$ & $\&$ sum & $u g / L$ & 8 sum & $u g / L$ & 8 sum & ug/L & 8 sum & \\
\hline Acetone & 100 & $\cdot 1$ & 100 & $\cdot$ & 100 & $\cdot$ & FP3 & $\cdot$ & 100 & $\cdot$ & 100 & & $\bullet$ \\
\hline Benzene & 50 & $\cdot$ & 50 & $\cdot$ & 50 & $\cdot$ & $5 U$ & $\cdot$ & 50 & $\cdot$ & 50 & & - \\
\hline Bromodichloromethane & 50 & $\cdot$ & 5u & $\cdot$ & $5 \mathrm{U}$ & $\cdot$ & 50 & $\cdot$ & 50 & $\cdot$ & 50 & & $\cdot$ \\
\hline 2-Butanone & 100 & $\cdot$ & FP1 & $\cdot$ & 100 & $\cdot$ & FP3 & $\cdot$ & 100 & $\cdot$ & FP3 & & $\cdot$ \\
\hline Carbon disulfide & 50 & $\cdot$ & 50 & $\cdot$ & 50 & $\cdot 1$ & 50 & $\cdot$ & 50 & $\cdot$ & 50 & & $\cdot$ \\
\hline Carbon tetrachloride & 50 & $\cdot$ & 5u & $\cdot$ & 50 & $\cdot$ & 50 & $\cdot$ & $\begin{array}{l}50 \\
50\end{array}$ & $\cdot$ & $\begin{array}{l}50 \\
5 v\end{array}$ & &. \\
\hline Chlorobenzene & 50 & $\cdot$ & 50 & $\cdot$ & 50 & $\cdot$ & $\begin{array}{l}50 \\
50\end{array}$ & $\bullet$ & 50 &. & 50 & & $\dot{.}$ \\
\hline Chloroform & 50 & $\cdot$ & $5 \mathrm{U}$ & $\cdot$ & $\begin{array}{l}50 \\
50\end{array}$ & $\cdot$. & 50 & . & 50 &. & 50 & & . \\
\hline 1,1-Dichloroethane & 50 & $\cdot$ & 50 & $\cdot$ & $\begin{array}{l}50 \\
50\end{array}$ &. & 50 &. & 50 &. & 50 & & 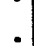 \\
\hline 1,2-Dichloroethane & 50 & $\cdot$ & 50 & $\cdot$ & 50 &. & 5u &. & 50 &. & 50 & & . \\
\hline 1,1-Dichloroethene & 50 & $\cdot 1$ & 50 & $\cdot$ & 50 &. & 50 &. & 50 &. & 50 & & 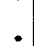 \\
\hline 1,2-Dichloroethene & 50 & $\cdot$ & $\begin{array}{l}50 \\
50\end{array}$ & $\cdot$ & 50 & $\cdot$ & 50 & $\cdot$ & 50 &. & 50 & & . \\
\hline $\begin{array}{l}\text { Ethylbenzene } \\
\text { 2-Hexanone }\end{array}$ & $\begin{array}{r}50 \\
100\end{array}$ &. & $\begin{array}{r}30 \\
100\end{array}$ &. & 100 &. & 100 & . & 100 & $\cdot$ & 100 & & $\bullet$ \\
\hline $\begin{array}{l}\text { 2-Hexanone } \\
\text { 4-Methy1-2-pentanone }\end{array}$ & 100 &. & FP1 &. & 100 & $\cdot 1$ & 100 & . & 100 & $\cdot$ & FP3 & & 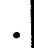 \\
\hline $\begin{array}{l}\text { 4-Methyl-2-pentanone } \\
\text { Methylene chloride }\end{array}$ & 50 &. & $5 v$ &. & 50 &. & FP3 & - & 50 & $\cdot$ & 5u & & $\bullet$ \\
\hline $\begin{array}{l}\text { Methylene chloride } \\
\text { Styrene }\end{array}$ & $5 \mathrm{v}$ &. & 50 & $\cdot$ & 50 & $\cdot$ & 50 &. & $5 U$ &. & 50 & & \\
\hline $\begin{array}{l}\text { Styrene } \\
\text { Tetrachloroethene }\end{array}$ & 50 & $\cdot$ & 50 &. & 50 &. & $5 U$ & . & 50 & $\cdot$ & 50 & & - \\
\hline $\begin{array}{l}\text { Tetrachloroethene } \\
\text { Toluene }\end{array}$ & 50 & $\cdot$ & 50 & $\cdot$ & 50 &. & 50 & $\cdot$ & 50 &. & 50 & & • \\
\hline $\begin{array}{l}\text { Toluene } \\
1,1,1 \text {-Trichloroethane }\end{array}$ & 50 & $\cdot$ & $5 v$ & $\cdot$ & 50 & $\cdot$ & 50 & $\cdot$ & 50 & $\cdot$ & 50 & & • \\
\hline $1,1,2$-Trichloroethane & $5 v$ & $\cdot$ & 50 & $\cdot$ & 50 & $\cdot$ & 50 & $\cdot$ & 50 & $\cdot$ & 50 & & $\bullet$ \\
\hline Trichloroethene & 50 & $\cdot$ & 50 & $\cdot$ & 50 & $\cdot$ & 50 & $\cdot$ & 50 & $\cdot$ & su & & $\bullet$ \\
\hline vinyl acetate & 100 & $\cdot$ & 100 & $\cdot$ & 100 & $\cdot$ & 100 & $\cdot$ & 100 & $\cdot$ & 100 & & - \\
\hline vinyl chloride & 100 & $\cdot$ & 100 & $\cdot$ & 100 & $\cdot$ & 100 & $\cdot$ & 100 & $\cdot$ & 100 & & - \\
\hline Xylenes & 50 & $\cdot$ & 50 & $\cdot$ & 50 & $\cdot$ & 50 & $\cdot$ & 50 & $\cdot$ & 50 & & • \\
\hline Summed vocs & 0 &. & 0 & $\cdot$ & 0 & $\cdot$ & 0 & $\cdot$ & o) & $\cdot$ & 0 & & • \\
\hline
\end{tabular}

(CONTINUED) 
APPENDIX J

Validated voC Results, 1993

\begin{tabular}{|c|c|c|c|c|c|c|c|c|c|c|c|c|}
\hline \multirow{4}{*}{$\begin{array}{l}\text { Sampling Point } \\
\text { Location } \\
\text { Date Sampled }\end{array}$} & \multicolumn{4}{|c|}{ GW-794 } & \multicolumn{8}{|c|}{ GW-795 } \\
\hline & \multicolumn{4}{|c|}{ AGLLSF } & \multicolumn{8}{|c|}{ AGLLSF } \\
\hline & \multicolumn{2}{|c|}{$07 / 11 / 93$} & \multicolumn{2}{|c|}{$10 / 18 / 93$} & \multicolumn{2}{|c|}{$03 / 02 / 93$} & \multicolumn{2}{|c|}{$05 / 25 / 93$} & \multicolumn{2}{|c|}{$07 / 12 / 93$} & \multicolumn{2}{|c|}{$10 / 18 / 93$} \\
\hline & ug/L & 8 sum & ug/L & sum & ug/I & $8 \mathrm{sum}$ & ug/L & 8 sum & ug/t & 8 sum & $u g / L$ & 8 sum \\
\hline Acetone & 100 & & FP1 & $\cdot$ & FP3 & $\cdot$ & 100 & $\cdot$ & 100 & $\cdot$ & FP1 & \\
\hline Benzene & so & & 50 & $\cdot$ & 50 & $\cdot$ & 5u & $\cdot$ & 5U & $\cdot$ & 50 & \\
\hline Bromodichloromethane & su & & 50 & $\cdot$ & $5 \mathbf{v}$ & $\cdot$ & 5u & $\cdot$ & 5u & $\cdot$ & 50 & \\
\hline 2-Butanone & 100 & & FP1 & $\cdot$ & 100 & $\cdot$ & 100 & $\cdot$ & 100 & $\cdot$ & FP1 & \\
\hline Carbon disulfide & 50 & & 50 & $\cdot$ & 5u & $\cdot$ & 50 & $\cdot$ & 50 & $\cdot$ & 50 & \\
\hline Carbon tetrachloride & 5u & & 50 & $\cdot$ & 50 & $\cdot$ & 50 & $\cdot$ & 50 & $\cdot$ & 50 & \\
\hline Chlorobenzene & 50 & & 5u & $\cdot$ & 50 & $\cdot$ & 5u & $\cdot$ & 50 & $\cdot$ & 50 & \\
\hline Chloroform & 50 & & 5u & $\cdot$ & 50 & $\cdot$ & 50 & $\cdot$ & 50 & $\cdot$ & $5 \mathbf{0}$ & \\
\hline 1,1-Dichloroethane & 5u & & 50 & $\cdot$ & $5 \mathbf{u}$ & $\cdot$ & 50 & $\cdot$ & 50 & $\cdot$ & 50 & \\
\hline 1,2-Dichloroethane & 50 & & 50 & $\cdot$ & 50 & $\cdot$ & 50 & $\cdot$ & 50 & $\cdot$ & 50 & \\
\hline 1,1-Dichloroethene & 50 & & 5v & $\cdot$ & 5u & $\cdot$ & 5u & $\cdot$ & 5u & $\cdot$ & 50 & \\
\hline 1,2-Dichloroethene & 5u & & 50 & $\cdot$ & 50 & $\cdot$ & $5 \mathbf{0}$ & $\cdot$ & 50 & $\cdot$ & 50 & \\
\hline Ethylbenzene & $5 \mathrm{U}$ & & 5u & $\cdot$ & 50 & $\cdot$ & 5u & $\cdot$ & 50 & $\cdot$ & $5 \mathbf{U}$ & \\
\hline 2-Hexanone & 100 & & 100 & $\cdot$ & 100 & $\cdot$ & 100 & $\cdot$ & 100 & $\cdot$ & 100 & \\
\hline 4-Methyl-2-pentanone & 100 & & 100 & $\cdot$ & FP1 & $\cdot$ & 100 & $\cdot$ & 100 & $\cdot$ & 100 & \\
\hline Methylene chloride & $5 v$ & & FP1 & $\cdot$ & FP1 & $\cdot$ & 5u & $\cdot$ & 50 & $\cdot$ & FP1 & \\
\hline Styrene & 50 & & 50 & $\cdot$ & 5u & $\cdot$ & 50 & $\cdot$ & 50 & $\cdot$ & 50 & \\
\hline Tetrachloroethene & 50 & & $5 \mathbf{v}$ & $\cdot$ & 50 & $\cdot$ & 50 & $\cdot 1$ & 50 & $\cdot$ & 50 & \\
\hline Toluene & 50 & & 5u & $\cdot$ & su & $\cdot$ & 50 & $\cdot$ & 50 & $\cdot$ & 5u & \\
\hline $1,1,1$-Trichloroethane & 50 & & 50 & $\cdot$ & $5 \mathrm{U}$ & $\cdot$ & 50 & $\cdot$ & 50 & $\cdot$ & 50 & \\
\hline 1,1,2-Trichloroethane & 50 & & 50 & $\cdot$ & 5v & $\cdot$ & 50 & $\cdot$ & $5 v$ & $\cdot$ & 5u & \\
\hline Trichloroethene & 5v & & 50 & • & 50 & $\cdot$ & 50 & $\cdot$ & 50 & $\cdot$ & 50 & \\
\hline vinyl acetate & 100 & & 100 & $\cdot$ & 100 & $\cdot$ & 100 & $\cdot$ & 100 & $\cdot$ & 100 & \\
\hline vinyl chloride & 100 & & 100 & $\cdot$ & 100 & $\cdot$ & 100 & $\cdot$ & 100 & $\cdot$ & 100 & \\
\hline xylenes & 50 & & su & $\cdot$ & 5u & $\cdot$ & 50 & $\cdot$ & 50 & $\cdot$ & $5 U$ & \\
\hline Summed vOCs & 0 & & 0) & • & 0 & • & o) & $\cdot$ & 0 & $\cdot$ & 0 & \\
\hline
\end{tabular}

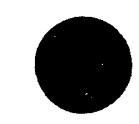


Validated voc Results, 1993

\begin{tabular}{|c|c|c|c|c|c|c|c|c|c|c|c|c|c|}
\hline \multirow{4}{*}{$\begin{array}{l}\text { Sampling Point } \\
\text { Location } \\
\text { Date Sampled }\end{array}$} & \multicolumn{8}{|c|}{$B C X-00.63$} & \multicolumn{5}{|c|}{ BCK-04.55 } \\
\hline & \multicolumn{8}{|c|}{$\operatorname{ExP}$} & \multicolumn{5}{|c|}{ EXP } \\
\hline & \multicolumn{2}{|c|}{$03 / 09 / 93$} & \multicolumn{2}{|c|}{$05 / 03 / 93$} & \multicolumn{2}{|c|}{$08 / 16 / 93$} & \multicolumn{2}{|c|}{$11 / 08 / 93$} & \multicolumn{2}{|c|}{$03 / 09 / 93$} & \multicolumn{3}{|c|}{$05 / 03 / 93$} \\
\hline & ug $/ \mathrm{L}$ & 8 sum & ug/L & 8 sum & ug/L & 8 sum & $\operatorname{ug} / \mathrm{L}$ & 8 sum & $u g / L$ & 8 sum & $\mathrm{ug} / \mathrm{L}$ & 8 sum & \\
\hline Acetone & 100 & $\cdot 1$ & 100 & $\cdot$ & 100 & $\cdot$ & 100 & $\cdot$ & FP3 & - & 100 & & $\cdot$ \\
\hline Benzene & 5u & $\cdot$ & su & $\cdot$ & 50 & $\cdot$ & 50 & $\cdot$ & 50 & $\cdot$ & 50 & & $\cdot$ \\
\hline Bromodichloromethane & 50 & $\cdot$ & 50 & $\cdot$ & 50 & $\cdot$ & 50 & $\cdot$ & 5u & $\cdot$ & 50 & & $\cdot$ \\
\hline 2-Butanone & 100 & $\cdot$ & 100 & $\cdot$ & 100 & $\cdot$ & 100 & $\cdot$ & 100 & $\cdot$ & 100 & & $\cdot$ \\
\hline Carbon disulfide & 50 & $\cdot$ & $5 U$ & $\cdot$ & 50 & $\cdot$ & $5 v$ & $\cdot$ & 50 & $\cdot$ & 50 & & $\cdot$ \\
\hline Carbon tetrachloride & $5 v$ & $\cdot$ & $5 \mathrm{U}$ & $\cdot$ & 5u & $\cdot$ & 5u & $\cdot$ & 50 & $\cdot$ & 50 & & $\cdot$ \\
\hline Chlorobenzene & 50 & $\cdot$ & $5 U$ & $\cdot$ & 5u & $\cdot$ & 5u & $\cdot$ & 50 & $\cdot$ & 50 & & $\cdot$ \\
\hline Chloroform & 50 & $\cdot$ & $5 \mathrm{v}$ & $\cdot$ & 50 & $\cdot$ & $5 \mathrm{U}$ & $\cdot$ & 50 & $\cdot$ & 50 & & $\cdot$ \\
\hline 1,1-Dichloroethane & 50 & $\cdot$ & 50 & $\cdot$ & 50 & $\cdot$ & 50 & $\cdot$ & 50 & $\cdot$ & 50 & & $\cdot$ \\
\hline 1,2-Dichloroethane & 5u & $\cdot 1$ & $5 \mathbf{u}$ & $\cdot$ & 50 & $\cdot$ & 50 & $\cdot$ & 50 & $\cdot$ & so & & $\cdot$ \\
\hline 1,1-Dichloroethene & 50 & $\cdot$ & 50 & $\cdot$ & sv & $\cdot$ & 50 & $\cdot$ & 50 & $\cdot$ & 50 & & $\cdot$ \\
\hline 1,2-Dichloroethene & 5u & $\cdot$ & 50 & $\cdot$ & 50 & $\cdot 1$ & 50 & $\cdot$ & 50 & $\cdot$ & 50 & & $\cdot$ \\
\hline Ethylbenzene & 50 & $\cdot$ & $5 u$ & $\cdot$ & 50 & $\cdot$ & 5U & $\cdot$ & 50 & $\cdot$ & $\begin{array}{r}50 \\
100\end{array}$ & & $\cdot$ \\
\hline 2-Hexanone & 100 & $\cdot$ & 100 & $\cdot$ & 100 & $\cdot$ & 100 & $\cdot$ & 100 & $\cdot$ & 100 & & $\cdot$ \\
\hline 4-Methy1-2-pentanone & FP1 & $\cdot$ & FP3 & $\cdot$ & 100 & $\cdot$ & 100 & $\cdot$ & FP1 & $\cdot$ & FP1 & & $\cdot$ \\
\hline Methylene chloride & FP1 & $\cdot$ & FP3 & $\cdot$ & 50 & $\cdot$ & FP1 & $\cdot$ & $\begin{array}{r}\text { FP1 } \\
50\end{array}$ & $\cdot$ & $\begin{array}{r}501 \\
50\end{array}$ & & $\dot{.}$ \\
\hline Styrene & $\begin{array}{l}5 \mathrm{U} \\
5 \mathrm{U}\end{array}$ & $\cdot \dot{1}$ & $\begin{array}{l}5 \mathbf{5 u} \\
5 \mathrm{u}\end{array}$ & $\cdot$ & $\begin{array}{l}50 \\
50\end{array}$ & $\cdot$ & $\begin{array}{l}50 \\
50\end{array}$ & $\cdot$ & 50 &. & 50 & & . \\
\hline $\begin{array}{l}\text { Tetrachloroethene } \\
\text { Toluene }\end{array}$ & $\begin{array}{l}50 \\
50\end{array}$ & $\cdot$ & $\begin{array}{l}50 \\
50\end{array}$ & $\cdot$ & 50 & $\cdot$ & 5v &. & 50 &. & 50 & & . \\
\hline 1,1,1-Trichloroethane & 50 &. & 50 & $\cdot$ & 50 &. & 50 & $\cdot$ & 50 &. & 50 & & $\cdot$ \\
\hline 1,1,2-Trichloroethane & $5 v$ & $\cdot$ & 50 & $\cdot$ & 50 & $\cdot$ & 50 & $\cdot$ & 50 & $\cdot$ & 50 & & $\cdot$ \\
\hline Trichloroethene & 50 & $\cdot$ & su & $\cdot$ & 50 & $\cdot$ & 50 & $\cdot$ & 50) & $\cdot$ & 50 & & $\cdot$ \\
\hline vinyl acetate & 100 & $\cdot$ & 100 & $\cdot$ & 100 & $\cdot$ & 10. & $\cdot$ & 100 & $\cdot$ & 100 & & • \\
\hline vinyl chloride & 100 & $\cdot$ & 100 & $\cdot$ & 100 & $\cdot 1$ & 100 & $\cdot$ & 100 & $\cdot 1$ & 100 & & $\cdot$ \\
\hline Xylenes & 50 & $\cdot$ & 50 & $\cdot$ & 50 & $\cdot$ & 50 & $\cdot$ & 50 & $\cdot$ & 50 & & • \\
\hline Summed vocs & 0 & $\cdot$ & 0 & $\cdot$ & 0 & $\cdot$ & 0 & $\cdot$ & 0 & $\cdot$ & 0 & & - \\
\hline
\end{tabular}

(CONTINUED) 
APPENDIX $J$

Validated VOC Results, 1993

\begin{tabular}{|c|c|c|c|c|c|c|c|c|c|c|c|c|}
\hline \multirow{4}{*}{$\begin{array}{l}\text { Sampling Point } \\
\text { Location } \\
\text { - } \\
\text { Date Sampled }\end{array}$} & \multicolumn{4}{|c|}{ BCK-04.55 } & \multicolumn{8}{|c|}{ BCK -09.40} \\
\hline & \multicolumn{4}{|c|}{ EXP } & \multicolumn{8}{|c|}{ EXP } \\
\hline & \multicolumn{2}{|c|}{$08 / 16 / 93$} & \multicolumn{2}{|c|}{$11 / 08 / 93$} & \multicolumn{2}{|c|}{$03 / 09 / 93$} & \multicolumn{2}{|c|}{$05 / 03 / 93$} & \multicolumn{2}{|c|}{$08 / 16 / 93$} & \multicolumn{2}{|c|}{$11 / 08 / 93$} \\
\hline & $\mathrm{ug} / \mathrm{L}$ & 8 sum & $\mathrm{ug} / \mathrm{L}$ & 8 sum & ug/L & 8 sum & $u g / L$ & 8 sum & ug/L & 8 sum & $\operatorname{ug} / \mathrm{L}$ & 8 sum \\
\hline Acetone & 100 & - & 100 & • & 100 & . & 100 & - & 100 & - & 100 & 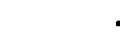 \\
\hline Benzene & 50 & • & 50 & • & 5U & . & 50 & - & 50 & - & 50 & . \\
\hline Bromodichloromethane & 50 & • & 50 & - & $5 U$ & - & 50 & - & 50 & - & 50 & . \\
\hline 2-Butanone & 100 & • & 100 & • & 100 & • & 100 & - & 100 & - & 100 & . \\
\hline Carbon disulfide & 50 & - & 50 & - & 50 & - & $\mathbf{5 u}$ & - & 50 & - & 50 & . \\
\hline Carbon tetrachloride & 50 & • & 50 & • & 50 & - & 50 & - & 50 & - & 50 & . \\
\hline Chlorobenzene & 5u & - & 50 & - & 50 & - & 50 & - & 50 & - & $5 \mathbf{v}$ & . \\
\hline Chloroform & 50 & • & 50 & • & 5u & - & 50 & - & 50 & - & 50 & . \\
\hline 1,1-Dichloroethane & $5 U$ & • & 50 & • & 50 & - & 50 & - & 5u & - & 50 & . \\
\hline 1,2-Dichloroethane & 50 & • & 50 & • & 50 & $\cdot$ & 50 & - & 50 & - & 50 & . \\
\hline 1,1-Dichloroethene & $5 v$ & • & 50 & • & 50 & • & 50 & - & 50 & - & 50 & 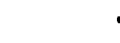 \\
\hline 1,2-Dichloroethene & 50 & $\cdot$ & 50 & • & 8 & 88.9 & 9 & 90 & 1 & 37 & 50 & 。 \\
\hline Ethylbenzene & 50 & - & 50 & - & $5 \mathbf{U}$ & - & so & - & 50 & - & 50 & . \\
\hline 2-Hexanone & 100 & - & 100 & • & 100 & • & 100 & $\cdot$ & 100 & - & 100 & . \\
\hline 4-Methyl-2-pentanone & 100 & - & 100 & - & 100 & - & FP1 & - & 100 & - & 100 & . \\
\hline Methylene chloride & 50 & - & FP1 & • & 50 & • & FP1 & - & 1 & 37 & 50 & . \\
\hline Styrene & 50 & . & 50 & . & $5 \mathbf{u}$ & . & 50 & - & 50 & - & 50 & . \\
\hline Tetrachloroethene & 50 & - & 50 & • & 1 & 11.1 & 1 & 10 & 50 & - & 50 & . \\
\hline Toluene & $5 v$ & • & 50 & - & 50 & $\cdot$ & 50 & $\cdot$ & 50 & - & 50 & 。 \\
\hline $1,1,1$-Trichloroethane & 5u & - & 50 & • & 50 & $\cdot$ & 5u & $\cdot$ & 50 & • & 50 & . \\
\hline 1,1,2-Trichloroethane & 50 & $\cdot$ & 50 & • & 50 & $\cdot$ & 50 & $\cdot$ & 50 & - & 50 & 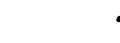 \\
\hline Trichloroethene & 50 & - & 50 & $\cdot$ & 5u & $\cdot$ & 5u & $\cdot$ & 0.7 & 25.9 & 50 & . \\
\hline Vinyl acetate & 100 & $\cdot$ & 100 & $\cdot$ & 100 & • & 100 & - & 100 & - & 100 & . \\
\hline Vinyl chloride & 100 & - & 100 & - & 100 & $\cdot$ & 100 & $\cdot$ & 100 & $\cdot$ & 100 & . \\
\hline xylenes & 50 & - & 50 & - & 50 & . & 50 & - & 50 & - & 50 & . \\
\hline Summed vOCs & 0 & - & $\mathbf{0}$ & - & 9 & 100 & 10 & 100 & 2.7 & 99.9 & 0 & . \\
\hline
\end{tabular}

(CONTINuEd) 
Validated VOC Results, 1993

\begin{tabular}{|c|c|c|c|c|c|c|c|c|c|c|c|c|c|}
\hline \multirow{4}{*}{$\begin{array}{l}\text { Sampling Point } \\
\text { Location } \\
\text { Date Sampled }\end{array}$} & \multicolumn{8}{|c|}{ NT-01 } & \multicolumn{5}{|c|}{ NT-13 } \\
\hline & \multicolumn{8}{|c|}{ EXP } & \multicolumn{5}{|c|}{ EXP } \\
\hline & \multicolumn{2}{|c|}{$03 / 09 / 93$} & \multicolumn{2}{|c|}{$05 / 03 / 93$} & \multicolumn{2}{|c|}{$08 / 16 / 93$} & \multicolumn{2}{|c|}{$11 / 08 / 93$} & \multicolumn{2}{|c|}{$03 / 09 / 93$} & \multicolumn{3}{|c|}{$05 / 03 / 93$} \\
\hline & ug/L & 8 sum & ug/L & 8 sum & ug/L & 8 sum & ug $/ \mathrm{L}$ & 8 sum & $u g / L$ & 8 sum & $\mathrm{ug} / \mathrm{L}$ & 8 sur & \\
\hline Acetone & 100 & - & FP3 & . & 100 & . & 100 & - & FP3 & . & 100 & & $\cdot 1$ \\
\hline Benzene & 50 & • & 50 & $\cdot$ & 50 & $\cdot$ & 50 & - & 50 & - & 50 & & $\cdot$ \\
\hline Bromodichloromethane & 50 & . & 5u & . & 50 &. & 5u & - & 5u & - & 50 & & $\cdot$ \\
\hline 2-Butanone & 100 & • & 100 & . & 100 & $\cdot$ & 100 & . & 100 & - & 100 & & $\cdot$ \\
\hline Carbon disulfide & 50 & . & 5u & $\cdot$ & 5u & $\cdot$ & 50 & - & 50 & - & 5v & & $\cdot$ \\
\hline Carbon tetrachloride & 50 & - & 50 & $\cdot$ & 50 & $\cdot$ & so & - & 50 & - & 50 & & $\cdot$ \\
\hline Chlorobenzene & 50 & - & $5 v$ & $\cdot$ & 50 & $\cdot$ & 50 & $\cdot$ & 50 & $\cdot$ & 50 & & $\cdot$ \\
\hline Chloroform & 5u & - & 50 & $\cdot$ & 50 & $\cdot$ & 50 & - & 50 & - & 50 & & $\cdot$ \\
\hline 1,1-Dichloroethane & 50 & • & 5u & $\cdot$ & 50 & $\cdot$ & 5v & • & 50 & - & 5U & & $\cdot$ \\
\hline 1,2-Dichloroethane & 5u & • & su & $\cdot$ & 50 & $\cdot$ & 5u & - & 50 & - & 50 & & $\cdot$ \\
\hline 1,1-Dichloroethene & 50 & • & 50 & $\cdot$ & 50 & $\cdot$ & 50 & • & 50 & - & 50 & & $\cdot$ \\
\hline 1,2-Dichloroethene & su & - & $5 \mathbf{v}$ & $\cdot$ & 50 & $\cdot$ & 50 & • & 5u & - & 50 & & $\cdot$ \\
\hline Ethylbenzene & 50 & - & 50 & $\cdot$ & 50 & $\cdot$ & 50 & - & 50 & - & 50 & & $\cdot$ \\
\hline 2-Hexanone & 100 & - & 100 & $\cdot$ & 100 & $\cdot$ & 100 & - & 100 & - & 100 & & $\cdot$ \\
\hline 4-Methy1-2-pentanone & 100 & - & FP1 & $\cdot$ & 100 & $\cdot$ & 100 & - & FP1 & - & FP1 & & $\cdot$ \\
\hline Methylene chloride & FP1 & • & FP1 & $\cdot$ & FP1 & $\cdot$ & 50 & - & FP1 & - & FP1 & & $\cdot$ \\
\hline Styrene & 50 & - & 50 & $\cdot$ & 50 & $\cdot$ & 5u & • & 50 & - & 50 & & $\cdot$ \\
\hline Tetrachloroethene & 4 & 100 & 1 & 100 & 2 & 100 & 3 & 100 & 50 & - & 50 & & $\cdot$ \\
\hline Toluene & 50 & . & 50 & $\cdot$ & 50 & $\cdot$ & 50 & $\cdot$ & 50 & - & 50 & & $\cdot$ \\
\hline $1,1,1$-Trichloroethane & 5u & - & 50 & $\cdot$ & 50 & $\cdot 1$ & 50 & $\cdot$ & 50 & - & 50 & & $\cdot$ \\
\hline 1,1,2-Trichloroethane & 50 & - & 50 & $\cdot$ & 50 & $\cdot$ & 50 & $\cdot$ & 50 & - & 50 & & - \\
\hline Trichloroethene & 5u & - & 50 & $\cdot$ & 50 & $\cdot$ & 50 & $\cdot$ & 50 & - & 50 & & - \\
\hline vinyl acetate & 100 & • & 100 & $\cdot$ & 100 & $\cdot 1$ & 100 & - & 200 & - & 100 & & - \\
\hline vinyl chloride & 100 & - & 100 & - & 100 & $\cdot$ & 100 & $\cdot$ & 100 & - & 100 & & - \\
\hline Xylenes & 5u & - & 50 & - & 50 & $\cdot$ & 50 & $\cdot$ & 50 & - & 50 & & - \\
\hline Summed vOCs & 4 & 100 & 1 & 100 & 2 & 100 & 3 & 100 & 0 & - & 0 & & $\bullet$ \\
\hline
\end{tabular}

(CONTINUED) 
APPENDIX $J$

Validated VOC Results, 1993

\begin{tabular}{|c|c|c|c|c|c|c|c|c|c|c|c|c|}
\hline \multirow{4}{*}{$\begin{array}{l}\text { Sampling Point } \\
\text { - } \\
\text { Location } \\
\text { Date Sampled }\end{array}$} & \multicolumn{4}{|c|}{ NT-13 } & \multicolumn{8}{|c|}{ ss-1 } \\
\hline & \multicolumn{4}{|c|}{ EXP } & \multicolumn{8}{|c|}{ EXP } \\
\hline & \multicolumn{2}{|c|}{$08 / 16 / 93$} & \multicolumn{2}{|c|}{$11 / 08 / 93$} & \multicolumn{2}{|c|}{$03 / 09 / 93$} & \multicolumn{2}{|c|}{$05 / 03 / 93$} & \multicolumn{2}{|c|}{$08 / 16 / 93$} & \multicolumn{2}{|c|}{$11 / 08 / 93$} \\
\hline & ug/L & 8 sum & ug/L & sum & $\mathrm{ug} / \mathrm{L}$ & \& sum & $u g / L$ & 8 sum & $\mathrm{ug} / \mathrm{L}$ & s sum & $u g / L$ & 8 sum \\
\hline Acetone & 100 & $\cdot 1$ & 100 & . & 100 & $\cdot$ & 100 &. & 100 & .1 & 100 & • \\
\hline Benzene & 50 & $\cdot$ & 50 & - & 50 & . & 50 & . & 5u & $\cdot$ & 50 & • \\
\hline Bromodichloromethane & sv & $\cdot$ & 50 & - & 50 & . & 50 & . & $5 v$ &. & 50 & - \\
\hline 2-Butanone & 100 & $\cdot$ & 100 & - & 100 & $\cdot$ & 100 & $\cdot$ & 100 & $\cdot 1$ & 100 & • \\
\hline Carbon disulfide & 5u & $\cdot 1$ & 50 & - & 50 & $\cdot$ & 50 & . & 50 & $\cdot$ & 50 & • \\
\hline Carbon tetrachloride & su & $\cdot$ & $5 v$ & - & 50 & $\cdot$ & 5u & $\cdot$ & so & $\cdot$ & 5u & • \\
\hline Chlorobenzene & su & $\cdot$ & 50 & - & 5u & $\cdot$ & 50 & $\cdot$ & 50 &. & 5u & • \\
\hline Chloroform & 50 & $\cdot$ & $5 \mathrm{U}$ & - & FP3 & $\cdot$ & 50 & . & 50 & $\cdot$ & 50 & - \\
\hline 1,1-Dichloroethane & 50 & $\cdot$ & 5u & . & 50 & $\cdot$ & 50 &. & 50 & $\cdot$ & so & - \\
\hline 1,2-Dichloroethane & $5 u$ &. & 50 & $\cdot$ & 50 & $\cdot$ & 50 & $\cdot$ & 50 &. & 50 & - \\
\hline 1,1-Dichloroethene & $5 \mathrm{v}$ & $\cdot 1$ & 50 & . & 50 & $\cdot$ & 50 &. & 50 & $\cdot$ & 50 & • \\
\hline 1,2-Dichloroethene & su &. & 50 & $\cdot$ & 5u & $\cdot$ & 50 & $\cdot$ & 50 & $\cdot$ & 50 & - \\
\hline Ethylbenzene & su & $\cdot$ & 50 & $\cdot$ & 5u & $\cdot$ & 50 & $\cdot$ & 50 & $\cdot$ & 50 & . \\
\hline 2-Hexanone & 100 & $\cdot$ & $\because \quad 100$ & $\cdot$ & 100 &. & 100 &. & 100 & $\cdot 1$ & 100 & - \\
\hline 4-Methyl-2-pentanone & 100 & $\cdot$ & 100 & $\cdot$ & 100 & $\cdot$ & FP1 & $\cdot$ & 100 & $\cdot$ & 100 & - \\
\hline Methylene chloride & 50 & $\cdot$ & FPI & $\cdot$ & FP1 & $\cdot$ & FP1 & $\cdot$ & 50 & $\cdot$ & 50 & - \\
\hline Styrene & 50 & $\cdot$ & so & $\cdot$ & 50 & $\cdot$ & 5u & $\cdot$ & 5u & $\cdot$ & 50 & - \\
\hline Tetrachloroethene & so &. & 50 & $\cdot$ & 2 & 100 & 3 & 100 & 50 & $\cdot$ & 1 & 100 \\
\hline Toluene & 50 & $\cdot$ & 5u & $\cdot$ & 50 & $\cdot$ & 5v & $\cdot$ & 50 & $\cdot$ & 50 & - \\
\hline 1,1,1-Trichloroethane & 50 & $\cdot$ & 50 & $\cdot$ & 50 &. & 5u & $\cdot$ & 50 & $\cdot 1$ & 50 & - \\
\hline 1,1,2-Trichloroethane & 50 & $\cdot$ & 5u & $\cdot$ & su & $\cdot$ & so & $\cdot 1$ & 5iv & $\cdot$ & 5v & • \\
\hline Trichloroethene & 50 & $\cdot$ & su & $\cdot$ & so & .1 & 5u & $\cdot$ & 50 & $\cdot$ & $5 v$ & . \\
\hline vinyl acetate & 100 &. & 100 & $\cdot$ & 100 & $\cdot$ & 100 & $\cdot$ & 100 & $\cdot$ & 100 & 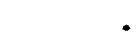 \\
\hline Vinyl chloride & 100 & $\cdot$ & 100 & $\cdot$ & 100 & $\cdot$ & 100 &. & 100 & $\cdot$ & 100 & - \\
\hline Xylenes & $5 \mathbf{u}$ & $\cdot$ & 30 & $\cdot$ & 50 & $\cdot$ & 50 & $\cdot$ & 5v & $\cdot$ & 50 & • \\
\hline Summed vOCs & 0 &. & 0 &. & 2 & 100 & 3 & 100 & $\dot{0}$ &. & 1 & 100 \\
\hline
\end{tabular}

(CONTIMUED) 
APPE:IDIX $J$

Validated VOC Results, 1993

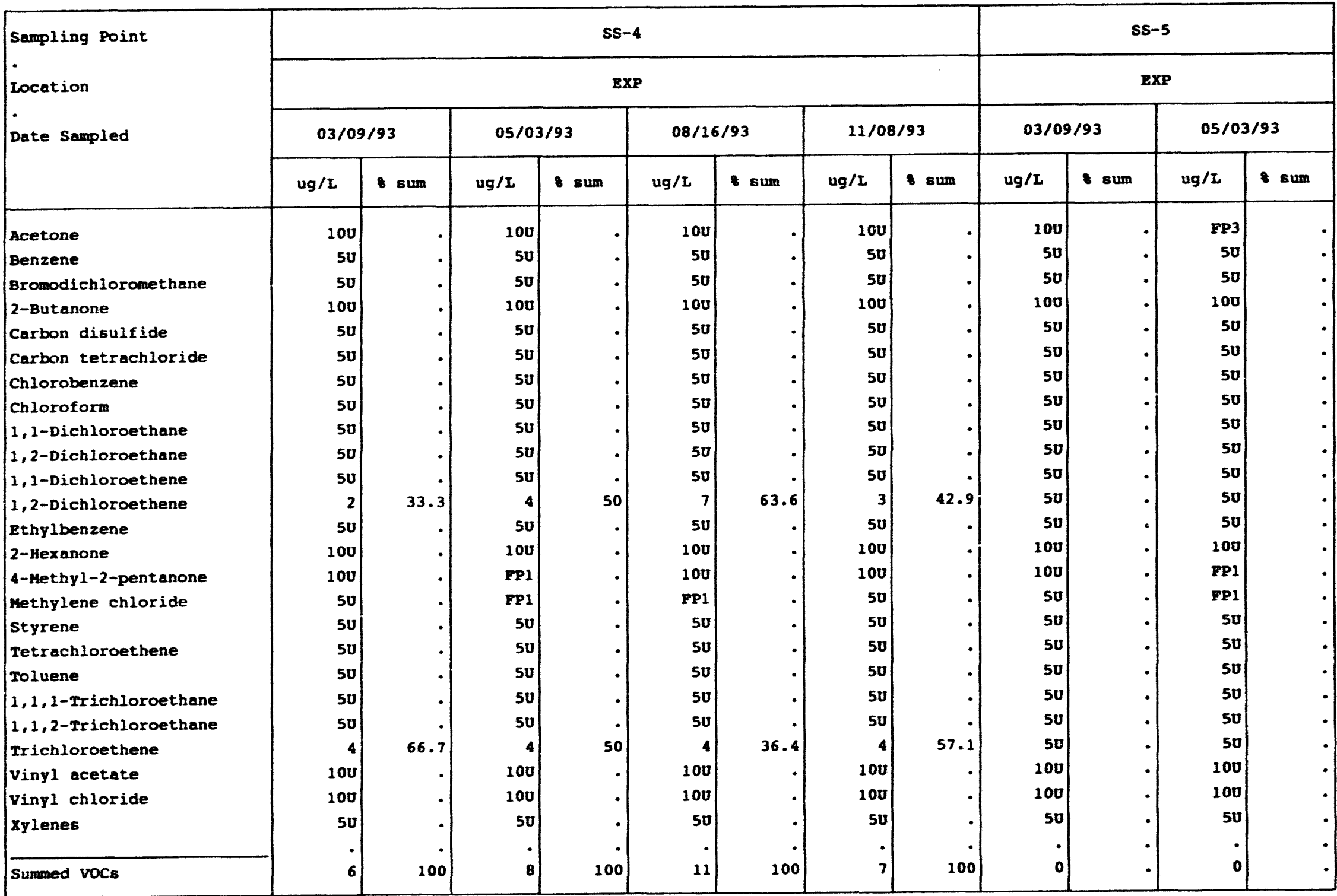

(CONrImUED) 
APPEINDIX J

Validated VOC Results, 1993

\begin{tabular}{|c|c|c|c|c|c|c|c|c|c|c|c|c|}
\hline \multirow{4}{*}{$\begin{array}{l}\text { Sampling Point } \\
\text { Location } \\
\text { Date Sampled }\end{array}$} & \multicolumn{4}{|c|}{ SS-5 } & \multicolumn{8}{|c|}{ SS-6 } \\
\hline & \multicolumn{4}{|c|}{$\operatorname{EXP}$} & \multicolumn{8}{|c|}{$\operatorname{ExP}$} \\
\hline & \multicolumn{2}{|c|}{$08 / 16 / 93$} & \multicolumn{2}{|c|}{$11 / 08 / 93$} & \multicolumn{2}{|c|}{$03 / 09 / 93$} & \multicolumn{2}{|c|}{$05 / 03 / 93$} & \multicolumn{2}{|c|}{$08 / 16 / 93$} & \multicolumn{2}{|c|}{$11 / 08 / 93$} \\
\hline & ug $/ \mathrm{L}$ & sum & ug $/ \mathrm{L}$ & sum & ug $/ \mathbf{L}$ & sum & ug/L & 8 sum & $u g / L$ & sum & $u g / I$ & 8 sum \\
\hline Acetone & 100 & - & 100 & $\cdot 1$ & 100 & $\cdot$ & 100 & $\cdot 1$ & 100 & - & 100 & • \\
\hline Benzene & $5 v$ & $\cdot$ & 50 & $\cdot$ & 5u & $\cdot$ & 50 & $\cdot$ & 50 & $\cdot$ & 50 & . \\
\hline Bromodichloromethene & 50 & $\cdot$ & 50 & $\cdot 1$ & 50 & $\cdot$ & sv & $\cdot$ & 5v & $\cdot 1$ & 50 & . \\
\hline 2-Butanone & 100 & $\cdot$ & 100 & $\cdot$ & 100 & $\cdot$ & 100 & $\cdot$ & 100 & $\cdot$ & 100 & . \\
\hline Carbon disulfide & 50 & $\cdot$ & 50 & $\cdot$ & 50 &. & so & $\cdot$ & su & $\cdot$ & su & . \\
\hline Carbon tetrachloride & 50 & $\cdot$ & su & $\cdot$ & su & $\cdot$ & 50 & $\cdot$ & 50 & $\cdot$ & 50 & . \\
\hline Chlorobenzene & 50 & $\cdot$ & sv & $\cdot$ & 50 & $\cdot$ & 50 &. & 50 & $\cdot$ & 50 & • \\
\hline Chloroform & su &. & sv & $\cdot$ & 50 &. & 50 &. & 50 & $\cdot$ & 50 & • \\
\hline 1,1-Dichloroethane & 50 & $\cdot$ & so & $\cdot$ & 50 & . & 5v & . & 50 & . & 50 & . \\
\hline 1,2-Dichloroethane & su & $\cdot$ & 50 & $\cdot$ & 50 & $\cdot$ & 50 & $\cdot$ & 50 & $\cdot$ & 50 & • \\
\hline 1,1-Dichloroethene & su & $\cdot$ & 50 & $\cdot$ & 50 & $\cdot$ & so & $\cdot$ & su & $\cdot$ & 50 & • \\
\hline 1,2-Dichloroethene & FP3 & $\cdot$ & so & $\cdot$ & 50 & $\cdot$ & 50 & $\cdot$ & 50 & $\cdot$ & FP3 & • \\
\hline Ethylbenzene & 5u & $\cdot$ & 5u & $\cdot$ & 50 &. & su & $\cdot$ & 5u &. & 50 & • \\
\hline 2-Hexanone & 100 & $\cdot$ & 100 & $\cdot$ & 100 & $\cdot$ & 100 & $\cdot$ & 100 & $\cdot$ & 100 & • \\
\hline 4-Methyl-2-pentanone & 100 & $\cdot$ & 100 & $\cdot$ & FP1 & $\cdot$ & 100 & $\cdot$ & 100 & $\cdot$ & 100 & • \\
\hline Hethylene chloride & so &. & 50 &. & $\mathbf{F P 1}$ & $\cdot$ & FP1 & - & 5u &. & 50 & • \\
\hline Styrene & 50 & $\cdot$ & 50 & $\cdot$ & 50 &. & 5v & $\cdot$ & so & $\cdot$ & 50 & • \\
\hline Tetrachloroethene & 50 & • & 50 &. & su & $\cdot$ & 50 &. & 50 & $\cdot$ & FP3 & • \\
\hline Toluene & 5u & - & 50 &. & 50 & $\cdot$ & 50 & $\cdot$ & 5u & $\cdot$ & 50 & • \\
\hline 1,1,1-Trichloroethane & 50 & • & 50 & $\cdot$ & so & $\cdot$ & 50 & $\cdot$ & 50 & $\cdot$ & so & • \\
\hline 1,1,2-Trichloroethane & 5u & $\cdot$ & 50 & $\cdot$ & su & $\cdot$ & 50 & $\cdot$ & 50 & $\cdot$ & 50 & • \\
\hline Irichloroethene & 2 & 100 & 1 & 100 & 50 & $\cdot$ & 50 & $\cdot$ & 50 & $\cdot$ & FP3 & • \\
\hline vinyl acetate & 100 & $\cdot$ & 100 & $\cdot$ & 100 & $\cdot$ & 100 & $\cdot$ & 100 & $\cdot$ & 100 & • \\
\hline vinyl chloride & 100 & $\cdot$ & 100 & $\cdot$ & 100 & $\cdot$ & 100 & $\cdot$ & 100 & $\cdot$ & 100 & • \\
\hline xylenes & 5v & $\cdot$ & so &. & 50 & $\cdot$ & so & $\cdot$ & 50 & $\cdot$ & 50 & $\cdot$ \\
\hline summed vocs & $\dot{2}$ & 100 & $\dot{1}$ & 100 & $\dot{0}$ & $\cdot$ & $:$ & . & $\dot{0}$ & $\cdot$ & $\dot{0}$ & • \\
\hline
\end{tabular}

(CONTINUED) 
Validated Voc Results, 1993

\begin{tabular}{|c|c|c|c|c|c|c|c|c|c|}
\hline \multirow{4}{*}{$\begin{array}{l}\text { Sampling Point } \\
\text { Location } \\
\text { Date Sampled }\end{array}$} & \multicolumn{9}{|c|}{ Ss-8 } \\
\hline & \multicolumn{9}{|c|}{$\mathbf{E X P}$} \\
\hline & \multicolumn{2}{|c|}{$03 / 09 / 93$} & \multicolumn{2}{|c|}{$05 / 03 / 93$} & \multicolumn{2}{|c|}{$05,16 / 93$} & \multicolumn{3}{|c|}{$11 / 08 / 93$} \\
\hline & ug/L & sum & ug/L & sum & $د g / L$ & s sum & $u g / x$ & sum & \\
\hline Acetone & 100 & - & 100 & - & 100 & • & 100 & & • \\
\hline Benzene & 50 & - & 50 & - & 50 & - & 50 & & - \\
\hline Bromodich loromethane & 5v & - & 50 & - & 50 & • & $5 \mathbf{v}$ & & - \\
\hline 2-Butanone & 100 & - & 100 & - & 100 & - & 100 & & - \\
\hline Carbon disulfide & 50 & - & 50 & - & 50 & • & 50 & & - \\
\hline Carbon tetrachloride & so & - & 50 & - & 50 & - & 50 & & - \\
\hline Chlorobenzene & 50 & - & 50 & • & 50 & - & 50 & & • \\
\hline Chloroform & 50 & - & 50 & - & 50 & - & 5v & & • \\
\hline 1,1-Dichloroethane & 50 & - & 5v & - & 5u & - & 50 & & - \\
\hline 1,2-Dichloroethane & 50 & . & su & - & 50 & - & 50 & & - \\
\hline 1,1-Dichloroethene & 50 & - & 50 & - & 5u & - & 50 & & • \\
\hline 1,2-Dichloroethene & su & - & 5u & - & 50 & - & 50 & & - \\
\hline Ethylbenzene & 50 & - & 50 & • & 50 & - & 50 & & • \\
\hline 2-Hexanone & 100 & - & 100 & $\cdot$ & 100 & - & 100 & & • \\
\hline 4-Methyl-2-pentanone & FP1 & • & FP1 & • & 100 & - & 100 & & • \\
\hline Methylene chloride & FP1 & - & FP1 & - & 50 & • & FP1 & & • \\
\hline styrene & 50 & - & 50 & • & 50 & - & 5u & & • \\
\hline Tetrachloroethene & 50 & - & 5u & • & 50 & - & 50 & & • \\
\hline Toluene & 5v & - & 50 & • & 50 & • & 50 & & • \\
\hline 1,1,1-Trichloroethane & 50 & - & 50 & $\cdot$ & 50 & - & 50 & & • \\
\hline 1,1,2-Trichloroethane & 50 & • & 5u & - & 50 & - & 50 & & • \\
\hline Trichloroethene & 50 & - & 5u & • & 50 & - & 50 & & $\cdot$ \\
\hline Vinyl acetate & 100 & - & 100 & $\cdot$ & 100 & • & 100 & & • \\
\hline vinyl chloride & 100 & - & 100 & • & 100 & - & 100 & & - \\
\hline xylenes & su & - & 50 & • & 50 & • & 50 & & $\bullet$ \\
\hline ammed VOCs & 0 & - & 0 & • & 0 & • & 0 & & • \\
\hline
\end{tabular}




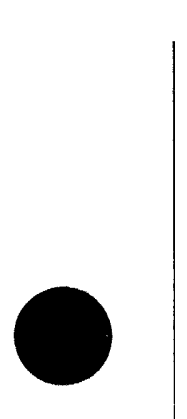

•

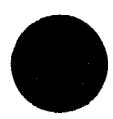


APPENDIX K

RADIOCHEMICAL DATA SUMMARY 


\section{EXPLANATION}

SAMPLING POINT:

$\begin{aligned} \text { BCK } & \text { - Bear Creek Kilometer } \\ \text { GW } & - \text { Monitoring Well } \\ \text { NT } & - \text { North Tributary to Bear Creek } \\ \text { SS } & - \text { Spring }\end{aligned}$

\section{LOCATION:}
AGLLSF - Above Grade Low Level Storage Facility
BG - Bear Creek Burial Grounds WMA
EXP - Exit-Pathway Monitoring
OLF - Oil Landfarm WMA
RS - Rust Spoil Area
S3 - S-3 Site
SPI - Spoil Area I

\section{NOTES:}

$\mathrm{pCi} / \mathrm{L}$ - picoCuries per Liter

CE ( \pm ) - Counting Error (two standard deviations)

- Not sampled

Mean counting error (e) calculated using the following formula from Evans (1955):

$$
e=\sqrt{\frac{E_{1}^{2}}{n^{2}}+\frac{E_{2}^{2}}{n^{2}}+\frac{E_{3}^{2}}{n^{2}}+\ldots}
$$

where $\mathbf{E}_{1}, \mathbf{E}_{2}, \ldots \mathbf{E}_{\mathbf{x}}$ are the individual errors, and $\mathbf{n}$ is the number of observations. 
Gross Alpha and Gross Beta Activity Data Summary, 1993

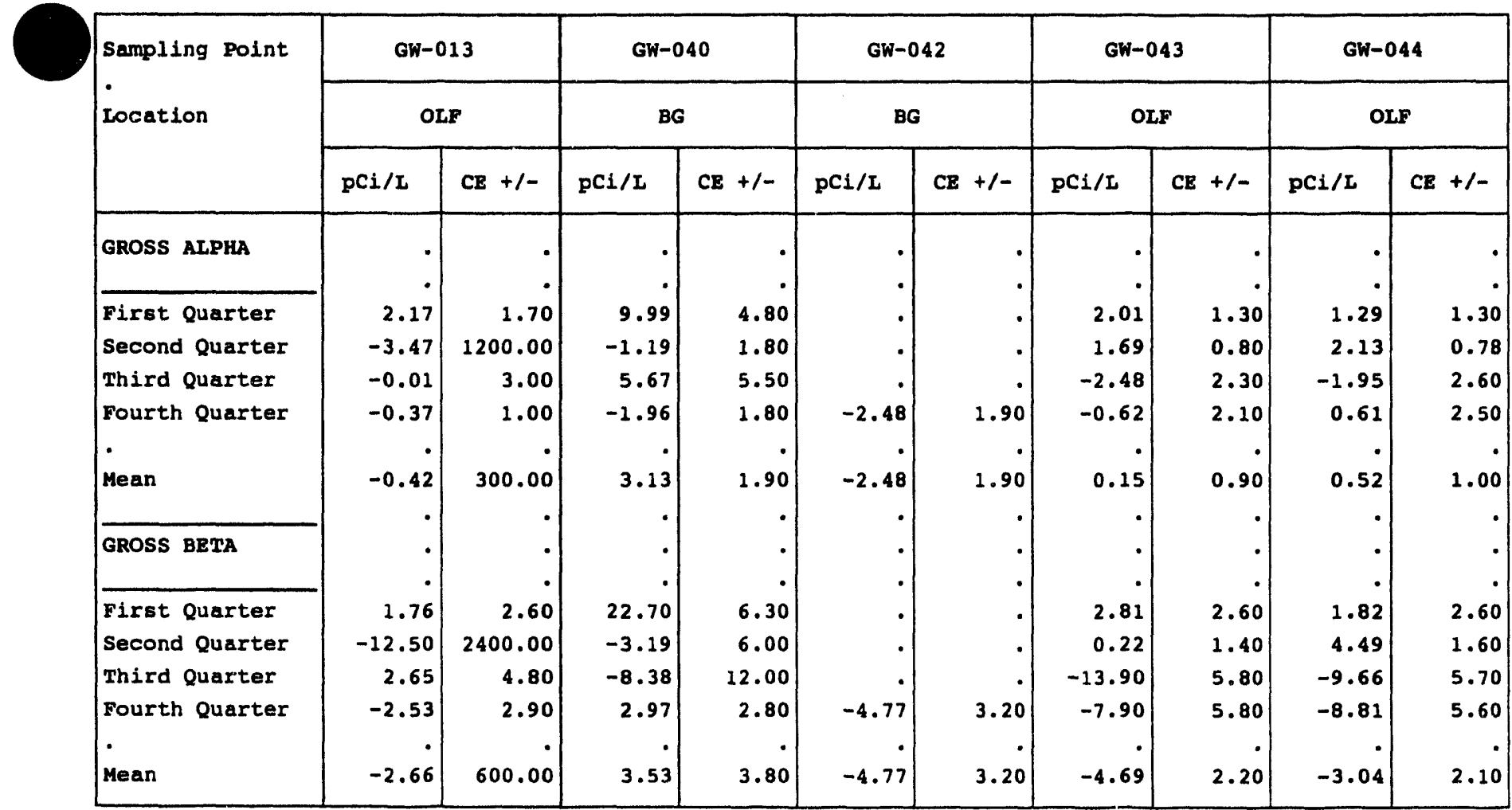

(CONTINUED)

\begin{tabular}{|c|c|c|c|c|c|c|c|c|c|c|}
\hline \multirow{3}{*}{$\begin{array}{l}\text { Sampling Point } \\
\text { Location }\end{array}$} & \multicolumn{2}{|c|}{ GW-052 } & \multicolumn{2}{|c|}{ Gพ-056 } & \multicolumn{2}{|c|}{ GW-057 } & \multicolumn{2}{|c|}{ GW-063 } & \multicolumn{2}{|c|}{ GW-064 } \\
\hline & \multicolumn{2}{|c|}{ BG } & \multicolumn{2}{|c|}{ BG } & \multicolumn{2}{|c|}{$\operatorname{ExP}$} & \multicolumn{2}{|c|}{ OLF } & \multicolumn{2}{|c|}{ OLF } \\
\hline & $\mathrm{pCi} / \mathrm{L}$ & $\mathrm{CE}+1-$ & $\mathrm{pCi} / \mathrm{L}$ & $\mathrm{CE}+/-$ & $\mathrm{pCi} / \mathrm{L}$ & $\mathrm{CE}+1-$ & $\mathrm{pCi} / \mathrm{L}$ & $\mathrm{CE}+1-$ & $\mathrm{pci} / \mathrm{L}$ & $\mathrm{CE}+/-$ \\
\hline GROSS ALPHA & . & $\cdot 1$ & $\cdot 1$ & . & . & $\cdot 1$ & $\cdot$ & $\cdot 1$ & . & \\
\hline First Quarter & 20.00 & 4.10 & 3.82 & 2.00 & 6.76 & 2.80 & 1.53 & 2.10 & 0.67 & 2.90 \\
\hline Second Quarter & 32.70 & 7.00 & 2.70 & 2.50 & 1.60 & 3.80 &. &. & -2.70 & 4.20 \\
\hline Third Quarter & . & $\cdot$ & 4.54 & 2.90 & 1.98 & 2.70 & . & . & -1.87 & 3.10 \\
\hline Fourth Quarter & $\cdot$ & $\cdot$ & 1.19 & 2.50 & 1.93 & 2.80 & . & $\cdot$ & -0.20 & 1.30 \\
\hline - & $\cdot$ & $\cdot$ & $\cdot$ & $\cdot$ & & & $\cdot$ & $\cdot$ & . & • \\
\hline Mean & 26.35 & 4.10 & 3.06 & 1.20 & 3.07 & 1.50 & 1.53 & 2.10 & -1.02 & 1.50 \\
\hline GROSS BETA & $\cdot$ & $\cdot$ & $\cdot$ & $\cdot$ & $\dot{.}$ & $\cdot$ & $\cdot$ & $\cdot$ & $\cdot$ & • \\
\hline First Quarter & 30.30 & 4.10 & 7.04 & 3.50 & 8.14 & 3.60 & 5.84 & 3.00 & 11.90 & 3.60 \\
\hline Second Quarter & 44.40 & 7.50 & 4.33 & 2.90 & 6.43 & 3.20 & . & . & 15.40 & 6.50 \\
\hline Third Quarter &. & . & 6.70 & 3.10 & 4.92 & 3.10 & . &. & 4.71 & 6.20 \\
\hline Fourth Quarter & . & . & 4.71 & 3.20 & 3.23 & 3.10 & . &. & 13.80 & 3.40 \\
\hline . & $\cdot$ & $\cdot$ & $\cdot$ &. & & - & $\cdot$ & $\cdot$ & $\cdot$ & • \\
\hline Mean & 37.35 & 4.30 & 5.69 & 1.60 & 5.68 & 1.60 & 5.84 & 3.00 & 11.45 & 2.60 \\
\hline
\end{tabular}

(CONTINUED) 
APPENDIX $\mathbf{K}$

Gross Alpha and Gross Beta Activity Data Summary, 1993

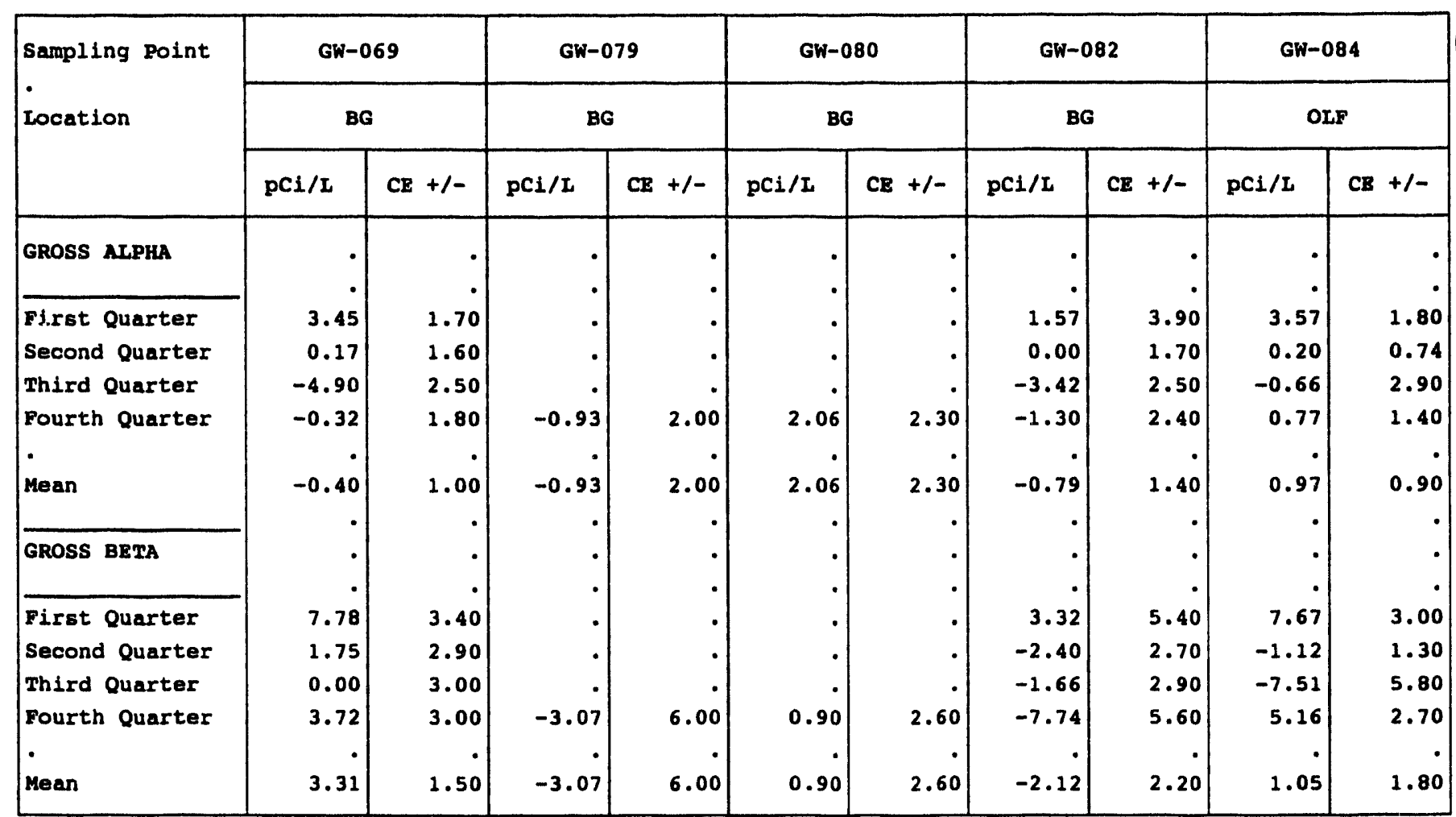

(CONTINUED)

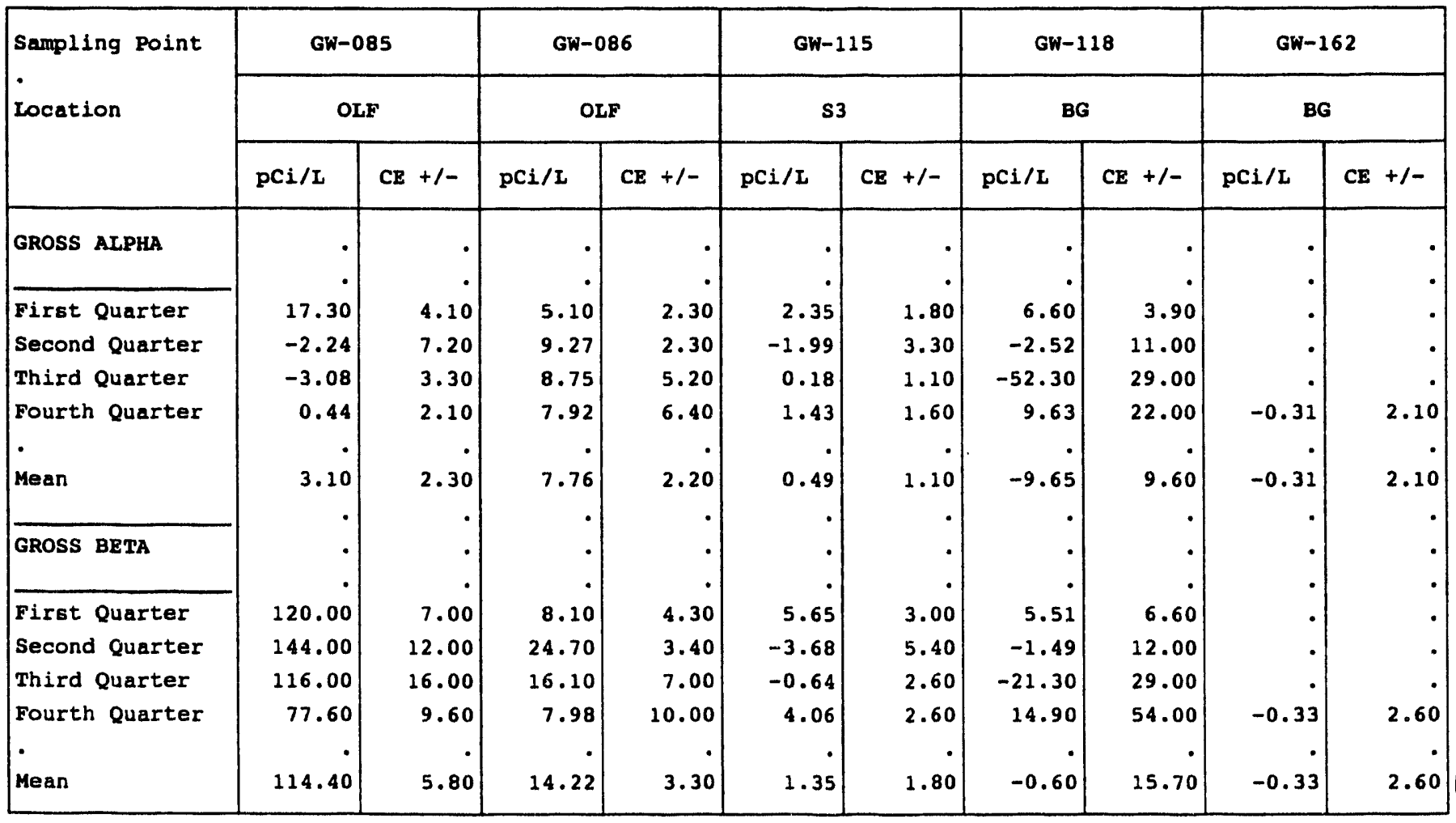


Gross Alpha and Grosø Beta Activity Data Summary, 1993

\begin{tabular}{|c|c|c|c|c|c|c|c|c|c|c|}
\hline \multirow{3}{*}{$\begin{array}{l}\text { Sampling Point } \\
\text { Location }\end{array}$} & \multicolumn{2}{|c|}{$G W-286$} & \multicolumn{2}{|c|}{$G W-287$} & \multicolumn{2}{|c|}{$G W-311$} & \multicolumn{2}{|c|}{ GW-312 } & \multicolumn{2}{|c|}{$G W-315$} \\
\hline & \multicolumn{2}{|c|}{ BG } & \multicolumn{2}{|c|}{ BG } & \multicolumn{2}{|c|}{ RS } & \multicolumn{2}{|c|}{ RS } & \multicolumn{2}{|c|}{ SPI } \\
\hline & $\mathrm{pCi} / \mathrm{L}$ & $\mathrm{CE}+/-$ & $\mathrm{pCi} / \mathrm{L}$ & $\mathrm{CE}+/-$ & $\mathrm{pCi} / \mathrm{L}$ & $\mathrm{CE}+1-$ & $\mathrm{pCi} / \mathrm{L}$ & $\mathrm{CE}+/-$ & $\mathrm{pCl} / \mathrm{L}$ & $\mathrm{CE}+/-$ \\
\hline GROSS ALPHA & - & $\cdot$ & - & - & . & $\cdot$ & - & - & - & $\bullet$ \\
\hline First Quarter & -0.33 & 1.50 & -0.48 & 1.40 & -0.74 & 2.20 & -0.55 & 3.30 & 5.35 & 3.40 \\
\hline Second Quarter & 0.87 & 1.70 & 5.38 & 1.20 & -0.00 & 0.56 & -0.35 & 0.55 & -2.46 & 4.60 \\
\hline Third Quarter & 1.64 & 1.70 & 1.48 & 1.70 & -2.75 & 2.60 & -1.88 & 3.10 & 0.33 & 1.50 \\
\hline Fourth Quarter & 4.59 & 2.50 & 0.00 & 1.70 & 1.00 & 1.50 & -0.20 & 1.40 & 2.54 & 2.60 \\
\hline & & $\cdot$ & • & & $\cdot$ & - & $\cdot$ & - & $\cdot$ & • \\
\hline Mean & 1.69 & 0.90 & 1.59 & 0.80 & -0.62 & 0.90 & -0.75 & 1.20 & 1.44 & 1.60 \\
\hline GROSS BETA & $\cdot$ &. & - &. & . &. & . & - & . & - \\
\hline First Quarter & -0.70 & 2.60 & 0.17 & 2.60 & 10.10 & 3.40 & 7.36 & 3.40 & 12.90 & 3.40 \\
\hline Second Quarter & -1.66 & 2.70 & 7.54 & 1.40 & -1.65 & 1.40 & 1.43 & 1.40 & 15.70 & 7.10 \\
\hline Third Quarter & -0.08 & 2.60 & 0.33 & 2.60 & -7.51 & 5.70 & -5.53 & 5.70 & 31.30 & 5.00 \\
\hline Fourth Quarter & 7.39 & 3.20 & 2.22 & 2.90 & -0.41 & 2.40 & 7.27 & 2.90 & 53.80 & 4.90 \\
\hline & & $\cdot$ & & & - & $\cdot$ & $\cdot$ & $\bullet$ & $\cdot$ & \\
\hline Mean & 1.24 & 1.40 & 2.57 & 1.20 & 0.13 & 1.80 & 2.63 & 1.80 & 28.42 & 2.60 \\
\hline
\end{tabular}

(CONTINUED)

\begin{tabular}{|c|c|c|c|c|c|c|c|c|c|c|}
\hline \multirow{3}{*}{$\begin{array}{l}\text { Sampling Point } \\
\text { Location }\end{array}$} & \multicolumn{2}{|c|}{ Gw-316 } & \multicolumn{2}{|c|}{$G W-317$} & \multicolumn{2}{|c|}{$G W-323$} & \multicolumn{2}{|c|}{$G W-324$} & \multicolumn{2}{|c|}{$G W-325$} \\
\hline & \multicolumn{2}{|c|}{ SPI } & \multicolumn{2}{|c|}{ SPI } & \multicolumn{2}{|c|}{ SPI } & \multicolumn{2}{|c|}{ s3 } & \multicolumn{2}{|c|}{ s3 } \\
\hline & $\mathrm{pCi} / \mathrm{L}$ & $\mathrm{CE}+1-$ & $\mathrm{pCi} / \mathrm{L}$ & $\mathrm{CE}+1-$ & $\mathrm{pCi} / \mathrm{L}$ & $\mathrm{CE}+/-$ & $\mathrm{pCi} / \mathrm{L}$ & $\mathrm{CE}+/-$ & $\mathrm{pCi} / \mathrm{L}$ & $C E+1-$ \\
\hline GROSS ALPHA & $\cdot$ & $\cdot$ & - & - & - & - & - & $\cdot$ & - & $\bullet^{\bullet}$ \\
\hline First Quarter & 2.21 & 1.50 & 1.55 & 1.40 & 2.20 & 1.50 & 1.59 & 1.30 & 1.12 & 1.60 \\
\hline Second Quarter & 1.75 & 3.60 & 2.94 & 3.80 & 2.08 & 3.70 & 0.00 & 3.10 & 0.56 & 3.20 \\
\hline Third Quarter & -0.01 & 0.95 & 0.16 & 1.40 & 0.68 & 1.20 & 0.33 & 1.10 & 0.29 & 0.94 \\
\hline Fourth Quarter & -0.45 & 2.10 & -1.64 & 1.90 & 0.75 & 2.30 & -0.32 & 2.20 & 10.80 & 3.20 \\
\hline - & & - & . &. & - & - & . & - & - & \\
\hline Mean & 0.88 & 1.10 & 0.75 & 1.20 & 1.43 & 1.20 & 0.40 & 1.00 & 3.19 & 1.20 \\
\hline GROSS BETA &. &. &. &. &. & . & . & . &. & • \\
\hline First Quarter & 2.16 & 2.60 & 3.58 & 2.70 & 2.16 & $2.6 \dot{0}$ & 0.75 & $2.6 \dot{0}$ & 4.41 & 2.70 \\
\hline Second Quarter & 5.42 & 5.00 & 7.40 & 5.10 & 6.92 & 5.10 & -3.77 & 5.20 & -1.63 & 5.30 \\
\hline Third Quarter & -0.97 & 2.60 & -1.43 & 2.30 & -1.21 & 2.60 & -2.66 & 2.50 & -0.10 & 2.90 \\
\hline Fourth Quarter & 2.64 & 2.70 & 1.98 & 2.70 & 1.81 & 2.60 & -7.50 & 5.70 & 15.50 & 3.50 \\
\hline - & & - & & $\cdot$ & $\cdot$ & $\cdot$ & - & . & $\cdot$ & • \\
\hline Mean & 2.31 & 1.70 & 2.88 & 1.70 & 2.42 & 1.70 & -3.30 & 2.10 & 4.55 & 1.90 \\
\hline
\end{tabular}

(CONTINUED) 
APPENDIX $\mathrm{K}$

Gross Alpha and Grose Beta Activity Data Summary, 1993

\begin{tabular}{|c|c|c|c|c|c|c|c|c|c|c|c|}
\hline \multirow{3}{*}{$\begin{array}{l}\text { Sampling Point } \\
\text { Location }\end{array}$} & \multicolumn{2}{|c|}{$G w-342$} & \multicolumn{2}{|c|}{$G W-345$} & \multicolumn{2}{|c|}{$G W-347$} & \multicolumn{2}{|c|}{ GW-348 } & \multicolumn{3}{|c|}{$G w-364$} \\
\hline & \multicolumn{2}{|c|}{ BG } & \multicolumn{2}{|c|}{ s3 } & \multicolumn{2}{|c|}{83} & \multicolumn{2}{|c|}{83} & \multicolumn{3}{|c|}{ OLP } \\
\hline & $\mathrm{pCl} / \mathrm{L}$ & $\mathrm{CE}+1-$ & $\mathrm{pC} 1 / \mathrm{L}$ & $\mathrm{CB}+/-$ & $\mathrm{pCi} / \mathrm{L}$ & $\mathrm{CE}+1-$ & $\mathrm{pCl} / \mathrm{L}$ & $\mathrm{CB}+1-$ & $\mathrm{pCl} / \mathrm{L}$ & $\mathrm{CE}$ & $+1-$ \\
\hline GROSS ALPHA & - & $\cdot$ & - & $\cdot$ & $\cdot$ & - & - & - & - & & - \\
\hline Firat Quarter & - & - & 3.33 & 2.00 & 1.81 & 1.90 & 2.42 & 2.50 & 1.65 & & 2.10 \\
\hline Second Quarter & - & - & $-1 \cdot 70$ & 2.90 & 0.85 & 3.40 & 0.00 & 3.40 & $-1 \cdot 34$ & & 3.00 \\
\hline Third Quarter & - & - & 0.47 & 1.40 & 2.50 & 1.60 & 1.42 & 1.40 & 2.77 & & 3.60 \\
\hline Fourth Quarter & 0.00 & 2.00 & -14.00 & 27.00 & 3.64 & 2.70 & 0.31 & 2.30 & 3.73 & & 2.00 \\
\hline - & $\cdot$ & & & & $\cdot$ & & & - & - & & - \\
\hline Mean & 0.00 & 2.00 & -2.98 & 6.80 & 2.20 & 1.30 & 1.04 & 1.30 & 1.70 & & 1.40 \\
\hline GROSS BETA & . & - & - & - & $\cdot$ & - & - & • & - & & • \\
\hline First Quarter & - & - & 25.70 & 3.90 & 4.71 & 2.70 & $37 \cdot 30$ & 4.40 & 5.26 & & 2.90 \\
\hline Second Quarter & . & - & 8.99 & 5.20 & 5.24 & 4.90 & 31.60 & 6.50 & 1.25 & & 5.00 \\
\hline Third Quarter & - & - & 15.10 & 3.60 & 5.53 & 3.10 & $18 \cdot 40$ & 4.00 & $22 \cdot 30$ & & 6.30 \\
\hline Fourth Quarter & 1.97 & 2.70 & 13.70 & 27.00 & 11.10 & 3.20 & 29.80 & 4.00 & 4.16 & & 3.20 \\
\hline - & • & - & - & & $\cdot$ & . & & - & • & & - \\
\hline Hean & 1.97 & 2.70 & 15.87 & 7.00 & 6.64 & 1.80 & 29.27 & 2.40 & 8.24 & & 2.30 \\
\hline
\end{tabular}

(CONTINUED)

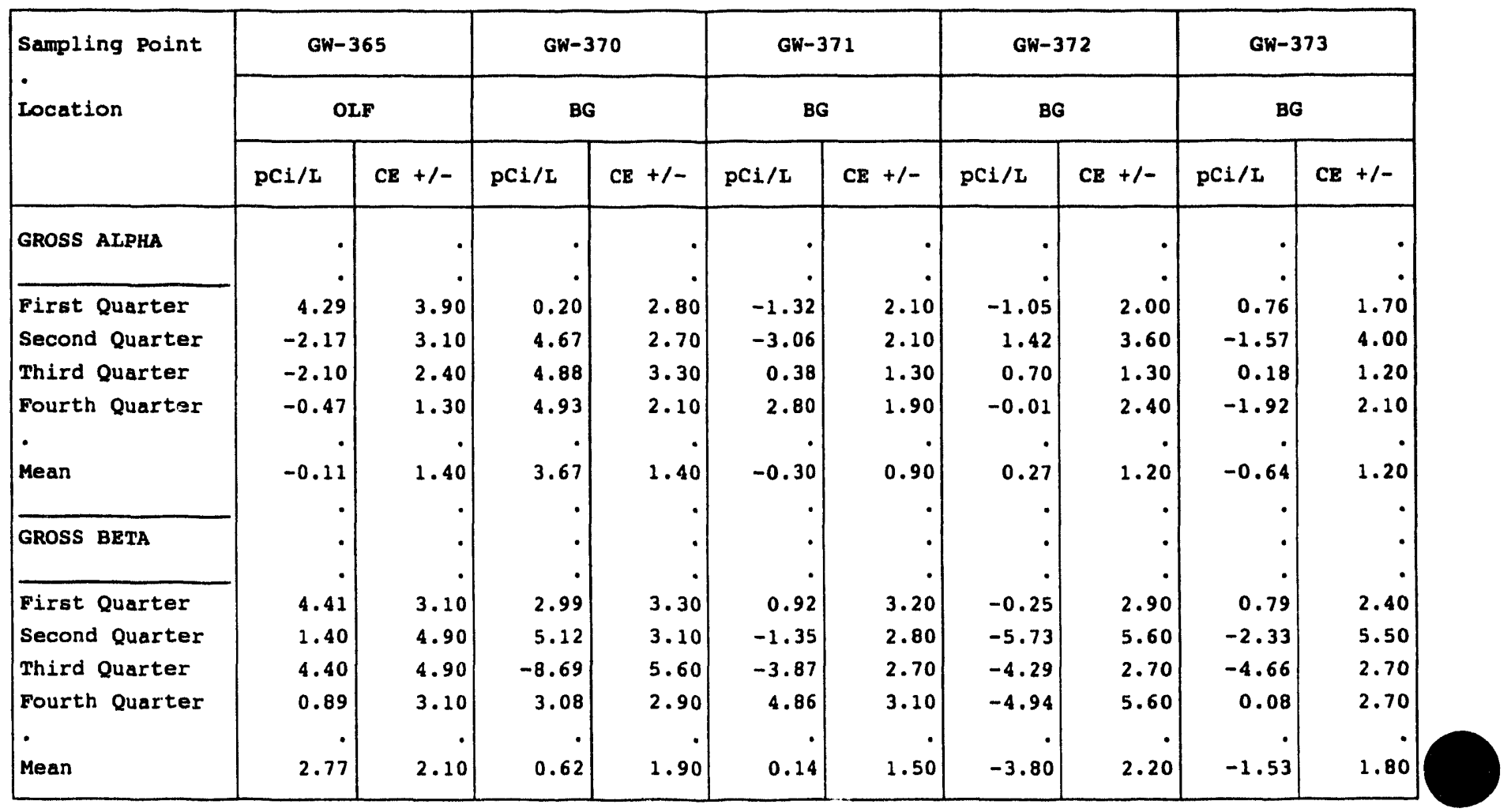

(CONTINUED) 
APPENDIX $k$

Gross Alpha and Gross Beta Activity Data summary, 1993

\begin{tabular}{|c|c|c|c|c|c|c|c|c|c|c|}
\hline \multirow{3}{*}{$\begin{array}{l}\text { Sampling Point } \\
\text { Location }\end{array}$} & \multicolumn{2}{|c|}{ GW-526 } & \multicolumn{2}{|c|}{$6 W-537$} & \multicolumn{2}{|c|}{$G W-601$} & \multicolumn{2}{|c|}{ ow-613 } & \multicolumn{2}{|c|}{ OW-614 } \\
\hline & \multicolumn{2}{|c|}{83} & \multicolumn{2}{|c|}{ OLP } & \multicolumn{2}{|c|}{ OLF } & \multicolumn{2}{|c|}{83} & \multicolumn{2}{|c|}{83} \\
\hline & $\mathrm{pc1} / \mathrm{L}$ & $C B+1-$ & $\mathrm{pCl} / \mathrm{L}$ & $\mathrm{CB}+1-$ & $\mathrm{pCl} / \mathrm{L}$ & $\mathrm{CE}+1-$ & $\mathrm{PCi} / \mathrm{L}$ & $\mathrm{CB}+/-$ & $\mathrm{pci} / \mathrm{L}$ & $C n+1-$ \\
\hline GROSS ALPHA & $\cdot$ & - & $\cdot$ & $\cdot$ & $\cdot$ & $\cdot$ & $\cdot$ & $\cdot$ & - & $\bullet$ \\
\hline First Quarter & 2.04 & 1.40 & 39.80 & 14.00 & 1.48 & 2.70 &. & . & . & . \\
\hline second Quarter & 121.00 & 80.00 & 0.00 & 20.00 & -2.15 & 3.00 &. & . & . & • \\
\hline Third Quarter & -14.20 & 24.00 & 16.60 & 33.00 & 0.33 & 3.10 & $\cdot$ & . & - & • \\
\hline Fourth Quarter & 0.79 & 2.40 & 19.70 & 12.00 & 2.08 & 1.80 & 1.79 & 1.60 & 1.23 & 1.50 \\
\hline & & & & & & & & & & • \\
\hline Mean & 27.41 & 20.90 & 19.02 & 10.70 & 0.43 & 1.30 & 1.79 & 1.60 & 1.23 & 1.50 \\
\hline GROSS BETA & & & $\cdot$ &. & . &. &. & $\cdot$ & $\cdot$ & • \\
\hline Pirst Quarter & 3.53 & 2.70 & 287.00 & 23.00 & 15.50 & 3.50 & $\dot{.}$ & • & $\cdot$ & $\stackrel{\bullet}{\bullet}$ \\
\hline Second Quarter & 287.00 & 120.00 & 373.00 & 42.00 & 18.70 & 6.20 & . & . & . & • \\
\hline Third Quarter & -22.10 & 46.00 & 426.00 & 80.00 & 23.40 & 6.40 & .1 & . & . & • \\
\hline Fourth Quarter & 44.70 & 4.60 & 432.00 & 27.00 & 21.80 & 4.20 & 2.82 & 2.50 & 2.92 & 2.60 \\
\hline . & & & & & & & & & $\cdot$ & \\
\hline Mean & 78.28 & 32.20 & 379.50 & 24.30 & 19.85 & 2.60 & 2.82 & 2.50 & 2.92 & 2.60 \\
\hline
\end{tabular}

(CONTINUED)

\begin{tabular}{|c|c|c|c|c|c|c|c|c|c|c|}
\hline \multirow{3}{*}{$\begin{array}{l}\text { Sampling Point } \\
\text { Location }\end{array}$} & \multicolumn{2}{|c|}{$G W-621$} & \multicolumn{2}{|c|}{ GW-626 } & \multicolumn{2}{|c|}{$G W-627$} & \multicolumn{2}{|c|}{$G W-636$} & \multicolumn{2}{|c|}{$G W-637$} \\
\hline & \multicolumn{2}{|c|}{ BG } & \multicolumn{2}{|c|}{ BG } & \multicolumn{2}{|c|}{ BG } & \multicolumn{2}{|c|}{ OLF } & \multicolumn{2}{|c|}{ OLF } \\
\hline & $\mathrm{pCi} / \mathrm{L}$ & $\mathrm{CE}+1-$ & $\mathrm{pCl} 1 / \mathrm{L}$ & $\mathrm{CB}+1-$ & $\mathrm{pCl} / \mathrm{L}$ & $\mathrm{CE}+1-$ & $\mathrm{pCi} / \mathrm{L}$ & $\mathrm{CB}+1-$ & $\mathrm{pC1} / \mathrm{L}$ & $\mathrm{CB}+1-$ \\
\hline GROSS ALPHA & $\cdot$ & $\cdot$ & $\cdot$ & $\cdot$ & . & $\cdot$ &. & $\bullet$ & & . \\
\hline First Quarter & 0.00 & 1.80 & 2.38 & 3.90 & 1.25 & 1.30 & 0.00 & 1.60 & -0.19 & 1.80 \\
\hline Second Quarter & 1.47 & 4.90 & 0.16 & 1.40 & -0.38 & 3.10 & -2.87 & 2.50 & -2.42 & 2.60 \\
\hline Third Quarter & 3.51 & 4.50 & $-22 \cdot 30$ & 33.00 & -6.24 & 3.60 & -0.72 & 2.80 & -0.72 & 2.80 \\
\hline Fourth Quarter & 2.41 & 1.70 & -1.59 & 2.30 & -0.82 & 3.20 & -0.60 & 1.00 & 1.39 & 1.50 \\
\hline - & & & & & . & $\cdot$ & • & . & • & • \\
\hline Mean & 1.85 & 1.80 & -5.34 & 8.30 & -1.55 & 1.50 & -1.05 & 1.10 & -0.48 & 1.10 \\
\hline GROSS BETA & $\cdot$ & $\cdot$ & $\cdot$ & - & - & - & $\cdot$ & $\cdot$ & $\cdot$ & • \\
\hline First Quarter & 5.13 & 2.80 & 2.97 & 5.30 & $5.84^{\circ}$ & 3.40 & 6.37 & 2.90 & $2 . \dot{89}$ & 2.70 \\
\hline Second Quarter & 7.89 & 6.60 & 0.26 & 2.60 & 1.70 & 2.90 & -1.61 & 4.80 & -2.73 & 4.80 \\
\hline Third Quarter & 24.10 & 6.30 & 17.90 & 52.00 & -0.96 & 3.00 & 3.44 & 4.80 & 0.46 & 4.60 \\
\hline Fourth Quarter & 5.79 & 3.30 & $-8 \cdot 32$ & 5.70 & -6.67 & 5.50 & -1.66 & 2.90 & -2.56 & 2.90 \\
\hline . & & & & & & & & & - & ${ }^{\circ}$ \\
\hline Mean & 10.73 & 2.50 & 3.20 & 13.20 & -0.02 & 1.90 & 1.63 & 2.00 & -0.49 & 1.90 \\
\hline
\end{tabular}

(CONTINUED) 
APPENDIX $\mathrm{K}$

Cross Alpha and Gross Beta Activity Data summary, 1993

\begin{tabular}{|c|c|c|c|c|c|c|c|c|c|c|c|}
\hline \multirow{3}{*}{$\begin{array}{l}\text { Sampling Polnt } \\
\text { Location }\end{array}$} & \multicolumn{2}{|c|}{$\theta w-638$} & \multicolumn{2}{|c|}{$c w-639$} & \multicolumn{2}{|c|}{$a w-640$} & \multicolumn{2}{|c|}{$0 w-641$} & \multicolumn{3}{|c|}{$a w-642$} \\
\hline & \multicolumn{2}{|c|}{ orF } & \multicolumn{2}{|c|}{ BO } & \multicolumn{2}{|c|}{$\mathbf{B G}$} & \multicolumn{2}{|c|}{ BG } & \multicolumn{3}{|c|}{ BO } \\
\hline & $\mathrm{pCl} / \mathrm{L}$ & $\mathrm{Cs}+1-$ & $\mathrm{pC} 1 / \mathrm{L}$ & $\mathrm{Cs}+1-$ & $\mathrm{pCl} / \mathrm{L}$ & $\mathrm{CB}+1-$ & $\mathrm{pCl} / \mathrm{L}$ & $\mathrm{CB}+1-$ & $\mathrm{pcl} / \mathrm{L}$ & $\mathbf{C B}$ & $+1-$ \\
\hline GROSS ALPHA & & & & & & & • & $\cdot$ & - & & - \\
\hline Flret Quarter & 5.01 & 4.20 & 0.46 & 2.20 & 0.59 & 1.50 & 1.00 & 1.50 & -1.00 & & 1.60 \\
\hline Second Quarter & 5.67 & 4.30 & 0.00 & 3.80 & -0.56 & 3.00 & -0.84 & 2.90 & -0.62 & & 0.43 \\
\hline Third Quarter & 8.70 & 4.40 & 0.43 & 1.50 & -0.16 & 0.93 & 0.15 & 0.99 & $-2 \cdot 38$ & & 2.40 \\
\hline Fourth Quarter & 9.13 & 3.00 & -0.77 & 3.00 & 0.44 & 1.30 & 1.27 & 2.10 & 2.10 & & 2.40 \\
\hline - & & & & & $\cdot$ & & $\cdot$ & $\cdot$ & $\cdot$ & & $\bullet$ \\
\hline Mean & 7.13 & 2.00 & 0.03 & 1.40 & 0.08 & 0.90 & 0.40 & 1.00 & -0.47 & & 0.90 \\
\hline GROSS BETA & $\cdot$ & $\cdot$ & $\cdot$ & $\cdot$ & $\cdot$ & $\cdot$ & $\cdot$ & $\cdot$ & $\cdot$ & & - \\
\hline Firot Quarter & 15.60 & 5.90 & 0.72 & 2.80 & 7.60 & 3.00 & 1.11 & 2.70 & 0.08 & & 2.80 \\
\hline Second Quarter & 8.17 & 5.50 & 0.17 & 5.60 & -1.47 & 5.40 & -2.28 & 5.30 & -2.64 & & 1.40 \\
\hline Third quarter & 13.50 & 5.70 & -4.58 & 2.80 & -4.22 & 2.90 & -4.96 & 3.00 & 0.64 & & 5.10 \\
\hline rourth Quarter & 2.14 & 3.10 & -7.74 & 5.40 & 0.26 & 2.80 & $-1 \cdot 31$ & 2.70 & 3.30 & & 2.80 \\
\hline & & & $\cdot$ & & & & $\bullet$ & & $\cdot$ & & \\
\hline Mean & 9.85 & 2.60 & -2.86 & 2.20 & 0.54 & 1.80 & -1.86 & 1.80 & 0.35 & & 1.70 \\
\hline
\end{tabular}

(CONTINUED)

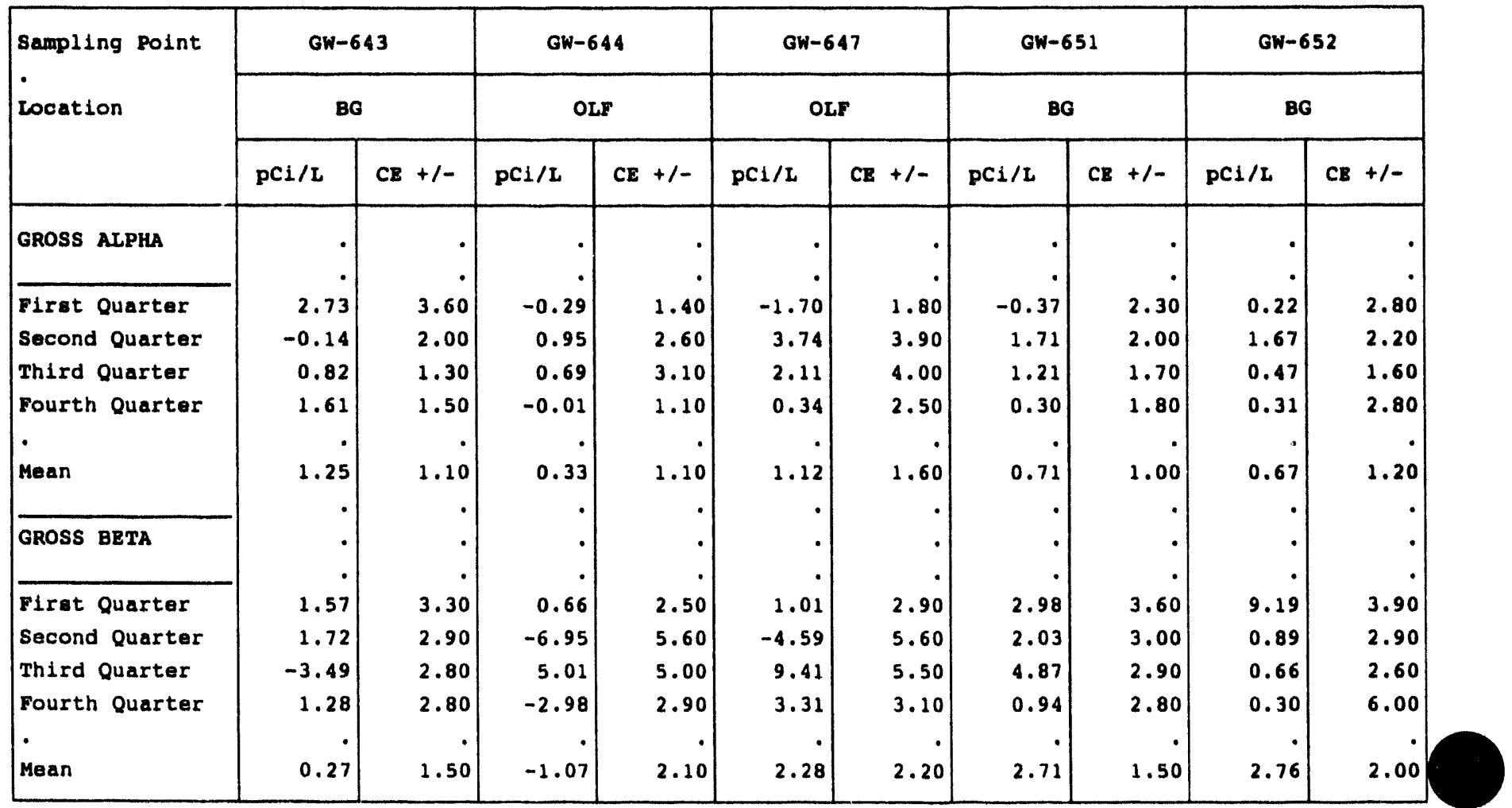

(CONTINUED) 
APPINDIX $\mathrm{K}$

Orose Alpha and Groes Deta Activity Data Summary, 1993

\begin{tabular}{|c|c|c|c|c|c|c|c|c|c|c|}
\hline \multirow{3}{*}{$\begin{array}{l}\text { 8ampling Point } \\
\text { Location }\end{array}$} & \multicolumn{2}{|c|}{$a w-653$} & \multicolumn{2}{|c|}{$a w-634$} & \multicolumn{2}{|c|}{$a w-655$} & \multicolumn{2}{|c|}{ CW-683 } & \multicolumn{2}{|c|}{$a w-684$} \\
\hline & \multicolumn{2}{|c|}{80} & \multicolumn{2}{|c|}{$\mathbf{B C}$} & \multicolumn{2}{|c|}{$B O$} & \multicolumn{2}{|c|}{$\mathbf{E X P}$} & \multicolumn{2}{|c|}{$\mathbf{E X P}$} \\
\hline & $\mathrm{pcl} / \mathrm{L}$ & $C 2+1-$ & $\mathrm{pCl} / \mathrm{L}$ & $\mathrm{CE}+1-$ & $p C 1 / L$ & $\mathrm{Cr}+1-$ & $\mathrm{pCl} / \mathrm{L}$ & $\mathrm{CE}+1-$ & $\mathrm{pCl} / \mathrm{L}$ & $\mathrm{CB}+1-$ \\
\hline Cross ntph & - & $\cdot$ & $*$ & $\cdot$ & $\cdot$ & - & $\cdot$ & $\cdot 1$ & • & - \\
\hline Fret Quarter & 12.90 & 5.60 & 0.31 & 1.50 & 2.18 & 1.60 & 7.31 & 2.30 & 9.05 & 2.50 \\
\hline 8econd Quarter & 3.93 & 3.50 & 3.52 & 1.00 & 14.10 & 3.90 & 5.33 & 2.30 & 5.89 & 2.50 \\
\hline Th1rd Quartar & 6.36 & 3.80 & -2.58 & 2.60 & -2.03 & 2.40 & 12.50 & 5.50 & $21 \cdot 20$ & 4.00 \\
\hline Pourth Quarter & 8.56 & 8.00 & 3.44 & 2.30 & 0.43 & 1.70 & 35.80 & 3.10 & 38.20 & 3.20 \\
\hline - & $\cdot$ & $\cdot$ & & $\cdot$ & $\dot{0}$ & i & , & , & 18,58 & 90 \\
\hline Maan & 7.94 & 2.80 & 1.17 & 1.00 & 3.07 & 1.30 & $13.4 \%$ & 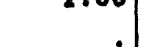 & $+\infty$ & 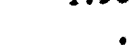 \\
\hline CROS8 BB2A & $\cdot$ & $\cdot$ & $\cdot$ & - & $\cdot$ & $\cdot$ & - & - & $\cdot$ & - \\
\hline Firet quarter & 11.60 & 7.20 & 1.89 & 2.70 & -4.35 & 3.70 & 15.80 & 3.80 & 20.90 & 4.00 \\
\hline Second Quarter & -3.09 & 5.30 & 1.31 & 1.30 & 13.70 & 5.90 & 10.60 & 3.20 & 27.50 & 4.00 \\
\hline Th1rd Quarter & 6.73 & 5.60 & -3.20 & 4.70 & -1.65 & 5.00 & 26.20 & 7.70 & 46.60 & 4.70 \\
\hline Fourth Quarter & 16.00 & 12.00 & 4.14 & 3.00 & 2.21 & 2.70 & 64.40 & 5.50 & 63.60 & 5.50 \\
\hline 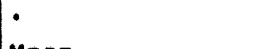 & i & & $\begin{array}{r}1 \\
1.03\end{array}$ & & , & , & 20 &, 20 & 39.65 & 2. \\
\hline Maan & 7.81 & 00 & 1.03 & 1.60 & 2.48 & 2.20 & 29.25 & 2.10 & 30.03 & 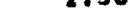 \\
\hline
\end{tabular}

(CONTINUED)

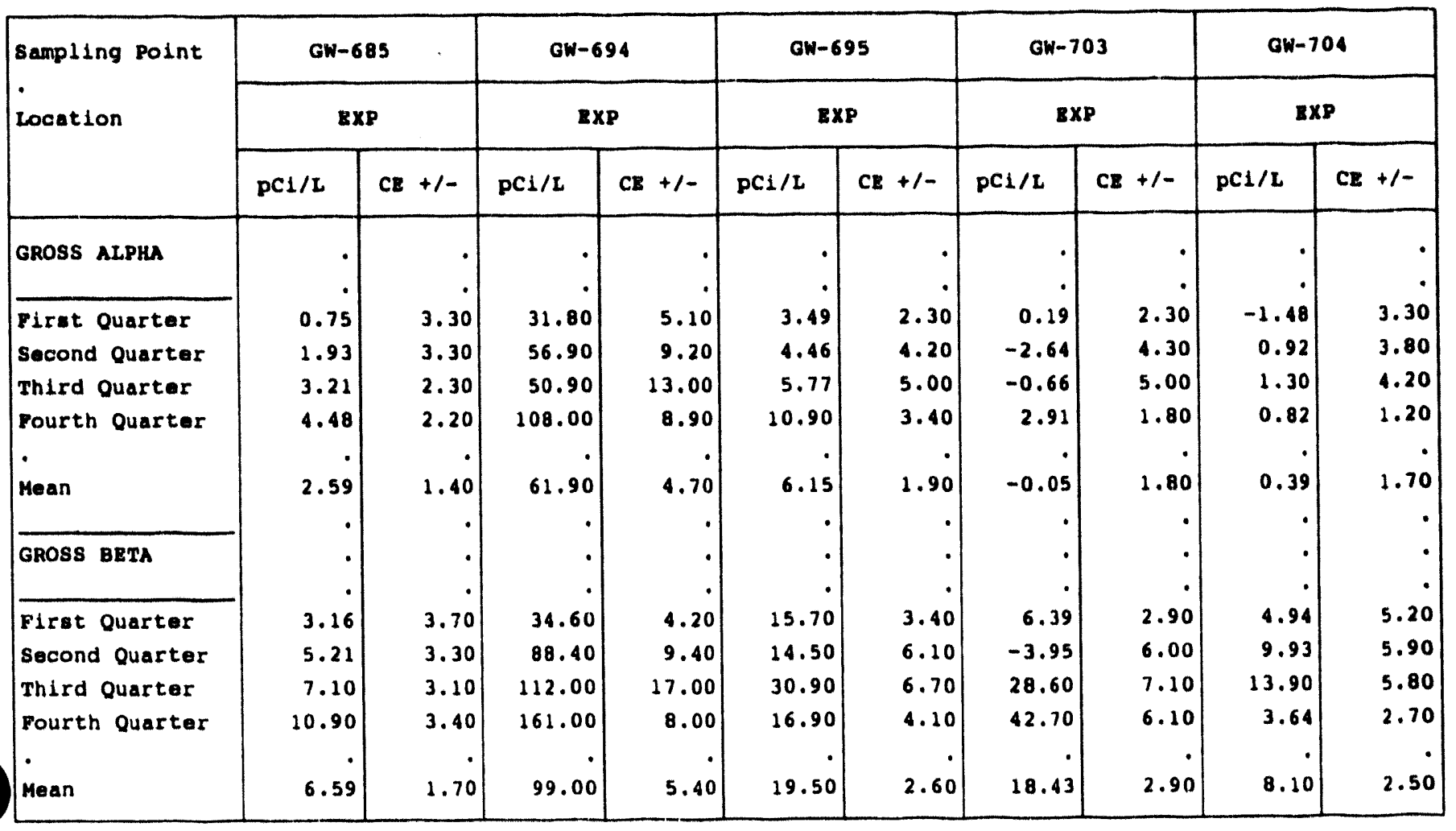

(CONTINUED) 
NPPINDTX $x$

Grose Alpha and Grose Beta Activity Duta summary, 1993

\begin{tabular}{|c|c|c|c|c|c|c|c|c|c|c|}
\hline \multirow{3}{*}{$\begin{array}{l}\text { Bampling point } \\
\text { Location }\end{array}$} & \multicolumn{2}{|c|}{ OW-706 } & \multicolumn{2}{|c|}{ ON-710 } & \multicolumn{2}{|c|}{ eN-711 } & \multicolumn{2}{|c|}{$a v-712$} & \multicolumn{2}{|c|}{$O N-713$} \\
\hline & \multicolumn{2}{|c|}{$\mathbf{E x p}$} & \multicolumn{2}{|c|}{$\exp$} & \multicolumn{2}{|c|}{$\operatorname{sxp}$} & \multicolumn{2}{|c|}{$\mathbf{E x p}$} & \multicolumn{2}{|c|}{$\mathbf{E x p}$} \\
\hline & $\mathrm{pCl} / \mathrm{L}$ & $c=+1-$ & $\mathrm{pCl} / \mathrm{L}$ & $\mathrm{Cz}+1-$ & $\forall 1 / 2$ & CE $+1-$ & $\mathrm{pCl} / \mathrm{L}$ & $c x+1-$ & $\mathrm{pcl} / \mathrm{L}$ & $\mathrm{cs}+1-$ \\
\hline OnOss ALPH & ' & $\cdot$ & • & - & $\cdot$ & - & - & $\cdot$ & - & • \\
\hline First Quarter & 0.00 & 1.50 & 1.00 & 4.20 & 1.45 & 4.60 & 1.71 & 1.80 & -0.33 & 2.00 \\
\hline second Quarter & 34.40 & 9.00 & -4.53 & 3.10 & 3.21 & 3.80 & 5.37 & 2.50 & -1.16 & 2.00 \\
\hline Th1rd Quarter & 11.70 & 7.40 & -6.39 & 41.00 & 3.52 & 47.00 & -2.80 & 37.00 & -22.30 & 33.00 \\
\hline Fourth Quarter & 99.20 & 8.50 & -6.99 & 22.00 & -6.49 & 28.00 & -0.29 & 2.50 & -0.17 & 2.20 \\
\hline & & & & & & & & & & • \\
\hline man & 41.32 & 3.60 & -4.23 & 11.70 & 0.43 & 13.80 & 1.02 & 9.30 & -6.04 & 8.30 \\
\hline$r \quad 8$ BมxA & - & • & . & • & $\cdot$ & $\cdot$ & • &. & $\cdot$. & • \\
\hline Firet Quarter & 7.42 & $2.9 \dot{0}$ & $7.8 \dot{4}$ & 3.20 & $16.0 \dot{0}$ & $3.2 \dot{0}$ & 3.37 & 2.90 & 3.30 & 2.70 \\
\hline second Quarter & 97.90 & 9.80 & -11.80 & 2.90 & 0.84 & 3.40 & -8.64 & 2.70 & -8.13 & 2.80 \\
\hline Third Quarter & 0.00 & 11.00 & 15.00 & 53.00 & 18.60 & 54.00 & 31.00 & 53.00 & -17.90 & 50.00 \\
\hline rourth Quarter & 150.00 & 7.70 & $-20 \cdot 10$ & 28.00 & 3.59 & 31.00 & 1.15 & 3.10 & 1.13 & 3.00 \\
\hline - & & & $\cdot$ & • & $\cdot$ & $\cdot$ & • & $\cdot$ & - & • \\
\hline mean & 63.83 & 1.20 & -2.27 & 13.00 & 11.96 & 15.60 & 6.72 & 13.30 & -5.40 & 12.60 \\
\hline
\end{tabular}

(COMTIMURD)

\begin{tabular}{|c|c|c|c|c|c|c|c|c|c|c|}
\hline \multirow{3}{*}{$\begin{array}{l}\text { Sampling Point } \\
\text { Location }\end{array}$} & \multicolumn{2}{|c|}{$0 w-714$} & \multicolumn{2}{|c|}{ ow-715 } & \multicolumn{2}{|c|}{$6 w-723$} & \multicolumn{2}{|c|}{$6 w-724$} & \multicolumn{2}{|c|}{$0 w-725$} \\
\hline & \multicolumn{2}{|c|}{ ExP } & \multicolumn{2}{|c|}{$\exp$} & \multicolumn{2}{|c|}{$\mathbf{E x p}$} & \multicolumn{2}{|c|}{ Exp } & \multicolumn{2}{|c|}{$\mathbf{E x P}$} \\
\hline & $\mathrm{pCl} / \mathrm{L}$ & $\mathrm{CE}+1-$ & $\mathrm{pCl} 1 / \mathrm{L}$ & $\mathrm{CE}+1-$ & $\mathrm{pCi} / \mathrm{L}$ & $\mathrm{CE}+1-$ & $\mathrm{pCl} / \mathrm{L}$ & $\mathrm{CE}+1-$ & $\mathrm{pc} 1 / \mathrm{L}$ & $\mathrm{Cr}+1-$ \\
\hline GROSS ALPHA & • & . & • & $\cdot$ & • & $\cdot$ & - & - & $\cdot$ & • \\
\hline Firat Quartor & 2.23 & 2.40 & -0.35 & 2.10 & 0.54 & 7.80 & 4.97 & 2.80 & 0.26 & 3.70 \\
\hline second Quarter & -3.05 & 1.60 & -3.15 & 1.80 & 3.24 & 4.00 & 2.08 & 3.80 & 2.06 & 3.80 \\
\hline Th1rd Quarter & -2.80 & 37.00 & -33.80 & 32.00 & -1.51 & 3.50 & 0.92 & 3.40 & 0.63 & 3.80 \\
\hline Fourth Quarter & 0.93 & 2.20 & 0.17 & 1.30 & 0.64 & 2.40 & -0.36 & 2.60 & 4.61 & 3.50 \\
\hline & & & - & & . & $\cdot 1$ & $\cdot$ & $\cdot$ & $\cdot$ & • \\
\hline Mean & -0.67 & 9.30 & -9.28 & 8.00 & 0.73 & 2.40 & 1.90 & 1.60 & 1.89 & 1.90 \\
\hline GROSS BETA & . &. & - & . & - &. &. &. & . & . \\
\hline -t Ounetor & & & & $\dot{1}$ & & & . & & $\cdot$ & \\
\hline it Quarter & 2.94 & 2.70 & 5.66 & 2.90 & 4.96 & 6.90 & 11.10 & 3.50 & 24.20 & 4.40 \\
\hline nd Quarter & -3.17 & 3.00 & -4.22 & 2.90 & 10.70 & 6.50 & 40.40 & 7.90 & 41.60 & 7.90 \\
\hline “Lrd Quarter & -22.80 & 49.00 & -11.40 & 50.00 & 9.41 & 5.50 & 41.80 & 4.90 & 46.50 & 5.20 \\
\hline Fourth Quarter & 6.28 & 3.30 & 2.65 & 2.50 & 9.78 & 3.40 & 32.00 & 4.40 & 53.70 & 5.30 \\
\hline - & & & - & • & & • & $\cdot$ & - & . & \\
\hline Mean & -4.19 & 12.30 & -1.83 & 12.60 & 8.71 & 2.90 & 31.32 & 2.70 & 41.50 & 2.90 \\
\hline
\end{tabular}


APRENDIX $\mathrm{x}$

Growe Alpha and Orose Bete Activity Dete Sumary, 1993

\begin{tabular}{|c|c|c|c|c|c|c|c|c|c|c|c|}
\hline \multirow{3}{*}{$\begin{array}{l}\text { Sampling Polnt } \\
\text { rooution }\end{array}$} & \multicolumn{2}{|c|}{$0: 1-736$} & \multicolumn{2}{|c|}{$01-737$} & \multicolumn{2}{|c|}{$0 N-738$} & \multicolumn{2}{|c|}{$0 N-739$} & \multicolumn{3}{|c|}{$a W-740$} \\
\hline & \multicolumn{2}{|c|}{$\mathbf{E X P}$} & \multicolumn{2}{|c|}{$\mathbf{E x P}$} & \multicolumn{2}{|c|}{ E4P } & \multicolumn{2}{|c|}{$\mathbf{E x P}$} & \multicolumn{3}{|c|}{$\mathbf{E x P}$} \\
\hline & $p c 1 / 2$ & $\mathrm{CE}+1-$ & $\mathrm{pCl} / \mathrm{L}$ & $\mathrm{cs}+1-$ & $\mathrm{pCl} / \mathbf{L}$ & $\mathrm{CE}+1-$ & $\mathrm{pCl} / \mathrm{L}$ & $\mathrm{CE}+1-$ & $p c 1 / 2$ & as & $+1=$ \\
\hline CRO88 A.PU & - & & . & - & - & - & - & . & - & & - \\
\hline rlret quarter & 7.80 & 4.50 & 9.66 & 4.80 & 7.81 & 3.30 & 1.57 & 1.50 & 0.17 & & 1.30 \\
\hline second Qunrter & 2.04 & 3.70 & 0.95 & 3.50 & 0.59 & 3.50 & $2 \cdot 33$ & 3.80 & 0.28 & & 3.40 \\
\hline Third Quarter & 3.51 & 4.00 & 0.00 & 3.70 & 0.33 & 4.10 & -1.22 & 3.60 & 1.85 & & 4.10 \\
\hline Fourth Querter & 4.12 & 3.40 & 5.85 & 3.60 & 0.18 & 2.60 & -0.96 & 2.20 & 0.65 & & 2.40 \\
\hline . & & & & & & & & - & - & & - \\
\hline Mean & 4.37 & 2.00 & 4.12 & 2.00 & 2.23 & 1.70 & 0.43 & 1.50 & 0.74 & & 1.50 \\
\hline CROS8 BETA & - & - & - & - & $\cdot$ & - & . & - & - & & - \\
\hline Plret quarter & 22.90 & 3.90 & 23.60 & 3.90 & 41.40 & 4.80 & 1.08 & 2.80 & 0.17 & & 2.80 \\
\hline second Quarter & 45.20 & 8.00 & 42.00 & 4.90 & 30.20 & 7.40 & 7.72 & 6.30 & 0.33 & & 5.90 \\
\hline Th1rd Quarter & 57.90 & 5.50 & 52.00 & 5.40 & 47.50 & 7.50 & 14.20 & 5.80 & 16.90 & & 5.90 \\
\hline Fourth quarter & 52.00 & 5.20 & 60.80 & 3.50 & 60.10 & 5.40 & 9.51 & 3.40 & 0.40 & & 3.30 \\
\hline . & & & & & $\cdot$ & - & & & & & • \\
\hline Man & 44.50 & 2.90 & 44.80 & 2.50 & 44.80 & 3.20 & 0.13 & 2.40 & 6.45 & & 2.30 \\
\hline
\end{tabular}

(CONTINUED)

\begin{tabular}{|c|c|c|c|c|c|c|c|c|c|c|c|}
\hline \multirow{3}{*}{$\begin{array}{l}\text { Sampling point } \\
\text { Location }\end{array}$} & \multicolumn{2}{|c|}{ CW-793 } & \multicolumn{2}{|c|}{$\sigma w-794$} & \multicolumn{2}{|c|}{ Gw-795 } & \multicolumn{2}{|c|}{$a w-800$} & \multicolumn{3}{|c|}{$88-1$} \\
\hline & \multicolumn{2}{|c|}{ NGLLSF } & \multicolumn{2}{|c|}{ AOLLSF } & \multicolumn{2}{|c|}{ NCLLSF } & \multicolumn{2}{|c|}{ OLF } & \multicolumn{3}{|c|}{$\mathbf{B X P}$} \\
\hline & $\mathrm{pCl} / \mathrm{L}$ & $\mathrm{CB}+1-$ & $\mathrm{pCl} / \mathrm{L}$ & $C E+1-$ & $\mathrm{pCl} / \mathrm{L}$ & $\mathrm{Cz}+1-$ & $\mathrm{pCl} / \mathrm{t}$ & $\mathrm{CB}+1-$ & $\mathrm{pCl} / \mathrm{L}$ & $\mathbf{C s}$ & $+1-$ \\
\hline GROSS ALPHA & $\cdot 1$ & - & - & - & $\cdot 1$ & $\cdot$ & - & . & - & & $\cdot$ \\
\hline First Quartar & 0.49 & 1.40 & 2.55 & 2.00 & 0.35 & 1.50 & . & - & 12.90 & & 3.50 \\
\hline second Quarter & -5.55 & 4.40 & -5.92 & 4.70 & -6.19 & 4.30 & -0.18 & 0.57 & 15.10 & & 3.60 \\
\hline Third Quarter & -4.03 & 7.90 & -4.51 & 7.60 & 0.00 & 8.30 & -2.26 & 3.00 & 18.90 & & 4.10 \\
\hline Fourth Quarter & -0.15 & 2.20 & 2.20 & 2.60 & 3.24 & 2.70 & 1.88 & 1.70 & 18.20 & & 4.50 \\
\hline$\cdot$ & & & & & $\cdot$ & $\cdot$ & - & - & $\cdot$ & & - \\
\hline Mean & -2.31 & 2.40 & -1.42 & 2.40 & -0.65 & 2.50 & -0.19 & 1.20 & 16.27 & & 2.00 \\
\hline GROSS BETA & - & $\cdot$ & - & - & - & - & - & - & . & & - \\
\hline Plret Quarter & 0.95 & 2,70 & 4.57 & 2.90 & 2.28 & 2.80 & - & - & 27.10 & & 4.50 \\
\hline second Quarter & 3.98 & 5.50 & 1.00 & 5.40 & 2.32 & 5.40 & 0.31 & 1.40 & 178.00 & & 8.30 \\
\hline Thlrd Quarter & -0.98 & 11.00 & -3.27 & 11.00 & -8.51 & 11.00 & -6.00 & 5.70 & 52.60 & & 5.00 \\
\hline Fourth Quarter & 3.21 & 3.10 & 7.15 & 3.40 & 10.00 & 3.50 & 4.08 & 2.70 & 63.60 & & 5.40 \\
\hline - & & & & & & & & - & - & & - \\
\hline Mean & 1.79 & 3.20 & 2.36 & 3.30 & 1.52 & 3.30 & -0.54 & 2.20 & 80.32 & & 3.00 \\
\hline
\end{tabular}

(CONT INUED) 
APPADIX

Orose Alphe and Oroes Bete Aotlvity Data 8umary, 1993

\begin{tabular}{|c|c|c|c|c|c|c|c|c|c|c|}
\hline \multirow{3}{*}{$\begin{array}{l}\text { sepling point } \\
\text { Location }\end{array}$} & \multicolumn{2}{|c|}{$88-4$} & \multicolumn{2}{|c|}{$88-5$} & \multicolumn{2}{|c|}{$88-6$} & \multicolumn{2}{|c|}{$88-8$} & \multicolumn{2}{|c|}{$\operatorname{BCK}-00.63$} \\
\hline & \multicolumn{2}{|c|}{$\operatorname{Exp}$} & \multicolumn{2}{|c|}{$\mathbf{E x p}$} & \multicolumn{2}{|c|}{$\mathbf{E x p}$} & \multicolumn{2}{|c|}{$\operatorname{Exp}$} & \multicolumn{2}{|c|}{$\exp$} \\
\hline & $\mathrm{pci} / \mathrm{L}$ & $\mathrm{Cs}+1-$ & $\mathrm{PCl} / \mathrm{L}$ & CE $+1-$ & $\mathrm{pCl} / \mathrm{L}$ & $\mathrm{Cs}+1-$ & $\mathrm{pcl} / \mathrm{l}$ & cs $+1-$ & $\mathrm{pCl} / \mathrm{L}$ & Cx $+1=$ \\
\hline OROS ALPM & & & - & • & • & • & $\cdot$ & - & - & • \\
\hline Firat Quarter & 29.80 & 5.00 & 0.69 & 3.00 & -0.13 & 1.60 & 0.00 & 1.70 & 6.99 & 2.70 \\
\hline 8eoond Quarter & 29.60 & 6.20 & 6.11 & 2.70 & -0.90 & 1.30 & 0.48 & 1.70 & 3.06 & 2.40 \\
\hline Thlrd Quarter & 57.00 & 14.00 & 30.60 & 11.00 & 2.29 & 6.10 & 6.91 & 6.90 & 18.30 & 8.70 \\
\hline Pourth guarter & ss.10 & 9.10 & 32.80 & 6.10 & 54.30 & 8.70 & -0.66 & 0.81 & 13.80 & 3.60 \\
\hline & & & & & & - & & & - & - \\
\hline mean & 41.88 & 4.60 & 19.55 & 3.30 & 13.89 & 2.70 & 1.68 & 1.00 & 11.04 & 2.50 \\
\hline anoss bern & $\cdot 1$ & . & . & . & . & . &. & . & . & . \\
\hline First Quarter & 37.20 & 4.50 & 12.50 & 3.40 & 0.82 & 2.70 & 1.65 & 2.80 & 10.50 & 3.30 \\
\hline seoond Quarter & 41.30 & 4.80 & 25.70 & 3.50 & 0.34 & 2.50 & -1.38 & 2.40 & 10.20 & 3.10 \\
\hline ThLrd Ouarter & 118.00 & 17.00 & 65.20 & 15.00 & -1.70 & 11.00 & 6.47 & 11.00 & 21.50 & 12.00 \\
\hline Fourth Quarter & 143.00 & 16.00 & 73.30 & 9.20 & 126.00 & 14.00 & -1.70 & 2.70 & 32.10 & 5.30 \\
\hline - & & & & & - & $\cdot$ & & & - & $\bullet$ \\
\hline man & 85.60 & 6.10 & 11.67 & 4.60 & 31.37 & 4.50 & 1.26 & 3.00 & 18.65 & 3.50 \\
\hline
\end{tabular}

(Comrrnued)

\begin{tabular}{|c|c|c|c|c|c|c|c|c|}
\hline \multirow{3}{*}{$\begin{array}{l}\text { Sampling Point } \\
\text { Location }\end{array}$} & \multicolumn{2}{|c|}{ BCK-04.55 } & \multicolumn{2}{|c|}{ BCK-09. 40} & \multicolumn{2}{|c|}{ MT-01 } & \multicolumn{2}{|c|}{$N T-13$} \\
\hline & \multicolumn{2}{|c|}{$\mathbf{E x p}$} & \multicolumn{2}{|c|}{$\mathbf{E X P}$} & \multicolumn{2}{|c|}{$\mathbf{E x p}$} & \multicolumn{2}{|c|}{$\mathbf{E X P}$} \\
\hline & $\mathrm{pCl} / \mathrm{L}$ & $\mathrm{CB}+1-$ & $\mathrm{pCl} / \mathrm{L}$ & $\mathrm{CE}+1-$ & $\mathrm{pCl} / \mathrm{L}$ & $C z+/-$ & $\mathrm{pCl} / \mathrm{L}$ & $\mathrm{CB}+1-$ \\
\hline OROS8 ALPHA & . &. & & . &. & - & $\cdot$ & - \\
\hline Plrst Quarter & 7.94 & 2.90 & 26.80 & 4.80 & 8.78 & 5.10 & 0.28 & 1.60 \\
\hline second Quarter & 3.81 & 2.60 & 19.10 & 4.40 & 16.30 & 7.10 & 0.00 & 1.40 \\
\hline Thlrd Quarter & 11.70 & 7.80 & 41.00 & 12.00 & 45.50 & 7.20 & 1.73 & 6.00 \\
\hline Fourth Quarter & 12.20 & 3.40 & -0.01 & 1.00 & 72.80 & 8.70 & 0.81 & 1.30 \\
\hline & & & & & & & . & \\
\hline Maan & 9.39 & 2.30 & 21.72 & 3.40 & 35.85 & 3.60 & 0.71 & 1.60 \\
\hline GROSS BETA & . &. & . &. & . & . &. & \\
\hline Firat Quarter & $13.90^{\circ}$ & 3.40 & 41.30 & 4.60 & 63.60 & 6.00 & 1.55 & $2.7 \dot{0}$ \\
\hline second Quarter & 8.17 & 3.00 & 43.80 & 4.70 & 64.40 & 8.60 & 2.46 & 2.60 \\
\hline Third Quarter & 21.80 & 12.00 & 95.50 & 16.00 & 384.00 & 12.00 & -2.04 & 11.00 \\
\hline Fourth Quarter & 30.50 & 5.10 & -1.47 & 2.70 & 318.00 & 11.00 & 0.23 & 2.80 \\
\hline & & & & . & . & . &. & \\
\hline Mean & 18.59 & 3.50 & 44.78 & 4.40 & 207.50 & 4.80 & 0.55 & 3.00 \\
\hline
\end{tabular}


APPENDIX L

ANNUAL AVERAGE NITRATE ( $\$$ N) CONCENTRATIONS 


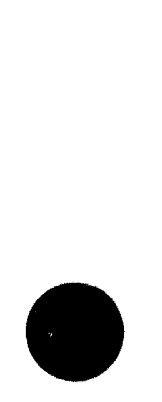

•

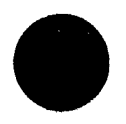




\section{EXPLANATION}

\section{SAMPLING POINT:}

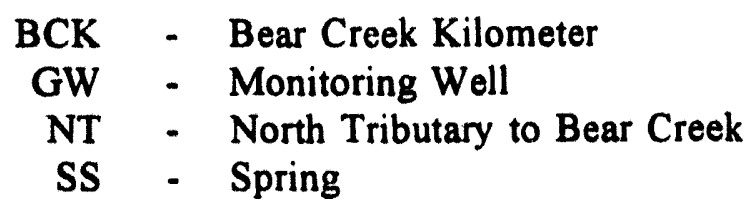

\section{LOCATION:}

AGLLSF - Above Grade Low Level Storage Facility

BG - Bear Creek Burial Grounds WMA

EXP - Exit-Pathway Monitoring

OLF - Oil Landfarm WMA

RS - Rust Spoil Area

S3 - S-3 Site

SPI - Spoil Area I

\section{NOTES:}

All concentrations reported as nitrate (as $\mathrm{N}$ ) in milligrams per Liter (mg/L).

Results reported as less than the detection limit were not used to calculate mean concentrations.

ND - Not detected, annual average

- Not sampled

$<\quad-\quad$ Not detected at the reported minimum attainable detection limit. 
APPENDIX L

Annual Average Nitrate Concentrations, 1993

\begin{tabular}{|c|c|c|c|c|c|c|}
\hline $\begin{array}{l}\text { Sampling } \\
\text { Point }\end{array}$ & Location & $\begin{array}{c}1993 \\
\text { Average }\end{array}$ & First & $\begin{array}{l}\text { terly C } \\
\text { second }\end{array}$ & $\begin{array}{l}\text { ntration } \\
\text { Third }\end{array}$ & $\begin{array}{l}\text { (mg/L) } \\
\text { Fourth }\end{array}$ \\
\hline$G W-013$ & OLF & ND & $<0.2$ & $<0.2$ & $<0.2$ & $<0.2$ \\
\hline$G W-040$ & BG & ND & $<0.2$ & $<0.2$ & $<0.2$ & $<0.2$ \\
\hline$G W-042$ & BG & ND & . & - & - & $<0.2$ \\
\hline GW-043 & OLF & ND & $<0.2$ & $<0.2$ & $<0.2$ & $<0.2$ \\
\hline GW-044 & OLF & ND & $<0.2$ & $<0.2$ & $<0.2$ & $<0.2$ \\
\hline GW-052 & BG & 9.18 & 6.3 & 12.06 & - & • \\
\hline GW-056 & BG & 0.3 & $<0.2$ & 0.3 & $<0.2$ & $<0.2$ \\
\hline$G W-057$ & EXP & 0.28 & 0.28 & 0.27 & $<0.2$ & 0.3 \\
\hline$G W-063$ & OLF & 1.8 & 1.8 & • & . & . \\
\hline GW-064 & OLF & 7.42 & 8.3 & 4.7 & 7.67 & 9 \\
\hline GW-069 & BG & 0.26 & $<0.2$ & $<0.2$ & $<0.2$ & 0.26 \\
\hline GW-079 & BG & ND & • & • & . & $<0.2$ \\
\hline$G W-080$ & BG & ND & • & - & - & $<0.2$ \\
\hline$G W-082$ & BG & ND & $<0.2$ & $<0.2$ & $<0.2$ & $<0.2$ \\
\hline$G W-084$ & OLF & ND & $<0.2$ & $<0.2$ & $<0.2$ & $<0.2$ \\
\hline GW-085 & OLF & 748.9 & 2139 & 240 & 304 & 312.6 \\
\hline$G W-086$ & OLF & 4.54 & 4.5 & 3.1 & 4.97 & 5.6 \\
\hline GW-115 & s3 & ND & $<0.2$ & $<0.2$ & $<0.2$ & $<0.2$ \\
\hline$G W-118$ & BG & ND & $<23$ & $<0.2$ & $<0.2$ & $<22.6$ \\
\hline$G W-162$ & BG & ND & . & • & . & $<0.2$ \\
\hline$G W-286$ & BG & ND & $<0.2$ & $<0.2$ & $<0.2$ & $<0.2$ \\
\hline$G W-287$ & BG & ND & $<0.2$ & $<0.2$ & $<0.2$ & $<0.2$ \\
\hline GW-311 & RS & 0.49 & 0.42 & 0.5 & 0.54 & 0.5 \\
\hline GW-312 & RS & 0.24 & $<0.2$ & 0.26 & 0.26 & 0.2 \\
\hline$G W-315$ & SPI & 12.88 & 7.8 & 8.9 & 15 & 19.8 \\
\hline$G W-316$ & SPI & 0.36 & 0.46 & 0.33 & 0.3 & 0.35 \\
\hline$G W-317$ & SPI & 0.23 & 0.26 & $<0.2$ & $<0.2$ & 0.2 \\
\hline$G W-323$ & SPI & ND & $<0.2$ & $<0.2$ & $<0.2$ & $<0.2$ \\
\hline GW-324 & s3 & ND & $<0.2$ & $<0.2$ & $<0.2$ & $<0.2$ \\
\hline GW-325 & s3 & ND & $<0.2$ & $<0.2$ & $<0.2$ & $<0.2$ \\
\hline$G W-342$ & BG & ND & . & . & . & $<0.2$ \\
\hline$G W-345$ & s3 & 24.28 & 7.7 & 3.4 & 20 & 66 \\
\hline$G W-347$ & s3 & ND & $<0.2$ & $<0.2$ & $<0.2$ & $<0.2$ \\
\hline$G W-348$ & s3 & 9.75 & 9.73 & 8.5 & 9.35 & 11.4 \\
\hline$G W-364$ & OLF & 3.83 & 1.21 & 1.12 & 10.2 & 2.8 \\
\hline$G W-365$ & OLF & 4 & 2.9 & 4.04 & 5.17 & 3.9 \\
\hline$G W-370$ & BG & ND & $<0.2$ & $<0.2$ & $<0.2$ & $<0.2$ \\
\hline GW-371 & BG & ND & $<0.2$ & $<0.2$ & $<0.2$ & $<0.2$ \\
\hline GW-372 & BG & ND & $<0.2$ & $<0.2$ & $<0.2$ & $<0.2$ \\
\hline$G W-373$ & BG & ND & $<0.2$ & $<0.2$ & $<0.2$ & $<0.2$ \\
\hline$G W-526$ & s3 & 962.25 & 826 & 1006 & 846 & 1171 \\
\hline$G W-537$ & OLF & 697.65 & 782 & 692 & 525 & 791.6 \\
\hline$G W-601$ & OLF & 34.03 & 32.1 & 41.6 & 30.5 & 31.9 \\
\hline GW-613 & s3 & ND & . & • & • & $<0.2$ \\
\hline$G W-614$ & s3 & ND & . & • & - & $<0.2$ \\
\hline
\end{tabular}


APPENDIX I

Annual Average Nitrate Concentrations, 1993

\begin{tabular}{|c|c|c|c|c|c|c|}
\hline \multirow{2}{*}{$\begin{array}{l}\text { Sampling } \\
\text { Point }\end{array}$} & \multirow[b]{2}{*}{ Location } & \multirow{2}{*}{$\begin{array}{c}1993 \\
\text { Average }\end{array}$} & \multicolumn{3}{|c|}{ Quarterly concentration } & \multirow{2}{*}{$\begin{array}{l}\text { (mg/I ) } \\
\text { Fourth }\end{array}$} \\
\hline & & & First & second & Third & \\
\hline GW-621 & BG & 6.14 & 4.45 & 6 & 10.12 & 4 \\
\hline GW-626 & BG & ND & $<0.2$ & $<0.2$ & $<0.2$ & $<0.2$ \\
\hline$G W-627$ & BG & 0.2 & $<0.2$ & $<0.2$ & 0.2 & $<0.2$ \\
\hline$G W-636$ & OLF & ND & $<0.2$ & $<0.2$ & $<0.2$ & $<0.2$ \\
\hline GW-637 & OLF & ND & $<0.2$ & $<0.2$ & $<0.2$ & $<0.2$ \\
\hline$G W-638$ & OLF & 0.34 & 0.23 & 0.38 & 0.4 & $<0.2$ \\
\hline GW-639 & BG & ND & $<0.2$ & $<0.2$ & $<0.2$ & $<0.2$ \\
\hline$G W-640$ & BG & ND & $<0.2$ & $<0.2$ & $<0.2$ & $<0.2$ \\
\hline$G W-641$ & BG & ND & $<0.2$ & $<0.2$ & $<0.2$ & $<0.2$ \\
\hline$G W-642$ & BG & ND & $<0.2$ & $<0.2$ & $<0.2$ & $<0.2$ \\
\hline GW-643 & BG & 0.29 & 0.29 & $<0.2$ & $<0.2$ & $<0.2$ \\
\hline$G W-644$ & OLF & ND & $<0.2$ & $<0.2$ & $<0.2$ & $<0.2$ \\
\hline GW-647 & OLF & ND & $<0.2$ & $<0.2$ & $<0.2$ & $<0.2$ \\
\hline$G W-651$ & BG & ND & $<0.2$ & $<0.2$ & $<0.2$ & $<0.2$ \\
\hline$G W-652$ & BG & 0.45 & $<0.2$ & $<0.2$ & $<0.2$ & 0.45 \\
\hline$G W-653$ & BG & ND & $<0.2$ & $<0.2$ & $<0.2$ & $<0.2$ \\
\hline$G W-654$ & BG & ND & $<0.2$ & $<0.2$ & $<0.2$ & $<0.2$ \\
\hline GW-655 & BG & ND & $<0.2$ & $<0.2$ & $<0.2$ & $<0.2$ \\
\hline$G W-683$ & EXP & 11.75 & 2.7 & 2.3 & 10.01 & 32 \\
\hline$G W-684$ & EXP & 9.4 & 4.3 & 4.1 & 10.3 & 18.9 \\
\hline$G W-685$ & EXP & 2.36 & 1.7 & 1.63 & 2.1 & 4 \\
\hline$G W-694$ & EXP & 24.86 & 10 & 29.25 & 31 & 29.2 \\
\hline GW-695 & EXP & 8.9 & 5.7 & 16 & 6.2 & 7.7 \\
\hline GW-703 & EXP & 19.09 & 22.36 & 9.6 & 21.8 & 22.6 \\
\hline GW-704 & EXP & 11.63 & 14.28 & 15 & 17 & 0.23 \\
\hline GW-706 & EXP & 30.45 & $<0.2$ & 32 & $<0.2$ & 28.9 \\
\hline GW-710 & EXP & 74.9 & $<11.3$ & 74.9 & $<0.2$ & $<0.2$ \\
\hline GW-711 & EXP & ND & $<11.3$ & $<23$ & $<2$ & -22.59 \\
\hline GW-712 & EXP & 0.21 & $<0.2$ & 0.24 & 0.2 & 0.2 \\
\hline GW-713 & EXP & 1.4 & $<0.2$ & 1.4 & $<0.2$ & $<0.2$ \\
\hline GW-714 & EXP & 4.05 & 4.16 & 4.8 & 4.03 & 3.2 \\
\hline GW-715 & EXP & 0.8 & 0.6 & 0.82 & 1.1 & 0.66 \\
\hline GW-723 & EXP & 11.65 & 20 & 6.39 & 9.6 & 10.6 \\
\hline$G W-724$ & EXP & 48.88 & 62 & 37 & 50.1 & 46.4 \\
\hline GW-725 & EXP & 53.03 & 61 & 55 & 49.9 & 46.2 \\
\hline$G W-736$ & EXP & 47.3 & 61 & 42 & 43.8 & 42.4 \\
\hline GW-737 & EXP & 47.05 & 57 & 37 & 49.9 & 44.3 \\
\hline$G W-738$ & EXP & 25.55 & 22 & 15 & 40.4 & 24.8 \\
\hline GW-739 & EXP & 7.23 & 7.4 & 4.17 & 8.6 & 8.76 \\
\hline$G W-740$ & EXP & 4.1 & 3.5 & 3.32 & 4.8 & 4.78 \\
\hline GW-793 & AGLLSF & ND & $<0.2$ & $<0.2$ & $<0.2$ & $<0.2$ \\
\hline GW-794 & AGLLSF & 0.34 & $<0.2$ & $<0.2$ & 0.3 & 0.37 \\
\hline GW-795 & AGLISF & 5.2 & $<0.2$ & $<0.2$ & $<0.2$ & 5.2 \\
\hline$G W-800$ & OLF & ND & . & $<0.2$ & $<0.2$ & $<0.2$ \\
\hline ss-1 & EXP & 68.03 & 31 & 142 & 44.2 & 54.9 \\
\hline
\end{tabular}


APPENDIX I

Annual Average Nitrate Concentrations, 1993

\begin{tabular}{|c|c|c|c|c|c|c|}
\hline $\begin{array}{l}\text { Sampling } \\
\text { Point }\end{array}$ & Location & \multirow{2}{*}{$\begin{array}{c}1993 \\
\text { Average }\end{array}$} & \multicolumn{3}{|c|}{ Quarterly concentration } & $\begin{array}{l}\text { (mg/L) } \\
\text { Fourth }\end{array}$ \\
\hline ss -4 & EXP & & 9.3 & 15 & 41.3 & 67.6 \\
\hline ss-5 & $\mathbf{E X P}$ & 11.75 & 2.5 & 4.5 & 15.2 & 24.8 \\
\hline ss-6 & EXP & 37.8 & $<0.2$ & $<0.2$ & $<0.2$ & 37.8 \\
\hline ss-8 & EXP & 0.55 & 0.36 & 0.32 & 1.2 & 0.3 \\
\hline $\mathrm{BCK}-00.63$ & EXP & 4.8 & 2 & 2.2 & 5.1 & 9.9 \\
\hline BCK -04.55 & EXP & 5.38 & 2.3 & 2.6 & 6.3 & 10.3 \\
\hline BCK -09.40 & EXP & 14.27 & 11 & 13 & 18.8 & $<0.2$ \\
\hline NT -01 & $\operatorname{ExP}$ & 172.03 & 112 & 31 & 286.1 & 259 \\
\hline NT-13 & $\operatorname{ExP}$ & ND & $<0.2$ & $<0.2$ & $<0.2$ & $<0.2$ \\
\hline
\end{tabular}



APPENDIX M

GROUNDWATER ELEVATIONS 


\section{EXPLANATION}

\section{LOCATION:}

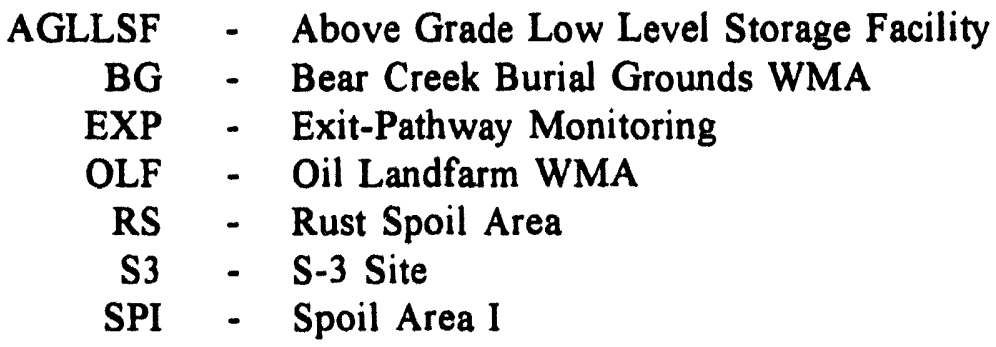

\section{SAMPLED IN 93:}

Y - Wells included in the 1993 groundwater monitoring program.

\section{NOTES:}

CTP Elev. - The elevation of the centerpoint of the monitored interval.

January - Measuring event from January 10 to 14, 1993.

August - Measuring event from August 31 to September 3, 1993.

Depth - The distance in $\mathrm{ft}$ from the top-of-casing measuring point to the static water level.

Elev. - The groundwater elevation in $\mathrm{ft}$ above mean sea level.

+/ Ft - The calculated drop in water level from the January to the August measuring events. Negative results show a rise in water level.

- No information available. 
APPENDIX $M$

Groundwater Elevations, 1993

\begin{tabular}{|c|c|c|c|c|c|c|c|}
\hline 8 ampl1ng & & sampled & CTP & January & & t & $+1-$ \\
\hline Pt. & Location & $\ln 93$ & Elev. & Depth & Depth & slev. & $\mathbf{F t}$ \\
\hline
\end{tabular}

Water Table Interval

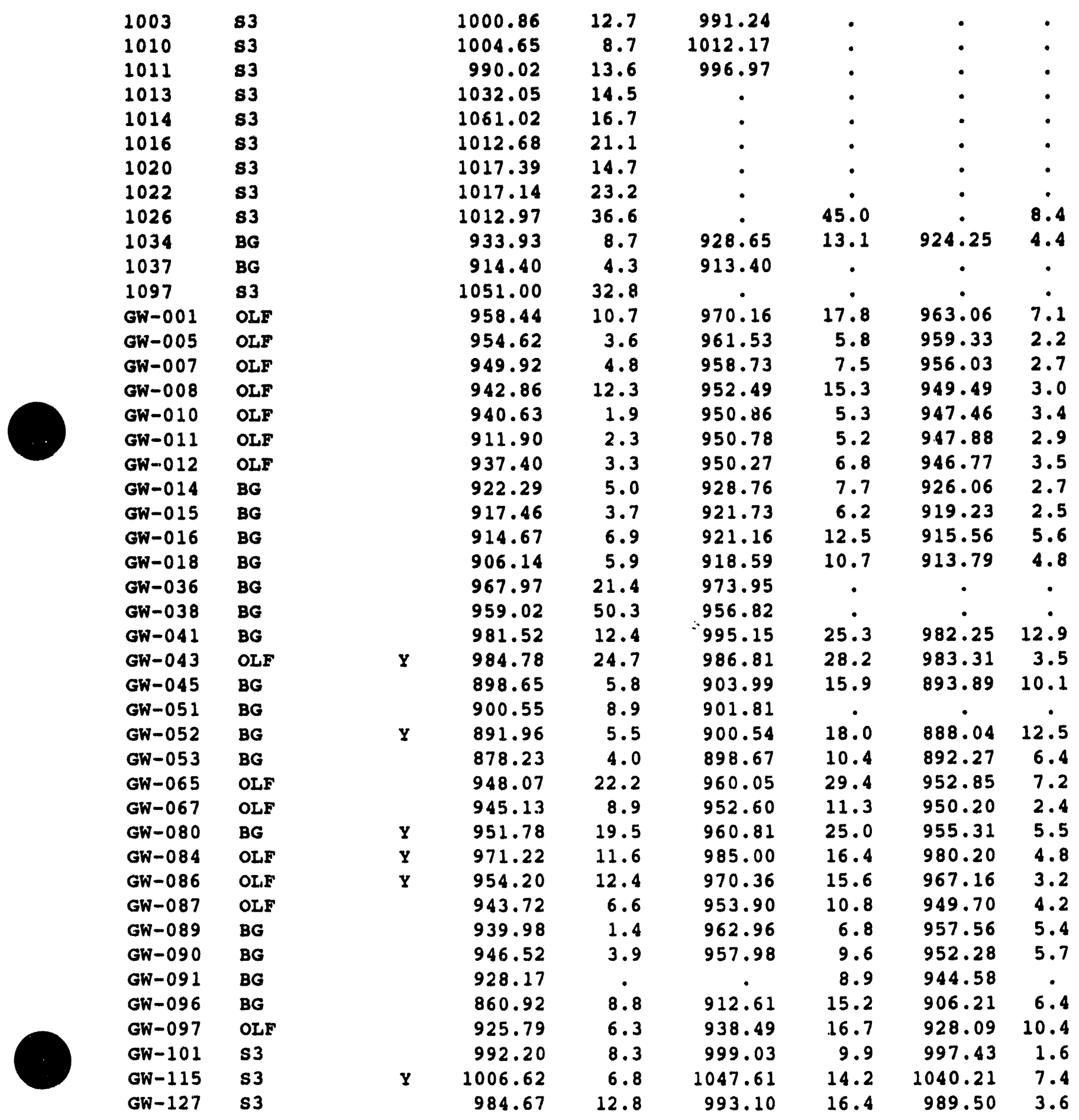


APPENDIX $M$

Oroundwater Elevations, 1993

\begin{tabular}{|c|c|c|c|c|c|c|c|c|}
\hline \multirow{2}{*}{$\begin{array}{c}\text { sampling } \\
\text { pt. }\end{array}$} & \multirow[b]{2}{*}{ Location } & \multirow{2}{*}{$\begin{array}{c}\text { sampled } \\
\text { in } 93\end{array}$} & \multirow{2}{*}{$\begin{array}{l}\text { CTP } \\
\text { Elov. }\end{array}$} & \multicolumn{2}{|c|}{ January } & \multicolumn{2}{|c|}{ Auguet } & \multirow{2}{*}{$+1-$} \\
\hline & & & & Dopth & Elev. & Depth & Elov. & \\
\hline Oพ-133 & 83 & & 695.57 & 80.8 & 945.06 & 80.4 & 945.46 & -0.4 \\
\hline OW-136 & OBR & & 436.47 & 3.0 & 856.41 & 7.6 & 851.81 & 4.6 \\
\hline GW -237 & $B G$ & & • & 9.0 & • & 16.1 & . & 7.1 \\
\hline OW-243 & 83 & & 948.48 & 14.4 & 997.15 & 14.9 & 996.65 & 0.5 \\
\hline$G W-244$ & 83 & & 946.93 & 12.3 & 996.95 & 14.8 & 994.45 & 2.5 \\
\hline$G W-245$ & 83 & & 955.41 & 12.8 & 996.28 & 15.0 & 994.08 & 2.2 \\
\hline$O W-246$ & 83 & & 950.97 & 12.9 & 996.29 & 15.0 & 994.19 & 2.1 \\
\hline Gw-249 & $\mathbf{B G}$ & & 956.82 & 21.7 & 969.45 & . & - & - \\
\hline$G W-257$ & BC & & 932.86 & 25.7 & 935.98 & . & - & . \\
\hline$G W-276$ & 83 & & 983.90 & 5.4 & 995.87 & 8.4 & 992.87 & 3.0 \\
\hline Gw-277 & 83 & & 929.25 & 4.9 & 996.86 & 7.6 & 994.16 & 2.7 \\
\hline OW-287 & BG & $\mathbf{Y}$ & 915.55 & 8.2 & 918.67 & 9.6 & 917.27 & 1.4 \\
\hline Gw-289 & BG & & 911.47 & 7.7 & 940.77 & . & - & - \\
\hline OW-291 & BG & & 931.89 & 6.4 & 941.96 & 11.9 & 936.46 & 5.5 \\
\hline $6 w-307$ & $\mathbf{R s}$ & & 955.86 & 26.5 & 966.64 & 35.1 & 958.04 & 8.6 \\
\hline GW-308 & RS & & 961.27 & 27.1 & 966.70 & 37.1 & 956.70 & 10.0 \\
\hline GW-309 & $\mathbf{R s}$ & & 954.27 & 19.9 & 968.27 & 24.0 & 964.17 & 4.1 \\
\hline GW-310 & $\mathbf{R s}$ & & 969.10 & 19.7 & 975.65 & 22.4 & 972.95 & 2.7 \\
\hline GN-311 & $\mathbf{R B}$ & $\mathbf{Y}$ & 963.48 & 31.0 & 968.35 & 41.6 & 957.75 & 10.6 \\
\hline$G W-312$ & $\mathbf{R s}$ & $\mathbf{Y}$ & 958.83 & 30.0 & 966.49 & 39.4 & 957.09 & 9.4 \\
\hline$G W-316$ & SPI & $\mathbf{Y}$ & 970.98 & 49.5 & 997.67 & 62.7 & 984.47 & 13.2 \\
\hline GW-323 & SPI & $\mathbf{Y}$ & 1026.41 & 85.7 & 1044.41 & 86.1 & 1044.01 & 0.4 \\
\hline GW-325 & $\mathbf{s 3}$ & $\mathbf{Y}$ & 989.03 & 7.2 & 995.80 & 18.3 & 984.70 & 11.1 \\
\hline$G W-345$ & 83 & $\mathbf{Y}$ & 974.18 & 17.7 & 981.76 & 20.5 & 978.96 & 2.8 \\
\hline GW-347 & 83 & $\mathbf{Y}$ & 979.11 & 16.7 & 984.86 & 19.6 & 981.96 & 2.9 \\
\hline$G W-370$ & $\mathbf{B G}$ & $\mathbf{x}$ & 930.84 & 12.0 & 948.62 & 15.8 & 944.82 & 3.8 \\
\hline$G W-372$ & BG & $\mathbf{Y}$ & 934.17 & 15.9 & 967.63 & 26.5 & 957.03 & 10.6 \\
\hline$O W-443$ & GBR & & 784.00 & 5.5 & 823.87 & 10.3 & 819.07 & 4.8 \\
\hline$G W-445$ & GBR & & 802.15 & 5.4 & 841.13 & 8.6 & 837.93 & 3.2 \\
\hline$G W-531$ & LD & & 971.94 & 13.6 & 991.01 & 19.7 & 984.91 & 6.1 \\
\hline GW-532 & LD & & 974.30 & 13.6 & 983.30 & 16.2 & 980.70 & 2.6 \\
\hline GW -533 & LD & & 977.36 & 12.3 & 991.52 & 18.6 & 985.22 & 6.3 \\
\hline GW-537 & OLF & $\mathbf{Y}$ & 960.14 & 5.7 & 970.54 & 8.2 & 968.04 & 2.5 \\
\hline$G W-538$ & LD & & 960.12 & 17.0 & 979.89 & 19.0 & 977.89 & 2.0 \\
\hline GW-613 & $\mathbf{s 3}$ & $\mathbf{Y}$ & 974.17 & 9.8 & 1003.57 & 16.5 & 996.87 & 6.7 \\
\hline$G W-621$ & BG & $\mathbf{y}$ & 891.17 & 8.7 & 916.54 & 21.2 & 904.04 & 12.5 \\
\hline$G W-622$ & BG & & 907.89 & 6.3 & 917.86 & 14.4 & 909.76 & 8.1 \\
\hline$G W-624$ & BG & & 898.72 & 4.8 & 917.16 & 12.2 & 909.76 & 7.4 \\
\hline$G W-626$ & BG & $\mathbf{Y}$ & 869.45 & 25.4 & 917.21 & 27.7 & 914.91 & 2.3 \\
\hline$G W-637$ & OLF & $\mathbf{Y}$ & 917.26 & 5.7 & 936.13 & 15.2 & 926.63 & 9.5 \\
\hline GW-638 & OLF & $\mathbf{Y}$ & 931.14 & 5.7 & 936.07 & 13.8 & 927.97 & 8.1 \\
\hline GW-641 & BG & $\mathbf{Y}$ & 926.36 & 16.5 & 930.16 & 22.3 & 924.36 & 5.8 \\
\hline$G W-642$ & BG & $x$ & 984.23 & - & . & 23.2 & 991.47 & . \\
\hline$G W-644$ & OLF & $\mathbf{Y}$ & 939.93 & 7.6 & 952.15 & 12.4 & 947.35 & 4.8 \\
\hline$G W-645$ & OLF & & 931.80 & 69.4 & 937.00 & 79.8 & 926.60 & 10.4 \\
\hline$G W-648$ & RS & & 953.93 & 62.7 & 966.47 & 71.2 & 957.97 & 8.5 \\
\hline$G W-649$ & OLF & & 966.46 & 6.8 & 976.86 & 10.8 & 972.86 & 4.0 \\
\hline
\end{tabular}


APPENDIX $M$

oroundwater Elevations, 1993

\begin{tabular}{|c|c|c|c|c|c|c|c|c|}
\hline \multirow{2}{*}{$\begin{array}{l}\text { sampling } \\
\text { Pt. }\end{array}$} & \multirow[b]{2}{*}{ Location } & \multirow{2}{*}{$\begin{array}{l}\text { samplod } \\
\text { in } 93\end{array}$} & \multirow{2}{*}{$\begin{array}{l}\text { CTP } \\
\text { alev. }\end{array}$} & \multicolumn{2}{|c|}{ January } & \multicolumn{2}{|c|}{ Auguat } & \multirow{2}{*}{$+1-$} \\
\hline & & & & Dopth & Elev. & Depth & Elov. & \\
\hline $0 x-651$ & BO & $\mathbf{Y}$ & 854.15 & 4.4 & 898.72 & 10.9 & 892.22 & 6.5 \\
\hline ON-652 & BO & 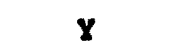 & 875.68 & 8.4 & 892.43 & 12.2 & 888.63 & 3.8 \\
\hline OW-653 & $B C$ & $\mathbf{y}$ & 896.20 & 20.4 & 911.20 & 24.5 & 907.10 & 4.1 \\
\hline ON-654 & $B C$ & $\boldsymbol{y}$ & 928.51 & 6.2 & 934.59 & 9.3 & 931.49 & 3.1 \\
\hline ow-715 & $\mathbf{E X P}$ & $\mathbf{y}$ & 834.17 & 26.0 & 848.72 & 30.0 & 844.72 & 4.0 \\
\hline OW-795 & ACLL8F & $\mathbf{Y}$ & 909.12 & - & - & 11.5 & 914.48 & - \\
\hline
\end{tabular}

Bedrock Interval

\begin{tabular}{|c|c|c|c|c|c|c|c|c|}
\hline $\begin{array}{l}1033 \\
42-D C\end{array}$ & $\begin{array}{l}B C \\
G B R\end{array}$ & & $\begin{array}{l}813.28 \\
870.65\end{array}$ & $\begin{array}{r}7.4 \\
27.4\end{array}$ & $\begin{array}{c}942.79 \\
.\end{array}$ & 33.6 & : & 6.2 \\
\hline ow-002 & OLF & & 928.91 & 9.2 & 973.22 & 16.8 & 965.62 & 7.6 \\
\hline OW-037 & $B C$ & & 946.97 & 41.1 & 964.50 & • & . & - \\
\hline ON-044 & OLF & $x$ & 954.05 & 26.6 & 986.89 & 30.4 & 983.09 & 3.8 \\
\hline OW-054 & $\mathbf{B G}$ & & 855.66 & 8.9 & 884.43 & 11.7 & 881.63 & 2.8 \\
\hline $6 x-055$ & BG & & 871.71 & 14.8 & 877.73 & 12.3 & 880.23 & -2.5 \\
\hline OW-056 & BC & $\mathbf{y}$ & 834.50 & 3.1 & 887.61 & 8.3 & 882.41 & 5.2 \\
\hline OH -057 & $\operatorname{ExP}$ & $\mathbf{y}$ & 866.68 & 3.7 & 885.66 & 6.8 & 882.56 & 3.1 \\
\hline GW-058 & BO & & 867.77 & 18.5 & 894.46 & 25.9 & 887.06 & 7.4 \\
\hline OW-059 & BG & & 887.72 & 17.9 & 894.58 & 25.0 & 887.48 & 7.1 \\
\hline GW-060 & BG & & 856.61 & 11.1 & 893.31 & 18.0 & 886.41 & 6.9 \\
\hline GW-061 & BG & & 879.73 & 11.2 & 893.24 & 16.0 & 888.44 & 4.8 \\
\hline ow-062 & OLF & & 911.23 & 25.9 & 936.26 & 32.3 & 929.86 & 6.4 \\
\hline GW-063 & OLF & $\mathbf{y}$ & 928.69 & 25.8 & 936.13 & - & - & - \\
\hline GW-064 & OLF & $\mathbf{Y}$ & 929.42 & 21.9 & 959.66 & 28.1 & 953.46 & 6.2 \\
\hline GW-066 & OLF & & 904.87 & 7.3 & 954.42 & 11.4 & 950.32 & 4.1 \\
\hline GW-069 & BG & $\mathbf{Y}$ & 835.15 & 7.5 & 919.51 & 11.0 & 916.01 & 3.5 \\
\hline GW-070 & BG & & 793.36 & 7.0 & 919.39 & 9.1 & 917.29 & 2.1 \\
\hline GW-071 & BG & & 718.42 & 6.6 & 921.82 & 8.9 & 919.52 & 2.3 \\
\hline GW-072 & BG & & 834.97 & 9.3 & 920.61 & 12.3 & 917.61 & 3.0 \\
\hline GW-075 & OLF & & 774.05 & 2.0 & 962.31 & 4.4 & 959.91 & 2.4 \\
\hline GW-076 & OLF & & 888.53 & 3.3 & 962.71 & 5.4 & 960.61 & 2.1 \\
\hline GW-077 & BG & & 820.57 & 4.8 & 913.59 & 11.5 & 906.89 & 6.7 \\
\hline$G W-078$ & BC & & 897.98 & 3.7 & 913.63 & 10.3 & 907.03 & 6.6 \\
\hline GW-079 & $B G$ & $\mathbf{Y}$ & 919.74 & 18.1 & 962.40 & 24.6 & 955.90 & 6.5 \\
\hline GW-082 & BG & $\mathbf{Y}$ & 927.59 & 19.4 & 943.09 & 20.3 & 942.19 & 0.9 \\
\hline GW-083 & BG & & 909.49 & 9.5 & 928.33 & 14.0 & 923.83 & 4.5 \\
\hline$G W-085$ & OLF & $\mathbf{Y}$ & 926.22 & 12.4 & 970.47 & 15.5 & 967.37 & 3.1 \\
\hline GW-094 & BG & & 808.51 & 6.1 & 907.02 & 11.2 & 901.92 & 5.1 \\
\hline GW-095 & BG & & 764.88 & 2.6 & 908.27 & 8.5 & 902.37 & 5.9 \\
\hline GW-098 & OLF & & 851.54 & 10.4 & 934.70 & 21.9 & 923.20 & 11.5 \\
\hline GW-112 & GC & & 640.88 & 23.2 & 854.28 & 29.8 & 847.68 & 6.6 \\
\hline$G W-113$ & GC & & 726.57 & 20.7 & 854.09 & 43.9 & 830.89 & 23.2 \\
\hline GW-114 & BCR & & 720.06 & 23.2 & 805.07 & 26.8 & 801.47 & 3.6 \\
\hline$G W-118$ & BG & $\mathbf{Y}$ & 359.37 & 14.0 & 898.39 & 4.7 & 907.69 & -9.3 \\
\hline$G W-119$ & BG & & 433.12 & 2.0 & 919.91 & - & - & - \\
\hline$G W-120$ & OLF & & 789.19 & 3.4 & 943.87 & 9.5 & 937.77 & 6.1 \\
\hline
\end{tabular}


APPENDIX $M$

Groundwater Elevations, 1993

\begin{tabular}{|c|c|c|c|c|c|c|c|c|}
\hline \multirow{2}{*}{$\begin{array}{l}\text { sampling } \\
\text { pt. }\end{array}$} & \multirow[b]{2}{*}{ Location } & \multirow{2}{*}{$\begin{array}{c}\text { sampled } \\
\text { in } 93\end{array}$} & \multirow{2}{*}{$\begin{array}{l}\text { CTP } \\
\text { slov. }\end{array}$} & \multicolumn{2}{|c|}{ January } & \multicolumn{2}{|c|}{ auguat } & \multirow{2}{*}{$+1-$} \\
\hline & & & & Depth & Elev. & Depth & Elev. & \\
\hline$O N-122$ & 83 & & 887.15 & 12.6 & 994.60 & 20.6 & 986.60 & 8.0 \\
\hline$O N-123$ & 83 & & 457.43 & 9.1 & 998.35 & 4.9 & 1002.55 & -4.2 \\
\hline$a n-124$ & 83 & & 878.51 & 8.9 & 997.88 & 22.2 & 984.58 & 13.3 \\
\hline$O W-125$ & 83 & & 476.98 & 4.9 & 1001.95 & 2.8 & 1004.05 & -2.1 \\
\hline$O W-162$ & 80 & $\mathbf{X}$ & 929.56 & 18.8 & 1021.64 & 21.8 & 1018.64 & 3.0 \\
\hline$O W-211$ & $\mathbf{E X P}$ & & 386.14 & 10.2 & 786.06 & - & - & • \\
\hline$O w-225$ & OLF & & 765.21 & 8.0 & 935.11 & 22.0 & 921.11 & 14.0 \\
\hline$O W-226$ & orf & & 890.56 & 7.5 & 935.90 & 22.1 & 921.30 & 14.6 \\
\hline$O H-227$ & OrF & & 908.91 & 7.8 & $9 \mathrm{~J} 8.66$ & 19.4 & 927.06 & 11.6 \\
\hline$O W-228$ & orf & & 853.85 & 9.1 & 937.37 & 22.0 & 924.47 & 12.9 \\
\hline on-229 & or: & & 898.21 & 5.5 & 943.50 & 32.8 & 916.20 & 27.3 \\
\hline ON-248 & BC & & 903.27 & 25.2 & 936.05 & - & - & • \\
\hline$O N-250$ & $B O$ & & 932.80 & 24.9 & 966.35 & - & - & - \\
\hline$O W-286$ & $\mathbf{B C}$ & $\mathbf{y}$ & 898.62 & 8.9 & 917.93 & 10.4 & 916.43 & 1.5 \\
\hline$G W-288$ & $B G$ & & 893.07 & 7.2 & 941.16 & . & - & - \\
\hline CN-290 & $B G$ & & 916.46 & 5.2 & 943.26 & 9.2 & 939.26 & 4.0 \\
\hline ON-306 & $R 8$ & & 937.09 & 29.7 & 962.08 & 37.7 & 954.08 & 8.0 \\
\hline$C W-313$ & BPI & & 944.77 & 63.4 & 989.12 & 75.9 & 976.62 & 12.5 \\
\hline OW -314 & 8PI & & 939.20 & 53.2 & 996.61 & 65.9 & 983.91 & 12.7 \\
\hline$G W-315$ & SPI & $\mathbf{Y}$ & 947.84 & 50.0 & 997.28 & 63.2 & 984.08 & 13.2 \\
\hline$O N-317$ & SPI & $\mathbf{y}$ & 936.30 & 66.8 & 996.18 & 79.3 & 983.68 & 12.5 \\
\hline$O W-324$ & 83 & $\mathbf{y}$ & 928.19 & 5.9 & 997.93 & 18.3 & 985.53 & 12.4 \\
\hline$C N-346$ & 83 & & 936.88 & 17.0 & 978.65 & 18.6 & 977.05 & 1.6 \\
\hline$O W-348$ & 83 & $\mathbf{y}$ & 923.50 & 19.9 & 980.22 & 26.1 & 974.02 & 6.2 \\
\hline ow-363 & OLF & & 892.91 & 4.6 & 953.31 & 6.8 & 951.11 & 2.2 \\
\hline$O N-364$ & oLf & $\mathbf{x}$ & 879.74 & 7.4 & 928.55 & 24.4 & 911.55 & 17.0 \\
\hline GW-365 & OLF & $\mathbf{y}$ & 794.68 & 4.1 & 931.48 & 17.4 & 918.18 & 13.3 \\
\hline ON-366 & orf & & 891.16 & 56.3 & 932.49 & 74.0 & 914.79 & 17.7 \\
\hline $6 N-367$ & OLF & & 848.71 & 58.8 & 929.36 & 75.5 & 912.66 & 16.7 \\
\hline OW-368 & OLF & & 763.63 & 62.5 & 938.03 & 73.9 & 926.63 & 11.4 \\
\hline$G W-369$ & OLF & & 864.86 & 66.8 & 932.92 & 82.6 & 917.12 & 15.8 \\
\hline OW-371 & $\mathbf{B C}$ & $\mathbf{Y}$ & 840.63 & 15.8 & 944.42 & 18.7 & 941.52 & 2.9 \\
\hline GW -373 & $B C$ & $\mathbf{Y}$ & 840.25 & 18.6 & 964.20 & 20.3 & 962.50 & 1.7 \\
\hline$G W-375$ & BG & & 776.23 & 5.7 & 915.13 & 10.7 & 910.13 & 5.0 \\
\hline CW-520 & OLF & & 911.03 & 56.1 & 931.28 & 67.5 & 919.88 & 11.4 \\
\hline ON-526 & 83 & $\mathbf{Y}$ & 883.34 & 11.6 & 985.85 & 13.7 & 983.75 & 2.1 \\
\hline$G W-534$ & LD & & 959.61 & 17.8 & 983.05 & 20.7 & 980.15 & 2.9 \\
\hline$G W-601$ & OLF & $y$ & 661.84 & 61.7 & 940.50 & 73.2 & 929.00 & 11.5 \\
\hline GW-602 & OLF & & 870.76 & 76.6 & 1001.17 & 79.7 & 998.07 & 3.1 \\
\hline GW-614 & 83 & $\mathbf{Y}$ & 928.49 & 4.2 & 1008.02 & 9.7 & 1002.52 & 5.5 \\
\hline CW-615 & 53 & & 780.42 & 11.6 & 1005.15 & 13.6 & 1003.15 & 2.0 \\
\hline CW-616 & 83 & & 765.81 & 9.2 & 1002.61 & 10.9 & 1000.91 & 1.7 \\
\hline$G W-623$ & BC & & 665.86 & 3.9 & 921.31 & 5.9 & 919.31 & 2.0 \\
\hline GW-627 & BG & $\mathbf{Y}$ & 678.39 & 23.5 & 919.35 & 25.3 & 917.55 & 1.8 \\
\hline CW-629 & BG & & 637.27 & 4.4 & 922.76 & 6.2 & 920.96 & 1.8 \\
\hline$O W-636$ & OLF & $\mathbf{Y}$ & 830.33 & 1.9 & 940.02 & 10.6 & 931.32 & 8.7 \\
\hline GW-639 & BG & $\mathbf{Y}$ & 827.48 & 10.3 & 930.40 & 13.7 & 927.00 & 3.4 \\
\hline
\end{tabular}


APPENDIX $\mathrm{H}$

Groundwater Elovatıone, 1993

\begin{tabular}{|c|c|c|c|c|c|c|c|c|}
\hline \multirow{2}{*}{$\begin{array}{l}\text { sampling } \\
\text { pt. }\end{array}$} & \multirow[b]{2}{*}{ Location } & \multirow{2}{*}{$\begin{array}{c}\text { sampled } \\
\text { In } 93\end{array}$} & \multirow{2}{*}{$\begin{array}{l}\text { CTP } \\
\text { Elov. }\end{array}$} & \multicolumn{2}{|c|}{ January } & \multicolumn{2}{|c|}{ Auguet } & \multirow{2}{*}{$+1-$} \\
\hline & & & & Depth & Elov. & Dopth & Elev. & \\
\hline$O N-640$ & BO & $\boldsymbol{y}$ & 901.39 & 15.1 & 930.28 & 21.3 & 924.08 & 6.2 \\
\hline$O N-646$ & OLF & & 932.41 & 39.7 & 964.76 & 40.6 & 963.86 & 0.9 \\
\hline ON-647 & ory & $\mathbf{Y}$ & 945.64 & 68.8 & 964.47 & 69.2 & 964.07 & 0.4 \\
\hline$O N-683$ & $\mathbf{E x P}$ & $y$ & 804.10 & 88.4 & 883.66 & 88.9 & 883.16 & 0.5 \\
\hline ON-684 & $\operatorname{ExP}$ & $y$ & 778.13 & 15.1 & 883.56 & 15.7 & 882.96 & 0.6 \\
\hline OW-685 & $\operatorname{ExP}$ & $y$ & 775.88 & 6.9 & 884.61 & 8.8 & 882.71 & 1.9 \\
\hline$O M-694$ & $\mathbf{E x P}$ & $y$ & 759.33 & 23.0 & 918.38 & 31.4 & 909.98 & 8.4 \\
\hline OW-695 & $\operatorname{ExP}$ & $x$ & 880.62 & 23.1 & 916.27 & 31.6 & 907.77 & 8.5 \\
\hline OH-703 & $\mathbf{E x P}$ & $\mathbf{x}$ & 793.30 & 38.1 & 916.59 & 26.6 & 928.09 & $-11 \cdot 5$ \\
\hline ON-704 & $\mathbf{E x P}$ & $\mathbf{Y}$ & 690.99 & 27.2 & 917.53 & 37.3 & 907.43 & 10.1 \\
\hline ow-70s & $\mathbf{E x P}$ & & 652.96 & 160.7 & 767.77 & 32.8 & 895.67 & -128 \\
\hline Ow-706 & EXP & $\mathbf{y}$ & 756.03 & 10.6 & 918.07 & 19.0 & 909.67 & 8.4 \\
\hline ON-710 & $\operatorname{Exp}$ & $\mathbf{y}$ & 265.93 & 63.5 & 847.76 & 69.1 & 842.16 & 5.6 \\
\hline ON-711 & $\operatorname{Exp}$ & $\mathbf{Y}$ & 260.86 & 61.1 & 844.10 & 67.3 & 837.90 & 6.2 \\
\hline ON-712 & EXP & $\mathbf{Y}$ & 424.11 & 31.5 & 845.59 & 35.2 & 841.89 & 3.7 \\
\hline OW-713 & EXP & $\mathbf{Y}$ & 567.73 & 35.0 & 845.63 & 38.6 & 842.03 & 3.6 \\
\hline OW-714 & $\mathbf{E X P}$ & $x$ & 742.25 & 27.5 & 847.58 & 31.6 & 843.48 & 4.1 \\
\hline OW-723 & EXP & $\mathbf{y}$ & 626.76 & 70.0 & 952.23 & 76.7 & 945.53 & 6.7 \\
\hline OW-724 & $\operatorname{EXP}$ & $\mathbf{y}$ & 681.02 & 26.5 & 952.77 & 35.4 & 943.87 & 8.9 \\
\hline OW-725 & $\operatorname{ExP}$ & $\mathbf{Y}$ & 820.76 & 7.6 & 953.45 & 15.9 & 945.15 & 8.3 \\
\hline OW-736 & $\operatorname{Exp}$ & $y$ & 859.05 & 6.5 & 953.62 & 14.8 & 945.32 & 8.3 \\
\hline ow-737 & EXP & $\mathbf{y}$ & 873.25 & 6.4 & 953.51 & 14.7 & 945.21 & 8.3 \\
\hline OW-738 & $\operatorname{ExP}$ & $\mathbf{y}$ & 904.61 & 25.8 & 957.28 & 31.6 & 951.48 & 5.8 \\
\hline ow-739 & $\mathbf{E x P}$ & $\mathbf{Y}$ & 716.06 & 70.3 & 953.44 & 78.8 & 944.94 & 8.5 \\
\hline ow -740 & EXP & $y$ & 839.15 & 66.3 & 953.33 & 74.4 & 945.23 & 8.1 \\
\hline CW-800 & OLF & $\mathbf{y}$ & 936.00 & - & - & 21.2 & 943.16 & $\cdot$ \\
\hline
\end{tabular}



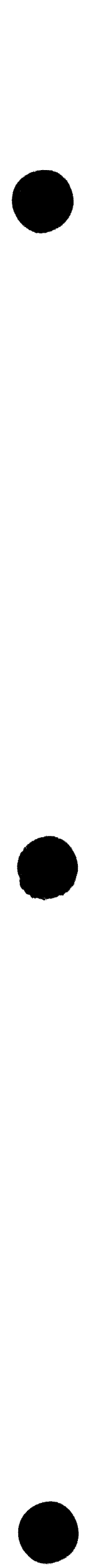


\section{DISTRIBUTION}

DEPARTMENT OE ENERGY

G. W. Bodenstein

W. G. McMillan

P. J. Gross/L. L. Radcliffe

HEALTH. SAEETY, ENVIRONMENT, AND

ACCOUNTABILITY ORGANIZATION

D. E. Bohrman

C. C. Hill

W. K. Jago

S. B. Jones

L. W. McMahon

J. E. Powell

E. B. Rundle

L. O. Vaughan

S. W. Wiley

GWPP-File

ENVIRONMENTAL RESTORATION PROGRAM

V. J. Brumback

J. T. Grumski

M. J. Norris

J. A. Vanderlan

C. S. Walker

UNIVERSTTY OF TENNESSEE

B. W. McMaster

G. K. Moore

UNIVERSITY OF NEVADA. RENO

L. A. Shevenell

QRISE

S. M. Field
ENVIRONMENTAL SCIENCES DIVISION

R. B. Dreier

T. O. Early

D. D. Huff

C. T. Rightmire

R. R. Turner

D. B. Watson

WASTE MANAGEMENT ORGANIZATION

K. D. Delius

C. W. Hutzler

I. W. Jeter

J. E. Stone

ENGINEERING ORGANIZATION

W. E. Manrod

QUALITY AND TECHNICAL SERVICES

P. Buckley

L. P. Burnett

HSW ENVIRONMENTAL CONSULTANTS

J. R. Walker

H\&R TECHNCIAL ASSOCIATES

P. M. Ezell

SCIENCE APPLICATIONS

INTERNATIONAL CORPORATION

S. Selecman/W. P. Kegley

Y -12 Central Files

A. K. Lee/DOE-OSTI (2) 


\section{DISCLAIMER}

This report was prepared as an account of work sponsored by an agency of the United States Government. Neither the United States Government nor any agency thereof, nor any of their employees, makes any warranty, express or implied, of assumes any legal liability of responsibility for the accuracy, completeness, of use. fulness of any information, apparatus, product, or process disclosed, or represents that its use would not infringe privately owned rights. Reference herein to any specific commercial product, process, or service by trade name, trademark, manufacturer, or otherwise, does not necessarily constitute or imply its endorsement, recommendation, or favoring by the United States Government or any agency thereof. The views and opinions of authors expressed herein do not necessarily state or reflect those of the United States Government or any agency thereof. 
$1 !$
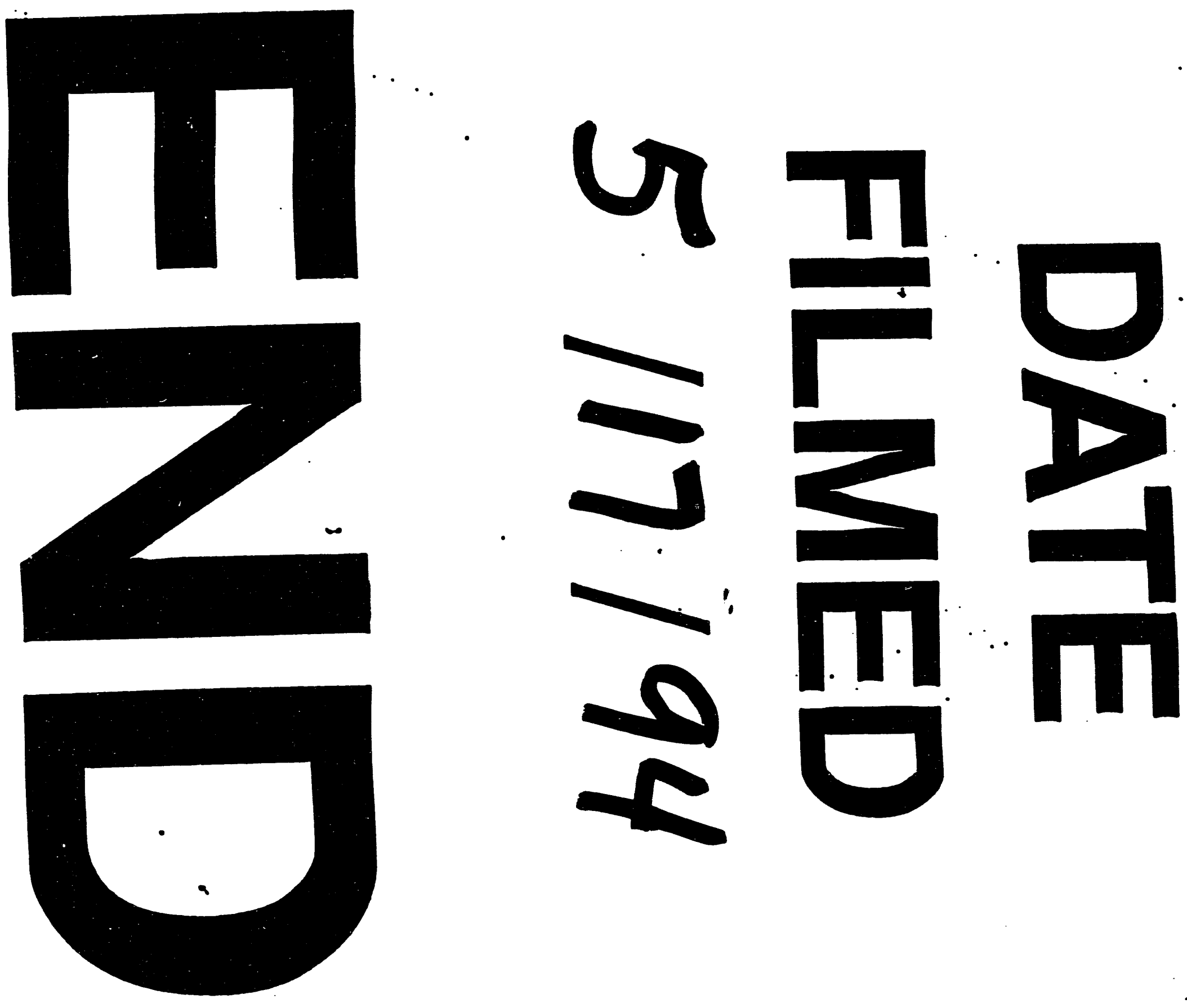


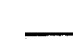

